\title{
NSS Program
}

N01: NSS Opening Plenary I

Monday, Oct. $31 \quad$ 08:30-10:15

Schweitzer

N01-1: Grand Welcome Opening

M. Titov

CEA, Saclay, France

Official Conference Opening Address

Welcome by:

- General Chair

- Strasbourg Eurometropole / EU Representatives

- City of Strasbourg

- ACAL Region Government

N01-2: International Research Cooperation - a View from Japan

A. Suzuki

Iwate Prefectural University and former KEK Director, Takizawa, Iwate, Japan

- Welcome by ESFRI Representative

- International Research Cooperation - a View from Japan

- Greetings from the Japanese Diet Members

Chaired by R.-D. Heuer, Member of the Advisor Committee for Research with the European Commissioner for Research,

Science and Innovation and former CERN Director-General

N01-3: Opening of the NSS Symposium

E. Elsen ${ }^{1}, \underline{\text { S. Kuehn }}{ }^{2,1}$

${ }^{I}$ CERN, Geneva, Switzerland

${ }^{2}$ University of Freiburg, Germany, Freiburg, Germany

Welcome Opening Welcome to all attendees by the NSS Program Chairs Opening of the NSS Program in Strasbourg

N01-4: Einstein's Gravitational Waves Observed

B. Barish

California Institute of Technology, California, US

Einstein predicted the existence of gravitational waves 100 years ago. They have been recently observed from a pair of merging Black Holes by the Laser Interferometer Gravitational-wave Observatory (LIGO). The physics of gravitational waves, the detection technique, the observation including latest results and its implications will all be discussed.

N02: NSS Opening Plenary II

Monday, Oct. $31 \quad$ 10:45-12:05 Schweitzer

N02-1: Awards Ceremony

C. Guazzoni

Sezione di Milano, Politecnico di Milano \& INFN Milano, Milano, Italy

On behalf of the RISC Awards Committee

Ceremony for Awards 2016 Radiation Instrumentation Early Career Award (NSS) 2016 Glenn F. Knoll Radiation Instrumentation Outstanding Achievement Award (NSS)

\section{N02-2: The Next Generation of Large Neutrino Oscillation Experiments}

M. Thomson

University of Cambridge, Cambridge, $U K$ 
Neutrinos are the most numerous type of matter particle in the Universe. However, these $\ddot{i}_{i}{ }^{1 / 2}$ ghostlyï $i^{1 / 2}$ particles are incredibly difficult to detect, mostly passing freely through matter. Nevertheless, as a result of a series of innovative large experiments in the last 20 years, we have learnt a great deal about neutrinos. For example, we now know that neutrinos have a mass, providing clear evidence for physics beyond the our current understanding. This achievement was recognised through the award of the 2015 Nobel prize for physics to the leaders of the SNO and Super-Kamiokande experiments for the conclusive establishment of the phenomenon of neutrino oscillations. Much of what we know about neutrinos comes from studying neutrino flavour oscillations, whereby one type of neutrino transforms into a different type as it propagates over a large distance.

The Deep Underground Neutrino Experiment (DUNE) is the next step in this decades long experimental programme. DUNE will address profound question in neutrino physics and particle astrophysics. DUNE consists of an intense neutrino beam fired a distance of $1300 \mathrm{~km}$ from Fermilab (near Chicago) to the 40,000 ton Liquid Argon DUNE detector, located deep underground in the Homestake mine. In this talk I will describe the scientific aims of DUNE, focussing on the experimental challenges in constructing an operating very large liquid argon time projection chamber detectors. I will also discuss the complementary experimental approaches being adopted by the Hyper-Kamiokande experiment in Japan and the JUNO experiment in China.

\section{N02-3: European Open Science Cloud}

\section{B. Jones}

CERN, Geneva, Switzerland

The work of Helix Nebula [1] has shown that is it feasible to interoperate in-house IT resources of research organisations, publicly funded e-infrastructures, such as EGI [3] and GEANT [4], with commercial cloud services. Such hybrid systems are in the interest of the users and funding agencies because they provide greater "freedom and choice" over the type of computing resources to be consumed and the manner in which they can be obtained. But to offer such freedom and choice across a spectrum of public and commercial suppliers, various issues such as service integration, intellectual property, legal responsibility and service quality need to be addressed as the next stage of a Science Cloud Strategic Plan [2]. Investigating these issues is the focus of the PICSE project within the Helix Nebula initiative [5]. Propelled by the growing IT needs of the Large Hardon Collider and the experience gathered through deployments of practical use-cases with Helix Nebula, CERN has proposed a model for a European Open Science Cloud [6] which has been further expanded by the EIROforum research organisations [7]. This presentation will explore the essential characteristics of a European Open Science Cloud if it is to address the big data needs of the latest generation of Research Infrastructures. A governance and financial model together with the roles of the stakeholders, including commercial service providers and downstream business sectors, that will ensure a European Open Science Cloud can innovate, grow and be sustained beyond the current project cycles is described. References: $1 \mathrm{http} / /$ www.helix-nebula.eu/ 2 http://www.egi.eu/ 3 http://www.geant.net/ 4 http://cdsweb.cern.ch/record/1374172/files/CERN-OPEN-2011-036.pdf 5 http://picse.eu/roadmap 6 http://dx.doi.org/10.5281/zenodo.16001 7 http://dx.doi.org/10.5281/zenodo.34264

\section{N03: Advanced computing and software for experiments - Poster session I}

\section{Monday, Oct. $31 \quad$ 14:00-16:00 Etoile}

N03-1: Development of the Application for Fitting Functions Using Expression Parsing K. Neichi

Department of business administration, Tohoku Gakuin University, Sendai, Miyagi, Japan

To develop an application for fitting functions to data for analysts who do not have an experience in programming, we propose applying an algorithm for expression parsing for evaluating the functions and the derivatives. In data analysis in high energy physics, fitting function models to measured data is one of the standard methods to obtain the estimates of the unknown parameters included in the function models. A ROOT is playing the important role in many analyses in high energy physics. However, it is not easy to use the ROOT for the analysts who do not have the experience in programming. The analyses should become easier for such analysts, so that the application with simpler interfaces for fitting functions with arbitrary expressions is desired. To construct the application, we can use an algorithm for expression parsing which is a technique for evaluating the expression tree of mathematical formula, for the recognition of functions. In fitting a function, we have to evaluate the first and second order derivatives with respect to the parameters involved in the function in the iterative process for minimizing the chi square or maximizing the likelihood. Giving the postfix form of the function in the expression parsing, the expression of the derivatives can be derived easily corresponding to the differential law. The code is written using Java because Java is one of the powerful languages for string manipulations.

N03-2: Magnetic Shield Optimization of PMTs Exposed to Constant Quadripolar Fringe Fields

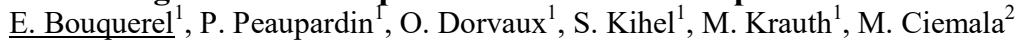

${ }^{I}$ IPHC, UNISTRA, CNRS, Strasbourg, France

${ }^{2}$ The Niewodnicza'nski Institute of Nuclear Physics, Kraków, Poland 
The Photon Array for the studies of Radioactive and Ion Stable beams (PARIS) is a multidetector of clusters. Each cluster is composed of 9 units two-shells phoswiches of $\mathrm{LaBr} 3 / \mathrm{NaI}$ scintillators optically coupled to one photomultiplier tube (PMT). PARIS will be used in combination with the VAMOS spectrometer at GANIL. During the experiment, PMTs will be exposed to the constant magnetic fringe fields produced by a quadrupole. Magnetic shielding is essential to efficiently lower the magnetic field inside the PMTs. ANSYS software allowed to simulate fringe fields produced by the quadrupole and to verify the impact of the shield initially foreseen. Relevant obtained and forthcoming results will be used to optimize and improve the geometry of the shield and to select the most efficient materials. They are an original illustration of the performance capability of ANSYS software within the fields of nuclear physics, high energy physics and medical imaging instrumentation.

\title{
N03-4: Geant4 Maintainability Assessed with Respect to Software Engineering References
} E. Ronchieri ${ }^{1}$, M. G. Pia ${ }^{2}$, T. Basaglia ${ }^{3}$, F. Giacomini $^{1}$

${ }^{I}$ INFN CNAF, Bologna, Italy

${ }^{2}$ INFN Genova, Genova, Italy

${ }^{3}$ CERN, Geneva, Switzerland

Maintainability is a critical issue for large scale, widely used software systems, characterized by a long lifetime. It is of great importance for a software toolkit, such as Geant4, which is a key instrument for research and industrial applications in many fields. Maintainability is related to a set of characteristics of the software, which have emerged from software engineering practice and have been distilled into established standards. Pertinent metrics are associated with these characteristics, which enable an objective assessment of the software maintainability. We present an extensive set of these metrics, gathered over recent Geant4 versions with multi-threaded execution capability: they include estimates of the software size, complexity and objectoriented design features. The collected metrics have been analyzed with various statistical methods to assess the status of Geant 4 code with respect to reference values established in software engineering literature, which represent thresholds for maintainability risk. The assessment has been detailed to a fine-grained level across Geant4 packages to identify potential problematic areas effectively, also taking into account specific peculiarities of different simulation domains. The evaluation of the metrics suggests preventive actions to facilitate the maintainability of the toolkit over an extended life cycle. The methodology developed for the objective assessment of Geant 4 maintainability is applicable to other large scale physics software systems as well, which could benefit from early identification of maintainability risks to elaborate effective mitigation strategies in critical areas.

\author{
N03-5: Optimised Lambda Architecture for Monitoring WLCG Using Spark and Spark Streaming \\ U. Suthakar ${ }^{1}$, L. Magnoni ${ }^{2}$, D. R. Smith ${ }^{1}$, A. Khan ${ }^{1}$ \\ ${ }^{I}$ College of Engineering, Design and Physical Sciences, Brunel University London, Kingston Lane, Uxbridge, Middlesex, UB8 \\ $3 P H, U K$ \\ ${ }^{2}$ Information Technology Department, European Organisation for Nuclear Research (CERN), Geneva, Switzerland
}

The Worldwide LHC Computing Grid (WLCG) serves over 170 computer centres and 3,000 physicists who execute over 2 million jobs daily, resulting in the movement of petabytes of data over the heterogeneous infrastructure. Monitoring the computing and user activities of the LHC experiments over such a tremendously complex infrastructure is remarkably demanding. Whereas the present solutions are traditionally based on the Oracle Relational Database Management System (RDBMS) for data storage and processing, recent developments evaluate Lambda Architecture, in particular, data storage and batch processing for processing large-scale monitoring datasets using Hadoop and its MapReduce framework. This paper presents an optimised Lambda Architecture using Apache Spark technology, which involves modelling an efficient way of joining batch computation and real-time computation without the need to add complexity to the UI. Three models were explored for use with WLCG Data acTivities (WDT): pure streaming, pure batch computation and the combination of both batch computation and streaming. The results of data analytics on the Hadoop cluster using Spark batch processing and Spark Streaming for WDT monitoring are presented, demonstrating how the new architecture can offer efficiency benefits by combining both batch and real-time processing to compensate for batch-processing latency.

\author{
N03-6: Metal Artefact Reduction in Spectral CT Imaging for the Inspection of Tyres \\ C. Fournier ${ }^{1,2}$, F. Mathy ${ }^{1,2}$, V. Moulin $^{1,2}$, V. Rebuffel ${ }^{1,2}$, L. Verger ${ }^{1,2}$ \\ ${ }^{I}$ Univ. Grenoble Alpes, Grenoble, France \\ ${ }^{2}$ CEA, LETI MINATEC Campus, Grenoble, France
}

X-ray imaging and especially CT is a suitable technique for the inspection of tyres. The problem to be solved is the distinction between closed materials such as rubbers containing more or less additives and plastic cords. The presence of metal and the $U$ shape of the tyre are two important constraints for tomographic reconstruction and induce so strong artefacts that the interface between two rubbers is no more detectable. In this paper we propose a method based on the new capabilities in counting mode of recently emerged semiconductor based X-ray detectors, which allows the inspection of tyres in a single shot and a reasonable time. The approach combines a material decomposition with an in-painting method to restore corrupted metal projections in the 
spectral sinogram. The entire process is applied on realistic simulated data (geometry, size, noise, ...) obtained with Sindbad software. Our results show that despite the fact that the material decomposition method increases noise, a clear contrast enhancement between various rubbers is obtained and the strong artefacts generated by the steel bead bundle are removed.

\author{
N03-7: A New Geant4 Modeling Solution Based on CAD and Unstructured Mesh Geometries \\ Y. Qiu, U. Fischer, L. Lu \\ Karlsruhe Institute of Technology, Eggenstein-Leopoldshafen, Germany
}

The design of nuclear facilities and devices nowadays is frequently based on detailed 3D CAD geometries linked with analysis codes for the provision of high fidelity spatial distributions of the physical quantities of interest. An advanced open source modeling system has been developed to process CAD models and unstructured mesh geometries for the Geant4 Monte Carlo (MC) toolkit. The processing of the CAD geometry data is achieved through the automatic MC geometry modeling program McCad which also supports the MC codes MCNPX and TRIPOLI. McCad converts the CAD geometry into a new half-space solid as well as tessellated solids. The Geometry Description Markup Language (GDML) format has been adopted and extended to support the new solid type. The modeling of unstructured meshes is achieved using tetrahedron, pyramid, wedge and hexahedron elements which have been developed as new Geant4 solid types. A superimposed mesh scoring capability has been developed for Geant 4 to enable the use of these mesh elements for the calculation of spatial distributions of physical quantities by overlaying the mesh on the actual geometry. The newly developed capabilities have been verified through several benchmark calculations and comparisons showing their reliability and efficiency.

N03-8: Photo-Nuclear Interaction Simulations with Geant4 and FLUKA: Validation and Comparison L. Quintieri ${ }^{1}$, M. G. Pia ${ }^{2}$, M. Augelli ${ }^{3}$, P. Saracco ${ }^{2}$, M. Capogni ${ }^{1}$, G. Guarneri ${ }^{4}$

${ }^{I}$ FSN-INMRI, ENEA Centro Ricerche Casaccia, Rome, Italy

${ }^{2}$ Sez. Genova, INFN, Genova, Italy

${ }^{3}$ CNES, Toulouse, France

${ }^{4} I C T$, ENEA Centro Ricerche di Portici, Portici, Italy

The simulation of photo-nuclear interactions is relevant for the design and optimisation of photo-neutron sources as well as in the shielding design of high energy particle accelerators. An assessment of the reliability of such simulations based on widely used Monte Carlo codes (with emphasis on Geant4 and FLUKA) is presented: it consists of quantitative validation tests, based on statistical data analysis methods, which compare simulated and measured observables in relevant scenarios whenever experimental data are available. Knowledge gaps due to lack of pertinent experimental data, preventing the validation of simulation models, are identified, especially for high energy range (above $150 \mathrm{MeV}$ ), and the need of further measurements is highlighted. This work is intended to perform the comparison of photo-nuclear predictions between the cited codes (and when possible the benchmarking with experimental data), for several elements (typically of medium and high atomic number) in an extended energy range (from few tens of $\mathrm{MeV}$ up to hundreds $\mathrm{GeV}$ ). Anyway, the first cycle of this work is focused on materials, such as natural lead and tungsten, commonly used for shielding purpose in radiation protection applications and in high energy particle bumps.

N03-9: Dynamic Derivative Convolution Algorithm for Prompt Gamma Neutron Activation Spectra M. J. Neuer ${ }^{1}$, T. Szczeniak ${ }^{2}$, H. Zastawny ${ }^{3}$, E. Jacobs ${ }^{1}$, M. Grodzicka ${ }^{2}$

${ }^{I}$ Research \& Development, innoRIID, Grevenbroich, Germany

${ }^{2}$ National Centre of Nuclear Research, Swierk, Poland

${ }^{3}$ Syskon, Wroclaw, Poland

A technique is presented to algorithmically evaluate prompt gamma neutron activation spectra, which were produced through excitation of specific material samples. The excitation is done with a neutron generator that provides a switchable, artificial form of neutron radiation. To evaluate these spectra, a mathematical transformation based on derivative convolution is proposed that dynamically incorporates the detector energy-resolution dependency over the full energy range. Based on this technique a series of measured response spectra are analyzed and the material composition of the samples are identified. Primary goal is a quick quantification of the $\mathrm{Ca}, \mathrm{Fe}, \mathrm{Al}, \mathrm{Mg}$ or $\mathrm{Si}$ contents of the samples. These elements are important for cement and coal industry as they are typical ingredients of the cement raw material and ashes in coal. Limits of the detection limits and confidence thresholds are derived. Due to its quick performance, the algorithm is capable also to run online, supporting a continuous analysis of moving material on a conveyor belts.

N03-10: Sensor Fusion of Spectroscopic Data and Gyroscope Accelerations for Direction Indication in a Handheld Radiation Detection Instrument

C. Henke, E. Jacobs, N. Teofilov, P. Henke, M. J. Neuer 
A method is presented that uses spectroscopic data and the sensorial output of a gyroscope to create a soft sensor for the incident direction of the radiation. The method can be applied by any handheld radiation detection equipment that is continuously moved by the operator in some regular waving or panning motion. The gyroscope output consists of acceleration values along the spatial coordinates. These values have to be smoothed and integrated for a certain time and a forget-module keeps the memory of acceleration data focused on the relevant, most recent parts needed for the direction assessment. Based on straightforward geometric considerations, the sensor fusion concept is described and the derived indicator solution is demonstrated. The system is tested in a field tests, training the localisation of vagabond sources.

N03-11: A Cluster Software Architecture Written in Python to Control and Monitor the Liquid Kripton Electromagnetic Calorimeter Level 0 Trigger System for the NA62 Experiment at CERN

R. Ammendola ${ }^{1}$, M. Barbanera ${ }^{2}$, M. Bizzarri ${ }^{3}$, V. Bonaiuto ${ }^{4}$, A. Ceccucci ${ }^{5}$, B. Checcucci ${ }^{2}$, N. De Simone ${ }^{5}$, R. Fantechi ${ }^{5}$, L. Federici ${ }^{1}$, A. Fucci ${ }^{5}$, M. Lupi ${ }^{2}$, G. Paoluzzi ${ }^{1}$, A. Papi ${ }^{2}$, M. Piccini ${ }^{2}$, V. Ryjov ${ }^{5}$, A. Salamon ${ }^{1}$, G. Salina ${ }^{1}$, F. Sargeni ${ }^{6}$, S. Venditti ${ }^{5}$

${ }^{I}$ Sezione Roma Tor Vergata, INFN, Roma, Italy

${ }^{2}$ Sezione Perugia, INFN, Perugia, Italy

${ }^{3}$ Dept. Physics, University of Perugia, Perugia, Italy

${ }^{4}$ Dept. Industrial Engineering, University of Roma Tor Vergata, Roma, Italy

${ }^{5}$ CERN, Geneva, Switzerland

${ }^{6}$ Dept. Electronic Engineering, University of Roma Tor Vergata, Roma, Italy

The NA62 experiment at CERN starts its second year of data taking to measure, with a precision never reached before, the branching ratio of the ultra-rare decay $\mathrm{K}+->$ pi + nu nubar. The accurate measure of the decays requires a high performance Trigger and Data Acquisition (TDAQ) system. Among the others, the high-precision liquid Kripton electromagnetic calorimeter is the detector that helps to reject background events related to photon production in the angular range between 1 and $8.5 \mathrm{mrad}$ and, in particular, those associated with the $\mathrm{K}+->$ pi+ pi0 decays. The LKR trigger processor is composed of 37 TEL62 electronic boards on which are mounted 221 high-performance FPGAs. These components have to be configured and the whole system has to be monitored during all the data taking operations. So, a tailored software architecture has been designed to handle, in real time, the system configuration, the monitoring of the performances and the interface toward the "Run Control System" of the experiment.

\section{N03-12: Implementation of Position and Angle Uncertainties in the Muon Reconstruction of the CMS Experiment and Impact on the Performance}

G. Abbiendi, S. S. Chhibra

INFN, Sezione di Bologna, Bologna, Italy

The alignment of muon chambers relative to each other and to the inner tracker is crucial to achieve the optimal performance for muon reconstruction at high momentum, in particular to improve the momentum resolution. With the energy and luminosity increase in LHC Run2, high-momentum muons become more important for searches of new particles with masses around the $\mathrm{TeV}$ scale.

The full alignment of the tracking and muon system is a complex task which needs analyzing a minimum statistics of about 1 $\mathrm{fb}^{\wedge}\{-1\}$ of collision events, and takes a few weeks to be run and validated. Before this is done, at startup of the 2016 running period, the performance is expected to be suboptimal, as a consequence of opening and closure of the detector during the winter shutdown and of magnetic field cycles.

The muon reconstruction of the CMS experiment has been recently improved and made more robust with the introduction of alignment uncertainties on the positions and angles of segments in the muon chambers. Not only does the momentum resolution improve at high energy, but, owing to the better quality of the track building and fit, also the trigger efficiency increases, especially for startup conditions.

N03-13: Efficient Implementation of Iterative Tomographic Reconstruction Routines

M. P. Pichotka ${ }^{1,2}$, J. Jakubek ${ }^{2}$, D. Vavrik ${ }^{1,2}$

${ }^{I}$ Experimental physics, IEAP, CTU Prague, Praha, CZ

${ }^{2}$ Czech Academy of Sciences, Praha, CZ

In particular cases iterative volume reconstruction in transmission tomography does not only obtain high quality results, but also is computationally very efficient. This for example is the case if a good initial guess of the volume can be provided beforehand. In this contribution implementations of iterative reconstruction routines exploiting the gradual changes in tomographies of 
dynamical processes or the coordinated motion of sample fragments in loading experiments are described. The iterative reconstruction routine applied is a TV-constrained OSEM implementation, dislocation analysis of the ROI is performed by image correlation within the volume.

\author{
N03-14: Underwater Nuclide Identification Strategy Using a Multi-Agent System with a Dedicated Scattering \\ and Attenuation Agent \\ M. J. Neuer, E. Jacobs, C. Henke \\ innoRIID, Grevenbroich, Germany
}

An approach for underwater nuclide identification is shown on the basis of a multi-agent system. A so-called scattering agent, capable of quickly combining multiple scattering and attenuation situations to a detector response matrix is used to establish a solid reference data set during the evaluation of the nuclide identification engine. The agent interacts with the nuclide agents by means of communication. The novel approach yields favourable results for the underwater identification. A series of test measurements with submerged instruments and sources was conducted to test and verify the quality of the concept. Therefore multiple sources were measured in an underwater situation. The distance to the source is crucial for successful identification. As result of the measurements, a clear recipe for the data acquisition under water is compiled.

\title{
N03-15: Control Command, DAQ \& Monitoring: Experience with Go and HTML5
}

\section{$\underline{\text { S. Binet }}$}

IN2P3/CNRS, CLERMONT-FERRAND, France

This paper explores the suitability of Go to deal with low level data acquisition, present this data in a (soft) real time fashion and control the physical device used to acquire and produce that data.

Traditionally, such a task is usually tackled by a mix of LabView, C/C++, Java or Python, with various levels of runtime performances and ease of deployment and portability.

The concurrency model of Go, a relatively new open source language, seemed a nice fit for handling all the moving parts of an application having to deal with acquiring data in (soft) real time, propagating user commands through various protocols (possibly over the network) and presenting monitoring data to the user.

After having presented the overall architecture of two applications, one ported from $\mathrm{C}++$ and the other written from scratch, this paper will report on their measured performances.

\author{
N03-16: Fads: a Go-Based, Concurrency Friendly, Fast Detector Simulation Toolkit \\ $\underline{\text { S. Binet }}$ \\ IN2P3/CNRS, CLERMONT-FERRAND, France
}

Current HENP control frameworks have been designed and written in the early 2000's, when multi-core architectures were not yet pervasive. As a consequence, an inherently sequential event processing design emerged. Evolving current frameworks' APIs and data models encouraging global states, non-reentrancy and non-thread-safety to a more concurrent friendly environment, in an adiabatic way, is a major undertaking, even more so when relying on the building blocks provided by $\mathrm{C}++$. This paper reports on the development of fads, a "FAst Detector Simulation toolkit." fads is written in Go, a relatively new programming language, tailored for concurrent programming. This allows fads to easily manage and expose concurrent work items from which parallelism can be extracted and thus leverage the compute power of multicore architectures. fads is the port of a single-threaded fast detector simulation toolkit (Delphes) to fwk. The paper will present benchmarks (CPU, RSS, I/O, scalability) of real Delphes use cases against fads. Performance tools and concurrency debugging tools shipped with the Go toolchain and used for this comparison will also be presented.

N04: Astrophysics and space - Poster session I

Monday, Oct. $31 \quad$ 14:00-16:00 Etoile

N04-1: Design of the Photomultiplier Tube Base with High Dynamic Range for LHAASO

X. Zhao, Z. Tang, C. Li, K. Jiang, M. Shao

University of Science and Technology of China, Hefei, China

The Large High Altitude Air Shower Observatory (LHAASO) project is scheduled to be built at Sichuan province in China. As a large scale complex of many kinds of detectors, the main scientific goals of LHAASO are exploring the origin of the galactic cosmic ray, searching for very high energy gamma ray sources, and the precise measurement of the components at the knee region. As one of the major components of LHAASO, the Water Cherenkov Detector Array (WCDA) is proposed to target gamma astronomy at energies between $100 \mathrm{GeV}$ and $30 \mathrm{TeV}$. The WCDA covers an area $90000 \mathrm{~m}^{\wedge} 2$, configured as $3120 \mathrm{small}$ 
ponds ( $5 \mathrm{~m} \times 5 \mathrm{~m} \times 4 \mathrm{~m}$ depth). A large area hemispherical PMT is located at the bottom-center of each cell, facing upward, to collect the Cherenkov light produced by shower particles in water. The PMT used requires a good single photoelectron resolution, good time performance, especially the transit time spread (TTS) and a charge dynamic range from 1 to 4000 photoelectrons (PE). Bases for the candidate PMTs Hamamatsu R5912, Beijing Hamamatsu CR365 and HZC Photonics XP1805 are designed, respectively. The voltage divider is a purely resistive chain and the base comprises two different outputs obtained by using the anode and the dynode. The results show that at the working gain of $3 \times 10^{\wedge} 6$, the characteristics of all the three PMTs satisfy the demands of WCDA. Additionally, a base of high dynamic range for Hamamatsu R5912 is designed for the underground Muon Detector (MD), which is another major detector in LHAASO and used to detect the muon components in the extensive air shower. The divider is designed to extract the signals from the 7th dynode (DY7) and the anode. The charge ratio between the anode and DY7 is around 160 and the equivalent anode peak current non-linearity of DY7 within 5\% is up to $1.87 \mathrm{~A}$, which satisfies the dynamic range requirement (1-15000 muons) in the MD.

\section{N04-2: Gamma-Ray Detection with a DSSSD in the MeV Range}

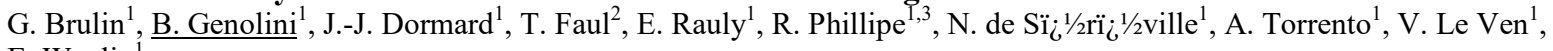
E. Wanlin ${ }^{1}$

${ }^{I}$ Institut de Physique Nuclï_1/2aire d'Orsay, 91406 Orsay Cedex, France

${ }^{2}$ Former member, Institut de Physique Nuclï ¿1/2aire d'Orsay, 91406 Orsay Cedex, France

${ }^{3}$ Present address, Institut de minï ${ }_{1}^{1 / 2}$ ralogie, de physique des matï ${ }^{1 / 2} / 2$ riaux et de cosmochimie, 75005 Paris, France

The measurement of gamma rays in the energy range $0.3 \dddot{i} i 1 / 230 \mathrm{MeV}$ with silicon detectors at room temperature via Compton scattering is being investigated for the next gamma-ray observatory in the MeV band. In this contribution, we show that $60-\mathrm{keV}$ gamma-ray detection with an energy resolution below $10 \mathrm{keV}$ is possible using a double-sided stripped, 1.5-mm thick silicon detector with a preamplifier made of discrete commercial components. Our study of the noise including the bias circuit and the connection between the detector and the electronics allowed us to design a system able to sustain a leakage current of $\sim 50$ $\mathrm{nA} /$ strip and a capacitance of $\sim 20 \mathrm{pF} /$ strip. These results encourage us to investigate the use of integrated electronics in view of a satellite mission.

N04-3: XIPE Mission Focal Plane Gas Mixture Optimization R. M. Curado da Silva ${ }^{1}$, J. M. Maia ${ }^{2}$, J. Escada ${ }^{1}$, B. Conceição ${ }^{1}$, T. H. Dias ${ }^{1}$, F. P. Santos ${ }^{1}$

${ }^{I}$ Laboratório de Instrumentação e Física Experimental de Partículas, Coimbra, Portugal

${ }^{2}$ Physics Department, University of Beira-Interior, Covilhã, Portugal

XIPE mission focal plane detectors are photoelectric polarimeters based on the Gas Pixel Detector (GPD) design, these devices are composed by Gas Electron Multipliers and multi-pixel anode readout. The filling gas mixture defines the ultimate intrinsic polarimetric sensitivity of the instrument, i.e. the modulation factor and the detector efficiency, which depends on gas parameters such as: the angular differential cross-section for photoionization by polarized X-rays, the total photoionization cross-section, the photoelectron and Auger electron practical ranges, the photoelectron multiple scattering, the electron transverse diffusion and the charge gain. Herein, we report simulation studies on the optimization of the GPD gas mixture. The best gaseous mixture solution will provide an accurate reconstruction of photoelectron emission direction and therefore to maximize the modulation factor, allowing optimal degree and angle of polarization determination. Noble gases like $\mathrm{He}$ or $\mathrm{Ne}$ as well as quenching additive gases like DME and iso-C4H10 gases will be studied and its polarimetric potential analyzed.

\section{N04-4: Evaluation of a Bread Board Model Gamma-Ray Burst Polarimeter Toward Installation on the International Space Station} $\underline{\text { Y. Oikawa }}^{1}$, S. Gunji ${ }^{1}$, T. Nakamori ${ }^{1}$, M. Takakura ${ }^{1}$, T. Ueda ${ }^{1}$, T. Kishikawa ${ }^{1}$, S. Daigle ${ }^{2}$, J. Gaskin $^{2}$, B. Ramsey ${ }^{2}$, C. Wilson-

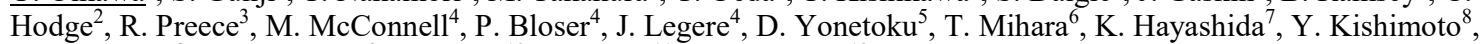
S. Kishimoto ${ }^{8}$, H. Takahashi ${ }^{9}$, Y. Yatsu ${ }^{10}$, K. Toma ${ }^{11}$, T. Sakamoto ${ }^{12}$

${ }^{I}$ Yamagata, Yamagata University, Yamagata, Japan

${ }^{2}$ Huntsville, NASA/MSFC, Alabama, U.S.A.

${ }^{3}$ Huntsville, University of Alabama Huntsville, Alabama, U.S.A.

${ }^{4}$ Durham, University of New Hampshire, New Hampshire, U.S.A.

${ }^{5}$ Kanazawa, Kanazawa University, Ishikawa, Japan

${ }^{6}$ Wako, Riken, Saitama, Japan

${ }^{7}$ Toyonaka, Osaka University, Osaka, Japan

${ }^{8}$ Tsukuba, KEK, Ibaraki, Japan

${ }^{9}$ Higashihiroshima, Hiroshima University, Hirohima, Japan

${ }^{10}$ Meguro, Tokyo kogyo University, Tokyo, Japan

${ }^{11}$ Aoba, Tohoku University, Miyagi, Japan

${ }^{12}$ Sagamihara, Aoyama Gakuin University, Kanagawa, Japan 
About fifty years ago, the history of the study on Gamma Ray Bursts (GRBs) started with its discovery by the Vela satellite. Nowadays, we can obtain much knowledge on the GRBs through various observations from space or on the ground. To explain the GRB primary emission mechanism, the fireball model has been developed and are widely accepted, though the emission mechanism of the gamma rays from the fireball was still unknown. Recently two primary theoretical models have been proposed as the radiation mechanism: synchrotron model and photosphere model. These models each predict a different degree of polarization. So in order to elucidate the radiation mechanism, which may be described by one of these or by a combination, the polarization must be measured at a relatively low uncertainty. Our plan is to install a GRB polarimeter with large effective area onto the International Space Station. This instrument is called LEAP, for LargE Area burst Polarimeter project and is led by the University of New Hampshire in partnership with Yamagata University and NASA Marshall Space Flight Center. LEAP will measure the degree of polarization for several tens of GRBs over a two year observation time. The LEAP baseline instrument is a Compton scattering polarimeter that utilizes a large array of plastic elements coupled to individual photomultiplier tubes (PMTs), surrounded by a layer of calorimeter material. We have constructed an alternate (but similar) configuration that utilizes MultiAnode PMTs to improve the performance of the baseline one. So we investigated the basic performance using polarized gamma rays at $60 \mathrm{keV}$ and $80 \mathrm{keV}$. We have confirmed that the modulation factor and the detection efficiency determined by the experiments agree with those by Monte Carlo simulation.

\author{
N04-6: Space X-Ray Polarimeter POLAR - Possible Fields for Cooperation \\ R. M. Marcinkowski \\ NUM, Paul Scherrer Institute, Villigen, Switzerland \\ On behalf of the POLAR
}

POLAR is a space instrument designed for measurements of the polarization of the hard X-rays from the GRBs. It is developed by the European-Chinese collaboration and will be launched on-board of the Chinese SpaceLab TG-2 in September 2016, a few weeks before IEEE Conference.

GRBs are flashes of hard X- and Gamma-rays, short in astronomical scale, and lasting roughly tenth of second to hundreds of seconds. But POLAR will be also able to detect the other transient objects like Solar flares and SGRs. Except of polarization, POLAR will provide perfect light curves and also will be able to provide coarse sources' positions as well as their approximate spectral characteristics, i.e. rough power-law index.

This presentation shows areas of cooperation of POLAR with the other existing and near future missions focusing on timing, spectrometry, localization and polarimetry.

POLAR will be a significant part of Inter-Planetary Network and, having almost $2 p$ srd field of view, will help to localize GRBs together with distant non-Earth orbiting mission: Konus-Wind and Mars Odyssey. It will also increase number of localized GRBs.

For proper calculation of polarization POLAR requires quite precise knowledge of position and spectrum of GRB. We will use results that came from the other instruments, i.e. Swift, INTEGRAL/IBIS, Fermi GBM and CALET.

POLAR is becoming the first, long operated instrument dedicated for X-ray polarimetry. We hope, that first successful and significant polarization measurement provided by POLAR will encourage the other missions (Integral IBIS/SPI, RHESSI) to reanalyze their data focusing on polarization as they reported promising results in the past.

The nearest years open new window in X-ray astronomy and collaboration with POLAR will increase the knowledge about uncovered part of Universe.

\author{
N04-7: Si and CdTe Detector Readout ASIC in 0.35 $\mu \mathrm{m}$ CMOS for Energetic Electron Spectroscopy for Space \\ Application \\ K. W. Wong ${ }^{1}$, G. Orttner ${ }^{1}$, O. Chassela ${ }^{1}$, M. Bassas $^{1}$, G. Roudil ${ }^{1}$, P. Devoto ${ }^{1}$, P. L. Blelly ${ }^{1}$, J. A. Sauvaud ${ }^{1}$, F. Bouyjou $^{2}$, \\ O. Bernal ${ }^{3}$, H. Tap ${ }^{3}$ \\ ${ }^{I}$ IRAP CNRS, toulouse, France \\ ${ }^{2}$ cea, gif sur yvette, france \\ ${ }^{3}$ LAAS CNRS, toulouse, France
}

The compact size and power consumption of the electron energy detector instrument IDEE TARANIS requires the use of a dedicated ASIC readout circuit instead of discret devices for energy measurement. The ASIC consists of $8 \mathrm{CdTe}, 4$ regular size Si and 1 small Si detectors readout. Each channel includes a charge amplifier, a shaper, a peak detector and an 8-bit ADC. Si type channel covers the energy detection range of $70 \mathrm{keV}$ up to $700 \mathrm{keV}$ while $\mathrm{CdTe}$ channel covers the $300 \mathrm{keV}$ to $4 \mathrm{MeV}$ range. For a 40-pF detector parasitic capacitances, low noise performances are achieved: 3120e- for Si type channels and 2335e- for CdTe type channels. Low power performance of $2 \mathrm{~mW}$ at $650-\mathrm{kHz}$ frequency per Si channel and $2.9 \mathrm{~mW}$ at $40-\mathrm{kHz}$ per CdTe channel is achieved. While both type of channel share a similar design, CdTe type channel analog front end had to include a pole zero cancellation in order to achieve the required frequency of operation. The ASIC has been tested in standalone as well as interfaced with the detectors. Finally, the ASIC has been qualified with a heavy ion test. The ASIC has been implemented in AMS $0.35 \mu \mathrm{m}$ HV CMOS technology. 
N04-8: Development of an Elpasolite Planetary Science Instrument

L. C. Stonehill, D. D. S. Coupland, K. E. Mesick

ISR-1, Los Alamos National Laboratory, Los Alamos, NM, USA

Planetary gamma-ray and neutron spectroscopy from orbiting spacecraft has become a standard technique to measure distinctive composition and abundance signatures for key elements relevant to planetary structure and evolution. Previous instrumentation that has led to the discovery of the concentration of many elements including hydrogen (a strong indicator of water) on planetary bodies, have used separate gamma-ray and neutron spectrometers. Elpasolite scintillators offer an opportunity to combine the gamma-ray and neutron spectrometer into a single instrument, leading to a significant reduction in instrument size, weight, and power (SWaP). We have developed an Elpasolite Planetary Ice and Composition Spectrometer (EPICS) instrument concept, which utilizes elpasolite scintillator and silicon photomultipliers to offer significantly reduced SWaP with similar neutron and gamma-ray detection efficiency but superior gamma-ray energy resolution compared to current scintillator-based instruments. We will present an overview and motivation for the EPICS instrument, preliminary conceptual design simulations that compare our instrument concept to current planetary science instruments, and discuss specific target missions that would benefit from the EPICS instrument.

N05: Circuits for readout and triggering - Poster session I

Monday, Oct. $31 \quad$ 14:00-16:00 Etoile

N05-1: CDS4C: A Novel CDS ASIC for a Multi-Readout X-Ray CCD with a 0.032\% INL

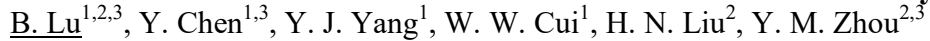

${ }^{I}$ Dept. of Key Laboratory of Particle Astrophysics, Institute of High Energy Physics, Chinese Academy of Sciences, Beijing, China

${ }^{2}$ Institute of Microelectronics, Chinese Academy of Sciences, Beijing, China

${ }^{3}$ University of Chinese Academy of Sciences, Beijing, China

This work presents the development of a four channel correlated double sampling (CDS) ASIC, named CDS4C, targeting the readout of a type of multi-readout swept charge device (SCD) for applications in the fields of both X-ray spectroscopy and imaging. Compared with conventional CDS architecture based on clamping technique, the innovation of the CDS4C ASIC is that it not only moves the clamping switch out of the signal path but also omits the sampling switch, eliminating the nonlinearity and thermal noise introduced by the these switches. Besides, it is implemented in a fully differential topology which suppresses the second-order harmonic distortion, improving the linearity further more. A theoretical noise analysis taking into consideration of the limited bandwidth of the operational-amplifier (OPA) highly coincides with the simulation results. The prototype of the CDS4C ASIC is fabricated with GlobalFoundries $0.35 \mu \mathrm{m} 2 \mathrm{P} 4 \mathrm{M}$ CMOS process, the preliminary experimental results show that, within the effective input dynamic range of $40 \mathrm{mV}$ which is equivalent to a wide range of soft X-ray energy band from $0 \sim 18.25 \mathrm{keV}$, a $0.032 \%$ integral nonlinearity (INL) and $35.5 \mu \mathrm{V}$ input-referred readout noise are achieved under $200 \mathrm{kHz}$ pixel rate with a $8 \times$ gain, whilst the total power consumption is only $8.8 \mathrm{~mW}$ from a single $3.3 \mathrm{~V}$ supply voltage.

\author{
N05-2: A Combination of Multiple Channels of FPGA Based Time-to-Digital Converter for High Time \\ Resolution \\ Y. Wang, Q. Cao, C. Liu \\ Modern Physics Department, University of Science and Technolgy of China, Hefei, Anhui, China
}

The full hardware solution introduced in our previous work could implement multi-channel time-to-digital converters (TDCs) in a Xilinx Kintex-7 FPGA with less than 10 ps RMS resolution and $710 \mathrm{MHz}$ measurement throughput. Based on these fundamental TDC blocks, we propose a method to improve the time resolution further by merging multiple TDC blocks, which is equivalent to increasing the number of TDC bins multiple times. Two merged TDC channels, each with four TDC blocks, are implemented in the Kintex-7 FPGA and the performance is evaluated. For fixed time intervals in the range from 0 to $22 \mathrm{~ns}$, the average RMS resolution measured by the two TDC channels reaches 4.0 ps. The test results show that the FPGA based multichannel TDC system can be flexibly configured as either more TDC channels with a low time resolution or fewer TDC channels with a high time resolution.

N05-3: Input Mezzanine Card for the Fast Tracker at ATLAS

T. Iizawa

Waseda University, Tokyo, Japan

On behalf of the ATLAS Collaboration 
The Fast Tracker (FTK) is an integral part of trigger upgrade program for the ATLAS experiment. At LHC Run 2, which started operations in June 2015 at a center-of-mass energy of $13 \mathrm{TeV}$, the luminosity could reach up to $2 * 10^{\wedge} 34 \mathrm{~cm}^{\wedge}-2 \mathrm{~s}^{\wedge}-1$ and an average of 40-50 simultaneous proton collisions per beam crossing will be expected. The higher luminosity demands a more sophisticated trigger system with increased use of tracking information. The Fast Tracker is a highly-parallel hardware system that rapidly finds and reconstructs tracks in the ATLAS inner-detector at the triggering stage. This paper focuses on the FTK Input Mezzanine Board that is input module of entire system. The functions of this board are to receive the insertable b-layer, pixel and micro-strip data from the ATLAS Silicon read-out drivers, perform clustering, and forward the data to its mother board. Mass production and quality control tests of Mezzanine Boards were completed, and staged installation and commissioning are ongoing. Details of its functionality, mass production, quality control tests, and installation as well as first results from data taking are reported.

\title{
N05-5: Design and Characterization of the Full Size AGIPD Readout Chips
}

$\underline{\text { X. Shi }}$

Paul Scherrer Institut, Villigen-PSI, Switzerland

On behalf of the AGIPD Consortium

The AGIPD (Adaptive Gain Integrating Pixel Detector) collaboration - consisting of DESY, University of Hamburg, University of Bonn and PSI - is currently developing a 2D hybrid pixel detector system capable to fulfill the requirements of the European XFEL (XFEL.EU) that is being built in Hamburg, Germany. At the XFEL.EU photon pulses will arrive in bunch trains every 100 $\mathrm{ms}$ (or at a rate of $10 \mathrm{~Hz}$ ). Each train consists of 2700 bunches with a spacing of $220 \mathrm{~ns}$, meaning $4.5 \mathrm{MHz}$ frame rate) followed by a break of $99.4 \mathrm{~ms}$ without pulses. Each single pulse consists of $1012 \mathrm{X}$-ray photons arriving in less than $100 \mathrm{fs}$ and having an energy range from $250 \mathrm{eV}$ up to $25 \mathrm{keV}$. In order to cope with the large dynamic range of photon pulse, the first stage of each pixel in the readout ASIC is a charge integration preamplifier with three different gains that can be dynamically switched during charge integration. Dynamic gain switching allows to have single photon resolution in the high gain setting and to cover a large dynamic range of 104 photons in the low gain mode with a linearity better than $1 \%$. The high frame rate $(4.5 \mathrm{MHz})$ requires to store the images in the pixels before the readout takes place during the gap between bunch trains. The first full scale readout chip (AGIPD1.0), received at the end of 2013, is a 64 x 64 pixel matrix. Each pixel with a size of $200 \times 200 \mu \mathrm{m} 2$ is equipped with 352 storage cells. After an extensive characterization, some design bugs were spotted although the performance fulfills the specifications of XFEL. The second full scale readout chip (AGIPD1.1), was submitted in the December 2015 and received in the March 2016. The preliminary measurement shows that all the major bugs seen on AGIPD1.0 disappeared on AGIPD1.1. The measurements are still ongoing. More results will be shown at the conference. This presentation will focus on the design and characteristics of the AGIPD1.0 and AGIPD1.1 chips.

\author{
N05-6: Ultra-Low Power Fast 10-Bit ADC for Multi-Channel Readout of Particle Physics Detectors \\ M. Idzik, S. Bugiel, R. Dasgupta, M. Firlej, T. Fiutowski, J. Moron, K. Swientek \\ Faculty of Physics and Applied Computer Science, AGH University of Science and Technology, Krakow, Poland
}

The architecture, design, and measurement results of multi-channel 10-bit SAR ADC ASICs for readout of particle physics detectors, developed in two CMOS $130 \mathrm{~nm}$ technologies, are presented. The developed ASICs comprise also a PLL-based data serialization and high speed data transmission. The ADC ultra-low power consumption scales with the sampling frequency up to $40 \mathrm{MS} / \mathrm{s}$. The measurement results of static and dynamic parameters as a function of sampling frequency, power consumption, performed on the ADC prototypes are presented. The measurements show an excellent effective resolution with ENOB above 9.5 bits and very good static linearity with INL, DNL below $0.5 \mathrm{LSB}$. The power consumption at $40 \mathrm{MS} / \mathrm{s}$ rate is below $700 \mu \mathrm{W}$ and it is is reflected by an excellent Figure of Merit (FOM) which, depending on input and sampling frequency, is in the range 20-28 $\mathrm{fJ} /$ conversion-step. This is the lowest, known to the authors, FOM obtained in $130 \mathrm{~nm}$ CMOS process for similar ADC resolution and sampling frequency.

This work was supported by Polish National Science Centre (NCN), grant reference number DEC-2012/07/B/ST7/01456.

N05-7: Development of an Asynchronous Readout ASIC for GEM Detectors

$\underline{\text { E. Malankin }}^{1}$, E. Atkin ${ }^{1}$, I. Bulbakov ${ }^{1}$, P. Ivanov ${ }^{1}$, D. Normanov ${ }^{1}$, V. Shumikhin ${ }^{1}$, I. Sagdiev ${ }^{1}$, O. Shumkin ${ }^{1}, S_{\text {. Vinogradov }}{ }^{1}$, A. Voronin ${ }^{1}$, V. Samsonov ${ }^{2}$, V. Ivanov ${ }^{2}$

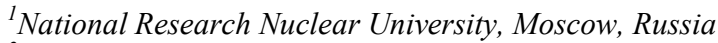

${ }^{2}$ Petersburg Nuclear Physics Institute National Research Centre Kurchatov Institute, Gatchina, Russia

Currently a multichannel readout chip with an asynchronous architecture is being developed for GEM detectors. The paper describes its prototype, which includes two full readout channels with analog front-end and digital back-end. The channel includes a preamplifier with fast and slow CR-RC shapers, discriminator with a differential threshold setup circuit, a 6 bit SAR ADC (40 Msps rate, $1.5 \mathrm{~mW}$ power consumption), digital peak detector and block of the time stamp registration. The digital peak 
detector has a feature preventing the false peak detection. The final chip version is considered to be compatible with the GBTx data processing board. Thus, the control data, clock and output data are supplied through SLVS transmitter and receiver. The slow and fast channels have a $1500 \mathrm{el}$ and $2000 \mathrm{el} \mathrm{ENC} \mathrm{accordingly} \mathrm{at} \mathrm{a} 50 \mathrm{pF}$ detector capacitance. Power consumption is 10 $\mathrm{mW} /$ channel.

N05-8: The Development of High-Performance Front-End Electronics for the ATLAS TileCal Upgrade A. P. White

Physics, University of Texas at Arlington, Arlington, TX, USA

On behalf of the ATLAS Tile Calorimeter System

We present the design of a new candidate front-end electronic readout system being developed for the ATLAS TileCal Phase 2 Upgrade. The system is based upon the QIE12 custom Application Specific Integrated Circuit. The chip features a least count sensitivity of $1.5 \mathrm{fC}$, more than 17 bits of dynamic range with logarithmic response, and an on-chip TDC with one nanosecond resolution. The design incorporates an on-board current integrator, and has several calibration systems. The new electronics will operate dead-timelessly at $40 \mathrm{MHz}$, pushing full data sets from each beam crossing to the data acquisition system that resides offdetector in the USA15 counting room using high-speed optical links. The system is one of three candidate systems for the Phase 2 Upgrade. We have built a "Demonstrator" - a fully functional prototype of the new system. Performance results from bench measurements and from a recent test beam campaign will be presented.

N05-9: FATALIC, a Very-Front-End ASIC for the ATLAS Tile Calorimeter in the Context of the HL-LHC A. White

Physics, University of Texas at Arlington, Arlington, TX, USA

On behalf of the ATLAS Tile Calorimeter System

The ATLAS Collaboration has started a vast program of upgrades in the context of the high-luminosity LHC (HL-LHC) forseen in 2024. The current readout electronics of every subdetector, including the Tile Calorimeter (TileCal), must be upgraded to comply with the new specifications aiming for the future operating conditions. The ASIC described in this document, named Front-end ATIAs tiLe Integrated Circuit (FATALIC), has been developed to fulfill the requirements of the TileCal upgrade. FATALIC is based on a $130 \mathrm{~nm}$ CMOS technology and performs the complete processing of the signal, including amplification, shaping and digitization. The first stage is a current conveyor which splits the input signal into three ranges, allowing to deal with a large dynamic range (from $25 \mathrm{fC}$ up to $1.2 \mathrm{nC}$ ). Each current conveyor output is followed by a shaper and a dedicated pipeline 12 bit ADC operating at $40 \mathrm{MHz}$. Measurements show a non-linearity at the percent level for the typical range of interest of the input charge. The noise of the whole chain is measured to be around $7 \mathrm{fC}$ in its nominal frequency band width.

\author{
N05-11: Building Blocks of a Read-Out Chip for a High Granularity Electromagnetic Calorimeter \\ J.-B. Cizel $^{1}$, R. Cornat ${ }^{2}$ \\ ${ }^{I}$ Weeroc, Palaiseau, France \\ ${ }^{2}$ LLR, Palaiseau, France
}

The particle flow methodology is used to develop new kind of calorimeters. The particle flow is designed to measure and track individual particles inside a jet. High granularity layered calorimeters are needed to efficiently use the particle flow algorithm. A high transversal and longitudinal resolution is a prerequisite. To handle this high resolution, electronics must be very compact and so integrated chips are needed to read-out millions of channels. This work takes place in the design of an electromagnetic calorimeter for the foreseen International Linear Collider, on which is expected 82 million of channels read-out. An ASIC prototype named SKIROC2 has already been developed as a proof of concept. It gives good results and has allowed proving the feasibility of such a project. There are still few issues with it, the most critical being the insufficient power supply rejection ratio which causes fake triggers on power supply noise. Moreover in the final chip a lot more digital will be integrated and the currently used technology (AMS s35d4) would not allow such integration. Trying to resolve these issues, new developments has been done in the X-Fab xt018 technology. Three preamplifiers have been designed to compare the noise and power supply rejection ratio of each. The full acquisition chain, including a bandgap reference, bandpass filters, discriminators, analog memories and a Wilkinson ADC have been designed and integrated in a 24 channels chip. There are 8 channels per preamplifier in order to measure crosstalk behavior.

\author{
N05-12: CAD-II: the Second Version Current-Mode Preamplifier-Dsicriminator ASIC for MRPC-TOF \\ Detectors


This report presents the second version of a fully current-mode front-end electronics, CAD-II, for MRPC detectors for TOF applications. Several upgrades have been made in this new version including: 1). Using differential input stage with input impedance down to $30 \mathrm{O}$ and LVDS compatible output; 2). Much higher current gain and bandwidth of $4.5 \mathrm{~A} / \mathrm{A}$ and $380 \mathrm{MHz}$ by simulation; 3). Fabricated in $0.18 \mu \mathrm{m}$ CMOS process instead of $0.35 \mu \mathrm{m}$ CMOS technology used in CAD-I. In this report, we will present a detailed analysis of the timing jitter for the current discriminator used, comparison between simulation and calculation will be given. Besides, we will present the first measurements of CAD-II covering its input impedance, power consumption, noise and timing performance. The single-end input impedance is as low as $32 \mathrm{O}$ with a $15 \mathrm{~mW} / \mathrm{channel}$ nominal power consumption. Input referred RMS noise current is about $0.56 \mu \mathrm{A}$. The input referred threshold current can be set as low as $4.5 \mu \mathrm{A}$. Sub-15ps timing resolution has been measured for input signal above $100 \mu \mathrm{A}$.

This work was supported in part by the National Basic Research 973 Program of China under Grant No.2015CB856905. We also thank ICC for providing the MPW service for prototype chip fabrication.

\author{
N05-13: High Performance Readout Module Based on ZYNQ with Giga Bit Ethernet \\ T. Xue, G. Gong, J. Li \\ Engineering Physics, Tsinghua University, Beijing, China
}

ZYNQ is now becoming a popular architecture of FPGA with dual high performance ARM Cortex-A9 processors in the application of data acquisition system of nuclear electronics. Many typical readout system use ZYNQ running embedded Linux and real time logic to deal with the data from high speed ADC or DAC. We have designed the first generation of readout module for HPGe detector's DAQ in the CJPL (China JingPing under-ground Lab) experiment based on ZYNQ XC7Z010 CLG225 footprint in the early of 2014. More than 10 readout system use this module, reach a fantastic performance and system flexibility. Now a new readout module based on ZYNQ XC7Z020 CLG400 footprint is develop recently for more LVDS IO channels to interface more $\mathrm{ADC}$ and DAC with more logic resources and in the same $56 \mathrm{~mm}$ plus $42 \mathrm{~mm}$ physics small size as the previous module. This paper will introduce how about the architecture of the new readout module. Also the throughput of the Giga Bit Ethernet which more than $600 \mathrm{Mbps}$ will be introduce and a sample of crystal neutron detector's data acquisition system based on the new module will also be introduced.

\title{
N05-14: Level-1 Data Driver Card of the ATLAS New Small Wheel Upgrade Compatible with the Phase II 1 MHz Readout Scheme
}

$\underline{\text { P. Gkountoumis }}$

NTU Athens, Athens, GREECE

On behalf of the ATLAS Muon Collaboration

In the ATLAS experiment, the present muon Small Wheels will be replaced by the New Small Wheels (NSW). The NSW is a set of precision tracking and trigger detectors able to work at high rates with excellent, even at the L1 trigger, spatial and time resolution. The new detectors consist of the resistive micromegas and the small-strip Thin Gap Chambers (sTGC). To readout the high number of electronics channels and in order to survive in such a harsh environment new electronics must be fabricated. The L1DDC is an intermediate board that aggregates and transmits the L1 data from multiple front-end boards to a network called Front End LInk eXchange (FELIX) and is fully compatible with the Phase-II $1 \mathrm{MHz}$ trigger rate. This is achieved using a number of high speed serializer/deserializer Application Specific Integrated Circuits (ASIC) called GigaBit Transceivers (GBTX) developed at CERN. Furthermore, the L1DDC distributes Timing, Trigger and Control (TTC) data from the network to the frontend and the ADDC (Address real time Data Driver Card) boards. Generally, the L1DDC combines three distinct paths: TTC, Data Acquisition and Slow Control (SC - configuration and monitoring data), into one bidirectional optical link at a rate of 4.8 Gbps. The L1DDC will be used also for the needs of the on-detector trigger boards (PAD and Routers) of the sTGC detectors.

N05-15: Experimental Results with TOFPET2 ASIC

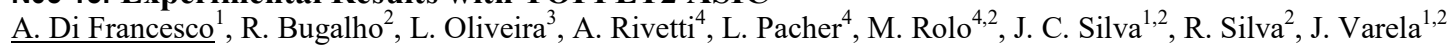

${ }^{1}$ LIP, Lisbon, Portugal

${ }^{2}$ PETsys Electronics, Oeiras, Portugal

${ }^{3}$ DEE, FCT-UNL, CTS-UNINOVA, Caparica, Portugal

${ }^{4} I N F N$, Torino, italy

We present the first experimental results obtained with TOFPET2, a readout and digitization ASIC for radiation detectors using Silicon Photomultipliers. The circuit is designed in CMOS $110 \mathrm{~nm}$ technology, has 64 independent channels and is optimized for time-of-flight measurement in PET or other applications. The chip has quad-buffered TDCs and charge integration ADCs in each channel. The chip tape-out was done in November 2015 and first tests started in end March 2016. Preliminary measurements of the timing performance obtained with test pulses show a resolution of $26 \mathrm{ps}$ r.m.s. Measurements with radioactive sources will be presented at the conference. 
N05-16: A Prototype 32 Channel Front-End Electronic Readout System for an UV Imaging Detector. $\underline{\text { A. Seljak }}^{1}$, G. S. Varner ${ }^{1}$, J. Vallerga ${ }^{2}$, R. Raffanti ${ }^{3}$, H. S. Cumming ${ }^{1}$

${ }^{I}$ Department of Physics and Astronomy, University of Hawai'i at Manoa, Honolulu, Hawaii

${ }^{2}$ Science and Space Laboratory, University of California,, Berkeley, California

${ }^{3}$ Techne Instruments, Oakland, California

NASA's Strategic Astrophysics Technology (SAT) program funded the development of an UV single photon sensitive imaging detectors $(50 \mathrm{~mm} \times 50 \mathrm{~mm}$ ). Part of the grant is dedicated to the improvement of the detector readout electronics system. The presently developed detector uses a readout system build of commercial components, however, for flight missions, the readout needs to be dramatically reduced in size, complexity and power consumption. The approach is to build dedicated Application Specific Integrated Circuit (ASIC) chips, which would meet the required constrains, and also increase the performance of the overall system. For this purpose two ASIC chips were designed. A programmable charge sensitive amplifier (CSA), and an 12bit, Giga Sample Per Second (GSPS) digitizing (ASIC) named HalfGRAPH. An FPGA Mezzanine Card (FMC) size board was produced to test these chips. This paper presents the development and evaluation of a 32 channel prototype readout system.

\section{N05-17: Performance Evaluation of Digital Pixel Readout Chip Architecture Operating at Very High Rate Through a Reusable UVM Simulation Framework}

$\underline{\text { E. Conti }}^{1}$, S. Marconi ${ }^{1,2,3}$, T. Hemperek ${ }^{4}$, J. Christiansen ${ }^{1}$, P. Placidi ${ }^{2,3}$

${ }^{I}$ CERN, Geneva, Switzerland

${ }^{2}$ Department of Engineering, University of Perugia, Perugia, Italy

${ }^{3}$ INFN Perugia, Perugia, Italy

${ }^{4}$ Institute of Physics, University of Bonn, Bonn, Germany

A large scale demonstrator pixel readout chip is currently being designed by the RD53 Collaboration, with the goal of proving the suitability of $65 \sim \mathrm{nm}$ technology for the extreme operating conditions associated to the High Luminosity upgrades of the ATLAS and CMS experiments at the Large Hadron Collider. The VEPIX53 simulation and verification environment was developed in order to support the chip design flow at different steps, from architectural modeling and optimization to final design verification, thanks to the flexibility and reusability of SystemVerilog and the Universal Verification Methodology (UVM) library.

In this work a test case of VEPIX53 is presented where an existing digital pixel architecture, already implemented in a small scale prototype chip, is simulated for evaluating whether it can comply to the specifications of the large scale demonstrator chip. The architecture inefficiency was measured by the analysis components of the environment, with respect to different models of analog front-ends and different pixel hit memory sizes, showing possible solutions for optimization.

N05-18: Upgrades to the CSC Cathode Strip Chamber Electronics for HL-LHC D. M. Morse

Physics Department, Northeastern University, Boston, Massachusetts, USA

On behalf of the CMS Collaboration

The luminosity, latency, and trigger rate foreseen at the High Luminosity LHC presents challenges to efficient readout of the Cathode strip chambers (CSCs) of the CMS end cap muon detector. Upgrades to the electronics are being planned to address these issues. These upgrades are targeted for the inner rings of CSCs in each station, which have the highest flux of particles. The existing cathode front end boards on the inner rings three stations will be replaced with new digital cathode front end boards that all for nearly deadtimeless operation and the capability to accommodate long latency requirements without loss of data. Also, new DAQ motherboards will be designed with optical output links with higher bandwidth to accept the higher data rate, and the Front End Driver system, which is the interface between the CSCs and the central DAQ of the CMS experiment, must be replaced with a system that can receive the higher input rates.

N05-19: Study of PMOS Front-End Solution with Signal Compression for XFEL MiniSDD X-Ray Detectors

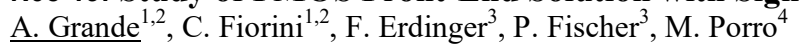

${ }^{I}$ Dipartimento Elettronica Informazione e Bioingegneria, Politecnico di Milano, Milano, Italy

${ }^{2}$ Sezione di Milano, INFN, Milano, Italy

${ }^{3}$ Institut für Technische Informatik, Universität Heidelberg, Heidelberg, Germany

${ }^{4}$ European XFEL, Hamburg, Germany

We present the study and the experimental results on different front-end stages for the MiniSDD pixel sensors of the DSSC detector for photon science applications at the European XFEL GmbH in Hamburg. The detector must be able to cope with an image frame rate up to $4.5 \mathrm{MHz}$ and must achieve a dynamic range up to $10^{\wedge} 4$ photons/pixel/pulse with a photon energy of 1 
$\mathrm{keV}$. In order to achieve this high dynamic range and single photon sensitivity at the same time, the system front-end must provide a nonlinear amplification. The DSSC system is designed in order to be operated with two different kind of sensors: the DEPFET arrays that provide intrinsic dynamic range compression and the MiniSDD pixel arrays that have a linear output response. In the last case a non-linear front-end on the readout ASIC is required. The front-ends we present are based on a PMOS input transistor. The non-linear response is obtained with a simple circuit that pushes the input PMOS into triode region as the input signal increases. Since the readout ASIC has more than four thousand channels operating in parallel, particular care was given to the homogeneity and the robustness of the implemented solution especially with respect to power supply rejection ratio and the cross talk among channels. First experimental results have shown that it is possible to achieve a noise of 52 electrons rms at an equivalent frame rate of $4.5 \mathrm{MHz}$. The simulated dynamic range, instead, is about $4700 \mathrm{ph}$ at $1 \mathrm{keV}$.

\author{
N05-20: The Waveform Digitizer System for the MU2E Experiment: Conceptual Design and First Prototype \\ Results \\ $\underline{\text { F. Spinella }}^{1}$, S. Donati ${ }^{2}$, S. Di Falco ${ }^{1}$, F. Cervelli ${ }^{1}$, L. Morescalchi ${ }^{3}$, E. Pedreschi $^{2}$, G. Pezzullo ${ }^{1}$ \\ ${ }^{I}$ Italian Institute for Nuclear Physics - section of Pisa, Pisa, Italy \\ ${ }^{2}$ Physics Department, University of Pisa, Pisa, Italy \\ ${ }^{3}$ Physics Department, University of Siena, Siena, Italy
}

The Mu2e experiment at Fermilab searches for the coherent muon conversion to electron in the Coulomb field of an $\mathrm{Al}$ nucleus, with the goal to improve the current experimental limit by 4 orders of magnitude. The Mu2e detector is composed of a straw tube tracker and an undoped CsI crystals electromagnetic calorimeter housed in a superconducting solenoid. In order to achieve the needed background suppression, the calorimeter provides a time resolution better than $500 \mathrm{ps}$ and an energy resolution of $\mathrm{O}(5 \%)$ (a) $100 \mathrm{MeV}$. To fulfill these requirements a digitizing system, composed of around 150 cards sampling a total of about 2700 channels at a frequency of $200 \mathrm{MHz}$, is currently being designed. The electronics will be located close to the calorimeter, inside the magnet cryostat and will be operated in vacuum. The harsh experimental conditions, with the presence of a high neutron flux, ionizing dose and magnetic field, make the design challenging. All the components and materials have been individually qualified and a first prototype has been tested in terms of performance and reliability. The design choices and the first tests results are presented.

N05-21: Performances of the Calorimetric Trigger Processor of the NA62 Experiment at CERN SPS R. Ammendola ${ }^{1}$, M. Barbanera ${ }^{2}$, M. Bizzarri ${ }^{3}$, V. Bonaiuto ${ }^{4}$, A. Ceccucci $^{5}$, B. Checcucci $^{2}$, N. De Simone ${ }^{5}$, R. Fantechi ${ }^{5}$, L. Federici $^{1}$, A. Fucci ${ }^{5}$, M. Lupi ${ }^{2}$, G. Paoluzzi ${ }^{1}$, A. Papi ${ }^{3}$, M. Piccini ${ }^{3}$, V. Ryjov ${ }^{5}$, A. Salamon ${ }^{1}$, G. Salina ${ }^{1}$, F. Sargeni ${ }^{6}$, S. Venditti ${ }^{5}$

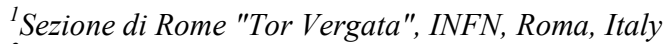

${ }^{2}$ Sezione di Perugia, INFN, Perugia, Italy

${ }^{3}$ Department of Physics, University of Perugia, Perugia, Italy

${ }^{4}$ Dept Industrial Eng., University Rome Tor Vergata, roma, Italy

${ }^{5}$ CERN, Geneve, Switzerland

${ }^{6}$ Dept Electronic Eng., University Rome Tor Vergata, Roma, Italy

The NA62 experiment aims to test the Standard Model by a measure of the branching ratio of the rare kaon decay $\mathrm{K}+->$ pi+ nu nubar at the CERN SPS (Super Proton Synchrotron). It makes use of a complex Trigger and Data Acquisition system (TDAQ) that has been installed and has taken first physics data in autumn 2014. In particular, the calorimeter L0 trigger (Cal-L0) is used to select events with a pi+ in the final state hadronic and to veto one of the most dominate background from events $\mathrm{K}+->$ pi+pi0. We present the design, performance and operation during the last two years (2015 and part of 2016) high intensity data taking of the calorimeter Level 0 trigger. The system is composed of 37 readout boards (TEL62) with dedicated daughter boards. These boards are arranged in three different layers of boards: Front-End, Merger (only for LKR calorimeter) and Concentrator. The CalL0 trigger manages 13520 physical channels and identifies electromagnetic clusters, with an instantaneous hit rate up to $30 \mathrm{MHz}$, providing information on time, position and energy.

\title{
N05-22: A 32-Channel Read-Out ASIC for PET Application
}

H. Xu, M. Perenzoni, N. Massari, A. Gola, A. Ferri, C. Piemonte, D. Stoppa

Integrated Radiation and Image Sensors Division, Fondazione Bruno Kessler, Trento, Italy

This paper presents an ASIC in 150nm CMOS technology, aiming at Silicon Photomultiplier (SiPM) readout for positron Emission Tomography (PET) application. The chip includes 32 channels, and each is composed of a current buffer, a validation block, a charge sensitive amplifier, a 10-bit ADC and a 12-bit TDC. The gamma characterization was conducted with $4 \times 4 \mathrm{~mm} 2$ SiPMs (FBK-NUV-HD) coupled to $3 \times 3 \times 5 \mathrm{~mm} 3$ LYSO scintillator, showing an energy resolution of $14.7 \%$ and Coincident Resolving Timing (CRT) of 433ps. 
N05-23: A MAPMT Compact Read-Out Based on CIAC, a Dedicated ASIC

$\underline{\text { M. Galasso }}^{1}$, A. Fabbri ${ }^{1}$, V. Orsolini Cencelli ${ }^{1}$, F. De Notaristefani ${ }^{2}$

${ }^{I}$ INFN Sezione Roma Tre, Roma, Italy

${ }^{2}$ Physics Department, Roma Tre University, Roma, Italy

A compact electronic board was developed to acquire the signals outgoing from a multi-anode photomultiplier tube (MAPMT). The board is based on two naked samples of CIAC (Charge Integrator Array Chip), an ASIC developed in our laboratories capable of acquiring individually 32 anode signals. Each acquisition channel of CIAC is composed of a charge sensitive amplifier with a variable integrating capacitance ranging from $5 \mathrm{pF}$ to $35 \mathrm{pF}$, a sample and hold and a variable gain amplifier to perform an hardware correction of the MAPMT gain non-uniformity. The ADCs, placed on the same board, provide as an output all the 64 digitized charge values. The size of the custom board is $50 \times 50 \times 10 \mathrm{~mm}^{3}$ and it can be coupled to one of the following MAPMTs: H8500, H10966 or H12700. The power consumption is of $0.9 \mathrm{~W}$ and the board detection efficiency is of $95 \%$ for a scintillation rate of $1 \mathrm{kHz}$ and of $72 \%$ for a scintillation rate of $30 \mathrm{kHz}$, thanks to a maximum acquisition frequency of $150 \mathrm{kHz}$ and a minimum dead time of 0.7 us.

N05-24: MATRIX: a Novel Two-Dimensional Resistive Interpolation 15 Ps Time-to-Digital Converter ASIC J. Mauricio, D. Gascon, D. Ciaglia, S. Gómez, G. Fernández, A. Sanuy

University of Barcelona, Barcelona, Spain

This paper presents a 4-chanel TDC chip demonstrator with the following features: 15-ps resolution, $1280 \mathrm{~ns}$ dynamic range, dead time $<20 \mathrm{~ns}$, up to $10 \mathrm{MHz}$ of sustained input rate per channel, around $60 \mathrm{~mW}$ of power consumption and very low area in a $180 \mathrm{~nm}$ technology. The main contribution of this work is the novel design of the clock interpolation circuitry which is based on a resistive interpolation mesh circuit (patented), a two-dimensional regular structure with outstanding performance in terms of power consumption, area and low process variability.

N05-25: SENSROC10: a 64-Channel Analog Front-End ASIC Dedicated to CZT Detector for SPECT Imaging W. Gao

Institute of Microelectronics, School of Computer, Northwestern Polytechnical University, Xi'an, Shaanxi Prov., China

This paper presents design techniques of an analog front-end ASIC dedicated to a pixilated CZT detector for single-photon emission computer tomography (SPECT) imaging applications. In our proposed scheme, the signals from 64 pixels are readout. However, only one pair of signals (peak voltage and time trigger) together with the hitting position are recorded and sent to storage. The topology of the single readout channel consists of a charge sensitive preamplifier, a CR-RC shaper followed by twostage Sallen-Key (SK) filters, a peak-detect-and-hold circuit, a time discriminator and a time controller. A 64-channel front-end readout ASIC is designed and implemented in TSMC $0.35 \mu \mathrm{m}$ CMOS process. The preliminary results are obtained. The input range of the ASIC is from 2000 e- to $130000 \mathrm{e}$-, which is suitable for the detection of the ? rays from $\sim 10 \mathrm{keV}$ to $600 \mathrm{keV}$. The linearity of the output voltage is less than $3 \%$. The gain of the readout channel is $60 \mathrm{mV} / \mathrm{fC}$. The static power dissipation is about $3 \mathrm{~mW} / \mathrm{channel}$. The above tested results show that the electrical performances of the ASIC can well satisfy SPECT imaging applications. The overall performance is concluded in Table 1. For the future wok, the ASIC will be tested with the connection of a pixilated CZT detector to obtain more tested results.

This work was supported in part by the National Natural Science Foundation of China under Grant No. 11475136, No. 61176094 and the National Key Scientific Instrument and Equipment Development Project under Grant No. 2011YQ040082. The autors would like to thank Dr. Christine Hu-Guo with IPHC, France for her valuable discussions on the circuit performance optimization and Prof. W. Jie and Prof. G. Zha with NPU, Xi'an, China for their discussions on the CZT detector charateristics.

\section{N05-26: Design, Characterization and Test of the Associative Memory Chip AM06 for the Fast TracKer System} R. Stamen

Kirchhoff-Institut für Physik, Heidelberg, Germany

On behalf of the FTK Collaboration

We will present performance of the new Associative Memory (AM) chip, designed and manufactured in $65 \mathrm{~nm}$ CMOS technology. The AM06 is the 6th version of a highly parallel ASIC processor for pattern recognition in high energy physics experiments. The AM06 is based on the XORAM cell architecture, which has been specifically designed to reduce power consumption and control complexity. The AM06 is a large chip, which contains memory banks that store all data of interest. The basic unit is a word of 18 bit. A group of 8 words (each of them related to a detector layer) is called a "pattern". Each AM06 chip stores 217 patterns. The AM06 integrates serializer and deserializer IP blocks (working up to $2.4 \mathrm{GHz}$ ), to avoid routing 
congestion at the board level. AM06 is a complex VLSI chip, designed combining full-custom memory arrays, standard logic cells and IP blocks. It occupies a silicon area of $168 \mathrm{~mm} 2$ and it contains about 421 millions transistors. The AM06 chip is able to perform a synchronous bitwise comparison of about $1 \mathrm{Mbit}$ per second. The parallel input data rate is $100 \mathrm{MHz}$. Thanks to the XORAM cell and to the design optimization, the AM06 consumes about $2 \mathrm{fJ} / \mathrm{bit}$ per comparison. The AM is tailored for on-line track finding in physics experiments; however, it is suitable also for interdisciplinary applications (i.e., general purpose image filtering and analysis). In future we plan to design a more powerful and flexible chip at $28 \mathrm{~nm}$ CMOS.

\section{N05-27: Design and Development of Radiation Hardened 2.5GHz Clock Multiplier Unit with $0.18 і{ }_{i}^{1 / 2 m}$ Technology for CBM-MUCH Detector Electronics}

H. K. Pandey ${ }^{1}$, T. K. Bhattacharya ${ }^{2}$, J. Saini $^{1}$

${ }^{I}$ ATDC, INDIAN INSTITUTE OF TECHNOLOGY, KHARAGPUR,WB, INDIA

${ }^{2}$ RIB, VARIABLE ENERGY CYCLOTRON CENTRE, KOLKATA, INDIA

A fast and radiation hardened detector system is required to detect the low momentum muons emanating from an environment of high particle densities. Hence a radiation hardened front-end electronics is required for that detector system. To read out the high flow data of Muon Chamber ( MUCH) which is expected at Compressed Baryonic Matter (CBM) experiment, it requires precise, time synchronized, compact, radiation tolerant and self triggered front-end electronics. The Clock Multiplier Unit (CMU) delivers a clock with minimum jitter to Serialize and De-serialize (SERDES) the data that comes with a high flow rate. A radiation hardened $2.5 \mathrm{GHz}$ clock multiplier unit (CMU) in $0.18 \ddot{i}_{i} 1 / 2 \mathrm{~m}$ standard CMOS process has been designed and developed as a sub-block of a 10 bit SERDES operating at $2.5 \mathrm{Gbps}$. The CMU design is based on phase lock loop and radiation hardening is achieved at the design level by carefully choosing architecture and topology. At the layout level guard rings are used to reduce the effect of radiation on circuits. This work focuses on the design and measurements of the developed CMU ICs. The effect of gamma-ray irradiation using a Co-60 source is examined and also with the neutron irradiation. The test results reveal that radiation tolerance is sufficient for the operation in an environment of the CBM-MUCH detector experiment.

\section{N05-28: BASIC64: a New Mixed-Signal Front-End ASIC for SiPM Detectors}

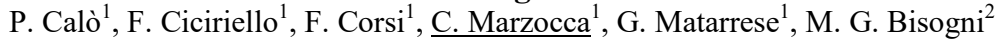

${ }^{I}$ Dept. of Electrical and Information Engineering, Politecnico di Bari, Bari, Italy

${ }^{2}$ Dept. of Physics, Università di Pisa, Pisa, Italy

In the framework of the INSIDE (INnovative Solutions for Dosimetry in hadrontherapy) project, aimed at the construction of a compact PET system and a fiber tracker to be used for in-beam dose monitoring and primary vertex reconstruction in hadrontherapy, a multichannel front-end ASIC, BASIC64, has been developed in a standard 0.35?m CMOS technology for interfacing the SiPM detectors used in the PET system. The self-triggering ASIC hosts 64 current-mode front-end channels, generates a trigger signal corresponding to the detection of a valid event and provides the associated energy information in the digital domain, by means of a 10-bit integrated ADC. The front-end architecture is based on a very simple open-loop current buffer, i.e. a common gate configuration, to avoid possible stability issues related to the application of negative feedback due to the large values of the equivalent capacitance of the detector. The input signal from the detector is split in two different branches directly at the input node, by means of two matched common gate transistors. A "fast" path is used to generate the trigger signal of the channel, while a "slow" path, which includes an integrator and a peak detector, produces an analog voltage proportional to the peak of the integrated charge, which is then A/D converted. The digital logic integrated in the ASIC manages autonomously the read out procedure, carried out in sparse or serial mode, controls the ADC and the data transfer via a LVDS link and resets the ASIC after the completion of the read out procedure. A test setup for the prototypes of the ASIC, based on a FPGA device, has been designed and realized and the characterization measurements of the chip are currently in progress.

\section{N05-29: Characterization of ASIC-Based Detectors for Limited Angle Tomography TOF-PET}

$\underline{A}_{\text {. Aguilar }}^{1}$, J. M. Monzo ${ }^{1}$, A. J. Gonzalez ${ }^{1}$, A. Gonzalez-Montoro ${ }^{1}$, G. Cañizares ${ }^{1}$, L. F. Vidal ${ }^{1}$, L. Hernandez ${ }^{1}$, R. Colom $^{1}$, D. Grau-Ruiz ${ }^{1}$, J. P. Rigla ${ }^{1,2}$, E. Diaz-Caballero, ${ }^{1,2}$, P. Bellido ${ }^{1}$, P. Conde ${ }^{1}$, A. Iborra ${ }^{1}$, L. Moliner ${ }^{1}$, M. J. Rodriguez-Alvarez ${ }^{1}$, S. Sanchez ${ }^{1}$, M. Seimetz ${ }^{1}$, A. Soriano ${ }^{1}$, F. Sanchez ${ }^{1}$, J. J. Garcia-Garrigos ${ }^{1}$, J. M. Benlloch ${ }^{1}$

${ }^{1}$ Institute for Instrumentation in Molecular Imaging, Valencia, Spain

${ }^{2}$ Tesoro Imaging S.L., Alicante, Spain

Time of Flight (TOF) functionality is primarily required to deal with limited angle tomography (LAT) scanners, where close geometry as the typical ring shape, cannot be implemented. It could, for instance, be the case for dedicated cardiac or prostate PETs. A PET imager with missing angular information produces non-uniformities and artifacts in the reconstructed image across the field of view. If TOF information is available, one could afford loosing part of this information reducing the artifacts on the final reconstructed image. Detectors based on SiPM technology have demonstrated that, in order to obtain a very accurate time resolution, it is required to process independently each SiPM element so that the time information related to the rise time of the signal is preserved. Application Specific Integrated Circuits (ASICs) are one of the most suitable solutions to carry out this work 
when SiPM arrays are used. ASICs can preserve a very good spatial and energy resolutions when compared to classical readout approaches. Unlike crystal arrays, monolithic scintillators allow one to preserve the light distribution of each impact. In this work, two readout options based on ASICs, coupled to SiPM arrays with different crystal types are studied in order to validate their use for TOF-PET applications. Crystal arrays have been used to initially optimize the experiments. Parameters such as spatial, energy and time resolution have been evaluated. In terms of Coincidence Resolving Time (CRT) promising results have been obtained, having times under $400 \mathrm{ps}$ FWHM. Spatial resolution is better than $1 \mathrm{~mm}$ according to the pixel resolving capability presented and energy resolution appears to be around $16 \%$. There is a huge room for improvement taking into account many remaining optimizations. The ultimate goal is to test monolithic crystals instead of crystal arrays to take advantage of their lower cost and their photon impact Depth of Interaction determination capabilities.

N05-30: A New Vertical JFET Technology for the Powering Scheme of the ATLAS Upgrade Inner Tracker P. Fernández-Martínez, D. Flores, S. Hidalgo, D. Quirion, M. Ullán

Instituto de Microelectrónica de Barcelona, IMB-CNM (CSIC), Barcelona, Spain

A new vertical JFET technology has been recently developed at the IMB-CNM (Barcelona). Mainly conceived to work as radhard switches for the renewed HV powering scheme of the ATLAS upgraded tracker, the new silicon V-JFET transistors take advantage of a deep-trenched 3D technology to achieve bulk conduction and low switch-off voltage. Together with the choice of a high-resistive p-type substrate, these features prospect suitable radiation hardness for the application. First V-JFET batches have been produced and characterized at the IMB-CNM clean room, with very promising pre-irradiated results, already meeting the specifications. A detailed compilation of the simulated and measured performance is shown in the contribution together with further aspects concerning the technology and design. To evaluate the radiation hardness, a thorough irradiation campaign, including gamma, neutron and proton irradiations, has been already scheduled and the results will be also presented in the final contribution.

N05-31: A 64 Channel Mixed-Signal ASIC for the Readout of GEM Detectors in the BESIII Experiment C. Leng ${ }^{1,2,3}$, J. Chai ${ }^{1,2,3}, \mathrm{H}_{\text {. Li }}^{2,4}$, A. Di Francesco ${ }^{5}$, R. Bugalho ${ }^{6}$, M. Maggiora ${ }^{2,7}$, S. Marcello ${ }^{2,7}$, A. Rivetti ${ }^{2}$, M. Rolo ${ }^{2}$, J. Varela ${ }^{5,6}$

${ }^{I}$ Dipartimento di Elettronica e Telecomunicazioni, Politecnico di Torino, Torino, Italy

${ }^{2}$ Sezione di Torino, Istituto Nazionale di Fisica Nucleare, Torino, Italy

${ }^{3}$ University of Chinese Academy of Sciences, Beijing, China

${ }^{4}$ IHEP, Chinese Academy of Sciences, Beijing, China

${ }^{5}$ LIP, Lisbon, Portugal

${ }^{6}$ PET-Sys Electronics, Oeiras, Portugal

${ }^{7}$ Dipartimento di Fisica, Università di Torino, Torino, Italy

A mixed-signal ASIC optimized for the readout of the cylindrical GEM detector of the BESIII experiment is presented. The chip consists of 64 analog front-ends, each featuring a charge-sensitive amplifier followed by a dual-shaper. A low-power TDC and a 10 bit Wilkinson ADC with derandomizing buffers allow to capture both the time-of-arrival of the event and the energy deposited by the impinging particles. The ASIC is designed for input capacitance up to $100 \mathrm{pF}$, event rates of $60 \mathrm{kHz}$ per channel and maximum power consumption of $10 \mathrm{~mW} /$ channel. The chip is fabricated in a $110 \mathrm{~nm}$ CMOS process.

N05-32: Development of a Low-Cost, Compact, Multi-Channel, FPGA-Based Digital Pulse Processor E. M. Becker ${ }^{1}$, A. T. Farsoni ${ }^{1}$, C.-S. Lee ${ }^{2}$, M. Mannino ${ }^{1}$

${ }^{I}$ School of Nuclear Engineering, Oregon State University, Corvallis, OR, USA

${ }^{2}$ BigML, Inc., Corvallis, OR, USA

As more complicated radiation detection systems are developed, the demand for compact, multi-channel pulse processing systems closely follows. While commercial multi-channel systems are available, they are often expensive, designed to fit into instrument crates rather than operate as a single unit, and do not allow the on-board digital pulse processor to be re-configured by the user. A low-cost, compact, single-unit digital pulse processor with eight-channels is being developed at Oregon State University that allows full control over the digital pulse processing algorithms implemented on the on-board high-performance FPGA. The channels are sampled using a 125 MSPS, 14-bit, eight-channel ADC to capture entire pulses and process them in real-time. The system was characterized for non-linearity, energy resolution, and timing resolution using both a pulse generator and an HPGe detector, and its performance in high count rate environments evaluated.

This work was funded in part by the Consortium for Verification Technology under the US Department of Energy National Nuclear Security Administration award number DE-NA0002534 and by the US Department of Homeland Security Domestic Nuclear Detection Office award number 2014-DN-077-ARI081-01.

N05-33: A Multi-Channel CCD Clock Driver ASIC for Space-Based Applications 
Q. Morrissey $^{1}$, S. Bell ${ }^{1}$, L. Jones ${ }^{1}$, N. Waltham ${ }^{2}$, M. Clapp ${ }^{2}$

${ }^{I}$ Technology, STFC Rutherford Appleton Laboratory, Didcot, Oxfordshire, United Kingdom

${ }^{2} R A L$ Space, STFC Rutherford Appleton Laboratory, Didcot, Oxfordshire, United Kingdom

A high voltage mixed signal ASIC is described that provides multiple fully programmable clock outputs capable of driving large format CCD capacitive electrodes. The COMET ASIC provides 6 independent clock buffering channels each with individually programmable rising/falling current drive and high/low voltage levels. Output voltage levels are controlled with integrated fast response regulators that operate over a $16.368 \mathrm{~V}$ range without the need for external decoupling capacitors. Clock drive currents can be adjusted for the load capacitance and voltage swing required over a $409.6 \mathrm{~mA}$ range, with edge speeds $<15 \mathrm{~ns}$ achievable for small loads. Setup and control of the ASIC is via a simple SPI interface with safety features to ensure correct sequencing of channel operation and prevent driver supply reverse biasing. The ASIC also features an under-voltage lock out circuit to safeguard the chip in the event of power loss. All necessary biases are generated internally and only supply decoupling, a single filtering capacitor, and a resistive divider are required to operate the device. The circuit is manufactured in a commercial $0.35 \mathrm{um}$ HV CMOS technology and uses established layout techniques to harden against both Total Ionising Dose (TID) and Single Event Latchup (SEL) radiation effects. The device has been manufactured and test results are shown relative to expected performance from simulation.

N05-34: Development and Evaluation of Multichannel Readout ASIC for the Development of SiPM Based PET $\underline{\text { K. Park }}^{1}$, Y. Choi ${ }^{1}$, J. Choi ${ }^{1}$, D. J. Kwak ${ }^{1}$, G.-C. Ahn ${ }^{2}$, J. Bong ${ }^{2}$, M. S. Kim ${ }^{2}$

${ }^{I}$ Molecular Imaging Research \& Education (MiRe) Laboratory, Department of Electronic Engineering, Sogang University, Seoul, Korea

${ }^{2}$ Mixed Signal Circuit Design Laboratory, Department of Electronic Engineering, Sogang University, Seoul, Korea

The purpose of this study was to develop and to evaluate the performance of the multichannel readout ASIC for the development of SiPM based PET. The multichannel readout ASIC implemented in a $0.18 \mu \mathrm{m}$ CMOS technology consisted of 4 preamplifiers, 4 peak-detect-hold stages (PDH), 4 cyclic ADCs, 2 comparators, a vernier TDC and a control interface. The output of comparator was used to detect arrival time for the sum of the pre-amplified signal and converted to time value by TDC. The PDH output was converted to energy value by ADC, which was synchronized by comparator output. An FPGA based data acquisition board was developed to evaluate the performance of the developed ASIC for the development of SiPM based PET. PET detector modules were composed of four $4 \times 4$ arrays of $3 \times 3 \mathrm{~mm}^{2}$ SiPM and four $4 \times 4$ arrays of $3 \times 3 \times 20 \mathrm{~mm}^{3}$ LYSO. 64 output signals from detector module were processed by the discretized positioning circuit (DPC) for channel reduction and position information and 4 output signals of the DPC were transferred to the developed ASIC to acquire position, energy and time information of an interacted gamma ray. The integral non-linearity and differential non-linearity of ADC was \pm 2 and \pm 1 LSB, respectively. The intrinsic resolution of TDC was $19.5 \mathrm{ps}$ and the total power consumption was $468 \mathrm{~mW}$. An acquired flood image showed that all 64 crystal pixels could be clearly resolved. The average energy resolution and the coincidence timing resolution was $18.6 \pm 2.2 \%$ (FWHM) and 724.5 ps (FWHM), respectively. The results of this study demonstrated that the developed multichannel readout ASIC could be utilized for the development of SiPM based PET. A further study will be performed to optimize internal control value of the ASIC and the performance of the multichannel readout ASIC for the development of SiPM based PET.

\author{
N05-35: An ASIC Architecture for Dead Time-Less, Multichannel Current Pulse Acquisition with Extended \\ Input Range \\ $\underline{\text { D. Mazur }}^{1}$, V. Herrero Bosch ${ }^{1}$, R. J. Aliaga ${ }^{2}$, J. M. Monzó Ferrer ${ }^{1}$, R. Gadea Gironés ${ }^{1}$, R. J. Colom Palero ${ }^{1}$ \\ ${ }^{I}$ I3M, Universitat Politècnica de València, Valencia, España \\ ${ }^{2}$ Instituto de Física Corpuscular, Paterna, Valencia, España
}

A multichannel IC architecture is presented which is able to process and digitize simultaneous current pulses in every input channel with no deadtime. The main part of the IC frontend is a continuous time integrator controlled by two comparators. When the frontend output voltage exceeds any of the comparator fixed thresholds a Charge Pulses System (CPS) is activated and brings the output voltage back inside the given range. As a consequence the frontend behaves as an asynchronous and self-regulating system. The CPS permits to increase the dynamic range of the input current by over two decades and improve at least $20 \mathrm{~dB}$ of SNR. The analog to digital conversion is performed in two steps: 6 MSBs are quantized by the CPS pulse counter and 8 LSBs are obtained from a later ADC. A total of 14 ENOB is achieved at the output for $1 \mathrm{MHz}$ of operation frequency. The IC is designed for sensors with fast pulse current responses such as SiPM. The CPS extended input range can take advantage of high gain sensors thus improving overall SNR of the detector. Energy resolution dependent applications such as PET might benefit from this novel DAQ architecture. The extracted layout simulations of the IC frontend will be presented at the conference.

N05-36: Analog Pixel Front-End for VIPIC-Large Detector

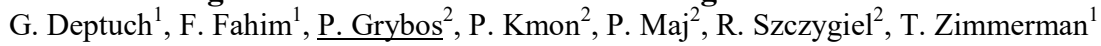


${ }^{I}$ PPD/EED, Fermi National Accelerator Laboratory, Batavia IL, USA

${ }^{2}$ Department of Measurement and Electronics, AGH University of Science and Technology, Krakow, Poland

We report on the design of the analog tier for VIPIC-L IC (Vertically Integrated Pixel Imaging Chip Large) designed for X-ray Photon Correlation Spectroscopy (XPCS) experiments. The VIPIC-L chip (with the area of $1,285 \times 1,285 \mathrm{~mm}^{2}$ ) is an array of 192 x 192 pixels with $65 \mu \mathrm{m}$ x $65 \mu \mathrm{m}$ pixel size and consists of two tiers: analog and digital. The single analog pixel cell consists of a charge sensitive amplifier (CSA) with a feedback discharge block (fedCSA), an amplifier I (AMP_I), two amplifier II (AMP_II) and two discriminators (DISCR_L and DISCR_H for setting the thresholds Low and High). Each pixel contains additional blocks, such as a simple test charge injection circuit, blocks for trimming the effective offset spread, a block for the recovery of sensitive analog references. The paper presents the detail of analog front-end pixel, including new low noise and very effective detector leakage current compensation circuit, blocks for adjustment of gain and trimming offset and the communication interface between analog and digital tier. 3D technologies open also new possibilities for distribution of power supply and biasing in large pixel matrix, which is effectively used in VIPIC-L design.

\title{
N05-37: Evaluation of the Spectroscopic Performance of the Integrated Multi-Channel Charge-Sensitive
} Preampli?er of TRACE with a Silicon Detector Prototype

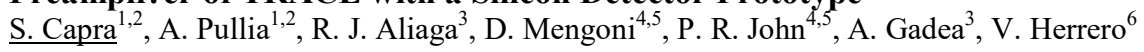

${ }^{I}$ Dpt. of Physics, University of Milano, Milan, Italy

${ }^{2}$ INFN of Milano, Milan, Italy

${ }^{3}$ Instituto de Fisica Corpuscolar, Paterna, Spain

${ }^{4}$ INFN of Padova, Padova, Italy

${ }^{5}$ Dpt. of Physics and Astronomy, University of Padova, Padova, Italy

${ }^{6}$ Universitat Politecnica de Valencia, Valencia, Spain

In this work the experimental results are presented showing the spectroscopic performance of the ASIC multi-channel chargesensitive preamplifier of TRACE (TRacking Array for light Charged particle Ejectiles). The results were obtained connecting a silicon pad detector to a custom-designed preamplifier board with eight ASIC CSPs. The detector and the board were put in a vacuum chamber with a triple $\mathrm{Am}-\mathrm{Cm}-\mathrm{Pu}$ alpha source. The output signals were digitized with four FPGA-powered $100 \mathrm{MHz}$ 14-bit resolution digitizer cards. The energy resolution obtained is around $22 \mathrm{keV}$ at $5486 \mathrm{keV}$. The results are very encouraging and pave the way for future developments.

\author{
N05-38: MuTRiG: a Mixed Signal Silicon Photomultiplier Readout ASIC with High Timing Resolution and \\ Gigabit Data Link \\ H. Chen, K. Briggl, P. Eckert, T. Harion, Y. Munwes, H.-C. Schultz-Coulon, W. Shen, V. Stankova \\ KIP, Heidelberg University, Heidelberg, Germany
}

MuTRiG is a mixed signal Application Specific Integrated Circuit (ASIC) developed in UMC 180nm CMOS technology. It is designed for the readout of Silicon Photomultiplier (SiPM) in ultra-precise timing and ultra-high event rate applications for high energy physics experiments and medical imaging. And it is dedicated to the readout of the tile detector and the fibre detector of Mu3e experiment, which is designed to search for the lepton-flavour violating decay of a positive muon into two positrons and one electron with a branching ratio sensitivity of $10^{\wedge}-16$. To reduce the combinatorial background at high rates and to facilitate event reconstruction, a good timing resolution of $100 \mathrm{ps}$ sigma and $500 \mathrm{ps}$ sigma is required for the Mu3e tile detector and the $\mathrm{Mu} 3 \mathrm{e}$ fibre detector, respectively. An event rate as high as $1.3 \mathrm{MHz} /$ channel poses another challenge for the development of MuTRiG. MuTRiG features 32 fully differential analog front end channels and 50 ps time binning TDCs, which both have been extensively characterized in STiCv3 ASIC and have been proven to provide an excellent timing resolution. The recorded event data are processed by the integrated digital circuits and transferred to the Data Acquisition system (DAQ) via a gigabit data link with $8 \mathrm{~b} / 10 \mathrm{~b}$ encoding. The gigabit data link is accomplished by a customized gigabit LVDS transmitter and a dedicated dualedge serializer. Preliminary tests show a performance of the gigabit data link which exceeds the requirements for Mu3e experiment. An external trigger functionality for event validation is also integrated, to reduce the load of data link. The design of the MuTRiG chip and the characterization results of the gigabit data link will be presented.

\section{N05-39: Design of Low Power and Low Area 12-Bit 40MSPS SAR ADCs with a Redundancy Algorithm and Digital Calibration for High Dynamic Range Calorimeter Readout \\ D. K. Dzahini, M. Zeloufi \\ Electronics, IN2P3-LPSC, France, France}

We present two versions of 12-bit 40MSPS SAR ADCs using a search algorithm so called generalized redundant. It offers the flexibility to relax the requirements on the DAC settling time. Two more bits of redundancy are included to allow a digital 
calibration based on a code density analysis to compensate the capacitors mismatching effects. A monotonic switching algorithm is used for these prototypes; hence $70 \%$ of dynamic power consumption is saved in comparison to a conventional switching algorithm. Our first prototype used a non segmented conservative scheme. The second prototype is segmented and offered a very aggressively low area feature. Both design are fully differential and was produced in a CMOS 130nm 1P8M process. The first design dissipates $11 \mathrm{~mW}$ with an area of $7 \mathrm{~mm} 2$, while the second dissipate only $6.5 \mathrm{~mW}$ for an area of only $0.35 \mathrm{~mm} 2$ and open the way for multichannel and multi-gain integrated readout circuits including high resolution converters.

N05-40: Design Considerations for Embedded Real-Time Processing for 3D Digital SiPMs with Multiple TDCs W. Lemaire, F. Nolet, A. Corbeil Therrien, J.-F. Pratte, R. Fontaine

Department of Electrical and Computer Engineering, Université de Sherbrooke, Sherbrooke, Québec, Canada

Recent developments in digital silicon photomultipliers (dSiPMs) detectors have placed them as serious candidates for replacing photomultiplier tubes (PMTs) in applications requiring high timing performance, such as positron emission tomography (PET). While offering excellent timing resolution, single photon avalanche diodes (SPADs) generate dark counts which can blur the beginning of scintillation events. A dSiPM architecture with multiple time-to-digital converters (TDCs) offers the advantage of timestamping individual photons, producing more detailed measurements of scintillation events. This opens up the possibility of applying dark count filtering. The individual detection of photons allows combining the timing information of multiple timestamps, and, with the appropriate estimator, improves the timing resolution. A dSiPM architecture with multiple TDCs offers many advantages, but if the TDCs are not perfectly uniform throughout the array, the timing resolution can be severely hindered. Acknowledging these design considerations, a real-time processing scheme is suggested to improve the timing resolution with dark count filter, multiple photon time estimation, and TDC uniformity corrections. With a $1 \times 1 \times 5 \mathrm{~mm}^{3}$ LYSO scintillator, the processing scheme improves the CTR by $45 \%$ or more when using at least 6 TDCs with the SPADs simulated.

N05-41: VIPRAM3D: a Multi-Tier 3D Architecture for Pattern Recognition-Based Track Finding J. R. Hoff, G. Deptuch, S. Joshi, T. Liu, A. Shenai

PPD/EED/ASIC, Fermilab, Batavia, IL, USA

Fermilab has recently conducted a vertically integrated wafer run for several projects. VIPRAM3D is a 3D integrated circuit constructed with two purposes in mind 1) High-speed associative memory-based track-finding pattern recognition, and 2) Basic research into multi-tiered vertical integration. Simply put, taking a known 2D design and converting it to 3D is a natural, obvious step in researching vertical integration. VIPRAM3D is a conversion of an existing 2D architecture (protoVIPRAM00) into a multi-tier stack. There are two types of tiers in the stack - CAM Tiers and Control Tiers. CAM tiers implement a 2-dimensional array of 15-bit Content Addressable Memories that search for matches from among event data from one particular detector layer. Control Tiers implement detector layer-by-detector layer pattern recognition of tracks. At least one CAM Tier must be vertically integrated below a Control Tier and up to four CAM Tiers can be accommodated by one Control Tier through additional vertical integration. A very simplified readout architecture is implemented so as to permit direct measurement of the performance characteristics (speed, power, etc.) of the CAM and Control Tiers. These direct measurements when compared to those made of protoVIPRAM00 will provide a comprehensive analysis of the effects of vertical integration.

N05-43: Pixel Back-End of the VIPIC-Large Chip for Dead-Time-Less Registration of X-Ray Photons G. W. Deptuch ${ }^{1}$, F. Fahim ${ }^{1}$, P. Grybos ${ }^{2}$, P. Kmon ${ }^{2}$, P. Maj $^{2}$, R. Szczygiel ${ }^{2}$

${ }^{I} P P D / E E D$, Fermilab, Batavia, IL, USA

${ }^{2}$ DMI, AGH-UST, Krakow, Poland

The concept of the Vertically Integrated Photon Imaging Chip (VIPIC) has been proposed to fill a niche need for a pixel detector for X-ray Photon Correlation Spectroscopy. The current realization is the successor of the first successful demonstration of a three-dimensionally integrated device, tested on the 8ID-I beam-line at the APS at ANL. The size of the new device has been increased, hence the name VIPIC "Large (L)", additionally its circuitry has been significantly enhanced. A single VIPIC-L chip has the external dimensions of $1.25 \times 1.25 \mathrm{~cm} 2$, with a matrix of $192 \times 192,65 \mu \mathrm{m}$ pixels. The planned experiments are characterized by relatively low occupancy of incoming photons, but require their dead-time-less registering with the timing resolution as good as less than $1 \mu \mathrm{s}$. Thus, the digital back-end of the VIPIC-L pixel was designed to operate with timing provided by an external clock and compatibly with a zero-supressed or, optionally, imaging mode. Furthermore, it avoids double counting of hits at the transitions of the time frames, and allows resetting of hit counters without losing new hits incoming even during the resetting. The details of the design are given.

N05-44: A GHz Waveform Recorder and Digitizer ASIC

J. Qin, L. Zhao, Y. Lu, B. Cheng, S. Liu, Q. An

University of Science and Technology of China, Hefei, 230026, China, Hefei, China 
Pulse waveforms from detectors carry the maximum possible information, and the high demands of fast waveform digitization led to the development of Switched Capacitor Arrays (SCAs). The Effective Number of Bits (ENOB) performance of current SCAs is quite limited by the sampling circuit. This paper focuses on research to improve the ENOB through circuit optimization, dual-gain structure design and new calibration method. And a GHz transient waveform digitization ASIC prototype has been designed to implement the above ideas and evaluate the performance. This SCA ASIC is fabricated in global foundry $0.18 \mu \mathrm{m}$ CMOS process, and each channel employs a SCA structure of 128 samples deep, while the high speed sampling clock is provided by an on-chip delay-locked loop (DLL). After waveform capture, the analog signal is fed into 128 parallel 12-bit rampcomparator Analog-to-Digital Convertors (ADCs), and then followed by a serializer with $200 \mathrm{MHz}$ rate. Based on the simulation results, input analog bandwidth is more than $300 \mathrm{MHz}$ and sampling speed can be adjusted from 0.5 to $2 \mathrm{GSa} / \mathrm{s}$. With the new amplitude and time correction, a full $1 \mathrm{~V}$ signal voltage range is available, and the ENOB reaches 9 bit at $200 \mathrm{MHz}$ input; combined with the dual-gain structure, an equivalent measurement accuracy can be further enhanced. The chip is in the process of fabrication and tests will be further conducted after fabrication of the ASIC is completed.

\title{
N05-45: The Front End Electronics of T0 Detector in the External Target Experiment of CSR in HIRFL P. Deng, L. Zhao, P. Xia, J. Lu, S. Liu, Q. An \\ University of Science and Technology of China, Hefei, China
}

Fully fledged front end electronics (FEE) modules are designed for the T0 detector in the External Experiment in CSR (Cooling Storage Ring) of HIRFL (Heavy Ion Research Facility in Lanzhou). Based on the NINO ASIC, two different front-end modules were designed for the internal and external MRPC of the T0 detector, which integrates 16 and 24 channels, respectively. Both the internal module and the external module can achieve high precision leading edge discrimination and Charge-to-Time Conversion, and can fit the mechanical structure for the detector. The output pulse of the front-end modules were digitized through digitization modules with FPGA-based TDC integrated inside. To evaluate the functionality and performance, we also conducted a series of tests in the laboratory. Test results indicate that these FEE modules function well, and the time precision of the frontend electronics is better than $30 \mathrm{ps}$, which satisfies the application requirement.

\author{
N05-46: Implementation of Broadband Mismatch Correction in a 1.6-Gsps TIADC System

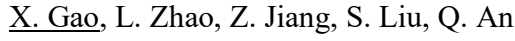 \\ University of Science and Technology of China, Hefei, China
}

Analog-to-Digital Converter (ADC) is widely used in both scientific experiment measurements and daily life such as communication. Unfortunately, high-resolution and high-speed are often incompatible in the current manufacturing technology. Although, Time-Interleaved ADC (TIADC) is a research hot spot, which can achieve much higher sampling rate beyond the speed limitation of single ADC chips. The offset, gain, and skew mismatches among different ADC channels would severally degrade the system performance, which can usually be described using ENOB. Efforts were devoted to the mismatch error correction algorithms. The perfect reconstruction algorithm is an easily implemented method to eliminate the effect of mismatches. However, the current methods mainly focus on correction with narrow bandwidth input signal, and real-time implementation of the algorithms is great challenge. This paper presents a broadband mismatch error correction method, which is implemented as real-time correction logic in FPGA devices. And we also tested and evaluated the correction performance with a 1.6 Gsps TIADC system. Test results indicate that this correction method fits a broadband range from 5 to $600 \mathrm{MHz}$, and the ENOB is enhanced to be better than 10 bit $(5 \sim 300 \mathrm{MHz})$ and 8 bit $(300 \sim 600 \mathrm{MHz})$ after correction, which is obvious enhancement compared with the traditional narrow band correction.

\author{
N05-47: Measurement of the Power Spectral Density of Noise Produced by a Large Integrated Feedback \\ Resistor for Charge-Sensitive Preamplifiers \\ $\underline{\text { S. Capra }}^{1,2}$, A. Pullia ${ }^{1,2}$ \\ ${ }^{I}$ Dpt. of Physics, University of Milan, Milan, Italy \\ ${ }^{2}$ INFN of Milan, Milan, Italy
}

Charge-sensitive preamplifiers (CSP) require high-valued feedback resistors as continuous-time reset devices: higher resistance values correspond to lower current noise and better spectroscopic performances. Designing integrated multi-channel CSP such resistors are generally left as external components or substituted with active transconductors. The former are bulky and not adequate for situations where a high degree of integration is required, the latter generally suffer from linearity and noise problems. A possible solution could be the use of large integrated polysilicon resistors. These ones, however, suffer from a very high distributed capacitive coupling to bulk, which tends to turn such devices into transmission lines. Simple resistor models are no longer adequate to describe both the impedance and the noise generators of such integrated resistors. We developed a closedform model which describes the current noise produced by a resistance with distributed capacitance. We realized a $100 \mathrm{Mohm}$ integrated polysilicon resistor and measured the power spectral density of noise produced by this device connecting it as a feedback resistor to a low-noise charge-sensitive preamplifier. 
N05-48: Evaluation of the Prototype Front End Electronics of WCDA in LHAASO

$\underline{\mathrm{S} . \mathrm{Chu}}$

University of Science and Technology of China, Hefei, China

Abstract: The Water Cherenkov Detector Array (WCDA) is one of the key parts of the Large High Altitude Air Shower Observatory (LHAASO). In WCDA, the high precision time and charge measurement are required for 3600 Photomultiplier Tubes (PMTs) scattered over a $90000 \mathrm{~m} 2$ area. With a distributed architecture, the 400 Front End Electronics (FEEs) placed near the PMTs are responsible for the time and charge measurement over a large dynamic range. The time measurement resolution is required to be better than $0.5 \mathrm{~ns}$ RMS and the required resolution for the charge measurement is better than 30\%@1 Photo Electron (P.E.) and 3\% @ 4000 P.E. Considering the large scale of the detector area, high precision clock distribution and automatic phase compensation over long distances is required. To simplify the electronics structure, clock, data and commands are transmitted together over the same optical fiber media. To evaluate the prototype FEE performance, we conducted a series of tests, including performance tests both with constant and changing ambient temperature. Test results indicate that time and charge measurement resolution and clock synchronization satisfy the application requirement.

N06: Data acquisition, trigger and analysis - Poster session I

Monday, Oct. $31 \quad$ 14:00-16:00 Etoile

N06-1: High-Speed Recorder Based on SCA Technology for Thomson Scattering Diagnostic on ITER E. A. Puryga $^{1}$, S. V. Ivanenko ${ }^{1,2}$, A. D. Khilchenko ${ }^{1,2}$, A. N. Kvashnin ${ }^{1}$, P. V. Zubarev ${ }^{1,2}$, D. V. Moiseev ${ }^{1}$

${ }^{1} 630090$, Budker Institute of Nuclear Physics, Novosibirsk, Russia

${ }^{2}$ 630092, Novosibirsk State Technical University, Novosibirsk, Russia

The Thomson scattering diagnostic are used for plasma parameter (density, temperature) measurements. For Thomson scattering diagnostic it is necessary to record the signals with short duration $(\sim \mathrm{ns})$ in a wide amplitude range of signal changes with high level of background. Using of switch capacitor array (SCA) technology for recording such signals is more attractive as compared to other methods, since it provides higher performance (up to several tens of GHz), amplitude acceptable dynamic range (10-14 bits) and high functional capacity (2-10 channels analog-digital conversion). This paper describes the prototype of the high-speed recorder based on SCA technology for Thomson scattering diagnostic developed by Budker Institute of Nuclear Physic (Russia, Novosibirsk) for ITER (Cadarache, France). Developed recorder is based on 8-channel crystal DRS4, created in PSI (Paul Scherrer Institut) for spectroscopic applications and FPGA with System of Cryslal (Cyclone V). Recorder allows recording signals of 8 channels with sampling rate up to $5 \mathrm{GHz}$ with a dynamic range of 10-12 bits.

N06-2: Hardware Tracking R\&D for the ATLAS at the High Luminosity LHC TDAQ System A. Tavares Delgado

Laboratorio de Instrumentacao e Fisica Experimental de Particulas, LIP, Lisboa, Portugal

On behalf of the ATLAS Collaboration

The unprecedented level of pile-up expected at the High Luminosity LHC, at $7.5 \mathrm{e} 34 / \mathrm{cm}^{\wedge} 2 / \mathrm{s}$, makes the role of tracking in the ATLAS Trigger system pivotal for exploiting the full physics potential of the delivered luminosity. ATLAS has been carrying out $\mathrm{R} \& \mathrm{D}$ to develop a hardware tracking system that will perform fast regional tracking at $1 \mathrm{MHz}$, with the pattern recognition based on custom-designed associative memory ASICS, contributing to a fast rejection of uninteresting events. We will present the performance of the pattern recognition at Hight Luminosity LHC pile-up conditions, and give estimates of the latency for the tracker regional readout and of the off-detector system.

N06-5: Trapezoidal Shaping Algorithm Based on Digital Penalized LMS Method Y. Huang ${ }^{1,2}$, H. Gong ${ }^{1,2}$, J. Li $^{1,2}$

${ }^{I}$ Key Laboratory of Particle \& Radiation Imaging, Tsinghua University, Beijing, China

${ }^{2}$ Dept. of Engineering Physics, Tsinghua University, Beijing, China

Trapezoidal shaping algorithm is widely used in the measurement of nuclear signal and spectroscopy, which contributes to reduce pile-up effect and achieve good resolution. Traditional trapezoidal filter is an IIR (Infinite Impulse Response) one, and strongly depends on the shape of the input pulse. In order to take into account the environmental noise and get high signal-to-noise ratio for dedicated systems, the Digital Penalized Least Mean Squares (DPLMS) method is employed to calculate optimum digital FIR filters. Implemented in FPGA and applied on X-ray diffraction system with the silicon drift detector, the DPLMS trapezoidal shaping algorithm acquired good resolution results.

N06-6: An IMPI-Compliant Control System for the ATLAS TileCal Phase II Upgrade PreProcessor Module 
$\underline{\text { A. White }}$

Physics, University of Texas at Arlington, Arlington, TX, USA

On behalf of the ATLAS Tile Calorimeter System

As a part of the Large Hadron Collider (LHC) upgrade program towards the High Luminosity LHC (HL-LHC), major changes are being introduced in the majority of the electronic subsystems of the ATLAS experiment. Amongst the many changes being incorporated, the on- and off-detector electronics of the TileCal will undergo a complete redesign process. It is expected that by the end of the ATLAS Phase II upgrade program, the detector and data acquisition system will be accommodated for the HLLHC requirements. The data generated by the beam collision activity will be digitized by the on-detector electronics and delivered to the PreProcessor (PPR) located in off-detector counting rooms. The total bandwidth of the readout system will, roughly, reach 80 Tbps. The PPR delivers pre-processed digital trigger information to Level 0/1 systems, therefore acting as a link between the on-detector electronics and the overall data acquisition system of ATLAS. The PPR also links the on-detector electronics with the so called Detector Control System (DCS), whose functionality is to monitor the high voltage distribution system.

With the purpose of efficiently monitor the PPR, a control system following the Intelligent Platform Management Interface (IPMI) specifications is implemented. The IPMI functionalities allows out-of-band access to the PPR in scenarios where the PPR firmware has not yet booted, or when the PPR's FPGAs are powered down or even in case of PPR failure. In this paper, the IPMI Module Management Controller (MMC) hardware and firmware incorporated into the PPR board are being described, together with the software and Graphical User Interface (GUI) developed to access, via the different IPMI-compliant boards, and visualize in real-time the state of the sensors and relevant control information regarding the PPR state and functions. Results about the performance of this setup are also shown and evaluated.

N06-7: Fast DAQ system for recording profiles of Thomson scattering ns pulses

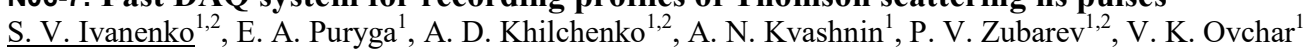

${ }^{I}$ Institute of Nuclear Physics, Novosibirsk, Russia

${ }^{2}$ Novosibirsk State Technical University, Novosibirsk, Russia

Thomson scattering is the most accurate and useful method of measuring plasma electron temperature and density in fusionoriented experiments. The small power $\left(\sim 10^{\wedge}-8 \mathrm{~W}\right)$ and short duration $(\sim 10 \mathrm{~ns}$ and less $)$ of scattering signal are the main technical problems of the method. That is why highest requirements are made to photodetection and recording systems. Developed by Budker Institute of Nuclear Physics (Novosibirsk, Russia) fast DAQ system allows recording profiles of short duration (3-5 ns) scattered pulses in $0-200 \mathrm{MHz}$ bandwidth with 2GSPS rate and 10-bit resolution. The system consists of photo detector modules, fast (2GSPS) and slow (10MSPS) ADC modules and synchronization subsystem. The 48-channel version of this system was developed as a prototype for ITER (Cadarache, France) divertor Thomson scattering diagnostic. The report includes the experimental results of using DAQ system for Thomson scattering diagnostic in GLOBUS tokamak (St. Petersburg, Russia).

N06-8: Development of a Fast Framing Detector for Electron Microscopy $\underline{\text { I. Johnson }}^{1}$, K. Bustillo ${ }^{2}$, J. Ciston ${ }^{2}$, E. Dart ${ }^{3}$, B. Draney ${ }^{4}$, P. Ercius ${ }^{2}$, E. Fong ${ }^{1}$, C. Grace ${ }^{1}$, J. Joseph ${ }^{1}$, J. Lee ${ }^{4}$, A. Minor ${ }^{2}$,

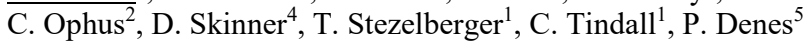

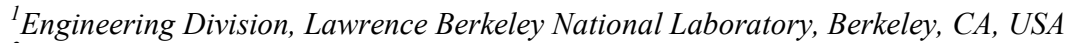

${ }^{2}$ National Center for Electron Microscopy, The Molecular Foundry, Lawrence Berkeley National Laboratory, Berkeley, CA, USA

${ }^{3}$ Energy Sciences Network, Lawrence Berkeley National Laboratory, Berkeley, CA, USA

${ }^{4}$ National Energy Research Scientific Computing Center, Berkeley, CA, USA

${ }^{5}$ Physical Sciences, Lawrence Berkeley National Laboratory, Berkeley, CA, USA

A high frame rate detector system is being developed to enable new scanning diffraction experiments in transmission electron microscopy. This detector will consist of a central monolithic active-pixel sensor that is surrounded by annular silicon diodes. Both components of the detector system will synchronously capture data at a $100 \mathrm{kHz}$ frame rate. The inner pixel detector will contain a 576 x 576 array of 10 um pixels. With 12 bits of amplitude information per pixel, this becomes approximately 400 Gbps of data. A team of scientists and engineers from National Center for Electron Microscopy (NCEM), National Energy Research Scientific Computing Center (NERSC) and the Engineering Division of Lawrence Berkeley National Laboratory have joined together to transport and analyze this data in real time. Data from the detector will be sent over 4 dedicated $100 \mathrm{GbE}$ links to the memory of the Cori supercomputer at NERSC that will perform the data analysis. First experiments will focus on convergent beam electron diffraction (CBED) data from the central pixel detector and the high-angle annular dark field (HAADF) signal from the surrounding diodes. The former method provides details about structure, composition, polarization, and three-dimensional defect crystallography; while the latter assesses the mass-thickness contrast of the sample. Rapidly recording and analyzing scanning diffraction patterns will make new research opportunities in electron microscopy possible.

\section{N06-9: Single-Chain 4-Channels High-Resolution Multi-Hit TDC in FPGA}


We present a TDC architecture designed for being implemented in a FPGA device with the highest regard to the trade-off among resource saving, resolution and input rate. In particular, 4 channels with a single physical measurement line guarantee resolution below 32 ps r.m.s. per channel at the input rate of events equal to $2 \mathrm{GHz}$. The system is composed of a single tapped-delay-line of length equal to the clock period where the 4 input channels merge, a register of the occurring events, and just one coarse counter.

\section{N06-10: The Real-Time Processor for the CMS Fast Beams Condition Monitor Implemented in FPGA}

\section{A. A. Zagozdzinska}

WUT (Warsaw University of Technology), Warsaw, Poland

On behalf of the CMS Collaboration

The Fast Beams Condition Monitor (BCM1F), upgraded for LHC Run II, is used to measure the online luminosity and machine induced background for the CMS experiment. The detector consists of sCVD diamond sensors mounted radially around the beam pipe that are read out with a custom fast front-end. The analogue signals are transmitted through the optical path to the dedicated back-end electronics. Since the signals from the sensors are used for real time monitoring of the LHC conditions they are processed to measure separately rates corresponding to LHC collision products, machine induced background and residual activation exploiting different arrival times. The back-end electronics system is built in MicroTCA technology. Signals are digitized using high speed analogue-to-digital converters synchronized to the LHC clock. The data acquisition module and online processor are implemented in a FPGA. The properties of the signals are measured and presented in the amplitude spectra to monitor the gain and in the occupancy histogram to reflect the beam structure of the LHC. Additional processing level is implemented in order to recognize the overlapping signals originating from pile-up. The high accuracy qualification of the hits is crucial to determine the luminosity and the machine induced background rates for the CMS experiment and the LHC.

N06-11: Modelling of DEPFET Based X-Ray Detectors for Athena's Wide Field Imager

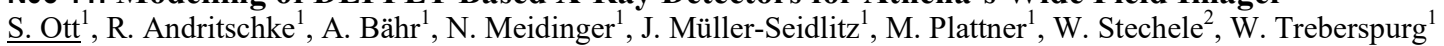

${ }^{I}$ Max-Planck-Institut für extraterrestrische Physik, Garching, Germany

${ }^{2}$ Technische Universität München, München, Germany

The X-ray observatory Athena is an ESA L-class mission to be launched in 2028. One of the two focal plane instruments is the Wide Field Imager. The WFI is an X ray camera for imaging and spectroscopic applications. Its detector consists of active pixel DEPFET sensors with front-end electronics. In this paper, mathematical models of the DEPFET detector are presented for use in an emulator system. This emulator system will be used for an end to end evaluation, to verify and characterize the detector, the detector electronics and further processing steps. The developed emulator models describe physical effects and the behavior of the DEPFET detector. The adjustable parameters of these models comprise detector architecture, characteristics and environmental influences. Furthermore models for generating an input data stack of X-ray photons with a particular energy, spatial distribution and hit time are explained. First results of an observed Fe 55 source show a good agreement of the simulated and measured spectra. Iterative adjustment of simulation models based on measurements of prototype DEPFET devices will lead to a continuous improvement of the emulator models. This manuscript will give a detailed summary of the emulator models of the WFI DEPFET detector. The key advantages of the WFI end to end evaluation with the detector emulator in view of the longterm development time for space applications are outlined.

\section{N06-12: Fully-Migratable TDC Architecture for FPGA Devices}

N. Lusardi, A. Palmucci, A. Geraci

DEIB, Politecnico di Milano, Milan, Italy

The demand for measuring time intervals by means of flexible, low-cost and programmable instruments is constantly growing. Always more frequently multi-channel and high-resolution features come up beside. In this sense, time-to-digital converters (TDCs) are very suited to be implemented into FPGA devices. Moreover a significant surplus value would be the availability of a TDC architecture that can be implemented in different FPGA devices with practically no re-design overhead. In this paper we present a " TDC component" that can be indifferently implemented in Xilinx and Altera FPGAs with minimum intervention on the design. We have experimentally verified the possibility of implementing the component in devices also very different in terms of structure and availability of resources.

N06-13: 99.26\% Gigabit Ethernet Link Efficiency for Distributed Data Acquisition Systems F. T. Abu-Nimeh, W.-S. Choong

Lawrence Berkeley National Laboratory, Berkeley, CA, USA 
Link efficiency, data integrity, and continuity for high-throughput and real-time systems is crucial. Most of these applications require specialized hardware and operating systems as well as extensive tuning in order to achieve high-efficiency. Here, we present an implementation of gigabit Ethernet data streaming which can achieve $99.26 \%$ link efficiency while maintaining no packet losses. A proof of concept is built for OpenPET, a data acquisition platform for nuclear medical imaging, where (a) a crate hosting multiple OpenPET detector boards uses a User Datagram Protocol over Internet Protocol (UDP/IP) Ethernet soft-core, that is capable of understanding PAUSE frames, to stream data out to computer, (b) the receiving computer uses Netmap API to allow the, user space, processing software (Python) to directly receive and manage the network card's ring buffers bypassing the operating system kernel's networking stack, and (c) a multi-threaded synchronous queue is implemented in the processing software (Python) to free up the ring buffers as quickly as possible while preserving data integrity and flow continuity.

The OpenPET platform is a development of the Lawrence Berkeley National Laboratory. Initial funding support was received by the U.S. Department of Energy under Contract No. DE-AC02-05CH1231. Subsequent work is supported by the National Institutes of Health under grant R01 EB016104.

\author{
N06-14: Development of Flexible, Scalable, Low Cost Readout for Beam Tests of High Granularity \\ Calorimeter for the CMS Endcap \\ $\underline{\text { P. Rubinov }}$ \\ EED/PPD, Fermi National Accelerator Laboratory, Batavia, IL, USA \\ On behalf of the CMS
}

Development of Flexible, Scalable, Low Cost Readout for Beam Tests of High Granularity Calorimeter for the CMS Endcap As part of the development of the High Granularity Calorimeter for the CMS Endcap at HL-LHC, a comprehensive series of beam test to be carried out at Fermilab and CERN have been planned. Here we describe the development of a low cost readout system that is simple to implement and is able to grow with the system under test. The first beam tests, with a single HGC module were carried out in March of 2016 at the Fermilab Test Beam Facility, continuing to a 28 module test (to be) carried out at FTBF in May of 2016 and growing to over one hundred modules in the Fall of 2016. The system is based on the low cost Zynq SoC, and allows simple DAQ development in a Linux environment. In our case we used the Digilent ZedBoard, allowing high speed LVDS links and Linux software development on a single commercial board. A small custom FPGA board designed to comply with the VITA 57 Field Programmable Mezzanine Card standard implements the interface to the SKIROC ASIC mounted on the HGC sensor modules and provides the LVDS links to the ZedBoard, either directly over the FMC connector or via the Downstream Dual Channel (DDC) carrier card. This architecture provides scalability, ease of development and low cost. We will share our experience with the system, both positive and negative.

N06-15: DQM4HEP : a Generic Data Quality Monitoring for High Energy Physics $\underline{\text { R. Eté }}^{1}$, A. Pingault ${ }^{2}$, L. Mirabito ${ }^{1}$

${ }^{I}$ Physics, CNRS/IN2P3, Villeurbanne, France

${ }^{2}$ Physics and Astronomy, Ghent University, Ghent, Belgium

With increasingly sophisticated experiment, online Data Quality Monitoring (DQM) is of a significant importance for the detector and operation efficiency. Monitoring data is also a first step to the certification of the recorded data for off-line physics analysis. Usually, monitoring systems are developed and built on top of the Event Data Model (EDM) and leads to a strong dependency to the data format and storage.

With this in mind, a generic online data quality monitoring system has been developed without any assumption on the event data model and data type to treat. In addition, a dedicated implementation was developed based on the LCIO Event Data Model for the Linear Collider Collaboration. This implementation will be put to real condition testing using a combined detector setup with the CALICE Semi-Digital Hadronic CALorimeter (SDHCAL) and Silicon Tungsten Electronic Calorimeter (SiWEcal) prototypes during coming test beam campaigns at CERN.

After introducing the key points of the framework, the software architecture is discussed with core technical aspects. Details on the dedicated implementation for the Linear Collider Collaboration are presented. Finally, foreseen tests on the CALICE SDHCAL and SiWEcal combined detector setup using this implementation are described.

N06-16: MADA Board: a 32 Channel, Open-Firmware, ASIC Readout System with Integrated MCA A. Abba, F. Caponio, A. Cusimano

Nuclear Instruments, Lambrugo, Italy

Complex high-energy physics measurement setups or medical imaging systems are based on thousands of detectors. Usually these systems are composed by complex analog front-ends (ASICs) that need to be read out by various multichannel readout boards. We developed a programmable fast 32 channels digitizer (called MADA Board) operating at 80 Msps with 12 bits resolution with integrated MCA. The system is supported via an open-firmware architecture where the user is able to generate its 
data acquisition and processing chain using a graphical tool. 96 digital I/Os could be programmed by the user as fast digital signals (such as trigger, gate, baseline hold, reset, ...) or the programming of the ASICs via I2C, SPI.

\author{
N06-17: Sub-Ns Detection System Emulator with Integrated Digital Pulse Processor \\ A. Abba, F. Caponio, A. Cusimano \\ Nuclear Instruments, Lambrugo, Italy
}

The Digital Detector Emulator is a device able to generate analog signals similar to the ones generated by charge preamplifier in real-time with a rise-time under $1 \mathrm{~ns}$. The Detector Emulator integrates an ADC with a programmable front-end electronics that is used to sample an external analog signal. This could be used for real-time signal mixing or as an MCA to initialize the emulation process. Moreover, it is possible to acquire the real-time signals and re-shape them, change the rate, remove or increase the noise contribution, providing the real-time processed signal to the output analog channel.

\title{
N06-18: DANTE, a Compact and Low-Power Digital Pulse Processor to Exploit CUBE Preamplifier Ultimate Energy Resolution and High-Count Rate Capability
}

L. Bombelli, M. Manotti, R. Alberti, T. Frizzi

XGLab S.R.L. - Spinoff del Politecnico di Milano, Milan, Italy

An increasing number of X-ray spectroscopy applications (e.g. using synchrotron facilities or adopting high-power X-ray tube generator) require detection systems operating with low energy resolution and high counting rate capability. In order to meet these requirements, Silicon Drift Detectors (SDDs) are widely used both for scientific and industrial applications. New preamplifier technologies have been designed in order to better exploit SDD characteristics, providing excellent energy resolution at short processing times. Nevertheless, in order to fully benefit from such advancements, the read-out electronics has to provide short processing time adding a negligible contribution to the overall measurement noise. A new digital pulse processor (DPP), named DANTE, has been developed taking these considerations into account. DANTE can be used coupled to X and Gamma-ray detectors equipped with CUBE, the CMOS preamplifier developed by XGLab, or with different pulsed-reset preamplifiers. DANTE is implemented on a single printed circuit board (10 cm x $6 \mathrm{~cm}$ in size), it is low-power (2.5W) and it is scalable to multi-channel (by daisy-chain) configuration. Thanks to a 32ns minimum peaking-time and to an accurate low-noise design, DANTE provides excellent spectroscopic performance even at very high count-rates with a throughput exceeding $1.4 \mathrm{Mcps}$ output count rate. The remarkably good energy resolution of $123.8 \mathrm{eV}$ has been achieved with a close-to-optimum peaking-time of 1.6 us. Moreover, excellent energy resolutions of $138 \mathrm{eV}$ and $159 \mathrm{eV}$ FWHM have been achieved with peaking times of 100ns and $32 \mathrm{~ns}$ respectively. The combination of ultra-short peaking-time and effective pile-up rejection system enables to efficiently detect pile-up and to maximize the throughput even when the input count-rate exceeds the 4Mcps. The performances of the DPP in such critical measurement conditions, will be presented with experiments performed at different synchrotron beamlines.

N06-19: Integration of GBTx Emulator with XYTER and Data Processing Board (DPB) for CBM Experiment S. Mandal ${ }^{1}$, S. Sau ${ }^{2}$, J. Saini ${ }^{1}$, A. Byszuk ${ }^{3}$, W. F. J. Mueller ${ }^{4}$, A. Chakrabarti ${ }^{2}$, W. Zabolotny ${ }^{3}$, S. Chattopadhyay ${ }^{1}$

${ }^{1}$ High Energy Experimental Physics, Variable Energy Cyclotron Center, Kolkata, India

${ }^{2}$ A.K.Choudhury school of IT, University of Calcutta, Kolkata, India

${ }^{3}$ Advance Electronics, Warsaw University of Technology, Wrasaw, Poland

${ }^{4}$ GSI, Frankfurt, Germany

The Compressed Baryonic Matter (CBM) experiment is a part of the Facility for Antiproton and Ion Research (FAIR) at Darmstadt, Germany. The challenge in CBM experiment is to measure all the particles generated in nuclear collisions with unprecedented precision and statistics. To capture the data from each collision a highly time synchronized fault tolerant selftriggered electronics is required for Data Acquisition (DAQ) system which can support high data rate (up to several TB/s). Basic readout chain for CBM consists of a frontend Application Specific Integrated Circuit (ASIC) also known as XYTER board, a radiation hardend high speed optical transceiver board also known as GBTx ASIC followed by a Data Processing Board (DPB) and First Level Event Selector Interface Board (FLIB). As the first step towards the development of the readout chain, FPGA prototype of GBTx ASIC and XYTER ASIC also known as GBTx emulator and XYTER emulator are used. GBTx will be connected to the XYTER through Low Voltage Differential Signalling (LVDS) electrical line also known as E-link and in the backend connected to DPB at 4.8 Gbps optical line. XYTER will send the data to GBTx at 320 Mbps in uplink direction and GBTx will send data and control signal to XYTER in down link direction at $160 \mathrm{Mbps}$. In this work, FPGA based readout chain upto DPB is developed as a testing prototype where control and configuration signal of XYTER will be sent from DPB through GBTx emulator. A python script is used to generate the control information and transferred to DPB via Ethernet using IPBus protocol.

\section{N06-20: X-Ray Digital Radiography of Operating Aero-Engines with a Universal Trigger Module}




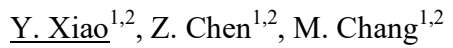

${ }^{I}$ Department of Engineering Physics, Tsinghua University, Beijing, China

${ }^{2}$ Key Laboratory of Particle \& Radiation Imaging (Tsinghua University), Ministry of Education, Beijing, China

We present a X-ray digital radiography system that can be used for obtaining exact internal structure changes from radiographic images of the operating aero-engine on test beds, which use a flexible UTM(Universal Tirgger Module) to control the image data acquisition process on the specified rotary position of the operating aero-engine. The radiography system with a UTM can improve the radiographic image quality and measurement accuracy by trigger the linear accelerator x-ray source expose exactly at the same rotary position during the image frame period. In this work, the X-ray digital radiography imaging system of the operating aero-engine is described together with the image data acquisition methods. The design of Universal Tirgger Module is also discussed and experimental results is presented.

\section{N06-21: Dual Threshold Time-over-Threshold Nonlinearity Correction for PET Imaging}

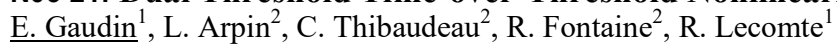

${ }^{I}$ Department of Nuclear Medicine and Radiobiology, Université de Sherbrooke, Sherbrooke, Canada

${ }^{2}$ Department of Electrical and Computer Engineering, Université de Sherbrooke, Sherbrooke, Canada

Submilliter spatial resolution in PET imaging can be achieved using highly pixelated detectors with individual readout. To process the signals from such dense detector arrays, advanced integration of the front-end electronics is required. The Time-overThreshold (ToT) analog-to-digital signal processing approach provides a power-efficient, cost-effective technique to extract all relevant information for PET. However, the technique is limited by its inherent signal nonlinearity. The aim of this study is to demonstrate the feasibility of simple non-linearity correction for dual-threshold ToT-based detectors that can be used for daily quality control in a PET scanner.

LabPET II detector arrays and electronics were used in this study. The signals from each APD pixel in the LabPET II detectors are individually processed using parallel dual-threshold ToT channels implemented in a mixed-signal ASIC. Three calibration approaches were investigated: (1) using ?-ray sources of various energies (300, 511, 662 and $909 \mathrm{keV}$ ); (2) varying the ASIC internal electronic gain in combination with a single energy ?-ray source; and (3) varying the ASIC internal gain, but replacing the ?-ray source with an internal pulser.

The electronic gain calibration technique (2) was shown to achieve equivalent accuracy as the ?-ray sources calibration (1). Furthermore, this approach has the advantage of allowing a faster calibration requiring only one single gamma-ray source (e.g., $511 \mathrm{keV}$ ) and a quick automated routine to switch the internal gain. The internal pulser technique would be even simpler, but requires more complex correction procedures due to a different pulse shape.

\section{N06-22: Bootstrapped Uncertainties in Coded-Aperture Images}

M. C. Fleenor ${ }^{1}$, K. P. Ziock ${ }^{2}$, M. A. Blackston ${ }^{2}$

${ }^{I}$ Physics Group, Roanoke College, Salem, VA, USA

${ }^{2}$ Nuclear Security \& Isotope Technology Division, Oak Ridge National Laboratory, Oak Ridge, TN, USA

Images generated with coded-aperture cameras utilize the same detector data to determine the image value in every pixel of the reconstructed image. This raises the specter of coupled uncertainties, which makes it difficult when fitting the shape of an extended object in the image. To quantify uncertainty properly, many observations are required to map the uncertainty distribution, which is usually unfeasible and impractical. In this paper, the feasibility of bootstrap sampling was explored as an alternative means to significantly expand the number of trials without requiring an increase of list-mode data. Several questions were explored as to the validity of bootstrap sampling with simulated data, by comparing uncertainties obtained with the bootstrapping method and those obtained through independent measurements. The goal was to determine the bootstrapped distribution for a particular measurement variable, and then to evaluate if that distribution was a valid representation of the uncertainty for a shorter measurement time of the same source configuration. By utilizing simulated, coded-aperture observations of radioactive disks, fit comparisons were made between bootstrap and independent realizations for a variety of input conditions. While the mean fit parameter values like radius maintained an offset between one another, the independent and bootstrap uncertainties seemed to contain no systematic shifts, indicating they were valid error estimates. In summary, our results indicate that bootstrap sampling is a valid technique to obtain uncertainty estimates for extended objects that are imaged with gammarays.

N06-23: Fast Algorithms for Multi-Level Threshold Dispersion and Gain Corrections P. Maj, P. Kmon, A. Koziol, A. Lisicka

Measurement and Electronics, AGH University of Science and Technology, Krakow, Poland 
We report on the new algorithms for fast threshold dispersion correction and gain correction for single photon counting hybrid pixel detector. Placing the detector in new measuring environment cause different operation conditions including different temperature, humidity or power supply voltage. All those conditions may influence the quality of the measuring device, namely the uniformity among pixels, which in almost all devices available on market today, is realized with additional trimming circuits inside each pixel. To overcome the problem of good matching between pixels not only the threshold dispersion is trimmed, but also the gain of the input stage can be in-pixel unified for better performance of the detector. We present detailed description of correction algorithm of threshold dispersion and gain. As the example the UFXC32k IC, hybrid pixel detector readout integrated circuit fabricated in $130 \mathrm{~nm}$ CMOS technology is choosen. This work presents considerations on different trimming approaches taking into account the possibility of in-pixel offset and range change. Optimization of the trimming for the noise separation is also taken into account as well as temperature dependence of offset spread and gain measured in wide range (-25 deg $\mathrm{C}$ to $60 \mathrm{deg}$ C).

This work was supported by the National Center for Research and Development, Poland PBS1/A3/12/2012 in the years 2012-2015.

N06-24: High-Resolution TDL-TDC System for MTCA.4 Standard

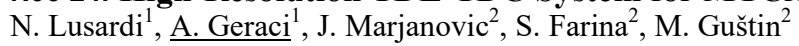

${ }^{I}$ DEIB, Politecnico di Milano University, Milan, Italy

${ }^{2}$ CAEN ELS d.o.o., Sežana, Slovenia

In this contribution, we present a high-resolution, multi-channel time measuring system (TDC) on an AMC board for MTCA.4 standard. The system allows to measure the inputs with a resolution of less than $25 \mathrm{ps} \mathrm{r.m.s.} \mathrm{The} \mathrm{TDC} \mathrm{implemented} \mathrm{into} \mathrm{the}$ FPGA (Xilinx Virtex5 70T in 67-nm technology, 10000 slices) receives signals from an analog front-end connected via HighPin-Count (HPC) FMC interface and composed of 32 comparators whose thresholds are user-programmable via software. The measurement is totally performed and decoded into the FPGA device. The configuration of the TDC and the communication of the measurements are performed via PCI-Express bus.

N06-26: Single Photon Counting Through Multi-Channel TDC in Programmable Logic

N. Lusardi ${ }^{1}$, F. Garzetti ${ }^{1}$, G. Bulgarini ${ }^{2}$, R. B. M. Gourgues ${ }^{2}$, J. W. N. Los ${ }^{2}$, A. Geraci ${ }^{1}$

${ }^{I}$ DEIB, Politecnico di Milano, Milan, Italy

${ }^{2}$ Single Quantum B.V., Delft, The Netherlands

We present a system for reading out signals from an array of 8 superconducting single photon detectors, which implements a high-performance architecture of multi-channel TDC in programmable logic with resolution guaranteed below 22 ps referred to each single channel. Each one of the 8 channels of the system consists of a detector, an analog conditioning stage, a comparator, and one channel of the TDC. Moreover, the TDC is equipped with an edge detector able to sense the position of the transition propagating along the delay line in the TDC layout within eight clock cycle, i.e. $20 \mathrm{~ns}$. The host device is the programmable logic part of a Xilinx ZYNQ-7020 SoC device, even if also different FPGA devices could be used just with a simple migration at no cost. The system is interfaced outside by means of a USB 3.0 communication gate.

N06-27: Towards a Low-Resources 10 Ps FPGA Time-Marker for (S)PE(C)T Applications

N. Chevillon, B. Humbert, C. Fuchs, F. Boisson, D. Brasse

IPHC / CNRS-IN2P3, Universite de Strasbourg, Strasbourg, FRANCE

To date, several methods dedicated to Positon Emission Tomography developments have been proposed to assess coincidence resolutions, Time-of-Flight measurements or Time-over-Threshold energy measurements. These methods often involve FPGAs, which are usually dedicated to digital applications. However, previous works have demonstrated the use of solely FPGA in asynchronous and analogue domains of Time-to-Digital Converters (TDC). Three different methods have been reported: the tapped delay lines, the Vernier delay lines and the multi-phase methods. In the present study, the time measurement tapped delay lines method has been implemented. We used carry chains as tapped delay lines associated to a $800 \mathrm{MHz}$ clock. We characterized the time behavior of the carry chain and developed a new non-statistical approach to reduce its inherent differential non linearity. We would like to emphasize the original aspect of our FPGA-independent approach. We have already designed one time-marker channel with a LSB of $39.1 \mathrm{ps} \pm 0.6 \mathrm{LSB}$. The low-resources property of our design allows us to easily consider 64 channels per FPGA. We are currently investigating the use of several carry chains instead of one, which will decrease the LSB down to 20 ps. In addition, our implementation of the time-marker in another device of the same family (Xilinx Zynq) and same speed grade gives us the ability to reduce the time bin size by a factor 2 . The combination of these two solutions will allow us to reach a FPGA-based time-marker of $10 \mathrm{ps}$.

N06-28: Signal Processing for MicroBooNE Experiment 
$\underline{\mathrm{Y} . \mathrm{Li}}$

Physics Department, Brookhaven National Laboratory, Upton, NY, 11973

On behalf of the MicroBooNE collaboration

The MicroBooNE experiment is a Liquid Argon Time Projection Chamber (LArTPC) detector located in the Booster Neutrino Beamline (BNB) at Fermilab at a baseline of $470 \mathrm{~m}$ and contains an active mass of 89 tons. The detector consists of a $2.5 \mathrm{~m} \times$ $2.3 \mathrm{~m} \times 10.4 \mathrm{~m}$ TPC and includes an array of 32 PMTs used for triggering purposes. The inner TPC is housed in an evacuable and foam insulated cryostat vessel with a fiducial mass of 60 ton of LAr. Primary electrons from an ionizing track drift along a uniform electric field to the sensitive wire planes composed with two induction wire planes (U, V planes) and one collection wire plane (Y plane) in sequence with $3 \mathrm{~mm}$ pitch for a total of 8,256 signal channels. Small bipolar signals are induced on the U and $\mathrm{V}$ induction planes, and a large unipolar signal is induced on the collection Y plane. All current signals formed on the sensitive wires are processed and read out by the front end electronics. We present the process of converting the input raw digitized current waveforms with the beam data which are a convolution of detector field response, electronics response, and noise, to the deconvoluted signal (in charge and time). These ingredients are critical for the correct event reconstruction which is essential for the future physics analysis with LArTPCs.

N06-29: List Mode Regression for Low Count Detection

$\underline{\text { J. Jin }}^{1}$, K. Miller ${ }^{1}$, D. J. Sutherland ${ }^{1}$, S. Labov ${ }^{2}$, K. Nelson ${ }^{2}$, A. Dubrawski ${ }^{1}$

${ }^{I}$ Auton Lab, Carnegie Mellon University, Pittsburgh, PA, USA

${ }^{2}$ Lawrence Livermore National Laboratory, Livermore, CA, USA

Background suppression and detection of a threatening radiation sources of a known type using spectral measurements is a commonplace problem in modern radiation monitoring scenarios. The challenges compound when small portable sensors are used in searches of urban areas with highly variable background radiation patterns. These sensors inject noise into the measured energy of each photon due to the Compton Effect. Additionally, total background photon counts are low, typically around 30-60 counts per second. In this paper we explore using List Mode Regression to improve upon current detection algorithms such as Censored Energy Windowing. We propose three improvements. First, we take the small sensor response model into account, which allows us to reason probabilistically about the true photon energies. Second, we represent the measured photons individually in a list format so the distribution-based regression can be learned directly from their non-parametrically represented energy distribution, rather than learning a standard multivariate regression using binned vector data representation, as in the standard Censored Energy Window model. Third, we use a powerful kernel to leverage non-linearities in data. We show that these changes result in extending the range of usability of Censored Energy Window methodology to low-count applications.

\author{
N06-30: Respiratory Motion Gating Using True Event Distribution in PET/CT \\ $\underline{\text { J. He }}^{1,2}$, L. Fu ${ }^{1}$, R. Cui ${ }^{1}$ \\ ${ }^{I}$ Kunming University of Science and Technology, Kunming, China \\ ${ }^{2}$ The University of Chicago, Chicago, US
}

In PET/CT imaging, respiratory motion may degrade the quality of PET images. A number of solutions for the motion compensation has been investigated and developed. Gating techniques are used widely in nuclear medicine practice for reducing the effect of respiratory motion on PET image. Data-driven gating is one of the gating techniques that exploits the correlation between acquisition data of PET scan and the respiratory motion. Specifically, a data-driven method compensates for the respiratory motion by use of the system sensitivity feature of a PET scanner to estimate the correlation between the respiratorymotion induced position variation and the geometric sensitivity distribution of the scanner. In this study, we investigate a respiratory-motion gating method that is based on the changes of true events detected by detector rings for different motion phases. This method is motivated by the observation that the number distribution of true coincidences detected by individual detector ring varies along with the respiratory motion positions within the scanner FOV. The effectiveness of the method for reducing motion artifacts and improving image quality is demonstrated by use of simulation studies in which data were generated by use of GATE and NCAT software packages.

N06-31: High-Rate Gamma Spectroscopy: A Sensitivity Study A. J. Gilbert, J. E. Fast, M. J. Myjak, B. A. VanDevender, L. S. Wood

Pacific Northwest National Lab, Richland, WA, USA

Many applications require the generation of gamma spectra at event rates in excess of $10^{6} \mathrm{~s}^{-1}$ as well as very good energy resolution, e.g., safeguards, emergency response, and nondestructive assay. Good energy resolution is especially important when lower activity isotopes are sought among a large background (or foreground) that would otherwise dominate the spectrum, such as the minor actinides present in spent fuel after a long cool down time. To this end, we anticipate that high energy resolution 
detectors, such as high purity germanium, can be adapted to high-rates at a small cost to energy resolution, rather than starting with a detector with high-rate capability and medium energy resolution, e.g., $\mathrm{LaBr}_{3}$. Here, we present recent design improvements of the ultra high-rate germanium (UHRGe) detection system to allow for a 24-channel spectrum generation output. Further, we present a sensitivity study to determine how uncertainties in parameters of the detection system response affect the resulting spectra. A preamplifier simulator is developed that can emulate the output of the system at various event rates, including very high rates in excess of $10^{6} \mathrm{~s}^{-1}$. Here, we show how various levels of uncertainty in choosing the DC offset of the preamplifier output can significantly affect the full width at half max (FWHM) of the resulting spectrum.

PNNL-SA-117873

N06-32: Digital Strategies for Time and Energy Measurement for Ultra Fast Scintillators

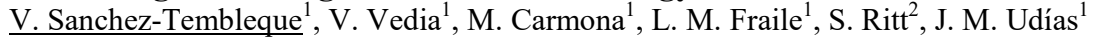

${ }^{I}$ Nuclear Physics Group, Universidad Complutense de Madrid, Madrid, Spain

${ }^{2}$ Paul Scherrer Institute, Villigen, Switzerland

Ultra fast inorganic scintillators, like LaBr3(Ce) are key detectors in Nuclear Physics and medical applications. On the other hand, fully digital acquisition systems, for which the pulses from the detectors are sampled at high rates, are being increasingly used. We present new digital pulse processing algorithms to extract time and energy signals from nuclear pulses produced by ultrafast detectors. We compare the results of the fully digital acquisition chain (DDAQ) to state-of-the-art results obtained with traditional electronics, based upon constant fraction discriminators (CFD), time to digital converters and multichannel analyzers. For this purpose, we have performed coincidence measurements using relatively large (1"x1.5"x1") truncated cone and cylindricalLaBr3(Ce) and $\mathrm{CeBr} 3$ crystals coupled to ultra fastphotomultipliers (PMTs). Coincidence measurements with Co-60 and Na-22 sources are acquired. The main advantage of fully digital processing is that many different algorithms may be applied to the same raw data set. Pulses from PMTs optimized for timing measurements were digitized to a switched capacitor array with a speed sampling of $5 \mathrm{Gs} / \mathrm{s}$ and a resolution of 16 bits, and to a fast digital oscilloscope able of $4 \mathrm{Gs} / \mathrm{s}$ and 8 bits resolution. With an in silico version of the analog CFD we obtain coincidence resolving times below 150 ps for Co-60, outperforming the standard acquisition system.

N07: High energy physics instrumentation - Poster session I

Monday, Oct. $31 \quad$ 14:00-16:00 Etoile

N07-1: TPX Luminosity Measurement of LHC Proton-Proton Collisions at 13 TeV

$\underline{\text { A. Sopczak }}^{1}$, J. Begera ${ }^{1}$, B. Bergmann ${ }^{1}$, T. Billoud ${ }^{2}$, P. Burian ${ }^{1}$, I. Caicedo ${ }^{1}$, D. Caforio ${ }^{1}$, J. Janecek ${ }^{1}$, C. Leroy $^{2}$, P. Manek $^{1}$,

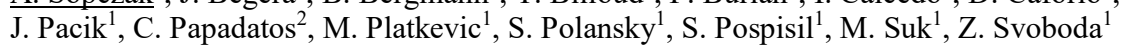

${ }^{I}$ Institute of Experimental and Applied Physics, Czech Technical University, Prague, Czech Republic

${ }^{2}$ Group of Particle Physics, University of Montreal, Montreal, Canada

A network of TPX detectors installed in the ATLAS cavern precisely measures the LHC luminosity as a function of time. Measurements are performed with hit and cluster counting, as well as energy deposits. As a proof of principle it is demonstrated that the TPX network has the capability to study the underlying mechanisms of the reduction of LHC luminosity with recorded data from $13 \mathrm{TeV}$ proton-proton collisions taking in 2015. The LHC luminosity reduction is mainly caused by beam-beam interactions (burning-off the proton bunches) and beam-gas (single bunch) interactions by the protons of the circulating beams with remaining gas in the vacuum pipe. The particle loss rate due to proton burn-off in collision is proportional to the number of protons in the second power as protons are lost in both colliding bunches. In the case that the protons in a beam collide with remaining gas in the vacuum pipe, the particle loss rate is proportional to the number of protons in the beam. As a stand-alone system the TPX network achieves a measurement precision of $0.3 \%$ for time intervals of one second.

N07-2: Testbeam Results from Pre and Post Irradiated Modules for the Upgrade of the ATLAS Strip Tracking Detector

A. J. Blue

SUPA School of Physics and Astronomy, University Of Glasgow, Glasgow, Scotland

On behalf of the ATLAS Collaboration

The planned HL-LHC (High Luminosity LHC) in 2025 is being designed to maximise the physics potential of the LHC through a sizeable increase in the luminosity, reaching $1 \times 10^{35} \mathrm{~cm}^{-2} \mathrm{~s}^{-1}$ after 10 years of operation. A consequence of this increased luminosity is the expected radiation damage at a integrated luminosity of $3000 \mathrm{fb}^{-1}$, requiring the tracking detectors to withstand hadron fluencies to over $1 \times 10^{16} 1 \mathrm{MeV}$ neutron equivalent per $\mathrm{cm}^{2}$. With the addition of increased readout rates, a complete redesign of the current ATLAS Inner Detector (ID) is being developed as the Inner Tracker (ITk), which will consist of both strip and pixelated silicon detectors. 
The silicon strip tracker exploits the concept of modularity. Fast readout electronics, deploying 130nm CMOS front-end electronics is glued on top of a silicon sensor. These so-called modules are glued on carbon structures and will span about $200 \mathrm{~m}^{2}$ of active area. A broad R\&D program is ongoing to develop and prototype many detector components.

The modules are extensively tested electronically and recent tests of the prototype strip sensors and associated binary readout electronics have been performed at the DESY II testbeam of 3-6 GeV electrons. The DURANTA telescope with an additional pixel layer was used to improve timing resolution. Results will be shown on the tracking performance of both silicon sensors for the central and forward region of the future silicon strip tracker. An irradiation programme was subsequently conducted on both module components (ASICs, sensors and hybrids) and a full central region module, with devices irradiated with both X-rays (up to $10 \mathrm{MRad})$ and hadrons $\left(\sim 3 \times 10^{15} \mathrm{p} / \mathrm{cm}^{2}\right)$. Results will then be shown comparing the performance of the modules before and after irradiation, using data obtained from both lab tests and testbeam.

N07-3: Silica Aerogel Radiator System for Belle II RICH Counter

I. Adachi ${ }^{1,2}$, R. Dolenec ${ }^{3}$, K. Hataya ${ }^{4}$, S. Iori ${ }^{5}$, S.-I. Iwata ${ }^{4}$, H. Kakuno ${ }^{4}$, R. Kataura ${ }^{6}$, H. Kawai ${ }^{7}$, H. Kindo ${ }^{2}$, T. Kobayashi ${ }^{6}$,

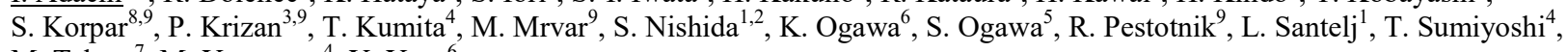
M. Tabata ${ }^{7}$, M. Yonenaga ${ }^{4}$, Y. Yusa $^{6}$

${ }^{1}$ KEK, Tsukuba, Japan

${ }^{2}$ SOKENDAI, Tsukuba, Japan

${ }^{3}$ University of Ljubljana, Ljubljana, Slovenia

${ }^{4}$ Tokyo Metropolitan University, Hachioji, Japan

${ }^{5}$ Toho University, Funabashi, Japan

${ }^{6}$ Niigata University, Niigata, Japan

${ }^{7}$ Chiba University, Chiba, Japan

${ }^{8}$ University of Maribor, Maribor, Slovenia

${ }^{9}$ Jozef Stefan Institute, Ljubljana, Slovenia

As a new forward particle identification device for the Belle II experiment, a ring imaging Cherenkov (RICH) counter based on silica aerogel radiator has been developed. In this RICH counter, two layers of the aerogel tiles with different refractive index are arranged. After intensive studies on optical properties of silica aerogels, a refractive index combination of 1.045 and 1.055 was chosen for this radiator system. Each tile has square dimensions of $180 \mathrm{~mm} \times 180 \mathrm{~mm} \times 20 \mathrm{~mm}$, and it will be cut into 4 types of wedge-shaped blocks to assemble the radiator system.

Mass-production of aerogel blocks started in November 2013. All tile productions were completed in May 2014. More than 200 tiles for each refractive index were successfully manufactured. Their optical properties have been measured as a quality check. The average transmission length at $400 \mathrm{~nm}$ wave-length was obtained to be $\sim 45 \mathrm{~mm}$ and $\sim 35 \mathrm{~mm}$ for $\mathrm{n}=1.045$ and $\mathrm{n}=1.055$, respectively. These results demonstrate that high transparency was kept for all the tiles. The refractive index was also extracted using the Fraunhofer method, and the resultant values are consistent with our expectation.

After checking an optical quality, the aerogel tile is cut into the wedge shape to arrange the RICH radiator system. This was done using a water jet cutter device. It is emphasized that hydrophobic feature of aerogel tiles enables us to take this machining technique. Optical degradation in aerogel blocks due to this step was found to be quite small.

The mechanical structure of the radiator container was fabricated in 2015. The most of components in the container are made of aluminum. An installation work for the radiator system will begin shortly and will be completed by this summer.

In this contribution, details of Belle II radiator system, putting emphasis on optical quality measurement of aerogel tiles will be reported. The present status of the radiator system construction will be also described.

\author{
N07-4: The Construction of the Fiber-SiPMT Beam Monitor System of the R484 and R582 Experiments at \\ RiKEN RAL Muon Facility \\ M. Bonesini ${ }^{1}$, R. Bertoni ${ }^{1}$, F. C. Chignoli ${ }^{1}$, R. Mazza ${ }^{1}$, T. Cervi ${ }^{2}$, A. De Bari $^{2}$, A. Menegolli ${ }^{2}$, M. Rossella ${ }^{2}$, L. Tortora $^{3}$, \\ R. Carbone ${ }^{4}$, D. Guffanti ${ }^{4}$, E. Mocchiutti ${ }^{4}$, A. Vacchi ${ }^{4}$, E. Vallazza ${ }^{4}$ \\ ${ }^{I}$ Sezione INFN Milano Bicocca, Dipartimento di Fisica G. Occhialini, Milano, Italy \\ ${ }^{2}$ Sezione INFN Pavia, Dipartimento di Fisica, Pavia, Italy \\ ${ }^{3}$ Sezione INFN Roma Tre, Roma, Italy \\ ${ }^{4}$ Sezione INFN Trieste, Trieste, Italy
}

Two different detectors have been developed to monitor the low energy muon beam at the RiKEN-RAL muon facility for the R484 and R582 experiments, that aim to study the muon transfer rate in various gases. The experiments are the first steps towards the precise determination of the Zemach radius of the proton, through the measurement of the hyperfine splitting in the ground state (1S) of the muonic hydrogen atom. Both beam monitors are based on square scintillating fibers from Bicron ( $1 \mathrm{or} 3 \mathrm{~mm}$ side) along $\mathrm{x} / \mathrm{y}$ coordinates read at one side by $1 \mathrm{x} 1 \mathrm{~mm} 2$ or $3 \times 3 \mathrm{~mm} 2$ SiPMT from Advansid, for a total of 64 channels each. They are designed to deliver position, shape and timing of the muon beam entering the experiment target. The two systems have different readout systems: one is based on custom boards, developed inside the INFN TPS project, with regulation of HV for individual SiPMT and signal amplification/shaping and discrimination using CAEN QADC for the readout, while the other uses a 
custom-made solution for SiPMT powering and a readout based on CAEN V1742 FADC. Details on the construction and assembly will be provided. Testbeam results for both systems and first results with the RAL muon beam will be shown.

\title{
N07-5: CMS Resistive Plate Chambers Performance at 13 TeV
}

\section{B. Pavlov}

University of Sofia, Sofia, Bulgaria

On behalf of the CMS RPC collaboration

During 2015 Large Hadron Collider (LHC) at CERN has reached the record-breaking center-of-mass energy of $13 \mathrm{TeV}$ for proton-proton collisions. The LHC restarted successfully after a two-year technical stop, know as Long Shutdown 1 (LS1), needed for servicing and consolidating the CERN accelerator complex. The Compact Muon Solenoid detector, a general-purpose detector at LHC, benefited from LS1 by performing crucial tasks necessary to operate the detector at higher energies. In particular, the Resistive Plate Chamber (RPC) system, one of the three muon detector technologies in CMS, was serviced, recommissioned, and upgraded with 144 new chambers to enhance muon trigger efficiency. The CMS RPC system confers robustness and redundancy to the muon trigger. A total of 1056 double-gap chambers cover the pseudo-rapidity region $|?|=1.6$. The CMS RPC collaborations has exploited early data samples at $13 \mathrm{TeV}$ for detector performance studies. These data allowed for a first characterization of the newly installed chambers. The results obtained are presented here.

\section{N07-6: Neutron Detection in the SoLid Experiment}

$\underline{\text { S. Vercaemer }}$

University of Antwerp, Antwerp, Belgium

On behalf of the SoLid collaboration

The SoLid experiment aims to make a measurement of very short baseline neutrino oscillations using reactor anti-neutrinos. For this purpose, a highly segmented detector was build out of PVT cubes lined with a ${ }^{6} \mathrm{LiF}: \mathrm{ZnS}(\mathrm{Ag})$ layer.

Unlike neutrino experiments conducted deep underground, neutrino detectors used in a reactor environment need to operate in high levels of background radiation. Therefore, a reliable distinction between the neutrons produced by neutrinos in inverse beta decay events and signals caused by other background interaction is crucial.

This poster presents a unique neutron detection method and its performance: The composite of scintillation materials with different time constants enables the efficient use of pulse-shape analysis to discriminate against electromagnetic signals.

\author{
N07-7: Development of Resistive Plate Chambers for the Upgrade of the CMS Muon System \\ $\underline{\text { I. Laktineh }}$ \\ On behalf of the CMS RPC collaboration
}

CMS is a general-purpose experiment running on CERN Large Hadron Collider. High pseudo-rapidity region of CMS muon system is covered only by Cathode Strip Chambers (CSC) and lacks redundant coverage despite the fact that it is a challenging region for muons in terms of backgrounds and momentum resolution. In order to maintain good efficiency for the muon trigger in this region additional RPC are planned to be installed in stations RE31 and RE41. The new stations will use RPCs with higher granularity but with a better timing resolution, compared to those of the current CMS RPCs, to mitigate background effects and enhance the redundancy of the trigger system. R\&D activities will be presented in the talk.

\section{N07-9: Slow Scintillation Component and Radiation Induced Readout Noise in CsI Crystals} F. Yang, L. Zhang, R. Zhu

Crystal Lab, HEP, California Institute of Technology, Pasadena, USA

Because of its fast decay time and low cost, pure CsI is widely used in HEP experiment for fast crystal calorimetry in the intensity frontier. CsI crystal samples from three vendors were characterized for the Mu2e experiment at Fermilab with data used to define crystal specification. In addition to the fast scintillation component peaked at $310 \mathrm{~nm}$, a slow scintillation component was found with a peak at $450 \mathrm{~nm}$, and may be eliminated spectroscopically by using optical filters. It was also found that the slow scintillation component and the radiation induced readout noise in CsI crystals are highly correlated. The nature of the slow scintillation component is believed to be defects and/or impurity related.

N07-10: Module Assembly Procedures for the Silicon Vertex Detector of the Belle II Experiment H. Jeon

Department of Physics, Kyungpook National University, Daegu, South Korea

On behalf of the Belle II SVD collaboration 
The Belle II experiment at SuperKEKB in Japan aims to precisely test the standard model (SM) predictions as well as to search for new physics beyond the SM. As one of the main components of the Belle-II detector, a silicon vertex detector (SVD) provides information of vertexing and low- momentum tracking of various particles emanating from the decays of beauty and charm mesons. The SVD is composed of four layers with a ladder structure comprising double-sided silicon strip detectors (DSSDs). A novel chip-on-sensor concept, dubbed $\ddot{i}_{i} 1 / 2$ Origamiï $\underset{i}{1} 2$, is developed to reduce the multiple Coulomb scattering and capacitive noise. All strips of the DSSDs with the Origami concept can be individually read out, however the assembly of Origami modules involves challenging procedures. The outermost layer, L6, of the SVD has three Origami modules out of five DSSD modules. In this paper, we present L6 assembly procedures and its electrical performances.

\author{
N07-11: Strip Detector for the ATLAS Detector Upgrade for the High-Luminosity LHC \\ D. Sperlich \\ HU Berlin, Berlin, Germany \\ On behalf of the ATLAS Collaboration
}

The planned HL-LHC (High Luminosity LHC) in 2025 is being designed to maximise the physics potential of the LHC through a sizeable increase in the luminosity, reaching $11 i 1 \frac{1}{i} 10^{35} \mathrm{~cm}^{2} \mathrm{~s}^{-1}$ after 10 years of operation. A consequence of this increased luminosity is the expected radiation damage at an integrated luminosity of $3000 / \mathrm{fb}$, requiring the tracking detectors to withstand hadron fluencies to over $1 \ddot{i}_{i}^{1 / 2} 10^{16} 1 \mathrm{MeV}$ neutron equivalent per $\mathrm{cm}^{2}$. With the addition of increased readout rates, a complete redesign of the current ATLAS Inner Detector (ID) is being developed as the Inner Tracker (ITk), which will consist of both strip and pixelated silicon detectors. $\ddot{i}_{i}^{1} / 2$ The physics motivations, required performance characteristics and basic design of the proposed upgrade of the strip detector will be a subject of this talk. Present ideas and solutions for the strip detector and current research and development program will be discussed.

N07-12: CLAWS - a Plastic Scintillator / SiPM Based Detector to Measure Backgrounds at SuperKEKB M. Gabriel, M. Kattau, C. Kiesling, F. Simon, H. Windel

Max-Planck-Institut für Physik, Munich, Germany

The SuperKEKB collider at KEK, which has started its commissioning earlier this year, is designed to achieve unprecedented luminosities, with a factor 40 higher than the record-breaking luminosity of the KEKB machine. For the operation of the Belle $\backslash, \mathrm{II}$ detector, in particular of its pixel vertex detector, a precise understanding of the background conditions at the interaction point of the accelerator is crucial. To study backgrounds, a dedicated detector setup consisting of different subsystems has been installed for the first commissioning phase of the accelerator. Among those systems is CLAWS, consisting of 8 scintillator tiles with directly coupled SiPMs, read out by computer-controlled oscilloscopes with very deep buffers. CLAWS will in particular study the background connected to the continuous injection of the accelerator, by monitoring the background levels of individual particle bunches in the machine continuously over ms time-frames. This contribution will present the technology of the CLAWS detectors, the overall installation and the detector performance including the time resolution. It will also discuss the performance in dedicated background runs to be carried out at KEK in May 2016.

\title{
N07-13: The Phase-II ATLAS Pixel Tracker Upgrade: Layout and Mechanics
}

A. Sharma

University of Oxford, Oxford, United Kingdom

On behalf of the ATLAS Collaboration

The ATLAS experiment will upgrade its tracking detector during the Phase-II LHC shutdown, to better take advantage of the increased luminosity of the HL-LHC. The upgraded tracker will consist of silicon-strip modules surrounding a pixel detector, and will likely cover an extended eta range, perhaps as far as $\mid$ eta $\mid<4.0$. A number of layout and supporting-structure options are being considered for the pixel detector, with the final choice expected to be made in early 2017. The proposed supporting structures are based on lightweight, highly-thermally-conductive carbon-based materials and are cooled by evaporative carbon dioxide. The various layouts will be described and a description of the supporting structures will be presented, along with results from testing of prototypes.

N07-14: DSiMPI - Utilisation of the SiMPI Concept for Tracking Applications in Particle Physics Experiments $\underline{\text { S. Petrovics }}{ }^{1}$, L. Andricek ${ }^{1}$, I. Diehl ${ }^{2}$, K. Hansen ${ }^{2}$, K. Krueger ${ }^{2}$, R. Lehmann ${ }^{1}$, J. Ninkovic ${ }^{1}$, C. Reckleben ${ }^{2}$, R. Richter $^{1}$, G. Schaller $^{1}$, F. Schopper ${ }^{1}$, F. Sefkow ${ }^{2}$, X. Wang ${ }^{2}$

${ }^{\text {I}}$ Semiconductor Laboratory of the Max-Planck Society, Munich, Germany

${ }^{2}$ DESY, Hamburg, Germany 
Silicon Photomultipliers (SiPMs) are a promising candidate for replacing conventional photomultiplier tubes in many applications, thanks to ongoing developments and advances in their technology. At the Semiconductor Laboratory of the MaxPlanck Society (HLL) a novel detector concept was developed integrating the quench resistor directly into the silicon bulk of the device resulting in a free entrance window. The feasibility of the concept was already confirmed by simulations and extensive studies of first prototype productions. Recently SiPMs were also considered as an attractive alternative for tracking applications in vertex detectors. The requirements for a fast response, simple design and high fill factor can all be met by SiPMs. In addition the increased trigger probability for an avalanche by MIPs allows device operations at lower overbias voltages, resulting in decreased noise. The concept can be evolved further towards an imaging photo-detector. A new design for an application of these SiPM devices as vertex detectors with active quenching developed by HLL and DESY as well as first simulation results will be presented. Also, first measurements of the trigger efficiency as a function of the applied overbias voltage will be shown, as well as characterisation measurements of the first prototypes for the particle tracking application.

\section{N07-15: SiPM Readout for the SHiP Timing Detector}

$\underline{\text { R. Bruendler }}$

Physics Institute, University of Zurich, Zurich, Switzerland

On behalf of the SHiP Collaboration

The SHiP experiment (Search for Hidden Particles) is a new general purpose fixed target experiment proposed at the CERN SPS accelerator. It will make measurements of tau neutrinos and is aimed at exploring the domain of hidden particles predicted by a large number of models beyond the Standard Model. A dedicated timing detector is used in order to reduce the background from combinatorial di-muon events. We report on results from a timing detector consisting of scintillating bars readout by silicon photomultipliers (SiPMs), with a resolution of less than $100 \mathrm{ps}$.

\section{N07-16: Instrumentation of the Detectors and DAQ Performance in the NOvA Experiment}

\section{J. Zalesak}

Elementary Particle Division, Institute of Physics, CAS, Prague, Czech Republic

On behalf of the NOvA Collaboration

The NOvA experiment studies neutrino oscillations with 2 functionally identical detectors separated by a baseline of $810 \mathrm{~km}$. The $14 \mathrm{kT}$ far detector in Ash River, Minnesota, comprises 344,064 channels of liquid scintillator detection cells read out via wavelength-shifting fiber into 32-channel Avalanche Photo Diodes (APD). A custom designed Front End Board (FEB) continuously digitizes and zero-suppresses the output signals from each APD. The smaller near detector located at Fermilab has 20,192 channels which are readout in an identical manner. Both detectors are internally synchronized by a GPS-based timing system with maintains a readout-to-readout synchronization to better than $15.6 \mathrm{~ns}$ across the full detector. The timing system also provides a universal time base, which is used provide synchronization and correlation between the geographically separated detectors and the Fermilab accelerator complex. The NOvA Data Acquisition (DAQ) system combines the 10,752 front end readout for the far detector with 168 power-PC based custom computers for reading out and collating data the raw data. The far detector site utilizes a farm of 196 Linux nodes for buffering the raw detector data for trigger decisions and event building, along with another 10 compute nodes and storage systems for dedicated DAQ functions such as run control, data logging, and DAQ system monitoring.

\section{N07-17: The LHCb VELO Upgrade}

\section{$\underline{\text { P. Collins }}$}

CERN, Geneva, Switzerland

The upgrade of the LHCb experiment, planned for 2018, will transform the experiment to a trigger-less system reading out the full detector at $40 \sim \mathrm{MHz}$ event rate. All data reduction algorithms will be executed in a high-level software farm with access to the complete event information, enabling the detector to run at luminosities of $\$ \backslash$ mathrm $\left\{2 \backslash\right.$ times $\left.10^{\wedge}\{33\} \mathrm{cm}^{\wedge}\{-2\} \mathrm{s}^{\wedge}\{-1\}\right\} \$$ with greatly enhanced efficiency.

The Vertex Locator (VELO) is the silicon vertex detector surrounding the interaction region. The current detector will be replaced with a hybrid pixel system equipped with electronics capable of reading out at $40 \sim \mathrm{MHz}$, designed to withstand the irradiation expected at an integrated luminosity of $50 \$ \backslash \operatorname{mathrm}\left\{\mathrm{fb}^{\wedge}\{-1\}\right\} \$$ and beyond. The upgraded VELO will form an integral part of the software trigger, and must provide fast pattern recognition and track reconstruction. The detector will be composed of silicon pixel sensors with $\$ \backslash$ mathrm $\left\{55 \backslash\right.$ times $55 \sim$ mu $\left.\mathrm{m}^{\wedge}\{2\}\right\} \$$ pitch, read out by the VeloPix ASIC which is being developed based on the TimePix/MediPix family. The hottest region will have pixel hit rates of $900 \mathrm{Mhits} / \mathrm{s}$ yielding a total data rate more than $3 \mathrm{Tbit} / \mathrm{s}$ for the upgraded VELO.

The module is a double sided construction, with two hybrid pixel assemblies and their PCB circuits mounted on either side of the central backbone; a 400 um thick silicon plate incorporating cooling microchannels for the circulation of evaporative CO\$2\$. Operation is at a sufficiently low temperature to avoid thermal runaway of the irradiated sensors. Thermal stability is an 
important issue and the design is optimised to minimise movements.

The material budget will be minimised by the use of evaporative $\$ \backslash$ mathrm CO_2\$ coolant circulating in microchannels within $400 \$ \backslash$ mathrm $\{\backslash \mathrm{mu} \mathrm{m}\} \$$ thick silicon substrates.

The design and development of the module and microchannel cooling will be described, and the latest results presented.

\section{N07-18: Cosmic Ray Telescop at Orsay}

\section{Burmistrov}

SERDI, LAL, Orsay, France

In Nuclear Physics and High Energy Physics the tests of new detector systems especially in the first stages of dewelopments is possible using cosmic muons. CORTO - the COsmic Ray Telescope at Orsay, located at Linear Accelerator Laboratory in France. CORTO - reconstructs muon track and its time. Measured position resolution (RMS) of the telescop is $7 \mathrm{~mm}$ (X direction), $15 \mathrm{~mm}$ (Y dirrection) and $90 \mathrm{ps}$ time resolution. CORTO consists of three positional sensitive strip gas detectors, called Multigap Resistive Plate Chamber redouted by USB-Wavecatcher electonics. Effective size of each chember is $180 \mathrm{x} 80$ $\mathrm{cm}^{\wedge} 2$. We use NARVAL software for data aquisition system.

N07-19: 3D Electron Tracking and Vertexing with Single Plane Pixel Detectors

G. Blaj, A. Dragone, P. Hansson, C. Hast, R. Herbst, C. Kenney, T. Smith

SLAC National Accelerator Laboratory, Menlo Park, CA, U.S.A.

Silicon strip and pixel detectors are present in most detectors for particle tracking and vertexing in particle physics. ePix100a, a low noise detector designed for soft x-ray imaging, provides a significantly improved signal to noise ratio (SNR) of $\sim 500-1000$, allowing tracking with improved subpixel and 3D angular resolution than that suggested by the pixel size and sensor thickness. This might lead to improved tracking and vertexing detectors, using fewer or even single detector layers.

\section{N07-20: 3D Avalanche Pixel for Precision Vertexing}

N. D'Ascenzo, Q. Xie

Huazhong University of Science and Technology, Wuhan, Cina

The development of an innovative position sensitive pixelated sensor to detect and measure with high precision the coordinates of the ionizing particles is proposed. The silicon avalanche pixel sensors (APiX) is based on the 3D integration of the avalanche pixels connected in pairs and operated in coincidence in fully digital mode and with processing electronics embedded on the chip. The APiX sensor addresses the requirements of minimize of the material budget and related multiple scattering effect in the precise tracking system for High Energy Physics Experiments. The expected features of the operation of the new APiX sensor: low noise, low power consumption and suitable radiation tolerance. The APiX sensors provides on-chip digital information of coordinate of the charge particles and can be the building block of the modular large scale tracking system. The technological challenges are the 3D integration of the device under CMOS processes and integration of the processing electronics on chip. In order to demonstrate experimentally the validity of the APiX principle, a very preliminary prototype of a avalanche pixel structure was assembled as the collinear pair of SiPM sensors. The prototype was tested at the CERN SPS test beam.

\section{N07-21: Production and Testing of a High-Performance, Low-Cost Readout System for the Belle II Upgrade:} KL and Muon (KLM) Scintillator Sub-Detector

I. Mostafanezhad, G. Varner, B. Edralin

Department of Physics, University of Hawaii at Manoa, Honolulu, HI, USA

In a decade of operation, the Belle experiment operating at KEKB accomplished all its technical milestones by accumulating data equivalent to an integrated luminosity of $1000 \mathrm{fb}-1$. The Belle II detector is designed to operate at 20 times higher background level and 50 times higher event rate. We present performance test results for implementation of a subdetector readout system that is capable of reading 20k channels using 1250 16-channel waveform sampling ASICs placed on 144 9U motherboards. Gain adjustment is provided for every channel of Geiger-mode avalanche photodetectors, while high efficiency triggering and ns-level timing are obtained. Waveform sampling permits tuning the thresholds to 7.5 photoelectrons. All 144 modules for the entire detector has been fabricated and tested. Production testing features an in-house inventory tracking software which interfaces with the calibration software. Readout tests were performed with scintillator modules and high voltage supplies installed in the upgraded Belle II detector. Integration with the new upgraded Belle II Trigger and Data Acquisition system is done and preliminary data demonstrates meeting the Key Performance Parameter requirements for the project.

\section{N07-22: Design of Data Aggregation Unit for High Energy Physics Experiment}


$\underline{\text { J. Mitra }}^{1}$, T. K. Nayak ${ }^{2}$

${ }^{I}$ VECC, PhD Scholar, KOLKATA, INDIA

${ }^{2}$ VECC, ALICE India Co-ordinator, KOLKATA, INDIA

This paper presents a prototype design for a basic configurable Data Aggregation Unit. This dedicated unit is required to gather and organize the bulk amount of detector data of High-Energy Physics (HEP) experiments before data processing. It discusses how the data traffic manager handles data based on information content. We then introduce three modes of operation, that allows HEP experiment to optimize resource cost with data reliability. It is followed by result section, which includes signal quality of incoming and outgoing links, Bit Error Rate, Throughput and Resource Occupancy.

N08: Instrumentation for Security - Poster session I

Monday, Oct. $31 \quad$ 14:00-16:00 Etoile

N08-1: Design of the Rapidly Relocatable Tagged Neutron Inspection System of the C-BORD Project

$\underline{\text { A. Sardet }}{ }^{1}$, B. Perot ${ }^{1}$, C. Carasco ${ }^{1}$, G. Sannie ${ }^{2}$, S. Moretto ${ }^{3}$, G. Nebbia ${ }^{3}$, C. Fontana ${ }^{3}$, M. Moszynski ${ }^{4}$, P. Sibczynski ${ }^{4}$, K. Grodzicki ${ }^{4}$, L. Swiderski ${ }^{4}$, A. Iovene ${ }^{5}$, C. Tintori ${ }^{5}$

${ }^{I}$ Nuclear Measurement Laboratory, CEA, DEN, Cadarache, Saint-Paul-lez-Durance, France

${ }^{2}$ CEA, DRT, LIST, Gif-Sur-Yvette, France

${ }^{3}$ Dipartimento di Fisica e Astronomia, INFN, Padova, Italy

${ }^{4}$ National Centre for Nuclear Research, Otwock-Swierk, Poland

${ }^{5}$ CAEN S.p.A, Viareggio, Italy

Within the framework of the European H2020 C-BORD project, aiming at improving container inspection technologies, a compact and "Rapidly Relocatable Tagged Neutron Inspection System", called RRTNIS, is being developed taking into account past EURITRACK experience with a portal TNIS, and the latest technologies in terms of associated particle neutron generator and data acquisition electronics. A dedicated shield surrounding the neutron generator has been designed with MCNP6 to limit the size of the restricted area and the count rate on gamma detectors, which are located very close to the generator. This new design with "reflection" detectors only, i.e. in backscattering position, is indeed more efficient to detect suspect items, like explosives or illicit drugs, in bottom regions of the container, compared to EURITRACK detectors which were mainly located above the container. It also allows designing a relocatable system for different inspection sites like seaports, borders, or other checkpoints. Dose and count rate calculations are presented to determine the restricted area and facilitate the design of the data acquisition electronics, respectively. Several test case scenarios are also simulated to assess the capability of the new RRTNIS to characterize, in a few minutes, suspicious items previously identified by X-ray radiography.

N08-2: Characterization and Simulation of Soft Gamma-Ray Mirrors for Their Use with Spent Fuel Rods at Reprocessing Facilities

$\underline{\text { J. Ruz }^{1}}$, M. J. Pivovaroff ${ }^{1}$, M.-A. Descalle ${ }^{1}$, D. L. Chichester ${ }^{2}$, S. M. Watson ${ }^{2}$, K. P. Ziock ${ }^{3}$

${ }^{I}$ Physics and Life Sciences, Lawrence Livermore National Laboratory, Livermore, CA, United States of America

${ }^{2}$ Physics and Life Sciences, Idaho National Laboratory, Idaho Falls, ID, United States of America

${ }^{3}$ Physics and Life Sciences, Oak Ridge National Laboratory, Oak Ridge, TN, United States of America

The use of a grazing incidence optic to selectively reflect K-shell fluorescence emission and isotope-specific lines from Special Nuclear Materials (SNM) is a highly desirable non-destructive analysis method for use in reprocessing fuel environments.

Preliminary measurements have been performed and a simulation suite has been developed to give insight into the design of the X-ray optics system as a function of source emission, multilayer coating characteristics and general experimental configurations. This talk will show experimental results and compare them to the predictions from our simulation toolkit to illustrate the raytracing capability and explore the effect of modified optics in future measurement campaigns.

\section{N08-3: Sensitivity Image Compensation of ASIC Artifacts and Event Loss in Pixelated 3-D Position Sensitive CdZnTe Detectors}

B. Williams, Z. He

Nuclear Engineering and Radiological Sciences, University of Michigan, Ann Arbor, MI, United States

The maximum-likelihood expectation maximization (MLEM) algorithm has been applied to reconstruct gamma-ray images and accurately estimate source activity based on list-mode data reconstructed from events recorded from position-sensitive CdZnTe detectors with pixelated anodes. Conventional methods for activity estimation require a simulated system sensitivity image and empirical correction factors to offset model mismatch in the simulation. In this paper, an analytical method for compensating the model mismatch in simulated sensitivities is proposed as an alternative to empirical corrections factors. Model mismatch manifests itself in the simulated sensitivity images by several means, most of which are due to the absence of the 129-channel 
self-triggered application specific integrated circuit (ASIC) readout electronic noise in the simulation model. Two dominant means of model mismatch - invalid ASIC time amplitude and low charge induction events - are considered in this work. By monitoring ASIC timing and event reconstruction performance from calibration measurements, event and detector-specific compensation factors are derived and applied in a modified sensitivity image calculation. The results suggest that the compensation method more accurately models the system sensitivity than conventional methods, as evidenced by the activity estimation accuracy to within 5\% using list-mode data from nine independent CdZnTe detectors with pixelated anodes.

This material is based upon work supported by DOE NA-22 grant award number DE-NA0002131.

N08-4: The Method to Improve the Angular Resolution of the Portable Gamma Camera with Pinhole Collimator

O. Ivanov, V. Potapov, I. Semin

National Research Centre Kurchatov Institute, Moscow, Russia

The use of portable gamma camera for different works at nuclear facilities has become common in recent years. In all application, it is desirable to have a gamma-ray image with a sufficiently large field of view and the good angular resolution. For available modern gamma cameras, these quantities are related to each other because of the method of formation of the gammaimage on the detector - the principle of the camera obscura (pinhole) or coded aperture. In addition, due to the angular collimation of gamma rays by the real pinhole it happens that signal drops to the edge of the field of view. Therefore, virtually the entire field of view of the instrument is not used for imaging. We have developed a way to restore the image obtained by the pinhole camera, which allows to increase the angular resolution by about 3-4 times and to obtain uniform sensitivity in all field of view without a noticeable increase in noise in the gamma-ray image. The method is based on the use of the inverse problem solution for finding the distribution of incident photons with the instrumental functions of the camera. The problem is reduced to a Fredholm equation of the first kind. The equation is effectively solved by an iterative method developed by Tarasco.

Previously, the method was used to reconstruct the spectra of neutrons using a neutron detector apparatus function. The method is implemented for the pinhole gamma camera "Cartogam", manufactured by Canberra. Camera Model "Cartogam", which was used in the measurements has a field of view $-40^{\circ}$, the angular resolution of $5^{\circ}$. The method of reconstruction is described. Search of apparatus function for point source is presented. The application of the method acquisition and processing of test images and gamma-images of packages of radioactive waste, superimposed on a video image are presented.

N08-5: The Image Processing for Improvement of Angular Resolution and Sensitivity of the Portable Gamma Camera with Medipix Detector

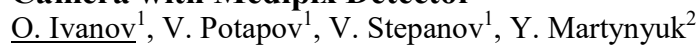

${ }^{I}$ National Research Centre Kurchatov Institute, Moscow, Russia

${ }^{2}$ Scientific and Producing Company Doza, Moscow, Russia

Imaging of gamma-ray sources and the hard X-ray sources is a recognized experimental method for mapping the distribution of radioactive elements, in plasma physics research, in high-energy astrophysics. In all studies, it is desirable to have a gamma-ray image with a sufficiently large field of view and the good angular resolution. The recently developed gamma cameras have a semiconductor position-sensitive detector Medipix with crystal CdTe and transverse size of the detector is $14 \mathrm{x} 14 \mathrm{~mm}$, and mask pattern used is of the order of $10 \times 10$ elements, the corresponding FoV - 30deg, angular resolution - 3deg . Therefore, improving the angular resolution can significantly improve the performance of the system. We have developed a method for reconstructing of images obtained using a gamma camera, which can improve angular resolution of about $2-3$ times when using the mask with large holes and align the sensitivity in the field of view without noticeable increase of noise in gamma image. The method is based on solving the inverse problem to find the distribution of incident photons with the instrumental functions of the camera. The problem is reduced to a Fredholm equation of the first kind for a function of two variables. The equation is effectively solved by an iterative method of Tarasko. Experimental shadow picture obtained by gamma camera developed by SPC "Doza". This camera has a coded mask of MURA type pattern with a base pattern of 11x11 elements and detector with CdTe crystal of $2 \mathrm{~mm}$ thick. In addition, we consider the question of the pre-treatment of shadowgramms to eliminate the signal from the meson tracks. This method increases the sensitivity of the system in the imaging of sources with low energy of gamma rays. Filtering of tracks is performed by a special program, which filters the signals from the events that have an elongated shape on the detector.

N08-6: Design Study on Differential Die-Away Technique in an Integrated Active Neutron NDA System for Non-Nuclear Proliferation

A. Ohzu, M. Maeda, M. Komeda, H. Tobita, M. Kureta, M. Koizumi, M. Seya

Japan Atomic Energy Agency, Ibaraki, Japan 
Since 2015, in the Japan Atomic Energy Agency, an advanced non-destructive analysis (NDA) mainly consisting of four active neutron analysis techniques, which are DDA (Differential Die-Away Analysis), PGA (Prompt Gamma-ray Analysis) combined with NRCA (Neutron Resonance Capture Analysis), NRTA (Neutron Resonance Transmission Analysis) and DGS (Delayed Gamma Spectroscopy) is under development for the measurement of Special Nuclear Materials (SNM) and Minor Actinides (MA) in low and high radioactive nuclear materials. Those different analysis are integrated and build in one measurement device using a pulsed neutron generator, so that each analysis become practicable independently. The design study on the device has been performed with Monte Carlo simulation code (MCNP) to evaluate the performance of those analysis. The simulation result of the performance test related to the DDA section shows that the 239Pu mass (contained in MOX fuel) of as low as of $10 \mathrm{mg}$ is detectable. The additive effects on the measurement are expected by combining those analysis.

N08-7: Development of a nasal monitor to evaluate an activity of plutonium in the nasal cavity

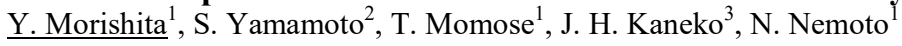

${ }^{I}$ Japan Atomic Energy Agency, Tokai-mura, Naka-gun, Ibaraki, Japan

${ }^{2}$ Nagoya University Graduate School of Medicine, Nagoya, Aichi, Japan

${ }^{3}$ Hokkaido University Graduate School of Engineering, Sapporo, Hokkaido, Japan

Plutonium dioxide $(\mathrm{PuO} 2)$ is used to fabricate mixed oxide fuel for fast breeder reactors. When airborne contamination of $\mathrm{Pu}$ occurs, the presence of Pu in the nasal cavity of a worker should be instantly evaluated. The nasal smear method is usual method to immediately evaluate the intake of $\mathrm{PuO} 2$ at a work site. However, this method cannot evaluate the activity of Pu quantitatively because the removable factor of $\mathrm{PuO} 2$ can be changed by the nasal swab and the pressure of a finger. An alpha-particle detector that can directly measure the nasal cavity has not yet been developed. Therefore, we developed a nasal monitor that can directly measure the activity of $\mathrm{Pu}$ in the nasal cavity in order to exactly evaluate the internal exposure dose of a worker. For our device, a $\mathrm{ZnS}(\mathrm{Ag})$ scintillator was attached to the surface of a $3 \mathrm{~mm} \times 3 \mathrm{~mm} \times 20 \mathrm{~mm}$ prismatic-shaped light guide. A Silicon Photomultiplier (SiPM) array was used to detect the scintillation light from the $\mathrm{ZnS}$ (Ag) scintillator. 241Am source was collimated to $2 \mathrm{~mm} \times 5 \mathrm{~mm}$ and was used to evaluate the efficiency of the nasal monitor. Alpha particles were irradiated from three different faces to the scintillator for the left and right nasal cavities. In addition, the effects of gamma and beta rays on the nasal monitor were evaluated. The positions of both the left and right nasal cavities could be identified based on the twodimensional distribution of the alpha particles. The average efficiency was comparable to the $\mathrm{ZnS}(\mathrm{Ag})$ scintillation survey meter. The effect of the gamma and beta rays was negligible for Pu monitoring. A worker's internal exposure dose can be accurately evaluated using our developed nasal monitor.

N08-9: Simplified Simulation Method for Modelling Illicit Materials Detection System Based on EDXRD T.-Y. YangDai ${ }^{1,2}$, L. Zhang ${ }^{1,2}$

${ }^{I}$ Department of Engineering Physics, Tsinghua University, Beijing, China

${ }^{2}$ Key Laboratory of Particle \& Radiation Imaging, Beijing, China

With the two fold aim of analyzing the energy dispersive X-ray diffraction (EDXRD) system for illicit materials detection and of selecting optimal configurations prior to experimental tests, a simulation method for modelling the response of EDXRD system has been proposed. The simulation is done based on two orthogonal planes of the system: the diffraction plane (H-Plane) and its vertical plane (V-Plane). Simulated results showed a very good agreement with experimental data and the computational amount of the proposed method is much smaller than the simulation programs based on Monte Carlo method or other complete numerical simulation methods. The simulation results also showed that the ways in which the angular distributions of H-Plane and V-Plane influence the diffraction spectra are different. The spectra resolution is less sensitive to the broadening of the collimator apertures in the V-Plane. This suggests that wider collimator apertures in the V-Plane can be used to increase the detection efficiency without much loss of the angular resolution.

This work was supported by a grant from the National Natural Science Foundation of China (Pioneer Comprehensive Optimization Theory and Technology for Low-dose CBCT. NO.11435007).

\author{
N08-11: Application of LaBr3(Ce) Detectors in a Mobile Spectrometric System \\ F. Finkel, I. Krainukovs, V. Gostilo \\ Baltic Scientific Instruments, Riga, Latvia
}

The developed mobile spectrometric system is a monitoring vehicle equipped with spectrometric equipment that provide realtime and thorough estimate of dose rate level, amount and spatial distribution of radioactive pollution caused by known or unknown radionuclide constituents independently of the nature and age of the pollution. This mobile system can be used for radiation monitoring of large areas, for searching lost or stolen radiation sources, as well as for control of accumulation of radionuclides in the vicinity of a radiation-hazardous facility. Sending the data obtained to organizations performing radiation control by means of cellular network or satellite communication is possible. The mobile base of the mobile spectrometric system 
is an off-road electric vehicle featuring large ground clearance and a system enabling driving at an extremely low speed of ca 1 $\mathrm{km} / \mathrm{h}$. Thanks to this, an extremely detailed gamma radiation mapping is possible. The key component of the mobile spectrometric system is a spectrometer consisting of two gamma radiation $\mathrm{LaBr} 3(\mathrm{Ce})$ detectors mounted within thermostabilizing housings and controlled by a 2-tract multi channel analyzer. The detectors are mounted so, that their position relative to each other and the ground can be changed. This feature enables investigation of radiation-hazardous objects in addition to performing in-situ measurements. The system indicates surpassing the preset threshold values of dose rate, surface or specific activity by means of light and sound alarm. The GPS equipment and the related software and methodology provide mapping of measurement results with the accuracy of up to $0.5 \mathrm{~m}$.

\author{
N08-12: Detection of High-Energy Delayed Gamma-Rays and Delayed Neutrons from Photofission Using \\ Large Size Plastic Scintillators and 3 He Counters \\ A. Grabowski, J.-M. Bourbotte, F. Carrel, G. Corre, H. Hamrita, V. Kondrasovs, F. Lain ̃̃ $\subset$, A. Sari \\ Sensors and Electronic Architectures Laboratory, CEA, LIST, Gif-sur-Yvette, France
}

Detection of hidden Special Nuclear Material (SNM) is one of the main topics of Homeland Security. One of the main goals of the European Horizon 2020 C-BORD project is to drastically improve SNM detection in containers to prevent illicit trafficking. One way to reach this goal is based on the photofission process: a high-energy photon beam generated by Bremsstrahlung in the conversion target of an electron accelerator (LINAC) is used to induce photofission reactions on nuclear materials such as uranium and plutonium.

High-energy delayed gamma-rays and delayed neutrons emitted after photofission reactions are a unique signature of the presence of SNM. Detection of these particles enable to detect, quantify, and identify the fissionable material irradiated. The use of He-3 is always a reference solution to detect delayed neutrons, despite the He-3 shortage. High-energy delayed gamma-rays can be detected in several different ways. However, their counting still remains a significant challenge due to several constraints related to the use of electronics and detector in a LINAC environment.

In this work, we study the performances of large size plastic scintillators for detecting high-energy delayed gamma-rays from photofission and use in parallel delayed neutron detectors in order to associate both of these detection systems. We compare performances obtained with two different sizes of EJ-200 plastic scintillators. We also compare results obtained associating the high-energy delayed gamma-ray signature obtained using plastic scintillators with delayed neutron detection resulting from He-3 counting. Measurements have been carried out with a $9 \mathrm{MeV}$ LINAC: variations of irradiation frequency, irradiation time and mass of uranium give a good overview of plastic scintillators performances to detect high-energy delayed gamma-rays from photofission. The coupling between delayed gamma-rays and neutrons is also a promising solution, especially for detecting shielded SNM.

\author{
N08-13: X-Ray Fluorescence Measurements of Toxic Metal Content in Ash from Municipal Solid Waste \\ Incineration

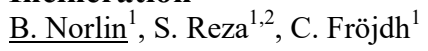 \\ ${ }^{I}$ Dept. of Electronics Design, Mid Sweden University, Sundsvall, Sweden \\ ${ }^{2}$ FS-DS, Deutsches Elektronen-Synchrotron (DESY), Hamburg, Germany
}

X-ray fluorescence is a powerful method to analyse the composition of various substances. In this project we applied the method to investigate the contents of toxic metals in fly ashes from municipal solid waste (MSW) incineration. The fluorescence yield is a complex function of the incident spectrum and matrix effects in the substance under investigation. In this paper we discuss optimisation of the setup for monitoring of different metals and the effect of variation in the composition of the ashes. The MSW incineration business has expanded rapidly, both in Europe and globally. But, although incineration is expected to have positive environmental impact, incineration will also increase the risk of spreading toxic metals to the environment. The vision of this project is to develop an online method for monitoring of toxic metals in ashes. With such measurements directly on site it is possible to optimize an ash washing process permitting the fly ash to be stored in a landfill for non-hazardous waste. The X-ray fluorescence measurement presented in this project assures that the measurement accuracy is sufficient for metal content monitoring. The actual measurement process is also fast enough to be possible to implement as an online measurement method. Different means to improve the measurement are discussed, such as adjusting X-ray source voltage, usage of source filtering and various detector material choices. The optimal setup is different for different metals, so it is essential to know which metal needs higher detection accuracy. The accuracy needed for toxic metal monitoring in incineration plants are estimated.

N08-14: Design of a Novel Instrument for Active Neutron Interrogation of Unknown Objects

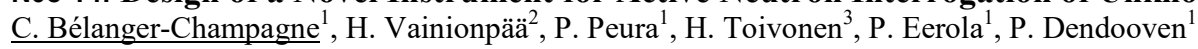

${ }^{I}$ Helsinki Institute of Physics, Helsinki, Finland

${ }^{2} J H V$ Physics Oy, Riihimaki, Finland

${ }^{3}$ HT Nuclear Oy, Hyvinkää, Finland 
Active neutron interrogation of unknown objects provides information about which isotopes are present through the detection of isotope-specific neutron-induced gamma radiation. It eliminates the safety risks associated with direct manipulation of the unknown object which can contain chemical, biological, nuclear, radioactive or explosive materials. We use GEANT4 Monte Carlo simulations to investigate the design of a novel instrument for active neutron interrogation that uses a high frequency intense pulsed neutron generator and fast scintillator detector. We demonstrate that it is possible to separate the fast neutroninduced gamma signal from the thermal neutron-induced gamma signal and use precise timing information to maximize signal significance and improve performance over existing commercial instruments.

\title{
N08-15: Simulation and Implementation of Mobile Sensor Networks for Radiation Detection
} J. Zhao, K. A. Roth, C. J. Sullivan

Dept. of Nuclear, Plasma, and Radiological Engineering, University of Illinois at Urbana-Champaign, Urbana, Illinois, USA

Detecting the presence of possible illicit radioactive materials in a large urban area is critical in national security applications. The concept of mobile radiation sensor networks have been put forward to solve this problem. Several factors, such as detector density, detector sensitivity, background radiation, radioactive source type and so on, are thought to influence the performance of the mobile sensor networks. It is necessary to study the influence of different factors before a large number of mobile detectors are actually deployed in large geographic regions. In this paper, the simulation of a mobile radiation sensor network system is presented. The simulation system, which takes into account the changing background radiation with position and time as well as the measured detector response, is used to simulate the scenario in which multiple geotagged mobile radiation detectors are deployed in a large area to detect and track the presence and movement of illicit radioactive materials. Quantitative results and deduced conclusions will be presented.

N08-16: Discrimination of High-Z Materials with Muon Scattering Tomography

L. Frazao $^{1}$, J. Velthuis ${ }^{1}$, C. Thomay ${ }^{1}$, C. Steer ${ }^{2}$

${ }^{I}$ University of Bristol, Bristol, UK

${ }^{2}$ AWE, Aldermaston, $U K$

We describe a method for distinguishing different high-Z materials inside a concrete volume, using muon scattering tomography. In a previous study, a method to find high- $Z$ materials was discussed and here this is extended in order to apply it to the areas of nuclear waste assay and treaty verifications. Material discrimination is a first step to identification, by comparing an unknown material to possible candidates, and finding the most likely material. Muons are highly penetrating particles, and undergo multiple Coulomb scattering in matter. In muon scattering tomography, cosmic muons are used as probes to scan containers, by measuring the muon tracks above and below the volume of interest, and obtaining a scatter angle distribution, as well as other correlated variables. A multivariate analysis was performed with these variables, in order to distinguish materials from different simulations with the same geometry. We know from previous work that it is possible to perform 3D imaging of concrete containers finding high- $Z$ materials inside, and their edges with a good resolution, so the analysis was performed by selecting the muon tracks that crossed the high- $Z$ volume. From these simulations, we show that it is possible to distinguish uranium blocks from lead, tungsten and plutonium blocks of the same size. The smallest blocks with a good discrimination were cubes with $2 \mathrm{~cm}$ side.

\author{
N08-17: Comparison of Prompt and Delayed Photofission Neutron Detection Techniques Using Different \\ Types of Radiation Detectors \\ $\underline{\text { P. Sibczynski }}{ }^{1}$, L. Swiderski ${ }^{1}$, M. Moszynski ${ }^{1}$, A. Syntfeld-Kazuch ${ }^{1}$, K. Grodzicki ${ }^{1}$, A. Dziedzic ${ }^{1}$, M. Matusiak ${ }^{1}$, T. Kosinski $^{1}$,

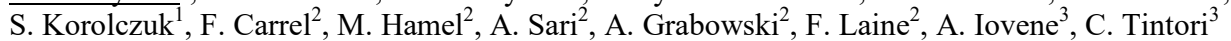 \\ ${ }^{I}$ National Centre for Nuclear Research, 05-400 Otwock, Poland \\ ${ }^{2}$ CEA LIST, F-91191 Gif-sur-Yvette, France \\ ${ }^{3}$ CAEN S.p.A., Via Vetraia 11, 55049 Viareggio (LU), Italy
}

For several years, detection of various threats on country borders plays a significant role in the frame of Homeland Security applications. One of this threat is the illicit trafficking of nuclear materials (especially including Special Nuclear Material - SNM ${ }^{235} \mathrm{U},{ }^{233} \mathrm{U}$ or ${ }^{239} \mathrm{Pu}$ ), which can be potentially used for production of nuclear weapon as well as radiological dispersal device (RDD) - known also as a "dirty bomb". In order to detect the potentially hidden nuclear material, systems using linear accelerators and a group of detectors are developed by several scientific groups around the world. Besides solutions focusing on detection of delayed ?-rays or neutrons, also the systems dedicated for prompt neutron detection were proposed. One of the possible prompt neutron detection technique is known as Threshold Activation Detection (TAD). This technique relies on activation of ${ }^{19} \mathrm{~F}$ nuclei in the scintillator medium by fast neutrons and registration of high-energy $\beta$ particles and ?-rays from the decay of reaction products (for example, ${ }^{19} \mathrm{~F}(\mathrm{n}, \mathrm{a}){ }^{16} \mathrm{~N}$ or $\left.{ }^{19} \mathrm{~F}(\mathrm{n}, \mathrm{p}){ }^{19} \mathrm{O}\right)$. Recent studies in the frame of the European Horizon 2020 C-BORD project showed that, despite the low ${ }^{19} \mathrm{~F}(\mathrm{n}, \mathrm{a}){ }^{16} \mathrm{~N}$ or ${ }^{19} \mathrm{~F}(\mathrm{n}, \mathrm{p}){ }^{19} \mathrm{O}$ reaction cross-section, the method could be a good solution for detection of shielded nuclear material. A benchmark of the TAD technique based on fluorine detectors with reference 
method focused on delayed neutron detection with ${ }^{3} \mathrm{He}$ detectors will be presented in this paper. These experimental results were obtained using a $9 \mathrm{MeV}$ linear accelerator.

This work was supported by the C-BORD H2020 project (Grant Agreement no 653323) for the inspection of large volume freight.

\section{N08-18: Peak Quantification with Neural Networks for Low-Resolution NaI Spectra}

M. M. Kamuda, J. Stinnett, C. J. Sullivan

NPRE, University of Illinois, Champaign, Il, USA

Gamma-ray photopeak detection for low resolution detectors is a key component of many isotope identification algorithms. Methods such as Gaussian fitting or derivative analysis are challenged by low count spectra, overlapping peaks, and Compton edges. We propose a neural network approach to peak detection and area quantification that can overcome these challenges while remaining computationally simple enough to be suited for handheld radioisotope identifier deployment.

\section{N08-19: Personal Dosimetry Geolocalized System for Radiation Monitoring}

$\underline{\text { R. Chil }}^{1}$, G. Konstantinou ${ }^{1}$, L. M. Fraile ${ }^{2}$, J. Vaquero ${ }^{3}$, C. Rodriguez ${ }^{3}$, S. Borromeo ${ }^{2}$, M. Desco ${ }^{1,4}$, J. M. Udías ${ }^{2}$, J. J. Vaquero ${ }^{1,4}$

${ }^{1}$ Dept. Bioingenieria e Ingenieria Aeroespacial, Universidad Carlos III de Madrid, Leganés, Madrid, Spain

${ }^{2}$ Grupo de Fïsica Nuclear, Universidad Complutense de Madrid, Madrid, Spain

${ }^{3}$ Dept. Tecnología Electrónica, Universidad Rey Juan Carlos, Móstoles, Madrid, Spain

${ }^{4}$ Instituto de Investigación Sanitaria Gregorio Marañón, Madrid, Spain

A geolocalized radiation monitoring system has been developed based on an small and portable personal dosimeter. This dosimeter uses a scintillator-SiPM combination for the radiation detection, it includes spectroscopy and isotope identification capabilities. The personal dosimeter uses a 9x9 $20 \mathrm{~mm} 3 \mathrm{CsI}: \mathrm{Tl}$ crystal and a $50 \mu \mathrm{M}$ SiPM. All measurements are time and location stamped, even for indoor locations. The system is designed in such a way that different wireless connectivity options are available. It can use Bluetooth, Wi-Fi, GSM, etc. depending on the application, and it also has the option of connecting to a mobile phone. When used for radiation monitoring over wide areas several networked dosimeters can provide a high sampling rate over an extended area simultaneously on fixed or mobile platforms. Three elements comprise the complete system: the dosimeters, a wireless gateway and a web-based remote storage, monitoring and management system (central system).

N08-20: Urban Source Detection with Mobile Sensor Networks Enhanced with Machine Learning Algorithms Z. Liu, C. J. Sullivan

Department of Nuclear, Plasma, and Radiological Engineering, University of Illinois at Urbana-Champaign, Urbana, IL, The United States

Mobile radiation sensor networks integrated with global positioning system coordinates provide an attractive option for the task of dynamically monitoring a region's background radiation and detecting the illicit movement of radioactive materials. It is important to estimate anomalous sources' position and location accurately. Both statistical and probabilistic approaches have been developed for stationary sensor networks to estimate source parameters. Due to the limitation of computation complexity, most of these algorithms assume background radiation is uniformly distributed and constant. These algorithms have low efficiency to solve multi-source problems. In our previous work, an algorithm called the BR-MLE algorithm was developed for mobile sensor networks to estimate the spatial and temporal distribution of background radiation. Following that work, a pre-filter framework for mobile sensor networks is presented in this paper that estimates multiple sources' positions and intensity under the fluctuating background radiation. The pre-filter framework combines the BR-MLE algorithm, a clustering algorithm, and a traditional maximum likelihood estimation algorithm together, and decomposes the computation intensive multi-source estimation problem into several single-source estimation problems. This offers an efficient alternative to traditional approaches where the multisource problems are difficult to solve and the background radiation distribution is not considered.

N08-21: Development of Nuclide Identifying Algorithm for PVT-Based Gate Monitor

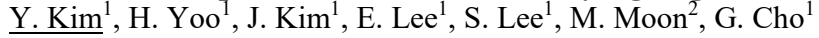

${ }^{I}$ Nuclear and Quantum Engineering, Korea Advanced Institute of Science and Technology, Daejeon, Republic of Korea

${ }^{2}$ Korea Atomic Energy Research Institute, Daejeon, Republic of Korea

Naturally occurring radioactive materials(NORM) such as bananas, fertilizer, cat litters and etc are major cause of nuisance alarm for radiation detection portal monitor(RPM) installed at the airports and harbours. The nuisance alarm caused by NORM wastes a lot of time and the careful inspection cost. To solve the problem, nuclide classifying algorithm to reduce nuisance alarms is essential. In this paper, conventional plastic scinitllator based RPM applicable nuclide classifying algorithm(NCA) is proposed. For the algorithm development, the gamma energy spectrum of the various gamma energy sources with the large- 
area(1800x720x50mm3) PVT(Polyvinyl-toluene) based plastic scintillator was measured. The obtained spectrum data was analyzed by the similarity analysis. For the evaluation of the developed NCA, the gamma energy spectra samples of the seven general calibration gamma sources were measured within less than 5.5 second because the speed of moving sources is specified $8 \mathrm{~km} / \mathrm{h}(2.2 \mathrm{~m} / \mathrm{s})$ at the ANSI for evaluation and performance of RPM for use in homeland security(N42.35). The obtained data was classified by developed NCA.

Measured sample spectra cannot be distinguish each other with the naked eye due to the similar tendency of the spectrum but they were classified exactly when the developed NCA was used. Although Ba-133, Co-57, Co-60 and Cs-137 spectra were measured during only 1 second, it was confirmed that they were exactly classified by developed NCA.

\author{
N08-22: Identification of ${ }^{90} \mathrm{Sr}$ and ${ }^{40} \mathrm{~K}$ Based on Cherenkov Radiation at Lower Background Suppressed \\ Cosmic Rays \\ $\underline{\text { H. Ito, }}$ A. Kobayashi, H. Kawai, S. Kodama, T. Mizuno, M. Tabata \\ Graduate School of Science, Chiba University, 1-33, Yayoicho, Inage, Chiba, Japan
}

A new detector, real-time ${ }^{90} \mathrm{Sr}$ counter, was developed with sensitivity of ${ }^{90} \mathrm{Sr}$ and insensitivity of ${ }^{137} \mathrm{Cs}$, ${ }^{40} \mathrm{~K}$ and Cosmic rays, based on Cherenkov radiation. The detector is threshold type Cherenkov counter using silica aerogel with a refractive index of 1.042. Since of the threshold energy of $1.31 \mathrm{MeV}$, the beta--ray from ${ }^{90} \mathrm{Y}$ can be identified. This detector would be applied for recovery Fukushima. By the Fukushima Nuclear Accident in 2011, particularly the fisheries were severely damaged due to radioactive contamination in Fukushima, Japan. Recent study is focus to radiation with longer half--life, ${ }^{90} \mathrm{Sr}$ and ${ }^{137} \mathrm{Cs}$, in the contaminated water. The performance of the detector was evaluated as an absolute efficiency using ${ }^{90} \mathrm{Sr}$, ${ }^{137} \mathrm{Cs}$ and $\mathrm{KCl}$ source; as the result, the efficiency of ${ }^{90} \mathrm{Sr},{ }^{137} \mathrm{Cs}$ and ${ }^{40} \mathrm{~K}$ are obtained ? $2.24 \pm 0.02 \times 10^{-3} \mathrm{~Bq}^{-1} \mathrm{sec}^{-1}, ? 1.27 \pm 0.23 \times 10^{-6} \mathrm{~Bq}^{-1} \mathrm{sec}^{-1}$ and less than $1.6 \times 10^{-4} \mathrm{~Bq}^{-1} \mathrm{sec}^{-1}$, respectively. The detector was estimated the detection limit of ${ }^{90} \mathrm{Sr}$ of $45 \mathrm{~Bq} / \mathrm{kg}$ for seafood and 1.6 $\mathrm{Bq} / \mathrm{kg}$ for seawater.

\author{
N08-23: Preliminary Results of a Nuclear Material Monitoring System Using Multiple Spectroscopes and \\ Anger Method

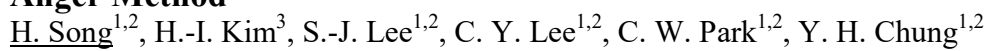 \\ ${ }^{I}$ Department of Radiological Science, College of Health Science, Yonsei university, Wonju, Gangwon-do, Korea \\ ${ }^{2}$ Institute of Health Science, Yonsei University, Wonju, Gangwon-do, Korea \\ ${ }^{3}$ Korea Institute of Nuclear Safety, Daejeon, Korea
}

Nuclear terrorism is recently considered as a critical threat in the international society. Therefore, diverse nuclear material monitoring system has been proposed to prevent the terrorism. To monitor nuclear materials in large area, we proposed a new nuclear material monitoring system using four gamma spectroscopy detectors and Anger method. The system can specify the position, energy and activity of the source. The performance of the system was evaluated using GATE (Geant4 Application for Tomographic Emission) simulation and the results were validated experimentally. In the simulation, $100 \mathrm{GBq}$ Cs-137 source was moved in the $X$ and $Y$ direction with $3 \mathrm{~m}$ intervals in $30 \ddot{i}_{i}^{1} 1 / 220 \ddot{i}_{i}^{1} 1 / 28 \mathrm{~m}^{3}$ volume. The four detectors were positioned at each upper corner of the measuring volume. In the simulation result, source position was specified with $2 \mathrm{~m}$ of accuracy and activity was agreed within $\ddot{i}_{i} 1 / 23 \%$ error range. The prototype system consisting of four $2 \ddot{i}_{i} 1 / 2 i{ }_{i} 1 / 2 \mathrm{NaI}(\mathrm{Tl})$ detectors was developed. The measured data was well matched with simulation result with $3 \%$ difference. The results demonstrated that the proposed system is expected to contribute to prevent nuclear terrorism.

N08-24: X-Ray Linear Laminography System for Void Inspection in Aluminum Welding

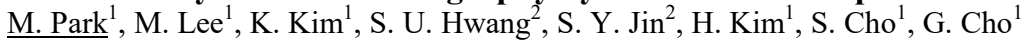

${ }^{I} 1$ Department of Nuclear and Quantum Engineering, Korea Advanced Istitute of Science and Technology, DAEJEON, KOREA

${ }^{2} 2$ Production Engineering Research Team, Samsung SDI, Yongin-Si, Gyeonhgi-Do, Korea

As in-line inspection of welded parts in various industrial fields is becoming crucial, there are requests for fast and efficient methodology for such nondestructive testing. 2D radiographic inspections, in general, suffer from poor image contrast and confounding noise due to the overlaid structures. An x-ray linear laminography system is proposed and implemented in order to overcome the challenges of digital radiography in void inspection of aluminum welding area. To validate the performance of our proposed system, we performed both Monte-Carlo simulation and real scan experiments. It has been demonstrated that the void detection up to an effective diameter of $50 \mu \mathrm{m}$ at critically important depth zones is possible for in-line inspection procedures.

N08-25: Development of Hybrid L-edge/XRF Densitometer: Fabrication and Evaluation $\underline{\text { S. Park }}^{1}$, U.-R. Park ${ }^{1}$, S.-W. Kwak ${ }^{1}$, A.-R. Lee ${ }^{1}$, J. Park ${ }^{2}$

${ }^{I}$ Korea Institute of Nuclear Nonproliferation and Control, Daejeon, Republic of Korea

${ }^{2}$ ISP Co., Ltd., Jeonju-si, Republic of Korea 
Assay of L-series of nuclear material solution is useful for determination of amount of nuclear materials and ratio of minor actinide in the materials. The hybrid system of L-edge densitometry and X-ray fluorescence spectrometry is one of the instruments for the assay. The hybrid L-edge/XRF densitometer (HLED) can be portable and compact from advantage of using low energy X-ray beams without heavy shielding systems and room temperature detector without liquid nitrogen cooling. The prototype of the equipment was fabricated and evaluated. In previous study, the equipment was designed and feasibility of measurement for nuclear material solution through Monte Carlo simulation. A surrogate material, such as led $(\mathrm{Pb})$ solution, is used for evaluation of performance of the instrument to avoid radiation effects from nuclear materials for evaluation of the equipment. The solution concentration could be obtained from L-edge densitometry and the characteristics of X-ray fluorescence of the material by incident low energy continuous X-ray spectrum $(<35 \mathrm{keV})$.

This work was supported by the Nuclear Safety Research Program through the Korea Foundation of Nuclear Safety (KOFONS), granted financial resource from the Nuclear Safety and Security Commission (NSSC), Republic of Korea.

N08-26: Neutron Activation in an Active Interrogation System

M. Cassinelli, C. Clemett, B. Campbell, C. Steer

NTR, AWE, Reading, Berkshire, UK

Active interrogation of cargo containers is a valid technique to screen for Special Nuclear Material. The use of a D2O converter allows a pure photon source to be augmented with photo-neutrons which can both cause fissions and activate cargo. The work presented here is a preliminary study into the neutron activation of cargo for such a system based on a Varian M9 LINAC. Results indicate that the neutron dose delivered to cargo ranges from $0.02 \%$ to $0.3 \%$ of the photon dose, and that the additional dose delivered due to activation products left in the cargo ranges from $0.0002 \%$ to $0.01 \%$ of this. In addition, it was concluded that the activation of food irradiated by such a system is unlikely to lead to significant levels of dose to those coming into contact with it, and that activated cargo is likely to lead to nSv levels of dose delivered to drivers transporting cargo around the country from ports.

ÂC British Owned Copyright 2016 / AWE

N08-27: Development of Portable SNMs Detection System Based on Threshold Energy Neutron Analysis

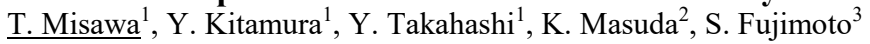

${ }^{I}$ Research Reactor Institute, Kyoto University, Kumatori, Osaka, Japan

${ }^{2}$ Institute of Advanced Energy, Kyoto University, Uji, Kyoto, Japan

${ }^{3}$ Pony Industry Co., Ltd., Kitakyuhoji-machi, Chuo-ku, Osaka, Japan

Detection of hidden special nuclear materials (SNMs) used for nuclear explosives such as U-235 is important issue for nuclear security against terrorism. Since U-235 emits only low intensity of gamma rays, several active interrogation systems used in seaports and airports have been proposed so far. However, those systems require highly intense neutron or photon sources and heavy shielding materials surrounding the detectors for reducing background neutrons and gamma-rays. To overcome those difficulties in active interrogation systems, we have been developing a portable interrogation system based on the Threshold Energy Neutron Analysis (TENA) technique. It is composed of a potable D-D neutron source by an IEC (Inertial Electrostatic Confinement) fusion system which is a compact and simple fusion device running by electrical discharge in D-D fuel gases and new neutron detectors called Tensioned Metastable Fluid Detector (TMFD). In TENA method, neutrons from a D-D source whose energy are $2.45 \mathrm{MeV}$ are injected into target suspicious object and neutron energy are measured by surrounding neutron detectors, then detection of neutron whose energy is more than $2.45 \mathrm{MeV}$ emitted by fission reaction means the existence of SNMs in the target. To detect high energy neutrons by rejecting $2.45 \mathrm{MeV}$ probing D-D neutrons and background gamma rays with high efficiency, newly developed TMFD is adopted, which is composed of organic liquid in supersaturation state by external tension and organic liquid is vaporized by detection of high energy neutron above threshold energy. The validity of those methods based TENA method was experimentally investigated by combination of a IEC D-D neutron source, enriched uranium and neutron detectors. The effectiveness of the methods also numerically investigated by MCNP Monte Carlo calculations to simulate a steel container with SNM which is surrounded by a D-D neutron source and neutron detectors.

N08-28: A New Method for the Determination of 241Am Activity for Large Site Contamination

E. Wilhelm $^{1}$, N. Arbor ${ }^{2}$, S. Gutierrez ${ }^{1}$, S. Ménard ${ }^{1}$, A.-M. Nourreddine ${ }^{2}$

${ }^{I}$ DIF, CEA, Bruyères le Châtel, France

${ }^{2} I P H C$, CNRS, Strasbourg, France

Airborne gamma-ray spectrometry system HELINUCTM is used for different missions like environmental control of radioactivity and nuclear emergency response. This type of equipment is well-developed for high energy emitters but less for low energy emitters. A new method for the determination of 241 Am activity for large site contamination is developed. This method is 
based both on statistical analysis of spectra and deconvolution of 241 Am signal with a reference library. Results are obtained on a site with artificial implemented contamination and on a real contaminated site. These results are compared to other method and show significant improvements in detection and estimation of 241 Am activity. This will improve the definition of the limits for contaminated area in case of accident or incident related to national security.

This work was supported by CEA - GIE Intra.

N08-29: Cylindrical Multi-Layer Neutron Monitor Using B4C Thin Film C. H. Lim 1 , J.-W. Park', J. H. Lee', J. Kim², S. Lee' ${ }^{2}$ J. H. Lee' ${ }^{2}$, M. K. Moon ${ }^{2}$

${ }^{I}$ Ocean System Engineering Research Division, Korea Research Institute of Ships \& Ocean Engineering, Daejeon, Republic of Korea

${ }^{2}$ Neutron Instrumentation Division, Korea Atomic Energy Research Institute, Daejeon, Republic of Korea

Nuclear material inspecting in the moving vehicle is carried out using neutron monitoring system and it is fabricated utilizing the $3 \mathrm{He}$ gas generally. However, necessity for alternative neutron reaction substance because of the lack of worldwide helium gas has increased. Therefore, developments of neutron detector of the alternative material by many research groups have been conducting and meaningful results are publishing in Journals.10B among alternative materials has many advantages as the high neutron cross-section, material stability, etc. So, we developed the neutron monitor with B4C thin film for monitoring of nuclear materials in the cargo truck. To increase the signal detection efficiency of the neutron monitoring system with B4C thin film for moving vehicle inspection, the monitoring system was designed to cylindrical feature. It is intended to minimize the change in signal collection efficiency according to the incident neutron direction. The neutron converter in the developed neutron monitor consist of B4C thin film of 6 layers. Before develop of monitor, we were calculated the neutron collection efficiency using Monte Carlo simulation. To perform the simulation, MCNP6 was used. Independent variables of the simulation model are the monitor height $(20 \sim 200 \mathrm{~cm})$ and the B4C thickness $(0.5 \sim 3.0 \mathrm{um})$. A B4C film was coated on a 200 um thickness aluminium substrate using Ar ion sputtering thin film coating machine. In order to estimate the performance of the neutron monitoring system, it was conducted using $252 \mathrm{Cf}$. The result of study, we confirmed the applicability of the cylindrical multi-layer neutron monitor with $\mathrm{B} 4 \mathrm{C}$ thin film for inspection of nuclear material in moving vehicle.

This research was a part of the project titled 'Development of Ubiquitous Technology based Security and Safety Systems for Shipping and Logistics' funded by the Ministry of Oceans and Fisheries, Korea.

N08-30: SPIR-Ace: a Novel Handheld Radio-Isotope Identifier

A. P. Fallu-Labruyere, F. Schulcz, J. Fellinger

Mirion Technologies (MGPI) SA, Lamanon, FRANCE

SPIR-Ace is a Novel Handheld Radio Isotope Identification Device (RIID) meant for Radioprotection, especially for Illicit Trafficking of Special Nuclear Material (SNM) protection in multi-modal fluxes of people and goods. It is based on proven core detection technologies deployed in the SPIR-ID instruments [1]. It comprises a NaI(Tl) scintillator (alternatively LaBr3(Ce)) coupled to a photomultiplier tube for spectroscopy. Dose rate is reported once the spectroscopy is saturated using a Geiger Muller tube. Neutron detector is achieved through novel LiZnS:Ag scintillators readout by a temperature compensated silicon photomultiplier, and instrumented by low power electronics. Spectrum is built using a two separate electronics operated in parallel for low power consumption, which also processes counts from the GM and nNeutron detectors. An ARM based processing board performs low range communication to the Android controller that performs gamma spectrum stabilization and Isotope Identification, through proprietary Spectrum Identification Algorithm (SIA). Local deconvolution is also performed for complex masking scenarios on sub-regions of the spectrum. Isotope libraries are loaded depending on the purpose of the inspection, namely post nuclear accident monitoring or illicit nuclear source trafficking protection. Instrument sensitivity exceeds those of the ANSI N42-34 [2] standard. In this paper, we report the instrument novel architecture and its key performance parameters.

References: [1]: SpiR-ID: a novel Handheld Radio-Isotope Identifier for Radioprotection and Illicit Trafficing Protection of Special Nuclear Material, poster presented at SORMA 2012. [2]: American National Standard Performance Criteria for Hand-Held Instruments for the Detection and Identification of Radionucleides, ANSI N42.34-2006 [3]: Technical Capability Standard for Handheld Instruments Used for the Detection and Identification of Radionucleides, Domestic Nuclear Detection Office (DNDO), October 2011.

N08-31: Surface Contamination Monitor, with Separate Alpha, Beta and Gamma Counting, Utilising Silicon Photomultipliers

I. C. Della-Rocca

Symetrica Security Ltd., Southampton, UK 
A compact surface contamination monitor (SCM) has been developed, which utilizes silicon photomultipliers (SiPMs), drastically reducing the volume and weight compared to currently available SCMs. The device uses a layered construction of scintillators to achieve separate alpha, beta and gamma ray counting. The lower layer consists of poly-vinyltoluene (PVT) and $\mathrm{ZnS}(\mathrm{Ag})$, whereas the upper layer consists only of PVT. Alpha particles are detected by their interactions in the $\mathrm{ZnS}(\mathrm{Ag}) \mathrm{layer}$ and separated from betas by pulse shape discrimination. Beta particles and gamma rays are differentiated on a rate comparison basis in the two separate layers of PVT. To achieve clear beta/gamma discrimination, a novel algorithm has been developed, which uses coincidence counting in the two PVT layers, and pulse height analysis. This leads to a beta/gamma discrimination ratio of better than $10^{-3}$. The utilization of SiPMs instead of the usual photomultiplier tubes, greatly reduces the volume and power consumption of the device. Other commercially available surface contamination monitors give either gamma only, or joint beta/gamma counts, owing to the similarity of their pulse-shape in scintillators. The advantage of giving separate alpha, beta and gamma counts is that in a high gamma flux environment, low activity beta emitting contamination should be clearly seen. This paper shows the results of early development work which demonstrates the alpha-beta and beta-gamma discrimination methods.

\author{
N08-32: Neutron Spallation to Enhance Muon Scattering Tomography \\ C. Eldridge \\ AWE plc, Reading, United Kingdom
}

Muon Scattering Tomography (MST) is a relatively young technology which relies on the Coulomb scattering undergone by cosmic ray muons as they pass through matter. If modules of detectors are placed around a volume then the particle tracks entering and exiting can be reconstructed. Using the scattering experienced by the muons passing through the volume, a three dimensional image of the space can be created. The scattering angle of a single interaction is dependent on the atomic number $(Z)$ of a material with the largest scattering occurring in very high $\mathrm{Z}$ material. This property has made the technique attractive for counter proliferation applications as it is particularly sensitive to heavy, fissile elements. The Atomic Weapons Establishment (AWE) has been pursuing research into muon scattering tomography with a focus on border security applications for the last six years.

Cosmic ray muons are known to stimulate both prompt and delayed neutron emission in heavy elements. The penetrative properties of these emitted neutrons along with their exclusivity to high $\mathrm{Z}$ material makes them a potential complement to existing MST imaging techniques for counter proliferation applications.

This work reports on the results of an experiment in which a muon scattering tomography system is utilised in combination with helium-3 detectors to correlate incident muons with the neutrons emitted from a tungsten mass. The potential of using neutrons to enhance the fissile material detection capabilities of muon scattering systems is also discussed.

\author{
N08-34: Strategies to Alleviate Aliasing in Coded Aperture Imagers \\ P. E. Vanier, I. Dioszegi, C. Salwen \\ NNS, Brookhaven National Laboratory, Upton, NY, USA
}

When a coded aperture imager is used to locate a single isolated source, it is desirable to identify the brightest pixel in the field of view and to calculate its statistical significance compared with the uncertainty in intensities of all the other pixels. Prior to deconvolution of the shadowgram to form an image, the detected events are usually binned into areas corresponding to the projected mask pixel areas. This binning process may result in misalignment of some events into areas that should be in shadow. In the worst case, the events transmitted by an open pixel may be shared by as many as four adjacent data bins. The statistical significance of the brightest spot could be reduced by a factor of four, unless offsets are used in the binning process to align the bins with the mask shadows. This effect is sometimes called aliasing. Choosing the offsets manually for each acquired image is tedious and somewhat subjective. We will describe two automated approaches to reduce the aliasing. In one method, all possible combinations of offsets are tested, and the combination that yields the maximum counts in the brightest pixel is chosen. For the second method, we subdivide the matrix representing the mask pattern into smaller areas, by factors of 4 or 16 . The shadow data are then binned into finer areas than before to match these subpixels.

\title{
N08-35: Development of Continuous Scanning for Fixed Type Container X-Ray Inspection System J. H. Lee, J. W. Park, C. H. Lim \\ KRISO, Daejeon, Korea
}

This study aims to develop a fixed type container inspection system (CIS) which is possible to scan continuously in order to enhance the efficiency of the system. The scanning time lagging due to standby time of vehicles is strongly related to the efficiency of the CIS, It is necessary to develop the continuous scanning fixed type CIS because the operation efficiency is proportional to the number of CIS sites and construction cost. Thus the continuous scanning fixed type CIS is designed and verified with ARENA simulation tool in order to enhance the whole operation time of the existing CIS. The parameters applied for the simulation are the times caused by building size, shielding door, vehicle transport system, and driver. As the result of the 
simulation, the extension of the Vehicle Transport System (VTS) rails in order to circle the VTS and 4 VTSs are required to realize the continuous scanning and the system is currently under construction.

\author{
N08-36: A Radiation Sensor Network with the Ability to Localize the Radiation Source \\ A. Kyriakis $^{1}$, G. Fragkos ${ }^{2}$, I. Kaissas ${ }^{3}$, K. Karafasoulis ${ }^{4}$, C. P. Lambropoulos ${ }^{5}$, C. Papadimitropoulos ${ }^{5}$, C. Potiriadis ${ }^{3}$ \\ ${ }^{I}$ Institute of Nuclear and Particle Physics, NCSR "DEMOKRITOS", Agia Paraskevi, Attica, Greece \\ ${ }^{2}$ R\&IT Directorate, Hellenic Army Genneral Staff, Cholargos, Attica, Greece \\ ${ }^{3}$ Greek Atomic Energy Commission, Agia Paraskevi, Attica, Greece \\ ${ }^{4}$ Hellenic Army Academy, Vari, Attica, Greece \\ ${ }^{5}$ Technological Educational Institute of Sterea Ellada, Psahna, Evia, Greece
}

A sensor network based on single crystal small factor CdZnTe or CdTe radiation detectors (non directional sensors) is described which has the ability to localize a radiation source in real time. In addition to the small factor detectors the network includes high performance $\mathrm{Cd}(\mathrm{Zn}) \mathrm{Te}$ as the directional detectors which can use either coded apertures or Compton imaging for the directional identification of a radioactive source. The capabilities of the non-directional sensor network have been studied using simulated data produced by SWORD (based on GEANT software). Using the simulation data, we have developed fusion algorithms for the localization of the source applying machine learning techniques, such as neural networks and boosted decision trees. Source location information from the directional detectors is used to restrict the search area and improve the localization accuracy. The effectiveness of the sensor network is evaluated using a testbed of $6 \mathrm{CdZnTe}$ sensors that transmit spectra data on a fusion host, where the fusion algorithms take place. The purpose of the network is to support homeland security and defend areas without specific entry or exit points even from potential radiological dispersion devices (RDD).

This research has been funded by the NATO SfP-984705, ESA Contract 4200014240 «CdTe crystallization and related compounds» and by the FP7-SEC-218000 COCAE project.

\title{
N08-37: Characterization of the $\$^{\wedge}\{11\} \$ B(d, n \$ \backslash$ gamma $\$) \$^{\wedge}\{12\} \$ C$ Monochromatic Photon Source for Active Interrogation
}

P. B. Rose Jr, A. S. Erickson

Nuclear and Radiological Engineering, Georgia Institute of Technology, Atlanta, Georgia, USA

Detecting special nuclear material (SNM), especially while in transit, is a challenge for homeland security. Shielding may further complicate detection of SNM when using traditional methods. Active interrogation can be utilized to probe through shielding uncovering illicit material. Monoenergetic radiation sources, such as ones based on low energy nuclear reaction, show promise for elevating SNM detection capabilities beyond traditional bremsstrahlung beam interrogation, in particular when elemental discrimination beyond the traditional low-, medium- and high- $Z$ is required. In this work we report advancements on the understanding of one of these monoenergetic sources, the accelerator driven $\mathbb{S}^{\wedge}\{11\} \$ B(\mathrm{~d}, \mathrm{n} \$ \backslash$ gamma $\$) \$^{\wedge}\{12\} \$ C$ reaction. This source is known to produce intense, highly penetrating gamma rays of $4.4 \mathrm{MeV}$ and $15.1 \mathrm{MeV}$, but it also produces an abundance of other high energy gamma rays. In addition, we analyze the effects of collimators and target mechanical parts on the produced signal. Detailed classification of the source is imperative for understanding of the physics taking place and how it can be exploited for active interrogation, paving the way for new possibilities in nuclear security and other fields where high energy monoenergetic photons are desired. We present a detailed analysis of experimentally observed gamma rays produced from this low energy nuclear reaction as well as their relative yields based on an incident $3.02 \mathrm{MeV}$ deuteron beam.

\author{
N08-38: Minimization of the Impact of Sensor Velocity on the Probability of Source Detection Using \\ Geographically Weighted Methods \\ M.-H. Jeong ${ }^{1}$, C. J. Sullivan ${ }^{2}$, M. Cheng ${ }^{1}$, S. Wang ${ }^{1}$ \\ ${ }^{l}$ CyberGIS Center, National Center for Supercomputing Applications, Urbana, IL, United States \\ ${ }^{2}$ Department of Nuclear, Plasma, and Radiological Engineering, University of Illinois at Urbana-Champaign, Urbana, IL, \\ United States
}

Location-aware mobile sensors have fundamentally changed the ways radiation levels are detected and tracked. These changes have raised many exciting research questions related to nuclear forensics. This paper investigates the relationship between a mobile sensors' velocity and radiation counts. The study compares the correlations of the sensors' velocity and measurment radiation counts with a source or without a source. Geographically weighted approaches such as a moving kernel are used to identify regional variations in the relationship between variables. The experimental results present that there is a negative correlation between sensors' velocity and radiation counts with a source, while there is no statistically significant correlation without a source. These results can be used to decrease false alarm rates in a geotagged sensor network. 
N08-39: A Comparison of Radiological Dispersal Device Deposition Characterization Techniques L. S. Erhardt ${ }^{1}$, L. E. Sinclair ${ }^{2}$, R. Fortin ${ }^{2}$

${ }^{I}$ Ottawa Research Centre, Defence Research and Development Canada, Ottawa, Ontario, Canada

${ }^{2}$ Geological Survey of Canada, Natural Resources Canada, Ottawa, Ontario, Canada

Following the detonation of a Radiological Dispersal Device (RDD), aerial gamma radiation surveys can provide a rapid determination of the extent and magnitude of the resulting radioactive contamination. Due to the near-homogeneous response of most gamma radiation survey instruments and the altitude of the aerial survey platform, the effective field of view of airborne gamma surveys can be hundreds of meters. The large field of view is used to advantage in aerial radiometric survey allowing for fastest possible full coverage of very large areas. However, it comes with the disadvantage of smearing out the spatial resolution of the measurements, resulting in over reporting of the spatial extent of the contamination and underestimation of peak concentration, which may have impact on tactical emergency response plans generated based on survey results. Aerial and ground-based survey data are compared for actual outdoor explosive dispersals of radioactive material. It is shown that although the derived peak contamination levels may vary by orders of magnitude, the measurements are consistent with each other. Understanding these fundamental differences in radiation survey techniques is important for situational awareness in the planning and conduct of an RDD emergency response.

\title{
N08-40: Compact Neutron Scatter Camera Search Applications
}

M. D. Gerling, J. E. M. Goldsmith, J. S. Brennan

Radiation and Nuclear Detection Systems, Sandia National Laboratories, Livermore, CA, USA

Neutron imaging has the potential to be a valuable tool in the search for localization and characterization of special nuclear material (SNM). In a recent campaign we attempted to demonstrate the ability of the Sandia developed mobile imager of neutrons for emergency responders (MINER) to discover SNM in a realistic environment consisting of several container stacks. MINER utilizes double scatter events in order to localize fission-energy neutrons, and with some additional limitations gammas. This bimodal imaging greatly increases the utility of the system, especially combined with neutron spectroscopy provided. Additionally, the system has a large volume of scintillator which provides singles rates (simple counting) well above most other even dedicated singles systems. These attributes combine to produce a versatile system that also provides unique contributions. We present results from a field test in and around a container stack under realistic conditions where MINER successfully located a variety of both neutron and gamma sources.

This work is supported by the National Nuclear Security Administration Office of Defense Nuclear Nonproliferation Research and Development. Sandia National Laboratories is a multi-program laboratory managed and operated by Sandia Corporation, a wholly owned subsidiary of Lockheed Martin Corporation, for the U.S. Department of Energy's National Nuclear Security Administration under contract DE-AC0494AL85000.

N08-41: Image Reconstruction of Radioactive Sources with a SCoTSS Compton Gamma Imaging Device $\underline{\text { P.-L. Drouin }}^{1}$, R. Ueno ${ }^{1}$, L. Erhardt ${ }^{1}$, J. Hovgaard ${ }^{2}$, B. Krupskyy ${ }^{2}$, A. MacLeod ${ }^{3}$, P. Saull ${ }^{3}$, L. Sinclair ${ }^{4}$, D. Waller ${ }^{1}$

${ }^{I}$ Defence Research and Development Canada, Ottawa, Ontario, Canada

${ }^{2}$ Radiation Solutions Inc, Mississauga, Ontario, Canada

${ }^{3}$ National Research Council Canada, Ottawa, Ontario, Canada

${ }^{4}$ Natural Resources Canada, Ottawa, Ontario, Canada

A Compton gamma imager is used for detecting, identifying and localizing sources of gamma-emitting radioactive material. This work follows the successful development of the SCoTSS (Silicon photomultiplier-based Compton Telescope for Safety and Security) laboratory prototype, aiming to develop a "mission-ready" imager for security, emergency response and military use. We have implemented and tested a number of image reconstruction techniques to determine their merits. Using a simulation of the SCoTSS imager performed with GEANT4 Monte Carlo, several image reconstruction algorithms were implemented: the twocone intersection method, List-Mode Maximum Likelihood Expectation Maximization method (LM-MLEM), and Stochastic Origin Ensembles method (SOE). The performances of the algorithms were quantified for several scenarios: a) a single point source, b) multiple point sources, c) a single moving source, and d) an extended source. All of the methods investigated show an improved precision on the localization of the radiation source for a single point source compared to the simple back-projection approach. Tracking of a source with a constant velocity was also demonstrated.

\author{
N08-42: Monte Carlo Simulations of a Physical Cryptographic Warhead Verification Protocol Using Nuclear \\ Resonance Fluorescence \\ J. R. Vavrek, A. Danagoulian, E. Immerman, R. S. Kemp, R. C. Lanza, R. R. Macdonald, B. Osmanov \\ Dept. of Nuclear Science and Engineering, Massachusetts Institute of Technology, Cambridge, MA, USA
}


Future multilateral nuclear arms reduction efforts will require technologies for the verification of treaty compliance. In particular, warheads slated for dismantlement will need to be verified for authenticity without revealing any sensitive weapons design information to international inspectors. Despite several decades of research, no technology has been able to meet these requirements simultaneously. Recent work at MIT has produced a novel physical cryptographic verification protocol that attempts to solve this treaty verification problem. The physical cryptographic protocol exploits the isotope-specific nature of nuclear resonance fluorescence (NRF) measurements to provide a strong indicator of the authenticity of a warhead. To protect against sensitive information leakage, the NRF signal from the warhead is convoluted with that of an encrypting foil containing the same isotopes as the warhead but in unknown amounts. The convoluted spectrum from a candidate warhead is then statistically compared against that from an authenticated template warhead to determine whether the candidate itself is authentic. This work presents the initial Geant4 Monte Carlo simulations of the physical cryptographic warhead verification protocol. Using a $2.7 \mathrm{MeV}$ endpoint bremsstrahlung beam, a template warhead is interrogated. Several hoax geometries are also compared against the template to show the protocol's robustness against cheating. In particular, an isotopic hoax in which weapons-grade plutonium is replaced with reactor-grade plutonium is shown to be detectable in realistic measurement times. An optimized geometric hoax that mimics the areal densities and attenuations of the authentic template warhead along one axis can also be detected. Results of the simulations as well as future research objectives will be presented and discussed.

This work is supported in part by the Consortium for Verification Technology under Department of Energy National Nuclear Security Administration award \#DE-NA0002534. This support does not constitute an express or implied endorsement on the part of the Government. The authors would like to thank Zachary Hartwig for his assistance with the computational infrastructure.

N08-43: MEBCIS: Multi-Energy-Betatron-Based Cargo Inspection System

$\underline{\text { A. Arodzero }}^{1,2}$, S. Boucher ${ }^{1}$, S. V. Kutsaev ${ }^{1}$, R. C. Lanza ${ }^{2}$, V. Palermo ${ }^{3}$, F. O'Shea ${ }^{1}$, V. Ziskin ${ }^{1,2}$

${ }^{I}$ RadiaBeam Technologies, LLC, Santa Monica, CA, USA

${ }^{2}$ Nuclear Science and Engineering, Massachusetts Institute of Technology, Cambridge, MA, USA

${ }^{3}$ Vertilon, Corp., Westford, MA, USA

The security market requirements for state-of-the-art mobile and portal radiography inspection system include high imaging resolution (better than $5 \mathrm{~mm}$ line pair), penetration beyond $300 \mathrm{~mm}$ steel equivalent, material discrimination (three groups of $Z$ ) at speeds up to $16 \mathrm{~km} / \mathrm{h}$ with $100 \%$ image sampling, low dose and small radiation exclusion zone. New research into radiography methods and systems has been actively pursued in order to achieve these challenging requirements. Recently, a significant fragment of the R\&D effort has been devoted to re-examining betatron based X-ray inspection systems. The advantages of the betatron-based inspection systems over conventional linac-based designs include small focal spot (which improves the resolution), low weight and form-factor, simpler control system and relatively low cost. The team lead by RadiaBeam Technologies is developing an innovative cargo X-ray scanning technique that promises to appreciably improve penetration and imaging capabilities. Our low dose mobile/portal Multi-Energy Betatron-based Cargo Inspection System, MEBCIS, will be able to provide radiographic scanning at speeds up to $17 \mathrm{~km} / \mathrm{h}$ with $100 \%$ sampling and material discrimination. The MEBCIS approach overcomes the limitations of cargo inspection systems with conventional dual energy interlaced betatron by use of the following key technologies: - Generation of X-ray pulses with lower- and higher- energies during the same betatron acceleration cycle, to avoid erroneous material discrimination due to cargo motion; - Real-time intelligent setting of energies of pulses depending on X-ray attenuation in cargo; - Scintillation-Cherenkov detectors with reduced sensitivity to scatter radiation; - Ultrafast detection with Silicon photomultipliers (SiPM) readout and automatic switching between photon-counting and integration operation modes depending on the attenuation in cargo. Application of these technologies maximizes material discrimination, penetration and contrast resolution while simultaneously reducing the dose.

N08-44: Uncertainty Quantification for Coded Aperture Quantitative Imaging A. M. Bevill, W. R. Martin

University of Michigan, Ann Arbor, United States

Radiation imaging systems provide a wealth of information for analyzing quantities of interest (enumeration, strength, dimensions) of an uncharacterized source. This capability is useful, for example, when measuring plutonium powder "held up" in unknown locations at reprocessing facilities. A characterization has far more impact when it is accompanied by a robust uncertainty estimate. In this work we analyze data from a coded-aperture imager to create a confidence interval for the total strength of a neutron source in a region of interest. The analysis approach relies on the convexity of the chi-squared residual; however the approach could be useful for some analyses in which the residual is not convex. The analysis uses barrier methods to solve the convex optimization problem. A solution bound and convergence criterion is calculated by evaluating the extrapolated chi-squared function with the Karush-Kuhn-Tucker conditions. A scripted implementation of the method solves problems with 500 datapoints and 1000 unknowns in 465 seconds on a typical laptop.

Profs. Brian Kiedrowski, Zhong He, and Jeffrey Fessler, and Drs. Keith Bledsoe, Paul Hausladen, and Matthew Blackston, provided mentorship and assistance on this work. 


\title{
N08-45: Design of a Rotational Modulation Collimator Utilizing Asymmetric Masks for the Gamma-
} Ray/Neutron Dual Imaging Technique

\section{H. S. Kim ${ }^{1}$, G. Lee ${ }^{2}$, S.-J. Ye ${ }^{1}$, G. Kim ${ }^{3}$}

${ }^{I}$ Program in Biomedical Radiation Sciences, Department of Transdisciplinary Studies, Graduate School of Convergence Science and Technology, Seoul National University, Seoul, Korea

${ }^{2}$ Electronic and IT Media Engineering, Seoul National University of Science and Technology, Seoul, Korea

${ }^{3}$ Department of Nuclear Engineering, Sejong University, Seoul, Korea

With the recent development of pulse shape discrimination (PSD)-capable scintillators, simultaneous detection and imaging of gamma rays and neutrons become feasible. As an attempt to develop gamma-ray/neutron dual imaging technique for the special nuclear material (SNM) detection, we build a fast and precise gamma-ray/neutron dual imager using RMC. Previous studies have shown that modulation patterns generated by utilizing RMC with symmetric masks will be identical if the source location is symmetric with respect to the rotational axis of the RMC, which makes distinction of symmetrically located two sources impossible. The purpose of this research is to solve the limitation of common RMC by designing an asymmetric mask, which can complement the drawbacks of the previous RMC design. We validated our asymmetric RMC design with Monte Carlo simulation (MCNP version 6.1). For the reconstruction of radiation images, the maximum-likelihood expectation maximization (MLEM) algorithm was employed. Based on the Monte Carlo simulation results, we generated the system matrix to implement the MLEM algorithm and the spatial distribution of the source was reconstructed. Based on the asymmetric design of the masks, we plan to fabricate RMC system using CLYC scintillation detector.

This work was supported by the Nuclear Safety Research Program through the Korea Nuclear Safety Foundation (KORSAFe), granted financial resource from the Nuclear Safety and Security Commission (NSSC), Republic of Korea (No. 1403024).

\author{
N08-46: Unfolding Large Plastic Scintillator Pulse-Height Data \\ N. E. Hertel ${ }^{1}$, E. A. Burgett ${ }^{2}$ \\ ${ }^{I}$ G. W. Woodruff School of Mechanical Engineering, Georgia Institute of Technology, Atlanta, GA, USA \\ ${ }^{2}$ Nuclear Engineering and Health Physics Department, Idaho State University, Pocatello, ID, USA
}

Large plastic scintillators are routinely used in portal monitors. Although the pulse-height spectra obtained are poorly resolved, plastic scintillator pulse-height spectra contain energy information. The potential to unfold the pulse-height spectra to obtain energy spectra and nuclide identification has been studied. A $30.48 \mathrm{~cm} \times 30.48 \mathrm{~cm} \times 5 \mathrm{~cm}$ plastic scintillator, coupled to a trapezoidal UVT light pipe $30.48 \mathrm{~cm}$ in length which in term is coupled to two PMTs, has been constructed at Georgia Tech. The signals from the PMTs are coincidenced to reduce noise and summed to provide the pulse-height spectrum. Pulse-height spectra were computed with MCNP for monoenergetic photons from $10 \mathrm{keV}$ to $2 \mathrm{MeV}$ in $50 \mathrm{keV}$ increments. From these monoenergetic photon responses, a response matrix incorporating the resolution of the plastic scintillator was constructed. Pulse-height spectra have been unfolded using the PTB MXD_MC33 unfolding algorithm employing the MAXED (Maximum Entropy Deconvolution Method) which requires a priori information, i.e. a starting spectrum. However, in the deconvolutions to be presented, the starting spectrum was input as a flat spectrum. The results of unfolding measured pulse-height spectra for the following radionuclides will be presented: Cs-137, Na-22, Co-60, Mn-54, and Am-241. The reconstructed gamma-ray energy spectra indicate that unfolding the pulse-height spectra from large plastic scintillators appears to be a viable approach to determining reasonable gamma-ray spectra without resorting to expensive high-resolution gamma-ray spectrometers.

\section{N08-47: Comparing the Performance of Portable Gamma Spectrometry Systems Based on the Quad-CdZnTe} Array

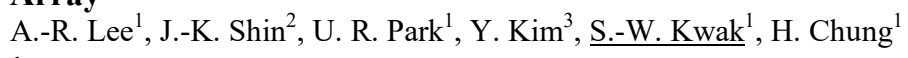

${ }^{I}$ Korea Institute of Nuclear Nonproliferation and Control, Daejeon, Republic of Korea

${ }^{2}$ Korea Institute of Nuclear Safety, Daejeon, Republic of Korea

${ }^{3} \mathrm{Nu}$ Care Medical Systems, Inc., Incheon, Republic of Korea

The present authors recently designed and built a new type of a portable gamma spectrometry system utilizing the Quad-CdZnTe (CZT) array, optimized for fast uranium enrichment verification of UF6 cylinders. The system consists of four CZT detectors, daisy-chained together and worked as a single detector, the related DAS (Data Acquisition System), and a detachable lead shield. Comparing to the previous system, the most featured improvements of this new system are to: 1) be placed extremely close to UF6 cylinders, thereby increasing the gamma flux incident on the system and 2) much easily transfer the system to any inspection points since the size and weight are relatively reduced. An algorithm to operate this new system and analyze the output signals were also upgraded in this study. The performance test was conducted with a $3.8 \%$ enriched uranium sample and the energy resolution was calculated using a Cs-137 source. The test results were compared to the results obtained from the previous system in order to provide an equal comparison of two systems. As a result, the new system showed higher energy resolution and efficiency than the previous system. 
N08-48: Assessment of near-Monoenergetic Photon Sources for Nuclear Security Applications

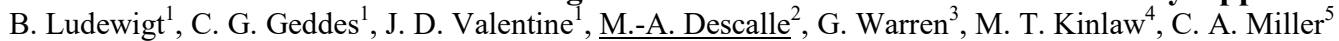

${ }^{I}$ ATAP Division, Lawrence Berkeley National Laboratory, Berkeley, CA, USA

${ }^{2}$ Lawrence Livermore National Laboratory, Livermore, CA, USA

${ }^{3}$ Pacific Northwest National Laboratory, Richland, WA, USA

${ }^{4}$ Idaho National Laboratory, Idaho Falls, ID, USA

${ }^{5}$ University of Michigan, Ann Arbor, MI, USA

Monoenergetic photon sources have the potential to provide significantly improved performance over existing bremsstrahlung based systems or enable new capabilities in radiography and active interrogation applications. Applications have been assessed including cargo screening and interdiction, detection of hidden SNM, treaty/dismantlement verification, nuclear safeguards, and emergency response. Estimates derived from simulations showed that dose reductions due to a narrow energy spread range from about $2 \mathrm{x}$ to $4 \mathrm{x}$ for radiography applications and can exceed a factor of 10 for photofission signatures. In particular, new laser Compton scattering photon sources currently under development promise unprecedented performance with selectable photon energies from $<1 \mathrm{MeV}$ to $20 \mathrm{MeV}$, energy spreads as low as $\sim 1 \%$, and $\sim 10^{\wedge} 8$ photons/pulse at repetition rates up to $10 \mathrm{~s}$ of $\mathrm{kHz}$. Main advantages include the ability to select the photon energy and to adjust energy spread, flux, and pulsing structure to deliver the photons needed for signature generation while suppressing extraneous dose and background that is associated with current bremsstrahlung sources. The narrow angular spread of the $\ddot{i}_{i}^{1} / 2$ pencili $i_{i}^{1} / 2$ beams makes it possible to deliver a high photon flux onto a target at a larger distance. Analysis has shown that scanning with a narrow beam can be of great benefit in radiography as it allows the scatter component in the radiograph of a thick object to be largely eliminated resulting in increased contrast and significant dose reduction beyond that due to narrow energy spread. Detailed simulation studies for quantifying capabilities and determining photon source requirements are in progress.

This work was supported by the Office of Nonproliferation and Verification Research and Development, National Nuclear Security Administration, and performed under the auspices of the U.S. Department of Energy by Lawrence Berkeley National Laboratory under Contract No. DE-AC02-05CH11231.

\section{N08-49: Detection Capabilities of KSr2I5:Eu2+}

E. Lukosi, C. Melcher, M. Rust

University of Tennessee, Knoxville, TN, USA

There is a current need for economical, yet high energy resolution gamma sensors for wide spread deployment in nuclear security applications. One promising new scintillator, KSr2I5:Eu2+, being developed at the University of Tennessee-Knoxville, has achieved excellent energy resolution of $2.4 \%$ at $662 \mathrm{keV}$ for a small crystal volume. Further, high quality crystal growth has been demonstrated many times faster for similarly performing scintillators, is very proportional, and has good detection efficiency. However, it also contains internal radioactivity due to the presence of $40 \mathrm{~K}$, which gives an internal radioactivity of $6.5 \mathrm{~Bq} / \mathrm{cm} 3$. To quantify the effects of the internal radioactivity on the detection and identification capabilities of KSr2I5:Eu2+, simulated and experimental data has been analyzed using two different approaches. The first method analyzed simulated external and internal background with a moving Cs-137 source to obtain proximity localization and time-to-detection information relative to a typical $\mathrm{NaI}(\mathrm{Tl})$ detector. Simulations also included $\mathrm{NaI}(\mathrm{Tl})$, CsI, and SrI2 with different energy resolutions and partial substitution of $40 \mathrm{~K}$ for its effect on its performance across a wide array of gamma-ray energies. Finally, laboratory investigations of various sources on these same detectors was used to benchmark simulations and provide tangible evidence on the performance of a moderately performing KSI detector. Results indicate that KSI has potential in nuclear security applications, but the intrinsic radioactivity limits its performance in certain energy ranges. Partial substitution of $40 \mathrm{~K}$ was found to dramatically improve performance and reduce the need for higher energy resolution to outperform other detectors.

\section{N08-50: Cylindrical Coded Moderator for Neutron Detection and Localization at Standoff}

\section{E. Lukosi, C. Haseler}

University of Tennessee, Knoxville, TN, USA

Due to the need for effective fast neutron detection at a large standoff with 360-degree localization, this work analyzes different coded moderator geometries using boron-coated straw thermal neutron detectors for the purpose of minimizing localization and time to detection while minimizing detection system complexity. Utilizing MCNP6 simulations, cylindrical moderator geometries with rotational symmetry about a central detector were demonstrated to have small uncertainty in a $360^{\circ}$ range while simultaneously decreasing the time-to-detection. By varying moderator thickness according to source angle and position on the detector axis, simulations have demonstrated the ability to locate a $1 \mathrm{MeV}$ neutron source equivalent to $8 \mathrm{~kg}$ of weapons-grade plutonium to $\pm 3^{\circ}$ at a $50 \mathrm{~m}$ standoff. Experimental verification of the simulated results was conducted by constructing a custom cylindrical coded moderator around a large $\mathrm{He} 3$ tube and used a $\mathrm{Pu} / \mathrm{Be}$ source. 
N08-51: Low Dose Source Trajectory Tracing Using Dynamic List Mode Reconstruction Algorithm Based on Dual-Modality Camera Setup

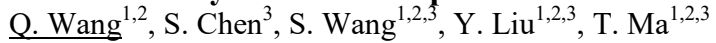 \\ ${ }^{I}$ Department of engineering physics, Tsinghua University, Institute of medical physics, Beijing, China \\ ${ }^{2}$ Ministry of Education (Tsinghua University), Key Laboratory of Particle \& Radiation Imaging, Beijing, China \\ ${ }^{3}$ Beijing Novel Medical Equipment Ltd, Beijing, China
}

Detection, localization, and characterization of radiation source has significance in the fields of nuclear safeguards and proliferation monitor. In large public space, limited system detection sensitivity and human motion lead to limited effectiveness for localization of low dose source carried by human. In this study, dedicated at low-count reconstruction and radiation source tracing, we propose human trajectory activity intensity reconstruction(TIR) algorithm based on dual-modality camera setup. Since we are mainly concerned with who emerges on site has maximum suspicion of carrying radiation source, trajectory with highest intensity reconstructed is reported with maximum suspicion of radiation source trajectory. TIR consists of two steps: 1) suspicious targets segmentation and their moving trajectories tracing; 2) trajectory intensity reconstruction based on dynamic list mode MLEM algorithm, while a derivate system matrix is implemented indicating possibility of a given detected event originating from a given trajectory. Not only trajectory number is relatively small compared to conventional static angle or voxel number, but also all detected events in certain time period are considered together in one reconstruction set of TIR. Thus TIR has better potential for low dose source tracing than conventional static reconstruction. Preliminary test includes: 1) analytical simulation of $100 \mu$ ci radiation source motion, with 3885 detected events in 10.5 seconds; 2) measurement of 1 mci human-carried radiation source motion, with 217 detected events in 22 seconds. Both studies show that compared to conventional static reconstruction, TIR has better low-dose source tracing performance with less bias and more effective convergence stability. Further study may concentrate on evaluation and optimization of TIR robust for tracing of source with different motion-model or lower dose.

N08-52: Optimization of a Silicon Beta Cell via Simulation

M. F. Mayer ${ }^{1}$, M. P. Foxe ${ }^{1}$, C. B. Sivels ${ }^{2}$, J. C. Hayes ${ }^{1}$, J. I. McIntyre ${ }^{1}$, R. Suarez ${ }^{1}$

${ }^{I}$ Pacific Northwest National Laboratory, Richland, WA, USA

${ }^{2}$ University of Michigan, Ann Arbor, USA

The measurement of radioactive xenon $\left({ }^{131 \mathrm{~m}} \mathrm{Xe},{ }^{133 \mathrm{~m}} \mathrm{Xe},{ }^{133} \mathrm{Xe},{ }^{135} \mathrm{Xe}\right)$ in the atmosphere is one of the primary approaches to monitoring for nuclear explosions. The ratios of these four radioxenon isotopes can provide the ability to discriminate between a nuclear explosion and other anthropogenic sources such as nuclear reactors. Measurements of these isotopes via beta-gamma coincidence can be improved by replacing more traditional scintillating plastic $\beta$-cells with silicon detectors that have a higher energy resolution. We report on simulations performed to optimize parameters such as cell volume and silicon thickness for increased detector sensitivity. The increased energy resolution will enable better isotopic identification and allow for more accurate source identification analysis. Initial simulations will be compared to experimental results using Canberra's PIPSBOX$2 \times 1200-500$.

\title{
N08-53: Low-Background Radioxenon Detector System
}

M. P. Foxe, J. L. Burnett, M. F. Mayer, J. I. McIntyre, C. B. Sivels

Pacific Northwest National Laboratory, Richland, WA, USA

The measurement of radioactive xenon (Xe-131m, Xe-133, Xe-133m, Xe-135) in the atmosphere is one of the main approaches to monitor nuclear explosions for verification. A low-background radioxenon beta-gamma detector is in operation in the shallow underground laboratory at Pacific Northwest National Laboratory. Measurements of the pure xenon isotopes allows for definitive determination of the region of interest and detection efficiency. Additional measurements of environmental samples provide insight into the potential limits of background for the radioxenon-monitoring regime. We report on the sensitivity limits of the low-background radioxenon detector system that is operated in the shallow underground laboratory at PNNL along with environmental measurement results.

N08-54: A New Square-Hole Based Coded Aperture Imaging Method

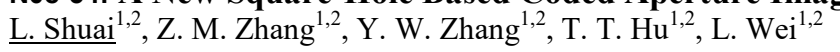

${ }^{I}$ Institute of High Energy Physics Chinese Academy of Sciences, Beijing, China

${ }^{2}$ Beijing Engineering Research Center of Radiographic Techniques and Equipment, Beijing, China

A square-hole based Coded Aperture Imaging (CAI) system is designed for far-field radiation source imaging. The CA is designed to be a $(4 \mathrm{~N}+1) \times(4 \mathrm{~N}+1)$ area mask with a $(2 \mathrm{~N}+1) \times(2 \mathrm{~N}+1)$ area square hole. In this method, a decoding function can be structured to ensure the point spread function been a d function. An imaging prototype is developed with a tungsten mask of 
$17 \times 17$ arrays and a BGO detector of $17 \times 17$ pixels. The mask is $8 \mathrm{~mm}$ thick with a $23.4 \mathrm{~mm} \times 23.4 \mathrm{~mm}(9 \times 9$ arrays $)$ hole.

Experimental result show that $28.8 \mu \mathrm{Ci}$ Cs-137 point source can be imaged within 100 s exposure time at 1 meter distance. Unlike conventional pinhole imaging, by sampling the projection of a large square hole as a coded arrays, a high resolution imaging can be restructured, meanwhile a highly detection efficiency is obtained. Compared to the widely used MURA array, the square-hole is more alterable. The coding numbers of a certain square-hole mask is decided by the pixels of detector. In addition the squarehole coded mask becomes more simple and easy to manufacture.

This work was supported by the Ministry of Science and Technology of China (2011YQ120096) and the National Natural Science Foundation of China (Grant no. 11205170, 11175200).

N08-55: Uranium Enrichment Determination Method for Quad_CZT Gamma-Ray Detector

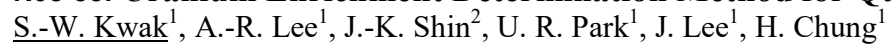

${ }^{I}$ Technology Research Division, Korea Institue of Nuclear Non-proliferation and Control, Daejeon, South Korea

${ }^{2}$ Technology Research Division, Korea Institue of Nuclear Safety, Daejeon, South Korea

The limitation of large-volume CZT crystal growth restricts detection efficiency of a CZT radiation detector and consequently increases measurement time for IAEA safeguards inspection activities. Four CZT crystals (named Quad-CZT array) are combined with an electronics to build a gamma-ray spectrometer with better detection efficiency than using a single CZT crystal. This new Quad-CZT array based gamma-ray spectrometer has prompted new analysis method development for uranium enrichment determination. Based on the enrichment meter principle, the developed uranium isotopic analysis method uses the $185.7 \mathrm{keV}$ of U-235 from spectral distribution obtained by the Quad-CZT array based gamma-ray spectrometer. The characteristics curve for uranium enrichment determination was derived using the net peak area of the U-235 $185.7 \mathrm{keV}$ which is dependent on the U-235 enrichment of measured nuclear materials. The relative error in a measured U-235 enrichment of UO2 pellets was about $2.0 \%$ when the suggested analysis method was applied. Using various nuclear materials, more detailed experiment will be conducted in future to characterize the developed CZT array-based detector and isotopic analysis method for it.

N09: Instrumentation for experimental reactors and nuclear power plants - Poster session I

Monday, Oct. $31 \quad$ 14:00-16:00 Etoile

N09-1: Development of a Gamma Detector Using Single Crystal Diamond for a Severe Environment

$\underline{\text { K. Ueno }}^{1}$, T. Tadokoro ${ }^{1}$, K. Sasaki ${ }^{2}$, M. Tsubota ${ }^{3}$, J. H. Kaneko ${ }^{3}$, R. Ohtani ${ }^{4}$, S. Koizumi ${ }^{4}$

${ }^{I}$ Research \& Development Group, Hitachi, Ltd., Hitachi, Ibaraki, Japan

${ }^{2}$ Services \& Platforms Business Unit, Hitachi, Ltd., Hitachi, Ibaraki, Japan

${ }^{3}$ Graduate School of Engineering, Hokkaido University, Sapporo, Hokkaido, Japan

${ }^{4}$ National Institute for Materials Science, Tsukuba, Ibaraki, Japan

After the accident at the Fukushima Daiichi Nuclear Power Station (NPS), the Japanese Government required reinforcement of heat and radiation resistances of gamma monitors used in NPSs. The reliability for severe environmental temperatures of more than $573 \mathrm{~K}$ can be improved by analyzing the dominant radionuclides in the atmosphere. Therefore, the authors have developed a gamma detector using a single crystal diamond with a wide band gap for this monitor. The dominant radionuclide in the atmosphere is estimated by the Compton distribution. The prototype detector was manufactured using a diamond element grown by Hokkaido University researchers. The element area and thickness were $5 \mathrm{~mm} \times 5 \mathrm{~mm}$ and $0.1 \mathrm{~mm}$, respectively. The electrodes were evaporated onto both sides by the electron beam evaporation method. The ohmic electrode was made of Ti/Au and the Schottky electrode was made of Pt. The element was installed in a detector housing of $20 \mathrm{~mm} \mathrm{f} \times 40 \mathrm{~mm}$ with a coaxial cable having high temperature resistance, and fixed with an epoxy also with high temperature resistance. The signal was analyzed by a preamplifier (PR16, EURORAD), an amplifier (572A, ORTEC), and a multichannel analyzer (MCA7700, SEIKO EG\&G). To evaluate the heat resistance, the temperature dependence of the count rate was measured from room temperature to $643 \mathrm{~K}$ using a ${ }^{137} \mathrm{Cs}$ gamma source. The count rate with the bias voltage of $-80 \mathrm{~V}$ was stable to $643 \mathrm{~K}$ within $3.0 \%$ full scale (F.S.). The Compton distributions were observed to $643 \mathrm{~K}$. No noise by heating was seen at $579 \mathrm{~K}$ or less; however, it appeared above 579 $\mathrm{K}$. To evaluate the radiation resistance, the dose dependence of the count rate was measured to 3 MGy using a ${ }^{60} \mathrm{Co}$ gamma source. The count rate at $3 \mathrm{MGy}$ was stable within $4.9 \% \mathrm{~F}$.S. Based on the results, the gamma detector with the diamond element was judged operable in a severe environment.

This paper includes some results obtained using the project "Development of diamond electric devices for nuclear power reactors with standing a severe accident" under the auspices of the Ministry of Education, Culture, Sports, Science, and Technology of Japan.

N09-2: The CdZnTe Detector with a Slit Collimator for Measure Distribution of the Specific Activity of Radionuclides in the Ground

V. E. Stepanov, A. G. Volkovich, V. N. Potapov, I. A. Semin, A. V. Stepanov, I. N. Simirskii 
Abstract: From 2011 in the NRC "Kurchatov Institute" carry out the dismantling of the MR multiloop research reactor. Now the reactor and all technological equipment in the premises of the reactor was dismantled. At the present time, measurements of radioactive contamination in the reactor premises are made. The most contaminated parts of premises - floor and the ground beneath it. To measure the distribution of specific activity in the ground the CdZnTe detector (volume 500? ? ${ }^{3}$ ) is used. The energy resolution of the detector on the line $662 \mathrm{keV}$ is $7.2 \mathrm{keV}$. Dimensions of detector housing: ø24mm, $\mathrm{L}=58 \mathrm{~mm}$. Detector placed in a lead shielding with a slit collimation hole. The upper part of shielding is made movable to close and open the slit of the collimator At each point carried out two measurements with open and closed collimator. For determination of specific activity of radionuclides in ground was developed software for calculation the coefficients of proportionality of specific activity to the corresponding the counting rates in the peaks of full absorption in the measured spectra. For determination of these coefficients the mathematical model of spectrometric system based on the Monte-Carlo method was used. Measurements of specific activity in ground are taken and are discussed. By results of measurements calculate the thickness of the removed layer of ground and the amount of radioactive waste.

\section{N09-3: Coordinated Control of HTR-PM Plant: From Design to Verification Z. Dong, X. Huang \\ Institute of Nuclear and New Energy Technology, Tsinghua University, Beijing, China}

HTR-PM plant is a multi-modular nuclear power plant based on pebble-bed modular high temperature gas-cooled reactor (MHTGR). Through adopting operation scheme of two nuclear steam supplying systems (NSSSs) driving one turbine set, the inherent safety feature of the MHTGR is applicable to the whole plant. Here, a NSSS is composed an MHTGR and a oncethrough steam generator (OTSG) that is side-by-side arranged by the MHTGR. The function of coordinated control is to regulate the nuclear power, helium temperature and steam temperature by adjusting the control rod positions, helium flowrate and feedwater flowrate so that the plant power supply can be well balanced with the demand. Since the two NSSSs of the HTR-PM plant are tightly coupled with each other by the commonly utilized turbine/generator, the variations of the state-variables of one NSSS can induce those of the other NSSS. Therefore, coordinated control is meaningful to provide safe, stable and efficient operation of the HTR-PM plant. In this paper, the design and verification of the coordinated controller for the HTR-PM plant is introduced, which lies in three aspects, i.e. theoretic analysis, numeric tuning and hardware-in-loop (HIL) verification. In the aspect of theoretic analysis, a novel method, i.e. the phyisically-based control design approach that provides globally asymptotically closed-loop stability for nuclear energy systems is introduced. In the aspect of numeric tuning, the code of numerical simulation and the process of controller tuning is given. Furthermore, in the aspect of HIL verification, both the simulation software and realtime simulation platform for of HTR-PM operation characteristics are introduced, and some preliminary HIL verification results are also shown.

\section{N09-5: Intense Fusion Neutron Source: DT Neutrons for Fusion and Beyond}

P. Agostini ${ }^{1}$, M. Angelone ${ }^{2}$, D. Bernardi ${ }^{1}$, P. Console Camprini ${ }^{1}$, M. Frisoni ${ }^{1}$, A. Pietropaolo ${ }^{2}$, M. Pillon ${ }^{2}$, A. Pizzuto $^{2}, \underline{\text { S. Fiore }}^{3}$ ${ }^{I}$ Department of Fusion and technologies for Nuclear Safety and Security, ENEA, Brasimone, Italy

${ }^{2}$ Department of Fusion and technologies for Nuclear Safety and Security, ENEA, Frascati, Italy

${ }^{3}$ Department of Fusion and technologies for Nuclear Safety and Security, ENEA, Rome, Italy

Within the framework of research and development on fusion technology, a $14 \mathrm{MeV}$ neutron source has long been considered as a key facility to perform irradiation tests supporting design and licensing of DEMO reactor. In this context, New Sorgentina Fusion Source (NSFS) project has been proposed taking advantage of well-established D-T generators technology, properly scaled in order to design a bright neutron source of some $10^{\wedge} 15 \mathrm{n} / \mathrm{s}$. It is based on multiple $200 \mathrm{keV}$ deuterium and tritium ion beams delivering several Amperes towards a deuterium-tritium enriched layer on a rotating target where fusion reactions take place. Tests on materials damage upon neutron irradiation are intended to be performed with the actual $14 \mathrm{MeV}$ neutron spectrum typical of fusion reactors. NSFS project is intended to be carried out within national as well as international collaborations in order to provide a fusion-related neutron source and possibly a multipurpose facility. In the present contribution, the main facility characteristics are highlighted along with expected irradiation performances. Possible implementations towards a multipurpose utilization in different research fields will be discussed.

\section{N09-6: Development of Compact Gamma Camera for Real-Time Radiation Monitoring at Nuclear Power Plants}


Gamma-imaging system is of great interest to monitor the contamination of radioactive substances in a wide range of areas at nuclear power plants. The purpose of this study was to develop a compact gamma camera capable of detecting and imaging the radioactive sources present in contaminated area. The proof-of-principle gamma camera with $3 \mathrm{~cm} \times 3 \mathrm{~cm}$ FOV consisted of pinhole collimator, SiPM based detector and dedicated electronics. Diameter, acceptance angle and channel height of the pinhole collimator were $1 \mathrm{~mm}, 25^{\circ}$ and $4.47 \mathrm{~mm}$, respectively. The detector block was composed of $2 \times 2$ matrix of detector modules, each of which consisting of a $4 \times 4$ array LYSO coupled to a $4 \times 4$ array SiPM. The 64:4 multiplexing circuit was designed to simplify the readout electronics and to reduce the number of channels being digitized. DAQ module equipped with 4 channel ADC and FPGA was also developed. The prototype gamma camera with $10 \mathrm{~cm} \times 10 \mathrm{~cm}$ FOV and improved energy resolution is also being constructed using newly designed pinhole collimator, GAGG scintillator having high light yield and no intrinsic radioactivity and 1024:4 multiplexing circuit. Flood image and energy spectrum were acquired to evaluate the performance of the proof-of-principle gamma camera. Spatial resolution and count rate as a function of distance from the radioactive source to pinhole collimator were also measured. High quality flood image was successfully acquired using the proof-of-principle gamma camera and each crystal pixel was clearly identified. Average energy resolution of 64 crystals was $18.1 \pm 2.0 \%$. Spatial resolution and count rate were $5.1 \mathrm{~mm}$ and $420 \mathrm{cps} / \mathrm{MBq}$ at a distance of $50 \mathrm{~mm}$ from the collimator. Preliminary experimental results indicate that compact gamma camera proposed in this study has the capability of real-time localization and imaging of radioactive sources.

N09-7: Compact HPGe Probe for Harsh Environment and in-Situ Spectroscopy

J. Clauss, M. Ginsz, J. Flamanc, B. Pirard, J.-B. Legras, V. Marian, P. Quirin, M.-O. Lampert

Canberra Specialty Detectors, Lingolsheim, France

This paper describes a novel HPGe-based spectroscopy system for in-situ measurement. It is able to withstand harsh environmental conditions with industrial reliability and fast deployment. This solution targets any application in which measurements under various weather conditions, contaminated areas, underground or submarine location are required. The detector is encapsulated within an ultra-high vacuum canister, coupled to a compact electric cryo-cooler and placed within a ruggedized external housing. A state-of-the-art DSP analyzer is used for the readout.

N09-8: Asymptotic Limits of a Realistic Oklo Reactor Model Criticality $\underline{\text { S. Bentridi }}{ }^{1}$, B. Gall ${ }^{2}$, F. Gauthier-lafaye ${ }^{1}$

${ }^{I}$ UDBKM, Laboratory of Energy and Smart Systems, University of KHEMIS MILIANA, Khemis-Miliana, Algeria

${ }^{2} \mathrm{CNRS} / \mathrm{UDS}$, Institut Pluridisciplinaire Hubert Curien, Strasbourg, France

The natural occurrence of criticality in the 2 billion years old uranium deposit of Oklo (Gabon) discussed and explained in previous works by the use of isocritical lines 1,2,3), representing the realistic critical configurations probably ignited in their geological environment, showed an asymptotic behaviour near low porosities (fig.1). Extended simulations with MCNPX were carried out on the basis of realistic critical configurations and interesting results were obtained on larger intervals of physical parameters to define the asymptotic limits of the critical radii of the critical initial cores.

N09-9: On the Design of a Remotely-Deployed Detection System for Reactor Assessment at Fukushima Daiichi $\underline{\text { A. R. Jones }}^{1}$, A. Griffiths ${ }^{2}$, M. J. Joyce ${ }^{1}$, S. Kamada ${ }^{3}$, J.-I. Katakura ${ }^{4}$, M. Katoh ${ }^{3}$, B. Lennox ${ }^{2}$, K. Okumura ${ }^{5}$, K. Nishimura ${ }^{3}$, D. Potts ${ }^{2}$, K.-I. Sawada ${ }^{3}$, S. Watson ${ }^{2}$

${ }^{1}$ Lancaster Univeristy, Lancaster, UK

${ }^{2}$ University of Manchester, Manchester, UK

${ }^{3}$ National Maritime Research Institute, Tokyo, Japan

${ }^{4}$ Nagoaka University of Technology, Niigata, Japan

${ }^{5}$ Japanese Atomic Energy Agency, Ibaraki, Japan

The premise behind this research is the design of a system that will allow fuel debris characterisation at Fukushima Daiichi. The precise location of the debris is not known for example as to whether it remains within the reactor pressure vessel or it has leaked through into the base of the pedestal below, additionally the state of the fuel is also in question as to whether this has corroded from within its encasing or if it is intact. The most likely scenario is a combination of all four of these situations. The flooding of the reactor floors immediately following the Fukushima accident adds an extra element of complexity for the detection system requiring it to be submersible and to hold any detector system in water tight confinement. The research carried out has involved extensive modifications to a previously-designed low-cost small-scale AVEXIS submersible inspection vehicle and the incorporation of a variety of radiation detectors. The latter has been designed to allow for mapping and determination of the situation that is present within the primary containment vessels. The challenges addressed with the detection system arise from the high dose rates that have been recorded around the reactor pressure vessels which can be as high as $1000 \mathrm{~Gy} / \mathrm{hr}$. In such a harsh environment not only will the radiation detectors struggle to operate but the components that make up the remote-operated vehicle are also likely to suffer radiation damage after only a relatively short period of time. The research presented here 
evaluates the components currently incorporated into the AVEXIS system in terms of their radiation tolerability as well as presenting the combination of detectors to be used in the remote probe for the investigation of the fuel debris.

This work was funded by the Engineering and Physical and Physical Sciences Research Council (EPSRC: EP/N017749/1) and MEXT (Japan).

N10: Nuclear Physics Instrumentation - Poster session I

Monday, Oct. $31 \quad$ 14:00-16:00 Etoile

N10-1: Hydrogen Beam Extraction of Penning Ion Source for Compact Neutron Generator

C. H. Lee ${ }^{1}$, D.-S. Chang ${ }^{2}$, B.-H. Oh ${ }^{2}$, J. Son ${ }^{1}$, Y.-K. Kim ${ }^{1}$

${ }^{I}$ Dept. of Nuclear Engineering, Hanyang University, Seoul, South Korea

${ }^{2}$ Korea Atomic Energy Research Institute, Daejeon, South Korea

Penning ion source was made for applying a compact neutron generator. Electrical and vacuum system was assembled to test the ion source. As a preliminary test, a hydrogen gas using a mass flow system was inserted to produce plasma into the ion source and the plasma was produced and the hydrogen ion beams were extracted successfully. Operation conditions such as anode voltage, pressure change, and extraction voltage were changed to extract a higher current of the hydrogen ion beams and the highest current was achieved to be $0.9 \mathrm{~mA}$ at $0.0021 \mathrm{mbar}(40 \mathrm{sccm})$ and $7.5 \mathrm{kV}$ anode biased voltage. Some details on the operation conditions of the penning ion source were discussed.

\author{
N10-3: Conversion Electron-Gamma Discrimination in Thick Silicon Detector by Signal Processing \\ B. Genolini, G. Andrea, V. David \\ IPN Orsay (CNRS-IN2P3-Univ. Paris South), Orsay, France
}

We show that we can achieve partial discrimination between gamma and conversion electrons events recorded using a 3-mm thick Silicon-Lithium detector with the analysis of the signals at the output of a charge preamplifier, using a method suitable for real time processing. We validated it with the particles emitted with a $207 \mathrm{Bi}$ source: we suppress more than $40 \%$ of the gamma events in the spectrum with the signal shape analysis. The method consists in calculating the decay time close to the leading edge with a linear regression, after subtracting the signal base line. As a secondary result, this algorithm distinguishes the double peak structures at 554 and $1048 \mathrm{keV}$. We also show that the combination of a low-pass filter and a simple trigger algorithm is sufficient to reach $5 \mathrm{~ns}$ RMS time resolution, which can improve the rejection by the coincidence with a reference detector.

N10-4: Silicon Pixel Telescope (SPT) Latest Developments and Results

$\underline{\text { J. M. Jose }}^{1}$, N. I. Rukhadze ${ }^{2}$, Y. A. Shitov ${ }^{2}$, L. Fajt ${ }^{1}$, I. Stekl ${ }^{1}$

${ }^{I}$ Institute of Experimental and Applied Physics, Prague, Czech Republic

${ }^{2}$ Joint Institute for Nuclear Research, Dubna, Moscow region, Russia

Experiment Silicon Pixel Telescope (SPT) aims to measure two neutrino double electron capture (2?EC/EC) in $106 \mathrm{Cd}$ using pixel detectors. One of the main challenges for double beta decay experiments is the background reduction. Pixel detector offer background reduction capabilities with its ability to identify the particle interaction (from the 2D signature it generates). SPT collaboration has been operating detector prototype (using eight Timepix pixel detectors) in the Modane underground laboratory. More than 8000 hours of data was collected and analyzed from the setup. A number of improvements on the detector system were also performed for the reduction of background events and improvement of data acquisition.

\title{
N10-5: Improvement of the performance of gamma-ray Broad Energy Germanium (BEGe) detectors using the pulse shape analysis for environmental applications \\ $\underline{\text { N. A. Ali }}$ \\ Physics Department, University of Liverpool, Liverpool, Merseyside, United Kingdom
}

The optimisation of High Purity Germanium (HPGe) detector performance for different gamma-ray interactions is an active area of research in the field of nuclear physics. For some commercial applications such as environmental sample counting, analysis of highly radioactive waste, the accurate characterisation of radionuclides within samples is required. HPGe detectors are the most often utilized detectors in such applications because of their excellent energy resolution. However, the shortcoming of these detectors is their poor Peak-to-Compton $(\mathrm{P} / \mathrm{C})$ background response that limits radionuclides with small activities in the sample to be easily detected. This drawback is mainly due to Compton scattering effect that leads to a significant continuum in the energy spectrum, which causes a considerable increase in the peak background. This project is, therefore, aimed at developing a direct method to improve the $\mathrm{P} / \mathrm{C}$ background response of germanium detectors, thus, increasing their sensitivity, which will benefit to different applications of these detectors. To achieve this, Digital Pulse Processing (DPP) techniques were applied to 
gamma spectroscopy measurements with a BEGe detector using environmental samples. Digitised detector traces from a digital acquisition (DAQ) system have been recorded for off-line analysis, which involved the application of rise time filtering techniques that allowed the $\mathrm{P} / \mathrm{C}$ ratios for the post-filtered energy spectra to be maximised.

\author{
N10-6: Diffusion Length of Rn-222 in Home-Stored CDs/DVDs -Influence on Rn-222 and Rn-220 \\ Measurement \\ I. S. Dimitrova $^{1}$, S. B. Georgiev ${ }^{1}$, D. S. Pressyanov ${ }^{1}$, K. K. Mitev ${ }^{1}$, T. A. Boshkova ${ }^{1}$, P. L. Vassileva ${ }^{2}$ \\ ${ }^{I}$ Sofia University, Faculty of Physics, Sofia, Bulgaria \\ ${ }^{2}$ National Center of Radiobiology and Radiation Protection, Sofia, Bulgaria
}

The CDs/DVDs stored indoors could serve as retrospective Rn-222 (radon) and Rn-222 (thoron) detectors. In the CD-method the disks are used as track-etch detectors of alpha-particles. The tracks at depths greater than $75 \mu \mathrm{m}$ below the disk surface are only due to radon that has diffused inside the material of the disks and can be used to measure radon. Because of its short half-life (55s), thoron does not diffuse in the disk volume, but can be measured using the track density in the interval $64-70 \mu \mathrm{m}$ below the disk surface, after a correction for the absorbed radon is made. This work studies the diffusion length of Rn-222 (average distance traveled by the atoms before they decay) in disks exposed in dwellings, which has influence on: 1) the calibration factor used in measurements of radon and 2) the detection limit and the estimate for the activity concentration of thoron in measurements by CDs/DVDs. The study is carried out with disks collected from dwellings and employs the in-depth decrease of the track density inside the disks to determine the effective diffusion length a posteriori (i.e. after the exposure in the dwellings). The observed values range from $45.4 \pm 2.0 \mu \mathrm{m}$ to $74.3 \pm 1.8 \mu \mathrm{m}$. The results are commented in view of their influence on the measurement of Rn-222 and Rn-220. Examples from the analysis of 62 disks collected from homes in an area with high natural radioactivity in Bulgaria are presented.

This work was supported by the European Atomic Energy Community's Seventh Framework Programme (FP7/2007-2011) under grant agreement Nr. 249689. The authors are grateful to Mr. D. Dimitrov for the help in disk processing.

N10-8: Module Architecture and Slow Control System of the FARCOS Telescopes

A. Castoldi ${ }^{1,2}$, C. Guazzoni ${ }^{1,2}$, T. Parsani ${ }^{1,2}$, D. L. Romeri ${ }^{1}$, C. Boiano ${ }^{2}$, G. Cardella ${ }^{3}$, G. Sacca' ${ }^{3}$, L. Acosta ${ }^{4,3}$, C. Serrano-Baza ${ }^{5}$ ${ }^{1}$ DEIB, Politecnico di Milano, Milano, Italy

${ }^{2}$ Sezione di Milano, INFN, Milano, Italy

${ }^{3}$ Sezione di Catania, INFN, Catania, Italy

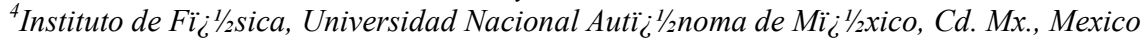

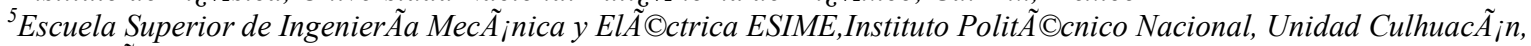
Culhuac $\tilde{A} ;$, Mexico

FARCOS (Femtoscope ARray for Correlation and Spectroscopy) is a novel modular detection system featuring high angular and energy resolution able to reconstruct the particle's momentum at high precision and capable of performing correlation measurements of LCPs and of LCPS and IMFs. The final system will be composed of 20 telescopes and the first prototypes will be ready to take data starting from 2017. A key point toward modularity and transportability is the module architecture together with its slow control. Each telescope features an active area of $6.4 \ddot{i}_{i} 1 / 26.4 \mathrm{~cm}^{2}$ and is composed of three detection stages: two silicon layers of DSSSDs acting as ?E stages and a calorimeter made of $\mathrm{CsI}(\mathrm{Tl})$ scintillators readout by a photodiode. A custom designed Kapton cable connects each face of each DSSSD to a custom board housing the frontend ASICs ( $2 \ddot{i}_{i}^{1 / 2} 16$ channel per side), the line-drivers and the slow control components. 4 of these boards are needed for each telescope and they form also the mechanical walls of the telescope. The local slow control is based on the microcontroller housed on the patch panel and performs the following monitoring and control functions: i) monitor ASIC and board temperature, ii) provides input test signals according to a given pattern for system calibration, iii) sets all the ASIC control bits. An 18 bits word is used for the slow control. All slow control bits are opto-coupled to prevent pick-ups from the digital section. A master microcontroller inside the vacuum chamber and communicating via IEEE 485 to the host PC oversees the slow control system.

This work has been supported by INFN in the framework of the NEWCHIM experiment

N10-9: A CMOS Frontend for CsI(TI) Scintillators Readout by Photodiodes for Nuclear Physics Experiments A. Castoldi ${ }^{1,2}$, C. Guazzoni ${ }^{1,2}, \underline{T}_{\text {. Parsani }}^{1,2}$

${ }^{1}$ DEIB, Politecnico di Milano, Milano, Italy

${ }^{2}$ Sezione di Milano, INFN, Milano, Italy

In the framework of the development of the fARCOS (Femtoscopy Array for Correlation and Spectroscopy) telescopes, we developed a CMOS frontend in C35B4C3 AMS technology for the readout of the photodiode current in CsI(Tl)-based calorimeters. The preamplifier features a continuous-reset feedback and an input PMOS transistor. The designed input dynamic 
range is $90 \mathrm{MeV}$ Silicon equivalent. The compensation capacitor at the gain node guarantees adequate GBWPs without shape distortion and a phase margin greater than $60^{\circ}$. The measured integral-non-linearity over the whole dynamic range is below $0.4 \%$. The preamplifer (intrinsic) rise-time $(20 \%-80 \%)$ is of the order of $10 \mathrm{~ns}$ over the entire dynamic range and keeps below $70 \mathrm{~ns}$ when coupled with the photodiode. The presentation will focus on the design of the charge preamplifier and on the results of the qualification of the circuit standalone and coupled with one of the FARCOS CsI(Tl) scintillator + photodiode assemblies.

This work has been supported by INFN in the framework of the NEWCHIM experiment.

N10-10: Radioprotection Study for Radioisotope Production with a Laser-Based Proton Accelerator $\underline{\text { P. Bellido }}^{1,2}$, M. Seimetz ${ }^{1}$, R. Lera ${ }^{3}$, A. Ruiz-de la Cruz ${ }^{3}$, M. Galán ${ }^{3}$, F. Sánchez ${ }^{2}, J$. M. Benlloch ${ }^{2}$, L. Roso $^{1}$

${ }^{1}$ Centro de Láseres Pulsados (CLPU), Salamanca, Spain

${ }^{2}$ Institute for Instrumentation in Molecular Imaging (I3M), Valencia, Spain

${ }^{3}$ Proton Laser Applications S.L., Barcelona, Spain

One of the most nearby applications of laser-based particle accelerators is the on-site production of radioisotopes. Laseraccelerated protons would be used as projectiles to induce nuclear reactions in the suitable target, similar to present cyclotron facilities.

Our group has studied in detail the different sources of radiological risk associated to a proposed, realistic production scenario. It comprises two parts. First, the laser-target interaction is considered, that involves the proton generation and the accompanying, mutli-MeV electrons. Then the laser-accelerated protons are guided to the secondary target to induce the specific nuclear reaction. An analytic-quantitative estimation of equivalent dose has been calculated. We have selected a set of different, broad electron spectra following a Boltzmann distribution for energies from $0.5 \mathrm{MeV}$ to $20 \mathrm{MeV}$. The total number of electrons is $10^{11}$ per shot. The scenario of radioisotope production requires 30 minutes of irradiation at $100 \mathrm{~Hz}$ repetition rate. In addition, a Montecarlo simulation has been performed simulating the same scenario in order to obtain a 3D dosimetric map of the system surrounding areas. The deposited dose due to the radiation activity of the radioisotope has been calculated analytically for the nuclear reaction ${ }^{11} \mathrm{~B}(\mathrm{p}, \mathrm{n}){ }^{11} \mathrm{C}$ assuming an activity growing linearly up to $0.5 \mathrm{GBq}$ during the irradiation time. This process will generate a total number of neutrons around $10^{14}$ which dosimetric impact has been adressed in a separate simulation. The results show that an equivalent dose of several $\mathrm{mSv}$ could be accumulated at one meter of distance during the irradiation period. Moreover, fast neutrons have to be stopped due to their large range in air and the high dose deposition due to the slow neutrons interactions have to been taken into account as well. Our study underlines the importance of radiation protection actions under these dosimetric conditions.

N10-11: Directional Reconstruction of Reactor Antineutrinos via Electron Scattering in Gd-Doped Water Cherenkov Detectors

D. Hellfeld $^{1}$, S. Dazeley ${ }^{2}$, A. Bernstein ${ }^{2}$, C. Marianno ${ }^{3}$

${ }^{I}$ Department of Nuclear Engineering, University of California, Berkeley, Berkeley, CA, USA

${ }^{2}$ Lawrence Livermore National Laboratory, Livermore, CA, USA

${ }^{3}$ Department of Nuclear Engineering, Texas A\&M Univeristy, College Station, TX, USA

Gadolinium-doped water Cherenkov antineutrino detectors at the kiloton and megaton scale are being considered for long-range remote monitoring of nuclear reactors. With such large sensitive radii, directional sensitivity in these detectors can be utilized to suppress backgrounds from multiple nearby reactors or possibly enable clandestine reactor search capabilities. We investigate the potential of the antineutrino-electron scattering interaction to reconstruct the direction of the incident nuclear reactor antineutrino flux. Signal and background were estimated assuming a $13 \mathrm{~km}$ standoff from a $3.758 \mathrm{GWt}$ light water nuclear reactor and detector response was modeled using a Geant4-based simulation package. The directional sensitivity was analyzed as a function of water-borne radon contamination, detector depth, and detector size. We present the experimental conditions required for $3 \mathrm{~s}$ significance.

N11: Scintillators I: Crystal growth

Monday, Oct. $31 \quad$ 16:30-18:15 Schweitzer

N11-1: In-Situ Diagnostics of Melting/Solidification and Segregation During Growth of Scintillator Crystals by Energy-Resolved Neutron Imaging

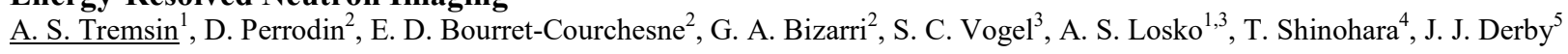

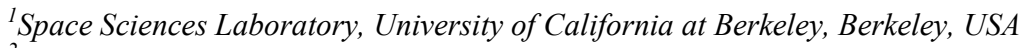

${ }^{2}$ Lawrence Berkeley National Laboratory, Berkeley, USA

${ }^{3}$ Los Alamos National Laboratory, Los Alamos, USA

${ }^{4}$ Japan Atomic Energy Agency, Naka-gun Ibaraki, Japan

${ }^{5}$ University of Minnesota, Minneapolis, USA 
Recent progress in high resolution energy-resolved neutron imaging provides unique possibilities to perform in-situ diagnostics of process parameters, which currently can be measured only indirectly (e.g. temperature, elemental composition and phase within the sample volume). Growth of gamma and neutron scintillator crystals can be optimized if parameters such as the shape of the solid/liquid interface or local dopant concentrations can be obtained in quasi-real time (in minutes or faster, since the crystal growth processes are typically very slow). This paper demonstrates the possibility to study in-situ the elemental distribution, shape and location of liquid/solid interface and structural defects in BaBrCl:Eu and Cs2LiLaBr6:Ce scintillator crystals grown by the Bridgman process. These compounds were chosen to represent two classes of halide materials currently under development for production as large crystals. The dopant concentration of the europium activator within a $\mathrm{BaBrCl}: \mathrm{Eu}$ ( $0.5 \%$ and $5 \%$ nominal doping concentrations per mole) scintillator as well as elemental distribution within Cs2 LaLiBr6:Ce crystal are mapped with sub- $\mathrm{mm}$ spatial resolution during crystal growth and cooling process. The strong tendency of the Eu dopant to segregate at the solid/liquid interface is observed in situ. Furthermore it is demonstrated that the Eu dopant distribution can be accurately quantified for low $(0.5 \%)$ and moderate $(5 \%)$ concentration levels in $\sim 12 \mathrm{~mm}$-thick samples. This new measurement technique enables optimization of growth parameters, such as thermal profile, growth and cooling rates and others. The finite element modeling of thermal profiles with feedback parameters measured in-situ can lead to a substantial improvement of resulting crystal quality and yield.

\author{
N11-2: Direct Interpretation of Bridgman Growth of Scintillator Crystals via Finite-Element Modeling and \\ Neutron Imaging \\ J. H. Peterson ${ }^{1}$, C. Zhang ${ }^{1}$, Y. Wu ${ }^{1}$, A. S. Tremsin ${ }^{2}$, D. Perrodin ${ }^{3}$, G. A. Bizarri ${ }^{3}$, E. D. Bourret ${ }^{3}$, A. S. Losko ${ }^{4}$, S. Vogel ${ }^{4}$, \\ M. Bourke ${ }^{4}$, J. J. Derby $^{1}$ \\ ${ }^{I}$ Chemical Engineering and Materials Science, University of Minnesota, Minneapolis, MN, USA \\ ${ }^{2}$ Space Sciences Laboratory, University of California, Berkeley, CA, USA \\ ${ }^{3}$ Lawrence Berkeley National Laboratory, Berkeley, CA, USA \\ ${ }^{4}$ Los Alamos National Laboratory, Los Alamos, NM, USA
}

For nearly a century, Bridgman crystal growth has been carried out in the dark. To be more precise, since the pioneering work of Bridgman in 1925, there have been no direct methods to observe what transpires within refractory crucibles that are moved through high-temperature furnaces during vertical Bridgman crystal growth. Indeed, we have only been able to infer the conditions of phase change and crystal growth indirectly, by measurement of discrete temperatures during a growth run, by observation of quenched interfaces after aborted runs, or via determination compositional profiles in the grown crystal. This latter approach has yielded particularly valuable understanding when interpreted in the context of crystal growth models and segregation theory. Even so, there has thus far been no means to directly validate the predictions of growth conditions provided by today's sophisticated computational models.

In companion research presented at this conference, Tremsin et al. discuss groundbreaking experiments that employ spallation neutrons to visualize, in situ, the compositional field that is developed during the growth of a mixed halide scintillator crystal via the Bridgman method. These measurements provide, for the very first time, a direct observation of melt crystal growth within a system large enough to be characteristic of an industrially relevant process. In this presentation, we apply finite element models to predict the macroscopic transport of heat, mass, and momentum along with phase-change phenomena in the crystal growth system employed in these neutron imaging experiments. Not only do the experimental observations serve to validate the modeling results, but, more importantly, the model provides a rigorous framework in which to understand the mechanisms that are responsible for the complicated evolution of interface shape and dopant distribution observed in the growth experiment.

Supported by U.S. DOE/NNSA DE-NA0002514 and U.S. DOE/NNSA/DNN R\\&D (LBNL subcontract AC0205CH11231); no official endorsement should be inferred.

\author{
N11-3: Impact of Post-Growth Temperature Treatment on the Triple Doped NaI: Tl, Eu and Ca Scintillation \\ Properties \\ I. Khodyuk, D. Perrodin, E. Bourret, G. Bizarri \\ Lawrence Berkeley National Laboratory, Berkeley, CA, USA
}

Co-doped NaI:Tl, Eu and $\mathrm{Ca}$ (NaI:TEC - $0.1 \%$ mole Tl, $0.1 \%$ mole Eu and $0.2 \%$ mole $\mathrm{Ca}$ ) is a promising alternative to existing low cost scintillation materials like NaI:Tl, CsI:Tl or CsI:Na in applications where better energy resolution and higher light output are important. We have previously shown that NaI:TEC exhibits greatly improved performance in comparison to that of a standard $\mathrm{NaI}$ (energy resolution below $5.0 \%$ at $662 \mathrm{keV}$ and light output above 52,000 $\mathrm{ph} / \mathrm{MeV}$ [1]). In this presentation, we will discuss the impact of post-growth annealing processes on NaI:TEC scintillation properties and how it relates to its performance. After annealing, a change in the x-ray excited emission spectrum is observed with the maximum of the emission band shifting from $470 \mathrm{~nm}$ to $450 \mathrm{~nm}$. This shift is connected with the perturbation and/or rearrangement of Eu/defects clusters in the NaI lattice. From the literature [2], the $450 \mathrm{~nm}$ emission is attributed to the presence of Eu2+-NaVac complexes while the $470 \mathrm{~nm}$ 
emission to that of Eu2+/(OH- or O2-) clusters. The spectral shift is paralleled to an improvement of the energy resolution and a degradation of the light output. This in time is followed by a slow process that tends to partly restore the initial state of the material. After more than 60 hours, a stable state is reached with constant light output and energy resolution. Crystals with initial "poor" performance benefit significantly from the treatment (improvement of about $10 \%$ in energy resolution and better luminosity) due to a probable homogenization of clusters and emitting centers throughout the materials. Our best as-grown materials, already uniform in distribution, didn't benefit as strongly from the treatment. This post-growth annealing process, and it potential to the control of the uniformity of the cluster and emitting center distribution, has the prospective to maximize the performance of large size crystals where non-uniformity is an important degradation mechanism.

This work was supported by the US Department of Homeland Security/DNDO and the US Department of Energy/NNSA/DNN R\&D and carried out at Lawrence Berkeley National Laboratory under Contract no. AC02-05CH11231. This work does not constitute an express or implied endorsement on the part of the government.

[1] Khodyuk et al. Journal of Applied Physics 118, 084901 (2015) [2] Shiran et al. Journal of Luminescence 164, 64-68 (2015)

\section{N11-4: High Throughput Growth of Scintillators by the EFG Method}

G. D. Calvert ${ }^{1}$, S. E. Swider ${ }^{2}$, M. R. Overholt ${ }^{2}$, R. S. Feigelson ${ }^{1}$

${ }^{I}$ Gebelle Labarotory, Stanford University, Stanford, CA, USA

${ }^{2}$ CapeSym, Inc., Natick, MA, USA

New scintillators such as SrI2(Eu) and Cs26LiYCl6(Ce) (CLYC) offer improvements in nuclear detection, such as highresolution gamma and dual-mode gamma-neutron detection, respectively. However, the high production cost and low throughput of the incumbent Bridgman method of crystal growth has restricted the availability of these materials, and impeded their adoption into mainstream detection technologies. Precursor and handling costs may be improved through scaling, but with the Bridgman technique, throughput becomes constricted by growth rates of less than 1 millimeter per hour. With edge-defined film-fed growth (EFG), crystals may be grown more rapidly. In this approach, crystal solidification proceeds from a free surface, which removes the possibility of defect formation at the ampoule wall. The lower density of surface defects in EFG-grown material allows the crystal to sustain higher levels of stress without cracking, as compared to the Bridgman method. High quality SrI2(Eu) crystals have been grown by EFG at rates 10 times faster than Bridgman-grown crystals. EFG also allows for near-net shape growth of cuboids that match the area of solid-state photomultiplier pads. We will share our latest results of EFG growth of SrI2(Eu) and CLYC. We will complement our discussion with 3D conjugate thermal models of the apparatus, and simulations of the stress fields in the growing crystals.

This work was supported by the U.S. Department of Homeland Security, Domestic Nuclear Detection Office, under the competitively awarded contract HSHQDC-15-C-B0040. This support does not constitute an express or implied endorsement on the part of the government.

N11-5: Bulk Crystal Growth and Scintillation Properties of 2 Inch Ce:La-GPS Single Crystal

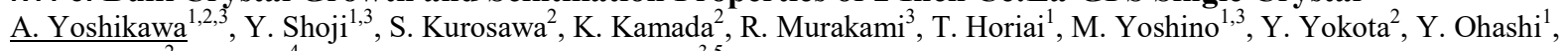
M. Arakawa ${ }^{2}$, M. Nikl ${ }^{4}$, V. V. Vladimir V.Kochurikhin ${ }^{3,5}$

${ }^{I} I M R$, Tohoku University, Sendai, Japan

${ }^{2}$ NICHe, Tohoku University, Sendai, Japan

${ }^{3}$ C\&A Corporation, Sendai, Japan

${ }^{4}$ Institute of Physics AS CR, Prague, Czech Republic

${ }^{5}$ General Physics Institute, Moscow, Russia

Ce-doped (La,Gd)2Si2O5 (La-GPS) crystals have high temperature stability of light output and FWHM energy resolution in the range from $0^{\circ} \mathrm{C}$ to $100-150^{\circ} \mathrm{C}$. Acceptable thermal stability of the luminescence was also demonstrated at temperatures up to $200^{\circ} \mathrm{C}$. Similarly, high thermal stability of this material synthesized in poly-crystalline form was noticed for the same temperature range. This property is very important for natural resource survey instruments and other radiation detection devices operated at elevated temperatures. In this study, we report the bulk crystal growth of 2 inch size single crystals of Ce:La-GPS. The composition of the host material was approximately equal to $\mathrm{La} 0.5 \mathrm{Gd} 1.5 \mathrm{Si} 2 \mathrm{O} 7$ and the concentration of the Ce3+-activator was either 0.5 or 1.5 at.\% with respect to the total content of the rare-earths forming the host crystal matrix. Effects of the hot zone construction including inductive coil position, presence/absence of the after-heater, rotation rate and other growth parameters on the crystal quality are discussed in some details. The crystals produced in optimized conditions were colorless, transparent, uniform in their shape, crack- and inclusions-free, and demonstrated smooth glass-like surface (What are the black points visible on the surfaces of several of the crystals). The length of the crystals' cylindrically-shaped body parts exceed $100 \mathrm{~mm}$. The growth results were well reproducible. It is found that the light yield of grown Ce :La-GPS is around 40,000 ph./MeV at room temparature and $35,000 \mathrm{ph} . / \mathrm{MeV}$ at $150 \mathrm{oC}$. 
This work is partially supported by Adaptable \& Seamless Technology Transfer Program through Target-driven R \& D (A- STEP), JST, Development of Systems and Technology for Advanced Measurement and Analysis, Japan Science and Technology Agency (JST) and New Energy and Industrial Technology Development Organization (NEDO).

\author{
N11-6: Directionally Solidified Ce Doped La(Br,Cl)3/AE(Br,Cl)2 (AE=Mg, Ca, Sr) Eutectic Scintillator for \\ High Resolution Radiation Imaging.

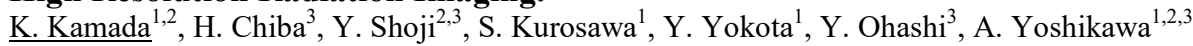 \\ ${ }^{I}$ NICHe, Tohoku University, Sendai, Japan \\ ${ }^{2}$ C\&A corp., Sendai, Japan \\ ${ }^{3} I M R$, Tohoku University, Sendai, Japan
}

Recently submicron-diameter phase-separated scintillator fibers (PSSFs) were reported and they possessed both the properties of an optical fiber and a radiation-to-light conversion. The PSSFs were fabricated using a directionally solidified eutectic (DSE) system. The DSE systems have been discovered in various materials for many applications. Up to now, $\mathrm{CsI} / \mathrm{NaCl}$ and GdAlO3/A12O3 have been already reported as PSSFs for high resolution X-ray imaging application. Ce:LaBr3 has attracted attention due to its high light yield of 61000 photons $/ \mathrm{MeV}$ and fast decay time of $25 \mathrm{~ns}$ with enough density of $5.1 \mathrm{~g} / \mathrm{cm} 3 \mathrm{for} x-$ ray and g-ray detection. In this research, exploration of PSSFs by directional crystal growth method will be reported. In this study, $\mathrm{Ce}$ doped $\mathrm{LaBr} 3 / \mathrm{CaBr} 2$ eutectics were explored. Crystal growth was performed by Bridgeman (BZ) method at the eutectic point. Investigations of their crystal structure and eutectic phase were performed. Luminescence and scintillation properties were also evaluated. Ce doped $\mathrm{LaCl} 3 / \mathrm{MgCl} 2, \mathrm{LaCl} 3 / \mathrm{CaCl} 2$, and $\mathrm{LaBr} 3 / \mathrm{CaBr} 2$ eutectics were grown by the $\mathrm{BZ}$ method in a quartz ampoule with $8 \mathrm{~mm}$ inner diameter. Mixed powders were induced into the ampoule under high purity Ar atmosphere in a glove box. Growth rate was $0.3 \mathrm{~mm} / \mathrm{min}$. Circular samples with $1-\mathrm{mm}$ thickness were obtained from the grown crystal. The eutectic showed well aligned eutectic structure with around $5 \mathrm{~mm}$ diameter $\mathrm{Ce}: \mathrm{La}(\mathrm{Ce}, \mathrm{Br}) 3$ fibers and optically transparent like bundled optical fibers. Grown Ce doped eutectics showed 350-360 nm emission ascribed to Ce $3+4 \mathrm{f}-5 \mathrm{~d}$ transition under X-ray excitation.Details on phase diagram, eutectic structure, scintillation properties and X-ray imaging result will be are showed in my presentation.

N11-7: High Granularity Scintillating Fibre Trackers Based on Silicon Photomultiplier

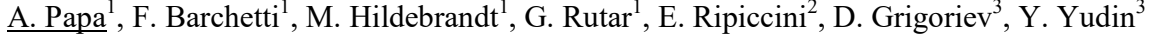

${ }^{I}$ Paul Scherrer Institute, 5232, Villigen, Switzerland

${ }^{2}$ CERN, 1211, Geneve, Switzerland

${ }^{3}$ Novosibirsk State University, 630090, Novosibirsk, Russia

Scintillating fibre detectors have already been realized in the past but the advent of Silicon Photomultipliers (MPPC) as photosensors and their coupling to thinner and thinner scintillating fibers opens a new era for such devices with dramatically improved performances and new challenges. Scintillating fibers coupled to MPPCs provide flexible, fast and high granularity detectors which are able to work in magnetic field and a high rate environment. We will report about the performances obtained with several detector prototypes (single and multi-layer array) based on $250 \mu \mathrm{m} \mathrm{BCF} 12$ multi-clad square scintillating fibers coupled to Hamamatsu MPPC S13360-1350C.

The aim of this work is to provide a tracker detecting minimum ionizing particles (m.i.p.) with a minimal amount of material (equivalent to a detector thickness below $0.4 \%$ of radiation length X_0) with full detection efficiency, timing resolutions below 1 ns and spatial resolutiona below $100 \mu \mathrm{m}$.

While expertise on scintillating fibers and MPPC has been around for a while the above demands put such a device at the detector frontiers. Current measurements show very promising results: a very high detection efficiency for m.i.p. with a single fiber layer $(=95 \%)$, and a full efficiency for multilayer configurations $(=99 \%)$; timing resolutions of the order of 500 ps (multilayer configuration); optical cross-talk between fibers at a negligible level $(<1 \%)$, for which spatial resolutions $<50 \mu \mathrm{m}$ are foreseen (multilayer configuration).

A comparison between the detector performances for m.i.p. and highly ionizing particle will be also given, showing the possibility of particle identification based on the large difference of the energy deposit on the scintillator by the two particles. We will show that this technology has the potential as a beam diagnostic tool at high beam intensity, providing beam profiles and rates in a single shot and appears to be a good candidate for a quasi-non-invasive monitoring device.

N12: High energy physics instrumentation I: Silicon

Monday, Oct. $31 \quad$ 16:30-18:30 Madrid

N12-1: Micro-Channel Cooling for Silicon Detectors

N. Flaschel

ATLAS, DESY, Hamburg, Hamburg 
In many high-energy experiments, the silicon sensors will need to be kept at very low temperatures. The main goals of cooling are to keep the leakage current low, to avoid thermal run-away as well as uncontrolled annealing of defects due to radiation damage in the sensors. This project aims at testing a method to reduce material and space needed for the cooling system. Microchannels etched into silicon are a very promising alternative to the current cooling strategy based on the use of bigger cooling pipes. DESY, in collaboration with IMB-CNM Barcelona, is investigating this method in a generic R\&D project.

A prototype micro-channel layout has been designed, produced and tested in collaboration with CNM. The micro-channels are etched into silicon using Deep-Reactive Ion Etching and are further fabricated using anodic and eutectic bonding. The samples have been implemented into a setup containing a Hydrofluoroether mono-phase coolant. To heat the samples, heaters matching the size of the sample surface were used. The temperature was measured using Pt100 temperature sensors for precise local measurements and an infrared camera to know more about the temperature distribution. To take effects of the ambient air into account, the tests were carried out in air and in vacuum and at a broad bandwidth of temperatures. At the same time the microchannels were simulated regarding the flow and the thermal properties.

\title{
N12-2: The SiD Detector for the International Linear Collider
}

$\underline{\text { R. Lipton }}$

Fermi National Accelerator Lab., Batavia, IL, USA

On behalf of the The SiD Consortium

The SiD Detector for the future International Linear Collider will deliver superb performance for high precision Higgs and Top measurements, and will have excellent sensitivity to a wide range of possible new phenomena. SiD features a compact, costconstrained design, with a robust silicon vertex and tracking system, which, combined with a 5 Tesla central solenoidal field, provides excellent momentum resolution. The highly granular calorimeter system is optimized for Particle Flow application to achieve very good jet energy resolution over a wide range of energies. Details of the proposed implementation of the SiD subsystems, as driven by the physics requirements, together with recent changes to the overall design and assembly procedures, will be given. Integration with the accelerator, the push-pull mechanism, and the assembly logistics at the Kitakami site will be described, with an estimated timeline for construction in relation to the overall ILC Project.

\author{
N12-3: The Timepix3 Telescope and Sensor Development for the LHCb VELO Upgrade \\ P. Collins \\ CERN, Geneva, Switzerland \\ On behalf of the $\mathrm{LHCb}$
}

The Vertex Locator (VELO) is the silicon detector surrounding the interaction region of the LHCb experiment. At the LHCb upgrade, planned for the LHC Long Shutdown 2, the VELO detector will be upgraded to a pixel system designed to withstand a radiation dose up to $370 \mathrm{MRad}$ or $\$ 8 \backslash$ times $10^{\wedge}\{15\} \$ 1 \mathrm{MeV} \mathrm{n} \$ \_\{\mathrm{eq}\} \$ \$ \mathrm{~cm}^{\wedge}\{-2\}$. An additional challenge is the non uniform nature of the radiation damage, which results in requiring a guard ring design with excellent high voltage control.

The Timepix 3 telescope is a high rate, data driven beam telescope created to study the performance of sensor prototypes for the VELO upgrade, and is also able to integrate external triggers in order to qualify other detector technologies. The telescope was installed in the SPS North hall at CERN, profiting from beam time provided by the AIDA2020 program.

The telescope consists of 8 planes with 300 um p-on-n silicon sensors read out by Timepix 3 ASICs. Tracks measured with the telescope have excellent temporal ( $\sim 1 \mathrm{~ns})$ and spatial resolution $(\sim 2 \mathrm{um})$. The telescope has been operated with a rate of tracks written to disk up to $5 \mathrm{MHz}$ - limited only by conditions at the SPS; we expect a rate capability up to approximately 30 Mtracks/s. At the center, where the spatial resolution is best, a central stage is provided which allows cooling and precise rotations and translations.

Sensors for the VELO upgrade have been produced with a variety of designs, including both \$n \$ and \$p\$-type, various guard ring designs and thicknesses, and from two vendors. The sensors have been irradiated to full fluence with both uniform neutron fluence and non uniform proton fluence, and tested with high energy particles at the CERN SPS. In addition the testbeam time precision has allowed detailed studies of timewalk in the various pixel designs.

The performance of the telescope and the software framework will be presented along with the latest results from sensor prototyping.

N12-4: FE65-P2: a Prototype Pixel Readout Chip in 65nm Technology for HL-LHC Upgrades

R. Carney ${ }^{1}$, M. Garcia-Sciveres ${ }^{1}$, D. Gnani ${ }^{1}$, C. A. Gottardo ${ }^{2}, \underline{T}$ Heim ${ }^{1}$, T. Hemperek ${ }^{2}$, L. Kashif $^{3}$, H. Krüger $^{2}$, A. Mekkaoui $^{1}$, V. Wallangen ${ }^{1}$

${ }^{1}$ Lawrence Berkeley National Lab, Berkeley, CA, USA

${ }^{2}$ University of Bonn, Bonn, Germany

${ }^{3}$ University of Wisconsin-Madison, Madison, WI, USA 
We present results of the FE65-P2 pixel readout test chip. This is a 64 by 64 pixel matrix on 50 um by 50 um pitch, produced in 65nm CMOS technology at the end of 2015. FE65-P2 was designed to demonstrate small pixel performance and stable operation down to 500 electron threshold even with the front end pixel amplifiers embedded in a synthesized logic environment. A layout scheme dubbed "analog islands in a digital sea" was implemented, where each set of four analog front ends (analog quad) is fully surrounded by synthesized logic, which is not a step and repeat layout, leading to a slightly different environment around each analog quad. A comprehensive substrate isolation strategy was used to achieve the required low stable threshold and excellent uniformity. FE65-P2 chips are being bump bonded to miniature matching sensors to allow full module performance studies. Test results will be presented including irradiation and beam tests. This work is carried out in the context of the RD53 collaboration, which is developing the pixel readout technology to cope with the high rate and radiation together with low noise and low power requirements for the High Luminosity LHC upgrades of the ATLAS and CMS experiments. The FE65-P2 results inform the ongoing design of a large format (400 by 192 pixels) demonstrator readout chip to be produced by RD53 in early 2017.

\title{
N12-5: The Phase-1 Upgrade of the CMS Pixel Detector
}

$\underline{\text { H. Weber }}$

Fermi National Lab, Chicago, USA

On behalf of the CMS Collaboration

The innermost layers of the CMS tracker are built out of pixel detectors arranged in three barrel layers (BPIX) and two forward disks in each endcap (FPIX). The original CMS detector was designed for the nominal instantaneous LHC luminosity of $1 \mathrm{x}$ $10^{\wedge} 34 \mathrm{~cm}^{\wedge}-2 \mathrm{~s}^{\wedge}-1$. Under the conditions expected in the coming years, which will see an increase of a factor two of the instantaneous luminosity, the CMS pixel detector will see a dynamic inefficiency caused by data losses due to buffer overflows. For this reason the CMS Collaboration has been building a replacement pixel detector which is scheduled for installation in an extended end of year shutdown during Winter 2016/2017. The Phase I upgrade of the CMS pixel detector will operate at full efficiency at an instantaneous luminosity of $1 \times 10^{\wedge} 34 \mathrm{~cm}^{\wedge}-2 \mathrm{~s}^{\wedge}-1$ with increased detector acceptance and additional redundancy for the tracking, while at the same time reducing the material budget. These goals are achieved using a new readout chip and modified powering and readout schemes, one additional tracking layer both in the barrel and in the disks, and new detector supports including a $\mathrm{CO} 2$ based evaporative cooling system, that contribute to the reduction of the material in the tracking volume. This contribution will review the design and technological choices of the Phase I detector, and discuss the status of the construction of the detector and the performance of its components as measured in system tests. The focus will be set on the onand off-detector electronics, reviewing the complete readout chain: beginning with the modules, the on-detector service electronics and power regulators, up to the frontend readout electronic boards. The discussion will include both dedicated studies of each component as well as tests of the full system readout. The detailed testing results, and the challenges encountered during the assembly will also be discussed.

N12-6: Module and Electronics Developments for the ATLAS ITK Pixel System C. Nellist

LAL-Orsay, Paris, France

On behalf of the ATLAS Collaboration

ATLAS is preparing for an extensive modification of its detector in the course of the planned HL-LHC accelerator upgrade around 2025 which includes a replacement of the entire tracking system by an all-silicon detector (Inner Tracker, ITk). The five innermost layers of ITk will comprise of a pixel detector built of new sensor and readout electronics technologies to improve the tracking performance and cope with the severe HL-LHC environment in terms of occupancy and radiation. The total area of the new pixel system could measure up to $14 \mathrm{~m}^{\wedge} 2$, depending on the final layout choice that is expected to take place in early 2017. An intense R\&D activity is taking place in the field of planar, 3D, CMOS sensors to identify the optimal technology for the different pixel layers. In parallel various sensor-chip interconnection options are explored to identify reliable technologies when employing 100-150 $\ddot{i}_{\zeta}^{1} \frac{1}{2} \mathrm{~m}$ thin chips. While the new read-out chip is being developed by the RD53 Collaboration, the pixel off detector read-out electronics will be implemented in the framework of the general ATLAS trigger and DAQ system. Simulations indicate that a readout speed of up to $5 \mathrm{~Gb} / \mathrm{s}$ per data link (FE-chip) is necessary in the innermost layers going down to $640 \mathrm{Mb} / \mathrm{s}$ for the outermost. Because of the very high radiation level inside the detector, the first part of transmission has to be implemented electrically with signals to be converted for optical transmission at larger radii. Extensive tests are being carried out to prove the feasibility of implementing serial powering chosen as the baseline for the ITK pixel system, given the reduced material in the servicing cables foreseen for this option.

\author{
N12-7: The Upgrade of the ALICE Inner Tracking System with the Monolithic Active Pixel Sensor ALPIDE \\ M. Mager \\ CERN, Geneva, Switzerland \\ On behalf of the ALICE Collaboration
}


The ALICE experiment at CERN LHC schedules a major upgrade of its apparatus for the long shutdown 2 of LHC in 2019/20. One of its pillars is the replacement of the inner tracking system by a detector entirely made of Monolithic Active Pixel Sensors fabricated in the $0.18 \mu \mathrm{m}$ TowerJazz process. It will comprise seven concentric layers around the beam pipe at radii from 2.2 to $40 \mathrm{~cm}$ and of lengths between 29 and $150 \mathrm{~cm}$, covering an ?-range $(90 \%$ most-luminous $)$ of \pm 1.22 , making a total active area of $10 \mathrm{~m}^{2}$ covered by 24,000 chips with $500 \mathrm{k}$ channels each. Its material budget is as low as $\mathrm{X} / \mathrm{X}_{0}=0.3 \%$ for the innermost three layers to limit the multiple scattering and to improve momentum and position resolutions especially for low-momentum tracks. It will be the first application of this technology at the LHC and only became feasible after an intensive R\&D program that led to the design of a new chip, the «ALPIDE», that pushes power consumption $\left(<35 \mathrm{~mW} / \mathrm{cm}^{2}\right)$, radiation tolerance (NIEL: $>1.7 \times 10^{13} 1 \mathrm{MeV} \mathrm{n}_{\mathrm{eq}} / \mathrm{cm}^{2}$, TID: $\left.>300 \mathrm{krad}\right)$, and integration/readout time $(<10 \mu \mathrm{s})$ to unprecedented values. Its latest full-scale prototype, «pALPIDE-3», includes all functionalities and has been thoroughly characterized at several institutes and test beam campaigns. It is now being integrated into larger detector building blocks.

This contribution summarizes the state of the key detector building blocks (mechanics, sensor, readout) and details results on the performance of the sensor as a standalone circuit as well as integrated into detector modules.

\section{N12-8: Characterization and Beam Test of the First Full-Sized DEPFET Modules for the Belle II Pixel Detector PXD \\ B. Paschen}

University of Bonn, Bonn, Germany

On behalf of the DEPFET Collaboration

The DEPFET collaboration is building a highly granular, ultra-transparent active pixel detector for high-performance vertex reconstruction at the Belle II experiment, KEK, Japan. A complete detector system is being developed, including solutions for ultra-thin sensors and their mechanical support, r/o ASICs, cooling, services, and a DAQ system capable of handling the huge amount of data coming from the pixel detector. The first production grade sensors have been finalized recently and modules with the latest set of read-out and steering ASICs have been assembled. The modules were extensively tested in the lab and two modules, one for each layer of the PXD detector, have been placed into an electron test beam at DESY. Together with the four double-sided strip detector layers of the SVD, the system under test was effectively a sector of the final vertex detector VXD of Belle II. This paper will focus on the lessons learned during the construction of the DEPFET pixel detector modules and present the test results obtained in the lab and in particular the performance of the system in a test beam environment.

\section{N13: Neutron detectors : Thermal Neutron Convertors}

Monday, Oct. $31 \quad$ 16:30-18:30 Londres

N13-1: Fast Neutron Detectors Based on Solid-State Single Crystalline and Multilayer Composite Scintillators

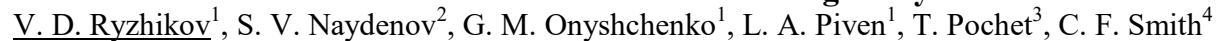

${ }^{I}$ Institute for Scintillation Materials, Kharkov, Ukraine

${ }^{2}$ Dept. Nonlinear Optic Crystals, Institute for Single Crystals, Kharkov, Ukraine

${ }^{3}$ DETEC-Europe, Vannes, France

${ }^{4}$ Naval Postgraduate School, Monterey, USA

We report on the development and analysis of two types of fast neutron and neutron-gamma detection media: (1) large volume inorganic oxide crystalline scintillators; and (2) large-area multilayer composite structures consisting of dispersed granules of small-crystalline scintillators contained in a transparent plastic matrix. The first type is based on solid-state single crystal ?WO, BGO and ZWO scintillators of large size (diameter 30-50 mm, length up to $100 \mathrm{~mm}$ ). For these large crystals, we have experimentally confirmed previous results demonstrating very high efficiency for fast neutron detection (up to 50\%), which were obtained earlier on small-sized samples of smaller $(10-12 \mathrm{~cm} 3)$ volume. Such larger-size scintillators are needed in order to increase detector sensitivity for the detection of very small quantities of neutron-emitting radioactive materials. To evaluate the possibility of suppressing the accompanying gamma-radiation when using these high-sensitivity neutron detectors, we have developed a spectrometric "windows" method for detection of fast neutrons against the background of intense gamma-radiation. In our subsequent search for new efficient and cost effective solid-state neutron detectors, we have developed and studied the second type of detection media: large area multi-layer composite detectors (named ZEBRA). We measured the sensitivity of a 40x100x100 mm multilayer ZEBRA detector (with a total thickness $40 \mathrm{~mm}$, and a scintillator cross sectional layer of area 100 ?m2) comprised of dispersed $\mathrm{GSO}(\mathrm{Ce})$ and found it to be comparable to the normal sensitivity of a 3??-detector with an area of $4000 ? \mathrm{~m} 2$. The efficiency of ZEBRA detectors is not lower than $50 \%$, i.e., approximately at the same level of detection achieved by single crystal scintillators. The cost of such composite detectors is projected to be substantially lower than that of neutron/gamma detectors based on conventional single crystals.

This research is supported by NATO's Emerging Security Challenges Division in the framework of the Science for Peace and Security Programme (Project SfP-984605). 


\section{N13-2: Development of a High Counting Rate 3He Curved MWPC for Neutron Diffraction}

B. Guerard, J.-C. Buffet, L. Chapon, J.-F. Clergeau, S. Cuccaro, A. Léandri, J. Marchal, G. Manzin, J. Pentenero, P. Van Esch, J.A. Rodriguez-Velamazan

ILL, Grenoble, France

The future XtremeD instrument, due to start operation at the ILL in 2019, will be equipped with a large area position sensitive detector able to sustain a global counting rate of $2 \mathrm{MHz}$, and a local counting rate of $150 \mathrm{kHz} / \mathrm{cm}-2$. A promising solution is to use a curved 3He 2D-MWPC made of independent sectors, each one equipped with individual readout electronics. This paper presents recent progress made in this way with a prototype of $14.4^{\circ} \mathrm{H} \mathrm{x} 17.8^{\circ} \mathrm{V}$ based on a new concept of curved cathode. The detector contains 96 horizontal curved Aluminum blades mounted with ceramic spacers at a pitch of $2.5 \mathrm{~mm}$, corresponding to a useful height of $24 \mathrm{~cm}$. The horizontal coordinate is measured with 96 anode wires mounted vertically at a pitch of $2 \mathrm{~mm}$; ceramic combs are used to define the position of the anode wires with a precision better than $0.1 \mathrm{~mm}$. The detector was tested with an Am-Be neutron source and with either CF4 or Ar-CO2 added to 3He; Ar-CO2 shows a clear advantage in terms of voltage operation compared to CF4, and our experience is that this gas is also more favourable concerning detector aging. For both gases, $99 \%$ of the events give an average time delay between $\mathrm{X}$ and $\mathrm{Y}$ around $50 \mathrm{~ns}$, indicating that the global counting rate capability per module will be $1 \mathrm{MHz}$. The TOT (Time-Over-Threshold) distributions show an average dead time around $1 \mu$ s. The average event multiplicity is 1.5 on the anodes and 4 on the cathodes. Combining the TOT average value together with the multiplicity average values, the expected local counting rate is $100 \mathrm{kHz}$ per cluster of 1.5 anode wires $\mathrm{x} 4$ cathode strips; this corresponds to $300 \mathrm{kHz} / \mathrm{cm} 2$. Preliminary results obtained with this prototype, and described in this paper, indicate that the proposed design fulfils the XtremeD detector requirements.

N13-3: Performance of a Large Area MCP Neutron Detector System $\underline{\text { R. A. Riedel }}{ }^{1}$, W. B. Feller ${ }^{2}$, V. N. Sedov ${ }^{1}$, X. Zhang ${ }^{1}$

${ }^{\text {I}}$ Oak Ridge National Lab, Oak Ridge, TN, USA

${ }^{2}$ NOVA Scientific Inc., Sturbridge, MA, USA

We report on the performance of a new large area $(15 \mathrm{~cm} \times 15 \mathrm{~cm})$ MCP based neutron detector. The neutron detector is comprised of a neutron sensitive MCP, a resistive plate anode and a delay line readout. The measurement electronics uses four readout channels to measure the time delay between signals in each of two dimensions. We measure an efficiency of about $10 \%$ for $4 \mathrm{~A}$ neutrons with a resolution of better than 500um. Pin cushion or barrel artifacts are not visible in distortion measurements.

N13-4: Cold Neutron Beam Conditioning with New Stacked Assemblies of Gd-Doped Glass Micron-Capillary Arrays for a Micron-Order Resolution Cold Neutron Microscope

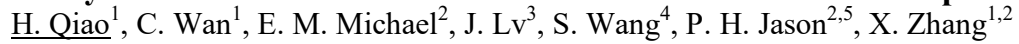

${ }^{I}$ School of Nuclear Science and Technology, Lanzhou University, Lanzhou, Gansu, china

${ }^{2}$ Nuclear Engineering Department, University of Tennessee, University of Tennessee, TN, USA

${ }^{3}$ School of Physics, Jilin Normal University, Siping, Jilin, China

${ }^{4}$ Institute of Nuclear Physics and Chemistry, CAEP, Mianyang, Sichuan, China

${ }^{5}$ Oak Ridge, Oak Ridge National Laboratory, TN, USA

Non-destructive, high spatial resolution, post-irradiation, nuclear fuel examination techniques are highly desirable for investigation of microstructure-level spent fuel properties in both the Chinese and American nuclear fuel R\&D campaigns. One particularly promising non-destructive nuclear fuel characterization technique is neutron radiography. However, for the current neutron radiography system two difficulties remain: 1) the reduced image quality caused by the use of thick shielding layers needed to mitigate the intense radiation emitted from used nuclear fuels, and 2) the limited spatial resolution of state-of-the-art neutron detectors. The end result is that resolution only slightly better than 100 microns has been achieved to date for this application. To overcome these difficulties, a next generation cold neutron microscope has been proposed and is under development at Lanzhou University in collaboration with North Night Vision Ltd. Co., Tsinghua University, and the University of Tennessee. This microscope is intended for use at the cold neutron beamline currently under construction at China's Spallation Neutron Source. It consists of three main components: a cold neutron beam collimator, cold neutron optics, and a micron-order resolution cold neutron detector. The neutron collimator is essential because the available cold neutron beams worldwide cannot fulfill the demands for realizing such a high resolution neutron imaging system due to their low L/D ratios. Our collimators are made of gadolinium-doped glass capillary arrays. The capillary arrays are made of different-sized (10, 6, and 3 microns) and shaped (circular and square) capillaries, each with lengths of $0.6,0.8$, and $12 \mathrm{~mm}$. With our new proposed stacking method of Gd-doped glass capillary arrays, even finer ( $=1$ micron) structural collimators can be made. Geant 4 simulation results and experimental validation of these results from measurements at ORNL's High Flux Isotope Reactor are described.

This work is supported by the National Natural Science Foundation of China (Grant no. 11275087, 10875054, and 11475077) and the U.S. Department of Energy, Office of Science, Office of Basic Energy Sciences, under Early Career Award no. DE-SC0010314. 
N13-5: Neutron Imaging Detector Based on Multiple Layers of Boron-Coated Straws ${ }_{\text {J. L. Lacy }}^{1}$, M. Regmi ${ }^{1}$, A. Athanasiades ${ }^{1}$, C. S. Martin ${ }^{1}$, G. J. Vazquez-Flores ${ }^{1}$, G. Ehlers ${ }^{2}$

${ }^{I}$ Proportional Technologies, Inc., Houston, TX, USA

${ }^{2}$ Oak Ridge National Laboratory, Oak Ridge, TN, USA

In previous projects funded by the DOE, Proportional Technologies, Inc. developed the basic design of a neutron imaging detector, based on the boron-coated straw technology, aimed to replace $3 \mathrm{He}$ tubes in large-scale neutron science instruments. Recent efforts have focused on automated production methods, including a critical 10B4C high volume sputter coating system, and automated in-line straw tube production system, in order to dramatically increase production capacity and reduce cost. A limited-scale prototype developed during Phase I of the project was operated successfully in the Cold Neutron Chopper Spectrometer (CNCS) at the SNS (ORNL) over a period of 6 months, with for more than 2500 hours logging more than 200 million events. The prototype demonstrated longitudinal spatial resolution of $5.5 \mathrm{~mm}$ (FWHM), and good image uniformity ( $2 \%$ ). A 5-layer deep, fully operational, imaging panel has been completed recently. The panel was installed in the CNCS instrument, and tested in real neutron scattering experiments. Results of detection efficiency over a range of neutron wavelengths, image uniformity, and time-of-flight distribution are reported.

This work was supported by the U.S. Department of Energy (DOE), under SBIR Award No. DE- SC0009615.

N13-6: The Multi-Blade Boron-10-based Neutron Detector for High Intensity Neutron Reflectometry at ESS

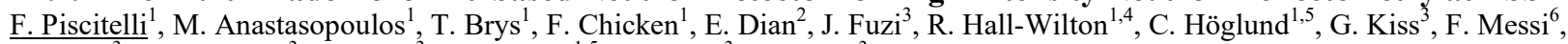

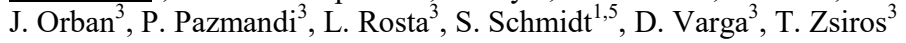

${ }^{I}$ European Spallation Source ERIC, Lund, Sweden

${ }^{2}$ Hungarian Academy of Sciences Centre for Energy Research, Budapest, Hungary

${ }^{3}$ Wigner Research Centre for Physics, Budapest, Hungary

${ }^{4}$ Mid-Sweden University, Sundsvall, Sweden

${ }^{5}$ Thin Film Physics Division, Linköping University, Linköping, Sweden

${ }^{6}$ Physics Department, Lund University, Lund, Sweden

The Multi-Blade is a Boron-10-based detector conceived to face the challenge of the counting rate capability arising from the neutron reflectometry at the European Spallation Source (ESS). The current detector technology is reaching fundamental limits in counting rate capability and position resolution. The problem with count rates is a general one, and the ESS solution could potentially be applied to existing instruments at other neutron sources. The work on the Multi-Blade began in 2011 at the Institut Laue-Langevin (ILL) where two technology prototypes were built and tested showing promising results. The European Union is now sponsoring the Multi-Blade detector through the BrightnESS project that aims to realise detectors optimized for these high rates. The Multi-Blade design has been improved and a new demonstrator has been built and tested within the collaboration of ESS, Lund University and the Wigner Research Centre for Physics, Hungary. It has been shown that aside from the improvement in counting rate capability, the Multi-Blade design also decreases the spatial resolution by about a factor three over state-of-theart helium-3-based reflectometer detectors. These and other results including the path ahead for this project will be presented. The Multi-Blade design is one of the favored as a development path to be pursued for reflectometry at ESS.

N13-7: Tailoring the Coating Properties for Use in Boron-Based Neutron Detector Applications

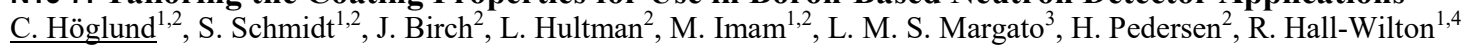

${ }^{I}$ European Spallation Source ERIC, Lund, Sweden

${ }^{2}$ Thin Film Physics Division, Linköping University, Linköping, Sweden

${ }^{3}$ Departamento de Física, Universidade de Coimbra, Coimbra, Portugal

${ }^{4}$ Department of Electronics, Mid-Sweden University, Sundsvall, Sweden

During the past few years, boron-10-based detectors were shown to be a competitive alternative to helium-3-containing neutron detectors. Here, high quality coatings are essential, where the primary material choice is boron-10 enriched boron carbide. We have previously reported these coatings to be long lasting, uniform in thickness, well adhering to aluminum substrates, and to contain a high amount of boron-10. With an increased number of detector designs and the vision to miniaturize the neutron detector technology, sets of new requirements have become important. We show that it is possible to tailor the growth parameters and the coating properties to fulfill the increased variety of needs. For this we expand the deposition toolbox to contain not only direct current but also high power impulse magnetron sputtering processes as well as high and low temperature chemical vapor deposition processes. The coatings are characterized using state-of-the-art coating diagnostics and neutron scattering techniques. Examples of solutions that are presented are how to lower deposition temperature for temperature sensitive substrates, how to minimize residual stresses to avoid substrate bending and to improve the film-to-substrate adhesion, and how to deposit onto previously unexplored substrate materials. Special focus is put into tailoring the surface resistivity, aiming to realize boron-10based resistive plate chambers for position sensitive neutron detectors with sub-millimeter resolution. 
N13-8: Design and Testing of the Multi-Grid Boron-10 Demonstrator on the Time-of-Flight Spectrometer CNCS

$\underline{\text { A. Khaplanov }}^{1}$, M. Anastasopoulos ${ }^{1}$, R. Bebb ${ }^{1}$, J. Birch ${ }^{2}$, T. Brys ${ }^{1}$, F. Chicken ${ }^{1}$, J.-F. Clergeau ${ }^{3}$, J.-C. Buffet ${ }^{3}$, G. Ehlers ${ }^{4}$, P. Van Esch $^{3}$, B. Guerard ${ }^{3}$, R. Hall-Wilton ${ }^{1,5}$, I. Lopes-Higuera ${ }^{1}$, L. Hultman ${ }^{2}$, C. Hoglund ${ }^{2}$, I. Iruretagoiena ${ }^{1}$, F. Issa ${ }^{1}$, F. Piscitelli ${ }^{1}$, L. Robinson ${ }^{1}$, S. Schmidt ${ }^{1,2}$, I. Stefanescu ${ }^{1}$

${ }^{I}$ Detector Group, European Spallation Source, Lund, Sweden

${ }^{2}$ Thin Films Group, Linkoping University, Linkoping, Sweden

${ }^{3}$ Institute Laue Langevin, Grenoble, France

${ }^{4}$ Spallation Neutron Source, Tennessee, USA

${ }^{5}$ Mid-Sweden University, Sundsvall, Sweden

The Multi-Grid (MG) detector for thermal neutrons has been introduced at ILL and developed by a collaboration between ILL, ESS and Linkoping University. It is aimed to provide a lower-cost alternative to the detectors based on the scarcely-available He3 for applications where a detector area of 10 s of square meters is needed, in particular, time-of-flight neutron spectrometers at the ESS. The MG utilises \$ $\backslash \mathrm{mu} \$ \mathrm{~m}$-layers of boron carbide enriched in B-10 as the neutron conversion medium, combined with the wire proportional chamber gas readout. In order to validate this new detector technology for the use at the instruments of the ESS, a demonstrator has been designed to operate in the Cold Neutron Chopper Spectrometer, CNCS at the SNS, ORNL. In this demonstration, data will be collected in parallel with the He-3 detector array, allowing a direct comparison between the old and the new technologies in terms of timing and position resolution, efficiency and background. This work is a part of a effort within the BrightnESS project aimed to enhance the performance of detectors available for neutron scattering science and to widen the available detector options.

\section{N14: Synchroton radiation and FEL instrumentation I}

Monday, Oct. $31 \quad$ 16:30-18:00 Churchill

N14-1: The DSSC Detector for the European XFEL: Overview and Experimental Results on the Prototype System

M. Porro

European X-Ray Free-Electron Laser Facility GmbH, Hamburg, Germany

On behalf of the DSSC Collaboration

Among the developments for the European XFEL, the DSSC will be the only 2D large-area high-speed detector able to achieve single photon resolution even in the low energy range down to $0.25 \mathrm{keV}$. The camera is based on Si-sensors and is composed of $1024 \times 1024$ hexagonal pixels for an active area of $210 \times 210 \mathrm{~mm}^{2} .256$ ASICs provide full parallel readout, comprising analog filtering, 8-bit digitization and data storage. The challenge of having high-dynamic range and single photon detection simultaneously requires a non-linear system front-end. The first mega-pixel camera for the day-zero of the European XFEL operation will be equipped with linear MiniSDD pixel arrays and the non-linear response for the dynamic range compression has to be provided by the ASIC front-end. The DEPFET pixels foreseen for the advanced version of the camera provide signal compression at the sensor level. All the electronics components are available and tested and the assembly of the first prototype cameras based both on DEPFETs and MiniSDDs has started. We will give an overview of the DSSC system with its main components from the sensor to the DAQ electronics. In the past a noise down to 18 electrons rms. was achieved with the DEPFETs, operating a small system demonstrator of $8 \times 8$ pixels at a speed of $1 \mathrm{MHz}$. A noise of about 50 electrons rms was obtained with MiniSDDs under the same operating conditions. We will now present experimental results on both types of large format sensors bump-bonded to the first full-format (64 x 64) readout ASICs, operating the full detector readout chain under realistic experimental conditions. The imaging capability of the system prototype at $4.5 \mathrm{MHz}$ with available sensors and the fullformat readout ASICs will be shown for the first time. Eventually, we will present the status of the fabrication of non-linear DEPFET pixels produced for the first time in an industrial CMOS foundry. They will be used for the advanced version of the mega-pixel camera.

This work is supported by the European X-Ray Free-Electron Laser Facility GmbH in the framework of the DSSC project.

\section{N14-2: PFM2: a 32x32 Readout Chip for the PixFEL X-Ray Imager Demonstrator}

\section{Lodola}

Dipartimento di Ingegneria Industriale e dell'Informazione, University of Pavia, Pavia, Italy

On behalf of the PixFEL Collaboration

A readout chip, consisting of 32x32 square cells, has been designed in a $65 \mathrm{~nm}$ CMOS technology. The circuit will be bump bonded to a slim/active edge pixel sensor to form the first demonstrator for the PixFEL X-ray imager, envisaged for applications to experiments at the next generation X-ray FELs. The pixel pitch is $110 \mathrm{um}$, for a total area of about $16 \mathrm{~mm}^{\wedge} 2$. Different 
solutions for the readout channel, which includes a charge preamplifier, a time variant filter and a 10 bit ADC, have been integrated in the chip. In particular, a couple of different versions for the time variant processor have been implemented. The charge preamplifier is provided with four different gain settings, therefore improving the system capability to comply with photon energies in the $1 \mathrm{keV}$ to $10 \mathrm{keV}$ interval. This work, besides discussing in detail the readout channel and array architecture, will present the results from the chip characterization.

The activity leading to the results presented in this paper was carried out in the framework of the PixFEL project, funded by the Italian Institute for Nuclear Physics (INFN). The PixFEL Collaboration members are with Universita' di Bergamo, Universita' di Pavia, Universita' di Pisa, Universita' di Trento and INFN, Italy.

N14-3: Soft X-Ray and High Resolution Imaging Using the 25 mum Pitch MÃ-NCH Detector

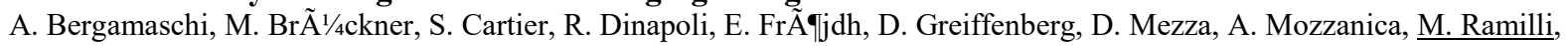
S. Redford, C. Ruder, L. Schaedler, B. Schmitt, X. Shi, D. Thattil, G. Tinti, J. Zhang

Paul Scherrer Institut, Villigen, Switzerland

$\mathrm{Mi}_{i}^{1} / 2 \mathrm{NCH}$ is a $25 \ddot{i}_{i}^{1 / 2} \mathrm{~m}$ pitch charge integrating hybrid silicon pixel detector aiming to combine the advantages given by high segmentation with the low noise and large dynamic range which are provided by adaptive gain analog detectors. In low flux conditions, charge sharing between neighboring pixels allows the spatial resolution to be improved beyond the pixel pitch using interpolation down to about a micrometer. Its performance promises to fulfill the specifications of many detector-limited synchrotron applications e.g. inelastic X-ray scattering (IXS), Laue diffraction, soft X-ray and high resolution imaging. We have demonstrated an outstanding bump-bond yield of better than $99.95 \%$ on the latest $1 \mathrm{x} 1 \mathrm{~cm} 2$ prototype $\left(160 \mathrm{k}\right.$ pixels at $25 \mathrm{i}_{i}{ }^{1 / 2 \mathrm{~m}}$ pitch). A low noise down to 35 electrons RMS has been measured, as well as a dynamic range up to about $100 \mathrm{x} 12 \mathrm{keV}$ photons, depending on the selected gain settings. The first imaging tests using soft X-rays down to $1.75 \mathrm{keV}$ will be shown. Moreover, the preliminary results obtained in a raster scan experiment using a $100 \mathrm{~nm}$ focused hard X-ray beam will be discussed in order to characterize the charge collection, precisely assess the spatial resolution and provide information concerning radiation hardness within the pixel. Finally, the challenges of developing develop the planned $3 \times 4 \mathrm{~cm} 2$ (1.9M pixels) system will be analyzed.

N14-4: The Large Pixel Detector for XFEL.EU - Build and Commissioning of the 1M System

M. D. Hart ${ }^{1}$, D. Beckett ${ }^{1}$, P. Brentnall ${ }^{1}$, B. Cline ${ }^{1}$, J. Coughlan ${ }^{1}$, M. French ${ }^{1}$, M. Kuster ${ }^{2}$, J. Lipp ${ }^{1}$, T. Nicholls ${ }^{1}$, P. Seller $^{1}$, D. Sole ${ }^{1}$, M. C. Veale ${ }^{1}$, P. Lang ${ }^{2}$

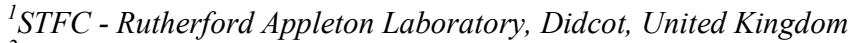

${ }^{2}$ The European XFEL, Hamburg, Germany

The Large Pixel Detector has been developed by The Science and Technology Facilities Council for use at the European XFEL. It is a high speed, high dynamic range, large area X-ray detector. We are nearing completion of the project that will see a $1 \mathrm{M}$ pixel system installed at XFEL. Over the year we have solved a number of engineering challenges including developments in the powering and cooling of the system. We have also completed x-ray testing of the system at the ESRF and Diamond Light Source synchrotrons. There we aimed to learn and tackle potential issues with the system. We present these developments, beam time results and some of the lessons learned.

\section{N14-5: The First Adaptive Gain Integrating Pixel Detector for the Beam Line SPB at the European XFEL} A. Allahgholi

Center for Free Electron Laser Science, Deutsches Elektronensynchrotron, Hamburg, Germany

On behalf of the AGIPD Colaboration

The European XFEL is an extremely brilliant Free Electron Laser Source with a very demanding pulse structure: It provides pulse trains at a frequency of $10 \mathrm{~Hz}$. Each train contains $2700 \mathrm{x}$-ray pulses which are separated by $220 \mathrm{~ns}$. Each pulse has a length of $=100 \mathrm{fs}$ and contains up to $10^{\wedge} 1212 \mathrm{keV}$ photons. The Adaptive Gain Integrating Pixel Detector (AGIPD) is a hybrid $1 \mathrm{M}$-pixel detector developed by DESY, PSI, and the Universities of Bonn and Hamburg to cope with these properties: AGIPD provides not only single photon sensitivity and a dynamic range up to $>104$ photons/pixel in the same image, but also an analogue memory which allows to record up to 352 individual pulses within a train, which are then read out in between the pulse trains. Each ASIC contains $64 \times 64$ pixels of $200 \mu \mathrm{m} \times 200 \mu \mathrm{m}$. The circuit of each pixel contains a charge-sensitive preamplifier with threefold switchable gain, a discriminator for an adaptive gain selection, and a correlated double sampling (CDS) stage to remove reset and low-frequency noise components. An AGIPD front end module (FEM) consists of 2x8 ASICS bump bonded to a 500 $\mu \mathrm{m}$ thick silicon sensor, giving a total of $128 \times 512$ pixels per module. The final 1 Mpix systems located at the beam lines SPB and MID at the European XFEL consist of 16 AGIPD FEMs, four each mounted on four independently movable quadrants which allow different hole and slit patterns to let the direct beam through. Each quadrant also acts as a cooling block which makes it possible to cool down the system in vacuum to its desired operating temperature of $-20^{\circ} \mathrm{C}$.

The final AGIPD 1Mpix detector will be delivered to the European XFEL in August 2016. We will present the aforementioned 
key features of AGIPD 1.0 and present results verifying the functionality of AGIPD. Furthermore we will present a general overview of the $1 \mathrm{M}$ detector and the status of the delivery and commissioning of the first $1 \mathrm{M}$ AGIPD system at the beam line SPB/EU-XFEL.

\author{
N14-6: Design and Characterization of the tPix Prototype: a Spatial and Time Resolving Front-End ASIC for \\ Electron and Ion Spectroscopy Experiments at LCLS \\ B. Markovic, P. Caragiulo, A. Dragone, C. Tamma, T. Osipov, C. Bostedt, M. Kwiatkowski, J. Segal, J. Hasi, C. Kenney, \\ G. Haller \\ SLAC National Accelerator Laboratory, Menlo Park, Ca, USA
}

tPix is the front-end ASIC for Tixel detector aimed for momentum spectroscopy experiments at LCLS. It has $100 \mu \mathrm{mx} 100 \mu \mathrm{m}$ pixels arranged in a 176x192 matrix (48x48 prototype) bump-bonded to a dedicated thin silicon sensor. The ASIC electronics measure the charge from the sensor and determine as well as digitize the timing information for each pixel of the detector with $100 \mathrm{ps}$ resolution. The timing information is extracted from the sensor signal with a charge-sensitive amplifier (CSA) followed by a discriminator. The time-to-digital conversion is performed in two steps: an 8 bit global counter performs the first 'coarse' measurement while an in-pixel delay-line performs a 6/10 bit high-resolution 'fine' measurement of the time-interval. The 14/18 bit conversion result is read out in between pulse intervals during the readout phase. The chip will be able to sustain a frame rate up to $1 \mathrm{KHz}$. A 48x48 pixel-array prototype has been fabricated in TSMC $0.13 \mu \mathrm{m}$ CMOS technology and its design and characterization will be reported in this talk.

\title{
N15: Scintillators II: Fundamentals \& Light collection
}

Tuesday, Nov. 1 08:00-10:00 Cassin

N15-1: First-Principles Investigation of the Structure, Mobility and Optical Properties of Self-Trapped Excitons in Alkali, Lanthanum and Barium Halide Scintillators

M. Del Ben, G. Bizarri, E. D. Bourret, A. Canning

Lawrence Berkeley National Laboratory, Berkeley, California, USA

The development of new and improved materials for gamma ray scintillator detectors requires a deep understanding of the optical and transport properties of the charge carriers at the atomistic level. In this respect, enormous progress has been made in the development of quantum mechanical methods that allow us to investigate quantitatively these mechanisms. Here we present first principles calculations at the hybrid density functional theory level for the structure, mobility and optical properties of selftrapped excitons and lattice defects (Vk centers) in three important families of scintillator materials, the alkali, lanthanum and barium halides. Alkali and lanthanum halides have been extensively characterized from an experimental point of view and serve in our studies as benchmark systems to assess the accuracy and reliability of our theoretical procedure. We show that hybrid density functional theory accurately predicts the different types of self-trapped excitons (on and off-center) found in these materials in agreement with EPR experiments. Moving further we used our methodology to perform preliminary studies of these defects in classes of new scintillator materials including the barium mixed halides that we will compare to new experimental results. The ultimate goal is then to set up a computational machinery with a predictive capability that will guide the design of new novel materials or modification of existing materials with enhanced scintillation performance.

This work is supported by the U.S. Department of Energy/NNSA/DNN R\&D and is carried out at Lawrence Berkeley National Laboratory under Contract No. AC02-05CH11231.

N15-2: Study of CsI:TI Scintillators with Different Concentration of $\mathrm{Tl}$ at the Temperature $\mathrm{Range}$ from $+30^{\circ} \mathrm{C}$ to $-70^{\circ} \mathrm{C}$

$\underline{\text { Z. Mianowska }}{ }^{1}$, M. Moszynski ${ }^{1}$, P. Sibczynski ${ }^{1}$, L. Swiderski ${ }^{1}$, A. Syntfeld-Kazuch ${ }^{1}$, T. Szczesniak ${ }^{1}$, A. Gektin ${ }^{2}$, S. Vasyukov $^{2}$, R. T. Williams ${ }^{3}$, S. Gridin ${ }^{3}$, X. Lu ${ }^{3}$, M. R. Mayhugh ${ }^{4}$

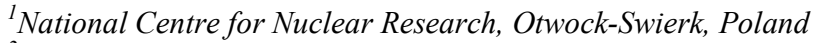

${ }^{2}$ Institute for Scintillation Materials, Kharkov, Ukraine

${ }^{3}$ Department of Physics, Wake Forest University, Winston Salem, NC, USA

${ }^{4}$ Faceted Development, LLC, Shaker Hts, OH, USA

A recent intensive modelling of the light generation process in caesium iodides activated by thallium requires precise experimental data concerning energy resolution, non-proportionality and decay time of the light pulses. CsI:Tl crystals activated by thallium in a range from 0.001 to $0.081 \%$ mole were investigated in temperature range from $+30^{\circ} \mathrm{C}$ down to $-70^{\circ} \mathrm{C}$. Each of tested scintillators were coupled to the XP5212/B photomultiplier from Photonics and placed into climatic chamber. Two experimental methods were used to characterize CsI:Tl properties. Initially, signal from PMT anode was fed to a charge sensitive preamplifier and then to a spectroscopy amplifier.All results were obtained by measuring the response of detection system to 
gamma-rays in energy range from 10 to $900 \mathrm{keV}$. The PC-based multichannel analyser was used to record energy spectra.Besides wide range of temperatures and $\mathrm{Tl}$ concentrations, two peaking times, equal $4.8 \mu$ s and $24 \mu \mathrm{s}$, were used to show large difference in obtained values of light output. Better understanding of light production processes was possible with second method, where PMT output was sent directly to digital oscilloscope. Signal was triggered by the full energy peak selected by the single channel analyzer from energy spectrum. Three components(fast, slow and tail) in the pulse light shape was quantified. The sum of three exponential terms was fitted to it. Strong slowing down of the light pulses below $-50^{\circ} \mathrm{C}$ was observed.

We hope that our experimental results will shed some light on luminescence phenomena and will give an input for theoretical modeling and interpretation.

\author{
N15-3: Modeling Temperature- and Energy-Dependent Scintillation Pulse Shape and the Proportionality of \\ Decay Components from 295 to $78 \mathrm{~K}$ in CsI and CsI:Tl \\ R. T. Williams ${ }^{1}$, X. Lu ${ }^{1}$, S. Gridin ${ }^{1}$, M. R. Mayhugh ${ }^{2}$, L. Swiderski ${ }^{3}$, Z. Mianowska ${ }^{3}$, W. Czarnacki ${ }^{3}$, T. Szczesniak $^{3}$, \\ T. Sworobowicz ${ }^{3}$, P. Sibczynski ${ }^{3}$, W. Klamra ${ }^{3}$, A. Syntfeld-Kazuch ${ }^{3}$, M. Moszynski ${ }^{3}$, A. V. Gektin ${ }^{4}$, S. Vasyukov $^{4}$, C. Piemonte $^{5}$, \\ A. Ferri ${ }^{5}$, A. Gola ${ }^{5}$ \\ ${ }^{I}$ Department of Physics, Wake Forest University, Winston-Salem, NC, USA \\ ${ }^{2}$ Faceted Development, LLC, Shaker Hts., OH, USA \\ ${ }^{3}$ National Centre for Nuclear Research, Otwock-Swierk, Poland \\ ${ }^{4}$ Institute for Scintillation Materials, Kharkov, Ukraine \\ ${ }^{5}$ Fondazione Bruno Kessler, Trento, Italy
}

Scintillation pulse shapes comprising a rise and three decay times along with their relative amplitudes in CsI:Tl have been measured versus temperature and gamma energy. Together with measurements of total light yield and proportionality including separate proportionalities of each decay component, and the ability to measure useful scintillation over a Tl concentration range including zero, this richness of observables makes CsI/CsI:Tl an excellent system for testing and refining computational models of scintillators. The purpose of this work is to apply a transport and rate equation model that was used last year to calculate proportionality in CsI and CsI:Tl including CsI at two temperatures, in order to examine how well it can account simultaneously for the entire array of observables listed above. Although there are many material parameters needed to specify the model equations, the many observables comprising pulse shape and component proportionality as well as independently variable conditions of gamma energy, temperature, and activator concentration constitute a sufficient number of target observables to enable refinement of material parameters not already measured independently. The goal is a computational material engineering tool in which properties, dopants, and defects can be varied and their predicted effects on scintillation explored.

Acknowledgments: WFU acknowledges support from the US Department of Homeland Security, Domestic Nuclear Detection Office, DNDO NSF ARI Grant 2014-DN-077-ARI-077. This support does not constitute express or implied endorsement on the part of the Government. Partial support is acknowledged from the Polish National Centre for Research and Development, "RaM-scaN" Grant PBS2/B2/11/2014, and European Union VII Framework Programme for research, technological development and demonstration under "TAWARA” grant No. 312713.

N15-4: Enhanced Scintillation Light Extraction Using Nanoimprinted Photonic Crystals

$\underline{\text { B. Singh }}^{1}$, M. S. J. Marshall ${ }^{1}$, S. Waterman ${ }^{1}$, C. Pina-Hernandez ${ }^{2}$, K. Munechika ${ }^{2}$, A. R. Knapitsch ${ }^{3}$, M. Salomoni ${ }^{3}$, P. Lecoq ${ }^{3}$, V. V. Nagarkar ${ }^{1}$

${ }^{I}$ Radiation Monitoring Devices, Inc., Watertown, MA, USA

${ }^{2}$ Abeam Technologies, Hayward, CA, USA

${ }^{3}$ CERN, Geneva, Switzerland

The extraction of scintillation light from a crystal with high efficiency and speed is vital for realizing the much needed gains in the performance of numerous radiation detection and imaging instruments that are vital in medical imaging, industrial, and homeland security applications for the detection, localization, and energy classification of X-rays, gamma rays or neutrons. Nanostructures such as photonic crystals (PhCs) maximize utilization of the scintillation light which is otherwise lost when a high refractive index (RI) scintillator is coupled to a photodetector with low RI window, thereby substantially improving the light extraction efficiency, and energy and timing resolution. Using nanoimprinted PhCs on the exit surface of LYSO, we had earlier experimentally demonstrated an $18 \%$ improvement in the light extraction compared to the current grease-coupling technique. However, to realize the full potential benefits offered by PhCs, a polymer with high RI and excellent optical transmission at the scintillation wavelength is needed. Currently, there are no such commercially available polymers. Here, we report on the successful demonstration of $41 \%$ improvement in the light extraction from a ceramic scintillator with RI $\sim 1.82$ and emitting at $540 \mathrm{~nm}$, using PhCs nanoimprinted in a custom developed 1.87 RI polymer with $98 \%$ transmission. Furthermore, we have demonstrated the low-cost scalability of the process by uniformly imprinting on substrates ranging from $10 \mathrm{~mm}-30 \mathrm{~mm}$ in diameter, limited only by the size of the imprinting template. Details of the design, fabrication and characterization of the PhCs, the high RI polymers, and their impact on scintillator performance will be presented. 
Acknowledgements: This work has been supported by the US Department of Homeland Security, Domestic Nuclear Detection Office, under competitively awarded contract HSHQDC-13-C-B0040. This support does not constitute an express or implied endorsement on the part of the Government.

\author{
N15-5: A Novel Ultra-Thin Multiple-Layer High-Reflector Film Directly Coated on LYSO Scintillators

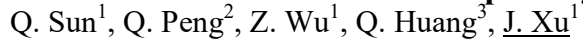 \\ ${ }^{I}$ School of Mechanical Science and Engineering, Huazhong University of Science and Technology, WuHan, China \\ ${ }^{2}$ Department of Molecular Biophysics and Integrated Bioimaging, Lawrence Berkeley National Laboratory, Berkeley, USA \\ ${ }^{3}$ Shanghai Jiaotong University, Shanghai, China
}

Optical dielectric coating is a technology commonly used to build reflectors. It is constructed from thin layers of materials such as magnesium/calcium fluoride and various metal oxides, which are deposited onto the optical substrate. By careful choice of the exact composition, thickness, and number of these layers, it is possible to tailor the reflectivity of the coating to produce almost any desired characteristic. Reflection coefficients of surfaces can be increased to greater than $99.99 \%$, producing a high-reflector (HR) coating.

In this study, we designed a novel high reflective film to match the entire emission spectrum of the LYSO (360-620nm) scintillator using three dielectric materials ( $\mathrm{HfO} 2, \mathrm{SiO} 2$ and $\mathrm{TiO} 2$ ). The simulation and design studies show that an average reflectivity higher than $99 \%$ at $360 \mathrm{~nm}-620 \mathrm{~nm}$ range could be achieved using 48 layers of the three dielectric materials to construct three quarter-wavelength reflective layers with the central wavelengths at $365 \mathrm{~nm}, 430 \mathrm{~nm}$ and $570 \mathrm{~nm}$. Experimental studies were performed to validate the simulation studies. Six LYSO samples were processed using high-precision polishing techniques and coated with the HR films using electron beam evaporation techniques. The Ra surface roughness of the LYSO samples were less than $0.57 \mathrm{~nm}$ and the total thickness of the HR coating was $3.4 \mu \mathrm{m}$. The reflective properties of the six samples were characterized and compared with a sample glued with a commonly-used enhanced spectrum reflector (ESR) film. The results show that six samples have a peak reflectivity higher than $99.76 \%$ at $420 \mathrm{~nm}$ and an average reflectivity higher than $98.79 \%$ at $360 \mathrm{~nm}-620 \mathrm{~nm}$ range.

In conclusion, this study demonstrates a novel HR film design which achieves better performances than the conventional ESR reflectors. The novel HR film $(3.03 \mu \mathrm{m})$ is much thinner than ESR film $(70 \sim 100 \mu \mathrm{m})$. One can achieve a much higher filling factor in clinic/pre-clinic PET detector designs by applying this novel technology.

\author{
N15-6: Reflectivity Quenching of ESR Multilayer Polymer Film Reflector in Optically Bonded Scintillator \\ Arrays \\ $\underline{\text { F. Loignon-Houle }}^{1}$, C. M. Pepin ${ }^{1}$, S. A. Charlebois ${ }^{2}$, R. Lecomte $^{1}$ \\ ${ }^{I}$ Sherbrooke Molecular Imaging Center of CRCHUS and Dept. of Nuclear Medicine and Radiobiology, Universite de \\ Sherbrooke, Sherbrooke, QC, Canada \\ ${ }^{2}$ Dept. of Electrical and Computer Engineering, Universite de Sherbrooke, Sherbrooke, QC, Canada
}

The 3M-ESR multilayer polymer film is a widely used reflector in scintillation detector arrays. As specified in the datasheet and confirmed experimentally by measurements in air, it is highly reflective ( $>98 \%)$ over the entire visible spectrum for all incidence angles. Despite these outstanding characteristics, it was previously found that light crosstalk between pixels in a bonded LYSO scintillator array with ESR reflector can be as high as $\sim 30 \%$. This unexplained crosstalk motivated further investigation of ESR optical performance. Analytical simulation of a multilayer structure emulating the ESR reflector showed that the film becomes highly transparent to incident light at high angle when surrounded on both sides by material of refractive index higher than air. Furthermore, Monte Carlo simulation indicated that a considerable fraction of scintillation photons were incident at these leaking angles in high aspect ratio LYSO crystals. The film transparency was investigated experimentally by measuring the scintillation light transmission through the ESR film sandwiched between a LSO crystal and an APD detector with or without layers of silicone grease. Strong light leakage $(\sim 30 \%)$ was measured through the reflector when coated on both sides with silicone, elucidating the major cause of light crosstalk in bonded arrays. This loss of reflectivity can be explained by the optical path difference between light reflected by adjacent interfaces in the multilayer film that raises the phase shift, reducing valuable constructive interferences. This unsuspected transparency can be beneficial for light sharing schemes, but is highly detrimental for scintillator arrays designed for individual pixel readout.

\author{
N15-7: Improved Scintillation Detector Performance Using Dye-Doped Coatings \\ S. R. Tornga ${ }^{1}$, D. T. Wakeford ${ }^{1}$, J. C. Adams ${ }^{2}$, O. C. Trautschold ${ }^{2}, \underline{\text { M. P. Hehlen }}^{2}$

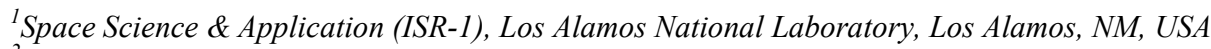 \\ ${ }^{2}$ Engineered Materials (MST-7), Los Alamos National Laboratory, Los Alamos, NM, USA
}

An increasing demand for better detection performance with a simultaneous reduction in size, weight and power consumption has motivated the use of compact semiconductors as photo-converters for many gamma-ray and neutron scintillators. The spectral response of devices such as avalanche photodiodes (APDs) however is poorly matched to many common high-performance 
scintillators. We are developing a method that matches the scintillator luminescence to the excitation spectrum of high quantum efficiency semiconductor detectors. This is accomplished by a series of high quantum yield, fast decay, wavelength shifting coatings. In initial experiments we have demonstrated a $28 \%$ increased photo-electron collection and a $10 \%$ improvement in energy resolution when applying a layered coating to a YSO:Ce scintillator coupled to an APD detector. We expect that improvements in the energy resolution of up to $40 \%$ can be achieved for some scintillators coupled to APDs. Likewise, scintillators with good pulse shape discrimination and other properties, but a poorly matching emission spectrum (e.g. LiCAF:Ce) may, with the application of this process, become indispensable neutron detectors. In this presentation, we will give details of the methodology for determining optimal coating properties, processes for applying coatings to scintillators, and experimental results to date.

\section{N15-8: Development of Wavelength-Shifting, Liquid-Filled Quartz Capillaries for Readout of Optically-Based Electromagnetic Calorimetry \\ B. W. Baumbaugh+, B. Dolezal, K. C. Ford, P. S. Link, C. M. Mohs, R. C. Ruchti, N. J. Siwietz, J. B. Taylor,} M. J. Vigneault, C. Jessop

Physics, University of Notre Dame, Notre Dame, Indiana, USA

Abstract: We are in the process of developing liquid Scintillator filled Capillaries for use in optically based Electromagnetic calorimetry. These capillaries are intended for use in the construction of a Shashlik detector using LYSO and tungsten as the basis for the calorimeter. The capillaries collect the scintillation light from the LYSO crystals and present this light to detectors based on SiPMs. These capillaries are $1.0 \mathrm{~mm}$ OD x $0.4 \mathrm{~mm}$ ID rad hard quartz. The work presented here will focus on the design, construction, and testing of capillaries, capillary materials, liquid scintillators, and filling/sealing techniques that optimize the linear response of the capillaries over the $135 \mathrm{~mm}$ active region of the Shashlik detector. We will discuss liquids including DSB1 and J2 regarding their light output, Resistance to radiation damage, and response time when excited. This paper will also describe the techniques used to optimize the linearity along the capillary including how the core light (light from the liquid scintillator directly) is blocked, and the light from the end farthest from the readout is optimized to maximize linearity.

N16: Instrumentation for Security I: Active and Imaging

Tuesday, Nov. $1 \quad$ 08:00-10:00 Madrid

N16-1: Requirements for Active Interrogation Systems

$\underline{\text { R. Kouzes }}^{1}$, G. Warren ${ }^{1}$, P. Chiaro ${ }^{2}$

${ }^{I}$ Pacific Northwest National Laboratory, Richland, WA, USA

${ }^{2}$ Oak Ridge National Laboratory, Oak Ridge, TN, USA

Active-interrogation (AI) systems for interdiction of radiological materials automatically determine if special nuclear material (SNM) is present in a conveyance (e.g., an intermodal cargo container) by observing the radiation emitted by the object when it has been exposed to an external radiation source. This is in contrast to passive detection in which the spontaneous decay of radioactive material is the signature that is detected. Because of the cost and complexity, AI systems are for the detection of SNM in shielded configurations that are not normally detectable by passive systems.

A performance standard is being developed for evaluating AI systems that detect SNM in unshielded and shielded configurations using surrogates for testing. The AI systems under consideration include those that detect high atomic number (High-Z) materials, fissionable materials, or specific SNM materials.

AI systems can use photons, neutrons, or muons as the interrogation particle, and detect photons, neutrons or muons as the emitted particle. Photofission, for example, uses energetic photons to induce fission in the interrogated object, and usually the neutron signature to identify material as fissionable. Further categorization is possible by subdividing the observed particles as either prompt or delayed. These distinctions are important for consideration of surrogate materials for use in testing. The AI system should also localize the suspect material to aid follow-on interdiction efforts. This talk will present the considerations that are applied to the development of standard testing requirements for AI systems.

This material is based upon work supported by the Department of Homeland Security. Battelle operates PNNL for the U.S. Department of Energy under contract DE-AC05-76RLO 1830.

N16-2: A Large Field-of-View Multimodal X-Ray Phase Contrast System for Security Scans and Other Applications

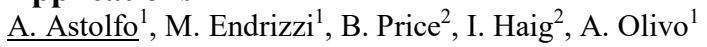

${ }^{I}$ Medical Physics and Biomedical Engineering, University College of London, London, UK

${ }^{2} X$-Tek Systems-Nikon, Tring, UK 
The use of X-ray Phase Contrast Imaging (XPCI) enables revealing sample properties that are invisible using conventional X-ray imaging techniques, which are sensitive to X-ray attenuation only. In addition to conventional contrast, XPCI improves object edges detection and is sensitive to subpixels microstructures. We have built an XPCI scanning system based on Asymmetric Edge Illumination to be initially employed for baggage screening, then translated to other applications. Our setup is capable of fast imaging over a large field of view using high-energy x-ray beams $(100 \mathrm{kVp})$. The XPCI multimodal sensitivity significantly improves the screening capabilities, in particular for the detection of weapons and explosives.

This project is funded under the Innovative Research Call in Explosives and Weapons Detection 2013 initiative. This is a Cross-Government programme sponsored by a number of Departments and Agencies under the UK Government's CONTEST strategy in partnership with the US Department of Homeland Security, Science and Technology Directorate.

N16-3: SCoTSS Modular Survey Spectrometer and Compton Imager

A. M. L. MacLeod ${ }^{1}$, L. E. Sinclair ${ }^{2}, \underline{\text { P. R. B. Saull }}{ }^{1}$, P. L. Drouin ${ }^{3}$, L. Erhardt ${ }^{3}$, J. Hovgaard ${ }^{3}$, B. Krupskyy ${ }^{4}$, R. Ueno $^{3}$, D. Waller ${ }^{3}$, A. McCann ${ }^{2,4}$

${ }^{I}$ Measurement Science and Standards, National Research Council, Ottawa, Canada

${ }^{2}$ Geological Survey of Canada, Natural Resources Canada, Ottawa, Canada

${ }^{3}$ Defence Research and Development Canada, Ottawa, Canada

${ }^{4}$ Physics Department, McGill University, Montreal, Canada

This work presents the development of a mobile survey spectrometer and fieldable Compton-scatter gamma-ray imager. The detector employs $\mathrm{CsI}(\mathrm{Tl})$ scintillator coupled to silicon photomultipliers and incorporates a unique modular design, where individual units can be employed separately in applications requiring a compact detector, e.g. military operations, or combined together for those requiring a more sensitive detector, e.g. aerial surveying. Each module is a fully functional imager, providing both mapping and imaging capabilities along with isotope identification.

We describe the overall performance of the imager in comparison to expectation based on Monte Carlo simulations with Geant 4 . The energy reconstruction is shown to perform well across the full spectrum of interest up to $3 \mathrm{MeV}$, with resolutions and noise levels suitable for low-energy measurement in both the scatter and absorber parts of the detector. We also present the performance of the custom readout electronics that digitize and process the SiPM pulses.

\section{N16-4: Imaging of Special Nuclear Material Using Coarse and Fine Spatial Resolution Arrays Coupled with Monochromatic Photon Sources} $\underline{\text { P. B. Rose Jr }}{ }^{1}$, J. Harms ${ }^{1}$, J. Nattress ${ }^{2}$, M. Mayer ${ }^{3,4}$, I. Jovanovic ${ }^{3}$, A. S. Erickson ${ }^{1}$

${ }^{I}$ Nuclear and Radiological Engineering, Georgia Institute of Technology, Atlanta, Georgia, USA

${ }^{2}$ Department of Nuclear Engineering and Radiological Sciences, University of Michigan, Ann Arbor, MI, USA

${ }^{3}$ Department of Mechanical and Nuclear Engineering, The Pennsylvania State University, University Park, PA, USA

${ }^{4}$ Also at Pacific Northwest National Laboratory, Richland, WA, USA

Special nuclear material (SNM) can be dangerous and have devastating consequences in the wrong hands. SNM can easily be shielded from passive detector systems, especially while in transit, resulting in it being referred to as "searching for a needle in a haystack." Active interrogation (AI) techniques can be employed to probe through shielding to uncover illicit material, but pose significant challenges for detection systems and can present with large doses to cargo and personnel. We report advancements in the proof-of-concept of a novel AI imaging technique using quasi-monoenergetic high-energy gamma rays to improve the detection and identification of SNM through Z\$_\{eff $\}$ determination of the cargo. Our system uses arrays of custom designed detectors to provide both coarse and fine spatial resolution capabilities using a combination of low and high energy resolution. Low energy nuclear reaction driven sources, such as $\mathbb{\$}^{\wedge}\{11\} \$ B(\mathrm{~d}, \mathrm{n} \$ \backslash$ gamma $\$) \mathbb{\$}^{\wedge}\{12\} \$ C$, produce a cascade of highly penetrating monoenergetic gamma rays which may reduce radiation dose to the cargo contents when compared to bremsstrahlung methods. These gamma rays have sufficient separation in energy to utilize Cherenkov detectors for imaging and spectroscopy. We show that monochromatic photons resulting from a low-energy nuclear reaction coupled with an array of Cherenkov detectors can be used to produce a sharp image of material while providing large and inexpensive detector coverage. We also report on the development of arrays leveraging tightly tiled SiPMs coupled with LYSO and Cherenkov crystals to increase spatial and energy resolution performance. Demonstrated here is the proof of concept of the system's capability to produce instantaneous high contrast planar images of interrogated material while also providing a "map" of the Z\$_\{eff $\}$ \$ of the cargo paving the way for practical deployment at ports and points of entry.

N16-5: Fun with Blocks, Gamma-Ray Images of Different Source Configurations K. P. Ziock, M. A. Blackston

Nuclear Science and Isotope Technology, Oak Ridge National Laboratory, Oak Ridge, TN, USA 
A coded-aperture, gamma-ray imager, based on a high-purity germanium detector has been used to collect data on a large number of configurations of up to $12 \mathrm{Pu}$ blocks. This provides a unique data set to explore the ability to determine the quantitative performance of gamma-ray imagers on different source geometries as a function of source shape and acquisition time. Quantitative results were obtained by fitting the images to square and rectangular source geometries built from complimentary error functions (erfc). Estimates of the uncertainties for fit results were obtained using bootstrap sampling techniques to generate data sets with different integration times and fitting these with the erfe shapes. The results will be used to provide examples of how such an instrument could be used in arms-control.

N16-6: Collimator-less 4p Gamma Imaging with 3-D Position-sensitive Detector

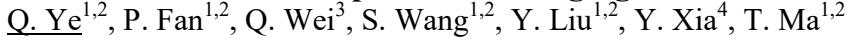

${ }^{I}$ Department of Engineering Physics, Tsinghua University, Beijing, China

${ }^{2}$ Key Laboratory of Particle \& Radiation Imaging, Ministry of Education (Tsinghua University), Beijing, China

${ }^{3}$ Department of Instrument Science, School of Automatic and Electrical Engineering, University of Science Technology Beijing, Beijing, China

${ }^{4}$ Beijing Institute of Spacecraft Environment Engineering, Beijing, China

Fast and accurate lost radioactive source positioning is of critical importance for the prevention of radiation damage in the accident. Traditional instruments with low detection efficiency cost lots of time and energy, and may lead to harms of the operators' health. A portable detector to directly determine the incident angle of gamma rays is promising for the homeland security applications. In this work, we proposed a collimator-less $4 \mathrm{p}$ gamma imaging with 3-D position-sensitive detector. The distribution of photon counts is dependent on the orientation angle of the point gamma source, and the angle is reconstructed with Maximum Likelihood Expectation Maximization (MLEM) algorithm. Angular resolution of this imaging system was calculated by Cramer-Rao lower bound (CRLB). We simplified the 3-D position-sensitive detector to 2-D angular discrimination design. This design is capable of positioning the incident angle of $511 \mathrm{keV}$ gamma rays. Excellent angular discrimination performance can be achieved except some particular angular positions at $0^{\circ}, 90^{\circ}, 180^{\circ}$ and $270^{\circ}$. And the angular discrimination performance can be improved with more counts. Besides, we performed a preliminary experiment with 4 BGO crystal blocks and for all the tested angular positions ranging from $15^{\circ}$ to $75^{\circ}$ with an increment of $15^{\circ}$, the maximum error was less than $6.5^{\circ}$. Besides, we are now developing the positioning technology in a monolithic crystal which can be applied to the gamma imaging method. From the analytical calculation and experimental results, we conclude that the proposed 4 p gamma imaging method with 3-D positionsensitive detector is effective and capable of searching for radioactive sources with excellent accuracy.

\author{
N16-7: Identification of Special Nuclear Material with Spectrum Isolation Using Stochastic Image \\ Reconstruction \\ M. C. Hamel, J. K. Polack, L. O. Supic, S. D. Clarke, S. A. Pozzi \\ Nuclear Engineering and Radiological Sciences, University of Michigan, Ann Arbor, MI, USA
}

Radiation imaging has proven to be a useful tool in the localization of special nuclear material (SNM) for non-proliferation applications. However, scenarios exist in which there are other radioactive sources present in the vicinity of the SNM. This work seeks to isolate the energy spectra from all sources localized in a measurement, which will allow for the identification or discrimination of SNM from other radioactive sources. To accomplish the isolation, we have reconstructed images using the stochastic origin ensembles (SOE) algorithm. SOE is based on Markov-chain Monte Carlo and reconstructs images through an iterative resampling of possible event origins. We have modified the mechanism used to find a solution such that each pixel in the image has an associated energy spectrum. We applied this method to an experiment performed with a dual-particle imager (DPI) that reconstructs images and spectra for both fast neutrons and gamma-rays. A 4.5-kg sphere of weapons grade plutonium (WGPu) and a 252Cf point source were measured simultaneously with the DPI. Using our modified version of SOE, we reconstructed both a neutron and gamma-ray image of the two sources. We showed that the isolated neutron energy spectra for both sources were similar as expected. However, the WGPu could be discriminated from the 252Cf because of the decay gammaray peak near $650 \mathrm{keV}$ from the $\mathrm{WGPu}$. These decay gamma-rays are not present in the $252 \mathrm{Cf}$ spectrum. This work demonstrates that the modified version of the SOE algorithm is promising for identification of SNM in a multi-source environment.

\author{
N16-8: Measurement of Time Distribution of Fissions in a Chain with Fast Scintillators \\ M. Monterial $^{1,2}$, P. Marleau ${ }^{2}$, S. A. Pozzi ${ }^{1}$ \\ ${ }^{I}$ Nuclear Engineering and Radiological Sciences, University of Michigan, Ann Arbor, MI, USA \\ ${ }^{2}$ Radiation and Nuclear Detection Systems, Sandia National Laboratories (CA), Livermore, CA, USA
}

Non-destructive assay (NDA) of fissile material is one the primary goals of non-proliferation and safeguards. For decades, multiplicity analysis, which requires large high-efficiency measurement systems, has played a key role in fissile material assay. Development of new systems of NDA of fissile material has shifted from thermal He-3 detectors to fast organic scintillators. We have developed a method for assaying fissile material by taking full advantage of the measured signatures available to fast 
organic scintillator systems. We have measured the timing distribution of fission events in a chain by recording the time between correlated gammas and neutrons, and the deposited energy of the incident neutron. We demonstrate how the combination of these signatures produces a distribution that is sensitive to underlying fission chain timing dynamics. Through a simple Monte Carlo based model of fission chain behavior it is possible to inversely solve for the underlying physical parameters that define the amount of fissile material and the presence of any moderator or reflector coupled to it. Finally, we demonstrate the technique through analysis of a set of parametric measurements of $4.5 \mathrm{~kg}$ of weapons grade plutonium metal in various configurations of moderator and reflector recorded during a 2015 campaign at the Device Assembly Facility.

This material is based upon work supported by the U.S. Department of Homeland Security Grant Award Number: 2012-DN-130-NF0001. The views and conclusions contained in this document are those of the authors and should not be interpreted as representing the official policies, either expressed or implied, of the U.S. Department of Homeland Security.

Sandia National Laboratories is multi-program laboratory managed and operated by Sandia Corporation, a wholly owned subsidary of Lockheed Martin Corporation, for the U.S. Department of Energy's National Nuclear Security Administration under contract DE-AC04-94AL85000.

This work was funded in-part by the Consortium for Verification Technology under Department of Energy National Nuclear Security Administration award number DE-NA0002534.

\section{N17: Data acquisition, trigger and analysis I (non-HEP)}

Tuesday, Nov. 1 08:00-10:00 Londres

N17-1: WaveDAQ: A New Generation of Integrated Trigger and Read Out System for the MEG II Experiment

L. Galli $^{1}$, D. Nicolo' ${ }^{1}$, F. Morsani ${ }^{1}$, M. Francesconi ${ }^{1}$, S. Ritt ${ }^{2}$, E. Schmid ${ }^{2}$, U. Hartmann $^{2}$

${ }^{I}$ Istituto Nazionale di Fisica Nucleare sezione di Pisa, Pisa, IT

${ }^{2}$ Paul Scherrer Institut, Villigen, $\mathrm{CH}$

A novel approach merging together high performance and high frequency waveform digitisers coupled with a hierarchical structure of FPGAs as real time processors has been designed to realise a multi-purpose device with integrated trigger and data acquisition capability. Detector signals are digitised by two independent, complementary and high resolution waveform digitisers: a faster one based on the DRS4 switched capacitor array chip, with a sampling speed in the programmable range from 1 to 5 GS and a depth of 1024 samples, to achieve an intrinsic time resolution of about 10 ps and about $1 \sim \mathrm{mV}$ baseline noise; a slower one based on a commercial $100 \sim \mathrm{MHz}$ FADC with a time depth up to $20 \$ \backslash$ mu $\$$ s. The samples of the latter are continuously sent to a hierarchy of FPGA and used in complex user defined algorithms for real-time event reconstruction suitable for trigger generation.

The basic version of the system large is enough for small and medium size detectors, hosting 16 waveform digitiser modules, each equipped with 16 channels each for a total number of 256 channels. A custom backplane provides high speed serial point-topoint connections between digitisers and two master modules to be used for trigger and data read-out, respectively. For larger applications the system can be composed of two or more crates in parallel, complemented by a central trigger concentration and a signal distribution crate, making the system scalable up to more than 10'000 channels. When more than one crate is required, trigger concentrators would be arranged in a hierarchical structure, where lower level boards (those connected to digitisers) would forward preliminary pieces of information to higher level ones, until the master trigger board gathers the data from the whole detector.

The system is in a advanced development phase and the first results from test beams are available. Results in terms of trigger capability and time and energy resolutions will be presented, complemented with a schedule towards a full system hosting 9'000 channels.

\section{N17-2: DRAC - a Fast Digitizer for the Mu2e Experiment}

\section{L. Rusu}

PPD, Fermilab, Batavia, IL, USA

On behalf of the mu2e collaboration

The mu2e tracking chamber is made of $\sim 20000$ thin wall straws to precisely measure the $\sim 100 \mathrm{MeV}$ electron characteristic of the coherent muon to electron conversion. Each straw is read out at both ends, in order to determine the position of the hit along the straw through time division. To meet the experimental requirement the time difference between the two ends has to be recorded to better than 50ps with a resolution on the absolute drift time measurement of 500ps. The total charge deposited in the straw is used for the $\mathrm{dE} / \mathrm{dx}$ measurement. The mu2e tracker is highly modular, with the basic unit being a panel, which contains 96 straws. A central board was designed to perform all those measurements for all 96 straws in a panel. This is the DRAC - Digitizer Recorder Assembler and Controller. This is a high density PCB that needs to fit the tracker tight space constraints and mounts on the outer perimeter of the panel and is therefore an integral part of the basic mu2e tracker module. The panel has to operate in vacuum, so we made an effort to limit the number of DRAC interfaces. Aside from the power port, the only other interface is a pair of duplex optical fibers for both data and slow control communications. At the heart of the DRAC are three radiation hard 
FPGAs that perform fast TDC measurements and record a slower version of the straw signal, used for charge integration. The latter is done with 50MS/s ADCs and there is one such ADC for each straw. All data is assembled into packets and is sent through the optical fiber to a host PC. The DRAC prototype is fully operational and we will present here a description of this powerful device together with the first results on performance.

N17-3: The Readout and Data Acquisition Design of the sPHENIX Detector at RHIC M. L. Purschke

Physics Department, Brookhaven National Lab, Upton, NY, USA

On behalf of the sPHENIX Collboration

The recently established sPHENIX Collaboration at RHIC is upgrading the PHENIX detector in a way that will enable a comprehensive measurement of jets in relativistic heavy ion collisions. The upgrade will give the experiment full azimuthal coverage within a pseudorapidity range of $-1.1<?<1.1$. In addition to measuring heavy-ion collisions, the new apparatus will provide enhanced physics capabilities for studying nucleon-nucleus and polarized proton collisions, and eventually allow a detailed study of electron-nucleus collisions at an envisioned Electron Ion Collider at Brookhaven.

The upgraded detector will be based on the former BaBar magnet and will include tracking detectors, a new electromagnetic calorimeter, and, for the first time at a RHIC experiment, a hadronic calorimeter. A new technology using a Tungstenscintillating fiber design for the electromagnetic calorimeter is what enables the full azimuthal coverage, as it achieves a radiation length of just about $7 \mathrm{~mm}$, which allows for a very compact design of the device.

The calorimeter signals are sampled with silicon photomultipliers and waveform digitizing electronics. The digitized waveforms are read out with custom PCIe boards that allow multiple streams with bandwidths of up to 5GBit/s. The goal is to have a sustained event rate to disk of about $15 \mathrm{KHz}$. Focusing on the calorimeters, we will describe the goals and design of the sPHENIX experiment, the design of the digitizers and other parts of the data acquisition system, and the results we got with current prototypes. By the time of the conference, we will have data from a test beam at FermiLab that will test the readout under beam conditions.

We will detail the design of the FPGA-based readout cards, and how we implement the so-called "multi-event buffering" in the front-end, which has traditionally enabled PHENIX to take data at rates rivaling, or exceeding, the LHC experiments.

N17-4: The SoLid anti-Neutrino Detector's Read-Out System

N. C. Ryder

Dept. of Physis, University of Oxford, Oxford, UK

On behalf of the The SoLid Collaboration

The SoLid collaboration will probe the reactor anti-neutrino anomaly by performing a search for anti-neutrino oscillations within $10 \mathrm{~m}$ from the BR2 nuclear reactor, with the first modules being deployed late in 2016. Anti-neutrino events are identified in the SoLid detector by reconstructing the positron and neutron emitted from an inverse beta decay. Performing anti-neutrino measurements at ground level and close to a nuclear reactor is particularly challenging due to the high rate of background signals that can mimic an anti-neutrino interaction. The SoLid collaboration have developed a novel, highly segmented, composite scintillator detector to overcome these challenges. The detector allows for a robust neutron identification and event selections based upon both the topology as well as relative timing of the positron and neutron signals. The SoLid collaboration have developed an intelligent read-out system to reduce their 3200 silicon photomultiplier detector's data rate by a factor of 10000 whilst maintaining high efficiency for storing data from anti-neutrino interactions. The system employs an FPGA-level waveform characterisation to trigger on neutron signals. Following a trigger, data from a space-time region of interest around the neutron will be read out using the IPbus protocol. This region of interest will be sufficiently large to contain any positron candidate signals. In this talk the SoLid detector concept will be introduced, the design of the read-out system will be explained and results showing the performance of prototype versions of the system will be presented.

N17-5: Development of Front-End Trigger System for COMET Experiment

Y. Fujii, S. Mihara, K. Ueno, M. Ikeno, T. Uchida, M. Shoji, E. Hamada

Ins. of Part. and Nucl. Studies, High Energy Accelerator Research Organization, KEK, Tsukuba, Japan

The COMET experiment searches for the muon-to-electron( $\mu$-e) conversion which is sensitive to many physics cases beyond the standard model with a sensitivity of $10^{-17}$. Since the $\mu$-e conversion is strictly forbidden in the standard model, it would be a clear evidence of new physics if it is found. In the COMET experiment, the front-end trigger system is required to send the trigger information within a $500 \mathrm{~ns}$ of the latency time and the system should be functional under the high radiation environment. The development of the trigger system to fulfill these requirements is essential for the success of the COMET experiment. The frontend trigger electronics, COTTRI (COmeT TRIger) is now being developed and tested by using a prototype board. The first prototype of the firmware was completed and implemented into the COTTRI prototype. The latency of COTTRI prototype was measured to be $150 \mathrm{~ns}$. 
The beam test was performed in March 2016 to evaluate the performance of the COTTRI prototype equipped on the prototype COMET detectors. In this beam test the COTTRI prototype was successfully operated to generate the trigger signal from crystal calorimeter signals as will be used in the COMET experiment.

Irradiation tests for neutrons and gamma-rays will be completed in summer 2016, allowing us to determine the final design and parts for the COTTRI front-end board.

\author{
N17-6: Synchronization of a Trigger-Less Data Acquisition for the PANDA Experiment \\ M. Kavatsyuk \\ KVI-CART, University of Groningen, Groningen, The Netherlands \\ On behalf of the PANDA collaboration
}

The PANDA experiment at the FAIR facility in Darmstadt, Germany, is aiming at precise measurement of hadron properties in the charmonium region and investigation of exotic-matter states. This will be possible because of employment of the annihilation of antiprotons with very low momentum spread of $2 \mathrm{e}-5$ and high luminosity, up to $1 \mathrm{e} 321 /(\mathrm{cm} \mathrm{s})$. At the highest luminosity the annihilation rate will rise up to $20 \mathrm{MHz}$. Moreover, the decay topology of events of interest is very similar to the one of background events. This excludes any reasonable performance of a classical triggered readout system. Therefore, a new readout has been developed where self-triggered front-end electronics is able to autonomously detect hits and extract all relevant physics information, a dedicated custom network builds events based on hit time-stamps, and an FPGA/GPU/CPU-based computing system, which selects events of interest on-line using the complete reconstruction, in order to achieve a data-reduction factor of about up to 1000 . Such a readout system is called "trigger-less". Since there is no trigger signal which forces simultaneous readout of acquired data from all front-ends, the electronics should use the same clock signal and should be synchronized. Therefore, a precise clock-distribution and -synchronization platform SODANET has been developed. The SODANET is a bidirectional data-transfer protocol for optical links which foresees transfer of detector- and slow-control data and synchronization messages with fixed latency. The synchronization messages have the highest priority and can be transmitted even if transmission of another packet is not yet finished. The synchronous operation is implemented only in the downstream direction and requires defined latency configuration of serializers/deserializers. A complete prototype of the readout system which employs the SODANET protocol has been constructed. During the presentation the SODANET platform and its performance will be discussed.

N17-7: System on Chip Achitecture for Auger PRIME

E. E. Lagorio

Electronic, CNRS, Grenoble, France

On behalf of the Pierre Auger Collaboration

The Pierre Auger Observatory, located near the town of Malargu"e, Argentina, is the world largest cosmic ray observatory. It has for objectives to probe the origin and characteristics of ultra high energy cosmic rays (UHECR). The results obtained by the Pierre Auger collaboration provide important information on key questions in the UHECR field, leading to new questions and open challenges which call for an upgrade of the Observatory. In the upgrade program, called AugerPrime, the 1660 Surface Detector stations will be improved in particular by implementing new electronics with higher performances. The new electronics aims to increase the number of analog channels and the dynamic range, to get a higher sampling rate and better timing accuracy.It is designed with a new generation of component, called SOC for System On Chip. A Linux system must be integrated for a higher access development. First prototypes of the new electronics have been built and are being tested .

The author thank the Auger collaboration for their contribution.

N17-8: Array of Superconducting Nanowire Single Photon Detectors Resolving the Number of Photons in a Weak Optical Pulse

N. Lusardi ${ }^{1}$, A. Geraci ${ }^{1}$, R. B. M. Gourgues ${ }^{2}$, J. W. N. $\operatorname{Los}^{2}$, G. Bulgarini ${ }^{2}$

${ }^{I}$ DEIB, Politecnico di Milano, Milan, Italy

${ }^{2}$ Single Quantum BV, Delft, The Netherlands

Determining the precise number of photons in a weak optical pulse is of crucial importance for imaging, computing or communication applications that use single photons or few photons to increase the performance of classical techniques. Single photons or pair of photons can for instance be used to enhance the resolution of optical imaging techniques, or to transmit information with ultimate security using quantum cryptography. In order to maintain this performance gain in such applications, the exact number of photons must be measured and monitored. In this contribution we present a method to measure the number of photons in an optical pulse by using 7 superconducting nanowire single photon detectors (SNSPDs) optically coupled to a weak laser via a fiber multiplexer. Since a single SNSPD is not sensitive to the number of incoming photons, the optical signal 
multiplexing is necessary to measure photon correlations among the channels and thus reconstruct the input photons statistics. The readout and counting of the photon arrivals is performed with a fast multi-channel Asynchronous-Correlated-Digital-Counter (ACDC) implemented in the Programmable Logic (PL) of a Xilinx ZYNQ-7020 SoC device. The optical input is given from a laser diode with wavelength $878 \mathrm{~nm}$, pulsed at a repetition rate of $100 \mathrm{kHz}$, with a pulse width of $2 \mathrm{~ns}$.

N18: Photodetectors I - SiPM radiation damage

Tuesday, Nov. $1 \quad$ 08:00-10:00 Curie 2

N18-1: Recent Progress in Silicon Photomultiplieres.

$\underline{\text { Y. Musienko }}$

University of Notre Dame/INR(Moscow), Geneva, Switzerland

This presentation reviews the latest developments in SiPMs, discusses the SiPM properties and problems and gives a speculative outlook on their future evolution. A special attention is paid to new developments in the field of radiation-hard SiPMs.

N18-2: Study of the Radiation Damage of SiPMs by Neutrons

M. Centis Vignali ${ }^{1}$, V. Chmill ${ }^{2}$, E. Garutti ${ }^{1}$, M. Nitschke ${ }^{1}$, R. Klanner ${ }^{1}$, J. Schwandt ${ }^{1}$

${ }^{1}$ Institute of Experimental Physics, University of Hamburg, Hamburg, Germany

${ }^{2}$ Samara State Aerospace University, Samara, Russian Federation

Silicon photomultipliers (SiPMs), thanks to their excellent performance, are becoming the photodetectors of choice for many applications. One major limitation, in particular for their use at high-luminosity colliders, is the radiation damage by hadrons. In this work, SiPMs with 4384 pixels of $15 \times 15 \mu \mathrm{m} 2$ size produced by KETEK have been irradiated by eactor neutrons to six fluences up to $1012 \mathrm{n}$ eq $/ \mathrm{cm} 2$ (1 MeV equivalent neutrons). Pulse-height, $\mathrm{I}-\mathrm{V}$, and $\mathrm{C}-\mathrm{V}$ easurements with and without illumination by a LED for temperatures between $-30^{\circ} \mathrm{C}$ and $+30^{\circ} \mathrm{C}$ have been performed. In this paper results from the $\mathrm{I}-\mathrm{V}$, and $\mathrm{C}-\mathrm{V}$ measurements are shown. The fluence and the temperature dependence of the current and of the SiPM electrical parameters like pixel capacitance, quenching resistance and breakdown voltage allows to better understand the origin of the dark current and find ways to reduce the radiation-induced dark-count rate. The results are compared to simulations.

\section{N18-3: A Comparison of Radiation Damage from Gamma Rays and Neutrons in SiPMs}

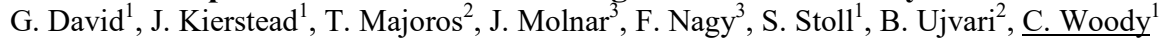

${ }^{I}$ Physics Department, Brookhaven National Lab, Upton, NY, USA

${ }^{2}$ Depatrment of Physics, University of Debrecen, Debrecen, Hungary

${ }^{3}$ Institute for Nuclear Research, Atomki, Debrecen, Hungary

The effects of radiation damage in SiPMs from gamma rays has been measured and compared with the damage produced by neutrons. SiPMs from several different manufacturers were exposed to Co-60 gamma rays at the Solid State Gamma Irradiation Facility at Brookhaven National Lab and the Atomki Institute for Nuclear Research in Debrecen, Hungary, and compared with similar devices exposed to neutrons Atomki and at the LENS Facility at Indiana University. The gamma ray exposures ranged from $1 \mathrm{krad}$ to more than $1 \mathrm{Mrad}$ and the neutron exposures ranged from $10^{\wedge} 9 \mathrm{n} / \mathrm{cm}^{\wedge} 2$ to $10^{\wedge} 13 \mathrm{n} / \mathrm{cm}^{\wedge} 2$. The main effect of gamma ray damage is an increase in the noise and leakage current in the irradiated devices, similar to what is seen from neutron damage, but the level of damage is considerably less at comparable high levels of exposure. In addition, the damage from gamma rays seems to saturate after a few hundred krad, while the damage from neutrons show no sign of saturation, suggestive of different damage mechanisms in the two cases. Damage due gamma rays from increased optical absorption in the epoxy window of the SiPMs will also be measured separately. Studies of damage and recovery, along with detailed materials analysis to study the effects of the damage, will be reported at the conference.

\section{N18-4: Impact of Local Defects on the Dark Count Rate of SiPM}

E. Engelmann $^{1}$, E. Popova ${ }^{2}$, F. Wiest ${ }^{3}$, P. Iskra ${ }^{3}$, S. Vinogradov ${ }^{4}$, R. Fojt ${ }^{3}$, E. Garutti ${ }^{5}$, W. Hansch ${ }^{1}$

${ }^{I}$ Institute for Physics, Universitaet der Bundeswehr Muenchen, Munich, Germany

${ }^{2}$ National Research University Moscow Engineering Institute, Moscow, Russia

${ }^{3}$ KETEK GmbH, Munich, Germany

${ }^{4}$ P.N. Lebedev Physical Institute, Moscow, Russia

${ }^{5}$ Institute for Experimental Physics, Universitaet Hamburg, Hamburg, Germany

The decreasing of the dark count rate (DCR) of Silicon-Photomultipliers (SiPM) is a modern challenge, which could lead to an enormous enhancement of the application range of this promising photo-detector. In this work we exploit the effect called "micro-plasma emission" in which light is emitted by radiative transitions of charge carriers generated during a micro-cell 
breakdown. Using a low light level camera, local defects ("hotspots") with an enhanced dark event rate within the active areas of the micro-cells could be identified and investigated separately. These spatially confined areas with a diameter of 3-5 $\mu \mathrm{m}$ are randomly distributed over the active area of the detector. A significant contribution up to $55 \%$ to the overall dark count rate could be attributed to $1-5 \%$ of the micro-cells. The aim of this work is to generate a deeper understanding of the origin of the hotspots in order to suppress their occurrence. So far the temperature dependence of their emitted light intensity revealed the generationrecombination mechanism to be the dominant hotspot-process. We expect the ion implantation to be responsible for the introduction of the majority of generation-recombination centers to the silicon crystal. Indeed, first experimental results show a clear dependence of the number and the emission intensity of hotspots on the ion implantation energy. In conclusion, our work indicates the necessity and provides possibilities for further research of local defects with regard to their suppression.

N18-5: Studies of 1400 Hamamatsu Production SiPM Arrays for the CMS HCAL Phase I Upgrade. Y. Musienko ${ }^{1,2}$, Y. Andreev ${ }^{2}$, A. Heering ${ }^{1}$, A. Karneyeu ${ }^{1,2}$, V. Postoev ${ }^{2}$, R. Ruchti ${ }^{1}$, M. Wayne ${ }^{1}$

${ }^{I}$ Physics, University of Notre Dame, Notre Dame, IN 46556, USA

${ }^{2}$ HEP, Institute for Nuclear Research (RAS), Moscow, Russsia

The CMS Barrel (HB) and Endcap (HE) Hadron Calorimeters are scintillator sampling calorimeters with embedded wavelength shifting fibers (WLS) in scintillator tiles. In 2012 the HCAL Phase I upgrade was approved for the increased luminosity $\left(5^{*} 10^{\wedge} 34\right)$ of SLHC. A key aspect of the HCAL upgrade is to add longitudinal segmentation to improve background rejection, energy resolution and scintillator radiation damage compensation. The increased segmentation can be achieved by replacing the hybrid photodiodes (HPDs) with silicon photomultipliers (SiPMs)). The SiPMs for the CMS HCAL upgrade have to operate in a very hostile HL LHC radiation environment (we expect a maximum total dose of $10^{\wedge} 12 \mathrm{n} / \mathrm{cm}^{\wedge} 2$ for an integrated luminosity of $3000 \mathrm{fb}^{\wedge}-1$ ). They should have good linearity for a wide range of scintillating signals and excellent reliability. During the last few years we have successfully completed the R\&D for instrumentation of SiPMs for the Phase 1 upgrade of HE/HB in 2017 and 2018. After successful production of 200 8-channel SiPM arrays Hamamatsu was selected as a vendor for the CMS HE Phase I upgrade project. Here we report the results of our measurements on 1400 production Hamamatsu 8-channel SiPM arrays . An overview of the results of our measurements of the most important SiPM parameters is reported in this presentation. Results from a study on the radiation hardness of silicon photomultipliers (SiPMs) are also presented. The SiPMs were exposed to neutrons at fluences up to $2 \times 10^{\wedge} 12 \mathrm{n} / \mathrm{cm}^{\wedge} 2$. The SiPM's main parameters were measured before and after irradiation. The effects of the neutron radiation on breakdown voltage, quenching resistance value, gain, photon detection efficiency and dark current for these devices are shown and discussed.

N18-6: Efficient Positioning of Silicon Photomultipliers on Large Scintillation Crystals P. R. Menge, K. Yang, M. McLaughlin, B. Brian

Saint-Gobain Crystals, Hiram, OH, United States

Silicon photomultipliers (SiPMs) are attractive replacements for photomultiplier tubes (PMTs) when compact geometry and low power draw are important. However, many radiation detector applications require large volumes of monolithic scintillator, which can pose a problem for the use of SiPMs in such applications. Single device SiPMs are smaller than $1 \mathrm{~cm} 2$ and large scintillators require an array of SiPMs or a large number of individual units to efficiently detect the light. When multiples of SiPMs are used, the dark count noise and cost increase proportionally with the surface area covered. As of 2016, SiPMs are roughly 5x the price of PMTs on a per $\mathrm{cm} 2$ basis. When too few SiPMs are used, non-uniformity of light collection degrades the energy resolution of the detector. Strategic placement of a limited number of SiPMs on a large scintillator can reduce the number of SiPMs necessary and decrease the cost-to-performance ratio. Simulations and experiments are being performed to find general guidelines regarding optimal positioning of SiPMs on large $\mathrm{NaI}(\mathrm{Tl})$ crystal scintillators. For example, if the SiPMs are placed near edges and vertices on a polished cuboid face, the number of SiPMs necessary to achieve adequate energy resolution need only cover $30 \%$ or less of the light output face.

\author{
N18-7: Ultra-High-Density Silicon Photomultipliers with Improved Effective-Fill-Factor and Detection \\ Efficiency \\ $\underline{\text { F. Acerbi }}{ }^{1}$, A. Gola ${ }^{1}$, A. Ferri ${ }^{1}$, G. Zappala ${ }^{1}$, Y. Musienko ${ }^{2}$, A. Heering ${ }^{2}$, G. Paternoster ${ }^{1}$, C. Piemonte ${ }^{1}$, R. C. Ruchti ${ }^{3}$, N. Zorzi ${ }^{1}$ \\ ${ }^{I}$ FBK, Trento, Italy \\ ${ }^{2}$ CERN, Genevra, Switzerland \\ ${ }^{3}$ University of Notre Dame, Paris, France
}

During last years, Fondazione Bruno Kessler (Trento) developed a "high-density" (HD) Silicon photomultipliers (SiPM) technology. HD-SiPM features narrow trenches to separate the SiPM microcells, obtaining small cells with high fill-factor (FF) and thus high Photon Detection Efficiency (PDE). We pushed this technology further and developed the ultra-high density RGB SiPM (RGB-UHD), with extremely low cell size. The devices have cell pitches between $7.5 \mu \mathrm{m}$ and $12.5 \mu \mathrm{m}$, arranged in a honeycomb configuration, corresponding to a cell density between 20500 and 7400 cells $/ \mathrm{mm} 2$, respectively. The main issue of 
such small cells was the active area border effect which dramatically reduces the effective FF. In this contribution we describe the new version of UHD SiPM, with a "new guard ring" (NGR) structure with engineered doping profiles leading to a reduction of the border transition region. Thanks to this technology we were able to have functioning SiPM with $5 \mu$ m-size pitch. This device features a cell density of 46190 cells $/ \mathrm{mm} 2$ with a FF of $\sim 4 \%$, and a PDE reaching $15 \%$. Another important feature of this $\mathrm{SiPM}$ is the recovery time constant which is shorter than $5 \mathrm{~ns}$. Such characteristics are of great interest in applications that require high dynamic range, like detection of high energy gamma rays, or applications requiring a good resistance to radiation damage, such as the CMS ECAL upgrade. In the conference we will describe in detail the performance of the new UHD SiPM technology.

N19: Circuits for readout of pixel detectors in photon science

Tuesday, Nov. 1 10:30-12:00 Cassin

N19-1: Characterization of a Photon Counting CHASE Jr. Chip in a 40nm CMOS Process with the C8P1 Charge Sharing Correction Algorithm Using a Collimated X-Ray Beam.

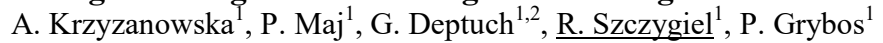

${ }^{1} A G H$ University of Science and Technology, Krakow, Poland

${ }^{2}$ ASIC Development Group of Electrical Engineering Department of Particle Physics Division, Fermi National Accelerator Laboratory, Batavia, USA

In hybrid pixel detectors charge sharing phenomenon occurs when charge generated in a detector as a result of photon interaction is collected by more than one pixel. This effect may introduce serious problems in detection systems such as missing some of the events or incorrect photon energy detection. The C8P1 algorithm for charge sharing compensation was implemented in the chip CHASE Jr. that was characterized for the first time using synchrotron radiation. The tests aimed at verification of charge sharing elimination possibilities. The influence of the global threshold settings, detector bias voltage as well as uniformity of the channels analog parameters on the algorithm performance is studied. The complex architecture of the chip, including the C8P1 algorithm implementation is described in details. The newest results from the synchrotron test prove that the charge sharing elimination algorithm enables retrieving total photon energy and registering proper number of hits even when events occur at pixel borders for the photon energy equal to $8 \mathrm{keV}$.

This work was supported by National Science Center, Poland under Contract UMO_2013/09/B/ST7/01627.

N19-2: IBEX: Versatile Readout ASIC with Spectral Imaging Capability and High Count Rate Capability M. Bochenek, S. Bottinelli, C. Brönnimann, T. Loeliger, R. Schnyder

DECTRIS Ltd., Baden-Daettwil, Switzerland

IBEX is a novel IC developed at DECTRIS Ltd. and dedicated for readout of Hybrid Photon Counting (HPC) semiconductor pixel detectors. The IBEX chip is a radiation tolerant design fabricated in the $110 \mathrm{~nm}$ CMOS technology. It has dimensions of $19.27 \mathrm{~mm} \times 19.76 \mathrm{~mm}$ and comprises of 65536 pixels. The pixel size is $75 \mu \mathrm{m} \times 75 \mu \mathrm{m}$. A so-called merging mode allows for an increased pixel size of $150 \mu \mathrm{m} \times 150 \mu \mathrm{m}$. The pixel readout electronics supports electron and hole collection, and consists of a charge sensitive preamplifier with a programmable gain, a shaper and two comparators that allow for two independent energy thresholds. In the merging mode the number of energy thresholds is increased up to four. To minimize the energy threshold dispersion at comparator inputs 6-bit trim DACs are available. The ASIC can operate in the continuous readout mode with two independent 16-bit counters or in the high dynamic range mode with one 32-bit counter following the comparator. The IBEX chip features counter overflow handling and an instant retrigger technology with an adjustable dead time for an improved highrate counting performance. The maximal count rate measured with the IBEX chip DC-coupled with a Silicon sensor is 1.7E7 photons per second and pixel. The ASIC offers a selectable external data bus width of 4, 8, or 16-bit.

\section{N19-3: A Time Interleaved, 10 Bit SAR ADC with Split Capacitor DAC for Diffraction Imaging at X-Ray} FELs

$\underline{\text { L. Lodola }}^{1,2}$, P. Malcovati ${ }^{1,2}$, L. Ratti ${ }^{1,2}$, C. Vacchi ${ }^{1,2}$

${ }^{I}$ Department of Electrical, Computer and Biomedical Engineering, Università di Pavia, Pavia, Italy

${ }^{2}$ INFN, Pavia, Italy

The PixFEL project aims at substantially advancing the state of the art in the field of 2D X-ray imaging for application at the next generation free electron laser (FEL) facilities, through the adoption of cutting-edge microelectronic technologies and innovative design and architectural solutions, building a large area focal plane detector based on an active edge sensor interconnected to a dual layer front-end chip with on board memory. For this purpose, the collaboration is developing the fundamental microelectronic building blocks for the readout channel including a low noise analog front-end with dynamic compression feature, a high resolution and low power ADC and high density memories. This work focuses on the design and characterization of the ADC. An interleaved SAR ADC architecture was adopted to obtain a good trade-off between power, conversion speed and 
area occupation. The design is being carried out in a $65 \mathrm{~nm}$ CMOS technology. Different test structures have been designed and tested, with an area going from $0.00563 \mathrm{~mm} 2$ to $0.0072 \mathrm{~mm} 2$. The maximum DNL and INL obtained from the most promising structure are $1 \mathrm{LSB}$ and 3.5 LSB, respectively. The input noise is around $0.4 \mathrm{LSB}$. From simulation, the power consumption is 85 $\mathrm{uW}$.

The activity leading to the results presented in this paper was carried out in the framework of the PixFEL project, funded by the Italian Institute for Nuclear Physics (INFN).

N19-4: Counting Integrating Pixel Readout Chip for Medical and Photon Science Applications R. Leys, R. Blanco, I. Peric

IPE, University of Karlsruhe, Eggenstein-Leopoldshafen, Germany

A multi purpose pixel readout ASIC has been implemented in UMC 180nm technology. The ASIC can both count the charge signals and measure the charge integral in a wide dynamic range. The chip has been implemented within a full mask run, which allows different post processing such as implementation of through silicon vias and redistribution layer routing. Different matrix connection options are presented for this production run, like the use of through silicon vias (TSV), enabling studying different connection schemes using multiple ASICs to create large sensor arrays. Ongoing Measurement results will be presented in the final talk.

\section{N19-5: A Pixel Readout with Asynchronous Approximation of a Center of Gravity of a Charge Distribution from a Radiation Conversion Event} P. Otfinowski, G. Deptuch, P. Maj

Department of Measurement and Electronics, AGH University of Science and Technology, Cracow, Poland

This work presents a new method in solving the negative effects of charge sharing phenomenon, which occurs when a charge generated by an x-ray photon inside the active volume of a semiconductor detector is divided by two or more readout channels. Unlike in existing solutions, where the hit position is determined through additional analog signal processing, the presented approach relies on algorithms known from binary image processing to find the center of gravity of an area affected by a single photon hit.

Presented approach has a few advantages over the already presented solutions. Most of the processing is done in the digital domain, therefore the required analog circuitry is significantly limited, consisting only of the typical blocks found in a conventional imaging system. The operation is fully asynchronous, therefore no extra clock signal is necessary and the impact on hit processing speed is negligible. Additionally, the algorithm yields valid results even when the area affected by a single charge cloud is larger than $2 \times 2$ pixels.

This work presents the details of the algorithm's implementation and performance metrics. For that purpose, the test chip has been designed in CMOS $55 \mathrm{~nm}$ process. It consists of a matrix of $16 \times 16$ pixels, containing only the implementation of the algorithm in digital domain - the signals corresponding to the discriminators outputs are generated off-chip, to simplify the verification procedure.

This work was supported by National Science Centre, Poland, according to the decision UMO-2014/13/B/ST7/01168.

N19-6: Ultra Fast Single Photon Counting Chip with Through Silicon Vias

$\underline{\text { P. Grybos }}^{1}$, K. Kasinski ${ }^{1}$, P. Kmon ${ }^{1}$, R. Szcztgiel ${ }^{1}, K^{2}$. Zischke ${ }^{2}$

${ }^{I}$ Department of Measurement and Electronics, AGH University of Science and Technology, Krakow, Poland

${ }^{2}$ Fraunhofer IZM, Berlin, Germany

Nowadays, single photon counting pixel hybrid detectors are becoming increasingly popular in high energy physics, X-ray detectors for synchrotron applications and medical imaging. Readout chips for these detectors usually have the area of a few $\mathrm{cm}^{2}$ and are designed to be abutted on 3 sides. However many applications require large area detectors without dead zone. The TSV option aims to minimize the dead area for a 4-sided buttable large area detector tile. AGH Krakow and IZM Berlin have undertaken a common effort to apply TSV technology to the readout integrated circuit called UFXC32k (Ultra Fast X-ray Chip with $32 \mathrm{k}$ channels) with pixel pitch of $75 \mu \mathrm{m}$, an area of $2 \mathrm{~cm}^{2}$, containing approximately 50 million transistors. Dead time in the front-end can be set as low as $85 \mathrm{~ns}$. In the continuous readout mode a user can select the number of bits read out from each pixel to optimize the UFXC32k frame rate. The TSVs were applied to the IO pads of UFXC32k wafers. Each wafer contains about 110 chips and each chip has 87 IO pads for TSV processing. The TSV diameter $20 \mu \mathrm{m}$ was chosen and the wafers were thinned to $100 \mu \mathrm{m}$. The redistribution layer and the array of pads for solder bumping were distributed at the bottom side of the chips. The UFXC32k chips with TSVs were bump bonded to silicon sensors of $320 \mu \mathrm{m}$ thick and the chip-detector modules were attached to LTCC boards. 
N20: Nuclear Physics Instrumentation I: Measurements and New Techniques

Tuesday, Nov. 1 10:30-12:00 Madrid

N20-1: Parallel-Plate Avalanche Counter with Optical Readout (O-PPAC): a New Detector Concept for Heavy-Ions Tracking and Imaging.

M. Cortesi, J. Yurkon, A. Stolz

National Superconducting Cyclotron Laboratory, Michigan State University, East Lansing, USA

We report the development of a novel gaseous detector concept consisting of a parallel-plate avalanche counter filled with highyield scintillating gas (i.e. CF4) and equipped with an optical readout (O-PPAC). The latter is based on arrays of small-area, collimated photo-sensors (SiPM or APD), positioned around the four sides of the PPAC's effective area. Compared to other more conventional charge-readout detector technologies, the O-PPAC has several noteworthy advantages, including a fast pulse propagation (good time resolution); insensitivity to electronic noise or RF pick-up problems because the readout is decoupled from the electron avalanche volume; no space charge limitations when operated in streamer mode (which allows high counting rate and large dynamic range with respect to the particle's mass and energy); good detection efficiency due to a large light yield; more uniform response and less angular/energy straggling due to a lower material budget. We present first experimental results with a test-bench detector system, comprising a small-area parallel-plate avalanche counter readout by a single SiPM; the detector was irradiated with $5.5 \mathrm{MeV}$ alphas. This simple prototype allows the investigation of the charge-light correlation in $\mathrm{CF} 4$, including dependence of electroluminescence yield on pressures and electric field applied to PPAC, time resolution measurement and effect of residual impurities on detection efficiency. In addition, we present a systematic Monte Carlo simulation study focused on O-PPAC geometry optimization and detector performance evaluation; we have found that the optimal detector assembling provides a sub-millimeter localization capability and sub-ns time resolution. Applications of the OPPAC include tracking, imaging and time-of-flight measurements for heavy-ions experiments, position-sensitive transmission detector for beam diagnostics in hadron-therapy, heavy-ions range radiography, and Compton scattering gamma-ray imaging camera.

N20-2: Design of a Mezzanine Card with Bandwidth Aggregation for HPGe Gamma Spectroscopy J. Collado, V. González, J. M. Blasco, E. Sanchis

Electronic Engineering, Universitat de Valencia - ETSE, Burjassot (VALENCIA), Spain

In experimental nuclear physics, HPGe segmented detectors are used to provide high energy resolution of the gamma rays. Besides, 4pi configuration is common to get a full coverage of the interaction point and detection of all the products of the collisions. In this type of experiments, the number of electronic channels can be high $(>100)$ and also the sampling frequency of the digitizing system ( $>100 \mathrm{Msps})$. This results in a high data rate per channel $(>2 \mathrm{Gbps})$ and thus a high aggregated bandwidth to process. In principle, this problem can be solved using a parallel data acquisition system where the input channels (commonly arriving through optical fibers) are read and processed accordingly to its bandwidth capacity. As a result of this limitation a high number of replicated electronics must be used that incur in high costs. However, Ethernet switch technology provides a way to compress the number of input channels taking profit of the high-performance FPGA devices presently available which can manage input link of up to $10 \mathrm{Gbps}$. So, data aggregation may increase the compactness of the processing system and reduce the number of replicated electronics.

This paper presents the design of an input data mezzanine with four SNAP12 optic links receiving $382 \mathrm{Gbps}$ channels. Inside the mezzanine, 1 to 4 multiplexing aggregation is implemented reducing the 38 input channels to ten 8Gbps output lines through a FMC connector. The FMC Mezzanine can be controlled from a FMC master by SPI with an internal low cost and low power FPGA. This FPGA is seen by the FMC master as a standalone intelligent device without the need of an active slow control.

\author{
N20-3: Characterization of New Generation Silicon Detector: SIRIUS Tunnel "Stripy-Pad" Detector \\ $\underline{\text { P. Brionnet }}$ \\ IPHC Strabourg, Strasbourg, France \\ On behalf of the SIRIUS collaboration
}

SIRIUS (Spectroscopy \& Identification of Rare Isotopes Using S3) is a detection system designed for the final focal plane of S3 (Super Separator Spectrometer) which is part of the SIPRAL2 facility. It is optimized for the study of very low production cross section such as exotic and super-heavy nuclei. This study presents the characterization of the tunnel silicon detector for SIRIUS. Based on the state-of-art in silicon detector, this new "Strippy-Pad" detector design benefits from ultra high resistivity and windowless technique from MICRON. The prototype and pre-series detectors were tested at Strasbourg on our innovative test bench with new fast preamplifiers (from CREMAT) and with our digital electronics (TNT2 Card). Beside the very good 
resolution obtained (better than $16 \mathrm{keV} @ 9 \mathrm{MeV}$ for alpha particles) those detectors allow us to perform online electron/alpha particle discrimination on the basis of pulse shape analysis with very good performance and very simple arguments.

\author{
N20-4: Experimental Evaluation of the Dynamic Range of the FARCOS Microstrip Frontend with a Pulsed \\ Proton Beam \\ A. Castoldi ${ }^{1}$, C. Guazzoni ${ }^{1}$, S. Maffessanti ${ }^{1}$, T. Parsani ${ }^{1}$, L. Carraresi ${ }^{2}$, C. Czelusniak ${ }^{2}$ \\ ${ }^{I}$ Politecnico di Milano, DEIB and INFN, Sezione di Milano, Milano, Italy \\ ${ }^{2}$ Università degli Studi di Firenze, Dip. Fisica e Astronomia and INFN, Sezione di Firenze, Italy, Firenze, Italy
}

FARCOS features high angular and energy resolution to reconstruct the particle's momentum at high precision and be capable of performing correlation measurements of LCPs and of LCPS and IMFs. In 2016 the first telescopes of FARCOS (Femtoscope ARray for Correlation and Spectroscopy) will be fully instrumented. A key feature of FARCOS is the capability to operate pulseshape analysis in order to fully identify the particles stopping even in the first detection layer. All the aforementioned requirements push a lot the frontend specs especially in terms of dynamic range (up to half $\mathrm{GeV}$ with 4 different selectable values) and of energy resolution (of the order of $10 \mathrm{keV} \mathrm{FWHM).} \mathrm{16-channel} \mathrm{charge} \mathrm{preamplifiers} \mathrm{are} \mathrm{integrated} \mathrm{in} \mathrm{a} \mathrm{single} \mathrm{chip}$ in AMS $0.35 \mu \mathrm{m} \mathrm{C35B4C3} \mathrm{technology.} \mathrm{A} \mathrm{dedicated} \mathrm{frontend} \mathrm{board} \mathrm{houses} 2$ ASICs and the linedrivers needed to provide a differential output and to drive the several-meter long connections. In order to probe experimentally the performance on beam and the achievable dynamic range of the FARCOS frontend system, we exploited the pulsed monoenergetic proton beam of one of the beamlines of the 3MV Tandetron accelerator of the LaBEC (Laboratorio di Tecniche Nucleari per i Beni Culturali of INFN), Sesto Fiorentino (FI), Italy. The bunch multiplicity can be increased up to cover the full designed dynamic range. From the measured histogram, by least square fitting of each proton peak one can derive the centroid, integral non linearity and resolution as a function of the deposited energy. The presentation will focus on the designed frontend system and on the results of the qualification of the system, together with the design of the final version of the frontend equipping the FARCOS telescopes.

This work has been supported by INFN in the framework of the EXOCHIM and NEWCHIM experiment.

N20-5: Spectroscopic Measurement of L X-Rays Emitted from a ${ }^{244} \mathrm{Cm}$ Source with a TES Microcalorimeter

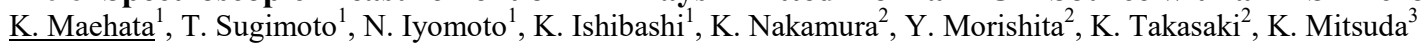

${ }^{I}$ Department of Applied Quantum Physics and Nuclear Engineering, Kyushu University, Fukuoka, Japan

${ }^{2}$ Japan Atomic Energy Agency, Ibaraki, Japan

${ }^{3}$ Japan Aerospace Exploration Agency, Sagamihara, Japan

Spectroscopic measurement of L X-ray is one of important techniques for the non-destructive assay of transuranium (TRU) elements. L X-rays of the energy ranging from $10 \mathrm{keV}$ to $25 \mathrm{keV}$ are emitted following internal conversion after the a-decay of TRU elements. High purity germanium semiconductor detectors have been used in spectroscopic measurement of L X-rays emitted from TRU elements so far. However, the accurate identification of L X-ray peaks is difficult due to the insufficient energy resolution of the detector and slightly differences between the L X-ray lines. Recently, transition-edge-sensor (TES) microcalorimeters have been developed for spectroscopic measurements of X-and gamma-rays with excellent energy resolution. In this work, a TES microcalorimeter was operated for spectroscopic measurement of Pu L X-rays emitted from a ${ }^{244} \mathrm{Cm}$ source. Energy spectrum of plutonium L X-rays was obtained by processing detection signal pulses with using an optimal filtering method. In the obtained energy spectrum, peaks of Pu L X-ray were clearly identified with the full-width at half maximum (FWHM) energy resolution better than $50 \mathrm{eV}$. In peak fittings, natural line width of L X-ray emission and an impulse response of the detector was approximated by Lorentzian distribution and Gaussian distribution, respectively. The FWHM energy resolution of the TES microcalorimeter was obtained to be $43 \mathrm{eV}$ at $\mathrm{Pu} \mathrm{L}_{\mathrm{a} 1} \mathrm{X}$-ray peak of $14.28 \mathrm{keV}$ with natural line width of $12.20 \mathrm{eV}$. Experimental result shows that L X-ray peaks of TRU elements can be identified by using the TES microcalorimter with a high precision.

This work was financially supported by a Grant-in-Aid for Scientific Research (B) (24360397) from the Japan Society for the Promotion of Science.

N20-6: Measurement of the 19F(alpha,n)22Na Reaction Cross Section for Nuclear Safeguards Science S. J. Thompson ${ }^{1}$, W. A. Peters ${ }^{2,3}$, M. S. Smith ${ }^{2}$, S. T. Pittman ${ }^{2}$, R. R. C. Clement ${ }^{4}$, S. D. Pain ${ }^{3}$, M. Febrarro ${ }^{3,5}$, K. Smith $^{3}$, K. A. Chipps ${ }^{2}$, C. Reingold ${ }^{6}$, W. P. Tan ${ }^{7}$, D. W. Bardayan ${ }^{7}$, S. Ilyushkin ${ }^{8}$, M. M. Grinder ${ }^{1}$, J. A. Cizewski $^{6}$

${ }^{I}$ Nuclear Nonproliferation, Idaho National Laboratory, Idaho Falls, ID, USA

${ }^{2}$ Physics, Oak Ridge National Laboratory, Oak Ridge, TN, USA

${ }^{3}$ University of Tennessee, Knoxville, TN, USA

${ }^{4}$ U.S. Airforce, Brevard, FL, USA

${ }^{5}$ University of Michigan, Ann Arbor, MI, USA

${ }^{6}$ Rutgers University, New Brunswick, USA 
${ }^{7}$ University of Notre Dame, Notre Dame, IN, USA

${ }^{8}$ Colorado School of Mines, Golden, CO, USA

Alpha particles emitted from the decay of Uranium in a UF6 matrix can interact with Fluorine and generate neutrons via the 19F(alpha,n)22Na reaction. These neutrons can be used to determine the Uranium content in a UF6 storage cylinder. The accuracy of this self-interrogating, non-destructive assay (NDA) technique is, however, limited by the large uncertainty of a critical input for NDA simulations: the probability (cross section) of the $19 \mathrm{~F}$ (alpha,n)22Na reaction. We have measured this cross section by bombarding thin LaF3 targets with 4He beams from an accelerator at the Nuclear Science Laboratory at the University of Notre Dame. We collected over 4000 neutron time-of-flight spectra with an array of plastic scintillator bars at 135 different alpha bombarding energies ranging from $4-6.7 \mathrm{MeV}$ with energy steps of $25 \mathrm{keV}$. This energy range covers what is needed for most NDA studies. We also performed a complementary measurement of this reaction over four targeted energy ranges with a 19F beam and a $4 \mathrm{He}$ gas target at Oak Ridge National Laboratory, using $5 \mathrm{keV}$-wide energy steps. From these data sets, we have determined an absolute cross section for $19 \mathrm{~F}$ (alpha,n)22Na to an average precision of $7 \%$ from $4-6.7 \mathrm{MeV}$ alpha bombarding energies. We will present details of our measurements and demonstrate its impact on NDA applications. This new cross section enables improved interpretations of NDAs of containers of any fluorinated actinide compound of arbitrary size and configuration.

Research supported by the Office of Defense Nuclear Nonproliferation R\&D in the National Nuclear Security Administration (NNSA), US Department of Energy under contract DE-AC05-00OR22725 with Oak Ridge National Laboratory, managed and operated by UT-Battelle, LLC.

N21: Calorimetry I - Calorimeters in lower-energy Experiments

Tuesday, Nov. $1 \quad$ 10:30-12:00 Londres

N21-1: Status and Test Beam Results for the sPHENIX Calorimeter Systems

A. M. Sickles

University of Illinois, Urbana, IL, US

On behalf of the sPHENIX Collaboration

The sPHENIX Collaboration is planning a major upgrade to the PHENIX experiment at RHIC that involves building an entirely new spectrometer based around the former BaBar solenoid magnet that will enable a comprehensive study of jets and heavy quarkonia in relativistic heavy ion collisions. It will include two new calorimeter systems, one electromagnetic and one hadronic, that will cover an acceptance of \pm 1.1 units in pseudorapidity and 2 ? in azimuth. The hadronic calorimeter (HCAL) will be a steel plate and scintillating tile design that is read out with wavelength shifting fibers and silicon photomultipliers. It will be divided into two sections: an Inner HCAL situated inside the magnet and an Outer HCAL located outside the magnet. The electromagnetic calorimeter will be a SPACAL design consisting of a tungsten powder epoxy matrix absorber with embedded scintillating fibers which are also read out with silicon photomultipliers. Prototypes of all three calorimeter detectors have been built and tested in the test beam at Fermilab in April of 2016. Results from those tests, along with a detailed comparison to Monte Carlo simulations, will be presented in this talk.

N21-2: The Light Collection Non-Uniformity of Strongly Tapered PWO Crystals and Its Impact on the Energy Resolution of the PANDA Electromagnetic Calorimeter in the Energy Region below $1 \mathrm{GeV}$

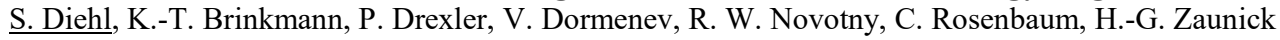

2nd Physics Institute, University Giessen, Giessen, Germany

The electromagnetic calorimeter (EMC) of the PANDA detector at the future FAIR facility comprises more than 15,000 lead tungstate (PWO) crystals. The barrel part will consist of 11 crystal geometries with different degree of tapering, which causes a non-uniformity in light collection as an interplay between the focusing and the internal absorption of the light. For the most tapered crystals the detected light is enhanced by $40 \%$, if the scintillation light is created in the front part of the crystal. Due to the shower development and its fluctuations the non-uniformity leads to a reduction of the energy resolution. To reduce this effect, one lateral crystal side face has been depolished to a roughness of $0.3 \mu \mathrm{m}$. Measurements confirm an increase of the light yield in the rear part of the crystal. In contrast, only a slight decrease can be observed in the front part. The overall non-uniformity is significantly reduced below 5\%. This paper will discuss the experimental studies based on GEANT4 and optical simulations to understand the impact of a de-polished side face on the light collection. For consequences on the future performance, a $3 \times 3$ subarray of de-polished crystals was directly studied using a tagged photon beam in the energy range from $50 \mathrm{MeV}$ up to $800 \mathrm{MeV}$, respectively, performed at the tagged photon facility at MAMI, Mainz. The comparison to an array composed of polished crystals confirms a significant improvement of the energy resolution in the region above $200 \mathrm{MeV}$, resulting in a reduction of the constant term of the energy resolution from more than $2 \%$ down to $0.5 \%$ and only a small increase of the statistical term. The results can be reproduced in GEANT4 simulations.

The project is supported by BMBF, GSI and HIC for FAIR. 
N21-3: The Combined Electromagnetic Calorimeter of the CMD-3 Detector.

V. F. Kazanin

Budker Institute of Nuclear Physics, Novosibirsk, Russian Federation

On behalf of the CMD-3 Collaboration

The Cryogenic Magnet Detector 3 (CMD-3) detector has been collecting data since 2010 at the $\mathrm{e}^{+} \mathrm{e}^{-}$accelerating complex VEPP2000 at the Budker Institute of Nuclear Physics in Novosibirsk, Russia. The complex VEPP-2000 uses the novel round beam technique and provides a high luminosity in the range from 0.3 to $2 \mathrm{GeV}$ in c.m. The main physics goal of the CMD-3 experiment is the study of the $\mathrm{e}^{+} \mathrm{e}^{-}$annihilation into hadrons. The CMD-3 is a general-purpose detector which with high efficiency for both charge and neutral particles.

One of the principal detecting system is a joint electromagnetic calorimeter that purposed to register photons and measure its parameters in all energy range. The calorimeter is unhomogenius with coverage of almost $4 \mathrm{p}$ solid angle and consists of the barrel calorimiter, based on Liquid Xenon and CsI crystals, and endcap calorimeter, based on BGO crystals. The main parameters and resolutions of the calorimeters are presented.

N21-5: Liquid Xenon Detector with VUV-Sensitive MPPCs for the MEG Experiment Upgrade S. Ogawa

The University of Tokyo, Tokyo, Japan

On behalf of the MEG II Collaboration

The MEG II experiment is an upgrade of the MEG experiment to search for the charged lepton flavor violating decay of muon, $\mu$ $\rightarrow \mathrm{e}$ ?. The MEG II experiment is expected to reach a branching ratio sensitivity of $4 \times 10^{-14}$, which is one order of magnitude better than the sensitivity of the current MEG experiment. The performance of the liquid xenon (LXe) ?-ray detector will be greatly improved with a highly granular scintillation readout realized by replacing 216 photomultiplier tubes (PMTs) on the ?-ray entrance face with 4092 Multi-Pixel Photon Counters (MPPCs). For this purpose, we have developed a new type of MPPC which is sensitive to the LXe scintillation light in vacuum ultraviolet (VUV) range, in collaboration with Hamamatsu Photonics K.K. The MPPC has been tested, and an excellent performance has been confirmed including high photon detection efficiency ( $>15 \%)$ for LXe scintillation light. An excellent performance of the LXe detector has been confirmed by Monte Carlo simulation based on the measured properties of the MPPC. The construction and the commissioning of the detector is in progress, aiming to start physics data taking in 2017. The performance of the VUV-sensitive MPPC will be reported, as well as the preliminary results of the detector commissioning.

N22: Gaseous detectors I: Development of Techniques I

Tuesday, Nov. $1 \quad$ 10:30-12:00 Curie 2

N22-1: Modeling Impurity Concentrations in Liquid Argon Detectors

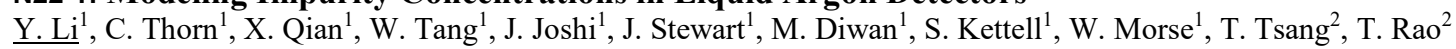

${ }^{I}$ Physics Department, Brookhaven National Laboratory, Upton, NY, 11973

${ }^{2}$ Instrumentation Division, Brookhaven National Laboratory, Upton, NY, 11973

We present a simple mathematical model of the dynamics of impurity distribution in liquid argon detectors. This model considers the dominant sources, sinks, and transport of the impurity between and within the gas and liquid phases of a detector. We use this model to extract Henry's coefficients for oxygen and water from the time dependence of the impurity concentration in the liquid phase, while the gas in the detector volume is being purified. We find that Henry's coefficient for oxygen in liquid argon is 0.95 , in agreement with previous measurements by other methods. For water, we obtain the first measured value of Henry's coefficient in liquid argon of $\sim 1.0$. This value is in rough agreement with the value estimated from the ideal solution theory using an extrapolation of the water vapor pressure.

\author{
N22-2: A Novel Technique for the Measurement of the Avalanche Fluctuation of Gaseous Detectors Using \\ Laser-Induced Tracks \\ T. Ogawa \\ Graduate University for Advanced Studies (SOKENDAI), Tsukuba, Japan \\ On behalf of the LCTPC-Asia Collaboration
}

Gas amplification of the electrons created by X-rays, UV photons, or charged particles plays an essential role in their detection with gaseous detectors. It acts as "preamplifier" with a sufficient gain. However, its gain fluctuates because of avalanche statistics, thereby degrading the energy resolution for monochromatic X-rays. For large Time Projection Chambers (TPCs) the 
azimuthal spatial resolution at long drift distances is partly limited by the relative variance of the gas gain for single drift electrons. Conventionally, avalanche fluctuations are estimated from the gas-amplified charge spectrum for single electrons created by a UV lamp or a laser. We have developed a novel technique for the measurement of the relative variance of avalanche fluctuation $(f)$ using laser-induced tracks, exploiting the fixed cluster size of one for each ionization act along the tracks. The primary electrons are multiplied by a gas amplification device, and then collected by several readout pad rows arranged along the laser beam. The signal charges on adjacent pad rows are compared for each laser shot. The value of $f$ is estimated from the width of the distribution of their differences using a straightforward relation. The technique is relatively simple and requires a short data-taking time of several tens of minutes. Furthermore, it is applicable for low gas gains. We present the experimental setup as well as the measurement principle, and the results obtained with a stack of Gas Electron Multipliers (GEMs) for several gas mixtures.

\section{N22-3: Performance of New High-Precision Muon Tracking Detectors for ATLAS}

H. K. Kroha, O. Kortner, E. Takasugi

Max-Planck-Institut fuer Physik, Munich, Germany

The goals of the ongoing and planned ATLAS muon detector upgrades are to increase the acceptance for precision muon momentum measurement and triggering and to improve the rate capability of the muon chambers in the high-background regions corresponding to the increasing LHC luminosity. Small-diameter Muon Drift Tube (sMDT) chambers have been developed for these purposes. With half the drift-tube diameter of the current ATLAS Muon Drift Tube (MDT) chambers with 30 mm drift tube diameter and otherwise unchanged operating parameters, the sMDT chambers share all the advantages of the MDTs, but have an about an order of magnitude higher rate capability and can be installed in detector regions where MDT chambers do not fit in. The construction of twelve chambers for the feet regions of the ATLAS detector (see Fig.1) is currently in progress for the installation in the winter shutdown 2016/17 of the LHC. The purpose is to increase the acceptance for three-point measurement which will substantially improve the muon momentum resolution in these regions. An unprecedentedly high wire positioning accuracy of 5 microns has been achieved in the construction of these chambers.

N22-4: Development of the Next-Generation Micro Pixel Chamber-Based Neutron Imaging Detector ( $\mu$ NID) for Energy-Resolved Neutron Imaging with High Rate and High Spatial Resolution at the J-PARC/MLF

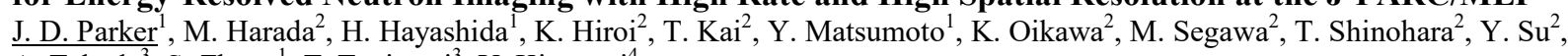
A. Takada ${ }^{3}$, S. Zhang ${ }^{1}$, T. Tanimori ${ }^{3}$, Y. Kiyanagi ${ }^{4}$

${ }^{I} R \& D$ Division, CROSS-Tokai, Tokai, Ibaraki, Japan

${ }^{2}$ J-PARC Center, Japan Atomic Energy Agency, Tokai, Ibaraki, Japan

${ }^{3}$ Graduate School of Science, Kyoto University, Kyoto, Japan

${ }^{4}$ Graduate School of Engineering, Nagoya University, Nagoya, Aichi, Japan

The Energy-Resolved Neutron Imaging System RADEN, located at beam line BL22 of the J-PARC Materials and Life Science Experimental Facility (MLF) in Japan, is the world's first dedicated high-intensity, pulsed neutron imaging beam line. In addition to conventional radiography and tomography, recently-developed energy-resolved neutron imaging techniques are used to directly image the macroscopic distribution of microscopic properties of bulk samples in situ, including crystallographic structure and internal strain (Bragg-edge transmission), nuclide-specific density and temperature distributions (resonance absorption), and internal/external magnetic fields (polarized neutron imaging). To carry out such measurements in the high-rate, high-background environment at RADEN, we use cutting-edge detector systems employing micropattern detectors and data acquisition systems based on Field Programmable Gate Arrays to provide the necessary sub- $\mu$ s time resolution for accurate measurement of neutron energy by time-of-flight, high counting rates, and event-by-event background rejection. One such detector, the Micro Pixel Chamber-based Neutron Imaging Detector $(\mu N I D)$, provides a spatial resolution of $120 \mu \mathrm{m}(\mathrm{s})$, time resolution of $0.6 \mu \mathrm{s}, 18 \%$ detection efficiency for thermal neutrons (He-3 for conversion), and effective gamma sensitivity of less than $10^{-12}$. We have recently increased the rate capacity from $600 \mathrm{kcps}$ to more than $4 \mathrm{Mcps}$ via an upgrade of the front-end electronics and tested a new gas mixture with increased electron drift velocity for improved rate performance, decreased electron diffusion for improved spatial resolution, and an increased He-3 fraction. We have also developed new readout planes with strip pitches of $280 \mu \mathrm{m}$ and $215 \mu \mathrm{m}$, down from $400 \mu \mathrm{m}$, with corresponding improvement in the spatial resolution expected. In this presentation, we will discuss the ongoing development of the $\mu$ NID and show results of recent on-beam tests performed at RADEN.

\section{N22-5: The RD51 Collaboration $\underset{i}{i \frac{1}{2}}$ Development of Micro Pattern Gas Detectors Technology}

T. Geralis

INPP, NCSR Demokritos, Athens, Greece

On behalf of the RD51 Collaboration

Driven by the availability of modern photolithographic techniques, Micro Pattern Gas Detectors (MPGD) have been introduced at the end of the 20th century by pioneer developments: Microstrip Gas Chambers (MSGC), Gas Electron Multipliers (GEM) and 
Micromegas, later followed by thick-GEM (THGEM), resistive GEM (RETGEM) and other novel micro-pattern devices. Nowadays intensive R\&D activities in the field of MPGDs and their diversified applications are pursued by the large CERNRD51 collaboration. The aims are to facilitate the development of advanced gas-avalanche detector concepts and technologies and associated electronic-readout systems, for applications in basic and applied research. MPGD systems now offer robustness, very high rate operation, high precision spatial resolution (sub 100-micron), and protection against discharges. MPGDs became important instruments in current particle-physics experiments and are in development and design stages for future ones. They are significant components of the upgrade plans for ATLAS, CMS, and ALICE at the LHC, exemplifying the beneficial transfer of detector technologies to industry. Beyond their design for experiments at future facilities (e.g. ILC), MPGDs are considered for rare-event searches, e.g. dark matter, double beta decay and neutrino scattering experiments. Detectors sensitive to x-rays, neutrons and light are finding applications in other diverse areas such as material sciences, hadron therapy systems, homeland security etc. The areas of research activities within the RD51 MPGD collaboration includes detector physics \& technology, model simulations, , readout electronics, production techniques, common test facilities, and applications. By this broad coverage RD51 brings together leading experts in the field of detector science and detectors users, resulting in effective progress over a wide array of applications. This talk will review the activities of the RD51, its major accomplishments so far, and future plans.

\author{
N22-6: Construction of Triple-GEM Detectors Using Commercially Manufactured Large GEM Foils \\ M. Posik, B. Surrow \\ Temple University, Temple University, Philadelphia, PA, United States
}

Many experiments are currently using or proposing to use large area GEM foils in their detectors, which is creating a need for commercially available GEM foils. Currently CERN is the only main distributor of GEM foils, however with the growing interest in GEM technology keeping up with the increasing demand for GEMs will be difficult. Thus the commercialization of GEMs up to $50 \$$ times $\$ 50 \mathrm{~cm} \$^{\wedge} 2 \$$ have been established by Tech-Etch Inc. of Plymouth, MA, USA using the single-mask technique. The electrical performance and optical quality of the single-mask GEM foils have been found to be on par with those produced by CERN. The next critical step towards validating the Tech-Etch GEM foils is to test their performance under physics conditions. These measurements will allow us to quantify and compare the gain and efficiency of the detector to other tripleGEM detectors. This will be done by constructing four single-mask triple-GEM detectors, using foils manufactured by TechEtch, which follow the design used by the STAR Forward GEM Tracker (FGT). The stack is formed by gluing the foils to the frames and then gluing the frames together. The stack also includes a Tech-Etch produced high voltage foil and a 2D \$r-lphi\$ readout foil. While one of the four triple-GEM detectors will be built identically to the STAR FGT, the other three will investigate ways in which to further decrease the material budget and increase the efficiency of the detector by incorporating perforated Kapton spacer rings rather than G10 spacing grids to reduce the dead area of the detector.

The materials and tooling needed to assemble the triple-GEM detectors have been acquired. The GEM foils have been electrically tested, and a handful have been optically scanned. We found these results to be consistent with GEM foils produced by CERN. With the success of these initial tests, construction of the triple-GEM detectors is now under way.

\title{
N23: Accelerator technologies and beam line instrumentation - Poster session II
}

Tuesday, Nov. 1 14:00-16:00 Etoile

N23-1: QBeRT: an Innovative Instrument for the Real-Time Qualification of a Particle Beam.

D. Lo Presti $^{1,2}$, D. L. Bonanno ${ }^{2}$, F. Longhitano ${ }^{2}$, D. G. Bongiovanni ${ }^{2}$, G. Gallo ${ }^{2}$, N. Randazzo ${ }^{2}$, E. Leonora ${ }^{2}$, V. Sipala $^{3,4}$, S. Reito ${ }^{2}$

${ }^{\text {I} P h y s i c s ~ a n d ~ A s t r o n o m y, ~ U n i v e r s i t y ~ o f ~ C a t a n i a, ~ C a t a n i a, ~ I t a l y ~}$

${ }^{2}$ Sezione di Catania, Istituto Nazionale di Fisica Nucleare, Catania, Italy

${ }^{3}$ University of Sassari, Sassari, Italy

${ }^{4}$ Sezione di Cagliari, Istituto Nazionale di Fisica Nucleare, Cagliari, Italy

The QBeRT (Qualification of BEam Real-Time) detector is an innovative set of detectors designed for the real-time imaging and monitoring of particle beams for hadrontherapy. It is composed by a tracker and a residual range detector (RRD) based on scintillating optical fibers and an innovative read-out strategy and reconstruction algorithm, patented by the Istituto Nazionale di Fisica Nucleare (INFN), which advantageously reduces the cost of the devices and associated electronics. The tracker, $2 \mathrm{~mm}$ water equivalent thick, is composed by four layers of 500 micron scintillating fibers, read-out by two MPPC matrixes, and exhibits a $9 \times 9 \mathrm{~cm}^{\wedge} 2$ sensitive area and about 100 micron spatial resolution and is capable of measuring in real time the fluence of the particle beam in therapy conditions (up to $10^{\wedge} 9$ particles per spill) and the projections of the beam spot at the coordinates $\mathrm{x}$ and $y$ and reconstruct the position and size of the spot. The tracker is also capable of performing real time imaging of the beam spot, in low intensity particle beam conditions up to $10^{\wedge} 7$ particle per second. The RRD, $3.5 \mathrm{~cm}$ water equivalent range expandable up to $30 \mathrm{~cm}$, is composed by a stack of 500 micron scintillating fibers layers, read-out by a MPPC matrix, and is able to measure real time the dose distribution deposited in its volume and the energy distribution of the beam. The RRD has a FOV 
of $9 \times 9 \mathrm{~cm}^{\wedge} 2$ and can be also used, in imaging conditions, in combination with the tracker to perform real time radiography of an object interposed betweeen the beam and the detector. A prototype has been built and characterized with proton and carbon ions at CATANA with $62 \mathrm{MeV}$ protons, at CNAO with up to $250 \mathrm{MeV}$ protons and up to $400 \mathrm{AMeV}$ carbon ions and at TIFPA with up to $250 \mathrm{MeV}$ protons. This prototype, scalable and modular, was made with low-cost technologies and is compact, robust and lightweight in order to be easily integrated into hadrontherapy facilities. The working principle of the detector and the results of the measurements compared to reference detectors measurements are presented.

N23-3: Performance Study of Little Ionization Chambers at the Large Hadron Collider M. Kalliokoski $^{1}$, B. Dehning ${ }^{1}$, E. Effinger ${ }^{1}$, V. Grishin ${ }^{1}$, E. B. Holzer ${ }^{1}$, M. Kastriotou ${ }^{1,2,3}$, E. Nebot Del Busto ${ }^{1,2}$

${ }^{I}$ Beams, CERN, Geneve, Switzerland

${ }^{2}$ Physics, University of Liverpool, Liverpool, United Kingdom

${ }^{3}$ Cockcroft Institute, Warrington, United Kingdom

The main detector type used to monitor beam losses in the LHC is a 1.51, 1.1 bar nitrogen filled parallel plate Ionization Chamber (IC). In the locations where the beam losses can saturate the read-out electronics of the ICs, two other monitor types, Little Ionization Chambers (LIC) and Secondary Emission Monitors, have been installed next to the ICs. LICs have the same gas composition and pressure as the ICs, but with an active volume 30 times smaller. This reduction in sensitivity allows the LICs to stay below saturation during high loss events, such as injection of the beam into the LHC. In total there are 109 LICs installed. In this contribution we study the non-scaling gas gain with the volume of the LICs, summarize results from various beam measurements where LICs were used, and analyse the LIC response in the LHC during 2015 operation.

N23-5: A Beam Pulser at the TTT-3 Tandem Accelerator of the University of Naples F. Di Capua, L. Campajola

Università degli Studi di Napoli Federico II, Napoli, Italy

The continuous beam provided by the TTT- 3 accelerator of the University of Naples have to be pulsed with 5 ns length bunch to allow some experiments in many fields of the fundamental and applied physics. A beam pulser is designed to produce bunch of particles in a wide range of masses and energies. The pulse length can be varied from about $5 \mathrm{~ns}$ to $100 \mathrm{~ns}$ and the pulse repetition rate is adjustable in a very large range from few $\mathrm{Hz}$ to $100 \mathrm{kHz}$. The beam pulser is based on two electrostatic deflectors, one located before the accelerator and one after the analyzer magnet. The main feature of this device is the great flexibility in its use and the cost of the system that is based on a standard low-cost electronic instrumentation. The characteristics of the first low energy chopper prototype and the results obtained during preliminary tests with a proton beam are presented.

N23-7: A Smart Adjustable Nuclear Interactions Counter Based on Compact Arduino Control System and Readout

F. Iacoangeli

INFN, Roma, Italy

We realized a new, fine, easy to install detector for nuclear interaction based on twin plastic scintillators, ArduSiPM prototype readout, and remote handling system for adjustment of pair gap. The system made up of two small scintillators $(5 \times 10 \times 25 \mathrm{~mm} 3$ of BC-408) as nuclear interaction counters. Signal readout is provided by the ArduSiPM prototype system, which makes available four pairs of bias voltage channel and analog readout channel for as many $3 \times 3 \mathrm{~mm} 2$ SiPMs. Two Hamamatsu SiPMs are coupled with each scintillator to get readout redundancy and to use coincidence strategy for minimizing the system noise. A constant fraction system allows getting fine timing coincidence both between scintillators and between beam's trigger signal and scintillators to minimize the fake counts on radiative environment. Remote gap adjustment make possible to set the nuclear scattering angle of measurement as well as to make an angular scan. The detector was proposed as new nuclear interaction counter for the H8 telescope of the UA9 experiment.

N23-8: An Electron Spectrometer for Proton Driven Plasma Accelerated Electrons at AWAKE: Predicted Resolution of Energy and Emittance Measurements $\underline{\text { L. Deacon }}^{1}$, S. Jolly 1 , F. Keeble ${ }^{1}$, A. Goldblatt ${ }^{2}$, S. Mazzoni ${ }^{2}$, A. Petrenko ${ }^{2}$, B. Biskup ${ }^{2,3}$, M. Wing ${ }^{1,4,5}$

${ }^{I}$ Physics and Astronomy, University College London, London, UK

${ }^{2}$ CERN, Geneva, Switzerland

${ }^{3}$ Czech Technical University, Prague, Czech Republic

${ }^{4}$ DESY, Hamburg, Germany

${ }^{5}$ University of Hamburg, Hamburg, Germany 
The Advanced Wakefield Experiment (AWAKE), to be constructed at CERN, will be the first experiment to demonstrate protondriven plasma wakefield acceleration. The $400 \mathrm{GeV}$ proton beam from the CERN SPS will excite a wakefield in a plasma cell several meters in length. To probe the plasma wakefield, electrons of $10--20 \mathrm{MeV}$ will be injected into the wakefield following the head of the proton beam. Simulations indicate that electrons will be accelerated to $\mathrm{GeV}$ energies by the plasma wakefield. The AWAKE spectrometer is intended to measure both the peak energy and energy spread of these accelerated electrons. Under certain conditions it is also possible to use the spectrometer to measure the transverse beam emittance. The expected resolution of these measurements is investigated for various beam distributions, taking into account an optimised vacuum chamber and scintillator screen design and results of beam and optical tests.

\section{N23-9: Hardware/software Structure of LEETECH Test Beam Facility}

V. Krylov $^{1,2}$, S. Barsuk ${ }^{1}$, O. Bezshyyko ${ }^{2}$, L. Burmistrov ${ }^{1}$, O. Fedorchuk ${ }^{2}$, L. Golinka-Bezshyyko ${ }^{2}$, V. Kubytskyi ${ }^{1}$, R. Lopez $^{3}$, D. Sukhonos ${ }^{2}$, M. Titov ${ }^{4}$, D. Tomassini ${ }^{3}$

${ }^{1}$ Laboratoire de l'Accélérateur Linéaire, Orsay, France

${ }^{2}$ Taras Shevchenko National University of Kyiv, Kyiv, Ukraine

${ }^{3}$ CERN, Geneva, Switzerland

${ }^{4}$ IRFU, CEA, Saclay, France

The structure of newly commissioned test beam LEETECH is addressed with detailed description of key components design. The organization of low-level communication interfaces (Ethernet $+\mathrm{CAN}$ ) with embedded solutions is described indicating important peculiarities in the hardware design in accelerator working conditions. Based on piezoelectric motors, with developed controller the collimator system ensures the positioning precision of 20 mum, providing smooth adjustment of delivered beam intensity. The developed software allows to control the LEETECH parameters and monitor the data from the test detector, providing possibilities to auto-adjustment and automatic data acquisition. Using the ARM Cortex M4-based controllers and USBWavecatcher board makes the control and data taking system cheap, robust and scalable, allowing to connect and monitor the additional components such as detector positioning system or additional detector modules.

N24: Calorimetry - Poster session II

Tuesday, Nov. $1 \quad$ 14:00-16:00 Etoile

N24-1: Commissioning of Upgrade Forward Hadron Calorimeters of CMS

B. Bilki ${ }^{1,2}$

${ }^{I}$ Physics and Astronomy, University of Iowa, Iowa City, IA, USA

${ }^{2}$ Mathematics and Computing, Beykent University, Istanbul, Turkey

On behalf of the CMS Collaboration

The CMS experiment at the Large Hadron Collider (LHC) at CERN is upgrading the photo-detection and readout system of the forward hadron calorimeter (HF). During Long Shutdown 1, all of the original PMTs were replaced with multi-anode, thin window photomultipliers. At the same time, the back-end readout system was upgraded to micro-TCA readout. Here we report on the experience with commissioning and calibrating the HF front-end as well as the online operational challenges of the microTCA system.

N24-2: Power Pulsing of the CALICE Tile Hadron Calorimeter

M. Reinecke

DESY, Hamburg, Germany

On behalf of the CALICE Collaboration

A large scale prototype of a tile hadron calorimeter for the International Linear Collider detector is currently under development. The proposed calorimeter follows the particle flow concept, which requires high granularity and a compact detector design. This is accomplished by using scintillating tiles that are read out by novel Silicon Photomultipliers and the integration of the associated front-end electronics into the gaps between the absorber plates. In order to keep the calorimeter structure homogeneous and simple, no active cooling system is allowed for the inner detector. In consequence, the power dissipation of the front-end electronics has to be limited as far as possible with an aim of $25 \mu \mathrm{W}$ per channel. The key component to achieve this is switching off the consumers of the front-end electronics during the gaps in between the ILC bunch trains (power pulsing). In this contribution we show the first results for power pulsing with a full-extension prototype of $2.20 \mathrm{~m}$ length. Next to a description of the setup, we compare results for the detector performance with and without power pulsing. The challenges of switching huge supply currents of several amperes in $5 \mathrm{~Hz}$ rate to the front-end electronics and the experiment's power supplies are addressed as well as electromagnetic compatibility aspects. 
This project has received funding from the European Union's Horizon 2020 Research and Innovation programme under Grant Agreement no. 654168 .

N24-3: Construction and Testing of the First Prototype of the CMS High Granularity Calorimeter J. Freeman

Fermilab, Batavia, IL, USA

On behalf of the CMS HGCAL Collaboration

The CMS experiment has decided to construct a High Granularity Calorimeter (HGCAL) to meet the challenges of harsh radiation and unprecedented in-time event pileup projected by the High Luminosity LHC conditions. The HGCAL is realized as a sampling calorimeter, including an electromagnetic compartment comprising 28 layers of silicon pad detectors with pad areas of $0.5-1.0 \mathrm{~cm}^{\wedge} 2$ interspersed with absorbers made from tungsten and copper to form a highly compact and granular device. A silicon-based single-layer prototype has been constructed and tested at Fermilab Test Beam Facility. The prototype includes many of the features required for this challenging detector, including a complex PCB glued directly to the sensor, using throughhole wire-bonding for signal readout and $\sim 5 \mathrm{~mm}$ spacing between layers - including the front-end electronics and all services. We present results from first tests of this prototype with beams of electrons and pions of up to $32 \mathrm{GeV} / \mathrm{c}$ as well as $120 \mathrm{GeV} / \mathrm{c}$ protons, and describe the plans for more extensive prototypes to be tested in beams at future test beam campaigns.

N24-4: High Resolution Metallic Magnetic Calorimeters for Rare Event Search Experiments

$\underline{\text { S.-R. Kim }}{ }^{1,2}$, H.-S. Jo ${ }^{1}$, C. S. Kang ${ }^{1}$, G.-B. Kim ${ }^{1}$, H. Kim ${ }^{1}$, I. Kim ${ }^{1,2}$, Y.-H. Kim ${ }^{1,2}$, C. Lee ${ }^{1}$, H. Lee ${ }^{1}$, M. Lee ${ }^{2}$, S.-Y. Oh ${ }^{1,2}$, J. So ${ }^{1}$

${ }^{I}$ Institude for Basic Science, Daejeon, South Korea

${ }^{2}$ Korea Research Institute of standard and Science, Daejeon, South Korea

Low-temperature detectors have become an important tool of sensor technologies in rare event search experiments such as direct detection of dark matter and search for neutrinoless double beta decay. Metallic magnetic calorimeters (MMCs) are a type of those thermal calorimeters operating at millikelvin temperatures. We present the performance of the MMCs in high resolution measurement of alpha particles. Better than $1 \mathrm{keV}$ FWHM was obtained for an external alpha particles while $300 \mathrm{eV}$ FWHM was achieved for low energy gamma rays. Recently, the MMC sensors have been used to searching for the neutrinoless double beta decay of ${ }^{100} \mathrm{Mo}$ by scintillating calorimetric technique using a $\mathrm{CaMoO}_{4}$ scintillating crystals at low temperatures. We present the result of the alpha spectroscopy, and discuss an efficient way of heat (energy) transfer between crystal absorbers and the MMC sensors to be used in various applications.

N24-5: Separation of Two Overlapping Electromagnetic or Electromagnetic-Hadronic Showers in CALICE Highly Granular Physical Calorimeter Prototype

K. Shpak

LLR / Ecole polytechnique, PALAISEAU, FRANCE

On behalf of the CALICE Collaboration

CALICE collaboration is developping highly granular calorimeters for future high energy e+e- colliders. Using Particle Flow Algorithms for individual reconstruction of particles in the jets, they provide currently the best jet energy resolution. At high jet energies (typically above $70-100 \mathrm{GeV}$ in the proposed detectors), the jet particle showers start to overlap, and the resolution is determined by the ability to separate them.

Here, we present results on the separation power of 1) two overlapping electromagnetic showers with CALICE physical prototype of silicon-tungsten electromagnetic calorimeter (SiW ECAL) and 2) electromagnetic-hadronic showers with physical prototypes of SiW ECAL and analog scintillator-iron hadronic calorimeter (AHCAL). We use three available reconstruction programs and compare the results with a Monte Carlo simulation.

\author{
N24-7: Construction and Assembly of One Barrel Slice for the Electromagnetic Calorimeter of the PANDA \\ Detector \\ $\underline{\text { H.-G. Zaunick }}^{1}$, S. Diehl ${ }^{1}$, V. Dormenev ${ }^{1}$, P. Drexler ${ }^{1}$, T. Kuske ${ }^{1}$, R. W. Novotny ${ }^{1}$, P. Rosier ${ }^{2}$, A. Ryazantsev ${ }^{3}$, C. Rosenbaum $^{1}$, \\ P. Wieczorek ${ }^{4}$, A. Wilms ${ }^{4}$, B. Wohlfahrt ${ }^{1}$, K.-T. Brinkmann ${ }^{1}$ \\ ${ }^{I}$ 2nd Physics Institute, University Giessen, Giessen, Germany \\ ${ }^{2}$ IPN Orsay, Orsay, France \\ ${ }^{3}$ IHEP Protvino, Protvino, Russia \\ ${ }^{4}$ GSI Helmholtzzentrum für Schwerionenforschung, Darmstadt, Germany
}


The electromagnetic calorimeter (EMC) of the PANDA detector at the future FAIR facility is composed of two endcaps and a barrel covering the major part of the solid angle consisting of more than 11.300 tapered PbWO4 crystals. The individual scintillator modules are readout via two large area avalanche photo diodes. The signal processing is performed with a custom made ASIC-preamplifier providing a large dynamic range, low noise and reduced power consumption since the calorimeter will be operated at a temperature of $-25 \mathrm{oC}$. The first major assembly stage outlined in this paper is going to be conducted beginning in mid 2016 by assembling one single barrel slice segment. The construction of this segment comprises a full length slice beam holding a total of 18 module blocks, each one being a matrix of $4 \times 10$ crystals, in place. The assembly procedure of single detector modules, 40-crystal module blocks and the overall slice segment, respectively will be discussed and important findings during the procedure mentioned. In order to lay out and optimize the assembly procedure, the results and experiences gained with an earlier 80-crystal fully functional prototype detector were accounted for, which are reviewed in this contribution. Test results of single components and fully assembled detector modules will be discussed and compared with earlier prototype in-beam and lab tests as well as with the envisaged PANDA requirements.

The project is supported by BMBF, GSI and HIC for FAIR

N24-8: Development of Versatile Calibration Method for Electro-Magnetic Calorimeters Using a Stopped Cosmic-Ray Beam

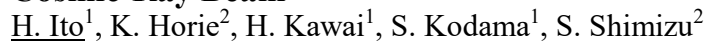

${ }^{I}$ Graduate School of Science, Chiba University, 1-33, Yayoicho, Inage, Chiba, Japan

${ }^{2}$ Graduate School of Science, Osaka University, 1-1, Machikaneyamacho, Toyonaka, Osaka, Japan

A new method using a stopped Cosmic-ray beam for a calibration of an electro-magnetic calorimeter which consists of several hundreds of modules has been established. Cosmic muons stop in the calorimeter, and positrons (and electrons) from the muon decays with the maximum energy of $53 \mathrm{MeV}$ are used for the energy calibration. These events could be identified as double pulses observed by a flash ADC. It has some advantages, (1) intermediate energy scale $\left(\mathrm{K}+? \mu^{+}{ }_{\mu},(2)\right.$ efficient procedure to calibrate all modules at the same time and (3) versatile method applied for many types of electro-magnetic calorimeters. Then, this method was checked in the J-PARC E36 experiment which was performed to precisely measure the ratio of $\mathrm{G}\left(\mathrm{K}^{+} \mathrm{e}^{+} ?_{\mathrm{e}}\right) / \mathrm{G}\left(\mathrm{K}^{+} ? \mu^{+} ?_{\mu}\right)$. Double pulses from the $\mathrm{CsI}(\mathrm{Tl})$ photon detector were successfully observed and the waveform function was carefully studied to decompose the second pulse generated by the decay positron. In this talk, (1) mechanism of this calibration procedure, (2) experimental method using Cosmic rays, (3) development of the waveform function to fit the flash ADC data, (4) experimental results and (5) confirmation of the new calibration method, will be presented.

N25: Gaseous detectors - Poster session II

Tuesday, Nov. 1 14:00-16:00 Etoile

N25-1: Experimental and Theoretical Investigation of the Collection Efficiency of Air-Filled Ionization Chambers in Pulsed Radiation Fields of High Pulse Dose M. Gotz ${ }^{1}$, L. Karsch ${ }^{1}$, J. Pawelke ${ }^{1,2}$

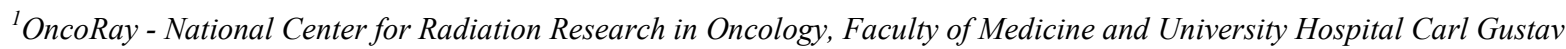
Carus, Technische Universität Dresden, Helmholtz-Zentrum Dresden - Rossendorf, Dresden, Germany

${ }^{2}$ Helmholtz-Zentrum Dresden - Rossendorf, Dresden, Germany

Ionization chambers are used in physical radiation research and are the most important dosimeters in radiation therapy. In this contribution an investigation of the collection efficiency by experiments and theoretical description is presented. The collection efficiency was measured for a plane-parallel advanced Markus ionization chamber (PTW 34045, 1 mm electrode spacing, $300 \mathrm{~V}$ nominal voltage), a chamber often used in clinical practice. The measurements were performed for collection voltages of $100 \mathrm{~V}$ and $300 \mathrm{~V}$ by irradiation with a pulsed electron beam of varied pulse dose up to approximately $600 \mathrm{mGy}(0.8 \mathrm{nC}$ liberated charge). These results are compared to existing descriptions of the collection efficiency and our own model which is based on a numeric solution by Euler method of a differential equation system modelling the processes within the chamber. While the existing models accurately describe the collection efficiency at the lower collection voltage $(100 \mathrm{~V})$ they fail to reproduce the experimentally observed behavior at the higher collection voltage $(300 \mathrm{~V})$ particularly at high pulse doses. In contrast, our own numeric solution reproduces the collection efficiency at all tested voltages and pulse doses. This illustrates the importance of considering additional effects such as electric shielding by the liberated charges and field strength dependent attachment of electrons which are not considered in the existing models. Subsequently, the developed more accurate numeric solution might provide a valuable tool for future investigations.

This project was supported by the BMBF grant number 03Z1N511.

N25-2: Construction of Large-Area Micro-Pattern Gaseous Detectors 
P. Bernhard ${ }^{1}$, A. S. Brogna ${ }^{1}$, S. S. Caiazza ${ }^{2,1}$, A. Düdder ${ }^{3}$, P. Gülker ${ }^{2,1}$, C. Kahra ${ }^{1}$, T. H. Lin ${ }^{3}$, M. Schott ${ }^{3} \underline{\text { Q. Weitzel }}^{1}$, E. Yildirim ${ }^{3}$

${ }^{I}$ Detector Laboratory, PRISMA Cluster of Excellence, Johannes Gutenberg University Mainz, 55128 Mainz, Germany

${ }^{2}$ Institute of Nuclear Physics, Johannes Gutenberg University Mainz, 55128 Mainz, Germany

${ }^{3}$ Institute of Physics, Johannes Gutenberg University Mainz, 55128 Mainz, Germany

Particle physics experiments often comprise tracking detectors with areas of up to a few square meters. If a spatial resolution of the order of $100 \mu \mathrm{m}$ and high-rate capability are required, Micro Pattern Gaseous Detectors (MPGD) are a cost-effective solution. However, the construction of large-area MPGDs is challenging, since tight fabrication tolerances have to be met to guarantee a stable and homogeneous performance. A precision granite table and an automated 3-D positioning system with an attached laser sensor, both inside a laminar flow box, have therefore been set up in the PRISMA Detector Lab at Mainz. Currently, this infrastructure is used to produce drift panels for the upgrade of the ATLAS muon spectrometer at CERN with Micro Mesh Gaseous Structure (Micromegas) detectors. To parallelize production steps, movable vacuum table boards with an average planarity of about $20 \mu \mathrm{m}$ have been designed and built. We present preliminary results on the achieved precision of drift panel prototypes and, in addition, first studies on the temperature stability of their surface planarity. These results are in particular relevant for future construction of large-area MPGDs, such as the Gas Electron Multiplier (GEM) detectors for experiments at the future MESA accelerator at Mainz.

N25-3: ORANGE: a High Sensitivity Particle Tracker Based on Optically Read Out GEM

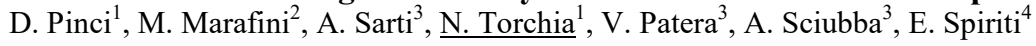

${ }^{I}$ INFN - Sezione di Roma, Roma, Italy

${ }^{2}$ Museo Storico della Fisica e Centro Studi e Ricerche, Roma, Italy

${ }^{3}$ Dipartimento di Scienze di Base e Applicate per Ingegneria, Sapienza Università di Roma, Roma, Italy

${ }^{4}$ Laboratori Nazionali dell'INFN di Frascati, Frascati, Italy

GEM-based detectors had a noticeable development in last years and have successfully been employed in different fields from High Energy Physics to imaging applications. Light production associated to the electron multiplication allows to perform an optical readout of these devices. The big progress achieved in CMOS-based photosensors makes possible to develop a high sensitivity, high granularity and low noise readout. In this paper we present the results obtained by reading out the light produced by a triple-GEM structure by means of a 4 mega-pixel CMOS sensor having a noise level less than two photons per pixel. The choice of a $\mathrm{CF}_{4}$ rich gas mixture $\left(\mathrm{He} / \mathrm{CF}_{4} 60 / 40\right)$ and a detailed optimisation of the electric fields allowed to reach a light-yield high enough to obtain very visible signals from minimum ionizing particles. In a test performed with $450 \mathrm{MeV}$ electron beam, 800 photons per millimeter were collected and a space resolution of about $75 \mu \mathrm{m}$ was obtained.

\section{N25-4: Twin GEM-TPC Prototype (HGB4) Beam Test at GSI and Jyväskylä - a Development for the Super- FRS at FAIR}

$\underline{\text { F. Garcia }}^{1}$, R. Turpeinen ${ }^{1}$, J. Äystö ${ }^{1}$, T. Grahn ${ }^{2}$, S. Rinta-Antila ${ }^{2}$, A. Jokinen ${ }^{2}$, P. Strmen ${ }^{3}$, M. Pikna ${ }^{3}$, B. Sitar ${ }^{3}$, B. Voss ${ }^{4}$, J. Kunkel $^{4}$, V. Kleipa ${ }^{4}$, A. Gromliuk ${ }^{4}$, H. Risch ${ }^{4}$, C. Caesar ${ }^{4}$, C. Simons ${ }^{4}$, A. Prochazka ${ }^{4}$, C. J. Schmidt ${ }^{4}$, J. Hoffmann $^{4}$, I. Russanov ${ }^{4}$, N. Kurtz

${ }^{I}$ Detector Laboratory, Helsinki Institute of Physics and Department of Physcal Sciences, University of Helsinki, Helsinki, Finland

${ }^{2}$ Department of Physics, University of Jyväskylä, Jyväskylä, Finland

${ }^{3}$ FMFI Bratislava, Comenius University, Bratislava, Slovakia

${ }^{4}$ GSI Helmholtzzentrum für Schwerionenforschung, Darmstadt, Germany

The GEM-TPC detector will be part of the standard Super-FRS detection system, as tracker detectors at several focal stations along the separator and its three branches.

In order to satisfy the requirements of the Super-FRS a GEM-TPC working group was created. From 2009 different designs were proposed and after two generations of GEM-TPCs [1,2] were built and tested, one of the main requirements, which was not yet completely tested was a close to $100 \%$ tracking efficiency at high rates, therefore the twin configuration was introduced. The main idea behind this new design is to place two GEM-TPCs one close to the other one and flipped in the middle horizontal plane, in such a way that the electric field of the field cages will be in opposite directions. Results from simulations indicate that this configuration can potentially achieve a close to $100 \%$ tracking efficiency at up to $1 \mathrm{MHz}$ rate.

The twin prototype called HGB4, shown in Fig. 1 was built at the GSI Detector Laboratory in 2014 and the full precomissioning has been carried out at Helsinki Institute of Physics

The results of the beam test at Jyväskylä with the irradiation of protons at $50 \mathrm{MeV}$ and at GSI with Ca and U beam at $550 \mathrm{MeV} / \mathrm{a}$ will be presented. 


\title{
N25-5: Design of the ATLAS New Small Wheel Gas Leak Tightness Station for the Micromegas Detector
} Modules

$\underline{\text { A. Bruni }}$

INFN Bologna, Bologna, Italy

On behalf of the ATLAS Muon Collaboration

In this work we describe advanced data processing and analysis techniques to be used in the gas tightness station at CERN for quality testing of the New Small Wheel (NSW) Micromegas Multiplets. Because this test is crucial for the stable operation of the Micromegas modules, we have combined two methods: a conventional one based on the Pressure Decay Rate (PDR) and an alternative, but novel one, based on the Flow Rate Loss (FRL). A prototype setup based on a Lock-in Amplifier device available at the NTUA Laboratory has been implemented by using in conjunction with the gas leak test via the FRL method. Both methods have been tested by using emulated leak branches based on the idea of using specific-low cost medical hypodermic needles. The gas leak rate of a certain number of such leak branches has measured by connecting them to the flow stream pipe. Additionally, a semi- automatic data analysis procedure and algorithms have been studied for both methods as well as atmospheric and temperature compensation. A data acquisition, monitoring and processing system based on WinCC is also proposed and described. The resulting performance of the observed signals is also presented.

\author{
N25-6: Studies of MicroMegas Chambers Using Cosmic Muons for the New Small Wheel \\ T. Klapdor-Kleingrothaus \\ University of Freiburg, Freiburg, Germany \\ On behalf of the ATLAS Muon Collaboration
}

Micromesh Gaseous (MicroMegas) Detectors will be implemented in the ATLAS detector in the framework of the New Small Wheel Upgrade during the long shut down II in 2019/20. These detectors are used for position measurement and have a high spatial resolution of $100 \ddot{i}_{i}^{1 / 2 \mathrm{~m}}$. In parallel to the ongoing constructions of the later modules, additional performance studies with small MicroMegas prototypes of a size of $10 \ddot{i}_{i}^{1 / 2} 10 \mathrm{~cm} 2$ are performed. The studies include a cosmic muon test stand in combination with a scalable readout system, such that the influences of variations in the pressure of the operation gas or changes in the humidity at the lower ppm level to the detector performance are investigated. These parameters will impact the later design of detector slow control system at the New Small Wheel in ATLAS.

\author{
N25-7: The INFN MicroMegas Module-0 Prototype for the Muon Spectrometer Upgrade of the ATLAS \\ Experiment \\ M. Del Gaudio \\ INFN Cosenza, University of Calabria, Cosenza, Italy \\ On behalf of the ATLAS Muon Collaboration
}

Large size resistive MicroMegas detectors will be employed for the first time in high-energy physics experiments for the Muon Spectrometer upgrade of the ATLAS experiment at CERN. The MicroMegas system will provide both trigger and tracking capabilities. The current innermost stations of the muon endcap system, the Small Wheel, will be upgraded in 2019 to retain the good precision tracking and trigger capabilities in the high background environment expected for LHC with the upcoming luminosity increase. Along with the small-strip Thin Gap Chambers (sTGC), the $\ddot{i}_{i}^{1} \frac{1}{2} \mathrm{New}$ Small Wheelï ${ }^{1 / 2}$ (NSW) will be equipped with eight layers of MicroMegas (MM) detectors, arranged in two quadruplets, for a total of about $1200 \mathrm{~m} 2$ detection planes. Four different types of MM quadruplets modules (SM1, SM2, LM1, LM2), built by different Institutes, will compose the NSW. They all have trapezoidal shapes, with surface areas between 2 and $3 \mathrm{~m} 2$. In April 2016 the first full size SM1 prototype (Module-0) has been completed by INFN Italy, passing throughout a validation phase. The INFN Module-0 construction procedures will be reviewed along with the description of the quality control steps, and the final validation tests obtained with $\mathrm{X}$ rays, cosmic tracks, and high-energy particle beams at CERN.

\author{
N25-8: Construction and QA/QC of the MicroMegas Pavia Readout Panels for the Muon Spectrometer \\ Upgrade of the ATLAS Experiment \\ A. Kourkoumeli-Charalampidi \\ INFN Pavia, Pavia, Italy \\ On behalf of the ATLAS Muon Collaboration
}

In order to cope with the required precision tracking and trigger capabilities during Run III in ATLAS experiment, the innermost layer of the Muon Spectrometer endcap (Small Wheels) will be upgraded. The New Small Wheel (NSW) will be equipped with eight layers of MicroMegas (MM) detectors and eight layers of small-strip Thin Gap Chambers (sTGC), both arranged in two 
quadruplets. MM detectors of large size (up to $2 \mathrm{~m} 2$ ) will be employed for the first time in HEP experiments. Four different types of MM quadruplets modules (SM1, SM2, LM1, LM2), built by different Institutes, will compose the NSW. Italian INFN is responsible for the construction of the SM1 modules. The construction is shared among different INFN sites. In particular, readout panels are built in Pavia. Due to the challenging mechanical specifications (with precisions of tens microns over meters), the construction procedure has been optimized to obtain the required strip alignment precision in the panel. A number of data quality checks on both the components and the assembled panels are also needed to meet the required specifications.

N25-9: Performance of MRPC Detector for the BESIII Endcap-TOF Upgrade

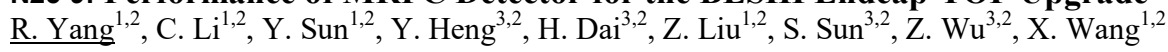

${ }^{I}$ Department of Modern Physics, University of Science and Technology of China(USTC), Hefei,Anhui, China

${ }^{2}$ State Key Laboratory of Particle Detection and Electronics, USTC \& IHEP, China

${ }^{3}$ Institute of High Energy Physics(IHEP), Chinese Academy of Sciences, Beijing, China

In order to improve the particle identification capability, The Beijing Spectrometer (BESIII) has just upgraded its end-cap timeof-flight (ETOF) with multi-gap resistive plate chamber (MRPC) technology, aiming at an overall time resolution of 80ps for MIPs. Due to the latest data taken during spectrometer's runs in 2016, it shows the MRPCs for ETOF have an overall time resolution around 60ps and an effeciency greater than $96 \%$ for bhabha's.

N25-10: Gain Uniformity and Characteristic Study of a Triple GEM Detector

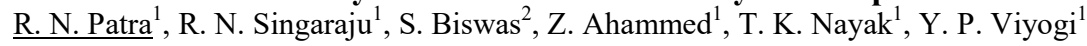

${ }^{I}$ EXHEP, Variable Energy Cyclotron Centre, Kolkata, India

${ }^{2}$ Department of Physics and CAPSS, Bose Institute, Kolkata, India

Gas Electron Multiplier (GEM) detectors are widely used in modern high energy physics experiments because of their high rate handling capability, fast timing, good position resolution and for offering suppression of ions. The ALICE experiment at LHC CERN plans to use GEM as the future readout for their Time Projection Chamber (TPC) for Run-3. We present a detailed study of the performance of a $10 \times 10 \mathrm{~cm}^{2}$ triple GEM detector, built by single mask foil and operated using a gas mixture of $70 \%$ Argon and $30 \% \mathrm{CO}_{2}$. To study the detector performance, a voltage scan was first carried out using cosmic rays and subsequently with ${ }^{106} \mathrm{Ru}-\mathrm{Rh}$ radioactive source, using a series of scintillators as trigger detectors. The two studies produced efficiency values very close to each other near the plateau region and the value being $\sim 95 \%$. For the gain uniformity study, the detector surface was divided into 16 zones. For each zone, gain was measured using a ${ }^{55} \mathrm{Fe} X$-ray source. It is found that the gain is almost uniform and the gain variation has an RMS of $6.6 \%$. We also measured the efficiency at plateau for the 16 zones. The efficiency is found to be quite uniform across the detector, with a RMS of only $1.9 \%$.

N25-11: Study of Non-linear Response of a GEM Read Out with Radial Zigzag Strips A. Zhang ${ }^{1}$, M. Hohlmann ${ }^{1}$, S. Colafranceschi ${ }^{1}$, B. Azmoun ${ }^{2}$, M. L. Purschke ${ }^{2}$, C. Woody ${ }^{2}$

${ }^{I}$ Department of Physics and Space Sciences, Florida Institute of Technology, Melbourne, FL, US

${ }^{2}$ Physics Department, Brookhaven National Laboratory, Upton, NY, US

Zigzag strips have been studied for reading out large-area GEM detectors for tracking purposes at future experiments, such as forward tracking at the Electron Ion Collider (EIC). With zigzag strips, the number of electronic channels and related cost can be reduced significantly, e.g. by a factor of three, over regular straight strips while good spatial resolutions can be maintained.

However, we observe a non-linear relation between incident particle position and hit positions in a GEM detector measured from the charge sharing among zigzag readout strips. We study this non-linear positional response of readout strips with different zigzag strip designs using a $10 \mathrm{~cm}$ by $10 \mathrm{~cm}$ triple-GEM detector with a strongly collimated X-ray gun on a 2D motorized stage at Brookhaven National Lab. All readout strips run radially and measure the azimuthal phi-coordinate of incident particles. The various zigzag boards feature different geometrical parameters for the zigzag strip design. For example, the $\ddot{i}_{i}{ }^{1 / 2 z i g s i} i{ }_{i}^{1 / 2}$ and $\ddot{i}_{i} 1 / 2$ zagsi $i_{i}^{1 / 2}$ on one strip interleave into neighboring strips by different amounts on different boards, and consequently they show different non-linear responses. We find that the more the $\ddot{i}_{i} 1 / 2$ zigsï $\dot{i}^{1 / 2}$ and $\ddot{i}_{i} 1 / 2$ zagsï $i^{1} / 2$ interleave into neighboring strips, the more linear the response. However, it is more difficult to produce such strongly interleaved zigzag strips in industry due to small dimensions and sharp angles. Consequently, we expect to have a small remaining non-linear response in reality with a zigzag structure design in which the $\ddot{i}_{\dot{c}}^{1} 1 / 2$ zigsi $i_{i}^{1 / 2}$ and $\ddot{i}_{i}^{1 / 2 z a g s i} \ddot{i}_{i}^{1 / 2}$ on one strip interleave to the center of its neighboring strips. We quantify this non-linearity so that it can be corrected for.

This work is supported by Brookhaven National Laboratory (BNL) under the EIC eRD-6 consortium.

N25-12: Study of Boron Coated Straw Detectors for Small-Angle Neutron Scattering of Compact Pulse Hadron Source 
Neutron scattering and diffraction methods are of utmost importance for probing the structure and dynamics of condensed matter. $3 \mathrm{He}$ detector has been widely used as instrument in neutron scattering application. However, due to $3 \mathrm{He}$ supply crisis, alternative thermal neutron detection technique is studied. A boron coated straw detector has been developed for small-angle neutron scattering (SANS) instrument of Compact Pulse Hadron Source (CPHS). The detector is proportional counter with diameter of $8 \mathrm{~mm}$ which has advantage in $\mathrm{B} 4 \mathrm{C}$ deposition process compared to the previous $4 \mathrm{~mm}$ diameter tube. Charge division was used as position readout method. Preliminary test has been made on the single tube in terms of its position resolution and detection efficiency. It is found that the average position resolution along the axis of the single tube is about $11.7 \mathrm{~mm}$, which could meet the demand of SANS application. A detection efficiency of about $6.6 \%$ was achieved, it is believed that the 8 mm diameter detector could be employed to build the $1 \mathrm{~m} \ddot{i} i^{1 / 2} 1 \mathrm{~m}$ boron coated straw neutron detector module with enough detection efficiency, position resolution and mechanical stability used in SANS of the CPHS in Tsinghua University.

N25-13: Characterization of Neutron Beam Monitors for the European Spallation Source

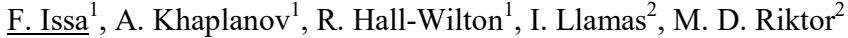

${ }^{I}$ European Spallation Source ERIC, Lund, Sweden

${ }^{2}$ Institute for Energy Technology, Kjeller, Norway

The European Spallation Source aspires to become the world's leading neutron source for the studies of materials during the next decade. At ESS 16 different instruments will be built. These instruments will require neutron beam monitors with high precision, low gamma sensitivity, high time and space resolution. Such neutron beam monitors are essential in various neutron experiments to continuously diagnose the delivered beam. In this work different types of neutron beam monitors from different suppliers have been characterized using the R2D2 beamline at IFE in Norway and using a Be-based neutron source. For the gamma sensitivity measurements different gamma sources have been used. The evaluation of these monitors includes the study of their efficiency, attenuation, uniformity, stability, and their sensitivity to gamma. In this work we report the results of this characterization.

\section{N25-15: A New Slow Control and Run Initialization Byte-Wise Environment (SCRIBE) for the Quality Control of Mass-Produced CMS GEM Detectors}

$\underline{\text { S. Colafranceschi }}$

Dept. of Physics \& Space Sciences, Florida Institute of Technology, Melbourne, Florida

On behalf of the CMS Collaboration

The CMS collaboration aims at improving the muon trigger and tracking performance at the HL-LHC by installing new Gas Electron Multiplier (GEM) detectors in the endcaps of the CMS experiment. Construction and commissioning of GEM detectors for the first muon endcap stations is ramping up in several laboratories using common quality control protocols. The SCRIBE framework is a scalable and cross-platform web-based application that controls data acquisition and analyzes data in near real time. It has been developed mainly to simplify and standardize measurements of the GEM detector response uniformities with Xrays across all production sites. SCRIBE works with zero suppression of raw SRS pulse height data. This has increased acquisition rates hundredfold to $5 \mathrm{kHz}$ for $10 \backslash \mathrm{deg}$ trapezoidal triple-GEM chambers, each covering $1.6<\mid$ eta $\mid<2.2$ with 3072 strips. The system allows strip-by-strip response comparisons with a few hours of data taking. SCRIBE also manages parallel data analysis to provide near real-time feedback on the detector response to the user. Preliminary results on the response performance of prototype and possibly first production chambers commissioned with SCRIBE will be presented.

\section{N25-17: A High Pressure Gaseous Detector for a Compton Camera Application}

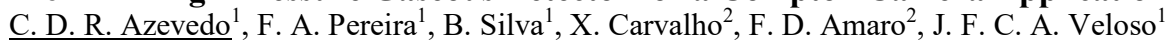

${ }^{I} I 3 N-P h y s i c s$ Department, University of Aveiro, Aveiro, Portugal

${ }^{2}$ LIBPhys - Department of Physics, University of Coimbra, Coimbra, Portugal

The progress on the development of a gaseous Compton Camera envisaging Nuclear Medical Imaging and radiation monitoring, will be presented. The proposed detector is composed by a High Pressure Gas Proportional Scintillation Counter (HP-GSPC) read-out by a large area Gaseous Photomultiplier (GPM).

The GPM is composed by a Thick-GEM (THGEM) covered with a CsI layer in the top electrode and a position sensitive THCOBRA operating in $\mathrm{Ar} / 5 \% \mathrm{CH}_{4}$ atmosphere.

The HP-GSPC was first characterized in Xenon pressures up to 5 bar with $59.5 \mathrm{keV}$ and $122 \mathrm{keV}$ photons provided by a ${ }^{241} \mathrm{Am}$ and a ${ }^{57} \mathrm{Co}$ sources, respectively. Light signal amplitude and energy resolution measured with a PhotoMultiplier Tube (PMT) will be presented.

Recent results in a similar GPM operating in $\mathrm{Ne} / 5 \% \mathrm{CH}_{4}$ have shown gain $\sim 10^{6}$ at about $100 \mathrm{kHz}$ readout rate. Position 
resolution better than $100 \mu \mathrm{m}$ have been measured in VUV single-photon mode with a full photoelectron collection efficiency fitting the requirements for the present application.

The GPM is assembled and coupled to the HP-GSPC. Characterization of the proposed detector using the GPM will be presented.

Acknowledgments:

This work was partially supported by project PTDC/FIS-NUC/2525/2014 through COMPETE, FEDER and FCT (Lisbon) programs.

N25-18: Measurements of Fe-55 in Radioactive Waste with GEMPix

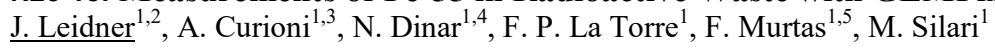

${ }^{I}$ CERN, Geneva, Switzerland

${ }^{2}$ RWTH Aachen, Aachen, Germany

${ }^{3}$ Politecnico di Milano, Milano, Italy

${ }^{4}$ Universite de Paris VII, Paris, France

${ }^{5}$ INFN, Frascati, Italy

GEMPix is a detector obtained by coupling a small triple Gas Electron Multiplier (GEM) detector to a quad Timepix ASIC. Systematic improvements on stability are presented here, as an example of the extensive characterization of the detector performed in view of its use for the determination of the Fe-55 content in radioactive waste. Comparison with standard radiochemical analysis shows linear dependence of GEMPix counts on specific activity. Detection limits and sensitivity are also presented.

N25-19: Experimental Ion Mobility Measurements in Nitrogen Based Mixtures P. M. C. C. Encarnação ${ }^{1,2}$, A. F. V. Cortez ${ }^{1,2}$, P. N. B. Neves ${ }^{3}, \underline{\text { F. P. Santos }}^{1,2}$, F. I. G. M. Borges ${ }^{1,2}$

${ }^{I}$ Department of Physics, Faculty of Science and Technology, University of Coimbra, P-3004-516 Coimbra, Portugal, Coimbra, Portugal

${ }^{2}$ LIP - Departamento de Física, Universidade de Coimbra, Rua Larga, 3004-516 Coimbra, Portugal, Coimbra, Portugal

${ }^{3}$ Closer Consultoria Lda Avenida Engenheiro Duarte Pacheco, Torre 2, 14o-C, 1070-102 Lisboa, Portugal, Lisboa, Portugal

Data on efective ion mobility for mixture is scarce, although important to improve the performance of large volume gaseous detectors, such as ALICE TPC or in the NEXT Experiment. In the present work the method, experimental setup and results of the ion mobility measurements in nitrogen gas based mixtures are presented. The preliminary results of this mixture show the presence of two peaks for different gas ratios of Ne-N2 and Ar-N2, low reduced electric fields, E/N (10-20 Td), low pressures (610 Torr), at room temperature. In the experimental setup used, the ions are originated in a GEM, inside the parent gas and are collected in a grid after travelling a known distance inside the gas chamber. Knowing the drift time, obtained from the peaks' centroid of the ions' signal, the drift velocity and mobility can then be calculated. The mobility values were seen to roughly follow the theoretical expected values.

This work was supported by the RD51 Collaboration/CERN, through the common project "Measurement and calculation of ion mobility of some gas mixtures of interest". André F.V. Cortez received a PhD scholarship from FCT-Fundação para a Ciência e Tecnologia (SFRH/BD/52333/2013).

\author{
N25-20: A Novel Encoding Readout Method Based on Graph Theory Model with Both Time and Spatial \\ Resolution

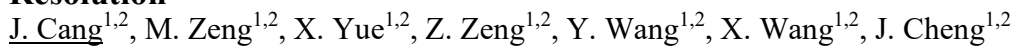 \\ ${ }^{1}$ Department of Engineering Physics, Tsinghua University, Beijing, China \\ ${ }^{2}$ Key Laboratory of Particle \& Radiation Imaging (Tsinghua University), Ministry of Education, Beijing, China
}

Encoding readout methods have been used widely to reduce the channels of electronics. Especially for muon tomography with cosmic ray muons, which requires large-scale, high-efficiency, high spatial resolution detectors to track the muon. However, there is no general methods about how to design the encoding readout. Recently, a mathematical modelling, based on graph theory, of the encoding readout has been proposed, which can illustrate several existing encoding readout, such as fine-fine method in the MAMA detector and the genetic multiplexing for the Micromegas. Moreover, according to the new mathematical model, a totally new encoding readout called "Eulerian Trail Approach (ETA)" was proposed. Recently, an encoding scheme prototype has been constructed, and verified with a $70 \mathrm{~cm} \times 70 \mathrm{~cm}$-scale, $6 \mathrm{~mm}$-pitch MRPC detector. With only 15 channels readout and the ETA method, 733ps time resolution and 1360um spatial resolution can be achieved at the same time. A newer MRPC detector with much smaller readout strip pitch was built as well, to achieve higher time and spatial resolution at the same time. In addition, details about statistical error and working condition of the prototypes are discussed as well. 
This work is supported by the National Natural Science Foundation of China (No.11322548 and No.11305093) and Tsinghua University Initiative Scientific Research Program (No.2014Z21016).

N25-21: Density-Normalized First Townsend Ionization Coefficients in a Methane-based Tissue-Equivalent Gas Mixture

$\underline{\text { A. R. Petri }}^{1}$, A. Mangiarotti ${ }^{2}$, J. A. C. Gonçalves ${ }^{1,3}$, C. C. Bueno ${ }^{1}$

${ }^{I}$ Centro de Tecnologia das Radiações, Instituto de Pesquisas Energéticas e Nucleares, São Paulo, SP, Brazil

${ }^{2}$ Dept. de Física Experimental, Instituto de Física - Universidade de São Paulo, São Paulo, SP, Brazil

${ }^{3}$ Dept. de Física, Pontifícia Universidade Católica de São Paulo, São Paulo, SP, Brazil

Since 1950 methane-based tissue-equivalent gas (TEG) mixtures have been employed at low pressure in gaseous microdosimeters, such as tissue-equivalent proportional counters. Nevertheless, values of the first Townsend ionization coefficient (a) for these mixtures are scarce in the literature. In this work measurements of a as a function of the densitynormalized electric field $(\mathrm{E} / \mathrm{N})$ were carried out in a methane-based TEG $\left(\mathrm{CH}_{4}-64.4 \%, \mathrm{CO}_{2}-32.4 \%\right.$ and $\left.\mathrm{N}_{2}-3.2 \%\right)$ in the range 100-300Td. The values of a were obtained using the Pulsed Townsend Technique by measuring the current growth as a function of the electric field strength in a Resistive Plate Chamber structure. Because of the parallel plate geometry, the field is uniform in our setup. As far as the authors are aware, there is only one set of experimental data on a for methane-TEG published by Schmitz and Booz for reduced field strengths between 110V.cm ${ }^{-1} \cdot \operatorname{torr}^{-1}$ ( 315Td) and 1780V $\cdot \mathrm{cm}^{-1} \cdot$ torr $^{-1}(\sim 5045 \mathrm{Td})$.

However, they employed a cylindrical proportional counter where a $/ \mathrm{p}$ values were gathered from measurements of the gas gain resulting from an interval of electric field strengths. Because there are no data on a in methane-based TEG in the E/ $\mathrm{N}$ range herein investigated, our results were compared with those expected from Magboltz simulations. Good agreement was found between experimental and simulated values of density-normalized a.

The authors express their appreciation to Dr. M. M. R. Fraga (in memoriam) from LIP-Coimbra, Portugal, for many helpful discussions on the low-pressure gas system. A. R. Petri would like to thank CNPq for the award of a scholarship. This work was co-financed by FAPESP under contract 02/04697-1 and by CNPq via contracts 478859/2009-0 and 479079/2010-2.

N25-22: Quantum Efficiency Dependence of a CsI Photocathode with Photon Incidence Angle

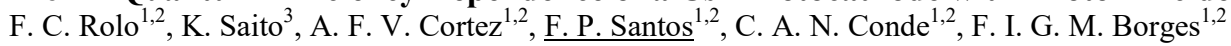

${ }^{1}$ Department of Physics, Faculty of Science and Technology, University of Coimbra, P-3004-516, Coimbra, Portugal

${ }^{2}$ LIP - Departamento de Física, Universidade de Coimbra, Rua Larga, 3004-516, Coimbra, Portugal

${ }^{3}$ High Energy Accelerator Research Organization (KEK), Tsukuba, Japan

Cesium iodide (CsI) photocathodes are widely used as VUV photo-sensors because of their high quantum efficiency and good stability. One important issue for photocathodes is that the effective quantum efficiency of the photocathodes when used in gaseous atmosphere is reduced due to photoelectron backscattering. Furthermore, quantum efficiency depends on the photon incidence angle. Although the properties of CsI photocathodes are well known, there is little information on how the effective quantum efficiency of the photocathodes used in gaseous atmosphere depends on the photon incidence angle. The purpose of our study is to measure the quantum efficiency of a CsI photocathode in gaseous xenon at several different pressures and for different photon incidence angles. The system consists on a stainless steel chamber divided in two regions separated by a quartz window. In the upper region, in vacuum, there is a deuterium lamp (photon source), and the collimators, while in the lower, filled with gas, is located a photocathode and above it a photoelectron collecting grid, both mounted on a Teflon support, with the photocathode plate being connected to a linear motion feedthrough through a crank, so that the photon incident angle on the photocathode can be varied between 0-50 degrees. In the experimental setup the collimated photons enter through the quartz window, irradiate the photocathode and the photocurrent induced in the grid is measured with the help of an electrometer. In this work, we present the results of recent measurements using the method and experimental system described above for Xe as the filling gas and for pressures ranging from vacuum to $5.0 \mathrm{MPa}$.

\section{N25-23: Performance of the EEE Cosmic Ray Telescope Array} F. Pilo $^{1,2}$

${ }^{I}$ Museo Storico della Fisica e Centro Studi e Ricerche Enrico Fermi, Rome, Italy

${ }^{2}$ Sezione di Pisa, Istituto Nazionale di Fisica Nucleare, Frascati, Italy

On behalf of the EEE Collaboration

The Extreme Energy Events (EEE) Project is an experiment for the detection of Extensive Air Showers of energy greater than $10^{11} \mathrm{eV}$. EEE consists of an array of telescopes each made of three Multigap Resistive Plate Chambers, hosted in high schools spread over the Italian territory. The telescopes are constructed at CERN by teams of students and teachers: this peculiarity combines the scienti?c relevance of its goals with an effective outreach action. During coordinated data taking periods, more than thirty telescopes were operated at the same time and more than 20 billions of cosmic ray events have been collected. Data were 
transmitted to CNAF, the biggest Italian storage and computing centre managed by INFN, to be reconstructed and analysed. In this presentation, an overall description of the experiment will be given and the excellent performance of the array, including single and combined telescope detection efficiency, spatial and time resolution and tracking capability, will be described.

N25-24: Characterization of 200bar 4He Neutron Scintillation Detectors with Silicon Photomultipliers J. D. Sanders, J. T. Johnson, S. J. Thompson, D. L. Chichester

National and Homeland Security, Idaho National Laboratory, Idaho Falls, ID, USA

Characterization of high pressure (200-bar) $4 \mathrm{He}$ fast neutron scintillation detectors with integrated silicon photomultipliers was performed. Time-of-flight measurements were conducted with Cf-252 sources to characterize the neutron energy response and timing characteristics of the detectors in a variety of configurations. Results from the measurements were compared with Monte Carlo simulations. The intrinsic detection efficiency for spontaneous fission neutrons from Cf- 252 was determined to be $\sim 8 \%$.

N25-25: Characterization of a Transparent MSGC with Optical Readout

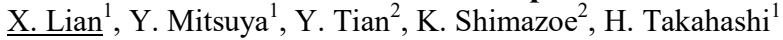

${ }^{I}$ Department of Nuclear Engineering and Management, School of Engineering, University of Tokyo, Tokyo, Japan

${ }^{2}$ Department of Bioengineering, School of Engineering, University of Tokyo, Tokyo, Japan

After the development of transparent Micro-strip Gas Chamber (MSGC), the optical readout of MSGC shows a possibility of new kind compact and cost-effective imaging gas detectors when combined with the LCD panel techniques. The indirect coupling of optical readout and MSGC electrodes which will avoid the spark damage problems about the readout electronics, and the high gain of gaseous detectors seems a promising feature compared with conventional flat panel detectors. In this work a transparent IZO MSGC with a fine pitch of $150 \mu \mathrm{m}$ was tested using compact optical readout design. Both the electric and optical signals under gas gain of 150 were measured for the future optimized design, which showed clearly energy difference under different sources.

N25-26: Calibration of TEPC System at HIMAC with Heavy Ions $\underline{\text { U. W. Nam }}^{1}$, J. J. Lee ${ }^{1}$, S. H. Kim ${ }^{2}$, J. H. Pyo ${ }^{1}$, B. K. Moon ${ }^{1}$, W. K. Park ${ }^{1}$, U. H. Kitamura ${ }^{3}$, S. Kodaira ${ }^{3}$

${ }^{1}$ Korea Astronomy and Space Science Institute, Daejeon, Korea

${ }^{2}$ Cheongju University, Cheongju, Korea

${ }^{3}$ National Institute Radiological Science, Chiba, Japan

The goal of this experiment is verifying the performance of new designed Tissue Equivalent Proportional Counter (TEPC) for monitoring space radiation. In space, human cells could be exposed by complex radiation sources, X-ray, Gamma ray, energetic electrons, protons, neutrons and heavy charged particles in a huge range of energies. These generate much larger range of Linear Energy Transfer (LET) than ground and cause unexpected effect on human cells. In order to measure the large range of LET, from 0.2 to $1000 \mathrm{keV} / \mu \mathrm{m}$, we developed a compact TEPC which measures ionized particles produced by collision between radiation sources and tissue equivalent (pure propane) gas in the detector. From measuring LET spectra, we can derive easily the equivalent dose from the complex space radiation field. In this HIMAC(Heavy Ion Medical Accelerator in Chiba, Japan) experiment, we intend to calibrate and demonstrate two active radiation detectors, multi-segmented TEPC and Benjamin type TEPC with C $400 \mathrm{MeV} / \mathrm{u}, \mathrm{Si} 490 \mathrm{MeV} / \mathrm{u}, \mathrm{He} 150 \mathrm{MeV} / \mathrm{u}$ and Fe $500 \mathrm{MeV} / \mathrm{u}$ ion beams.

This work was supported by the National Research Foundation of Korea (NRF) grant funded by the Ministry of Science, ICT \& Future Planning (MSIP) (No. 2011-0020918)

\section{N25-27: Development of WLS+SiPM Photo Detection System for Readout of THGEM in Noble Gas Filled} Detectors.

$\underline{\text { I. S. Alexandrov }}^{1}$, D. Y. Akimov ${ }^{2}$, A. V. Akindinov ${ }^{3}$, V. A. Belov ${ }^{3}$, A. A. Burenkov ${ }^{3}$, M. A. Kirsanov ${ }^{2}$, A. G. Kovalenko ${ }^{3}$, V. N. Stekhanov ${ }^{3}$, N. M. Surin ${ }^{4}$, S. A. Zav'yalov', M. Y. Yablokov', M. ?. Danilov²

${ }^{1}$ Tomsk Polytechnic University, Tomsk, Russia

${ }^{2}$ National Nuclear Research University «MEPHI», Moscow, Russia

${ }^{3}$ State Scientific Centre of Russian Federation Institute for Theoretical and Experimental Physics (ITEP), Moscow, Russia

${ }^{4}$ Enikolopov Institute of Synthetic Polymer Materials, Moscow, Russia

${ }^{5}$ State Scientific Centre of Russian Federation Karpov Institute of Physical Chemistry, Moscow, Russia

A newly developed by CPTA LTD, Russia "blue sensitive" multipixel avalanche Geiger photodiode (MGPD) has been tested in LXe with a wavelength shifter (WLS) to detect scintillation. WLS was vacuum deposited to a sapphire window and specially protected from pollution to the LXe medium. A Photon Detection Efficiency (PDE) of $\sim 10 \%$ has been obtained for the MGPD + 
WLS system. Possible design of a high precision detection system with THGEM + WLS + MGPD is considered for the use in two-phase noble gas detectors for Dark Matter experiments and for detection of reactor antineutrino coherent scattering off nuclei. Such system will be able to measure 2D coordinates of single electron events with a mm-accuracy.

N26: New concepts in solid-state detectors and radiation damage effects - Poster session II

Tuesday, Nov. $1 \quad$ 14:00-16:00 Etoile

N26-1: A Radiation-Tolerant, High Performance SPAD for SiPMs Implemented in CMOS Technology

Y.-D. Li ${ }^{1}$, C. Veerappan ${ }^{2}$, L. Myung-Jae ${ }^{2}$, L. Wen ${ }^{1}$, Q. Guo ${ }^{1}$, E. Charbon ${ }^{2}$

${ }^{I}$ Material physics \& chemistry, Xinjiang Technical Inst.of Physics and Chemistry of Chinese Academy Sciences, Urumqi, China

${ }^{2}$ Micro-Electronic Systems \& Technology Association, Delft University of Technology, delft, The Netherlands

We investigate the effects of radiation on single-photon avalanche diodes (SPADs) fabricated in CMOS technology. The SPADs retain good performance in terms of dark counts and photon detection probability upon irradiation doses from $10 \mathrm{kRad}(\mathrm{Si})$ to $50 \mathrm{kRad}(\mathrm{Si})$, with a slight increase of dark count rates and stable dark current, breakdown voltage, and sensitivity. This feature makes our device especially suitable to be integrated in SiPMs for applications in radiation-rich environments, where high radiation tolerance and low noise are essential.

N26-2: Time-of-Flight Ion Mass Spectrometry Using Avalanche Photodiodes K. Ogasawara, S. A. Livi, M. I. Desai, R. W. Ebert

Southwest Research Institute, San Antonio, TX, USA

This study reports on the performance of Avalanche Photodiodes (APDs) as a timing detector for ion Time-of-Flight (TOF) mass spectroscopy. We found that the fast signal carrier speed in a reach-through type APD enables an extremely short timescale response with a mass or energy independent $<2 \mathrm{~ns}$ rise time for $<200 \mathrm{keV}$ ions (1-40 AMU) under proper bias voltage operations. When combined with a Microchannel Plate (MCP) to detect start electron signals from an ultra-thin carbon foil, the APD comprises a novel TOF system that successfully operates with a $<0.8$ ns intrinsic timing resolution even using commercial offthe-shelf constant-fraction discriminators. By replacing conventional total-energy detectors in the TOF-E system, APDs offer a significant power and mass savings or an anti-coincidence background rejection capability in future space instrumentation.

N26-3: Tracking in 4 Dimensions

N. Cartiglia ${ }^{1}$, H. Sadrozinski ${ }^{2}$, A. Seiden ${ }^{2}$

${ }^{I} I N F N$, Torino, italy

${ }^{2}$ UCSC, Santa Cruz, United States

In this contribution we will review the progresses toward the construction of a tracking system able to measure the passage of charged particles with a combined precision r.m.s. of $\sim 10 \mathrm{ps}$ and $\sim 10 \mu \mathrm{m}$, either using a single type of sensor, able to concurrently measure position and time, or a combination of position and time sensors. The inclusion of timing information in the structure of a recorded event has the capability of changing the way we design experiments, as this added dimension dramatically improves the reconstruction process. Depending on the type of sensors that will be used, timing information can be available at different stages in the reconstruction of an event, for example (i) at tracking reconstruction, if timing is associated to each point or (ii) during the event reconstruction, if timing information is associated to each track. In the first case, the 4th dimension brings? a simplification already in the reconstruction algorithm as only time-compatible hits are used in the pattern recognition phase, however the electronics is very demanding as it needs to be able to accurately measure timing in each pixel. The second case is simpler as it requires the implementation of a dedicated timing layer, either inside or outside the main silicon tracker volume, to assign the timing information to each crossing track without changing the vast majority of the tracker hardware. The timing information can then be used to improve Level 1 trigger decisions, as it can be obtained faster than tracking reconstruction, and to separate events with overlapping vertices. We will present the research and development progresses in terms of sensors and read-out electronics, showing the current best limits and reviewing the possible technological choices currently developed.

N26-4: Neutron Radiation Hardness Tests of Timing Counter MEG SiPMs

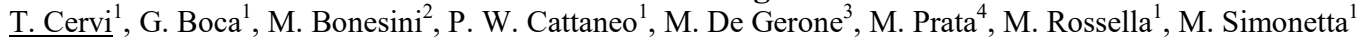

${ }^{I}$ INFN Sez. Pavia \& University of Pavia, Pavia, Italy

${ }^{2}$ INFN Sez Milano Bicocca, Milano, Italy

${ }^{3}$ INFN Sez. Genova \& University of Genova, Genova, Italy

${ }^{4}$ Laboratorio Energia Nucleare Applicata (L.E.N.A.) of the University of Pavia, Pavia, Italy 
Radiation hardness is an important requirement for readout elements operating in high radiation environments common in particle physics experiments. The MEG2 experiment, at PSI, Switzerland, investigates the forbidden decay $\mu+$ ? e + ? exploiting the most intense muon beam of the world. A significant flux of non-thermal neutrons (kinetic energy $\mathrm{E}_{\mathrm{k}}=0.5 \mathrm{MeV}$ ) is present in the experimental hall produced along the beamline and in the hall itself. A sample of the Silicon PhotoMultipliers (SiPMs) used in the experiment are irradiated with neutrons having a spectrum comparable to the experimental one at differences fluence up to a maximum of $10^{13} \mathrm{n} / \mathrm{cm}^{2}$ at the LENA reactor in Pavia, Italy. In this poster we report on the change of their most important electric characteristics: dark current, dark pulse frequency, gain, direct bias resistence, as a function of the integrated neutron fluence.

\section{N26-6: The Influence of Edge Effects on the Determination of the Doping Profile of Silicon Pad Diodes}

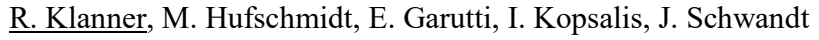

University of Hamburg, Hamburg, Germany

Edge effects for square $\mathrm{p}+\mathrm{n}$ pad diodes with guard rings fabricated on high-ohmic silicon are investigated. Using capacitancevoltage measurements of two pad diodes with different areas, the planar and the edge contributions to the diode capacitance are determined separately. Different methods of extracting the doping concentration are compared. In all cases it is found that the results with and without edge corrections differ significantly. After the edge corrections, the bulk doping of the pad diodes is found to be uniform within $\pm 1.5 \%$. The voltage dependence of the edge capacitance is compared to the predictions of two simple models.

N26-7: Geiger-Mode Avalanche Pixels in a $180 \mathrm{Nm}$ HV CMOS Process for a Dual-Layer Particle Detector

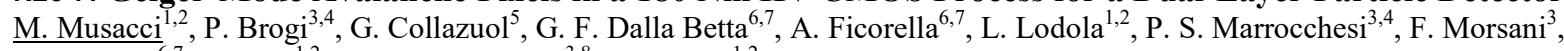
L. Pancheri ${ }^{6,7}$, L. Ratti ${ }^{1,2}$, A. Savoy-Navarro ${ }^{3,8}$, C. Vacchi ${ }^{1,2}$

${ }^{I}$ Department of Electrical, Computer and Biomedical Engineering, Università di Pavia, Pavia, Italy

${ }^{2}$ INFN, Pavia, Italy

${ }^{3}$ INFN, Pisa, Italy

${ }^{4}$ Università di Siena, Siena, Italy

${ }^{5}$ Università di Padova, Padova, Italy

${ }^{6}$ Università di Trento, Trento, Italy

${ }^{7}$ TIFPA INFN, Trento, Italy

${ }^{8}$ University Paris- Diderot/CNRS, Paris, France

An array of avalanche pixels has been designed in a $180 \mathrm{~nm}$ CMOS process with high voltage (HV) option. This is a single poly, up to six metal technology, tuned for applications to the automotive market. The array includes sensors with a pitch of 50 um $x$ $100 \mathrm{um}$, different size ( $20 \mathrm{um}$ x $20 \mathrm{um}, 30 \mathrm{um}$ x $30 \mathrm{um}$ and $40 \mathrm{um}$ x $36 \mathrm{um}$ ) and based on different process layers. Different versions of the front-end electronics, implementing a passive or active quenching technique to suppress the avalanche, have been monolithically integrated in the same substrate as the detector. The main purpose of this work is to investigate the characteristics of the technology in view of the fabrication of a dual-tier, low material budget sensor for charged particle detection. The paper will present the results from the chip characterization in terms of front-end electronics functionality, dark count rate, breakdown voltage and optical cross-talk.

The activity leading to the results presented in this paper was carried out in the framework of the APiX2 project, funded by the Italian Institute for Nuclear Physics (INFN).

N26-8: Performance Study of N-in-P Active Edge Planar Pixel Sensors for ATLAS Inner Detector Upgrade $\underline{\text { T. Rashid }}^{1,2}$, A. Lounis ${ }^{1,2}$, C. Nellist ${ }^{1,2}$

${ }^{I}$ ATLAS, Laboratoire de l'Accélérateur Linéaire (LAL), Orsay (91400), France

${ }^{2}$ Physics, University Paris-Sud, Orsay (91400), France

Active edge planar pixel sensors are promising candidates to instrument the inner layers of the new ATLAS pixel detector for HL-LHC, thanks to its radiation tolerant properties and the increased fraction of active area due to a distance as low as $50 \mu \mathrm{m}$ between the last pixel implants and the activate edge. This work addresses the study of active edge n-in-p planar sensors. It is mainly aimed to investigate the performance of the different design configuration of these sensors. Simulation based on TCAD of the doping profile as well as Secondary Ion Mass Spectroscopy (SIMS) measurements will be shown. Moreover, the electrical characterization of some irradiated samples will be discussed. This study lead to optimize the different fabrication parameters for silicon pixel detector for a better performance.

\section{N26-9: Interstrip Properties of Highly Segmented Double Metal Strip Sensors}




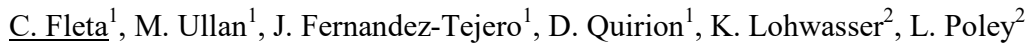

${ }^{I}$ Instituto de Microelectronica de Barcelona, IMB-CNM (CSIC), Barcelona, Spain

${ }^{2}$ DESY, Hamburg, Zeuthen, Germany

One of the challenges in designing highly segmented silicon detectors is to provide an easy interconnection of all sensor channels with the readout electronics, usually with a different geometrical distribution. A possible solution is the use of a double-metal technology, where the signals are routed on tracks fabricated on a second metal layer. However, the introduction of the secondmetal tracks may induce unwanted capacitive and resistive parasitics on the sensor: an increase in interstrip capacitance, signal integrity issues such as cross-talk and pick-up due to capacitive coupling between metal layers, and variations on track and interstrip resistance. A complete analysis of the influence of the second metal tracks on interstrip capacitance in a double-metal strip sensor has been performed considering all the different layers present in the device cross-section. A detailed equivalent model of the device has been considered taking into account all the parasitic elements introduced in the device by the second metal tracks. The model has been matched with precise interstrip capacitance and resistance measurements on actual sensors, including sensors irradiated with 60Co gammas to 50Mrad. This model will allow for future design and technology optimizations of double-metal sensors.

N26-10: CMOS MAPS Development Status for the ATLAS Inner Strip Tracker Upgrade at the LHC $\underline{\text { K. Kanisauskas }}$

Experimental Particle Physics, University of Glasgow, Glasgow, United Kingdom

On behalf of the Strip CMOS Collaboration

The most recent developments in CMOS active sensor technology, driven by the upcoming silicon strip tracker upgrade at the ATLAS for HL-LHC, demonstrate that commercially available high voltage (HV) and high resistivity (HR) processes could potentially be employed for tracking applications in high-energy physics experiments. Currently prototype chips manufactured in AMS HV-CMOS 350nm and TJ-180nm HR-CMOS processes are being investigated for their performance before and after irradiation to the fluence levels reaching $1 \times 10^{15} \mathrm{n}_{\mathrm{eq}} / \mathrm{cm}^{2}$. The key areas of interest are the response of active pixels to minimum ionizing particles (MIPs), edge-TCT studies, change in signal-to-noise ratio and speed of response. The results obtained so far from two different AMS HV-CMOS prototype chips suggest more than twofold increase in depletion depth after irradiation for the same reverse bias voltage, which manifested as a higher signal level induced by MIPs. On the other hand, the irradiation also led to the increase in noise and negative effects on in-built pre-amplifier gains. The investigation of HV/HR-CMOS technologies is not limited to laboratory measurements since TCAD models have been employed to simulate sensor characteristics. Some preliminary simulations of charge collection have already been done using HR-CMOS sensor model.

\author{
N26-12: Characterization of Lateral Spatial Resolution in High Purity Germanium Double-Sided Strip \\ Detectors as a Function of Strip-to-Gap Ratio for a Fixed Pitch \\ $\underline{\text { M. Folsom }}^{1}$, K. Ziock ${ }^{2,3}$, J. P. Hayward ${ }^{1,2}$ \\ ${ }^{I}$ Nuclear Engineering, University of Tennessee, Knoxville, Knoxville, TN, US \\ ${ }^{2}$ Nuclear Science and Technology Division, Oak Ridge National Lab, Oak Ridge, TN, US \\ ${ }^{3}$ Physics and Astrophysics, University of Tennessee, Knoxville, Knoxville, TN, US
}

Interpolation based on signals from strips neighboring the one directly over an event can be used to obtain spatial resolution in double sided strip detectors (DSSD) that is better than the width of a single strip. At a fixed pitch, the signal-to-noise ratio of the transient signals from the neighboring electrodes used to perform the interpolation should be a function of the ratio of the strip and gap widths, indicating that an optimum ratio may exist. However, typically such detectors minimize the inter-strip gap size in order to minimize incomplete charge collection. Results obtained using a finely collimated gamma-ray beam from a 133Ba source show improved spatial resolution from a DSSD with a $2.5 \mathrm{~mm}$ gap compared to one with a $500 \ddot{i}_{i}^{1} / 2 \mathrm{~m}$ gap. In both cases the strip pitch is $5 \mathrm{~mm}$. The results agree with calculated signals obtained using charge transport calculations and weighting potentials. Comparison of the experimental and calculated signals will be presented, together with implications for the optimum gap width.

N26-13: Current Progress on 3D Diamond Detector Development at UTK E. Lukosi ${ }^{1}$, T. Wulz ${ }^{1}$, B. Canfield ${ }^{2}$, L. Davis ${ }^{2}$, S. Spanier ${ }^{1}$

${ }^{I}$ University of Tennessee, Knoxville, TN, USA

${ }^{2}$ University of Tennessee Space Institute, Tullahoma, TN, USA

The use of lasers in fabricating 3D diamond detectors have faced challenges with noise and/or interface trapping. The issue lies in the phase transformation of the graphitic columns. We have focused on an alternative approach, using reactive ion etching techniques, to fabricate a 3D diamond detector. In this method, the wire electrodes or chromium over laser-created columns, 
which have shown non-ideal properties for low-noise, fast radiation sensing applications. We will present on current progress on detector design using this alternative approach along with radiation damage studies to planar diamond detectors.

\author{
N26-14: How to Build a Non-Depleted "fully Depleted" CMOS Monolithic Active Pixel Sensor \\ M. Deveaux \\ Institut für Kernphysik, Goethe University Frankfurt/M, 60438 Frankfurt/M, Germany \\ On behalf of the CBM-MVD collaboration
}

Thanks to their excellent performances for charged particle detection, CMOS Monolithic Active Pixel Sensors (MAPS) form the technology of choice for the tracking and vertex detectors of various particle and heavy ion physics experiments including STAR, NA61, ALICE, CBM.

To reach the radiation tolerance required for those applications, it is being tried to fully deplete the active medium of MAPS. This is done by reducing P-doping of the active volume of the sensor and by applying a "high" depletion voltage U to the tiny N-well implantations forming the collection diodes. According to the equation for flat $\mathrm{PN}$-junctions, one expects a depletion voltage of a few volts to deplete the pixel.

This ignores that the surface of the collection diode is regularly minimized to $10 \mu \mathrm{m}^{2}$ in order to reduce the input capacity of the on-pixel amplifier. The diode is therefore rather described by a hemisphere then by a flat junction. We study the properties of such a point-like diode and show that its depletion depth scales with $U^{\wedge}(1 / 6)$ only. Consequently, a voltage beyond $1000 \mathrm{~V}$ is required to deplete a pixel. Moreover, the diode capacity saturates after applying few volts, no matter if the active medium is depleted or not. Ignoring both features, one risks to build non-depleted sensors, which provide the illusion of a full depletion in $\mathrm{C} / \mathrm{V}$ measurements. This illusion is supported by the fact that naïve TCAD simulations may overestimate the depletion depth dramatically.

We discuss the origin of the above mentioned features and demonstrate the validity of our arguments by comparing our analytic calculations with elaborated TCAD simulations and measurements. Moreover, we propose design rules for fully depleted MAPS.

This work was supported by HIC for FAIR, GSI and the BMBF(05P12RFFC7).

\title{
N27: Photodetectors - Poster session II
}

Tuesday, Nov. 1 14:00-16:00 Etoile

N27-1: Development of Polycapillary Optics for a TES Microcalorimeter EDS System on a Scanning Transmission Electron Microscope

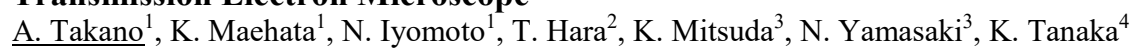

${ }^{I}$ Applied Quantum Physics and Nuclear Engineering, Kyushu University, Fukuoka-shi, Fukuoka, Japan

${ }^{2}$ National Institute for Materials Science, Tsukuba-shi, Ibaraki, Japan

${ }^{3}$ Institute of Space and Astronautical Science, Sagamihara-shi, Kanagawa, Japan

${ }^{4}$ Hitachi High-Tech Science Corporation, Sunto-gun, Shizuoka, Japan

Scanning transmission electron microscope (STEM) allows direct observation of the nanoscale structure with a high spatial resolution. An energy dispersive spectrometer (EDS) performed on a STEM plays an important role in a wide range of science. A $\mathrm{Si}$ (Li) semiconductor detector (SSD) is used for detecting X-rays in a typical EDS. An insufficient energy resolution of SSD results in overlaps between adjacent peaks and hinders high accuracy analysis. A superconducting transition edge sensor (TES) microcalorimeter system has been developed for improving the energy resolution of EDS performed on a STEM. The objective lens of the STEM generates a strong magnetic field in the specimen chamber, which makes it difficult to operate the TES microcalorimeter without degrading the energy resolution. Therefore, the TES microcalorimeter has been placed outside the STEM column. To increase the effective detection solid angle, we employed polycapillary optics. The X-ray transmission characteristics of polycapillary optics such as a focal spot size and an intensity gain depend on geometrical arrangements of the optics and the X-ray energy. The counting rate of $300 \mathrm{cps}$ was insufficient for a practical application to the STEM. To achieve the counting rate larger than $5000 \mathrm{cps}$, we are now conducting development of a 64-pixel TES microcalorimeter system operating on the STEM. In this work, the X-ray transmission characteristics of polycapillary optics for a single pixel TES microcalorimeter were evaluated by analyzing results of energy spectrum measurements of X-rays transmitted by the polycapillary optics installed in the STEM. Then we made a simulation code that reproduces experimental results. A polycapillary optics for a 64-pixel TES microcalorimeter was designed with using the simulation code for evaluating X-ray transmission characteristics. We will perform energy spectrum measurements of X-rays transmitted by the fabricated polycapillary optics for a 64-pixel TES microcalorimeter.

N27-2: Characterization of SiPM Properties at Cryogenic Temperatures

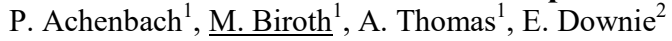

${ }^{1}$ Institut für Kernphysik, Johannes Gutenberg-Universität, D-55099 Mainz, Germany

${ }^{2}$ Physics Department, George Washington University, Washington, DC 20052, USA 
SiPM operation at cryogenic temperatures fails for many common devices. A particular type with deep channels in the silicon substrate instead of quenching resistors was tested at liquid helium temperature. Two similar types were characterized from room temperature down to liquid nitrogen temperature. The devices were mounted in vacuum with the temperature stabilized to allow long-term operation.

With these measurements a deeper investigation of the influence of cryogenic temperatures on the SiPM properties was possible. At cryogenic temperatures the SiPM signals have a fast rise-time, practically no after-pulses, and exhibit no increased crosstalk probability. The dark count rate dropped exponentially with temperature. In the full temperature range the voltage drop across the diode in forward direction was measured to compare doping properties that influence the reverse saturation current through the charge carrier density. SiPM signals from a LED pulser were acquired with single-pixel resolution. Charge collection spectra at different temperatures and light intensities were used to extract the shift in break-down voltage and quantum efficiency. The single pixel gain and the pixel gain variation were comparable to room temperature at the same over-voltage.

The characterized devices can be used for the read-out of an active polarized target at temperatures between 1 and $4 \mathrm{~K}$ for photonuclear experiments at the high-energy beam of the Mainz Microtron MAMI in Germany.

N27-3: Experimental Investigation of Excess Noise Factors in Silicon Photomultipliers

G. Kawata $^{1}$, J. Yoshida ${ }^{2}$, K. Sasaki ${ }^{2}$, R. Hasegawa ${ }^{1}$

${ }^{I}$ Transducer Technology Laboratory, Toshiba Corporate Research \& Development Center, 1 Komukai-Toshiba-cho, Saiwai-ku, Kawasaki-shi 212-8582, Japan

${ }^{2}$ Electron Devices Laboratory, Toshiba Corporate Research \& Development Center, 1 Komukai-Toshiba-cho, Saiwai-ku, Kawasaki-shi 212-8582, Japan

The linearity of optical response in silicon photomultipliers is limited by excess noise, which is caused by after-pulsing and optical cross-talk. Since these types of excess noise are correlated each other, it is difficult to determine the after-pulsing probability and optical cross-talk independently from the output signals of the silicon photomultiplier. Here we experimentally examined the gain recovery process in silicon photomultipliers and single-photon avalanche diodes. Since optical cross-talk does not affect the output signal of the single-photon avalanche diode, we can derive the after-pulsing probability directly from the experimental results. We confirmed that the number of after-pulses obeys a geometric distribution, and also that the after-pulsing probability is proportional to the square of the excess bias voltage in the low bias region.

\author{
N27-4: Two Dimensional Tetra Lateral Position-Sensitive Silicon Photomultiplier with Charge Division \\ Mechanism

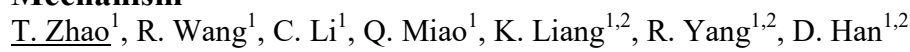 \\ ${ }^{I}$ Beijing Normal University, College of Nuclear Science and Technology, Beijing, China \\ ${ }^{2}$ Beijing Radiation Center, Beijing, China
}

Two-dimensional (2-D) tetra-lateral position-sensitive silicon photomultiplier (PS-SiPM), featured by epitaxial quenching resistors and an intrinsic continuous cap resistive layer for charge division has been developed for discrimination of both photon number and incident light position recently at Novel Device Laboratory (NDL, Beijing). It has attractive advantages of less signal output electrodes and simple readout electronics as a PS-SiPM, fast response, high gain, large dynamic range, compatible to regular SiPM with epitaxial quenching resistors and simple fabrication technology. In this report, we eliminate the effect of transmission spreading of signal pulses by recording pulse area instead of pulse height. In addition, we alleviate the barrel position distortion by introducing an improved positional calculation algorithm. The position measuring error is $90.1 \mu \mathrm{m} \pm 67.5 \mu \mathrm{m}$ for the device with a size of $2.2 \mathrm{~mm} \times 2.2 \mathrm{~mm}$ and $194 \times 226$ micro APD cells; A high position resolution of $58.5 \mu \mathrm{m} \sim 82.9 \mu \mathrm{m}$ is demonstrated as the mean photoelectron number is 706 and over voltage is $2.9 \mathrm{~V}$. The position resolution is improved with the increase of the mean photoelectron number and the over voltage.

Dejun Han is the corresponding author,whose emali is djhan@bnu.edu.cn.

N27-6: Characterization of AdvanSiD and Hamamatsu SiPMs for Novel Design Cryogenic Detectors $\underline{\text { T. Cervi }}{ }^{1}$, M. Bonesini ${ }^{2}$, A. Falcone ${ }^{3}$, A. Menegolli ${ }^{1}$, G. L. Raselli ${ }^{1}$, M. Rossella ${ }^{1}$, M. Torti

${ }^{I}$ INFN Sez. Pavia, Pavia, Italy

${ }^{2}$ INFN Sez. Milano Bicocca, Milano, Italy

${ }^{3}$ University of Texas, Arlington, United States of America

The detection of the scintillation light produced in liquified noble gases after the passage of ionizing particles is a major technological challenge. The realization of novel design photo-devices operating at cryogenic temperature is mandatory for a number of applications, ranging from neutrino and dark matter liquid argon and xenon Time Projection Chamber (TPC) detectors, to nuclear medicine imaging devices such as liquid xenon TPCs for Positron Emission Tomograpy (PET). Silicon Photomultipliers (SiPMs) are semiconductor-based photo-detectors able to reach gains of the order of $10^{6}$. This feature, together 
with the possibility to operate these devices under strong magnetic fields, makes SiPMs a realistic alternative to traditional PhotoMultiplier Tubes. Our work was devoted to test different SiPM models (AdvanSiD $40 \mu \mathrm{m}$ cells ASD-NUV1S-P, AdvanSiD 40 $\mu \mathrm{m}$ cells ASD-RGB1S-P, Hamamatsu S12571-050-P and Hamamatsu S12572-050-P) at cryogenic temperatures, in particular at the liquid argon $(87 \mathrm{~K})$ and xenon $(165 \mathrm{~K})$ ones. We present results from measurements of breakdown voltage, gain, noise, pulse shape and photon detection efficiency. This campaign of measurements is preliminary to choose the best device for the realization of a first large area cryogenic photo-detector made of SiPMs.

N27-7: Simulating Multi-Channel Vacuum Phototriodes Using COMSOL $\underline{\text { S. Zahid }}^{1}$, D. J. A. Cockerill ${ }^{2}$, P. R. Hobson ${ }^{1}$

${ }^{I}$ Dept. Electronic and Computer Engineering, Brunel University London, Uxbridge, UK

${ }^{2}$ Particle Physics Dept., STFC Rutherford Appleton Laboratory, Harwell, UK

Vacuum phototriodes (VPT) are single-gain-stage photomultiplier tubes which can operate in high quasi-axial magnetic fields. They have been used in a number of particle physics experiments and nearly 16000 are currently in operation, in a $3.8 \mathrm{~T}$ magnetic field, in the endcap calorimeter of the Compact Muon Solenoid experiment at the Large Hadron Collider, CERN. The simulation of such a VPT using the COMSOL multi-physics software environment is described. Their proximity focussing provides a fast signal and suggests that a multi-anode or multi-dynode version should be achievable. A VPT with a segmented dynode, to provide four independent channels in the same overall device envelope, is presented. The induced signal as a function of time and the effects of cross-talk in adjacent quadrants is calculated. Such a device has a number of potential applications such as reading out scintillating or wavelength-shifting fibres from "spaghetti" calorimeters.

This work was supported in part by the Science and Technology Facilities Council, UK

N27-8: 10 X 10 Micro Pixels with a Operational Voltage-Independent Bias Circuit for Positron Emission Tomography

H. Park, J. Burm

Dept of Electronic Engineering, Sogang University, Seoul, South Korea

To minimize the characteristic variation of SPADs (Single-Photon Avalanche Photo Diodes) with bias, a current mirror based bias circuit is implemented and tested for Positron Emission Tomography (PET) application. With the proposed quenching bias circuit, the operational bias variation of SPADs is successfully reduced. A SPAD and quenching bias circuit are integrated in a about $75 \mu \mathrm{m} X 75 \mu \mathrm{m}$ area to make a micro pixel. The optimized bias circuit maximizes the photon detection area to have more than $50 \%$ fill factor. This paper is based on a $0.35 \mu \mathrm{m}$ standard CMOS process with thick gate oxide option.

N27-9: Towards the Standardization of SiPM Characterization on the Example of Three Recent Blue Sensitive Devices

N. Otte

Georgia Institute of Technology, Atlanta, GA, USA

We present the characteristics of the latest generation of blue sensitive Silicon photomultipliers (SiPMs) from FBK, Hamamatu, and SensL. Measured parameters include the breakdown probability and the photon detection efficiency from $200 \mathrm{~nm}$ to $1000 \mathrm{~nm}$ as well as temperature dependent measurements between $-40^{\circ} \mathrm{C}$ and $+40^{\circ} \mathrm{C}$ of dark rates, afterpulsing, direct and delayed optical crosstalk, quench resistor values, recovery times, and cell capacitances over a wide range of overvoltages.

These measurements demonstrate the impressive advancement of SiPMs over the past ten years. For example, the photon detection efficiency has dramatically improved and reaches $55 \%$ with a peak response of blue sensitive devices that is now below $400 \mathrm{~nm}$. Also the temperature dependency of the photon detection efficiency and the gain of SiPMs is now at the same level or even outperforms classical photomultipliers, which had been a major drawback of SiPMs in the past. We give reasons why the latest generation of SiPMs is much more insensitive to temperature variations.

Based on our measurements we show how the temperature and overvoltage dependencies of the majority of the SiPM characteristics can be understood with a small set of physical parameters and thus provides a deeper insight into the device physics of SiPMs. The major advantage for a user, however, is that such a minimal set of parameters allows one to easily simulate the performance of an SiPM in an application over a wide range of operating voltages. It is thus not only possible to quickly find the optimal operating point of an SiPM for a specific application but also to significantly minimize design costs and time. Our work is in part meant to initiate a discussion within the community that aims at standardizing the characterization of SiPM measurements.

\section{N27-10: HEPS-BPIX, the Hybrid Pixel Detector System for High Energy Photon Source in China}




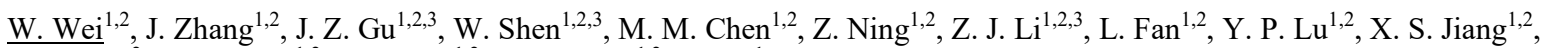
A. K. Lan ${ }^{2}$, Q. Ouyang ${ }^{1,2}$, K. J. Zhu ${ }^{1,2}$, Y. B. Chen ${ }^{1,2}$, P. Liu ${ }^{1}$

${ }^{1}$ Institute of High Energy Physics, CAS, Beijing, China

${ }^{2}$ State Key Laboratory of Particle Detection and Electronics, Beijing, China

${ }^{3}$ University of Chinese Academy of Sciences, Beijing, China

HEPS-BPIX is a dedicated hybrid pixel detector for the next generation of light source, High Energy Photon Source (HEPS) in China. The pixel readout chip works in the single photon counting mode. It was successfully designed and manufactured by a full mask tapeout. Aiming for Small Angle X-ray Scattering (SAXS) applications and other typical experiments, the chip contains a pixel array of $104 * 72$ pixels with a pixel size of $150 \mathrm{um} * 150 \mathrm{um}$. Its runs with tunable frame rate which can exceed $1.2 \mathrm{kHz}$. Based on the successful design of the chip, the detector module was assembled by Indium bump bonding with $2 * 4$ pixel chips and a single large sensor, achieving a sensitive area of about $4.5 \mathrm{~cm} * 3.6 \mathrm{~cm}$. Six detector modules grouped in a $2 * 3$ array were then mounted on an Aluminum supporting sketch as the final prototype system, covering an area of $9 \mathrm{~cm}^{*} 10 \mathrm{~cm}$ with $360 \mathrm{k}$ pixels. More detector modules can be further butted with proper mechanical supporting. The hybrid Printed Circuit Board was carefully designed with rigid and flexible sections, in order to achieve less dead area, low noise, good thermal conductivity and be convenient for every step of assemblies. The data acquisition is served with a single server, and each detector module communicates with the server through two $10 \mathrm{~Gb}$-Ethernet connections, achieving a full data rate of $862 \mathrm{MB} / \mathrm{s}$. The detector system consumes $60 \mathrm{~W}$ at the full speed and can run without water cooling, making it easily portable. A typical SAXS experiment, giving the X-ray image of the diffraction rings of a powder sample $\mathrm{CeO} 2$, shows good agreement with the theoretical calculation on ring positions and good peak-to-valley ratio of the normalized intensity peaks. Continuous animations taken from the known-frequency movements were also given, proving the frame rate of $1.2 \mathrm{kHz}$. Other experiments including those done on $\mathrm{X}$-ray tubes were discussed, including the flatfield corrections and the preliminary results on detector calibrations.

This work was supported by the State Key Laboratory of Particle Detection and Electronics of China and Institute of High Energy Physics of Chinese Academy of Sciences. It was also supported by the Test Facility of High Energy Photon Source in China.

N27-11: Test and Characterization of 20 Pre-Series Hamamatsu R5916-MOD Photomultiplier Tubes for the ICARUS T600 Detector.

G. L. Raselli ${ }^{1}$, C. Montanari ${ }^{1}$, M. Rossella ${ }^{1}$, T. Cervi ${ }^{1,2}$, M. Torti ${ }^{1,2}$, M. Spanu ${ }^{1,2}$, M. Bonesini ${ }^{3}$, M. Nessi ${ }^{4}$, U. Kose ${ }^{4}$, A. Zani $^{4}$,

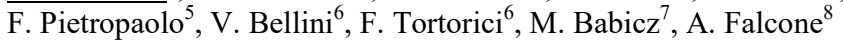

${ }^{I}$ INFN Sezione di Pavia, Pavia, Italy

${ }^{2}$ Università di Pavia, Pavia, Italy

${ }^{3}$ INFN Sezione di Milano Bicocca, Milano, Italy

${ }^{4}$ CERN, Geneva, Switzerland

${ }^{5}$ INFN Sezione di Padova, Padova, Italy

${ }^{6}$ Università di Catania and INFN, Catania, Italy

${ }^{7}$ AGH University of Science and Technology, Krakow, Poland

${ }^{8}$ University of Texas at Arlington, Arlington, U.S.A.

The ICARUS T600 detector is thelargest liquid argon time projection chamber ever built for neutrino oscillation study. It took data from 2010 to 2013 in Gran Sasso Laboratory (Italy), both with cosmic and with neutrino from CNGS beam from CERN. In future it will become the far detector of the SBN short baseline neutrino program at Fermilab, which foresees three liquid argon detectors along the Booster Neutrino beam line operating at surface condition. The apparatus is now under refurbishing at CERN in the framework of the WA104 activities. The most important aspect of this renewal is the new light detection system: it foreseen the use of four photomultiplier planes with 90 units, 8 in. diameter Hamamatsu R5916-MOD, suitable for cryogenic applications. A first pre-series of 20 photomultiplier tubes has been fully tested, both at room and at liquid argon temperature, to ensure the devices capability on allowing good performances of the detector. The main parameters of these photomultipliers namely gain vs. power supply, single electron response peak to valley ratio, dark count rate and response linearity - have been evaluated at room temperature. Same measurements have then performed with thedevice immersed in liquid argon. Results demonstrate the good behaviour of the tested devices, and led to the full series procurement.

\section{N27-12: Design, Fabrication and Testing of Sealed Microchannel Plate Photodetectors for Time-of-Flight and Imaging Applications} J. Wang

High Energy Physics, Argonne National Laboratory, Lemont, USA

Microchannel Plates (MCP) photodetectors feature picosecond level time resolution and sub-mm level position resolution, which makes them a perfect candidate to replace the traditional Photomultipier Tubes (PMTs). Argonne National Laboratory (ANL) has produced $6 \mathrm{~cm} \times 6 \mathrm{~cm}$, all-glass body, sealed photodetectors based on Microchannel Plates (MCPs) functionalized by Atomic 
Layer Deposition (ALD). A Small Single Tube Processing System (SmSTPS) was constructed for fabricating the photodetectors. We produced a number of first prototypes and have obtained satisfactory results from the laser test. Recently, we developed an improved detector design that allows for individually controlling the voltage of each stack component for best optimization. In addition, we have improved the quantum efficiency of the photocathode and report results from this work. In this paper, we report on the design, fabrication technology, and test results of production runs. The detectors are appealing for applications requiring precision timing and position resolution including time-of-flight and ring Cherenkov imaging detectors. Currently, the group is developing components for the MCP photodetector suitable for scintillation detection in cryogenic liquid detectors such as liquid argon TPCs (128nm scintillation) and xenon dark matter detectors (175nm scintillation). Status and results from this development work will also be discussed.

N27-13: Comparison of SensL and Hamamatsu 4x4 Channel SiPM Arrays M. Grodzicka, T. Szczesniak, M. Moszynski

National Centre for Nuclear Research, Otwock-Swierk, Poland

The market of silicon photomultipliers (SiPMs) consists of many manufacturers that produce their detectors in different technology. Hamamatsu (Japan) and SensL (Ireland) seems to be the most popular companies that produce large SiPM arrays. The aim of this work is characterization and comparison of $4 \times 4$ channel SiPM arrays produced by these two producers. The both tested SiPMs were made in through silicon via (TSV) technology, consist of 16, 3x3 mm pixels and have fill factor slightly above $60 \%$. The largest difference is single APD cell size and hence total number of APD cells (55424 for Hamamatsu, 76640 for SensL). In the case of SensL SiPM, its spectral response characteristics is shifted slightly toward shorter wavelengths with maximum at $420 \mathrm{~nm}$ (450nm for Hamamatsu). The presented measurements covered selection of the SiPM optimum operating voltage (in respect to energy resolution), verification of the excess noise factor and check of the linearity characteristics. Moreover, the gamma spectrometry with LSO, BGO and CsI:Tl scintillators together with pulse characteristics for these crystals (rise time and full time) is reported, as well as temperature dependence. The presented measurements show better performance of the SensL array comparing to the Hamamatsu detector.

\author{
N27-14: Design, Fabrication and Testing of a Novel Planar Microchannel Plate Photomultiplier with Extended \\ Photon Response

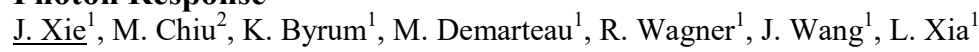 \\ ${ }^{I}$ Argonne National Laboratory, Lemont, IL, USA \\ ${ }^{2}$ Brookbaven National Laboratory, Upton, NY, USA
}

MCP-PMTs with glass window have a response range of $300 \mathrm{~nm}-600 \mathrm{~nm}$, limited by the window transmission and cathode materials. By replacing the glass window with fused silica, the detection range can be dramatically extended from $300 \mathrm{~nm}$ to 170 $\mathrm{nm}$, drawing great interests in experiments requiring time of flight (TOF) measurement and/or Cherenkov imaging at ultraviolet (UV) range.

The Argonne MCP-PMT detector group is working towards direct detection of the full VUV-UV-Vis spectra range by applying suitable windows and photocathodes with extended response. We have recently designed and fabricated $6 \mathrm{~cm} \times 6 \mathrm{~cm}$ MCP-PMTs with fused silica window and bialkali photocathode. Initial characterization indicates that the fused silica window photomultipier exhibits a transit-time spread of $57 \mathrm{psec}$ at single photoelectron detection mode and of $27 \mathrm{psec}$ at multi photoelectron mode (100 photoelectrons). Currently, we are testing the MCP-PMTs at Fermilab test beam facility for its particle detection performance and rate capability. The progress on new window exploration and characterization of the new MCP-PMT in beamline particle test will also be reported and discussed.

\author{
N27-15: Second Generation Prototype Silicon Photomultiplier Focal Plane Imaging Detector for the MAGIC \\ Telescopes

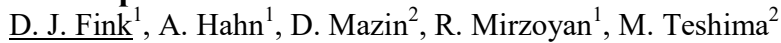 \\ ${ }^{I}$ Max Planck Institut fuer Physik, Munich, Germany \\ ${ }^{2}$ Institute for Cosmic Ray Research, Tokyo, Japan
}

The MAGIC Imaging Atmospheric Cherenkov Telescopes located on the Canary Island of La Palma are currently in operation using cameras based on photomultipliers as photodetectors. In order to asses the feasibility of upgrading the cameras to use silicon based photodetectors, a prototype detector module compatible with the photomultiplier based units was constructed and installed in the camera of MAGIC II in May of 2015. Based on the data gathered to date from this prototype, a second generation design has been completed which improves upon important parameters such as signal timing, optical fill factor, thermal management, and power consumption.

N27-16: Gain Stabilization of SiPMs 
$\underline{\text { I. Polak }}$

HEP, FZU, Institute of Physics ASCR, Prague, Prague, Czech republic

On behalf of the CALICE

The gain G of SiPMs depends both on bias voltage and on temperature. For stable operation in large detector systems, gain needs to be kept constant. This can be achieved by readjusting the bias voltage with temperature. We have built an adaptive bias voltage regulator (Adapower) that uses a linear algorithm to adjust the bias voltage $\mathrm{V}$ if the temperature $\mathrm{T}$ changes. We determined the $\mathrm{V}(\mathrm{T})$ dependence from measurements of the gain-versus-temperature dependence $(\mathrm{dG} / \mathrm{dT})$ for different bias voltage and the gainversus-bias-voltage dependence $(\mathrm{dG} / \mathrm{dV})$ for different temperatures in a climate chamber Spiral3 at CERN. We tested and studied 30 pieces of $\mathrm{SiPM}$ in groups of six types in temperature range of 5 to $40^{\circ} \mathrm{C}$ and found that the gain stabilization works excellently $(<1 \%)$.

The authors wish to thank to teams at the CERN QART and DT/LCD laboratories for the help provided. This work is conducted with support by the European Commission as INFRA project no. 262025, AIDA, with the support by the University of Bergen as well as the support by the Ministry of Education, Youth and Sports, Czech Republic, INGO project LG130131.

N27-17: Performance of SensL C-Series SiPM with High Photoelectron Resolution at Cryogenic Temperatures

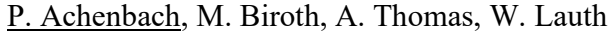

Institut für Kernphysik, Johannes Gutenberg-Universität, D-55099 Mainz, Germany

The C-Series of silicon photomultipliers (SiPM) from SensL provide a fast response and high performance at low cost. The device's ability to detect light at temperatures of liquid nitrogen $(77 \mathrm{~K})$ and liquid helium $(4 \mathrm{~K})$ with high photo-electron resolution was demonstrated. Results include photo-detection efficiency (PDE), gain, microcell capacitance, and cross-talk probability at different over-voltages, both at room and at cryogenic temperatures. At $77 \mathrm{~K}$ the SiPM demonstrated significantly improved operating characteristics while at $4 \mathrm{~K}$ the observed increase in break-down voltage, the reduction of PDE by a factor of $2-3$, and the extensively dropped microcell capacitance degraded the performance.

\author{
N27-18: Optimized SiPM for the Development of Intracerebral Beta Probes Dedicated to Neurosciences \\ Studies on Freely Moving Animals \\ A. Nagai ${ }^{1}$, N. Dinu ${ }^{1}$, M.-A. Verdier ${ }^{2}$, P. Lanièce ${ }^{3}$, L. Ménard ${ }^{2}$ \\ ${ }^{1}$ LAL CNRS/IN2P3, Univ. Paris Sud, Université Paris-Saclay, 91405 Orsay Cedex, France \\ ${ }^{2}$ Univ. Paris-Diderot, Sorbonne Paris Cité, IMNC CNRS/IN2P3, 91405 Orsay Cedex, France \\ ${ }^{3}$ IMNC CNRS/IN2P3, Univ. Paris Sud et Paris Diderot, 91405 Orsay Cedex, France
}

Behavioral assays and positron emission tomography (PET) neuroimaging in rodents are widely used in neuroscience. PET gives insights into the molecular processes of neuronal communication, and behavioral methods analyze the actions that are associated with such processes. Because PET imaging in animal requires general anaesthesia or strong restraint to immobilize the subject, this precludes combination with simultaneous behavioral studies. We propose the development of beta-sensitive intracerebral probes, allowing to measure the local concentration of radiolabelled molecules on awake and freely moving animal. In that context, SiPM photosensors offer the possibility to develop ultra-miniaturized scintillator-based intracerebral probes with high sensitivity. The probe prototype is based on low-noise Silicon photomultiplier (SiPM) photodetector coupled to a scintillating fiber and read-out by a dedicated miniaturized low-power consumption electronics. We present here the comparison between three SiPMs: two small KETEK devices of $0.5 \times 0.5 \mathrm{~mm}^{2}$ size with $50 \times 50 \mu \mathrm{m}^{2} \mu$ cell size specifically designed for our project (with and without optical trenches) and a standard Hamamatsu device of $1.3 \times 1.3 \mathrm{~mm}^{2}$ size with $50 \times 50 \mu \mathrm{m}^{2} \mu$ cell size. Gain, dark count rate and beta sensitivity were measured as a function of bias voltage and temperature for each SiPM. Results show that the small field of view and new structure of the KETEK devices allow a large decrease of the thermal and correlated noises but also lead to a reduced light collection due to the numerical aperture of the fiber. Because the beta sensitivity results of a tradeoff between photodetection efficiency and dark noise, KETEK SiPMs exhibit similar performances than the Hamamatsu device. This preliminary results demonstrate that the new KETEK devices should help to significantly improve the beta sensitivity future intracerebral probe when coupled to light focusing system.

N27-19: Investigation of Silicon Photomultipliers after Irradiation with Neutrons up to $2 \times 10^{\wedge} 13$ N/cm^2. A. Heering $^{1}$, A. Karneyeu ${ }^{1,2}$, Y. Musienko ${ }^{1,2}$, V. Postoev ${ }^{2}$, R. Ruchti ${ }^{1}$, M. Wayne ${ }^{1}$

${ }^{I}$ University of Notre Dame, Notre Dame, IN 46556, USA

${ }^{2}$ Institute for Nuclear Research RAS, Moscow, Russia 
Results on the radiation hardness of SiPMs to fast neutrons are presented. SiPMs from Hamamatsu and FBK were irradiated with reactor neutrons up to $1 \mathrm{MeV}$ equivalent fluence of $2 * 10^{\wedge} 13 \mathrm{n} / \mathrm{cm}^{\wedge} 2$. The effects of this radiation on many parameters such as gain, intrinsic dark current, photon detection efficiency and noise for these devices are shown and discussed.

N27-20: Timing Resolution of Monolithic Scintillators Coupled to Large SiPM Arrays

T. Szczesniak, M. Grodzicka, M. Moszynski, D. Wolski, M. Szawlowski

National Centre for Nuclear Research, Otwock-Swierk, Poland

Timing resolution is one of the most important parameters of scintillation detectors in many applications. One of the major fields in which timing resolution with SiPMs is studied is medical imaging and Time of Flight PET. In such case small scintillator pixels are used. However there are many applications in which timing information from large, monolithic crystals is needed. The aim of this work is to study the timing resolution of large SiPM arrays in readout of various, monolithic scintillators. The presented measurements are made with S12642-1616PB-50(X) Hamamatsu TSV MPPC array having a 50x50 $\mu \mathrm{m}^{2}$ cell size and effective active area of $48 \times 48 \mathrm{~mm}^{2}(16 \times 16$ channels). During the tests, MPPC is treated as a single, large detector. The different active area of MPPC is achieved by readout of subareas of the device. In the first part of the study, experiments are made with small 10x10x5 mm $\mathrm{mm}^{3}$ LSO scintillator and various number of readout channels in order to understand the influence of the detector area (or capacitance) on timing resolution. Next, the timing spectra are recorded for 4 types of scintillators: $20 \times 20 \times 20$

$\mathrm{mm}^{3}$ LYSO, 2 inch $\mathrm{CeBr}_{3}, 2$ inch $\mathrm{LaBr}_{3}$ and 2 inch $\mathrm{NaI}(\mathrm{Tl})$. The optimization steps and the analysis of the main aspects affecting the timing resolution of large MPPC arrays are discussed. The timing results are compared with data recorded previously with fast photomultipliers.

N27-21: Statistical Characterization of NUV-HD SiPMs at Low Temperature

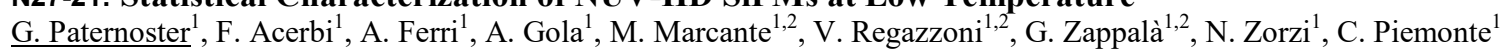

${ }^{I}$ Fondazione Bruno Kessler, Trento, Italy

${ }^{2}$ Department of Physics, University of Trento, Trento, Italy

In this contribution, we show, for the first time, a systematic characterization of SiPMs as a function of the temperature. This massive statistical measurement is crucial to assess the potential use of SiPMs at low temperatures in real applications, where a large number of devices is required. We performed reverse and forward IV measurements on a six-inch wafer containing about $15003 \times 3 \mathrm{~mm}^{2} \mathrm{NUV}-\mathrm{HD}$ SiPMs by means of an automatic prober with a temperature controlled chuck in the range between $40^{\circ} \mathrm{C}$ to $+24^{\circ} \mathrm{C}$. We will report on the uniformity of the temperature dependence of some important parameters like the Breakdown Voltage, Dark Current and Quenching Resistance. Furthermore, we will describe few anomalous cases $(<1 \%)$ in which the dark current shows the appearance of defects with decreasing temperature. By the conference, we will further extend the dataset including more wafers and the temperature range.

N27-22: A New Silicon Drift Detector Array for the Maia X-Ray Fluorescence Detector System W. Chen, D. Elliott, G. Giacomini, A. K. Rumaiz, D. P. Siddons, G. Smith

Instrumentation Division, Brookhaven National Lab, Upton, NY, USA

A new Maia detector is being currently developed, consisting of an array of 384 silicon drift detectors (SDDs), each having a surface area of $1 \mathrm{~mm} 2$. The detector has a new double metal scheme that supplies biases to the single pixel SDD rings. All 384 anodes of SDDs are connected to individual electronic channels. The detector will be referred to as the Drift Maia. This effort was motivated by improving the energy resolution and the throughput of existing Maia detectors whose pixels are diode based. In order to use the existing circuit boards and back-end electronics that have already developed, this new Maia detector was designed with a footprint identical to the original Maia, after replacing the diode sensors with SDDs, in order to significantly lower the capacitance of each readout channel and shorten the shaping times, thus permit improved energy resolution and reduced experimental times. The production of the detector is near the end. The bench test and the spectroscopic test will soon be carried out. The design, fabrication details, and test results will be presented in the conference.

N27-23: Single Photon Avalanche Diode Sensor Prototypes in Planar CMOS and 3D Integration Technologies D. Arutinov, M. Beer, W. Brockherde, Y. Celik, S. Dreiner, M. Figge, S. Gläsener, A. Göhlich, J. Heß, A. Schwinger, A. Schmidt

Fraunhofer Institute for Microelectronic Circuits and Systems, Duisburg, Germany

Due to high photon sensitivity and temporal resolution in the nanosecond range, photomultiplier tubes (PMT) are widely used as reliable single photon detectors in different industrial, medical, and scientific fields. However, substantial operating voltage requirements, low spatial resolution, and other PMT requirements can challenge their utilization in a variety of applications. With the advancement of microelectronics, the photo-detection concept used in PMTs has been moved to a silicon substrate, and 
empowered by a conventional CMOS technology enabling different functionality implementation alongside SPAD structures on a same silicon die. The new approach made possible overcoming many limitations peculiar to PMTs, opening the door to innovative solutions in the field of photo-detection. Based on existing 0.35 $\mu \mathrm{m}$ CMOS fabrication process, we have developed Single-Photon Avalanche Diode (SPAD) technology. In the CMOS SPAD process several front-side illuminated sensor prototypes have been implemented for various applications. However, some of the prototypes require a lot of electronics which occupy die space at the cost of an active sensor area. To solve the issue, we are currently developing a 3D integration technology to bond back-side illuminated SPAD sensors to standard CMOS wafers. As a result, the CMOS electronics can be partially or completely moved to another wafer without sacrificing SPAD performance. In this talk, we present our CMOS SPAD technology, recently implemented CMOS SPAD sensor prototypes, our new 3D bonding technique, and preliminary characterization results from both planar and 3D sensor prototypes.

N27-24: Operation of Silicon Photomultipliers as Photosensors of Liquid Xenon Detectors.

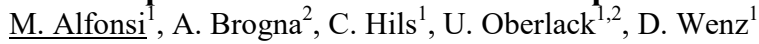

${ }^{I}$ Institut für Physik \& PRISMA Exzellenzcluster, Johannes Gutenberg Universität Mainz, Mainz, Germany

${ }^{2}$ PRISMA Detector Laboratory, Johannes Gutenberg Universität Mainz, Mainz, Germany

Silicon Photomultipliers as photosensors for liquid xenon detectors are an attractive alternative to Photomultiplier Tubes: liquid xenon detectors employed in low background experiments or compact Compton cameras can take advantage of the small sensor mass and dimensions, the larger light collection coming from a more packed tiling of the readout plane, and possibly a smaller cost per area. We investigate the operation in liquid xenon of samples developed by Ketek GmbH. To provide sensitivity to the liquid xenon scintillation light wavelength, $178 \mathrm{~nm}$, the inactive entrance layer of the device has been thinned. The operation of such devices at temperatures as low as $-100^{\circ} \mathrm{C}$ has been verified, characterizing dark count rate and after-pulsing as well. In order to not spoil the liquid xenon purity, we selected a substrate and a bonding adhesive measuring the outgassing properties of the parts. Finally, we realized a test station to assess the photon detection efficiency at a wavelength of $178 \mathrm{~nm}$. In a cryogenic vacuum-insulated vessel an ${ }^{241} \mathrm{Am}$ a-source and up to four samples are immersed in liquid xenon, allowing an event-by-event comparison of the response of three Silicon Photomultipliers and a 1" Photomultiplier Tube used as reference.

N27-25: Dead Time Investigation of SiPMs for Application at Pulsed Muon Sources

D. E. Pooley, S. P. Cottrell, M. S. Huzan, L. Pollastri, E. M. Schooneveld, N. J. Rhodes

STFC Rutherford Appleton Laboratory, ISIS, Harwell Oxford, UK

The paper reports on our investigation into the use of SiPMs where very high data rates need to be measured. Our specific application is for muon spectrometers at pulsed sources, where the instantaneous counting rate is extremely high immediately following muon implantation. However, our results can be generalised to any application where the expected count rate is comparable to the SiPM recovery time. For muon spectrometers, an understanding of the dead time characteristics of the detector is crucial if undistorted spectra are to be measured. To this end, the dead time performance of SensL and Hamamatsu SiPMs have been investigated and compared. Our investigation shows the complexity of the dead time properties of a SiPM are due to its multicellular architecture. We demonstrate that the intrinsic characteristics of a SiPM result in a detector with a performance inferior to that of a PMT based equivalent, the benchmark in our study. However, we show that providing the SiPM is operating in the appropriate optical conditions, signal processing (both in-silicon and using analogue electronics) makes it possible to attain dead time performance comparable to that of a PMT based detector. The study encompasses evaluation using a laser-driven optical characterisation facility, Monte Carlo simulation and on-beam measurements (performed at the ISIS pulsed muon source) where a figure-of-merit was defined to facilitate cross-evaluation of different detector technologies.

The support for this work provided by the European Commission under the 7th Framework Programme through the 'Research Infrastructures' action of the 'Capacities' Program, NMI3-II Grant Number 283883, is gratefully acknowledged.

\author{
N27-26: Study of a Relationship Between the Electric Field and Dark Count Rate in a SiPM for a PET-MR \\ Application \\ K. T. $\operatorname{Lim}^{1}$, M. Cho ${ }^{1}$, J. Kim ${ }^{1}$, G. Cho ${ }^{1}$, W. S. Sul ${ }^{2}$ \\ ${ }^{I}$ Nuclear and Quantum Engineering, Korea Advanced Institute of Science and Technology (KAIST), Daejeon, Korea, Republic of \\ ${ }^{2}$ National Nanofab Center, Daejeon, Korea, Republic of
}

In this study, an improved version of SiPM fabricated at National NanoFab Center is being investigated and evaluated. The temperature dependency of old version of SiPMs was first studied in order to determine the major source for DCR and this was done by finding the activation energy of defect states. The extracted value was found to be $0.44 \mathrm{eV}$, indicating that DCR was dominated by Shockley-Read-Hall recombination-generation enhanced by trap-assisted tunneling. Based on these results, a new version of SiPM was fabricated and tested to compare its performance with the previous version. The sensors were fabricated via a customized CMOS process with a micro-cell size of $65 \times 65 \mathrm{um} 2$ on $200 \mathrm{~mm}$ p-type epitaxial layer wafers. In contrast to the 
previous version, some modifications are made for plasma used in dry etching to reduce the process induced defect sites. Furthermore, the peak electric field was varied from $6.0 \times 106$ to $5.0 \times 106 \mathrm{~V} / \mathrm{cm}$ to reduce the noise mechanism caused by trapassisted tunneling where $6.0 \times 106 \mathrm{~V} / \mathrm{cm}$ was the peak value for the previous generation. Then, I-V characteristic curves of new type are plotted together with the old version to compare their performance. It was found that the dark current level was reduced to one tenth of the previous version and the extracted DCR was found to be $65 \mathrm{kHz}$ for a single micro-cell. This estimated to 7.1 $\mathrm{MHz} / \mathrm{Ch}$ for a $65 \mathrm{um}$ micro-cell. Chip-scale packaging is currently under the process for all samples. Once the samples are ready, dark count ratio and photon detection efficiency will be experimentally measured for a further analysis. By the poster submission, a study of a reduction of the maximum electric fields in terms of the trade-off relationship between DCR and PDE will be presented. Likewise, the power consumption will also be analyzed since a lower electric field indicates a higher breakdown voltage in a SiPM and thus an optimum electric field value at a given process will be suggested.

\title{
N28: Neutron detectors - Poster session II
}

Tuesday, Nov. 1 14:00-16:00 Etoile

N28-1: Neutron Counting Type Imaging Detector with Super-Resolution Technique

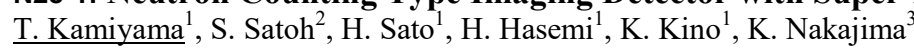

${ }^{I}$ Faculty of Engineering, Hokkaido University, Sapporo, Hokkaido, Japan

${ }^{2}$ Institute of Materials Structure Science, High Energy Accelerator Research Organization (KEK), Tsukuba, Ibaraki, Japan

${ }^{3}$ Research Reactor Institute, Kyoto University, Kumatori, Ohsaka, Japan

Neutron imaging with energy analysis is interested in many fields, because of its versatile ability for non-destructive material characterization. As an example of the method, neutron Bragg-edge transmission imaging with a pulsed neutron source, which is applied the time-of-flight (TOF) technique for energy analysis, can map the distributions of the crystal structure and texture of materials on the real object image. For such energy selective imaging, we have developed a neutron imaging device, which is a combination of a vacuum tube type neutron image intensifier and a high speed digital camera for TOF measurement. The device has advantages in the higher spatial resolution and count rate than the usual 2-dimensional neutron detector, but has still subjects in the linearity to the input neutrons and the rate of image data transfer. In this study, we shift the concept of the device to exchange the camera to a photomultiplier tube (PMT) array. Through the neutron image intensifier a neutron absorption signal is convert to a photon pulse, and it is counted by a pixel on the PMT array. The linearity of the input neutron intensity can be expected by the pulse counting and the counting rate becomes enough for pixelization. Although the problem is on the pixel size of the PMT array, which is 2 millimeters square, we improve it by applying the super-resolution technique with the center-ofmass calculation. The prototype device with a 4 inch neutron image intensifier and $16 \times 16$ photomultiplier tube array was trialed on the thermal neutron beamline at Hokkaido University Neutron Source (HUNS) facility, Japan. The result of the neutron spectrum measurement showed good agreement with the usual ones by the other detectors. The super-resolution calculation could applied to the imaging results, and 2 to 8 times higher spatial resolution images were obtained. The results showed fine images without large failure, but some disturbance appeared at the rim of the images.

The research is supported under the Development of Non-Destructive Methods Adapted for Integrity test of Next generation nuclear fuels project by the Ministry of Education, Culture, Sports, and Technology (MEXT), Japan.

\author{
N28-2: Spatial Resolution Enhancement of Neutron Radiogram by Cooperating with X-Ray Radiography

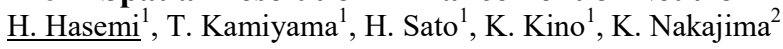 \\ ${ }^{I}$ Division of Quantum Science and Engineering, Faculty of Engineering, Hokkaido University, Sapporo, Hokkaido, Japan \\ ${ }^{2}$ Research Reactor Institute, Kyoto University, Kumatori, Osaka, Japan
}

Pulsed neutron imaging is the spectroscopic radiography technique based on the energy-analysis of neutrons by the time of flight (TOF) method. By analyzing the dip due to the neutron resonance absorption on a transmission spectrum, absolute values of nuclei densities and temperatures can be estimated non-destructively. We can obtain the distributions of these values using a position sensitive detector. However, the images obtained by the pulsed neutron imaging have low spatial resolution because of the performance limitations of the detector. On the other hand, X-ray radiography images have higher spatial resolution compared with the neutron images. To obtain higher-resolution neutron images, we studied the resolution enhancement method for neutron images by cooperating with the X-ray radiography. The resolution enhancement method consists of the image alignment and the mapping methods. As a first step, we tried mapping neutron transmission image into the X-ray image. At the Hokkaido University neuron source, we measured a neutron transmission image by a neutron detector dedicated for the pulsed neutron imaging. The X-ray image was obtained by the neutron image intensifier which have X-ray sensitivity combined with a digital camera. The image alignment method using the mutual information, which is a degree of mutual dependence between two images, was applied for the neutron image and the X-ray image. Next, the density information which was estimated by the analysis of neutron transmission was mapped into a high-resolution X-ray image. After all, we successfully obtained a higherresolution image of density distribution. 
The research is supported under the Development of Non-Destructive Methods Adapted for Integrity test of Next generation nuclear fuels project by the Ministry of Education, Culture, Sports, and Technology (MEXT), Japan.

\author{
N28-3: Improved Neutron-Gamma Discrimination for a 3He Neutron Detector Using Subspace Learning \\ Methods \\ C. L. Wang, L. L. Funk, R. A. Riedel, K. D. Berry \\ Instrument and Source Division, Oak Ridge National Laboratory, Oak Ridge, TN, USA
}

3He gas based neutron linear-position-sensitive detectors were applied for the majority of neutron scattering instruments at ORNL. Traditional Pulse-Height Analysis (PHA) gave a Neutron-Gamma Discrimination (NGD) ratio of about 3 x 106 . The NGD ratios of $3 \mathrm{He}$ detectors need to be improved to satisfy present and future neutron scattering instruments at $\mathrm{SNS}$. We proposed digital signal analysis methods of individual waveforms acquired from a $3 \mathrm{He}$ tube using: i). rising time; ii). charge integration; iii). pulse-amplitude (Amax) histograms; iv). two event parameters (a1, b0) obtained from a Wiener filter; and v). a ratio of cross-correlation (CC) coefficients between individual and reference waveforms of neutron and gamma events. Fisher Linear Discriminant Analysis (FLDA) was performed for seven feature parameters obtained above and a projected parameter (P6) in a feature subspace vs. Amax was used for the NGD. The NGD ratios were improved by about 30 times compared with the traditional PHA method. Further improvements may be possible using other subspace projection methods. Our results indicate the NGD capabilities of $3 \mathrm{He}$ tube detectors can be significantly improved using subspace-learning methods.

N28-4: A New Material for Slow and Fast Neutron Detection

L. Stuttgï $i \frac{1}{2}{ }^{1}$, A. Chietera ${ }^{1}$, L. Douce ${ }^{2}$, E. Bouajila ${ }^{2}$, J. Fouchet ${ }^{2}$, S. Kihel ${ }^{1}$, C. Mathieu ${ }^{1}$, B. Benoï $i^{1 / 2 t^{2}}$

${ }^{I}$ DRS, IPHC-CNRS/UDS, Strasbourg, France

${ }^{2} D R S, I P C M S-U D S$, Strasbourg, France

New scintillating components based on organic ionic compounds have been synthesized with the purpose to produce a new material able to detect and identify both slow and fast neutrons over a wide domain of energies. The material has to be solid in order to be usable under vacuum. These materials present some major advantages as they offer an excellent thermal and chemical stability. They are non-flammable and non-volatile. It is possible to act on the properties of these ionic compounds by changing either the anion or the alkyl chain or both. It is thus possible to incorporate boron for instance for slow neutron detection or/and increase the hydrogen concentration by increasing the length of the alkyl chain. Although these materials are still under development, promising results have been obtained both for slow and fast neutron detection. Characteristics of the new components have been compared to the performances of existing scintillators as 6Li glasses and BF3 gas counters for slow neutron detection and NE213/BC501A organic liquid scintillators for fast neutrons. The new materials can be adapted and configured in order to optimize their properties to any special use.

N28-5: A prototype system for real-time fast neutron multiplicity using liquid scintillation detectors R. Sarwar ${ }^{1}$, M. J. Joyce ${ }^{1}$, C. H. Zimmerman ${ }^{2}$

${ }^{I}$ Engineering Department, Lancaster Univeristy, Lancaster, Lancashire, LA1 4YW, United Kingdom

${ }^{2}$ The Central Laboratory, National Nuclear Laboratory, Sellafield, Cumbria, CA20 1PG, United Kingdom

The development of a SoC-FPGA based system for the real-time multiplicity analysis with organic liquid scintillation detectors is described. Helium-3 detectors, which are still the primary choice for scientists when carrying out neutron multiplicity analysis, exploit the large cross-section of Helium-3 for thermal neutrons and thus the neutron radiation has to be thermalized which prevents the use of small (i.e. $<100 \mathrm{ns)}$ coincidence gates. Small coincidence windows are essential for achieving the high levels of statistics needed to yield detect high-order multiplicity detection and to remove significant sources of accidentals. As such, there has been a renewed interest in the field of fast neutron multiplicity counting using liquid scintillation detector assay. This paper focuses on the development of a prototype real-time system capable of rapid data processing which is essential when using liquid scintillation detectors for coincidence counting and multiplicity assay. This has proven to be particularly difficult due to the small detection window, of the order of 150 to $200 \mathrm{~ns}$, that originates when radiation is detected. Moreover, considering the practical geometric possibilities of a typical experimental setup (i.e. several rings of detectors surrounding a source with a radius of 50 to $100 \mathrm{~cm}$ ) and the high speed at which neutrons travel, it is expected that the required coincidence windows will be in the range of 15 to $40 \mathrm{~ns}$. Using this prototype system, this paper will describe a system capable of undertaking the real-time determination of Rossi-a distribution, Feynman-Y distribution and neutron coincidence distribution using a californium-252 source. For validation, simulated results using MCNP6 for the corresponding experiments will be presented which demonstrate that the results bear a close resemblance to expectations.

The authors would like to thank Engineering and Physical Sciences Research Council (EPSRC, UK) and National Nuclear Laboratory (NNL, $\mathrm{UK}$ ) for funding the PhD research under DISTINCTIVE grant EP/L014041/1. This work was also supported by the EPSRC under ADRIANA grant, EP/L025671/1. 


\section{N28-6: Implementation of Gadolinium for Neutron Measurement Systems Based on Plastic Scintillators or}

Semi-Conductors

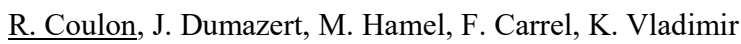

Sensors and Electronics Laboratory, CEA LIST, Gif-sur-Yvette, France

The CEA LIST works on the development of neutron sensors based on the use of gadolinium as thermal neutron convertor into sensors because it shows the best available cross-section for this purpose. However, the neutron measurement market is still dominated by Helium 3 or boron proportional counters. The realization of neutron systems based on gadolinium is limited and circumcised to some research works and is completely absent in commercially available products [Abdu]. The main reason of this anathema is the radiative nature of the signature of the interaction which suffers to be penetrative and not discriminable to gamma-rays background. The CEA LIST has decided to give a chance to this remarkable element for neutrons measurement. In one hand, we have detailed radiative and electronic signatures associated to the neutron capture by gadolinium nuclei. We have conclude that two domains of use can be distinguished: the low/medium energy domain characterized by the low penetration path of the deposited energy ( 0 to $200 \mathrm{keV}$ ), and the high energy domain (above $5 \mathrm{MeV}$ ) where radiations are very penetrative.

Regarding to these domains and the corresponding penetrative power, two scales of sensors could be respectively envisaged: The thin/ medium size sensors (portable systems), and the high volume systems (fixed monitors). In a second hand, we have discussed about strategies able to perform separation between neutron and gamma background signals. While a pulse high discrimination is suitable for the high volume system, the smaller concepts do not allow the particular discrimination. In front of this technological barrier, we have oriented our studies on compensated measurement techniques. The different approaches will be presented for high or small volume systems, with and without compensation, using plastic scintillation or semi-conductor technologies. The authors will present the obtained results; discuss the advantages and the limitations of the different concepts.

\section{N28-7: A Ceramic-Insulated Ball-Anode Element for Two-Dimensional Neutron Detector}

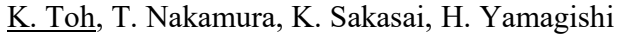

Japan Atomic Energy Agency, Tokai, Japan

A ceramic-insulated ball-anode $(\mathrm{CiB})$ element that consists of a ceramic insulator, cathode lines, ball-shaped anodes, and anode lines was developed, and an irradiation experiment was performed using a Cf-252 neutron source. When a gas-based detector is used in neutron scattering experiments, incident neutrons are usually scattered by both the measuring sample and the gas-based detector itself. The detector element usually utilizes a polymer as its gaseous amplification structure and/or the signal readout substrate, though the polymer acts as strong neutron scatterers. To suppress background noise from unintentionally scattered neutrons, we are currently developing a novel concept based on a two-dimensional neutron detector element using a ceramic insulator. An additional advantage of ceramics is that outgassing, which affects the long-term stability of the gas-based detector, can be reduced without the use of polymer materials. A simulation of the electric field was conducted to study the field behavior of the developed $\mathrm{CiB}$ element. Strong electric fields of more than $10^{7} \mathrm{~V} / \mathrm{m}$ was obtained by the calculation using the finite element method. Under neutron irradiation, $8 \times 8 \mathrm{ch}$ small-sized test element output neutron-induced signals in all pixels, though the intensity of each pixel was difference. It is estimated the non-uniformity is caused by the fabrication accuracy, and the problem needs to overcome in the near future.

N28-8: Improvement of Spatial Resolution of Time-Resolved MA-PMT Camera for Imaging of TRU Elements J. Koide $^{1}$, T. Uragaki ${ }^{1}$, N. Hagura ${ }^{2}$, J. Kawarabayashi ${ }^{2}$, K.-I. Mochiki ${ }^{2}$

${ }^{I}$ Cooperative Major in Nuclear Energy, Tokyo City University, Setagaya-ku, Tokyo, JAPAN

${ }^{2}$ Department of Nuclear Safety Engineering, Tokyo City University, Satagaya-ku, Tokyo, JAPAN

As a new technique the pulsed neutron transmission spectroscopic radiography is attractive in the research fields of energyresolved neutron radiography. We are developing a new time-resolved neutron imaging system for resonance absorption of transuranic (TRU) elements to investigate TRU alloy fuel. To achieve time resolution of less than $100 \mathrm{~ns}$ which is demanded to detect resonance absorption of U-238 a multi-anode (MA) - PMT is selected to detect fast scintillation light. The time-resolved neutron imaging system is installed at a compact accelerator-driven neutron source. Transmitted images of the sample are obtained by the time-resolved neutron imaging system. The system consists of a neutron scintillator, a $16 \times 16 \mathrm{ch}$ MA-PMT, a LiTA12 signal processing system and a personal computer. The neutron scintillator is a bundled $48 \times 48$ tiny scintillator column with $1 \mathrm{~mm} \times 1 \mathrm{~mm}$ cross section each. To achieve high detection efficiency for relatively high energy neutrons scintillators with the length of $10 \mathrm{~mm}$ were produced. The intrinsic spatial resolution is restricted by the cross section of $1 \mathrm{~mm}$ and it can be achieved by interpolating function of the LiTA12 system with the $16 \times 16 \mathrm{ch}$ MA-PMT which is viewing the area of $48 \mathrm{~mm} \times 48$ $\mathrm{mm}$. The spatial resolution of $1 \mathrm{~mm}$ is not enough to detect Plutonium spots with the size of $0.4 \mathrm{~mm}$ in diameter. To improve the spatial resolution subpixel-shift method was examined. A simulation X-ray experiment was made and four subpixel-shifted dummy image data were obtained. The ML-EM method was applied to obtain high-definition reconfigured image and the effectiveness of the method was confirmed. The subpixel-shift method with the solution of ML-EM algorithm was effectively 
applied for improving spatial resolution of low-definition dummy images. X-Z moving tables with stepping motors have been assembled into the stand on which a test object is set to obtain subpixel-shifted images.

\section{N28-9: Low Background Thermal Neutron Detector System}

Z. Zeng ${ }^{1}$, H. Gong ${ }^{1}$, Q. Yue ${ }^{2}$, J. Li $^{1}$

${ }^{I}$ Key Laboratory of Particle \& Radiation Imaging, Tsinghua University, Beijing, China

${ }^{2}$ Department of Engineering Physics, Tsinghua University, Beijing, China

The neutron flux is one of the important parameters characterizing an underground laboratory. In order to accurately measure the thermal neutron flux in underground laboratory and verify the effectiveness of the neutron shield design we implemented a low background thermal neutron detector system. The detector used is a He3 proportional counter tube made up oxygen-free copper with optimized parameters for low background performance. A He4 proportional counter tube with exactly the same parameters with the He3 one has also been made to study the background of the counter tube. The electronic readout system has also been developed which was designed for working in tough environment like an underground laboratory under construction. The system accumulated data for 16.3 days in CJPL(China JinPing underground Laboratory)and the background level is found to be four times lower than that of commercial stainless steel He3 proportional counter tube. We also find that it is the inner surface contamination condition for oxygen-free copper who determines the background count rate. The pulse shape analysis can distinguish micro-discharge and ? events effectively from thermal neutron events, but ROI with few a events can only be found at the cost of neutron sensitivity loss.

This project is funded in part by the National Natural Science Foundation of China under Grant no.11475094.

\section{N28-10: Diagnosis of Neutron Sensitivity Within a Scintillator / Wavelength-Shifting Fiber Coil Element by Using Collimated Pulsed Neutron Beam}

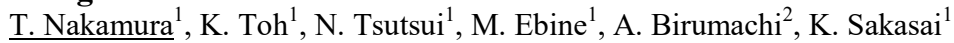

${ }^{1} J-P A R C / M L F$, Japan Atomic Energy Agency, Ibaraki, JAPAN

${ }^{2}$ Nuclear Science Research Institute, Japan Atomic Energy Agency, Ibaraki, JAPAN

We have been developing a position-sensitive tubular scintillation neutron detector as an alternative to $3 \mathrm{He}$ gas based detector. The detector comprised a number of individual neutron-sensitive elements, where a pair of wound wavelength-shifting fiber coils sandwiched with the rolled $\mathrm{ZnS} / 6 \mathrm{LiF}$ scintillator screens in each element. Position-dependent neutron sensitivity within an element of the scintillator / wavelength-shifting fiber coil (SFC) was evaluated by using a collimated pulsed neutron beam at the J-PARC/MLF. The collimated beam that has a size of $1 \times 1 \mathrm{~mm} 2$ was scanned over the SFC element. The neutron counts were recorded and plotted at each incident position. The scanning results showed less neutron sensitivity at the juncture of the rolled scintillator, suggesting less light collection efficiency or a lack of scintillator material due to imperfect manufacturing process.

N28-11: Analysis and Optimization of Spatial Resolution for a Neutron-Sensitive MicroChannel Plate Detector

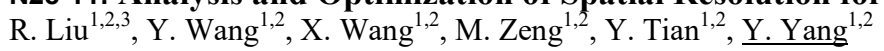

${ }^{I}$ Tsinghua University, Department of Engineering Physics, Beijing, P.R.China

${ }^{2}$ Ministry of Education, Key Laboratory of Particle \& Radiation Imaging (Tsinghua University), Beijing, P.R.China

${ }^{3} X i$ 'an Research Institute of Hi-Tech, Xi'an, P.R.China

Comparing with traditional X-ray radiography and Gamma-ray radiography, neutron radiography has great advantages and application prospects in special scientific experiments and engineering applications, such as the detection of hydrogen, carbon and their compounds. The neutron-sensitive microchannel plate (nMCP) detectors with delay line readout anodes can achieve a high spatial resolution, which is a crucial indicator for neutron radiography. In this paper, three main factors related to the spatial resolution are analyzed and optimized for the nMCP detector: geometric construction of the plate, physics process of neutron absorbing and working conditions. Considering the geometric construction of the plate, the diameter of pore (about $10 \mu \mathrm{m})$ limits the spatial resolution of the $\mathrm{nMCP}$ detector. The physic process of neutron absorbing in $\mathrm{nMCP}$ degrades the spatial resolution about $40 \mu \mathrm{m}$ because of the transport of conversion electrons. Adjusting the nMCP detector to perform excellent spatial resolution, the working conditions of nMCP detector need to be optimized. The forming process of induced current related to the working conditions is researched to improve the temporal resolution, and then improve the spatial resolution. The result can guide the choice of working conditions. In conclusion, the research is beneficial to improve the spatial resolution through three factors.

\section{N28-12: Position Sensitivity Within a Bar of Stilbene Coupled to Silicon Photomultipliers} $\underline{\text { M. L. Ruch }}^{1}$, P. Marleau ${ }^{2}$, S. A. Pozzi ${ }^{1}$ 
${ }^{I}$ Nuclear Engineering and Radiological Sciences, University of Michigan, Ann Arbor, MI, USA

${ }^{2}$ Radiation and Nuclear Detection Systems Division, Sandia National Laboratories, Livermore, CA, USA

A 6-mm by 6-mm by 50-mm bar of stilbene was coupled on both ends to silicon photomultipliers (SiPMs) to assess the detector's position sensitivity to interactions throughout the bar. A Na-22 gamma ray source was collimated with a pair of lead bricks to produce a source beam that was used to irradiate five positions along the length of the bar. A logarithmic relationship between the ratio of the pulse heights obtained from the two SiPMs and the position of the collimated source was established. The standard deviation of the distribution of ratios from each measurement was propagated through the functional form to determine position resolution. The position resolution along the length of the bar was determined to have an average value of $4.9 \mathrm{~mm}$.

N28-13: Optimization of a Fast Neutron Scintillator for Real-Time Pulse Shape Discrimination in the Transient Reactor Test Facility (TREAT) Hodoscope

J. T. Johnson, D. L. Chichester, S. M. Watson, S. J. Thompson

Idaho National Lab, Idaho Falls, Idaho, USA

Abstract -We present a multi-channel, fast neutron/photon detection system that utilizes $\mathrm{ZnS}(\mathrm{Ag})$ scintillator detectors. The system employs Field Programmable Gate Arrays (FPGAs) to do real-time all-digital neutron/photon differentiation, producing particle dependent pulse height and temporal distributions while allowing count rates in excess of 1,000,000 Hertz per channel. The system size is scalable in blocks of 16 channels.

Manuscript submitted April 30, 2016. This work was supported by the U.S. Department of Energy Nuclear Science User Facilities.

N28-14: Advanced Boron Coated Straw Manufacturing Techniques for Replacement 3He Detectors J. L. Lacy, C. S. Martin, S. Davenport, M. Regmi, G. J. Vazquez-Flores, M. Lacy, N. S. King, E. X. Zhang, A. Athanasiades Proportional Technologies, Inc., Houston, TX, USA

Proportional Technologies, Inc. (PTI) has developed advanced manufacturing techniques for the production of boron coated straw (BCS) in a wide variety of neutron detection applications. Similar to the industry standard 3He tubes they are designed to replace, BCS detectors maintain a high level of stability, reliability, and are highly scalable to meet the requirements of large area detection or portable handheld detectors. The core component of each detector is an enriched 10B4C lined copper straw formed in an automated laser welding station. This station continuously converts spools of coated copper foil into straws (diameter determined by interchangeable parts) and cuts them to a precise length at a rate of $3.0 \mathrm{~meters} / \mathrm{min}$. High sensitivity applications require a more complex straw geometry with a corrugate wall that increases boron content and neutron sensitivity while simultaneously improving the packing efficiency. Batches of round straws are reformed into this corrugated shape (Star3 design) using a semi- automated die forming station. Straws are guided down a mandrel and through the Star3 die by computer controlled grippers/translators at a rate of 1.1 meters/min. Once the round or corrugated straws are assembled into the final detector housing, a 20 micron wire is automatically threaded through each straw as the collection anode of the proportional neutron detector. This previously time consuming manual task has been replaced by a programmable wiring station that can perform the same precision task with a 15- fold improvement in speed. The straw welding, straw forming, and straw wiring stations have the flexibility to switch between different detector geometries with ease. Combining these automation advances into an in-line manufacturing flow increases the overall production rate by over an order of magnitude. Integration of automated control algorithms greatly reduces the labor and production cost of the critical manufacturing steps of BCS detectors.

This work was supported by DARPA SIGMA contract no. HR0011-14-C-0096, and by DTRA RIF contract no. HDTRA1-14-C-0047. The views, opinions, and/or findings expressed are those of the authors and should not be interpreted as representing the official views or policies of the Department of Defense or the U.S. Government.

N28-15: High Yield Low Cost Boron-10 Coating Process Development for Production of Neutron Detectors J. L. Lacy, M. Regmi, G. J. Vazquez-Flores, S. Davenport, A. Athanasiades, C. S. Martin

Proportional Technologies, Inc., Houston, TX, USA

The boron-coated straw (BCS) neutron detection technology pioneered by Proportional Technologies, Inc. (PTI) has been shown as one of the leading technologies for $3 \mathrm{He}$ replacement in many applications, such as homeland security, safeguards, and large area neutron science detectors. PTI has successfully developed and demonstrated a 10B coating technology and has been using it for production of pure and resilient coating with precise thickness over the last few years. Moreover, to address the increasing demand, PTI has been working to increase the production rate by several folds and to significantly reduce the coating cost per square meter of $\mathrm{Cu}$ foil without compromising the coating quality. This innovative, patented reel-to-reel 10B4C coating technology uses high power DC magnetron sputtering of enriched 10B4C tiles inside a clean Argon atmosphere, and can 
continuously run longer than a week without requiring to open in air. Produced coating can easily endure extreme thermal and mechanical stresses allowing the formation of complex structures such as corrugated, star or pie shaped straws. Recently, the overall efficiency of the 10B4C tiles has been increased from $15 \%$ to $39 \%$, with further prospects of reaching up to $60 \%$, an overall four-fold reduction in the materials cost. A new standalone 10B4C coating system with three 10B4C magnetron guns has been designed and built with better design of components for reduced maintenance time and labor. With this system online, PTI's production capacity increases by four times, to 14.8 square-meters per day for $1 \mu \mathrm{m}$ thick coating and reduces the overall cost per square meter of coating by up to five times.

This work was supported by DARPA SIGMA contract no. HR0011-14-C-0096, and by DTRA RIF contract no. HDTRA1-14-C-0047. The views, opinions, and/or findings expressed are those of the authors and should not be interpreted as representing the official views or policies of the Department of Defense or the U.S. Government.

\section{N28-16: Pie-Shaped Boron-Coated Straws with Enhanced Neutron Sensitivity}

J. L. Lacy, N. S. King, C. S. Martin, R. Nguyen, G. J. Vazquez-Flores, S. Davenport, E. X. Zhang, A. Athanasiades

Proportional Technologies, Inc., Houston, TX, USA

The boron-coated straw (BCS) neutron detection technology has been proposed as an attractive 3He replacement solution in a series of applications in homeland security, neutron science, and safeguards. In order to improve the neutron detection efficiency of the BCS, while maintaining manufacturability, Proportional Technologies, Inc. (PTI) is introducing a modified BCS with significant performance benefits. The proposed pie-like shape consists or any desired number of arc segments, and an equal number of septa walls, which are radially oriented, and extending a desired distance towards the center of the geometry. Each septum is essentially a segment of the wall that has been collapsed onto itself, resulting in a rigid surface, coated on both sides. The area coated on the septa is added to the area coated on the arc segments, thus offering a significant benefit in sensitivity. Compared to a round straw of the same diameter, the coated wall area of the proposed shape with 12 septa is 3 times larger, assuming the septa are equal in length to each of the arc segments. We present laboratory measurements with two prototype detectors that demonstrate stable operation, a neutron sensitivity that is nearly 3 times higher than that measured in a simple BCS with no septa walls, and adequate energy resolution to effectively discriminate against gamma rays.

This work was supported by the Defense Threat Reduction Agency (DTRA), under Contract No. HDTRA1-14-C-0047, and Alion subcontract no. SUB1213349. The views, opinions, and/or findings expressed are those of the authors and should not be interpreted as representing the official views or policies of the Department of Defense or the U.S. Government.

\section{N28-17: Neutron Coincidence Counters Based on Boron-Coated Straws}

J. L. Lacy, A. Athanasiades, C. S. Martin, N. S. King, G. J. Vazquez-Flores, M. Regmi

Proportional Technologies, Inc., Houston, TX, USA

Neutron coincidence counters, critical to ensure the nonproliferation of nuclear materials, are currently serviced by detectors based on $3 \mathrm{He}$ gas. A worldwide $3 \mathrm{He}$ shortage plus the need for significant improvements in performance, however, requires the development of a new class of neutron detectors. Previous studies have shown that boron-coated straw (BCS) detectors can successfully replace $3 \mathrm{He}$ tubes in small coincidence counters, like the commercially available High Level Neutron Coincidence Counter (HLNCC-II) without sacrificing performance or form factor. Recently, a full-scale, fully operational straw-based HLNCC prototype was tested at an international safeguards workshop, and was shown to perform better than its $3 \mathrm{He}-\mathrm{based}$ counterpart. Unlike most other replacement technologies, BCS detectors share many strong features of $3 \mathrm{He}$ tubes, including high reliability and robust operation under environmental extremes. Moreover, they are free of ageing components, such as photomultiplier tubes and light transmitting fibers or guides. BCS detectors have already been employed with success in many different configurations for neutron detection in defense and homeland security applications. We now introduce a new boroncoated straw detector element design that increases the neutron-sensitive 10B coating area by a factor of 3 . This new design brings significant benefits in the safeguards field and pushes straw performance to near equivalence with even $10 \mathrm{~atm} 3 \mathrm{He}$ safeguards systems as validated by modeling studies. The benefits of the new design will be demonstrated with a neutron coincidence collar development for fresh fuel assemblies having an Figure-of-Merit performance nearly 3 times higher than its $3 \mathrm{He}$-based commercial equivalent. Also this device is expected to have very high gamma ray tolerance extending its applicability well beyond what is possible with $3 \mathrm{He}$.

This work was supported by the Defense Threat Reduction Agency (DTRA), under Contract No. HDTRA1-14-C-0047, and Alion subcontract no. SUB1213349. The views, opinions, and/or findings expressed are those of the authors and should not be interpreted as representing the official views or policies of the Department of Defense or the U.S. Government.

\section{N28-18: Shielding Optimization Study for ${ }^{10}$ B-Based Large Area Neutron Detectors with Detailed Geant4 Model}


$\underline{\text { E. Dian }}^{1,2}$, K. Kanaki ${ }^{2}$, R. Hall-Wilton ${ }^{2,3}$, A. Khaplanov ${ }^{2}$, T. Kittelmann ${ }^{2}$

${ }^{I}$ Hungarian Academy of Sciences Centre for Energy Research, Budapest, Hungary

${ }^{2}$ European Spallation Source ESS ERIC, Lund, Sweden

${ }^{3}$ Mid-Sweden University, Sundsvall, Sweden

The European Spallation Source (ESS) aspires to be the world leading neutron source of the upcoming decades. Numerous large scale instruments will be installed. ESS sets the scope on replacing ${ }^{3} \mathrm{He}$ tube detectors where it is reasonably achievable, consequently advanced neutron detectors require a signal-to-noise $(\mathrm{S} / \mathrm{N})$ ratio high enough to be competitive with ${ }^{3} \mathrm{He}$ tubes and satisfy scientific requirements. Advanced local shielding could provide the improved S/N.

The objective of the current study is to create a tool that can be used during the shielding optimization process. The study is performed with Monte-Carlo simulations using a Geant4 version extended with NXSG4, that is capable to handle the crystal structure of specific materials, therefore the effects of neutron absorption, coherent and incoherent scattering could have been simulated. Validation of the extended Geant4 code, developed at ESS is also part of the current study by comparing the simulated results with analytical calculations. A detailed and realistic model of the state-of-the-art Multi-Grid detector has been implemented, as it is almost the only prototype with published data on scattering effects. Simulations were performed for appropriate shielding materials e.g. $\mathrm{Gd}_{2} \mathrm{O}_{3}, \mathrm{Cd}, \mathrm{B}$ and $\mathrm{B}_{4} \mathrm{C}$ and glue, estimated as acrylic or polyethylene, with various monoenergetic neutron beams and shielding thicknesses.

A robust tool has been developed that could be effectively used to arise the $\mathrm{S} / \mathrm{N}$ via optimizing the detector shielding for specific setups and requirements for all inelastic instruments.

\author{
N28-19: Assessment of the Photodetection Performance of Different Silicon Photomultiplier Technologies \\ under Irradiation with Cold Neutrons \\ $\underline{\text { D. Durini }}^{1}$, C. Degenhardt ${ }^{1}$, A. Feoktystov ${ }^{2}$, A. Palomino-Razo ${ }^{1}$, H. Frielinghaus ${ }^{2}$, H. Rongen ${ }^{1}$, M. Schlösser ${ }^{1}$, S. van $_{\text {Waasen }}{ }^{1}$ \\ ${ }^{I}$ Central Institute of Engineering, Electronics and Analytics ZEA-2 - Electronic Systems, Forschungszentrum Jülich GmbH, \\ Jülich, Germany \\ ${ }^{2} J u ̈ l i c h$ Centre for Neutron Science (JCNS) at Heinz Maier-Leibnitz Zentrum (MLZ), Forschungszentrum Jülich GmbH, \\ Garching, Germany
}

Cold neutrons are used for investigation of soft and condensed matter. They unchain nuclear reactions. The neutron magnetic moment simultaneously allows for investigation of the magnetic properties of matter. These properties are used e.g. in Small Angle Neutron Scattering (SANS) experiments. Traditionally, ${ }^{3} \mathrm{He}$ based detectors have been widely used for neutron detection. Current low world-wide availability of ${ }^{3} \mathrm{He}$ boosted research efforts looking for alternatives, e.g. using scintillation based detectors. Most of these use photomultiplier tubes to detect visible photons generated within scintillation materials. We propose using silicon photomultiplier technology instead, providing the possibility of using magnetic fields much higher than 5 Gauss on the detector level, much lower bias voltages, integrated logic that could increase neutron count rates and improve the neutrongamma discrimination, and lower costs. Nevertheless, it is a known fact that neutrons interacting with crystalline silicon induce nuclear transmutations that translate into impurity doping, and introduce defects that cause an increase of dark signals simultaneously diminishing the photon detection efficiency. We irradiated two analog and one digital SiPM arrays from three different manufacturers with $5 \AA$ wavelength cold neutrons at the KWS- 1 instrument of the Heinz Maier-Leibnitz Zentrum in Garching, Germany, up to a dose of $6 \mathrm{E} 12 \mathrm{n} / \mathrm{cm}^{2}$ and analyzed the dark signal changes in them. We came to a conclusion that all three technologies could be used in SANS experiments. In this occasion, we present the results of further opto-electrical characterization performed before and after irradiation of the three SiPM arrays including: gain and breakdown voltage changes, dark signal behavior at different temperatures, changes in the photodetection efficiency for visible photons, and the ability to still detect small amounts of photons after being irradiated with different neutron doses.

\title{
N28-20: A First Look at the Neutron Sensitive Scintillator LiNaI in an Anger Camera Detector
} $\underline{\text { R. A. Riedel }}^{1}$, M. J. More ${ }^{2}$, V. V. Nagarkar ${ }^{2}$, M. S. J. Marshall ${ }^{2}$

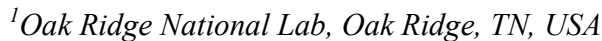

${ }^{2}$ Radiation Monitoring Devices, Inc, Watertown, MA, USA

We present results from the use of a promising new neutron sensitive scintillator, Lithium Sodium Iodide (LNI) fabricated in microcolumnar format using the physical vapor deposition (PVD) method. Microcolumnar structure minimizes lateral light spread in the film, thereby offering high resolution and high contrast neutron imaging. Here we report results from a 2" square LNI film coupled to a Anger Camera. Resolution is estimated at 650um FWHM. The detection efficiency of the approximately 375 um thick film was measured to be about $43 \%$ for $4.2 \mathrm{~A}$ neutrons. The potential for higher resolution due to the narrow light cone of the scintillator will also be discussed.

N28-21: Initial Results of a Microstrip Gas Detector for 2d Neutron Reflectometer Experiments D. M. Duxbury ${ }^{1}$, R. Dalgliesh ${ }^{2}$, N. J. Rhodes ${ }^{2}$, E. M. Schooneveld ${ }^{2}$, E. J. Spill ${ }^{1}$ 
${ }^{I}$ Technology Dept, Science and Technology Facilities Council, RAL, Oxfordshire, UK

${ }^{2}$ ISIS Dept, Science and Technology Facilities Council, RAL, Oxfordshire, UK

The development and testing of a $2 \mathrm{~d}$ neutron detector for the OffSpec reflectometer on the second target station of the ISIS spallation neutron source is reported. Based on a microstrip gas chamber (MSGC) the detector utilises a wire plane operating at zero gain to provide the second coordinate, which relaxes the tolerances on the wireplane necessary for stable operation. The detector operates within a pressurised atmosphere of $3 \mathrm{He}$, variable from 1 to 10 bars, with 4 bars of CF4 to keep the proton range from the neutron-helium reaction in the sub $\mathrm{mm}$ region. The detector has an active area of $80 \mathrm{~mm}$ by $80 \mathrm{~mm}$. The anodes of the MSGC are on a $0.5 \mathrm{~mm}$ pitch, and the wireplane is formed with wires which are also on a $0.5 \mathrm{~mm}$ pitch. These features should allow sub $\mathrm{mm}$ resolution to be readily achievable. Initial measurements have shown that $70 \%$ of the anode signal is induced on the wireplane, enabling the same charge sensitive preamplifiers to be used for both dimensions. An FPGA based readout solution is currently in development to process the detector signals which will interface directly with the ISIS data acquisition electronics. Previous measurements have shown these detectors capable of capturing data in the MHz range making such a device an attractive solution to replace the rate limited detector that is currently used on the OffSpec instrument.

\section{N28-22: A Sub-Millimeter Resolution Neutron Detector with Scintillator and Wavelength-Shifting Fibers Using a Center of Mass Reconstruction. \\ D. Roulier, B. Guerard}

Institut Laue-Langevin, Grenoble, France

Some instruments at the ILL need position-sensitive neutron detectors with sub-millimeter resolution, i.e. for neutron scattering or ultra-cold neutron spectroscopy. A prototype of a 1-D position sensitive neutron detector based on the combination scintillator/wavelength-shifting fibers is in development at the ILL for generic studies. Neutron conversion takes place in a thin $\mathrm{ZnS}: \operatorname{Ag}(6 \mathrm{Li})$ scintillator, and the scintillation light is collected by $1 \mathrm{~mm}$ diameter fibers and guided towards a multi-anode photomultiplier. We count the photoelectrons in each fiber during every event, and a center of mass reconstruction gives the neutron position with a FWHM resolution lower than the diameter of the fibers. The prototype tested at the ILL with a collimated neutron beam and has an average resolution of $0.7 \mathrm{~mm}$, which will be improved by increasing light collection and taking into account the position non-linear effects.

N28-23: Neutron Commissioning of the SPRINTER Detector D. M. Duxbury $^{1}$, C. J. Kinane ${ }^{2}$, N. J. Rhodes ${ }^{2}$, E. M. Schooneveld ${ }^{2}$, M. W. Skoda ${ }^{2}$, E. J. Spill ${ }^{1}$, R. J. Welbourn ${ }^{2}$

${ }^{I}$ Technology Dept, Science and Technology Facilities Council, RAL, Oxfordshire, UK

${ }^{2}$ ISIS Dept, Science and Technology Facilities Council, RAL, Oxfordshire, UK

The aim of the SPRINTER detector development is to produce a high counting rate detector capable of replacing the existing $3 \mathrm{He}$ tube currently used on the INTER reflectometer of the ISIS spallation neutron source. The detector utilises microstrip gas chamber (MSGC) technology as this affords a much greater rate capability compared to the single wire gas tube before saturation effects occur. The initial experimental testing of the detector on both the CRISP and INTER reflectometers at the ISIS spallation neutron source proved this indeed to be the case, but also highlighted a drop in neutron efficiency at neutron wavelengths greater than $2 \AA$. The cause of this drop has been isolated to the materials and design of the field shaping electrode. Further measurements made on CRISP along with some Monte Carlo simulation have shown that re-designing the field shaper of the detector should 'recover' the lost neutron events. The new field shaper has now been manufactured and once it's assembled into the detector the final commissioning of the detectors will be made on INTER.

\section{N28-24: Selective Doping of Lithium-Glass for Micron Resolution Neutron Radiography} M. E. Moore, X. Zhang, J. P. Hayward

Department of Nuclear Engineering, University of Tennessee, Knoxville, TN, United States

Higher-impact research at neutron science facilities worldwide is limited by the current spatial resolution of neutron detection devices. In response, the neutron imaging community has emphasized the need to develop detector technology capable of reaching $1 \mu \mathrm{m}$ spatial resolution. Currently, the spatial resolution of all cold neutron imaging methods in use and under development is fundamentally limited by the variance introduced by the charged particles emitted from neutron absorption. Thus, we are developing and experimentally evaluating designs for scintillating, lithium-glass microfiber arrays fabricated via optoelectronic methods, which are capable of particle tracking. In order to avoid light loss while preventing damage to our photosensor, we will directly couple a bent microfiber array to the photosensor. Because cold neutron scintillation interactions, along the length of the bent microfiber array, will create position confusion, selective doping will be accomplished through controlled diffusion of cerium into only the desired detection volume of the lithium-glass array. In this work, experimental results for thermal diffusion of cerium acetylacetonate $\mathrm{Ce}(\mathrm{AcAc}) 3$ into undoped lithium-glass are presented. 
This material is based upon work supported by the U.S. Department of Energy, Office of Science, Office of Basic Energy Sciences, under Early Career Award no. DESC0010314.

N28-26: Performance of a HLNCC Boron Coated Straw Detector for International Safeguards Applications A. T. Simone, S. Croft, J. P. Hayward

University of Tennessee Knoxville/ Oak Ridge National Laboratory, Knoxville, TN, USA

$3 \mathrm{He}$ gas has been used in various scientific and security applications for decades, but it is now in short supply. Alternatives to $3 \mathrm{He}$ detectors are now being integrated and tested in neutron coincidence counter designs. Neutron coincidence counters are used in safeguards practice for nuclear materials assay. A boron coated straw-based design similar to the High Level Neutron Coincidence Counter II (HLNCC-II) was built by Proportional Technologies Inc. (PTI) and has been tested by Oak Ridge National Laboratory at the JRC in Ispra. This paper presents the results of the measurements made with this coincidence counter replacement in comparison with other similar counters.

This work was supported by the DOE/NNSA Office of Nonproliferation and Arms Control (NA-24).

N28-27: Improved Fast Neutron Detector Based on Timepix and Plastic Scintillating Converter P. Masek $^{1,2}$, J. Jakubek ${ }^{3}$

${ }^{1}$ IEAP CTU in Prague, Prague, Czech Republic

${ }^{2}$ FEE CTU in Prague, Prague, Czech republic

${ }^{3}$ Advacam s.r.o, Prague, Czech republic

The detection of fast neutrons by recoiled protons from plastic scintillator situated very close to pixel detector Timepix was introduced [1]. The directional sensitivity of the detection was proven when signal generated by scintillating light collected by silicon photomultipliers (SiPM) was used for triggering and synchronization of the system. In the proposed contribution the technique moves further by measuring the analog value of the scintillation light from all segments of an array $4 \times 4$ of silicon photomultipliers. Position and energy sensitivity of the silicon pixel detector Timepix is supplemented by position and energy sensitivity of the plastic scintillator. Each of 16 SiPM segments is connected to independent channel of spectroscopic ASIC VATA64HDR16. The ASIC provides trigger signal for the system including pixel detector readout interface FITPix. Variable threshold allows changing the sensitive level and omitting SiPM dark noise or low-energetic particles. Sampled and hold analog value can be readout channel-by-channel through analog multiplex and converted by ADC for further digital processing. The whole activity is controlled by FPGA. The results of measurements with laboratory radioactive source (AmBe) and a Van de Graaff accelerator (D-T reaction) will be presented.

\section{N28-32: Digtal Pulse-Shape Analyzer Based on Multiple Constant-Fraction Time Intervals} V. T. Jordanov

Yantel, LLC, Los Alamos, NM, USA

A novel configuration for pulse-shape analysis and pulse-shape discrimination has been developed. The current pulses from a pulse-shape discriminating detector are applied to a fast charge sensitive preamlifier with a resistive feedback. The output of the preamplifier is sampled at $125 \mathrm{MHz}$ by a 16 -bit analog-to-digital converter. The digital data are processed in real time by first detecting the amplitude of the detector pulses. The measured amplitude of the pulses is used to create amplitude constant-fraction thresholds. In addition the digital data from the ADC are delayed (shifted) by a fixed number of samples which is larger than the number of samples to reach the peak of the measured pulses. Using the constant-fraction thresholds and amplitude discriminators a multiple time intervals between the leading and falling edges of the pulses are measured. A linear combination of the measured intervals is used to establish a pulse-shape discrimination threshold. The system was tested using a stilbene scintillator.

N28-28: Investigations and Optimizations of the Gamma Sensitivity of Gadolinium-GEM Neutron Detectors

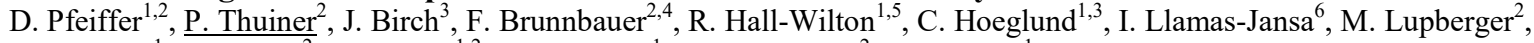
E. Oksanen ${ }^{1}$, E. Oliveri ${ }^{2}$, F. Resnati ${ }^{1,2}$, L. Robinson ${ }^{1}$, L. Ropelewski ${ }^{2}$, S. Schmidt $^{1}$

${ }^{I}$ European Spallation Source ERIC, Lund, Sweden

${ }^{2}$ CERN, Meyrin, Switzerland

${ }^{3}$ Linkoping, Linkkoping, Sweden

${ }^{4}$ Vienna University of Technology, Vienna, Austria

${ }^{5}$ Mid-Sweden University, Sundsvall, Sweden

${ }^{6}$ Institute for Energy Technology IFE, Kjeller, Norway 
Neutron detectors based on solid converters in combination with Micro Pattern Gaseous Detectors (MPGDs) are a promising option for ESS neutron scattering instruments that require excellent spatial resolution combined with time resolution, high-rate capabilities and a good neutron detection efficiency. Gas Electron Multipliers (GEMs) with a $250 \mu \mathrm{m}$ thick gadolinium converters have been used to detect cold neutrons at a wavelength of $2 \mathrm{~A}$ with an efficiency of $11.8 \%$ and a position resolution of better than $250 \mu \mathrm{m}$. Due to a substantial gamma background and the gamma sensitivity of the detector, a signal to background ratio of about 12 to 1 has been obtained. The present work summarizes the optimization efforts to decrease the gamma sensitivity in order to improve the signal to background ratio. To further improve the position resolution of the detector and to limit the scattering of neutrons, the amount of Kapton in the detector has been reduced.

\author{
N28-33: Field-Deployable LiF/ZnS Neutron Detectors for Fission Source Detection and Identification \\ D. V. Lewis \\ $R \& D$, Symetrica Security Ltd., Hampshire, UK
}

In-field fission source detection and identification using $\mathrm{LiF} / \mathrm{ZnS}$ neutron detectors has been investigated. A two-panel system has been constructed consisting of 8 detectors per panel in conjunction with the PTR-32 list-mode acquisition system (AEMI, Hungary). Signatures which may be suitable for in-field source detection and identification including $\mathrm{D} / \mathrm{S}$ v $\mathrm{S}$ have been simulated for a range of material types and isotopic compositions using MCNP-PTA. The D/S ratio can be used for detection of correlated neutron sources. In certain circumstances the $\mathrm{D} / \mathrm{S}$ v S signature permits the material type and quantity to be estimated. The $\mathrm{D} / \mathrm{S}$ ratio is dependent on the unknown detection efficiency and doubles gate fraction. Two methods have been used to estimate these quantities. Firstly, the use of two rows of blades allows an estimate of the amount of moderator surrounding the source by comparing the response of the front and rear rows. Secondly, individual channel readout allows the position of a localized source to be estimated with a resolution of less than $5 \mathrm{~cm}$ within the detection zone. These values can be used to select the appropriate efficiency and gate fraction for the in-field measurement. A set of predetermined normalised D/S curves are then scaled via the efficiency and gate-fraction before being compared with the $\mathrm{D} / \mathrm{S}$ measurement of the source in question. This technique has been tested with Pu metal, PuBe, AmLi and Cf 252 sources. A single row of blades centrally located in the moderator and a double-row configuration have been studied. The efficiencies of the single row and double row systems with their panels at 90 degrees, for a centrally located Cf 252 source are $3.7 \%$ and 3.2\% respectively. The MDAs of Pu metal ( $>90 \%$ $\mathrm{Pu} 239$ ) within 30 minutes are estimated to be $0.15 \mathrm{~g}$ for the single-row and $0.20 \mathrm{~g}$ for the double-row.

N28-30: Hybrid Gas-Filled Neutron Detector Using Microstrip Electrodes $\underline{\text { N. S. Edwards }}^{1}$, B. W. Montag ${ }^{1}$, L. C. Henson ${ }^{2}$, S. L. Bellinger ${ }^{1}$, D. S. McGregor ${ }^{1}$

${ }^{I}$ Mechanical and Nuclear Engineering, Kansas State University, Manhattan, KS, USA

${ }^{2}$ Radiation Detection Technologies, Inc., Manhattan, KS, USA

Microstrip electrodes have been fabricated and tested with a 10B4C-coated aluminum honeycomb sample. The microstrip electrodes were fabricated using standard metal lift-off photolithography techniques with dimensions of 0.875 " x 0.875 " on 100 mm diameter, $525 \mu \mathrm{m}$ thick low-resistivity silicon wafers with a $3 \mu \mathrm{m}$ thick layer of $\mathrm{SiO} 2$ on both surfaces of the silicon substrate. The metals used for the contacts were chromium, copper, and gold at thicknesses of $500 \AA, 3000 \AA$, and $1000 \AA$, respectively. The dimensions of the anode and cathode strips were $9 \mu \mathrm{m}$ and $90 \mu \mathrm{m}$, respectively, with a pitch of $200 \mu \mathrm{m}$ between neighboring like-features (i.e. distance between neighboring anodes). The operating voltage scheme was $-1000 \mathrm{~V}$ applied to the drift cathode and $235 \mathrm{~V}$ applied to the anode strips, with the cathode strips maintained at ground potential, and was determined using a collimated-241Am source. The 0.5 " thick, 1.625" diameter 10B4C-coated aluminum honeycomb sample was positioned between the microstrip electrodes, placed within an aluminum enclosure backfilled with 2 atm of UHP-Ar, and tested using a 252Cf source moderated with lead, steel, and high density polyethylene. The 10B4C-coating was applied to the array of hexagonal-shaped cells with a cell span of 1/16" by Exothermics, Inc. via magnetron sputtering and the measured coating thickness was $4.68 \pm 1.25 \mu \mathrm{m}$. The measured count rate from a $29 \mathrm{ng} 252 \mathrm{Cf}$ source positioned 7 " from the sample was 0.45 counts per second. Finally, the 10B4C-coated aluminum honeycomb sample was also simulated using MCNP6 to compare to experimental results.

\author{
N28-31: Ultra-Low Power, Modular Thermal Neutron Counter Based on Microstructured Semiconductor \\ Neutron Detectors (MSND) \\ $\underline{\text { R. G. Fronk }}^{1}$, S. L. Bellinger ${ }^{2}$, L. C. Henson ${ }^{2}$, D. E. Huddleston ${ }^{3}$, T. R. Ochs ${ }^{1}$, T. J. Sobering ${ }^{3}$ \\ ${ }^{1}$ Kansas State University - S.M.A.R.T. Laboratory, Manhattan, KS, USA \\ ${ }^{2}$ Radiation Detection Technologies, Inc., Manhattan, KS, USA \\ ${ }^{3}$ Kansas State University - Electronics Design Laboratory, Manhattan, KS, USA
}

Modular thermal neutron counting sensors have recently been of interest with the increasing attention for scalable neutron detector instruments. 3He proportional counters have long held the market from small, hand-held portable instruments to largearea stationary panel arrays. However, with the increasing cost of $3 \mathrm{He}$ gas, alternatives are being sought for instruments at any 
scale. A mass-producible, ultra-low power, compact, modular thermal neutron counter has been developed in an attempt to meet many requirements for populating detector instruments with sensors that can compete with $3 \mathrm{He}$. The present-generation Domino thermal neutron detector package is populated with four 1-cm2 active-area microstructured semiconductor neutron detectors (MSNDs) and self-contained signal processing electronics. The detectors can be calibrated with an intrinsic thermal neutron detection efficiency of $30 \%$ over the $4-\mathrm{cm} 2$ active area. The total device dimensions are $2.5 \mathrm{~cm}$ wide by $3.8 \mathrm{~cm}$ tall and $0.47 \mathrm{~cm}$ thick, and the detector package has a gamma-ray rejection ratio of better than 1:106. Individual Dominoes can be tiled together into strings or arrayed laterally to form large-area instruments. In this form, the small sensor can populate instruments up to $1 \mathrm{~m} 2$, with a cost that is comparable to a similar $3 \mathrm{He}$-based array. The recent addition of I2C-based bias and threshold controllers that reduces sensor cost and complexity over the previously-reported Domino versions and allows for user-end calibrations.

Furthermore, the present-generation Domino has realized a 3x reduced power consumption, to nearly $300 \mu \mathrm{W}$ when supplied with 5 volts, and reduced sensitivity to thermal and capacitance-induced noise from the MSNDs, making the Domino an ideal candidate for replacement of $3 \mathrm{He}$ counters in future instruments.

\section{N29: Scintillators - Poster session II}

Tuesday, Nov. 1 14:00-16:00 Etoile

N29-1: Development of a Novel DOI Detector Using Laser Manufacturing

H. Yamauchi, H. Uchida, T. Sakai, K. Hakamata, K. Shimizu

Development Center, HAMAMATSU PHOTONICS K.K., Hamamatsu-City, Japan

A novel single-ended readout depth of interaction (DOI) detector using laser manufacturing is proposed for PET application. A crystal unit of this detector is "U" shaped transform of a dual-ended readout detector using a scintillator pillar segmented by Subsurface laser engraving (SSLE) technique, where the scintillator pillar is virtually separated in two pieces and coupled with optical glue at the top segment, while an ESR film is inserted from the second segment to the bottom. By placing crystal units in two dimensional and coupled with an MPPC array, a block detector can be constructed. We developed a dedicated new MPPC array having $8 \times 8$ channels, each of which had $2.0 \times 2.0 \mathrm{~mm} 2$ active area and be placed with $2.2 \mathrm{~mm}$ pitch in both directions. The crystal unit was made of two $2.1 \times 2.1 \times 20 \mathrm{~mm} 3$ LYSOs, each of which was segmented in six rooms by SSLE. Using these devices, a six-layer DOI detector was fabricated and clearly separated flood histograms were obtained.

N29-2: Lutetium Fine Silicate (LFS): a New Scintillator for Range-Verification Applications in Oncology $\underline{\text { K. E. Roemer }}^{1}$, G. Pausch ${ }^{2}$, D. Bemmerer ${ }^{1}$, K. Fahmy ${ }^{3}$, C. Golnik ${ }^{4}$, F. Hueso-Gonzalez ${ }^{2}$, M. Iltzsche ${ }^{1}$, B. Lutz ${ }^{1}$, J. Petzoldt ${ }^{4}$, D. Weinberger ${ }^{1}$, F. Fiedler ${ }^{1}$

${ }^{1}$ Institute of Radiation Physics, Helmholtz-Zentrum Dresden-Rossendorf, Dresden, Saxony, Germany

${ }^{2}$ Institute of Radiooncology, Helmholtz-Zentrum Dresden-Rossendorf, Dresden, Saxony, Germany

${ }^{3}$ Institute of Resource Ecology, Helmholtz-Zentrum Dresden-Rossendorf, Dresden, Saxony, Germany

${ }^{4}$ OncoRay - National Center for Radiation Research in Oncology, Faculty of Medicine and University Hospital Carl Gustav Carus, Technische Universität Dresden, Dresden, Saxony, Germany

Proton therapy facilities are built all around the world for optimized cancer treatments. In order to fully exploit the advantages of this therapy, a proton range verification is mandatory. The most promising advances use either the positron emission tomography (PT-PET) approach or the prompt gamma emissions caused by nuclear reactions (by means of passive collimation, Compton cameras or prompt gamma timing). Gamma ray detectors in this field face unique requirements: Good properties with respect to energy, time and spatial resolution even when operated with high counting rates in rather high background environments. Lutetium Fine Silicate (LFS) is a novel scintillation material which is especially designed for PET applications. Thus, in this work, the characteristics of LFS are determined for energy ranges which match the requirements of the prompt gamma ray monitors (i.e. up to $8 \mathrm{MeV}$ ). Additionally, the usage of different photodetectors (i.e. PMT or Silicon-based read-out), depending on the particular application, is discussed.

N29-3: Growth and Scintillation Performance of $\mathrm{Cs}_{2}{ }_{6}^{\mathrm{LiYCl}: \mathrm{Ce} \text { Crystals Without Enrichment of } 6}{ }_{\mathrm{Li}}$ G. Ren, Y. Li, Q. Wang, J. Shi, H. Li, X. Chen

$R \& D$ Center, Shanghai Institute of Ceramics, Shanghai, China

The Cs2LiYCl6:Ce (shorten as CLYC) crystal is a kind of promising scintillator because of its capability to identify ?-rays and fast/thermal neutrons. In this paper, a crystal with formula Cs2 LiY 0.95Cl6:5\%Ce was grown by Bridgman method from fused silica crucible loaded with natural $\mathrm{LiCl}, \mathrm{CsCl}, \mathrm{YCl} 3$ and $\mathrm{CeCl} 3$. It was cut and polished into two samples with size of $\mathrm{f} 25 \times 10 \mathrm{~mm}$ and $10 \times 10 \times 5 \mathrm{~mm}$ and then they packaged into aluminum can with a glass window. The crystal structure was analyzed with powder X-Ray Diffractionmeter and was proved to be composed of single phase of CLYC with elpasolite structure, space group of Fm3 m and cell constants of $\mathrm{a}=1.0481 \mathrm{~nm}$ and $\mathrm{V}=1.1515 \mathrm{~nm} 3$. Its luminescence excited with X-ray, ?-rays and thermal neutrons were measured at room temperature. In the X-ray excited luminescence spectrum, a strong emission band between 350 - 
$450 \mathrm{~nm}$ can be fitted into two emission peaks, $374 \mathrm{~nm}$ and $397 \mathrm{~nm}$, which are corresponding to the transitions of electron from $5 \mathrm{~d} 1$ $2 \mathrm{~F} 5 / 2$ and $5 \mathrm{~d} 1-2 \mathrm{~F} 7 / 2$ of $\mathrm{Ce} 3+$ respectively. In addition, a very weak luminescence band around $300 \mathrm{~nm}$ was also observed and is suggested to result from the core-valence luminescence (CVL). Their decay curve excited with ?-rays from 137Cs can be fitted into two components, 58.36ns and 580.56ns, which originate from the luminescence center of Ce3+ and STE in the crystals, but no component as fast as $1 \mathrm{~ns}$ was found. Its energy resolution at $662 \mathrm{keV}$ is about $6.8 \%$, not as good as those reported in previous literature, this phenomenon may be caused by some defects, such as little bubbles or non-enriched 6Li in the crystals.

This work was supported by the Natural Science Foundation of China (NSFC) with project numbers of 51272263 and 51372256.

\title{
N29-4: GAGG:Ce Crystal Array Based Compact Radio-TLC Scanner
}

S. J. Jeon, K. M. Kim, J. G. Kim

Molecular Imaging research center, Korea Institute of Radiological and Medical science, Seoul, Korea

Radio-TLC scanner, which is named after one of radio-detectors based on the technology of thin-layer chromatography, is generally the standard QC(Quality Control) equipment for analyzing the radiochemical purity in production and research of radio-pharmaceuticals, in parts of nuclear medicine. The system showed as graphs of counting gamma radioactivity with position information, emitting by radiopharmaceutical including a specific radioisotope, so it can guarantee quantitative accuracy and reproducibility for chemically stability and change of a targeting radiopharmaceutical. In present, the most commonly used radioTLC scanners are made into a gas-filled proportional counter, and it operate with continuous supply of P10 gas. These gas detector based radiation detectors need continuous maintenance cost and periodic calibration. In this study, we evaluated a compact with cost-effective Radio-TLC(Thin Film Chromatograph) scanner. We adapted a scintillation detector, which is 1.4 $\mathrm{mm}(\mathrm{W}) \times 20 \mathrm{~mm}(\mathrm{D}) \times 3 \mathrm{~mm}(\mathrm{H}) \times 64$ pixels of the GAGG:Ce crystal array coupled with Hamamatsu Si PIN diode. The scintillator array based Radio-TLC was compared with AR-2000, the commercial gas detector based device made by Bioscan. We scanned $1 \mu \mathrm{Ci} /$ ? of Tc- $99 \mathrm{~m}$ and $\mathrm{F}-18$ on each device. The ROI count ratios and the peak position differences are less than $1.2 \%$ and $4 \%$. The GAGG:Ce based radio-TLC scanner has enough position resolution and sensitivity to be used in the measurement of radiochemical purity test with several advantages of size, maintenance and price.

\author{
N29-5: Influence of the Secondary Fluorophore and the Volume in a Plastic Scintillator Aiming at \\ Discriminating Fast Neutrons from Gamma Rays \\ E. Montbarbon $^{1,2}$, M. Hamel $^{1}$, R. B. Pansu ${ }^{2}$, A. Grabowski ${ }^{1}$ \\ ${ }^{I}$ Sensors and Electronic Architectures Laboratory, CEA, LIST, Gif-sur-Yvette, France \\ ${ }^{2}$ Ecole Nationale Supérieure de Cachan, CNRS UMR8531, Cachan, France
}

Pulse Shape Discrimination (PSD) is one of the popular techniques to identify ionizing particles, and is particularly used for discrimination between fast neutrons and gamma rays. Since the 1950's, it has been demonstrated that plastic scintillators could discriminate fast neutrons from gamma rays.

In this context, we address the issue of the influence of the secondary fluorophore in a plastic scintillator aiming at discriminating fast neutrons and gamma rays. We then studied the influence of the volume of a plastic scintillator on its neutron/gamma discrimination abilities. For the need of these studies, plastic scintillators were lab-made.

In the first work, we analyzed 8 different plastic scintillators (dimensions $\varnothing 120 \mathrm{~mm}$ x $12 \mathrm{~mm}$ ) in terms of decay times, slow and fast relative intensities, which represent the proportion of the tail or the fast component according to the whole mean signal, and Figure-Of-Merit (FOM). Our first observations concerned the nature of the secondary fluorophore. Indeed, some fluorophores enable a better neutron/gamma discrimination than others. Besides, a high concentration of the secondary fluorophore increases the FOM. However, these plastic scintillators are subject to self-absorption. An experiment, which consisted of cutting centimeter-large slices of plastic scintillators has given rise to this phenomenon. A last experiment proved that it was effectively self-absorption occurring in these tested plastic scintillators. This effect was quantified and results will be presented at the meeting.

N29-6: Comparison of damage effects in plastic scintillators due to irradiation with ?-quanta, $150 \mathrm{MeV}$ and 24 GeV/c protons $\underline{\text { V. Dormenev }}^{1}$, E. Auffray ${ }^{2}$, K. T. Brinkmann ${ }^{1}$, M. Korjik ${ }^{3}$, M. T. Lucchini ${ }^{2}$, V. Mechinsky ${ }^{3}$, R. W. Novotny ${ }^{1}$, H. G. Zaunick $^{1}$

${ }^{I}$ II. Physical Institute, Justus-Liebig-University, Giessen, Germany

${ }^{2}$ CERN, Geneve, Switzerland

${ }^{3}$ Institute for Nuclear Problems, Minsk, Belarus

Plastic scintillation materials play a crucial role in the construction of large area detectors in high energy physics experiments. Further concepts of the detectors at HEP experiments, particularly at collider facilities, will require an unique combination of the material features and affordable price. Crucially important becomes a minimal level of radiation damage effects under electromagnetic part of the ionizing radiation and high energy hadrons as well as low deterioration of the optical transmittance, 
low level of the scintillation mechanism damage and the radio-luminescence due to radio-nuclides generated by nuclear reactions in the material of the detector. A systematic study of the radiation hardness of inorganic optical and scintillation materials has been performed by us since several years. It resulted in the understanding of the damage effects in particular in self activated, $\mathrm{Ce}^{3+}$ doped and cross-luminescent crystalline materials. Here we report on first results for the industrially produced plastic scintillation material EJ260 (ELJEN). This plastic is a bright, fast, green light emitting scintillator with properties similar to other materials such as BC428 and NE103. Samples of the thickness $1.25 \mathrm{~cm}$ were investigated before and after irradiation with ?quanta (1.2 MeV, Giessen) and protons of $150 \mathrm{MeV}$ (KVI-CART, Groningen) and $24 \mathrm{GeV} / \mathrm{c}$ (CERN, PS), respectively. No damage of the material properties was observed after irradiation with ?-quanta up to absorbed doses of 100 Gy consistent with similar studies. However, first the exposure to $150 \mathrm{MeV}$ protons with a fluence of $5 \times 10^{13} \mathrm{p} / \mathrm{cm}^{2}$ showed a large reduction of the transmittance and a slowing down of the scintillation kinetics. In this study we also focused on the impact of high energetic protons. We obtained evidence that light fragments of the $\left(\mathrm{p},{ }^{12} \mathrm{C}\right)$ reactions play a significant role in the damage of the matrix and the luminescent organic dye centers.

Acknowledgment: We would like to thank the company Eljen Technology (Sweetwater, USA) for providing samples.

N29-7: Time-of-Flight Detection of Al Ions from Laser Produced Plasma

M. Seimetz ${ }^{1}$, P. Bellido ${ }^{1,2}$, A. Peralta Conde ${ }^{2}$, J. I. Apiñaniz ${ }^{2}$, A. V. Carpentier ${ }^{2}$, M. Sanchez Albaneda ${ }^{2}$, F. Valle Brozas $^{2}$, C. Mendez ${ }^{2}$, J. Lozano ${ }^{2}$, J. M. Alvarez ${ }^{2}$, R. Lera ${ }^{3}$, A. Ruiz-de la Cruz ${ }^{3}$, M. Galan ${ }^{3}$, L. Vidal ${ }^{1}$, A. Soriano ${ }^{1}$, S. Sanchez ${ }^{1}$, F. Sanchez ${ }^{1}$, M. J. Rodriguez-Alvarez ${ }^{1}$, J. P. Rigla ${ }^{1}$, L. Moliner ${ }^{1}$, A. Iborra ${ }^{1}$, L. Hernandez ${ }^{1}$, A. J. Gonzalez ${ }^{1}$, P. Conde ${ }^{1}$, A. Aguilar ${ }^{1}$, L. Roso ${ }^{1}$, J. M. Benlloch ${ }^{1}$

${ }^{I}$ Instituto de Instrumentación para Imagen Molecular (I3M), Valencia, Spain

${ }^{2}$ Centro de Láseres Pulsados (CLPU), Salamanca, Spain

${ }^{3}$ Proton Laser Applications SL, Olèrdola, Spain

Background and objectives: The acceleration of ions in laser-induced plasmas covers a wide range of phenomena over several orders of magnitude in laser intensity. Time-of-flight measurements provide accurate reconstruction of the ion energy spectra. Multi-channel plates or Faraday cups have often been applied in related experiments. We report on the observation of aluminium ions with energies around $1 \mathrm{keV}$ by a scintillator-based detector with adjustable dynamic range.

Methods: Data have been taken at a 30 fs, 200 TW pulsed Ti:Sapphire laser focused on aluminium foils with 1.8 and $12.5 \mu \mathrm{m}$ thickness. A time-of-flight detector consisting in a plastic scintillator with fibre-optic coupling to a PMT has been mounted $50 \mathrm{~cm}$ behind the target. The PMT output pulses have been recorded on a fast oscilloscope.

Results: After an initial peak caused by prompt X-rays and relativistic electrons, a second peak has been observed in the time-offlight spectra after several microseconds. It corresponds to $\mathrm{Al}$ ions with energies of the order $1 \mathrm{keV}$. Quantitative energy spectra have been reconstructed.

Discussion: The observations confirm the feasibility of our detection method. At the given ion energies the detection mechanism, based on electrostatic charge transfer, is different to the usual ionisation process in scintillators. Our detector may thus be applied for more detailed studies of plasma dynamics in laser-solid interactions.

Project funded by Centro para el Desarrollo Tecnológico Industrial (CDTI, Spain) within the INNPRONTA program, grant no. IPT-20111027.

N29-8: Scintillation Detectors Constructed with an Optimized 2x2 Silicon Photomultiplier Array F. Liang ${ }^{1}$, H. Brands ${ }^{1}$, L. Hoy ${ }^{1}$, J. Preston ${ }^{2}$, J. Smith ${ }^{1}$

${ }^{I}$ FLIR Systems Inc., Oak Ridge, TN, USA

${ }^{2}$ Consolidated National Security LLC., Oak Ridge, TN, USA

Silicon photomultipliers (SiPMs) are a good alternative to photomultiplier tubes (PMTs) because the gain and quantum efficiency of SiPMs are comparable to PMTs. However, the largest single-chip SiPM is still less than $10 \mathrm{~mm}$. In order to use SiPMs with scintillators that have reasonable sensitivity, it is necessary to use an array of SiPMs. In this work, scintillation detectors are constructed and tested with a custom $2 \times 2$ SiPM array. The layout of the SiPMs and the geometry of the scintillator were determined by performing Geant4 simulations. Cubic NaI, CsI and CLYC with $18 \mathrm{~mm}$ sides have been tested. The output of the scintillation detectors are stabilized over the temperature range between -20 and $50{ }^{\circ} \mathrm{C}$ by matching the gain of the SiPMs in the array. The resolution for these detectors have been measured as a function of temperature. All three detectors have resolution of $6.8 \%$ at $20^{\circ} \mathrm{C}$. Furthermore, neutron identification for the CLYC detector was studied in the same temperature range. Using pulse-shape discrimination, neutrons can be cleanly identified without contribution from gamma photons. As a result, these detectors are suitable for deploying in a spectroscopic personal radiation detector (SPRD) such as the identiFINDER R200.

N29-9: Response of GAGG:Ce, LuAG:PR and LYSO:Ce Coupled to SiPM

B. Seitz, N. Campos Rivera, R. Gray, R. A. Montgomery, F. Thomson

School of Physics and Astronomy, University of Glasgow, Glasgow, U.K. 
Inorganic scintillation crystals coupled to solid-state soft photon counters (SiPM) provide a way to construct compact radiation sensors with good energy and time resolution. We report on recent measurements performed with novel SensL SiPM and Philips dSIPM coupled to GAGG:Ce, LuAG:Pr and LYSO:Ce crystals, studying the energy and coincidence time resolution of the coupled system for gamma rays between $100 \mathrm{keV}$ and $1 \mathrm{MeV}$ in energy for both. We study the repsonse for the fast and normal output of the SensL SiPM as well as exploiting the added features offered by dSiPM. In addition, the noise characteristics and photon detection efficiency of the SiPM were evaluated in an experimental setup by our group.

\author{
N29-10: Temperature Dependence of $\mathrm{CdMoO}_{4}$ Scintillation Properties for the Search of ${ }^{100} \mathrm{Mo}$ and ${ }^{116} \mathrm{Cd}$ \\ Neutrinoless Double Beta Decay

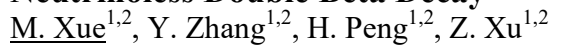 \\ ${ }^{I}$ Dept. of Modern Physics, University of Science and Technology of China, Hefei, China \\ ${ }^{2}$ State Key Laboratory of Particle Detection and Electronics, Hefei, China
}

Abstract-Cryogenic phonon-scintillation detectors already play a major role in the search of neutrinoless double beta decay, while cadmium molybdate $\left(\mathrm{CdMoO}_{4}\right)$ is a very attractive material in this field. A single crystal of $\mathrm{CdMoO}_{4}$ was grown by NingBo University, and the temperature dependence of $\mathrm{CdMoO}_{4}$ scintillation properties have been investigated over a wide temperature range from $22 \mathrm{~K}$ to $300 \mathrm{~K}$. The $\mathrm{CdMoO}_{4}$ crystal excited with laser of $355 \mathrm{~nm}$ exhibits a broad emission bands peaked at $551 \mathrm{~nm}$. The main decay time constant is found to be $1.2 \mu \mathrm{s}$ at room temperature $(\mathrm{T}=300 \mathrm{~K})$. During cooling to $22 \mathrm{~K}$ the scintillation decay time increases up to $170 \mu \mathrm{s}$. The measurements of scintillation properties using a-source $\left({ }^{241} \mathrm{Am}, 5.5 \mathrm{MeV}\right)$ and ?-source $\left({ }^{137} \mathrm{Cs}, 0.662 \mathrm{MeV}\right)$ will be discussed here as well. The MC-study has demonstrated that $\mathrm{CdMoO}_{4}$ crystal is a promising scintillator for use in search of neutrinoless double beta decay based on both ${ }^{100} \mathrm{Mo}$ and ${ }^{116} \mathrm{Cd}$, two target nuclides.

\title{
N29-11: Radiation Damage Studies of New Intrinsically Radiation-Hard Scintillators
}

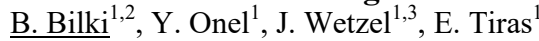

${ }^{I}$ Physics and Astronomy, University of Iowa, Iowa City, IA, USA

${ }^{2}$ Mathematics and Computing, Beykent University, Istanbul, Turkey

${ }^{3}$ Physics, COE College, Cedar Rapids, IA, USA

Following the development of the intrinsically radiation-hard scintillators, we exposed various scintillators tiles to the gammas from a ${ }^{137} \mathrm{Cs}$ source at the University of Iowa Hospitals and Clinics up to 1 and $10 \mathrm{MRad}$. The results are within expectations and exhibit sufficiently high performance for implementations in the future/upgrade hadron/lepton collider detectors. Here we report on the nature of the irradiation tests and present the results of the laboratory measurements performed continuously for more than 60 days following the irradiation.

\section{N29-12: Single Crystalline and Composite Scintillators for Hadron Calorimetry at High Luminosity LHC} M. Lucchini ${ }^{1}$, E. Auffray ${ }^{1}$, A. Fedorov ${ }^{2}$, J. Houžvicka ${ }^{3}$, M. Korjik ${ }^{2}$, D. Kozlov², V. Mechinsky ${ }^{2}$, M. Nikl ${ }^{4}$, S. Ochesanu ${ }^{3}$

${ }^{I}$ CERN, Geneva, Switzerland

${ }^{2}$ RINP, Minsk, Belarus

${ }^{3}$ CRYTUR, Turnov, Czech Republic

${ }^{4}$ Institute of Physics, Prague, Czech Republic

Further physical programs at the LHC will require a significant increase of the accelerator luminosity throughout the High Luminosity phase of LHC. During this period, charged hadrons with fluences higher than $1014 \mathrm{p} / \mathrm{cm} 2$ per year in the largest pseudo-rapidity regions of the detectors will have a non-negligible influence on the radiation damage of materials. Moreover, with the increasing activation of the experimental equipment, it will become more difficult to periodically replace and maintain the detector components. Therefore, the selection of materials for new detectors to be used at the upgrade of experimental setup requires a more reliable assessment of the risks of detector failures due to severe radiation damage. During last few years we performed systematic studies of the damage effects in inorganic scintillation materials. The results obtained confirmed that Y3A15O12:Ce (YAG:Ce) crystal has a very low optical transmission damage after irradiation with $24 \mathrm{GeV}$ protons up to the fluence of $5 \times 1014 \mathrm{p} / \mathrm{cm} 2$. In addition to the single crystalline option we developed and tested a composite scintillation material consisting of YAG:Ce grains compacted by gluing (Sylgard -184) in a translucent body with density up to $65 \%$ of the single crystal, i.e. around $3 \mathrm{~g} / \mathrm{cm} 3$. As all the components of the composite module are selected to be radiation hard, such technology can be considered a suitable option to replace plastic scintillators in the region of the detector where high radiation hardness is required. In this study, we report the results of a comparative tests of the YAG:Ce single crystals and YAG:Ce/quartz composite modules scintillation properties and radiation tolerance.

N29-13: Temperature Response of PWO:F Single Crystal

R. Mao, Y. Wang, Y. Li 
Flouride doped lead tungstate (PWO:F) single crystal was grown by using the modified Bridgman method. Optical and scintillation properties were investigated in the temperature range $80-320 \mathrm{~K}$. Two scintillation emission bands, peaking at $430 \mathrm{~nm}$ $(2.88 \mathrm{eV})$ and $555 \mathrm{~nm}(2.23 \mathrm{eV})$, respectively, were found. By going from $320 \mathrm{~K}$ to $80 \mathrm{~K}$ the overall light yield increases by about a factor of 50 while the intensity of the $430 \mathrm{~nm}$ and $555 \mathrm{~nm}$ bands increase by a factor of 150 and 8 , respectively. The light output for the PWO:F is determined to be $1800 \pm 100$ photons/MeV at room temperature with an energy resolution of $33 \%$ (FWHM). The scintillation time constants were found to be $9 \mathrm{~ns}$ and $452 \mathrm{~ns}$ at room temperature. This work will address also the mechanism on the temperature response for $430 \mathrm{~nm}$ and $555 \mathrm{~nm}$ bands in the PWO:F crystal.

N29-14: High-Performance Large Diameter $\mathrm{SrI}_{2}: \mathrm{Eu}^{2+}$ Crystals

$\underline{\text { R. Hawrami }}{ }^{1}$, C. Ji ${ }^{1}$, E. Ariesanti ${ }^{1}$, J. Glodo ${ }^{1}$, H. Wei ${ }^{1}$, K. S. Shah ${ }^{1}$, N. J. Cherepy ${ }^{2}$, S. A. Payne ${ }^{2}$

${ }^{I}$ Radiation Monitoring Devices, Inc., Watertown, MA, USA

${ }^{2}$ Lawrence Livermore National Laboratory, Livermore, CA, USA

In this presentation, we will discuss crystal growth and scintillation properties of large diameter $\operatorname{SrI}_{2}: \mathrm{Eu}^{2+}$ single transparent crystals. The crystals were grown successfully using the vertical Bridgeman technique. Crystals with different diameters from 1 inch to 2 inches will be discussed. $\mathrm{SrI}_{2}: \mathrm{Eu}^{2+}$, a scintillator discovered a half century ago by Hofstadter, was recently re-discovered as an outstanding material for gamma ray-spectroscopy. Its key properties include good non-proportionality, high light yield (from 85,000 to 120,000 photons/MeV) and excellent energy resolution $(2.8 \%$ at $662 \mathrm{keV}$ ). Due to its good properties our immediate focus has been on scaling up and development of this material for commercial use. Other properties of $\mathrm{SrI}_{2}: \mathrm{Eu}$ include $Z_{\text {eff }}$ of 50, a mono-exponential decay with a time constant of $1 \mu \mathrm{s}$ to $5 \mu \mathrm{s}$ (depending on crystal size and dopant concentration), an emission band at 410-450 nm (2.7-3.0 eV). $\mathrm{SrI}_{2}$ :Eu also shows no intrinsic radioactivity unlike lanthanide compositions.

\author{
N29-15: Mg Co-Doping Effects on Multi Component Garnet of Ce:(Lu,Gd,Y)3(Ga,Al)5O12 Single Crystal \\ Scintillators. \\ $\underline{\text { K. Kamada }}^{1,2}$, H. Yamaguchi ${ }^{3}$, Y. Shoji ${ }^{1,2}$, S. K. Kurosawa ${ }^{1}$, Y. Yokota ${ }^{1}$, Y. Ohashi ${ }^{3}$, M. Nikl ${ }^{4}$, A. Yoshikawa ${ }^{1,2,3}$ \\ ${ }^{I}$ NICHe, Tohoku University, Sendai, Japan \\ ${ }^{2} C \& A$ corp., Sendai, Japan \\ ${ }^{3} I M R$, Tohoku University, Sendai, Japan \\ ${ }^{4}$ Physics AS CR, Praque, Czech republic
}

Mg co-doping effects on scintillation properties of multi component garnet Ce:(Lu,Gd,Y)3(Ga,Al)5O12 (LGGAG) were investigated. Mg 200 ppm co-doped garnet single crystals were prepared by micro pulling down method. Absorption and luminescence spectra were measured together with several other scintillation characteristics, namely the scintillation decay and light yield to reveal the effect of $\mathrm{Mg}$ co-doping. Ce4+ charge transfer absorption was observed below 300nm in Mg,Ce:LGGAG which is in good agreement with previous reports. The scintillation decay times were accelerated by Mg co-doping. Light yield was increased in Gd free samples. Details on crystal structure and scintillation properties will be shown in my presentation.

N29-16: Growth and Scintillation Properties of Eu Doped LiSrI3/LiI Eutectics

$\underline{\text { K. Kamada }}^{1,2}$, H. Chiba ${ }^{1}$, Y. Shoji ${ }^{1,2}$, S. Kurosawa ${ }^{1}$, Y. Yokota ${ }^{1}$, Y. Ohashi $^{1}$, A. Yoshikawa ${ }^{1,2}$

${ }^{I}$ Tohoku University, Sendai, Japan

${ }^{2}$ C\&A corp., Sendai, Japan

In this study, as a candidate for novel neutron detectors, Eu activated LiSrI3/LiI eutectic scintillators were developed. Here we propose submicron-diameter phase-separated scintillator fibers (PSSFs) with both the properties of an optical fiber and a neutronto-light conversion. The PSSFs were fabricated using the DSE system. In PSSFs, the light emitted from the scintillator fibers is confined and transported along the fiber direction by a total reflection mode, so that high-resolution neutron imaging can be achieved. Eu doped LiSrI3/LiI eutectics were grown by the BZ method in a quartz ampoule with $8 \mathrm{~mm}$ inner diameter. Mixed powder were induced into the ampoule under Ar atmosphere in a glove box. Growth rate was 0.2-1.5 $\mathrm{mm} / \mathrm{min}$. The eutectic showed well aligned eutectic structure and optically transparent. Grown Eu doped LiSrI3/LiI eutectic shows $390 \mathrm{~nm}$ emission ascribed to Eu2 + 4f-5d transition under X-ray excitation. The light yield was around 1.2 times higher than that of Ce:Lu2SiO4 (LYSO) standard and 37000 photon/MeV. Details of the results about eutectic growth, phase identification, optical, luminescence and scintillation properties will be discussed in the presentation.

N29-17: In Situ Stoichiometry Monitoring During Processing and Crystal Growth of Halide Scintillators (Case Study - Strontium Iodide) 
A. Datta, S. Lam, S. Swider, S. Motakef

CapeSym, Inc., Natick, MA, US

In recent years, there has been an elevated research effort towards the development of halide-based scintillator and semiconductor gamma detectors. Due to the high vapor pressure and volatile nature of most halide melts, it is extremely difficult to maintain the desired stoichiometry during processing and crystal growth of these materials. Also, hygroscopic and reactive nature of most halide materials poses an extreme unreliability in the crystal growth processes and the properties of the resulting crystals. These issues are overcome after several years of developmental research which significantly impedes the overall impact of the material. In this paper, we demonstrate a technique to monitor the stoichiometry of the melt atmosphere during processing and crystal growth of halide materials. This method is demonstrated for a high resolution scintillator (Europium-doped Strontium Iodide) and a semiconductor (Thallium Bromide). The crystal growth of SrI2:Eu has been impaired for a long time due to cracking problems, which makes it highly unreliable and nonreproducible. This significantly increases the cost of the material which in turn impedes wide scale deployment and limits its advantages over other scintillators such as NaI and CsI. Using the feedback information from in situ monitoring technique, the stoichiometry of the melt was corrected and multiple crack-free SrI2:Eu crystals of diameters 1.5 inches were repeatedly grown using Bridgman configuration with no visible inclusions, bubbles or defects whatsoever.

N29-18: CsI:TI Scintillation Pulse Shapes Measured with a SiPM Photodetector in a Liquid Nitrogen Cryostat L. Swiderski ${ }^{1}$, M. Moszynski ${ }^{1}$, W. Czarnacki ${ }^{1}$, Z. Mianowska ${ }^{1}$, P. Sibczynski ${ }^{1}$, T. Sworobowicz ${ }^{1}$, T. Szczesniak ${ }^{1}$, A. Syntfeld-

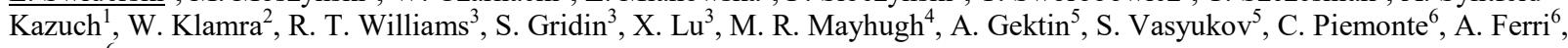
A. Gola ${ }^{6}$

${ }^{I}$ National Centre for Nuclear Research (NCBJ), Otwock, Poland

${ }^{2}$ Royal Institute of Technology, Stockholm, Sweden

${ }^{3}$ Department of Physics, Wake Forest University, Winston Salem, USA

${ }^{4}$ Faceted Development, Shaker Heights, USA

${ }^{5}$ Institute for Scintillation Materials (ISMA), Kharkov, Ukraine

${ }^{6}$ Fondazione Bruno Kessler (FBK), Trento, Italy

A custom designed cryostat was constructed in order to measure the response of a CsI:Tl scintillator in temperature close to the liquid nitrogen (LN2) boiling point. The scintillator was coupled to an RGB-HD SiPM from FBK with $4 \times 4 \mathrm{~mm}^{2}$ area and $25 \times 25$ $\mu \mathrm{m}^{2}$ cell pitch used for scintillation readout. The crystal size was $5 \times 5 \times 7 \mathrm{~mm}^{3}$. All surfaces except the one facing the SiPM were covered with Teflon tape to enhance light collection by the photodetector. The performance of the experimental setup was verified at room temperature using analog electronics for signal processing. The energy resolution of the full energy absorption peak at $662 \mathrm{keV}$ is $(9.0 \pm 0.5) \%$. High gain of the SiPM device allows for recording events with energies as low as $14.4 \mathrm{keV}$ (gamma rays from a ${ }^{57} \mathrm{Co}$ source) and $22.1 \mathrm{keV}$ (KX rays from a ${ }^{109} \mathrm{Cd}$ source). Thanks to good quality spectroscopic response of the CsI:Tl crystal coupled to the SiPM at room temperature and low noise performance of the photodetector at LN2 temperatures we expect that we will be able to record the scintillation pulse shapes with an oscilloscope gated for specifically selected energies. Recorded pulses will be fitted to extract the information about the intensity of scintillation decay components as a function of energy deposited in the scintillator. The measurements will be carried out at temperatures as low as $160 \mathrm{~K}$ or lower (depends on the heat dissipation inside the cryostat). We expect that the outcome of this experiment will be a valuable source of information that can be used in the development of models predicting scintillators response to gamma-rays.

WFU acknowledges support from the US Department of Homeland Security, Domestic Nuclear Detection Office, DNDO NSF ARI Grant $2014-$ DN-077-ARI-077. This support does not constitute express or implied endorsement on the part of the Government.

\section{N29-19: Crystal Identification Technique and Advanced Imaging Algorithm for 4p Gamma-ray Imager with} Stacked Scintillator Rods

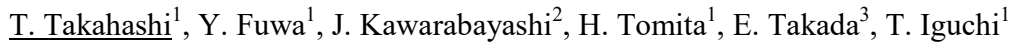

${ }^{I}$ Dept. of Quantum Engineering, Nagoya University, Nagoya, Aichi, Japan

${ }^{2}$ Dept. of Nuclear Safty Engineering, Tokyo City University, Tokyo, Japan

${ }^{3}$ Dept. of Electrical and Control System Engineering, National Institute of Technology, Toyama College, Toyama, Japan

$4 p$ Field of View (FOV) gamma-ray imaging is promising method for real time measurement of distribution of radioactive materials which released from nuclear reactor in severe accident and also detection of unanticipated radiation materials such as nuclear nonproliferation materials or dirty bombs. We have been developing a prototype of the $4 \mathrm{p}$ FOV gamma-ray imager based on stacked scintillator rods. To enhance the imaging performance of the imager, shaper scintillator rods should be used. Although increasing the number of scintillator rods could remain the sensitive volume of the imager, it would make the readout system complicated. For simple readout system, we have proposed Small Area Center of Gravity (SACG) method to readout each scintillator rod by photo detector with less channels. We have demonstrated the rod identification with SACG method for 
coincident events with a prototype detector composed of $16 \times 16 \mathrm{Ce}$ :GAGG scintillator rods and two multi-anode photomultipliers with $8 \times 8$ channels. The direction of $137 \mathrm{Cs}$ was correctly estimated by back projection method. In addition, a novel weighted imaging algorithm for real time measurement in the $4 p$ gamma-ray imager. We demonstrated that signal to noise ratio in the image was improved by the proposed algorithm.

N29-20: Characterization of a Fast Timing and Energy Spectroscopy System for Real-Time Range Verification in Particle Therapy

A. Rinscheid $^{1}$, J. Berthold ${ }^{2}$, W. Enghardt ${ }^{2,3,4,5,6}$, C. Golnik ${ }^{3}$, F. Fiedler ${ }^{7}$, F. Hueso-Gonzalez ${ }^{4}$, T. Kormoll ${ }^{3}$, J. Petzoldt ${ }^{3}$, K. Roemer ${ }^{7}$, K. Ruhnau ${ }^{8}$, J. Stein ${ }^{8}$, T. Werner ${ }^{3}$, A. Wolf ${ }^{8}$, D. Reichert ${ }^{1}$, G. Pausch ${ }^{3}$

${ }^{I}$ Institute of Physics, Martin Luther University Halle-Wittenberg, Halle (Saale), Germany

${ }^{2}$ Technische Universitaet Dresden, Dresden, Germany

${ }^{3}$ Oncoray - National Center for Radiation Research in Oncology, Faculty of Medicine and University Hospital Carl Gustav Carus, Dresden, Germany

${ }^{4}$ Institute of Radiooncology, Helmholtz-Zentrum Dresden-Rossendorf, Dresden, Germany

${ }^{5}$ German Cancer Consortium (DKTK), Dresden, Germany

${ }^{6}$ German Cancer Research Center (DKFZ), Heidelberg, Germany

${ }^{7}$ Institute of Radiation Physics, Helmholtz-Zentrum Dresden-Rossendorf, Dresden, Germany

${ }^{8}$ Target Systemelektronik, Wuppertal, Germany

A compact high-throughput gamma-ray timing and energy spectroscopy system has been characterized with respect to time and energy resolution at detector loads up to 2.4 Mcps by using 0.1-12.5 MeV bremsstrahlung photons as well as a common radioactive source. The detection system, developed for range assessment in particle therapy based on the Prompt Gamma-Ray Timing (PGT) technique, consists of a commercial Ø2" $\times 1$ " or $\varnothing 2 " \times 2 " \mathrm{CeBr}_{3}$ scintillation detector with photomultiplier readout (Scionix), coupled to a digital plug-on spectrometer (U100) from Target Systemelektronik. The excellent time structure of the bremsstrahlung beam at ELBE (HZDR) allowed measuring the system time resolution in a broad energy range between 0.1-12.5 $\mathrm{MeV}$. Furthermore, the beam current and thus the detector load could be varied in a wide range. The energy resolution was determined in parallel with a ${ }^{60} \mathrm{Co}$ source attached to the detector. Source and bremsstrahlung photons were separated by means of suitable regions of interest in the timing spectra, i.e. detector time relative to the accelerator radio frequency. For the detection system, a time resolution of $<260 \mathrm{ps}$ (FWHM) for deposited energies above $3 \mathrm{MeV}$ could be demonstrated with the smaller crystal. Moreover, an energy resolution of $<3.7 \%$ at $1.173 \mathrm{MeV}$ was found for both crystals. Timing and energy resolution was validated at detector loads up to $1.4 \mathrm{Mcps}$, corresponding to a system throughput of $\sim 600 \mathrm{kcps}$. This fits well with the design goals of the detection system. Corresponding units shall be used for measuring PGT spectra during patients treatments with proton beams, in order to verify the proton beam range for single beam spots in Pencil Beam Scanning (PBS) mode. Range verification is considered a key for reducing safety margins, and for improving precision and health outcome of particle therapy.

\section{N29-21: CeBr3 Scintillator for Imaging Application: Comparison Between MAPMT and SiPMs Array}

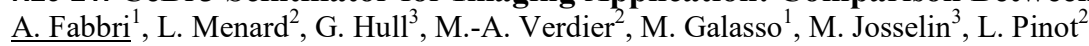

${ }^{\text {I}}$ Roma3 Section, INFN, Roma, Italy

${ }^{2}$ Laboratory of Imaging and Modelisation in Neurobiology and Cancerology, CNRS-IN2P3, Orsay, France

${ }^{3}$ Istitut de Physique Nucleaire, CNRS-IN2P3, Orsay, France

In this communication we will present the imaging properties of a gamma camera based on a CeBr3 crystal. This material represents a very promising scintillator for medical imaging applications; as a matter of fact, $\mathrm{CeBr} 3$ provides detection features close to these of $\mathrm{LaBr} 3(\mathrm{Ce})$ at a considerably reduced production cost, with the additional advantage of having no self-activity. In this work we compare the imaging capabilities of a $51 \times 51 \times 6 \mathrm{~mm} 3 \mathrm{CeBr} 3$ scintillator when optically coupled to a 64-channel H10966 Hamamatsu MAPMT and to a 256-channels SiPM array based on S11828-3344M modules. Both photodetector systems are read-out by a dedicated electronics specifically developed for medical imaging applications. For both CeBr3-based gamma cameras we evaluated the energy resolution in the range between $60 \mathrm{keV}$ and $662 \mathrm{keV}$ and we performed a spot scanning with a highly collimated $57 \mathrm{Co}$ source to estimate linearity and spatial resolution. The same measurements have been performed with a same sized-LaBr3(Ce) crystal, for comparison. The obtained preliminary results demonstrate the effectiveness of $\mathrm{CeBr} 3$ as scintillator material for medical imaging.

N29-22: High Efficient Ce doped TI based Elpasolite Scintillator for ?-rays Detection

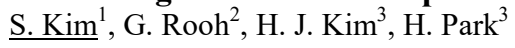

${ }^{I}$ Radiological Sceicne, Chungju University, Cheongju, South Korea

${ }^{2}$ Physics, Abdul Wali Khan University, Mardan, Pakistan

${ }^{3}$ Physics, Kyungpook National University, Daegu, South Korea 
In this paper, crystal growth, luminescence and scintillation properties of new $\mathrm{Tl}_{2} \mathrm{LiLuCl}_{6}$ single crystal doped with $1.0 \%$ $\mathrm{Ce}^{3+}$ concentration (mole\%) are presented. The studied crystals were grown by the Bridgman method using a two-zone vertical furnace. The material was synthesized using the reaction $2 \mathrm{TlCl}+\mathrm{LiCl}+\mathrm{LuCl}_{3} \rightarrow \mathrm{Tl}_{2} \mathrm{LiLuCl}_{6}$. Under X-ray excitation, the emission of crystal was observed in the range of 360 to $560 \mathrm{~nm}$ peaking at $420 \mathrm{~nm}$ and $460 \mathrm{~nm}$. The observed emission was due to the $5 \mathrm{~d}->4 \mathrm{f}$ transitions in $\mathrm{Ce}^{3+}$ ion. The energy resolution of the crystal measured under $662 \mathrm{keV}$ ?-ray was found to be $5.6 \%$ (FWHM). Scintillation decay time profile showed three components characterized by $72 \mathrm{~ns}(8 \%), 366 \mathrm{~ns}(30 \%)$ and $1.5 \mu \mathrm{s}$ $(62 \%)$. The light yield of the crystal was estimated to be $27,000 \pm 2,700$ photons/MeV. The effective $\mathrm{Z}$ number of the $\mathrm{Tl}_{2} \mathrm{LiLuCl}_{6}: \mathrm{Ce}^{3+}$ scintillator was about 70.3 .

Index Terms - $\mathrm{Tl}_{2} \mathrm{LiLuCl}_{6}$, cerium, scintillation, emission, decay time, energy resolution.

This work is supported by the Ministry of Science and Technology, Korea (MEST) (No.2015R1A2A1A13001843).

N29-23: Temporal Imaging: Observation and Localization of a Compton Effect Inside a 20 Mm Monolithic LYSO Plate with a Philips Digital Si-PM

$\underline{\text { A. Iltis }}^{1}$, H. Snoussi ${ }^{2}$, L. Rodrigues de Magalhaes ${ }^{1}$, C. Morel $^{3}$

${ }^{1}$ R\&D Department, Damavan Imaging, Troyes, France

${ }^{2}$ Laboratory of Systems Modelling and Dependability, University of Technology of Troyes, Troyes, France

${ }^{3}$ CPPM, Aix Marseille Université, Marseille, France

Recently, temporal imaging has been shown to be very promising for enhancing the accuracy of spatio-temporal localization of a scintillation events within a monolithic scintillator. The objective of this communication is to show that, besides scintillation localization performances, Compton events could be efficiently detected and characterized inside a monolithic plate. A timebased robust Compton event reconstruction could lead to the implementation of a cost effective single plate Compton camera based on monolithic scintillating crystals.

N29-24: The Origin of Afterglow in GGAG:Ce Scintillation Materials

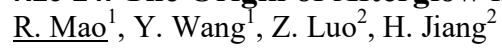

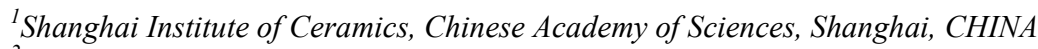

${ }^{2}$ Ningbo Institution of Materials Technology and Engineering, Chinese Academy of Sciences, Ningbo, CHINA

Because of high density and high light output (L.O.) cerium activated Gd3Ga3A12O12 (GGAG:Ce) is a promising scintillator for radiation detection. The GGAG:Ce crystal, however, suffer from strong afterglow which limits its usage in High Energy Physics (HEP) experiments and Medical Imaging (MI) society. Our previous study showed that $\mathrm{Ga} 2 \mathrm{O} 3$ tend to evaporate from melting matrix in the process of single crystal growth resulting in the as grown crystal nonstoichiometric. To prevent as far as possible the evaporation of the Ga2O3 the Transparent Ceramic Technology (TCT) was applied and transparent GGAG:Ce scintillation ceramics were made. The properties of the single crystal and the ceramic GGAG:Ce scintillation materials were investigated. The origin of the scintillation afterglow in these materials will be discussed.

\author{
N29-25: Investigation into Neutron Damage of EJ-299 and EJ-200 Plastic Scintillators \\ M. P. Taggart, C. Payne, P. J. Sellin \\ Dept. of Physics, University of Surrey, Guildford, Surrey, United Kingdom
}

The advent of new plastic scintillator formulations opens avenues of application in terms of neutron-gamma discrimination. As a consequence, the likelihood that these detectors will be subject to regions of significant neutron flux is high. In order to study the tolerance to radiation damage a number of samples of both EJ-299 and EJ-200 plastic scintillator were irradiated with fast neutrons using a neutron flux of up to $2.2 \times 10^{12}(+/-5.6 \%) \mathrm{n} / \mathrm{cm}^{2} / \mathrm{s}$. All samples were cut to a size of $30 \mathrm{~mm} \times 30 \mathrm{~mm} \times 15 \mathrm{~mm}$ and polished prior to irradiation.

The radiation response of the samples were measured in terms of gamma spectroscopy and, for the EJ-299, pulse shape discrimination performance. The optical emission spectra were analysed by irradiating each sample with a $50 \mathrm{kV} X$-ray beam from an Amptek Mini-X X-ray tube. Optical spectroscopy of the scintillation light was recorded using an Ocean Optics QE Pro spectrometer. The effect on the peak emission wavelength, optical intensity, and spectral shape of the scintillation was determined as a function of neutron fluence.

N29-26: CRY-018, CRY-019 and LaBr3:Ce Spectrometric Response Evaluation and Comparison

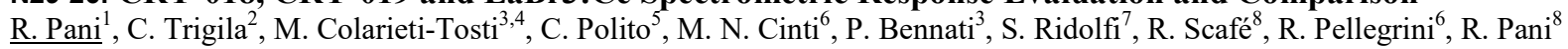

${ }^{I}$ Department of Physics, Sapienza University, Rome, Italy

${ }^{2}$ Departiment of Physics, Roma Tre University, Rome, Italy

${ }^{3}$ Department of Medical Engineering, School of Technology and Health, KTH Royal Institute of Technology, Stockholm, Sweden 
${ }^{4}$ Clintec, Division of Medical Imaging and Technology, Karolinska Institutet, Stockholm, Sweden

${ }^{5}$ SAIMLAL Department, Sapienza University, Rome, Italy

${ }^{6}$ Department of Molecular Medicine, Sapienza university, Rome, Italy

${ }^{7}$ Ars Mensurae, Rome, Italy

${ }^{8}$ Department of Medico-Surgical Sciences and Biotechnologies, Sapienza University, Rome, Italy

Crytur enterprise recently released two new scintillation crystals, CRY-018 and CRY-019. These two crystals, basing on what declared by Crytur, seem to overcome principal problems in modern scintillation crystal used in medical imaging applications. They are both non hygroscopic, with a short decay time, and low refraction index. The aim of this work is to study two cylindrical shaped samples of CRY-018 and CRY-019, both with a 52.0 $\pm 0.1 \mathrm{~mm}$ diameter and $6.25 \pm 0.05 \mathrm{~mm}$ thickness, coated with a white diffusive paint. Radiation detection properties are investigated throughout the comparison with a cylindrical sample of $\mathrm{LaBr}: \mathrm{Ce} 50.0 \pm 0.1 \mathrm{~mm}$ of diameter and $6.0 \pm 0.1 \mathrm{~mm}$ of thickness with a reflective coating of $\mathrm{MgO}$. Calibrated point sources of $241 \mathrm{Am}, 57 \mathrm{Co}, 133 \mathrm{Ba}, 22 \mathrm{Na}$ and $137 \mathrm{Cs}$ were used. A XRF analysis has been executed on both crystals in order to determine the principal chemical elements of the mixture, which turned out to be Yttrium for CRY-018 and Yttrium and Lutetium for CRY019. XRF analysis identified the diffusive coating to be a TiO white paint $0.2 \mathrm{~mm}$ thick. Both crystals present traces of Gadolinium probably used as doping. Crystals have slightly the same light yield of about 45.5\% of $\mathrm{LaBr} 3: \mathrm{Ce}$ one and are non proportional as far as concerned the pulse height uniformity response. The percentage non proportionality of the light yield has been estimated of about $10 \%$ for CRY- 018 and $30 \%$ for CRY- 019 for energies around $30 \mathrm{keV}$ and lower than $1 \%$ for energies higher than $60 \mathrm{keV}$. They are characterized by an energy resolution of about 15\% for CRY-018 and 17\% for CRY-019 at 122 $\mathrm{keV}$ and an intrinsic energy resolution of about $13 \%$ and $15 \%$ at the same energy respectively. Compared to their class of scintillators they seem to represent the best trade off between the intrinsic energy resolution and light yield. Preliminary studies on gamma imagers based on CRY-018 and CRY-019, coupled to a multi-anode PMT, report an intrinsic spatial resolution of about $1.5 \mathrm{~mm}$ for both crystals.

\author{
N29-27: Anion Impurity Quenching of $\mathrm{SrI}_{2}$ :Eu Scintillation Efficiency and Criteria of Raw Material Purity \\ Selection \\ E. Galenin $^{1}$, V. Taranyuk ${ }^{1}$, V. Romanchuk ${ }^{1}$, S. Vasyukov ${ }^{1}$, N. Shiran ${ }^{1}$, O. Sidletskiy ${ }^{1}$, A. Gektin ${ }^{1}$, C. Dujardin ${ }^{2}$ \\ ${ }^{I}$ Institute for Scintillation Materials NAS of Ukraine, Kharkiv, Ukraine \\ ${ }^{2}$ Institut Lumière Matière, UMR5306 CNRS, Universite de Lyon 1, Villeurbanne CEDEX, France
}

Nevertheless to 50 years history of $\mathrm{SrI}_{2}:$ Eu and 10 years renaissance of the interest to this scintillator upgrade the technology of industrial growth and cost efficient production does not exists till now. The goal of this study relates to evaluation of criteria for high quality scintillation crystal growth and initial components selection. The ability to study harmful impurities anion contribution to crystal emission due to the luminescence difference emission wavelength and decay kinetics for Eu $\mathrm{Eu}^{2+}(420 \mathrm{~nm}$ and 900-1000 ns) and these impurities (broad, complex peak from 400 to $640 \mathrm{~nm}$ and $500 \mathrm{~ns}$ ) is shown. Basing on this approach, $\mathrm{SrI}_{2}$ crystals doped by oxygen containing impurities (in particular - carbonate, oxide, nitrate, hydroxide) were grown by Bridgman techniques and their luminescence and scintillation parameters were studied. Some traces of oxygen containing impurities were revealed by IR spectroscopy tests. Multiple effects of impurities do not allow separating contribution of each of them and claiming for unique criteria selection. Finally typical chemical parameter - PH was chosen for preliminary evaluation to initial raw material acidity. We have not succeed to grow the crystals with the energy resolution 5. It is impossible to grow the single crystals under $\mathrm{PH}>9$. Therefore, $\mathrm{PH}$ of the water solution can be selected at an important criterion of the raw material quality.

The work was partially supported by the NATO NUKR.SFPP984958 Project.

N29-28: Growth and Scintillation Properties of 2 inchf $\times 2$ inch SrI2(Eu) Single Crystal

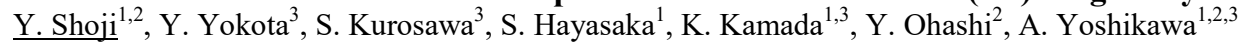

${ }^{I}$ C\&A Corporation, Sendai, Japan

${ }^{2}$ Institute for Materials Research, Tohoku University, Sendai, Japan

${ }^{3}$ New Industry Creation Hatchery Center, Tohoku University, Sendai, Japan

Eu doped SrI2 (Eu) single crystals have been investigated intensively for possible application in radiation detection. It shows such as high light output of over 80,000 photons/MeV and energy resolution of $\sim 3 \%$ at $662 \mathrm{keV}$ (FWHM)[1]. Usually, SrI2(Eu) scintillator crystals have been grown by the Vertical Bridgman (VB) method using a quartz ampoule [2]. on the other hand, we have developed the modified micro pulling down $(\mu-\mathrm{PD})$ furnace which have removable chamber for large diameter bulk single crystals growth [3]. There are some advantages of the novel VB method using $\mu$-PD furnace compared to the conventional VB method using quartz ampoule. One of the main reasons is that we can grow SrI2 crystal without sealing process of quartz ampoule. When the required diameter of the crystal increases, the sealing process will become more difficult. The idea in this study is to apply existing $\mu$-PD apparatus designed and produced for the halide fiber crystal growth for realization of the VB 
process without considerable modifications of the existing hardware. We'll report the result of the developed SrI2(Eu) bulk single crystals with diameters of up to 2 inch by the VB method using the $\mu$-PD furnace and investigated the scintillation properties in this study.

References [1] N. J. Cherepy, G. Hull, A. D. Drobshoff, S.A. Payne, E. van Loef, C. M. Wilson, K. S. Shah, U. N. Roy, A. Burger, L. A. Boatner, W. S. Choong, and W. W. Moses, Strontium and Barium Iodide High Light Yield Scintillators, Appl. Phys. Let., 92 (2008) 83508-83510. [2] M. Takabea, J. Kataokaa, S. Sakuragi, et.al., Nucl. Instr. \& Meth. A (in press) [3]Y. Yokota, A. Yoshikawa, et.al., J. Cryst. Growth 401(2014)343346

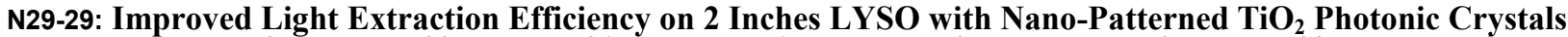
$\underline{\text { S. Zanettini }}{ }^{1}$, V. Gâté ${ }^{2}$, E. Usureau ${ }^{2,3}$, J. Ruscica, ${ }^{2,4}$, F. Hamouda ${ }^{4}$, K. Nomenyo ${ }^{3}$, L. O. Le Cunff ${ }^{3}$, H. Kadiri' ${ }^{2,3}$, G. Lerondel ${ }^{3}$, M. Salomoni ${ }^{5}$, R. Plots 5 , E. Auffray ${ }^{5}$, P. Lecoq ${ }^{5}$, J. Alamo ${ }^{6}$, A. Iltis ${ }^{1}$, D. Turover ${ }^{1,2}$

${ }^{I}$ Napa-Technologies, Troyes, France

${ }^{2}$ SILSEF, Archamps, France

${ }^{3}$ ICD/LNIO, Université de Technologie de Troyes (UTT), Troyes, France

${ }^{4}$ Institut d'Electronique Fondamentale, CNRS - Université Paris Sud, Orsay, France

${ }^{5}$ CERN, Geneva, Switzerland

${ }^{6}$ Oncovision, Valencia, Spain

We have manufactured a two-dimensional photonic crystal of $\mathrm{TiO}_{2}$ onto a 2 inches LYSO (Lutetium Yttrium Silicate) surface to enhance light extraction from the high refractive index inorganic scintillator. Such a large area nano-patterning is a real breakthrough and it has been made possible by using the high resolution and cost effective nano-imprint lithography technique. The photonic crystals coated on LYSO enable scintillation photons impinging the exit surface beyond the critical angle to overcome total internal reflection (TIR) and be out-diffracted into higher-order modes towards the photo sensor [1]. Different structures of nano-pillars and nano-holes have been manufactured and their enhanced light extraction has been tested by laser excited photoluminescence measurements. The highest optical gain (external quantum efficiency) was observed with a periodic array of $\mathrm{TiO}_{2}$ nano-pillars $300 \mathrm{~nm}$ high with a pitch of $700 \mathrm{~nm}$. This result is in line with simulations. The light output measured with $\mathrm{Cs}^{137}$ source on thick LYSO crystals before and after patterning will also be presented. This large-area surface treatment will allow improved performances in standard PET-scans and Time of Flight PET systems.

This work is financially supported by the Eurostars Turbo-PET program E! 8974.

[1] A. Knapitsch and P. Lecoq, "Review on photonic crystal coatings for scintillators,” Int. J. Mod. Phys. A, vol. 29, no. 30, p. 1430070, Nov. 2014.

N29-30: Ce Concentration Dependence of Quenching Effects in Ce:CaF2 Small Size Dosimeters

$\underline{\text { Y. Hirata }}^{1}$, K. Watanabe ${ }^{1}$, A. Uritani ${ }^{1}$, A. Yamazaki ${ }^{1}$, S. Yoshihashi ${ }^{1}$, Y. Koba ${ }^{2}$, N. Matsufuji ${ }^{2}$, T. Yanagida ${ }^{3}$, K. Fukuda $^{4}$

${ }^{I}$ Nagoya University, Nagoya, Japan

${ }^{2}$ National Institute for Quantum and Radiological Science and Techinology, Chiba, Japan

${ }^{3}$ Nara Institute of Science and Technology, Nara, Japan

${ }^{4}$ Tokuyama Corp., Shunan, Japan

Heavy ion therapies can form fine and complex dose distributions and reduce undesired irradiation into normal organs. Misalignment of an irradiation position may cause a significant accident. In order to accurately estimate the irradiation dose, it is desired that dosimeters are inserted into an affected region in a patient body. We, therefore, are developing a small size dosimeter consisting of an optical fiber and an optically stimulated luminescence (OSL) element. We evaluated the characteristics of the OSL used small size dosimeters with high energy carbon beams. OSL materials showed quenching effects when irradiated by high linear energy transfer (LET) particles. We assumed the quenching effect was caused by local occupation of trap centers due to high density ionization. The quenching effect, therefore, depends on the density of trap centers. $\mathrm{Ce}: \mathrm{CaF}_{2}$ is one of the most useful OSL elements. The OSL signal intensity is increased with Ce concentration. This means high Ce concentration make high density of trap centers. In this paper, we evaluate the quenching effect of $\mathrm{CaF}_{2}$ doped with different Ce concentration. We prepared different $\mathrm{Ce}$ concentration as 0.1, 0.5 and 5.0 \% doped $\mathrm{CaF}_{2}$. Carbon ions with an energy of $290 \mathrm{MeV} / \mathrm{u}$ were irradiated to the dosimeters at Heavy Ion Medical Accelerator in Chiba (HIMAC). The fabricated dosimeters were set behind the water equivalent acrylic phantom. The LET value of carbon ions were varied by changing the phantom thickness. The dosimeters showed different level quenching. The luminescence efficiencies increased with the Ce concentration. We confirmed that the quenching effect depended on the density of the trap centers.

N29-31: Composite Scintillators for Neutron and X-Ray Detection

A. Boyarintsev, A. Bobovnikov, A. Gektin, Y. Gerasimov, S. Kovalchuk, T. Nepokupnaya, Y. Onufriyev, V. Tarasov

The Institute for Scintillation Materials of National Academy of Sciences of Ukraine, Kharkov, Ukraine 
The goal of the study is the development of the composite detectors for neutron and X-ray detection based on composite scintillators. Composites consist of the scintillation granules distributed in the optical silicone compound and has such advantages as cheap production of large area detectors, variability of design, simple manufacturing.

6LiI(Eu), $\mathrm{CsI}(\mathrm{Tl})$ and GAGG $(\mathrm{Ce})$ scintillators were selected like objects for the simulations and for the manufacturing of the composite detectors because of their good sensitivity to neutron and X-ray radiation.

The simulation of light collection efficiency of $6 \mathrm{LiI}(\mathrm{Eu}), \mathrm{CsI}(\mathrm{Tl})$ and GAGG (Ce) composites by the Monte Carlo method were carried out. Optical characteristics for above scintillators and silicon compounds were used in the calculation. It was shown that the light collection coefficient for composites increases with increasing of effective thickness at the irradiation of the $6 \mathrm{LiI}(\mathrm{Eu})$ by thermal neutrons $(\mathrm{E}=0,005 \mathrm{eV})$ and $\mathrm{CsI}(\mathrm{Tl})$ by gamma-particles $(\mathrm{E}=59,6$ and $122 \mathrm{keV})$.

It was found that light collection coefficient and relative light output values for the manufacturing composites are in the good agreement with the simulation results. So composite scintillators on the base of $\mathrm{LiI}(\mathrm{Eu}), \mathrm{CsI}(\mathrm{Tl})$ and GAGG (Ce) can be used for the X-ray and neutron detections. It needs to carry out additional experimental research to increase the light collection efficiency in the composite detectors.

\section{N29-32: Scintillation Properties of Hafnium-Based Chloride Scintillator}

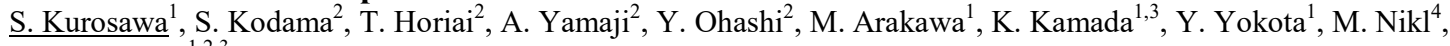
A. Yoshikawa ${ }^{1,2,3}$

${ }^{I}$ NICHe, Tohoku University, Sendai, Miyagi, Japan

${ }^{2} I M R$, Tohoku University, Sendai, Miyagi, Japan

${ }^{3} C \& A$, Sendai, Miyagi, Japan

${ }^{4} A S C R$, Prague, Czech Republic

Gamma-ray scintillation crystals were required to have gamma-ray stopping power which depends on the effective atomic number, and Gd or Lu-based scintillators are used in several applications due to high atomic numbers, and good light output. On the other hand, Lu has a large number of intrinsic gamma-ray backgrounds, and Gd has smaller atomic number than Lu. Hafnium has a higher atomic number of 72 compared with $\mathrm{Lu}$ and $\mathrm{Gd}$, and its intrinsic-gamma-ray-background is negligible. Moreover, Chloride materials tend to have smaller band gap than oxide scintillators, and we expected good scintillation properties for Hafnium Chloride Scintillator. Indeed, some groups reported the scintillation properties for pure $\mathrm{CsHfCl}_{6} \mathrm{crystal}_{\text {, and this }}$ scintillator has good light output of over 50,000 photons/MeV. ON the other hand, the decay time was slow due to the emission origin: charge-transfer emissions. Thus, we grew new materials Tl-doped $\mathrm{CsHfCl}_{6}$ crystals grown by the Bridgman technique, and Tl play role in the activator. After the crystal growth, powder X-ray diffraction was operated to check the crystal strictures and single phase, and photo-luminescence and radio luminescence were investigated for these samples. Moreover, we measured the gamma-ray response with ${ }^{137} \mathrm{Cs}$ souse, and these samples had good light outputs of over 40,000 photons/MeV and good energy resolutions of less than 5\% at $662 \mathrm{keV}$ (FWHM), and decay times were shorter than pure samples.

\section{N29-33: Composite Halide Scintillators}

$\underline{\text { S. Lam }}{ }^{1}$, J. Fiala ${ }^{1}$, I. Jovanovic ${ }^{2}$, S. Motakef ${ }^{1}$

${ }^{1}$ CapeSym, Inc., Natick, MA, USA

${ }^{2}$ University of Michigan, Ann Arbor, MI, 48109

While SrI2(Eu)'s excellent scintillation performance and CLYC's dual mode detection capability make these halides ideal candidates for nuclear radiation detection, large diameter crystals of these materials ( $>3$-inches) are costly due to lower crystal growth yield. However, the cost of encapsulating a large array of small diameter crystals into a large plastic matrix (e.g. made of PVT or PMMA) is fairly low, and the plastic matrix can be even shaped to improve light collection. Thus, composite scintillators hold the promise of high performance and low-cost gamma/neutron detectors in sizes that would be otherwise prohibitively expensive or impossible to produce. In this presentation, we share the scintillation performance of SrI2(Eu)-PVT and CLYC-PVT composite scintillators. The experimental results are accompanied by GEANT4 simulations to characterize the generation, transport, and collection of photons, and their dependence on surface conditions, index of refraction of the host, among other factors.

This work has been supported by the U.S. Department of Homeland Security under competitively awarded contract HSHQDC16-R-00012. This support does not constitute an express or implied endorsement on the part of the government.

N29-34: Cs2HfCl6: A Non-Hygroscopic, High-Performance Scintillator $\underline{\text { S. Lam }}{ }^{1}$, A. Burger ${ }^{2}$, S. Motakef ${ }^{1}$

${ }^{1}$ CapeSym, Inc., Natick, MA, USA

${ }^{2}$ Fisk University, Nashville, TN, USA

Cesium hafnium chloride ( $\mathrm{Cs} 2 \mathrm{HfCl}, \mathrm{CHC})$ is a recently discovered material with superior radiation detection properties relative to the incumbent detectors $\mathrm{NaI}(\mathrm{Tl})$ and $\mathrm{CsI}(\mathrm{Tl})$. Some of the major advantages of $\mathrm{CHC}$ include an excellent energy resolution of 
$3.3 \%$, lack of self-absorption, absence of self-activity, and its non-hygroscopic nature. Thus, unlike $\mathrm{SrI} 2(\mathrm{Eu}) \mathrm{and} \mathrm{LaBr} 3(\mathrm{Ce})$, the handling and crystal growth of $\mathrm{CHC}$ is not complicated by adsorption of water in the starting charge, which even in miniscule amounts can result in formation of hydroxides that react with the quartz growth ampoule to cause cracking of the crystal. Our first-grown crystals are characterized by a light yield of 30,000-54,000 ph/MeV and an energy resolution of 3.3\%. CHC has a decay time close to $4 \mu \mathrm{s}$, which makes it suitable for low-count scenarios encountered in homeland security applications. In this presentation, we discuss the crystal growth of 1-inch boules of $\mathrm{CHC}$ and the importance of purification in improving crystal quality and scintillation performance. The effect of doping/alloying will also be discussed.

N29-35: Scintillation Properties of Gadolinium Pyrosilicate Crystals in High Temperature

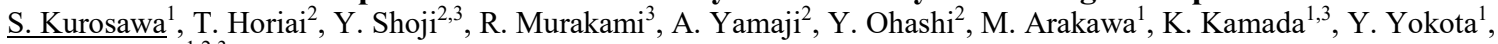
A. Yoshikawa ${ }^{1,2,3}$

${ }^{I}$ NICHe, Tohoku University, Sendai, Miyagi, Japan

${ }^{2} I M R$, Tohoku University, Sendai, Miyagi, Japan

${ }^{3} C \& A$, Sendai, Miyagi, Japan

Recently, the pyrochlore crystals described with a $\mathrm{A}_{2} \mathrm{Si}_{2} \mathrm{O}_{7}$ formula, have been studied as scintillation materials, where the $\mathrm{A}$ is rare-earth atoms. The $\mathrm{Gd}_{2} \mathrm{Si}_{2} \mathrm{O}_{7}$ (GPS) and $\mathrm{Lu}_{2} \mathrm{Si}_{2} \mathrm{O}_{7}$ (LPS) are the recently most investigated systems. These materials have good light output or energy resolution. However, they do not melt congruently (e.g. $\mathrm{Gd}_{2} \mathrm{O}_{3}-\mathrm{SiO}_{2}$ system), and therefore the growth from the melt is difficult. In order to obtain the congruent composition, the material was doped with approximately $10-50 \%$ La. Then the Ce:( $\mathrm{La}, \mathrm{Gd})_{2} \mathrm{Si}_{2} \mathrm{O}_{7}$ (Ce:La-GPS) scintillator crystals with a good energy resolution (FWHM) of $5 \%$ at $662 \mathrm{keV}$ were obtained. Moreover, good scintillation properties are expected to be stable up to $450 \mathrm{~K}$, as has been reported earlier for Ce:LPS and Ce:La-GPS crystals. Although Ce:LPS cannot be applied to oil well logging due to its intrinsic background, there is not such a limitation for the Ce:La-GPS. In this presentation, we show the temperature dependence for several Ce-doepd $(\mathrm{Gd}$, $\mathrm{La})_{2} \mathrm{Si}_{2} \mathrm{O}_{7}$ samples.

N29-36: Effect of Annealing on Scintillation Characteristics of Ce-Doped Gadolinium Silicate Single Crystals Y. Anzai, K. Nagao, S. Takekawa, Y. Furukawa, H. Ishibashi

Oxide Corporation, Hokuto, Yamanashi, Japan

Ce-doped gadolinium silicate single crystal (GSO) is widely used for radiation detectors for PET and oil well loggings. To get a better scintillation performance, post-growth annealing under O2-containing atmosphere e.g. under the air is applied for many oxide scintillators. But GSO is drop in scintillation characteristics such as light output by annealing with O2-containing atmosphere due to coloring which is thought to be by the change of $\mathrm{Ce} 3+$ to $\mathrm{Ce} 4+$ in the crystal during annealing. In-line annealing with O2-containing atmosphere is only useful for preventing cracks during GSO growth. In this work, we have investigated the post-growth annealing for GSO using a tubular furnace and metallic furnace. Both furnaces are air tight and are able to flow N2 and O2 gases during annealing. A GSO specimen with $12 \mathrm{~mm}$ in diameter and $12 \mathrm{~mm}$ in length was put in a furnace and was heated up under different temperature and different atmosphere. As a result, all of GSO annealed with the tubular furnace shows better light output than before. The light output of GSO annealed with the temperature of $1,450{ }^{\circ} \mathrm{C}$ and 60 120 hours under the $\mathrm{N} 2$ flow increased $15-17 \%$ as a best result in these experiments. In this case, oxygen concentration was around $3 \mathrm{ppm}$ as a results. In contrast, light output of GSO decreased slightly by annealing with the metallic furnace even 3 hours at $1,450{ }^{\circ} \mathrm{C}$. This degradation is assumed to create oxygen defects due to too small oxygen concentration during annealing in the GSO. Other scintillation characteristics such as energy resolution and decay time will be discussed in the conference. Result of other Ce-doped rare-earth silicate such as Ce doped Lu2-xGdxSiO5 (LGSO) will be compared.

N29-37: Large, High performance CLLB detectors for dual gamma and neutron detection. J. Lejay $^{1}$, P. Menge ${ }^{2}$, S. Blahuta ${ }^{1}$, K. Yang ${ }^{2}$, J. Frank ${ }^{2}$

${ }^{I}$ Saint-Gobain Recherche, Aubervilliers, France

${ }^{2}$ Saint-Gobain Crystals, Hiram, USA

Cs2LiLaBr6:Ce (CLLB) crystals containing 6Li belong to elpasolite compositions family A2BReX6 (A = Cs, $\mathrm{Rb}, \mathrm{K}, \mathrm{Ba}$; $\mathrm{B}=\mathrm{Na}$, $\mathrm{Li} ; \mathrm{Re}=$ rare earth, $\mathrm{X}=\mathrm{F}, \mathrm{Cl}, \mathrm{Br}, \mathrm{I})$. It is a very attractive candidate for dual gamma and thermal neutron detection systems [1] based on the modern pulse-shape discrimination technique. This material is well known as a difficult crystal to grow, to significant commercial sizes $(>100 \mathrm{~cm} 3)$. Saint-Gobain Crystals has developed a reliable process to produce Elpasolites detectors with large dimensions (more than 2,5”), with improvements in quality, mechanical properties and scintillation performance. We propose to show the performance we can achieve today with detector sizes ranging from 1.5" to close to 3" in diameter. The latest improvements in terms of process, performance and dimensions allow Saint-Gobain to propose CLLB at industrial scale in 2017. 
Bibliography [1] C.W.E. van Eijk, J.T.M. de Haas, P. Dorenbos, K.W. Kramer, H.U. Gudel, Development of Elpasolite and Monoclinic Thermal Neutron Scintillators 2005 IEEE Nuclear Science Symposium Conference Record N12-3, pp 249-243. [2] K.Yang, P.R. Menge, J.Lejay, V.Ouspenski. Improving the Neutron and Gamma-Ray Response of Cs2LiLaBr6:Ce3+. 2013 IEEE Nuclear Science Symposium Conference Record NP01-73, p123.

\author{
N29-38: Improved Light Collimation for Scintillators Crystals Using a Photonic Crystal \\ D. Costantini, S. Chatel, S. Le Roy, P. Trinh \\ Optics group, Saint Gobain Recherche 39 Quai Lucien Lefranc, Aubervilliers, France
}

There is a great interest in improving light extraction from scintillator crystals for fundamental research, security and medical applications. This would allow increasing image contrast or reducing the radiation dose to obtain an equal result. In particular in medical applications patients could be less irradiated decreasing health issues related to radioactivity. Light coming out from a scintillator generally has a quasi-Lambertian distribution, meaning that the angular distribution is very large. Typically the scintillator and photosensor are close coupled, but in some applications, as the high energy radiography with an electron accelerator, IRM-PET, the reactor core imaging and human PET imaging, standoff between the scintillator and the detector is required. Possible solutions are use of fibers and lenses which need a somewhat collimated beam. In these cases the efficiency can be increased by narrowing the angular distribution of the light extracted from the scintillator.

In order to improve collimation of the extracted light, we propose the use of a Photonic Crystal (PhC). A PhC is a periodically patterned structure whose the typical dimensions are about the wavelength size. Light crossing a $\mathrm{PhC}$ is not anymore obeying to the Snell law and can be redirected thanks to interference effects. We numerically demonstrated the focusing effect obtaining an improvement of $50 \%$ in a solid angle of apex $30^{\circ}$. Further we experimentally realized a high-index $(\mathrm{n}=1,85) \mathrm{PhC}$ trough embossing a matrix containing $\mathrm{TiO} 2$ nanoparticles. This technique paves the way to large scale fabrication.

N29-39: PEN Scintillator Films for the Ionizing Radiation Identification and Spectroscopy A. Plukis, J. Garankin, E. Lagzdina, V. Kovalevskij, M. Gaspariunas, R. Plukiene, V. Remeikis Center for Physical Sciences and Technology, Vilnius, Lithuania

Industry grade polyethylene 2,6-naphthalate (PEN) films were investigated as scintillator material for detection and spectroscopy of ionizing radiation particles. A wide range of particles as alphas, betas, gammas, protons, neutrons were detected and spectra were measured using PEN scintillation. The energy deposition in the samples was calculated by Monte Carlo computer modelling. Separate neutron/gamma and alpha/beta pulse shapes were obtained, using pulse shape discrimination. The figure of merit was used to determine quality of discrimination of particles. The results of study showed that PEN film could be used as polymer scintillation detector without wavelength shifters for ionizing radiation detection and is suitable for discrimination between high and low LET particles.

N29-40: Study of the Response of a CsI(TI) - SiPM Detector to Low Energy Protons

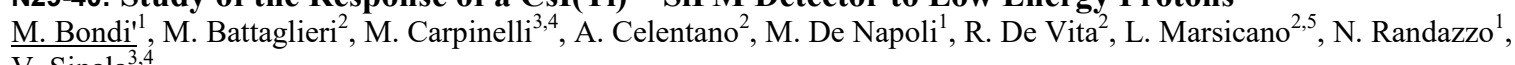
V. Sipala ${ }^{3}, 4$

${ }^{1}$ INFN - Sezione di Catania, Catania, Italy

${ }^{2}$ INFN - Sezione di Genova, Genova, Italy

${ }^{3}$ INFN - LNS, Catania, Italy

${ }^{4}$ Università degli Studi di Sassari, Sassari, Italy

${ }^{5}$ Università degli studi di Genova, Genova, Italy

Silicon Photomutiplier is a successful solid-state light detector. Thanks to its characteristics, especially the high quantum efficiency comparable to that of PMTs and the much higher gain compared to APDs, it can play an important role in many physics fields. Scintillator crystals coupled to SiPMs are widely used as radiation detectors but they have been scarcely characterized as low energy ion detectors. In the present work we report a study of the light response of a CsI(Tl) crystal read out by SiPMs to low energy protons, accelerated by the Tandem Van der Graaf at the Laboratori Nazionali del Sud (LNS) - INFN, Catania, Italy. Two types of Hamamatsu MPPCs were used: one with a pixel size of $25 \mu \mathrm{m}$ and 14400 pixels, and the other with a pixel size of $50 \mu \mathrm{m}$ and 3600 pixels. The light yield was measured for protons of different incident energies, from $2.5 \mathrm{MeV}$ to 24 $\mathrm{MeV}$. Moreover, we searched for and quantified how the light yield changes as a function of the point where the beam hit crystal. Light yield, energy resolution and timing "jitter" were measured, for each energy and for each position. The experimental results were also compared with Geant4 Monte Carlo simulations. The results of this work indicate that a slow CsI coupled with a SiPMbased modern readout solution allows for the detection of low energy signals with a good time and energy resolution.

N29-41: Engineering LYSO Single Crystals for Performance

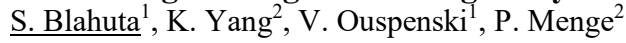


${ }^{I}$ Saint-Gobain Recherche, Aubervilliers, France

${ }^{2}$ Saint-Gobain Crystals, Hiram, OH, USA

Cerium-doped Lu2(1-x)Y2xSiO5 (LYSO:Ce) is known to be an efficient material for Positron Emission Tomography (PET) application. However materials with faster timing and higher light output are required for future Time-Of-Flight PET systems. In LYSO as in other oxide scintillators oxygen vacancies are the main defects and limit performance [1]. During the last decade we have studied and developed different techniques that allow controlling the impact of these vacancies or even preventing their formation during the growth process. Besides the well know oxygen containing atmosphere annealing post treatment, applying controlled co-doping or the new oxidizing agent method are powerful tools to enhance materials performance. When the appropriate conditions are applied LYSO can show a light output up to 43,000 photons/MeV together with a good $8 \%$ energy resolution at $662 \mathrm{keV}$ excitation. This makes it a competitive industrial material for medical imaging applications. These improvements are explained by a complex charge compensation mechanism combined with a partial substitution of Cerium ions in their $\mathrm{Ce} 4+$ state [2]. When co-doping is pushed to its limits very fast decay time below $33 \mathrm{~ns}$ can be obtained. However this strong co-doping has also a dramatic impact on the crystal growth stability [3]. The newly developed oxidizing agent technique allows recovering crystal growth yields while maintaining good scintillation performance. In order to achieve faster timing a good control of the dopant / co-dopant relative contents has been proven to be an efficient approach to engineer scintillation materials.

* corresponding author e-mail: samuel.blahuta@saint-gobain.com

References [1] S. Blahuta, A. Bessière, B. Viana, V. Ouspenski, E. Mattmann, J. Lejay and D. Gourier, Materials 4, no. 7, 1224-1237 (2011). [2] S. Blahuta, A. Bessière, B. Viana, P. Dorenbos and V. Ouspenski, IEEE Transactions On Nuclear Science 60, 3134-3141 (2013). [3] M. Spurrier, P. Szupryczynski, H. Rothfuss, K. Yang, A. A. Carey and C. L. Melcher, J. Crystal Growth 310, 2110-2114 (2008).

N29-42: Evaluation of Structured Scintillator Films for Synchrotron Applications

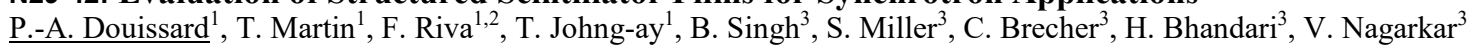

${ }^{I}$ ISDD/Detector Unit, ESRF, Grenoble, France

${ }^{2}$ Institut Lumière Matière, Villeurbanne, France

${ }^{3}$ Radiation Monitoring Devices Inc, Watertown, USA

In the frame of the upgrade program phase II, the European Synchrotron Radiation Facility will build a new storage ring in the period 2015-2022, which will deliver an X-ray beam of higher brightness and coherence. In this context, diffraction and X-ray imaging experiments will benefit from X-ray detectors covering a large dynamic range (above 16 bits), large field of view (at least $10 \mathrm{~cm} \times 10 \mathrm{~cm}$ ), while operating at high X-ray energies (between $30 \mathrm{keV}$ and $100 \mathrm{keV}$ ) and high frame rates (above 500 frames per second). In the case of X-ray diffraction, indirect detection scheme using a scintillator to absorb and convert X-ray photons into light is important at high X-ray energies (starting from $30 \mathrm{keV}$ ). Concerning X-ray imaging, indirect detection is the only scheme able to cope with the huge intensities of the X-ray beams while providing micrometer spatial resolution. Therefore we plan to build flat panels optimized for those high-energy applications, based on specially developed CMOS sensors coupled to a structured scintillator. The scintillating screen will be a key of such detection system, as it will determine the final X-ray absorption efficiency, speed, precision as well as stability of the system under high energy and intense X-ray flux. To help define the final specifications of the sensor, we evaluated some commercially available scintillating screen, including CsI:T1 microcolumnar films, Tb-doped scintillating face plates and $\mathrm{Ba}_{2} \mathrm{CsI}_{5}: \mathrm{Eu}$ film, in terms of their spatial uniformity, spatial resolution, afterglow, hysteresis and stability under intense and long X-ray exposure. We will present the results in this paper.

N29-43: Scintillation Properties of $\mathrm{Mg}^{2+} \mathrm{Co}$-Doped Multicomponent GdGaLuAG:Ce Garnet Scintillators

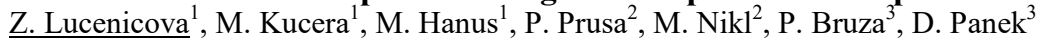

${ }^{I}$ Faculty of Mathematics and Physics, Charles University, Prague, Czech Republic

${ }^{2}$ Institute of Physics, Academy of Sciences, Prague, Czech Republic

${ }^{3}$ Faculty of Biomedical Engineering, Czech Technical University, Kladno, Czech Republic

Scintillation materials with short decay time and low afterglow are necessary in applications where fast response is required. Slow components can be almost completely suppressed by optimal $\mathrm{Gd}^{3+}$ and $\mathrm{Ga}^{3+}$ co-doping, and additional $\mathrm{Mg}^{2+}$ doping can shorten the prompt $\mathrm{Ce}^{3+}$ decay time due to creation of the stable $\mathrm{Ce}^{4+}$ centres. In this paper, we focus on the study of optical, luminescence, decay kinetics, and scintillation properties of multicomponent $(\mathrm{LuGd})_{3}(\mathrm{GaAl})_{5} \mathrm{O}_{12}: \mathrm{Ce}, \mathrm{Mg}$ single crystalline films co-doped with various content of divalent $\mathrm{Mg}^{2+}$ ions. The films with thickness of $17 \mu \mathrm{m}$ were grown from the $\mathrm{BaO}_{-} \mathrm{B}_{2} \mathrm{O}_{3}$ $\mathrm{BaF}_{2}$ flux by the method of liquid phase epitaxy. We observed that Ce-related scintillation decay is significantly accelerated to 25 - $40 \mathrm{~ns}$ at optimized Mg co-doping and simultaneously the slow components in the decays are almost completely suppressed. The studied materials have potential to shorten the decays even more, however, at the expense of the light yield.

N29-44: Scintillation Properties of Optimized YSO:Ce Single Crystalline Films 


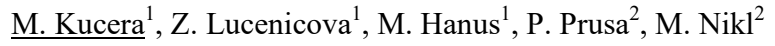

${ }^{I}$ Charles University in Prague, Prague, Czech Republic

${ }^{2}$ Institute of Physics, AS CR, Prague, Czech Republic

The scintillation and luminescence properties of single crystalline films Y2SiO5:Ce (YSO:Ce) orthosilicates grown by liquid phase epitaxy from the $\mathrm{PbO}-\mathrm{B} 2 \mathrm{O} 3$ flux were studied. The detrimental effect $\mathrm{Pb} 2+$ impurities coming from the flux was significantly reduced by suitable flux composition and adjusting optimized growth conditions. Due to the charge compensation, both $\mathrm{Ce} 3+$ and $\mathrm{Ce} 4+$ ions were identified in films. The stable $\mathrm{Ce} 4+$ centers form fast parallel radiative channel and the slow components in the scintillation decay were largely eliminated. The timing properties of studied epitaxial films are very good. The light yield, determined by pulse height measurements using the alpha particle excitation, is independent on the shaping time within 0.5 to $10 \mu \mathrm{s}$. The timing properties were further improved by intentional $\mathrm{Zr} 4+$ co-doping. This was tentatively explained by forming $\mathrm{Pb} 2+-\mathrm{Zr} 4+$ self-compensating pairs and reducing thus surplus $\mathrm{Ce} 4+$ ions. The integrated radioluminescence signal under X-ray excitation was $10 \times$ higher as compared to BGO crystal.

\author{
N29-45: Applications of SSLE on Monolithic Scintillator Crystals: Novel Pixel Geometries and Depth of \\ Interaction

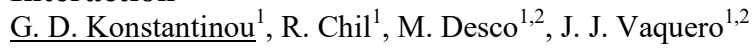 \\ ${ }^{I}$ Departamento de Bioingeniería e Ingeniería Aeroespacial, Universidad Carlos III de Madrid, Madrid, Spain \\ ${ }^{2}$ Instituto de Investigación Sanitaria Gregorio Marañon, Madrid, Spain
}

Pixelated scintillator crystals are commonly used in gamma radiation detectors for PET scanners. The size of these pixels, the crystal surface treatment and the thickness of the reflector inserted between crystals directly affects energy and spatial resolution as well as the sensitivity of the detector, while increasing the signal to noise ratio by channeling the visible light produced by the gamma photon. Pixel fabrication can be greatly simplified by the application of sub-surface laser engraving (SSLE), where instead of external reflectors, optical barriers are created inside the scintillator itself. Apart from cost effectiveness and ease of application, this technique is free from any geometrical limitations, allowing different shapes or mixed pixel topologies to be used according to the particular application or readout system. In this study we showcase this function by simulating, engraving in LYSO and testing an array of hexagonal pixels. Furthermore, we propose a novel concept of depth-of-interaction, which can be applied on any kind of pixel topologies and demonstrate its application on the hexagonal array. Addition of such characteristics, as well as light sharing or edge compensation for anger logic readouts, is made easily, without any additional cost to the monolithic scintillator, greatly reducing the cost of a gamma detector.

This work was partially funded by the TOPUS S2013/MIT-3024 project from the regional government of Madrid, the TEC2014-56600-R from the Spanish Ministerio de Economía y Competitividad, the INFIERI Network (Marie Curie ITN EU FP7, grant agreement no. 317446), and the Human Frontier Science Program grant R GP0004/2013. G. Konstantinou is with the Departamento de Bioingeniería e Ingeniería Aeroespacial, Universidad Carlos III de Madrid, Madrid, Spain (telephone: +34916248201, e-mail: gkonstantinou@hggm.es). R. Chil, M. Desco, and J.J. Vaquero are with Departamento de Bioingeniería e Ingeniería Aeroespacial, Universidad Carlos III de Madrid, Madrid, Spain and the Instituto de Investigación Sanitaria Gregorio Marañon, Madrid, Spain.

\title{
N29-46: A New Method for Quantifying Pulse Shape in Organic Scintillator Pulse Shape Discrimination
} $\underline{\text { P. Schuster }}$

University of California, Berkeley, Berkeley, CA, US

Organic crystal scintillators serve as valuable radiation detectors in nuclear security applications, providing excellent scintillation characteristics and neutron-gamma discrimination. Numerous techniques exist for quantifying the pulse shape into a onedimensional parameter, but limitations exist in pulse shape discrimination performance. This paper reports a new method for quantifying the pulse shape that improves neutron-gamma discrimination in materials with slow decay times, such as anthracene.

N29-47: Crystal Growth and X-Ray Luminescence of MgO-Ta2O5 Compounds

D. Smiadak, Y. Ma, D. Perrodin, G. Bizarri, E. Bourret

Materials Sciences Division, Lawrence Berkeley National Laboratory, Berkeley CA, USA

While the luminescence of tantalate compounds has been extensively studied by Blasse et al.[1] there are few reports on single crystal growth of these materials for use as scintillators. The phase diagram of MgO-Ta2O5 system is still somewhat elusive: only two forms are known with certainty, $\mathrm{Mg} 4 \mathrm{Ta} 2 \mathrm{O} 9$ (density $=6.17 \mathrm{~g} / \mathrm{cm} 3$ ) and MgTa2O6 (density = 8.03 g/cm3). The existence of an $\mathrm{O} 8$ or an $\mathrm{O} 15$ compound is still being debated. We will report on the synthesis, crystal growth and characterization of the $\mathrm{Mg} 4 \mathrm{Ta} 2 \mathrm{O} 9, \mathrm{MgTaO} 6$ and $\mathrm{Mg} 3 \mathrm{Ta} 2 \mathrm{O} 8$ compounds. Growth of single crystals was achieved using the micro-pull down technique. Measurements of the $\mathrm{x}$-ray excited luminescence shows that Mg4Ta2O9 is an efficient scintillator at room temperature. We also confirmed the beneficial role of $\mathrm{Nb}$ to increase the quenching temperature of $\mathrm{MgTaO6}$. For the 
Mg3Ta2O8, we were able to obtained and confirmed by x-ray diffraction the existence of the structure. However, attempts to produce this phase by solid state reaction gave a compound of formula $\mathrm{Mg} 3.48 \mathrm{Ta} 2.52 \mathrm{O} 10$ adding fuel to the controversy. Moving forward to grow large pixel size crystals, $\mathrm{Mg} 4 \mathrm{Ta} 2 \mathrm{O} 9$ is being grown by the float-zone technique. The conditions to get a pure phase of $\mathrm{Mg} 4 \mathrm{Ta} 2 \mathrm{O} 9$ and its impact on the crystal growth and the x-ray luminescence will be presented. [1] G.Blasse and A.Bril Journal of Luminescence 3 (1970) 109-131 and later publications

This work is supported by the U.S. Department of Homeland Security/Domestic Nuclear Detection Office and carried out at Lawrence Berkeley National Laboratory under Contract No. AC02-05CH11231. Y. Ma is supported by the National Natural Science Foundation of China (No.11374031) and China Scholarship Council under No. 201406540016.

N29-48: Development of Large-Area Charged Particle Detectors with High Position Resolution and Low Cost T. Mizuno, H. Ito, N. Kaneko, H. Kawai, A. Kobayashi, S. Kodama, M. Tabata

Graduate School of Science, Chiba University, Chiba-city, Chiba-prefecture, Japan

We have developed two types of large-area charged particle detectors with high position resolution and low cost. One is a scintillation fiber detector of $2 \mathrm{~m} \mathrm{x} 2 \mathrm{~m}$. A detector is composed of 4000 scintillating fibers of $1 \mathrm{~mm}$ in diameters. The position resolution is $0.13 \mathrm{~mm}$ (RMS). 14 clear fibers of $0.25 \mathrm{~mm}$ in diameter are attached to each end surface of a scintillating fiber and 7 clear fibers are bundled. Thus, each scintillating fiber has 4 bundles. A four-digit octal number is assigned to each scintillating fiber. First bundle of 512 fibers of which the first digit are same number are gathered and attached to a photo detector and so on. The fired fibers are identified by only 32 photo-detectors. The other is a scintillating plate detector of $15 \mathrm{~m} \mathrm{x} 5 \mathrm{~m}$. It contains about 26,000 plastic scintillator of $5 \mathrm{~mm} \times 7.5 \mathrm{~mm}$ x $700 \mathrm{~mm}$. The position resolution is $1 \mathrm{~mm}$. 600 clear fibers of $0.25 \mathrm{~mm}$ in diameters are attached on each end surface of a plastic scintillator. At the other end, they are attached on the side surface of a wave length shifting fiber of $0.5 \mathrm{~mm}$ in diameter. On each end of the WLSF, a clear fiber of which the diameter is $0.5 \mathrm{~mm}$ and the length is $10 \mathrm{~m}$ is attached. Thus each scintillating plate connects 4 clear fibers. A four-digit bace- 13 number is assigned to each scintillating plate and the fired plates are identified by only 52 photo-detectors.

\section{N29-49: Bulk Crystal Growth and Scintillation Properties of 3 Inch Ce:GAGG and Mg Co-Doped Ce:GAGG} Single Crystal

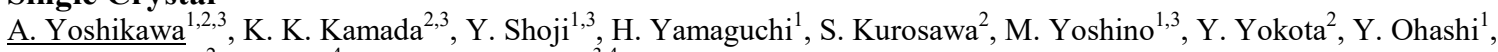
M. M. Arakawa ${ }^{2}$, M. Nikl ${ }^{4}$, V. V. Kochurikhin ${ }^{3,4}$

${ }^{I}$ IMR, Tohoku University, Sendai, Japan

${ }^{2}$ NICHe, Tohoku Universiry, Sendai, Japan

${ }^{3}$ C\&A Corporation, Sendai, Japan

${ }^{4}$ Institute of Physics AS CR, Prague, Czech Republic

After a decade of R\&D of the Lu3A15O12 (LuAG) -based single crystal scintillators, new material concept was defined, based on multicomponent $(\mathrm{Gd}, \mathrm{RE}) 3(\mathrm{Ga}, \mathrm{Al}) 5 \mathrm{O} 12$ host, $\mathrm{Re}=\mathrm{Lu}, \mathrm{Y}$. Doped by $\mathrm{Ce} 3+$, the Gd- and Ga rich host compositions showed amazingly high light yield up to almost 50,000 phot/MeV which is the value exceeding by $30-40 \%$ the best Ce:LYSO materials ever seen. Recently, our group reported about Ce:Gd 3A12Ga3O12 (GAGG) single crystal and scintillation response of about $~ 90$ $\mathrm{ns}$ at emission around $520 \mathrm{~nm}$, excellent light yield of about 56000 photon/MeV, and density of $6.63 \mathrm{~g} / \mathrm{cm} 3$. In the silicate, perovskite and garnet scintillators the slow tunneling-driven radiative recombination between Ce emission centers and nearby lying electron traps can deteriorate scintillation performance. Improvement of scintillation performance by co-doping with alkali earth AE2+ ions in Ce activated scintillators such as, LSO:Ce,Ca, LYSO:Ce,Ca, and LuAG:Ce,Mg. Such an approach has been used in $\mathrm{Mg}$ or Ca co-doped Ce:GAGG single crystals the scintillation decay time of which was enormously accelerated and the presence of Ce4+ was clearly identified by its characteristic charge transfer (CT) absorption in the near UV range below $350 \mathrm{~nm}$. In this study, we report the bulk crystal growth of 3inch size single crystals of Ce:GAGG and Mg co-doped Ce:GAGG. we investigated the relationship between Ce concntration, $\mathrm{Mg}$ concentration, and optical, luminescence and scintillation properties of Ce:GAGG and Mg,Ce:GAGG crystal grown by the Czochralski method. Light yield of Mg 0.1\% co-doped sample showed $79 \%$ of light output comparing with Ce:GAGG standard with 56000 photon/MeV and around 44,000 photon/MeV. Non co-doped GAGG showed decay time of $92.2 \mathrm{~ns}(81 \%)$ and $296 \mathrm{~ns}(19 \%)$. The scintillation decay time was accelerated by Mg co-doping and decay time value decreased down to $45.2 \mathrm{~ns}(58 \%)$ and $135 \mathrm{~ns}(42 \%)$ at $\mathrm{Mg} 0.1 \%$ sample.

This work is partially supported by Adaptable \& Seamless Technology Transfer Program through Target-driven R \& D (A- STEP), JST, Development of Systems and Technology for Advanced Measurement and Analysis, Japan Science and Technology Agency (JST) and New Energy and Industrial Technology Development Organization (NEDO).

N29-50: Effect of Digital DAQ System Properties on the Pulse Shape Discrimination Performance of CLYC

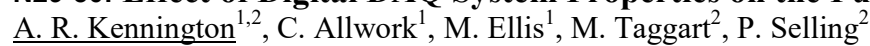


${ }^{I} A W E$, Reading, UK

${ }^{2}$ Department of Physics, University of Surrey, Guildford, UK

The need for simultaneous neutron and gamma detection continues to motivate the development of dual mode radiation detectors. One promising candidate is the elpasolite inorganic scintillator CLYC. Previous studies, with other dual neutron-gamma detectors (EJ299/EJ309), have shown that the choice of digital data acquisition (DAQ) system can significantly affect pulse shape discrimination (PSD) performance. In this work, results are presented from a 2" CLYC crystal, with PMT pulses captured by Caen V1720, V1751 and V1730 digitisers. Through offline analysis, PSD performance was explored as a function of digitisation parameters. Particular attention was given to assessing where diminishing returns may lie, so that reductions to the size and power requirements can be made for use with compact CLYC detector systems in the future.

\section{N29-51: Micro-Pulling down Method; from Materials Screening of Novel Scintillator to Bulk Crystal Growth} of Scintillator Crystal

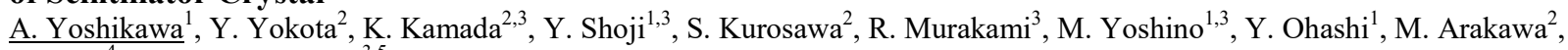
M. Nikl ${ }^{4}$, V. V. Kochurikhin ${ }^{3,5}$

${ }^{I}$ IMR, Tohoku University, Sendai, Japan

${ }^{2}$ NICHe, Tohoku Universiry, Sendai, Japan

${ }^{3} C \& A$ Corporation, Sendai, Japan

${ }^{4}$ Institute of Physics AS CR, Prague, Czech Republic

${ }^{5}$ General Physics Institute, Moscow, Russia

A scintillator works as a spectral and energy transformer: it converts a high energy photon from the X- or gamma-ray range into a bunch of ultraviolet-visible (UV/Vis) ones, i.e., to a flash of light. Alternatively, accelerated charged particles (electrons, protons or heavier ions) or even neutrons can be detected through their energy deposit in interaction with a scintillator host, which is again converted into a flash of light. Inorganic scintillators are probably the most used in practical applications nowadays and intense research and development (R\&D) activities have continuously taken place in last few decades with big market potential and prospects in coming years as well. Micro-Pulling-Down ( $\mu$-PD) method is a useful tool to search for new materials. This method allows us to prepare single crystalline materials quickly and relatively inexpensively. Grown crystals are of sufficient dimensions for all the necessary characterization by optical and luminescence methods, including photo- and radioluminescence, decay kinetics and light yield measurements. In this study, the detail of micro-pulling down method is shown together with the example of material screening for multi-component garnet (Ce,Gd, $\mathrm{Y}, \mathrm{Lu}) 3(\mathrm{Al}, \mathrm{Ga}) 5 \mathrm{O} 12$. Moreover, bulk crystal growth of Eu:SrI2 is also shown using the micro-pulling down furnace.

This work is partially supported by Adaptable \& Seamless Technology Transfer Program through Target-driven R \& D (A- STEP), JST, Development of Systems and Technology for Advanced Measurement and Analysis, Japan Science and Technology Agency (JST) and New Energy and Industrial Technology Development Organization (NEDO).

\section{N29-52: New Radiation-Hard Wavelength Shifting Fibers}

B. Bilki ${ }^{1}$, D. Winn ${ }^{2}, \underline{\text { Y. Onel }}{ }^{1}$

${ }^{I}$ Physics and Astronomy, University of Iowa, Iowa City, IA, USA

${ }^{2}$ Physics, Fairfield University, Fairfield, CT, USA

R\&D on new radiation-hard wavelength shifting fibers is gaining crucial importance as the radiation conditions projected for the High Luminosity LHC and future hadron and lepton colliders reach unprecedented levels. We have identified materials with proven radiation resistance, long Stokes shifts to enable long self-absorption lengths, with decay constants $\sim 10 \mathrm{~ns}$ or less. Here we describe two strong candidates Doped $\mathrm{ZnO}: \mathrm{Zn} / \mathrm{Mg}$ and $3 \mathrm{HF}$ (3-hydroxyflavone) properties and report on basic performance characteristics of recent prototypes.

\section{N30: Simulation and prototyping for detector development - Poster session II}

Tuesday, Nov. 1 14:00-16:00 Etoile

N30-1: Simulations and Test Results of Large Area Continuous Position Sensitive Diamond Detectors

M. Ciobanu $^{1}$, M. Pomorski ${ }^{2}$, E. Berdermann ${ }^{3}$, A. Braeuning-Demian ${ }^{3}$, C. Bunescu ${ }^{3}$, V. Constantinescu ${ }^{1}$, M. Kišs ${ }^{3}$, O. Marghitu ${ }^{1}$, M. Mayr ${ }^{4}$, M. Schreck ${ }^{4}$, M. Träger ${ }^{3}$, K. O. Voss $^{3}$

${ }^{I}$ ISS, Bucharest, Romania

${ }^{2}$ CEA-LIST, Saclay, France

${ }^{3}$ GSI, Darmstadt, Germany

${ }^{4}$ University of Augsburg, Augsburg, Germany 
We report on the development of Continuous Position Sensitive Diamond Detectors (CPSDD). The first CPSDD was realized in 2009 on a homoepitaxial single crystal (sc) base material of an area of (4 x 4) $\mathrm{mm}^{2}$ and a thickness of $300 \mu \mathrm{m}$. The high efficiency of sc diamond detectors (sc DD) provided high enough Signal to Noise (S/N) ratio enabling tests of the CPSDD with alpha particles. The first Large Area CPSDD (LACPSDD) was fabricated in 2012 on a $(30 \times 30) \mathrm{mm}^{2}$ polycrystalline (pc) diamond plate of $350 \mu \mathrm{m}$ thickness. In this case, in beam tests with ${ }^{54} \mathrm{Ni} 1.7 \mathrm{AGeV}$ showed an ion rate limitation due to the significantly higher detector time constant (RDCD) resulting from the much larger capacitance CD of such a large diamond plate compared to that of the homoepitaxial sc DD. In addition, the reduced Charge Collection Efficiency (CCE 0.1) of the pc DD resulted in much lower signal amplitude and thus a lower $\mathrm{S} / \mathrm{N}$ ratio. In 2015 we tested two new LACPSDD realized on pc basis materials: one of $(10 \times 10) \mathrm{mm}^{2}$ area and thickness of $110 \mu \mathrm{m}$ and the other one of $(20 \times 20) \mathrm{mm}^{2}$ and $180 \mu \mathrm{m}$, respectively. Each detector consisted of DLC layers deposited on both faces and each layer is equipped with two parallel metallic charge collection electrodes (on one side along the $\mathrm{x}$ and on the other side and along y direction). Finally in 2016 we produced the same structure on the basis of heteroepitaxial single-crystal diamond grown on iridium (DOI). The impact position is obtained via charge division measurements using four charge sensitive amplifiers (CSA). A careful tuning of the electronics is required in order to meet the challenge of the reduced $\mathrm{S} / \mathrm{N}$ ratio and the high RDCD constant, affecting directly the accuracy of the position reconstruction. We present preliminary simulations intended to optimize the LACPSDD read out, as well as experimental results of tests with alpha particles and ${ }^{12} \mathrm{C}$ micro-beam ions of $11.4 \mathrm{AMeV}$.

N30-2: Femtosecond Resolution Timing Extraction Study for a Waveform Sampling ASIC P. Orel, G. S. Varner

Dept. Phys. and Astr., University of Hawaii at Manoa, Honolulu, HI, USA

Timing resolution on the femtosecond scale enables the possibility of a novel approach to vertex detectors for high luminosity particle colliders. Present efforts are centered on the development of an application specific integrated circuit (ASIC) that can measure signal arrival times with timing resolution in the range of $100 \mathrm{fs}$ or less. Timing resolutions on the picosecond scale have been reported using the waveform sampling technique. The purpose of this paper is to study and analyze the viability of this technique in the femtosecond regime. A synthetic waveform generator has been developed to emulate detector response and a simulation environment has been set up to allow for decoupling of hardware and software related effects. This has made it possible to evaluate and quantify their respective contributions. Results from the simulation indicate deviations in the overall timing resolution from simple extrapolation. Possible causes for this deviations are identified and discussed, thus providing important guidelines for the design of the ASIC.

N30-3: Systematic Error of Counting Efficiency Estimation due to Spectral Conformity Assessment in Geant4 Based Liquid Scintillation Counter Simulation

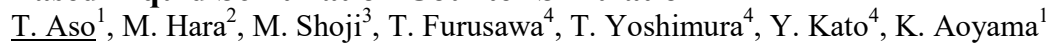

${ }^{I}$ Electronics and Computer Engineering, National Institute of Technology, Toyama College, Toyama, JAPAN

${ }^{2}$ Hydrogen Isotope Research Center, University of Toyama, Toyama, JAPAN

${ }^{3}$ Life Science Research Center, University of Toyama, Toyama, JAPAN

${ }^{4}$ Hitachi, Ltd., Tokyo, JAPAN

Liquid scintillation counter (LSC) is the most powerful tool for a quantitative measurement of radioactivity in the field of low level and low energy beta decay radionuclide. LSC counts the number of flushes of scintillation lights which are generated by the charged particles of decay products, e.g. electrons in C-14 and H-3 decay. However, due to the influence of quench, part of the nuclear decay energy and the generated scintillation photons may escape the detection. Therefore, the compensation of the count rate loss is essential to determine the sample's activity. We previously investigated the performance of Geant4 Monte Carlo simulation for estimating counting efficiencies of C-14 and H-3 samples, respectively. In the simulation, the counting efficiency of each quench sample was estimated by adjusting the scintillation photon yield to reproduce the measured LSC spectrum. Since the conformity assessment of LSC spectra between a simulation and a measurement directly influences the counting efficiency, the assessment protocol need to be verified. In this paper, the conformity of simulated and measured LSC spectra was examined by introducing three feature points, which were defined at $50 \%, 20 \%$ and $5 \%$ of the peak counts in the spectrum. The deviation of pulse height at each feature point and the detection efficiency were obtained by varying the scintillation photon yield. Then, the relations among the pulse height deviations, detection efficiencies and scintillation photon yields were analyzed. As a result, the most feasible feature point was the $5 \%$ of the peak counts in the spectrum for both $\mathrm{C}-14$ and $\mathrm{H}-3$, respectively. The differences of counting efficiencies between simulations and measurements were within the measurements' uncertainty, when the simulated spectrum coincides with the measured spectrum at the feature point.

This work was supported in part by JSPS KAKENHI Grand Numbers 26610065.

N30-4: Fissile Mass Quantification in Radioactive Waste Packages Using Photofission Delayed Gamma Rays E. Simon $^{1}$, F. Jallu ${ }^{1}$, B. Pérot ${ }^{1}$, S. Plumeri ${ }^{2}$ 
${ }^{I}$ DEN/CAD/DTN/SMTA/LMN, CEA, Cadarache, France

${ }^{2}$ Andra, Chatenay-Malabry, France

This paper reports a feasibility study, performed by numerical simulation with MCNPX, for fissile mass characterization in dense, large volume, long-lived and medium activity radioactive waste packages, using photofission delayed gamma-rays. Highenergy photon interrogation with a $15 \mathrm{MeV}$ LINAC has been modelled for two types of cemented waste packages, coupled to high resolution spectroscopy of photofission delayed gamma rays. The study was carried out by assessing the passive and active backgrounds respectively due to the waste package gamma emission, and to material activation during irradiation, in view to determine the detection limits for the main delayed gamma rays of interest. The obtained detection limits are lower than the expected uranium mass for the two waste packages, but the sensibility to the waste matrix density and uranium localization, which imposes to get an accurate measurement of these parameters by other means like high-energy X-ray imaging and photofission tomography. On the other hand, as the photofission signal results from both fissile ${ }^{235} \mathrm{U}$ and fertile ${ }^{238} \mathrm{U}$ isotopes, a method for uranium isotopes discrimination based on gamma-ray ratios has been evaluated, showing that photofission delayed gamma rays can be used to assess the fissile mass as well as the total uranium mass.

N30-5: Characterization of Monolithic SDD Arrays and SFERA ASIC for Siddharta Experiment $\underline{\text { A. D. Butt }}^{1,2}$, G. Bellotti ${ }^{1,2}$, M. Carminati ${ }^{1,2}$, C. Fiorini ${ }^{1,2}$

${ }^{I}$ Dipartimento di Elettronica, Informazione e Bioingegneria, Politecnico di Milano, Milano, Italy

${ }^{2}$ Sezione di Milano, Instituto Nazional di Fisica, Milano, Italy

This work deals with the first characterization results of detection system developed within the scope of SIDDHARTA experiment. SIDDHARTA is a nuclear physics experiment aimed at the study of strong nuclear interactions using exotic atoms. This is achieved using monolithic arrays of Silicon Drift Detectors (SDDs), each consisting of eight SDD elements arranged in a $2 \times 4$ format (total area $18 \times 34 \mathrm{~mm} 2$ ). A total of $48 \mathrm{SDD}$ arrays arranged on a gantry structure are required to perform the experiment. Each SDD element is coupled to a CUBE pre-amplifier with the consequent shaping amplifier and complementary analog electronic stages implemented in a custom developed 16-channel SFERA chip. During the experiment, SDD arrays must be cooled down to cryogenic temperature $(100 \sim 150 \mathrm{~K})$ to perform X-ray spectroscopy to understand spread/shift of energy levels of exotic Kaonic atoms. Alongside the cryogenic operation, the X-ray spectrometer needs to operate with a linearity of around 1 $\%$ with an output stability of a few eV/day. This work describes the preliminary characterization results of cryogenically cooled $2 \times 4$ SDD arrays readout by SFERA chip. These include X-ray spectroscopy, output yield stability and output linearity performances.

N30-6: Gamma Beam Characterization System for ELI-NP: the Absorption Calorimeter $\underline{\text { R. Borgheresi }}$

Università degli studi di Firenze and INFN Firenze, Firenze, Italy

On behalf of the ELI-NP Gamma Beam Characterization Team

The ELI-NP Gamma Beam System will deliver an intense gamma beam with unprecedented specifications in terms of brilliance, photon flux and energy bandwidth in an energy range from 0.2 to $20 \mathrm{MeV}$. A characterization system providing a measurement of the energy spectrum, intensity, space and time profile of the beam is essential for the commissioning and development of the source, as well as to demonstrate the performance achieved. The specification of the gamma pulse prevents to use any traditional gamma spectroscopic detector, so a specific system equipped with four basic elements is under development: a sampling calorimeter, for a fast combined measurement of the beam average energy and its intensity; a Compton spectrometer, for photon energy spectrum measuring and monitoring; a nuclear resonant scattering spectrometer, for an absolute beam energy calibration of the other detectors; and finally a beam profile imager to be used for alignment and diagnostics purposes. An overview of the ELI -NP gamma characterisation system will be shown in this work, with a focus on the calorimeter working principle, its expected performances and the preliminary prototype tests.

\section{N30-7: Development of Cherenkov Light Imaging System for Studies of Radiocesium Dynamics in Plant} $\underline{\text { K. Kurita }}^{1}$, N. Suzui ${ }^{1}$, Y. Y-G. Yin ${ }^{1}$, S. Ishii ${ }^{1}$, H. Watabe ${ }^{2}$, S. Yamamoto ${ }^{3}$, N. Kawachi ${ }^{1}$

${ }^{I}$ Department of Radiation-Applied Biology, National Institutes for Quantum and Radiological Science and Technology, Takasaki, Gunma, Japan

${ }^{2}$ Cyclotron and Radioisotope Center, Tohoku University, Sendai, Miyagi, Japan

${ }^{3}$ Graduate School of Medicine, Nagoya University, Nagoya, Aichi, Japan

High resolution images of radiocesium (Cs-137) distribution are required to research cesium kinetics in plants. Cherenkov light imaging can visualize fine distributions of radionuclides that emit beta particles using an optical camera. We have developed an imaging system with this method to study radiocesium movements in intact plants. The system was constituted of a high 
sensitivity cooled charge coupled device camera and a bright lens placed in a black box. To evaluate a linearity of the system, an imaging test was performed with point sources of Cs-137 which have 10 2000 kBq radioactivity. As a result, the data indicated that the system has a good linearity between the image intensity and the radioactivity of Cs-137 and which has a range of the order of $10^{3}$. To demonstrate an imaging ability of radiocesium in a plant, an imaging experiment was performed with an intact soybean plant for 7 days. First, the root of the 13 days-old soybean had been dipped in $17 \mathrm{~mL}$ of the culture solution containing $10 \mathrm{MBq}$ of Cs-137 without potassium. After one day, the solution was replaced to the no Cs-137 solution with potassium. As a result, the high resolution serial images indicated that Cs-137 was transported to the shoot and accumulated into node. Above two results, Cherenkov light imaging may be concluded to be as the promising method for imaging of radiocesium in intact plants.

This study was supported in part by JSPS KAKENHI no. 15H06851, 16H03908.

N30-8: Silicon Pixel Sensor Processing on 8" (200 mm) FZ and MCZ Wafers

J. J. Kalliopuska, K. Lavanti, S. Vähänen, J. Salmi

Semiconductors, Advacam Oy, Espoo, Finland

The processing of Si pixel sensors has been mostly done on 4"-6" (100-150 mm) high resistivity wafers for the past 10 years. Recently, both the Float Zone (FZ) and Magnetic Czochralski (MCZ) silicon have become available in 8" (200 mm) wafers with higher resistivity than $5000 \mathrm{Ohm}-\mathrm{cm}$ and lifetimes of few milliseconds. The main advantage in transferring the production to 8 " wafers is the doubled process area compared to 6" wafers. This enables either manufacturing of large-area sensors up to $15 \times 15$ $\mathrm{cm} 2$ or doubling the number of produced sensors per wafer.

Advacam is prototyping fabrication of n-on-p and p-on-n polarity, fine pitch, pixel sensors on 8" FZ and MCZ wafers. The pixel sensors with $55 \mathrm{um}$ pitch will be flip chip bonded to Medipix readout ASICs and constructed to cameras to characterize the suitability of the sensor materials for X-ray imaging and particle tracking. All the 8 " wafers received from the suppliers are Single Side Polished (SSP) having thickness of $725 \mu \mathrm{m}$. At the beginning of the processing these wafers are thinned and polished to varied thicknesses of $300,380,500$ and $675 \mu \mathrm{m}$.

The presentation will describe the designs used for the sensors and their processing sequence on 8 " wafers. Comparison of the processing on 6" and 8" wafers will be given in terms of the overall yield, electrical characterization and X-ray imaging properties. The electrical characterization comprises I-V and $\mathrm{C}-\mathrm{V}$ measurements performed across the wafer. The X-ray imaging properties comprise characterization of the large area $1 \times 5$ and 2x5 Medipix cameras for X-ray inspection of lightweight materials. The following imaging quality related properties are presented: flip chip-bonding yield of the cameras, uniformity of the sensor materials in terms of resistivity and full charge collection efficiency; charge sharing between the pixels and between the Medipix ASICs at their boundaries; and overall stability of the cameras.

N30-9: Experimental Assessment of Electron Ionization Cross Sections

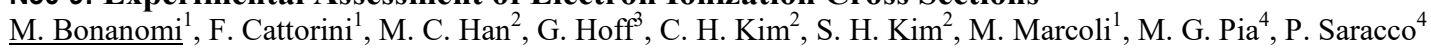

${ }^{I}$ University of Milano Bicocca, Milano, Italy

${ }^{2}$ Hanyang University, Seoul, Korea

${ }^{3}$ CAPES, Brasilia, Brazil

${ }^{4}$ INFN Genova, Genova, Italy

Accurate simulation of ionization by electron impact is of great importance in Monte Carlo simulation in many experimental domains, as this process - involving either primary or secondary electrons interacting with materials - contributes to determine the observed detector signal in a wide variety of experimental scenarios. We report an extensive investigation of theoretical and semi-empirical calculations of electron impact ionization cross sections, detailed by individual shells: they encompass the popular tabulations of the EEDL data library (also distributed within ENDF/B-VII) used by Geant4, MCNP and other codes, recent calculations used in Penelope, as well as other models not yet used in general-purpose Monte Carlo transport codes. All models have been subject to a rigorous validation test against a wide collection of experimental measurements. Special attention has been devoted to possible sources of systematics affecting the validation process, both of physical and mathematical origin. As most of the data reported in the literature as experimental measurements of ionization cross sections actually derive from X-ray production measurements, the systematic effect of different compilations of fluorescence yields has been quantitatively assessed. The compatibility of calculated and experimental cross sections has been further examined with categorical analysis methods to determine whether the observed differences across the various models are statistically significant. The results of this validation process identify objectively and quantitatively the state of the art in modeling electron impact ionization; they are relevant for the improvement of ionization modeling in Monte Carlo codes.

N30-10: Response of the MST Microdosimeter to Low-Energy Carbon Ions

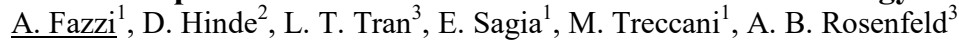


${ }^{I}$ Dept of Energy, Politecnico di Milano, Milano, Italy

${ }^{2}$ Dept of Nuclear Physics, Australian National University, Canberra, Australia

${ }^{3}$ Centre for Medical Radiation Physics, University of Wollongong, Wollongong, NSW, Australia

Monolithic Silicon Telescope microdosimeter has proved to assay the radiation quality of therapeutic beams reproducing the response of reference Tissue Equivalence Proportional Counters at medium and high lineal energy. It has been studied the response to very low energy carbon ions $(5.83 \mathrm{MeV} / \mathrm{u})$, fragmentation of which is negligible, as a reference for a comparison with therapeutic conditions, where fragmentation plays an important role. The measurement campaign has been performed at the Heavy Ion Accelerator Facility at the Australian National University in Canberra.

N30-11: Characterization of a Commercial CMOS Imager for Laser-Driven Accelerated Particles Diagnostics

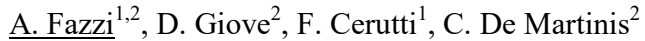

${ }^{I}$ Dept. of Energy, Politecnico di Milano, Milano, Italy

${ }^{2}$ Milan Section, INFN, Milano, Italy

In order to develop a diagnostic tool for charged particles (mainly protons) produced in laser-driven acceleration experiments, a commercial CMOS imager, the Rad-Eye by Teledyne, has been characterized. The main parameters affecting the response of the imager to visible light like thermal generation (leakage), reading noise, linearity and dynamic range have been addressed. A series of measurements with Alpha particles of different energy allowed to study the response of the imager to charged particles. Results can be reproduced by a simple model of the device charge collection that has to take into account charge diffusion from the bulk. The model can thus supply the response to monoenergetic light particles as required by the diagnostic tool.

N30-12: Mapping Tool for Investigation of Component-Level PCB Compatibility in Multimodal MRI/SPECT G. L. Montagnani ${ }^{1,2}$, M. Occhipinti ${ }^{1,2}$, M. Carminati ${ }^{1,2}$, C. E. Fiorini ${ }^{1,2}$

${ }^{I}$ Dipartimento di Elettronica, Informazione e Bioingegneria, Politecnico di Milano, Milano, Italia

${ }^{2}$ Sezione di Milano, INFN, Milano, Italia

A maneuverable test bench device has been developed to evaluate the compatibility of a gamma ray detector and MRI magnetic switching gradients. The device was designed to characterize the sensitivity to magnetic gradients of the detector modules of INSERT, a SPECT ring compatible with commercial MRI machines. The MRI gradients switching has revealed to be the most aggressive source of pick-ups to the electronics boards, as, contrary to RF pulses, shielding results quite ineffective. Spatial mapping of board sensitivity is obtained with sub-cm resolution. i.e. with single component assessment capability. The timevarying magnetic field generated with our tool (up to a slope of $100 \mathrm{~T} / \mathrm{s}$ from $10 \mathrm{kHz}$ to $1 \mathrm{MHz}$ ) can be tailored to be identical in amplitude and frequency to the one utilized in common MRI sequences. Differently form the in-field case, it can be localized in such a way that sensitive points inside the hardware under test can be identified. The device has been utilized to locate weaknesses inside the ASIC board of the INSERT system, and it was then used to evaluate the improvement obtained with a new board designed to reduce the coupling to the magnetic field, tested with real DWI sequences in a hospital facility. In this work we describe the development of this tool and, as an example of its successful employment, how the tool has been used to investigate the compatibility of the electronics board of the INSERT detector.

\section{N30-13: Online Cluster Finding Algorithms for the PANDA Electromagnetic Calorimeter} M. Tiemens

KVI-CART, University of Groningen, Groningen, Netherlands

On behalf of the PANDA collaboration

Three algorithms, designed to search for clusters in the electromagnetic calorimeter (EMC) of the future PANDA experiment, are described and evaluated. The high rate of $2 \mathrm{E} 7$ interactions/s between the antiproton beam and the proton target leads to a high probability for pile-up and event mixing, complicating the search for clusters. To reduce the amount of data produced by the experiment, the detector will preprocess as much data as possible online, and select events of interest based on online reconstruction. To perform these reconstructions, input from the cluster finding algorithm, which is part of the online preprocessing, is essential. The algorithms are tested using simulated data for both their efficiency and processing time using two 5000 event data samples in the high rate case, a lower rate case (factor 100 lower rate), and the case with completely disjoint events. The first sample is a low photon-multiplicity sample of direct two-photon production events. The efficiency, i.e. the number of successful reconstructions, is similar for all methods, around $90 \%$. The algorithm that is most eligible for online usage runs the fastest, as expected. The second sample tests the high photon-multiplicity channel where the hc meson decays to 7 photons, and will also be expanded with background events. More detailed results on the performance evaluation will be presented. 
N30-14: GATE Simulation of a High Performance Stationary SPECT System for Cardiac Imaging D. Uzun Ozsahin $^{1}$, L. Blackberg ${ }^{2}$, G. El Fakhri ${ }^{1}$, N. Moghadam ${ }^{3}$, H. Sabet ${ }^{1}$

${ }^{I}$ NMMI Radiology Department, Massachusetts General Hospital, Harvard Medical School, Boston, MA 02129, USA

${ }^{2}$ Physics and Astronomy, Uppsala University, Uppsala, 75120, Sweden

${ }^{3}$ Electrical Engineering, Electronic Engineering, Engineering Physics, Université de Sherbrooke, Québec, Canada

We are developing a stationary cardiac SPECT platform that can provide high-resolution images. Unlike the two state of the art commercial systems, GE's NM-530 and Spectrum-Dynamic's D-SPECT, that are based on expensive CZT detector modules, our design is based on low cost scintillator detectors (such as CsI:Tl or NaI:Tl) pixelated by using laser-induced optical barriers technique. We report here the GATE simulation results of various versions of our cardiac SPECT platform, DC-SPECT in comparison with dual head gamma camera (DHGC) as well as other mainstream cardiac SPECT systems. We used NEMA-NU2001 protocol in the simulations. We have optimized our system design as a function of number of detector modules, detector area, magnification, detector resolution, and sensitivity at fixed resolution. In DC-SPECT system, each laser processed CsI:T1 detector is backed by a pinhole collimator (with no projection overlap) where each pinhole can provide a complete view of the heart. In one design of the system, there are 45 detector modules arranged in 3 rows providing 225 degree detector arc. The result show that the proposed system can provide 0.073 system geometric sensitivity (SGE) at $10 \mathrm{~mm}$ FWHM system spatial resolution (SR) without resolution recovery at a $20 \mathrm{~cm}$ pinhole-FOV distance. SGE and SR are 0.12 and $8 \mathrm{~mm}$ at $15 \mathrm{~cm}$ pinholeFOV distance. This translates to $\sim 10 \mathrm{x}$ larger sensitivity compared with DHGC encouraging a lower patient dose, larger patient throughput, or the possibility of dynamic cardiac studies.

The author acknowledges support from the The Scientific and Technological Research Council of Turkey (TUBITAK).

N30-15: Design and Optimization of the Cherenkov TOF Full-Body PET Scanner

M. Alokhina $^{1,2}$, C. Canot ${ }^{1}$, O. Kochebina ${ }^{1,3}$, O. Bezshyyko ${ }^{2}$, I. Kadenko ${ }^{2}$, G. Tauzin ${ }^{1}$, D. Yvon ${ }^{1}$, V. Sharyy ${ }^{1}$

${ }^{I}$ IRFU, CEA, Saclay, France

${ }^{2}$ Nuclear Physics Department, Taras Shevchenko National University of Kyiv, Kyiv, Ukraine

${ }^{3}$ SHFJ, CEA, Orsay, France

In this presentation we discuss the simulation of the time-of-flight (TOF) full-body PET scanner using GATE software. The proposed device will use the lead fluoride crystalline Cherenkov radiator coupled to the fast micro-channel-plate photomultiplier. This simulation is motivated by the on-going development of the fast and efficient $511 \mathrm{keV}$ gamma detector at IRFU, CEA. We demonstrated that the photon coincidence resolving time of about $150 \mathrm{ps}$ (FWHM) or better could be achieved with the reasonable efficiency. Cherenkov detectors don't provide any resolution in energy, but have intrinsic capability to reject Compton scattered events, which allows to keep the scattering contribution under control. The spatial resolution of this detector depends on the size of the crystals in a same manner as for the conventional PETs. We consider different geometrical configurations of the elementary detectors, different crystal coatings and different options for the optical interfaces. We optimize the scanner configuration using the noise equivalent count rate, modified to account for the TOF benefits of the proposed device.

\section{N30-16: Alpha Contamination Assay, Dosimetry and Spectrometry Using Charge Coupled Devices}

$\underline{\text { R. Newton }}^{1}$, M. J. Joyce ${ }^{1}$, M. J. Scott ${ }^{2}$

${ }^{I}$ Engineering Department, Lancaster University, Lancaster, UK

${ }^{2}$ BIC Technology Ltd., Liverpool, UK

The potential of using charge coupled devices (CCDs) to perform in-situ spectroscopy of alpha contamination has been investigated. Literature and previous research shows that alpha particles give a distinctive readout from CCDs when compared with other types of radiation. TRIM (Transport of Ions in Matter) simulations have been performed to identify optimum features of CCDs for detecting alpha particles. It has been shown that thinner gate structures are preferable for low-energy alpha particles (less than $2 \mu \mathrm{m}$ thickness). For high-energy particles a sensitive region of at least $56 \mu \mathrm{m}$ is required for maximum charge collection. More than $90 \%$ of the ionisation has been shown to occur in the sensitive region for a wide range of alpha particle energies using typical CCD specifications and 78\% useful ionization occurred for a thick structure at an energy of $2.232 \mathrm{MeV}$. An experimental investigation into the response of CCDs to alpha particles is described to focus on the relationship between the size of the pixel clusters, the corresponding extent of blooming and the incident alpha particle energy.

This work was supported by the Next Generation Nuclear Centre for Doctoral Training and the Engineering and Physical Sciences Research Council (EPSRC).

N30-17: Wraparound Conductive Cables

A. Tomada, C. Kenney, J. Segal 
Wraparound Conductive Traces Astrid Tomada, Chris Kenny, Julie Segal

Most microsystem interconnections are formed using wire bonds or bump bonds, both of which have enabled cutting-edge science such as nEXO, LHC, Fermi, LCLS, CDMS, etc. Wire bonding is an intrinsically one-dimensional process, while bump bonding is two-dimensional, but limited to connecting a pair of parallel substrates. Several national laboratories -concurrently with Tezzaron Inc- have been jointly pursuing a partially 3D interconnect technology, which they have the potential to increase the amount of circuitry per pixel by a factor of 2-3. This development effort has taken considerable time and resources, while producing very modest results due to the intrinsically difficult nature of the processing involved. While increasing the amount of circuitry per pixel by a factor of 2-3 it clearly represents a very marginal gain given the increased cost. In contrast, the technology presented here is less expensive, simpler, and can increase the circuitry per pixel by a factor between 10 and 100 . Fabricating wraparound conductive traces is a key element of the success of this technology. In this work we demonstrate the ability to form metal traces that wrap around a chip edge. Tests have shown that all these traces are electrically conducting around the corner and that there are no shorts between neighboring traces. This technique is less challenging than fabricating TSV on silicon substrates, especially if dealing with quartz-like substrates. In this paper we will show the process and results of fabricating traces that wrap around the edge of substrates.

\author{
N30-19: Realistic Hit Reconstruction in the CBM Silicon Tracking System \\ H. Malygina $^{1,2,3}$, V. Friese ${ }^{1}$, M. Zyzak ${ }^{1,2,4}$ \\ ${ }^{I}$ GSI, Darmstadt, Germany \\ ${ }^{2}$ Goethe University, Frankfurt, Germany \\ ${ }^{3}$ KINR, Kyiv, Ukraine \\ ${ }^{4}$ FIAS, Frankfurt, Germany
}

The Silicon Tracking System (STS) is the main tracking appliance of the future Compressed Baryonic Matter (CBM) experiment (a) FAIR. The STS employs double-sided silicon micro-strip sensors with double metal layers. An accurate detector model is a prerequisite for proper design and correct reconstruction of physical signals. In this report, we describe the hit reconstruction in the STS. Our detector response model includes the charge creation, its drift, collection, the cross-talk, and digitization. The unbiased cluster position finding algorithm, which we use, simplifies the hit error estimation and yields a spatial resolution close to that obtained with the Centre-Of-Gravity algorithm. We have developed a method to estimate the hit error, which includes the non-uniformity of an incident particle energy loss, the detector noise, the signal discretisation, and the error introduced by the cluster position finding algorithm. A reliable estimate of the hit position error is required to obtain a valid $\chi^{2}$ of the track, which is further used to discard ghost track candidates. This improves the signal-to-background ratio of the reconstructed physical signals. Both the pull (residual/error) and the $\chi^{2}$ distributions verify the viability of the method: the pull width is about 1 , its shape reproduce the shape of the residual distribution, the $\mathrm{p}$-value of $\chi^{2}$ is flat.

\title{
N30-20: Validation of Geant4 Electron Pair Production by Photons
} M. Begalli ${ }^{1}$, G. Hoff ${ }^{2}$, M.-G. Pia ${ }^{3}$, P. Saracco ${ }^{3}$, C. Choi $^{4}$

${ }^{I}$ Physics Institute, State University of Rio de Janeiro (UERJ), Rio de Janeiro, Brazil

${ }^{2}$ Politecnic Institute of Rio de Janeiro (IPRJ), State University of Rio de Janeiro (UERJ), Nova Friburgo, Brazil

${ }^{3}$ INFN-Sezione di Genova, Istituto Nazionale di Física Nucleare (INFN), Genova, Italy

${ }^{4}$ Hanyang University, Seoul, Korea

We present a comparison between experimental data collected in the literature and models implemented in the Geant 4 toolkit for the simulations of photon conversion; we also discuss how other widely used Monte Carlo codes deal with this process. Geant4 describes pair production in the nuclear field and in the atomic electron field (more commonly known as triplet production); it accounts for the Landau-Pomeranchuk-Migdal (LPM) effect, which is responsible for the suppression of pair production at high energies. Geant4 provides a variety of models for this purpose; nevertheless limited documentation is available in the literature regarding their validation and their relative characteristics of physical accuracy and computational performance. The available experimental database cover a wide energy range, from $1.02 \mathrm{MeV}$ (threshold) to $147.794 \mathrm{GeV}$ and atomic numbers from 1 to 92 .

Quantitative comparison results, based on statistical methods, are reported.

\author{
N30-21: Pixel Discrimination Using Artificial Neural Network for Gamma Camera Module \\ D. Kim, K. B. Kim, S. Lee, D. Jang \\ Molecular Imaging Research \& Education (MiRe) Laboratory, Sogang University, Seoul, Korea
}

Radiation interaction position in gamma camera is generally estimated using Anger logic. However, the position accuracy could be hampered by nonlinnearities and noise properties especially at the edges of scintillation crystal. The goal of this study was to 
determine a gamma ray interaction position using artificial neural network (ANN). Gamma camera detector module was composed of 4 × 4 matrix of $3 \times 3 \times 20 \mathrm{~mm}^{3}$ LYSO (Siniceramics, China), 4 × 4 array SiPM (Sensl, Ireland) and Anger circuit with four outputs. The peak values of output signals were used as position estimator in ANN consisted of 4 input nodes and 1 hidden layer with hyperbolic tangent activation functions at every node. Training was performed using delta-rule method of backpropagation algorithm and training dataset was obtained by irradiating a radiation source along a line parallel to the $\mathrm{x}$-axis and $\mathrm{y}$ axis. ANN generated highly precise pixel position map of the detector module. The performance of trained ANN was evaluated by root mean-squared-error (RMSE) and peak signal-to-noise ratio (PSNR) and the measured values were 0.022 and 0.024 RMSE and 32.21 and 29.37 PSNR for each axis, respectively. The results demonstrated that the trained ANN proposed in this study can be reliably used to estimate radiation interaction position in gamma camera and the training procedure can be easily applied to a expanded detector module. Further study will be performed with a gamma camera having large number of channels.

\section{N31: Synchroton radiation and FEL instrumentation - Poster session II}

Tuesday, Nov. 1 14:00-16:00 Etoile

N31-1: A Theoretical Model of the Pixel Response to X-Rays in Photon Counting Detectors

P. Zambon, V. Radicci, M. Rissi, C. Broennimann

DECTRIS Ltd., Baden-Daettwil, Switzerland

We present a simple, first-principles theoretical model that describes the pixel response to monochromatic x-rays in single photon counting pixelated detectors that takes into account the effects of the charge sharing and of the electronic noise on the photon counting process. The model is based on only geometrical and physical parameters such as the pixel size, the charge cloud size at the pixel depth and the total electronic noise of the front-end circuitry. The equations describing the pixel point spread function and the integral pulse height spectrum are derived preserving the genuine $2 \mathrm{D}$ nature of the charge collection process while the randomness introduced by the electronic noise in the discrimination process is treated with statistical arguments and a probabilistic interpretation is provided. The model has been qualified by fitting a set of experimental integral pulse height spectra measured with the new-generation IBEX photon counting ASIC developed at DECTRIS Ltd., coupled with a Silicon sensor 450 $\mu \mathrm{m}$ thick and $75 \mu \mathrm{m} \times 75 \mu \mathrm{m}$ pixel size, irradiated with monochromatic $\mathrm{x}$-rays in the energy range $6 \mathrm{keV}-20 \mathrm{keV}$. The fitting function adheres with excellent accuracy to the experimental curves and a comparison with a previously used empirical model shows the improvement. Moreover, the actual contributions of the charge sharing and of the electronic noise are correctly identified and estimated. Thus, the model can be used to draw precise predictions of the behavior of photon counting detection systems as a function of the design parameters.

\section{N31-2: Carbon Nanotube Yarn Field Emitter for Micro-Focus X-Ray Generation}

C. H. Lee, H. Kim, E. J. D. Castro

RIC for Next Generation Industrial Radiation Technology, Wonkwang University, Iksan,Jeonbuk, S.Korea

In this work, the field emission (FE) characteristics of multi-walled carbon nanotube (MWCNT) yarn and its contribution in the X-ray generation have been investigated. Dry spinning method was used to fabricate the MWCNT yarn from super MWCNTs, which was fabricated by MW-PECVD. The FE behavior of the MWCNT yarn followed the Fowler-Nordheim model. The MWCNT yarn displayed a significant FE capability in both diode and the triode X-ray generation structure compared to a MWCNT. The low-voltage-FE of the MWCNT yarn can be attributed to the field enhancing effect of the yarn due to its shape and the contribution of the high-aspect-ratio nanotubes that protrude from the sides of the yarn. The effect of filters on the development of X-ray images has been also demonstrated, and the amount of exposure time of the samples to the X-ray was manipulated. The MWCNT yarn can be a good candidate for use in the low voltage FE application for X-ray imaging. (1) MWCNT Yarning (2) OPERA 3D Simulation (3) Design of Diode and Triode Field Emission X-ray tubes using MWCNT Yarn (4) Images of Leaf and Spatial resolution line pattern

\section{N31-3: DSSC Ladder Camera: First Results}

M. Donato

European X-Ray Free-Electron Laser Facility GmbH, Hamburg, Germany

On behalf of the DSSC Consortium

The European X-ray Free Electron Laser (XFEL.EU) will provide every $0.1 \mathrm{~s}$ a train of 2700 coherent X-ray ultrashort-pulses at 4.5 MHz to six scientific instruments. The DEPFET Sensor with Signal Compression (DSSC) detector has been developed to meet the requirements set by the XFEL.EU soft X-ray $(0.5 \mathrm{keV}-6 \mathrm{keV})$ instruments. The DSSC imager is a 1 mega-pixel camera able to store up to 800 single-pulse images per train.

The ASIC read-out electronics provide a pixel-wise amplification and filtering, an up-to 9 bit ADC and a digital memory. The electronics has a double front-end to allow to use either the DEPFET sensors or the Mini-SDD (Silicon-Drift Detector) sensors. In the first case the signal compression is a characteristic of the sensor; in the second case the compression is generated at the 
first amplification stage. The goal of signal compression is to meet the requirement of single-photon detection capability and wide dynamic range $\left(\sim 10^{4}\right)$.

The so-called DSSC Ladder camera is the basic unit of the DSSC. It is the single unit of sixteen identical-units composing the DSSC-megapixel camera. Therefore, from the electronic point-of-view, a single DSSC Ladder contains all representative components of the full-size system and allows testing the full electronic chain including the control and the data-acquisition system, power-cycling as well as veto capabilities and timing synchronization. Each DSSC Ladder has a focal plane equipped with two sensor-modules, for a total of $64 \mathrm{k}$ pixels.

Due to the requirements of soft-X-rays beamlines, the detector will be operated under high vacuum conditions. A vacuum vessel (FENICE) designed to operate the DSSC Ladder in vacuum has been commissioned. There, the ladder can be operated at the planned operating temperature $\left(\sim-20^{\circ} \mathrm{C}\right)$ and can be moved on stages to scan the whole active area.

The DSSC Ladder camera is the first DSSC-detector demonstrator usable for scientific experiments at the beamlines. We present the first results of the DSSC Ladder under real operating conditions.

N31-4: X-Ray Elemental Mapping Using an Advanced SDD and Ultra-Fast Pulse Processing $\underline{\text { S. Barkan }}^{1}$, V. D. Saveliev ${ }^{1}$, Y.-N. Wang ${ }^{1}$, L. Feng ${ }^{1}$, M. Zhang ${ }^{1}$, E. V. Damron ${ }^{1}$, B. J. Goolsby ${ }^{1}$, R. Goldsbrough ${ }^{2}$, L. O'Ryan $^{2}$

${ }^{I}$ Hitachi High-Technologies Science America, Inc., Northridge, CA, USA

${ }^{2}$ Quantum Detectors Ltd., Harwell, Oxford, UK

With the availability of high brightness from synchrotron beam lines and new imaging techniques, scientists have a need for $\mathrm{x}-$ ray spectrometers capable of analyzing high $\mathrm{x}$-ray flux for chemical analysis applications as well as for high quality $\mathrm{x}$-ray mapping.

To address that requirement, a multi-element Silicon Drift Detector (SDD), was developed, which, combined with an advanced Digital Pulse Processor (DPP), resulted is a spectroscopic system capable of collecting quality x-ray images in short time. This work presents a significant improvement in the high count-rate capability of the 4-element SDD, the "Vortex ME-4", equipped with ASIC electronics, when combined with an advanced DPP, the "Xspress3", developed by Quantum Detectors (Harwell Oxford, UK).

The new combined system of the Vortex ME-4 and the Xspress 3 produces a dramatic increase in count rate capability and enhances the efficiency of measuring methods whenever high count rate and good energy resolution play an important role. The new spectrometer was evaluated at the Advance Photon Source (APS), located at the Argonne National Laboratories, by collecting a wide range of data using mapping and tomography techniques.

Several high quality images, collected at GSECARS, APS 13-ID-E by Matt Newville will be presented. Among the images are that of a polished thin section of garnet with zoning in yttrium, and an XRF image extracted from a zircon grain (574 pixels). Total count rate (throughput) was around 5.5 Mcps, with Iron Ka contributing around 1.1 Mcps and Zirconium Ka around 2.2 Mcps.

N31-5: Performance of the LBNL FastCCD for the European XFEL

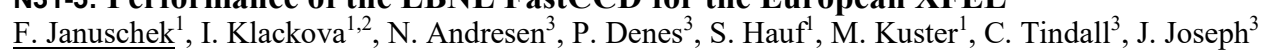

${ }^{I}$ European XFEL GmbH, Hamburg, Germany

${ }^{2}$ Institute of Nuclear and Physical Engineering, FEEIT, Slovak University of Technology, Bratislava, Slovak Republic

${ }^{3}$ Lawrence Berkeley National Laboratory, Berkeley, USA

The European X-ray Free Electron Laser (XFEL.EU) is currently being constructed in Hamburg, Germany. From 2017 onwards it will provide spatially coherent X-rays of energies between $0.25 \mathrm{keV}$ and $25 \mathrm{keV}$ with a unique timing structure. One of the detectors foreseen at XFEL.EU for the soft X-ray regime (energies below $6 \mathrm{keV}$ ) is a quasi column-parallel readout FastCCD designed by Lawrence Berkeley National Lab (LBNL) specifically for the XFEL.EU requirements. Its sensor has $1920 x 960$ $30 * 30 \mu \mathrm{m}^{2}$ pixels with a beam hole in the middle of the sensor. The camera can be operated in full frame and frame store mode. With the FastCCD a frame rate of up to $120 \mathrm{fps}$ can be achieved, but at XFEL.EU the camera settings are optimized for $10 \mathrm{~Hz}$ XFEL bunch-mode. The detector is now ready for operation at XFEL.EU. The results of the detailed performance tests and calibration done using the XFEL.EU detector calibration infrastructure and at LBNL will be presented quantifying e.g. noise levels, gains and CTI. In addition, it will be shown how the integration of the FastCCD at the beamline at XFEL.EU is planned.

\section{N31-6: Initial Results from Planar Active/Slim-Edge Pixel Sensors for XFEL Applications} $\underline{\text { G.-F. Dalla Betta }}^{1,2}$

${ }^{I}$ University of Trento, Trento, Italy

${ }^{2}$ TIFPA INFN, Trento, Italy

On behalf of the PixFEL Collaboration

Within the INFN PixFEL project, we are developing active- and slim-edge pixel sensors for X-ray imaging applications at future Free Electron Laser facilities. We have designed several different termination structures aimed at the best trade off between high 
breakdown voltage, as required to counteract plasma effects, and small dead area at the sensor edge. We report on the initial results from the characterization of the first prototypes fabricated at FBK (Trento, Italy) on 6" SiSi Direct Wafer Bonded wafers. Electrical tests have shown good results in terms of breakdown voltage, with values spanning from $\sim 400 \mathrm{~V}$ to $\sim 600 \mathrm{~V}$ depending on the oxide charge density, in good agreement with TCAD simulations. Further tests are under way to assess the charge collection behavior in response to laser pulses and radioactive sources, as well as the radiation hardness.

The PixFEL project is funded by INFN. The members of the PixFEL Collaboration are affiliated with Università di Bergamo, Università di Pavia, Università di Pisa, Università di Trento and INFN, Italy.

N31-7: Afterglow Artifacts Correction for Ultra-Fast Tomography Acquisition by Synchrotron Radiation $\underline{\text { K. Zarei Zefreh }}^{1}$, F. Marone Welford ${ }^{2}$, W. van Aarle ${ }^{1}$, J. Sijbers ${ }^{1}$

${ }^{1}$ iMinds-VisionLab, University of Antwerp, Wilrijke, Belgium

${ }^{2}$ X-ray Tomography Group, Swiss Light Source, PSI Villigen, switzerland

Thanks to the ultra-fast endstation of the TOMCAT beamline, it is possible to do a tomographic scan with 50 milisecond temporal resolution which allows following dynamic processes in 4D (3D space + time). This ultra- high-rate tomography acquisition, exploiting the distinctive peculiarities of synchrotron radiation, provides nondestructive investigation of many dynamic processes which were not possible in the past. For example a continuous tensile test has been conducted recently in-situ for the first time with a frequency of 20 tomograms per second ( $20 \mathrm{~Hz}$ acquisition frequency). In the ultra-fast endstation a scintillator is used to convert X-ray to visible photons that can be detected by the camera. However, this conversion is not ideal and the scintillator decays exponentially with afterglow. Afterglow can cause resolution degradation and artifacts (such as ring and band) especially with high rotation speed. On the other hand, to achieve a higher scan speed, thicker scintillators are more common because they result in higher emission intensities that can compensate the short exposure time in fast scans. However, the resolution deteriorates as the scintillator's thickness increases and thicker scintillators show higher afterglow. Performing many ultra-fast scans at the TOMCAT beamline with different acquisition rate, we demonstrate how the rotation speed effects on the projection data and reconstructed images. Using different thicknesses of LAG scintillator we also investigate the afterglow artifacts for different acquisition rate and exposure time. Developing a realistic model for afterglow we propose a correction method to remove afterglow from the projections which improves the reconstruction visually and quantitatively.

N31-8: The Calibration and System Simulation Software Package for the European XFEL DSSC Detector G. Weidenspointner $^{1}$, S. Schlee ${ }^{1}$, A. Castoldi ${ }^{2,3}$, F. Erdinger ${ }^{4}$, C. Guazzoni ${ }^{2,3}$, K. Hansen ${ }^{5}$, M. Kirchgessner ${ }^{4}$, S. Maffessanti ${ }^{2,3}$,

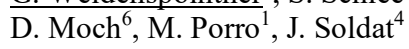

${ }^{I}$ XFEL, Hamburg, Germany

${ }^{2}$ Politecnico di Milano, Milano, Italy

${ }^{3}$ Sezione di Milano, INFN, Milano, Italy

${ }^{4}$ Universitaet Heidelberg, Heidelberg, Germany

${ }^{5}$ DESY, Hamburg, Germany

${ }^{6}$ Universitaet Stuttgart, Stuttgart, Germany

The DSSC (DEPFET Sensor with Signal Compression) is a new instrument with non-linear compression of the input signal and with parallel signal processing (filtering, linear amplification, and 8/9-bit digitization) for all pixels. The DSSC will serve as ultra fast, megapixel sized imaging detector at the European XFEL (X-ray Free Electron Laser) currently under construction in Hamburg, Germany. The DSSC design goal for initial Day-1 operation at the beamline is to achieve a high dynamic range of about 3000 photons, both for photon energies down to $0.7 \mathrm{keV}$ and read-out speeds up to $4.5 \mathrm{MHz}$. We present an overview of the DSSC detector calibration and system simulation software package, and first calibration results for DSSC prototype systems comprising $8 \times 8$ or $64 \times 64$ pixel mini-SDD sensors connected to our current prototype read-out ASICs. The system simulation package features all relevant sensor physics and read-out electronics signal processing properties. It can be used to generate realistic calibration data, for electrical input signals as well as for external input signals such as X rays, optical light, or charged particles. It can also be applied to simulate the DSSC output for an incident photon distribution for a given scientific experiment. The calibration software comprises algorithms and functionalities for all analysis steps relevant for the calibration of the DSSC response. The calibration tools are verified using simulated data. The combination of system simulation and calibration tools allows us to optimize calibration methods and sources, and to assess the performance of the DSSC system for a given scientific experiment.

This work was supported by European XFEL GmbH in the framework of the DSSC project.

N31-9: First Operation of a DSSC Hybrid 2D Soft X-Ray Imager with 4.5 MHz Frame Rate J. Soldat $^{1}$, F. Erdinger ${ }^{1}$, C. Fiorini ${ }^{2}$, P. Fischer ${ }^{1}$, A. Grande ${ }^{2}$, K. Hansen ${ }^{3}$, P. Kalavakuru ${ }^{3}$, M. Kirchgessner ${ }^{1}$, M. Manghisoni ${ }^{4}$, B. Nasri ${ }^{2}$, M. Porro ${ }^{5}$, D. Comotti ${ }^{4}$, C. Reckleben ${ }^{3}$, J. Szymanski ${ }^{3}$ 
${ }^{I}$ Institute of Computer Engineering, Heidelberg University, Heidelberg, Germany

${ }^{2}$ Dipartimento di Elettronica e Informazione, Politecnico di Milano, Milano, Italy

${ }^{3}$ Deutsches Elektronen-Synchrotron DESY, Hamburg, Germany

${ }^{4}$ Dipartimento di Ingegneria Industriale, Universita di Bergamo, Dalmine (BG), Italy

${ }^{5}$ European XFEL GmbH, Hamburg, Germany

The DSSC (DEPFET Sensor with Signal Compression) collaboration develops a hybrid pixelated X-Ray photon detector with 4.5 $\mathrm{MHz}$ frame rate and immediate amplitude digitization for experiments at the European XFEL.

We present measurements of the first full format $14.9 \times 14 \mathrm{~mm}^{\wedge} 2$ pixel readout ASIC assembly for the DSSC detector. The readout architecture is specially adapted to the burst structure of the XFEL (bursts of 2880 pulses spaced by down to $221 \mathrm{~ns}$ at a rate of $10 \mathrm{~Hz}$ ) by in-pixel digitization and digital hit data storage and data transfer during the burst gaps. The readout ASIC contains $64 \times 64$ pixels of $229 \times 204 \mathrm{um}^{\wedge} 2$ size and includes per pixel two low noise front-end versions for DEPFET and silicon drift detectors (SDD), a single-slope 8-bit ADC and local memory.

New results from measurements with both DEPFET and SDD sensors will be shown, demonstrating the low noise, high dynamic range and fast operation. First results on the trimming of pixel parameters throughout the matrix for homogenous operation will be presented.

\author{
N31-10: Timepix3 Readout System for Time Resolved Experiments at Synchrotron Radiation Facilities \\ G. Crevatin, D. Omar, I. Horswell, H. Yousef, E. Gimenez-Navarro \\ Science, Diamond Light Source Ltd, Didcot, UK
}

Demand for time resolved experiments, of which the time information related to the detected events is an essential requirement, is rapidly increasing in synchrotron research environments.

Timepix3 [1] is a read-out chip for pixelated detectors that places a time stamp to every detected event with a nominal resolution of $1.5625 \mathrm{~ns}$ and provides a measure of the energy of the same event by the Time over Threshold technique. Timepix 3 can then be a promising readout chip for time resolved experiments.

A prototype of a Timepix3 readout system [2], based on a modified version of the MERLIN readout system [3], has already been developed by the detector group of Diamond Light Source (DLS). This prototype has been used to run a first set of

characterization measurements using synchrotron radiation of the Timepix3 chip [4].

The results of the characterization measurements proposed additional modifications to optimize the Timepix 3 detector system to be used for time resolved experiments. The Timepix 3 detector system needs to allow longer measurable time period for the Time of Arrival since it is limited by the small amount of bits dedicated. There is a need as well for locking the clock of the readout system to the source clock in order to avoid beats due to independent frequencies and track eventual deviations from nominal clock values.

Development on the readout system, mainly on the FPGA firmware, has been carried out in order to increase the number of bits dedicated to the Time of Arrival. The possibility to drive the detector system via external clock was added.

The contribution is focused on describing the new specifications and their implementation in the readout system. Furthermore, results from experiments run at DLS using the developed Timepix3 readout system will be briefly presented.

[1] T. Poikela et al., Timepix3: a 65K channel hybrid pixel readout chip with simultaneous ToA/ToT and sparse readout, 2014 JINST 9 C05013

[2] G. Crevatin et al., Development of a Timepix3 readout system, 2015 JINST 10 C03042, doi:10.1088/1748-0221/10/03/C03042

[3] R. Plackett et al., Merlin: a fast versatile readout system for Medipix3, 2013 JINST 8 C01038

[4] H. Yousef et al., Timepix3 as X-ray detector for time resolved synchrotron experiments, 2016 Nucl. Instrum. Methods A, doi:10.1016/j.nima.2016.04.075

N31-11: Safety-Interlock System of the DSSC X-Ray Imager

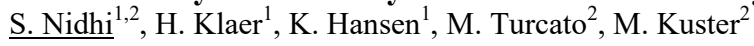

${ }^{I}$ FEC, DESY, Hamburg, Germany

${ }^{2}$ WP75, European XFEL, Hamburg, Germany

A stand-alone microcontroller-based safety-interlock board (SIB) has been designed to ensure the DSSC Mega-pixel X-ray imager operation in a safe mode and detection of operational failures followed by prompt action. The camera head consists of 4 quadrants, each controlled by a single SIB. Each SIB monitors the signals of 75 temperature sensors and 2 pressure sensors, the connectivity of 16 low-voltage and high-voltage cables, and controls further 8 channels of external equipment like cooling system, pressure gauge, power crate and experimental environment. Furthermore, the SIB generates the startup and shutdown sequences. The current detector-system state is provided to the user per bunch train. Full information is recorded permanently and can be accessed offline. The SIB prototype has been developed, programmed and tested. This test also comprises the evaluation of the detector-decision matrix with critical failure and warning cases. All the sensors were quantified separately in the temperature chamber. We present the design, hardware implementation and test results. 
N31-13: Calibration Sources and Techniques for Large Format X-Ray Imagers at XFEL

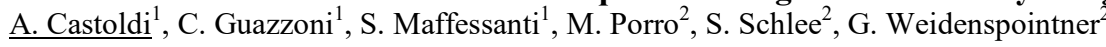

${ }^{I}$ DEIB, Politecnico di Milano and INFN, Milano, Italy

${ }^{2}$ European XFEL GmbH, Hamburg, Germany

The availability of sources able to probe the X-ray imaging detectors in conditions close to the foreseen XFEL experiments is a key issue for a twofold reason: i) the optimization of the final architecture of sensor and electronics, ii) the implementation of the appropriate strategies aiming at the calibration of each individual readout channel with the required degree of accuracy. The complexity of the active pixel design makes large-format detector calibration a further challenge - typically less visible during the design phase - due to the large number of parameters per channel involved. In this work we compare different calibration sources and focus on their combination to optimize the calibration of large-format X-ray imagers over a wide range. This activity is carried out in the framework of the DSSC project and the primary aim is the calibration of the DSSC camera. We considered pulsed IR laser, radioactive sources, X-ray tube, electrical injection devices, X-ray synchrotron beam, low-energy protons, LED sources. The relevant features (deposited energy and pulse width) are critically summarized and calibration strategies will be discussed.

\section{N31-14: 2-D Mapping of the Response of SDD Cells of Different Shape in Monolithic Arrays for XRF} Spectroscopy

A. Castoldi ${ }^{1}$, C. Guazzoni ${ }^{1}$, G. V. Montemurro ${ }^{1}$, C. Liu $^{1}$, C. Piemonte ${ }^{2}$, I. Rashevskaya ${ }^{3}$, A. Rashevsky ${ }^{4}$, G. Zampa $^{4}$, N. Zampa $^{4}$, A. $\mathrm{Vacchi}^{4}$

${ }^{I}$ Politecnico di Milano and INFN, Milano, Italy

${ }^{2}$ Fondazione Bruno Kessler- FBK, Trento, Italy

${ }^{3}$ TIFPA-INFN, Trento, Italy

${ }^{4}$ INFN, Sezione di Trieste, Trieste, Italy

In the frame of the INFN experiment REDSOX/REDSOX2 and in collaboration with Fondazione Bruno Kessler (FBK), Trento, we are developing trapezoidal-shaped monolithic arrays of Silicon Drift Detector (SDD) cells for low energy X-ray fluorescence (XRF) experiments. The trapezoidal geometry is chosen in order to maximize the detection solid angle at the spectro-microscopy beamline TwinMic at the Elettra Synchrotron Trieste, Italy. The shape of the individual SDD cell must be carefully selected in order to find the optimal compromise between spectroscopic performance and the number of SDD cells. We conducted an experimental campaign with a pulsed IR laser in order to map in detail the transport properties inside square SDD cells (3.1 mm side) and inside triangular SDD cells $(3.1 \mathrm{~mm}$ side). The main parameters investigated are i) collected amplitude, ii) drift time and iii) the rise time at the charge-sensing preamplifier output, the last being a very sensitive parameter related to size of the charge cloud. The expected anisotropy of charge transport close to the corners, particularly evident also inside a single cell, has been studied in detail. Moreover, the drift field in this design decreases with the distance from the anode because of the uniform junction on the back side and is mostly driven by the bulk doping, which makes the "corner effect" more critical. The collected experimental data are a precious guide for the optimization of the final detector prototype.

\section{N31-15: Use of Silicon Drift Detectors at the LCLS}

G. Blaj, G. Carini, M. Chollet, G. Dakovski, P. Hart, G. Haller, S. Herrmann, C. Kenney, S. Nelson, J. Pines, J. Thayer, A. Tomada, S. Song

SLAC National Accelerator Laboratory, Menlo Park, CA, U.S.A.

Silicon drift detectors (SDDs) are a well-established technology that revolutionized spectroscopy. For a subset of the experiments at FELs, silicon drift detectors can make a substantial contribution. Many measurements involve only several distinct photon energies known a priori. Studies have been performed to investigate the utility of silicon drift detectors at X-ray FELs. In particular we studied the ability to deconvolve the spectrum that results from various combinations of a few wavelengths and the possibility of separately recording photons that are absorbed at different radii (thus having varying drift times). Results for LCLS beam time data are shown, discussed, and analyzed. In standard spectroscopy applications there is often a complex spectrum within which a multitude of peaks needs to be resolved and matched to a specific element. In contrast, at an x-ray FEL there are often only a few photon energies involved in an experiment. Such a situation greatly simplifies the measurement. Two classes of experiments will be described: ultra-low occupancy with an unknown background and low-occupancy with an a priori known spectrum. We will show that the usefulness of silicon drift detectors will continue into the x-ray FEL era of science.

N31-16: High Speed Multi-Element SDD X-Ray Spectrometers

Y. J. Wang, S. Barkan, V. Saveliev, L. Feng, M. Zhang, B. Goolsby

RD, Hitachi High Tech Science America, Northridge, United States 
A state-of-art multi-element silicon drift detector (SDD) with improved solid angle is developed for advanced X-ray spectroscopy, such as x-ray fluorescence micro imaging, fluorescence computed microtomography (fCMT), XANES (x-ray absorption near-edge structure) and EXAFS (extended x-ray absorption fine structure). A 3- and 4-element SDD spectrometers are based on 0.5 and $1 \mathrm{~mm}$ thick SDDs integrated with advanced front-end ASIC preamplifiers. They feature superior energy resolution at very short shaping times. For each channel, output count rate is even higher than 1Mcps with the energy resolution better than $160 \mathrm{eV}$ with the latest adaptive pulse processing electronics. The option of $1 \mathrm{~mm}$ thick SDDs provides significant increased quantum efficiency for high energy X-ray. The superior result has been achieved in multiple synchrotron light source. In BNL HXN beamline, a focused 3 element SDD spectrometer measured an image of the Pt L-edge clearly shows the individual circular structures of $20 \mathrm{~nm}$ wide array using a fly-scanning method.

N31-17: An Improved Method for Energy Calibration of Photon Counting X-Ray Detectors

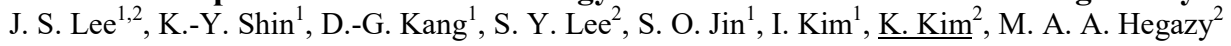

${ }^{I}$ Dept. of Applied Electromagnetic Wave Research Center, Korea Electrotechnology Research Institute, Ansan, Gyeonggi, Republic of Korea

${ }^{2}$ Dept. of Biomedical Engineering, Kyung Hee University, Yongin, Gyeonggi, Republic of Korea

Photon counting X-ray detectors with energy discrimination capabilities are able to discriminate the energy of incident X-ray photon, thereby spectral images can be obtained simultaneously. Energy calibration is a process to find a linear mapping function between the amplitudes of electric pulses and their corresponding photon energy. For practical and efficient energy calibration, $\mathrm{X}$-ray tube voltages can be used as a reference. However, as the exposure time increases, threshold giving $50 \%$ of OFF pixels also increases without converging to a point. To reduce calibration errors due to this non-convergent behavior, we present an improved method for energy calibration and apply it to two commercial photon counting X- ray detectors, i.e., CdTe Medipix-2 detector and CdTe Pixirad2 detector with PIXIE-II read-out ASIC. With the improved mapping function by the proposed method, we could better identify iodine component in a phantom consisting of iodine and other components for both detectors

\author{
N31-18: Characterisation of a Novel 3D Silicon Strip Detector for Microbeam Radiation Therapy (MRT) \\ Quality Assurance \\ M. Cameron ${ }^{1}, \underline{\text { M. Lerch }}^{1}$, S. Guatelli ${ }^{1}$, M. Petasecca ${ }^{1}$, J. Davis ${ }^{1}$, A. Dipuglia ${ }^{1}$, V. Perevertaylo ${ }^{2}$, A. Rozenfeld ${ }^{1}$ \\ ${ }^{I}$ Centre for Medical Radiation Physics, University of Wollongong, Wollongong, NSW, Australia \\ ${ }^{2} S P A-B I T$, Kiev, Ukraine
}

Microbeam Radiation Therapy (MRT) is a preclinical radiotherapy modality characterised by the use of multiple micron sized, spatially fractionated, high dose rate synchrotron x-ray fields and is promising for the treatment of inoperable and paediatric head and neck tumours. Dosimetry and Quality Assurance of MRT requires a detector with high spatial resolution, large dynamic range, and fast real-time readout. CMRP has developed novel 3D MESA silicon Single Strip Detector (SSD) as successor to Edge-on MOSFET and EPI SSD in order to improve spatial resolution of small silicon detectors. 3D MESA is produced via Ion Plasma Etching away silicon surrounding the sensitive volume $10 \mu \mathrm{m}$ down to SiO insulating layer to produce a smaller, isolated sensitive volume. Electrical characterisation has shown less than $0.2 \mathrm{nA}$ leakage current and full lateral depletion at $-3 \mathrm{~V}$ bias. MRT dose profiles were measured at Australian Synchrotron Imaging and Medical Beamline for a multi-slit collimator setup of slit-width $50 \mu \mathrm{m}$ and pitch $400 \mu \mathrm{m}$. multiple samples produced similar FWHMs of 46-47 $\mu \mathrm{m}$ and Peak-Valley-Dose-Ratios of 60-100 as expected. Supplementary Ion Beam Induced Charge Collection study and geant4/TCAD simulations to model expected dose and charge collection efficiency to follow.

CMRP wishes to thank AIIM and the Australian Synchrotron IMBL team (particularly Andrew Stevenson, Jayde Livingston, Chris Hall, and Daniel Hausermann) for the use of their facilities and their assistance in the characterisation of the 3D SSD. CMRP wishes to acknowledge the financial support of NHMRC (Development Grant APP1093256) and the Australian Synchrotron (Beamtime Grant AS153/IM/10045).

\author{
N31-19: Readout of Multi-Element HPGe-Detectors with the PIXIE ASIC for High-Resolution X-Ray \\ Spectroscopy Applications \\ P. Seller ${ }^{1}$, M. French ${ }^{1}$, M. C. Veale ${ }^{1}$, J. Lipp ${ }^{1}$, L. L. Jones ${ }^{1}$, A. Hardie ${ }^{1}$, U. Spillmann ${ }^{2}$, T. Krings ${ }^{3}$

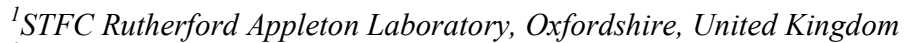 \\ ${ }^{2}$ GSI Helmholtzzentrum für Schwerionenforschung, Darmstadt, Germany \\ ${ }^{3}$ Forschungszentrum Jülich GmbH, Jülich, Germany
}

Since several years planar, structured $\mathrm{Si}(\mathrm{Li})$ - and High-Purity Germanium (HPGe) detectors are routinely used for the measurement of high-energy x-rays (up to several $100 \mathrm{keV}$ ). The individual detector-elements are connected to charge-sensitive preamplifiers, which are typically placed outside the cryostat and thus operated at room temperature. To improve the energy resolution the preamplifiers used so far have to be replaced by preamplifiers which can be mounted next to the detector-elements on the one hand and allow to be cooled on the other hand. - For the first time a PIXIE ASIC - developed at the Rutherford 
Appleton Laboratory (RAL) - has been coupled to a single-sided structured HPGe-detector (strip-detector). The chip houses 9 independent charge-sensitive preamplifiers which are connected to the detector-elements (strips). This setup has been tested with different radioactive sources to cover an energy range up to several $100 \mathrm{keV}$. The results of the first test measurements will be presented.

N32: Photodetectors II - Cryogenic and Novel SiPM Designs

Tuesday, Nov. $1 \quad$ 16:30-18:00 Cassin

N32-1: Trend of Photo-Detector Technologies for Operation at Cryogenic Temperatures

A. Cardini

INFN Sezione di Cagliari, Monserrato, Italy

In the last decade many experiments, in particular those dedicated to the search of Dark Matter and for Neutrino Physics, have been using liquid noble gases as the active detection medium, collecting not only the charge produced by ionization but also the scintillation light produced by excitation. These liquid noble gas volumes have traditionally been readout using photomultipliers, and an important optimization work was required to improve the performance of these devices at cryogenic temperatures. In more recent years, thanks to their increased availability, large-area silicon photomultipliers arrays have started to become a very attractive option, and both running and new experiment are considering the use these new photo-detectors in their design. In this talk I will review the trend of photo-detectors used in experimental apparatus operating at cryogenic temperatures by comparing advantages and disadvantages of both vacuum tube and solid-state light-detector technologies.

\section{N32-2: Development of Large Area VUV-Sensitive Silicon Photomultipliers for Operation in Liquid Xenon F. Retiere \\ TRIUMF, Vancouver, Canada \\ On behalf of the nEXO collaboration}

Large area vacuum ultra-violet (VUV) sensitive SiPMs are being developed for the nEXO experiment. The aim of the experiment is the search of neutrino-less double beta decay of 136Xenon. It is a 5 ton liquid Xenon detector requiring 4 to $5 \mathrm{~m} 2$ of photodetectors that have to be very efficient at $175 \mathrm{~nm}$ and carry very small amount of radioactive elements. SiPMs appear ideally suited for this application yet they have never been used in very large plane and their efficiency in the VUV is marginal. We will report on the characterization of SiPMs manufactured by Fondazione Bruno Kessler (FBK), Hamamatsu Photonics and KETEK specifically for the detection of VUV light in liquid Xenon. FBK RGD-HD and Hamamatsu VUV3 SiPMs fulfill most of the nEXO specifications. The latest devices produced in 2016 by FBK NUV-HD and Hamamatsu VUV4 are expected to be event better. Promising performances have also been achieved with KETEK SiPMs coated with a wavelength shifting material. In addition to reporting about the individual device performances we will also discuss scaling up from $<1 \mathrm{~cm} 2 \mathrm{SiPM}$ to $100 \mathrm{~cm} 2$ and eventually to $\mathrm{m} 2$ using analog readout technology as well as digital 3-dimensionally integrated technology

N32-3: SiPM Timing at Low Light Intensities R. Dolenec $^{1,2}$, S. Korpar ${ }^{1,3}$, P. Krizan ${ }^{1,2}$, R. Pestotnik ${ }^{1}$

${ }^{I}$ Experimental Particle Physics Department, J. Stefan Institute, Ljubljana, Slovenia

${ }^{2}$ Faculty of Mathematics and Physics, University of Ljubljana, Ljubljana, Slovenia

${ }^{3}$ Faculty of Chemistry and Chemical Engineering, University of Maribor, Maribor, Slovenia

Silicon photomultiplier (SiPM) is becoming the photodetector of choice in many applications where good efficiency and precise timing are required. However, their timing performance at very low light intensities is still slightly limited, especially for SiPMs with at least moderately large active surface. Extracting the best timing information from single photon level pulses would be especially desired for the detection of Cherenkov photons in ring imaging Cherenkov counters (RICH) or very fast time-of-flight positron emission tomography (TOF PET). To improve the understanding SiPM timing at low light intensities, we examined the performance of devices produced by four different manufacturers with a picosecond laser. The experiments were performed at controlled temperatures down to $-25^{\circ} \mathrm{C}$, while the laser pulses could be focused to a few micrometer spot size and scanned over the SiPM surface. We report on the timing performance of different SiPMs with special emphasis on the details of the double cell signals, where important effects that degrade the timing are best visible. By taking this events into account in the data analysis, some improvement in the time resolution was achieved, while detailed simulations of the processes in the SiPM microcell could improve the understanding of the source of degrading effects.

N32-4: Sherbrooke's First 3D Digital SiPM: Measurements, Recommendations and Future Work

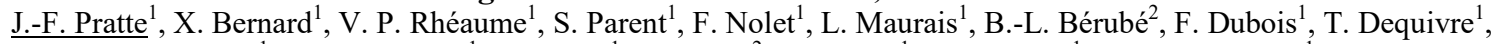

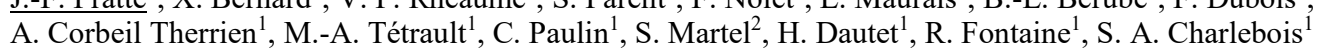


${ }^{I}$ Interdisciplinary Institute for Technological Innovation - 3IT, Universite de Sherbrooke, Sherbrooke, QC, Canada

${ }^{2}$ Teledyne DALSA - TDSI, Bromont, QC, Canada

This paper presents Sherbrooke's first functional vertically integrated 3D digital SiPM. The motivation of this work is to establish a proof of the 3D digital SiPM concept. First, using a digital readout for SPAD array takes advantage of the inherently binary nature of the device. Second, by moving the readout electronics under the SPAD instead of next to it, we can improve the fill factor, hence photodetection efficiency, and select the optimal process for the SPAD and the CMOS independently. For this objective, 3D vertical integration is the key. The current 3D digital SiPM is composed of two tiers: the top tier is the SPAD array fabricated in Teledyne DALSA $0.8 \mu \mathrm{m}$ CMOS HV (with process modifications) and the bottom tier is the CMOS readout realized in standard Teledyne DALSA $0.8 \mu \mathrm{m}$ CMOS HV. Design choices were made to ease the realization of a proof of concept and consequently did not aim at ultimate performance. Initial characterization shows that the devices are functional, but with some issues. For instance, a fraction of the SPAD are not well connected to the CMOS. Most of the issues are understood and will not be a problem in the future as we are developing a 3D integration process with industrial partners. At the conference, the following measurements will be presented: single photon timing resolution, PDE, afterpulsing, optical crosstalk studies, photon counting capability, as well as the impact of the fabrication process on the dark count rate. Finally we will report openly on learned experience from the 3D integration and future work.

N32-6: VSiPMT: an Hybrid Approach to High Resolution Photodetedector

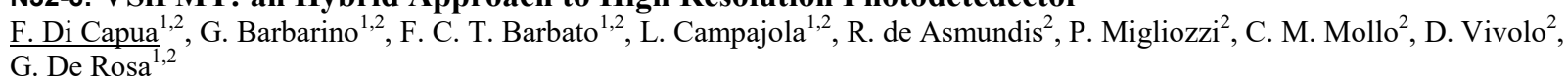
${ }^{I}$ Dipartimento di Fisica, Università degli Studi di Napoli Federico II, Napoli, Italy

${ }^{2}$ Sezione di Napoli, Istituto Nazionale di Fisica Nucleare, Napoli, Italy

The VSiPMT (Vacuum Silicon PhotoMultiplier Tube) is an innovative design for a revolutionary hybrid photodetector. The idea, consists in replacing the classic dynode chain of a classic PMT with a SiPM. This new device aims at extending SiPM technology to large detection volumes. In this configuration, we match the large sensitive area of a photocathode with the performances of the SiPM technology, which therefore acts like an electron detector and current amplier. The excellent photon counting capability, fast response, low power consumption and great stability are among the most attractive features of the VSiPMT. In order to realize such a device we first studied the feasibility of this architecture by studying the response of a special nonwindowed Multi Pixel Photon Counter (MPPC) by Hamamatsu with an electron beam. Thanks to this result Hamamatsu realized two VSiPMT industrial prototypes with a photocathode of $3 \mathrm{~mm}$ diameter. In this work we present an overview of the full characterization of the VSiPMT industrial prototypes with their advantage. We also present the progress on the realization of a 1inch prototype based on CsI photocathode and the preliminary tests we are performing on it.

\section{N33: Neutron detectors : Thermal Capture Scintillation Detectors}

\section{Tuesday, Nov. $1 \quad$ 16:30-18:30 Madrid}

N33-1: Novel Neutron Detector Material: Microcolumnar Li1-xNaxI:Eu

M. S. Marshall ${ }^{1}$, M. J. More ${ }^{1}$, H. B. Bhandari ${ }^{1}$, R. A. Riedel ${ }^{2}$, S. Waterman ${ }^{1}$, J. Crespi ${ }^{1}$, P. Nickerson ${ }^{1}$, V. V. Nagarkar ${ }^{1}$

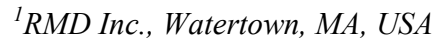

${ }^{2}$ Neutron Scattering Science Division, Oak Ridge National Laboratory, Oak Ridge, TN, USA

Current neutron detection relies on He-3 detectors, which are prone to material shortages, and suffer from poor spatial resolution. Such shortages and inadequacies make He-3 unsuitable for new applications in state-of-the-art spallation sources such as the Spallation Neutron Source (SNS) at Oak Ridge National Laboratory (ORNL). In this manuscript, we demonstrate the use of a novel scintillator material for neutron detection: a mixed halide compound, Eu-doped Li1-xNaxI (LNI). LNI:Eu is a bright, efficient, scintillator that can be grown in large format, thin films that exhibit microcolumnar structure with a resolution of approximately $50 \mu \mathrm{m}$. The ability to grow in a thin film format confers advantages over solid neutron scintillators such as CYLC, making a key advantage of LNI:Eu thin films the ability to make large area detectors, which is important for detecting specular reflections. The use of enriched lithium, Li-6, increases the neutron absorption cross section of the thin films, while preserving the brightness of the scintillation response. The films were tested at ORNL at HFIR, and an efficiency was measured of approximately $43 \%$ for $4.2 \AA$ neutrons was measured for a $375 \mu \mathrm{m}$ thick film of LNI. The vapor deposition techniques used to fabricate the scintillator screens in a cost-effective approach will be discussed.

This work was supported by Department of Energy under grants DE-SC0011311 and DE-SC0008291.

N33-2: A Portable Fast-Neutron Imager with ${ }^{6}$ Li-Containing Scintillators

T. Matsumura, T. Shinkawa

Dept. of Applied Physics, National Defense Academy in Japan, Yokosuka, Japan 
A portable fast-neutron imager was designed for the purpose of nuclear security. The imager can identify the positions of neutron sources and make an accurate measurement in a short time based on a moderated-neutron-detection method. We use squareshaped lithium-containing scintillators to detect moderated neutrons.

Eighteen scintillator plates arranged in a cage shape are entirely covered by a 7-cm-thick polyethylene as a moderator. According to a Geant4 simulation, the response of each scintillator shows strong angular dependence since the most of the incident neutrons tend to be detected by the scintillators located near the incident surface. Hence, the neutron-source direction can be reconstructed from a measured neutron-hit distribution by using an unfolding method. The positional resolution to search for 5-kg weapongrade plutonium located $3 \mathrm{~m}$ away from the imager was estimated to be about 15 degrees within one minute. MPPC with an active area of $6 \times 6 \mathrm{~mm}^{2}$ and tapered acrylic light-guide are employed to detect scintillation photons. We checked the response of scintillation counters to thermal neutrons with two types of scintillator: LiCAF and Li glass. From this measurement we confirm that light yield of the counter is enough to detect thermal neutrons although the active area of the MPPC is small compering to the area of the scintillators.

\author{
N33-4: ZnO:Zn/6LiF - a Low Afterglow Alternative to ZnS:Ag/6LiF for Thermal Neutron Detection \\ G. J. Sykora, E. M. Schooneveld, N. J. Rhodes \\ ISIS, STFC, Harwell Oxford, United Kingdom
}

Recent advances in neutron scattering facilities have led to a search for detectors that can cope with increasing count rates. Current $\mathrm{ZnS}: \mathrm{Ag} /{ }^{6} \mathrm{LiF}$ based detectors are count rate limited by the long lifetime afterglow in the scintillator. Despite this drawback, new instruments are still considering use of $\mathrm{ZnS}$ :Ag due to the simplicity of detector design and relatively inexpensive production. It is becoming clear that these new instruments will be limited by the count rate capability of the detectors so an alternative scintillator technology with equivalent simplicity is being sought. $\mathrm{ZnO}: \mathrm{Zn} /{ }^{6} \mathrm{LiF}$ is being investigated here as a low afterglow alternative to $\mathrm{ZnS}: \mathrm{Ag} /{ }^{6} \mathrm{LiF}$. $\mathrm{ZnO}$ based scintillators have previously been considered for thermal neutron detection with the majority of the work being focused on the fast (100s of ps) UV component of the ZnO emission. The main disadvantage of this is that neutron-gamma discrimination has to be done by pulse height separation methods. In this work, neutron-gamma discrimination is investigated through pulse shape discrimination offered by the blue-green emission due to Oxygen vacancies in $\mathrm{ZnO}: \mathrm{Zn}$. Pulse shape discrimination using single photon counting methods is shown. Results from $\mathrm{ZnO}: \mathrm{Zn} /{ }^{6} \mathrm{LiF}$, directly and fibre coupled to a PMT, demonstrate improvements in count rate capability over ZnS:Ag based neutron detectors.

\author{
N33-5: Neutron-Gamma Discrimination Using a Combined EJ299/SiPM System and Fast Digital Acquisition \\ M. P. Taggart, C. Payne, P. J. Sellin \\ Dept. of Physics, University of Surrey, Guildford, Surrey, United Kingdom
}

We present the neutron/gamma discrimination performance of EJ299 plastic scintillator detectors that use SiPM read-out based on SensL C- and J-series silicon photomultiplier technologies with fully digital pulse processing. We contrast the performance of the latest SensL J-series SiPM sensor when used with EJ299 scintillator, and show that the dark noise, time response, and pulse shape discrimination (PSD) performance is significantly improved compared to the earlier C-series sensor. The detection components allow for a more compact, and less hazardous, neutron-gamma discrimination package than has previously been available for deployment.

The use of EJ-299 enables the discrimination of neutron and gamma signals on a pulse-by-pulse basis and the construction of PSD histograms through the charge-integration method. Data acquisition was performed via Caen digitisers with sample rates sufficient that the fast component of the emission can be adequately resolved. Initial work took place using a 10-bit digitiser with a $1 \mathrm{Gs} / \mathrm{s}(1 \mathrm{~ns})$ sample rate, with further investigations focused on the impact of increasing resolution to a 14-bit digitiser albeit with a reduction in sample rate to $500 \mathrm{Ms} / \mathrm{s}$.

All data was processed offline with a ROOT analysis code constructing the PSD histograms. The quality of the PSD abilities of EJ-299 was characterised by a common figure of merit (FoM) defined as the separation of the two (neutron and gamma) peaks divided by the sum of their respective full widths at half maximum. For energies ranging across $0.5-1.5 \mathrm{MeVee}$ we obtained FoM of at least 0.75 with the 10-bit system which increases to $>1.0$ for the 14-bit digitiser. The FoM performance of the J-series coupled to EJ299 was significantly better than that of the C-series in an otherwise identical configuration, achieving FoM=1.95 at $1.5 \mathrm{MeVee}$ compared to 1.45 for the $\mathrm{C}$-series sensor.

\title{
N33-6: A New Concept of 2D Scintillation Detector for Thermal Neutrons Based on a Light Sharing Approach J.-B. Mosset, A. Stoykov, M. Hildebrandt \\ Detector Group, Paul Scherrer Institute, Villigen-PSI, Switzerland
}

In this new concept of two-dimensional scintillation detector, the detector consists of an almost gapless assembly of individual and optically isolated $\mathrm{ZnS} /{ }^{6} \mathrm{LiF}$ bars with embedded and uniformly distributed WLS fibers running from one end of the bar to the other. On each extremity of a bar, the fibers are combined and readout together. The longitudinal position is determined from the asymmetry between the number of photons detected on both sides of the bar while the transverse position is simply given by the 
transverse position of the bar.

Simulations show that the attenuation length of the WLS fibers should be between $50 \%$ and $100 \%$ of the fiber length to achieve the best longitudinal resolution. Consequently, special Y11(400)M WLS fibers with a core doped with PMMA have been developed by Kuraray for this specific application. We received WLS fibers with different attenuation lengths ranging from 13 $\mathrm{cm}$ to $30 \mathrm{~cm}$.

As a first step, we used single WLS fibers illuminated in a pulsed mode with a collimated LED light source and we studied the dependence of the longitudinal resolution on the fiber length, the attenuation length of the fiber, the light yield, and the illumination position. In this contribution, we will present the measurements with single fibers and we will show the validation with the experimental data of a simple model describing the light attenuation in a WLS fiber.

As a second step, a Monte Carlo simulation based on this model has been developed for estimating the longitudinal resolution and the trigger efficiency of a single channel detection unit irradiated with thermal neutrons. Two different detection units with PMMA doped WLS fibers have been produced. Their performance in a neutron beam will be presented and compared to the simulation results.

\section{N33-7: Fast-Neutron and Gamma-Ray Survey Using Compact Plastic Scintillation Detectors R. M. Preston, J. R. Tickner}

CSIRO Mineral Resources, Lucas Heights, NSW, Australia

The sensitive detection and measurement of 1 to $20 \mathrm{MeV}$ fast-neutrons in mixed neutron/gamma-ray fields is crucial for both radiation protection and homeland security applications, particularly as common neutron sources primarily emit fast-neutrons. While organic scintillators allow for the efficient detection and identification of both fast-neutrons and gamma-rays, they have yet to find widespread use for dose survey in mixed-radiation fields. By employing a solid-state Silicon Photomultiplier (SiPM) photodetector in place of the conventional photomultiplier tube (PMT), compact scintillation detectors better suited for portable applications can now be realized. We present results on the design, calibration and characterisation of a prototype survey meter based on a $1.5 \times 2 \times 7 \mathrm{~cm}$ EJ-299-34 plastic scintillator with SiPM optical readout. Custom digital pulse processing hardware was used to perform on-the-fly pulse shape discrimination (PSD) and energy measurement, allowing the real-time measurement of separated fast-neutron and gamma-ray spectra. Ambient dose equivalent $\mathrm{H}^{*}(10)$ was calculated on-the-fly by means of two energy-dependant "G-functions". The SCIRESP and EGSnrc Monte Carlo codes were used to characterise the detector response and optimise the G-functions. The calibrated detector has a mean sensitivity of between 0.4 and $0.8 \mathrm{cps} /(\mu \mathrm{Sv} / \mathrm{hr})$ for neutrons between $2 \mathrm{MeV}$ and $16 \mathrm{MeV}$. The dose from gamma-rays between $150 \mathrm{keV}$ to $10 \mathrm{MeV}$ could also be measured, with a mean sensitivity of $75 \mathrm{cps} /(\mu \mathrm{Sv} / \mathrm{hr})$ at $1 \mathrm{MeV}$. The high sensitivity and lightweight nature of this detector makes it interesting for rapid survey of mixed-radiation fields, even those with dose rates approaching regulatory limits for public occupancy.

\section{N33-8: The Source Testing Facility at Lund University}

J. Scherzinger $^{1,2}$, J. R. M. Anand ${ }^{3}$, K. G. Fissium ${ }^{1,2}$, R. Hall-Wilton ${ }^{2,4}$, R. Jebali ${ }^{3}$, F. Messi ${ }^{1}$, H. Perrey ${ }^{1,2}$, E. Rofors ${ }^{1}$

${ }^{1}$ Lund University, Lund, Sweden

${ }^{2}$ European Spallation Source ERIC, Lund, Sweden

${ }^{3}$ University of Glasgow, Glasgow, UK

${ }^{4}$ Mid-Sweden University, Sundsvall, Sweden

The Source Testing Facility at Lund University is jointly operated by the Division of Nuclear Physics and the Detector Group of the European Spallation Source ERIC. It was developed as a cost-efficient testbed for neutron-detector development and shielding studies based on a/Be-9 mixed-field neutron/gamma sources and standard gamma calibration sources. The facility provides a tagged-neutron beam for an energy-dependent characterizations in the fast neutron range and a moderated neutron beam for characerizations in the thermal energy range. A general overview will be presented together with selected results.

\section{N34: Data acquisition, trigger and analysis II (HEP)}

Tuesday, Nov. $1 \quad$ 16:30-18:45 Londres N34-1: The Evolution of the ATLAS Region of Interest Builder: from Custom to Commodity $\underline{\text { J. Love }}$

Argonne National Lab, Chicago, USA

The ATLAS trigger system is deployed to reduce the event rate from the Large Hadron Collider bunch crossing frequency of 40 $\mathrm{MHz}$ to $1 \mathrm{kHz}$ for permanent storage using a tiered system. In the PC trigger farm decisions are seeded by Regions of Interest found by the custom hardware trigger system. The Regions of Interest are collected and distributed to the farm at $100 \mathrm{kHz}$ by the ATLAS Region of Interest Builder. The Evolution of the Region of Interest Builder from a crate of custom VME-based electronics to a commodity PC hosting a single custom PCIe card has been undertaken to increase the system performance, flexibility, and ease maintenance. The functionality and performance of the Region of Interest Builder previously only possible 
using FPGAs and a custom backplane VME Crate, has now been implemented in a multi-threaded C++ software library interfaced to a single PCIe card with one Xilinx Vertex 6 FPGA. The PC-based system was installed in the ATLAS Data Acquisition system between the 2015 and 2016 data taking periods. This presentation describes the requirements and functionality of the ATLAS Region of Interest Builder, and the PC-based implementation as well as measurement results from controlled test setups and in situ operation of the ATLAS experiment.

\section{N34-2: A Fully Digital Trigger and Data Acquisition System for the NA62 Kaon Factory at the CERN SPS} E. Pedreschi $^{1,2}$, J. Pinzino ${ }^{1,2}$, R. Piandani ${ }^{2}$, M. Sozzi ${ }^{1,2}$, F. Spinella ${ }^{2}$

${ }^{I}$ Physics Dept., University of Pisa, Pisa, Italia

${ }^{2}$ Pisa Section, National Institute of Nuclear Physics, Pisa, Italia

CERN's long tradition in kaon physics is carried out by NA62. The NA62 experiment at CERN SPS is a high-intensity facility designed to perform precision measurements of rare kaon decays, in particular the decays into a pion and two neutrinos. The nominal performance of the detector in terms of data quality and quantity is so good that the experiment can undeniably play the role of a kaon factory. The experiment has chosen to use high momentum kaons $(75 \mathrm{GeV} / \mathrm{c})$ from the accelerator with a technique of in-flight-decay. The intense flux of NA62 dictates the need for a high-performance triggering and data acquisition system, which must minimize dead time while maximizing data collection reliability. A unified fully digital trigger and data acquisition (TDAQ) system was designed in order to address such requirements in a simple and cost-effective manner: readout uniformity of sub-detectors, scalability, efficient online selection and lossless high rate readout are key issues. The TDCB and the TEL62 boards are the common blocks of the TDAQ: TDCB measure hit times for sub-detectors, TEL62 process and store them in a buffer, extracting only those requested by the trigger system, which merges trigger primitives produced from different detectors by TEL62. NA62 was commissioned in 2014 and 2015, and it is now in the middle of a first long phase of data taking. The common trigger and data acquisition architecture, the complete dataflow, the firmware organization and the performance of the system are described.

N34-3: Operation and Performance of a New microTCA-Based CMS Calorimeter Trigger in LHC Run 2 P. R. Klabbers

University of Wisconsin - Madison, Madison, WI, USA

On behalf of the CMS Collaboration

The Large Hadron Collider (LHC) at CERN is currently increasing the instantaneous luminosity for p-p collisions. In LHC Run 2, the center-of-mass energy has gone from 8 to $13 \mathrm{TeV}$ and the instantaneous luminosity will approximately double for proton collisions. This will make it even more challenging to trigger on interesting events since the number of interactions per crossing (pile-up) and the overall trigger rate will be significantly larger than LHC Run 1. The Compact Muon Solenoid (CMS) experiment has installed the second stage of a two-stage upgrade to the Calorimeter Trigger to ensure that the trigger rates can be controlled and the thresholds kept low, so that physics data will not be compromised. The stage-1, which replaced the original CMS Global Calorimeter Trigger, operated successfully in 2015. The completely new stage-2 has replaced the entire calorimeter trigger in 2016 with AMC form-factor boards and optical links operating in a microTCA chassis. It required that updates to the calorimeter back-ends, the source of the trigger primitive data, were also fully installed and operational. The stage- 2 system's boards use Xilinx Virtex 7690 FPGAs and have hundreds of links operating at up to 10 Gbps to maximize data throughput. In addition, a new trigger time-multiplexed architecture was implemented and extensive firmware and software development was necessary. The final commissioning, operation, and performance of the stage- 2 calorimeter trigger in 2016 proton collisions will be presented, as well as the expectations of CMS for the remainder of LHC Run 2.

N34-4: The ATLAS Tile Calorimeter DCS for Run 2

A. White

Physics, University of Texas at Arlington, Arlington, USA

On behalf of the ATLAS Tile Calorimeter System

TileCal is one of the ATLAS subdetectors operating at the Large Hadron Collider (LHC), which is taking data since 2010. Seventy thousand (70000) parameters are used for control and monitoring purposes, requiring an automated system. The Detector Control System (DCS) was developed to ensure the coherent and safe operation of the whole ATLAS detector. The TileCal DCS is mainly responsible for the control and monitoring of the high and low voltage systems but it also supervises the detector infrastructure (cooling and racks), calibration systems, data acquisition and safety. During the first period of data taking (Run 1, 2010-12) the TileCal DCS allowed a smooth detector operation and should continue to do so for the second period (Run 2) that started in 2015. The TileCal DCS was updated in order to cope with the hardware and software requirements for Run 2 operation. These updates followed the general ATLAS guidelines on the software and hardware upgrade but also the new requirements from the TileCal detector. A report on the upgrade and status of the TileCal DCS system will be presented. 
N34-5: Soft-Errors in FPGAs at the SuperKEKB Interaction Point

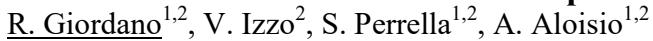

${ }^{\text {I}}$ Università di Napoli 'Federico II', Naples, Italy

${ }^{2}$ INFN Sezione di Napoli, Naples, Italy

In February 2016, the SuperKEKB positron-electron high-luminosity collider of the KEK laboratory (Tsukuba, Japan) started being commissioned. A dedicated commissioning detector, named BEAST2, is being used to characterize beam backgrounds before the Belle2 detector is rolled into the beams and to provide tuning parameters for Monte Carlo simulations. BEAST2 consists of a fiberglass support structure and several subdetectors mounted onto it, including time projection chambers (TPCs) and He-3 tubes.

In this work, we present direct measurements of radiation-induced single event upsets in a SRAM-based FPGA device installed in BEAST2 at a distance of $\sim 1 \mathrm{~m}$ from the beam interaction point. Our goal is to provide experimental results of the expected radiation-induced configuration upset rate and power consumption variation at Belle 2 and at other experiments operating in similar radiation conditions. For this study, we designed a dedicated board hosting a Xilinx Kintex-7 325T FPGA without additional active components, in such a way to be able to decouple FPGA failures from those of other devices. During the commissioning of the collider, we periodically read back the FPGA configuration in order to detect errors and we logged the power consumption on the different power domains of the device. Currents for both electron and positron rings spanned a range between 50 and $500 \mathrm{~mA}$, therefore providing data about the FPGA operation in different radiation conditions. BEAST2 subdetectors provided valuable information about the radiation environment. This work is part of the ROAL SIR project funded by the Italian Ministry of Research (MIUR).

\author{
N34-6: Latest Frontier Technology and Design of the ATLAS Calorimeter Trigger Board Dedicated to Jet \\ Identification. \\ E. Rocco \\ J. Gutenberg University, Mainz, Germany
}

To cope with the enhanced luminosity of the beam delivered by the Large Hadron Collider (LHC) in 2020, the A Thoroidal LHC ApparatuS (ATLAS) experiment has planned a major upgrade. As part of this, the trigger at Level-I based on calorimeter data, will be upgraded to exploit fine-granularity readout using a new system of Feature Extractors, which differ in the physics objects for the trigger selection. The presentation is focused on the jet Feature EXtractor (jFEX) prototype, one of the three Feature Extractors. In few hundreds nanoseconds latency budget, up to $2 \mathrm{~TB} / \mathrm{s}$ have to be processed to provide jet identification (even large area jets) and measurements of global variables. This requires the use of large Field Programmable Gate Array (FPGA) with the largest Multi Giga Transceiver available on the market. The jFEX board prototype hosts four large FPGAs from the Xilinx Ultrascale family with 120 Multi Giga Transceivers each, connected to 24 opto-electrical devices, resulting in a densely populated high speed signals board. For the 24 layers jFEX board stack-up, the MEGTRON6 material was chosen for its property of low transmission loss with high frequency signals (GHz range) and to further preserve the signal integrity, special care has been put into the design accompanied by simulation to optimise the voltage drop and minimise the current density over the power planes. An integrated test has been installed at the ATLAS test facility to perform numerous tests and measurements with the JFEX prototype.

\title{
N34-7: Electronics Development for the ATLAS Liquid Argon Calorimeter Trigger and Readout for Future LHC Running
}

W. Lampl

University of Arizona, Tucson, AZ, USA

On behalf of the ATLAS LAr Calorimeter Group

The upgrade of the LHC will provide up to 7.5 times greater instantaneous and total luminosities than assumed in the original design of the ATLAS Liquid Argon (LAr) Calorimeters. The radiation tolerance criteria and the improved trigger system with higher acceptance rate and longer latency require an upgrade of the LAr readout electronics. In the first upgrade phase in 20192020, a trigger-readout with up to 10 times higher granularity will be implemented. This allows an improved reconstruction of electromagnetic and hadronic showers and will reduce the background for electron, photon and energy-flow signals at the first trigger level. The analog and digital signal processing components are currently in their final design stages and a fully functional demonstrator system is operated and tested on the LAr Calorimeters. In a second upgrade stage in 2024-2026, the readout of all 183,000 LAr Calorimeter cells will be performed without trigger selection at $40 \mathrm{MHz}$ sampling rate and 16 bit dynamic range. Calibrated energies of all cells will be available at the second trigger level operating at $1 \mathrm{MHz}$, in order to further mitigate pile-up effects in energy reconstruction. Radiation tolerant, low-power front-end electronics optimized for high pile-up conditions is currently being developed, including pre-amplifier, ADC and serializer components in $65-180 \mathrm{~nm}$ technology. This talk will give an overview of the future LAr readout electronics and present research results from the two upgrade programs. 
N34-8: Development of a Highly Selective Single Muon Trigger Exploiting Precision Muon Chamber Data for the ATLAS Experiment at the HL-LHC

S. Abovyan, V. Danielyan, M. Fras, P. Gadow, O. Kortner, S. Kortner, H. Kroha, F. Müller, S. Nowak, R. Richter, K. SchmidtSommerfeld

Max-Planck-Institut fuer Physik, Munich, Germany

The High-Luminosity LHC will provide the unique opportunity to explore the nature of physics beyond the Standard Model of strong and electroweak interactions. Highly selective first level triggers are essential for the physics programme of the ATLAS experiment at the HL-LHC where the instantaneous luminosity will exceed the LHC Run 1 instantaneous luminosity by almost an order of magnitude. The ATLAS first level muon trigger rate is dominated by low momentum muons, selected due to the moderate momentum resolution of the resistive plate and thin gap trigger chambers. This limitation can be overcome by including the data of the precision muon drift tube (MDT) chambers in the first level trigger decision. This requires the fast continuous transfer of the MDT hits to the off-detector trigger logic and a fast track reconstruction algorithm performed in the trigger logic.

We are presenting the reduction of the muon trigger rate that can be achieved by the proposed trigger scheme, a novel fast track reconstruction algorithm, and the demonstration of the scheme with test-beam data acquired at CERN's new Gamma Irradiation Facility.

\section{N35: Accelerator technologies and beam line instrumentation I}

Tuesday, Nov. $1 \quad$ 16:30-18:30 Curie 2

N35-1: LSP Simulations of Dielectric Laser Accelerators

P. K. Soin

AWE, Reading, UK

Simulations of a Dielectric Laser Accelerator (DLA) are presented. An electron beam is passed through a double grating structure of a dielectric material, whilst laser light polarised in the direction of travel of the electron beam applied to both sides. This results in acceleration of the electron beam. The LSP (Large Scale Plasma) code is a particle in cell code which is applied in a novel way to model the DLA. Ultimately, answers are needed to the questions: what is the acceleration gradient, how does this depend on the starting acceleration of the electrons and what is the current that can be accelerated through the DLA? DLAs have great potential in many fields, from providing compact table top size linear accelerators, $\mathrm{x}$-ray light sources and medical devices to application in the next high energy collider for particle research.

N35-2: Diagnosing Laser Plasma Accelerator Based Thomson Source in a Single Shot by Using CCD Electron Trackers $\underline{\text { Y. Zhang }}{ }^{1,2}$, B. J. Quiter ${ }^{1}$, K. Vetter ${ }^{1,2}$, C. G. R. Geddes ${ }^{3}$

${ }^{I}$ Nuclear Science Division, Lawrence Berkeley National Laboratory, Berkeley, CA, United States

${ }^{2}$ Nuclear Engineering, University of California, Berkeley, Berkeley, CA, United States

${ }^{3}$ Accelerator Technology \& Applied Physics Division, Lawrence Berkeley National Laboratory, Berkeley, CA, United States

Laser plasma accelerators generate $\mathrm{GeV}$ electrons within centimeters. MeV-level monochromatic photon sources can be produced by scattering laser photons off the $\mathrm{GeV}$ electrons. The $\mathrm{MeV}$ monochromatic photon sources can be used in radiography and nuclear resonance fluorescence to detect special nuclear materials for nonproliferation purposes. A diagnostic system is needed to characterize the energy and angular deviations of the photons. We propose a diagnostic scheme of using a thin scatterer to induce Compton electrons and using scientific CCDs to track the electrons' energy and direction. The electrons' energy and direction are used to compute the incoming photon's energy and position. A beam of $2 \times 10^{\wedge} 9$ photons at $1.73 \mathrm{MeV}$ incident upon the scatterer-tracker system is simulated in GEANT4. By selecting events in certain regions on the CCD, position resolution of $2.6 \mathrm{~mm}$ and energy resolution of $5.8 \%$ can be achieved.

This work is performed under the auspices of the US Department of Energy by Lawrence Berkeley National Laboratory under Contract DEAC02- 05CH11231. The project was funded by the US Department of Energy, National Nuclear Security Administration, Office of Defense Nuclear Nonproliferation Research and Development (DNN R\&D).

N35-3: Transverse and Longitudinal Beam Profile Monitoring for the AWAKE Experiment at CERN $\underline{\text { B. Biskup }}^{1,2}$, S. Burger ${ }^{1}$, A. Goldblatt ${ }^{1}$, L. Jensen ${ }^{1}$, O. R. Jones ${ }^{1}$, S. Mazzoni ${ }^{1}$, F. Roncarolo ${ }^{1}$, M. Turner ${ }^{1,3}$

${ }^{1}$ CERN, Geneva, Switzerland

${ }^{2}$ Czech Technical University, Prague, Czech Republic

${ }^{3}$ Graz University of Technology, Graz, Austria 
The Advanced Wakefield Experiment (AWAKE) is the first proton-driven plasma wakefield acceleration experiment worldwide. It will exploit the interaction between the CERN SPS $400 \mathrm{GeV}$ proton beam and a rubidium plasma to accelerate a low energy electron beam to over $1 \mathrm{GeV}$ over a very short distance after seeding with a high power pulsed laser. Due to the simultaneous presence of such beams, precise beam diagnostics is crucial. In this contribution we will present the instrumentation for the measurement of the transverse beam size, transverse alignment and temporal synchronisation of all three beams. These systems will be described with a focus on the challenges associated with measuring beams with very different characteristics. The setup optimisation studies together with the necessary changes to the conventional systems used at CERN will be explained. The installation and commissioning phases will also be covered giving details on system performance.

\author{
N35-4: Application of an X-Ray Flat Panel Sensor to a GeV Region Gamma-Ray Beam Profile Monitor \\ H. Kanda ${ }^{1}$, K. Honda $^{1}$, T. Ishikawa ${ }^{2}$, M. Kaneta ${ }^{1}$, K. Maeda $^{1}$, M. Miyabe ${ }^{2}$, Y. Muroi ${ }^{1}$, S. N. Nakamura ${ }^{1}$, A. Ninomiya ${ }^{1}$, \\ Y. Obara $^{3}$, K. Ozawa ${ }^{4}$, K. Ozeki ${ }^{1}$, T. Sasaki ${ }^{1}$, H. Shimizu ${ }^{2}$, A. O. Tokiyasu ${ }^{2}$ \\ ${ }^{I}$ Department of Physics, Tohoku University, Sendai, Miyagi, Japan \\ ${ }^{2}$ ELPH, Tohoku University, Sendai, Miyagi, Japan \\ ${ }^{3}$ Departiment of Physics, University of Tokyo, Tokyo, Japan \\ ${ }^{4}$ IPNS, KEK, Tsukuba, Ibaraki, Japan
}

A beam profile monitor for the $1 \mathrm{GeV}$-region bremsstrahlung gamma-ray beams was developed using an $\mathrm{X}$-ray flat panel sensor taking advantage of its high pixel granularity, portability and remote-controllability. It consists of a thin inorganic scintillator sheet for the absorption of X-ray photons and CMOS area sensors to detect visible light emitted by the scintillator. Both of them are not effective in detecting gamma-ray photons with energies of several to one thousand $\mathrm{MeV}$. A converter plate made of lead was set before the effective area for the conversion of photons into electron and position which produce signals in the CMOS area sensor. Though it does not have the signal multiplication mechanism, monitoring of the beam profile as an integrated image of electrons and positrons was possible. We tested the spatial resolution using a step response to the edge of the converter. Brightness distribution of the edge was spread from the step function and dependent on the thickness of the converter. The sharpest image was obtained by a lead plate with $0.5 \mathrm{~mm}$ thick. The spread was translated as $0.3 \mathrm{~mm}$ in Gaussian sigma. We compared the beam profile with the one obtained by using a scintillating fiber based beam profile monitor for the test of the integrity of the measured profile. The obtained beam profiles were horizontally and vertically projected and Gaussian functions were fitted to them. The obtained sigmas were consistent within errors. We also irradiated a positron beam of $400 \mathrm{MeV}$ to it after penetrating through a gas electrons multiplier (GEM) based particle tracker which detected each position of penetrating positron. The obtained profiles also exhibited consistent spread. By considering these results, we concluded that the X-ray flat panel sensor coupled with a 0.5 -mm-thick lead converter measures reasonably correct beam profiles. This is a first attempt to utilize the X-ray flat panel sensor to the gamma-ray monitoring.

\title{
N35-5: Cherenkov Detector for Proton Flux Measurement in SPS
}

$\underline{\text { S. Dubos }}^{1}$, L. Burmistrov ${ }^{1}$, D. Breton ${ }^{1}$, S. Conforti Di Lorenzo ${ }^{1}$, J. Maalmi ${ }^{1}$, V. Puill ${ }^{1}$, V. Chaumat ${ }^{1}$, A. Stocchi ${ }^{1}$, J.-F. Vagnucci ${ }^{1}$,

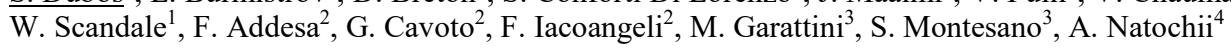

${ }^{l}$ CNRS/IN2P3 - Universite Paris-Sud, Laboratoire de l'Accelerateur Lineaire (LAL), Orsay, France

${ }^{2}$ INFN - Sezione di Roma I, Rome, Italy

${ }^{3}$ CERN, Geneva, Switzerland

${ }^{4}$ Taras Shevchenko National University of Kyiv, Kiev, Ukraine

In the scope of the upgrade of the LHC (Large Hadron Collider) collimation system - with an improved cleaning efficiency of the beam halo and a reduced impedance - the UA9 project is investigating how bent crystals can be used as primary collimators or deflectors. Bent silicon crystals directs the beam halo onto beam stoppers in such way beam losses are well localized along the beam pipe. Investigation of this channeling process close to a circulating beam requires detectors which could be located inside the vacuum pipe itself. The CpFM - Cherenkov detector for proton Flux Measurements - is a 3-year R\&D effort to develop a calibrated detector fitting these requirements, with the aim of counting the number of deflected protons or ions with a precision of about 5\% for 100 incoming particles in the LHC environment. After a campaign of design, prototyping and calibration, the CpFM has been recently directly installed in the Super Proton Synchrotron (SPS) at CERN - without the use of Roman pot - as a fully working system. This detector integrates Cherenkov light produced by relativistic charged particle penetrating a quartz radiator. Report about last two years activity which leads to successful use of this new device by UA9 collaboration is presented. We measured a $15 \%$ resolution for 100 particles, with data-taking done with $500 \mathrm{MeV} / \mathrm{c}$ electrons and $270 \mathrm{GeV} / \mathrm{c}$ protons. Latest results from SPS data taking in July 2015 are also discussed, with a clear CpFM signal without electromagnetic perturbations and a sensitivity resolving the single particle.

N35-6: An ATCA Based Accelerator Controls \& RF Detector Platform

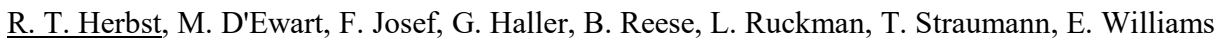

SLAC National Accelerator Laboratory, Menlo Park, CA, United States 
SLAC is currently developing an upgrade of its Linac Coherent Light Source (LCLS) that will be at the forefront of X-ray science. This upgrade will replace the first $1 \mathrm{KM}$ of its existing accelerator tunnel with a superconducting $1 \mathrm{Mhz}$ accelerator requiring a major upgrade to the existing control infrastructure. This new control system moves operations and computations previously performed in software and EPICs into FPGA firmware capable of operating at 1Mhz. SLAC has developed an ATCA based control platform to facilitate this high rate control operation while providing a data acquisition platform for RF based detectors. This platform hosts Beam Position Monitors (BPMs), Machine Protection System (MPS), Bunch Length Monitors (BLEN), Beam Charge Monitor (BCM) and serves as the base platform for the accelerator timing generator.

N35-7: Low-Energy Electron Test Beam at LAL

V. Krylov $^{1,2}$, S. Barsuk ${ }^{1}$, O. Bezshyyko ${ }^{2}$, L. Burmistrov ${ }^{1}$, A. Chaus ${ }^{3}$, P. Colas ${ }^{3}$, O. Fedorchuk ${ }^{2}$, L. Golinka-Bezshyyko ${ }^{2}$,

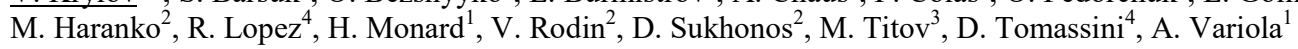

${ }^{1}$ Laboratoire de l'Accélérateur Linéaire, Orsay, France

${ }^{2}$ Taras Shevchenko National University of Kyiv, Kyiv, Ukraine

${ }^{3}$ Commissariat à l'énergie atomique, Institut de Recherche sur les lois Fondamentales de l'Univers, Saclay, France

${ }^{4}$ CERN, Geneva, Switzerland

A new test beam facility providing low energy electrons bunches named LEETECH with adjustable energy and intensity was commissioned at LAL. With the range of possible energies between few $100 \mathrm{keV}$ and $5 \mathrm{MeV}$ it fills the low-cost gap in existing test beam facilities. Among other innovative instrumentation techniques, LEETECH will be used for testing various gaseous tracking detectors and studying new Micromegas/InGrid concept. Covering the MIP region for electrons it provides the possibility of precise $\mathrm{dE} / \mathrm{dx}$ measurement in gaseous medium. In the current research we present the results of preliminary test measurements and detailed Geant4 simulations of LEETECH system in precision mode - dependence between various setup parameters and output beam characteristics. The technique for reducing the time of the full simulation is addressed. After optimization the time spread about 10 ps and energy resolution of $0.3 \%$ are expected according to calculation results. Research was conducted in the scope of the IDEATE International Associated Laboratory (LIA).

\section{N35-8: Luminosity Determination at the ALICE Experiment at the Large Hadron Collider} $\underline{\text { A. Konevskikh }}$

INR RAS, Moscow, Russian Federation

Luminosity determination in ALICE at the Large Hadron Collider is performed via the visible cross sections measured in dedicated calibration experiments (van der Meer scans). In 2015, van der Meer scans were performed for pp collisions at sqrt(s)= 5 and $13 \mathrm{TeV}$, and for $\mathrm{Pb}-\mathrm{Pb}$ collisions at sqrt(s_NN)=5.02 TeV. Visible cross sections have been measured using the ALICE forward detectors, and used to determine the integrated luminosity corresponding to the various data samples collected in 2015. The description of the measurements and analysis is presented. The results and their uncertainties are discussed

N36: Scintillators III: Characterization

Wednesday, Nov. $2 \quad 08: 00-10: 00 \quad$ Madrid
N36-1: Emerging New Ternary Halides as Scintillators for Radiation Detection
K. Biswas
K. Biswas

Dept. of Chemistry \& Physics, Arkansas State University, State University, USA

Recently there has been a discernible shift from simple binary halide scintillators (e.g., NaI, CsI) toward compounds that are structurally and electronically more complex. Besides SrI2 and LaBr3, several pseudobinary, ternary and quaternary halides have shown promise as scintillators for radiation detection. It is an interesting development: on one hand there are inherent difficulties associated with growing and characterizing these crystals, while on the other they may allow more flexibility and configurational degrees of freedom to optimize properties. Within this context, we survey our recent first-principles based computational studies of doped and self-activated ternary halides. Among the doped compounds we consider a family of halides of the type $\mathrm{AB} 2 \mathrm{I} 5: \mathrm{Eu} 2+(\mathrm{A}=\mathrm{K}, \mathrm{Cs} ; \mathrm{B}=\mathrm{Sr}, \mathrm{Ba})$. After briefly discussing their thermodynamic and electronic properties and formation of ordered quaternary phase $\mathrm{A}(\mathrm{BaSr}) \mathrm{I} 5$, we segue to a set of ns2 containing ternaries. These are an analogous group of materials, e.g., InBa2I5 or TlBa2I5 where the ns2 ion is part of the crystal framework, replacing the alkali ion. Here, the valence and conduction band edges have contributions from the ns 2 ions' $s$ and $p$ orbitals which is crucial in carrier localization. Possibility of fast electron capture at the ns2 sites and the prospect of self-activated scintillation via ns2-p????? or ns2-p?ns2-s transitions may draw interest in related applications.

This material is based upon work supported by the U.S. Department of Homeland Security under Grant Award Number, 2014-DN-077-ARI07503. 
N36-2: New Heavy Ce-Activated Scintillator for X- and G-Rays Detection

$\underline{\text { H. Kim }}^{1}$, G. Rooh ${ }^{2}$, H. Park 1 , S. Kim ${ }^{3}$

${ }^{I}$ Phyiscs, Kyungpook National University, Daegu, KOREA

${ }^{2}$ Physics, Abdul Wali Khan University, Mardan, Pakistan

${ }^{3}$ Radiological Science, Cheongju University, Cheongju, KOREA

This study presents the scintillation properties of the new T12LaCl5 single crystal doped with $10 \% \mathrm{Ce} 3+$-concentration (mole\%). This scintillation material is highly hygroscopic and grown by vertical Bridgman technique. Melting point is found to be $530 \mathrm{oC}$. $\mathrm{X}$-ray induced luminescence study is performed at room temperature and the emission spectrum exhibits broad bands between 358 to $435 \mathrm{~nm}$ wavelengths. The maximum emission peak is observed at $389 \mathrm{~nm}$. The obtained emission spectrum is assigned to the $\mathrm{Ce} 3+$ ion due to its $5 \mathrm{~d}$ ? $4 \mathrm{f}$ transition. Pulse height spectrum of $\mathrm{Tl} 2 \mathrm{LaCl} 5: 10 \% \mathrm{Ce} 3+$ obtained under $662 \mathrm{keV}$ ?-rays excitation from a $137 \mathrm{Cs}$ source showed an energy resolution of $6.9 \%$ (FWHM). Highest light yield of 51,000 $\pm 5,000 \mathrm{ph} / \mathrm{MeV}$ is obtained at room temperature using $137 \mathrm{Cs}$ source. Under ?-ray excitation, two exponential decay time constants are observed. The values of the two decay time constants are $31 \mathrm{~ns}$ and $111 \mathrm{~ns}$ with the total light yield contribution of $87 \%$ and $13 \%$, respectively. On the basis of the studied scintillation properties of $\mathrm{T} 12 \mathrm{LaCl} 15$ : $\mathrm{Ce} 3+$, we expect that this material has the potential to be used in medical imaging techniques. Improvement in the scintillation properties such as energy resolution is expected with better crystal quality and the growth optimization of this material.

This work is supported by the Ministry of Science and Technology, Korea (MEST) (No.2015R1A2A1A13001843).

N36-3: Effects of Cerium Concentration in $\mathrm{Tl}_{2} \mathrm{LiYCl}_{6}$ Scintillation Detectors

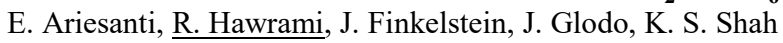

Radiation Monitoring Devices, Inc., Watertown, MA, USA

$\mathrm{Tl}_{2} \mathrm{LiYCl}_{6}$ : $\mathrm{Ce}$ (TLYC), a new, thallium-based elpasolite scintillation crystal, equivalent to $\mathrm{Cs}_{2} \mathrm{LiYCl}_{6}$ :Ce (CLYC), was recently discovered, grown and analyzed at RMD. The exchange of $\mathrm{Tl}^{-}$for $\mathrm{Cs}^{-}$improves the stopping power and photo-fraction of TLYC because of an increase in physical density and effective $Z$ number or $Z_{\text {eff. }}$ TLYC crystals with $0 \%, 0.5 \%, 3 \%, 5 \%$, and $9 \%$ cerium concentrations will be reported in this presentation. Emission spectra of TLYC with $0 \% \mathrm{Ce}, 0.5 \% \mathrm{Ce}$, and $3 \% \mathrm{Ce}$ concentrations peak between 430 and $445 \mathrm{~nm}$. Energy resolution of $6.8 \%, 5.2 \%$, and $4.2 \%$ at $662 \mathrm{keV}$ (FWHM) are measured for samples of TLYC with $0 \% \mathrm{Ce}, 0.5 \% \mathrm{Ce}$ and $3 \% \mathrm{Ce}$, respectively. Comparison with a ${ }^{137} \mathrm{Cs}$ spectrum collected with a NaI:Tl results in gammaray induced light yields of 18,000 ph./MeV for TLYC:0\%Ce, 27,000 ph./MeV for TLYC:0.5\%Ce and 27,000 ph./MeV for TLYC:3\%Ce. A peak from single thermal neutron interactions is resolved at gamma equivalent energy (GEE) of approximately $1.87 \mathrm{MeVee}$ for TLYC:0\%Ce, $1.91 \mathrm{MeVee}$ for TLYC:0.5\%Ce, and $1.89 \mathrm{MeVee}$ for TLYC:3\%Ce. Non-proportionality data, gamma-ray and neutron decay time measurements, and pulse shape discrimination (PSD) results from TLYC:0\%Ce, TLYC:0.5\%Ce, and TLYC:3\%Ce will be reported. Scintillation properties of TLYC:5\%Ce and TLYC:9\%Ce will also be investigated and compared with TLYC grown with lower cerium concentrations.

\section{N36-4: Performance Comparison Between Ceramic Ce:GAGG and Single Crystal Ce:GAGG with Digital-} SiPM

$\underline{\text { C. Park }}^{1}$, C. Kim ${ }^{1}$, J. Kim ${ }^{2}$, Y.-S. Lee ${ }^{1}$, Y. Na ${ }^{2}$, J.-Y. Yeom ${ }^{1,3}$

${ }^{I}$ Bio-Convergence Engineering, Korea University, Seoul, South Korea

${ }^{2}$ Chosun Refractories co. Ltd., Daejeon, South Korea

${ }^{3}$ Biomedical Engineering, Korea University, Seoul, South Korea

We investigated the performance of ceramic $\mathrm{Gd}_{3} \mathrm{Al}_{2} \mathrm{Ga}_{3} \mathrm{O}_{12}$ (Ce:GAGG) and single crystal Ce:GAGG for ?-ray spectrometry coupled to the digital silicon photomultiplier (dSiPM). To compare two different scintillators, we prepared the different samples with $3 \times 3 \times 20 \mathrm{~mm}^{3}$ in size and were coupled to the dSiPM along the long edge. The single crystal Ce:GAGG showed an energy resolution of $9.9 \%$ FWHM, while the ceramic Ce:GAGG demonstrated $10.0 \%$ energy resolution at $511 \mathrm{keV}$ photopeak with corrected for energy resolution. The ceramic Ce:GAGG showed the promising result in spite of its lack of transparency compared to the Ce:GAGG single crystal. A more detailed study with thinner samples and timing performance tests will be carried out.

N36-5: Improvement of Scintillation Properties and Radiation Tolerance of the Ce- Doped Garnet Crystals by Mg Co-Doping

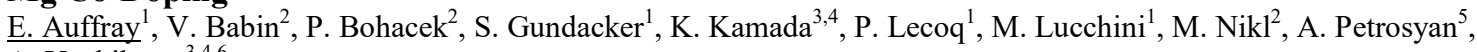

A. Yoshikawa ${ }^{3,4,6}$

${ }^{I} E P / C M X, C E R N$, Geneva, Switzerland

${ }^{2}$ Institute of Physics AS CR, Prague, Czech Republic

${ }^{3}$ Tohoku University, New Industry Creation Hatchery Center, Sendai, Japan 
${ }^{4}$ C\&A Corporation, T-Biz, Sendai, Japan

${ }^{5}$ Institute for Physical Research, Ashtarak, Armenia

${ }^{6}$ Tohoku University, Institute for Material Research, Sendai, Japan

Yttrium and lutetium aluminium garnet doped with cerium, Y3A15O12, (YAG) and Lu3A15O12 (LuAG) scintillating crystals were developed for radiation detectors in early 1990's and 2000's, respectively, presenting high light yield, relatively fast decay, and good radiation tolerance to gamma irradiation. The relatively low density of YAG $(4.5 \mathrm{~g} / \mathrm{cm} 3)$, presence of radioactive lutetium isotope in LuAG and enhanced presence of shallow electron traps deteriorating noticeably their scintillation performance, have motivated the development of multicomponent garnet crystals with higher density, limited intrinsic radioactivity and suppressed trapping effects. One of the main outcome of this research is the discovery of Ce-doped Gd3Al2Ga3O12 (GAGG) single crystal scintillator. With the increasing demand of very fast scintillators for TOF-PET applications and for use in future high luminosity accelerators for the search of rare events, the research has been carried out to accelerate the scintillation decay in the garnet scintillators without decreasing the light yield and deteriorating the radiation tolerance. It has been recently demonstrated that the co-doping with divalent $\mathrm{Me}$ cations $(\mathrm{Me}=\mathrm{Ca}, \mathrm{Mg})$ allows to significantly accelerate the decay characteristics without worsening the other properties of garnet scintillators.

In this contribution we present the improvements of the optical and scintillation properties in terms of light yield, decay time, rise time and coincidence time resolution as well as on the radiation resistance to both gamma and hadron radiation of the Me-codoped garnet crystals YAG:Ce, LuAG:Ce and GAGG:Ce.

This work was performed in the framework of the Crystal Clear Collaboration and received funding from the European Union's Horizon 2020 research and innovation program under the Marie Skodowska-Curie Grant agreement no. 644260 (Intelum) and from the ERC Advanced Grant no. 338953 (TICAL). Support has been received also from ASCIMAT project under Grant agreement no. 690599 and from COST Action TD1401 (FAST). We also acknowledge the support received by the Czech Science foundation 16-15569S project. This work is partially supported by Adaptable \& Seamless Technology Transfer Program through Target-driven R \& D (A- STEP), JST, Development of Systems and Technology for Advanced Measurement and Analysis, Japan Science and Technology Agency (JST) and New Energy and Industrial Technology Development Organization (NEDO).

N36-6: High-Quality Lead Tungstate Crystals Available for EM-Calorimetry in High-Energy Physics ${ }_{\text {R. W. Novotny }}^{1}$, K.-T. Brinkmann ${ }^{1}$, V. Dormenev ${ }^{1}$, J. Houzvicka ${ }^{2}$, M. Korjik ${ }^{3}$, H.-G. Zaunick ${ }^{1}$

${ }^{I}$ 2nd Physics Institute, Justus-Liebig-University, Giessen, Germany

${ }^{2}$ CRYTUR spol. s.r.o., Turnov, Czech Republic

${ }^{3}$ Institute for Nucelar Problems, Minsk, Belarus

There is a strong interest and demand for high quality lead tungstate crystals (PbWO4, PWO) for electromagnetic (EM) calorimetry. PWO has been implemented into the EM calorimeter of the CMS-ECAL detector at LHC and is required for the completion of the PANDA EMC. In spite of moderate radiation hardness in an environment of high hadron fluences, PWO represents an ideal material for EM-calorimetry on electron accelerators and/or experiments with primarily electromagnetic probes. The compactness, sufficient light yield and various recovery options combined with a moderate price require the opportunity for mass production. The Czochralski method has been proven to be the optimum growing technology. However, after bankruptcy of the Bogoroditsk Technical Chemical Plant in Russia as the major producer so far, a new manufacturer had to be found. The company CRYTUR (Turnov, Czech Republic) with good experience in the development and production of different types of inorganic oxide crystals has re-started end of 2014 in a common effort the development of lead tungstate for the mass production based on the Czochralski method. An impressive progress of the R\&D was achieved since then. The growing technology was optimized to produce full size samples with the quality meeting the PANDA EMC specifications for PWO-II. The presentation will give a detailed progress report on the research program in collaboration with groups at Orsay and JLab. The full size crystals will be characterized with respect to optical performance, light yield, kinetics and radiation hardness. Presently, the stringent quality limits of PWO have been achieved for $200 \mathrm{~mm}$ long crystals. The report will give a status on the ongoing pre-production run of tapered crystals to be completed end of 2016.

supported by BMBF, GSI and EU

N36-7: Development of BaF2 Crystals for Future HEP Experiments at the Intensity Frontiers F. Yang ${ }^{1}$, J. Chen ${ }^{2}$, L. Zhang ${ }^{1}$, R. Zhu ${ }^{1}$

${ }^{I}$ Crystal Lab, HEP, California Institute of Technology, Pasadena, USA

${ }^{2}$ Shanghai Institute of Ceramics, Shanghai, China

Barium fluoride $(\mathrm{BaF} 2)$ is a fast inorganic crystal scintillator. Because of its fast scintillation with sub nanosecond decay time it is considered as a candidate crystal for a very fast crystal calorimetry for future HEP experiments at the intensity frontier. Two crucial issues of BaF2 application are its radiation hardness and its slow scintillation component with $600 \mathrm{~ns}$ decay time, which 
causes pile-up. BaF2 crystals produced by different vendors were irradiated by ?-rays up to $200 \mathrm{Mrad}$, and show good radiation hardness. A rare earth doped $\mathrm{BaF} 2$ crystal grown by BGRI shows promising performances in slow component suppression.

N37: Astrophysics and space I

Wednesday, Nov. $2 \quad$ 08:00-10:00 Londres

N37-1: The DAMPE Silicon-Tungsten Tracker

$\underline{\text { R. Asfandiyarov }}$

DPNC, University of Geneva, Geneva, Switzerland

On behalf of the DAMPE Collaboration

The DAMPE (DArk Matter Particle Explorer), one of the five satellite missions in the framework of the Strategic Pioneer Research Program in Space Science of the Chinese Academy of Science (CAS), was successfully launched on December 17 last year. DAMPE is a powerful space telescope which has as main scientific objective the identification of possible Dark Matter signatures thanks to the capability to detect electrons and photons in a wide range of energy from $5 \mathrm{GeV}$ up to $10 \mathrm{TeV}$ and with unprecedented energy resolution. Moreover, the DAMPE satellite will contribute to a better understanding of the origin and propagation mechanisms of high energy cosmic rays thanks to the measurement of the flux of nuclei up to $100 \mathrm{TeV}$. Hints in the sector of high energy gamma astronomy are also expected thanks to its excellent photon detection capability. After the launch DAMPE has shown excellent performance in orbit. Currently, data is being analyzed and the first results will be published soon. Here we present a detailed description and the current status of the DAMPE tracker: the Silicon-Tungsten tracKer-converter (STK). The STK was developed by an international collaboration composed of groups from University of Geneva, INFN Perugia, INFN Bari, INFN Lecce and Institute of High Energy Physics in Beijing. The STK is made of 192 ladders of about $10 \mathrm{~cm}$ by 40 $\mathrm{cm}$, each of them is made up of four single-sided silicon strip detectors. The tracker is capable of precise track reconstruction of charge particles with precision of 50um. It plays a crucial role in particle identification, $\mathrm{e} / \mathrm{p}$ separation, ion charge measurement and gamma identification. The STK behavior in orbit is very stable and it performs as on the ground and according to the specification. It exhibits excellent detection capabilities in terms of charge and position measurements.

N37-2: From PoGOLite to PoGO+ - the Development of a Balloon-Borne Hard X-Ray Polarimetry Mission M. Pearce

Dept. of Physics, KTH Royal Institute of Technology, Stockholm, Sweden

On behalf of the PoGO+ Collaboration

Polarimetry has long been a routine probe of sources within radio, optical and infra-red astronomy. The application to X-and gamma-ray astronomy has not evolved as rapidly due to the relatively long integration times required for measurements - thereby requiring a dedicated mission - and the particular attention which must be paid to systematic effects due to the positive definite nature of measurements. Advances in the field are instead currently driven by spectroscopy, imaging and timing studies. Many astrophysical X-ray sources are dominated by non-thermal emission with radiation transferred in highly asymmetric systems. A measurement of the linear polarisation of the emitted radiation therefore constitutes a key observable and diagnostic for sources which cannot be spatially resolved. PoGO+ is a balloon-borne hard X-ray polarimeter operating in the $20-\sim 200 \mathrm{keV}$ energy band. Polarisation is determined using coincident Compton scattering and photo-absorption in a segmented array of plastic scintillators surrounded by a BGO anticoincidence system and a polyethylene neutron shield. The design is development of the PoGOLite mission which measured the polarisation of hard X-rays from the Crab during a balloon-flight in 2013. This is the first measurement in the hard X-ray band using a purpose built polarimeter which had been characterised with both polarised and unpolarised beams prior to flight. The PoGO+ mission is scheduled for launch from the Esrange Space Center in July 2016 with the Crab and Cygnus X-1 (hard state) as the primary observation targets. The design of the PoGO+ mission and the outcome of the 2016 flight opportunity will be reviewed.

\author{
N37-3: POLAR: Final Calibration and In-Flight Performance of a Dedicated GRB Polarimeter \\ M. Kole \\ DPNC, University of Geneva, Geneva, Switzerland \\ On behalf of the POLAR Collaboration
}

Despite being the brightest events in the universe since the Big Bang, a proper understanding of Gamma-Ray Bursts is still missing. The POLAR instrument, built by a Chinese-European collaboration, aims to provide new insights in the physics of GRBs using the first instrument to accurately measuring the polarization parameters of several of these objects per year. The polarimeter is optimized for dedicated measurements of the polarization parameters of the gamma-ray component of the prompt emission of GRBs. The polarization parameters of a photon flux can be extracted by measuring the Compton scattering angles when the photons interact in a detector. In POLAR these Compton scattering angles are measured using a finely segmented plastic scintillator array consisting of 1600 bars. The bars, with a surface area of 6 by $6 \mathrm{~mm}$ and a length of $176 \mathrm{~mm}$, are read out 
in groups of 64 by 25 flat-panel multi-anode photomultipliers. Due to the relatively fine segmentation, POLAR can measure the photon interaction locations, and therefore the scattering angles, with a high precision resulting in a high sensitivity to polarization. The instrument furthermore has a large effective area and a field of view of $1 / 3$ of the sky. The instrument was extensively calibrated during both 2015 and 2016. Through Geant4 simulations, validated using calibration data, the instrument was shown to be capable of performing measurements with a minimum detectable polarization below $10 \%$ for several GRBs per year. The instrument is foreseen to be launched in September 2016 on-board of the second Chinese Space Lab, the Tiangong-2. The design of this new space-based gamma-ray polarimeter will be presented along with the instrument response measured during calibration measurements and during the first weeks of flight. Finally based on the instrument performance the achievable science goals of instrument will be discussed.

\section{N37-4: The Spectrometer/Telescope for Imaging X-Rays (STIX) of the ESA Solar Orbiter Mission D. Casadei}

Fachhochschule Nordwestschweiz (FHNW), Windisch, Switzerland

On behalf of the STIX Collaboration

The Solar Orbiter spacecraft is a Sun-observing mission led by the European Space Agency (ESA), to be launched in 2018 for addressing the interaction between the Sun and the heliosphere. Among its ten instruments, the Spectrometer/Telescope for Imaging X-rays (STIX) will observe solar flares in order to determine the intensity, spectrum, timing, and location of thermal and accelerated electrons near the Sun through their bremsstrahlung X-ray emission. STIX performs indirect imaging with the moiré technique while measuring the energy of individual photons in the range $4-150 \mathrm{keV}$ with CdTe-based detectors. This report illustrates the configuration and expected performance of the STIX flight model, which shall be delivered to ESA by the end of October 2016.

N37-5: Implementation of the First Level Trigger of JEM-EUSO: Results of the First Tests

A. Jung $^{1}$, S. Bacholle ${ }^{1}$, P. Barrillon ${ }^{2}$, M. Bertaina ${ }^{3,4}$, S. Blin-Bondil ${ }^{5}$, M. Casolino ${ }^{6,7}$, A. Cummings ${ }^{8}$, S. Dagoret-Campagne ${ }^{2}$,

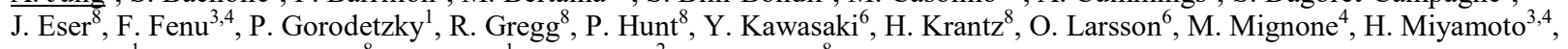
E. Parizot ${ }^{1}$, L. W. Piotrowski ${ }^{8}$, G. Prévôt ${ }^{1}$, J. Rabanal $^{2}$, L. Wiencke ${ }^{8}$

${ }^{I}$ APC, AstroParticule et Cosmologie, Université Paris Diderot, CNRS/IN2P3, CEA/lrfu, Observatoire de Paris, Sorbonne Paris Cité, Paris, France

${ }^{2}$ Laboratoire de l'Accélérateur Linéaire, Université Paris Sud-11, CNRS/IN2P3, Orsay, France

${ }^{3}$ Universita degli studi di Torino, Torino, Italy

${ }^{4}$ INFN Torino, Torino, Italy

${ }^{5}$ OMEGA, Ecole polytechnique, Palaiseau, France

${ }^{6}$ RIKEN Advanced Science Institute, Wako, Japan

${ }^{7}$ INFN Roma Tor Vergata, Roma, Italy

${ }^{8}$ Department of physics, Colorado School of Mines, Golden, Colorado, USA

The JEM-EUSO Collaboration is developing a series of pathfinders for a space-based mission to detect Ultra-High-Energy Cosmic Rays (UHECRs). They partly share the same electronics, in particular the central data acquisition system. We report on the successful test of the implementation of the first-level trigger within the FPGA of the photo-detection module (PDM) board, which processes the signals from 36 64-pixel MAPMTs with a time unit of $2.5 \mu \mathrm{s}$.

\section{N37-6: Overview and Status of the Cherenkov Telescope Array}

J. Cortina

Institut de Física d'Altes Energies (IFAE), Cerdanyola del Valles, Spain

On behalf of the CTA Consortium

Imaging Atmospheric Cherenkov Telescopes (IACTs) have opened a new astronomical window at photon energies exceeding tens of $\mathrm{GeV}$ during the last decades. The technique relies on the detection of Cherenkov light produced by electromagnetic showers in the Earth's atmosphere using telescope mirrors with diameters of a few to tens of meters. A world-wide community is behind the design and construction of the next generation Cherenkov Telescope Array (CTA), consisting of two arrays of IACTs at the northern and southern hemispheres. CTA will feature IACTs of three different sizes optimized to cover different energy ranges. The design incorporates a significant number of innovations in all mirror, photo detection, readout, trigger or data processing technologies. This presentation will cover the design characteristics of the instrument and the status of the project.

N37-7: A Solid-State Pixelated X-Ray Detector for Solar Flare Observations

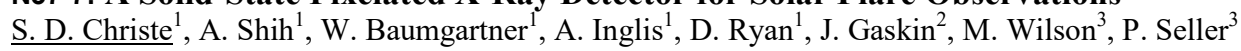


${ }^{I}$ NASA Goddard Space Flight Center, Greenbelt, MD, USA

${ }^{2}$ NASA Marshall Space Flight Center, Huntsville, AL, USA

${ }^{3}$ Rutherford Appleton Laboratory, Oxford, UK

High resolution X-ray spectroscopic imaging has the potential to provide new and ground-breaking opportunities to study the poorly understood high energy processes in the solar corona such as solar flares, coronal heating, etc. However, such observations require a new breed of solid-state detectors sensititve to high energy X-rays with fine independent pixels to subsample the point spread function (PSF) of the X-ray optics. They must also be capable of handling very high count rates from bright and impulsive events such as solar flares. The Rutherford Appleton Laboratory (RAL) has developed a Cadmium Telluride (CdTe) detector system, dubbed HEXITEC (High Energy X-ray Imaging Technology). It is an 80x80 array of 250 micron independent pixels sensitive in the $4--80 \mathrm{keV}$ band and capable of a high full frame readout rate of $10 \mathrm{kHz}$. HEXITEC provides the smallest independently read out pixels currently available, and are well matched to the few arcsecond PSF produced by the current and next generation hard X-ray focusing optics. NASA's Goddard and Marshall Space Flight Centers are collaborating with RAL to develop these detectors for use on future space-borne hard X-ray focusing telescopes. In this presentation we show the latest results on HEXITEC's imaging capability, high read out rate, and energy sensitivity and explore it's application to solar observations.

This work is supported by NASA grant NNH13ZDA001N-APRA.

N37-8: Operation and Calibration of MAXI/GSC Xe Gas Counters on the International Space Station for over 6 Years since 2009

M. Sugizaki

Riken, Saitama, Japan

On behalf of the MAXI GSC Team

MAXI (Monitor of All-sky X-ray Image) is an X-ray astronomical mission performed on the International Space Station (ISS), Since the payload was activated on the ISS in August 2009, the main instrument, GSC (Gas Slit Camera) has been sanning the whole sky every 92-minute orbital cycle. The GSC utilizes 12 Xe-gas proportional counters as the X-ray detectors. They employ resistive carbon anodes to achieve the position sensitivity. Passing through the in-orbit operation for over the 6 years, some gas counters now expose significant performance degradation. Three out of the 12 counters are considered to have a broken anode wires, which is thought to be caused by repeated heavy particle irradiation and discharge. We operate these counters carefully by activating only their alive segments. Another counter showed a sudden gain increase on 2013 June 10. Its gas gain reached the 1.5 times higher by a month. From the diagnostic data analysis, it is suggested that the gas pressure began to decrease at that time with the exponential decay time of $\sim 1.5$ year. We operate the counter by turninig the anode voltage so that the gas gain keep constant. In this paper, we summarize the 6-year operation and the detail performance calibration for the science analysis.

N38: Simulation and prototyping for detector development I

Wednesday, Nov. $2 \quad$ 08:00-10:00 Curie 1

N38-1: Identification of Background Components with the SoLid anti-Neutrino Detector I. Piñera-Hernández

University of Antwerp, Antwerp, Belgium

On behalf of the SoLid collaboration

The SoLid experiment aims to measure the anti-neutrino energy spectrum 5.5-10m from the core of the BR2 nuclear reactor at SCK-CEN in Mol, Belgium. The goal is to provide the most sensitive search for short-baseline neutrino oscillations and to resolve the reactor neutrino anomaly. In fact, the proposed detector technology will be very useful for anti-neutrino detection in other settings as well, such as nuclear safeguard and non-proliferation monitoring of nuclear reactors.

The experiment utilizes a novel, highly segmented composite scintillator detector. The detector unit is based on $5 \mathrm{~cm}$ polyvinyl toluene scintillator cubes, thin neutron sensitive ${ }^{6} \mathrm{LiF}: \mathrm{ZnS}(\mathrm{Ag})$ sheets and a reflective Tyvek layer wrapping them for light tight. The first $288 \mathrm{~kg}$ detector prototype based on this technology was deployed at the BR2 reactor in November 2014. The main purpose was to study the capability of the detector design to discriminate background. Due to the low overburden and proximity to a nuclear reactor, efficient background reduction is crucial for a successful experiment.

This work will presenting the advantages of the SoLid detector design for background reduction. The background components studied include muons, fast neutrons induced by cosmic rays, fission neutrons, and gamma rays. The results are based on a full chain GEANT4 based Monte Carlo simulations of a 1.5 tonne SoLid detector, and when applicable, also their comparison with data recorded using the prototype detector.

N38-2: Simulation Validation Epistemics in a Geant4 Case Study 


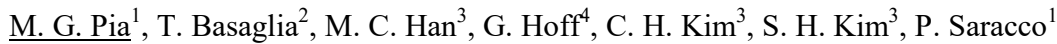

${ }^{I}$ INFN Genova, Genova, Italy

${ }^{2}$ CERN, Geneva, Switzerland

${ }^{3}$ Hanyang University, Seoul, Korea

${ }^{4}$ CAPES, Brasilia, Brazil

This presentation summarizes the outcome of a three year long project concerning the validation of Geant4-based simulation of electron backscattering. Accurate simulation of backscattering is of great importance in many experimental scenarios - from calorimetry to medical dosimetry, as it affects the pattern of energy deposition in detectors or other kinds of sensitive volumes. All Geant4 multiple and single scattering models, as well as Geant4 recommended settings for PhysicsLists, have been involved in a validation test against several thousand experimental measurements documented in the literature. Comparison with experiment is carried out through rigorous statistical methods. The effect of dependencies of physics modeling on other parts of the software has been investigated. Recent unpublished results, which also concern two new Geant 4 multiple and single scattering models first released in Geant4 10.2, are presented and critically discussed. Differences in the capability of reproducing experiment associated with physics modeling options are quantitatively evaluated by means of categorical data analysis methods. The presentation of this validation test, its methodology and its statistical analysis tools offer the opportunity to review the foundations of simulation validation epistemology and provide concrete guidelines to the experimental community to assess the reliability of simulation applications in experiments.

N38-3: The Design of Time Projection Chamber for Fission Cross-Section Measurements

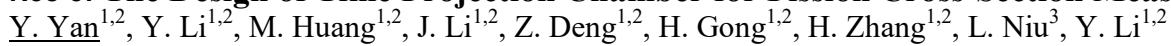

${ }^{I}$ Tsinghua University, Beijing, China

${ }^{2}$ Key Laboratory of Particle \& Radiation Imaging (Tsinghua University), Beijing, China

${ }^{3}$ High-Tech Institute of Xi'an, Xi'an, China

In order to measure cross-sections of the major actinides with high precision, a TPC is proposed taking advantage of its ability of particle track reconstruction. In this study, a Monte Carlo simulation was developed to optimize the design of the fission Time Projection Chamber(fTPC) system. Based on the simulation, we determined the size of the pad and the parameters of the readout electronics which have a great influence on the system performance.

N38-4: SPAD Array Simulator: Release and Distribution

A. C. Therrien, V. Libioulle, S. A. Charlebois, R. Fontaine, J.-F. Pratte

Institut Interdisciplinaire d'Innovation Technologique, Sherbrooke, Canada

SPAD array design is a complex endeavour and requires a long turnaround time between prototypes. Selecting a readout strategy for these arrays is also difficult. To aid in these tasks, we developed a SPAD array simulator for both analog and digital arrays. This simulator has recently been upgraded and converted to $\mathrm{C}++$ and has been packaged for distribution to the community. It uses scintillation photons from GEANT4 simulations, to which it can be interfaced directly, however, the simulator core was designed to be flexible and can be used with different photon source files and different readouts.

This simulator emulates the non-linear behavior of SPAD arrays by tracking the status of each SPAD independently. In addition it tracks the trigger of each SPAD avalanche, listing how many were caused by primary photons (scintillation, Cerenkov, etc.), noise (dark counts, crosstalk, afterpulsing, etc.) and how many photons were not detected because the SPAD was not available (quenched). Other features of this new version include an enhanced crosstalk module and more shape options for the SPADs in the array. Most notably, the execution speed of the simulator was dramatically improved, taking less than 5 seconds to simulate 1000 PET events in the digital mode, and about 5 minutes for 1000 events in the analog mode, making it 100 times faster than previous versions.

\section{N38-5: Multivariate Techniques for Energy Reconstruction in Highly Granular Calorimeters}

\section{B. Bilki ${ }^{1,2}$}

${ }^{I}$ Physics and Astronomy, University of Iowa, Iowa City, IA, USA

${ }^{2}$ Mathematics and Computing, Beykent University, Istanbul, Turkey

The overall trend in calorimetry is towards high granularity both laterally and longitudinally. This trend is a requirement for the full exploitation of the Particle Flow Algorithms, which reconstruct individual particles using the subdetector that provides the best resolution for this specific particle. The increased level of detail in the event topologies due to higher segmentation of the calorimeter provides additional handles for energy reconstruction. Here, the power of using multivariate statistical techniques using various topological variables in the reconstruction of the energy of single particles in highly granular calorimeters will be demonstrated. 
N38-6: GeantV: Particle Transport Spanning CPUs and Accelerators J. Apostolakis

EP Dep., CERN, Geneva, Switzerland

On behalf of the GeantV Collaboration

The aims of the GeantV project are to research and develop the next generation particle transport simulation, targeting not only modern CPU architectures, but also accelerators including GPGPU, Intel@ Xeon Phi, and more exotic resources using Atom or ARM processors, which have potential for future use in HEP computing. The first, proof of concept GeantV prototype has been engineered for CPU threads with hardware vector support, but planning from early stages included joint development for such accelerators. A key goal is to access the improved performance of vector instructions and GPU threads, but maintain common source code in order to ensure maintainability. We describe how we achieve this, using a set of 'backend' classes. A number of components have been created to demonstrate the performance potential of this approach, starting from single geometry shapes and single physics processes, and moving to the full navigation of tracks in complex geometries using simplified physics. We summarize the progress in creating a complete, stand-alone transport engine for electron-gamma physics, and survey the performance of the parts of the code that have been vectorized.

N38-7: Optimization of a Spherical Active Coded Mask Gamma-Ray Imager $\underline{\text { D. Hellfeld }}^{1}$, P. Barton ${ }^{2}$, D. Gunter ${ }^{2}$, L. Mihailescu ${ }^{2}$, K. Vetter ${ }^{1,2}$

${ }^{I}$ Department of Nuclear Engineering, University of California, Berkeley, Berkeley, CA, USA

${ }^{2}$ Lawrence Berkeley National Laboratory, Berkeley, CA, USA

Gamma-ray imaging allows for efficient detection, characterization, and localization of compact radioactive sources in cluttered environments. Fieldable planar detector systems employing active coded masks demonstrate broad energy sensitivity via both coded aperture and Compton imaging modalities. Because planar configurations suffer from a limited field-of-view (FOV), we introduce a novel design by rearranging the detectors into an active coded spherical configuration, thereby facilitating both coded aperture and Compton imaging with an isotropic 4p FOV. The Portable Radiation Imaging Spectroscopy and Mapping (PRISM) detector system under development at Lawrence Berkeley National Laboratory consists of $961 \mathrm{~cm}^{3} \mathrm{CdZnTe}$ coplanar grid (CPG) detectors distributed among 192 positions on the inner surface of a sphere with an outer diameter of $14 \mathrm{~cm}$. This report investigates only the low-energy coded aperture mode of PRISM. Uniform system response over $4 \mathrm{p}$ can be achieved by adjusting the locations of the 96 detectors on the sphere. The detector locations are optimized using a number of metrics, including the autocorrelation of the system response matrix with uniform diagonal and minimal sidelobes, and the maximum likelihood expectation maximization based image reconstruction of simulated data. We also explore the imaging benefits associated with the additional depth-of-interaction information obtained from each CPG detector. The results of this work provide an optimal configuration of the detectors in PRISM.

\section{N38-8: A New Large Calorimeter Based on Lanthanum Bromide Coupled to Silicon Photomultipliers: Monte Carlo Simulation Predictions}

A. Papa, P. Schwendimann

Paul Scherrer Institute, 5232, Villigen, Switzerland

In the last few years, lanthanum halide scintillators are interesting the scientific community because of their almost ideal scintillator properties. Recent studies have shown that both $\mathrm{LaBr} 3(\mathrm{Ce})$ and $\mathrm{LaCl} 3(\mathrm{Ce})$ are very attractive materials due to its ultra high light output and its fast response, resulting in good candidates as a crystal for a calorimeter able to provide simultaneously very high energy and timing performances. The $\mathrm{LaBr} 3(\mathrm{Ce})$ and $\mathrm{LaCl} 3(\mathrm{Ce})$ crystals are commercially available with cylindrical shapes and sizes up 3 inch. (diameter) $\times 3$ inch. length. Crystals with reduced diameter and length up 8 inch. can be obtained under a custom production.

We performed detailed Monte Carlo simulations in order to study the response of a new large calorimeter based on $\mathrm{LaBr} 3(\mathrm{Ce})$ coupled to Silicon Photo- multipliers (MPPC). It is made of an array of $4 \times 4$ crystals of $2.5 \times 2.5 \times 20 \mathrm{~cm} 3$ coupled to Hamamatsu MPPC S12573-025C (active area $6 \times 6 \mathrm{~mm} 2$; pixel size $25 \times 25 \mu \mathrm{m} 2$ ). The simulation is supported by measurements done with an available 3 inch. (diameter) $\times 3$ inch. length $\mathrm{LaBr} 3(\mathrm{Ce})$ crystal coupled to a photomultiplier tube and the characterization of the MPPC response. It is based on GEANT4 and a custom code which includes the simulation of the MPPC response, the full electronic chain and the reconstructed algorithms. The analysis is based on the simulated waveforms sampled at 5 GSample/s. Very promising results have been obtained: for ?s with an energy of $55 \mathrm{MeV}$ an energy resolution of s_E /E $\sim 2 \%( \pm 0.2 \%)$ and a timing resolution of $\mathrm{s}_{-} \mathrm{t} \sim 30( \pm 1)$ ps have been predicted.

Such results pose this calorimeter at the detector frontiers and turns out to be very interesting in particular for future charged lepton flavour experiments, for which high detector performances are required for ?s in the energy range of 50-100 MeV.

N39: Circuits for readout of pixel and strip detectors in HEP and nuclear physics 


\title{
Wednesday, Nov. $2 \quad$ 08:00-10:00 Curie 2
}

N39-1: TOFFEE: a Fully Custom Amplifier-Comparator Chip for Silicon Detectors with Internal Gain. N. Cartiglia ${ }^{1}, \underline{\text { F. Cenna }}^{1}$, A. Rivetti ${ }^{1}$, M. Da Rocha Rolo ${ }^{1}$, J. Varela $^{2}$, A. Di Francesco ${ }^{2}$, J. Olave ${ }^{1,3}$

${ }^{1}$ INFN, Torino, italy

${ }^{2}$ LIP, Lisbon, Portugal

${ }^{3}$ Politecnico di Torino, Torino, Italy

In this contribution we present the design of a 8-channel amplifier-comparator chip specifically optimized to match the signals produced by Ultra-Fast Silicon Detectors (UFSD). The time resolution of the TOFFEE $\ddot{i} ; \frac{1}{2}$ UFSD system is expected to be around $30 \mathrm{ps}$, which is the required time resolution of the next generation of track-timing detectors at HL-LHC. The chip is designed in UMC 110nm CMOS technology, it has a 2x2 mm area and it requires $40 \mathrm{~mW}$ per channel. It features LVDS outputs and the signal dynamic range matches the requirements of the HPTDC system.

The TOFFEE-UFSD sensor has been designed to match the timing requirements of the CT-PPS detector at CMS, and it is schedule for installation during the winter 2016 winter shutdown.

\author{
N39-2: SALT - a Dedicated Readout ASIC for Upstream Tracker in the Upgraded LHCb Experiment \\ T. Fiutowski \\ Faculty of Physics and Applied Computer Science, AGH University of Science and Technology, Krakow, Poland \\ On behalf of the LHCb UT Collaboration
}

The present Large Hadron Collider beauty $(\mathrm{LHCb})$ detector performance is limited by readout electronics and data acquisition architecture. After the upgrade of LHC machine it will be capable to deliver more than one order of magnitude higher luminosity than presently used by the $\mathrm{LHCb}$ detector. To achieve this goal various detectors will need a new faster front-end electronics with the read-out running at the bunch-crossing rate of $40 \mathrm{MHz}$.

SALT is a new 128-channel readout ASIC for silicon strip detectors in the upgraded Upstream Tracker of the LHCb experiment. It will extract and digitize analogue signals from the sensor, perform digital processing and transmit serial output data. It is designed in CMOS $130 \mathrm{~nm}$ process and uses a novel architecture consisting of an analogue front-end and ultra-low power $(<0.5$ $\mathrm{mW}$ ) fast sampling (40 MSps) 6-bit ADC in each channel.

Prototypes of all important SALT blocks, i.e. 8-channel analogue front-end, 8-channel 6-bit ADC, PLL, DLL, and SLVS I/O, were designed, fabricated and tested, showing very good performance. An 8-channel SALT prototype comprising all important functionalities was produced and found, in tests, to be fully operational. The 128-channel version of ASIC has been just submitted. In this paper we report on the design and tests results of the 8-channel prototype and first results of the 128-channel version.

\author{
N39-3: STS/MUCH-XYTER2, a Full-Size Prototype Readout Chip for Silicon Strip and GEM Detectors

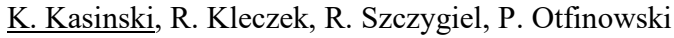 \\ Department of Measurement and Electronics, AGH University of Science and Technology, Cracow, Poland
}

New fixed-target experiments at Facility for Antiproton and Ion Research (FAIR) using high intensity beams from SIS 100 accelerator are currently being at the final stage of design. Most of the readout electronics are expected to suffer high flux of radiation and have to be radiation hard. Particles under investigation will produce little charge on their way across the Silicon Tracking System (STS) detector stations which, in conjunction with relatively large sensor and interconnecting cable capacitances and series resistances and tight power requirements, makes a challenging task to provide amplitude measurements with signal-to-noise ratio high enough for efficient track recovery. The STS/MUCH-XYTER2, a full-size chip for time and amplitude measurement in the STS and Muon Chamber (MUCH) detectors is presented. Specification and architecture of the analog front-end and digital back-end are shown. This 128-channel chip is able to measure amplitude (5-bit resolution) and time ( $3.125 \mathrm{~ns}$ resolution) and meet the specified average input rate of $250 \mathrm{kHit} / \mathrm{s} / \mathrm{channel}$. The front-end is capable to operate with double-sided silicon microstrip sensors of the STS and gas detectors at MUCH thanks to the flexibility and configurability of the amplifier and the shapers. As a mature prototype, the chip incorporates all the testability features providing means for fast and effective wafer-level, during assembly process (via pogo probing) and in-system functional testing, calibration and performance tuning during system's lifetime which will be also presented.

\section{N39-4: New Development on Digital Architecture for Efficient Pixel Readout ASIC at Extreme Hit Rate for HEP Detectors at HL-LHC}


A novel digital architecture has been designed and implemented in a 65nm CMOS pixel readout ASIC prototyped by the CHIPIX65 project. The architecture has a high efficiency (above 99\%) at pixel hit rate up to $3 \mathrm{GHz} / \mathrm{cm} 2$, a trigger rate of $1 \mathrm{MHz}$ and a trigger latency of $12.5 \mathrm{usec}$; the overall inefficiency is dominated by analog dead-time. The digital architecture is organized in pixel regions where the hit is stored locally and output to the end of Column only if correspond to triggered events, strategy also used in other working chips, but in order to have a very compact and low power architecture a larger $4 \times 4$ pixels pixel regions has been used, allowing an improved sharing of resources and overcoming freezing problems seen in early work. A 5-bit ToT charge is stored in a centralised latency buffer and at the arrival of a trigger from the periphery, a trigger matching logic selects eventually the right memory location and send the information to the End of Column logic and therefore to the chip periphery. Detail simulation results are discussed and shown.

N39-5: Design and Test of a Zero Dead Time Analog Front-End for Next Generation Pixel Detectors

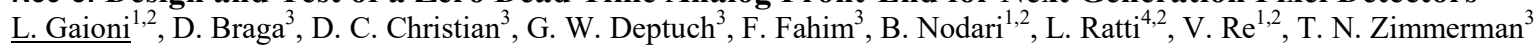

${ }^{I}$ University of Bergamo, Dalmine (BG), Italy

${ }^{2}$ INFN Pavia, Pavia, Italy

${ }^{3}$ Fermilab, Batavia, IL, USA

${ }^{4}$ University of Pavia, Pavia, Italy

Future pixel chips at the High-Luminosity (HL) Large Hadron Collider (LHC) will be exposed to unprecedented levels of radiation and particle rates. New tracker detectors, complying with the very demanding operating conditions and the delivered luminosity (up to $5 \times 10^{\wedge} 34 \mathrm{~cm}^{\wedge}-2 \mathrm{~s}^{\wedge}-1$ ), will be required in the Phase II upgrade at ATLAS and CMS. This work is concerned with the design and the test of a synchronous analog processor with zero dead time developed in a $65 \mathrm{~nm}$ CMOS technology, conceived for pixel detectors at the HL-LHC experiment upgrades. It includes a low noise, fast charge sensitive amplifier featuring a detector leakage compensation circuit, and a compact, single ended comparator that guarantees very good performance in terms of channel-to-channel threshold dispersion without needing any pixel-level trimming. A 2-bit flash ADC is exploited for digital conversion immediately after the charge amplifier. A prototype chip including front-end channels arranged in an $8 \times 8$ matrix and other test structures will be submitted by the end of May 2016. A thorough discussion on the design and on the characterization of the readout channel will be provided in the conference paper.

N39-6: The VeloPix ASIC for the LHCb VELO Upgrade

$\underline{X . \text { Llopart }}$

CERN, Geneva, Switzerland

The LHCb upgrade, scheduled for LHC Run-3, will enable the experiment to be read out at $40 \mathrm{MHz}$ in triggerless mode, with full event selection being performed offline. The Vertex Locator (VELO) will be upgraded to a pixel device with a new dedicated ASIC, the VeloPix, a $130 \mathrm{~nm}$ technology chip with data driven and zero suppressed readout. The sensors are positioned with an innermost radius at just $5.1 \mathrm{~mm}$ from the LHC beams and the hottest ASICs will experience rates of more than $900 \mathrm{Mhits} / \mathrm{s}$. The recently submitted ASIC will be presented along with the first test results.

N39-7: The CHESS-2 prototype in AMS 0.35 $\mu$ m process: a high voltage CMOS monolithic sensor for ATLAS detector.

C. Tamma

SLAC National Accelerator Laboratory, Menlo Park, CA, U.S.A.

On behalf of the Strip CMOS Collaboration

CHESS-2 (CMOS (HV) Evaluation for Strip Sensors) is a novel ASIC strip architecture designed to explore the feasibility of using high voltage CMOS MAPS (Monolithic Active Pixel Sensors) as alternative sensors for the ATLAS Phase-II Strip Tracker Upgrade. The ASIC is optimized to characterize several crucial performance aspects of sensors with different substrate resistivities by signal processing and hit pixel position encoding and readout. The ASIC is made of three independent groups of 128 strips composed of 32 subpixels each. The pixel size is $630 \mu \mathrm{m}$ x $40 \mu \mathrm{m}$ and includes a charge sensitive amplifier and the first stage of a comparator inside the collecting well. The second stage, the configuration, the hit encoding and the readout architectures are located at the periphery of the strips. A novel "fast skip" hit encoding logic identifies the first 8 hit pixel positions with a single-bunch timing resolution and sends the data to a fast readout circuit for serialization and transmission on 14 LVDS channels at $320 \mathrm{MHz}$. The ASIC architecture functionalities and performances will be presented at the meeting.

N39-8: A Prototype of a New Generation Pixel Readout ASIC in CMOS 65nm for Extreme Rate HEP Detectors at HL-LHC 
E. Monteil ${ }^{1}$, L. Pacher ${ }^{1}$, A. Paterno ${ }^{2}$, N. Demaria ${ }^{3}$, A. Rivetti ${ }^{3}$, M. Da Rocha Rolo ${ }^{3}$, G. Dellacasa ${ }^{3}$, G. Mazza ${ }^{3}$, F. Ciciriello ${ }^{4}$, C. Marzocca ${ }^{4}$, F. Loddo ${ }^{5}$, F. Liciulli ${ }^{5}$, S. Mattiazzo ${ }^{6}$, F. De Canio ${ }^{7}$, L. Gaioni ${ }^{7}$, V. Re ${ }^{7}$, G. Traversi ${ }^{7}$, L. Ratti ${ }^{8}$, S. Marconi ${ }^{9}$, G. Magazzu ${ }^{10}$, A. Stabile ${ }^{11}$, P. Placidi ${ }^{12}, \underline{\text { S. Panati }^{2}}$

${ }^{I}$ University of Torino, Department of Physics and with INFN Torino, Torino, Italy

${ }^{2}$ Politecnico of Torino and with INFN Torino, Torino, Italy

${ }^{3}$ INFN Torino, Torino, Italy

${ }^{4}$ Politecnico di Bari and with INFN Bari, Bari, Italy

${ }^{5}$ INFN Bari, Bari, Italy

${ }^{6}$ Dipartimento di Ingegneria dell'Informazione, University of Padova, Padova, Italy

${ }^{7}$ University of Bergamo and with INFN Pavia, Bergamo, Italy

${ }^{8}$ University of Pavia and with INFN Pavia, Pavia, Italy

${ }^{9}$ University of Perugia, with CERN and with the INFN Perugia, Perugia, Italy

${ }^{10}$ INFN Pisa, Pisa, Italy

${ }^{11}$ INFN Milano and Universit 'a degli Studi di Milano, Milano, Italy

${ }^{12}$ University of Perugia and with the INFN Perugia, Perugia, Italy

A first prototype of a readout ASIC in CMOS $65 \mathrm{~nm}$ for a pixel detector at HL-LHC is described. The pixel cell is of 50 um x 50 um and the matrix consists of $64 \times 64$ pixels. The chip has been designed to guarantee high efficiency at extreme data rate, for very low signals and low power consumption. Two different analog very front end designs, one synchronous and one asynchronous, are implemented both having: area of $35 \mathrm{um}$ x35 um; ENC of $100 \mathrm{e}$-; in-time threshold below 1000 e-; leakage current compensation up to 50nA; power consumption below $5 \mathrm{uW}$. A 5-bit charge digitisation is foreseen adopting ToT technique, using a $40 \mathrm{MHz}$ clock or a local Fast Oscillator going up to $500 \mathrm{MHz}$, to perform charge sampling.

Internal 10-bit DACs are used for biasing, and monitoring is provided by 12-bit ADC. A novel digital architecture have been developed, maintaining high efficiency (above 99.5\%) at pixel hit rate up to $3 \mathrm{GHz} / \mathrm{cm} 2$, trigger rate of $1 \mathrm{MHz}$ and trigger latency of $12.5 \mathrm{usec}$, with a power consumption of about $5 \mathrm{uW}$ per pixel. Analog dead-time is below 1\%. Data are sent via serialiser connected to a CMOS-to-SLVS transceivers working at $320 \mathrm{MHz}$. All IP-blocks and very front ends used are silicon proven and tested also after high irradiation of 500-800 MHz.

This chip have been designed by CHIPIX65 project and will be submitted in June 2016. Early results will be presented in this paper.

\title{
J01: Joint Session I - MIC-NSS-RTSD
}

\author{
Wednesday, Nov. $2 \quad$ 10:30-12:00 Schweitzer \\ J01-1: Evolution of Diamond Based Microdosimetry \\ $\underline{\text { J. A. Davis }}^{1}$, K. Ganesan ${ }^{2}$, D. A. Prokopovich ${ }^{3}$, M. Petasecca ${ }^{1}$, S. Guatelli ${ }^{1}$, D. N. Jamieson ${ }^{2}$, M. L. F. Lerch ${ }^{1}$, A. B. Rosenfeld ${ }^{1}$ \\ ${ }^{I}$ Centre for Medical Radiation Physics, wollongong, NSW, Australia \\ ${ }^{2}$ University of Melbourne, Parkville, VIC, Australia \\ ${ }^{3}$ Australian Nuclear Science and Technology Organisation, Lucas Heights, NSW, A
}

The requirements for solid state microdosimetry particularly within the context of medical and space environments necessitate a tissue equivalent and radiation hard material, for which diamond is uniquely suited. An overview of the current status of diamond based microdosimetry is given. This overview will explore previous and current technologies including but not limited to those produced internally at the Centre for Medical Radiation Physics (CMRP). The main contribution to this body of work by the CMRP is that of 3D pixelated detectors. The overview will analyse technologies in terms of their advantages and disadvantages within the context of microdosimetry. Using this as a basis, recommendations with respect to fabrication methodologies/techniques are provided in order to direct the course and progress of development so as to achieve true diamond based microdosimetry.

\author{
J01-2: Tackling the Count Rate Problem in Spectral CT by Means of a GaAs-Based Medipix3RX Detector \\ Operated in Edge-on Geometry

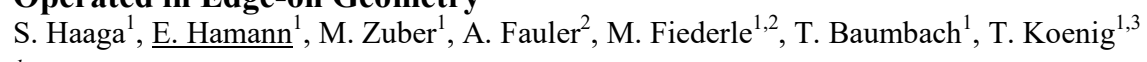 \\ ${ }^{I}$ IPS/LAS, Karlsruhe Institute of Technology, Karlsruhe, Germany \\ ${ }^{2}$ FMF, University of Freiburg, Freiburg, Germany \\ ${ }^{3}$ Ziehm Imaging GmbH, Nürnberg, Germany
}

Spectroscopic CT using photon counting detectors is considered a significant advancement to methods based on varying X-ray spectra. A common drawback to the method, however, is the relatively low flux tolerance of photon counting detectors, which must be able to separate individual photon events in time to give meaningful results. This currently prevents a good spectral resolution in high flux clinical applications, where the pixel pitch needs to be reduced to lower the photon flux per pixel, typically resulting in a degradation of energy resolution due to effects such as charge sharing. Here, we present a detector module 
operating in edge-on geometry which distributes the incoming X-ray flux across a large number of depth segments. Being based on a Medipix3RX ASIC and a high resistivity, chromium compensated gallium arsenide sensor, the attenuation of high energy photons along its depth of $1.4 \mathrm{~cm}$ is perfectly suited to distributing the high photon flux across so many detector channels that only very few require being operated under pile-up conditions. This approach allows performing spectral and photon counting CT scans at fluxes exceeding $10^{8} \mathrm{~mm}^{-2} \mathrm{~s}^{-1}$, while still using the detector's charge summing functionality to full extent and thus guarantee a good spectral resolution. Our results open a way out of the dilemma of having to trade energy resolution for high count rates, and pave the road to building medical scanners operated beyond tube voltages of $120 \mathrm{kVp}$ at high absorption efficiencies.

\author{
J01-3: CZT Sensor - Readout ASIC Interfaces for High-Flux Photon Counting Systems \\ $\underline{\text { K. Iniewski }}$ \\ Redlen Technologies, BC, Canada
}

CZT sensors are primary candidates for many next-generation X-ray photon counting imaging systems. We have conducted a study to evaluate performance tradeoffs and limitations of CZT sensors and readout ASICs for security, non-destructive testing (NDT) and medical imaging applications. We have found that performance of the photon counting systems depends critically on the nature of the CZT-ASIC interface including interposer capacitance and sensor dark leakage currents. We have built a mathematical model for the energy resolution (ER) and count rate (CR) using analytical models for the pile-up and chargesharing. The model can be used to optimize sensor geometry (pixel pitch, sensor thickness), detector fabrication (injecting vs. non-injecting contacts) and operating conditions (HV bias, count rate) for the optimum performance of the high flux X-ray imaging systems.

The author gratefully acknowledges support numerous people at Redlen Technologies in particular special thanks go to Uri El-Hanany, Adam Densmore, Georgios Prekas, and Glenn Bindley. Several external collaborators contributed greatly towards obtaining experimental results: Michael Rissi (Dectris), Valeria Radicci (Dectris), Pietro Zambon (Dectris), Matthias Schneebeli (Dectris), Jan Iwanczyk (DxRay), William Barber (DxRay), Phil Butler (MARS Bioimaging), Eric Johnson (SureScan) and Carl Bosch (SureScan). Kris Iniewski is with Redlen Technologies, 1763 Sean Heights, Saanichton,, BC V8M 1X6S, Canada (e-mail: kris.iniewski@redlen.com).

\author{
J01-4: Breast Microcalcification Classification Using Energy Dispersive X-Ray Coherent Scatter Computed \\ Tomography \\ B. Ghammraoui, L. M. Popescu \\ Division of Imaging, Diagnostics and Software Reliability, U.S Food and Drug Administration, Silver Spring, Maryland, United \\ States
}

We investigate the application of Coherent Scatter Computed Tomography (CSCT) using photon counting energy resolving detectors as a non-invasive diagnosis method for breast microcalcifications classification. The breast microcalcifications can be categorized in type I, consisting of calcium oxalate dihydrate, or weddellite (WD), which are mainly associated with benign tumors, and type II, composed of calcium phosphates or calcium hydroxyapatite (HA), which are preponderantly associates with malignant tumors. In a preliminary study using an energy dispersive x-ray diffraction experimental arrangement, we have determined the form factors for HA and WD. The coherent scatter cross-sections of HA and WD have distinct profiles allowing for potential differentiation. With the experimental form-factors introduced into the MC-GPU Monte Carlo simulation code, we have carried out simulation tests using a breast phantom consisting of a uniform main background equivalent to $50 \%$ glandularity, and additional regions of interest of varying background composition in which were inserted HA and WD grains of different sizes. The energy resolved coherent scatter projection data was generated considering a scanning pencil beam configuration. We used a maximum-likelihood estimation of scatter components algorithm which takes into account the polychromatic source, the detector response function and the energy dependent attenuation. Adipose, glandular, HA and CO cross sections were used as basis scatter components. The classification of type I or II calcification was done using the relative contrasts of their components as criterion. Simulation tests were carried out for different doses and energy resolutions for multiple realizations. The results were analyzed using the relative operating characteristic (ROC) methodology showing good discrimination ability at medium and higher doses. The noninvasive CSCT technique shows potential for further improving the breast diagnostic accuracy.

J01-5: Scintillator-Based Photon Counting Detector: Is It Feasible? L. Bläckberg ${ }^{1,2}$, N. Moghadam ${ }^{3}$, D. Uzun-Ozsahin ${ }^{1}$, G. El Fakhri ${ }^{1}, \underline{\text { H. Sabet }}^{1}$

${ }^{I}$ Department of Radiology, Massachusetts General Hospital, Harvard Medical School, Boston, MA, United States

${ }^{2}$ Department of Physics and Astronomy, Uppsala University, Uppsala, Sweden

${ }^{3}$ GRAMS Lab, Institut interdisciplinaire d'innovation technologique (3IT), Université de Sherbrooke, Shebrooke, QC, Canada 
By utilizing finely pitched scintillator arrays where the scintillator has high atomic number and density, fast decay time, and high light output, realizing a scintillator-based PCD is conceptually feasible. Fabrication of fine-pitched scintillator arrays however, has been the bottleneck for realizing such PCDs. Combining the novel scintillator fabrication technique called laser-induced optical barriers (LIOB) where optical barriers can be placed inside transparent crystal acting as reflector without removing the material, with laser ablation (LA) method, we are now able to overcome the obstacles for developing scintillator-based PCD. In this regard, we are developing an LSO-based PCD where the LSO crystal is laser pixelated to sub-mm pixels. The scintillator array will be coupled to an application specific integrated circuit (ASIC) where each ASIC pixel has built-in photodiode, amplifiers and 3-4 energy windows and their associated counters. We have simulated different scenarios where the crystal is pixelated by a combination of LIOB and LA techniques where the $2 \mathrm{~mm}$ thick crystal is first pixelated by LIOB to a depth and then the rest is pixelated by the LA technique. We also simulated the fraction of collected light in the same scintillator pixel by modeling various surface properties of the pixel cuts as well as optical barrier surface roughness and refractive index (RI). Simulation results show that up to $\sim 67 \%$ of the scintillation light will be contained in the same pixel when only using LIOB technique with barrier refractive index of 1.0. This value is $\sim 55 \%$ with barrier RI of 1.2 . These results suggest that laser processed arrays can potentially change the paradigm in PCD development as they can replace the traditional array production and thus allow for scintillator-based PCD development in a more robust and cost-effective manner.

This work is supported in part by the U.S. National Institute of Health under grant R03EB020762-01. Lisa Blackberg acknowledges support from Swedish Research Council (VR).

J01-6: Scintillating Glass GEM Detector for High Resolution X-ray Imaging

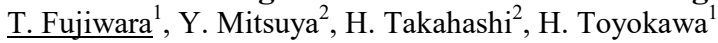

${ }^{I}$ National Institute of Advanced Industrial Science and Technology (AIST), Tsukuba, Japan

${ }^{2}$ The University of Tokyo, Tokyo, Japan

A high resolution X-ray imaging gaseous detector has been successfully developed with Scintillating Glass GEM. The imaging system consists of a chamber filled with scintillating gas ( $\mathrm{Ar} / \mathrm{CF} 4, \mathrm{Kr} / \mathrm{CF} 4, \mathrm{Xe} / \mathrm{CF} 4)$, inside of which Glass GEM (G-GEM) is mounted for high gain gas multiplication. Since the gas gain of the G-GEM is much higher compared to conventional GEMs, ultra high yield scintillation photons are produced during the avalanche process. These photons can be easily detected by a mirror-lens-CCD-camera system and a high resolution X-ray radiograph is formed. In this study, we demonstrate X-ray imaging performance of our digital X-ray imager with 20 - $110 \mathrm{kV}$ X-ray tube, and combination of various gas (Ar/CF4, Kr/CF4, $\mathrm{Xe} / \mathrm{CF} 4)$. The imaging system shows high sensitivity to low-energy X-rays, which results in a high contrast radiograph for elements with low atomic numbers. In addition, high spatial resolution and high speed image forming is demonstrated with taking $\mathrm{X}$-ray movies in $10 \mathrm{~ms}$ integration time.

N40: Calorimetry II - Radiation-hard Calorimeters

Wednesday, Nov. $2 \quad$ 10:30-12:00 Madrid

N40-1: Radiation High Dose Rate Tolerant Scintillation Materials for Future Experiments at High Luminosity LHC and FCC M. Korjik ${ }^{1}$, E. Affray ${ }^{2}$

${ }^{I}$ RINP, Minsk, Minsk, Belarus

${ }^{2}$ CERN, Geneva, Switzerland

This report gives of a review the last results of the radiation damage effects in scintillation materials used in high energy physics experiments, that are caused by ?-quanta and high energy hadrons, the two main causes of irradiation in the High Luminosity LHC and future FCC. The creation and recovery of color centers, induced radioactivity and harmful radio-luminescence in organic and inorganic materials under hadron irradiation are described. An approach to select scintillation materials suitable to operate in the different parts of the detectors, particularly in the forward part of calorimeters will be proposed. Report also considers approaches to construct detection modules capable to survive during the full operation period of the High Luminosity LHC.

\section{N40-2: Energy Resolution and Timing Performance Studies of a W-CeF3 Sampling Calorimeter with a} Wavelength-Shifting Fiber Readout

P. Meridiani ${ }^{1}$, R. Becker ${ }^{2}$, L. Bianchini ${ }^{2}$, G. Dissertori ${ }^{2}$, L. Djambazov ${ }^{2}$, M. Donegi $_{i}^{1} 2^{2}$, L. Brianza $^{3}$, D. Del Re ${ }^{4}, N_{i}$ Chiodini $^{5}$, N. Pastrone ${ }^{6}$, G. Della Ricca ${ }^{7}$, N. Akchurin ${ }^{8}$, M. Droge ${ }^{1}$, C. Haller ${ }^{1}$, U. Horisberger ${ }^{1}$, T. Klijnsma ${ }^{1}$, W. Lustermann ${ }^{1}$, A. Marini $^{1}$, D. Meister ${ }^{1}, \underline{\text { F. Micheli }}{ }^{1}$, F. Nessi-Tedaldi ${ }^{1}$, F. Pandolfi ${ }^{1}$, M. Peruzzi ${ }^{1}$, U. Roeser ${ }^{1}$, M. Schoenenberger ${ }^{1}$, A. Ghezzi ${ }^{3}$, A. Martelli $^{3}$, S. Pigazzini ${ }^{3}$, T. Tabarelli de Fatis ${ }^{3}$, P. Govoni ${ }^{3}$, M. Fasoli $^{5}$, A. Vedda ${ }^{5}$, F. Cavallari ${ }^{1}$, I. Dafinei ${ }^{1}$, M. Diemoz ${ }^{1}$, F. Pellegrino ${ }^{1}$, M. Nuccetelli ${ }^{1}$, R. Paramatti ${ }^{1}$, C. Rovelli ${ }^{1}$, G. D'imperio ${ }^{4}$, S. Gelli ${ }^{4}$, G. Organtini ${ }^{4}$, F. Santanastasio ${ }^{4}$, L. Soffi ${ }^{4}$, V. Monti ${ }^{6}$, P. P. Trapani ${ }^{6}$, V. Candelise ${ }^{7}$, F. Vazzoler ${ }^{7}$, J. Faulkner ${ }^{8}$ 
${ }^{I}$ Sezione di Roma, INFN, Roma, Italy

${ }^{2}$ Institute for Particle Physics, ETH, Zurich, Switzerland

${ }^{3}$ Dipartimento di Fisica, Universitï $i^{1 / 2}$ di Milano Bicocca and INFN, Milano, Italy

${ }^{4}$ Dipartimento di Fisica, Universitï ${ }^{1} / 2$ di Roma La Sapienza and INFN, Roma, Italy

${ }^{5}$ Dipartimento di Scienza dei Materiali, Universitï ${ }^{1} 1 / 2$ di Milano Bicocca and INFN, Milano, Italy

${ }^{6}$ Sezione di Torino, INFN, Torino, Italy

${ }^{7}$ Dipartimento di Fisica, Universiti ${ }^{1 / 2} 2$ degli Studi di Trieste and INFN, Trieste, Italy

${ }^{8}$ Texas Tech University, Lubbock, Texas, USA

An innovative sampling calorimeter prototype matrix of $5 \times 3$ channels has been built and evaluated, that uses tungsten as absorber and Cerium Fluoride as the scintillating medium, while light is read out by wavelength-shifting fibers running along each channels chamfers. An in-depth study of the energy resolution and of the response to electrons as a function of the impact position and angle has been performed and compared with expectations from simulation. The time resolution has been studied using different photodetectors and wavelength-shifting fibres, showing that a resolution better than $100 \mathrm{ps}$ is achievable with SiPMs. CeF3 has been used since it is a fast scintillator, very well suited for radiation hard applications, which spontaneously recover from hadron damage at room temperature and that can be produced resistant to ionising radiation.

\title{
N40-3: Precision Crystal Calorimetry at High Energy and High Luminosity: CMS ECAL Performance at 13 TeV and Upgrade Test Beam Studies F. Micheli
}

ETH, Zurich, Switzerland

On behalf of the CMS Collaboration

The electromagnetic calorimeter (ECAL) of the Compact Muon Solenoid Experiment (CMS) is operating at the Large Hadron Collider (LHC) in 2016 with proton-proton collisions at $13 \mathrm{TeV}$ center-of-mass energy and at a bunch spacing of $25 \mathrm{~ns}$. The instantaneous luminosity during this LHC Run II is expected to exceed $10^{34} \mathrm{~cm}^{-2} \mathrm{~s}^{-1}$ in routine operation. In this talk we present detailed performance results from the 2016 data taking period. At the higher center of mass energy and with the rapidly growing data set the performance at higher electron and photon energies becomes crucial. The CMS ECAL design ensures that its superb performance extends over a very wide range of energies up to electron and photon energies of $1 \mathrm{TeV}$ and beyond. This is of crucial importance for physics searches beyond the standard model. We discuss how the triggering, event reconstruction and calibration of the ECAL detector is performing in this new regime. The impact of the ECAL performance on resonance searches in the mass range up to $1 \mathrm{TeV}$ will be discussed. The running conditions in 2016 give a first impression on the challenging conditions we expect at the high luminosity LHC (HL-LHC). We review the design and R\&D studies for the CMS ECAL crystal calorimeter upgrade and present first test beam studies. Particular challenges at HL-LHC are the harsh radiation environment, the increasing data rates and the extreme level of pile-up events, up to 200 simultaneous proton-proton collisions. We present test beam results on hadron irradiated PbWO crystals up to fluences expected at HL-LHC and the status of the new readout and trigger electronics R\&D. The pile-up mitigation may be substantially improved by means of precision time tagging of calorimeter clusters, by associating them to primary vertices via 4D triangulation. We present test beam results on the precision timing potential of the CMS PbWO crystal calorimeter and discuss how the readout electronics may be adapted to exploit this performance in CMS.

\author{
N40-4: Upgrade of the ATLAS Liquid Argon Calorimeters for the HL-LHC \\ T. McCarthy \\ Foehringer Ring 6, Max-Planck-Institut fuer Physik, 80805 Muenchen, Germany \\ On behalf of the ATLAS Liquid Argon Group
}

The increased particle flux at the high luminosity phase of the Large Hadron Collider (HL-LHC) with instantaneous luminosities of up to 7.5 times the original design value will have an impact on many sub-systems of the ATLAS detector. In particular, in the liquid Argon forward calorimeter. The increase in the ionization load poses a number of problems that can degrade its performance like beam heating and space charge effects in the liquid Argon gaps and high-voltage drop due to increased current drawn over the current-limiting resistors. This contribution will discuss possible solutions to these problems including the construction of a new forward calorimeter. To further mitiage the effects of pile-up, it is also considered to install a highgranularity timing detector at the front face of the end-cap cryostat. Several different sensor technologies for this timing device are currently under investigation.

N40-5: Longitudinally Segmented Shashlik Calorimeters with SiPM Readout: the SCENTT Experiment A. Berra ${ }^{1,2}$, C. Brizzolari ${ }^{1,2}$, S. Cecchini ${ }^{3}$, F. Cindolo ${ }^{3}$, C. Jollet ${ }^{4}$, A. Longhin ${ }^{5}$, L. Ludovici ${ }^{6}$, G. Mandrioli ${ }^{3}$, N. Mauri $^{3}$, A. Meregaglia ${ }^{4}$, A. Paoloni ${ }^{5}$, L. Pasqualini ${ }^{3,7}$, L. Patrizii ${ }^{3}$, M. Pozzato ${ }^{3}$, F. Pupilli ${ }^{5}$, M. Prest ${ }^{1,2}$, G. Sirri ${ }^{3}$, F. Terranova ${ }^{2,7}$, E. Vallazza ${ }^{8}$, L. Votano ${ }^{5}$ 
${ }^{I}$ Universita' degli Studi dell'Insubria, Como, Italy

${ }^{2}$ INFN Milano Bicocca, Milano, Italy

${ }^{3}$ INFN Bologna, Bologna, Italy

${ }^{4}$ Institut Pluridisciplinaire Hubert Curien, Strasbourg, France

${ }^{5}$ INFN Laboratori Nazionali di Frascati, Frascati (RM), Italy

${ }^{6}$ INFN Roma, Roma, Italy

${ }^{7}$ Universita' degli Studi di Bologna, Bologna, Italy

${ }^{8}$ INFN Trieste, Trieste, Italy

Shashlik calorimeters are sampling calorimeters which use wavelength shifting fibers running perpendicularly to the scintillating/absorber plates for the light readout. These calorimeters are cost effective, easy to assemble, and characterized by a good flexibility in terms of energy resolution, which can be tuned with the proper choice of scintillating/absorber thickness and fiber sampling. On the contrary, the perpendicular optical fiber readout and the resulting fiber bundling to the photosensor pose a strong limitation to the longitudinal segmentation of these detectors. Recently, the fast development of solid state photosensors allowed for the integration of the readout system directly in the bulk of the calorimeter, opening new possibilities in terms of longitudinal segmentation. The SCENTT collaboration is a R\&D project funded by INFN with the goal to develop longitudinally segmented shashlik calorimeters readout by new generation Silicon PhotoMultipliers for applications in high granularity devices for collider physics, beam dumps and non-conventional neutrino facilities. In particular, the SCENTT collaboration is developing an ultra-compact module where every single fiber segment is directly connected to a non-amplified SiPM; the SiPMs are arranged in arrays on custom PCBs and readout by a fast electronics based on waveform digitizers. This contribution presents the results in terms of linearity, energy resolution and e/pi discrimination obtained with a prototype of longitudinally segmented shashlik calorimeter, readout with SiPMs embedded in the calorimeter bulk. The tests have been performed at the CERN PS-T9 beamline in the 1-5 GeV energy range in 2015. The final ultra-compact modules of the SCENTT calorimeter have been produced and are being tested with cosmic rays before moving them to CERN for the data taking with particles in summer 2016. In the talk we will present the results and a detailed performance assessment of the novel ultra-compact design.

N40-6: A Finely-Segmented Radiation-Tolerant Shashlik Electromagnetic Calorimeter A. Ledovskoy

Department of Physics, University of Virginia, Charlottesville, VA, USA

On behalf of the Shashlik Calorimetry Development Group

A design for a sampling EM calorimeter option suitable for HL-LHC operations, consisting of two 31,000-module W/LYSO plate Shashlik endcaps, has been developed and prototyped within the CMS experiment at the LHC. These detectors would not only provide superb energy resolution for photons and electrons of $10 \mathrm{GeV}=\mathrm{ET}=0.5 \mathrm{TeV}$, but when coupled with existing endcap hadron calorimetry, could deliver excellent hadronic resolution for jets in the harsh environment of the end cap region $(1.47=?=3.0)$ of LHC collider experiments. We present the Shashlik configuration that has been proposed and the results of beam tests, radiation damage studies, and simulations of performance of the actual configuration.

\section{N41: New concepts in solid-state detectors and radiation damage effects I}

\section{Wednesday, Nov. $2 \quad$ 10:30-12:00 Curie 1}

N41-1: Estimation of the Energy Resolution Limit for Particle Detectors with Schottky-Barrier Based on VPE GaAs

A. V. Chernykh ${ }^{1}$, S. V. Chernykh ${ }^{1}$, F. M. Baryshnikov ${ }^{1}$, S. I. Didenko ${ }^{1}$, N. Burtebayev ${ }^{2}$, G. I. Britvich ${ }^{3}$, A. P. Chubenko ${ }^{4}$, Y. N. Glybin ${ }^{5}$, T. Zholdybayev ${ }^{2}$, Z. Kerimkulov ${ }^{2}$ J. T. Burtebayeva ${ }^{2}$

${ }^{I}$ National University of Science and Technology «MISIS», Moscow, Russia

${ }^{2}$ Institute of Nuclear Physics, Almaty, Kazakhstan

${ }^{3}$ Institute of High Energy Physics, Protvino, Moscow Region, Russia

${ }^{4}$ P.N. Lebedev Physical Institute of the Russian Academy of Sciences, Moscow, Russia

${ }^{5}$ LLC «SNIIP Plus», Moscow, Russia

Radiation damage-resistant semiconductor detectors are needed in experiments on measurements of crosssections of nuclear reactions in the interactions of heavy ions with energies close to the Coulomb barrier for astrophysical applications [1]. The low yield of such nuclear reactions is accompanied by enormous Rutherford scattering crosssections that can quickly lead to failure of the conventional Si detectors. In [2] it was shown that surface barrier detectors based on HP VPE GaAs can efficiently replace Si detectors in such applications. This work is focused on characterization of such detectors and estimation of their energy resolution limit.

The detectors with an active area of 25 and $80 \mathrm{~mm}^{2}$ were fabricated on HP GaAs epilayers. A $12 \mathrm{~nm}$ Pt Schottky barrier was formed by ion-plasma sputtering. The detectors demonstrated high FWHM of 14.2 and $15.5 \mathrm{keV}$ on for 25 and $80 \mathrm{~mm}^{2}$, respectively (5.5 MeV a-line), which is at the level of Si spectrometric detectors. 
Various components of the FWHM were analyzed in order to estimate the energy resolution limit of the detectors. On the basis of the carried out measurements and simulation it was shown that the FWHM of the detectors is mainly determined by the fluctuation in the number of collected electron-hole pairs. It was shown that the charge losses caused not only by recombination in the detectors volume, but also by recombination in the surface layer of a thin Schottky contact. Performed calculations show that the obtained FWHM can be improved (by about 1-2 keV) by optimizing the thin Schottky barrier formation process. Characteristics of the Schottky contact detectors fabricated by optimized process will also be presented and discussed.

1. S. Hamada and N. Burtebayev. INT J MOD PHYS E, vol. 24, no. 06, p. 1550047, 2015.

2. S. Chernykh et al. GaAs detectors with an ultra-thin Schottky contact for spectrometry of charged particles. The 14th VCI. Vienna, Austria, 2016.

N41-2: 3D Silicon and Passive CMOS Pixel Detectors for Radiation Hard Environments

$\underline{\text { D.-L. Pohl }}{ }^{1}$, C. da Via ${ }^{2}$, M. Daas ${ }^{1}$, L. Gonella ${ }^{3}$, T. Hemperek ${ }^{1}$, F. Hügging ${ }^{1}$, J. Janssen ${ }^{1}$, H. Krüger ${ }^{1}$, A. Macchiolo ${ }^{4}$, L. Vigani $^{5}$, N. Wermes ${ }^{1}$

${ }^{I}$ Physikalisches Institut der Universität Bonn, Bonn, NRW, Germany

${ }^{2}$ University of Manchester, School of Physics and Astronomy, Manchester, England, United Kingdom

${ }^{3}$ University of Birmingham, School of Physics and Astronomy, Birmingham, England, United Kingdom

${ }^{4}$ Max-Planck-Institut für Physik, München, Bayern, Germany

${ }^{5}$ University of Oxford, Department of Physics, Oxford, England, United Kingdom

The high-luminosity upgrade for the Large Hadron Collider at CERN requires a new inner tracking detector for the ATLAS experiment. About $200 \mathrm{~m}^{2}$ of silicon detectors are needed with very radiation hard detectors for the inner pixel layer and low-cost detectors with a high production throughput for the outer layers. Promising approaches are the usage of 3D-silicon sensors for a hybrid pixel detector design for the inner layers and the utilization of commercial CMOS technologies for the sensors of the outer layers. 3D-silicon sensors from two different vendors (FBK, CNM) and passive CMOS pixel sensors in $150 \mathrm{~nm}$ technology from LFoundry have been characterized. A detailed charge-collection efficiency (CCE) study of 3D-sensors has been carried out using an actual pixel-readout chip, the ATLAS FE I4. The CCE of the 3D-sensors are compared to the mature and well known n in-n planar pixel sensors from CiS. For the novel passive CMOS sensors key properties have been determined like the leakage current density, the depletion depth, and the particle detection efficiency. All studies have been carried out at different fluences according to their proposed location in the future ATLAS pixel detector.

N41-3: New 3D Mushroom Microdosimeter for RBE Studies in Passive Scattering and Pencil Beam Scanning Heavy Ion Therapy

L. Chartier ${ }^{1}$, L. T. $\operatorname{Tran}^{1}$, D. A. Prokopovich ${ }^{2}$, D. Bolst ${ }^{1}$, A. Pogossov ${ }^{1}$, S. Guatelli ${ }^{1}$, M. Petasecca ${ }^{1}$, M. L. F. Lerch ${ }^{1}$, M. I. Reinhard ${ }^{2}$, A. Kok ${ }^{3}$, M. Povoli ${ }^{4}$, A. Summanwar ${ }^{3}$, M. Jackson ${ }^{5}$, T. Kanai ${ }^{6}$, N. Matsufuji ${ }^{7}$ A. B. Rosenfeld ${ }^{1}$

${ }^{I}$ Centre for Medical Radiation Physics, University of Wollongong, Wollongong, NSW, Australia

${ }^{2}$ Nuclear Stewardship Platform, Australian Nuclear Science and Technology Organisation, Lucas Heights, NSW, Australia

${ }^{3}$ Microsystems and Nanotechnology, SINTEF, Oslo, Norway

${ }^{4}$ Centre for Material Science and Nanotechnology, University of Oslo, Oslo, Norway

${ }^{5}$ Faculty of Medicine, University of New South Wales, Kensington, NSW, Australia

${ }^{6}$ Physics Division, Gunma University Heavy Ion Medical Center, Maebashi, Gunma, Japan

${ }^{7}$ Research Centre for Charged Particle Therapy, National Institute of Radiological Science, Inage, Chiba, Japan

A new microdosimeter with freestanding 3D sensitive volumes, known as the 3D Mushroom Microdosimeter, is described and utilized in passively scattered $400 \mathrm{MeV} / \mathrm{u}$ oxygen and $180 \mathrm{MeV} / \mathrm{u}$ nitrogen ion beams. In addition, the first microdosimetric application in an active scanning $290 \mathrm{MeV} / \mathrm{u}$ carbon ion beam used in modern treatment facilities is presented. For each ion species, the dose-mean lineal energy was measured along the depth of the pristine Bragg peaks along the central axis with high precision at the Bragg peak edge. Maximum dose-mean lineal energies for oxygen and nitrogen ion beams at the Bragg peak were measured to be $330 \mathrm{keV} / \mathrm{i}_{i} 1 / 2 \mathrm{~m}$ and $420 \mathrm{keV} / \mathrm{i} i{ }^{1} / 2 \mathrm{~m}$, with maximum RBE10 values of 2.9 and 3.1 , respectively. For the scanning carbon ion beam, a maximum dose-mean lineal energy was found to be $140 \mathrm{keV} / \mathrm{i} \mathrm{i}^{1 / 2 \mathrm{~m}}$ and maximum RBE10 value of 2.4. This work demonstrates the ability of the 3D Mushroom microdosimeter to investigate field quality and determine RBE10, giving great promise to its utility as a microdosimetric QA device in passive and active delivery heavy ion therapy facilities.

N41-4: Proton Induced Radiation Damage in Fast Crystal Scintillators

F. Yang ${ }^{1}$, L. Zhang ${ }^{1}$, R.-Y. Zhu' ${ }^{1}$, J. Kapustinsky ${ }^{2}$, R. Nelson ${ }^{2}$, Z. Wang ${ }^{2}$

${ }^{I}$ California Institute of Technology, Pasadena, CA 91125, USA

${ }^{2}$ Los Alamos National Laboratory, Los Alamos, NM 87545, USA 
Future high energy physics experiments at the energy and intensity frontiers will face challenges of a severe radiation environment from both ionization dose and charged and neutral hadrons. In this paper, we report a continued investigation on charged hadron induced radiation damage in crystal scintillators (BGO, LYSO and PWO) by using $800 \mathrm{MeV}$ and $24 \mathrm{GeV}$ protons of up to $8 \times 10^{15} \mathrm{p} / \mathrm{cm}^{2}$ at LANL and CERN respectively. Degradations in both transmittance and light output are reported. The results show an excellent radiation hardness of LYSO crystals against charged hadrons.

\author{
N41-5: Profile SP (P-Type) HPGe Detectors - Premium Resolution at Low to Medium Energies \\ G. Geurkov, E. Roth, K. Schmitt, T. Underwood \\ Detectors, ORTEC, Oak Ridge, TN, USA
}

The resolution performance of a High Purity Germanium (HPGe) gamma-ray detector is a function of three factors: crystal quality and geometry, electronic noise and any microphonic contribution. Improving the detector resolution solves peak overlapping problems in complicated multi-peak spectrums and improves the performance of peak identification algorithms yielding fewer false positive results in spectrometry analysis. A new Low Noise Back Contact (LNBC) has been developed by ORTEC for P-type semi-planar HPGe detectors. This LNBC minimizes electronic noise, while semi-planar p-type crystal geometry offers premium efficiency and charge collection performance for the $3 \mathrm{keV}$ to $1 \mathrm{MeV}$ energy region, where the majority of nuclides reside. Additionally, semi-planar crystal geometry lowers the Compton region caused by higher energy gammas, allowing lower Minimum Detectable Activity (MDA) levels. The Profile SP with commercially unmatched premium resolution results will be presented for various energies and shaping times.

\author{
N41-6: Beam Test Results of the Dependence of Signal Size on Incident Particle Rate in Diamond Pixel and \\ Pad Detectors \\ D. Hits \\ ETH, Zurich, Switzerland \\ On behalf of the RD42 Collaboration
}

We will present beam test results on the dependence of signal size on incident particle rate in charged particle detectors based on single-crystal and poly-crystalline CVD diamond. The detectors were tested over a range of particle fluxes from $2 \mathrm{kHz} / \mathrm{cm} 2$ to 20 $\mathrm{MHz} / \mathrm{cm} 2$. The pulse height of the sensors was measured with pad and pixel readout electronics at shaping times from $10 \mathrm{~ns}$ to 1us, as a test of possible charge carrier lifetime dependences. We will present data from the 2014 and 2015 beam tests at PSI indicating the pulse height of non-irradiated single-crystal CVD diamond sensors is independent of flux, while the pulse height of irradiated single-crystal CVD diamond sensors decreases with increasing particle flux. The sensitivity to flux is similar in both diamond pad sensors constructed using diamonds from the Pixel Luminosity Telescope (PLT) irradiated during its pilot run in CMS experiment and in neutron irradiated diamond pad sensors, from the same manufacturer, irradiated to the same fluence. The pulse height for non-irradiated and irradiated poly-crystalline CVD diamond pad sensors was observed to be independent of particle flux. Correlations between the spatial uniformity of the charged particle pulse-height in polycrystalline diamond and any dependence on the flux are also being studied. The latest test beam results for both single-crystal and poly-crystalline CVD diamond will be shown.

\title{
J02: NSS/MIC Joint Session 2: Hadrontherapy
}

\author{
Wednesday, Nov. $2 \quad$ 14:00-16:00 Schweitzer \\ J02-1: Novel Imaging for Ion Beam Therapy \\ K. Parodi \\ Department of Medical Physics, Ludwig-Maximilians Universität München, Munich, Germany
}

Owing to the continued evolution of modern radiation therapy for high precision delivery of large therapeutic doses to the tumour with optimal sparing of surrounding healthy tissue, imaging is becoming a crucial component in the entire treatment chain, from pre-treatment identification and characterization of the tumour up to proper patient positioning at the treatment site and, in more advanced research applications, visualization of the treatment delivery. This contribution will review the main role of imaging in modern external beam radiation therapy with emphasis on emerging ion beam technologies, which aim at exploiting the favourable properties of ion interaction in matter for unprecedented ballistic accuracy in dose delivery. In particular, it will address the role of multi-modal imaging for ion therapy treatment planning, outline the progress of in-room anatomical image guidance and its potential for new workflows of adaptive radiotherapy, and, finally, give a broad overview of new imaging modalities which can contribute to a reduction of the main challenge of ion beam range uncertainty in tissue. The latter techniques feature pre-treatment transmission imaging of multi-energy X-ray sources or energetic ion beams for refined assessment of the ion stopping power relative to water, or utilize acoustic and nuclear-based emission processes induced by ion interaction in tissue, which can be detected during or shortly after treatment for in vivo verification of the beam range. Preclinical studies as well as initial clinical experience gathered with first prototype systems will be highlighted, together with the 
prospects of new dedicated instrumentation under development with the final goal to enable full clinical exploitation of the ballistic advantages of ion beams for high precision tumour therapy.

\author{
J02-2: A Real-Time Dosimetric System Using CMOS Sensors for Secondary Neutrons in Radio/Hadron \\ Therapy

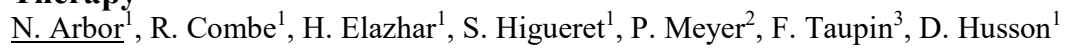 \\ ${ }^{I}$ Institut Pluridisciplinaire Hubert Curien, CNRS UMR7178 Universite de Strasbourg, 67037 Strasbourg, France \\ ${ }^{2}$ Division of Medical Physics, Department of Radiotherapy, Paul Strauss Center, Strasbourg, France \\ ${ }^{3}$ Department of Medical Physics, Centre Hospitalier Lyon Sud, Pierre Benite, France
}

The new CMOS sensor AlphaRad has been designed at the IPHC Strasbourg for real-time monitoring of thermal and fast neutrons over a full energy spectrum. Completely integrated, highly transparent to photons and optimized for low power consumption, this chip is particularly appropriate to the survey of secondary neutrons in the context of medical applications like radio or hadron therapy. Fast and slow neutrons are converted respectively into recoil protons with a polyethylene foil and 1.4 $\mathrm{MeV}$ alpha particles by means of a 10B converter. The use of a CMOS sensor for neutron dosimetry requires a precise analysis of specific effects related to the metal sub-structures of the CMOS and the charge collection process. A detailed characterization of the AlphaRad chip in the $\mathrm{MeV}$ energy range has been done using proton and alpha micro-beams experiments (AIFIRA facility CENBG, Bordeaux) and a complete Geant4 Monte Carlo simulation. We performed then the first CMOS measurements of outof-field neutrons production around two Varian 15 and $16 \mathrm{MV}$ accelerators in a radiotherapy vault. The field size dependence of the neutron emission rate is presented for various primary and multi-leaf collimators openings. The results are compared to the outputs of a GATE v7.1 Monte Carlo simulation and passive neutron detectors (CR-39) measurements. The next step consists in using the AlphaRad CMOS on-line measurements as inputs parameters of a 3D patient dose algorithm.

\title{
J02-3: Development of a High-Intensity Photon-Beam Profile Monitor T. Ishikawa \\ Research Center for Electron Photon Science, Tohoku University, Sendai, Miyagi 982-0826, Japan
}

A beam-profile monitor consisting of scintillating fibers has been developed for high-energy photon beamlines at the Research Center for Electron Photon Science, Tohoku University, Japan. The position of the photon converted into an electron-positron pair in a metal plate is measured using two layers of 16 scintillating-fiber hodoscopes with a $3 \times 3 \mathrm{~mm}^{2}$ cross-section. The effective area is $48 \times 48 \mathrm{~mm}^{2}$. Events in which charged particles are produced upstream are rejected with a charge veto plastic scintillator placed in front of the plate, while pair-production events are identified with a trigger plastic scintillator placed behind the plate. The position is determined by a logic circuit with a field-programmable gate array. The dead time for processing an event is several tens of nanoseconds ( $35 \mathrm{~ns}$ ), and a high data acquisition efficiency can be achieved for high-intensity photon beams (approximately $400 \mathrm{MHz}$ ). A new monitor is under development to reduce the detector size, and a metal-packaged photomultiplier tube, Hamamatsu Photonics H9500 (256 anodes), is proposed for the position measurement. Secondary electrons produced at the photoelectric surface by the electron-positron pair are utilized. The developed photon-beam profile monitor and prospects for the new monitor will be reported.

\author{
J02-4: The INSIDE Bi-Modal System for in-Vivo Particle Range Monitoring Toward Clinical Validation \\ M. G. Bisogni ${ }^{1,2}$ \\ ${ }^{I}$ Department of Physics, University of pisa, Pisa, Italy \\ ${ }^{2}$ Sezione di Pisa, INFN, Pisa, Italy \\ On behalf of the INSIDE Collaboration
}

Positron Emission Tomography (PET) is the in-vivo non-invasive range monitoring technique most used for particle therapy quality assurance. PET monitoring exploits the correlation between the particle range and the distribution of the positron emitters induced by nuclear reactions in the irradiated tissues. The most advanced in-beam PET technique consists of a dedicated scanner integrated in the gantry and operated during the irradiation to provide a prompt feedback of the particles range. A rather recent invivo range monitoring method relies on the the detection of prompt radiation such as photons and secondary protons. This method is known as single particle imaging. PET imaging and single particle imaging have always been considered as competitive techniques. However, in 2013 the INSIDE (INnovative SolutIons for DosimEtry in hadrontherapy) collaboration proposed an innovative bi-modal imaging concept that combines an in-beam PET scanner with a particle tracking. INSIDE was born from the collaboration of Italian Universities (Pisa, Roma "La Sapienza", Torino and Polytechnic University of Bari) and the Italian National Institute for Nuclear Physics (INFN). The INSIDE system is designed to detect simultaneously the annihilation gammas and the prompt charged particles with kinetic energies higher than $20 \mathrm{MeV}$ for the monitoring of head and neck tumors. The particle tracker is under assembling while the in-beam PET scanner has been recently installed at the Italian National Center of Oncologic Hadronterapy (CNAO) in Pavia, Italy, and it is currently in the commissioning phase. Tests with clinical proton and carbon beams on phantoms clearly show the capability of the in-beam PET to operate during the beam 
delivery and to reconstruct on-line the beam-induced activity map. The accuracy and precision in the activity distal fall-off is submillimetric for therapeutical doses ( 2 Gy). These results open the way to the clinical validation of the INSIDE system at CNAO.

This work has been supported by Ministero dell'Istruzione, dell'Università e della Ricerca of the Italian government under the program PRIN 2010-2011 project nr. 2010P98A75 and by European Union's Seventh Framework Program for research, technological development and demonstration under grant agreement no 317446 (INFIERI)

J02-5: A New Hadron Radiography Method Based on Time-of-Flight Residual Energy Measurement W. A. Worstell ${ }^{1}$, K. Grogg ${ }^{2}$, B. W. Adams ${ }^{1}$, C. A. Craven ${ }^{1}$, T. L. Cremer ${ }^{1}$, M. R. Foley ${ }^{1}$, A. Lyashenko ${ }^{1}$, M. J. Minot ${ }^{1}$, M. A. Popecki ${ }^{1}$, H.-M. Lu ${ }^{3}$, H. Paganetti ${ }^{3}$, G. El Fakhri ${ }^{2}$

${ }^{1}$ Research \& Development, Incom, Inc., Charlton, MA, US

${ }^{2}$ Department of Radiology, Massachusetts General Hospital, Boston, MA, US

${ }^{3}$ Department of Radiation Oncology, Massachusetts General Hospital, Boston, MA, US

We propose a novel data acquisition method for hadron radiography and hadron $\mathrm{CT}$ in support of hadron therapy patient imaging. The proposed method makes use of precision time-of-flight measurement of proton or light ion residual energies for particles which have traversed a patient. Our new method allows for a compact, cost-effective, and high performance detector design which might be more readily incorporated into clinical geometry and workflow than designs incorporating the calorimeters and range stacks which have been studied previously for proton residual energy measurement. In addition to providing for very highrate and high-resolution imaging data acquisition using individual hadrons, our new method is also compatible with particle bunch measurements for increasingly popular compact proton and light ion accelerator designs which have a low duty-cycle pulsed rather than a continuous beam time structure. We present a design for a proton radiograph based on proton residual energy measurement using time-of-flight techniques, incorporating paired Large Area Picosecond Photon Detectors (LAPPD ${ }^{\wedge}$ TM) operating in ionization mode. Estimates of expected device performance and plans for future experimental validation are described.

\author{
J02-6: A Comparative Study of Energy-Loss Proton Radiography (ElpRad) Based on High-Spatial Resolution \\ or Ultrafast Methods \\ Z. Wang $^{1}$, C. L. Morris ${ }^{1}$, L. P. Neukirch ${ }^{1}$, D. J. Clark ${ }^{1}$, M. Gaowei ${ }^{2}$, F. G. Mariam ${ }^{1}$, E. Ramberg ${ }^{3}$, A. Saunders ${ }^{1}$, S. K. Sjue ${ }^{1}$, \\ J. Smedley ${ }^{1}$, D. Tupa ${ }^{1}$, R. Zhu ${ }^{4}$ \\ ${ }^{I}$ Physics Division, Los Alamos National Laboratory, Los Alamos, NM, USA \\ ${ }^{2}$ Brookhaven National Laboratory, Upton, NY, USA \\ ${ }^{3}$ Fermilab, Batavia, IL, USA \\ ${ }^{4}$ California Institute of Technology, Pasedena, CA, USA
}

Precise measurement of proton energy loss has been recognized as a potentially powerful approach to radiographic imaging of thick (areal density above $10 \mathrm{~g} / \mathrm{cm} 2$ ) objects, overcoming certain limitations of X-ray and high-energy photon techniques. A high spatial resolution magnetic spectrometer technique is compared with two ultrafast techniques: one is based on the ultrafast detection of proton-induced image charge, the other is based on detection of proton-induced Cherenkov light emission. All three methods can deliver an energy resolution in the range of $1.0 \mathrm{e}-4$ to $1.0 \mathrm{e}-5$ for $800 \mathrm{MeV}$ protons, allowing high-resolution proton radiography based on energy loss. In additional to the design principles of new energy-loss proton radiography systems, experimental progress using stripline detectors is also highlighted.

We would like to thank Science Campaign 3 of US DoE/NNSA for their kind support

\author{
J02-7: Helium Beam Radiography Using a Silicon Pixel Detector for Particle Tracking and Identification \\ $\underline{\text { T. Gehrke }}^{1,2,3,4}$, S. Berke ${ }^{1,3,4}$, G. Arico ${ }^{1,2,3,4}$, J. Jakubek ${ }^{5}$, O. Jaekel ${ }^{1,2,3,4}$, M. Martisikova ${ }^{1,2,3,4}$ \\ ${ }^{I}$ German Cancer Research Center (DKFZ), Heidelberg, Germany \\ ${ }^{2}$ Heidelberg University Hospital, Heidelberg, Germany \\ ${ }^{3}$ National Center for Radiation Oncology (NCRO), Heidelberg, Germany \\ ${ }^{4}$ Heidelberg Institute for Radiation Oncology (HIRO), Heidelberg, Germany \\ ${ }^{5}$ Advacam, Prague, Czech Republic
}

Ion beam therapy provides potential for an improved dose confirmation to the tumor in comparison to the standard radiation therapy with photons. This can be achieved only if a precise target positioning is ensured. Thus, the determination of the ion stopping power inside the patient and a monitoring of the patient anatomy during treatment is desirable. For this purpose, ion beam radiography (IBR) is attractive due to the possibility of direct measurements of ion stopping power, the potentially high soft-tissue contrast and the lower dose to the patient compared to x-ray imaging. However the limited spatial resolution due to multiple Coulomb scattering (MCS) and the lack of suitable detectors are still challenges, which have to be addressed. This 
contribution introduces a method of IBR using a compact silicon pixel detector for particle tracking and identification. Imaging with accelerated helium ions, which traverse the object to be imaged with less pronounced MCS than protons, was performed at the Heidelberg Ion-Beam Therapy Center (HIT). The deployed Timepix detector has a high spatial resolution, which allows single ion detection with energy sensitivity in each pixel. Plastic phantoms of patient head size (170 mm) with fine structures were imaged. Multiple layers of detectors facilitated to track the ions. Additionally the energy loss and the ion type was determined. The combination of these information was used for reconstructing helium radiographies. Relative thickness difference below $1.2 \%$ could be resolved. The contrast to noise ratio and spatial resolution of the radiography was shown to improve by distinguishing between different ions species and taking into account the direction of every single impinging ion. In conclusion, it could be shown that helium radiography with the Timepix detector provides a high thickness resolution of the imaged object. The distinction of different ion species and the direction information improves the image quality.

\title{
N42: High energy physics instrumentation II
}

\author{
Wednesday, Nov. $2 \quad$ 14:00-16:00 Madrid \\ N42-1: The Herschel Forward Shower Counters for LHCb \\ M. Ravonel \\ CERN, Geneva, Switzerland \\ On behalf of the $\mathrm{LHCb}$
}

Herschel $(\{\mathrm{bff} H\}$ igh $\{\backslash \mathrm{bf} R\}$ apidity $\{\mathrm{bbf} \mathrm{S}\}$ hower $\{\backslash \mathrm{bfC}\}$ ounters for $\backslash$ lhcb) is a new subdetector which has been installed for the LHCb experiment for LHC Run II. It consists of scintillator plates installed in the LHC tunnel symmetrically around the interaction region, which are designed to identify showers from high rapidity particles interacting with beam-pipe elements. The primary purpose is as a rapidity gap detector which complements the LHCb coverage, of particular interest for Central Exclusive Production processes. The detector can also be used to tag forward showers in low mass diffractive events, and for the study of single and double diffractive processes and studies of beam related processes including background and luminosity studies. The system exploits the low pileup running conditions of LHCb and operates continuously during LHCb data taking. Herschel has already accumulated more than $350 \mathrm{pb} \$^{\wedge}\{-1\} \$$ in low pile up conditions and is fully integrated in the LHCb data taking for 2016 . The Herschel performance and the impact on physics results will be presented.

\author{
N42-2: SciFi - a Large Scintillating Fibre Tracker for LHCb \\ $\underline{\text { S. Bachmann }}$ \\ Physikalisches Institut, Heidelberg University, Heidelberg, Germany \\ On behalf of the LHCb Collaboration
}

The $\mathrm{LHCb}$ detector will be upgraded during the Long Shutdown 2 (LS2) of the LHC in order to cope with higher instantaneous luminosities and to read out the data at $40 \mathrm{MHz}$ using a trigger-less read-out system. All front-end electronics will be replaced and several sub-detectors must be redesigned to cope with higher occupancy. The current tracking detectors downstream of the LHCb dipole magnet will be replaced by the Scintillating Fibre (SciFi) Tracker. Concept, design and operational parameters are driven by the challenging LHC environment including significant ionising and neutron radiation levels. Over a total active surface of $360 \mathrm{~m} 2$ the SciFi Tracker will use scintillating fibres $\left(\ddot{i} i^{1 / 2} 0.25 \mathrm{~mm}\right)$ read out by Silicon Photomultipliers (SiPMs). State-of-theart multi-channel SiPM arrays are being developed to read out the fibres and a custom ASIC will be used to digitise the signals from the SiPMs. The project is now at the transition from R\&D to series production. We will present the evolution of the design and the latest lab and test beam results.

N42-3: The Liquid Hole-Multiplier: a Novel Local Dual-Phase Element for Noble-Liquid TPCs L. Arazi, E. Erdal, Y. Korotinsky, M. Rappaport, A. Roy, S. Shchemelinin, D. Vartsky, A. Breskin

Dept. of Astrophysics \& Particle Physics, Weizmann Institute of Science, Rehovot, Israel

Large-volume noble-liquid time projection chambers are a leading tool in rare-event searches and neutrino-physics experiments. However, scaling up existing designs to the multi-ton regime calls for new innovative detector solutions. We propose a new concept, the bubble-assisted Liquid Hole-Multiplier (LHM) - a "local dual-phase" detection element, designed for the detection of both ionization electrons and primary scintillation photons induced by particle interactions within the noble liquid. The LHM comprises a perforated micro-pattern electrode (e.g. Thick Gas Electron Multiplier - THGEM, or Gas Electron Multiplier GEM) immersed in the liquid, with a bubble of the noble gas supported underneath. Ionization electrons and scintillation-induced photoelectrons extracted from a cesium iodide (CsI) photocathode drift through the electrode's holes and induce electroluminescence (EL) in the bubble, with up to several hundred EL photons emitted per drifting electron. Accurate imaging of the EL patterns, resulting in precise reconstruction of the event topology, can be performed with small-pixel photon detectors, e.g. SiPMs or Gaseous Photomultipliers - GPMs. We will present the principle of the new concept, as well as measurements of ionization electrons and VUV scintillation photons using CsI-coated THGEM and GEM-based LHM prototypes in liquid xenon 
(LXe). The mechanism, stable over months of operation, yields energy resolutions surpassing those of current dual-phase LXe TPCs, with a time resolution on the nanosecond scale for events comprising a few hundred photoelectrons. In addition, we will present our parallel work on the development on cryogenic GPMs. Potential applications are discussed within the framework of dark matter searches, neutrino physics experiments and neutron and gamma radiography.

N42-4: Radiation Hard Composite Element for High Energy Physics

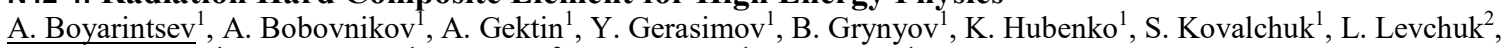
T. Nepokupnaya ${ }^{1}$, Y. Onufriyev ${ }^{1}$, V. Popov ${ }^{2}$, O. Sidletskiy ${ }^{1}$, V. Tarasov ${ }^{1}$

${ }^{I}$ National Academy of Sciences, The Institute for Scintillation Materials, Kharkov, Ukraine

${ }^{2}$ National Science Center "Kharkov Institute of Physics and Technology, Kharkov, Ukraine

The goal of the work is development of radiation hard detector with the stable parameters up to 30 Mrad and even higher doses. Composite scintillators were selected as an alternative to currently used crystals and plastics. Such material/detector consists of the small size scintillation crystal granules uploaded to an optically transparent medium (radiation stable optical gel). YSO:Ce scintillator was selected as the scintillation component for composite. Technology allows receiving large area flexible composites with thickness 1- $2 \mathrm{~mm}$ that still sustain transparency of detector. Radiation test at electron energy $8.3 \mathrm{MeV}$ were carried out till the dose $50 \pm 0.5 \mathrm{Mrad}$. It is shown that at such irradiation dose scintillation output decreased for about $2 \%$ only. The transparency was reduced for 5\% in 300-700 $\mathrm{nm}$ range. Transmission and light scattering simulations allows to optimize the YSO:Ce size and uploading concentration for the use with fiber light guide. It is shown that detector is an efficient for calorimeter application in HEP.

\section{N42-5: ARICH - Particle Identificantion in the Forward End-Cap of Belle II} $\underline{\text { R. Pestotnik }}$

Experimental Particle Physics Department, Jožef Stefan Institute, Ljubljana, Slovenia

On behalf of the Belle II ARICH group

Particle identification in the forward end-cap of the Belle II spectrometer will be accomplished by a proximity focusing Ring Imaging Cherenkov counter with an aerogel radiator. The design has to ensure the excellent performance which should allow at least a 4 sigma separation of pions from kaons in the experiment kinematic region from $0.5 \mathrm{GeV} / \mathrm{c}$ to $4 \mathrm{GeV} / \mathrm{c}$. In the magnetic field of $1.5 \mathrm{~T}$ the detector is positioned in the small space between the drift chamber and the electromagnetic calorimeter. The proximity focusing type of RICH consists of an aerogel radiator, an expansion volume and a photon detector. The single Cherenkov photons are registered by segmented 144 channel Hamamatsu Hybrid Avalanche Photo Detector (HAPD). The output signals of 420 HAPDs are amplified, shaped and discriminated in the front end electronics. The digitized data are collected by the FPGA and send to the Belle II common data acquisition system. All detector components have been delivered and tested and in 2016 installed in the spectrometer. In the presentation the requirements and the latest design challenges will be presented. We will show the first rings obtained during the cosmic tests of the final detector.

N42-6: Borehole Muon Tomography

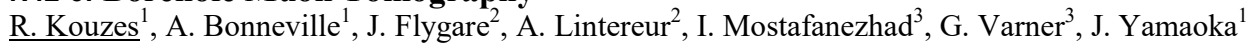

${ }^{I}$ Pacific Northwest National Laboratory, Richland, WA, USA

${ }^{2}$ University of Utah, Salt Lake City, UT, USA

${ }^{3}$ University of Hawaii, Honolulu, HI, USA

Muons can be used to image the density of materials through which they pass, including geological structures. Increasing atmospheric $\mathrm{CO} 2$ concentrations have spurred investigation into carbon sequestration methods, where $\mathrm{CO} 2$ is stored in underground reservoirs. Monitoring of the $\mathrm{CO} 2$ during, and after, injection is of utmost importance. Current monitoring options for sequestered $\mathrm{CO} 2$ are active methods, such as gravimetric data collection and seismic detection. One alternative for monitoring is the development of a muon detector that is compact and robust enough for deployment in a borehole under a $\mathrm{CO} 2$ sequestration reservoir. Such a muon detector would provide 4-D tomographic data to detect small changes in density at depths of 1-2 km. A detector with these capabilities is being developed by a collaboration supported by the U.S. Department of Energy. Monte Carlo modeling methods are being used for detector simulations. Current simulation results predict the incoming muon angle can be resolved with an error of approximately two degrees using underground muon spectra. The robustness of the design comes primarily from the use of scintillating rods as opposed to drift tubes that are used in many large muon detectors. The polystyrene scintillating rods are arrayed in alternating layers to provide a coordinate scheme. Preliminary testing and measurements are being performed to test and enhance the performance of the scintillators, in both a laboratory and a shallow underground facility at Pacific Northwest National Laboratory. The simulation predictions and data from the experiments are guiding the design and improvement of this borehole muon detector, and the results will be presented. 
This material is based upon work supported by the Department of Energy under Award Number FWP-66844. Battelle operates PNNL for the US Department of Energy under contract DE-AC05-76RLO 1830.

N42-7: Upgrades of the CMS Muon System

L. Guiducci

Universita' di Bologna \& INFN, Bologna, Italy

On behalf of the CMS Collaboration

The present CMS muon system operates three different detector types: in the barrel drift tubes (DT) and resistive plate chambers (RPC), along with cathode strip chambers (CSC) and another set of RPCs in the forward regions. In order to cope with increasingly challenging conditions various upgrades are planned to the trigger and muon systems. New detectors will be added to improve the performance in the critical forward region: large-area triple-foil gas electron multiplier (GEM) detectors will already be installed in LS2 in the pseudo-rapidity region $1.6<$ eta $<2.4$, aiming at suppressing the rate of background triggers while maintaining high trigger efficiency for low transverse momentum muons. For the HL-LHC operation the muon forward region should be enhanced with another large area GEM based station, called GE2/1, and with two new generation RPC stations, called RE3/1 and RE4/1, having low resistivity electrodes. These detectors will combine tracking and triggering capabilities and can stand particle rates up to few $\mathrm{kHz} / \mathrm{cm} 2$. In addition to take advantage of the pixel tracking coverage extension a new detector, ME0 station, behind the new forward calorimeter, covering up to $\mid$ ? $\mid=3$.

N42-8: Large Area GEM Chambers for Muon Tracking in CBM Experiment at FAIR A. Kumar

Variable Energy Cyclotron Centre, Kolkata, Kolkata, West Bengal, India

On behalf of the CBM collaboration

The CBM-Experiment is one of the core experiments of the upcoming FAIR facility in Darmstadt, Germany. It will explore the phase diagram of hadronic matter in the region of highest baryon densities with various rare probes including light vector mesons and charmonium decaying into di-muon pairs. CBM has been designed to handle unprecedented interaction rates of $10 \mathrm{MHz}$ $\mathrm{Au}+\mathrm{Au}$ collisions in an energy range of up to $11 \mathrm{AGeV}$. The Muon Chamber (MUCH) system consists of layers of instrumented muon absorbers with detector stations sandwiched between them. We will discuss the concept of the MUCH stations, which rely on large area and novel schematic of segmented absorbers. Another constraint in MUCH is that the first few stations will have to handle a high particle density about $400 \mathrm{kHz} / \mathrm{cm}^{\wedge} 2$ for central collisions. Thus a high rate, trapezoidal shaped, large size, Gas Electron Multiplier (GEM) detectors would be employed to perform the job of tracking in these first stations. Signals will be readout using self triggered electronics, which is a unique feature for all CBM detectors. Several triple GEM prototype detectors with surfaces ranging from $100 \mathrm{~cm} 2$ to $2000 \mathrm{~cm}^{\wedge} 2$ (real size) have been built and tested with particle beams. The design challenges in the GEM based modules, granularity optimization and FEB layout will be discussed in detail. Moreover, the test results of $2000 \mathrm{~cm}^{\wedge} 2$ real size detector with $2.3 \mathrm{GeV} / \mathrm{c}$ proton beams, readout in a self triggered mode will be highlighted. Issues on a novel HV biasing scheme, foil design and the challenging mechanical integration of the chambers will be presented and discussed.

N43: New concepts in solid-state detectors and radiation damage effects II

Wednesday, Nov. $2 \quad$ 14:00-16:00 Curie 1

N43-1: Irradiation induced effects in the FE-I4 front-end chip of the ATLAS IBL detector

A. La Rosa

Max-Planck-Institut fuer Physik, Munich, Germany

On behalf of the ATLAS Collaboration

The ATLAS Insertable B-Layer (IBL) detector was installed into the ATLAS experiment in 2014 and has been in operation since 2015. During the first year of IBL data taking an increase of the low voltage currents associated with the FE-I4 front-end chip was observed and this increase was traced back to the radiation damage in the chip. The dependence of the current on the totalionising dose and temperature has been tested with X-ray and proton irradiations and will be presented in this talk together with the detector operation guidelines.

N43-2: Radiation Tolerance of Straw-Tracker Read-Out System for COMET Experiment

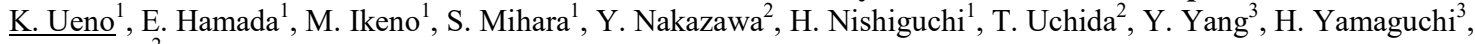
H. Yoshida ${ }^{2}$ 
${ }^{I}$ KEK, Tsukuba, Ibaraki, Japan

${ }^{2}$ Osaka University, Toyonaka, Osaka, Japan

${ }^{3}$ Kyushu University, Fukuoka, Fukuoka, Japan

The COMET experiment at J-PARC aims to search for the charged lepton flavor violating process of neutrinoless mu-e conversion, which indicates the new physics beyond the standard model. The goal sensitivity is to be improved by a factor of 10000 to the current limit given by SINDRUM-II at PSI. When the mu-e conversion occurs, almost all the energy of the muon mass is carried out by the electron which is expected to have the monochromatic energy of about $105 \mathrm{MeV}$. In order to achieve the goal sensitivity, the measurement of the electron with momentum resolution of better than $200 \mathrm{keV} / \mathrm{c}$ is needed. We plan to use a straw tube tracker as an electron detector, which can reduce multiple scattering effect strongly. To read out the signal from the tracker precisely, an optimal front-end electronics is also needed, and we have developed the readout electronics board called ROESTI. For the ROESTI, we need to consider radiation tolerance because the board will be installed in neutron and gamma-ray rich environment. We calculated radiation level with PHITS and it was found that neutron fluence of $\sim 10^{12} \mathrm{n} / \mathrm{cm}^{2}$ and gamma-ray absorbed dose of $\sim 1 \mathrm{kGy}$ with the safety factor of 10 are estimated. On the basis of these results, radiation tolerant parts were selected and countermeasure against single event upset (SEU) in FPGA was considered, then we have improved and developed the new version of ROESTI prototype, which has Artix-7 FPGA and no flush memory. Using this prototype, neutron and gammaray irradiation tests have been performed and radiation tolerance has been investigated. Based on those results, the design of final version of ROESTI has began. In this presentation, we describe the radiation study with PHITS calculation and the design of the ROESTI based on that study. Then, the results of irradiation tests of the ROESTI prototype with neutron and gamma-ray are described. The prospect of final version of ROESTI is also presented.

\title{
N43-3: Radiation Damage Effects and Operations of the LHCb Vertex Locator
}

A. Oblakowska-Mucha

AGH University of Science and Technology, Krakow, Poland

On behalf of the LHCb VELO group

The LHCb Vertex Locator (VELO) is a silicon micro-strip detector operating extremely close to the LHC proton beams. During nominal data-taking the innermost active strips are as close as $\sim 8 \mathrm{~mm}$ to the beams. In addition to providing the vertex measurements that allow the LHCb experiment to select signal events containing b and c quarks, this proximity makes the LHCb VELO an ideal laboratory to study radiation damage effects in silicon detectors.

Two particular features of the VELO make it even more interesting to closely study the effects of radiation damage in this detector: the second metal layer and the direct comparison of n-in-n and n-in-p technologies.

After accumulating some significant radiation damage during LHC Run 1, the analysis of charge collection efficiency (CCE) data showed that there is a correlation of cluster finding efficiency (CFE) with the distance of strip to a second metal layer routing line. The CCE scans require colliding beams and therefore have to be well prepared and negotiated in order not to cut unnecessarily into the data-taking efficiency of the LHCb experiment. This results in about four CCE scans taken per year; the aim is to scale the frequency of these scans with integrated luminosity rather than time. The CCE scans provide the best handle on to project the bias voltages needed to operate the VELO efficiently throughout LHC Run 2.

Run 2 of the LHC exceeds the radiation damage requirements the LHCb VELO was originally designed for. There are numerous challenges resulting from this, affecting how the VELO has to be operated. Furthermore the VELO will be taking data in Pb-Pb collisions. This has been determined not to be a risk to the detector but still poses some operational challenges. The latest results from radiation damage studies and their impact on the operation of the LHCb VELO in LHC Run 2 will be presented.

\author{
N43-4: Ionizing Radiation Effects on the Noise of $65 \mathrm{~nm}$ CMOS Transistors for Pixel Sensor Readout at \\ Extreme Total Dose Levels

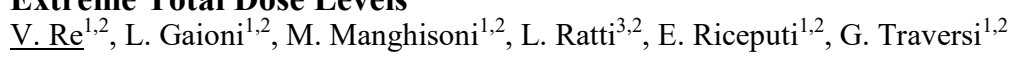 \\ ${ }^{1}$ Dipartimento di Ingegneria e Scienze Applicate, Università di Bergamo, Bergamo, Italy \\ ${ }^{2}$ Sezione di Pavia, INFN, Pavia, Italy \\ ${ }^{3}$ Dipartimento di Ingegneria Industriale e dell'Informazione, Università di Pavia, Pavia, Italy
}

Integrated circuits for the readout of silicon pixel sensors are required to stand extremely high levels of ionizing radiation in future experimental environments such as the innermost detector layers of the ATLAS and CMS experiments at the High Luminosity LHC and the X-ray imaging cameras at XFEL. In these applications, analog circuits have to preserve adequate noise performance at total ionizing dose levels of several hundreds of MRad, up to 1 GRad. This paper presents the experimental results of irradiation tests at extremely high levels of total ionizing dose on transistors and analog circuits fabricated in a $65 \mathrm{~nm}$ CMOS technology, which is a promising candidate for the readout of a next generation of pixel sensors at HL-LHC and XFEL. The analysis of the noise behavior after irradiation provides further insight into the radiation-induced damage mechanisms that may affect the performance of $65 \mathrm{~nm}$ CMOS pixel front-ends during their operational lifetime in these very harsh environments. 
N43-5: Comparison of the Radiation Hardness of Silicon Mach-Zehnder Modulators for Different DC Bias Voltages

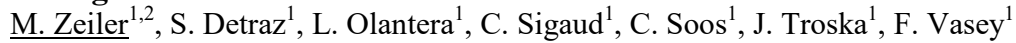 \\ ${ }^{I}$ EP-ESE, CERN, Geneva, Switzerland \\ ${ }^{2}$ School of Electronic Engineering, Dublin City University, Dublin, Ireland
}

Radiation hard optical links are the backbone of read-out systems in high-energy physics experiments at CERN. The optical components have to withstand large doses of radiation and provide high data rates. Silicon photonics is currently being considered a promising technology to replace electrical and optical links in future experiments. It has already been demonstrated that integrated silicon Mach-Zehnder modulators can withstand a high neutron fluence and large total ionizing doses. Before read-out systems based on these components can be taken into consideration, it has to be determined how biasing affects their radiation hardness. For this reason we prepared bonded and fiber-pigtailed prototypes and irradiated them with x-rays. We found that under reverse-bias the radiation hardness of the tested components is reduced in comparison to un-biased samples. However, we were able to show that one device type can withstand the radiation without phase shift degradation up to 1MGy despite the accelerated degradation due to biasing.

N43-6: HV-CMOS Detectors for High Energy Physics: Characterization of BCD8 Technology and Controlled Hybridization Technique.

A. Andreazza ${ }^{1}$, A. Castoldi ${ }^{2}$, G. Chiodini ${ }^{3}$, M. Citterio ${ }^{1}$, G. Darbo ${ }^{4}$, G. Gariano ${ }^{4}$, A. Gaudiello ${ }^{4}$, C. Guazzoni ${ }^{2}$, V. Liberali ${ }^{1}$, S. Passadore ${ }^{1}$, F. Ragusa ${ }^{1}$, A. Rovani ${ }^{3}$, E. Ruscino ${ }^{3}$, C. Sbarra ${ }^{5}$, A. Sidoti ${ }^{5}$, H. Shrimali ${ }^{6}$, E. Zaffaroni ${ }^{1}$

${ }^{I}$ Università di Milano and INFN Sezione di Milano, Milano, Italy

${ }^{2}$ Politecnico di Milano and INFN Sezione di Milano, Milano, Italy

${ }^{3}$ INFN Sezione di Lecce, Lecce, Italy

${ }^{4}$ INFN Sezione di Genova, Genova, Italy

${ }^{5}$ INFN Sezione di Bologna, Bologna, Italy

${ }^{6}$ Indian Institute of Technology Mandi, Mandi, India

Radiation detectors built in high-voltage and high-resistivity CMOS technology are an interesting option for the large area pixeltrackers sought for the upgrade of the Large Hadron Collider experiments. A possible architecture is a hybrid design, where CMOS sensors are readout by front-electronics coupled through a thin dielectric layer. A critical requirement is the radiation hardness of both the sensor and interconnection technology up to a dose of 0.1-1 Grad, depending on the distance from the interaction region. A characterization of the BCD8 technology by STMicroelectronics process has been performed to evaluate its suitability for the realization of CMOS sensors. The shift of transistor threshold and transconductance has been evaluated after irradiation with? rays and protons up to $36 \mathrm{Mrad}$, showing limited performance degradation. Sensors featuring $50 \times 250 \mu \mathrm{m} 2$ pixels on a $125 \mathrm{Ocm}$ substrate have been characterized showing a uniform breakdown at $70 \mathrm{~V}$ and a capacitance of about $80 \mathrm{fF}$ at $50 \mathrm{~V}$ reverse bias voltage. At this bias, the depletion is suitable for charged particle detection, as demonstrated with radioactive source and X-ray measurements. A hybridization process for capacitive coupling has been developed. It consists of gluing the CMOS sensor to the readout electronics using a dielectric epoxy, whose thickness is controlled by SU8 pillars deposited on the readout chip surface. Uniformity of better than $100 \mathrm{~nm}$ on the pillar surface has been obtained. Assemblies have been performed using the ATLAS FE-I4 readout ASIC and prototype CMOS sensors. Measurements show a planarity better than $1.5 \mu \mathrm{m}$ peak-topeak on the $5 \mathrm{~mm}$ length of the HV-CMOS chip. To evaluate more precisely the achievable uniformity dummy chips of FE-I4 sizes have been made. The measurement of the 24 capacitors on each chip is expected to achieve a precise estimation of the real thickness uniformity. The goal is to achieve less then $10 \%$ variation on the glue thickness $(\sim 0.5 \mu \mathrm{m})$.

Reasearch funded by the INFN HVR_CCPD project

\author{
N43-7: Simulation Studies for the Optimization of a Neutron Shield for the SciFi Tracker in the LHCb \\ Upgrade \\ M. Karacson, G. Corti \\ CERN, Geneva, Switzerland
}

FLUKA Monte Carlo simulations have been performed for the upgrade of the LHCb experiment at CERN to determine the radiation environment the detector will operate in. A large fraction of the studies focus on the expected radiation field around various components of the new SciFi Tracker. The SciFi is made of scintillating fibers and uses newly developed silicon based multichannel array photon detectors (SiPM): these components are particularly sensitive to radiation damage through Total Ionizing Dose (TID) and $1 \mathrm{MeV}$ neutron equivalent fluence. Using a very detailed geometry description of the current LHCb experiment, simulation results initially provided useful estimates for the viability assessment of the new tracker. Further, a modified version of the geometry using some already agreed specifications for the LHCb upgrade confirmed a significant impact on the radiation field and highlighted the need for additional neutron shielding for the SiPMs. A preliminary design for this 
shielding has been established reducing the fluence at the relevant locations by a factor ranging from 2.5 to 3.5. Simulation results for TID and $1 \mathrm{MeV}$ n-equivalent fluence in the $\mathrm{LHCb}$ experiment have been validated using various on-site detectors over the course of the first 3 years of operation of the experiment and are expected to correctly predict the expected radiation field within a factor of 2 .

N43-8: GigaRad Total Ionizing Dose and Post-Irradiation Effects on 28 nm Bulk MOSFETs C.-M. Zhang ${ }^{1}$, F. Jazaeri ${ }^{1}$, A. Pezzotta ${ }^{1,2}$, C. Bruschini ${ }^{1}$, G. Borghello ${ }^{3,4}$, F. Faccio ${ }^{3}$, S. Mattiazzo ${ }^{5}$, A. Baschirotto ${ }^{2}$, C. Enz ${ }^{1}$ ${ }^{I}$ ICLAB, Ecole Polytechnique Federale de Lausanne, Neuchatel, Switzerland

${ }^{2}$ Microelectronic Group, INFN \& University of Milano-Bicocca, Milano, Italy

${ }^{3}$ EP-ESE, CERN, Geneva, Switzerland

${ }^{4}$ University of Udine, Udine, Italy

${ }^{5}$ Information Engineering Department, University of Padova, Padova, Italy

Nowadays, the large majority of ASICs included in CERN's Large Hadron Collider (LHC) detector systems are built in a commercial $250 \mathrm{~nm}$ CMOS technology. The systems will need a major upgrade around 2020 in order to increase the LHC's luminosity by a factor of ten. This will require new radiation-tolerant tracking detectors and radiation-tolerant front-end (FE) circuits. More advanced CMOS technologies are expected to withstand high level of total ionizing dose (TID) effects up to 1 Grad over ten years of operation. In this perspective, we have investigated 1 Grad TID levels and post-irradiation effects on nMOSFETs fabricated in a commercial $28 \mathrm{~nm}$ bulk CMOS process to assess its potential use for the upgrade of CERN's LHC systems. Radiation-induced charges building-up in field and gate oxides are expected to degrade the device performance. This oxide and interface traps charge leads to a threshold voltage shift of maximum $-80 \mathrm{mV}$ which remains within the process tolerances and a drive current reduction of maximum $25 \%$ which remains acceptable for most applications. A degraded subthreshold slope less than $38 \mathrm{mV} / \mathrm{dec}$ and radiation-induced DIBL effect as well as free electron carrier mobility reduction are also observed. The leakage current increases by three orders of magnitude. However, despite this significant increase of leakage current, the Ion/Ioff ratio remains larger than three orders of magnitude which should be sufficient for most applications. Postirradiation annealing recovers part of the overall performance degradation even at room temperature. In order to complete the experimental study, pMOSFETs are currently under investigation.

This work has been developed within the Scaltech 28 and GigaRadMOST projects funded by INFN and SNSF respectively (SNSF N $200021-$ 160185). The authors would like to thank the CERN EP-ESE Group for their contribution, especially Alessandro Marchioro for the worthful collaboration, as well as the University of Padova, namely Dario Bisello for the Physics Department and Alessandro Paccagnella for the Information Engineering Department.

N44: Calorimetry III - High Granularity Calorimeters

Wednesday, Nov. $2 \quad$ 16:30-18:30 Madrid

N44-1: Analysis of Hadronic Showers in the Physics Prototype of the CALICE Silicon Tungsten Electromagnetic Calorimeter - Si-W ECAL R. Pöschl

LAL Orsay, Orsay, France

On behalf of the CALICE Collaboration

A detailed study of hadronic interactions is presented using data recorded with the highly granular CALICE silicon-tungsten electromagnetic calorimeter. The predictions of several Monte Carlo Geant4 models are compared with experimental data, taken at FNAL in 2008. The contribution recaps results published in 2015 and a set of new results available since the beginning of 2016. The published results present a detailed analysis of hadronic showers in terms of radial and longitudinal hit and energy distributions. For the most recent analysis a simple track-finding algorithm was developed to study tracks left by secondary particles, emerging from hadronic interactions in the highly granular electromagnetic calorimeter prototype.

Present Monte Carlo simulations provide a good description of the experimental data in terms of new observables, available through the detailed analysis of secondary particles; the Monte Carlo predictions are within $20 \%$ of the data, and for many observables much closer. Main systematic effects were studied using electron and muon samples.

\section{N44-2: Technological Prototype of the CALICE / ILD Silicon-Tungsten Electromagnetic Calorimeter} $\underline{\text { K. Shpak }}$

LLR / Ecole polytechnique, PALAISEAU, FRANCE

On behalf of the ILD SiW ECAL group

Particle Flow Algorithm (PFA) and highly granular calorimeters provide currently the best jet energy resolution, which is of primary importance for precise physics measurements at future colliders. In the electromagnetic calorimeter, the best granularity 
and performance can be achieved with the silicon-tungsten technology. After years of R\&D within the CALICE collaboration this concept is a primary candidate for electromagnetic calorimetry at a future e+e- collider, and it was also chosen for the upgrade of the CMS calorimeter endcaps (HGCAL project). Here, we report on the recent development of the technological prototype of ILD detector with fully embedded front-end electronics and the first results obtained at the CERN test beam.

\section{N44-3: High Granularity Calorimeter for the CMS Endcap at HL-LHC}

\section{R. Rusack}

School of Physics and Astronomy, University of Minnesota, Minnesota, USA

On behalf of the CMS Collaboration

Calorimetry at the High Luminosity LHC (HL-LHC) faces two enormous challenges, particularly in the forward direction: radiation tolerance and unprecedented in-time event pileup. To meet these challenges, the CMS experiment has decided to construct a High Granularity Calorimeter (HGCAL), featuring an unprecedented transverse and longitudinal segmentation in a collider detector, both for electromagnetic and hadronic compartments. This will enable the optimal utilization of the Particle Flow Algorithms, with which the fine structure of showers can be measured and used to enhance particle identification, energy resolution and pileup rejection. The majority of the HGCAL will be based on robust and cost-effective hexagonal silicon sensors with $\sim 1 \mathrm{~cm}^{\wedge} 2$ or $0.5 \mathrm{~cm}^{\wedge} 2$ hexagonal cell size, with the final 5 interaction lengths of the hadronic compartment being based on highly segmented plastic scintillator with a SiPM readout. Here, we present an overview of the HGCAL project, including the motivation, engineering design, readout/trigger concept and simulated performance

\section{N44-4: Investigation of Fast Timing Capabilities of Silicon Sensors for the CMS High Granularity Calorimeter at HL-LHC \\ $\underline{\text { Y. Onel }}$}

Physics, University of Iowa, Iowa City, Iowa, USA

On behalf of the CMS Collaboration

The High Granularity Calorimeter (HGCAL) is the technology choice of the CMS collaboration for the endcap calorimetry upgrade planned to cope with the harsh radiation and unprecedented in-time event pileup projected at the High Luminosity-LHC era. In this context, profiting from fast-timing information ( tens of picoseconds) embedded in the calorimeter would represent a unique capability for resolving information from individual collisions at the HL-LHC. This will enhance the reconstruction and physics capabilities of the CMS detector in terms of pileup mitigation and particle identification. The HGCAL is realized as a sampling calorimeter, including 40 layers of silicon pad detectors with pad areas of $0.5-1.0 \mathrm{~cm}^{\wedge} 2$ and three active thicknesses: 320, 200 and $120 \mu \mathrm{m}$. Prototype p-in-n and n-in-p 5x5mm^2 silicon pads, with thicknesses of 285, 211 and $133 \mu \mathrm{m}$, were tested with high-energy electrons at the CERN SPS. We present the motivation for this study including the concept and use of fasttiming in the HGCAL, and the measured intrinsic timing capabilities for electromagnetic showers and minimum-ionising particles.

N44-5: Comparative Test Beam Studies of Precision Timing Calorimeter Technologies A. Bornheim, D. Anderson, C. Pena, A. Apresyan, M. Spiropulu, S. Xie, J. Duarte, A. Ronzhin, S. Los HEP, CALTECH, Pasadena, CA, USA

High energy particle colliders experiments are facing ever more challenging conditions, operating at todays accelerators capable of providing instantaneous luminosities of $10^{34} \mathrm{~cm}^{-2} \mathrm{~s}^{-1}$ and above. The high center of mass energy, the large number of simultaneous collision of beam particles in the experiments and the very high repetition rates of the collision events pose huge challenges. They result in extremely high particle fluxes, causing very high occupancies in the particle physics detectors operating at these machines. A precise timing information with a precision of around $10 \mathrm{ps}$ and below is seen as a major aid in the reconstruction of the physics events under such challenging conditions. We present beam test results from different detector concepts which can provide time measurements for charged particles and photons with a precision in the range of a few 10 ps. These detectors utilize micro channel plates (MCPs) with and without photo cathode, scintillating crystals read out with silicon photo multipliers (SiPM) and MCPs as well as semiconductor based sensors such as silicon pads. We will discuss how detectors could be equipped with a precision timing calorimeter based on these technologies.

\section{N44-6: The SDHCAL Prototype Status: Present and Future}

M. C. Fouz

CIEMAT, Madrid, Spain

On behalf of the SDHCAL group of the CALICE Collaboration 
The first technological SDHCAL prototype having been successfully tested, a new phase of R\&D to validate completely the SDHCAL option for the International Linear Detector (ILD) project of the ILC has started with the conception and the realization of a new prototype to host few but large active layers of the future SDHCAL. The new active layers made of GRPC with sizes larger than $2 \mathrm{~m} 2$ will be equipped with a new version of the electronic readout fulfilling the requirements of the future ILD detector. The new GRPC are conceived to improve the homogeneity with a new gas distribution scheme. Finally the mechanical structure will use the electron beam welding. The progress realized will be presented and future steps will be discussed.

\author{
N44-7: Calorimetric Measurements with Extremely Fine Spatial Resolution \\ $\underline{B . ~ B i l k i ~}^{1,2}$ \\ ${ }^{I}$ Physics and Astronomy, University of Iowa, Iowa City, USA \\ ${ }^{2}$ Mathematics and Computing, Beykent University, Istanbul, Turkey \\ On behalf of the CALICE Collaboration
}

In special tests, the active layers of the CALICE Digital Hadron Calorimeter prototype, the DHCAL, were exposed to low energy particle beams, without being interleaved by absorber plates. The thickness of each layer corresponded approximately to 0.29 radiation lengths or 0.034 nuclear interaction lengths, defined mostly by the copper and steel skins of the detector cassettes. With its extremely fine segmentation of the readout based on $1 \times 1 \mathrm{~cm}^{\wedge} 2$ pads, the DHCAL provides unprecedented details of both electromagnetic and hadronic showers. This paper reports on measurements performed with this device in the Fermilab test beam with positrons and pions in the energy range of 1 to $10 \mathrm{GeV}$. The measurements are compared to simulations based on GEANT4 and a standalone program to emulate the detailed response of the active elements.

\title{
N44-8: R\&D with Very Forward Calorimeters for Linear Colliders
}

$\underline{\text { M. Idzik }}$

AGH University of Science and Technology, Cracow, Poland

On behalf of the FCAL Collaboration

The luminosity of the International Linear Collider will be measured by counting Bhabha scattering events, recognized by coincident detection of two low angle electrons in two calorimeters located symmetrically on the opposite side of the interaction point. The calorimeters are designed as sampling calorimeters with tungsten absorber disks interspersed with silicon sensors. For a precise measurement, it is essential to achieve a small Moliii $i^{1} / 2$ re radius. The design thus foresees ultra-thin sensors that allow 1 $\mathrm{mm}$ spacing between the absorbers. In 2014, a first multi-layer prototype of the calorimeter was tested in a beam of $5 \mathrm{GeV}$ electrons and muons at CERN. Excellent performance of the sensors was demonstrated and development of the showers has been studied by comparing the data to simulations. In 2015, different silicon technologies have been explored allowing to reach sensor thickness of $800 \mathrm{um}$. Four thin layers, fully equipped with front-end electronics, have been succesfully tested in a $5 \mathrm{GeV}$ electron beam at DESY, Hamburg. The different steps of the construction of the thin sensor assemblies and the test Beam results will be presented.

N45: Gaseous detectors II: Development of Techniques II

\author{
Wednesday, Nov. $2 \quad$ 16:30-18:30 Londres \\ N45-1: GridPix Detectors - Developments and Applications \\ C. Krieger ${ }^{1}$, Y. Bilevych ${ }^{1}$, K. Desch ${ }^{1}$, J. Kaminski ${ }^{1}$, M. Lupberger ${ }^{2}$, T. Schiffer ${ }^{1}$ \\ ${ }^{I}$ University of Bonn, Bonn, Germany \\ ${ }^{2}$ CERN, Geneva, Switzerland
}

GridPix detectors are a promising new detector technology featuring the combination of a Micromegas gas amplification stage with an integrated readout structure of matched high granularity, implemented by the Timepix ASIC. The Micromegas mesh is produced directly on top of the Timepix ASIC by means of photolithographic postprocessing techniques resulting in each mesh hole well aligned with one pixel. Thereby, the gas amplification avalanche is collected by one pixel resulting in a high detection efficiency of single primary electrons. Allowing to resolve the structure of tracks or X-ray conversions both energy and spatial resolution are improved. A wafer-based production of GridPix detectors has been set up and a scalable readout system has been developed allowing the construction and simoultaneous operation of large numbers of GridPixes in experimental setups. Different applications will be presented: A small GridPix detector applied in the solar axion search at a low-rate experiment benefitting from the good energy resolution (down to $3.85 \%$ possible) and precise event shape inspection. Another application covered in this presentation will be tests of a GridPix based transition radiation detector in a magnetic field. Finally, a test beam campaign with a large volume TPC at DESY demonstrating the smooth operation of 160 GridPix detectors recording tracks of up to $50 \mathrm{~cm}$ length will be presented. 
N45-2: Optimization of Resistive Micromegas for Sampling Calorimetry at High Rates

T. Geralis ${ }^{1}$, M. Chefdeville ${ }^{2}$, M. Titov ${ }^{3}$

${ }^{I}$ INPP, NCSR Demokritos, ATHENS, GREECE

${ }^{2} L A P P$, Universite de Savoie, ANNECY-LE-VIEUX, FRANCE

${ }^{3} I R F U$, Saclay CEA, GIF-SUR-IVETTE, FRANCE

Micromegas, a micro pattern gaseous detector, is proposed as an active medium for sampling calorimetry. Future linear collider experiments or the High Luminosity LHC experiments can profit from those developments for Particle Flow Calorimetry. Micromegas possesses remarkable properties concerning gain stability, reduced ion feedback, response linearity, adaptable sensitive element granularity, fast response and high rate capability. Recent developments on Micromegas with a protective resistive layer present excellent results, resolving the problem of discharges caused by local high charge deposition, thanks to its RC slowed charge evacuation. Higher resistivity though, causes loss of the response linearity. We have scanned five orders of magnitude in resistivities and performed laboratory tests with X-rays that demonstrate excellent response linearity up to rates of about $1 \mathrm{MHz} / \mathrm{mm} 2$, with simultaneous mitigation of discharges. Beam test studies at SPS/CERN with hadrons and electrons have also shown a remarkable stability of the resistive Micromegas and low currents for rates up to $5 \mathrm{MHz} / \mathrm{cm} 2$. We will be presenting results from the aforementioned studies confronted with MC simulation as well as efforts for modeling the Micromegas operation concerning the signal formation, the spark suppression and the charge evacuation in avalanches. This is mandatory for the detector optimization under different conditions of operation in terms of rate and occupancy.

N45-4: Beam Test Results with a Large Prototype of TPC for the ILD Detector at ILC $\underline{\text { S. Ganjour }}$

SPP, CEA/Scalay/IRFU, Gif-sur-Yvette, France

On behalf of the LCTPC Collaboration

A Linear Collider (LC) in Japan is the major large scale accelerator project in High Energy Physics today, which is aimed to address unresolved mysteries of particle physics. The International Large Detector (ILD) is one of the detector concepts at the ILC. The ILD central tracker comprises a Time Projection Chamber (TPC) embedded in a 3.5 Tesla solenoidal field. An extensive research and development program for a TPC has been carried out by the LCTPC collaboration in the past. A large prototype of TPC, equipped with up to seven identical modules in a 1 Tesla magnetic field has been tested with a $6 \mathrm{GeV}$ electron beam at DESY. Track reconstruction performance was measured for three different detector technologies: Gas Electron Multiplier, Resistive Micromegas and GridPix. It was demonstrated that current technologies allow us to meet the ILD tracker requirements.

\section{N45-5: The COMPASS New Hybrid GEM-Micromegas Pixelized Detectors and Their Performance in High Particle Flux Conditions \\ D. Neyret \\ IRFU / SPhN, CEA Saclay, Gif sur Yvette, France \\ On behalf of the IRFU COMPASS Micromegas group}

Large size Micromegas gaseous detectors $\left(40 \times 40 \mathrm{~cm}^{2}\right.$ active area) were developed in view of the forthcoming COMPASS new physics programs which uses the CERN high intensity muon and hadron beams of a few hundred GeV scattered on thick fixed targets. The new detectors are now active in the beam area, where intensities are as high as a few hundred of $\mathrm{kHz} / \mathrm{mm}^{2}$, with the same performances ( $96 \%$ efficiency, $70 \mu \mathrm{m}$ spatial resolution) compared to the other positions thanks to a pixelized read-out on this area. In such a hot detector environment, with highly ionising particles, spark generation is a major issue for MPGD detectors. The new hybrid technics, where a pre-amplifying GEM foil is added to a non resistive Micromegas detector, is used in the new detectors to strongly reduce the spark rate in presence of high particle flux. The delicate technology of making "bulk" detectors (monolithic assembly of a micro-mesh and a board) has been transferred to industry in the framework of this project, leading to high production rate and lower cost. The detector are installed and in use in the hard conditions of the COMPASS experiment since 2015, with very good performance in term of gain, efficiency and spatial resolution.

The key characteristics of the detectors will be presented, with an overview of their performance in nominal conditions from the 2015 and 2016 runs. The different aspects of the technology transfer and the industrialization of the production will also be presented.

N45-6: Development of a Double-Grid-Type MSGC with Two-Dimensional Readout Using LCD Technology

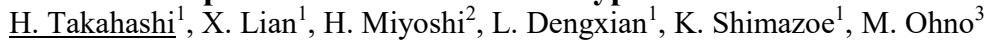

${ }^{I}$ Department of Nuclear Engineering and Management, The University of Tokyo, Tokyo, Japan

${ }^{2}$ SHARP Corporation, Osaka, JAPAN

${ }^{3}$ Nuclear Professional School, The University of Tokyo, Ibaraki, JAPAN 
The LCD technology intends to integrate many small pixels, as a result, the latest technology could form a very fine pixel of $\sim 100 \mathrm{um} \times 100 \mathrm{um}$. Although the display technology is thought to be quite different from sensor technology, the device itself is similar each other except for the signal level. Thus, the LCD technology is successfully applied to the Flat Panel Detector, where the sensor pixel is scanned by the switching amorphous transistors. We are thinking about another compatibility of the LCD technology with the micro-pattern gaseous detectors. Very fine pitch electrode pattern realized in the LCD display on a glass substrate is what we aimed at the MSGC (Micro Strip Gas Chamber). Therefore, we think it is possible to employ the latest LCD technology for a very sophisticated and high-technology MSGC. We have fabricated and tested a small test device with the anode pitch of 500um and the same cathode pitch (their orientations are orthogonal) using the LCD technology. We successfully measured X-ray pulses from an Fe-55 source, where the second grid was kept at the same potential as the anode (i.e. single-gridmode operation) as an initial measurement.

N45-7: VUV Xenon Scintillation Wavelength Shift by Trimethylamine

F. O. P. Santos, A. M. F. Trindade, J. M. D. Escada, F. I. G. M. Borges, A. F. V. Cortez

Departamento de Física, Universidade de Coimbra, Coimbra, Portugal

Gaseous xenon has been widely used as detection medium in detectors, either in charge or scintillation amplification modes, in spite of the small caveats it reveals. To circumvent these caveats, several molecular additives have been tentatively used, in small percentages. Among them trimethylamine (TMA) has been one of the most promising, expected to increase electrons drift velocity, decrease diffusion coefficients and even to shift to a higher wavelength the challenging xenon VUV emission. Other possible attractive features are Penning effect with Xe. In this work the possible wavelength shifting role of TMA will be investigated. In order to assess the absorption of xenon scintillation $(172 \mathrm{~nm})$ by TMA molecules and possible reemission in another wavelength $(300 \mathrm{~nm}$, the peak of the emission spectra), a simple experimental system has been built. It consists of a cylindrical chamber of fixed length, with a xenon lamp at the top and a quartz spectrosil window on the bottom, to which an external photomultiplier tube (PMT) is assembled. Preliminary experiments have already been made under vacuum and with gas at various pressures and different compositions. To assess the possible reemission of xenon scintillation by TMA, measurements were taken with and without a special bandpass filter (EO U-330 UV - transmission band between 200 and $400 \mathrm{~nm}$ ) placed between the window and the PMT. The analysis of the results showed that there seems to be absorption of Xe scintillation but no reemission. Further experiments are under way and results will be presented.

N45-8: Live Event Reconstruction and Scintillation Studies in an Optically Read Out GEM-Based TPC $\underline{\text { F. M. Brunbauer }}^{1,2}$, C. Bault ${ }^{1}$, D. Gonzalez Diaz ${ }^{3}$, E. Oliveri ${ }^{1}$, F. Resnati ${ }^{1}$, L. Ropelewski ${ }^{1}$, C. Streli ${ }^{2}$, P. Thuiner ${ }^{1,2}$, M. van Stenis ${ }^{1}$

${ }^{I}$ CERN, Geneva, Switzerland

${ }^{2}$ Technische Universität Wien, Vienna, Austria

${ }^{3}$ Uludag University, Bursa, Turkey

Optically read out MicroPattern Gas Detectors (MPGDs) allow for highly sensitive and accurate track reconstruction and imaging applications by combining strong signal amplification with the precise spatial readout made possible by modern pixelated image sensors. Augmenting the 2D projection of particle tracks with timing information obtained from scintillation signals, a versatile Time Projection Chamber (TPC) based on a triple Gas Electron Multiplier (GEM) is presented. Taking advantage of the high scintillation yield of an $\mathrm{Ar} / \mathrm{CF}_{4}$ gas mixture, primary scintillation signals of alpha particles were observed and allowed for unambiguous $3 \mathrm{D}$ track reconstruction. The light yield in various gas mixtures and pressure regimes is studied and used to enhance optically read out GEM detectors. Drift and amplification fields were exploited to optimize the detector response to a range of ionizing particles including alpha particles, lower energetic electrons and cosmic muons. Long term studies conducted in the ultra-high purity TPC revealed low light signal amplitude degradation, effectively paving the way for sealed mode operation, which may prove to be of paramount importance for future applications. The remarkable stability of the presented detector even in sealed mode and the insensitivity to electronic noise inherent to the optical readout presents a promising approach for universal tracking and imaging detectors, which can be employed in unfavorable environments and even as portable devices. Moreover, the pixelated readout inherent to CCD and CMOS cameras avoids the need for X-Y matching and ambiguity issues associated with pileup. Thus, optical readout of MPGDs and TPCs based upon it may prove useful for the study of nuclear reactions including photo-absorption and two-proton decay and for particle physics experiments aiming at the detection of rare events such as nuclear recoils associated with weakly interacting massive particles or neutrinoless double beta decay.

N46: Astrophysics and space II

Wednesday, Nov. $2 \quad 16: 30-18: 30 \quad$ Curie 1
N46-1: Status and Progress of the International Axion Observatory (IAXO)
E. Ferrer Ribas
CEA, Université Paris-Saclay, IRFU, Gif sur Yvette, France


The International Axion Observatory (IAXO) is a fourth generation axion helioscope that aims to go beyond the current state of the art and achieve a sensitivity on the axion-photons coupling ga? down to a few $\times 10-12 \mathrm{GeV}-1$ for a wide range of axion masses up to $\sim 0.25 \mathrm{eV}$. IAXO will probe a large fraction of the currently unexplored axion and axion-like-particle parameter space. This represents an improvement over the currently best (3rd generation) axion helioscope, the CERN Axion Solar Telescope (CAST), of about 5 orders of magnitude in signal strength, corresponding to a factor $\sim 20$ in the axion photon coupling. We will present the conceptual design of IAXO, which follows the layout of an enhanced axion helioscope, based on a purpose-built $20 \mathrm{~m}$-long 8-coils toroidal superconducting magnet. All the eight $60 \mathrm{~cm}$-diameter magnet bores are equipped with focusing x-ray optics, able to focus the signal photons into $\sim 0.2 \mathrm{~cm} 2$ spots that are imaged by ultra-low-background x-ray detectors. The magnet is built into a structure with elevation and azimuth drives that will allow for solar tracking for $\sim 12 \mathrm{~h}$ each day. The progress and plans towards the Technical Design Report will be shown.

\author{
N46-2: Pulsed Neutron Generator - Gamma Ray Spectrometer Measurements of Venus' Bulk Elemental \\ Composition \\ $\underline{\text { A. M. Parsons }}^{1}$, J. Grau ${ }^{2}$, D. Lawrence ${ }^{3}$, T. P. McClanahan ${ }^{1}$, J. Miles ${ }^{2}$, P. Peplowski ${ }^{3}$, L. Perkins ${ }^{4}$, J. Schweitzer ${ }^{5}$, R. D. Starr ${ }^{6}$ \\ ${ }^{I}$ Solar System Exploration Division, NASA/ Goddard Space Flight Center, Greenbelt, MD, USA \\ ${ }^{2}$ Schlumberger Doll Research Center, Cambridge, MA, USA \\ ${ }^{3}$ Johns Hopkins University Applied Physics Laboratory, Laurel, MD, USA \\ ${ }^{4}$ Schlumberger Princeton Technology Center, Princeton Junction, NJ, USA \\ ${ }^{5}$ Department of Physics, University of Connecticut, Storrs, CT, USA \\ ${ }^{6}$ Department of Physics, The Catholic University of America, Washington, DC, USA
}

Bulk elemental composition measurements are difficult on the surface of Venus since all of a lander's instruments have to be housed inside a pressure vessel with sufficient thermal control systems to protect the equipment from the harsh surface conditions $\left(462{ }^{\circ} \mathrm{C}, 93\right.$ bars pressure) for at least one hour. The Probing In situ with Neutrons and Gamma rays (PING) instrument combines a Pulsed Neutron Generator (PNG) with a Gamma Ray Spectrometer (GRS) placed inside a Venus lander pressure vessel. PING allows the measurement of the bulk elemental composition over a large volume beneath a landed probe $(\sim 30 \mathrm{~cm}$ deep, $\sim 50 \mathrm{~cm}$ radius). The combination of a high intensity PNG $(5 \times 108$ isotropic neutrons per second) and a scintillator GRS with a fast light decay time (16 ns for $\mathrm{LaBr} 3$ ) allows for gamma ray count rates in excess of $2 \mathrm{MHz}$. This extremely high gamma ray throughput is necessary to provide the counting statistics needed for the $\sim 1$ hour measurement times currently available for Venus landers. Simulations demonstrate the precision and accuracy of Venus elemental composition measurements possible with PING in the brief time available on the planet's surface. The excellent sensitivity of PING to Venus basaltic rock compositions illustrated by these model results will be described in detail. Results for simulation validation experiments and additional simulations for various lander configurations will also be presented.

N46-3: The High-Energy Particle Detector Onboard the CSES Satellite $\underline{\text { V. Scotti }}$

INFN, Naples, Italy

On behalf of the CSES-Limadou Collaboration

The China Seismo Electromagnetic Satellite (CSES) aims to contribute to the monitoring of earthquakes from space. This space mission will study electromagnetic phenomena and their correlation with the geophysical activity. The satellite will be launched in 2017 and will host several instruments onboard: two magnetometers, an electrical field detector, a plasma analyzer, a Langmiur probe and an High Energy Particle Detector (HEPD). The High-Energy Particle Detector (HEPD), developed by the Italian Collaboration, detects electrons, protons and light nuclei. The main objective is to measure the increases of the electron and proton fluxes due to short-time perturbations of the radiation belts caused by solar and terrestrial phenomena. Before building the Flight Model of the instrument, three different models have been realized and tested in laboratory to assess the compliance of the HEPD characteristics and to verify the detector performances. The last model, identical to the Flight Model, has also been tested at the Beam Test Facility of Frascati with electron beams of energies comprised between 30 and $150 \mathrm{MeV}$. In this paper a description of the HEPD apparatus, its main characteristics and preliminary results of the calibration will be presented.

N46-4: The SENSER CLYC Experiment

D. D. S. Coupland, L. C. Stonehill, K. E. Mesick, J. P. Dunn

Los Alamos National Laboratory, Los Alamos, NM, USA

Los Alamos National Laboratory (LANL) has designed, built, and supported the operation of satellite payloads for monitoring of nuclear test ban treaties for over 50 years, since the launch of the first Vela satellite. New technologies can provide performance 
improvements and reduction of size, weight, and power (SWaP), but are riskier than tried-and-true methods and must be evaluated in the space environment before they can be considered for operational use. The Space and Endo-Atmospheric Nudet Surveillance Experimentation and Risk-Reduction (SENSER) project, scheduled to launch to geosynchronous orbit in 2019, contains several experimental and demonstration systems developed by LANL and Sandia National Laboratories to assess nextgeneration technologies for the United States Nuclear Detonation Detection System (USNDS). The CLYC experiment in SENSER will examine the Cs2LiYCl6 (CLYC) scintillator in the space environment, which enables neutron and gamma-ray detection in a single instrument with better energy resolution than traditional scintillators. The CLYC experiment will also investigate silicon photomultiplier (SiPM) photodetectors for additional SWaP reduction. In this presentation, we present the CLYC experiment design, Monte Carlo simulations of the expected response to the space environment, and results of initial laboratory tests and calibrations of the fully-built system.

\author{
N46-5: EPI-Hi: A New Instrument for Measuring Energetic Nuclei and Electrons in the MeV Range on \\ NASA's Solar Probe Plus Mission \\ M. E. Wiedenbeck \\ Jet Propulsion Laboratory, California Institute of Technology, Pasadena, CA, USA \\ On behalf of the Integrated Science Investigation of the Sun Collaboration
}

The Integrated Science Investigation of the Sun is a suite of two energetic-particles instruments, EPI-Hi and EPI-Lo, that is to be flown on the Solar Probe Plus (SPP) spacecraft as it investigates particles and fields of solar origin to within 10 AU of the Sun's surface after launch in 2018. The EPI-Hi instrument includes three energetic-particle telescopes that identify particles using the $\mathrm{dE} / \mathrm{dx}$ versus total energy technique in stacks of silicon state detectors. The telescopes observe energetic nuclei from $\sim 1 \mathrm{MeV} / \mathrm{nuc}$ to $>100 \mathrm{MeV}$ /nuc and electrons from $\sim 0.5$ to $\sim 6 \mathrm{MeV}$, providing high-cadence measurements of composition, spectra, and angular distributions that will be used to investigate energetic-particle acceleration and transport close to the Sun. Construction and calibration of the flight instrument is being completed in 2016 in preparation for delivery to the spacecraft in 2017 . Tests using radioactive sources and accelerator beams of energetic heavy nuclei are being performed to show that the instrument satisfies measurement requirements and to provide calibrations that will be used in interpreting data collected in flight. We will describe the instrument design and present accelerator test data that demonstrate its measurement capabilities.

N46-6: Developments in Atomic Layer Deposited Microchannel Plates

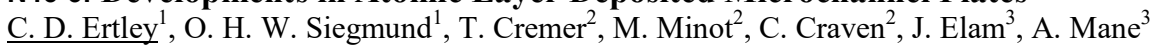

${ }^{I}$ Space Sciences Laboratory, The University of California - Berkeley, Berkeley, CA, USA

${ }^{2}$ Incom, Inc., Charlton, MA, USA

${ }^{3}$ Argonne National Laboratory, Lemont, IL, USA

Microchannel plate (MCP) imaging electron multipliers are now being manufactured using borosilicate microcapillary array substrates functionalized by the application of resistive and secondary emissive layers using atomic layer deposition (ALD) techniques. Unlike traditional MCP manufacturing, the substrate and the active layers are independent allowing custom nanofabrication and optimization of parameters like gain and resistance through application of high secondary emissive materials. Borosilicate glass substrates are more robust than traditional lead glass MCPs, allowing them to be produced in large formats $(400 \mathrm{~cm} 2)$ with high open area ratios (up to $85 \%$ ). ALD MCPs have no lead and low alkali metal content, promoting a low intrinsic background ( $7 \mathrm{C} \mathrm{cm}-2$ charge extraction after preconditioning (vacuum bake and burn-in). The UV quantum efficiency of ALD MCPs exceeds conventional MCPs and application of standard alkali halide photocathode layers results in the same improvements expected for MCP devices. The robust nature of ALD MCPs means they can also withstand the processing temperatures required for high temperature deposition of III-V materials, such as Gallium Nitride (GaN), for UV photocathodes with high QEs in the Near-UV ( 200-300nm). Here we present significant progress in the development of ALD MCPs. The newest generation of substrates in $20 \mu \mathrm{m}$ and $10 \mu \mathrm{m}$ pores are more uniform than previous generations, have lower background, and are more stable during life testing. We have tested the QE of bare ALD MCPs and compared them to historical values. We also present the progress of our opaque photocathode deposition efforts.

N46-7: MUSIC: an ASIC for SiPM Array Signal Summation and Processing

D. Gascon, S. Gomez, G. Fernandez, A. Sanuy, J. Mauricio, D. Ciaglia, R. Graciani, D. Sanchez

Dept. FQA, ICCUB. University of Barcelona, Barcelona, Spain

SiPMs are replacing PMTs in many applications. Still, limitations due to SiPM high capacitance exist when large photo-detection areas are required. Dedicated ASICs adding the signals of several SiPM covering the same area alleviate this drawback. The Multiple Use SiPM Integrated Circuit (MUSIC) has been designed to serve this purpose, i.e. summation of SiPM signals, but also for individual channel readout (analog or ToT) or photon counting. This paper presents an 8 channel ASIC for SiPM anode readout based on a novel low input impedance current conveyor. The current division scheme at the very front end part of the circuit splits the input current into differently scaled copies which are connected to independent current mirrors. The circuit 
contains a tunable pole zero cancellation of the SiPM recovery time constant to deal with sensors from different manufacturers. Decay times up to $100 \mathrm{~ns}$ are supported covering most of the available SiPM devices in the market. MUSIC offers three main features: (1) differential output of the sum of (selectable) individual input channels; (2) 8 individual single ended analog outputs and; (3) 8 individual binary outputs. The digital outputs encode the amount of collected charge in the duration of digital signals using a time over threshold (ToT) technique. For each individual channel, the user must select the analog or digital output. Each functionality, the signal sum and the $8 \mathrm{~A} / \mathrm{D}$ outputs, includes a selectable dual-gain configuration. Moreover, the signal sum implements dual-gain output providing a 15 bit dynamic range. MUSIC is designed using a $0.35 \mu \mathrm{m} \mathrm{SiGe} \mathrm{BiCMOS}$ technology. Post-layout simulation results are presented: $500 \mathrm{MHz}$ bandwidth for channel sum and $150 \mathrm{MHz}$ for A/D channels, low input impedance ( 30 ?), single photon output pulse width at half maximum (FWHM) between 5 and $10 \mathrm{~ns}$, cable driving capabilities and a power consumption between 15 and $30 \mathrm{~mW} / \mathrm{ch}$. Characterization will occur from May 2016.

N46-8: Capability of Thin Dead-Layer Silicon Photomultipliers to Count Low-Energy Electrons K. Ogasawara, S. A. Livi, M. I. Desai, R. W. Ebert, F. Allegrini, M. A. Dayeh

Southwest Research Institute, San Antonio, TX, USA

The low-energy threshold level and the response characteristics of thin surface-layer silicon Photomultipliers (SiPMs) to count sub-keV secondary electrons are investigated. In this study, we show that a novel thin dead-layer SiPMs can count electrons $>1000 \mathrm{eV}$ using secondary electron clouds produced by one-stage MCP. The future application of these types of SiPMs for counting secondary electrons instead of conventional electron multipliers can mitigate most of the limitations described above.

N47: Circuits for readout of SiPM and timing

Wednesday, Nov. $2 \quad$ 16:30-18:30 Curie 2

N47-1: A 16-Channel Readout System for Analog and Digital SiPMs

E. Venialgo $^{1}$, N. Lusardi ${ }^{2}$, A. Geraci ${ }^{2}$, E. Charbon ${ }^{1}$

${ }^{I}$ Electrical Engineering, Delft University of Technology, Delft, Netherlands

${ }^{2}$ DEIB, Politecnico di Milano, Milano, Italy

In this paper we present a readout system capable of converting any commercial analog silicon photomultipliers onto a 16channel digital SiPM. Multi-channel digital SiPMs can capture multiple photon timestamps per scintillation to reconstruct the gamma-event timemark more robustly than if a single timestamp is used. The reconstruction is performed optimally with the knowledge of order statistics governing the scintillation. The readout system was implemented though discrete components in combination with an array of TDCs implemented and validated on a field-programmable gate array. The achieved overall resolution of each channel was 56ps (FWHM) or better, while crosstalk was undetectable.

N47-2: Triroc, a Versatile 64-Channel SiPM Readout ASIC for Time-of-Flight PET

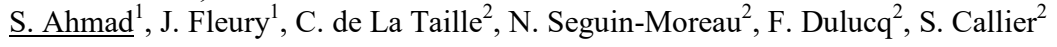

${ }^{1}$ Weeroc $S A S$, Palaiseau, France

${ }^{2}$ Omega - CNRS/IN2P3/Ecole Polytechnique, Palaiseau, France

Triroc is a highly configurable SiPM readout ASIC intended for PET prototyping with time-of-flight capability. This 64-channel ASIC incorporates a dual triggering system that will flag the arrival photons and provides energy cut-off at a specified level. Time and charge measurements are performed internally by 40ps-bin TDC and 10-bit ADC.

The analog design of this ASIC has been optimized for accepting positive and negative polarity inputs while maintaining nearly similar performances for both input polarities. Channel-by-channel input DC level adjustment is also available in order to correct the non-uniformity of SiPM array and fine tuning each pixel gain. Additionally, the digital conversions are assignable to other analog components, which extends the suitability of Triroc for any application requiring SiPM readout.

The design of Triroc and the characterization results will be presented in this work.

\section{N47-3: PETIROC2A, a 32-Channel 20 GHz GBW Readout ASIC for Accurate Time Resolution and Precise} Charge Measurements

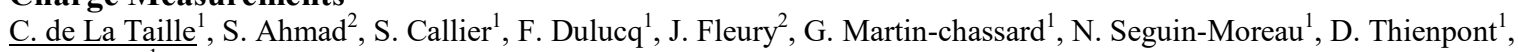
S. Conforti ${ }^{1}$

${ }^{I}$ OMEGA Ecole Polytechnique - CNRS/IN2P3, Palaiseau, FRANCE

${ }^{2}$ Weeroc SAS, Palaiseau, France

Petiroc2a is a 32-channel front-end ASIC designed in $0.35 \mu \mathrm{m}$ SiGe AMS technology to read out Silicon Photo Multipliers (SiPMs) for particle time-of-flight measurement applications and for physics experiments that both require accurate time 
resolution and precise energy measurement over a large dynamic range. Each channel of Petiroc2A integrates a High Frequency preamplifier with a Gain Bandwidth Product of $20 \mathrm{GHz}$ followed by a fast discriminator and a TDC. The first incident photons can be then detected and measured with a time resolution better than 100ps. The energy measurement is made by a variable low gain shaper to ensure a good linearity over a large dynamic range that goes up to $400 \mathrm{pC}$. The time and charge measurements of each channel are internally digitized by a 10-bit ADC. The chip was fabricated in 2015. Architecture and test bench measurements will be presented as well as the measurements performed with SiPM matrixes using a dedicated board.

\author{
N47-4: PACIFIC: SiPM Readout ASIC for LHCb Upgrade \\ J. Mazorra de Cos \\ Instituto de Física Corpuscular (CSIC-UV), Valencia, Spain \\ On behalf of the LHCb Scintillating Fibre Tracker Group
}

PACIFIC is a 64 channel mixed-signal ASIC designed for the readout of the Scintillating Fiber (SciFi) Tracker developed for the LHCb upgrade in 2018/19. This tracker will replace the current Tracking Stations, since the increased hit occupancy and higher radiation dose imposed by the upgrade exceeds the capabilities of the current detector. The SciFi Tracker uses as active material $250 \mu \mathrm{m}$ scintillating fibres, stacked in 6 layer mats and sensed by custom designed 128 channel silicon photomultiplier (SiPM) arrays. It will be comprised of 12 planes, each covering an area of $5 \times 6 \mathrm{~m} 2$.

PACIFIC takes on the readout of the SiPM signal, producing a digital representation of it at a rate of $40 \mathrm{MHz}$. The ASIC is connected directly to them without any interface components, using a current conveyor to acquire the SiPM current pulses. These pulses have the typical shape, with a very fast rising edge and a much slower the falling edge, modelled as a double exponential decay and largely extending over the clock period. For that reason, the next stage of the analog processing is a fast shaper with a fully configurable double pole-zero cancellation. Subsequently, the signal is accumulated with a gated integrator, using two interleaved units to maximize the integration time. Finally, the digitization is performed with a 2 bit non-linear flash ADC, bundling together the data from two channels and outputting them at $160 \mathrm{MSa} / \mathrm{s}$. Altogether, the target power consumption is below $10 \mathrm{~mW}$ per channel.

Several prototypes have been produced and tested so far. The first two prototypes were produced in IBM 130nm technology, allowing the validation of the proposed architecture. PACIFICr3 is the first full 64 channel prototype and its design has been migrated to TSMC 130nm. Furthermore, a new version of the ASIC is planned for submission during spring 2016.

N47-5: SPACIROC3: a Low Power 100MHz Photon Counting ASIC for Cosmic Ray Observatory

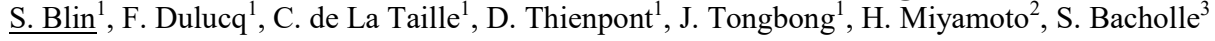

${ }^{I}$ OMEGA/Ecole Polytechnique/CNRS, France, Palaiseau

${ }^{2}$ Universita di Torino/INFN, Italy, Torino

${ }^{3}$ APC/Université Paris Diderot, France, Paris

SPACIROC (Spatial Photomultiplier Array Counting and Integrating ReadOut Chip) is the front-end ASIC designed for the space-borne fluorescent telescope JEM-EUSO (Extreme Universe Space Observatory onboard Japanese Experiment Module). This ASIC is dedicated to readout 64-channel Multi Anode Photo Multiplier Tube (MAPMT). The two main features are the photon counting for each input and the charge conversion for each 8 -channel sum. In the photon counting mode, $100 \%$ trigger efficiency is achieved for $1 / 3$ photo-electron (pe) input charges. In order to detect pile-up in case of a large flux of photons, the double pulse separation must be shorter than $10 \mathrm{~ns}$. The high-speed performance is obtained using low power (1 mW/channel) thanks to the SiGe technology and by integrating photo-electron trigger counters and a dedicated design to improve the double pulse separation up to $100 \mathrm{MHz}$. For the charge measurement, the ASIC should operate with a large dynamic range (1 pe to 100 pe per pixel). SPACIROC3 has been designed to improve the double pulse separation and to increase the charge dynamic range thanks to a new front-end architecture compared to the previous version, which was extensively used in the EUSO balloon campaign of 2014. The architecture and the performance of the photon counting part will be detailed in this presentation.

\author{
N47-6: ?-PET-V1.0: a Novel and Low-Cost Electronics for Large Scale SiPM Array Readout and Advanced \\ PET Applications

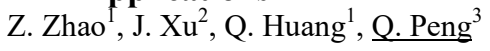

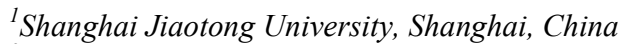 \\ ${ }^{2}$ School of Mechanical Science and Engineering, Huazhong University of Science and Technology, WuHan, China \\ ${ }^{3}$ Department of Molecular Biophysics and Integrated Bioimaging, Lawrence Berkeley National Laboratory, Berkeley, USA
}

Silicon photomultiplier (SiPM) becomes a choice of photon sensors for advanced radiation detector development. However, reading out large-scale SiPM arrays is still a fundamental technical obstacle. We present a revolutionary new method (named pPET electronics) to address this issue. Very different from conventional front-end electronics design, the key innovation of the new electronics is to include almost all functions of front-end readout electronics inside a low-cost FPGA. That not only simplifies the analog components and reduce the cost (with only one linear amplifier) but also provides powerful and flexible 
signal processes to enable applying different algorithms to both enhance the performance and add new real-time dark current measurement and calibration features.

We have successfully developed the first generation of 100-channel p-PET v1.0 electronics. p-PET v1.0 is able to readout 100 channels of SiPM signals simultaneously or 768 channels of SiPM signals with crosswire multiplexing. The size, full-speed power consumption and hardware cost of the p-PET v1.0 electronics are $6 \mathrm{~cm} \times 6 \mathrm{~cm} \times 1 \mathrm{~cm}, 3 \mathrm{~W}$ (analog board: 1W, FPGA board: $2 \mathrm{~W})$ and $\sim \$ 500$, respectively. The p-PET electronics was tested comprehensively in term of energy measurement, crystal decoding ability $(0.5 \mathrm{~mm})$, SiPM dark currents $(\sim 100 \mathrm{pA})$ measurement, gain measurement, and timing performance.

We conclude that p-PET electronics is a high-performance, low-cost, low-power consumption, compact, scalable, versatile (e.g. capable for adapting to different device and design requirement), and relatively easy to implement readout electronics for SiPMbased PET system and imaging applications. It has the potential to change the current research and development landscape by enabling most researchers with the capability to design and implement advanced SiPM-based imaging detectors and systems.

\title{
N47-7: The ARAGORN Front-End - FPGA Based Implementation of a Time-to-Digital Converter
} M. Buechele, H. Fischer, F. Herrmann, C. Schaffer

Dep. of Physics, University of Freiburg, Freiburg, Germany

The ARAGORN front-end offers high-performance readout capabilities for high-luminosity experimental environments. The design constraints aim to develop a cost efficient Time-to-Digital Converter (TDC) platform with considerable channel density at smallest form factor possible. The logic of 384 input channels on a single module is implemented by four Xilinx Artix-7 FPGAs with a time resolution smaller than 200 ps to allow for precise time-of-flight or drift-time measurements. Another FPGA acts as data concentrator and masters the communication with the remaining onboard devices. A multi-channel optical transceiver slot provides interconnection with seven other boards through a star topology. This design feature makes it possible to read out eight boards yielding 3072 input channels via a single optical fiber with a bandwidth of $6.6 \mathrm{~Gb} / \mathrm{s}$. Accordingly, the required infrastructure interfacing with the subsequent readout system is strongly reduced.

\author{
N47-8: A compact size, 64-channel, 80 MSPS, 14-bit dynamic range ADC module for the PANDA \\ Electromagnetic Calorimeter

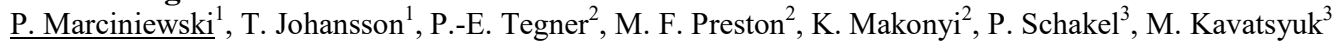 \\ ${ }^{I}$ Physics and Astronomy, Uppsala University, Uppsala, Sweden \\ ${ }^{2}$ Physics, Stockholm University, Stockholm, Sweden \\ ${ }^{3}$ KVI-CART, University of Groningen, Groningen, The Netherlands
}

A compact size, 64-channel, 80 MSPS, 14-bit dynamic range ADC modules for the scintillating electromagnetic calorimeter of PANDA were developed and used for testing in various detector readout set-ups [1]. To minimize cabling bulk, the modules are planned to be placed inside of the PANDA detector volume, where they will be exposed to magnetic field of $2 \mathrm{~T}$ and a nonnegligible radiation flux. The module performs signal filtration, extracts important signal parameters and allows for resolving and parametrizing overlapping pulses. A dual FPGA structure and a hardwired arbitration circuit allows for resolving potentially catastrophic situations caused by radiation-induced (SEU) configuration damages. The FPGAs are prepared for self-detection and recovery from SEU. Processed data are pushed to the optical link running at $2 \mathrm{Gbit} / \mathrm{s}$. The ADC module is compliant with a "Synchronization Of Data Acquisition" (SODA) System, which allows for obtaining defined latencies with a reference time accuracy of $50 \mathrm{ps}$ [2]. The paper describes construction details and test environments. The results of performance test, including dynamic range, linearity, magnetic field and preliminary radiation sustainability are also presented.

N48: High energy physics instrumentation III

Thursday, Nov. 3 08:00-10:00 Madrid

N48-1: DOM, the Digital Optical Module of the KM3NeT Neutrino Telescope E. Leonora

INFN, sezione di Catania, Catania, Italy

On behalf of the KM3NeT Collaboration

The KM3NeT collaboration started building a new neutrino telescope in the depths of the Mediterranean Sea, which will consist of cubic kilometer sized 3-dimensional arrays of Digital Optical Modules (DOMs), suspended in the sea by vertical string structures, called detection units (DUs). The KM3NeT telescope will comprise two detectors with different density of the optical modules: KM3NeT/ARCA at the KM3NeT-It site dedicated to high-energy neutrino astronomy and KM3NeT/ORCA, a denser detector located at the KM3NeT-Fr site and dedicated to the study of neutrino mass hierarchy. The optical modules, which represent the sensitive part of the neutrino telescope, are pressure-resistant 17-inch diameter transparent glass vessels, each hosting 31 photomultiplier tubes with a photocathode diameter of 3 inch and all associated electronics. The multi-PMT solution represents an innovative design, considering that the optical modules of all the other neutrino telescopes -ANTARES, Baikal and 
IceCube - have a design with a single large photomultiplier. This novel solution allows for accurate photon counting and offers directional information and the capability of background rejection at the DOM detection level. The use of many small-area photomultipliers increases the total detection area of the optical module compared to a design with a single 10-inch photomultiplier, also taking advantages from the weaker sensitivity to the Earth's magnetic field. The DOM design has been demonstrated in-situ by several prototypes. Since end 2015, 18 DOMs are operational in the first full size detection unit of $\mathrm{KM} 3 \mathrm{NeT}$ installed at a depth of $3500 \mathrm{~m}$. The next two detection units are already assembled and scheduled to be deployed in May 2016. The proposed contribution focuses on the design and on the main components of the Digital Optical Modules, discussing the enabling technologies and the integration procedures including the characterization and calibration performed before deployment.

\author{
N48-2: The SHiP Experiment at CERN \\ C. Betancourt \\ Physics Institute, University of Zurich, Zurich, Switzerland \\ On behalf of the SHiP Collaboration
}

SHIP is a new general purpose fixed target facility, whose Technical Proposal has been recently reviewed by the CERN SPS Committee, who recommended that the experiment proceeds further to a Comprehensive Design phase. This recommendation was recently endorsed by the CERN Research Board. In its initial phase, the $400 \mathrm{GeV}$ proton beam extracted from the SPS will be dumped on a heavy target with the aim of integrating $2 \times 10^{\wedge} 20$ pot in 5 years. A dedicated detector, based on a long vacuum tank followed by a spectrometer and particle identification detectors, will allow probing a variety of models with light long-lived exotic particles and masses below $\mathrm{O}(10) \mathrm{GeV} / \mathrm{c}^{\wedge} 2$. The main focus will be the physics of the so-called Hidden Portals, i.e. search for Dark Photons, Light scalars and pseudo-scalars, and Heavy Neutrinos. The sensitivity to Heavy Neutrinos will allow for the first time to probe, in the mass range between the kaon and the charm meson mass, a coupling range for which Baryogenesis and active neutrino masses could also be explained. Another dedicated detector will allow the study of neutrino cross-sections and angular distributions. tau neutrino deep inelastic scattering cross sections will be measured with a statistics 1000 times larger than currently available, with the extraction of the F4 and F5 structure functions, never measured so far and allow for new tests of lepton non-universality with sensitivity to BSM physics. This second detector will also allow direct dark matter detection produced in the decay of the dark photons

\title{
N48-3: The NA62 GigaTracker Detector
}

E. Gamberini

University of Ferrara, INFN Ferrara, Ferrara, Italy

On behalf of the GigaTracker working group

The GigaTracker (GTK) is a magnetic spectrometer made of 3 hybrid silicon pixel detectors built for the NA62 experiment. NA62 aims to measure the branching fraction of the ultra-rare kaon decay $\mathrm{K}^{+}$? $\mathrm{p}^{+}$??bar at the CERN SPS. The detector has to track particles in a $75 \mathrm{GeV} / \mathrm{c}$ beam with non-uniform rate of $\sim 750 \mathrm{MHz}$, reaching an expected peak flux of $1.3 \mathrm{MHz} / \mathrm{mm}^{2}$ around the centre, and provide single-hit timing with 200 ps RMS resolution for a total material budget of less than $1.5 \% \mathrm{X}_{0}$. The tracker comprises three $60.8 \mathrm{~mm} \times 27 \mathrm{~mm}$ stations installed in vacuum $\left(\sim 10^{-6}\right.$ mbar $)$ and cooled with liquid $\mathrm{C}_{6} \mathrm{~F}_{14}$ circulating through micro-channels etched inside few hundred of microns thick silicon plates. Each station is composed of a $200 \mu \mathrm{m}$ thick silicon sensor readout by $2 \times 5$ custom $100 \mu \mathrm{m}$ thick ASIC designed in $130 \mathrm{~nm}$ CMOS technology, called TDCPix. Each chip contains $40 \times 45$ asynchronous pixels, each $300 \mu \mathrm{m} \times 300 \mu \mathrm{m}$ and is instrumented with 720 time-to-digital converters with 100 ps bin. In order to cope with the high rate, the TDCPix is equipped with four $3.2 \mathrm{~Gb} / \mathrm{s}$ serialisers sending continuously data to custom-made FPGA off-detector read-out boards (GTK-RO) placed outside the experimental area. We will describe the detector and the results from the 2015 NA62 run.

\section{N48-4: Simulations and First Measurements of the Radiation Field in the New Gamma Irradiation Facility} (GIF++) at CERN

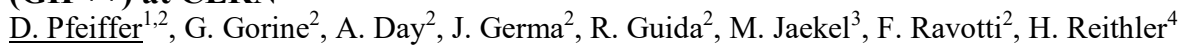

${ }^{I}$ Detector Group, European Spallation Source ERIC, Lund, Sweden

${ }^{2}$ CERN, Geneva, Switzerland

${ }^{3}$ University of Oslo, Oslo, Norway

${ }^{4}$ RWTH Aachen, Aachen, Germany

At CERN, the increase in luminosity at the LHC will produce a particle background in the gas-based muon detectors that is ten times higher than under present conditions. The detailed knowledge of the detector performance in the presence of such a high background is crucial for an optimised design and efficient operation after the HL-LHC upgrade. A precise understanding of possible ageing effects of detector materials and gases is of extreme importance. To cope with these challenging requirements, a 
new Gamma Irradiation Facility (GIF++) was designed and built at the CERN SPS North Area (EHN1, building 887) during the Long Shutdown 1 (LS1) period. Operational since spring 2015, the facility is used at present by all the major LHC experiments for detector studies. Starting already in the design phase of the facility, extensive simulations of the photon field have been carried out. These results are presented here together with first measurements in the facility.

N48-5: Measuring the Magnetic Flux Density with Flux Loops and Hall Probes in the CMS Magnet Flux Return Yoke

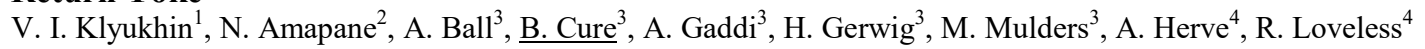

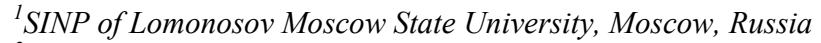

${ }^{2} I N F N$ Turin and the University of Turin, Turin, Italy

${ }^{3}$ CERN, Geneva, Switzerland

${ }^{4}$ University of Wisconsin, Madison, USA

The Compact Muon Solenoid (CMS) is a general purpose detector, designed to run at the highest luminosity at the CERN Large Hadron Collider (LHC). Its distinctive features include a $4 \mathrm{~T}$ superconducting solenoid with 6-m-diameter by 12.5-m-length free bore, enclosed inside a 10,000-ton return yoke made of construction steel. The flux return yoke consists of five dodecagonal three-layered barrel wheels and four end-cap disks at each end comprised of steel blocks up to $620 \mathrm{~mm}$ thick, which serve as the absorber plates of the muon detection system. To measure the field in and around the steel, a system of 22 flux loops and $823-\mathrm{D}$ Hall sensors is installed on the return yoke blocks. A TOSCA 3-D model of the CMS magnet is developed to describe the magnetic field everywhere outside the tracking volume measured with the field-mapping machine. The voltages induced in the flux loops by the magnetic flux changing during the CMS magnet standard ramps up and down are measured with six 16-bit DAQ modules USB-1608G of Measurement Computing. The off-line integration of the induced voltages reconstructs the magnetic flux density in the yoke steel blocks at the operational magnet current of $18.164 \mathrm{kA}$. The results of the flux loop measurements during the magnet ramps up and down are presented and discussed.

N48-6: Development of a Prototype Portable Muography Detector for Exploration of Underground Cavities K. Chaiwongkhot, T. Kin, H. Ohno, K. Kondo, H. Sato, Y. Watanabe

Department of Advance Energy Engineering Science, Faculty of Engineering Science,, Kyushu University, Fukuoka, Japan

A prototype portable muography detector was developed for exploration of underground cavities and some performance tests were conducted. The detector consists of two muon position sensitive detectors (mu-PSDs) having an area size of $140 \mathrm{~mm} \times 140$ $\mathrm{mm}$ which are placed in a compact light shielding box. Each mu-PSD was fabricated using plastic scintillating fibers (PSFs) connected to multi-pixel photon counters (MPPCs). The pixel size of $8.75 \mathrm{~mm}$ x $8.75 \mathrm{~mm}$ and the distance of $100 \mathrm{~mm}$ between the two mu-PSDs were determined so as to satisfy the solid angle resolution of $30 \mathrm{msr}$ required from subsidence risk cavity exploration. The detector system worked stably by using a Peltier cooling and heating module for temperature control function. Zenith and azimuth angular differential fluxes of cosmic-ray muons on the ground were measured using the muography detector, and the measured zenith angular differential flux was found to be in good agreement with the well-known empirical formula of cosine square. An imaging test of interior structure of a seven-story concrete building was successfully performed using the muography detector placed on the basement.

\author{
N48-7: The S-CVD Radiation Monitoring and Beam Abort System of the Belle-II Vertex Detector \\ C. La Licata \\ INFN and Univ. Trieste, Trieste, Italy \\ On behalf of the Belle II SVD collaboration
}

The Belle-II VerteX Detector (VXD) is a 6 layers silicon tracker device that will cope with an unprecedented luminosity of $8 \times$ $10^{\wedge} 35 \mathrm{~cm}-2 \mathrm{~s}-1$ achievable by the new SuperKEKB e+e- collider, now under commissioning at the KEK laboratory (Tsukuba, Japan).

A radiation monitoring and beam abort system has been developed based on single-crystal s-CVD diamond sensors. The sensors will be placed in 20 key positions in the vicinity of the interaction region. The severe space limitations require a remote readout of the sensors.

In this contribution we present the system design, along with the sensor characterization procedure and the prototype of the readout electronics. We present also the preliminary results with the prototype system during the first SuperKEKB commissioning phase in February-June 2016.

\author{
N48-8: System Architecture and Data Processing Capabilities of the Beam Profile Monitor for the CERN \\ IRRAD Facility

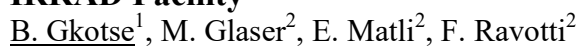


${ }^{I}$ CERN and Télécom Bretagne, Geneva, Switzerland

${ }^{2}$ CERN, Geneva, Switzerland

Materials and devices employed at the large CERN High Energy Physics (HEP) experiments and accelerators need to be first evaluated for their tolerance to radiation damage. IRRAD is one of the CERN facilities used for this purpose. In IRRAD, samples are exposed to a Gaussian $12 \times 12 \mathrm{~mm}^{2}$ wide high-energy proton beam $(24 \mathrm{GeV} / \mathrm{c})$ to given fluence levels. Custom-made Beam Profile Monitor (BPM) devices are used for the proton beam monitoring and steering. The proton beam delivered by the CERN Proton Synchrotron (PS), in spills of $\sim 400 \mathrm{~ms}$ and crosses four BPM detector elements placed in different locations along the irradiation area. The BPM acquisition system includes an Arduino Yún that performs the data acquisition from the BPM hardware and sends the data on a server for archiving, processing and on-line beam display. In this paper we describe the architecture of the BPM system, its advanced data processing capabilities together with its performance and perspective for utilization in other irradiation facilities infrastructures.

This project has received funding from the European Commission project AIDA, as well as from the European Union's Horizon 2020 Research and Innovation program under Grant Agreement no. 654168.

N49: Synchroton radiation and FEL instrumentation II

Thursday, Nov. 3 08:00-10:00 Londres

N49-1: The Detection Module of ARDESIA: a New Versatile Array of SDDs for X-Ray Spectroscopy Synchrotron Applications.

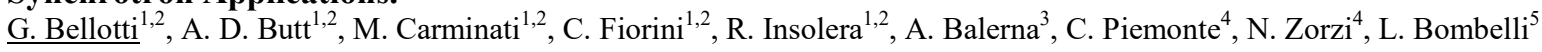

${ }^{I}$ Elettronica Informazione e Bioingegneria, Politecnico di Milano, Milan, Italy

${ }^{2}$ Sezione di Milano, INFN, Milan, Italy

${ }^{3}$ Laboratori Nazionali di Frascati, INFN, Frascati, Italy

${ }^{4}$ Fondazione Bruno Kessler, Trento, Italy

${ }^{5} X G L A B$ srl, Milan, Italy

This work presents the first results of ARDESIA, a novel, SDD-based, multichannel X-ray detector. ARDESIA has been optimized for synchrotron applications which require a high-rate, high-resolution soft $\mathrm{X}$-ray detector. The main applications here considered are X-ray fluorescence (XRF) and x-ray absorption fine structure (XAFS) measurements. The structure of the ARDESIA detector is modular i.e. it is composed by single detection elements that can be juxtaposed together with small dead area. Thus, several detection configurations can be obtained with this versatile unit that consists of a 4-channel, $25 \mathrm{~mm} 2$ singleunit, monolithic SDD matrix, produced by FBK (Trento, Italy). The SDD matrix is connected to a 4-channel, monolithic, CUBE preamplifier chip. SDD and CUBE chips are assembled on a compact ceramic carrier. To achieve good XRF and XAFS performances the detector need to fulfil three main requirements: solid angle, energy resolution and count rate capability. ARDESIA covers a solid angle large enough to collect a high fluorescence signal while minimizing the collected background signal (in $90^{\circ}$ geometry). A good energy resolution is mandatory to reduce background intensity and to increase signal to noise ratio. In synchrotron measurements, high count rate capability is needed to reduce measurement time. CUBE preamplifier allows reaching high count rates ( $>1 \mathrm{Mcps}$ ), while keeping good spectroscopic performances as its extremely low white series noise guarantees good energetic resolution, even at short pulse processing times. SDD matrix is produced with a low-leakage technology available at FBK, which allows achieving high energy resolutions, even with moderate cooling. The ARDESIA preamplifier output signal can be processed with both an analog ASIC and a digital pulse processor. Experimental measurements of the first produced prototype are presented in this work.

\author{
N49-2: Silicon Avalanche-Photodiode Linear-Array X-Ray Detector of 64 or 128 Pixels with 0.5-Ns Time-Bin \\ Multichannel Scaler \\ $\underline{\text { S. Kishimoto }}^{1}$, R. Haruki ${ }^{1}$, T. Mitsui ${ }^{2}$ \\ ${ }^{I}$ Photon Factory, Institute of Materials Structure Science, High Energy Accelerator Research Organization, Tsukuba, Ibaraki \\ 305-0801, Japan \\ ${ }^{2}$ Kansai Photon Science Institute, National Institutes for Quantum and Radiological Science and Technology, Sayo, Hyogo 679- \\ 5148, Japan
}

We developed a silicon avalanche photodiode (Si-APD) linear-array X-ray detector to be used for time-resolved X-ray scattering experiments using synchrotron radiation, for example, nuclear resonant scattering. The Si-APD linear array consists of 64 or 128 pixels (pixel size: $100 \times 200 \mu \mathrm{m}^{\wedge} 2$ and $100 \times 400 \mu \mathrm{m}^{\wedge} 2$ for 128 pixel array) with a pixel pitch of $150 \mu \mathrm{m}$ and a depletion depth of $10 \mu \mathrm{m}$. The multichannel scaler (MCS) of the 64-pixel detector system was designed by using one chip of field programmable gate arrays. The MCS counted X-ray pulses over continuous 2046 time bins for every $0.5 \mathrm{~ns}$ and recorded a time spectrum of $14.41 \mathrm{keV}$ photons for each pixel. Using the detector system of 64 pixels, we were able to observe linear images of the nuclear resonant scattering emitted from a sample including Fe-57. Time structure of decaying ? rays were recorded in each pixel with 
sub-nanosecond time resolution. We confirmed that 2-ns interval 14.41-keV X-ray peaks clearly separated along the time with a time resolution of $0.5 \mathrm{~ns}$ (FWHM) in the hybrid operation mode of the Photon Factory synchrotron ring. Test measurements for the detector system of 128 pixels (sensitive length: $19.2 \mathrm{~mm}$ ) is now in progress.

N49-3: MIMOSA-22SX - A Monolithic Active Pixel Sensor for Low Energy X-Ray Counting Applications M. Kachel $^{1,2}$, J. Baudot ${ }^{1,2}$, G. Bertolone ${ }^{1,2}$, A. Dawiec ${ }^{3}$, F. Guezzi-Messaoud ${ }^{1,2,3}$, J. Heymes ${ }^{1,2}$, A. Himmi ${ }^{1,2}$, C. Hu-Guo $^{1,2}$, L. A. Perez-Perez ${ }^{1,2}$, M. Winter ${ }^{1,2}$

${ }^{I}$ Université de Strasbourg, IPHC, 23 rue du Loess 67037 Strasbourg,, France

${ }^{2}$ CNRS, UMR7178, 67037 Strasbourg, France

${ }^{3}$ SOLEIL Synchrotron, L'Orme des Merisiers, 91190 Saint-Aubin, France

MIMOSA-22SX is a prototype Monolithic Active Pixel Sensor dedicated to low energy X-Ray counting applications at the Synchrotron SOLEIL. The circuit is fabricated in a $180 \mathrm{~nm}$ CMOS technology and features a matrix of 128 x 256 pixels, which are $22 \times 22 \mu \mathrm{m}^{2}$ large. The chip was produced over two types of substrates differing in sensitive layer thickness. The first wafer includes a high resistive $(>1 \mathrm{k} \mathrm{O} \cdot \mathrm{cm})$ epitaxial layer of $18 \mu \mathrm{m}$, while the second is produced by Czochralski method to reach high resistivity offers a potentially $200 \mu \mathrm{m}$ thick sensitive layer.

The efficient X-Ray detection requires the ability to fully deplete the sensitive volume. In each pixel of MIMOSA-22SX, the collecting diode is AC coupled to a low noise amplifier in order to apply moderate voltages $(=20 \mathrm{~V})$. The excellent charge collection properties and equivalent noise charge below $20 \mathrm{e}$-, yield an energy resolution between 100 and $200 \mathrm{eV}$ for X-Rays energies of a few keV. MIMOSA-22SX exploits this energy resolution with two discriminators providing the possibility to select $\mathrm{X}$-Rays within an energy window. These performances make the sensor suitable for the detection over the energy range from several $100 \mathrm{eV}$ up to $5 \mathrm{keV}$.

In order to reach photon counting rates interesting for synchrotron applications, the fast column-parallel rolling shutter readout was implemented. With $100 \mathrm{~ns}$ row access time, MIMSOSA-22SX can count up to $40 \times 10^{4} \mathrm{photons} / \mathrm{pixel} / \mathrm{s}$. The circuit is controlled through JTAG protocol, which allows setting all the necessary polarization signals and circuit parameters. In this paper we review the design and main features of MIMOSA-22SX. Functional tests of the charge collection and readout chain are presented to demonstrate the maximum reachable photon counting rate per pixel. We also report the results of the laboratory measurements performed with X-Ray sources on the two types of substrate and discuss the sensor detection performances. We conclude with the prospect of a larger surface sensor, $1 \mathrm{~cm}^{2}$, based on the same architecture.

N49-4: The Percival 2 Megapixel Soft X-Ray Imager

C. B. Wunderer ${ }^{1,2}$, P. Göttlicher ${ }^{1}$, I. Shevyakov ${ }^{1}$, J. Supra ${ }^{1}$, Q. Xia ${ }^{1}$, M. Zimmer ${ }^{1}$, J. Viefhaus ${ }^{1}$, F. Scholz ${ }^{1}$, J. Seltmann ${ }^{1}$,

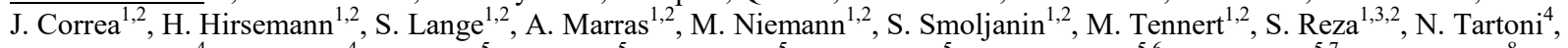
U. K. Pedersen ${ }^{4}$, H. Yousef ${ }^{4}$, R. Menk ${ }^{5}$, L. Stebel ${ }^{5}$, G. Cautero ${ }^{5}$, D. Giuressi ${ }^{5}$, A. Khromova ${ }^{5,6}$, G. Pinaroli ${ }^{5,7}$, A. D. Jewell ${ }^{8}$, T. J. Jones 8 , M. E. Hoenk ${ }^{8}$, S. Nikzad ${ }^{8}$, S. Rah ${ }^{9}$, H. Hyun ${ }^{9}$, K. Kim ${ }^{9}$, R. Turchetta ${ }^{10}$, I. Sedgwick ${ }^{10}$, D. Das ${ }^{10}$, N. Guerrini ${ }^{10}$, B. Marsh ${ }^{10}$, T. Nicholls ${ }^{10}$, S. Klumpp ${ }^{11}$, C. Laubis ${ }^{12}$, H. Graafsma ${ }^{1,3,2}$

${ }^{I}$ DESY, Hamburg, Germany

${ }^{2}$ CFEL, Hamburg, Germany

${ }^{3}$ Mid Sweden University, Sundsvall, Sweden

${ }^{4}$ Diamond Light Source, Didcot, UK

${ }^{5}$ Elettra, Trieste, Italy

${ }^{6}$ Universita degli Studi di Trieste, Trieste, Italy

${ }^{7}$ Universita degli Studi di Udine, Udine, Italy

${ }^{8}$ NASA Jet Propulsion Laboratory, Pasadena, CA, USA

${ }^{9}$ Pohang Accelerator Laboratory, Pohang, Republic of Korea

${ }^{10}$ STFC / RAL, Didcot, UK

${ }^{11}$ University of Hamburg, Hamburg, Germany

${ }^{12}$ PTB, Berlin, Germany

With the advent of advanced storage rings and Free Electron Lasers comes the need for a new generation of X-ray detectors capable of combining a large 2D imaging area with many pixels with a large dynamic range, starting from single photon discrimination and extending to $10^{\wedge} 5$ photons per pixel per frame or more, as well as with high frame rates. Fulfilling this need is particularly challenging in the soft X-ray regime where the desire for single-photon discrimination corresponds to low noise requirements at high read speed. Percival ("Pixellated Energy Resolving CMOS Imager, Versatile and Large") is a development by a collaboration of DESY, RAL/STFC, Elettra, DLS, PAL, and JPL aiming to meet these soft X-ray imaging needs. Following a series of prototypes, in spring 2016 we have submitted the Percival 2Megapixel ("P2M"), a stitched sensor with $4 \times 4 \mathrm{~cm} 2$ active imaging area, for fabrication. We will summarize the soft X-ray performance achieved by our prototypes, describe the full P2M system, and discuss expected performance.

N49-5: Eiger 9M: High Frame Rate, Large Area Photon Counting Detector for Synchrotron Applications 
$\underline{\text { E. Fröjdh }}^{1}$, A. Bergamaschi ${ }^{1}$, M. Bruckner ${ }^{1}$, S. Cartier ${ }^{1,2}$, D. Greiffenberg ${ }^{1}$, D. Mayilyan ${ }^{1}$, D. Mezza ${ }^{1}$, A. Mozzanica $^{1}$, M. Ramilli ${ }^{1}$, S. Redford ${ }^{1}$, C. Ruder ${ }^{1}$, L. Schadler ${ }^{1}$, B. Schmitt ${ }^{1}$, X. Shi ${ }^{1}$, D. Thattil ${ }^{1}$, G. Tinti ${ }^{1}$, J. Zhang $^{1}$

${ }^{I}$ Paul Scherrer Institut, Villigen, Switzerland

${ }^{2}$ Institute for Biomedical Engineering, University and ETH Zurich, Zurich, Switzerland

EIGER is a single photon counting hybrid pixel detector developed at Paul Scherrer Institute (PSI), Switzerland, for applications at synchrotron light sources. The pixel size is $75 \times 75 \mu \mathrm{m}^{2}$ and the pixel matrix of the ASIC consists of $256 \times 256$ pixels. Each pixel has a counter which can be configured in 4,8 or 12 bit mode. The counter features also double buffering for continuous readout. One global threshold is applied to the ASIC, and an on-pixel 6-bit DAC is provided for threshold equalization. One module consists of $2 \times 4$ ASICs covered by a single silicon sensor, and several modules can be tiled to form big area systems.

The Eiger 9M detector ( $3 \times 6$ modules) is at present under development at PSI and will replace the Pilatus $2 \mathrm{M}$ detector at the cSAXS beamline of the Swiss Light Source. The improvements in detector performance include smaller pixels, higher incoming photon rate per unit area and a significantly higher frame rate. In fact, in 4 bit mode the maximum frame rate is $23 \mathrm{kHz}$ with a $3 \mu \mathrm{s}$ dead time between frames. Due to its parallel design the performance of the PSI Eiger detector does not depend on the number of modules in a system.

In this paper we present an overview of the Eiger $9 \mathrm{M}$ detector including the data backend. We also present calibration, threshold trimming and characterization of the detector module as well as count rate performance and online rate correction.

\title{
N49-6: Ultra Fast X-Ray Detector for Synchrotron Applications
}

P. Maj ${ }^{1}$, A. Dawiec ${ }^{2}$, G. Deptuch ${ }^{3}$, E. M. Dufresne ${ }^{4}$, P. Grybos ${ }^{1}$, P. Kmon ${ }^{1}$, S. Narayanan ${ }^{4}$, A. R. Sandy ${ }^{4}$, R. Szczygiel ${ }^{1}$, Q. Zhang ${ }^{4}$

${ }^{I}$ Measurement and Electronics, AGH University of Science and Technology, Krakow, Poland

${ }^{2}$ Division Expériences / Groupe Détecteurs, Soleil, Saint Aubin, France

${ }^{3}$ Particle Physics Division, Fermi National Accelerator Laboratory, Batvia, USA

${ }^{4}$ Time Resolved Research, Argonne National Laboratory, Lemont, USA

We report on the setup realization and experimental results of the single photon counting, ultra-fast X-ray detector for synchrotron applications. The hybrid pixel detector consisting of a UFXC32k chip and 320um thick silicon sensor together with dedicated FPGA readout is used for time-resolved experiments at the Advanced Photon Source (APS) in Argonne National Laboratory as well as at Soleil in Paris showing a very good performance. The core parameters of the hybrid pixel detector allowing its operation with synchrotron radiation are: area of $9.64 \mathrm{~mm} \times 20.15 \mathrm{~mm}$, small pixel size $(75 \mu \mathrm{m} 2)$, selectable and tunable gain of the analog front-end and its low noise of only $123 \mathrm{e}$ - rms (for the peaking time of $40 \mathrm{ns)} \mathrm{allowing} \mathrm{uniform}$ response of the whole detector. The high-count rate expressed as a dead-time in ultra-high speed mode can be set as low as $85 \mathrm{~ns}$. Different modes of operation, namely standard mode, high dynamic range and a zero-dead time mode with different counter depth (2, 4, 8 and 14-bit) allow for additional non-standard configurations described in more details (e.g. 100 ns gate time mode). Current experiments at the APS and Soleil were confirmed for $100 \mathrm{MHz}$ serial clock allowing $11.7 \mathrm{kfps}$ acquisition. Our laboratory tests confirm proper operation of the detector with $500 \mathrm{MHz}$ serial clock and therefore the detector can be read out with the speed of 50000 frames per second. Final tests confirming this speed in real experiments are already scheduled for June and July 2016.

AGH University of Science and Technology was supported by the National Center for Research and Development, Poland PBS1/A3/12/2012 in the years 2012-2015.

\author{
N49-7: Edgeless Digital Tier of the 3D Development for the Vertically Integrated Photon Imaging Chip - \\ Large (VIPIC-L) \\ $\underline{\text { F. Fahim }}^{1}$, G. Deptuch ${ }^{1}$, A. Shenai ${ }^{1}$, P. Grybos ${ }^{2}$, P. Kmon ${ }^{2}$, P. Maj ${ }^{2}$, R. Szczygiel ${ }^{2}$, D. P. Siddons ${ }^{3}$, J. Mead ${ }^{3}$, A. Kuczewski $^{3}$, \\ A. Rumaiz ${ }^{3}$, J. Weizeorick ${ }^{4}$, R. Bradford ${ }^{4}$ \\ ${ }^{I}$ PPD/EED, Fermi National Accelerator Laboratory, Batavia, IL, USA \\ ${ }^{2}$ DMI, AGH-UST, Cracow, Poland \\ ${ }^{3}$ Detector Development Group, Brookhaven National Laboratory, Upton, NY, USA \\ ${ }^{4}$ APS, Argonne National Laboratory, Lemont, IL, USA
}

The VIPIC-L large area, edgeless, vertically integrated multi-tier detector comprising of a sensor, an analog and a digital ASIC, is built for X-ray photon counting. A full custom analog pixel is $65 \mu \mathrm{mx} 65 \mu \mathrm{m}$. It is connected to a sensor pixel of the same size on one side, and on the other side it has approximately 40 connections to the digital pixel. VIPIC-L has no peripheral functional blocks, and hence the active area extends to the edge of the detector. The Digital ASIC consists of approximately 100million transistors and 1.5million inter-pixel connections to the Analog ASIC. The $1.25 \mathrm{~cm} x 1.25 \mathrm{~cm}$ ASIC is subdivided into a $6 \times 6$ array of an indivisible functional unit (sub-chip). A sub-chip contains 1024 pixels, with adequate area to contain the pixel hit processing logic along with a priority encoder, an output serializer, LVDS drivers and receivers and $21510 \mathrm{~b}$ serial configuration 
register for trimming and setup of the analog pixels. Each ASIC has 720 bump-bond I/O connections, on the back of the digital tier to a PCB. The detector can be configured in a sparsified or imaging readout mode, the data packet can be programmed to optimize the output data rate vs. packet length. A novel technique for ensuring that high priority data is not corrupted during frame changes has been implemented. It is required due to the different propagation delays of asynchronous signals across the ASIC, the one that selects and enables a pixel for readout and the second that changes the readout frame. It allows for minimal unavoidable readout dead time. The subchip implementation requires the use of EDA tools in a specialized approach, due to various placement and functional constraints. An iterative strategy allows for the placement, routing and timing of full-custom, semi-custom and high-speed circuitry such that the user maintains control over routing, parasitics and placement of the fullcustom blocks and the tool manages the clock distribution and timing of the high speed functionality.

\section{N49-8: The Use of Single-Crystal CVD Diamond X-Ray Beam Diagnostics for Synchrotron Beamline Commissioning and Operation at Diamond Light Source Ltd.}

\section{Bloomer}

Diagnostics Group / Technical, Diamond Light Source Ltd., Didcot, Oxfordshire, OX11 0DE, UK

We report on the use of single-crystal CVD diamond X-ray beam diagnostics at Diamond Light Source Ltd. for beamline commissioning and day-to-day beamline operation. Such detectors offer beamlines a measurement of X-ray beam position with resolutions of a few $10 \mathrm{~nm}$ at $\mathrm{kHz}$ bandwidths. It is straightforward to extract extremely valuable spectral information of both beam motion and intensity, aiding beamlines in determining the source of vibrations or other beam instabilities. In this paper we discuss the motivation behind the use of single-crystal diamond detectors for monitoring monochromatic X-ray beams, and advantages over other diagnostic techniques are described. In order to absolutely quantify the performance of the diamond detectors in use at Diamond Light Source Ltd. an experiment has been conducted where three diamond X-ray beam position monitors were installed on a beamline, observing an incident X-ray beam of less than 25 microns in size at $40 \mathrm{kHz}$ bandwidth. Measurements from each of the three diamond detectors were synchronously acquired. Other, independent, diagnostics techniques were employed to verify the performance of the three X-ray beam position monitors. The results from this experiment are presented. The uncorrelated beam position noise recorded by the synchronous measurements is shown to be less than $1 \%$ of beamsize at this bandwidth. Longer integration times resulted in correspondingly less noise. Experimental evaluations of a range of X-ray beam diagnostic techniques for beamlines at Diamond Light Source Ltd. have shown single-crystal CVD diamond detectors to be superior in almost all respects.

\section{N50: Advanced computing and software for experiments I}

Thursday, Nov. 3 08:00-10:00 Curie 1

N50-1: Datasets for Radiation Network Algorithm Development and Testing

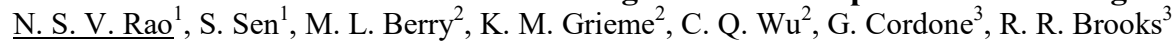

${ }^{I}$ Computer Science and Mathematics Division, Oak Ridge National Laboratory, Oak Ridge, TN, USA

${ }^{2}$ Department of Computer Science, New Jersey Institute of Technology, Newark, NJ, USA

${ }^{3}$ Department of Electrical and Computer Engineering, Clemson University, Clemson, SC, USA

Networks of radiation detectors are increasingly being deployed to detect and localize radiation sources in urban areas, special events, and border crossings. Their performances critically depend on the algorithms used to aggregate the measurements from individual detectors. Several network algorithms have been developed based on a variety of approaches, including Bayesian methods, particle filters, spectral template matching, statistical tests, stochastic approximation, and others. Currently, however, there is a lack of appropriate experimental datasets to support objective, comprehensive and comparative testing of these algorithms in varied scenarios. Domestic Nuclear Detection Office's Intelligence Radiation Sensors Systems (IRSS) program supported the development of networks of commercial-off-the-shelf detectors, which were used in multiple characterization tests conducted with different source strengths and types, background profiles, and various source/detector movements. These experiments generated a rich collection of datasets that can be used for testing the current and next-generation radiation network algorithms, including the ones (to be) developed by broader R\&D communities such as distributed detection, information fusion, and sensor networks. We describe twelve canonical datasets that are distilled from IRSS database and packaged with supplemental contextual information for public release. They represent increasingly challenging baseline scenarios for robustly testing radiation network algorithms. We also present border and portal monitoring scenarios that are re-constructed from these datasets to assess the performance of network detection algorithms.

This work has been supported by the US Department of Homeland Security, Domestic Nuclear Detection Office, under competitively awarded IAA HSHQDC-13-X-B0002. This support does not constitute an expressed or implied endorsement on the part of the Government.

\section{N50-2: Simulation of Beam Backgrounds from the LHC Ring to the ATLAS Experiment} $\underline{\text { A. Manousos }}$ 
European Organization for Nuclear Research (CERN), Geneva, Switzerland

On behalf of the ATLAS Collaboration

We present results of dedicated Fluka simulations of the Large Hadron Collider (LHC) beam backgrounds in the ATLAS experiment. In 2012 these backgrounds arose from losses of the $4 \mathrm{TeV}$ protons on beam cleaning collimators of the LHC and from beam-gas interactions. The latter are normalised with corresponding pressure distributions in the ring in order to arrive at rate predictions on absolute scale. The simulation results are compared with beam background measurements by ATLAS, primarily using data taken in 2012 - the last year of LHC Run-1. Through these comparisons the origins of the backgrounds leading to different observables in the ATLAS detectors are analysed and normalisation factors between the rate of proton loss from the beam and the backgrounds observed in ATLAS are derived. This work is the first time that LHC beam backgrounds in experiments are compared with simulations at a quantitative level.

N50-3: Online Monte-Carlo Generator Validation in a HEP Environment T. Harenberg ${ }^{1}$, T. Kuhl ${ }^{2}$, N. Lang ${ }^{1}$, P. Mï $i^{1 / 2 t^{2} g^{1}, \text { M. Sandhoff }}{ }^{1}$, C. Schwanenberger ${ }^{2}$, F. Volkmer ${ }^{1}$

${ }^{I}$ Bergische Universitï ${ }_{i}^{1} / 2 t$, Wuppertal, Germany

${ }^{2}$ DESY, Hamburg, Germany

Petabytes of data are being processed and stored requiring millions of CPU-years within the LHC Grid to simulate high energy physics events. These significant resources require a high quality and efficient production and the early detection of potential errors. A novel monitoring technique is presented to collect quality measures already during job execution, which allows early detection of possible configuration errors or inappropriate settings of simulation parameters and therefore is able to save time and resources. This monitoring has been successfully applied within ATLAS using some 350 million events to validate simulations at the generator level. During these first runs, safe validation was proven and it was shown that the online feature of JEM is useful for further validation campanigns. The structure of the validation program and operational experience will be presented.

N50-4: Development and Deployment of a Fully Parameterized Fast Monte Carlo Simulation in LHCb B. G. Siddi

Sezione Ferrara, INFN, Ferrara, Italy

On behalf of the LHCb Collaboration

Faster alternatives to a full, GEANT4-based simulation are being pursued within LHCb experiment. In this context the integration of the Delphes toolkit in the LHCb simulation framework is intended to provide a fully parameterized option. Delphes is a modular software designed to perform fast simulations by propagating stable particles using a parametric approach and providing reconstructed physical objects as output. It includes propagation inside a magnetic field and parameterized response for tracking and muon systems as well as calorimetric response. Particle energies are computed by smearing the initial visible particles momenta according to detector resolution in a highly-customizable way. Delphes was initially designed for generalpurpose experiments such as ATLAS and CMS where is already used. The present contribution illustrates the different aspect of the integration of the Delphes toolkit in Gauss, the LHCb simulation framework. Technically the particle transport performed by GEANT4 and subsequent mimicking of detector response and reconstruction has been replaced with a parametric response of the various detector elements. The implementation required extension to Delphes itself to constrain the particle transport inside the spectrometer acceptance, in order to match the LHCb dipole magnetic field. The configuration of various parameterizations of resolution and efficiency (charged particle tracking, mis-identification rate of particle identification algorithms, calorimeter, trigger response) is also a major aspect of the work to provide a fully functional simulation system. The output of the resulting fast simulation is formatted in such a way that can be used in the LHCb physics analysis framework. The status of the integration and an outline of the use of Delphes to evaluate future detector upgrade options is given.

N50-5: UFO - a Scalable Platform for High-Speed Synchrotron X-Ray Imaging

A. Kopmann, S. Chilingaryan, M. Vogelgesang, T. Dritschler, A. Shkarin, R. Shkarin, T. Farago, T. dos Santos Rolo, T. van de Kamp, M. Balzer, M. Caselle, M. Weber, T. Baumbach

Karlsruhe Institute of Technology, Karlsruhe, Germany

New imaging stations aim for high spatial and temporal resolution and are characterized by ever increasing sampling rates and demanding data processing workflows. Key to successful imaging experiments is to open up high-performance computing resources. This includes carefully selected components for computing hardware and development of advanced imaging algorithms optimized for efficient use of parallel processor architectures. We present the novel UFO computing platform for online data processing for imaging experiments and image-based feedback. The platform handles the full data life cycle from the $\mathrm{X}$-ray detector to long-term data archives. Core components of this system are an FPGA platform for ultra-fast data acquisition, the GPU-based UFO image processing framework, and the fast control system "Concert". Reconstruction algorithms 
implemented in the UFO framework are optimized for the latest GPU architectures and provide a reconstruction throughput in the GB/s-range. The control system "Concert" integrates high-speed computing nodes and fast beamline devices and thus enables image-based control loops and advanced workflow automation for efficient beam time usage. Low latencies are ensured by direct communication between FPGA and GPUs using AMDs DirectGMA technology. Time resolved tomography is supported by cutting edge regularization methods for high quality reconstructions with a reduced number of projections. The new infrastructure at ANKA has dramatically accelerated tomography from hours to second and resulted in new application fields, like high-throughput tomography, pump-probe radiography and stroboscopic tomography. Ultra-fast X-ray cine-tomography for the first time allows one to observe internal dynamics of moving millimeter-sized objects in real-time.

The UFO project has been gratefully funded by the German BMBF.

N50-6: Event Building Process from Time Stream Data

$\underline{V}_{\text {. Singhal }}{ }^{1}$, S. Chattopadhyay ${ }^{1}$, V. Friese $^{2}$

${ }^{I}$ Experimental High Energy Application Group, Variable Energy Cyclotron Centre, Kolkata, WB, India

${ }^{2}$ CBM, GSI, Darmstadt, Germany

Up-to now all the High Energy Experiments are based on the hardware triggered approach, which means a specific set of hardware, signals the entire electronics to collect the data, this is known as triggered electronics. This signal define the event boundary, therefore all the available simulation and reconstruction software are working on the event by event basis. On the contrary due to unprecedented interaction rate at the CBM experiment at FAIR conventional triggered electronics will not work, therefore building of trigger-less electronics has been under development. In this scenario, the event boundary will not be defined by any hardware. This implies a novel read-out and data acquisition concept with self-triggered front-end electronics which will generate free-streaming data. To do the analysis from this time stamped free steam data, event association must be performed in software. To simulate the free stream data, work has been under development to change the CBMROOT framework in the direction of putting timestamp on each and every digi (digi is the smallest piece of raw data known as one hit which detected by trigger less electronics). To use all the available software for processing of free stream time stamped data, generated by CBM experiment, an event association package has to be introduced at the appropriate place in the simulation, reconstruction and analysis chain which will separate one event to another and also this should cater to actual experiment data processing. This work describes that how we are going to address this issue. Time stamped raw data stream have been generating via simulation and each detector will generate separate raw data stream like STS data stream, MUCH data stream etc and all will be stored in a ROOT Tree with time stamp. As a first step towards the reconstruction of such free-streaming data, we have introduced an eventbuilding process which tags physical events based on the time information of the raw data. This work shows that event can be reconstructed with the reasonable efficiency.

I would like to express my sincere gratitude to the CBM Collaboration to work in CBM experiment.

N50-7: Application of Econometric Data Analysis Methods to Physics Software

M. G. Pia ${ }^{1}$, E. Ronchieri ${ }^{2}$

${ }^{1}$ INFN Genova, Genova, Italy

${ }^{2} I N F N C N A F$, Bologna, Italy

We report an investigation of data analysis methods derived from disciplines other than physics, which we applied to physics software systems. They concern the analysis of inequality, trend analysis and the analysis of diversity. The analysis of inequality exploits statistical methods originating from econometrics; trend analysis is typical of economics and environmental sciences; the analysis of diversity is based on concepts derived from ecology and treats software as an ecosystem. To the best of our knowledge, this is an innovative exploration, as we could not find track of previous use of these methods in the experimental physics domains within the scope of the IEEE Nuclear Science Symposium. We applied these methods in the context of Geant4 physics validation and Geant 4 maintainability assessment. We illustrate the conceptual characteristics of these statistical methods, document their application and discuss the lessons learned.

N50-8: Data Knowledge Base as Metadata Catalog for Scientific Experiments

V. V. Osipova ${ }^{1}$, A. A. Alekseev ${ }^{1}$, M. A. Ivanov ${ }^{1}$, M. A. Grigorieva ${ }^{2}$, M. Y. Gubin ${ }^{1}$, A. A. Klimentov ${ }^{3}$

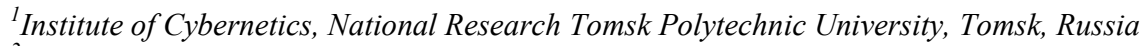

${ }^{2}$ Laboratory of Information Integration, National Research Centre "Kurchatov Institute", Moscow, Russia

${ }^{3}$ Brookhaven National Laboratory, Upton, NY, USA

Large volumes of data produced as a result of scientific experiments are needed to be processed and analyzed in the proper way. These data should come with auxillary metadata whichare available from experiments and are recorded at each phase of their lifecycle. In science experiments likein High Energy and Nuclear Physics there are a lot of experiment results representedas 
production steps, tasks in order to transform them into datasetsneeded for further analysis and constantly recorded as metadata. Describing the lifecycle of the data processing and publications as a result of analyzing scientific data, metadataoperates separately. Such integrated metadata will be performed for analysis and mining to develop the Data Knowledge Baseas a central part of the information system, acceptinguser requests to combine them into queries. Thus it presents as essential source of aggregating scientific big data, producing new knowledgefor the scientific field.

N51: Circuits for triggering and calorimetry readout

Thursday, Nov. 3 08:00-10:00 Curie 2

N51-1: The Electronics for Data Acquisition and Data Transport for the KM3NeT Towers

C. A. Nicolau

Roma, INFN, Rome, Italy

On behalf of the KM3NeT-Italy Collaboration

The KM3NeT European project has entered the production stage of a large volume neutrino telescope that is going to be deployed at about $100 \mathrm{~km}$ off the Sicily coast. The forthcoming installation includes 24 strings, equipped with multi-PMT optical modules, and 8 towers, equipped with single-PMT optical modules. The KM3NeT tower design is based on the NEMO Phase-2 prototype tower, deployed in 2013. In order to optimize production costs, power consumption, usability and to simplify calibration procedures, the whole electronics chain has been re-engineered and partially redesigned by taking advantage of the previously gained experience and technological progress. The aim of this contribution is to give a description of the electronics, including the PMT front-end, and data transport and clock distribution systems of the KM3NeT towers.

N51-2: VIPRAM_L1CMS: a 2-Tier 3D Architecture for Pattern Recognition for Track Finding

J. R. Hoff, G. Deptuch, S. Joshi, T. Liu, J. Olsen, A. Shenai

PPD/EED/ASIC, Fermilab, Batavia, IL, USA

In HEP tracking trigger applications, flagging an individual detector hit is not important. Rather, the path of a charged particle through many detector layers is what must be found. Moreover, given the increased luminosity projected for future LHC experiments, this type of track finding will be required within the Level 1 Trigger system. This means that future LHC experiments require not just a chip capable of high-speed track finding but also one with a high-speed readout architecture. VIPRAM_L1CMS is 2-Tier Vertically Integrated chip designed to fulfill these requirements. It is a complete pipelined Pattern Recognition Associative Memory (PRAM) architecture including pattern recognition, result sparsification, and readout for Level 1 trigger applications in CMS with 15-bit wide detector addresses and eight detector layers included in the track finding. Pattern recognition is based on classic Content Addressable Memories with a Current Race Scheme to reduce timing complexity and a 4bit Selective Precharge to minimize power consumption. VIPRAM_L1CMS uses a pipelined set of priority-encoded binary readout structures to sparsify and readout active road flags at frequencies of at least 100MHz. VIPRAM_L1CMS is designed to work directly with the Pulsar2b Architecture.

N51-3: The Prototype of Global Calorimetric Hardware Trigger for ATLAS at High Luminosity LHC H. Chen

Brookhaven National Laboratory, Upton, NY, USA

The ATLAS will follow the upgrade steps of Large Hadron Collider, which will undergo a series of upgrades to increase the luminosity in next ten years. During the Phase-I upgrade, a new component - global feature extractor (gFEX) will be designed to maintain the trigger acceptance against the increasing luminosity for the ATLAS Level-1 calorimeter trigger system. The gFEX is intended to identify patterns of energy associated with the hadronic decays of high momentum Higgs, W, \& Z bosons, top quarks, and exotic particles in real time at the LHC crossing rate. A pre-prototype with one single system-on-chip processor, ZYNQ, and one Xilinx Vertex-7 FPGA for technologies validation has been designed and tested last year. With the lessons learned from pre-prototype, a prototype with three Xilinx Ultra-scale FPGAs and one ZYNQ is implemented in an ATCA module. This board will receive coarse-granularity information from the entire ATLAS calorimeters on 276 optical fibers at the speed up to $12.8 \mathrm{~Gb} / \mathrm{s}$ synchronized to $40 \mathrm{MHz}$ LHC clock frequency. The ZYNQ will be used to control and configure all the three processor FPGAs, monitor board health, and interface to Gigabit Ethernet and UART. Now, the prototype board is on the way to fabrication. We will bring it up in July and will present the performance on the meeting.

N51-4: The Level-1 Topological Trigger of ATLAS: Commissioning and Operations E. Simioni

Johannes-Gutenberg-Universitaet Mainz, Mainz, Germany

On behalf of the The ATLAS Collaboration 
In order to be able to keep or even improve the physics potential reach in ATLAS, during the LHC shutdown after Run 1, the Level-1 trigger system was upgraded at hardware, firmware and software levels. In particular, a new electronics sub- system was introduced in the real-time data processing path: the Topological Processor System (L1Topo). It consists of a single AdvancedCTA shelf equipped with two Level-1 topological processor blades. They receive real-time information from the Level1 calorimeter and muon triggers, which is processed by four individual state-of- the-art FPGAs. It deals with a large input bandwidth of up to $6 \mathrm{~Tb} / \mathrm{s}$, optical connectivity and low processing latency on the real-time data path. The L1Topo firmware includes algorithms calculating angles or invariant masses between objects and many other kinematic variables based on lists of selected or sorted trigger objects, which need to be done within $200 \mathrm{~ns}$. Over one hundred VHDL algorithms are producing trigger outputs and are used by the logic of the central trigger processor, responsible of generating the Level- 1 acceptance signal. The system has been installed and commissioning started during 2015 and continued during 2016. As part of the firmware commissioning, the physics output from individual algorithms needs to be simulated and compared with the hardware response. An overview of the design, commissioning and operations process during Run 2 will be illustrated.

\title{
N51-5: Frontend and Backend Electronics for the ATLAS New Small Wheel Upgrade
} $\underline{\text { V. I. Martinez Outschoorn }}$

Department of Physics, University Illinois, Urbana-Champaign, USA

On behalf of the ATLAS Muon Collaboration

The Phase-I and Phase-II upgrades of the LHC accelerator will increase the LHC instantaneous luminosity to $2 \ddot{i} \iota^{1 / 2} 1034 \mathrm{~cm}-2 \mathrm{~s}-1$ and $7.5 \ddot{i}_{i} 1 / 21034 \mathrm{~cm}-2 \mathrm{~s}-1$, respectively. The luminosity increase drastically impacts the ATLAS trigger and readout data rates. The present ATLAS small wheel muon detector will be replaced with a New Small Wheel (NSW) detector in 2019. The NSW will feature two new detector technologies, Resistive Micromegas (MM) and small strip Thin Gap Chambers (sTGC) conforming a system of $\sim 2.4$ million readout channels. Both detectors will be used for muon triggering and precision tracking. A common readout path and two separate trigger paths are developed for these two detector technologies. The frontend electronics will be implemented in about 8000 boards including the design of 4 custom ASICs capable of driving trigger and tracking primitives to the backend trigger processor and readout system. The readout data flow is designed through a high-throughput network approach. The large number of readout channels, short time available to prepare and transmit trigger data, large volume of output data, harsh radiation environment, and the need of low power consumption all impose greati $i_{i}^{1} / 2$ challengesi $i_{i}^{1 / 2}$ on the system design. We will present the overall design along with the status of all ASIC and board prototypes.

\author{
N51-6: LAUROC: "A New Electronically Cooled Line-Terminating Preamplifier for the ATLAS Liquid \\ Argon Calorimeter Upgrade" \\ G. Martin-Chassard $^{1}$, F. Dulucq ${ }^{1}$, C. de La Taille ${ }^{1}$, N. Seguin-Moreau ${ }^{1}$, N. Morange ${ }^{2}$, L. Serin ${ }^{2}$, S. Simion ${ }^{2}$ \\ ${ }^{I}$ OMEGA Ecole Polytechnique-CNRS/IN2P3, Palaiseau, France \\ ${ }^{2}$ LAL Université Paris Sud-CNRS/IN2P3, Orsay, France
}

The readout electronics of the ATLAS Liquid Argon (LAr) Calorimeter (for the phase II of the high luminosity Large Hadron Collider at CERN) will be replaced and integrated in a single chip in order to reduce the power dissipation by an order of magnitude and to provide fully digital data. The cornerstone of the circuit is the preamplifier which is very demanding in terms of low noise, large dynamic range (at least 16 bits) and precise input impedance ( 25 or $50 \mathrm{Ohms}$ ) to terminate the cables from the detector. An innovative architecture is proposed to fulfill these requirements: a current conveyer and a single resistor are imbedded as feedback of a low noise amplifier. This architecture ensures accurate input impedance over a large frequency range $(100 \mathrm{MHz})$ as well as input current range $(10 \mathrm{~mA})$. The noise remains low thanks to this architecture which acts as an "electronically cooled" resistor out of an ultra-low noise amplifier $(0.4 \mathrm{nV} / \mathrm{sqrtHz})$. This design provides at the same time a current and a voltage output which could be used as high and low gain paths. An anti-saturation system is embedded to switch off the high gain when is saturated. "LAUROC", which stands for Liquid Argon Upgrade Read-Out Chip, represents the first step of this new chip development designed in TSMC 130nm technology by OMEGA. The chip was sent in fabrication in April and is expected during summer to be tested in lab on a test board developed by LAL group.

\section{N51-7: Development of xTCA Compliant Processor Board for future Trigger Upgrade}

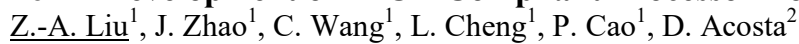

${ }^{I}$ Experimental Physics Division, Inst. of High Energy Physics, Chinese Academy of Sciences, Beijing, China

${ }^{2}$ University of Florida, Gainesville, US

The CMS trigger system will be further upgraded in phase II to be effectively working with a luminosity of $7.5 \times 10^{\wedge} 34 \mathrm{~Hz} / \mathrm{cm} 2$ by Introducing crystal granularity at Level- 1 for ECAL barrel with ???? $=0.0175 \times 0.0175$ vs. $0.0875 \times 0.0875$ for better spike rejection and EM shower identification, and incorporating Phase-2 forward muon detectors into muon Track-Finder trigger (GEM, iRPC) for increased redundancy, more bending angles, in addition to the Major Phase II task of incorporation of tracking at Level-1 from the silicon tracker with correlation of tracks with other Level-1 objects, and the xTCA architecture is under 
consideration for the development for the upgrade, especially for the silicon tracker trigger prototyping. This presentation will be focused on the development of a common processing unit including the carrier board, power board, and the double height AMC processor card which will be working with a $10 \mathrm{Gbps}$ links for data input/output and inter transmission.

N51-8: ICECAL: a 4 Channel ASIC for the Upgrade of the LHCb Calorimeter

D. Gascon $^{1}$, E. Picatoste ${ }^{1}$, J. Mauricio ${ }^{1}$, C. Beigbeder ${ }^{2}$, O. Duarte ${ }^{2}$, F. Machefert ${ }^{2}$, L. Garrido ${ }^{1}$, E. Grauges ${ }^{1}$, Y. Guz ${ }^{3}$, J. Lefrançois ${ }^{2}, X$. Vilasis ${ }^{4}$

${ }^{I}$ Dept. FQA, ICCUB. University of Barcelona, Barcelona, Spain

${ }^{2}$ Laboratoire de l'Accelerateur Lineaire, Orsay, France

${ }^{3}$ Institute for High Energy Physics (IHEP), Protvino, Russia

${ }^{4}$ GR-SETAD, La Salle. Universitat Ramon Llull, Barcelona, Spain

ICECAL, an integrated circuit for the Upgrade of the LHCb Calorimeter front end electronics is presented. The circuit is based on a current mode input stage followed by two fully differential interleaved channels with a switched integrator and a track and hold. A pole-zero cancellation circuit is used to achieve stringent spill-over requirements. ICECAL includes four analog channels, a Delay Locked Loop (DLL) for signal phase synchronization for all channels and an SPI communication protocol based interface. Results corresponding to a test beam performed in November 2015 show that ICECAL fulfills linearity (1\%), dynamic range $(>120 \mathrm{GeV})$, spill-over $(<1 \%)$, noise (about $1 \mathrm{LSB})$ and peak stability requirements $(1 \%$ stability during $4 \mathrm{~ns})$. A radiation qualification campaign is foreseen for 2016 summer. The chip has been designed in AMS SiGe BiCMOS 0.35um technology.

N52: Scintillators IV: Timing properties

Thursday, Nov. 3 10:30-12:00 Londres

N52-1: State of the Art Scintillation Based Detectors for Precise Timing in High Energy and Medical Physics $\underline{\text { S. Gundacker }}{ }^{1}$, A. Benaglia ${ }^{2}$, M. Lucchini ${ }^{1}$, A. Para ${ }^{3}$, K. Pauwels ${ }^{4}$, E. Auffray ${ }^{1}$, P. Lecoq ${ }^{1}$

${ }^{1}$ CERN, Geneva, Switzerland

${ }^{2}$ Princeton University, New-Jersey, USA

${ }^{3}$ Fermilab National Accelerator Laboratories, Batavia, USA

${ }^{4}$ University Milano-Bicocca, Milano, Italy

Precise timing capability will be a key aspect of particle detectors at future high energy colliders, as the time information can help in the reconstruction of physics events at the high collision rates expected. Furthermore, a precise time of flight (TOF) estimation of the coincident $511 \mathrm{keV}$ annihilation gammas in positron emission tomography (PET) can give a more than tenfold improvement in image signal to noise ratio. Fast scintillating crystals coupled to compact silicon photomultipliers (SiPMs) constitute a versatile system with exceptional timing capabilities for PET and can as well be exploited to realize an ad-hoc timing device to be hosted in a larger high energy physics detector. In this work we present the timing performance of LYSO:Ce and LSO:Ce codoped with $0.4 \%$ Ca crystals coupled to SiPMs measured with $150 \mathrm{GeV}$ muons at the CERN SPS H2 extraction line. Small crystals, with lengths ranging from $5 \mathrm{~mm}$ up to $30 \mathrm{~mm}$ and transverse size of $2 \times 2 \mathrm{~mm}^{2}$ or $3 \times 3 \mathrm{~mm}^{2}$, were exposed to a 150 $\mathrm{GeV}$ muon beam. SiPMs from two different producers (Hamamatsu and FBK) were used to detect the light produced in the crystals. The best coincidence time resolution (CTR) value of $34 \pm 1.2 \mathrm{ps}$ FWHM ( 10ps sigma single detector time resolution) has been measured for 5mm long LSO:Ce,Ca crystals coupled to FBK NUV-HD SiPMs, when time walk corrections are applied. The same detector gives a CTR of $83 \pm 4$ s FWHM if measured with $511 \mathrm{keV}$ gammas in TOF-PET. This confirms the potential of this technology for precise time measurements, not only in TOF-PET, but also in high energy physics. In addition, results obtained with Cherenkov radiators and different type of crystals (e.g. LuAG, GAGG) will be discussed in terms of the factors influencing the time resolution, e.g. SiPM photon detection efficiency, SiPM single photon time resolution, light production and transport in the crystal.

This work has been carried out in the frame of the Crystal Clear Collaboration and received funding from the ERC Advanced Grant Agreement no. 338953-TICAL and from Grant Agreement 289355-PicoSEC-MCNet. Support has also been received from COST Action TD1401 (FAST).

N52-2: Comparative Study on the Time Resolution of Co-Doped LSO:Ce, LYSO:Ce and LFS

$\underline{S . ~ E . ~ B r u n n e r ~}^{1}$, A. Ferri ${ }^{2}$, A. Gola ${ }^{2}$, C. Piemonte ${ }^{2}$, D. R. Schaart ${ }^{1}$

${ }^{I}$ Department of Radiation Science and Technology, TU Delft, Delft, The Netherlands

${ }^{2}$ Center for Materials and Microsystems, Fondazione Bruno Kessler, Trento, Italy

Electron-positron annihilation photon coincidence detection with high time resolution is required for applications such as TOFPET and PALS. The scintillator L(Y)SO doped with Ce has become the state-of-the-art material in TOF-PET over the years. Nevertheless, several manufacturers now offer new types of L(Y)SO:Ce with various types and amounts of co-doping. We 
present a direct comparison of the time resolution of LSO:Ce co-doped with $0.2 \% \mathrm{Ca}$ (Agile), LYSO:Ce co-doped with $0.075 \%$ Ca (Saint Gobain), and LFS type 3 (Zecotek). All samples had the same size of $3 \mathrm{~mm}$ x $3 \mathrm{~mm}$ x $5 \mathrm{~mm}$ and the same surface treatment, i.e., 5 sides ground and the side attached to detector polished. Prototypes of the new NUV-HD silicon photomultiplier (SiPM) developed by Fondazione Bruno Kessler (FBK), which have high photodetection efficiency (PDE), high single-photon time resolution (SPTR), and a spectral sensitivity ranging from the UV to the visible range, were optically coupled to the crystal samples. The SiPMs were read out using fast amplifier boards comprising pile-up suppression for time stamping with an electronic time jitter of a few tens of picoseconds in coincidence (FWHM). To obtain best results, the measurements were repeated with increasing bias voltage in steps of $0.5 \mathrm{~V}$ and at 3 different temperatures $\left(-20^{\circ} \mathrm{C}, 0^{\circ} \mathrm{C},+20^{\circ} \mathrm{C}\right)$. Coincidence resolving times (CRT) in the range of 80 ps to $90 \mathrm{ps} \mathrm{FWHM} \mathrm{were} \mathrm{found} \mathrm{for} \mathrm{all} \mathrm{the} \mathrm{samples.} \mathrm{The} \mathrm{best} \mathrm{value} \mathrm{found} \mathrm{equals} 79.2 \mathrm{ps}$ $\pm 0.6 \mathrm{ps}$ FWHM. To our knowledge, this represents the best measured time resolution for $3 \mathrm{~mm} \times 3 \mathrm{~mm} \times 5 \mathrm{~mm} \mathrm{~L}(\mathrm{Y}) \mathrm{SO}$ :Cebased crystals to date.

\author{
GFAG \\ H. V. Wachter ${ }^{1}$, F. R. Schneider ${ }^{2}$, L. Ferramacho ${ }^{3}$, S. Tavernier ${ }^{3}$, J. Varela ${ }^{3}$, R. Fojt ${ }^{2}$, F. Kreupl $^{1}$ \\ ${ }^{I}$ Department of Hybrid Electronic Systems, Technical University Munich, Munich, Germany \\ ${ }^{2}$ KETEK GmbH, Munich, Germany \\ ${ }^{3}$ PETsys Electronics SA, Lisbon, Portugal
}

N52-3: Study on Coincidence Time Resolution with SiPM and TOFPET-ASIC Utilizing LYSO, GAGG and

The performance of a detection system is defined by the interaction of scintillator, detector and readout electronics. KETEK silicon photomultipliers in combination with PETsys TOFPET-ASICs provide a high-performance detection chain, which will be studied in combination with $3 \times 3 \times 5 \mathrm{~mm}^{3}$ LYSO, GFAG and GAGG crystals. As a detector, a $3 \times 3 \mathrm{~mm}^{2}$ timing-optimized KETEK PM3350T silicon photomultiplier with $50 \mu \mathrm{m}$ cell pitch was used. The TOFPET-ASIC provides 64 channels, each with $25 \mathrm{ps}$ r.m.s intrinsic time resolution and $300 \mathrm{pC}$ dynamic range. First measurements with LYSO yield a CTR of 294 ps FWHM in a temperature stabilized setup environment at $18^{\circ} \mathrm{C}$. The studies with GFAG amd GAGG showed, that the time-over-threshold principle is sensitive towards the longer decay time constants and therefore different pulse shapes of GFAG and GAGG. A CTR of 660 ps FWHM for GFAG and 743 ps FWHM for GAGG could be achieved, which has been benchmarked on a dedicated reference setup to be 329 ps for GFAG and 663 ps for GAGG. Both materials could not fully exploit their intrinsic performance. The first results of this setup are very promising, as the performance for LYSO with 294 ps FWHM is close to the reference measurements with 248 ps FWHM. Forthcoming work will utilize a new generation ASIC and temperature stabilized setup environment, which overcomes the current limitations to handle any scintillator at maximum performance.

\title{
N52-4: Light Production in Scintillators and Nanocrystals During the First 5 Ns Using X-Ray Excitation.
} $\underline{\text { R. Martinez Turtos }}^{1}$, S. Gundacker ${ }^{2}$, J. Grim ${ }^{3}$, M. Salomoni ${ }^{1}$, E. Auffray ${ }^{2}$, P. Lecoq ${ }^{2}$, M. Paganoni ${ }^{1}$

${ }^{I}$ Physics, Università degli Studi di Milano Bicocca, Milano, Italy

${ }^{2} E P-C M X, C E R N$, Geneve, Switzerland

${ }^{3}$ Research Naval Laboratory, Washington, USA

Light production kinematics in terms of rise and decay time at the very first nanoseconds of the scintillating pulse constitute key parameters in order to achieve ultimate time resolution in radiation detectors systems. This contribution presents time spread measurements of the energy transfer coming from pulsed picosecond X-rays excitation; aiming to identify new sources of intense prompt signal which could be used as time tag for time of flight coincidence measurements. The study comprises conventional lutetium orthosilicates: $\mathrm{Ce}^{3+}$ scintillators (with and without $\mathrm{Ca}$ co-doping) and a new generation of scintillating materials: nanocrystals. Two different nanocrystals were measured: 1. CdSe colloidal nanoplatelets on glass and 2. ZnO:Ga nanopowders on $\mathrm{SiO}_{2}$ substrate. For all conventional scintillator samples, (with and without Ca co-doping), 2 rise time components are seen. A fast one, which agrees with the rise time obtained by laser direct excitation of the Cerium centers of around $10 \mathrm{ps}$. We conclude on a first direct excitation of the luminescent centers by thermalized electrons and a second energy transfer mechanisms coming from the decay of a trapped population with a lifetime which is around $300 \mathrm{ps}$. This could be explained by free or self-trapped excitons, which are also seen in wide band gap semiconductors and is in line with the excitonic lifetime present in nanocrystals where the oscillator strength allows stable excitons at room temperature. For CdSe nanoplatelets, the first decay component overlaps with the system IRF which points towards multiexcitonic population. However the exciton lifetime is preserved at 320 ps. ZnO:Ga nanopowders show near zero rise time and 2 fast decay components around $150 \mathrm{ps}$ and 590 ps with a light yield comparable to LYSO crystal. Both materials constitute a promising route to a strong prompt signal due to interaction of ionizing radiation.

This work was carried out in the frame of Crystal Clear collaboration and supported by a Marie Curie Early Initial Training Network Fellowship of the European Community's $\$ 7^{\wedge}\{$ th $\} \$$ Framework Programme under contract number (PITN-GA-2011-289355-PicoSEC-MCNet), COST Action Fast TD1401 and ERC Advanced Grant TICAL \#338953. 
N52-5: Fast Luminescence Response in Self-Activated and Ce-Doped Scintillation Materials G. Tamulaitis $^{1}$, E. Auffray ${ }^{2}$, R. Augulis ${ }^{3}$, A. Fedorov ${ }^{4}$, V. Gulbinas ${ }^{3}$, M. Korjik ${ }^{2}$, M. T. Lucchini ${ }^{2}$, V. Mechinsky ${ }^{4}$, S. Nargelas $^{1}$, E. Songaila $^{3}$, A. Vaitkevicius ${ }^{1}$

${ }^{1}$ Vilnius University, Vilnius, Lithuania

${ }^{2}$ CERN, Geneva, Switzerland

${ }^{3}$ Center for Physical Sciences and Technology, Vilnius, Lithuania

${ }^{4}$ Research Institute for Nuclear Problems, Minsk, Belarus

Our study is aimed at verifying the potential of commonly used and prospective scintillation materials for fast timing in highenergy physics experiments, positron emission tomography, and positron annihilation lifetime spectroscopy. Time-resolved photoluminescence spectroscopy and free carrier absorption at band-to-band and selective excitation with femtosecond laser pulses have been used to study two types of scintillation materials: self-activated lead tungstate $\left(\mathrm{PWO}, \mathrm{PbWO}_{4}\right)$ and $\mathrm{Ce}$-doped garnets - gadolinium aluminum gallium garnet ( $\left.\mathrm{GAGG}, \mathrm{Gd}_{3} \mathrm{Al}_{2} \mathrm{Ga}_{3} \mathrm{O}_{12}\right)$ and yttrium aluminum gallium garnet (YAGG, $\mathrm{Y}_{3} \mathrm{Al}_{1} \mathrm{Ga}_{4} \mathrm{O}_{12}$ ). A sub-picosecond photoluminescence (PL) rise time is observed in PWO, while longer processes in the PL response in GAGG:Ce are detected. The mechanisms responsible for the PL rise time in self-activated and Ce-doped scintillators are under discussion. A sub-picosecond rise time of luminescence in self-activated PWO scintillation crystal is demonstrated for both intrinsic blue and defect-related green emission components in the crystal. Two luminescence decay components with time constants of 4-6 ps and 600-800 ps, i.e., substantially faster than the "standard" decay component with the decay time of $\sim 10 \mathrm{ns,}$ are explicitly revealed in PWO. Meanwhile, the rise of the luminescence response after a short-pulse excitation in GAGG:Ce was found to be in the range of several nanoseconds even at the direct resonant excitation of $\mathrm{Ce}_{3+}$ ions. Moreover, it was found that free carriers appear in the conduction band of $\mathrm{PbWO}_{4}$ and YAGG:Ce crystals in the sub-picosecond time domain, whereas free holes appear in the valence band via ${ }^{8} \mathrm{~S}^{6} \mathrm{D}_{7 / 2,9 / 2}$ intra-center transition of $\mathrm{Gd}^{3+}$ ions in few picoseconds.

N53: New concepts in solid-state detectors and radiation damage effects III

Thursday, Nov. 3 10:30-12:00 Curie 1

N53-1: Radiation Hardness of Monolithic Active Pixel Sensors for the ALICE Inner Tracking System Upgrade H. Hillemanns

EP, CERN, Geneva, Switzerland

On behalf of the ALICE Experiment

The features of the 180nm TowerJazz CMOS Technology have enabled the ALICE experiment to chose Monolithic Active Pixels Sensors (MAPS) as the technology for the upgrade of the Inner Tracking System (ITS), foreseen to be installed in 2020, in order to match the requirements in terms of material budget, readout speed and low power consumption. A comprehensive R\&D program has been carried out over the last three years, resulting in the development of full size preproduction pixel sensor prototypes that have been extensively tested at various test beam facilities. The radiation levels expected in particular for the inner layers of the upgraded ITS require to assess the radiation hardness of the pixel sensors in order toensure the full functionality of the pixel sensors over the detector lifetime. In this paper we present and discuss the results of TID, NIEL, SEU and SEL irradiation tests carried out on pAlpide-3 sensor prototypes at various irradiation facilities.

\title{
N53-2: HVCMOS Pixel Detectors - First Measurements on the Reticle Size Prototype for the ATLAS Pixel
} Layers

$\underline{\text { F. Ehrler }}^{1}$, I. Peric ${ }^{1}$, R. Schimassek ${ }^{2}$

${ }^{I}$ Institute for Data Processing and Electronics (IPE), Karsruhe Institute of Technology, Karlsruhe, Baden-Württemberg, Germany

${ }^{2}$ Institute of Experimental Nuclear Physics (IEKP), Karsruhe Institute of Technology, Karlsruhe, Baden-Württemberg, Germany

High-Voltage CMOS (HVCMOS) pixel sensors are depleted active pixel sensors implemented in standard commercial CMOS processes. Thanks to its radiation hardness and fast signal generation HVCMOS pixel sensors will be used, or are proposed, for several experiments: Mu3e, ATLAS, CLIC. In 2015 a reticle size HVCMOS sensor was fabricated for the first time on high resistive substrates. This sensor is part of the ATLAS pixel demonstrator project. Measurement results will be presented.

\author{
N53-3: Characterization of Fully Depleted CMOS Active Sensors on High Resistive Substrate for High \\ Radiation Environment

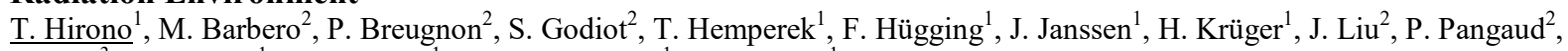 \\ I. Peric ${ }^{3}$, D.-L. Pohl ${ }^{1}$, A. Rozanov ${ }^{1}$, P. Rymaszewski ${ }^{1}$, N. Wermes ${ }^{1}$ \\ ${ }^{\prime}$ Physikalisches Institute, University of Bonn, Bonn, Germany \\ ${ }^{2}$ CPPM, Aix-Marseille Universite, Marseille, France \\ ${ }^{3}$ IPE, Karlsruher Institut für Technologie, Karlsruhe, Germany
}


Depleted CMOS active sensors (DMAPS) are being developed for high energy particle physics experiments in high radiation environments, such as ATLAS HL-LHC. Since charge collection by drift is mandatory for high radiation tolerance, high bias voltage on high resistive sensor volumes are needed. Thus, a prototype of a DMAPS (CCPD_LF) has been fabricated in a $150 \mathrm{~nm}$ CMOS process on $>2 \mathrm{k}$ ? $\cdot \mathrm{cm}$ substrate, and its substrate has been thinned to $100 \mu \mathrm{m}$. Full depletion occurs around $20 \mathrm{~V}$ which is far below the breakdown voltage of $110 \mathrm{~V}$. One of the features of the CCPD_LF is the capability to connect it to the existing readout chip of ATLAS pixel detector (FE-I4) enabling fast trigged readout. Since the pixel size of the CCPD_LF chip is $1 / 3$ of the pixel size of the FE-I4, signals from three CCPD_LF pixels are pulse-height encoded into one and sent to one FE-I4 pixel. Decoding of the three sub-pixels is achieved in the FE-I4 by time-over-threshold evaluation. The basic concept of this scheme is confirmed by electrical charge injections with the charge injection circuitry and using a $3 \mathrm{GeV}$ electron beam. The outer pixel layers in ATLAS HL-HLC will be irradiated up to total ionization dose of $50 \mathrm{Mrad} / \mathrm{cm}^{2}$ and a total fluence of $10^{15} \mathrm{n}_{\mathrm{eq}} / \mathrm{cm}^{2}$. Since the radiation tolerance is one of the largest concerns of DMAPS, the CCPD_LF has been irradiated with X-ray and neutrons up to these values. The detailed characteristics of the irradiated CCPD_LF will be shown and discussed.

N53-4: The INVESTIGATOR - an Efficient Tool to Optimize Design Parameters of a CMOS Pixel Sensor J. W. van Hoorne ${ }^{1}$, G. Aglieri ${ }^{1}$, C. Gao ${ }^{2}$, H. Hillemanns ${ }^{1}$, A. Junique ${ }^{1}$, M. Keil ${ }^{1}$, D. Kim ${ }^{3}$, M. Kofarago ${ }^{1}$, T. Kugathasan ${ }^{1}$,

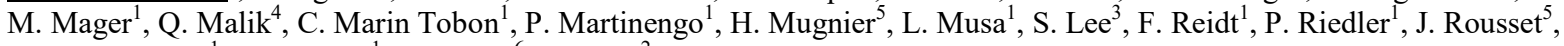
K. M. Sielewicz ${ }^{1}$, W. Snoeys ${ }^{1}$, M. Suljic ${ }^{6}$, P. Yang ${ }^{2}$

${ }^{I}$ CERN, Geneva, Switzerland

${ }^{2}$ Central China Normal University, Wuhan, China

${ }^{3}$ Dongguk and Yonsei University, Seoul, Korea

${ }^{4}$ COMSATS, Islamabad, Pakistan

${ }^{5}$ Mind, Archamps, France

${ }^{6}$ University and INFN, Trieste, Italy

A monolithic silicon pixel chip, the ALPIDE, is being developed for the upgrade of the Inner Tracking System (ITS) of the ALICE experiment at CERN's LHC. It will be fabricated in the TowerJazz $180 \mathrm{~nm}$ CMOS imaging-sensor process, on wafers with a high-resistivity epitaxial layer. In the design of the ALPIDE chip particular focus was put on the ratio of the collected charge on a single pixel and the pixel input capacitance, the $\mathrm{Q} / \mathrm{C}$ ratio of the sensor. A small-scale prototype with analogue readout, the INVESTIGATOR, was designed to optimize this $\mathrm{Q} / \mathrm{C}$ ratio and to study the characteristics of the sensitive layer and the charge-collection electrode. The Investigator contains 134 small pixel matrices with different pixel designs, varying the layout of the charge-collection electrode, and the pixel pitch. The chip has been produced on various wafers with different epitaxial-layer thickness and resistivity. For studies of the influence of bulk damage, samples were irradiated with a fluence of $1 \times 10^{13} 1 \mathrm{MeV} \mathrm{n} \mathrm{n}_{\mathrm{eq}} / \mathrm{cm}^{2}$. This contribution presents results, obtained from measurements with X-rays from an ${ }^{55} \mathrm{Fe}$ source, on the charge-collection time, depletion volume, pixel- input capacitance, and the charge-collection efficiency before and after irradiation. The results are presented as function of several pixel design parameters, the reverse-substrate bias voltage, and epitaxial-layer thickness. Devices recently fabricated in a modified process to enhance the depletion of the sensitive layer are functional. First results of this novel development are discussed.

N53-5: HVCMOS Pixel Detectors - Methods for Enhancement of Time Resolution $\underline{\text { R. Schimassek }}^{1}$, F. Ehrler ${ }^{2}$, I. Peric ${ }^{2}$

${ }^{I}$ Institute of Experimental Nuclear Physics (IEKP), Karlsruhe Institute of Technology (KIT), Karlsruhe, Baden-Württemberg, Germany

${ }^{2}$ Institute for Data Processing and Electronics (IPE), Karlsruhe Institute of Technology (KIT), Karlsruhe, Baden-Württemberg, Germany

High-Voltage CMOS (HVCMOS) pixel sensors are depleted active pixel sensors implemented in standard commercial CMOS processes. HVCMOS pixel sensors will be used, or are proposed, for several experiments: Mu3e, ATLAS, CLIC. Thanks to charge collection with a high electric field, HVCMOS sensors potentially have a very high time resolution. But the high time resolution is not achieved automatically as the it is limited by the rise time of the amplifier. This contribution describes methods for the enhancement of the time resolution.

\author{
N53-6: Enhancement of the Radiation Tolerance of High Resolution CMOS Pixel Sensors Using Strong \\ Depletion in a High Resistivity Epitaxial Layer for Charged Particle Tracking \\ $\underline{\text { A. Perez Perez }}$ \\ IPHC - IN2P3/CNRS, Strasbourg, France \\ On behalf of the PICSEL-IKF collaboration
}

CMOS Pixel Sensors (CPS) have a rather unique potential to comply with demands of micron level spatial resolutions combined with material budget requirements of a few per-mill of radiation length per detection layer. These achievements are however 
accompanied with a radiation tolerance and a read-out speed which do not comply with the needs of future subatomic physics experiments dealing with particularly high interaction rates. To improve the read-out speed and the tolerance to non-ionising radiation, the PICSEL group of IPHC develops a new generation of CPS which include the possibility to deplete the sensitive volume over dimensions exceeding substantially those prevailing in the sensors it has developed previously.

Prototypes were fabricated in 2015 and 2016 via a $0.18 \mu \mathrm{m}$ CMOS process relying on a $18 \mathrm{um}$ thick, high resistivity $(>1 \mathrm{kO} . \mathrm{cm})$, epitaxial layer, to test the depletion capacity associated to a new pixel design. Within the latter, the sensing node is connected to a standalone biasing voltage and is only capacitively coupled to the pre-amplification stage. A depletion voltage exceeding $10 \mathrm{~V}$ is then made possible. The talk will show that strong depletion was achieved with pixels which remain at a low noise level. It will examin the influence of the sensing node geometry on its charge collection performances when illuminating the sensors with an $55 \mathrm{Fe}$ source. It will next describe irradiation tests of the prototypes. The sensor response to fluences in excess of $10^{\wedge} 141 \mathrm{MeV}$ equivalent neutrons per square centimetre will be discussed for various temperatures and sensing node designs, making evidence of an improvement of the tolerance to non-ionising radiation going well beyond previous achievements. The performances observed will be confronted to the requirements of the Micro-Vertex Detector of the CBM experiment at FAIR/GSI, which is presently a major motivation for this development, in collaboration with the Frankfurt University.

\title{
N54: Instrumentation for Security II
}

Thursday, Nov. 3 10:30-12:00 Curie 2

N54-1: Why Is Nuclide Identification so Difficult? - Lessons from the RASE Program. N. Martin-Burtart

Radiation Solutions Inc, Mississauga, ON, Canada

Correct nuclide identification is critical for first responders. It is the final result of the complex chain occurring in a detector: a spectrum with poor statistics in a variable environment is recorded then analyzed. Industries are facing a complex challenge, as they have to design instruments that can identify materials that for the most part are difficult to obtain. Modelling a source is a viable option to skirt this issue, or participating in data collection events organized by governmental agencies such as DNDO with their RASE program. Modelling a source means spending a lot of processing time for a result that is not likely to be an exact replica of the real world, especially when it comes down to backscattering. Data collection events are only a small excerpt of the real world: performance evaluation based on only that may not reflect the true performances of the instrument. Nuclide identification can fail due to poor instrument performance, a template or peak database that is not optimized. The conditions of the experiments strongly influence the final spectrum and can throw the identification off. Some examples of source modelling (infinite volume sources, planar sources), experiments and database will be presented in this paper

\author{
N54-2: A Cost Effective Means of Extending the Lifetime of Plastic Scintillators in Portal Monitors \\ M. R. Kusner, P. R. Menge \\ Saint-Gobain Crystals, Hiram, Ohio, USA
}

Large plastic scintillators $(5000$ to $>35000 \mathrm{cc}$ ) deployed as portal monitors and subjected to environmental extremes have been shown to suffer performance degradation due to water vapor penetration of the base material. Low temperatures can cause the absorbed vapor to condense and manifest itself as a fog within the scintillator that can dissipate as the material warms. Repeated cold cycles may lead to a permanent residual haze. Both Polyvinyltoluene and Polystyrene based scintillators are susceptible to this effect. The presence of internal haze cause scattering of scintillation light and results in reduced detector sensitivity. An obvious, but expensive solution would be to hermetically seal the plastic as one would a hygroscopic scintillating crystal. In this study we examine the performance of 12 plastic scintillation detectors packaged in various configurations and exposed to $>30$ days storage at $55 \mathrm{C}$ and $85 \%$ relative humidity. The detectors were cycled to $25 \mathrm{C}, 0 \mathrm{C}$ and $-30 \mathrm{C}$. Pulse height spectra were acquired continuously during the experiment. Count rate information along with the pulse height spectra are used to gauge the effectiveness of the various packaging configurations in preventing water vapor penetration and subsequent fogging at low temperatures. An effective packaging solution will be presented.

N54-3: Design of a Compact Radioxenon Detection System Using CdZnTe, Plastic Scintillator, and SiPMs S. A. Czyz, A. T. Farsoni, E. M. Becker, H. R. Gadey

School of Nuclear Science Engineering, Oregon State University, Corvallis, OR, USA

The measurement and identification of radioxenon isotopes in the atmosphere using beta-gamma coincidence techniques has been effectively utilized by the Comprehensive Test Ban Treaty Organization (CTBTO) to verify the nuclear nature of clandestine explosions. In an effort to mitigate conversion electron backscatter effects observed in the dual-CdZnTe (CZT) coincidence radioxenon detection system previously designed at Oregon State University, a prototype detection system consisting of a well-type plastic scintillator and a coplanar CZT crystal has been developed. The plastic scintillator gas cell is used to measure the full energy deposition of beta particles and conversion electrons without significantly attenuating gamma and X- 
rays. The inner wall of the scintillator is coated with a very thin layer of $\mathrm{Al} 3 \mathrm{O} 2$ in order to reduce the diffusion of radioactive gas into the plastic (memory effect). The base of the scintillator is coupled to an array of silicon photomultipliers (SiPMs) for light readout, chosen for their size, low cost, and ruggedness. A coplanar CZT crystal, chosen for its excellent energy resolution and room temperature operation, is placed beside the scintillator in order to detect gamma and X-rays. The detector components are mounted on a PCB for real-time digital signal processing and readout. Characterization will be performed using check sources and radioxenon isotopes produced via irradiation of stable and enriched xenon isotopes in the OSU TRIGA reactor. The detection system offers a compact and inexpensive alternative to other state-of-the-art radioxenon detection systems, while still achieving a minimum detectable concentration in accordance with CTBTO standards.

\author{
N54-4: ?Performance of a Wearable Neutron Search Instrument with Direction-Finding Capability \\ M. Foster, D. Ramsden \\ Symetrica Security Ltd., Southampton, Hampshire, UK
}

A wearable detector system has been developed comprising up to four neutron detector modules, that, when worn on the torso can provide a sensitivity comparable to that of backpack systems but with a much reduced weight and bulk.

Each module comprises a thin $\mathrm{LiF} / \mathrm{ZnS}$ thermal neutron detector viewed by a silicon photomultiplier (SiPM) array, pulse-shape discrimination logic, internal health monitoring, and an internal battery providing over twenty hours of operation from a fourhour charge. Data is transmitted from each individual module via Bluetooth Low Energy and collated by an Android smartphone. By running advanced anomaly detection and background monitoring algorithms on the smartphone, it is possible to achieve source detection performance meeting the ANSI N42.53-2013 and IAEA NSU-NSD (v3) standards. Gamma-ray rejection of each module has been measured at 1:10E8 and again meets the ANSI and IAEA standards.

By analysing the relative count rates it is possible to provide directional information in the horizontal plane to aid searches for the location of a source, with an accuracy of 45 degrees. The search for a source is further aided by fusion of this directional count rate information with GPS and time stamps to produce a radiation map.

This paper will present the results of performance tests against the ANSI and IAEA standarda, including dynamic source detection, gamma-ray rejection and false alarm measurements. Results of environmental tests and direction-finding performance will also be presented.

\author{
N54-5: Initial Characterization of a Silicon Photomultiplier Based System for Neutron Detection with \\ Enhanced Gamma Discrimination

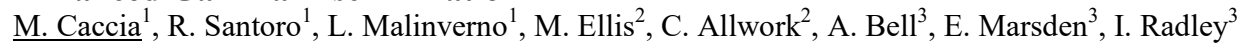 \\ ${ }^{I}$ Dipartimento di Scienza e Alta Tecnologia, Universiti ${ }_{i}^{1 / 2}$ dell'Insubria, Como, Italy \\ ${ }^{2}$ AWE, Aldermaston, United Kingdom \\ ${ }^{3}$ KROMEK, Sedgefield, United Kingdom
}

Silicon Photomultipliers (SiPM) are a new class of solid-state photon detectors, featuring single photon sensitivity, low bias voltage and power consumption, extended dynamic range, magnetic field immunity and an extreme design flexibility. The SiPM concept appeared about 15 years ago and benefitted from the tremendous development of the silicon technology to quickly achieve its maturity, making it a viable alternative to PhotoMultiplier Tubes (PMT) for an increasing number of applications. This paper reports the initial study of a SiPM based system for neutron detection with gamma/neutron discrimination, the first step towards the design and engineering of a hand-held instrument that has applications in nuclear security. The study is based on sensors by two producers, coupled to two scintillators sensitive to fast and thermal neutrons: the EJ-299-34 (a fast neutron sensitive organic scintillator by Eljen Technologies) and the CLYC (a thermal neutron sensitive scintillator provided by CAPESYM and RMD Inc). The sensors have been characterized with pulses of blue light. The performance assessed in terms of gamma/neutron separation based on Pulse Shape Discrimination (PSD), related to the different time profile of the emitted scintillation light depending on the nature of the detected particle. The results obtained with $6 \times 6 \mathrm{~mm}$ SiPM show the relevance of the sensor signal time development in the PSD figure. Preliminary outcome, obtained with larger array of SiPM, show a good discrimination capability with a figure of merit (FOM) exceeding 2.2 for both scintillators. This result is comparable with published data obtained with PMT and confirms the potential of SiPM in this field.

N54-6: Recent Advances in Hard X-Ray and Soft Gamma-Ray Multilayer Mirrors Applications M.-A. Descalle, J. Alameda, N. F. Brejnholt, T. A. Decker, M. J. Pivovaroff, J. Ruz-Armendariz, R. Soufli PLS/Physics, Lawrence Livermore Nat. Lab., Livermore, CA, USA

Measurements in environments with low signal-to-noise ratio characteristics due to high radiation fields or weak signals can present a challenge to the radiation detection community. In addition, the need to resolve and identify distributed radiation sources imposes stringent requirements on imaging systems spatial and energy resolution. Multilayer X-ray mirrors, which are an integral component of complex optic systems used in grazing incidence X-ray telescopes for astrophysics satellites, potentially offer a solution to selectively capture and reflect an emission line of interest in a shielded detector. Lawrence Livermore National 
Laboratory has been developing expertise and capabilities from the modeling and design of thin d-spacing multilayer coatings to the assembly of highly nested optics. Measurement campaigns at the European Synchrotron Radiation Facility demonstrated multilayer optics designed with d-spacing $<20 \mathrm{~nm}$ can selectively reflect photons up to $650 \mathrm{keV}$, within the energy range of interest to gamma ray astrophysics, plasma physics and safeguards applications. Recent advances in the use of hard X-ray mirrors for the non-destructive assay of spent fuel and plasma matter interactions are described. Practical challenges and limitations are addressed and possible future applications are presented.

This work was performed under the auspices of the U.S. Department of Energy by Lawrence Livermore National Laboratory under Contract DEAC52-07NA27344. The project is funded by the U.S. Department of Energy Office/LLNL LDRD office, through grant 13-ERD-048.

\section{N55: New concepts in solid-state detectors and radiation damage effects IV}

Thursday, Nov. 3 14:00-16:00 Madrid

N55-1: Development of Thin Active Region Sensors on 8" Wafers for for Particle Physics

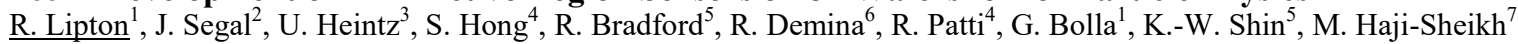

${ }^{I} P P D$, Fermilab, Batavia, Ill, USA

${ }^{2} S L A C$ National Accelerator Laboratory, Menlo Park, Ca, USA

${ }^{3}$ Physics Department, Brown University, Providence, RI, USA

${ }^{4}$ Tezzaron Inc., Naperville, Ill, USA

${ }^{5}$ Advanced Photon Source, Argonne National Laboratory, Lemont, Ill, USA

${ }^{6}$ Physics Department, University of Rochester, Rochester, NY, USA

${ }^{7}$ Electrical Engineering, Northern Illinois, Dekalb, Ill, USA

Increased sensor wafer diameters have the prospect of reducing the $\operatorname{cost} / \mathrm{cm}^{2}$ and reducing the number of modules needed for large area detector systems. A central problem in moving from the current $150 \mathrm{~mm}$ wafers to $200 \mathrm{~mm}$ diameter wafers is fabrication and handling of devices with thin active depth necessary for radiation hardness. Thin active layers can be fabricated by physical thinning, deep diffusion of the backside, or wafer bonding either using Silicon on Insulator (SOI) or direct $\mathrm{Si}-\mathrm{Si}$ bonded wafers. Silicon detectors on $200 \mathrm{~mm}$ high resistivity p-type float zone wafers have been fabricated at Tezzaron/Novati. The initial run, completed in July of 2015 consisted on p-stop plus p-spray and p-stop isolated wafers. In this run wafers were delivered both at full thickness (725 microns) and with one thinned to 500 microns. A second run, aimed at lower active thickness was delivered in March of 2016. This was composed of two float zone wafers thinned to 500 microns and four SOI wafers with active thickness of 200 microns. Process splits included p-stop implantation dose and oxidization variants. We will discuss process flow, test structure results, and extension of this work to Si-Si bonded wafers.

N55-2: Two-Tier Pixelated Avalanche Sensor for Particle Detection in 150nm CMOS

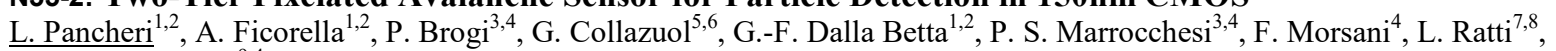
A. Savoy-Navarro 9,4

${ }^{I}$ Dipartimento di Ingegneria Industriale, Università di Trento, Trento, Italy

${ }^{2}$ TIFPA - INFN, Trento, Italy

${ }^{3}$ DFSTA, Università di Siena, Siena, Italy

${ }^{4}$ INFN Pisa, Pisa, Italy

${ }^{5}$ Dipartimento di Fisica e Astronomia, Università di Padova, Padova, Italy

${ }^{6}$ INFN Padova, Padova, Italy

${ }^{7}$ Dipartimento di Ingegneria Industriale e dell'Informazione, Università di Pavia, Pavia, Italy

${ }^{8}$ INFN Pavia, Pavia, Italy

${ }^{9}$ Laboratoire APC, University Paris-Diderot/CNRS, Paris, France

In this work, we present a two-layer Geiger-mode avalanche sensor designed for direct particle detection. In the proposed device, each pixel is formed by two vertically-aligned avalanche detectors, exploiting the coincidence between two simultaneous avalanche events to discriminate between particle-triggered detections and dark counts. A two-tier sensor was designed and fabricated in a commercial 150nm CMOS process in the framework of INFN project APiX2. The core of the sensor is a 48x16 pixel array, with a pixel size is $50 \mu \mathrm{m} \times 75 \mu \mathrm{m}$ and a maximum fill factor of $51.6 \%$. Several sensor samples have been verticallyintegrated through bump bonding. The operation of the vertically integrated sensors has been validated with dark count measurements, showing a median and a mean coincidence rate per pixel of $0.17 \mathrm{~Hz}$ and $1.43 \mathrm{~Hz}$, respectively, with $1 \mathrm{~V}$ overvoltage and $10 \mathrm{~ns}$ coincidence time at room temperature, corresponding to an average dark count rate of $380 \mathrm{~Hz} / \mathrm{mm}^{2}$. An extensive measurement campaign is currently being carried on to assess the effectiveness of the proposed approach, and the characterization results will be presented at the conference.

The research activity presented in this work has been carried out in the framework of the APiX2 experiment funded by Istituto Nazionale di Fisica Nucleare (INFN), CSN5. 
N55-3: New Thin 3D Pixel Sensors for HL-LHC: First Results

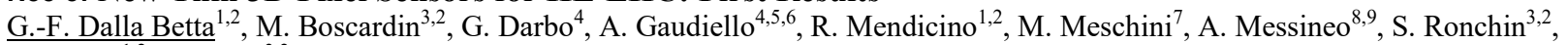
D. Sultan ${ }^{1,2}$, N. Zorzi ${ }^{3,2}$

${ }^{I}$ University of Trento, Trento, Italy

${ }^{2}$ TIFPA INFN, Trento, Italy

${ }^{3}$ Fondazione Bruno Kessler, Trento, Italy

${ }^{4}$ INFN Sezione di Genova, Genova, Italy

${ }^{5}$ University of Genova, Genova, Italy

${ }^{6}$ CERN, Geneva, Switzerland

${ }^{7}$ INFN Sezione di Firenze, Firenze, Italy

${ }^{8}$ INFN Sezione di Pisa, Pisa, Italy

${ }^{9}$ University of Pisa, Pisa, Italy

We report on new 3D pixel sensors oriented to the Phase 2 Upgrades at the High-Luminosity LHC (HL-LHC). These sensors have increased pixel granularity (e.g., $50 \times 50$ or $25 \times 100 \mu \mathrm{m}^{2}$ pixel size), thinner active layer $(\sim 100 \mu \mathrm{m})$ with columnar electrodes having narrower size $(\sim 5 \mu \mathrm{m})$ and reduced spacing $(\sim 30 \mu \mathrm{m})$, as required for high radiation hardness (up to a fluence of $2 \times 10^{16} \mathrm{n}_{\mathrm{eq}} \mathrm{cm}^{-2}$ ). Measurements from the first sensor batch, fabricated at FBK (Trento, Italy) on 6" SiSi Direct Wafer Bonded wafers, have shown very promising results in terms of low leakage current ( $\sim 1 \mathrm{pA} /$ column $)$, high breakdown voltage $(\sim 150 \mathrm{~V})$, and low capacitance $(\sim 50 \mathrm{fF} /$ column $)$, in good agreement with TCAD simulations.

This work was supported by the Autonomous Province of Trento, by the Italian National Institute for Nuclear Physics (INFN), Projects ATLAS, CMS, RD-FASE2 (CSN1), and by AIDA-2020 project EU-INFRA proposal no. 654168.

N55-4: Properties of DEPFET Active Pixel Sensors Fabricated in an Industrial CMOS Foundry

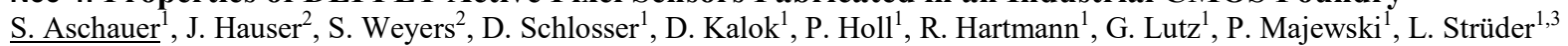

${ }^{I}$ PNSensor GmbH, Munich, Deutschland

${ }^{2}$ Fraunhofer-Institut für Mikroelektronische Schaltungen, Duisburg, Deutschland

${ }^{3}$ University of Siegen, Siegen, Deutschland

Detector fabrication in a modern state-of-the-art CMOS foundry offers a wide spectrum of advantages compared to research laboratory style fabrications, provided that the physical key parameters needed for a good detector performance are preserved: low leakage currents, defect free double sided handling, and a good Si-SiO2 interface quality to mention some of the relevant parameters. We developed a fabrication process for an industrial CMOS foundry which fulfills the above boundary conditions and facilitates the production of backside-illuminated DEPFET active pixel sensors on $725 \mu \mathrm{m}$ thick, double sided polished, high resistivity float-zone silicon. The CMOS foundry offers high process stability, small feature sizes, a high throughput in a short time and the option to include more complex additional circuitry in the future. After a diode test fabrication, we produced first real DEPFET prototype devices, which are now available and which are the very first DEPFETs ever built in an industrial-scale CMOS fab. This presentation will give a comprehensive overview about the performance of these new DEPFET devices. In addition, it will give an outlook on the future applications of such CMOS based DEPFETs for X-ray and electron detection.

\section{N55-5: Optimization of Thin N-in-P Planar Pixel Modules for the ATLAS Upgrade at HL-LHC} A. Macchiolo, J. Beyer, R. Nisius, A. La Rosa, N. Savic

Max-Planck-Institut fuer Physik, Munich, Germany

The ATLAS experiment will undergo around the years 2023-2025 a major upgrade of the tracker system in view of the high luminosity phase of the LHC (HL-LHC). The most severe challenges are to be faced by the innermost layers of the pixel detector which will have to withstand a radiation fluence up to $1.4 \times 10^{* *} 161 \mathrm{MeV}$ neq. Thin planar pixel modules are promising candidates to instrument this region of the new pixel system, thanks to the reduced contribution to the material budget and their high charge collection efficiency after irradiation. 100-200 um thick sensors, interconnected to FE-I4 read-out chips, have been characterized with radioactive source scans and beam tests at the CERN-SPS and DESY. Their performance up to a fluence of $10 * * 161 \mathrm{MeV}$ neq will be compared in terms of charge collection and hit efficiency. New designs of the pixel cells, with an optimized bias structure, have been implemented in n-in-p planar pixel productions, and the possible gain in the hit efficiency investigated as a function of the received irradiation fluence. The outlook for future planar pixel sensor productions will be discussed, with a focus on sensor design at the pixel pitches (50x50 and 25x100 $\left.\mu \mathrm{m}^{2}\right)$ foreseen for the RD53 Collaboration readout chip in $65 \mathrm{~nm}$ CMOS technology. Highly segmented sensors will represent a challenge for the tracking in the forward region of the pixel system at HL-LHC. In order to reproduce the performance of 50x50 $\mu \mathrm{m}^{2}$ pixels at high eta, FE-I4 compatible planar pixel sensors have been studied before and after irradiation in beam tests at high incidence angle $\left(80^{\circ}\right)$ with respect to the short pixel direction. Results on cluster shapes, charge collection and hit efficiency will be shown. 
N55-6: Measurement of the Time Resolution of Ultra-Fast Silicon Detectors

H. Sadrozinski

Univ. of California Santa Cruz, Santa Cruz Institute for Particle Physics, Santa Cruz, CA, USA

On behalf of the UFSD Collaboration

Measurement of the Time Resolution of thin Ultra-Fast Silicon Detectors

H. F.-W. Sadrozinski *, Senior Member IEEE, J. Chen, V. Fadeyev, P. Freeman, Z. Galloway, H. Grabas, B. Gruey, L. Hibbard, C. Labitan, R. Losakul, A. Seiden, N. Woods, A. Zatserklyaniy Santa Cruz Institute for Particle Physics, UC Santa Cruz, Santa Cruz, CA 95064, USA

N. Cartiglia, F. Cenna, M. Ferrero, F. Ravera INFN Torino, Torino, Italy

M. Baselga, P. Fernández-Martínez, D. Flores, S. Hidalgo, A. Merlos, G. Pellegrini, D. Quirion Centro Nacional de

Microelectrónica, IMB-CNM-CSIC, Barcelona, Spain

?Abstract- We report the measurements of the time resolution of Ultra-Fast Silicon Detectors (UFSD). UFSD are silicon sensors based on the Low-Gain Avalanche Diodes (LGAD) design and, due to internal gain, exhibit a signal which is a factor of $\sim 10$ larger than standard silicon detectors. The internal gain allows obtaining fast and large signals, ideal for time applications. The time resolution was measured with $1064 \mathrm{~nm}$ laser signals and with charged particles in beam tests and with a $90 \mathrm{Sr}$ beta source. The pulse shapes are recorded in a digital scope and analyzed off-line.

While previous measurements were done on $300 \mu \mathrm{m}$ thick sensors, we report here the first timing measurements on thin LGAD with thickness $<100 \mu \mathrm{m}$, showing the advantage of thin sensors because of a large "slew-rate" $\mathrm{dV} / \mathrm{dt}$ and small Landau fluctuations. Preliminary results from the $90 \mathrm{Sr}$ beta source are a timing resolution of about $50 \mathrm{ps}$ for the time difference between LGAD and a trigger counter consisting of a quartz radiator read out by a fast SiPM.

Finally we compare the results with the prediction of the simulation program Weightfield 2.

Part of this work was performed in the framework of the CERN RD-50 collaboration. It was (partially) financed by the Spanish Ministry of Economy and Competitiveness through the Particle Physics National Programs FPA2013-48308-C2-2-P and FPA2014-55295-C3-2-R, Horizon 2020, ERC Adv Grants INFN CSN5 (project UFSD), the Autonomous Province of Trento and INFN (framework agreement MEMS3), and the United States Department of Energy, grant DE-FG02-04ER41286

\author{
N55-7: Investigation of Radiation Damage Due to Particle Irradiation on Silicon Drift Detector for \\ Chandrayaan-2 Mission \\ S. M. ${ }^{1}$, V. S. V. ${ }^{1}$, P. A. ${ }^{1}$, G. S. K. ${ }^{1}$, L. T. ${ }^{1}$, P. S. ${ }^{2}$, N. V. ${ }^{2}$ \\ ${ }^{I}$ Department of space, Physical Research Laboratory, Ahmedabad, Gujarat, India \\ ${ }^{2}$ Department of atomic energy, Tata Institute of Fundamental Research, Mumbai, Maharastra, India
}

It is well known that the Earth is surrounded by extremely intense radiation regions known as Van Allen radiation belts which consists of highly energetic electrons and protons trapped within the Earth's magnetosphere. Such harsh radiation environment is known to adversely affect the performance of X-ray detectors. Forth coming Chandrayaan-2 mission, the second Indian mission to the Moon will have two X-ray spectrometer experiments using Silicon Drift Detectors (SDD) namely Solar X-ray Monitor (XSM) and Alpha Particle X-ray Spectrometer (APXS). Silicon detectors are sensitive to displacement damage which is due to the Non Ionizing Energy Loss (NEIL) of charged and neutral particles. The displacement damage leads to increase in the detector leakage current and thus degrades the energy resolution. Thus in order to estimate the end-of-life performance, it is necessary to carry out the radiation damage tests.

\author{
N55-8: Validation Strategy for the Simulation of Highly Irradiated Silicon Pixel Sensors \\ J. Schwandt, E. Fretwurst, E. Garutti, R. Klanner, G. Steinbrueck \\ Institute of Experimental Physics, University of Hamburg, Hamburg, Germany
}

For the high-luminosity phase of the Large Hadron Collider (HL-LHC), at the expected position of the innermost pixel detector layer of the CMS and ATLAS experiment, the estimated equivalent neutron fluence after $3000 \mathrm{fb}^{-1}$ is $2 \times 10^{16} \mathrm{n}_{\mathrm{eq}} / \mathrm{cm}^{2}$, and the IEL (Ionizing Energy Loss) dose in the $\mathrm{SiO}_{2}$ is $5 \mathrm{MGy}$. The optimization of the pixel sensors and the understanding of their performance as a function of fluence and dose makes a radiation damage model for TCAD simulations, which describes the available experimental data, highly desirable. The currently available bulk-damage models are not able to describe simultaneously the measurements of dark current (I-V), capacitance-voltage (C-V) and charge collection efficiency (CCE) of pad diodes for fluences $=1 \times 10^{15} \mathrm{n}_{\mathrm{eq}} / \mathrm{cm}^{2}$. For the development and validation of an accurate bulk damage model we use I-V, C-V and CCE measurements on pad diodes available within the CMS-HPK campaign and data from samples irradiated recently with 24 $\mathrm{GeV} / \mathrm{c}$ protons. For the determination of the radiation-induced damage parameters we utilize the "optimizer" of Synopsys TCAD, which allows the minimization of the difference between the measured and simulated I-V and C-V. A validation of the parameters obtained is done by comparing the CCE simulation to the measurements. By this method accurate fits for the I-V and 
$\mathrm{C}-\mathrm{V}$ can be achieved and a systematic study of the number and type of the defect levels, which are required for a consistent description of the bulk damage, can be achieved.

N56: Instrumentation for experimental reactors and nuclear power plants I

Thursday, Nov. 3 14:00-15:45 Londres

N56-1: Electronic Support System Enhancements for Micro-Pocket Fission Detectors

M. A. Reichenberger ${ }^{1}$, T. J. Sobering ${ }^{2}$, D. M. Nichols ${ }^{1}$, J. A. Geuther ${ }^{1}$, D. S. McGregor ${ }^{1}$, P. B. Ugorowski ${ }^{1}$, T. C. Unruh $^{1}$

${ }^{1}$ SMART Laboratory, Kansas State University, Manhattan, KS, USA

${ }^{2}$ Electronics Design Laboratory, Kansas State University, Manhattan, KS, USA

Micro-Pocket Fission Detectors (MPFDs) continue to be an important development in advancing nuclear research capabilities. Commonly, nuclear reactor cores are located far from the location which is desired for re-out electronics. Specifically, the core of the TRIGA Mk II research nuclear reactor at Kansas State University is submerged under approximately $16 \ddot{i}_{i} 1 / 2$ of water. Additionally, research nuclear reactor facilities often possess un-anticipated sources of noise due the aging facilities. A robust method to transmit MPFD pulse signals from the MPFD sensors to the readout electronics is under development. The results of pulse calculations were used to determine that the pre-amplifier being used for MPFD testing required further adjustment. An MPFD emulator was developed which enables the optimization of the pre-amplifier circuit for future MPFD sensor testing. Finally, a lab-top MPFD substitute has made electronics testing far more reliable by eliminating many of the variables that are inherent to the research nuclear reactor facility at Kansas State University.

N56-2: $\boldsymbol{\mu G e : ~ a ~ M i n i a t u r e ~ G e r m a n i u m ~ D e t e c t o r ~ f o r ~ I m m e d i a t e ~ O p e r a t i o n ~}$

$\underline{\text { V. Marian }}^{1}$, J. Clauss ${ }^{1}$, R. Abou-Khalil ${ }^{2}$, J.-O. Beau ${ }^{1}$, B. Pirard ${ }^{1}$, J. Flamanc ${ }^{1}$, M.-O. Lampert ${ }^{1}$

${ }^{I}$ Canberra Specialty Detectors, Lingolsheim, France

${ }^{2}$ AREVA Corporate Research \& Development, Paris, France

This paper describes a novel ultra-miniature electrically-cooled HPGe detector, designed for use in very tight spaces, as well as for field applications, such as nuclear material characterization and decommissioning. The lightweight design (1.2kg) and low power consumption $(\sim 10 \mathrm{~W})$ makes it possible to embark the detector onto remote-controlled robotic platforms (terrestrial or aerial) in order to perform high resolution radiation measurements in highly contaminated areas. The ?-ray version of the detector exhibits excellent spectroscopic performance (750eV@122keV, 1.95keV@1.33 MeV) and has almost immediate availability (cool-down time below 15 minutes), making it an ideal alternative to Room Temperature Semiconductor Detector (RTSD) solutions with inferior spectroscopic and count-rate capabilities. An x-ray version is under prototyping and is expected to deliver performance levels superior to state-of-the-art SDD detectors, with unmatched stopping power above $20 \mathrm{keV}$.

\author{
N56-3: Passive Neutron Coincidence Counting with Plastic Scintillators for the Characterization of \\ Technological Radioactive Waste Drums

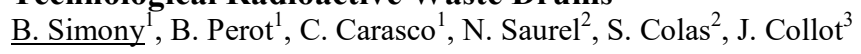 \\ ${ }^{I}$ Nuclear Measurement Laboratory (LMN), building 224, CEA Cadarache, Saint Paul lez Durance, France \\ ${ }^{2}$ DAM, CEA Valduc, Is-sur Tille, France \\ ${ }^{3}$ Laboratoire de physique subatomique et de cosmologie, CNRS IN2P3, Grenoble, France
}

This paper reports a feasibility study, performed by numerical simulation with MCNPX PoliMi v2.0, for plutonium characterization in radioactive waste packages by passive neutron coincidence counting with plastic scintillators. These last could indeed constitute a good alternative to $3 \mathrm{He}$ proportional counters, now too costly because of $3 \mathrm{He}$ shortage, owing to their low cost and good detection efficiency for fast neutrons. However, their high sensitivity to gamma rays and cross talk might be overwhelming drawbacks. Two types of $120 \mathrm{~L}$ technological waste drums have been modelled with organic and metallic matrixes contaminated with plutonium in different localization, and MCNP PoliMi output data have been processed with ROOT. A $5 \mathrm{~cm}$ thick lead shield is defined to attenuate $\mathrm{Pu}$ and Am gamma radiation level on the detectors. For the studied cases, triple coincidences due to $240 \mathrm{Pu}$ spontaneous fissions represent more than $90 \%$ of the counts when using cross talk rejection algorithms, with respect to parasitic counts due to $(\mathrm{a}, \mathrm{n})$ reactions, or to Am and $\mathrm{Pu}$ gamma rays. This fraction is not as high for doubles, but it remains larger than $57 \%$. Cross-talk rejection strongly reduces counting statistics, but this last is still a few thousand triples for $1 \mathrm{~g}$ of $\mathrm{Pu}$ in $25 \mathrm{~min}$. On the other hand, localization uncertainties remain acceptable for both the metallic and organic matrices. First experimental tests are also presented, using $252 \mathrm{Cf}, \mathrm{Pu}$ (spontaneous fission) and $\mathrm{AmBe}(\mathrm{a}, \mathrm{n})$ neutron sources, inside inactive organic and metallic matrixes.

N56-4: Self-Powered Detectors for Test Blanket Modules in ITER P. Raj ${ }^{1}$, M. Angelone ${ }^{2}$, U. Fischer ${ }^{1}$, A. Klix ${ }^{1}$ 
${ }^{I}$ Karlsruhe Institute of Technology, Eggenstein Leopoldshafen, Germany

${ }^{2}$ ENEA CR, Frascati, Italy

Various types of nuclear detection instruments will be installed in the Test Blanket Modules (TBM) of ITER, the fusion reactor under construction at St. Paul lez Durance in France. In this work, experimental tests and computational modelling are undertaken to check the possibility of using Self Powered Detectors (SPD) for the measurement of neutron and/or gamma flux in European ITER TBMs. Such detectors are commonly used in fission reactors for neutron monitoring due to their compactness and high-reliability. Commercial Self Powered Neutron Detectors (SPND) are tailored to the application in thermal fission reactors and are thus, sensitive to low energy neutrons. To make it responsive to fast neutrons (up to D-T neutron energy) present in TBM, new materials were selected to replace the central emitter of SPND. A test detector with a flexible design was prepared in-house and used for experiments with $14-\mathrm{MeV}$ neutron generator at the Technical University of Dresden. Direct current in the range of 1E-13 to 1E-11 Ampere was measured, which varied proportionally with neutron flux. In case of the beryllium-emitter based SPND, multiple factors were found to affect the small current output. An alternate method using plastic scintillator based counting was used to compare the electron emission rates from different material foils, which could make emitter-collector combinations. Furthermore, a multi-step Monte Carlo particle transport method for the estimation of the sensitivity of these detectors to the relevant neutron and gamma spectra is used to check the appropriate combinations of materials and their sizes.

The work leading to this publication has been funded partially by Fusion for Energy under the Specific Grant Agreement F4E-FPA-395-1. This publication reflects the views only of the authors, and Fusion for Energy cannot be held responsible for any use which may be made of the information contained therein.

N56-5: Neutron Activation System for the European ITER Test Blanket Modules

A. Klix ${ }^{1}$, U. Fischer ${ }^{1}$, D. Gehre ${ }^{2}$, B. Ghidersa ${ }^{1}$, K. Tian ${ }^{1}$

${ }^{I}$ Karlsruhe Institute of Technology, Eggenstein-Leopoldshafen, Germany

${ }^{2}$ Technical University of Dresden, Dresden, Germany

Test Blanket Modules (TBM) will be installed in the experimental reactor ITER with the aim to investigate the nuclear performance of different breeding blanket designs for future power fusion reactors. A very important parameter for many TBM experiments is the neutron flux density inside the TBM since the neutron flux determines other parameters such as tritium production rate, nuclear heating, material activation including production of mobile radioisotopes. Currently there is no fully qualified neutronics instrumentation available for the TBMs which would be able to withstand the harsh environment conditions such as high temperature (up to $650 \mathrm{oC}$ ) and, depending on the operation scenario, intense radiation. KIT is developing a neutron activation system for the measurement of neutron flux densities in selected positions in the TBM based on pneumatic activation probe transport. A preliminary engineering assessment of the pneumatic transport system has been performed and extensive irradiation tests of selected activation materials and $14 \mathrm{MeV}$ neutrons from an intensive DT neutron generator were done. Here we summarize the results of the engineering assessment and focus in particular on the results of the irradiation tests and the uncertainty of the measurement method.

The work leading to this publication has been funded partially by Fusion for Energy under the Specific Grant Agreement F4E-FPA-395-1 and OMF-331-02-01-02. This publication reflects the views only of the authors, and Fusion for Energy cannot be held responsible for any use which may be made of the information contained therein.

N56-6: Fast Megavoltage X-Rays Radioscopy

N. Estre, E. Payan, L. Berge

CEA, St Paul Lez Durance, FRANCE

As part of its R\&D programs on severe accidents, CEA is commissioning a major upgrade of its radioscopic X-ray setup in the cell named KROTOS, at Cadarache. This radioscopic tool allows high-energy radiography acquisition on a large phosphor surface $(30 \times 40 \mathrm{~cm} 2)$ with fine resolution (1920x2560 pixels), high frame-rate (200 fps) and during few minutes. Since these performances aren't available with any commercial detector, a prototype has been built with state-of-the-art components. The Xray source is a $9 \mathrm{MeV}$ LINAC which produces short pulses of Bremsstrahlung X-ray ( $15 \mathrm{~Gy} / \mathrm{min}$ at 1 meter in the beam axis). The phosphor screen is made with GADOX deposition on a brass plate. In order to obtain the expected framerate, four low-noise scientific-CMOS cameras are running simultaneously. Each camera output (1920x2560 16 bits-pixels at $50 \mathrm{~Hz}$ ) provides large data flow (3.7 Gbit/s per camera). This data flow is buffered in computer RAM, then saved on fast solid state drives. The setup can sustain image acquisition during $1.5 \mathrm{~min}$, corresponding to $18 \mathrm{k}$ images and $1.3 \mathrm{Tbit}$. The last step is the four-camera-output merging, accounting for parallax correction and lens distortion.

The multi-camera radioscopy principle and synchronization constraints are presented. Image merging methods are then discussed. Experimental performances on phantoms are presented and compared to simulation results. 
N56-7: A Combined Compton Camera and LIDAR System for 3D Imaging of Near-Field Gamma Sources A. C. Caffrey ${ }^{1}$, L. J. Harkness-Brennan ${ }^{1}$, D. S. Judson ${ }^{1}$, A. J. Boston ${ }^{1}$, H. C. Boston ${ }^{1}$, J. R. Cresswell ${ }^{1}$, P. J. Nolan ${ }^{1}$, A. Patel ${ }^{1}$, G. Randall $^{1}$, C. Reid ${ }^{1}$, E. A. Rintoul ${ }^{1}$, C. Unsworth ${ }^{1}$, T. F. Woodroof ${ }^{1}$, K. D. Atkinson ${ }^{2}$

${ }^{I}$ Dept. of Physics, University of Liverpool, Liverpool, UK

${ }^{2}$ Nuclear Dept., Defence Academy, Gosport, UK

An imaging modality is described which integrates radiometric positional information from a Compton camera into a $3 \mathrm{D}$ image of the environment as produced by a Light Detection and Ranging (LIDAR) system. The aim of this project is to image nuclear control rods in order to determine the presence, identity and location of radioactive substances. The Compton camera used in this work was a 3-tier system located at the University of Liverpool which has a good efficiency up to $\mathrm{E}_{?}=2 \mathrm{MeV}$. Compton cameras can determine the location of a gamma-ray source to the position on the surface of a cone. The number of conic overlaps in a $2 \mathrm{D}$ slice of the imaging space from the front of the detector was calculated by a novel analytical reconstruction code. This information was used by a dedicated $\mathrm{C}++$ algorithm to produce point-cloud representations of radioactive sources by gating on the slice intensity. Integration of these point clouds into LIDAR images has been found to be accurate to within $1 \mathrm{~cm}$ of the known source location. The technique is fast and not computationally intensive. Further work is being conducted to determine the potential of this method for imaging distributed gamma-ray sources and gamma-ray sources at greater stand-off distances. The method to achieve the data coupling is presented here, along with the performance of the system for a series of test data.

\section{N57: Photodetectors III - Other photodetectors}

Thursday, Nov. 3 14:00-16:00 Curie 1

N57-1: Characterization of the IDP2 SPAD Array Chip

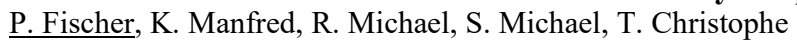

Circuit Design and Simulation, Institute for Computer Engineering, Heidelberg University, Mannheim, Germany

The IDP2 chip contains an array of $88 \times 88$ single photon sensitive avalanche photo diodes which can be read out in full frame mode with up to 400.000 frames per second. Each pixel has an area of $\sim 56$ um x 56um with $\sim 55 \%$ fill factor. The chip contains a low-skew self triggering logic with selectable (column) multiplicities of up to $>3$ hits within an adjustable coincidence time window. The chip is being characterized with a precisely focussed fast pulsed laser. Results on measured fill factor, crosstalk, dark count rates and timing resolution will be presented. As an example, a preliminary response time spread in a single pixel of sigma $<70$ ps and a similar dispersion of average response times across the array have been determined.

N57-2: Microscale Mapping of the Photon Detection Probability of SPADs

$\underline{\text { E. Gros-Daillon }}^{1}$, L. Verger ${ }^{1}$, D. A. B. Bonifacio ${ }^{1,2}$, E. Charbon ${ }^{3}$, C. Bruschini ${ }^{4}$, L. H. C. Braga ${ }^{5}$, L. Gasparini ${ }^{5}$, N. Massari ${ }^{5}$,

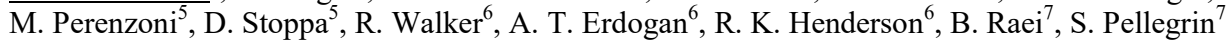

${ }^{l}$ MINATEC Campus, CEA - LETI, Grenoble, France

${ }^{2}$ IRD/CNEN, Institute of Radioprotection and Dosimetry, Rio de Janeiro, Brazil

${ }^{3}$ Delft University of Technology, Delft, The Netherlands

${ }^{4}$ EPFL, Lausanne, Switzerland

${ }^{5}$ Integrated Radiation and Image Sensors (IRIS), Fondazione Bruno Kessler, Trento, Italy

${ }^{6}$ CMOS Sensors and Systems (CSS) Group, School of Engineering, the University of Edinburgh, Edinburgh, United Kingdom

${ }^{7}$ Imaging Division, STMicroelectronics, Edinburgh, United Kingdom

A setup based on a microscope, a spectrograph and a servo positioning system is used to map the homogeneity of the Photon Detection Probability (PDP) within Single Photon Avalanche Diodes (SPADs), along the anode and the guard ring. A $1 \mu \mathrm{m}$ light spot is focused onto the sensor surface and the SPAD triggering rate is recorded with respect to the spot position. Varying the wavelength of the incoming light from $420 \mathrm{~nm}$ to $600 \mathrm{~nm}$ enables to probe the device volume from the P-WELL to the N-WELL. The obtained maps are compared to the electric field computed by Technology Computer-Aided Design (TCAD). The PDP is homogenous with the SPAD surface with a loss of efficiency around the edges, which is due to the implanted PWELL guard ring width around the $\mathrm{P}+$ breakdown region and to the field curvature at the edge of the $\mathrm{P}+$ region.

N57-3: Capacitive Signal Coupling Through the Vacuum Package in LAPPD(TM) Detectors $\underline{B}_{\text {. W. Adams }}^{1}$, M. R. Foley ${ }^{1}$, M. J. Minot ${ }^{1}$, E. J. Angelico ${ }^{2}$, T. M. Seiss ${ }^{2}$, A. Elagin ${ }^{2}$, H. J. Frisch ${ }^{2}$, M. J. Wetstein ${ }^{1}$

${ }^{1}$ Incom, Inc., Charlton, MA, U.S.

${ }^{2}$ Physics, University of Chicago, Chicago, IL, U.S.

LAPPD is a photon-detector technology based upon amplification of photoelectrons in large-area microchannel plates and fast waveform-sampling electronics. In the next-generation design, the signals will be coupled capacitively through the vacuum 
package, thus enabling flexible readout configurations on an external printed-circuit card, improving signal bandwidth, and eliminating all but one electric feedthrough.

\section{N57-4: LAPPDTM Manufacturing Test \& Performance Results}

M. J. Minot ${ }^{1}$, C. A. Craven ${ }^{1}$, A. Lyashenko ${ }^{1}$, J. W. Elam², A. A. Mane ${ }^{2}$, O. H. W. Siegmund ${ }^{3}$, C. Ertley ${ }^{3}$, H. J. Frisch ${ }^{4}$, A. Elagin $^{4}$, B. W. Adam ${ }^{1}$, M. J. Aviles ${ }^{1}$, J. L. Bond ${ }^{1}$, T. Cremer $^{1}$, M. R. Foley ${ }^{1}$, M. A. Popecki ${ }^{1}$, M. E. Stochaj ${ }^{1}$, W. A. Worstell ${ }^{1}$

${ }^{I}$ Research \& Development, Incom Inc., Charlton, MA, USA

${ }^{2}$ Energy Systems Division, Argonne National Laboratory, Lemont, IL, USA

${ }^{3}$ Space Sciences Laboratory, University of California, Berkeley, CA, USA

${ }^{4}$ HEP, Enrico Fermi Institute, University of Chicago, Chicago, IL, USA

The LAPPD ${ }^{\mathrm{TM}}$ is a microchannel plate (MCP) based large area picosecond photodetectors, capable of imaging with single-photon sensitivity at high spatial and temporal resolutions in a hermetic package with an active area of 400 square centimeters for applications including particle and astro-particle physics, Cherenkov imaging, medical imaging, fast imaging, homeland security systems and neutron imaging. The "baseline" LAPPD"M employs an all-glass hermetic package with top and bottom plates and sidewalls made of borosilicate float glass. Signals are generated by a bi-alkali Na2KSb photocathode and amplified with a stacked chevron pair of MCPs produced by applying resistive and emissive atomic layer deposition coatings to borosilicate glass capillary array (GCA) substrates. Signals are collected on RF strip-line anodes applied to the bottom plates which exit the detector via pin-free hermetic seals under the side walls. Early fully functional but dynamically pumped prototypes were shown to achieve gains greater than $10^{\wedge} 7$, gain variability $<15 \%$, a time response for single photons of better than 50 ps (less than $10 \mathrm{ps}$ for charged particles), and a spatial resolution of $700 \mu \mathrm{m}$ in each of the two lateral dimensions. Initial attempts to produce fully functional, self-contained LAPPD ${ }^{\mathrm{TM}}$ encountered mechanical and process issues effectively addressed in the pilot production manufacturing environment described here. Early prototype test and performance results are reviewed as well as the current status of early manufacturing

This paper was submitted for review on May 3, 2016. This work was supported by the US Department of Energy Phase II SBIR TTO Contract award DE-SC0009717 with additional funding provided by the National Geospatial Intelligence Agency.

\section{N57-5: The Tynode: a New Vacuum Electron Multiplier for Ultra Fast Pixelised Particle Detectors}

H. van der Graaf

Detector R\&D, Nikhef, Amsterdam, Netherlands

On behalf of the MEMBrane Group

By placing, in vacuum, a stack of transmission dynodes (tynodes) on top of a CMOS pixel chip, a single free electron detector could be made with outstanding performance in terms of time and spatial resolution. Its essential element is the tynode: an ultra thin membrane, which emits, at the impact of an energetic electron on one side, a multiple of electrons at the other side. The electron yields of tynodes have been calculated by means of GEANT-4 Monte Carlo simulations, applying special low-energy extensions. The results are in line with another simulation based on a continuous charge-diffusion model. By means of MEMS technology, tynodes and test samples have been realised. The secondary electron yield of several samples have been measured in three different stations. Our best result so far is a transmission secondary electron yield of 2.7, obtained with an Alumina membrane. Several possibilities to improve the yield are presented.

This work is supported by the ERC-Advanced Grant 2012 nr. 320764 MEMBrane.

N57-6: The Novel ABALONE Photosensor Technology

D. Ferenc ${ }^{1}$, A. Chang ${ }^{1}$, M. Segedin Ferenc ${ }^{2}$

${ }^{I}$ Physics, University of California, Davis, CA, USA

${ }^{2}$ PHOTONLAB Inc., Davis, CA, USA

The fields of astroparticle physics, nuclear medical imaging and nuclear security — which all rely on large-area photosensorshave been virtually stuck with the expensive 80-year old photomultiplier tube (PMT) manufacture. PMTs comprise more than 180 hand-made components, created out of $\sim 8$ disparate materials. That compexity necessitates a very slow, labor-intensive and expensive vacuum processing, incompatible with low-cost mass-production. Our search for a modern mass-production technology has therefore started with a fundamental redefinition of allowed processes and materials. The patented-and by now fully proven-ABALONE technology for inexpensive mass-production of large-area photosensors is based on only 3 vacuumprocessed components - exclusively mass-producible of glass. Metals, silicon, microchannel plates, ceramics and other materials have been strictly excluded from our consideration. That also means - the absence of electrodes and feedthroughs. The 3 glass components are processed by the common thin-film deposition technique (equivalent to CDs), and bonded using our novel 
vacuum encapsulation method, based on a new thin-film material, synthesized in our laboratory. The production process has been thoroughly proven in our pilot production plant by 2012. We report on the unique performance results of an ABALONE Photosensor prototype, tested by now continuously for 3 years. The unprecedentedly low, and even constantly dropping afterpulsing rate proves the vacuum integrity. We will report on an exceptional single-photon resolution, the resolution of backscattered photoelectrons, very wide dynamic range, excellent timing resolution, and the prospects for an unprecedentedly low radioactivity, the unique suitability for large neutrino detectors, and inexpensive modular arrays and large-area detectors, including the ultrasensitive, low-radiation whole-body-enclosing ABALONE-PET scanner for preventive screening of a symptom-free population.

\section{N57-7: FASPAX: a Fast, Integrating Detector for the APS-Upgrade}

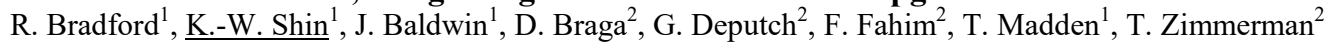

${ }^{I} X$-Ray Science Division, Argonne National Laboratory, Argonne, IL, USA

${ }^{2}$ Particle Physics Division, Fermi National Accelerator Laboratory, Batavia, IL, USA

FASPAX (Fermi-Argonne Semiconducting Pixel Array X-ray detector) has been planned as a fast integrating area detector with wide dynamic range for time resolved applications at the upgraded Advanced Photon Source (APS). The detector will achieve a burst image rate of $13 \mathrm{MHz}$, matching the bunch rate of the proposed storage ring timing mode. A unique passive adoptive gain control integration circuit will permit wide dynamic range - from single photon sensitivity to 10 to 5 photons/pixel $(100 \mu \mathrm{m}$ by $100 \mu \mathrm{m})$ in a single exposure. Incorporation of a novel interposer layer in the hybrid stack will permit large area sensors without the usual coverage gaps associated with hybrid pixel devices. The detector will provide access to the full temporal resolution of the APS, and enable novel science, such as pump-probe studies of irreversible systems.

The APS Upgrade is currently funding development of small prototypes $(5.5 \mathrm{~cm}$ by $5.5 \mathrm{~cm})$ that will be tested in the next few years. This presentation will present an overview of the detector, and test results of the first prototype hybrid detectors.

Acknowledgement Work at Argonne National Laboratory was supported by the U. S. Department of Energy, Of?ce of Science, Of?ce of Basic Energy Sciences, under Contract No. DE-AC02-06CH11357.

N57-8: A New Spectroscopic Imager for X-Rays from $0.5 \mathrm{keV}$ to $150 \mathrm{keV}$ Combining a pnCCD and a Columnar CsI(TI) Scintillator

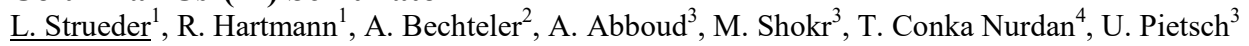

${ }^{I}$ PNSensor GmbH, Munich, Germany

${ }^{2}$ PNDetector GmbH, Munich, Germany

${ }^{3}$ Department of Physics, University of Siegen, Siegen, Germany

${ }^{4}$ Türkisch-Deutsche Universität, Istanbul, Turkey

By combining a low noise fully depleted pnCCD detector with a columnar CsI(Tl) scintillator an energy dispersive spatial resolving detector can be realized with a high quantum efficiency (QE) in the range from below $0.5 \mathrm{keV}$ to above $150 \mathrm{keV}$. The columnar $\mathrm{CsI}(\mathrm{Tl})$ scintillator increases the pulse height of the Gamma rays converted in the $\mathrm{CsI}(\mathrm{Tl})$, due to focusing properties of the columnar scintillator structure. This also reduces the event size in indirect detection mode (conversion in the scintillator). In direct detection mode (conversion in the silicon of the pnCCD) the relative energy resolution is $0.7 \%$ at $122 \mathrm{keV}(820 \mathrm{eV}$ FWHM) and the spatial precision is less than $75 \mu \mathrm{m}$. In indirect detection mode the relative energy resolution integrated over all event sizes is about $10 \%$ at $122 \mathrm{keV}$ with a spatial precision extracted from simulations of less than $30 \mu \mathrm{m}$ with the help of centroiding the scintillator light cone. Measurements performed at the BESSY synchrotron around $80 \mathrm{keV}$ will be shown.

N58: Gaseous detectors III: Applications in Large Experiments

Thursday, Nov. 3 14:00-16:00 Curie 2

N58-1: First Results from a Prototype Combination TPC Cherenkov Detector with GEM Readout

B. Azmoun ${ }^{1}$, T. Hemmick ${ }^{2}$, R. Majka ${ }^{3}$, M. Phipps ${ }^{4}$, M. L. Purschke ${ }^{1}$, N. Smirnov ${ }^{3}$ C. Woody ${ }^{1}$, A. Zhang

${ }^{I}$ Physics Department, Brookhaven National Lab, Upton, NY, USA

${ }^{2}$ Physics Department, Stony Brook University, Stony Brook, NY, USA

${ }^{3}$ Physics Department, Yale University, New Haven, CT, USA

${ }^{4}$ Physics Department, University of Illinois, Urbana-Champaign, IL, USA

${ }^{5}$ Physics Depoartment, Florida Institute of Technology, Melbourne, FL, USA

A multipurpose detector that combines the functions of a Time Projection Chamber for particle tracking and a Cherenkov detector for particle identification is being developed for possible applications at a future Electron Ion Collider. The TPC portion consists of a $10 \times 10 \times 10 \mathrm{~cm}^{\wedge} 3$ drift volume where the charge is drifted to a $10 \times 10 \mathrm{~cm}^{\wedge} 2$ quadruple GEM detector and measured on a readout plane consisting of $2 \times 10 \mathrm{~mm}^{\wedge} 2$ chevron pads. The Cherenkov portion consists of a second $10 \times 10 \mathrm{~cm}^{\wedge} 2$ quadruple 
GEM with a photosensitive CsI photocathode on the top layer that measures Cherenkov light produced in the drift volume of the TPC. CF4 was used as the drift gas due to its high transparency into the deep UV in order to provide high efficiency for detecting Cherenkov light. The GEM detectors were also operated in the same gas volume, thus providing a windowless configuration for the Cherenkov in order to maximize its efficiency. A prototype of this detector was tested in a test beam at Fermilab in April of 2016 and the results from those tests will be presented in this talk. Applications for such a detector at a future Electron Ion Collider will also be discussed.

\author{
N58-2: The KLOE-2 Cylindrical GEM Inner Tracker: Detector Operation, Calibration and Performance \\ E. De Lucia \\ INFN-LNF, Frascati, Rome, Italy \\ On behalf of the KLOE-2 Inner Tracker Group
}

KLOE-2 at the e+e- DAPHNE $\$$ Phi $\$$-factory is the main experiment of the INFN Frascati National Laboratories (LNF) and represents the continuation of the KLOE experiment, upgraded with state of the art technology to improve its discovery potential. KLOE-2 is the first high-energy experiment using the GEM technology with a cylindrical geometry, a novel idea that was developed at LNF exploiting the kapton properties to build a light and compact tracking system. Four concentric cylindrical triple-GEM detectors, for a total material budget below $2 \backslash \%$ of the radiation length $\$ X \_\$$, are inserted around the interaction region and before the inner wall of the pre-existing KLOE Drift Chamber, at distances from $130 \mathrm{~mm}$ to $205 \mathrm{~mm}$. For this project, state-of-the-art solutions have been expressly developed or tuned: single-mask GEM etching, multi-layer XV patterned readout circuit, PEEK spacer grid, GASTONE front-end board, a custom 64-channel ASIC with digital output, and the Global Interface Board for data collection, with a configurable FPGA architecture and Gigabit Ethernet. The dedicated XV strips patterned readout allows space coordinates to be reconstructed. Alignment and calibration of a cylindrical GEM detector was never done before and represents one of the challenging activities of the experiment. Preliminary results obtained with cosmic-ray muons and Bhabha scattering events are within expectations for the Inner Tracker resolution. During 2015 both KLOE-2 and DAPHNE successfully demonstrated the feasibility of a long term acquisition program with the first data taking campaign, started in November 2014 and ended in July 2015 with $1 \mathrm{fb} \$^{\wedge}\{-1\} \$$ integrated luminosity. The second new data taking campaign started in September 2015 and KLOE-2 is presently taking data. The Inner Tracker detector operation, calibration and performance will be presented.

N58-3: Performance Tests of a Resistive Micromegas Detector Quadruplet $\underline{\text { J. Bortfeldt }}$ EP, CERN, Geneva, Switzerland

Resistive Micromegas have been developed in recent years with the aim of making this technology usable in HEP experiments where the high sparking rate of classical Micromegas is not tolerable. A resistive Micromegas with four layers with active surface of $0.4 \mathrm{~m} 2$ each has been designed and built at CERN as prototype of the detectors to be used for the upgrade of the ATLAS experiment. The performances of the detector have been deeply studies with cosmics, X-rays and high-energy beams. Detector efficiency higher than $98 \%$, spatial resolution of the order of $100 \ddot{i}_{i}^{1} / 2 \mathrm{~m}$ and good amplification homogeneity have been measured. The detector has been exposed to an intense gamma source $(14 \mathrm{TBq})$ showing a rate capability higher than 15 $\mathrm{KHz} / \mathrm{cm} 2$.

\author{
N58-4: Design, Construction, and Performance of the Slice Test Detectors for the Forward Muon Upgrade of \\ the CMS Detector \\ B. L. Dorney \\ EP/CMX, CERN, Genève, Genève, Switzlerand \\ On behalf of the CMS Collaboration
}

The Forward Muon Upgrade of the CMS detector is entering a critical stage; with the slice test installation planned for the first quarter of 2017, followed by the full installation during the second long shutdown of the LHC, the detector mass production has begun in earnest. After six years of R\&D on Gas Electron Multiplier (GEM) technology, the CMS Collaboration has developed a system to instrument the vacant high-eta region of the CMS muon system using large-area triple-GEM detectors. These new large-area detectors are 1200 -by- $446 \mathrm{~mm}^{\wedge} 2$ at maximum acceptance and cover a psuedorapidity range of $1.6<\mid$ eta $\mid<2.2$. Here we present techniques involved in the construction and characterization of the slice test detectors. Measurements of detector gain and response uniformity collected with charge-sensitive front-end electronics will be presented. Additionally expectations on the system performance within CMS will be shown based on cosmic-ray data-taking campaigns.

\author{
N58-5: Development and Test of a microTPC Cluster Reconstruction for a Triple GEM Detector in Strong \\ Magnetic Field
}


G. Cibinetto

INFN Ferrara, Ferrara, Italy

On behalf of the BESIII GEM group

Performance of triple GEM prototypes has been evaluated by means of a muon beam at the H4 line of the SPS test area at CERN. The data from two planar prototypes have been reconstructed and analyzed offline with two readout methods: charge centroid and micro-Time-Projection-Chamber.

GEM prototype performance evaluation, performed with the analysis of data from a TB, showed that two-dimensional cluster efficiency is above $95 \%$ for a wide range of operational settings. Concerning the spatial resolution, the charge centroid cluster reconstruction performs extremely well with no magnetic field: the resolution is well below 100 microns. Increasing the magnetic field intensity, the resolution degrades almost linearly as effect of the Lorentz force that displaces, broadens and asymmetrizes the electron avalanche. Tuning the electric fields of the GEM prototype we could achieve the unprecedented spatial resolution of 190 microns at 1 Tesla.

In order to boost the spatial resolution with strong magnetic field and inclined tracks a microTPC cluster reconstruction has been investigated. Such a readout mode exploits the good time resolution of the GEM detector and electronics to reconstruct the trajectory of the particle inside the conversion gap. Beside the improvement of the spatial resolution, information of the track angle can be also extracted.

The new clustering algorithm has been tested with diagonal track with no magnetic field showing a resolution between 100 microns and 150 microns for the incident angle ranging from 10? to 45?. Preliminary studies show similar performance with 1 Tesla magnetic field. This is the first use of a microTPC readout with a triple GEM detector in magnetic field.

This study has shown that a combined readout is capable to guarantee stable performance over a broad spectrum of particle momenta and incident angles, up to a 1 Tesla magnetic field.

N58-6: The MPGD-Based Photon Detectors for the Upgrade of COMPASS RICH-1

$\underline{\text { S. Dalla Torre }}$

Sezione di Trieste, INFN, Trieste, Italy

On behalf of the The COMPASS RICH group

The RICH-1 Detector of the COMPASS Experiment at CERN SPS is undergoing an important upgrade for the physics run 2016: four new Photon Detectors, based on MPGD technology and covering a total active area larger than 1.2 square meters will replace the actual MWPC-based photon detectors in order to cope with the challenging efficiency and stability requirements of the new COMPASS measurements. The new detector architecture consists in a hybrid MPGD combination: two layers of THGEMs, the first of which also acts as a reflective photocathode (a CsI layer is deposited on its top face) are coupled to a bulk Micromegas on a pad segmented anode; the signals are read-out via capacitive coupling by analog F/E based on the APV25 chip. The related R\&D is shortly recalled. All aspects of the COMPASS RICH-1 Photon Detectors upgrade are presented and large emphasis is dedicated to the engineering aspects, the mass production and the quality assessment of the MPGD components. In particular, the design and production of the detector components, the assembling and the validation tests of THGEMs and Micromegas and the engineering challenges of the detector installation are presented together with the expected performance of the upgraded COMPASS RICH-1. Preliminary performance figures are provided.

\author{
N58-7: Performance of the RICH Detector of the NA62 Experiment \\ V. Duk \\ INFN Perugia, Perugia, Italy \\ On behalf of the NA62 collaboration
}

The NA62 experiment at CERN is aimed at measuring the ultra-rare charged kaon decay into a charged pion and two neutrinos with a $10 \%$ uncertainty. The main background comes from the charged kaon decay into a muon and a neutrino which is suppressed by applying specific selection criteria on kinematic variables and taking use of the different stopping power of muons and pions in the calorimeters. A RICH detector is needed to further suppress the $\mu+$ contamination in the $\mathrm{p}+$ sample by a factor of at least 100 in the momentum range between 15 and $35 \mathrm{GeV} / \mathrm{c}$, to measure the pion crossing time with a resolution of about 100 ps and to produce the trigger for a charged track. The detector consists of a $17 \mathrm{~m}$ long tank (vessel), filled with Neon gas at atmospheric pressure. The Cherenkov light is reflected by a mosaic of 20 spherical mirrors with $17 \mathrm{~m}$ focal length, placed at the downstream end, and collected by 1952 photomultipliers placed at the upstream end. The RICH detector installation was completed in the summer of 2014 and the detector was operating during the pilot run at the end of 2014 and during the NA62 Commissioning Run in 2015. The RICH will be used in the 2016 Physics Run. During the winter shutdown 2015-2016 the detector maintenance was carried out in order to improve the performance, especially the mirror alignment, which was found to be crucial for the efficient pion-muon separation. In this talk the construction of the detector will be described and the performance during the 2014-2015 data taking period will be discussed. Preliminary results of the 2016 data-taking will also be presented. 
N58-8: The Straw Tube Tracker for the Mu2e Experiment

$\underline{\text { V. L. Rusu }}$

PPD, Fermilab, Batavia, IL, USA

On behalf of the mu2e collaboration

The Mu2e experiment will search for neutrinoless conversion of muons into electrons In the field of an aluminum nucleus. The signature of this process is an electron with energy nearly equal to the muon mass. Precise and robust measurement of the outgoing electron momentum is an essential element to the experiment. We describe the design of a low mass tracking system to meet this requirement. The tracker must operate in a vacuum and a 1 Tesla magnetic field. We have chosen to use about 20,000 thinwall Mylar straws held under tension to avoid the need for supports within the active volume. In addition to measuring distance from the wire by drift time, subnanosecond measurement of signal propagation time will be used to measure position along the wire. Charge will be measured using ADCs to provide particle identification capability. To minimize the number of vacuum penetrations, digitization will be performed on the detector. Readout will use a triggerless, streaming architecture with data transmitted on optical fiber.

N59: Instrumentation for Security III

Thursday, Nov. 3 16:30-18:30 Madrid

N59-1: Detection of Special Nuclear Materials with Tagged Neutrons

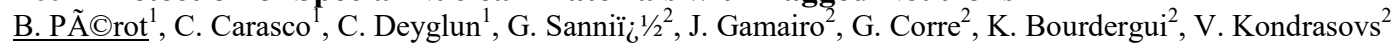

${ }^{I}$ DEN, Cadarache, CEA, Saint-Paul-lez-Durance, France

${ }^{2}$ LIST, Saclay, CEA, Gif-sur-Yvette, France

The Associated Particle Technique can be used to detect Special Nuclear Materials in cargo containers. A DT neutron generator, based on the $3 \mathrm{H}(2 \mathrm{H}, \mathrm{n}) \mathrm{i}$

embedded alpha detector. Prompt neutrons and gamma rays emitted during neutron-induced fissions are detected by plastic scintillators, in coincidence with the alpha particle. Nuclear materials are differentiated from non-nuclear materials and cross-talk events using high multiplicity coincidences. The data acquisition electronics is made of compact FPGA boards. Experiments performed with a mock-up of the measurement system are reported and compared to calculations to validate numerical simulation and post-processing tools. Measurements have been performed with different targets made of iron, lead, or uranium, placed in metallic or wood cargo matrixes. The performances of a full scale cargo container inspection system, with a larger number of detectors optimized for container inspection, are also studied by numerical simulation. Random background due to neutrons which are not correlated with an alpha particle, counting statistics, time and energy resolutions of the data acquisition system, are all taken into account to model the measurements as realistically as possible. A wide range of inspections, with the suspect item in different positions in different cargo matrixes, have been studied.

N59-2: Recent Results on Complementing the CRIPT Muon Tomography Detector with Passive Neutron and Gamma-Ray Systems for the Detection and Imaging of Shielded Special Nuclear Materials

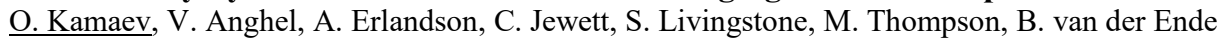

Canadian Nuclear Laboratories Ltd, Chalk River, ON, Canada

A unique muon tomography system with a capability of measuring muon momentum, the Cosmic Ray Inspection and Passive Tomography (CRIPT) detector, has been in operation at Canadian Nuclear Laboratories since 2013. The system demonstrated the ability to detect high-density/high-Z special nuclear materials (SNM) inside shielded containers and is promising for border security and non-proliferation applications. Recently, passive neutron and gamma-ray detectors were added to complement CRIPT muon measurements. Results from blind tests to examine integration of these multiple technologies for the detection of shielded SNM will be presented.

N59-3: High-Resolution Imaging of Nuclear Waste Containers with Muon Scattering Tomography $\underline{\text { L. Frazao, }}$ S. Maddrell-Mander, J. Velthuis, C. Thomay

University of Bristol, Bristol, UK

We have developed an algorithm to define edges of uranium blocks embedded in concrete. There is a need to characterise large containers of nuclear waste without having to open it. Muon scattering tomography uses the natural occurring cosmic muons as probes, which does not introduce additional radiation and can be used to scan large volumes. Muons undergo multiple Coulomb scattering when passing through matter, and the amount of scattering depends on the atomic number $\mathrm{Z}$ of the material. So it is possible to perform imaging of different materials by measuring the incoming and outgoing muon tracks. We had previously developed a $3 \mathrm{D}$ imaging algorithm to find lumps of high- $\mathrm{Z}$ materials in concrete. This algorithm divided the volume in bins, 
where the most likely scatter positions were reconstructed as single vertices. A distribution of a weighted distance between each pair of vertices was obtained for each bin. In order to achieve a better resolution, a method of defining the edges was created by studying that distribution in the transition bins between concrete and uranium. We performed simulations of uranium objects of different lengths, enclosed in concrete. These lengths were measured with the new method and compared with the simulated lengths, resulting in a resolution of $0.7 \mathrm{~mm}$, with a $0.1 \mathrm{~mm}$ error. The smallest length measured was a uranium sheet with a width of $2 \mathrm{~mm}$.

\author{
N59-4: Portable Muon Scattering Tomography Detectors for Security Imaging Applications \\ J. Burns \\ AWE plc, Reading, United Kingdom
}

Muon scattering tomography techniques can be used to image the contents of an enclosed volume by measuring the scattering angle of cosmic ray muons as they pass through a volume using particle tracking detectors. The magnitude of the observed muon scatter varies with the density of the objects within a volume, therefore the technique is well suited to distinguishing dense (highZ) materials from lower density (low-Z) materials. AWE has been investigating muon scattering tomography techniques for use in the identification of special nuclear material (SNM) in transit cargo for a number of years. As part of this work AWE and our partner institutions have developed a number of prototype muon tomography systems, based on drift chamber and resistive plate chamber technology, and a software suite of reconstruction tools and Monte Carlo simulations that we have used to quantify the capability and limitations of muon tomography techniques for SNM identification.

Recent work at AWE has focused on the development of portable detector technologies for the imaging of objects and packages at unfixed locations. It is envisaged that portable detectors will be positioned to the sides of the screened objects rather than at the top and bottom with the object in the centre. This work has involved the further development of our Monte Carlo models to provide a more detailed of the horizontal muon flux and the implementation of new tracking algorithms to identify muons coming from either side of the tracking system. Part of this work has also involved investigating the use of portable wire chamber and silicon tracking detectors. Our work has also focused on examining the potential improvements to image quality afforded by the detection of spallation neutrons from muon interactions inside an object. The work presented will provide an overview of these recent developments and draw conclusions on the use of muon scattering tomography techniques for deployable security imaging applications.

N59-5: Dual-Particle Transmission Spectroscopy Using a ${ }^{11} B(d, n ?)^{12} C$ Source $\underline{\text { J. Nattress }}^{1}$, M. Mayer ${ }^{2}$, P. Rose ${ }^{3}$, M. Wonders ${ }^{4}$, K. Wilhelm ${ }^{4}$, A. Erickson ${ }^{3}$, I. Jovanovic ${ }^{1}$

${ }^{I}$ The University of Michigan, Ann Arbor, MI, United States

${ }^{2}$ Pacific Northwest National Laboratory, Richland, WA, United States

${ }^{3}$ Georgia Institute of Technology, Atalanta, GA, United States

${ }^{4}$ The Pennsylvania State University, State College, PA, United States

Detection of shielded special nuclear material (SNM) persists as one of the greatest unresolved security problems, specifically in areas of nuclear nonproliferation, safeguards, and material interdiction at ports of entry. Active interrogation (AI) may be used to increase the signal-to-background and penetrate shielding, providing a more viable method to detect SNM. High-energy neutron and photon transmission measurements are promising for rapid scanning and decision making that is needed in time-sensitive SNM detection and verification applications. In this work, we report on a new AI approach that integrates the neutron and gamma transmission characteristics to deduce the material properties that can aid SNM identification. The approach uses a nuclear reaction-based AI source and an imaging system based on a single, particle-discriminating detector type. We demonstrate a practical implementation of this method using the ${ }^{11} \mathrm{~B}(\mathrm{~d}, \mathrm{n} \text { ? })^{12} \mathrm{C}$ gamma- and neutron-producing reaction and an array of eight EJ309 liquid scintillators. Neutron and gamma spectroscopic transmission is used in tandem to perform material identification. We discuss the results of transmission spectroscopy of various materials, compare the experimental results with simple models based on published nuclear data, and demonstrate improved material identification of high-Z materials with this dual-particle measurement approach.

The work was supported by the National Science Foundation, the Department of Homeland Security, and by the Deparment of Energy, National Nuclear Security Administration. The research of J.N. was performed under appointment to the Nuclear Nonproliferation International Safeguards Graduate Fellowship Program sponsored by the National Nuclear Security Administration's Next Generation Safeguards Initiative (NGSI).

N59-6: Development and Testing of the Multi-Sensor Interdiction System Testbed (MIST) Advanced Technology Demonstration (ATD) for Detection and Tracking of Vehicle-Borne Radiation Sources $\underline{\text { D. A. Cooper }}^{1}$, R. J. Ledoux ${ }^{1}$, W. Franklin ${ }^{1}$, K. Kamieniecki ${ }^{1}$, S. E. Korbly ${ }^{1}$, J. Costales ${ }^{1}$, R. Niyazov ${ }^{1}$, D. Hempstead ${ }^{1}$, M. Gallagher ${ }^{1}$, C. Monnier ${ }^{2}$, R. Wronski ${ }^{2}$, A. Ost ${ }^{2}$ 
${ }^{I}$ Passport Systems, Inc., N. Billerica, MA, USA

${ }^{2}$ Charles River Analytics, Inc., Cambridge, MA, USA

The threat represented by loose radioactive materials in the form of radiation dispersal devices (RDDs) and special nuclear materials (SNM) has continued to increase in recent years. Passport Systems, Inc. and Charles River Analytics, Inc. have developed the prototype Multi-sensor Interdiction System Testbed (MIST) to monitor highways and other similar paths without affecting the flow of commerce. The system consists of a distributed network of heterogeneous detectors - specifically radiation detectors, video cameras, and license plate readers - designed to detect and identify radioactive threats, track source vehicles, and provide information to support interdiction by law enforcement. The system is being developed as part of the Radiation Awareness and Interdiction Network (RAIN) Advanced Technology Demonstration (ATD) program sponsored by the US Dept. of Homeland Security, Domestic Nuclear Detection Office (DHS/DNDO). The modular system architecture makes it both rapidly deployable and inherently scalable to any operational scenario. Several advanced data fusion techniques have been developed to utilize the multi-modal data. Specifically, data-level fusion of spatially diverse radiation detector measurements is used to enhance detection and tracking of radiation sources, while extracted feature-level data from the video sensors is used both to improve computational speed and accuracy, and to provide relevant attribution data. An overview of the system design and performance will be provided with special emphasis on algorithmic performance during vendor development testing. Additionally, the lessons learned from the prototype system will be discussed in terms of the technology's operational readiness. This work has been supported by the US Dept. of Homeland Security, Domestic Nuclear Detection Office, under competitively awarded contract HSHQDC-14-C-B0049. This support does not constitute an express or implied endorsement on the part of the Government.

N59-7: Post-Blast Radiological Dispersal Device Source Term Estimation D. L. Chichester, J. T. Johnson, S. M. Watson, S. J. Thompson, N. R. Mann, K. P. Carney

Idaho National Laboratory, Idaho Falls, Idaho, USA

Research is underway to develop instruments and methods to determine the activity of radionuclides present in the fallout debris from the detonation of a radiological dispersal device (RDD). Handheld instruments, including commonly used health physics survey instruments, have been incorporated into a portable telemetry kit containing a global positioning system receiver, WiFi and radio communications, and a small microcomputer to facilitate data processing, logging, and transmission. An operator carries the system and walks through the RDD post-blast environment, real-time radiological data is logged, stored locally, and transmitted to a base station outside of the RDD hot zone. A map of the distributed radiological dispersal is generated, subdividing the world into $1-\mathrm{m}^{2}$ squares. Separate measurements of ground activity taken at a finite number of discrete locations is used to cross-correlate the survey data, transforming the health physics data (e.g., mrem hr ${ }^{-1}$ ) to surface activity $\left(\mathrm{Bq} \mathrm{m}^{-2}\right)$. The map data, smoothed using standard Kriging approaches, is then analyzed by summing each discrete square area, producing an estimate for the total dispersed ground activity. The instrumentation and method have been tested multiple times at Idaho National Laboratory during field exercises using short-lived radionuclides detonated in small-scale experiments.

N59-8: Operation and Sensitivity of the Radioxenon Laboratory at PNNL M. P. Foxe, J. M. Mendez, M. F. Mayer, I. M. Cameron, D. A. Haas, J. C. Hayes, T. W. Bowyer Pacific Northwest National Laboratory, Richland, WA, United States

The measurement of radioactive xenon (Xe-131m, Xe-133, Xe-133m, Xe-135) in the atmosphere is one of the fundamental approaches to monitoring nuclear explosions. One key aspect of radioxenon detection is the ability to process archived samples of interest. Pacific Northwest National Laboratory (PNNL) has developed a laboratory for the purpose of radioxenon processing and measurement of radioxenon samples that have been extracted and concentrated down to pure xenon from atmospheric samples. In this presentation, we will discuss the operation of the beta-gamma radioxenon laboratory at PNNL, and the means of achieving the optimal sensitivity for the four-radioxenon isotopes.

N60: Scintillators V: Applications

Thursday, Nov. 3 16:30-18:30 Londres

N60-1: A Novel Scheme of Compton Imaging for Nuclear Medicine

G. Pausch ${ }^{1}$, A. Schulz ${ }^{1}$, W. Enghardt ${ }^{1,2,3}$

${ }^{1}$ Institute of Radiooncology, Helmholtz-Zentrum Dresden - Rossendorf, Dresden, Germany

${ }^{2}$ OncoRay, Technische Universität Dresden, Dresden, Germany

${ }^{3}$ DKTK - German Cancer Consortium, Dresden, Germany

The paper introduces a novel concept of gamma imaging, the Single-Plane Compton Imager (SPCI), and proofs its applicability for reconstructing distributed sources by basic simulations. An SPCI is a compact array of scintillation crystals with separate light 
readouts, arranged for example in a checkerboard configuration. The passive collimation of conventional gamma cameras is replaced by kind of a "soft" electronic collimation derived from the Compton kinematics. In contrast to Compton cameras, where individual scattering angles are determined event-by-event from coincident energy depositions in separate detector planes, the SPCI reconstructs activity distributions from accumulated "conditional" spectra by using Maximum Likelihood Expectation Maximization algorithms. The condition is a coincident energy deposition in two (adjacent) detector elements of a single plane, which occurs most likely due to Compton scattering in one element followed by absorption in the other one. The SPCI could overcome drawbacks of Anger and Compton cameras: (i) Image resolution and detection efficiency are no competing factors. Both improve with increasing detector area. (ii) The fraction of valid events per hit exceeds that of passively collimated systems or Compton cameras by orders of magnitude. (iii) A single detector construction could fit to a wide range of gamma energies. Last not least, optimal SPCI pixels sizes fit with pixel dimensions in PET scanners. SPCI could thus be based on recent detector developments for PET-MRI featuring individual Si-based pixel readouts.

This work was supported in part by by the German Federal Ministry of Education and Research, grant number BMBF-03Z1NN12.

\author{
N60-2: Development of a SiPM-Based Detection Module for Large LaBr3:Ce Scintillators for Nuclear Physics \\ Applications \\ $\underline{\text { G. Cozzi }}^{1,2}$, J. Agostini ${ }^{1}$, M. Carminati ${ }^{1}$, C. Fiorini ${ }^{1,2}$, C. Piemonte $^{3}$, A. Gola ${ }^{3}$, V. Regazzoni ${ }^{3,4}$ \\ ${ }^{I}$ Dipartimento di Elettronica, Informazione e Bioingegneria, Politecnico di Milano, Milano, Italy \\ ${ }^{2}$ Sezione di Milano, INFN, Milano, Italy \\ ${ }^{3}$ Fondazione Bruno Kessler, Trento, Italy \\ ${ }^{4}$ Università degli studi di Trento, Trento, Italy
}

Aim of the work is to develop a compact and temperature stabilized SiPM-based gamma-ray imaging module that can be adopted in nuclear physics experiments. Silicon PhotoMultipliers (SiPMs) are an emerging solid-state technology as to be considered a promising alternative to the consolidated PhotoMultiplier Tubes (PMTs) in many gamma-ray applications thanks to their excellent timing spectroscopy performances. Nowadays the research efforts are focused on coupling these devices with fast and thick state-of-the-art scintillators like Cerium doped Lanthanium Bromide ( $\mathrm{LaBr} 3$ :Ce) for high energy applications as nuclear physics measurements. The module proposed allows to read the crystals typically adopted in nuclear physics experiments such as 1 " and 2" thick LaBr3:Ce, giving spectroscopic results in line with PMTs standards (3\% at $662 \mathrm{keV})$. New High-Density cell SiPM technology for Near UltraViolet detection (NUV-HD SiPM) (Fondazione Bruno Kessler, Italy) have been studied as optimal choice for the detector. They shows high Photo Detection Efficiency (PDE $=55 \%$ at $400 \mathrm{~nm}$ ) and low Dark Count Rate $(\mathrm{DCR}<100 \mathrm{kHz} / \mathrm{mm} 2)$. The prototype we are developing is based on an array of 5 x $5 \mathrm{SiPMs}$, each one having an active area of $6 \mathrm{~mm} \times 6 \mathrm{~mm}$. This array is used for 1" scintillator readout and it is foreseen as basic unit for the assembly of larger array to read 2" scintillator. Preliminary spectroscopic measurements were performed with an array of $2 \times 2$ NUV-HD SiPMs coupled to a 8 $\mathrm{mm} \times 9 \mathrm{~mm} \mathrm{LaBr} 3: \mathrm{Ce}$. An energy resolution of $7.85 \%$ and $3.96 \%$ has been achieved at $122 \mathrm{keV}$ and $662 \mathrm{keV}$, respectively. Results are promising and in line with the best ones available in literature to our knowledge. Theoretical studies predict negligible worsening of performances by increasing of the detection area for 1" and 2" scintillators readout, thanks to the very low DCR proper of this new technology. Experimental results of 1" $\mathrm{LaBr} 3$ :Ce readout will be presented to validate the theoretical energy resolution.

\author{
N60-3: Development of a Less Expensive Gamma-Ray Detector Sensitive to a Source Direction Using \\ GAGG(Ce) Scintillators and MPPCs \\ $\underline{\text { K. Kojima }}{ }^{1}$, T. Nakamori ${ }^{1}$, S. Gunji ${ }^{1}$, M. Takebe ${ }^{1}$, H. Sato $^{2}$, S. Ito ${ }^{2}$, S. Kato ${ }^{2}$, M. Yoshino ${ }^{2}$, Y. Usuki ${ }^{2}$, J. Kataoka $^{3}$ \\ ${ }^{1}$ 1-4-12 Kojirakawa-machi, Faculty of Science, Yamagata University, Yamagata, Yamagata, Japan \\ ${ }^{2}$ 1-25-13 Kannondai, Furukawa Co., Ltd.,, Tsukuba,Ibaraki, Japan \\ 3-4-1 Okubo, Waseda University, Shinjuku,Tokyou, Japan
}

After the disaster at the Fukushima Daiichi nuclear power plant, serious amounts of radioactive isotopes, such as ${ }^{137} \mathrm{Cs}$ and ${ }^{134} \mathrm{Cs}$, became widespread in the environment. Gamma-ray imaging technique plays a crucial role in localizing radiation hotspots and then assisting in efficient decontamination work. Compton cameras that offer excellent imaging capability and detection efficiency are commercially available. However, the high cost of detector materials and a complex reconstruction system make the cameras very expensive. We have developed a simpler and smaller, and thus a less expensive gamma-ray detector that can roughly determine the incident direction of a radiation source with moderate angular resolution, aiming for personal use beyond that afforded by conventional survey meters. The detector consists of six GAGG(Ce) scintillators with dimensions of $20 \times 20 \times 6$ $\mathrm{mm}^{3}$ on each face of a $3-\mathrm{cm}$ cubic lead block. Signals from each scintillator are read by a $3 \times 3 \mathrm{~mm}^{2}$ Hamamatsu MPPC. We then applied $?^{2}$ fit to the observed count number set in order to determine the incident direction. We evaluated the angular resolution as $20^{\circ} ? 24^{\circ}$ for one-minute exposure to a ${ }^{137} \mathrm{Cs}$ source that gives $0.1 \mu \mathrm{Sv} / \mathrm{h}$. We also examined the capability for detecting two sources. 
N60-4: Li co-doped NaI:Tl - a Large Volume Neutron-Gamma Dual Mode Scintillator with Exceptional Pulse Shape Discrimination

K. Yang, P. R. Menge

Saint-Gobain Crystals, Hiram, OH, USA

Saint-Gobain Crystals has been actively working on scintillator performance engineering for the past several years. In this paper, we continue reporting on our researches of engineered NaI. We have demonstrated that Li can be substantially incorporated into the matrix of $\mathrm{NaI}$ under an optimized crystal growth process. The incorporation of Li introduces efficient neutron detection capability into an already successful commercial gamma scintillator. Exceptional gamma-neutron pulse shape discrimination (PSD) has been demonstrated in all Li co-doped NaI crystals with up to 8\% Li concentration. PSD Figure-of-Merits are between 1.5 and 4.4 depending on the Li content. Single crystals of Li co-doped NaI has gamma performance on par with standard NaI:Tl. Creation of large $(>1000 \mathrm{~cm} 3)$, low cost, dual-gamma spectroscopy/neutron detectors are likely possible with this technology. Updates on $\mathrm{Sr}^{2+}$ and $\mathrm{Ca}^{2+}$ co-doped NaI:Tl with improved energy resolution and decay time will also be reported.

N60-5: New Pulse Shape Discrimination Algorithms for Application on Digitized Scintillation Pulses A. Enqvist, H. Wang, K. Stadnikia, R. Kelley, K. Jordan

University of Florida, Gainesville, United States

This work details 2 new pulse shape discrimination (PSD) algorithms suitable for digitized pulse waveforms, obtained with high sampling rate and high bit-resolution. The first method tries to isolate single or few scintillation photon initiated electric pulse variations $i$ the photo-multiplier and analyses the pulse jitter in the pulse tail region. The method has a couple of parameters of that can be optimized depending on scintillation time constants and signal noise. The tail-jitter method is found to be promising for low amplitude pulses where the standard charge integration method is exhibiting degraded performance. However at large pulse amplitudes the excellent separation of standard PSD methods means that the PSD method which is slightly more computationally intense might be best implemented as a complementing method on low amplitude pulses. However for relatively long-lived slow decay constants, such as the case of He-4 scintillation detectors where single scintillation photon events are more discernible in the long tail this method has an improved appeal vs standard organic liquid scintillators. Additionally, a second method of pulse shape discrimination suitable for digitized waveforms is based off of the third moment of a probability distribution, skewness. While the pulses are not a probability distribution, the shape is nevertheless similar with a distinct skewness in the forward time-direction. This skewness depends on the particle type (mass) generating the scintillation material excitations. The PSD methods are evaluated vs current standard PSD methods and as a suggested combined algorithm approach using multiple algorithms depending on pulse amplitude, classification confidence and other considerations.

\author{
N60-6: New Approaches for a 2D Neutron Detector Based on ZnS:6LiF Scintillator Readout with WLS Fibers \\ and SiPMs \\ M. Hildebrandt, J.-B. Mosset, A. Stoykov \\ Paul Scherrer Institut, 5232 - Villigen PSI, Switzerland
}

Recently we reported on a high efficiency, low gamma-sensitivity, high count rate $1 \mathrm{D}$ neutron detector consisting of $\mathrm{ZnS}$ :6LiF scintillator readout with WLS-fibers coupled to SiPMs which achieved a performance comparable with a 3He-based detector. This remarkable performance was achieved through the optimization of the light collection from the scintillator using WLS-fibers combined with the high photon detection efficiency of a SiPM.

Here we discuss the approaches to new detector designs which extend the developed technology to highly segmented 2D detectors with pixel sizes of about $(3 \times 10) \mathrm{mm}^{\wedge} 2$ suitable e.g. for large-area neutron powder diffraction instruments. In these approaches the scintillation light from each pixel is collected by a single WLS-fiber (diameter $=0.25 \mathrm{~mm}$ ) placed in loops within the pixel volume. The two ends of the WLS-fiber are connected to two SiPMs for an XY-coding to reduce the number of readout and processing channels. Feasibility studies on such a detector including estimation of the neutron absorption probability and measurements on the trigger efficiency, gamma-sensitivity, and the count-rate capability are reported.

N60-7: Laboratory Tests for X-Rays Crystal Detectors with SiPMT Array Readout

$\underline{\text { M. Bonesini }}{ }^{1}$, R. Bertoni ${ }^{1}$, M. Clemenza ${ }^{1}$, R. Mazza ${ }^{1}$, M. Nastasi ${ }^{1}$, T. Cervi ${ }^{2}$, A. deBari ${ }^{2}$, A. Menegolli ${ }^{2}$, M. Rossella ${ }^{2}$

${ }^{\text {I}}$ Sezione INFN Milano Bicocca, Dipartimento di Fisica G. Occhialini, Milano, Italy

${ }^{2}$ Sezione INFN Pavia, Dipartimento di Fisica, Pavia, Italy

New scintillating crystals for X-rays spectroscopy applications, such as Pr:LuAG and Ce CAAG have been studied and compared to more common crystals, such as $\mathrm{LaBr} 3$ :Ce and $\mathrm{NAI}(\mathrm{Tl})$ using different radioactive sources and SiPMT arrays readout. Both Pr:LuAG and CeCAAG represent a cheaper solution with respect to the Germanium detectors, but with remarkable properties which make them useful for many applications, ranging from fundamental physics measurements to the PET imaging for medical 
purposes: high density, elevate light yield, fast response, high energy resolution, no hygroscopicity. A sample of Pr:LuAG, CeCAAG, LaBr3:Ce and NaI crystals with 0.5 " x 0.5" surface area and $\sim 13 \mathrm{~mm}$ thickness (26 mm for NaI) have been characterized with several radioactive sources, emitting photons in the range 100-1000 keV. Different SiPMT arrays were adopted for these studies, including new arrays from Hamamatsu with TSV technology.sensitive to PrLuAG ultraviolet emission (peak at $310 \mathrm{~nm}$ ). The goal is the detection of the characteristic X-rays (around $130 \mathrm{keV}$ ) emitted during the de-excitation processes of the muonic hydrogen after the excitation with an IR laser with wavelength set at the resonance of the hyperfine splitting, to measure the muonic atom proton radius with unprecedented precision at the RiKEN-RAL facility .

\section{N61: Simulation and prototyping for detector development II}

Thursday, Nov. 3 16:30-18:30 Curie 1

N61-1: Benefits for nEXO of a Fully Digital Large Area Photon Detector Made of 3D Digital SiPMs

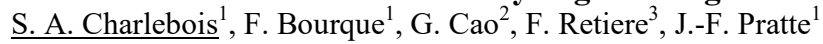

${ }^{I} 3 I T$, Université de Sherbrooke, Sherbrooke, Canada

${ }^{2}$ Institute of High Energy Physics, Beijing, China

${ }^{3}$ Detector Development Group, TRIUMF, Vancouver, Canada

We propose a triggering and summing algorithm, validate it with LXe scintillation simulations from the nEXO experiment and show that no $0 \mathrm{v} ß \beta$ decay events are missed and power consumption can be reduced. Low background experiments requiring large area detectors are now considering SiPMs for photon detection. To study neutrino less double beta decay (0vßß) in liquid xenon (LXe), the nEXO experiment expects using a $5 \mathrm{~m}^{2}$ cylindrical wall of low-noise and low-power SiPM based detectors. We propose using a fully digital photon detector based on 3D digital SiPM (3DdSiPM) all linked through control electronics. In this architecture, each SPAD (Single Photon Avalanche Diode) is sensed and quenched individually and a digital trigger is sent to the processing electronics. The simulation of $2.5 \mathrm{MeV} 0 \mathrm{v} \beta ß$ decay events LXe scintillation photon is done in GEANT4 (NEST) for the baseline design of the nEXO vessel. The photon detector comprises an array of $92 \times 3003 \mathrm{DdSiPMs}$ each $1.34 \times 1.34 \mathrm{~cm} 2$. Various combinations of DCR (50 and $100 \mathrm{~s}-1 \mathrm{~mm}-2)$ and of PDE (5, 15 and 20\%) are used to represent the LXe operation conditions. We use a detection algorithm in a which a short coincidence window (50-150 ns) applied on clusters of 3DdSiPMs is used to discriminate the real events from the dark counts. A detection occurs when at least one cluster reaches the threshold value. A longer summation window (150-500 ns) then gives the total number of counts (photons + DCR) of all 3DdSiPMs to the data acquisition system. We have studied the impact of the threshold, the cluster size $(1,4,16)$ and the various PDE and DCR values and found optimized threshold that enable 1) identifying all real 0vßß events and 2) reducing by orders of magnitude the dark counts. The reduction of dark counts also reduces the power consumption related to data communication. This is a critical goal for liquid noble gas detectors such as the nEXO experiment.

N61-2: New Developments in the Design and Production of Low-Gain Avalanche Detectors N. Cartiglia ${ }^{1}$, G. F. Dalla Betta ${ }^{2}$, M. Boscardin ${ }^{3}$, G. Paternoster ${ }^{3}$, L. Pancheri ${ }^{2}$, F. Cenna ${ }^{1,4}, \underline{\text { M. Ferrero }}^{1,4}$, V. Sola $^{1,4}$,

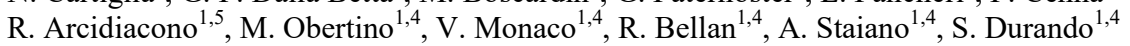

${ }^{I} I N F N$, Torino, italy

${ }^{2}$ Universita' di Trento, Trento, Italy

${ }^{3}$ Fondazione Bruno Kessler, Trento, Italy

${ }^{4}$ Universita' di Torino, Torino, Italy

${ }^{5}$ Universita' Piemonte Orientale, Torino, Italy

In this contribution we report on the design, fabrication and performances of the first fully double-sided production of Low-Gain avalanche detectors on 6-inch wafers. The production was carried on at the FBK facility in Trento (Italy), in collaboration with the Trento University and INFN, Torino. LGAD are a novel type of silicon detectors with internal multiplication that due to their low noise and large signals are ideal for timing applications (the so called Ultra-Fast Silicon Detectors) and low material budget tracking systems. LGAD are considered in the CMS and ATLAS upgrades for HL-LHC, therefore the capability of large scale production is particularly important.

\section{N61-3: Testbeam Studies of Silicon Microstrip Sensor Architectures Modified to Facilitate Detector Module Mass Production \\ L. Poley \\ DESY, Zeuthen, Germany \\ On behalf of the ATLAS Collaboration}

For the High Luminosity Upgrade of the LHC, the Inner Detector of the ATLAS detector will be replaced by an all-silicon tracker, consisting of pixel and strip sensor detector modules. Silicon strip sensors are being developed to meet both the tracking requirements in a high particle density environment and constraints imposed by the construction process. Several thousand wire 
bonds per module, connecting sensor strips and readout channels, need to be produced with high reliability and speed, requiring wire bond pads of sufficient size on each sensor strip. These sensor bond pads change the local sensor architecture and the resulting electric field and thus alter the sensor performance.

These sensor regions with bond pads, which account for up to $10 \%$ of a silicon strip sensor, were studied using both an electron beam at DESY and a micro-focused X-ray beam at the Diamond Light Source.

This contribution presents measurements of the effective strip width in sensor regions where the structure of standard parallel silicon strips is altered to accomodate aluminium bond pads. The data is compared to simulations, which support the hypothesis that the geometry change impacts the sensor strip performance.

\title{
N61-4: Compact Pixel Module with Through-Silicon Vias, TSV
}

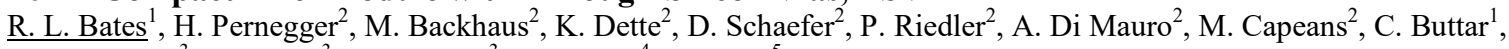
A. Macchiolo ${ }^{3}$, R. Nisius ${ }^{3}$, A. La Rosa ${ }^{3}$, A. Lounis ${ }^{4}$, G. Pares ${ }^{5}$

${ }^{I}$ Physics and Astronomy, The University of Glasgow, Glasgow, UK

${ }^{2}$ CERN, Geneva, Switzerland

${ }^{3}$ Max Planck Institute for Physics, Munich, Germany

${ }^{4}$ LAL, Laboratoire de l'Accélérateur Linéaire, Paris, France

${ }^{5}$ CEA LETI, Grenoble, France

Hybrid pixel modules have become ubiquitous in particle physics experiments over the past few decades due to their true space point information coupled with low noise and high radiation tolerance. The standard module with wire bond connections from the edge of the readout chip to the PCB on the back of the sensor has several limitations introduced by the wire bonds and their bond pads.

This paper presents the technology developments to remove the wire bonds from the module with the use of through-silicon-vias, TSVs. The electrical connections to the readout chip pass through the chip itself and are re-routed on the back side of the chip with a redistribution layer. Using this new module concept makes four-side buttable detector arrangements possible with very small inactive areas in large detector surfaces. The connection of the redistribution layer pads to the PCB is made via laser soldering.

The module development performed at CEA LETI with the FEI4B chip are presented. The TSV last processed used requires a readout chip thickness of 100 micrometers or less which therefore produces a very low radiation length module. Fabrication details of the TSV, redistribution layer and the laser solder are discussed. Characterisation of the chip and also the pixel assembly after TSV fabrication and after laser soldering to a flex are shown.

\author{
N61-5: Development of the Fast and Efficient Gamma Detector Using Cherenkov Light

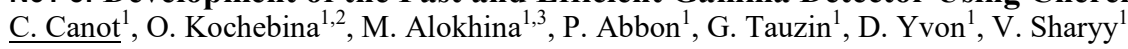 \\ ${ }^{I} I R F U, C E A$, Gif-sur-Yvette, France \\ ${ }^{2}$ SHFJ, CEA, Orsay, France \\ ${ }^{3}$ Taras Shevchenko National University of Kyiv, Kyiv, Ukraine
}

We describe the development and tests of the gamma detector using Cherenkov light. This device is designed for the detection of $511 \mathrm{keV}$ photons from the positron annihilation and provides simultaneously the high resolution in time and a large efficiency. The main application for such detector is the positron detection using the time-of-flight (TOF) technique, which plays an important role in the positron emission tomography (PET). The gamma detector consists of a lead fluoride crystal coupled to a micro-channel-plate photo-multiplier with a sapphire window. Using the radioactive positrons source Na22, we tested the efficiency and time resolution of the prototype detector and compared the obtained results with the Geant 4 simulation.

\section{N61-6: Dispersed SiPM Readout for PVT}

M. Meshkian ${ }^{1}$, U. Gendotti ${ }^{1}$, R. Chandra ${ }^{1}$, P. Schotanus ${ }^{2}$

${ }^{I}$ Arktis Radiation Detectors Ltd., Zurich, Switzerland

${ }^{2}$ Scionix Holland B.V., La Bunnik, The Netherlands

For detection of illicit trafficking of radionuclides, different systems have been developed employing plastic scintillators. Silicon Plastic Readout Gamma Detection Technology (SiPR), sponsored by EUROSTARS-PROJECTS (E!9828), utilizes a plastic scintillator with an embedded Silicon Photomultiplier (SiPM)-based light read-out solution. The main applications of SiPR could include radiation portal monitors as well as hand contamination monitoring systems.

The performance of SiPR has been studied with Geant4 Monte Carlo simulations and actual experiments, including different scintillator thicknesses combined with different wrapping materials to compare their efficiency. Consequently, similar measurements were also performed for different scintillator thicknesses combined with different wrapping materials. In this study, a novel gamma detection device has been demonstrated, in terms of detection efficiency and light collection efficiency, performing measurements and simulations, respectively. The work presented here reports the results obtained in the 
early prototype phase using a small plastic scintillator with dimensions of $10 \times 20 \times 4 \mathrm{~cm} 3$. The prototype detector was irradiated with different gamma sources. The obtained results from the simulations are comparable to those of the measurements. The detector demonstrates an intrinsic detection efficiency of $19 \%$ for Co- 60 .

\author{
N61-7: High Precision Timing Measurement in the CALICE Analogue Hadronic Calorimeter \\ E. Brianne \\ DESY, Hamburg, Germany \\ On behalf of the CALICE Collaboration
}

The CALICE Collaboration is developing highly granular calorimeters for a future $\mathrm{e}^{+} \mathrm{e}^{-}$linear collider. The development of the calorimeters is driven by the Particle Flow approach (PFA) in order to achieve a jet energy resolution of 3-4 \%. The Analog Hadronic Calorimeter (AHCAL) is one of the detector concepts based on $3 \times 3 \times 0.3 \mathrm{~cm}^{3}$ scintillating tiles read out by novel Silicon Photomultipliers (SiPM) and processed by an ASIC (SPIROC2b) capable to measure the time of individual calorimeter hits. A second generation engineering prototype of the AHCAL is developed to focus on the full scalability of the detector and features, in addition to amplitude measurements, timing measurements. This prototype is composed of 14 active layers corresponding to 3744 channels inserted into an absorber structure of 4 ? depth. Alternative designs for the scintillating tiles with and without wavelength-shifting fibres and tiles individually wrapped with a reflector foil were used as well as different types of SiPMs. It was tested at the CERN Super Proton Synchrotron (SPS) in summer 2015 with steel and tungsten absorbers, in muon, electron and hadron beams. First results of the AHCAL prototype concerning timing capabilities will be shown including timing calibration procedure and comparison to simulation.

\author{
N61-8: Development of PET Detector Module Using Capacitive Multiplexing Circuit for Time-of-Flight (TOF) \\ PET

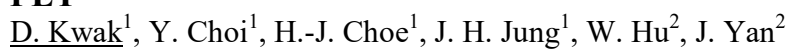 \\ ${ }^{I}$ Molecular Imaging Research \& Education (MiRe) Laboratory, Department of Electronic Engineering, Sogang University, \\ Seoul, South Korea \\ ${ }^{2}$ FMI Medical Systems Co., Ltd., Shaoxing, China
}

It has been reported that capacitive multiplexing circuit can provide superior timing performance such as sharp rise time and less baseline fluctuation. In our previous study, a capacitive multiplexing circuit for a SiPM array was developed, and demonstrated the coincidence resolving time (CRT) with the multiplexing circuit was comparable to that of individual channel readout. The objective of this study was to develop a prototype TOF-PET detector module consisted of scintillator, SiPM array, capacitive multiplexing circuit, analog front-end (AFE) and data acquisition (DAQ) electronics in a single compact PCB. The AFE consisted of 64:4 capacitive multiplexing circuit, wide-bandwidth current feedback preamplifiers, and single-to-differential amplifiers. The quenching resistor value of the SiPM array was also adjusted to prevent positive overshoot for capacitive multiplexing circuit. Position outputs of the capacitive multiplexing circuit were digitized using 4 channel high-speed analog-todigital converter. Deserialization, peak detection, and time-to-digital converter (TDC) modules were implemented in a FPGA in order to acquire energy and arrival time information. The FPGA was also used to generate event packet and transfer the packet to PC via USB3.0 interface. Flood histograms and energy spectra of four $4 \times 4$ arrays of $3 \times 3 \times 20 \mathrm{~mm}^{3}$ LYSO crystals coupled to an $8 \times 8$ array of $4 \times 4 \mathrm{~mm}^{2}$ SiPMs were measured. The CRT of two single LYSO crystals was measured using two detector modules located at opposite side each other. All 64 LYSO crystals were clearly resolved in the flood histograms. The average energy resolution of the pixels was $17.3 \pm 1.2 \%$. The CRT measured using the crystals was $425 \pm 10 \mathrm{ps}$ FWHM. A compact TOF-PET detector module integrated with capacitive multiplexing circuit, AFE and DAQ electronics was successfully developed and evaluated. Further study to increase the field of view of the detector module and improve the CRT will be performed.

N62: Neutron detectors : Semiconductor Devices and Neutron Sources

Thursday, Nov. 3 16:30-18:30 Curie 2

N62-1: Rugged, Compact, Diamond-Based Fast Neutron Detector Operating at up to $200^{\circ} \mathrm{C}$ $\underline{\text { O. Philip }}^{1}$, F. Gicquel ${ }^{1}$, V. Ernst ${ }^{1}$, Z. Zhou $^{2}$

${ }^{I}$ Princeton Technology center, Schlumberger, Princeton Junction, NJ, USA

${ }^{2}$ Doll Research Center, Schlumberger, Cambridge, MA, USA

Recent improvements in the production of artificial single crystal diamonds have made it possible to produce commercial radiation detectors. However, the availability of diamond detectors for high temperature, high shock applications is limited. We have developed a compact solid state diamond detector for high temperature applications which require compact size, stable performance and extreme ruggedness. It is used as a fast neutron detector for monitoring the output of a pulsed neutron generator in oil well logging tools. The sensor consists of a high purity mono-crystalline diamond wafer with metallized electrodes on both faces. One of the metallized surfaces is mounted on a metallic plate resting on a ceramic substrate. The metallized diamond 
assembly is mounted in a TO-257 metal housing, which has pins connected to the two electrodes on each face of the diamond. The detector package, consisting of the diamond sensor and the electronics assembly, has been qualified for operation in the harsh downhole environment of oil fields. This includes high temperature and repeated shocks and vibration. However, this detector has applications beyond well logging, wherever there is a need for a compact, reliable detector for fast neutrons in an environment where ruggedness, exposure to high temperature, compactness and radiation hardness are essential.

\title{
N62-2: High Temperature Operation of Single Crystal Diamond Detectors
}

M. Angelone ${ }^{1}$, R. Pilotti ${ }^{2}$, F. Sarto ${ }^{1}$, S. Loreti ${ }^{1}$, G. Pagano ${ }^{1}$, M. Pillon ${ }^{1}$, M. Marinelli ${ }^{2}$, E. Milani ${ }^{2}$, C. Verona ${ }^{2}$, G. Prestopino ${ }^{2}$, G. Verona-Rinati ${ }^{2}, \underline{\text { S. Fiore }}{ }^{1}$

${ }^{I}$ Department of Fusion and Technology for Nuclear Safety and Security, ENEA, Frascati, Italy

${ }^{2}$ Dipartimento di Ingegneria Industriale, Universita degli Studi, Roma, Italy

Diamond is studied since many years as potential radiation detector material. In the last years there has been an increasing claim about the capability of diamond to withstand harsh working conditions including high temperature. The latter capability depends upon the large band gap and the excellent thermal properties of diamond. At ENEA C.R. Frascati diamond detectors at high temperature are studied since 2010 as possible neutron monitors for nuclear fusion reactors. The paper reports the performances and discusses the main issues of single crystal diamond detectors designed to operate at high temperatures. An innovative detector layout was studied which avoids the use of critical components such as glue, welding, etc. Diamond metallization was realized using several metals (e.g. $\mathrm{Cr}, \mathrm{Ti} / \mathrm{Pt}, \mathrm{Ag}$ ) deposited on both side of commercial SCD films 500 micron thick. The maximum working temperature, under $14 \mathrm{MeV}$ neutron irradiation and in spectrometric mode, was around $220-230 \ddot{i}_{i}^{1} / 2 \mathrm{C}$ for all the different metal contacts used. However, the best performance was obtained using Ag metal contacts post annealed after deposition. Stable and reproducible operation was observed up to $240 \ddot{i}_{i} 1 / 2 \mathrm{C}$. It was also found that once cooled below the maximum working temperature the detectors performances were perfectly reproduced. All the above detectors were characterized by double metal-diamond (Schottky) contacts. It was observed that metallization can be damaged by long lasting operation at high temperature. In the attempt to overcame this issue, a new detector was realized having a Schottky Cr-diamond metal contact on one side and an ohmic, not metal, contact on the other side. A commercial film 100 micron thick was used. This detector shown excellent performances well above $300 \ddot{i} i \frac{1}{2} \mathrm{C}$.

\author{
N62-3: Microstructured Semiconductor Neutron Detector (MSND)-Based Direct Helium-3 Replacement \\ (HeRep) for High Pressure Helium-3 Detectors \\ $\underline{\text { T. R. Ochs }}^{1}$, S. L. Bellinger ${ }^{2}$, R. G. Fronk ${ }^{1}$, L. C. Henson², D. E. Huddleston ${ }^{3}$, T. J. Sobering 3 , D. S. McGregor ${ }^{1}$ \\ ${ }^{I}$ Mechanical and Nuclear Engineering Department, Kansas State University S.M.A.R.T. Laboratory, Manhattan, KS, United \\ States \\ ${ }^{2}$ Radiation Detection Technologies, Inc., Manhattan, KS, United States \\ ${ }^{3}$ Kansas State University Electronics Design Laboratory, Manhattan, KS, United States
}

Small diameter, low-power, compact 3He replacement (HeRep) instruments have been designed, fabricated, and populated with Microstructured Semiconductor Neutron Detectors (MSNDs) to compete with expensive, high-pressure 3He gas-filled neutron proportional counters. MSNDs offer an inexpensive, high-efficiency, solid-state alternative to thermal-neutron detection. MSNDs with straight trenches etched into one side of a diode and backfilled with $6 \mathrm{LiF}$ have realized intrinsic thermal-neutron detection efficiencies greater than 30\%. Dual-sided MSNDs (DSMSNDs), fabricated by etching trenches on both-sides of the detector diode, increases the intrinsic thermal-neutron detection efficiency to greater than 50\%. Numerous MSND or DSMSND diodes with $1-\mathrm{cm} 2$ or $4-\mathrm{cm} 2$ active area can then be arranged to form larger and more complex neutron detection instruments. The HeRep Mk I and HeRep Mk II utilized single-sided MSNDs and were designed to replace 2-in. diameter 3He detectors. The 2-in. diameter by 6 -in. long HeRep Mk II previously reported $95.15 \pm 9.04 \%$ and $102.71 \pm 2.65 \%$ of the count rate of a similarly sized 4 atm $3 \mathrm{He}$ detector for bare detector and moderated detector (6-in. diameter by 9 -in. long cylindrical HDPE moderator cask) measurements, respectively. The 0.75 -in. diameter by 4.50-in. long HeRep Mk III was designed to serve as test bed instrument for the more efficient DSMSNDs and to compete with similarly sized 6-atm 3He detectors. The HeRep Mk III draws a maximum of $15-\mathrm{mW}$ power and reported a net count rate of $0.23 \pm 0.01 \mathrm{cps}$ and $8.59 \pm 0.12 \mathrm{cps}$ for a 32-ng $252 \mathrm{Cf}$ bare source at a distance of $0.25 \mathrm{~m}$ for bare detector and the detector in a 3 -in. diameter by 5.75 -in. cylindrical HDPE moderator cask, respectively. The DSMSND-based HeRep technology can be further improved my implementing detector packages with more intimate MSND configurations to compete with 0.75 -diameter 10 -atm 3 He detectors.

N62-4: Conception of a New Recoil Proton Telescope for Real-Time High-Energy Neutron Spectrometry R. Combe, N. Arbor, Z. El Bitar, S. Higueret, D. Husson

RaMsEs, IPHC, Strasbourg, France

A new model of high-energy real-time neutron spectrometer based on a Recoil Proton Telescope technology has been developed at the IPHC. The Bonner Sphere Spectrometer, the current reference method for the measurement of neutron energy gives a 
precise reconstruction of the neutron energy spectrum but it requires a painful work of signal deconvolution, that cannot be done in real time. The developed Recoil Proton Telescope enables a fast and direct reconstruction of the neutron spectrum from the measurement of the recoil proton trajectory and energy. A new fast-readout CMOS sensor, called FastPixN, has been developed at the IPHC for this specific purpose. The geometry has been optimized using extensive Geant4 Monte Carlo simulations in order to reach the best compromise between efficiency and energy resolution. A first prototype has been developed for the detection of neutrons in the 3-20 MeV energy range. It has been carefully validated with the AMANDE facility at CEA Cadarache. We are currently developing a new RPT for secondary neutron detection in proton therapy applications. This prototype will use a new system to measure higher energies and an optimized collimator, allowing to reach neutron energies as high as $200 \mathrm{MeV}$.

\section{N62-5: The ATLAS-TPX Detector Network}

B. Bergmann ${ }^{1}$, J. Begera ${ }^{1}$, P. Burian ${ }^{1}$, I. Caicedo ${ }^{1}$, D. Caforio ${ }^{1}$, E. Heijne ${ }^{1}$, J. Janecek $^{1}$, P. Manek ${ }^{1}$, Y. Mora ${ }^{1}$, M. Platkevic ${ }^{1}$, J. Pacik $^{1}$, S. Polansky ${ }^{1}$, S. Pospisil ${ }^{1}$, M. Suk ${ }^{1}$, Z. Svoboda ${ }^{1}$, C. Leroy ${ }^{2}$, T. Billoud ${ }^{2}$, A. Sopczak ${ }^{1}$

${ }^{I}$ Institute of Experimental and Applied Physics, Czech Technical Uinversity in Prague, Prague, Czech Republic

${ }^{2}$ Groupe de la Physique des Particules, Universite de Montreal, Montreal, Quebec, Canada

A network of 16 Medipix detectors has proven valuable for the investigation of the ATLAS machine's luminosity, the activation of surrounding material during and after collisions, the composition of the radiation fields and the dose rates at different positions within the cavern. During the 2013-2014 shutdown period the network was upgraded to a two layer Timepix design (ATLASTPX) with a faster readout. Two silicon sensor layers (thicknesses $300 \mu \mathrm{m}$ and $500 \mu \mathrm{m}$ ) are interlaced by neutron converter foils (Li-6, polyethylene (PE) and PE+aluminum) sensitive to neutrons of different energies. The two layer design allows an improved discrimination of charged particles and gammas against neutrons and provides information about the directionality of the fast neutron component. Detector responses were calibrated at the neutron spallation source in the Los Alamos Neutron Science Center, at the Heidelberger Ion Therapy Center and in CERN SPS beams. This way, we have obtained detection efficiencies for charged particles with energies up to $1 \mathrm{GeV}$ and for neutrons up to $800 \mathrm{MeV}$ including their angular dependencies. The methodology for the data evaluation has arisen from this calibration effort and the experiences with the previously installed detector network. A dedicated readout system based upon a Raspberry Pi was designed for configuring and remotely controlling the ATLAS-TPX. Data are taken continuously, pre-processed, visualized and stored in a database for offline data analysis. We present the basic evaluation techniques, the design of the detector control and readout system, the data transfer scheme and the web application for almost realtime data visualization and offline data evaluation.

N62-6: Web-Shaped Microstructured Silicon Hybrid Detectors of Thermal Neutrons

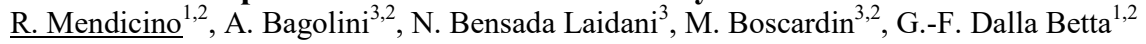

${ }^{I}$ University of Trento, Trento, Italy

${ }^{2}$ TIFPA INFN, Trento, Italy

${ }^{3}$ Fondazione Bruno Kessler (FBK), Trento, Italy

We report on the development of hybrid detectors of thermal neutrons based on microstructured silicon sensors coupled to ${ }^{10} \mathrm{~B}$ converter. We designed, simulated and fabricated a new MEMS based device with high aspect ratio cavities allowing for a good detection efficiency ( $20 \%$ as predicted by Geant 4 simulations) while minimizing the process complexity. A prototype batch was recently fabricated at Fondazione Bruno Kessler (FBK) in Trento, Italy, showing good results from electrical measurements. Tests on wafers indicated full depletion voltages between 80 and $100 \mathrm{~V}$ (depending on the sensor's geometry) and breakdown voltages in excess of $500 \mathrm{~V}$, with leakage currents as low as $\sim 4 \mathrm{nA} / \mathrm{cm}^{2}$. Preliminary functional tests with an ${ }^{241} \mathrm{Am}$ a-source have shown full charge collection efficiency. Both electrical and functional test results are in good agreement with TCAD simulations. At the conference we will report on selected results from the sensor characterization, including thermal neutron detection and gamma-ray discrimination tests.

This work was supported in part by the Autonomous Province of Trento and by the Italian National Istitute for Nuclear Physics (INFN) HYDE Project.

N62-7: The Frascati Neutron Generator: Present Activities and Future Upgrades

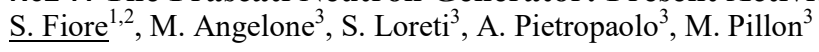

${ }^{I}$ Department of Fusion and Technology for Nuclear Safety and Security, ENEA, Rome, Italy

${ }^{2}$ Sezione di Roma, INFN, Rome, Italy

${ }^{3}$ Department of Fusion and Technology for Nuclear Safety and Security, ENEA, Frascati, Italy

The Frascati Neutron Generator FNG, of the ENEA Frascati research center in Italy, is a highly versatile active source of 14 and $2.5 \mathrm{MeV}$ neutrons, with a peak intensity of $1 \times 10^{11}$ neutron/s in steady state or pulsed mode. The generator exploits the $\mathrm{T}(\mathrm{d}, \mathrm{n}) \mathrm{a}$ and the $\mathrm{D}(\mathrm{d}, \mathrm{n})^{3} \mathrm{He}$ fusion reactions, obtained by deuteron beam interaction with a tritiated or deuterated target. The FNG facility was designed to conduct neutronics experiments in the framework of the research activity on controlled thermonuclear fusion, 
starting its operation in the 90's. Over the years, many other research activities have been performed: these include the development of Single Crystal Diamond detectors for Plasma Fusion diagnostics and in-core fast fission reactor diagnostics; Gas Electron Multipliers (GEM) for 2.5 MeV (D-D) and 14.0 MeV (D-T) neutron detection; self powered neutron detectors for ITER TBM. A number of scientific collaborations exploited the FNG for neutron detection experiments and radiation hardness evaluation: among these, silicon photodetectors (APDs, SiPMs) and front-end electronics have been recently tested by FNG research staff in collaboration with several foreign research institutes for experiments at JLAB, Fermilab and KEK. FNG is also available as a user facility for testing and irradiation of electronics and material samples for nuclear, aerospace and life sciences applications. Recently FNG has been included in the list of European Space Agency compliant facilities for neutron irradiation. A description of the FNG characteristics, ancillary laboratories and current activities will be presented; moreover, a recent characterisation of the FNG emission spectrum has been performed and will be described, together with upgrades that will extend its capabilities to the generation of thermal neutrons and higher fluxes of fast neutrons.

\author{
N62-8: Photoneutron Interrogation Measurements with a $9 \mathrm{MeV}$ Electron Accelerator for the Detection of \\ Fissile Materials

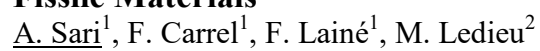 \\ ${ }^{I}$ CEA LIST, 91191 Gif-sur-Yvette, France \\ ${ }^{2}$ CEA DEN, 13108 St Paul lez Durance, France
}

Neutron interrogation is a widely used technique that enables to detect nuclear materials such as uranium and plutonium contained in nuclear waste packages. Deuterium-tritium neutron generators are traditionally used on such interrogation measurement setup. Carrying out high-energy imaging of nuclear waste packages would provide highly useful information on the content of packages in order to interpret accurately neutron interrogation measurement results. This technique can be performed using a linear electron accelerator. Moreover, neutrons are generated by photonuclear reactions in the accelerator's conversion target. Therefore, an electron accelerator could be used as a neutron source instead of a traditional neutron generator, and enable to achieve both techniques with a single device, thereby reducing cost and improving characterization of nuclear waste packages. In this study, we evaluate performances of a $9 \mathrm{MeV}$ electron accelerator to carry out neutron interrogation measurements on nuclear waste packages. First, we study whether to use photoneutrons generated by the accelerator's conversion target, or by a secondary target made of beryllium or heavy water. A photoneutron flux characterization study has been conducted at the $9 \mathrm{MeV}$ electron accelerator of the SAPHIR facility (CEA Saclay). We encountered simulation underestimation problems due to poor knowledge of photonuclear reaction cross-sections in the energy range of interest. Then, we design by simulation a photoneutron source based on a $9 \mathrm{MeV}$ electron accelerator using beryllium or heavy water as secondary targets. Finally, we evaluate experimentally the feasibility of this method by carrying out neutron interrogation measurements on uranium and plutonium samples using a secondary target of heavy water. We show that prompt neutron signals enable to quantify actinide mass. Ratios between prompt and delayed neutron signals enable to differentiate ${ }^{235} \mathrm{U}$ and ${ }^{239} \mathrm{Pu}$.

N63: Data acquisition, trigger and analysis III

Friday, Nov. $4 \quad$ 08:00-10:00 Madrid

N63-1: A Scalable Data Acquisition for the CALICE Tile Hadron Calorimeter

J. Kvasnicka $^{1,2}$

${ }^{I}$ Institute of Physics of the Czech Academy of Sciences, Prague, Czech Republic

${ }^{2}$ Deutsches Elektronen-Synchrotron (DESY), Hamburg, Germany

On behalf of the CALICE Collaboration

The data acquisition system (DAQ) for a highly granular analogue hadron calorimeter (AHCAL) for the future International Linear Collider (ILC) will be presented. The developed DAQ chain has several stages of aggregation and scales up to 4 million channels in the main barrel foreseen for the AHCAL design. The front-end electronics will be embedded in the detector layers in between absorber plates without active cooling. The ILC has a specific timing of $<1 \mathrm{~ms}$ active recording followed by $199 \mathrm{~ms}$ of slow readout, which allows the detector to be operated in power-pulsing mode in order to meet the power dissipation budget. This timing is reflected in the architecture of the DAQ from the very front-end ASIC embedded in the detector layers between absorber plates to the largest link aggregation device, the LDA (Link data aggregator), which has 96 HDMI connectors matching the AHCAL steel absorber plate spacing, four Kintex7 FPGAs and a central Zynq SoC (System-On-Chip). The LDA meets the ILC timing and bandwidth, which will be shown. The same DAQ is used also for AHCAL prototype beam tests, which require rather continuous data taking. DAQ architecture, data flow, timing and performance will be presented in detail. The performance has been demonstrated in recent beam tests at CERN at the PS in 2014 and at the SPS in 2015.

N63-2: The ATLAS Trigger Algorithms for General Purpose Graphics Processor Units A. Tavares Delgado

Laboratorio de Instrumentacao e Fisica Experimental de Particulas, LIP, Lisboa, Portugal 
We present the ATLAS Trigger algorithms developed to exploit Generalï $i^{1} / 2$ Purpose Graphics Processor Units. ATLAS is a particle physics experiment located on the LHC collider at CERN. The ATLAS Trigger system has two levels, hardwareï ${ }^{1} / 2$ based Level 1 and the High Level Trigger implemented in software running on a farm of commodity CPU. Performing the trigger event selection within the available farm resources presents a significant challenge that will increase future LHC upgrades. are being evaluated as a potential solution for trigger algorithms acceleration. Key factors determining the potential benefit of this new technology are the relative execution speedup, the number of GPUs required and the relative financial cost of the selected GPU. We have developed a trigger demonstrator which includes algorithms for reconstructing tracks in the Inner Detector and Muon Spectrometer and clusters of energy deposited in the Calorimeter. The $\ddot{i}_{i}^{1} / 2$ clienti $i_{j}^{1} / 2$ serveri $i_{i}^{1 / 2}$ architecture is used to integrate the GPUi $i \frac{1}{2}$ accelerated code running on the server side with multiple clients running the remaining serial code on the CPUs. We give a brief overview of the algorithms implemented and present measurements that compare the performance of various configurations exploiting different GPU cards.

N63-3: Real-Time Track Reconstruction During Readout Using an Artificial Retina Architecture M. J. Morello ${ }^{1,2}$, G. Punzi ${ }^{2,3}$, R. Cenci, ${ }^{1,2}$, P. Marino ${ }^{1,2}$, S. Stracka ${ }^{2,3}$, L. Ristori ${ }^{4}$, J. Walsh ${ }^{2}$, F. Bedeschi ${ }^{2}$, F. Spinella ${ }^{2}$

${ }^{I}$ Scuola Normale Superiore, Pisa, Italy

${ }^{2}$ INFN-Pisa, Pisa, Italy

${ }^{3}$ University of Pisa, Pisa, Italy

${ }^{4}$ Fermilab, Batavia, USA

We present the results of a study of the feasibility of implementing real-time track reconstruction within the framework of a preexisting readout system, using the "Artificial Retina" approach. This is the first attempt at building a complete medium-size prototype of this kind (comprising about $8 \mathrm{M}$ Logic Elements, distributed on several separate boards), operating it continuously at its limit speed. This study emphasizes logic architecture, correct operation, size, and compatibility with a standard readout framework, to explore its useability as an add-on to a conventional readout system. For this reason we have chosen for implementation a moderate-cost, moderate-speed board already in use in the data acquisition of an existing HEP experiment. Our choice fell on the readout boards currently in use by the NA62 experiment (TEL62), each equipped with 5 Altera Stratix III FPGAs and $4 \mathrm{Gbit} / \mathrm{s}$ ethernet interface. We have reprogrammed the board firmware in two different ways, to make the boards behave as the two main blocks of a AR system (switch system, and cellular processor farm). They are interfaced via the front panel, inverting their normal data flow path, by custom interconnection boards, and devoped in internal sytem for continuous feeding of data in order to test them at their maximum achievable speed. We report the results of extensive tests perfomed with this prototype, and discuss their implications regarding the applicability of the "retina architecture" to faster, and customdeveloped real-time processors.

N63-4: A Modular Data Acquisition System for (S)PE(C)T Applications N. Chevillon, C. Fuchs, J. Sahr, R. Sefri, X. Fang, V. Bekaert, F. Boisson, D. Brasse IPHC / CNRS-IN2P3, Universite de Strasbourg, Strasbourg, FRANCE

A Data Acquisition System (DAQ) dedicated to Positron Emission Tomography (PET) systems has been developed at the Institut Pluridisciplinaire Hubert Curien. This new electronic architecture is based on modules. Each module integrates four identical front-end boards of 64 independent channels, linked to a Synchro_fmc board, which splits and buffers the signals between the front-end boards and a commercial digital board driving the data acquisition. A front-end board integrates one homemade ASIC dedicated to triggerless charge and time measurements and an ADC chip for the digitization of the charge information. The Synchro_fmc board also acts as a relay for the clock and the synchronisation signals shared from one module to another. Data are read by System-on-Chips (SoCs) and send to acquisition computers using standard gigabit TCP/IP data transmissions with a sustained rate up to $97 \mathrm{MiB} / \mathrm{s}$ per 256 channels. Here we present a modular DAQ system with a high count-rate of $100 \mathrm{kHz}$ per channel, which allows us to consider an unlimited number of independent channels. The DAQ has recently been used to read both photomultiplier tubes and Silicon-photomultipliers coupled to a $3 \times 3 \times 5 \mathrm{~mm}^{3}$ LYSO crystal.

\section{N63-5: Robust Detection of Radiation Threat Using Uncertain Censored Energy Windows} E. Lei $^{1}$, K. Miller ${ }^{2}$, P. Huggins ${ }^{2}$, A. Dubrawski ${ }^{2}$

${ }^{I}$ Machine Learning Department, Carnegie Mellon University, Pittsburgh, PA, United States

${ }^{2}$ Auton Lab, Robotics Institute, Carnegie Mellon University, Pittsburgh, PA, United States

Given gamma-ray spectrometer data collected from noisy background environments, a popular method for detecting threats of known types is the Censored Energy Window (CEW). CEW aggregates features of the target energy window where we expect to see the source signatures most clearly, potentially losing information in the process. In addition, CEW is sensitive to the quality of selected target energy window, which may be an issue in practice when the optimal energy window is not perfectly known a 
priori. In this work, we develop a classifier of measured photon count spectra for detecting a source signature that exploits the individual energy bins in the target window and is robust to the choice of this window. To reduce the loss of potentially useful information, we apply canonical correlation analysis (CCA), a well-known statistical method for finding structured correlations. We empirically demonstrate that our method is more robust to the quality of the energy window on a dataset of gamma-ray measurements recorded in an urban area. This study is joint work at Carnegie Mellon University by Eric Lei of the Machine Learning Department and Kyle Miller, Peter Huggins, and Artur Dubrawski of the Auton Lab.

\author{
N63-6: Liquid Contrabands Classification with Mixed XRD Spectra \\ T.-Y. YangDai ${ }^{1,2}$, L. Zhang ${ }^{1,2}$ \\ ${ }^{I}$ Department of Engineering Physics, Tsinghua University, Beijing, China \\ ${ }^{2}$ Key Laboratory of Particle \& Radiation Imaging, Beijing, China
}

The energy dispersive $x$-ray diffraction (EDXRD) is a novel identification technique suited to security screening. The validity of liquid classification method based on EDXRD and hybrid discriminant analysis (HDA) is demonstrated by previous work. As the spatial resolution of the EDXRD system is relatively low, the measured spectrum from one detection unit might be a mixed spectrum of two objects. The actual spectra could be very complex with hundreds of objects combinations. The changes of the mixed spectra patterns influence the statistical analysis results of HDA, which might lead to classification mistakes. In order to solve this problem, the spectra of the contrabands and daily supplies in the original data set were mixed up to extend the training set in a specified way. Experiment results showed that the classification method with extended training set are more effective when dealing with complex mixed spectra. The proposed method would be very practical in liquid classification based on EDXRD.

This work was supported by a grant from the National Natural Science Foundation of China (Pioneer Comprehensive Optimization Theory and Technology for Low-dose CBCT. NO.11435007).

N63-7: Null-Hypothesis Testing Using Distance Metrics for Verification of Arms-Control Treaties M. Khalil $^{1}$, E. M. Brubaker ${ }^{1}$, N. R. Hilton ${ }^{1}$, M. A. Kupinski ${ }^{2}$, C. J. MacGahan ${ }^{2}$, P. A. Marleau ${ }^{1}$

${ }^{1}$ Sandia National Laboratories, Livermore, California, USA

${ }^{2}$ College of Optical Sciences, The University of Arizona, Tucson, Arizona, USA

We investigate the feasibility of constructing a data-driven distance metric for use in null-hypothesis testing in the context of arms-control treaty verification. The distance metric will be used in testing the hypothesis that the available data are representative of a certain object or otherwise, as opposed to binary-classification tasks that have been studied previously. The metric, being of strictly quadratic form, is essentially computed using projections of the data onto a set of optimal vectors. These projections can be accumulated in list mode. The relatively low number of projections hampers the possible reconstruction of the object and subsequently the access to sensitive information. The projection vectors that channelize the data are optimal in capturing the Mahalanobis squared distance of the data associated with a given object under varying nuisance parameters. The vectors are also chosen such that the resulting metric is insensitive to specific data that are deemed to contain sensitive information and thus providing some desirable flexibility for treaty verification tasks. Data used in this study were generated using the GEANT4 toolkit to model gamma transport through Monte Carlo simulations. For numerical illustration, synthetic data were obtained using custom models for plutonium inspection objects. The resulting metric based on a relatively low number of channels shows moderate agreement with the Mahalanobis distance metric while being insensitive to predefined data.

\title{
N63-8: Photon Counting Energy Resolved Transmission Spectroscopy for Real-Time Mineral Analysis
} P. A. B. Scoullar, L. Grundy, F. Linnane

Southern Innovation, Melbourne, Australia

X-ray transmission (XRT) techniques are increasingly used in ore processing to provide a means of material discrimination based on bulk X-ray transmission characteristics. However, despite their increasing use, the potential of these techniques suffers from the limitations of conventional saturated detection systems.

Our presentation will detail a photon counting, energy resolved, X-ray transmission spectroscopy technique that overcomes many of the limitations of traditional XRT systems. The photon counting method allows us to capture transmission information at much higher detector count rates and thereby increase the translation speed and resolution of the material scanned. We also use a full spectrum technique to convert this transmission information to effective atomic number (Zeff) at high data rates. This combined transmission and effective atomic number (Zeff) information acquired at high resolution gives us the ability to sample and analyse in more detail than has been traditionally possible with XRT.

We demonstrate the effectiveness of this technique with images from coal samples demonstrating our ability to discriminate between high and low grade coal. We also present data from tests on Iron ore demonstrating a discrimination ability to better than $5 \%$. The results that we present demonstrate the potential of our techniques to effectively discriminate at high speeds in both Coal 
and Iron ore industries. This has the potential to significantly increase the range of applications for this enhanced XRT method across a range of ores and processes.

N64: Nuclear Physics Instrumentation II: Experiments

Friday, Nov. $4 \quad$ 08:00-10:00 Curie 1

N64-1: The Status of AMoRE-Pilot Experiment

C. S. Kang

Center for Underground Physics, Institute for Basic Science, Daejeon, Republic of KOREA

On behalf of the AMoRE collaboration

The AMoRE (Advanced Mo-based Rare process Experiment) aims to search for neutrinoless double beta decay of 100 Mo. The international project uses Mo-based scintillating crystals based on simultaneous detection of heat and light signals at mK temperatures. Scintillating crystals of $40 \mathrm{Ca} 100 \mathrm{MoO} 4$ synthesized with radio-pure elements in $48 \mathrm{Ca}$-depletion and $100 \mathrm{Mo}-$ enrichment are used as the main target material in the source-equal-to-detector concept. Metallic magnetic calorimeters are adapted as the sensor technology to measure the thermal calorimetric signals. We have built a pilot detector with five crystals with the total mass of about $1.5 \mathrm{~kg}$ installed in the YangYang Underground Laboratory (Y2L). We present the current statue of the pilot experiment together with scheduled future upgrades of the project.

N64-2: Searching Neutrinoless Double Beta Decay with EXO-200

T. Michel

Erlangen Centre for Astroparticle Physics, Erlangen, Germany

On behalf of the EXO-200 Collaboration

Neutrinoless double beta decay is a hypothetical nuclear decay in which two neutrons decay in a nucleus to two protons and two electrons without neutrino emission. Neutrinoless double beta decay is considered to be the most practical way to answer the question whether neutrinos and antineutrinos are their own antiparticles, thus Majorana fermions. The half-life of this decay would be related to the masses of neutrino mass eigenstates and the neutrino mixing parameters and therefore could contribute to determine the neutrino masses and their hierarchy. Several experiments in the world - using different nuclides and detector concepts - are looking for this decay. EXO-200, the Enriched Xenon Observatory 200, is an ultra-low background single-phase time projection chamber filled with $175 \mathrm{~kg}$ of liquid xenon enriched to $80 \%$ with ${ }^{136} \mathrm{Xe}$. The measurement of charge induction and collection signals in two tilted planes of wires at both lids of the cylindrical vessel together with the measurement of the produced scintillation light using two planes with 234 large area avalanche photodiodes allows to locate the positions of the energy depositions and the determination of the total deposited energy. EXO-200 has demonstrated excellent energy resolution and background rejection capabilities by exploiting its position resolving capabilities. With the first two years of data, EXO-200 has set a lower limit of $1.1 \times 10^{25}$ yrs at $90 \%$ C.L. on the neutrinoless double-beta decay half-life of ${ }^{136}$ Xe. After a two-year long interruption, EXO-200 is close to resume data taking with hardware improvements like for example an upgraded signal processing electronics to improve energy resolution further. In this talk, we will present the detector principle with a special view on light detection and charge measurement and the results of the search for neutrinoless beta decay with EXO-200.

N64-3: nEXO - an experiment to search for neutrinoless double beta decay

A. Schubert

Standford University, Stanford, CA, USA

On behalf of the nEXO collaboration

The successor of the neutrinoless double beta decay search experiment EXO-200 is the "next Enriched Xenon Observatory" nEXO. nEXO is currently in a research and development phase. The baseline detector concept assumes 5 tons of liquid xenon, enriched in ${ }^{136} \mathrm{Xe}$, within a cylindric time projection chamber. The detector is being designed to reach a half-life sensitivity of $>5$ $\mathrm{x} 10^{27}$ yrs for the neutrinoless double beta decay of ${ }^{136}$ Xe covering the inverted neutrino mass hierarchy with 5 years of data. The half-life sensitivity depends on energy resolution and background rate. VUV scintillation light detection will be performed with silicon photomultipliers at the mantle of the TPC to fulfill the demands of the experiment concerning background radiation level and photon number resolution. In contrast to EXO-200, nEXO is a single-sided time-projection chamber. Another difference to EXO-200 is the electrode configuration to detect the charge released in the liquid xenon by the two decay electrons. The baseline nEXO concept assumes $2.1 \times 2.1 \mathrm{~mm}^{2}$ wide charge collecting pads connected along the diagonals of quadratic matrices of electrodes. In this contribution, we will present the $\mathrm{nEXO}$ detector design along with the physics case and the current status of R\&D efforts.

\section{N64-4: RED-100 Two-Phase Xenon Emission Detector to Search for Coherent Neutrino Scattering}




\author{
$\underline{\text { A. Bolozdynya }}$ \\ National Research Nuclear University MEPhI, Moscow, Russia \\ On behalf of the RED Collaboration
}

The elastic coherent scattering of neutrinos off nuclei is a process allowed in the Standard Model of particle interactions that was predicted 50 years ago, but so far has not been observed. This process plays an important role in the formation of the universe and the evolution of stars and can be a sensitive probe of New Physics beyond the Standard Model. Experimental observation of the effect is very difficult because of the extremely low energy deposition in a massive detector medium. The paper describes the first technical run of the RED-100 two-phase liquid xenon emission detector constructed to detect the elastic coherent neutrino scattering off xenon nuclei when the detector is installed practically on the Earth's surface. The detector RED-100 cryostat is made of titanium and includes 38 low-background HAMAMATSU R11410-20 photomultipliers operating with active bases and viewing $220 \mathrm{~kg}$ LXe detector medium. The experimental setup includes gas storage and purification system, "break-out-box" interface unit containing all feedthroughs and gas connectors of the detector that operate at room temperature and can be placed outside a passive shielding, based on thermosiphon technology cryogenic system, slow control and data acquisition systems.

\title{
N64-5: The Neutron Induced Fission Fragment Tracking Experiment: High-Precision Fission Cross Section Measurements with a Time Projection Chamber
}

N. Bowden

Lawrence Livermore National Laboratory, Livermore, CA, USA

On behalf of the NIFFTE Collaboration

The goal of the Neutron Induced Fission Fragment Tracking Experiment (NIFFTE) is to measure fission cross sections with unprecedented precision. The NIFFTE Collaboration has designed and built a Time Projection Chamber (TPC) for this purpose. The tracking capabilities of this device allow for the full reconstruction of charged particles produced by neutron beam induced fissions from a thin central target. The wealth of information gained from this approach will allow cross section systematics studied in detail, particularly those relating to particle identification and target and beam spatial uniformity. Here we present the current status of the NIFFTE TPC, describe the wide variety of systematic studies being performed, and outline recent results from measurements performed at the Los Alamos Neutron Science Center (LANSCE) facility.

LLNL-ABS-690608. This work was performed under the auspices of the U.S. Department of Energy by Lawrence Livermore National Laboratory under Contract DE-AC52-07NA27344

N64-6: Active Target Time Projection Chamber (AT-TPC) at the NSCL

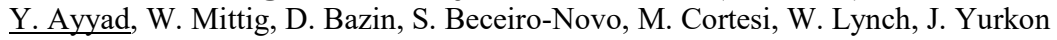
MSU, NSCL, East Lansing, USA

Active targets are versatile devices well adapted for reactions with the most exotic beam species, providing high luminosity without loss of resolution. Particles detected inside the detector gas target volume are tracked and reconstructed with high efficiency. The Active Target Time Projection Chamber (AT-TPC) [1] detector is a novel active target particularly designed for performing direct and resonant reactions with the radioactive beams available in the NSCL/FRIB facilities within an energy range of around 5 to $100 \mathrm{~A} \mathrm{MeV}$. One of the main features of this detector is the possibility of coupling a superconductive MRI solenoid ( $2 \mathrm{~T}$ ) to allow the measurement of the magnetic rigidity of the particles inside the volume. This novel technique will be used together with thick Gas Electron Multipliers (thick GEMs) designed to operate the detector with pure gases such as hydrogen, deuterium or helium [2]. This is the first active target operating under such conditions.

In this work we will discuss the experimental program regarding direct and resonant reactions for the AT-TPC active targets. In particular, the AT-TPC has proven to be very successful in determining exotic cluster structures in $10 \mathrm{Be}, 14 \mathrm{C}$ and $12 \mathrm{Be}$ as well as in investigating isobaric analog states in $47 \mathrm{~K}$. The current scientific program will involve reactions of astrophysical interests, 2-proton radioactivity and the measurement of fission barriers of neutron deficient nuclei. Current results and future perspectives will be presented in this talk. In addition, novel and sophisticated reconstruction algorithms suited for this setup (Active Target+Solenoid) will be presented.

[1] D. Suzuki et al., Nucl. Instrum. Meth. A 691 (2012) 3954 [2] M. Cortesi et al., Conference: 4th Conference on Micro-Pattern Gaseous Detectors - MPGD2015, At Trieste (Italy), on October 12 - 15, (2015)

N64-7: DSSSD Based Spectrometer of the Dubna Gas-Filled Recoil Separator to Search for ER-Alpha Sequences in a Real-Time Mode

Y. S. Tsyganov, A. N. Polyakov, A. A. Voinov

FLNR, JINR, Dubna, Moscow region, Russia 
Recently, in the DGFRS related experiments the discoveries of new superheavy elements Z=114-118 were successfully performed. As concerns to a background radical suppression in those experiments it was namely "active correlat6ions" method which was applied to this goal. Note, that application of DSSSD detector allows one to apply this method more effectively, although with some difficulties due to neighbor strips edge effects. Present version of the DGFRS spectrometer is considered. First results of its application in the $240 \mathrm{Pu}+48 \mathrm{Ca}-->114^{*}$ complete fusion nuclear reaction are presented too.

\section{N64-8: SIRIUS Project Spectroscopy \& Identification of Recoiling Ions Using S3}

N. Karkour

CNRS/IN2P3/CSNSM, Orsay, France

On behalf of the SIRIUS Project Collaboration

SIRIUS Project (Spectroscopy \& Identification of Recoiling Ions Using S3) SIRIUS is a state of the art detector system for nuclear decay spectroscopy that is mounted at the focal plane of S3 (Super Separator Spectrometer), which is part of the new SPIRAL2 facility at GANIL, Caen. Such a system requires high performance as it is dedicated to the study of very exotic nuclei. It is the result of collaboration between IRFU, IPHC, GANIL and CSNSM. SIRIUS is financed by the CPIER (Contrat de Plan Interrégional Etat-Régions Vallée de le SEINE). It is composed of a succession of detectors (Trackers, Si DSSD and Tunnel plus an array of five clover Ge detectors). This set-up is mounted in a compact geometry using new advanced technologies. The Energy range measurement varies from $50 \mathrm{keV}$ to over $500 \mathrm{MeV}$ with high precision $(2 \times 10-3)$ on low energies and $1 \%$ for the heavy ions. A major challenge has been the development of new electronics with a very large dynamic range maintaining an adequate resolution for the measured particles. The electronic readout used for these tracker detectors is based on the GET system funded by the ANR (ANR-09-BLAN-0203-01). The GET system consists of 256 channels front-end boards called AsAd (Asic Adc board) connected to back-end boards called CoBo (Concentration Board). The 256 DSSD detector channels are processed by double gain ASIC chip FPCSA (Floating Point Charge Sensitive Amplifiers). The 96 tunnel detector channels are processed using DFCSP (Digital Feedback Charge Sensitive Preamplifiers). The charge pulses from the Si and Ge detector preamplifiers are digitized at $200 \mathrm{MHz}$ by 14bits Specific flash ADCs-Digital to Analog Converter (FADC-DAC) in the NUMEXO2 boards from EXOGAM2 project. The Trigger system will use the Advanced GAmma Tracking Array (AGATA) Global Trigger System (GTS) and a BEAST interface philosophy for event handling with a common clock distribution, timestamping and triggerless acquisition. The acquisition system uses the GANIL standard NARVAL and GECO software installed @ GANIL for other facilities.

\section{NCP: NSS Closing Plenary}

Friday, Nov. $4 \quad$ 10:30-13:00 Curie 1+2

NCP-1: Instrumentation, diagnostic and measurement challenges and opportunities for JHR testing reactor and ITER fusion Device

R. Reichle $^{1}$, G. Bignan ${ }^{2}$

${ }^{I}$ ITER organisation, Saint Paul lez Durance, France

${ }^{2}$ CEA-DEN, Saint Paul lez Durance, France

Challenges, requirements and latest developments will be presented. In southern France two worldwide technological and scientific challenging devices/machines are under construction dealing with advanced and future nuclear energy: JHR Material Testing Reactor and ITER Fusion Device. The JHR reactor objectives, principles and main characteristics, specific experimental devices associated to measurement techniques and methodology, their performances, their limitations and field of applications will be presented and discussed. And the presentation will also give a short update on the general status of the ITER project.

\section{NCP-2: Instrumentation Challenges for Future Colliders}

\section{Demarteau}

Argonne National Laboratory, Argonne, Illinois, US

The field of particle physics is exploring different options for a new collider to follow the Large Hadron Collider, currently operating at CERN. Linear and circular electron-positron colliders have been proposed as next machines, as well as protonproton and electron-proton colliders running at various center of mass energies. These next generation machines create tremendous challenges for the detectors, their data acquisition systems and analysis frameworks. The stringent requirements arise from the technical aspects of the experimental environment as well as from the physics requirements. An overview will be given of these instrumentation challenges for the various experimental domains and a path to adequately address these issues will be indicated.

NCP-3: Student Awards Ceremony for Best Student Oral Presentation and Poster $\underline{\text { S. Kuehn }}^{1,2}$, E. Elsen ${ }^{2}$ 
${ }^{1}$ University of Freiburg, Germany, Freiburg, Germany

${ }^{2}$ CERN, Geneva, Switzerland

Student Awards Ceremony for best student oral presentation and poster. A selected committe judges on all abstract submitted for the student awards.

NCP-4: Presentation from the IEEE Radiation Instrumentation Steering Committee

P. Le Du

CEA, Saclay, France

Presentation from the Radiation Instrumentation Steering Committee Chair Patrick Le Du Details on the conference survey and experience

NCP-5: NSS Closing Ceremony and Happy Hour

$\underline{\mathrm{S} . \mathrm{Kuehn}}^{1,2}$, E. Elsen ${ }^{2}$

${ }^{I}$ University of Freiburg, Germany, Freiburg, Germany

${ }^{2}$ CERN, Geneva, Switzerland

Closing of the NSS Symposium

Thank you for your participation in the NSS Symposium 2016! Please don't hesitate to contact us with your feedback, suggestions, and questions, by sending an email to nss2016program AT physik.uni-freiburg.de. We sincerely hope you enjoy the exciting 2016 IEEE NSS/MIC/RTSD symposium in Strasbourg, France, in the heart of Europe, in autumn 2016.

\section{MIC Program}

M01: MIC Plenary 1

Wednesday, Nov. 2

08:00-10:00

Schweitzer

M01-1: Introduction

D. Visvikis ${ }^{1}$, S. Surti ${ }^{2}$

${ }^{I}$ INSERM UMR 1101, CHRU Brest, INSERM, LaTIM, Brest, France

${ }^{2}$ Department of Radiology, University of Philadelphia, Philadelphia, USA

This presentation will concern the introduction of the second MIC plenary session, as well as information related to the rest of the MIC schedule and events

M01-2: Radiomics: Mining images to make better decisions in cancer therapy A. Dekker

Department of Radiation Oncology (MAASTRO), MAASTRO Clinic, Maastricht, The Netherlands

The rise of radiomics, the high-throughput mining of quantitative image features from (standard-of-care) medical imaging for knowledge extraction and application within clinical decision support systems (please watch the animation:

https://youtu.be/Tq980GEVP0Y) to improve diagnostic, prognostic, and predictive accuracy, has significant and substantial implications for the medical community. Radiomic analysis exploits sophisticated image analysis tools and the exponential growth of medical imaging data to develop and validate powerful image-based signatures/models. This lecture describes the process of radiomics, its pitfalls, challenges, opportunities, and its capacity to improve clinical decision making (presently primarily in the care of patients with cancer, however, all imaged patients may benefit from quantitative radiology). Finally, the field of radiomics is emerging rapidly, however, the field lacks standardized evaluation of both the scientific integrity and the clinical significance of the numerous published radiomics investigations resulting from this growth. The lecture will discuss the need for rigorous evaluation criteria and reporting guidelines in order for radiomics to mature as a discipline.

\section{M01-3: Adventures in the nuclear medical imaging wonderland}

S. Cherry

Department of Biomedical Engineering and Department of Radiology, University of California, Davis, Davis, CA, USA 
Adventures in the nuclear medical imaging wonderland. The presentation will be given as part of the IEEE Marie Curie award for Prof Simon Cherry

J01: Joint Session I - MIC-NSS-RTSD

Wednesday, Nov. $2 \quad$ 10:30-12:00 Schweitzer

See listing in the NSS section.

M02: Parametric Imaging / kinetic modeling

Wednesday, Nov. $2 \quad$ 10:30-12:00 Cassin

M02-1: Hybrid Whole-Body Dynamic TOF PET Imaging for Simultaneous Estimation of Compartmental and Patlak Parametric Maps from Continuous Bed Motion Data

$\underline{\text { F. A. Kotasidis }}^{1,2,3}$, V. Garibotto ${ }^{3}$, H. Zaidi ${ }^{3,4,5}$

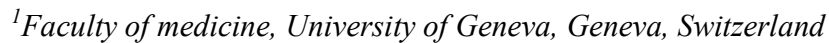

${ }^{2}$ Wolfson Molecular Imaging Centre, University of Manchester, Manchester, United Kingdom

${ }^{3}$ Department of Nuclear Medicine and Molecular Imaging, Geneva University Hospital, Geneva, Switzerland

${ }^{4}$ Geneva Neuroscience Centre, University of Geneva, Geneva, Switzerland

${ }^{5}$ Department of Nuclear Medicine and Molecular Imaging, University of Groningen, Groningen, The Netherlands

A number of studies have highlighted the clinical role of kinetic modelling following dynamic PET acquisition, since it is able to provide quantitative parameters, more closely related to the underlying pathology than semi-quantitative indices, such as SUV. However, due to the limited axial FOV of current PET scanners, temporally continuous dynamic data are only available for a single bed position. Therefore, full compartmental modelling is axially limited as opposed to whole body imaging protocols which are used in oncology, limiting its applicability in this field. Furthermore, if the blood activity concentration is derived from the images, similar dynamic data of a large blood pool are needed. Lately a new approach to clinical dynamic whole-body imaging has been proposed, consisting of an initial blood pool (cardiac) scan, followed by a number of whole body passes, to estimate whole-body Ki Patlak parametric images. While such an approach improves upon previous whole-body protocols for kinetic parameter estimation, it doesn't fully exploit the information that could be extracted, as well as the benefits of continuous bed motion (CBM) acquisition in dynamic imaging. In this work, we propose a modified CBM whole-body dynamic protocol to simultaneously perform full compartmental modelling in the FOV covering the initial blood pool scan as well as whole-body Patlak analysis. Furthermore, the new protocol does not restrict the initial blood pool scan, for which full compartmental modelling can be performed, to be limited over the heart region but to be chosen based on the localization of the disease within the body. Initial clinical whole-body dynamic scans are reported, along with the estimated micro- and macro-parameter maps. Initial results suggest that not only Ki images from full modelling provide superior tumor to background contrast and improved variance to Patlak Ki but additional micro-parameters are made available to the referring clinician, enabling multi-parametric evaluation of the disease within a clinically feasible dynamic imaging protocol.

M02-2: Direct Parametric Imaging of Reversible Tracers Using Partial Dynamic Data K. Kim, G. El Fakhri, Q. Li

Radiology, Massachusetts General Hospital and Harvard Medical School, Boston, MA, USA

Direct reconstruction of parametric image in positron emission tomography (PET) computes the voxel-wise kinetic parameters directly from raw measurement, and thus can obtain more accurate physiological information of tracer uptake. Conventional direct estimation usually has two steps in iterative process: 1) the update of dynamic images and 2) the voxel-wise nonlinear least square (NLS) parameter fitting. Recently, a penalized direct estimation method using a total-variation (TV) algorithm has been proposed to estimate parametric images from complete dynamic time series, which is demonstrated to be able to significantly improve the image quality. However, it is difficult to acquire dynamic data from tracer injection to steady state in clinical applications. This difficulty is one of major obstacles of wide use of direct parametric imaging. In this project, we explore the feasibility to estimate voxel-wise kinetic parameters using partial dynamic data, particularly the first and last 10 minutes of a typical dynamic scan. We propose a novel penalized direct estimation of parametric images in which the cost function contains the Poisson likelihood, ridge regression, a TV penalty of dynamic images, and a patch-based joint similarity penalty of kinetic images. Considering the fact that usually the K1 parameter dominates at the first 10 minutes of tracer dynamics and therefore can be accurately estimated, we utilize the updated K1 image in each iteration as a structural prior to facilitate the estimation of other kinetic parameters. In addition, we exploit an alternating direction method of multipliers (ADMM) combining with a separable quadratic surrogate (SQS) to solve the optimization problem. We also perform a simulation with a brain phantom to evaluate the proposed method. Our results demonstrate that the proposed method outperforms the conventional direct estimation methods.

M02-3: 18F-FDG:18F-NaF PET/MR Multi-Parametric Imaging with Kinetics-Based Bone Segmentation for Enhanced Dual-Tracer PET Quantification 


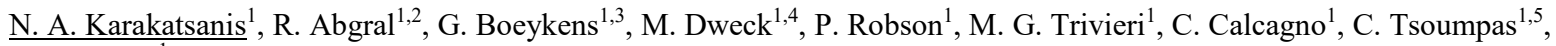
Z. A. Fayad ${ }^{1}$

${ }^{I}$ Translational and Molecular Imaging Institute, Icahn School of Medicine at Mount Sinai, New York, NY, United States

${ }^{2}$ Department of Nuclear Medicine, University Hospital of Brest, Brest, France

${ }^{3}$ Academisch Medisch Centrum, Amsterdam, Netherlands

${ }^{4}$ British Heart Foundation/University Centre for Cardiovascular Science, University of Edinburgh, Edinburgh, United Kingdom

${ }^{5}$ Division of Biomedical Imaging, University of Leeds, Leeds, United Kingdom

Dual-tracer Positron Emission Tomography (PET) imaging involves the administration of two tracers for their co-registered evaluation within the same imaging subject and session. Previously, the concept of mixing 18F-Fluorodeoxyglucose (18F-FDG) and $18 \mathrm{~F}$-sodium fluoride (18F-NaF or $18 \mathrm{~F}-$ Fuoride) tracers had been proposed in oncology to enable characterization of a wider spectrum of tumor types from a single PET/CT exam. In this study, we are proposing an optimized F18-FDG:F18-NaF cocktail PET/MR imaging framework to enable multi-parametric PET/MR assessments of enhanced quantification. Our aims are: (i) to benefit from the superior soft tissue characterization from MR and, thus, enable more accurate PET lesion localization, (ii) significantly reduce radiation exposure, therefore allowing for multiple low-dose exams to closely monitor treatment response in oncology, (iii) facilitate multi-parametric evaluations for cardiovascular diseases too, and iv) exploit the high bone uptake rate of the cocktail NaF compound to improve PET/MR attenuation correction. For that purpose, we introduced a clinically feasible single-bed dynamic 18F-FDG:18F-NaF PET/MR protocol employing the same recommended dosage as for single-tracer PET studies. Subsequently, the dynamic PET images were robustly analyzed with the Patlak method to produce tracer uptake rate Ki parametric images. Then, the bone regions were segmented from the Ki and PET images and included as a fifth tissue class in the co-registered MR Dixon-based 4-class attenuation map. Our series of rabbit dual-tracer PET/CT and PET/MR exams, as well as the subsequent human carotid PET/MR studies, suggested a maximum FDG:NaF ratio of 4:1. This cocktail synthesis yielded sufficient signal for the robust segmentation of the major bone tissues, while FDG count statistics remained high. Finally, an average 15\% improvement was observed for PET FDG:NaF bone quantification with the proposed 5-tissue class attenuation correction method.

This work was supported by NIH/NHLBI R01HL071021 grant and Siemens Medical Solutions.

M02-4: A Practical SUV-Guided Approach to Parametric Image Reconstruction via Mixture Models F. O'Sullivan ${ }^{1}$, R. Xiu ${ }^{1}$, M. Muzi ${ }^{2}$, J. Huang ${ }^{1}$, T. Mou ${ }^{1}$

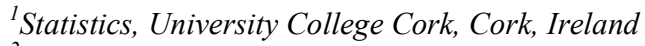

${ }^{2}$ Radiology, University of Washington, Seattle, Washington, USA

In the residue function approach to parametric imaging, the local tissue residue (or impulse response function) is represented by a mixture of basis residue functions corresponding to a collection underlying physiologic characteristics represented in the field of view. This formulation allows the estimation/reconstruction problem to be described in terms of standard constrained programming. In this setting parametric imaging can be formulated as a problem of estimating local coefficients of the nonnegative sum of the temporal time-course of the basis residues. The normalisation of these temporal time-courses can ensure that the sum of the local spatial coefficients at the voxel equates to the normalised integrated activity (SUV). Two broad strategies for parametric imaging reconstruction with mixture models can be considered:(i) Voxel-level mixture modelling of reconstructed data, or (ii), Direct reconstruction of the mixture model. The work explores both of these strategies and also considers a hybrid approach in which direct reconstruction of local integrated activity (SUV) is combined with a segmentation based scheme to obtain an implementation of the direct reconstruction approach. The efficiency of the proposed approach relies on a novel image domain approximation of the ML reconstruction. In this technique the time-consuming projection and back-projection steps are implemented using FFT techniques. Numerical simulations are used to explore these alternative strategies. The methods are further illustrated with data from 3-D dynamic imaging studies with FDG, FLT and H2O. While there are identifiable benefits associated with direct reconstruction techniques, the 2-step hybrid model formulation is found to be a highly effective and practical implementation.

Supported by SFI under PI-11/1027 and NIH under NCI CA-42045.

M02-5: Quantitative Myocardial Perfusion from Static Cardiac CT Informed by Timing Bolus Kinetics M. Bindschadler ${ }^{1}$, K. R. Branch ${ }^{2}$, A. M. Alessio ${ }^{1}$

${ }^{I}$ Radiology, University of Washington, Seattle, WA, United States

${ }^{2}$ Cardiology, University of Washington, Seattle, WA, United States

Qualitative perfusion assessment using cardiac CT angiography (CTA) imaging has been demonstrated, but, like all qualitative methods, cannot detect perfusion abnormalities in balanced ischemia and has limited ability to follow disease progression. We propose to estimate myocardial blood flow (MBF) quantitatively using only data collected for a typical CTA exam by exploiting 
the dynamic information in the initial timing bolus image sequence. Methods: Through simulation studies of myocardial iodine exchange, we derive a functional relationship between contrast injection parameters, the myocardial input function, true MBF, and resulting myocardial enhancement at the time of CTA acquisition. The timing bolus sequence is used to determine the patient-specific venous transfer function between the contrast injection site and ascending aorta. Convolution of a larger contrast bolus used for the CTA with the transfer function enables prediction of the myocardial input function. This input function and the soft-tissue enhancement difference between the timing bolus and CTA images yield a patient-specific mapping between myocardial CT number and estimated MBF. We demonstrate this method on seven patient CTA studies. Results: Simulation studies demonstrate an exponential mapping between CT number and flow and suggest that patient-specific input function parameters of time to peak and area lead to unique mappings. Results of the method applied to seven patients with normal CTAs and no evidence of ischemia produced MBF estimates of $0.92+/-0.53 \mathrm{ml} / \mathrm{min} / \mathrm{g}$, consistent with normal resting MBF.

Conclusion: The new method could have considerable advantages over existing quantitative methods in radiation dose savings and ease of clinical adoption. Initial results suggest that timing-bolus informed MBF estimation from CTA images has promise, but needs further refinement and validation.

M02-6: Quantitative Myocardial Blood Flow Estimation from Dynamic Contrast Enhanced Cardiac CT

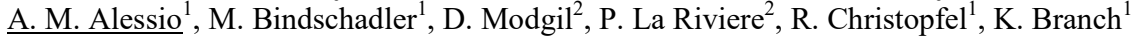

${ }^{I}$ University of Washington, Seattle, WA, USA

${ }^{2}$ University of Chicago, Chicago, IL, USA

Several studies have shown that myocardial blood flow (MBF) can be estimated from dynamic contrast enhanced (DCE) cardiac $\mathrm{CT}$ acquisitions. This estimation is challenging considering a) frame-to-frame motion, b) the high level of image noise resulting from the need to acquire relatively low-dose data, and c) the non-quantitative nature of CT numbers. In this work, we present processing steps to address these challenges and compare our DCE-CT flow estimates to PET flow estimates in patient studies. Methods: We developed a segmentation method that morphs a single frame segmentation to each dynamic frame in order to minimize the impact of motion. We also developed a 2-compartment model that estimates myocardial perfusion, volume of the interstitial fluid, input delay, and CT number baseline. The estimation of the baseline value is intended to minimize the impact of inaccurate $\mathrm{CT}$ numbers. We tested the processing in a patient trial with 13 patients receiving a Rb82 PET exam and a research rest+stress DCE-CT exam within 2 months of their PET study. DCE-CT exams were performed using $120 \mathrm{kVp}, 18 \mathrm{mAs}$ with prospective ECG-triggering on a wide-angle CT system with full heart coverage at each frame. The proposed processing was performed on the 12-18 image volumes taken at end inspiration. Results: Three stress exams were excluded due to unsuccessful stress response and one CT was excluded due to poor timing. For the remaining 12 rest and 9 stress studies, the estimation of regional flow at a 17-segment level yielded a CT to PET correlation of $0.6+/-0.2$, with an error between modalities of $-0.2+/-0.9$ $\mathrm{ml} / \mathrm{g} / \mathrm{min}$ and a root mean square error of $0.9+/-0.6 \mathrm{ml} / \mathrm{g} / \mathrm{min}$. Each dynamic CT scan was performed with an effective dose of less than $4 \mathrm{mSv}$. Conclusion: Although flow estimates have a high variance in both the CT and PET domains, this study demonstrates viable processing steps in order to generate CT-derived MBF estimates that correlate well to quantitative PET imaging.

J02: NSS/MIC Joint Session 2: Hadrontherapy

Wednesday, Nov. $2 \quad$ 14:00-16:00 Schweitzer

See listing in the NSS section.

M03: Quantitative imaging techniques \& image analysis I

Wednesday, Nov. $2 \quad$ 14:00-16:00 Cassin

M03-1: Multi-Center Evaluation of Eleven PET/MRI Brain Attenuation Correction Methods

C. N. Ladefoged ${ }^{1}$, I. Law ${ }^{1}$, U. Anazodo ${ }^{2}$, K. St. Lawrence ${ }^{2}$, D. Izquierdo-Garcia ${ }^{3}$, C. Catana ${ }^{3}$, N. Burgos ${ }^{4}$, J. Cardoso $^{4,5}$, S. Ourselin ${ }^{4,5}$, B. Hutton ${ }^{6}$, I. Mérida ${ }^{7,8}$, N. Costes ${ }^{7}$, A. Hammers ${ }^{7,9}$, D. Benoit ${ }^{1}$, S. Holm ${ }^{1}$, M. Juttukonda ${ }^{10}$, H. An $^{10}$, J. Cabello $^{11}$, M. Lukas ${ }^{11}$, S. Nekolla ${ }^{11}$, S. Ziegler ${ }^{1}$, M. Fenchel ${ }^{12}$, B. Jakoby ${ }^{12,13}$, M. E. Casey ${ }^{14}$, T. Benzinger ${ }^{15}$, L. Højgaard ${ }^{1}$, A. E. Hansen ${ }^{1}$, F. L. Andersen ${ }^{1}$

${ }^{1}$ Clinical Physiology, Nuclear Medicine and PET, Rigshospitalet University Hospital, Copenhagen, Denmark

${ }^{2}$ Lawson Health Research Institute, London, Ontario, Canada

${ }^{3}$ Department of Radiology, Athinoula A. Martinos Center for Biomedical Imaging, Charlestown, Massachusetts, United States of America

${ }^{4}$ Translational Imaging Group, Centre for Medical Image Computing, University College London, London, United Kingdom

${ }^{5}$ Dementia Research Centre, Institute of Neurology, University College London, London, United Kingdom

${ }^{6}$ Institute of Nuclear Medicine, University College London, London, United Kingdom

${ }^{7}$ CERMEP imaging Centre, LILI-EQUIPEX - Lyon Integrated Life Imaging: hybrid MR-PET, Lyon, France

${ }^{8}$ Siemens Healthcare France SAS, Saint-Denis, France

${ }^{9}$ King's College London \& Guy's and St Thomas' PET Centre, Division of Imaging Sciences and Biomedical Engineering, King's College London, London, United Kingdom 
${ }^{10}$ Joint Department of Biomedical Engineering, The University of North Carolina at Chapel Hill and North Carolina State University, Chapel Hill, North Carolina, United States of America

${ }^{11}$ Nuklearmedizinische Klinik und Poliklinik, Klinikum rechts der Isar, Technische Universitaet Muenchen, Munich, Germany

${ }^{12}$ Siemens Healthcare GmbH, Erlangen, Germany

${ }^{13}$ University of Surrey, Guildford, Surrey, United Kingdom

${ }^{14}$ Siemens Medical Solutions USA, Inc., Knoxville, Tennessee, United States of America

${ }^{15}$ Mallinckrodt Institute of Radiology, Washington University, St. Louis, Missouri, United States of America

AIM: In order to further improve the current vendor-implemented MR attenuation correction (AC) for more accurate quantification, a number of MRAC methods have been proposed in the literature. Here, we evaluate a selection of novel methods in a large subject cohort.

METHODS: Eleven MRAC methods were evaluated on $201\left[{ }^{18} \mathrm{~F}\right] \mathrm{FDG}, 48\left[{ }^{11} \mathrm{C}\right] \mathrm{PiB}$, and $88\left[{ }^{18} \mathrm{~F}\right]$ florbetapir scans, both globally and regionally. Methods were vendor-implemented: Dixon and UTE; atlas/template-based: Segbone (Siemens), Ontario, Boston, UCL, and MaxProb (Lyon); simultaneous reconstruction of attenuation and emission-based: MLAA (Copenhagen); imagesegmentation-based: Munich, CAR-RiDR (UNC), and RESOLUTE (Copenhagen).

RESULTS: The average \pm SD global percentage bias in PET FDG uptake for each method was: Dixon (-11.3 \pm 3.5$) \%$, UTE ($5.7 \pm 2.0) \%$, Segbone $(-1.7 \pm 3.6) \%$, Ontario $(-4.3 \pm 3.6) \%$, Boston $(-0.3 \pm 1.8) \%$, UCL $(0.7 \pm 1.2) \%$, MaxProb (-0.4 \pm 1.6$) \%$, MLAA ($1.9 \pm 2.6) \%$, Munich $(3.7 \pm 2.1) \%$, CAR-RiDR $(-0.4 \pm 1.9) \%$, and RESOLUTE $(0.3 \pm 1.7) \%$. The best performing methods (Boston, UCL, MaxProb, RESOLUTE, CAR-RiDR) showed regional average errors within $\pm 3 \%$ of PET with CT-AC in all regions of the brain with FDG, and the same five methods showed that for $95 \%$ of the patients, $95 \%$ of brain voxels had an uptake that deviated by less than $15 \%$ from the reference. Comparable performance was obtained with $\mathrm{PiB}$ and florbetapir.

CONCLUSIONS: All of the proposed novel methods have an average global performance within limits likely to be acceptable, and the main difference between the methods is in the robustness, clinical feasibility, and number of outliers. We conclude that the problem of MRAC in the brain has been solved to a quantitatively acceptable degree, and that methods are ready for final clinical validation.

\author{
M03-2: Evaluation of Zero-TE-Based Attenuation Correction Methods on PET Quantification of PET/MRI \\ Head and Neck Lesions \\ K. S. Lee, G. Zaharchuk, P. K. Gulaka, C. S. Levin \\ Radiology, Stanford University, Stanford, CA, United States
}

Quantitative PET image reconstruction requires an accurate map of photon attenuation coefficients ( $\mu$-map) in order to correct the PET emission data. Current PET/MR imaging systems use methods based on MR image segmentation with subsequent assignment of empirical attenuation coefficients. In this study we examine the differences in the quantification of $18 \mathrm{~F}-\mathrm{FDG}$ standardized uptake values (SUV) in head and neck cancer, using two different MR imaging sequences for MR-based attenuation correction (MRAC): a zero echo time (ZTE) sequence which can image bone directly (ZTE-MRAC), and a vendor-provided 2point Dixon sequence that neglects bone (Dixon-MRAC). The $\mu$-maps from each MRAC techniques were compared to CT-based attenuation correction (CTAC) maps. Percent SUV-mean and SUV-max differences in relevant regions of interest (ROIs) were calculated for three patients. Relative to Dixon-MRAC, we observed $15 \pm 7 \%$ and $14 \pm 8 \%$ increase of SUV-mean and SUV-max, respectively, when ZTE-based bone information was incorporated in the attenuation map and using Dixon-based attenuation map, respectively. We also observed that use of Dixon-MRAC led to $7 \pm 7 \%$ and $8 \pm 8 \%$ underestimation of SUV-mean and SUVmax, respectively, whereas with ZTE-MRAC led to $6 \pm 8 \%$ and $5 \pm 8 \%$ higher SUV-mean and SUV-max, respectively, compared to with CTAC. This study is the first demonstration of ZTE-based attenuation correction in the head and neck region and compared with CTAC as a gold standard with the goal of improving PET quantitation. The study shows that incorporation of bone information on $\mu$-maps has a significant impact on SUV quantitation in head and neck cancer lesions.

Authors would like to thank Dr. Gaspar Del Paso for providing tools to get the attenuation map from ZTE and Dr. Garry Chinn for sharing insights about the methods.

M03-3: Joint Estimation of Activity and Attenuation: Application to Non-FDG TOF PET/MR Clinical Data L. Cheng ${ }^{1}$, S. Ahn ${ }^{1}$, D. D. Shanbhag ${ }^{2}$, H. Qian ${ }^{1}$, T. Deller ${ }^{3}$, F. Wiesinger ${ }^{4}$

${ }^{I}$ GE Global Research, Niskayuna, NY, USA

${ }^{2}$ GE Global Research, Bangalore, India

${ }^{3}$ GE Healthcare, Waukesha, WI, USA

${ }^{4}$ GE Global Research, Munich, Germany 
Attenuation correction is critical to accurate PET quantitation. Accurate and robust attenuation correction remains challenging in hybrid PET/MR because it is difficult to segment bones, internal air and lungs accurately in MR images and MR often suffers from artifacts due to metal implants and signal shading. Joint estimation (JE) of activity and attenuation from TOF PET data has recently gained substantial interest because the JE approach shows a promise to address the challenges in MR based attenuation correction. We have previously implemented a JE algorithm with MR-based priors and demonstrated its feasibility using FDG PET/MR clinical data. However, JE algorithms have rarely been evaluated on non-FDG tracers which often have spatially more specific uptake patterns with little activity in background tissue, and hence may pose challenges to JE. In this study, we evaluate the JE algorithm using non-FDG PET/MR clinical data. We demonstrate that the JE algorithm improves the accuracy and robustness of MR-based attenuation correction not only for FDG as previously shown but also for non-FDG tracers such as $68 \mathrm{Ga}-\mathrm{DOTATOC}$ and Fluoride. In addition, we demonstrate the critical role of TOF information in JE algorithms.

\author{
M03-4: Non-Rigid Event-by-Event Respiratory Motion Compensation for PET with Motion Information \\ Derived from Matched Attenuation Corrected Gated Data

Respiratory motion compensated PET reconstruction methods typically require non-rigid registration among respiratory-gated PET images, which are often reconstructed with mismatched CT-based attenuation correction (CTAC) due to misalignment between CT and gated PET. Such mismatch can cause substantial artifacts in the gated PET reconstructions, and can substantially affect the accuracy of motion estimate. The artifacts and inaccuracy will propagate into the final motion compensated reconstruction. In this work, we investigated the impact of mismatched CTAC on motion estimation and proposed to utilize MLACF (maximum-likelihood activity and ACF estimation) to generate phase-matched gated PET reconstructions to facilitate motion estimation and correction. Both XCAT phantom and human data of 18F-FPDTBZ tracer were investigated in this study. We compared the emission data reconstructed by MLACF approach to the mismatched CTAC and data without AC (NAC), in terms of motion field estimation and final event-by-event respiratory motion compensated reconstructions using a previously developed approach (Chan, MIC 2014 abstract). The results showed that gated images reconstructed using the MLACF approach led to more accurate motion estimation, while both CTAC and NAC could result in motion estimation errors. The final motioncompensated reconstruction demonstrated that MLACF-derived motion estimation reduced the artifact in the lower-lung regions and maintained the accuracy of bone marrow activity estimation. Our study suggests that it is critical for motion compensated image reconstruction to utilize motion information estimated from match attenuation corrected gated images.

\author{
M03-5: Modelling the Motion Dependent Point Spread Function in Motion Corrected Small Animal PET \\ Imaging \\ G. I. Angelis $^{1}$, J. E. Gillam ${ }^{1}$, A. Z. Kyme ${ }^{1,2}$, R. R. Fulton ${ }^{1,3}$, S. R. Meikle ${ }^{1}$ \\ ${ }^{I}$ Brain and Mind Centre, Faculty of Health Sciences, The University of Sydney, Sydney, Australia \\ ${ }^{2}$ Department of Biomedical Engineering, University of California Davis, Davis, USA \\ ${ }^{3}$ School of Physics, The University of Sydney, Sydney, Australia
}

Motion corrected images from awake and freely moving animals often exhibit reduced resolution when compared to their stationary counterparts. This could be attributed to the combination of brief periods of fast animal motion and insufficient motion sampling speed. In this paper we hypothesise that we can measure the motion dependent point spread function of a given study and mitigate the motion blurring artefacts in the reconstructed images, in a similar way that a measured system response point spread function can improve resolution due to geometric effects (e.g. parallax errors). We investigated this hypothesis on a set of experimentally measured phantom data, which underwent a series of distinctively different motion patterns, ranging from slow to fast. Preliminary results showed that motion corrected images have reduced resolution compared to the stationary image and noticeable motion blurring artefacts, particularly for fast speed/acceleration settings. In addition, images deconvolved after reconstruction with the measured motion dependent PSF appear to be sharper compared to their unprocessed counterparts, yet without completely eliminating the motion blurring artefacts. Work is in progress to refine the methodology, by decomposing the geometric and motion components of the PSF, as well as including the deconvolution within the reconstruction algorithm.

M03-6: Joint Respiratory and Cardiac Motion Compensation for Cardiac PET Imaging J. Cal-Gonzalez $^{1}$, M. L. Lassen ${ }^{1}$, K. Schäfers ${ }^{2}$, M. Hacker ${ }^{3}$, T. Beyer ${ }^{1}$

${ }^{I}$ QIMP group, Center for Medical Physics and Biomedical Engineering, Medical University of Vienna, Vienna, Austria ${ }^{2}$ European Institute for Molecular Imaging, University of Münster, Münster, Germany

${ }^{3}$ Division of Nuclear Medicine, Department of Biomedical Imaging and Image-guided Therapy, Medical University of Vienna, Vienna, Austria 
Respiratory and cardiac motion adds blurring to the PET activity distribution in the heart. We propose a novel method for a joint respiratory and cardiac motion compensation of PET data. We assume that cardiac triggers are available from an Electrocardiogram (ECG). The respiratory motion signals are obtained by using data-driven respiratory motion detection. Once the respiratory signal is extracted from the PET raw data, it will be used to create respiratory-gated sinograms, which are then coregistered to a reference frame obtaining MVFs in the projection space for each gated sinogram. These MVF are used for motioncompensation of each event in the listmode data. The transformed data is then stored in a new "respiratory motion-free" listmode file together with the original cardiac triggers. Finally, the cardiac-gated sinograms are reconstructed using a cardiac MoCo reconstruction. Acquisitions of a torso phantom were used for the evaluation of the method. Single respiratory ( 8 gates), cardiac (4 gates) and dual (6 respiratory +4 cardiac) gating approaches were evaluated. Further, respiration-only, cardiac-only MoCo and joint respiratory + cardiac MoCo methods were evaluated using the same data sets. Single-gated approaches lead to noisy images with an incomplete recovery of the motion blur. Dual-gated approaches lead to a better motion compensation at the expense of increased image noise. Respiration-only and cardiac-only MoCo approaches resulted in incomplete motion compensation, especially in cardiac-only MoCo. The proposed joint respiratory and cardiac MoCo lead to images with better contrast and lower noise. In summary, we developed a joint respiratory and cardiac motion compensation method for PET data, applicable to cardiac PET/CT and PET/MR imaging. The application of this MoCo method in clinical ${ }^{18} \mathrm{~F}-\mathrm{NaF}$ cardiac datasets is work-in-progress and the results will be presented at the conference.

M03-7: Real-Time Data-Driven Rigid Motion Detection and Correction for Brain Scan with Listmode PET

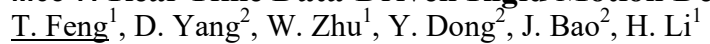

${ }^{1}$ United Imaging Healthcare America, Inc, Houston, US

${ }^{2}$ United Imaging Healthcare, Shanghai, China

Voluntary patient motion may cause blurring effects and mismatch of attenuation map with emission image during a brain PET scan. The goal of this study is to present a data-driven method that automatically detects and corrects rigid patient motion in real time, without any additional external device. The translational motion information over time was calculated by acquiring the center of mass $(\mathrm{COM})$ of the 3D tracer distribution in the image domain, while the rotational information was calculated by acquiring one of the eigenvector matrixes of the covariance matrix of the $3 \mathrm{D}$ tracer distribution in image domain. To acquire the above-mentioned image domain parameters within each time interval ( 1 second), the corresponding first and second moments (expectation and variance) of the dimensions in projection domain for each time interval were calculated using listmode data. The mathematical relationship of the calculated values in projection domain and the desired values in image domain were then derived. Using these steps, translational and rotational information over time were directly estimated from listmode data without image reconstruction. The motion information was then applied on the listmode data to reduce motion effects. Several brain scans were carried out with 18 F-FDG tracer to evaluate this method. Conventional method using dynamic frames was also implemented to compare with the new method. The measured translational and rotational information over time using both new listmode based approach and conventional dynamic frame approach matched well. The motion corrected images using the new method shows much reduced blurring effects with almost no additional computational cost post scan. This result demonstrated the feasibility of applying data-driven approach for detection and correction of patient head movement during PET scans without adding additional hardware, or additional post processing time.

M03-8: MRI-Dixon-Based Lean Body Mass Estimation in PET/MRI Using a Single Bed-Position Scan $\underline{\text { A. Olin }}^{1}$, C. N. Ladefoged ${ }^{1}$, I. Rausch ${ }^{2}$, J. Löfgren ${ }^{1}$, B. M. Fischer ${ }^{1}$, A. Kjær ${ }^{1}$, T. Beyer ${ }^{2}$, F. L. Andersen ${ }^{1}$, S. Holm ${ }^{1}$

${ }^{I}$ Dept. of Clinical Physiology, Nuclear Medicine \& PET, Rigshospitalet, University of Copenhagen, Copenhagen, Denmark ${ }^{2}$ Center for Medical Physics and Biomedical Engineering, Medical University of Vienna, Vienna, Austria

AIM: In quantitative PET, SUV normalized by lean body mass (LBM) has been shown to be more consistent across patients than SUV normalized by body weight. We propose an MRI-Dixon-based method for LBM estimation using data acquired from a single bed-position in PET/MRI. Our findings were compared to an empirical equation for LBM estimation.

MATERIALS AND METHODS: Whole-body PET/MRI was performed on 31 subjects with Dixon acquisition for the derivation of tissue classifying MR-based attenuation correction (MR-AC) maps. From these MR-AC maps the whole body composition was determined as the relative amount of lean tissue in the body and served as gold standard. For each subject, six artificial single bed-position MR-AC maps (head, head/thorax, thorax, thorax /abdomen, abdomen, pelvis) were extracted and from these the regional body compositions were calculated. For each bed-position multiple regression analysis was proposed as a method to model the relationship between the regional and whole body composition, along with other variables like subject weight and height. These models were evaluated by a cross-validation. Using the resulting LBM estimates the mean square error (MSE) was assessed.

RESULTS: The $\mathrm{R}^{2}$ between the regional and whole body composition ranged from 0.88 (head) to 0.97 (pelvis). For the empirical equation $\mathrm{R}^{2}=0.67$. The MSE (in $\mathrm{kg}^{2}$ ) of the cross-validation was 3.4 (head), 1.7 (head/thorax), 1.5 (thorax), 2.8 (thorax/abdomen), 1.5 (abdomen), and 1.0 (pelvis). The MSE of the empirical equation was $33 \mathrm{~kg}^{2}$.

CONCLUSION: The high $\mathrm{R}^{2}$ and low MSE of our method relative to the empirical equation imply that improved LBM estimates can be obtained, regardless of the anatomical location. The method is straightforward and relies only on easily assessable data, 
such as intrinsic MR-AC maps from combined PET/MRI examinations. Thus, using this method for SUV normalization, a potentially more reliable quantitative PET measure can be obtained, which is especially important in tumor response evaluation.

M04A: Posters: Radiotherapy I

\author{
Wednesday, Nov. $2 \quad$ 16:30-18:30 Etoile \\ M04A-1: Construction and Tests of an in-Beam PET-like Demonstrator for Hadrontherapy Beam Ballistic \\ Control \\ G. Montarou $^{1}$, M. Bony ${ }^{1}$, E. Busato ${ }^{1}$, R. Chadelas ${ }^{1}$, D. Donnarieix ${ }^{2}$, P. Force ${ }^{1}$, C. Guicheney ${ }^{1}$, C. Insa ${ }^{1}$, D. Lambert ${ }^{1}$, L. Lestand ${ }^{1}$, \\ M. Magne ${ }^{1}$, F. Martin ${ }^{1}$, M. Nivoix ${ }^{1}$, F. Podlyski ${ }^{1}$, A. Rozes ${ }^{1}$ \\ ${ }^{\text {I} C l e r m o n t ~ U n i v e r s i t y, ~ L a b o r a t o i r e ~ d e ~ P h y s i q u e ~ C o r p u s c u l a i r e ~ C l e r m o n t ~ F e r r a n d, ~ C l e r m o n t-F e r r a n d, ~ F r a n c e ~}$ \\ ${ }^{2}$ Service de Physique Médicale, Centre Jean Perrin, Clermont-Ferrand, France
}

We present the first results obtained with a detector, called Large Area Pixelized Detector (LAPD), dedicated to study the control the ballistics of the beam delivered to the patient by in-beam and real time detection of secondary particles, emitted during hadrontherapy treatments. These particles could be high energy photons (prompt ?) or $511 \mathrm{keV}$ ? from the annihilation of a positron issued from the $\beta+$ emitters induced in the patient tissues along the beam path. These methods require being able to detect with a huge efficiency, and with a minimum dead time, these secondary particles emitted when the beam hits the patient. The LAPD is similar to a PET camera. The $511 \mathrm{keV}$ ? are detected and the reconstructed line of responses allows measuring the $ß+$ activity distribution. When trying to use $511 \mathrm{keV}$ annihilation ? for the ballistic control in hadrontherapy, the large ? prompt background should be taken into account and properly rejected. This detector is made of two half-rings of 120 channels each. Each channel consists of a LYSO crystal glued to a PMT. The PMT signal is sent to an Analog Sampling Module. This VME board is based on the Switch Capacitor Array technology and was specially designed for the LAPD detector. The first part is devoted to the description of the detector and its electronics. The selection of the coincident $511 \mathrm{keV}$ ? is discussed, and the reconstruction using an iterative MLEM algorithm is presented. Few results from an experiment with one third of the detector, using proton and carbon ion beams at the Heidelberg Ion-Beam Therapy Center in 2014, are also described, and the Coincidence Resolution Time and energy resolution are given. First reconstruction results, obtained with a phantom filled with a high intensity FDG source are also shown. This detector will be installed at the Lacassagne hadrontherapy center (Nice, France), on the 65 $\mathrm{MeV}$ line (Medicyc), and on the future $230 \mathrm{MeV}$ line (S2C2 from IBA) in 2017

M04A-2: Luminescence Imaging of Water During Proton-Beam Irradiation for Dose Estimation

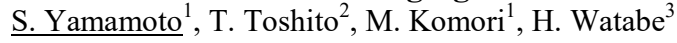

${ }^{I}$ Nagoya University Graduate School of Medicine, Nagoya, Japan

${ }^{2}$ Nagoya Proton Therapy Center, Nagoya, Japan

${ }^{3}$ Tohoku University, CYRIC, Sendai, Japan

Since proton therapy has the ability to selectively deliver a dose to a target tumor, the dose distribution should be accurately measured. A precise and efficient method to evaluate the dose distribution is desired. Recently we found that luminescence was emitted from water during proton irradiation and thought this phenomenon could be used for estimating the dose distribution. For this purpose, we placed water phantoms set on a table with a spot-scanning proton therapy system, and luminescence images of these phantoms were measured with a high-sensitivity cooled charge coupled device (CCD) camera during proton-beam irradiation. The luminescence images of water phantoms during the proton-beam irradiations showed clear Bragg peaks, and the measured proton ranges from the images were almost the same as those obtained with an ionization chamber. The image of the pure-water phantom also showed almost the same distribution as the tap-water phantom, indicating that the luminescence image was not related to impurities in the water. The spectrum for the luminescence of water distributed wide wavelengths which peaked at $450 \mathrm{~nm}$ to $550 \mathrm{~nm}$. To obtain the 3-dimensional distribution of the proton-beams, we reconstructed transverse images from projection images by the use of the filtered back projection algorism. Reconstructed images were compared to estimate the proton-beam widths with those obtained from the projection images and the reconstructed image showed better accuracy. Luminescence imaging during proton-beam irradiation has the potential to be a new method for dose estimation in proton therapy.

\author{
M04A-3: Emulsion Cloud Chamber: Measurements of 12-C Ions Beam Fragmentation at Large Angle \\ M. C. Montesi ${ }^{1,2}$, G. De Lellis ${ }^{1,2}$, A. Lauria ${ }^{1,2}$ \\ ${ }^{I}$ Dipartimento di Fisica, Università di Napoli, Federico II, Napoli, Italy \\ ${ }^{2}$ INFN, sezione di Napoli, Napoli, Italy
}

Nowadays, the use of carbon beam is the new frontier of radiotherapy and several facilities were built all over the world. The energy deposited in the Bragg peak, at the end of the Carbon ions range, allows to have a sharp and focused beam irradiating the cancer volume while preserving the healthy tissues. At the present time, the dose released by secondary particles is still a crucial 
problem: experimental data and full characterization of secondary fragments are necessary to validate Monte Carlo models which are at the basis of $\mathrm{C}$ ion treatment planning systems. Moreover, protons produced by carbon beam fragmentation can be useful for dose monitoring. We have constructed an Emulsion Cloud Chamber (ECC) detector for studying the large angle fragments produced by $400 \mathrm{MeV} / \mathrm{n} 12-\mathrm{C}$ ions beam impinging on a composite target. The ECC is composed by 61 nuclear emulsions (thickness $300 \mu \mathrm{m}$ ), showing a sub-micrometric tridimensional spatial resolution, excellent multi-particle separation and large angle track recognition, alternated with 55 lead (thickness $1 \mathrm{~mm}$ ) as passive material to stop the particles therein and measure their momentum by range. Two ECC detectors were exposed at the GSI laboratory in Germany in the framework of the FIRST (Fragmentation of Ions Relevant for Space and Therapy) experiment and the analysis of the recorded data are reported here. The exposure geometry allowed to collect fragments with a continuous angular distribution in the range $34^{\circ} \div 81^{\circ}$ with respect to the beam axis. Results on the angular distribution of fragments as well as their momentum estimations have been produced. The measured momentum of secondary particles ranges from 120 to $640 \mathrm{MeV} / \mathrm{c}$ with a relative error from $18 \%$, after 1 lead layer, to less than 1\%, after 25 lead layers. These data might be used to cross-check the model implemented in the Monte Carlo codes.

M04A-4: Monitoring and Imaging of the Hadron Mini-Beams for Spatially Fractionated Radiation Therapy $\underline{\text { A. Chaus }}^{1}$, S. Brons ${ }^{2}$, M. Campbell ${ }^{3}$, C. Granja ${ }^{4}$, V. Iakovenko ${ }^{1,5}$, O. Kovalchuk ${ }^{1}$, X. L. Cudie ${ }^{3}$, I. Martínez-Rovira ${ }^{6}$, E. Momot $^{1}$, S. Pospisil $^{4}$, Y. Prezado ${ }^{6}$, V. Pugach ${ }^{1}$, I. Sorokin ${ }^{1}$

${ }^{1}$ High energy physics department, Institute for Nuclear Reserch, Kyiv, Ukraine

${ }^{2}$ Heidelberg University Clinic, Heidelberg Ion Beam Therapy Center (HIT), Heidelberg, Germany

${ }^{3}$ CERN, Geneva, CERN, Switzerland

${ }^{4}$ Department of experimental physics, Institute of Experimental and Applied Physics, Prague, Czech Republic

${ }^{5}$ Princess Margaret Cancer Centre, University Health Network (UHN), Toronto, Canada

${ }^{6}$ Laboratoire Imagerie et Modélisation en Neurobiologie et Cancérologie (CNRS), Orsay, France

Metal as well as hybrid micro-detectors were explored for 1D and 2D monitoring and imaging of spatially distributed hadron minibeams in real-time. Minibeams were shaped by slit and matrix collimators to study the feasibility of fractionated radiation therapy at the Heidelberg Ion Therapy Center (HIT). Holes (1 mm diameter) or slits ( $1 \mathrm{~mm}$ wide) were made in aluminum, brass or tungsten plates to provide different multi-beam structures in the dose field of $15 \times 15 \mathrm{~mm}^{2}$. Studies were carried out with protons, carbon and oxygen ion beams $(80-250 \mathrm{AMeV})$. Metal Microstrip Detectors (MMD, 64 x 64 strips) and micropixel detector TimePix (256 x 256 pixels, $55 \times 55 \mu \mathrm{m}^{2}$ pixel area, metal and hybrid modes) were successfully applied for the overall beam profile monitoring as well as for the measurement of spatial minibeams intensity distribution. A low thickness of the MMD in combination with its high radiation tolerance allows for non-destructive beam profile monitoring. Peak-to-Valley Dose Ratios measured by microdetectors in real time are in a good agreement with the gafchromic films data evaluated off-line.

\author{
M04A-5: Prompt Gamma Imaging with a Multi-Knife-Edge Slit Collimator for Large FOV Monitoring of \\ Scanned Proton Pencil Beams \\ ${ }^{I}$ Department of Engineering Physics, Tsinghua University, Beijing, China \\ ${ }^{2}$ Key Laboratory of Particle \& Radiation Imaging, Ministry of Education (Tsinghua University), Beijing, China
}

Proton therapy is superior to conventional radiation therapy due to dose deposition sharply increasing at Bragg peak. However, an uncertainty in Bragg peak position as large as $15 \mathrm{~mm}$ could occur due to a variety of uncertainties in proton therapy treatment delivery. One solution is to monitor the treatment during delivery by prompt gamma imaging. Pencil beam scanning mode is one of the widely adopted modes in proton therapy treatment. In this mode, two sets of magnets scan the proton beams in a $2 \mathrm{D}$ pattern, and therefore the emitted gamma photons are focused to a small region, and the position of this region are rapidly changing with time. This is different with conventional nuclear medicine imaging, when the gamma source are distributed over the entire patient body, and slowly changing with time. To meet the requirement of $40 \times 40 \times 30 \mathrm{~cm}^{3} \mathrm{FOV}$ for proton pencil beam scanning mode, we propose a prompt gamma imaging system design with a multi-knife-edge slit collimator based on the optimization of a single-knife -edge slit collimator design. Monte Carlo simulation was performed to evaluate the positioning accuracy of Bragg peak and the spatial resolution of the imaging system. The prompt gamma distribution was generated via Monte Carlo simulation of proton interaction with water in GATE. The generated prompt gamma distribution was subsequently used to evaluate the system performance. For the single-knife-edge slit collimator-based imaging system, a spatial resolution superior to $2 \mathrm{~mm}$ and a positioning accuracy in Bragg peak better than $3 \mathrm{~mm}$ were achieved. For the multi-knife-edge slits collimator-based imaging system, preliminary evaluation results show that it can provide a large FOV size of $40 \times 40 \times 30 \mathrm{~cm}^{3}$ and $\sim 3 \mathrm{~mm}$ positioning accuracy in Bragg peak. Based on the preliminary results, we conclude that the proposed multi-knife-edge slits collimator-based prompt gamma imaging system design is very promising and could potentially facilitate precise proton therapy.

M04A-6: Kinect Surface Filtering During Gantry Motion for Radiotherapy Applications

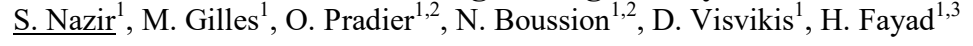




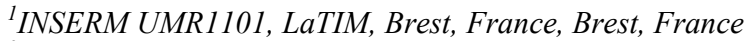

${ }^{2}$ Radiotherapy department, CHRU Morvan, Brest, France

${ }^{3}$ Université de Bretagne Occidentale, Brest, France

Kinect is a motion sensing device developed by Microsoft for Xbox 360 video game console. Since releasing the Windows SDK, Kinect is no more being used for video games but in much more complex applications of motion sensing technologies. Moreover, this system was lately suggested to be used in a variety of medical fields such as radiotherapy. In radiotherapy, Kinect, and especially its second version the Kinect 2 including time of flight information, is used for patient repositioning, patient respiratory motion tracking and collision detection that may occur between the patient, the table and the moving gantry. While repositioning the patient is done without any gantry motion, the other two applications are usually affected by the large surface errors caused by the reflections associated with the gantry motion. This is presented by the occurrence of a significant noise that affects depth measurements. In this study, we aim to reduce this effect by filtering the respiratory 1D signal extracted from the Kinect 2 surfaces, and then, in a second step, we aim to filter the Kinect surfaces directly in order to decrease surface information errors. Filters used for this study include polynomial fitting and median filtering for the 1D and the 2D cases respectively. This study was validated by placing a radiotherapy phantom used for dose verification on the Varian TrueBeam accelerator table and introducing a patient $1 \mathrm{D}$ respiratory signal for the table in research mode. This allows to simulate a respiratory motion to the phantom and to image the phantom using the Kinect 2 with or without gantry motion. Our results show significant noise compensation after surface filtering and therefore a potential use of the Kinect 2 camera during radiotherapy delivery.

\title{
M04A-7: Design of a High Dynamic Range Integrated Charge to Digital Converter for Online Dosimetry in Radiotherapy \\ L. Gallin-Martel, O. Rossetto, Y. Arnoud, B. Boyer, R. Delorme, R. Fabbro, M.-L. Gallin-Martel, O. Guillaudin, A. Pélissier Laboratoire de Physique Subatomique et de Cosmologie (LPSC) CNRS/IN2P3, Grenoble, France
}

The Intensity Modulated Radiotherapy allows a more efficient dose delivery in the tumour leading to a reduction of the dose deposited in the nearby healthy organs. However, this technique requires additional resources to perform on line dosimetry and insure patient safety. A Charge to Digital Converter has been designed in a CMOS $0.35 \mu \mathrm{m}$ process to read-out a 2D dedicated gaseous detector designed at LPSC. The Charge Balancing Integration technique coupled to an internal 6- bit Analog to Digital Converter allows high resolution and high dynamic range to be reached. This 16-channel, 16-bit converter exhibits a $6 \mathrm{fC}$ LSB value, a nonlinearity better than $0.1 \mathrm{LSB}$ and a RMS noise lower than $0.5 \mathrm{LSB}$.

\author{
M04A-8: Charged Particle Imaging: Comparison Between Different Particles \\ C. Bopp $^{1}$, D. Brasse ${ }^{2}$, N. Matsufuji ${ }^{1}$ \\ ${ }^{I} Q S T$ - NIRS, Chiba, Japan \\ ${ }^{2}$ IPHC - CNRS/Université de Strasbourg, Strasbourg, France
}

Charged particle radiography and tomography are being developed for range verification and treatment planning purposes in the context of hadron therapy. Lighter charged particles, such as protons, present some advantages in terms of dose deposit with regard to the achievable resolution on the stopping power in reconstructed images. However, they are also more subject to multiple scattering, leading to a degradation of the spatial resolution. This is a great drawback, but can be somewhat lessened in the context of tomographic image reconstruction by making use of a detection system recording particle-by-particle information and accounting for the trajectory of each particle. Through Monte Carlo simulations of charged particle computed tomography scan data, this study aims at illustrating and quantifying the trade-offs in terms of dose, spatial resolution and stopping power resolution in the case of proton, helium, carbon and oxygen particles at different energies. In order to evaluate the advantage of single particle tracking, which puts challenging requirements on the detection system of a scanner, images reconstructed using algorithms accounting for the most likely path and not accounting for it will be compared for each particle type.

\author{
M04A-9: Large Area Polycrystalline Diamond Detectors for Online Hadron Therapy Beam Tagging \\ Applications \\ M.-L. Gallin-Martel ${ }^{1}$, A. Bes ${ }^{1}$, A. Boukhémiri ${ }^{1}$, G. Bosson ${ }^{1}$, J. Collot ${ }^{1}$, D. Dauvergne ${ }^{1}$, M. Fontana ${ }^{2}$, L. Gallin-Martel ${ }^{1}$, \\ A. Gorécki ${ }^{1}$, J.-Y. Hostachy ${ }^{1}$, J. Krimmer ${ }^{2}$, A. Lacoste ${ }^{1}$, J. Morse ${ }^{3}$, J.-F. Muraz ${ }^{1}$, F. Rarbi ${ }^{1}$, M. Salome ${ }^{3}$, E. Testa $^{2}$, M. Yamouni ${ }^{1}$, \\ Y. Zoccarato ${ }^{2}$ \\ ${ }^{I}$ Laboratoire de Physique Subatomique et de Cosmology (LPSC) CNRS / IN2P3, Grenoble, France \\ ${ }^{2}$ Institut de Physique Nucléaire de Lyon (IPNL), Lyon, France \\ ${ }^{3}$ European Synchrotron Radiation Facility (ESRF), Grenoble, France
}

The MoniDiam project is part of the French national collaboration CLaRyS for the on-line monitoring of hadron therapy. It relies on the imaging of nuclear reaction products that is related to the ion range. The goal here is to provide large area detectors with a 
high detection efficiency for carbon or proton beams giving time and position measurement at count rates greater than $100 \mathrm{MHz}$. High radiation hardness and intrinsic electronic properties make diamonds reliable and very fast detectors with a good signal to noise ratio. Commercial Chemical Vapor Deposited (CVD) diamonds are studied starting with plasma etch thinning and contact metallization done at the LPSC laboratory. Their applicability as particle detector is being investigated using a and $\beta$ radioactive sources, $95 \mathrm{MeV} / \mathrm{u}$ carbon beams and short-bunched $8.5 \mathrm{keV}$ photons from the European Synchrotron Radiation Facility (ESRF). This facility offers unique capability of highly focused beams. The energy deposition is uniform in the irradiated detector volume. It permits us to mimic the interaction of single ion beams. The possible application of diamonds as a position sensitive detector will be discussed from these radiation tests results. Finally, we focused on the diamond time response measurement. The reported study prefigures dedicated microelectronics developments.

\author{
M04A-10: Monitoring of Hadrontherapy Treatments with a Novel Tracking Device Based on Charged Particle \\ Detection \\ $\underline{\text { S. Muraro }}^{1}$, G. Battistoni ${ }^{1}$, F. Collamati ${ }^{2}$, E. De Lucia ${ }^{2}$, R. Faccini ${ }^{3,4}$, M. Marafini ${ }^{4,5}$, I. Mattei ${ }^{1}$, R. Paramatti ${ }^{4}$, V. Patera ${ }^{4,5,6}$, \\ D. Pinci ${ }^{4}$, A. Rucinski ${ }^{4,5}$, A. Russomando ${ }^{3,4,7}$, A. Sarti ${ }^{4,5,6}$, A. Sciubba ${ }^{4,5,6}$, E. Solfaroli Camillocci ${ }^{3,4}$, M. Toppi $^{2}$, G. Traini $^{3,4}$, \\ C. Voena ${ }^{4}$

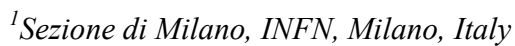 \\ ${ }^{2}$ Laboratori Nazionali di Frascati, INFN, Frascati, Italy \\ ${ }^{3}$ Dipartimento di Fisica, Universita La Sapienza di Roma, Roma, Italy \\ ${ }^{4}$ Sezione di Roma, INFN, Roma, Italy \\ ${ }^{5}$ Museo Storico della Fisica e Centro Studi e Ricerche E. Fermi, Roma, Italy \\ ${ }^{6}$ Dipartimento di Scienze di Base e Applicate per Ingegneria, Universita La Sapienza di Roma, Roma, Italy \\ ${ }^{7}$ Center for Life Nano Science@Sapienza, Istituto Italiano di Tecnologia, Roma, Italy
}

Hadrontherapy is a technique for cancer treatment that exploits ion beams. A critical issue is the accuracy that is achievable when monitoring the dose released by the beam to the tumor and to the surrounding tissues. We present here a new tracking device, developed in the framework of the INSIDE project, with the aim of performing on-line range monitoring of ion therapy by means of the detection of charged particles produced by the beam interacting in the patient tissues. This approach can be profitably adopted in treatments using nucleon projectiles heavier than protons. This device is able to provide a fast measurement of the longitudinal distribution of the reconstructed emission point of charged particles (mainly protons) detected at large angle with respect to the primary beam. The measured emission profile can be correlated to the Bragg peak position. Charged tracks are detected using 6 planes of scintillating fibers $\left(0.5 \times 0.5 \mathrm{~mm}^{\wedge} 2\right)$ with orthogonal views, readout by Silicon PM, followed by a plastic scintillator and by a small calorimeter made of a pixellated LFS crystal. The sensitive area of the tracking planes is $20 \times 20$ $\mathrm{cm}^{\wedge} 2$. The achievable spatial resolution for single charged particle has been studied by means of a full simulation of the device, benchmarked against experimental data, and a dedicated reconstruction code. In order to develop a suitable strategy for the analysis of the dose profile during treatments, the device response has been studied for different setup configurations and clinical scenarios in the framework of carbon ion particle therapy. Charged particle yield as a function of primary energy, thickness of material and device positions with respect to the patient have been considered in order to establish a possible procedure for realtime comparison of measurements with expectations in the actual clinical operation.

M04A-11: Commissioning of a Compton Camera for Particle Beam Range Monitoring $\underline{\text { P. G. Thirolf }}^{1}$, S. Aldawood ${ }^{1,2}$, M. Böhmer ${ }^{3}$, J. Bortfeldt ${ }^{4}$, I. Castelhano ${ }^{1,5}$, G. Dedes ${ }^{1}$, W. Enghardt ${ }^{, 7}$, F. Fiedler $^{8}$, R. Gernhäuser $^{3}$, C. Golnik $^{6}$, S. Helmbrecht ${ }^{8}$, F. Hueso-González ${ }^{7}$, H. van der Kolff ${ }^{1,9}$, T. Kormoll ${ }^{6}$, C. Lang ${ }^{1}$, S. Liprandi ${ }^{1}$, L. Maier ${ }^{3}$, T. Marinšek ${ }^{1}$, A. Miani ${ }^{1,10}$, G. Pausch ${ }^{6}$, J. Petzoldt ${ }^{6}$, M. Pocevicius ${ }^{1}$, K. Römer $^{8}$, D. R. Schaart ${ }^{9}$, I. Valencia-Lozano ${ }^{1}$, K. Parodi ${ }^{1}$

${ }^{I}$ Faculty of Physics, Department of Medical Physics, Ludwig-Maximilians-Universität München, Garching b. München, Germany

${ }^{2}$ Department of Physics and Astronomy, King Saud University, Riyadh, Saudi Arabia

${ }^{3}$ Physics Department E12, Technical University Munich, Garching b. München, Germany

${ }^{4}$ Faculty of Physics, Department of Elementary Particle Physics, Ludwig-Maximilians-Universität München, Garching b.

München, Germany

${ }^{5}$ Faculty of Science, University of Lisbon, Lisbon, Portugal

${ }^{6}$ National Center for Radiation Research in Oncology "OncoRay", Dresden, Germany

${ }^{7}$ Institute of Radiooncology, Helmholtz-Zentrum Dresden-Rossendorf, Dresden, Germany

${ }^{8}$ Institute of Radiation Physics, Helmholtz-Zentrum Dresden-Rossendorf, Dresden, Germany

${ }^{9}$ Delft University of Technology, Delft, The Netherlands

${ }^{10}$ Università degli Studi di Milano, Milano, Italy

A Compton camera prototype for the position-sensitive detection of prompt ? rays from nuclear reactions between therapeutic proton (or ion) beams and organic targets is being commissioned in Garching. The detector system is designed to allow for reconstructing the ?-source position from the Compton scattering kinematics of the primary photon as well as from tracking the Compton-scattered electron trajectory. The camera consists of a monolithic $\mathrm{LaBr} 3(\mathrm{Ce})$ scintillation absorber crystal, read out by a 
256-fold segmented multi-anode PMT and preceded by a stacked array of 6 double-sided silicon strip detectors acting as scatterers. The detector system has been calibrated and characterized in the laboratory as well as at different accelerator facilities, including clinical proton beams. Results from online commissioning runs will be presented, demonstrating very good agreement between experimental prompt-? energy spectra and Monte-Carlo simulations. Particular effort was dedicated to characterize the spatial resolution achievable with the monolithic $\mathrm{LaBr} 3(\mathrm{Ce})$ scintillator when targeting the primary photon interaction position. Intense, tightly collimated $137 \mathrm{Cs}$ and $60 \mathrm{Co}$ sources were used for $2 \mathrm{D}$ irradiation scans as prerequisite for studying the performance of the "k-Nearest Neighbour"-algorithm developed in Delft and extending its applicability into the energy range beyond $511 \mathrm{keV}$. Systematic results of the monolithic scintillator's spatial resolution will be presented as a function of the k-NN parameters (e.g. events per irradiation position) and the PMT segmentation, resulting in the realization of the presently optimum spatial resolution (3.8(2) mm @1.3 MeV) already for a reduced PMT segmentation (8x8). An outline of ongoing and planned further experimental activities and upgrade plans will be presented.

This work is supported by the DFG Cluster of Excellence MAP (Munich-Centre for Advanced Photonics) and by King Saud University, Riyadh, Saudi Arabia.

\author{
M04A-12: Monitoring Tumor Lung Irradiation with OrthoCT (Orthogonal Ray Imaging): a Full System \\ Simulation Study \\ $\underline{\text { H. Simões }}^{1,2}$, P. Crespo ${ }^{1,2}$ \\ ${ }^{I}$ Laboratório de Instrumentação e Física Experimental de Partículas, Coimbra, Portugal \\ ${ }^{2}$ Departamento de Física, Universidade de Coimbra, Coimbra, Portugal
}

The OrthoCT (standing for orthogonal computed tomography) imaging technique consists in detecting radiation dispersed in the patient and emitted at right angles in respect to the beam axis. Since photon scattering in the patient occurs with higher intensity in tissues of higher density, a detection system (constituted by a multi-slice collimator and a photon detector) positioned perpendicularly to the beam axis yields a signal proportional to the photons that escaped the patient (i.e., a signal correlated with patient morphology). This low-dose morphological imaging can potentially be useful to (1) on-board imaging for assisting radiotherapy, or (2) real time radiotherapy monitoring. In this work we report Geant4 simulation results with an anthropomorphic phantom to analyze the capability of OrthoCT to detect pertinent and clinically-relevant lung tumor deviation and lung tumor regression/progression. Tumor deviation or progression potentially leads to the tumor being partially not irradiated. On the other hand, the detection of tumor shrinkage in lung irradiation may allow for a reduction of the dose field, which represents an important relief of the dose burden to the surrounding healthy lung. The counts distributions obtained with a realistic full system (including the multi-slice collimator, the scintillator crystals, and the electronic readout mode) show a very high visual correlation both with the simulated, prescribed dose, and with the tumor location/size, as well as with the phantom structures. The capability of OrthoCT to obtain morphological images with no X-ray source rotation potentially allows to minimize dose in healthy tissues and organs at risk. Therefore, this technique is likely to represent a high potential asset for on-board imaging (i.e. imaging after patient positioning, just before the treatment), or eventually, during the treatment.

M04A-13: A Prototype System for Portal Imaging for Intensity Modulated Neutron Therapy $\underline{\text { S. St. James }}{ }^{1}$, G. Moffitt ${ }^{1}$, D. Argento ${ }^{1}$, D. DeWitt ${ }^{2}$, R. Miyaoka ${ }^{2}$, R. Stewart ${ }^{1}$

${ }^{I}$ Radiation Oncology, University of Washington, Seattle, WA, USA

${ }^{2}$ Radiology, University of Washington, Seattle, WA, USA

Fast neutron therapy is offered at the University of Washington Medical Center for the treatment of selected cancers. The hardware and control systems of the UW Clinical Neutron Therapy System are undergoing upgrades to enable delivery of intensity modulated neutron therapy (IMNT). To clinically implement IMNT, dose verification tools need to be developed. We propose a portal imaging system that relies on the creation of positron emitting isotopes $\left({ }^{11} \mathrm{C}\right.$ and $\left.{ }^{15} \mathrm{O}\right)$ through $(\mathrm{n}, 2 \mathrm{n})$ reactions with a PMMA plate placed below the patient. After field delivery, the plate is retrieved from the vault and imaged using a reader that detects the annihilation photons. The pattern of activity produced in the plate provides information to reconstruct the neutron fluence map that can be compared to fluence maps from Monte Carlo (MCNP) simulations to verify treatment delivery. We have previously performed Monte Carlo simulations of the portal imaging system (GATE simulations) and the beam line (MCNP simulations). In this work, initial measurements using a prototype system are presented. Custom electronics were developed for BGO detectors read out with photomultiplier tubes (previous generation PET detectors from a CTI ECAT 953 scanner). Two detectors were placed in coincidence, with a detector separation of $2 \mathrm{~cm}$. Custom software was developed to create the crystal look up tables and perform a limited angle planar reconstruction with a stochastic normalization. To test the initial capabilities of the system, PMMA squares were irradiated with neutrons at a depth of $1.5 \mathrm{~cm}$ and read out using the prototype system. Doses ranging from 10-200 cGy were delivered. Using the prototype system, dose differences in the therapeutic range could be determined. The prototype portal imaging system is capable of detecting neutron doses as low as 10-50 cGy and shows great promise as a patient QA tool for IMNT 
M04A-14: Image-Based Internal Dosimetry of Cu-64 Labeled Radiopharmaceuticals Using Monte Carlo Method

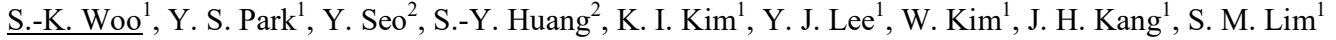 \\ ${ }^{I}$ Molecular Imaging research center, Korea Institute of Radiological and Medical science, Seoul, Korea \\ ${ }^{2}$ Department of Radiology and Biomedical Imaging, University of California, San Francisco, San Francisco, CA, USA
}

The purpose of this study was to evaluate the image-based internal dosimetry of $\mathrm{Cu}-64$ labeled radiopharmaceuticals using Monte Carlo code in mice. The tumor bearing mouse was injected with $3.7 \mathrm{MBq}$ of $\mathrm{Cu}-64$ labeled radiopharmaceuticals. At 1, 2, 6, 24, 48, and 70 hours after the radiotracer administration, the mice images were acquired using a dedicated small animal PET/CT scanner (Inveon ${ }^{\mathrm{TM}}$, Siemens Healthcare, Malvern, PA). Anatomic and density information of organs and tumor were obtained using CT image. The tumor and organs region were segmented by mean-based region growing 3D segmentation method and manually. Residence times were calculated via time activity curves of the segmented PET image. The CT density and PET radioactivity information were used for input data of Monte Carlo simulation. S-values and Does map of organs and tumor were calculated using Geant4 Monte Carlo simulation. The organs and tumor region were segmented by using CT and PET, respectively. Dose map of organs and tumor were obtained using Monte Carlo simulation. Representative residence time of the heart, river, and tumor were 2.64, 1.47, and 0.47 MBq-h/MBq, respectively. Monte Carlo simulated S-values of the heart, river, and tumor were 1.05E-02, 2.11E-02, and 3.28E-01 MBq-h/MBq, respectively. An Estimated absorbed dose of the heart was 2.79E-01, liver was 2.79E-01 and tumor was $1.56 \mathrm{E}-01 \mathrm{mGy} / \mathrm{MBq}$. We performed internal dose calculation of Cu-64 labeled radiopharmaceuticals organs and tumor in mice. The result of this study would be expected to improve accuracy of radiopharmaceuticals characteristic evaluation in animal model.

\author{
M04A-15: Developing an Optimum Protocol for Thermoluminescence Dosimetry with GR-200 Chips, in \\ Radiotherpy, Using Taguchi Method \\ M. Sadeghi ${ }^{1}$, R. Faghihi ${ }^{1}, \underline{\text { S. Sina }^{2}}$ \\ ${ }^{I}$ Radiation Medicine department, School of Engineeing, Shiraz university, Shiraz, Iran, Shiraz, Iran \\ ${ }^{2}$ Radiation Research center, School of Engineeing, Shiraz university, Shiraz, Iran, Shiraz, Iran
}

Introduction: Thermoluminescence dosimetry is a powerful technique with wide applications in personal, environmental, and clinical dosimetry, especially in radiotherapy. The optimum annealing, storage, and reading protocols are very effective in accuracy of TLD response. The purpose of this study is to obtain an optimum protocol for GR-200; $\mathrm{LiF}: \mathrm{Mg}, \mathrm{Cu}, \mathrm{P}, \mathrm{by}$ optimizing the effective parameters, to increase the reliability of the TLD response using Taguchi method.

Materials and Methods: Taguchi method has been used in this study for optimization of annealing, storage, and reading protocols of the TLDs. A number of 108 GR-200 chips were divided into 27 groups, each containing 4 chips. The TLDs were exposed to three different doses, and stored, annealed and read out by different procedures as suggested by Taguchi Method.

Results: By comparing the signal to noise ratios the optimum dosimetry procedure was obtained. Using the optimum protocol, an efficient glow curve with low residual signals can be achieved. According to the results obtained this study, the optimum protocol for thermoluminescence dosimAnnealing Temperature $\left({ }^{\circ} \mathrm{C}\right): 240$ Annealing Time (s): 90; Time of Annealing to Exposure (d):1; Time of Exposure to Readout (d):2; Pre-heat Temperature $\left({ }^{\circ} \mathrm{C}\right): 50$; Pre-heat Time (s):0; Heating Rate $\left({ }^{\circ} \mathrm{C} / \mathrm{s}\right): 15$; Maximum Temperature of Readout $\left({ }^{\circ} \mathrm{C}\right): 240$; Time $(\mathrm{s}): 13$; Storage Temperature $\left({ }^{\circ} \mathrm{C}\right)$ etry is as follows: -20 ;

Conclusion: Using optimum protocol obtained by Taguchi method, the radiotherapy dosimetry can be effectively performed with great accuracy.

M04A-16: Impact of Tumor Contrast in Orthogonal Ray Imaging: a Prostate Irradiation Study

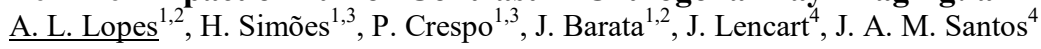

${ }^{1}$ Laboratório de Instrumentação e Física Experimental de Partículas, Coimbra, Portugal

${ }^{2}$ Universidade da Beira Interior, Covilhã, Portugal

${ }^{3}$ Universidade de Coimbra, Coimbra, Portugal

${ }^{4}$ Instituto Português de Oncologia do Porto FG EPE, Porto, Portugal

Orthogonal ray imaging (OrthoCT) is a potential new technique under investigation by our group that consists in operating a dedicated X-ray detection system specially built for collecting selected patient-scattered radiation. This imaging technique does not require rotational irradiation of the target because it is based on the detection of photons emitted at almost right angles in respect to the incoming photon flux. Modern IGRT techniques allow for a more accurate position of the patient, consequently improving the treatment accuracy. However these techniques have some limitations like poorly resolution in soft tissues and possible side effects. One way to increase the contrast enhancement in the lesion is to inject a contrast agent. To study the influence of an iodinated contrast in prostate cancer OrthoCT imaging, our investigation will comprise three different scenarios according to the diffusion flow of the contrast in the body. First, we will evaluate the Hounsfield units in the early arterial phase when the contrast has not enhanced the organs and other soft tissues. Secondly, we will study the late arterial phase of the contrast when the tumor already incorporated the iodine. Finally, to evaluate the dose in the tumor and surrounding irradiated 
tissues with contrast we will study the delayed phase that occurs 6-10 minutes after the injection. The main purpose is to evaluate through simulation the benefit of iodinated-contrast agent administration in cases of prostate cancer in OrthoCT, using Geant 4 simulation toolkit and an anthropomorphic phantom. We will also study the display of gold fiducial markers implanted into the prostate gland.

A. L. Lopes and H. Simões contributed equally to this work.

M04A-17: Development of a SiPM-Based Detection Module for Prompt Gamma Imaging in Proton Therapy G. Cozzi $^{1,2}$, D. Giarrusso ${ }^{1}$, M. Carminati ${ }^{1}$, C. Fiorini ${ }^{1,2}$, A. Gola ${ }^{3}$, C. Piemonte ${ }^{3}$, V. Regazzoni ${ }^{3,4}$

${ }^{I}$ Dipartimento di Elettronica, Informazione e Bioingegneria, Politecnico di Milano, Milano, Italy

${ }^{2}$ Sezione di Milano, INFN, Milano, Italy

${ }^{3}$ Fondazione Bruno Kessler, Trento, Italy

${ }^{4}$ Università degli studi di Trento, Trento, Italy

We provide a description of a new SiPM-based prototype of gamma camera for prompt gamma imaging detection in proton therapy, supported by first experimental results. Treatments delivered by proton therapy are affected by uncertainties on the range of the beam within the patient. To reduce these margins and deliver safer treatments, different projects are currently investigating real-time range control by imaging prompt gammas emitted along the proton tracks in the patient. The potential of this concept for real-time range monitoring has been confirmed, also with Silicon PhotoMultiplier (SiPM) gamma cameras. The prototype is based on a II-dimensional projection of the beam on a pixelated detector module to improve count rate capability thanks to the detector segmentation. The photodetector is produced with a new High-Density (HD) SiPMs technology available at Fondazione Bruno Kessler (FBK, Italy) and designed to improve the dynamic range at high energy. The detector is made of an array of $8 \times 8$ SiPMs: a single SiPM has an active area of 4 × $4 \mathrm{~mm} 2,15$ x $15 \mu \mathrm{m} 2$ microcells, for a total of 70000 microcells for SiPM. Each SiPM is coupled to a $4 \times 4 \times 22 \mathrm{~mm} 3 \mathrm{LYSO}$, enhancing the detection module segmentation. Signals are processed by two ASICs, improving the compactness of the system, and acquired by an external Data Acquisition System (DAQ) based on FPGA logic. The camera should also implement an automatic temperature stabilization strategy.

M04A-18: Large-Area Segmented Polycrystalline CVD Diamond for Dose Mapping in Advanced Radiotherapy Techniques

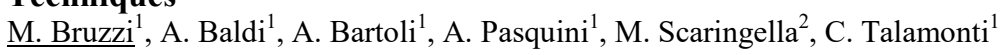

${ }^{I}$ University of Florence, Firenze, Italy

${ }^{2}$ INFN Firenze, Firenze, Italy

Diamond has a potential for dosimetry in advanced radiotherapy techniques, being a solid-state nearly tissue equivalent material, with the possibility to be grown in large diameter wafers by Chemical Vapour Deposition (CVD). A large-area bidimensional dosimeter made of up to two detector-grade adjacent $2.5 \times 2.5 \mathrm{~cm} 2$ polycrystalline Chemical Vapour Deposited (pCVD) diamond samples, each equipped with a $12 \times 12$ matrix of contacts and connected to a custom-made electronic read-out system, has been manufactured in view to achieve fast dynamics as well as spatial and time resolutions required in such advanced radiotherapic techniques. The device has been used to extract a 2D map of an IMRT prostate cancer plan and a Volumetric Modulated Arc Therapy (VMAT) lung plan, showing promising performances.

M04A-19: Development of a Scanner for Proton Computed Tomography

Y. Karakaya, M. Vanstalle, M. Rousseau, C. Finck

IPHC, UNISTRA, Strasbourg, France

Recently, there is renewed interest for proton therapy around the world. In clinical routine, treatment plans are based on X-ray Computed Tomography (CT) images. In X-ray CT scan, Hounsfield Unit are converted into Relative Stopping Powers (RSP) by a calibration curve that introduces uncertainties. It has been suggested that proton computed tomography (pCT) has the potential to improve the accuracy of proton therapy treatment planning by direct measurement of the RSP. However, the clinical application of pCT is limited by some technical constraints mainly due to the high counting rate that the scanner has to sustain. Thus, there is no prototype used in clinical routine yet. Recently our team has proposed a new approach based on the reconstruction of each proton bunch trajectory and RSP rather than single proton reconstruction. This implies the development of a new prototype adapted to this technique. This work presents the optimization of this prototype, carried out with Monte Carlo simulations. This device will consist of a forward tracker, reconstructing the position and the spread of the beam, and a rangemeter located after the patient, reconstructing the Water Equivalent Thickness (WET) by an unfolding approach. The resolution on beam position of the optimised tracker, composed of optical fibers embedded in plastic scintillator, has been found to be 0.2 $\mathrm{mm}$. The unfolding method that we adapted allows to obtain a resolution on the WET below $1 \mathrm{~mm}$, which is better than existing prototypes $(\sim 3 \mathrm{~mm})$. 
M04A-20: Evaluation of Proton CT as a Low-Dose Modality for Image-Guided Proton Therapy F. R. Cassetta Junior ${ }^{1}$, R. Schulte ${ }^{2}$, V. Bashkirov ${ }^{2}$, G. Baroni $^{1}$, M. Riboldi ${ }^{1}$, R. Johnson ${ }^{3}$, H. Sadrozinski ${ }^{3}$

${ }^{I}$ DEIB, Politecnico di Milano, Milan, Italy

${ }^{2}$ Division of Radiation Research, Loma Linda University, Loma Linda, CA, USA

${ }^{3}$ Santa Cruz Institute for Particle Physics, UC Santa Cruz, Santa Cruz, CA, USA

The clinical benefit of proton therapy can be compromised by water equivalent path length (WEPL) variations, e.g., due to patient's anatomical changes between the planning CT and treatment and due to intra-fraction motion. In addition, treatment planning in proton therapy based on a CT data set of the patient can lead to considerable proton range uncertainties, due to ambiguity in the non-linear conversion of CT numbers into relative stopping power (RSP). A prototype proton CT (pCT) scanner has been developed to reduce this uncertainty and the imaging dose delivered to the patient. The purpose of this work was to evaluate the potential of $\mathrm{pCT}$ for in-room image guidance. The performance of a 3D registration algorithm was tested with a realistic pediatric head phantom for two types of image reconstruction (FBP only, FBP followed by iterative reconstruction) and imaging dose, which was adjusted according to the number of proton histories used in reconstruction. An experimental pCT scan of the phantom was modified by prescribing random $3 \mathrm{D}$ errors and applying the algorithm to correct them. The proton CT images were reconstructed with $100 \%, 50 \%, 25 \%$ and $12.5 \%$ of the total dose of a standard treatment planning pCT scan to simulate low-dose imaging for patient setup. After rigid registration procedures, the scale invariant feature transform (SIFT) was used to extract features and to calculate the residual distance between corresponding histogram-based landmarks. Residual errors were mostly of sub-millimeter magnitude even for sub-mGy doses. Proton CT 3D head image registration is allows low dose and high positioning accuracy.

The author is supported in part by the Brazilian institution of education CAPES. The proton CT research at Loma Linda University was supported by National Institute of Biomedical Imaging and Bioengineering (NIBIB), and the National Science Foundation (NSF), award Number R01EB013118.

M04B: Posters: New radiation detectors I

Wednesday, Nov. $2 \quad$ 16:30-18:30 Etoile

M04B-1: In-Beam PET with Pulse Shape Discrimination at a Clinical Cyclotron Facility

T. Kormoll $^{1}$, W. Enghardt ${ }^{1,2,3}$, F. Fiedler ${ }^{4}$, M. Iltzsche ${ }^{4}$, G. Pausch ${ }^{1}$, C. Tintori ${ }^{5}$, S. Helmbrecht ${ }^{4}$

${ }^{I}$ Technische Universität Dresden, OncoRay - Radiation Research in Oncology, Dresden, Germany

${ }^{2}$ Deutsches Konsortium f ür Translationale Krebsforschung (DKTK), Dresden, Germany

${ }^{3}$ Institute of Radiooncology, Helmholtz-Zentrum Dresden-Rossendorf, Dresden, Germany

${ }^{4}$ Institute of Radiation Physics, Helmholtz-Zentrum Dresden-Rossendorf, Dresden, Germany

${ }^{5}$ CAEN SpA, Viareggio, Italy

Positron emission tomography can yield in-vivo range information in hadron tumor therapy. Measurements are preferably done during the irradiation to minimize the loss of information due to physical decay and a metabolic transport of the nuclides. One challenge is the high number of background events due to prompt photons and neutron induced radiation. In this work, pulse shape discrimination has been applied to conventional Anger logic block detectors to enable an in-beam acquisition at a cyclotron generated beam. Images could be acquired with clinical dose rates in phantom experiments. This technique could pave the way towards more cost effective in-beam PET hardware.

M04B-2: Luminescence Imaging of Water During Alpha-Particle Irradiation

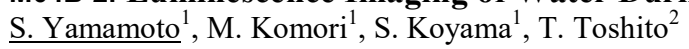

${ }^{I}$ Nagoya University Graduate School of Medicine, Nagoya, Japan

${ }^{2}$ Nagoya Proton Therapy Center, Nagoya, Japan

The luminescence imaging of water using the alpha-particle irradiation of several MeV energy range is thought to be impossible because this alpha-particle energy is far below the Cerenkov-light threshold and the secondary electrons produced in this energy range do not emit Cerenkov-light. Contrary to this consensus, we found that the luminescence imaging of water was possible with 5.5-MeV alpha-particle irradiation. We placed a 2-MBq of Am- 241 alpha source in water, and luminescence imaging of the source was conducted with a high-sensitivity, cooled charge-coupled device (CCD) camera. We also carried out such imaging of the alpha source in three different conditions with that in air, with a plastic scintillator, and an acrylic plate, to compare the photon productions of that with water. The luminescence imaging of water was observed from 10 - to 20 -s acquisition, and the intensity was linearly increased with time. The intensity of the luminescence with the alpha irradiation of water was $0.05 \%$ of that with the plastic scintillator, $4 \%$ with air, and $15 \%$ with the acrylic plate. The spatial resolution of the luminescence image of water was better than $0.25-\mathrm{mm}$ FWHM. The light spectra showed the luminescence of water had wide distribution peaked from $450 \mathrm{~nm}$ to $550 \mathrm{~nm}$. We conclude that alpha particles of 5.5-MeV energy emit luminescence in water. Although the intensity of the 
luminescence was smaller than that in air, it was clearly observable. The luminescence of water with alpha particles will be a new method for alpha particle detection and distribution measurements in water.

\section{M04B-3: A New PET Detector Concept for Preclinical Hybrid MR/PET}

A. Berneking ${ }^{1}$, A. Gola ${ }^{2}$, A. Ferri ${ }^{2}$, F. Finster ${ }^{1}$, G. Paternoster ${ }^{2}$, C. Piemonte ${ }^{2}$, C. Lerche ${ }^{1}$, N. J. Shah ${ }^{1}$

${ }^{I}$ Medical Imaging Physics (INM-4), Institute of Neuroscience and Medicine, Forchungszentrum Jülich GmbH, Jülich, Germany

${ }^{2}$ Fondazione Bruno Kessler, Trento, Italy

This work presents a new PET detector concept for preclinical hybrid MR/PET. The detector concept is based on LinearlyGraded SiPM produced with latest FBK RGB-HD technology. One $8 \mathrm{~mm}$ by $8 \mathrm{~mm}$ large quadrant of the sensor chip is coupled to a black coated $8 \mathrm{~mm}$ by $8 \mathrm{~mm}$ large and $3 \mathrm{~mm}$ thick Cerium-doped Lutetium Yttrium Orthosilicate (LYSO) monolithic crystal with optical grease. The readout of this quadrant is realized by four readout channels with the linear encoding based on integrated resistors and the Center of Gravity approach.

To characterize the new detector concept, the measurement setup was prepared to focus a collimated beam to 25 different points perpendicular on the monolithic scintillator crystal, starting in the center point of the crystal at $0 \mathrm{~mm} / 0 \mathrm{~mm}$ and sampling a grid with a pitch of $1.75 \mathrm{~mm}$.

The measured intrinsic spatial resolution (FWHM) was $0.72+/-0.01 \mathrm{~mm}$ in $\mathrm{x}$ - and $0.61+/-0.01 \mathrm{~mm}$ in y-direction at the center of the detector. At the same point, an energy resolution (FWHM) was measured $13.01+/-0.05 \%$. Compared to detectors based on pixelated crystal arrays with the same thickness, the sensitivity of the presented detector concept is significantly increased thanks to the use of monolithic crystals. Combining compact design, high spatial resolution, and high sensitivity, the detector concept is particularly suitable for applications where the scanner bore size is limited and high resolution is required, as it is in small animal hybrid MR/PET.

The authors would like to thank Suzhou JT crystal technology Co., Ltd. for providing monolithic crystals.

This article is based upon work from COST Action (TD1401, FAST), supported by COST (European Cooperation in Science and Technology).

\section{M04B-4: Photon Interaction Position-Dependent Time Walk Measurement for PET Detectors Based on Continuous Crystal Coupled to a Multi-Anode PMT}

Y. Wang, Y. Xiao, X. Cheng

Modern Physics Department, University of Science and Technolgy of China, Hefei, Anhui, China

Relying on the single-end scintillation light detection for PET detectors based on continuous crystal, the annihilation photon interaction position inside of crystal can be determined by our 3D maximum likelihood position estimation algorithm, which provides a possibility to investigate the correlation between the time walk and interaction position to optimize the detector timing performance. Measurements were performed with a test detector consisting of a continuous LYSO crystal (18.1 mm? $18.1 \mathrm{~mm}$ ? $10 \mathrm{~mm}$ ) optically coupled to a multi-anode PMT (H7546B) and a coincidence detector constructed by a block of LaBr3 crystal coupled to R9800 PMT. The time walk, referring to the shift of the timing distribution peak, was experimentally measured as a function of the $3 \mathrm{D}$ interaction position. The maximum time walk for events in a (x,y) plane is measured as $140 \mathrm{ps}$, meanwhile it is $50 \mathrm{ps}$ for events with different depth-of-interaction (DOI). The (x,y)-dependent time walk is rooted in the timing signal readout structure of the MA-PMT, and the DOI-dependent time walk is consistent with the variation of the average propagation time from the interaction position to the surface of PMT. The time walk was corrected by the measured function, which results in the detector time resolution improvement from 374.5 ps to $342.3 \mathrm{ps}$.

M04B-5: Statistical Analysis of Time Resolution of the J-PET Scanner

L. Raczynski ${ }^{1}$, W. Wislicki ${ }^{1}$, P. Kowalski ${ }^{1}$, W. Krzemien ${ }^{2}$, D. Alfs ${ }^{3}$, T. Bednarski ${ }^{3}$, P. Bialas ${ }^{3}$, C. Curceanu ${ }^{4}$, E. Czerwinski ${ }^{3}$, K. Dulski ${ }^{3}$, A. Gajos ${ }^{3}$, B. Glowacz ${ }^{3}$, M. Gorgol ${ }^{5}$, B. Hiesmayr ${ }^{6}$, B. Jasinska ${ }^{5}$, D. Kaminska ${ }^{3}$, G. Korcyl ${ }^{3}$, T. Kozik ${ }^{3}$, N. Krawczyk ${ }^{3}$, E. Kubicz ${ }^{3}$, M. Mohammed ${ }^{3}$, M. Pawlik-Niedzwiecka ${ }^{3}$, S. Niedzwiecki ${ }^{3}$, M. Palka $^{3}$, Z. Rudy ${ }^{3}$, O. Rundel ${ }^{3}$, N. G. Sharma ${ }^{3}$, M. Silarski ${ }^{3}$, J. Smyrski ${ }^{3}$, A. Strzelecki ${ }^{3}$, A. Wieczorek ${ }^{3}$, B. Zgardzinska ${ }^{5}$, M. Zielinski ${ }^{3}$, P. Moskal ${ }^{3}$

${ }^{1}$ Department of Complex System, National Centre for Nuclear Research, Otwock-Swierk, Poland

${ }^{2}$ High Energy Physics Division, National Centre for Nuclear Research, Otwock-Swierk, Poland

${ }^{3}$ Faculty of Physics, Astronomy and Applied Computer Science, Jagiellonian University, Cracow, Poland

${ }^{4}$ Laboratori Nazionali di Frascati, Istituto Nazionale di Fisica Nucleare, Frascati, Italy

${ }^{5}$ Institute of Physics, Maria Curie Sklodowska University, Lublin, Poland

${ }^{6}$ Faculty of Physics, University of Vienna, Vienna, Austria 
The commercial Positron Emission Tomography (PET) scanners use inorganic crystal scintillators for the detection of gamma photons. The Jagiellonian-PET (J-PET) detector exhibits high time resolution due to use of fast plastic scintillators and dedicated electronics circuits. Since the time resolution of PET scanner is influenced by numerous factors, e.g. a type of photomultipliers attached to the scintillators, the optimal selection of components of the J-PET system requires detailed understanding of the method for calculation of time resolution. In this presentation we show the idea of this method, based on statistical analysis of the observed signals on the photomultiplier's output. We incorporate the ideas of signal processing from the Tikhonov regularization framework and Kernel Density Estimation method. We derive a simple, closed-form analytical solution. The method is tested using signals registered by means of the single detection module of the J-PET scanner built out from $30 \mathrm{~cm}$ long plastic scintillator strip. We show consistency of the experimental and theoretical results, obtained for J-PET scanner equipped with vacuum tube photomultipliers. Moreover, two main factors affecting the photon registration probability, photomultiplier's quantum efficiency and photomultiplier's transit time spread are investigated. It is demonstrated that the quantum efficiency of photomultipliers represents the most important factor influencing overall performance of the J-PET scanner.

M04B-6: Performance tests of TOF PET detectors with a strip-line based readout

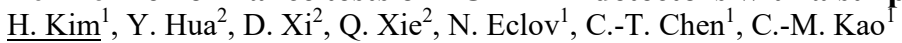

${ }^{I}$ Radiology, University of Chicago, Chicago, IL, United States

${ }^{2}$ Bio-medical Engineering, Huazhong University of Science and Technology, Wuhan, China

We are developing a time-of-flight (TOF) positron emission tomography (PET) scanner for brain imaging using SiPMs. In our development, we have adopted a strip-line based signal multiplexing method for efficient channel reduction for SiPMs; the method is using a single strip-line on which multiple SiPMs are connected for sharing readout, and the position of hit SiPM is decoded from the arrival time difference measured at the two ends of the strip-line. We have conducted experimental tests for evaluating the performance of the detectors based on the strip-line signal readout. Hamamatsu MPPC array (S12642-0404PA-50, $3.2 \mathrm{~mm}$ pitch) is employed in the detector module; 32 MPPC signals from two MPPCs arrays are routed to 4 strip-lines on the current strip-line board. For the tests, two detectors are used in a coincidence setup, and an array of 4x4 LYSO scintillator $\left(3 \times 3 \times 20 \mathrm{~mm}^{\wedge} 3,3.2 \mathrm{~mm}\right.$ pitch) is coupled to MPPC array in each detector. A $22 \mathrm{Na}$ source with $3 \mathrm{uCi}$ activity is used, and the waveform of the detectors signals are collected by using a Domino Ring Sampler (DRS4) evaluation board running at 2.5 giga sampling rate. The preliminary results show that the position on the strip-line is determined with $\sim 1.0 \mathrm{~mm}$ precision from the measured differential time on the strip-line. The average energy and coincidence time resolution are measured to be $\sim 13 \%$ FWHM and 726 ps FWHM, respectively, at $511 \mathrm{keV}$ photo-peak. A systematic investigation of the effects of strip-line on detector performance is carried out in parallel by using a specially designed strip-line board. The complete test results is presented in the paper.

M04B-7: A Novel 4-Layer DOI Detector Using Peak-to-Charge Discrimination R. Ogawara ${ }^{1}$, M. Ishikawa ${ }^{2}$

${ }^{I}$ Department of Medical Physics and Engineering, Hokkaido University Graduate school of Medicine. Research Fellow of Japan Society for the Promotion of Sciencs, Sapporo, Hokkaido, Japan

${ }^{2}$ Department of Health Sciences, Hokkaido University Graduate school of Health Sciences, Sapporo, Hokkaido, Japan

\section{Objective}

Recently depth-of-interaction (DOI) information is applied for a small PET device to improve an image quality. Generally, the DOI information is obtained by a unique reflector or pulse shape discrimination (PSD). We developed a novel PSD method with high accuracy, named peak-to-charge discrimination (PQD) method. In this study, we show the accuracy assessment of 4-layer DOI detector using the PQD method.

\section{Materials and Methods}

A ratio of peak value Vp and integral charge Q in PMT anode pulse was used for the PQD method. In this study, 4-layer DOI detector stacked with 4 types of GSO:Ce scintillators $\left(0.5,1.0,2.0,3.0 \mathrm{~mol} \%, 2.5 \times 2.5 \times 6 \mathrm{~mm}^{3}\right)$ was used with PMT R6231-100. We evaluated the discrimination accuracy of 4 stacked GSO:Ce scintillators quantitatively using the PQD method. Moreover, simulation study on the DOI detector was carried out by a developed simulation technique of PMT anode pulse using GEANT4 Monte Carlo code with the PMT response.

\section{Results}

We achieved the PSD accuracy of $99.57 \%, 99.27 \%, 98.07 \%$ and $97.46 \%$ using 4 stacked GSO:Ce (0.5, 1.0, 2.0, 3.0 mol\%) scintillators, respectively. The difference in the $\mathrm{Vp} / \mathrm{Q}$ value between the measurement and simulation was $<\sim 3 \%$. However, FWHM of the measured Vp/Q value was 2.5 3.8 times larger than the simulation value, since an electric circuit noise is absent in the simulation.

\section{Conclusion}


We demonstrated excellent PSD accuracy of the PQD method using the 4-layer DOI detector. Moreover, the PQD simulation reproduced exact measured values using developed PMT anode pulse simulation technique.

This work was supported by Grant-in-Aid for JSPS Fellows Grant Number 16J02014.

M04B-8: A Side-by-Side Phoswich PET Detector Configuration for Providing High Resolution of 0.4 mm J. Kang ${ }^{1}$, S. K. Han ${ }^{1}$, C.-H. Baek ${ }^{2}$

${ }^{I}$ Department of Biomedical Engineering, Chonnam National University, Yeosu, Korea

${ }^{2}$ Department of Radiological Science, Dongseo University, Busan, Korea

This study introduces a side-by-side phoswich PET detector consisting of currently widely used LYSO:Ce and recently newly developed GAGG:Ce for providing high spatial resolution of $<0.4 \mathrm{~mm}$. A Monte Carlo simulation was conducted to investigate the light collection efficiency (LCE) and the imaging performance of the proposed design. Crystal block consists of crystal assembly of $17 \times 17$ array arranged with a $0.9 \mathrm{~mm}$ pitch, and has a $15.5 \times 15.8 \times 10 \mathrm{~mm} 3$ volume. Each crystal assembly is consisted of a phoswich pair assembled with LYSO $(0.4 \times 0.8 \times 10 \mathrm{~mm} 3)$ and GAGG $(0.4 \times 0.8 \times 10 \mathrm{~mm} 3)$. They were optically coupled along one long side and covered with reflective film to other side surfaces and top surfaces. The X-Y position of each crystal assembly can be distinguished by a high reflectivity material and the specific interaction position of different crystals within phoswich pair was distinguished by pulse shape discrimination method. The 8x8 GAPD array is comprised of $1 \mathrm{x} 1 \mathrm{~mm} 2$ active area pixels arranged with a $2.0 \mathrm{~mm}$ pitch. The monolithic light guide was inserted with the optical grease between the crystal block and GAPD array, to distribute the scintillation light on the GAPD sensitive area. LCE value in the proposed crystal assembly was similar for LYSO and was improved from $26.7 \%$ to $34.6 \%$ for GAGG, compared to conventional high resolution PET detector designs with relatively narrow pixelated crystal. It should be noted that LCE value of $34.6 \%$ in the proposed crystal assembly with $0.4 \mathrm{~mm}$ pixel width was quite better than $29.6 \%$ for the relatively wide pixelated GAGG of $0.8 \mathrm{~mm}$ pixel width. Also, the flood histogram without the overlapping of each crystal position can be generated for side-by-side phoswich PET detectors using light guides with a thickness of 1-2 mm. Further experimental study will be performed through measurement of the discrimination error between different crystals using PSD method, the module's flood image, energy resolution, and timing resolution.

This work was supported by the National Research Foundation of Korea(NRF) grant funded by the Korea government(Ministry of Science, ICT \& Future Planning) (No. 2015R1C1A1A01051983).

\author{
M04B-9: Comparison and Performance Evaluation of a 0.9 Mm Pixelated Ce:GFAG with Ce:GAGG and \\ LYSO Array Coupled to dSiPM

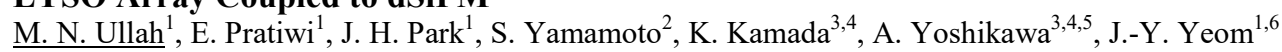 \\ ${ }^{1}$ Bio-Convergence Engineering, Korea University, Seoul, South Korea \\ ${ }^{2}$ Department of Medical Technology, Graduate School of Medicine, Nagoya University, Nagoya, Japan \\ ${ }^{3} \mathrm{New}$ Industry Creation Hatchery Center (NICHe), Tohoku University, Sendai, Japan \\ ${ }^{4} C \& A$ Corporation, Sendai, Japan \\ ${ }^{5}$ Institute for Material Research, Tohoku University, Sendai, Japan \\ ${ }^{6}$ Biomedical Engineering, Korea University, Seoul, South Korea
}

Abstract - Energy and time resolution are two important characteristics for achieving high spatial resolution in positron emission tomography (PET) system. In this work, we assessed the energy resolution performance of four different block detectors; Cerium doped Gd3Al2Ga3O12 (GAGG), gadolinium fine aluminum gallate (Ce:GFAG) and two types of silicate yttrium lutetium (Ce:LYSO) each with different reflector, BaSO4 and ESR. All four crystal block detectors consist of 12 x 12 arrays with 0.9 mm x $0.9 \mathrm{~mm}$ x $6 \mathrm{~mm}$ elements and a $1 \mathrm{~mm}$ thick acrylic resin as light guide. To assess energy resolution performance, these crystal blocks were coupled with DPC-3200-22-44, a digital silicon photomultiplier (dSiPM) for signal readout. All the measurements were conducted at sensor temperature of $\sim 15 \mathrm{oC}$, validation scheme 8 ( 52.2 photons avg.), validation length of $40 \mathrm{~ns}$ while the integration interval set as $85 \mathrm{~ns}$. The 2-dimensional position histogram for $22 \mathrm{Na}$ gamma photons showed that all pixels were clearly resolved for all block detectors. The average energy resolutions of Ce:GAGG (unpolished, BaSO_4reflector), Ce:GFAG (unpolished, BaSO_4reflector), Ce:LYSO (polished, BaSO_4reflector) and Ce:LYSO (polished, ESR reflector) were $15.0 \%$, $19.9 \%, 15.3 \%$ and $13.7 \%$ FWHM respectively without non-linearity correction. Ce:LYSO block detectors, with ESR reflector showed better energy resolution compared to BaSO4. More detailed studies including timing performance will be presented.

M04B-10: Real-Time Processing for an Adaptable SPECT System Based on CZT Detectors $\underline{\text { M. Bernard }}^{1}$, G. Montémont ${ }^{1}$, S. Stanchina ${ }^{1}$, L. Verger $^{1}$, S. Mancini ${ }^{2}$

${ }^{I}$ CEA LETI MINATEC Campus, F-38054 Grenoble, France

${ }^{2}$ TIMA laboratory, UGA, F-38031 Grenoble, France 
CdZnTe (CZT) detectors are increasingly used instead of NaI crystals in SPECT architectures first because of their good performances, second because systems based on it are more compact, since no photomultiplicator is needed. We have proposed some signal processing techniques to optimize spatial resolution/sensitivity trade-off, which enables precise 3D-positioning. Further enhancements are achievable by adapting the field of view of the system to the emitting object to be imaged in real-time. It could allow changing the configuration depending on the morphology of the patient and the kind of examination. This adaptability is challenging for two reasons: first, geometrical position of detectors is to be considered in order to interpret each detection properly. This dynamic configuration leads to more complex data than for a static system. Second, reconstruction must be computed on real-time to adapt accurately the configuration during the acquisition. We propose alternative reconstruction techniques to face challenges occurred by adaptability. This work firstly present HiSPECT module developed by our laboratory to process signals from CZT detector. Then we explain how adaptation enhances systems performances. Finally, this paper presents algorithmic adaptations of MLEM to real-time computing to empower such approach.

\author{
M04B-11: Simulations and Image Reconstruction for High Resolution CaLIPSO PET Scanner for Human \\ Brain and Preclinical Studies

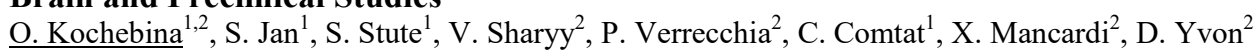 \\ ${ }^{I} I M I V-S H F J, C E A$, Orsay, France \\ ${ }^{2} S P P-I R F U, C E A$, Gif-sur-Yvette, France
}

The foreseen CaLIPSO (french acronym for Liquid Ionization Calorimeter, Scintillation Position Organometallic) Positron Emission Tomography (PET) scanner is expected to have simultaneously a fine image resolution, about $1 \mathrm{~mm} 3$, and a high contrast. Such performances are especially relevant in human brain imaging and preclinical researches. The CaLIPSO scanner uses the concept of double detection of the signal created by a photoelectron from the $511-\mathrm{keV}$ photon conversion in liquid trimethyl bismuth (TMBi) filling a cell of this innovative detector. The converted electron emits Cherenkov photons and ionizes the medium. Both light and free electrons are collected simultaneously and used for the reconstruction of the time and 3D position of the interaction as well as deposited energy. Enhancement of image contrast is possible thanks to the Time of Flight techniques with expected coincidence resolving time of 100-200ps. The preliminary results on simulations for a full PET scanner with the GATE platform are presented. New results obtained with a non-standard «cube» geometry of the scanner in terms of image resolution and Noise Equivalent Count rate and are compared to HRRT performance. We discuss the challenges for the reconstruction of data produced by this scanner with a high spatial resolution and present the first simulated brain images for 18F-FDG and 11C-PE2I tracer distributions. CaLIPSO is a promising ongoing project for designing a PET scanner with a high potential dedicated to small animal and brain imaging that could outperform the other detector technologies proposed for PET imaging such as scintillating crystals, high-Z semiconductors and liquid Xenon.

\author{
M04B-12: Evaluation of a 16 X 16 MPPC Array for Small-Animal PET \\ J. Du, X. Bai, E. Berg, S. R. Cherry \\ Biomedical Engineering, University of California, Davis, Davis, CA, USA
}

A $16 \times 16$ through silicon via multi pixel photon counters (MPPCs) array was evaluated for small-animal PET applications using a $16 \times 16$ array of $0.975 \mathrm{~mm} \times 0.975 \mathrm{~mm} \times 10 \mathrm{~mm}$ LYSOs (pitch size $1.05 \mathrm{~mm}$, ESR reflector). The MPPC array, with a surface area $19.25 \mathrm{~mm} \times 19.25 \mathrm{~mm}$, consists of 16 x 16 MPPC pixels. Each pixel has a $1.0 \mathrm{~mm}$ x $1.0 \mathrm{~mm}$ active area on a $1.2 \mathrm{~mm}$ pitch. Custom front-end electronics was designed to reduce the $256 \mathrm{SiPM}$ signals to five signals, four for position information, and one for timing information. We evaluated the performance of this MPPC array with a focus for its use in small-animal PET applications. Measurements of energy resolution and flood histograms were obtained at a bias voltage of $67.5 \mathrm{~V}$ and at a temperature of $20^{\circ} \mathrm{C}$. The flood histogram shows that all the crystal in the LYSO array can be clearly resolved, and that crystal arrays with even smaller pitch size might be resolved. The average energy resolution was $17.6+/-1.2 \%$. In future, a depth-ofinteraction detector module using dual-ended readout method will be built using two $16 \times 16$ MPPC arrays and a $38 \times 38$ array of $0.445 \mathrm{~mm} \times 0.445 \mathrm{~mm} \times 20 \mathrm{~mm}$ LYSOs (pitch size $0.5 \mathrm{~mm}$, Toray reflector), and performance of flood histogram, energy resolution, timing resolution and DOI resolution will be investigated.

\author{
M04B-13: Timing Performance of Wide Scintillator Crystal Elements for Super-Resolution Clinical PET \\ J. W. Cates ${ }^{1}$, G. Chinn ${ }^{1}$, C. S. Levin ${ }^{1,2,3,4}$ \\ ${ }^{I}$ Radiology, Stanford University, Stanford, CA, USA \\ ${ }^{2}$ Bioengineering, Stanford University, Stanford, CA, USA \\ ${ }^{3}$ Electrical Engineering, Stanford University, Stanford, CA, USA \\ ${ }^{4}$ Physics, Stanford University, Stanford, CA, USA
}

"Super-resolution PET" is a data acquisition technique that has been used in PET to boost reconstructed spatial resolution beyond a system's intrinsic capabilities by incremental sampling at bed positions finer than the crystal element width of the detectors. Another application of this technique for clinical PET is to achieve the same spatial resolution as current commercial systems $(\sim 5$ 
$\mathrm{mm}$ at system center) but with large area pixel elements (6-12 $\mathrm{mm}$ width). The benefits of larger crystal element width would be improved photon sensitivity owing to an overall reduced number of inter-crystal gaps, improved scintillation light collection efficiency due to higher light collection aspect ratio, reduced cost for cutting and preparing scintillation material, simplified electronic readout, and less sensitivity to inaccurate positioning of inter-crystal scatter events (which are more probable than photoelectric events). Since fine spatial information is achieved by translating the patient bed, sub-crystal positioning information is not required from the PET detector, and signal processing can be optimized for timing performance. Therefore, this technique of using a much larger crystal element width is a potential solution for a simple, low cost method to enhance timing performance. In this work, we present the achievable coincidence timing resolution (CTR) with LYSO:Ce with crystal element widths of 3-12 $\mathrm{mm}$ and lengths ranging from 10-20 mm 1:1 coupled to analog SiPMs and SiPM arrays, with timing and energy information encoded into a single readout channel. A sub-250 ps FWHM CTR is achievable with LYSO:Ce crystals as large as $12 \times 12 \times 20$ $\mathrm{mm}^{3}$ using a single channel of readout.

\author{
M04B-14: Timing Performance of Two PET Detector Designs Capable of Time-of-Flight and Depth-of- \\ Interaction Measurement: Phoswich and Offset Crystal Layers \\ C.-M. Chang ${ }^{1}$, C. S. Levin $2,3,4,5$ \\ ${ }^{I}$ Department of Applied Physics, Stanford University, Stanford, California, USA \\ ${ }^{2}$ Department of Radiology, Stanford University, Stanford, California, USA \\ ${ }^{3}$ Department of Bioengineering, Stanford University, Stanford, California, USA \\ ${ }^{4}$ Department of Physics, Stanford University, Stanford, California, USA \\ ${ }^{5}$ Department of Electrical Engineering, Stanford University, Stanford, California, USA
}

PET detectors capable of measuring $511 \mathrm{keV}$ photon time-of-fight (TOF) and depth-of-interaction (DOI) can improve resulting image quality and accuracy. In this work, we studied the timing performance of two TOF-DOI PET detector designs: (1) phoswich and (2) offset crystal layers. The phoswich detector comprises a $3 \times 3 \times 20 \mathrm{~mm}^{3}$ element made from a $3 \times 3 \times 10$ $\mathrm{mm}^{3}$ LYSO:Ce crystal with $42 \mathrm{~ns}$ decay time optically epoxied to a $3 \times 3 \times 10 \mathrm{~mm}^{3}$ calcium co-doped LSO:Ce, Ca( $\left.0.4 \%\right)$ crystal with 33 ns decay time. The offset crystal block comprises a $4 \times 4$ array of $3 \times 3 \times 10 \mathrm{~mm}^{3}$ LYSO crystals in the bottom layer and a $4 \times 3$ array of LYSO crystals of the same size in the top layer. The top array is offset by half pixel pitch from the bottom array. The coincidence timing resolution for photopeak events acquired by two opposing phoswich detectors ranges from $164.6 \pm 0.9 \mathrm{ps}$ to $183.1 \pm 4.2$ ps FWHM, depending on the interaction layer combinations between two identical, dual layer elements. The coincidence timing resolution for photopeak events acquired by the offset crystal layers against a reference detector comprising a single $3 \times 3 \times 20 \mathrm{~mm}^{3}$ LYSO crystal ranges from 177.8 ps to $272.6 \mathrm{ps}$ FWHM and averaged to $219.7 \pm 35.5$ ps FWHM. These results show that both designs are capable of TOF and DOI measurement.

\author{
M04B-15: Development of an Isotropic DOI Detector Based on a Dual-Ended Readout and Subsurface Laser \\ Engraving Technique

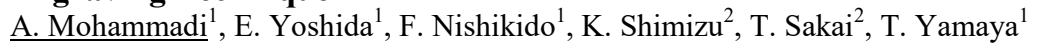 \\ ${ }^{I}$ Department of Radiation Measurement, Imaging and Dosimetry, National Institute of Radiological Sciences, Chiba, Japan \\ ${ }^{2}$ Hamamatsu Photonics K.K., Hamamatsu, Japan
}

Depth of interaction (DOI) information is indispensable to improve sensitivity and resolution of positron emission tomography (PET) systems. We have already developed a series of X'tal cube detectors for isotropic resolution. The isotropic resolutions of $0.77 \mathrm{~mm}$ and $3 \mathrm{~mm}$ were obtained for the detectors with six-sided readout and two-sided readout, respectively. However, it is still hard to apply the former detector for practical PET systems due to the high costs of six-sided readout electronics and carrying out the three-dimensional (3D) subsurface laser engraving (SSLE) technique. In this work, we tried to develop a more practical X'tal cube with the two-sided readout detector by using a crystal array of $1.5 \times 1.5 \times 20 \mathrm{~mm} 3$ crystal elements segmented to 13 DOI segments using the 1D SSLE technique. The objective was to obtain the best crystal identification by combination of different optical conditions including air and reflectors between the crystal elements. The 3D position maps of the detector without any reflectors and by inserting reflectors fully or partially between the crystals were obtained by the anger type calculation. Crystal identification performance was evaluated for the crystal arrays. Clear separation was obtained for the crystal array without reflectors and with full reflectors only for the crystals at the middle of the array. Good crystal identification and isotropic resolution of $1.5 \mathrm{~mm}$ were obtained for all crystal segments with partial reflectors between the crystals. The energy resolutions was also evaluated for the crystal array with partial reflectors. The energy resolutions of $9.6 \% \pm 0.2 \%$ and $12.4 \% \pm 1.3 \%$ were obtained for the segments at the top layer and at the central layer, respectively. The timing performance of the detector is going to be evaluated and discussed in the conference.

M04B-16: A Simulation Study on Lateral Readout Method Using Elongated Detector Module for TOF Axial PET

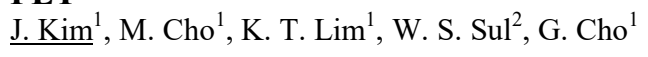


${ }^{I}$ Dept. of Nuclear and Quantum Engineering, Korea Advanced Institute of Science and Technology, Daejeon, South Korea

${ }^{2}$ National Nano Fab Center, Daejeon, South Korea

A Monte Carlo simulation using Geant4 Application for Tomographic Emission (GATE) was done to test the spatial, energy, and timing resolution of a new detector structure design for time-of-flight (TOF) positron emission tomography (PET) which is axially oriented. Conventional end-readout PET detectors have shown limitations in achieving high spatial, energy, and timing resolution simultaneously. Detectors using monolithic scintillators have appeared to have promising spatial, energy, and timing resolution but they suffer from varying DOI resolution along the depth direction and the edge artifacts. On the other hand, axial PET detectors with lateral readout have shown excellent spatial, energy, and timing resolution; however, those detectors require too many expensive components, which adds the production cost tremendously. Therefore, the objective of this simulation study is to test whether a TOF PET with fine spatial and energy resolution is achievable with a lateral readout axial PET detectors, using relatively small number of solid-state sensors such as silicon photomultiplier (SiPM). The proposed detector module has a $3 \times 3 \times 100 \mathrm{~mm}$ Lutetium Yttrium Orthosilicate (LYSO) crystal and ten $2 \times 2 \mathrm{~mm}$ sensors along the scintillation crystal. Without considering the limitations of a SiPM and its readout circuit, the initial simulation results showed around $0.6 \mathrm{~mm} \mathrm{FWHM} \mathrm{of} \mathrm{axial}$ resolution, around $13 \%$ of energy resolution, and 270 ps of coincidence resolving time (CRT). The optimization of time trigger scheme and the correction of time-walk were not applied yet in calculation of the CRT, which is why the CRT here seems worse than the results of previous research from other groups. After optimizing the trigger scheme and applying the time-walk correction in the near future, the simulated CRT would show certain improvements. Further studies will be done to verify the simulation results with a real test module using SiPM designed and fabricated in our lab until October 2016.

M04B-17: MONDO: a Fast Neutron Tracker for Particle Therapy Secondary Emission Fluxes Measurements.

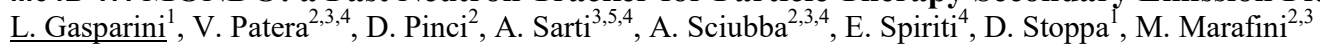

${ }^{I}$ Fondazione Bruno Kessler, Trento, Italy

${ }^{2}$ INFN Sezione di Roma, Roma, Italy

${ }^{3}$ Museo Storico della Fisica e Centro Studi e Ricerche "E. Fermi", Roma, Italy

${ }^{4}$ Dipartimento di Scienze di Base e Applicate per Ingegneria, Sapienza Universit l a di Roma, Roma, Italy

${ }^{5}$ Laboratori Nazionali di Frascati dell'INFN, Frascati (RM), Italy

In Particle Therapy, cancer treatments are performed using accelerated charged particles whose high irradiation precision and conformity permit to destroy the tumor while sparing the surrounding healthy tissues. Several secondary particles are produce during the treatments mainly photons, protons and neutrons. The reduced attenuation length of neutrons yields a secondary particle sample that is larger in number when compared to photons and charged particles. Since neutrons can release a significant dose far away from the tumor region, a precise measurement of their flux, production energy and angle distributions is eagerly needed in order to improve the Treatment Planning Systems (TPS) software, so to properly take into account not only the normal tissue toxicity in the target region, but also the risk of late complications in the whole body. The technical challenges posed by a neutron detector aiming for high detection efficiency and good backtracking precision will be addressed within the MONDO (MOnitor for Neutron Dose in hadrOntherapy) project. The MONDO main goal is to develop a tracking detector targeting fast and ultrafast secondary neutrons. The neutron tracking principle is based on the reconstruction of two consequent elastic scattering interactions of a neutron with a target material. Reconstructing the recoiling protons it is hence possible to measure the energy and incoming direction of the neutron using different therapeutical beams (protons, $12 \mathrm{C}$ ions and possibly $4 \mathrm{He}$ and $16 \mathrm{O}$ ions). The detector will be composed by a tracker, realized with squared scintillating fibers, readout by a dedicated CMOS based digital SPAD array matrix.

\section{M04B-18: Comparison of Columnar Scintillators and Pixelated Scintillators for Small Field of View Hybrid Gamma Camera Imaging}

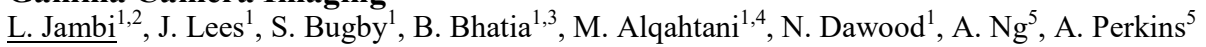

${ }^{I}$ Space Research Centre, Physics and Astronomy Department, University of Leicester, Leicester, UK

${ }^{2}$ Radiological Sciences Department, College of Applied Medical Sciences, King Saud University, Riyadh, Saudi Arabia

${ }^{3}$ Clinical Imaging Group, Sandwell and West Birmingham Hospital NHS Trust, Birmingham, UK

${ }^{4}$ Radiological Sciences Department, College of Applied Medical Sciences, King Khalid University, Abha, Saudi Arabia

${ }^{5}$ Radiological Sciences, School of Medicine, University of Nottingham, Nottingham, UK

Aims: In intraoperative medical imaging, the development of small field of view (SFOV) hybrid gamma cameras is an expanding research field. Combining both gamma and optical imaging in a co-aligned configuration is a new innovation that could provide high spatial resolution scintigraphic imaging and enhance the physical localisation of radiopharmaceutical uptake during critical surgical procedures such as in head and neck biopsies.

The aim of this study was to compare the performance characteristics of the Hybrid Gamma Camera (HGC) using thallium doped caesium iodide CsI(TI) columnar scintillator and pixelated gadolinium oxysulfide (GOS) ceramic scintillator following the schematic protocols provided by the Institute of Physics and Engineering in Medicine (IPEM).

Materials and Methods: The hybrid gamma camera (HGC) consists of an electron multiplying CCD coupled to a $1500 \mu \mathrm{m}$ thick 
scintillator (in this study both CsI(TI) and GOS scintillators were used). A tungsten pinhole collimator coupled with a mirror and optical camera.

A performance characterisation of the HGC has been completed using a columnar CsI(TI) and a pixelated gadolinium oxysulfide (GOS) ceramic scintillator. Characteristics investigated include spatial resolution (both intrinsic and extrinsic), intrinsic uniformity, intrinsic sensitivity and count rate capability.

Results: The performance characterisation allowed for quantitative comparisons of HGC with different scintillators installed. Intrinsic spatial resolution, for example, was $\sim 0.32 \mathrm{~mm}$ for the CsI(TI) and $\sim 1.09 \mathrm{~mm}$ for the GOS scintillator.

Conclusion: The performance characteristics of the HGC illustrate its capability to be used in a wide range of healthcare applications such as: diagnosis, surgical investigation and the visualisation of drug delivery. These studies encourage us to carry out further evaluation in preparation for its use in a surgical theatre setting

\title{
M04B-19: Magnetic Field Compatible Buck Converters and Optical Link
}

\section{R. Becker ${ }^{1}$, A. Buck ${ }^{2}$, C. Casella ${ }^{1}$, V. Commichau ${ }^{1}$, G. Dissertori ${ }^{1}$, J. Fischer ${ }^{1}$, A. S. Howard ${ }^{1}$, M. Ito ${ }^{1}$, P. Khateri ${ }^{1}$, K. Kramer $^{1}$,} W. Lustermann ${ }^{1}$, J. F. Oliver ${ }^{3}$, C. Ritzer ${ }^{1}$, U. Röser ${ }^{1}$, Q. Wang ${ }^{4}$, G. Warnock ${ }^{5}$, B. Weber ${ }^{5}$

${ }^{I}$ Institute of Particle Physics, ETH Zürich, Zurich, Switzerland

${ }^{2}$ Division of Nuclear Medicine, Zürich University Hospital, Zurich, Switzerland

${ }^{3}$ Instituto de Física Corpuscular, Valencia, Spain

${ }^{4}$ Institute of Medical Physics, Tsinghua University, Beijing, China

${ }^{5}$ Institute of Pharmacology and Toxicology, University of Zurich, Zurich, Switzerland

Within the Small Animal Fast Insert for mRi (SAFIR) project, optical links and power conversion electronics are evaluated with respect to their compatibility with the $7 \mathrm{~T}$ magnetic field of the target magnetic resonance tomograph. Results of the test of a commercially available optical link will be presented as well as the estimated performance of a custom development of two types (for high and low current) of synchronous buck converters with components suitable for usage inside the 7T magnetic field.

This work was supported by the ETH Zurich Foundation through ETH Research Grant ETH-30 14-2, and by an SSSTC Exchange Grant EG $02-$ 03201 .

\author{
M04B-20: Response Function Estimation for the XCounter Flite X1 Photon Counting Detector Using Monte \\ Carlo Method

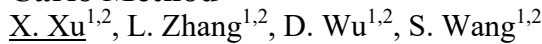 \\ ${ }^{I}$ Key Laboratory of Particle \& Radiation Imaging (Tsinghua University), Ministry of Education, Beijing, China \\ ${ }^{2}$ Department of Engineering Physics, Tsinghua University, Beijing, China
}

The introduction of photon counting detectors into the computed tomography (CT) has a lot of benefits. However, affected by a lot of effects such as charge sharing, K-escaping, the energy response functions are not ideal and may cause spectral distortion. It is vital to know the energy response functions for energy spectrum correction and system optimization. The goal of this paper is to build the energy response functions that fit the measured spectrums. In this work we estimate the response functions of the XCounter Flite X1 detector by measuring the X-ray fluorescence of mental samples. The Compton scattering and k-escape peak are simulated by Monte Carlo method using Geant4, while the charge sharing and other effects are simulated by the correction function. The energy response functions are based on Gaussian distribution and correction functions. The results show that the model is accurate and we can correct the measured spectrum based on response functions.

M04B-21: Development of a Monolithic Detector with 3D Positioning Capability and Readout Channel Number Compression

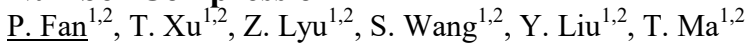

${ }^{1}$ Key Laboratory of Particle \& Radiation Imaging, Ministry of Education (Tsinghua University), Beijing, China

${ }^{2}$ Engineering Physics, Tsinghua University, Beijing, China

Monolithic PET detector design is promising for low cost, high packing fraction, intrinsic depth-of-interaction (DOI) capability as well as potentially better energy and timing resolution compared to the conventional segmented crystal-based detector design. In this work, we developed a monolithic detector with 3D positioning capability and readout channel number compression. The detector contains a monolithic LYSO crystal (size: $25 \times 25 \times 14 \mathrm{~mm}^{3}$ ) coupled to a $6 \times 6$ SiPM array (SensL FC30035). Two 3D positioning algorithms including statistical-based positioning (SBP) and artificial neural network (ANN) were developed to estimate gamma ray interaction positions inside the crystal. To simplify detector electronics design, two readout channel number compression strategies respectively based on Anger and principal component analysis (PCA) were proposed. With Anger, the readout channel number was compressed from 36 to 12 while with PCA, the readout channel number was respectively compressed to 12 (PCA12), 6 (PCA6) and 4 (PCA4) corresponding to using different number of principal component vectors. The results demonstrate that $3 \mathrm{D}$ positioning capability is achievable with both SBP and ANN and the positioning resolution in $\mathrm{x}$, 
$\mathrm{y}$ and $\mathrm{z}$ direction are respectively $2.58 \mathrm{~mm}, 2.56 \mathrm{~mm}$ and $4.79 \mathrm{~mm}$ with SBP, and $2.02 \mathrm{~mm}, 2.03 \mathrm{~mm}$ and $3.96 \mathrm{~mm}$ with ANN. Both strategies can effectively reduce the readout channel number without inducing degradations in detector positioning performance. With Anger and PCA12, almost no degradation is observed in positioning performance compared to no compression. With PCA6 or PCA4, deteriorations in positioning performance in z direction are observed, especially with PCA4, although there is no significant degradation in $\mathrm{x}$ or $\mathrm{y}$ direction. We conclude that the developed monolithic detector is promising for its 3D positioning capability as well as engineering application value with compressed readout channel number in PET system designs.

M04B-22: Development of PET Using Multi-Voltage Threshold and FPGA Only DAQ

K. B. Kim, Y. Choi, S. Lee, J. Jung, H. T. Leem, J. H. Jung

Molecular Imaging Research \& Education (MiRe) Laboratory, Department of Electronic Engineering, Sogang University, Seoul, Korea

We have reported the feasibility of Multi-Voltage Threshold (MVT) based front-end circuit and PET DAQ system using fieldprogrammable-gate-array (FPGA) without ADC and TDC. In this sampling method, MVT method has been employed to generate samples of an event waveform of PET with respect to the user defined thresholds to extract energy and time information of a PET event. The goal of this study was to extend the MVT based multi-channel front-end circuit and FPGA only DAQ for the development of a prototype PET system. A PET detector module was composed of a $4 \times 4$ matrix of $3 \times 3 \times 20 \mathrm{~mm}^{3} \mathrm{LYSO}$, a $4 \times 4$ array GAPD. Output charge signals of PET detector modules were amplified and fed into 4 comparators to generate trigger signals in analog circuit. The energy of the detected gamma ray was calculated by integrating the digitized pulse and the arrival time was determined from the time stamp of each trigger signal by FPGA. The calculated data packet containing energy, time and position information was formed into 64-bit single event data package and sent to the host computer using USB 3.0 control interface. A proof-of-principle PET system was configured with two detector blocks each of which consisted of $4 \times 2$ detector module (128 ch / detector block). Individual projections of the PET system were acquired by rotating the radiation source on a vertical axis in front of the detector blocks. The performance of PET system using 256 channel analog circuit and DAQ system was evaluated by measuring the energy resolution and time resolution and the values were $20.2 \%$ and $1 \mathrm{~ns}$, respectively. This study demonstrated that the proposed MVT based front-end circuit and DAQ system could be utilized for the development of PET with the advantage of simple design of electronics and DAQ and scalable design for PET system. Further study will be performed to acquire PET imaging and to develop a functional PET system using the MVT and DAQ proposed in this study.

M04B-23: Light Response Estimation and Gamma Events Reconstruction in Gamma Detectors Based on Continuous Scintillators M. Occhipinti ${ }^{1,2}$, P. Busca ${ }^{1,2}$, C. Fiorini ${ }^{1,2}$

${ }^{I}$ Dipartimento di Elettronica, Informazione e Bioingegneria, Politecnico di Milano, Milano, Italy

${ }^{2}$ Sezione di Milano, INFN, Milano, Italy

we present the implementation of a practical adaptive method for optical model estimation in Anger cameras for medical imaging. The optical model, also referred to as light response, represents a fundamental requirement for statistical event reconstruction in gamma detectors implementing monolithic scintillators. Despite we considered several approaches for the response calibration, based either on Monte Carlo optical modelling or experimental scanning of the detectors response, none of them was practical in producing a reliable optical model and a solution for our scintillation cameras calibration seemed not available. We therefore propose a by far more effective approach for optical model estimation. We designed and tested it on detectors based on Silicon PhotoMultipliers (SiPMs) and adopted in the INSERT project, aiming at the development of a nonrotational SPECT insert for MR scanners, composed by 10 detectors (preclinical version). The adaptive method calibrates the light response starting from an experimental acquisition of an uncollimated source. Thus, the model is reliable, keeping the calibration procedure fast and simple. This permits to estimate, within the same session, the optical models for every gamma camera populating the SPECT in few tens of minutes. The estimated optical model feeds a Maximum Likelihood algorithm for optimal event reconstruction, providing better uniformity and linearity together with recovery of the field of view. We tested the reconstructed images quality by acquiring irradiation profiles with a gamma-camera designed for the preclinical version of the INSERT SPECT. The maximum deviation from linearity results inferior to $1 \mathrm{~mm}$. Deviation from uniformity is less than the 15 $\%$ of the average number of counts. The optical model is also well adaptable to the variability of detection response thanks to the well uniform behavior of the SiPMs adopted.

M04B-24: Development and Performance Evaluation of Time over Threshold Digital PET (TODPET2) Using SiPM-Arrays Coupled with Pixelized Ce:GAGG Scintillators for Non-Invasive Measurement of Blood RI Concentrations

$\underline{\text { M. Yoshino }}^{1,2}$, K. Kamada ${ }^{1,3}$, Y. Shoji1 ${ }^{1,2}$, A. Yoshikawa ${ }^{1,2,3}$, K. Shimazoe ${ }^{4}$, A. Lipovec ${ }^{4}$, H. Takahashi ${ }^{4}$, K. Fujiwara ${ }^{5}$,

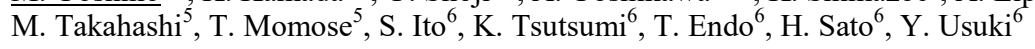


${ }^{I}$ C\&A Corporation, Sendai, Japan

${ }^{2}$ Institute for Material Research, Tohoku University, Sendai, Japan

${ }^{3}$ New Industry Creation Hatchery Center, Tohoku University, Sendai, Japan

${ }^{4}$ Department of Nuclear Engineering and Management, The University of Tokyo, Tokyo, Japan

${ }^{5}$ Department of Radiology and Nuclear Medicine, The University of Tokyo, Tokyo, Japan

${ }^{6}$ Tsukuba Development Center, Furukawa Co., Ltd, Tsukuba, Japan

PET detector using silicon photomultipliers ( $\mathrm{SiPM}$ ) arrays coupled with pixelized Ce: $\mathrm{Gd}_{3}(\mathrm{Al}, \mathrm{Ga})_{5} \mathrm{O}_{12}$ (GAGG) scintillators dedicated for non-invasive measurement of blood RI concentrations was developed. The detector consists of $1.57 \times 1.57$ $\mathrm{mm}^{2} \mathrm{SiPM}$ chips and $1.6 \times 1.6 \mathrm{~mm}^{2} \mathrm{Ce}$ GAGG scintillators arranged on a $12 \times 12$ channel, both working as individual read out systems. After development of the detector, we fabricated the PET gantry composed of 8 pieces of SiPM/Ce:GAGG detector array which signals were sent to the current-comparing type time-over-threshold (TOT) ASIC for individual readout of pixels. The spatial resolution of the PET gantry reconstructed with maximum likelihood estimation method (MLEM) algorithms is 0.98 $\mathrm{mm}$ full width at half maximum (FWHM) at the center of field of view (FOV). The sensitivity of the system is measured to be $1.31 \%$ using ${ }^{22} \mathrm{Na}$ point source. Finally, timing response to changes in RI concentration was also measured using $5 \mathrm{~mm}$ diameter syringe injected with several concentrations of ${ }^{18} \mathrm{FDG}$. As the result, this system is capable of identifying the differences of $0.13 \mathrm{MBq}$ and $0.065 \mathrm{MBq}$ within 1 -minute intervals.

This research is supported by Research on Development of New Medical Devices from Japan Agency for Medical Research and Development (AMED).

M04B-25: Characterisation of the SiPM-Based Detector Module of the TRIMAGE PET/MR Scanner N. Belcari $^{1,2}$, G. Sportelli ${ }^{1,2}$, M. G. Bisogni ${ }^{1,2}$, N. Camarlinghi ${ }^{1,2}$, A. Di Pasquale $^{1,2}$, S. Dussoni $^{1,2}$, J. Fleury $^{3}$, M. Morrocchi $^{1,2}$, S. Ahmad $^{3}$, A. Del Guerra ${ }^{1,2}$

${ }^{I}$ Department of Physics, University of pisa, Pisa, Italy

${ }^{2}$ INFN, Pisa, Italy

${ }^{3}$ Weeroc s.a.s., Paris, France

We present the first results that have been obtained with the PET detector module the trimodal PET/MRI/EEG TRIMAGE scanner. The PET component of TRIMAGE features a full ring made of 18 detectors each consisting of twelve $8 \times 8$ Silicon SiPMs matrices coupled to two staggered layers of LYSO, i.e., each crystal of the top layer is coupled to four crystals of the bottom layer that are one to one coupled to the SiPMs. This configuration allows performing a dichotomic depth of interaction reconstruction. The front-end electronics is made of four TRIROC ASICS of 64 channels each, mounted on a single board and connected to a FPGA. The four ASICS allow to read $256 \mathrm{SiPMs}$ in parallel. In order to test the performances of the acquisition system, an experimental setup has been developed that allow to record the full frames in a host computer. However, acquiring the full frame in normal operation condition would be infeasible due to the high data bandwidth required to transmit each SiPM output value to the host. Therefore some basic real time data processing is needed such as pixel identification, energy correction and synchronization. The readout strategy has been to record in the RAM of the FPGA a full image of the SiPM output values in order to allow the implementation of real-time (i.e., implemented in the front-end FPGA and performed at the same rate of single events) advanced pixel/layer identification techniques such as linear Support Vector Machine (SVM) algorithms. Flood maps, pixel identification and energy resolutions are shown for this setup. The pixel and layer identification capability of the TRIMAGE PET detection module in real-time are here evaluated. Results of the SVM algorithms are compared with those achieved with a standard layer and pixel separation performed with the adoption of the centroid information. A detector module prototype is studied. The analysis is performed using both a flood field irradiation and a collimated pencil beam of $511 \mathrm{keV}$ photons.

The research leading to these results has received funding from the European Union Seventh Framework Programme (FP7/2007-2013) under grant agreement $n^{\circ} 602621$ TRIMAGE.

M04C: Posters: preclinical I

Wednesday, Nov. $2 \quad$ 16:30-18:30 Etoile

M04C-1: Towards Dynamic Imaging of the Ac-225 Decay Chain

$\underline{\text { A. K. Robertson }}^{1,2}$, C. Rodriguez-Rodriguez ${ }^{1}$, C. F. Ramogida ${ }^{2}$, V. Sossi ${ }^{1}$, P. Schaffer ${ }^{2}$

${ }^{1}$ Physics and Astronomy, University of British Columbia, Vancouver, BC, Canada

${ }^{2}$ Life Sciences, TRIUMF, Vancouver, BC, Canada

The use of the Ac-225 decay chain in targeted alpha-radioimmunotherapy requires the retention of both Ac-225 and its progeny at the target site. Imaging-based tests of Ac-225 radiopharmaceuticals must therefore be capable of separately yet simultaneously imaging multiple isotopes that may not be co-localized despite being part of the same decay chain. We present first steps taken 
towards developing quantitative preclinical imaging tests of Ac-225 radiopharmaceuticals via dual-isotope SPECT of Ac-225 daughters Fr-221 (218 keV) and Bi-213 (440 keV) using a VECTor scanner (MILabs), a multi-modality imaging system with interchangeable collimators capable of sub-millimeter quantitative SPECT. An approximately mouse-sized phantom consisting of a small volume of activity ( $2 \mathrm{Ci}$ of Ac-225) submerged in water was imaged over 20 hours using both ultra-high sensitivity (UHS) and high energy ultra-high resolution (HEUHR) collimators. Separate Fr-221 and Bi-213 images were reconstructed for each collimator and assessed for quantitative accuracy, noise, and SNR. Image quality was limited by the small amount of activity available for these initial studies. For Fr-221 images, the UHS collimator showed lower noise $(0.54 \mathrm{Ci} / \mathrm{mL}$ vs. 1.10 $\mathrm{Ci} / \mathrm{mL})$ and greater SNR (10.59 vs. 3.26) compared to the HEUHR collimator. For Bi-213 images, the UHS collimator also showed lower noise $(0.70 \mathrm{Ci} / \mathrm{mL}$ vs. $0.84 \mathrm{Ci} / \mathrm{mL})$ but similar SNR (7.56 vs. 7.63$)$ compared to the HEUHR collimator. All images except the Fr-221 HEUHR image measured mean ( noise) activity concentrations consistent with the known value (5.9 $\mathrm{C} / \mathrm{mL}$ ). These preliminary results demonstrate the feasibility of simultaneously imaging Fr-221 and Bi-213 and suggest that continued exploration is warranted into the technique's ability to assess the targeting performance of Ac-225

radiopharmaceuticals. While the HEUHR images may improve when more activity is available, it is likely the UHS collimator may be the best option for future studies considering the difficulty and cost of obtaining large Ac-225 quantities.

\section{M04C-2: A Novel Generic Organ-PET for Small Animal Organs and Tissues}

L. Sensoy, J. J. Sunderland

Department of Radiology, University of Iowa, Iowa City, IA, USA

We designed, simulated, and constructed a novel, compact, high resolution, and cost-effective PET imaging device designed specifically for imaging of excised murine tissue and organs. The prototype scanner is a four-face parallel-plate PET scanner based on the SensL MatrixSM-9 SiPM detectors coupled with $2 \mathrm{~mm}$ thick LYSO crystals. We implemented a GATE/Geant4based Monte Carlo simulation of this scanner to validate and analyze its properties using several crystal configurations including individual crystals directly coupled to SiPM array pixels and monolithic crystals covering the SiPM arrays. STIR was used to perform reconstructions of both simulated and experimental acquisition data. Based upon simulation, we identified a central 40 $\mathrm{mm}$ diameter region as the scanner's field of view $(\mathrm{FoV})$, where resolution was only minimally impacted by parallax. The scanner system with pixelated LYSO crystals was able to separate small objects as close as $1.25 \mathrm{~mm}$ around the center and $1.50 \mathrm{~mm}$ at the periphery of the defined FoV. Simulations demonstrated the necessity of detector (or specimen) rotation to achieve acceptable image quality. A single $45^{\circ}$ rotation was sufficient for the monolithic crystal configuration, but more angular sampling was required for the pixelated crystal configuration. We measured precise crystal-photon interaction positioning information from collimated beam experiments with the monolithic crystals using the scintillated light energy sharing mechanism across the neighboring detector pixels. Using this approach we achieved approximately $0.5 \mathrm{~mm}$ positioning accuracy within the monolithic crystals. Incorporating this position distribution model in our Monte Carlo simulations we achieved a sub-millimeter spatial resolution in simulated reconstructed images of complex distributions.

M04C-3: Preclinical PET Detector with Temperature Gain Compensation $\underline{\text { A. Kolb }}^{1}$, E. Engelmann ${ }^{2}$, P. Major ${ }^{3}$, G. Patay ${ }^{3}$, B. Tölgyesi ${ }^{3}$, C. Parl ${ }^{1}$, T. Ganka ${ }^{4}$, M. J. Czeller ${ }^{3}$, G. Nemeth ${ }^{3}$, B. Pichler $^{1}$

${ }^{1}$ Werner Siemens Imaging Center, University of Tubingen, Tubingen, Germany

${ }^{2}$ University of the Bundeswehr, Institute for Physics, Munich, Germany

${ }^{3}$ Mediso Ltd., Budapest, Hungary

${ }^{4}$ Ketek GmbH, Hofer Str. 3, Munich, Germany

In preclinical imaging, several PET/MRI prototype systems have already been developed, and first results show a high impact in many fields of medical research. In this study, we present a PET detector based on silicon photomultipliers (SiPM) for a whole body small animal PET system to be integrated in a 7 T MRI system with on-board temperature compensation. The detector proposed for the PET insert is based on a custom 6x6 SiPM array with an on-board temperature compensation. It was assembled to readout a $15 \times 15$ crystal and a $20 \times 20$ crystal block with individual crystal sizes of $1.5 \times 1.5 \times 10 \mathrm{~mm} 3$ and $1.27 \times 1.27 \times 12 \mathrm{~mm} 3$. The 36 electronic channels of the SiPMs were multiplexed into 4 readout channels to simplify the readout electronics. The method of temperature compensation has been evaluated based on a single photon timing resolution with a single SiPM over a temperature range from $-15^{\circ} \mathrm{C}$ to $35^{\circ} \mathrm{C}$. The PET detector performance has been evaluated according to energy and timing resolution. Furthermore, the detector has been operated in a 7 T MRI to measure potential noise introduced by the PET and digital feedback electronics. All crystals could be resolved in the $15 \times 15$ configuration, whereas only $19 \times 19$ crystals could be resolved in the $20 \times 20$ configuration. The method of temperature compensation enabled a single photon timing resolution over a temperature range of $40^{\circ} \mathrm{C}$ with $300 \pm 8 \mathrm{ps}$ and furthermore a stable operation of the PET detector from $15^{\circ} \mathrm{C}$ to $35^{\circ} \mathrm{C}$ without any deformations in the crystal flood map. An overall energy resolution of $13.2 \pm 5.7 \%$ and a timing resolution of $1.65 \pm 0.69 \mathrm{~ns}$ have been achieved. The average energy resolution of the detector with $20 \times 20$ crystals was $21.0 \pm 3.0 \%$. The prototype detector with on-board temperature compensation enables a stable readout without any performance degradation between $15^{\circ} \mathrm{C}$ and $35^{\circ} \mathrm{C}$. First compatibility measurements of the detector operated in a 7 T MRI system showed no negative influences on the MRIs acquisition bandwidth. 
M04C-4: 3-D Position Sensitive CZT Positron Emission Tomography System: Current Status

$\underline{\text { S. Abbaszadeh, C. Levin }}$

Radiology, Stanford University, Stanford,CA, USA

We are developing a small animal PET system based on 3-D position-sensitive cadmium zinc telluride (CZT) detectors. The full system comprises four panels with an adjustable box-shaped field of view. This study presents results on two panels out of four panels of this system that are completed. Each panel consists of 24 CZT crystals, each with dimensions of $4 \mathrm{~cm} \times 4 \mathrm{~cm} \times 0.5 \mathrm{~cm}$, tightly stacked in an edge-on configuration to form CZT volume of $12 \mathrm{~cm} \times 4 \mathrm{~cm} \times 4 \mathrm{~cm}$. The CZT crystals have cross-strip electrodes pattern $(1 \mathrm{~mm}$ pitch anode strips with steering electrode strips on one face, and $5 \mathrm{~mm}$ pitch cathode strips on the other face). The cathodes, anodes, and steering electrodes' biases are $-500 \mathrm{~V}, 0 \mathrm{~V}$ and $-80 \mathrm{~V}$, respectively. The long-term stability of the current for CZT detectors was monitored over one year and it was shown that the leakage current of the detectors are very stable with a mean value of $270 \pm 20 \mathrm{nA}$ per CZT crystal. $68 \mathrm{Ge}$ and $137 \mathrm{Cs}$ radioactive isotopes were used to characterize the anode energy resolution. The mean measured global $511 \mathrm{keV}$ photopeak energy resolution over 902 anode channels of one panel was found to be $7.97 \pm 1.36 \%$ FWHM.

\section{M04C-5: Positron Emission Particle Tracking (PEPT) Extension to Hemodynamic Studies}

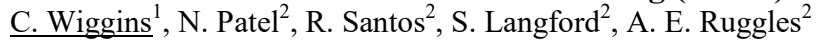

${ }^{I}$ Department of Physics and Astronomy, University of Tennessee-Knoxville, Knoxville, TN, United States

${ }^{2}$ Department of Nuclear Engineering, University of Tennessee-Knoxville, Knoxville, TN, United States

Positron Emission Particle Tracking (PEPT) is an established method, using positron-emitting radiotracers to examine flow and particulate behavior in engineering systems. Recent extensions in PEPT software are presented allowing many particles to be identified and tracked simultaneously. These extensions have facilitated Lagrangian specification of engineering flows using PEPT methods that are comparable to those obtained using high speed video particle tracking. PEPT data are presented for a pulsatile flow of frequency $1.77 \mathrm{~Hz}$ in an elastic tube with attributes typical of arterial hemodynamics. These data are collected using a Siemens Inveon scanner with energy window restricted to 425 to $625 \mathrm{keV}$ and external trigger to coordinate pulse cycle data collection. Radiotracer locations are established every millisecond during an 1800 second scan, and 948 particle traces are collected. The particle trace data are used to reconstruct 10 frames of spatiotemporal flow specification in a pinched-tube geometry. These data are offered in animation.

M04C-6: Evaluation of a Cross Strip Encoding DoI PET Detector F. P. Schmidt, A. Kolb, B. J. Pichler

Werner Siemens Imaging Center, University of Tubingen, Tubingen, Germany

This study evaluates a PET detector module for a dedicated breast PET insert for a clinical positron emission tomography and magnetic resonance imaging (PET/MRI) system. To guarantee a continuous spatial resolution over the field of view (FOV) the detector module uses a dual side readout for depth of interaction (DoI) information. The two detectors are made of silicon photomultipliers (SiPM) with a $25 \mu \mathrm{m}$ cell size arranged in a strip array. Further, a multiplexing approach offers a readout with four channels. Kolb et al. have previously shown that the prototype detector module performs well for pixelated scintillator blocks with a crystal pitch of $1.55 \mathrm{~mm}$. The aim of this study is to decrease the crystal pitch to $1.071 \mathrm{~mm}$ and thus enable a potential system with improved spatial resolution. Further, it is investigated if a light guide can compensate the saturation of the SiPM introduced by the reduced crystal size. The scintillator is a $18 \times 18$ pixelated LSO block with a length of $2 \mathrm{~mm}$ and a spectral reflector (ESR). Within this study it is demonstrated that the timing and energy characteristics show no significant degradation in comparison with the bigger crystal pitch block. However, the DoI resolution degrades from $3.05 \mathrm{~mm}$ to $6.93 \mathrm{~mm}$ for the setup with $1.55 \mathrm{~mm}$ crystal pitch and for the setup with $1.071 \mathrm{~mm}$ crystal pitch, respectively. The light guide leads to a small improvement of the DoI resolution from $6.93 \mathrm{~mm}$ to $6.06 \mathrm{~mm}$.

M04C-7: Compact, MR Compatible SiPM Small Animal PET DOI Detector

$\underline{\text { R. Chil }}^{1}$, G. Konstantinou ${ }^{1}$, M. Desco ${ }^{1,2}$, J. J. Vaquero ${ }^{1,2}$

${ }^{I}$ Universidad Carlos III de Madrid, Leganés, Madrid, España

${ }^{2}$ Instituto de Investigacion Sanitaria Gregorio Maranonn, Madrid, Spain

We introduce a compact preclinical PET detector that combines the output from 64 SiPM channels, forming an 8x8 matrix of pixels capable of efficiently encoding event position, with good energy and time resolution, as well as depth of interaction information. The scintillator matrices used to characterize the detector are a $16 \times 16$ matrix comprised of $1.3 \times 1.3 \times 12 \mathrm{~mm} 3 \mathrm{LYSO}$ crystals, and a $1.3 \times 1.3 \times 15 \mathrm{~mm} 3 \mathrm{GSO} / \mathrm{LYSO}$ phoswhich. Preliminary results regarding spatial resolution show that the detector has a resolvability index of 0.25 , an energy resolution below 14 for the $511 \mathrm{KeV}$ peak and a good separation between the two different phoswich crystal layers. Due to the compactness and MR compatibility of this detector it is proposed as a candidate to 
substitute those based on the R8900 PS-PMT in a full ring system that could be inserted into the MR bore of a preclinical PETMR imager.

\author{
M04C-8: New DOI Encoding Method with Single Layer Scintillator Array Using Depth-Dependent Reflector \\ Patterns

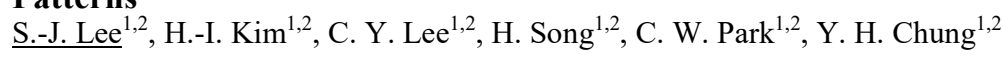 \\ ${ }^{I}$ Department of Radiological Science, College of Health Science, Yonsei university, Wonju, Gangwon-do, Korea \\ ${ }^{2}$ Institute of Health Science, Yonsei University, Wonju, Gangwon-do, Korea
}

We designed a depth of interaction (DOI) PET detector using depth-dependent reflector patterns in single layer scintillator array. By configuring the different reflector pattern in depth, light distribution of each depth can be altered. To evaluate the characteristics of DOI capability, we simulated the optical photon transport in the crystal array using DETECT2000. The crystal array consists of $6 \times 6$ array of $2 \mathrm{~mm} \times 2 \mathrm{~mm} \times 20 \mathrm{~mm}$ LSO crystals and 4 × 4 array of $3 \mathrm{~mm} \times 3 \mathrm{~mm} \mathrm{SiPMs}$. The $511 \mathrm{keV}$ gamma ray interactions were generated on the all depths and the flood images were acquired. The triangle and hexagonal shaped crystal arrays were also simulated. It was confirmed that DOI layers were distinguished in the obtained images. We verified that the number of DOI layers can be determined depends on the number of crystal sides.

M04C-9: IMIC - Needle-Shaped Low-Power Monolithic Active Pixel Sensors for Molecular Neuroimaging on Awake and Freely Moving Rats.

$\underline{\text { J. Heymes }}^{1,2}$, L. Ammour ${ }^{3}$, M. Bautista ${ }^{4}$, G. Bertolone ${ }^{1,2}$, S. Fieux ${ }^{5}$, F. Gensolen ${ }^{4}$, M. Goffe ${ }^{1,2}$, F. Guezzi-Messaoud ${ }^{1,2}$, C. Hu-

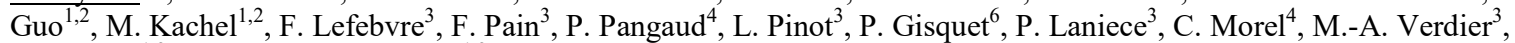
M. Winter ${ }^{1,2}$, L. Zimmer ${ }^{5}$, J. Baudot ${ }^{1,2}$

${ }^{I}$ Université de Strasbourg, IPHC, 23 rue Becquerel 67037 Strasbourg, France

${ }^{2}$ CNRS, UMR7178, 67037 Strasbourg, France

${ }^{3} I M N C, C N R S / I N 2 P 3$, Univ Paris-Sud/Paris Diderot, Orsay, France

${ }^{4} C P P M$, CNRS/IN2P3, Univ Aix-Marseille, Marseille, France

${ }^{5}$ LNRC, CNRS/INSERM, Univ Lyon 1, Lyon, France

${ }^{6}$ NeuroPSI CNRS/INSB, Univ Paris Sud, Orsay, France

IMIC A, B, and C circuits are Monolithic Active Pixel Sensors prototypes dedicated to local counting applications of the low energy Beta + rays, in the brain of an awake and freely-moving rat. Former experiments using the passive PIXSIC circuit validated the proof of concept with a need of improvement for signal to noise ratio, and spatial resolution. Each IMIC circuit is fabricated in a $180 \mathrm{~nm}$ CMOS Image Sensor Technology and features a matrix of $16 \mathrm{x} 128$ pixels, which are $30 \mathrm{x} 50 \mu \mathrm{m}^{2}$ large. The sensors have a needle-like aspect ratio $(610 \mu \mathrm{m}$ x $12000 \mu \mathrm{m})$. The chips were produced over two types of high-resistivity substrates: $18 \mu \mathrm{m}$ epitaxial layer, and $700 \mu \mathrm{m}$ Czochralski.

The foreseen application requires the high sensitivity to beta-rays while being immune to background gamma-rays. Another important constraint is the limited power dissipation in order to avoid perturbations in measurements caused by thermal-induced brain damage of the rat. IMIC A and C are fully analogue chips. In each pixel, the collecting diode is connected to a sourcefollower, which is read out using a rolling-shutter readout architecture. IMIC B is a fully-programmable digital sensor. The pixel design is based on the front-end architecture of the ALPIDE chip developed at CERN. However modifications have been made in order to store the information inside fired pixels between two readouts. The circuit is controlled through the SPI protocol, which allows for setting all the necessary polarization signals, sequencer parameters and selecting the operation mode.

The results of post-layout simulations show a high signal to noise ratio $(>40)$ and low power dissipation of $115 \mu \mathrm{W} / \mathrm{matrix}$ for IMIC B, and $280 \mu \mathrm{W} /$ matrix for IMIC A and C.

In this paper, we present a detailed design and main features of every IMIC version. We also give the functional test results along with the laboratory characterization measurements with X-rays and beta-rays.

\author{
M04C-10: Characterization of ?-eye: a Low Cost Bench Top Mouse Sized Gamma Camera for Dynamic and \\ Static Imaging Studies \\ $\underline{\text { M. Georgiou }}^{1}$, P. Papadimitroulas ${ }^{1}$, E. Fysikopoulos ${ }^{1}$, K. Mikropoulos ${ }^{1}$, G. Loudos $^{2}$ \\ ${ }^{I}$ BET Solutions, $R \& D$, Athens, Greece \\ ${ }^{2}$ Department of Biomedical Engineering, Technological Educational Institute of Athens, Athens, Greece
}

Small animal imaging, using nuclear medicine techniques, is a valuable tool in preclinical research. We present ?-eye: a new commercial, low cost system, suitable for scintigraphic small animal imaging. Design constraints include i) adoption to average user needs, ii) efficient performance, iii) minimization of dimensions and weight, iv) minimization of cost, v) user friendly environment and vi) robustness. The system is inspired by the facts that: a) many in vivo studies can be initially assessed with planar cameras; b) the purchase of a high cost system can be justified and exploited only by large centers; c) many groups can attract resources for a system that would cost 5 to 10 times less than the standard commercial ones and will give them the 
opportunity to shift from in vitro and ex vivo to in vivo. The ?-eye is a unique bench top system with $5 \mathrm{~cm} \times 10 \mathrm{~cm}$ field of view for whole-body mouse imaging. It provides static and fast dynamic images with user-selection time frame. We envisage that ?eye can be a low cost alternative to optical imaging for groups already working with radioactivity and will allow fast screening and initial evaluation of radiolabelled compounds.

M04C-11: A PET Block Detector Based on a 5x5 Array of 6x6 mm² SiPMs Read Out by TOFPET-ASIC

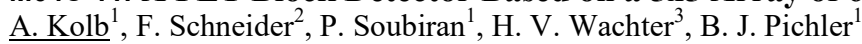

${ }^{I}$ Werner Siemens Imaging Center, University of Tubingen, Tubingen, Germany

${ }^{2}$ Ketek GmbH, Hoferstr. 3, Munich, Germany

${ }^{3}$ Department of Hybrid Electronic Systems, Technical University Munich, Munic, Germany

A complete detection system for a future PET (positron emission tomography) scanner is investigated. The PET block detector is based on a $5 \times 5$ array of $6 \times 6 \mathrm{~mm}^{2}$ KETEK PM6660 SiPMs (silicon photomultipliers) with $60 \mu \mathrm{m}$ cells. Their signals are digitized by the PETsys Electronics TOFPET-ASIC (time of flight PET application specific integrated circuit) and read out by the PETsys Electronics DAQ (data acquisition) system.

A $15 \times 15$ LSO (Lutetium Oxyorthosilicate) crystal block with an individual crystal size of $1.5 \times 1.5 \times 10 \mathrm{~mm}^{3}$ was attached to the 6x6 SiPM array by using a $2.7 \mathrm{~mm}$ light-guide, and coupled using optical grease. The light-guide was placed over 4x4 SiPMs, over which the crystal block is centered. To measure the time resolution of the block, an opposing KETEK PM3350 SiPM ( $3 \times 3$ $\mathrm{mm}^{2}, 50 \mu \mathrm{m}$ cells), coupled to a $3 \times 3 \times 5 \mathrm{~mm}^{3}$ LYSO crystal, was used. All measurements have been performed at a bias voltage of 31.0 V. A ${ }^{22} \mathrm{Na}$ point-like source $(970 \mathrm{kBq})$ was used for all measurements. From the computed flood maps, all of the crystals in the $15 \times 15$ array can be clearly identified. The average energy resolution computed over all crystals is $11.4 \pm 0.4 \%$ FWHM (not corrected for non-linearity). A line profile through the crystal flood map indicates a maximum and minimum peak to valley ratio of 4.2 and 2.1, respectively. The overall FWHM timing resolution of the block is $1066 \mathrm{ps}$. The investigated PET detector with the combination of SiPMs and the PETsys Electronics DAQ system could be a potential candidate for the use in a novel PET box design and will be further investigated.

\author{
M04C-12: Small Animal PET Insertable to 9.4T Preclinical MRI: Performance Evaluation and Simultaneous \\ Small Animal Imaging

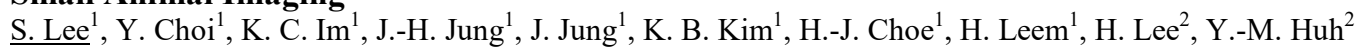 \\ ${ }^{I}$ Molecular Imaging Research \& Education (MiRe) Laboratory, Department of Electronic Engineering, Sogang University, \\ Seoul, South Korea \\ ${ }^{2}$ Department of Radiology, College of Medicine, Yonsei University, Seoul, South Korea
}

We have developed a light-weight small animal PET insert utilizing high resolution event positioning circuit having 144-to-4 multiplexing ratio and charge transmission method using $4 \mathrm{~m}$ long cable. The purpose of this study was to evaluate the performance and to demonstrate the imaging capability of the small animal PET insertable to 9.4T preclinical MRI. The PET insert consisted of 18 detector blocks comprising $65 \mathrm{~mm}$ TFOV and $41 \mathrm{~mm}$ AFOV. The output signals from the PET detectors were transmitted to readout electronics using $4 \mathrm{~m}$ long cables to minimize mutual interference between PET and MRI by separately locating PET detector and subsequent electronics. The PET gantry having only detector blocks was placed in MRI bore and RF coil was fitted inside the PET insert. The PET performance including energy, time, spatial resolution and sensitivity was measured in MRI. Interference between PET and MRI was assessed by measuring SNR and homogeneity of MRI and flood map of PET with simultaneous imaging setup. The simultaneous images of mice injected with 7-10 MBq [18F]FDG or $18 \mathrm{~F}$ were obtained to demonstrate the imaging performance of small animal PET-MR system. Energy and time resolutions were $13.2 \%$ and $8.1 \mathrm{~ns}$, respectively. The spatial resolution at the center of TFOV was $1.1 \mathrm{~mm}$ and a peak sensitivity at the center of AFOV was $2.6 \%$. No considerable degradation of MRI performance was observed and SNR and homogeneity of MR image were only slightly degraded $5 \%$ and $3 \%$ by the inserted PET, respectively. The acquired simultaneous images demonstrated high quality tracer uptake patterns and high resolution anatomy of small experimental animals. The results of this study indicate that the small animal PET insert is capable of excellent performance and high quality small animal PET-MR imaging. Moreover, the small animal PET insert offers light-weight design with high MR compatibility and cost effective solution using PET readout circuit having high multiplexing ratio.

\author{
M04C-13: A Two-Step Depth-of-Interaction PET Detector Using a Stair-Pattern Reflector Arrangement: \\ Feasibility Study with LYSO Arrays \\ J.-W. Son ${ }^{1,2}$, M. S. Lee ${ }^{1,3}$, J. S. Lee $e^{1,2}$ \\ ${ }^{I}$ Department of Nuclear Medicine, Seoul National University, Seoul, South Korea \\ ${ }^{2}$ Department of Biomedical Sciences, Seoul National University, Seoul, South Korea \\ ${ }^{3}$ Interdisciplinary Programs in Radiation Applied Life Science, Seoul National University, Seoul, South Korea
}


In this study, we propose a depth-of-interaction (DOI) PET detector based on reflectors inserted between pixelated crystals and arranged in a stair-pattern with a relative offset of one crystal pitch in both $\mathrm{X}$ and $\mathrm{Y}$ directions between the upper and lower segments of scintillators for two-step DOI encoding. The main advantage of the proposed method is simplicity because DOI information is easily decoded from a histogram of estimated 2-D gamma-ray interaction positions or a flood map and data can be simply acquired by using single-ended readout systems. Ideally, two-step DOI detectors based on the proposed method can generate flood maps where 2-D peak positions are evenly distributed on the map, maximizing the accuracy of DOI identification of the two segments. As a proof-of-concept study, we acquired a flood map through Monte Carlo simulation and the peak position in each scintillator was divided into two clearly separated peaks, providing two-step DOI information. Then, we conducted experimental studies using two-step DOI arrays (upper and lower segments with 8 and $12 \mathrm{~mm}$ heights) of $5 \times 5$ LYSO crystals with the cross-section of $1.7 \times 1.7 \mathrm{~mm}^{2}$ with three different detector configurations: a single-layer unpolished LYSO array (detector \#1), a single-layer polished LYSO array (detector \#2), and a stacked two-layer polished LYSO array (detector \#3). The scintillator arrays were coupled to the H9500 multi-channel PMT (pixel pitch of $3 \mathrm{~mm}$ ) and data were acquired using labbuilt FPGA-based data acquisition system. The average peak-to-valley ratio and distance-to-width ratio were $1.85 \pm 0.29$ and $1.47 \pm 0.13$ for detector $\# 1$, and $3.67 \pm 2.46$ and $1.79 \pm 0.73$ for detector $\# 3$. In detector \#2, peaks separation in each scintillator was insufficient to obtain DOI positions. The results demonstrate that the proposed DOI encoding method is a promising candidate for two-step DOI PET detectors based on single-ended readout systems and DOI decoding from the flood map.

\author{
M04C-14: A 0.7 Mm Spatial Resolution All-Digital Animal PET System Using Improved Trans-PET \\ Electronics

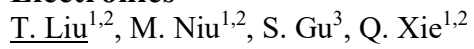 \\ ${ }^{1}$ Wuhan National Laboratory for Optoelectronics, Wuhan, China \\ ${ }^{2}$ Department of Biomedical Engineering, Huazhong University of Science and Technology, Wuhan, China \\ ${ }^{3}$ Raycan Technology Co., Ltd, Suzhou, China
}

Spatial resolution is one of the most important performance in PET system. In animal PET imaging, higher spatial resolution will give more information about the region of interest. In this work, we implemented a high resolution (approximate $0.7 \mathrm{~mm}$ ) all digital animal PET system using improved Trans-PET electronics. The improved basic detector module used has an overall energy resolution of $13.8 \%$ FWHM @ $511 \mathrm{keV}$ and a block-level coincidence timing resolution of 1.9 ns FWHM. The all digital system has got a micro-Derenzo phantom image which can resolve $0.7 \mathrm{~mm}$ diameter rods separated by $1.4 \mathrm{~mm}$.

This work was supported in part by the Natural Science Foundation of China (NSFC) Grant \#61425001, \#U1201256, \#61027006, in part by the National Key Scientific Instrument and Equipment Development Project of China \#2013YQ030923, in part by the Key Grant Project of Chinese Ministry of Education \#31302.

M04C-15: Firmware Architecture of the Data Acquisition System for the LabPET II Mouse Scanner

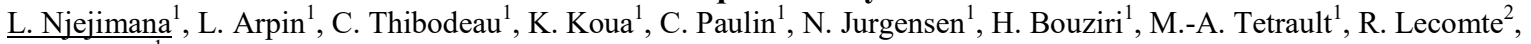
R. Fontaine ${ }^{1}$

${ }^{I}$ Institut Interdisciplinaire d'Innovation Technologique (3IT), Université de Sherbrooke, Sherbrooke, Québec, Canada ${ }^{2}$ Department of Nuclear Medicine and Radiobiology, Université de Sherbrooke, Sherbrooke, Québec, Canada

A new, highly pixelated, PET scanner dedicated to mouse imaging is being developed based on the LabPET II detector module. Each module includes four monolithic array of $4 \times 8$ avalanche photodiodes (APD) individually coupled to four $4 \times 8$ array of $1.12 \times 1.12 \mathrm{~mm}^{2}$ scintillator pixels aimed at achieving sub-mm spatial resolution. A PCB routes all APD to two 64-channel, mixed-signal, Application Specific Integrated Circuit (ASIC), forming a 128-channel readout unit. This paper focuses on the firmware developed to interface this novel detector module for a new mouse scanner with a geometry entailing a total of 6144 acquisition channels and an expected maximum of 3,000 events per channel. The firmware architecture consists of 2 types of custom FPGA-based electronic boards processing data from the detector module. An embedded signal processing unit based on an FPGA is in charge of detected data readout, corrections and sorting. It also features live energy histogramming and count rates computation. An FPGA-based coincidence, data communication and gating unit collects data from all embedded signal processing units and deals with coincidence detection together with random estimation and sends processed data to a host computer. In parallel to the detected events flow, a command and control flow was added to allow ASIC calibration, electronic boards parameters setting and readout from the host computer. This firmware allows a real-time processing of the expected $\sim 18$ $\mathrm{M}$ events without degrading the detector module performance. It is also easily reconfigurable to cope with a higher channels count.

M04D: Posters: simulation I

\author{
Wednesday, Nov. $2 \quad$ 16:30-18:30 Etoile \\ M04D-1: Does Energy Non-Linearity Affect Noise Estimates from Monte Carlo Simulations of X-Ray Imaging \\ Detectors?
}


$\underline{\text { A. Badano }}$

CDRH/OSEL/DIDSR, FDA, Silver Spring, MD, USA

Publicly available Monte Carlo simulation packages for light transport in scintillator-based x-ray imaging detectors utilize a linear yield model. However, scintillators are known to be non-linear in the lower end of the energy range. Is this assumption reasonable for $\mathrm{x}$-ray imaging simulations? We modified a freely available Monte Carlo package for modeling x-ray scintillators (mantis) to compare a linear versus a non-linear optical yield model. We report simulations in the diagnostic x-ray energy range for a CsI:Tl. To determine the effect of the light yield model on imaging performance, we calculated the distribution of signal outputs characterized by the information or Swank factor. We find that the choice of yield model plays a significant role in the outcome statistics increasing the intensity of lower energy peaks favored by the non-linear model. This observation is confirmed by the results for the Swank factor with larger values for the non-linear models for an increase with respect to the linear model results of 42 and $26 \%$ for a high-resolution and a high-light-output model respectively. Our findings indicate that the assumption of linear light yield in Monte Carlo simulations of imaging detectors might introduce a significant bias in the estimates of noise as expressed by the Swank factor. More research is needed to implement realistic non-linear yield models, to calculate the effect on realistic simulations including x-ray spectra of interest, and to experimentally validate these models.

\author{
M04D-2: A Simulation Study on Detection of a Cavity across a Therapeutic Carbon Beam Using Secondary \\ Electron Bremsstrahlung \\ M. Yamaguchi ${ }^{1}$, Y. Nagao ${ }^{1}$, T. Satoh ${ }^{1}$, T. Kamiya ${ }^{1}$, M. Sakai ${ }^{2}$, H. Sugai ${ }^{3}$, K. Arakawa ${ }^{1,2}$, N. Kawachi ${ }^{1}$ \\ ${ }^{I}$ Quantum Beam Science Research Directorate, National Institutes for Quantum and Radiological Science and Technology, \\ Takasaki, Gunma, Japan \\ ${ }^{2}$ Gunma University Heavy Ion Medical Center, Gunma University, Maebashi, Gunma, Japan \\ ${ }^{3}$ Fukushima Prefectural Centre for Environmental Creation, Miharu-machi, Fukushima, Japan
}

The purpose of this work was to study the feasibility of detection of a cavity across a therapeutic C-12 beam in a phantom by measuring low-energy (63-68 keV) secondary electron bremsstrahlung (SEB) emitted from a beam trajectory. Monte Carlo simulations were performed using the Particle and Heavy Ion Transport code System (PHITS). A C-12 beam (290 MeV/u) was injected on an acrylic target having an air cavity across the beam axis. To measure the depth dependence of the SEB intensity, a gamma camera consisting of a lead collimator having thirty-nine slits and a cadmium-telluride detector having thirty-nine elements was placed around the target. Both the slits and detector elements were arrayed along the beam axis and the position of the center of each slit was set to that of each detector element. Distributions of energy depositions in the detector elements were recorded. In addition, the distribution without SEB generation was calculated by performing a simulation again. These two simulations were repeated with the same setup other than without the cavity. We found that the yields having energy deposition of 63-68 keV with both the SEB generation and the air cavity have a valley in the cavity region, in contrast no valley appeared in the result without the cavity. The yields without SEB generation reached $40-50 \%$ of that with SEB generation and had no valleys. These results indicate that the SEB component can be utilized for monitoring of air cavities.

\title{
M04D-3: Complex 3D Printed Phantoms for Nuclear Medicine Applications
}

T. H. Farncombe

Radiology, McMaster University, Hamilton, ON, Canada

While many research projects in PET and SPECT utilize mathematical phantoms and simulations of the image systems, there is often a requirement to perform experimental imaging studies using radioactivity-containing phantoms. These phantoms normally consist of relatively simple geometric volumes (e.g., spheres) or simple anatomic structures (e.g., myocardium) due to manufacturing or filling complexities. While other methods have been developed to include more complex features, these methods are typically either 2D or still rely on fillable volumes, thus resulting in potentially time-consuming filling requirement with the potential for high radiation exposure. We present here an alternative technique to create complex $3 \mathrm{D}$ phantoms using desktop 3D stereolithography that directly incorporates the radioactive tracer within the print media. We have incorporated radioactive materials into photosensitive resins that are then used in a UV curable stereolithography system. The resulting phantoms are very high resolution (up to $0.025 \mathrm{~mm}$ feature size) and correspond very closely to modelled geometries. We have printed phantoms based on the Ultra Deluxe phantom for both PET and SPECT and have shown excellent agreement between the modelled geometry and the actual phantom. More complex phantoms based on animal CT scans (e.g., mouse skull) have also been created with excellent results. This technique allows for near perfect matching between simulated phantom geometries and experimental acquisitions and when used with long-lived radionuclides such as Co-57 or Ge-68, provides a long-term stable phantom for equipment quality control.

M04D-4: Simulating Functional Brain Images in Neurodegenerative Diseases

U. Vidal-Sanz, F. J. Martinez-Murcia, J. M. Górriz, J. Ramírez, I. A. Illán, F. Segovia, D. Salas-González

Dept. of Signal Theory, Networking and Communications, University of Granada, Granada, Spain 
Small sample size is a common issue in neuroimaging, where the number of features frequently exceed the number of samples by the thousands, thus reducing the statistical power of the analyses. This is an important issue that affects reproducibility, and therefore, a number of strategies to overcome this problem have been proposed. Whereas most of them focus on a refinement of the results by parsing out type I or II errors, or recruiting a larger population, in this work we propose an algorithm that generates a new set of functional images analog to a original set. The procedure involves modeling the original database with PCA, in order to project the original set to the eigenbrain space, and estimating the empirical statistical distribution of the projections.

Afterwards, new coordinates in the eigenbrain space can be obtained for each class using its probability distribution and a random number generator. The algorithm has been tested on a subset of the PET ADNI database, with a series of experiments that prove that the simulated images are independent from the original dataset and can effectively predict results in the real world, therefore they can be safely used to increase sample size, and even for educational purposes.

\title{
M04D-5: Geant4 Based X-Ray Tube Leakage Radiation Simulation for Developing Mobile Radiography
}

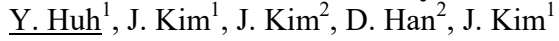

${ }^{I}$ CAE Group, Advanced R\&D Team, Health and Medical Equipment Business, Samsung Electronics Co., LTD., Suwon, South Korea

${ }^{2}$ DR R\&D Group, DR Business Team, Health and Medical Equipment Business, Samsung Electronics Co., LTD., Suwon, South Korea

In development a compact X-ray tube which is a major part of the mobile radiographic imaging apparatus, optimizing a shield against leakage radiation is significant because it effects on not only mobility of the apparatus but also additional radiation damage of the patient. In order to estimate the radiation leakage of the X-ray tube preliminarily, the Monte Carlo based simulator is a powerful method to estimate dose distributions of the X-ray and to verify the X-ray tube design. An X-ray tube model for mobile radiographic imaging was simplified by using a 3D modeling tool in this study. Tube's CAD models were imported into Geant 4 toolkit with a method which allows to the models to be directly loaded. To reduce simulation time, simulation tasks run in parallel modes and x-ray photons were emitted on the anode instead of electron beam flows. According to the IEC regulation, radiation leakage dose maps were acquired by each detector which was positioned in the left, right, upper, bottom, front and rear at 1 meter from the focal spot. To validate our simulation model, we compared with distributions influenced by the heel effect, emitted x-ray photons and electrons respectively and with dose values acquired with simulation and experimental results at 11 points. The simulation results showed a strong dependence on the shape, thickness and position of the lead shield blocks. Based on our preliminary results, the possibility of MC simulation tool for validation of radiation leakage of X-ray tube was proved. By using this method, the weight of X-ray tube can be optimized by adjusting the thickness and shape of shield structure.

\author{
M04D-6: An Improved Computational Method to Optimize the Stopping Power Calibration Curve for Patient- \\ Specific Proton Therapy Planning

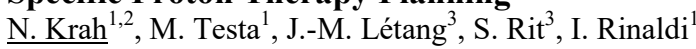 \\ ${ }^{I}$ Lyon University and CNRS/IN2P3, UMR 5822, Villeurbanne, France \\ ${ }^{2}$ Heidelberg Collaboratory for Image Processing, Heidelberg, Germany \\ ${ }^{3}$ Lyon University, INSA-Lyon, University Lyon 1, UJM-Saint Etienne, CNRS, Inserm, CREATIS UMR 5220, U1206, Lyon, France
}

We present an improved computational method to optimize the calibration curves to convert the Hounsfield Unit (HU) of the Xray planning computed tomography $(\mathrm{CT})$ to proton relative stopping powers (RSP) for patient-specific proton therapy treatment planning. The commonly used optimization method (Schneider et al. 2005 MP 32, Doolan et al. 2015 PMB 60) calculates a proton digitally reconstructed radiography (pDRR) by converting the HU of the X-ray planning CT into RSP and integrating along the line of beam penetration. The deviation of the $\mathrm{pDRR}$ from a measured proton radiography is minimized by optimizing the parametrization of the HU-RSP curve. This scenario assumes that all discrepancies between proton radiography and pDRR originate from uncertainties in the HU-RSP curve which is not satisfied if the pDRR is obtained by a simple projection along straight lines. Instead, multiple Coulomb scattering (MCS) and, in case of an active scanned beam delivery system, the finite beam profile lead to an imperfect representation of geometric structures ("blurring") in the measured proton radiography. We analyze these effects and propose to accurately model them to obtain an extended optimization method. We furthermore perform a thorough statistical treatment and propose an analytical formulation to determine the gained accuracy of the optimized HU-RSP curve. We demonstrate that without extending the optimization scheme, spatial blurring in the proton radiographies can cause up to $10 \%$ deviation between the optimized and the ground truth HU- RSP calibration curve. Instead, results obtained with our extended method reach $1 \%$ or better correspondence. Our contribution underlines the potential of a single proton radiography to generate a patient-specific calibration curve and to improve dose delivery by optimizing the HU-RSP

M04D-7: Impact of the Modelling of Charge Collection on the Simulation of SPECT Recordings from CZT Semiconductors Camera

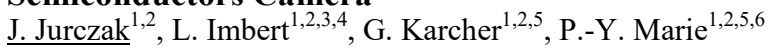


${ }^{I}$ Nancyclotep Molecular Imaging Platform, Nancy, FRANCE

${ }^{2}$ Department of Nuclear Medicine, CHRU Nancy, Nancy, FRANCE

${ }^{3}$ Institut de Cancérologie de Lorraine, Vandoeuvre-lès-Nancy, FRANCE

${ }^{4}$ University of Lorraine, CNRS UMR 7039, CRAN, Vandoeuvre-lès-Nancy, FRANCE

${ }^{5}$ Faculty of Medicine, University of Lorraine, Nancy, FRANCE

${ }^{6}$ U1116, INSERM, Nancy, FRANCE

Introduction: CZT cameras, such as the DSPECT one (Spectrum Dynamics $\left.{ }^{\circledR}\right)$, involve a principle of direct detection to convert ? photon into an electric signal. SPECT-recordings from the DSPECT camera were already simulated with the GATE platform [1], but without considering certain physical properties of semiconductors, in particular the partial collection of charges between anode and cathode leading to a low energy tailing effect. This study aimed to model this property by using the Hecht equation [2] putting in relation the amount of collected charge with the depth of interaction of the ? photon within the CZT crystal. Materials and methods: The Hecht equation was used to model the response of the 9216 pixels of the DSPECT camera. A comparison was planned between the energy spectra provided by simulated and actually recorded data from a source of $99 \mathrm{mTc}$ setting in the air or in a diffusing environment (water cylinder of a $20 \mathrm{~cm}$ diameter). Results: A good agreement was documented between the energy spectra from simulated and actually recorded data in the air as well as in the diffusing environment. An under-estimation was however documented for the low-energy photons obtained with the simulation, particularly in diffusing environment and in the lower part of the recording window of the $99 \mathrm{mTc}$ photopeak (relative decreases of $-7.5 \%$ in the air and of $-5.7 \%$ in the diffusing environment for the interactions simulated at $126 \mathrm{keV}$ and compared to actual data). Conclusion: The modelling of charge collection allows improving the Monte-Carlo simulation of SPECT-recording with energy spectra being close to those actually observed with CZT cameras. References: [1] L. Imbert, E. Galbrun, F. Odille, et al. "Assessment of a Monte-Carlo simulation of SPECT recordings from a new-generation heart-centric semiconductor camera: from point sources to human images", Phys. Med. Biol., vol. 60, pp. 1007-1018, 2015. [2] L. Chen, YX. Wei, "Monte Carlo simulations of the SNM spectra for CZT and NaI spectrometers", Appl. Radiat. Isot., vol.66, pp.1146-50, 2008.

\section{M04D-8: Imaging Device Functions in PTSIM for Irradiation Field Reconstruction in Particle Therapy}

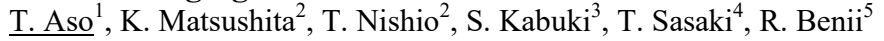

${ }^{I}$ Electronics and Computer Engineering, National Institute of Technology, Toyama College, Toyama, JAPAN

${ }^{2}$ Institute of Biomedical \& Health Sciences, Hiroshima University, Hiroshima, JAPAN

${ }^{3}$ Radiation Oncology, Tokai University, Kanagawa, JAPAN

${ }^{4} \mathrm{KEK}$, Computing Center, Ibaraki, JAPAN

${ }^{5}$ Advanced Course, National Institute of Technology, Toyama College, Toyama, JAPAN

PTSIM is a particle therapy system simulation framework based on Geant4 Monte Carlo simulation toolkit. It was originally developed as a reliable dose calculation tool to verify dose distributions inside patient and dose calculation algorithms in treatment planning systems. Besides such a reliable dose calculation, an irradiation field monitoring is desirable for delivering dose with more ideal distribution. It realizes a concept of a tailor made proton therapy which performs a specially optimized treatment for each patient. The irradiation field monitoring is performed by reconstructing images by detecting prompt gamma rays and annihilation gamma rays from produced radionuclides by nuclear interactions. The PTSIM has an important role to identify the mutual relationship among dose and radionuclide distributions inside a patient, and reconstructed images from signals in imaging devices. We previously reported on the relevant extending functions in PTSIM such as a pencil beam scanning, a material mapping of CT images and a data output interface for a hospital information system, and proposed the software design for imaging systems. In this paper, we report on the development of imaging device functions in PTSIM according to the software design in the previous work. The implementation provides functional capabilities for a flexible scoring and a detector signal flow. Because of the modulated structure, these functions are handled by users through online commands. For example, a user can choose score quantities and specify a signal flow for emulating a detector response. These capabilities were demonstrated with a simple tubular detector for a flexible scoring function and with PET detectors for a signal flow function, respectively. The simulated results show the correlation among dose, radionuclides, and coincide signals in PET detectors.

This work was partly supported by a grant program, Development of Advanced Measurement and Analysis Systems (SENTAN) in Japan Agency for Medical Research and Development (AMED).

\section{M04D-9: Simulation of Nanoparticle-Mediated near-Infrared Thermal Therapy Using GATE} V. Cuplov ${ }^{1}$, F. Pain ${ }^{2}$, S. Jan ${ }^{1}$

${ }^{I}$ DRF/I2BM/SHFJ/IMIV, Commissariat à l'Energie Atomique, Orsay, France

${ }^{2}$ CNRS/IN2P3, Imagerie et Modélisation en Neurobiologie et Cancérologie, Orsay, France

Nanotechnology for biomedicine is a novel approach for cancer therapy which allows direct delivery of anticancer agents to tumors. An example of such therapies is the nanoparticle-mediated near-infrared hyperthermia treatment. In order to investigate 
the influence of nanoparticle properties on the spatial distribution of heat in the tumor and healthy tissues, simulations might be a great asset. The Geant4 Application for Emission Tomography (GATE) open-source simulation platform, based on the Geant4 toolkit, is currently widely used by the research community involved in molecular imaging, radiotherapy and optical imaging. We present an extension of GATE that can model nanoparticle-mediated hyperthermal therapy as well as simple heat diffusion in biological tissues. This new hybrid Monte-Carlo and analytical simulation module has been validated against experimental data that consisted of the NIR illumination of a common thermoplastic polymer phantom with specific optical and thermal properties. This new feature of GATE combined with optical imaging allows for the simulation of a theranostic scenario in which the patient is injected with theranostic nanosystems that can simultaneously deliver therapeutic (i.e. hyperthermia therapy) and imaging agents (i.e. fluorescence imaging). GATE is the first ever theranostics in-silico modeling platform.

M04D-10: Sensitivity and Spatial Resolution Simulation of a PET-Compton Insert Imaging System

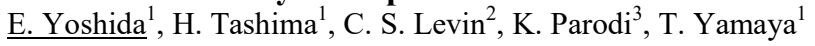

${ }^{I}$ National Institute of Radiological Sciences, Chiba, Japan

${ }^{2}$ Stanford School of Medicine, California, USA

${ }^{3}$ Ludwig-Maximilians Universität München, München, German

PET is the most sensitive imaging technique in the field of nuclear medicine. However, the single count rate is typically 10 times higher than the coincidence count rate. This means that there are a lot of pairs of undetected annihilation photons. For single gamma imaging, Compton imaging is known as the only method to obtain directional information of an incoming gamma ray without using collimators. Compton kinematics collimation suffers from poor angular resolution due to Doppler broadening, energy blurring, and position blurring. Therefore, Compton imaging is still challenging compared to PET imaging in general. However, non-coincidence single photons have the potential to contribute to improved imaging performance. Previously, we have proposed a system which combines PET and Compton imaging and we showed high sensitivity of this system using Compton kinematics collimation events. In this paper, we evaluate sensitivity and spatial resolution for feasibility of this system. This system modeled the PET ring and the inserted Compton scatter ring. The PET ring consisted of 48 DOI detectors arranged in five rings with a ring diameter of $80 \mathrm{~cm}$. The PET ring was made of GAGG with a size of $2.9 \times 2.9 \times 5 \mathrm{~mm}^{3}$ including 4-layered DOI capability. Ring diameters of the scatter ring were selected as 40, 50, 60 and $70 \mathrm{~cm}$. Detector materials of the scatter ring were $\mathrm{Si}$ with a pixel size of $0.5 \times 0.5 \times 6 \mathrm{~mm}^{3}$ or GAGG with a pixel size of $2.9 \times 2.9 \times 6 \mathrm{~mm}^{3}$. From point source measurements, images of PET and Compton events were individually reconstructed by the 3D-LM-OSEM algorithm. From point source measurements at the center of the field-of-view, spatial resolution with the Si scatter ring with $40 \mathrm{~cm}$ diameter was $5 \mathrm{~mm}$. Also, as the position of the point source was close to the detector, spatial resolution was improved. The proposed system had a potential of high spatial resolution for not only PET imaging but also Compton imaging with the small scatter ring.

M04D-11: A Monte Carlo Framework for Estimating Staff and Patient Dosimetric Quantities in Interventional Radiology Procedures

S. Higueret ${ }^{1,2}$, A.-M. Nourreddine ${ }^{1,2}$, T. Deschler $^{1,2,3}$, N. Arbor ${ }^{1,2}$, F. Carbillet ${ }^{3}$

${ }^{1}$ Université de Strasbourg, IPHC, Strasbourg, France

${ }^{2}$ CNRS, UMR7178, Strasbourg, France

${ }^{3}$ ALARA Expertise, Strasbourg, France

The practice of interventional radiology procedures keeps increasing since the last two decades. This X-ray guided medical act is source of exposure to ionizing radiation for the patient and the medical staff who stay near the source of radiation during the surgery. An efficient framework based on the Monte Carlo code dedicated to medical physics GATE v7.2 is presented. It relies on a precise modelization of the irradiation elements, patient and staff and on parameters determined through DICOM images of procedures. The joint use of a track length estimator variance reduction method and a new optimized dose calculation algorithm in voxelized geometry brings significant gain on computed dose uncertainty, up to a factor of 30 . This gain makes this framework execution time equivalent to widely used minimalist physics Monte Carlo frameworks and opens the way for its use on clinical practice.

\author{
M04D-12: Optimizing a Multi-Stage CdZnTe Compton Camera for Real-Time Proton Range Determination in \\ Proton Radiotherapy \\ L. Stothers, X. Hou, J. Tanguay, A. Celler \\ -Medical Imaging Research Group, -Department of Radiology, -University of British Columbia, Vancouver, BC, Canada
}

External beam radiotherapy using high-energy protons has the potential to precisely deliver high radiation dose to target tumour volumes. With the aim of improving proton therapy, a multi-stage CZT Compton camera was designed and characterized for real-time proton range determination. Monte Carlo simulation software GATE V7.1 was used to both investigate the prompt gamma energy spectra produced by a $150 \mathrm{MeV}$ proton beam in Lucite, bone and muscle, and to optimize the CZT Compton camera system efficiency. It was demonstrated that the prompt gamma energy spectra ranges from $1.0 \mathrm{MeV}$ to $10.0 \mathrm{MeV}$, and that 
a Compton camera system efficiency of $0.13 \% \pm 0.01 \%$ can be achieved, at these energies, using a triple CZT detector system with detector separations $=3.0 \mathrm{~cm}$ and with successive detector thicknesses $1.2 \mathrm{~cm}, 1.5 \mathrm{~cm}$, and $3.0 \mathrm{~cm}$, respectively. Assuming $1 \%$ energy and $1 \mathrm{~mm}$ spatial resolutions of the Compton camera system, a point source was reconstructed in air with a $1.1 \mathrm{~mm}$ spatial resolution using SOE reconstruction, and can be considered practical for clinical use.

Thank you NSERC and Redlen Technologies for their support of this research.

M04D-13: Analytic Modeling for Sensitivity of Tilted Pinhole Collimator

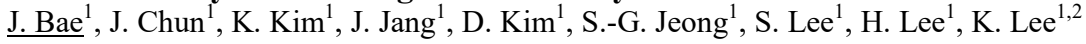

${ }^{I}$ Bio-convergence Engineering, Korea University, Seoul, Korea

${ }^{2}$ School of Biomedical Engineering, Korea University, Seoul, Korea

The major advantage of the tilted pinhole over the cylindrical shape is simple method of manufacturing. In this study, we have built analytic model of the tilted pinhole sensitivity for acquiring high uniformity gamma image. We approximate the tilted pinhole by modeling geometrical effect for low energy applicable system. The area of overlap between two ellipses was used for modeling the geometrical effect depending on the source location. Monte Carlo simulations were performed for evaluating the modeled pinhole sensitivity. The two types of pinhole considered in these simulations were a hole with a $0^{\circ}$ tilt and a hole with a $45^{\circ}$ tilt towards the center of the source. The Simulation results were compared with the results of our theoretical estimates.

Preliminary results suggest superior performance of ellipse based model. Therefore, the developed tilted pinhole collimator with cylindrical shape is suitable for applications in the low energy gamma source. In the future, we will continue to evaluate the various cases about the pinhole diameter, the source energy, the collimator thickness, the tilted angle, and the penetration effect.

Keywords : Pinhole collimator, analytic modeling, ellipse overlap area, collimator sensitivity

M04D-14: Advantages of Using MCNP6 Meshing Tools: Three Medical Cases Studies

B. Juste, S. Morató, R. Miró, G. Verdú

Polytechnic University of Valencia, Instituto de Seguridad Industrial, Radiofisica y Medioambiental (ISIRYM), Valencia, Spain

This work aims to improve the utility of Monte Carlo simulation within the clinical environment by developing techniques which enable faster and more accurate Monte Carlo simulation of radiotherapy geometries. This is achieved principally through the use new high performance computing tools. Los Alamos National Laboratory Monte Carlo N-Particle transport code (MCNP)

Version 6 (MCNP6) has been extended to include a new capability that permits tracking particles on an unstructured mesh that is embedded as a mesh universe within its constructive solid geometry capability. The mesh geometry of a Varian Clinac was created through Abaqus/CAE using its solid modeling capabilities. This model defined in detail, has been subsequently used in various applications with medical interests: - Spectrum reconstruction: Monte Carlo simulation of LinAcs depends on the accurate description of the spectrum beam and this data is not easily available to researchers. Instead, companies distribute phasespace files beams tallied at a plane located just upstream of the jaws. This works, uses the meshed model to unfold the initial spectrum from experimental depth dose curves and adapt the initial beam parameters to match measured dose curves. - Study the generation of photoneutrons and induced activity: The model has also included the transport of photoneutrons generated by primary photons and the $(n, ?)$ reactions, which result in activation of some isotopes. - Planning treatment tool: We have coupled an anthropomorphic 3D phantom model generated with MCNP mesh tools from a CT segmentation to simulate a treatment during LinAc irradiation, obtaining curve doses inside .

This work presents the advantages of using MCNP6 meshing tools by showing the good agreement of our results in these three LinAc cases studies.

The authors wish to thank the Hospital Universitari la Fe de Valencia for their kind support in doing this work.

M04D-15: Monte Carlo Simulation of DNA Damage Clustering Induced by a 24 MeV Proton Beam

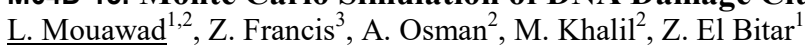

${ }^{I}$ Institut Pluridisciplinaire Hubert Curien, University of Strasbourg, Strasbourg, France

${ }^{2}$ Azm Center for Scientific Research, Lebanese University, Tripoli, Lebanon

${ }^{3}$ Dept. of Physics, Saint Joseph University, Beirut, Lebanon

Ionizing radiation induced DNA damage has been the subject of extensive research in the past twenty years. Monte Carlo simulations have provided a powerful tool to investigate the nature and frequency of DNA strand breaks as well as the relative biological effectiveness for double strand break induction as a function of particle energy and linear energy transfer. However, not much research has been dedicated to comparing the calculated microscopic DNA damage distributions to the macroscopic dose-depth distributions especially for proton beams. This work directly addresses this subject questioning the validity of 
absorbed-dose-based treatment planning and highlighting the need to incorporate DNA damage estimation in treatment plans. For that purpose, Monte Carlo simulations using the Geant4 toolkit with the Geant4-DNA processes are performed to compare the dose-depth distribution in a macroscopic water volume to the induced strand break yields in a microscopic water volume. A recently developed version of the Density Based Spatial Clustering for Applications with Noise algorithm adapted for DNA damage clustering calculations is used to group the events obtained with the simulations into clusters and calculate strand break yields. A computing grid is also used to reduce computing time for the large number of events extracted from the simulations. The results support the importance of using parameters directly linked to DNA damage in treatment planning to avoid the overestimation or underestimation of the actual damage that is caused at the DNA level.

Keywords - DNA damage clustering, DBSCAN, Monte Carlo, Geant4-DNA, proton therapy.

This work was done in the course of a thesis financed by "Institut de Recherche pour le développement" and "Institut National de Physique Nucléaire et de Physique des Particules". The authors also thank the International Project of Scientific Cooperation (Pics 6509) between the French and Lebanese CNRS, the Lebanese University and the Saint Joseph University.

M04D-16: Radioactive Ion Beam Studies Using FLUKA for Hadrontherapy Applications

$\underline{\text { R. S. Augusto }}^{1,2}$, A. Ferrari ${ }^{2}$, P. G. Ortega ${ }^{2,3}$, K. Parodi ${ }^{1}$, T. Tessonnier ${ }^{1,4}$

${ }^{I}$ Faculty of Physics, LMU Munich - Ludwig-Maximilians-Universität München, Munich, Germany

${ }^{2}$ EN/STI, CERN (European Organization for Nuclear Research), Geneva, Switzerland

${ }^{3}$ IFIC - Instituto de Física Corpuscular, University of Valencia, Valencia, Spain

${ }^{4}$ Heidelberg University Hospital, University of Heidelberg, Heidelberg, Germany

Hadrontherapy exploits charged hadrons advantages of a more localized dose delivery of higher biological effectiveness, making it ideal for radio-resistant, deep-seated tumors, otherwise untreatable by conventional methods.

However, in order to fully exploit the highly precise energy deposition of charged hadron beams, the accuracy of the dose delivery must be carefully monitored. Among other possibilities, this could be achieved with the use of PET instrumentation technologies.

The Monte Carlo particle transport code FLUKA was used to investigate the gain in imaging signal resulting from the possible use of $\beta^{+}$radioactive ion beams, while ensuring comparable dosimetric performance, with respect to stable ion beams.

First investigations confirm the higher imaging potential of radioactive beams, without additional absorbed dose drawback from the ensuing fragmentation. Also, it has been seen that reliable verification of the dose delivered could be achieved, along with an improvement of imaging quality, both during irradiation and afterwards.

\section{M04D-17: GGEMS: GPU GEant4-Based Monte Carlo Simulation Platform}

J. Bert, Y. Lemaréchal, D. Benoit, M.-P. Garcia, D. Visvikis

CHRU Brest - LaTIM - INSERM UMR1101, Brest, France

Monte Carlo Simulations (MCS) are associated with long execution times, which is one of the major issues preventing their use in routine clinical practice for both imaging and dosimetry applications. Recently, graphics processing units (GPU) have become in many different domains, especially in medical physics, a low cost alternative solution for the acquisition of high computation power. Within this context different Monte Carlo simulation codes have been used and implemented on GPU, targeting specific applications. Most of them are focused on dose calculation, and few of them on CT imaging applications. Each of these codes has different implementation strategies.

Although, on standard CPU programming several Monte Carlo frameworks gather a large spectrum of applications such as for example GATE, there is today no equivalent in GPU programming. The objective of this work was to develop a unique solution in terms of flexibility and coverage for both therapy and imaging applications. The proposed efficient MCS toolkit for GPU architectures named GGEMS (GPU GEant4-based Monte carlo Simulation) was based on the well-validated Geant4 toolkit. Among the different possibilities offered by GGEMS in terms of simulations two applications were considered in this study: one in $\mathrm{MeV}$ dosimetry and another in transmission imaging. GGEMS simulation results have shown a very good agreement against corresponding GATE/Geant4 simulations with substantial computational efficiency gains. The proposed platform has shown impressive capability to perform very fast and versatile MCS using GPU architectures.

M04D-18: Linac Modeling for External Beam Radiotherapy Quality Assurance Using a Dedicated 2D Pixelated Detector

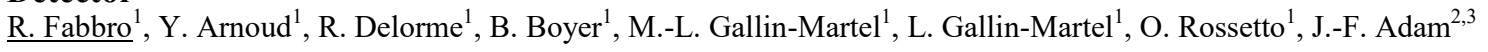

${ }^{I}$ Medical Application Physics, LPSC, Université Grenoble-Alpes, CNRS/IN2P3, Grenoble, France

${ }^{2}$ Equipe d'accueil Rayonnement Synchrotron et Recherche Médicale, Université Grenoble Alpes, ID17 European Synchrotron Radiation Facility, Grenoble, France

${ }^{3}$ Centre hospitalier universitaire de Grenoble, Grenoble, France 
Quality assurance is a key issue in intensity modulated radiotherapy. Errors can occur in the dose delivery process induces significant differences between the planned treatment and the delivered one. In this context, the Medical Application Physics group of the LPSC is developing TraDeRa (Transparent Detector for Radiotherapy), a 2D pixelated matrix of ionization chambers located upstream to the patient. The signal map obtained with TraDeRa has to be processed to provide medical observables to quantify the quality of the treatment delivery. This relies on accurate Monte Carlo simulations benchmarked with measurements performed under a linear accelerator (Linac). The work described in this paper lies in the optimization of the Linac head simulation and the development of an innovative Monte Carlo/measurements comparison method to perform an accurate enough model of the X-ray production device. An optimized parametrization of the particles transport allowed an increase of the simulation efficiency by a factor 3 . The characteristics of an electron beam of a reference Linac were matched with the simulation results by using dose deposition of the created X-ray beam in a water tank. Two parameters are particularly critical: the nominal energy of the electrons and the radial distribution of impact on the target. The innovative method was able to provide within minutes those two parameters for any Linac, achieving, for example, a $10 \mathrm{keV}$ precision on the energy determination for a 6 MV operating Linac.

This work was supported by the LABEX PRIMES (ANR-11-LABX-0063) of Université de Lyon, within the program "Investissements d'Avenir" (ANR-11-IDEX-0007) operated by the French National Research Agency (ANR).

\author{
M04D-19: A Compton Camera Prototype Simulation Study: Camera Performance and First Tests of Range \\ Monitoring Capabilities

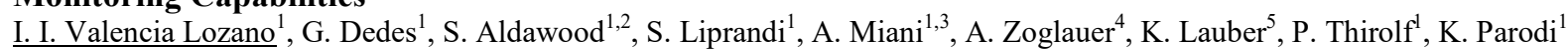 \\ ${ }^{I}$ Department of Medical Physics, Faculty of Physics, Ludwig-Maximilians-Universität München, Garching b. München, Bayern, \\ Germany \\ ${ }^{2}$ Department of Physics and Astronomy, King Saud University, Riyadh, Saudi Arabia \\ ${ }^{3}$ Department of Physics, Università degli Studi di Milano, Milan, ?Lombardy, Italy \\ ${ }^{4}$ Space Sciences Laboratory, University of California at Berkeley, Berkeley, California, USA \\ ${ }^{5}$ Department of Radiation Oncology, Ludwig-Maximilians-Universität München, München, Bayern, Germany
}

A Compton camera prototype is currently commissioned in the Department of Medical Physics in Garching. It is planned to be used for studying in-vivo proton range monitoring capabilities by means of prompt gamma rays. The latter are produced by the interaction of the proton or ion beam with the patient and, utilizing Compton kinematics, a prompt gamma based image correlated to the proton range can be reconstructed.

The camera consists of a monolithic $\mathrm{LaBr}_{3}(\mathrm{Ce})$ scintillation absorber crystal, read out by a 256-fold segmented multi-anode PMT and preceded by a stacked array of 6 double-sided silicon strip detectors acting as scatterers. Alongside the prototype commissioning, an intensive simulation/reconstruction work is being carried out in order to assess the design and performance of the constructed prototype under preclinical conditions.

In the current study, we have used the Geant4 [1] toolkit and the MEGAlib [2] software for simulating various prompt gamma sources, coupled with a realistic response of the Compton camera detectors, as well as for reconstructing prompt gamma images. We present the benchmarking of our prototype simulation against experimental data from laboratory photon sources and from clinical proton accelerator facilities. The impact on the reconstructed image of different detector effects, such as spatial and energy resolutions, are investigated.

Furthermore, we demonstrate range-monitoring studies of the prototype Compton camera, based on water phantom and mouse proton irradiation simulations in preparation of future application to preclinical investigations.

This work is supported by DFG Cluster of Excellence MAP (Munich-Centre for Advanced Photonics), by the International Max Planck Research School of Advanced Photon Science (IMPRS-APS) and by King Saud University.

[1] Agostinelli S. et al., "Geant4 - a simulation toolkit", Nucl. Instrum. Methods Phys. Res. A 506 250-303, 2003.

[2] Zoglauer A. et al., "MEGAlib The Medium Energy Gamma-ray Astronomy Library”, NewAR 50 (7-8), 2006.

\author{
M04D-20: An Anthropomorphic Phantom for Advance Image Processing of Realistic 18F-FDG PET-CT \\ Oncological Studies \\ $\underline{\text { F. Gallivanone }}^{1}$, M. Interlenghi ${ }^{1}$, D. D'Ambrosio ${ }^{2}$, D. Fantinato $^{2}$, L. Alberizzi ${ }^{3}$, G. Trifirò $^{3}$, I. Castiglioni ${ }^{1}$ \\ ${ }^{I}$ IBFM - CNR, Segrate (MI), Italy \\ ${ }^{2}$ Medical Physics Unit, IRCCS Fondazione S. Maugeri, Pavia, Italy \\ ${ }^{3}$ Nuclear Medicine Unit, IRCCS Fondazione S. Maugeri, Pavia, Italy
}

In recent years, great efforts have been devoted to develop advanced image processing methods for oncological PET/CT images (e.g. segmentation, quantification, texture analysis) able to extract from images tumor characteristics hidden at naked eye, e.g. intra-tumor phenotypic heterogeneity. In order to accurately estimate such imaging descriptors, image processing methods need to be validated on datasets closer to the real clinical conditions, e.g. including lesions described by functional signal of irregular spatial distribution and heterogeneous intensity. The aim of this work was to realize an experimental dataset of a torso 
anthropomorphic phantom suitable for the assessment of advanced image processing in 18F-FDG PET/CT oncological studies. The dataset consists into several 18F-FDG PET/CT measurements of the RSD Alderson Thorax phantom including 42 lesions of irregular shape, different volumes $(0.8-11.3 \mathrm{cc})$, and heterogeneous $18 \mathrm{~F}-\mathrm{FDG}$ uptake (L/BGS 3-40). Simulation of real oncological lesions was obtained deriving the shape of the lesions from 18F-FDG PET/CT images of real patient lesions and printing 3D shells by a 3D printer. Radioactive gels consisting in a fast-setting alginate powder at different concentration allowed the simulation of heterogeneous uptake within the shells. The lesions were inserted in the RSD Alderson Thorax phantom, in the thorax and breast compartments, and measured by a current generation PET-CT system. Such phantom image set could be downloaded from our Institutional web site and used by researchers for validation purposes.

\section{M04D-21: GamSim - a Windows Based Simulation Tool for Gamma Ray Detector Development} $\underline{\text { A. H. Walenta }}^{1}$, A. B. Brill ${ }^{2}$, T. Conka-Nurdan ${ }^{3}$, I. Fleck ${ }^{1}$, L. Furenlid ${ }^{4}$, T. E. Peterson ${ }^{5}$

${ }^{I}$ Physics Dept., University of Siegen, Siegen, Germany

${ }^{2}$ Radiology, Vanderbilt University, Nashville, USA

${ }^{3}$ Engineering, Turkish-German University, Istanbul, Turkey

${ }^{4}$ College of Optical Science, University of Arizona, Tucson, USA

${ }^{5}$ Radiological Science, Vanderbilt University, Nashville, USA

After the introduction of radiation simulation tools such as Geant4 or EGS in nuclear and high energy physics, the use in applied fields like nuclear medicine becomes increasingly important and a number of extensions have been introduced with particular focus on modeling PET and SPECT systems. Despite the very successful developments there are still a few fields where a different approach is needed: a very fast program that is easily configured in a windows environment allowing a detailed study of the essential properties of gamma ray detection with the emphasis on high flexibility for assessing a new detector design as well as techniques for improvement of position resolution, including depth of interaction determination or efficiency optimization. The presented software package GamSim is conceived to fulfill these requirements. In addition it is designed to test new imaging concepts such as the Compton Camera or detection of Cherenkov cones for gamma ray detection. For the latter task, to be discussed in more detail, it can be shown that a pattern recognition of the Cherenkov ring detection using a Hough transform can be easily implemented. The ease to optimize parameters leads to the conclusion that the concept could allow a depth of interaction measurement of $2 \mathrm{MeV}$ gamma rays with a precision better than $5 \mathrm{~mm}$.

M04E: Posters: signal processing I

Wednesday, Nov. $2 \quad$ 16:30-18:30 Etoile

M04E-1: A Multi-Channel High-Precision CMOS Time-to-Digital Converter for Medical Imaging Systems X. Fang, R. Sefri, F. Boisson, D. Brasse

iphc in $2 p 3$ cnrs, Strasbourg, France

A Multi-channel high-precision CMOS time-to-digital converter integrated circuit has been designed. Time interval measurement is based on a counter and two-level interpolation realized with stabilized delay lines. Three-level conversion structure enables first the use of a low frequency reference clock $(80 \mathrm{MHz})$ and a large dynamic measurement range with a high resolution. The counter and the DLLs (delay locked loop) in the two-level interpolation are common for all channels in order to well adapt to multi-channel applications. The third-level interpolation is based on a Vernier method to bypass the technology limitation. The key benefits of the proposed TDC are a reduction of the conversion jitter and a better design flexibility to enhance matching performances of delay cells. This approach reaches high conversion linearity regardless of the process technology. A prototype of 16 channels has been implemented in $0.35 \mu \mathrm{m}$ CMOS technology. Simulation results show that the TDC achieves a bin size of $24.4 \mathrm{ps}$ rms in a long $3.2 \mu$ s timing window. The differential and integral nonlinearities (DNL and INL) are 0.03 LSB and 0.26 LSB with a standard deviation of 0.08 LSB and 0.19 LSB respectively. Such a TDC will find useful applications in tomographic imaging systems, where several time-correlated single photon counting channels need to be used in a massively parallel configuration.

M04E-2: Accuracy of PET Partial Volume Correction in Tracer Uptake Measurements with Six Isotopes J. J. Hamill $^{1}$, A. T. Sjoeholm ${ }^{2}$, D. W. Townsend ${ }^{2}$, M. Conti $^{1}$

${ }^{I}$ Molecular Imaging, Siemens Healthcare, Knoxville, TN, USA

${ }^{2} A * S T A R-N U S$ Clinical Imaging Research Center, Singapore, Singapore

Aim. Soderlund et al. recently published a sphere-phantom study with six PET tracers, concluding that image quality was good but tracer concentration measurements based on mean uptake (A-mean) were strongly affected by partial volume errors (PVE). In this work we ask if a previously published PVE correction method (A-corr) is more accurate.

Methods. The A-corr method, described in 2002 by Hickeson and colleagues, may be appropriate in applications like lung-nodule $\mathrm{PET} / \mathrm{CT}$ when isolated hot structures, perhaps lung nodules, sit in a region of approximately uniform background, and the 
object's volume (V) is known from CT. A volume of interest in the PET image (VOI) is defined around the object. Background is estimated from voxels adjacent to the VOI. Tracer concentration (A-corr) in $\mathrm{Bq} / \mathrm{ml}$ is set to the background-corrected VOI total, divided by $\mathrm{V}$. We have shown that A-corr is only weakly affected by motion in the PET scan. Hot spheres had diameters 10,13 , 17 and $22 \mathrm{~mm}$. Cold spheres had diameters 28 and $37 \mathrm{~mm}$. The phantom was filled with positron-emitting 18F, 11C, 89Zr, 124I, $68 \mathrm{Ga}$, and $90 \mathrm{Y}$ activity with a sphere to background ratio of approximately $4: 1$. TOF PET data from a Siemens mCT PET/CT scanner were reconstructed with the TOF-PSF method. Using the A-mean and A-corr methods, we calculated contrast recovery (CR), defined by NEMA NU 2-2007, for hot and cold spheres.

Results. In hot spheres $13 \mathrm{~mm}$ or more in diameter, CR based on A-corr was between $90 \%$ and $105 \%$ for all but one measurement with $18 \mathrm{~F}, 11 \mathrm{C}, 89 \mathrm{Zr}, 124 \mathrm{I}, 68 \mathrm{Ga}$. In the $10 \mathrm{~mm}$ sphere, CR was $82 \%$ on average. In these spheres and these isotopes, CR based on A-mean varied between 0.3 and 0.8 . In the cold spheres, recovery based on A-corr was between 0.7 and 1.0 for these isotopes. Reconstructions of $90 \mathrm{Y}$ were less accurate.

Conclusions. In this phantom study with substantial PVE, A-corr improved contrast recovery measurements, compared to Amean.

M04E-3: A Constrained Feature-based Cardiac Motion Estimation Method for Cardiac PET

J. Wang, T. Feng, J. Xu, B. Tsui

Radiology, Johns Hopkins University, Baltimore, MD, USA

The goal is to develop and evaluate a new constrained feature-based cardiac motion estimation (ME) method for cardiac gated (CG) myocardial perfusion (MP) PET images to improve the accuracy of the estimated cardiac motion vector field (MVF). CGMP PET projection data were generated from the 4D XCAT phantom with realistic anatomical structures and cardiac MVF models, and reconstructed using the STIR simulation and reconstruction software. The interventricular sulcus (IS) was extracted from each CG-MP PET image by applying B-spline extrapolation and interpolation methods to the extracted edges of the left and right ventricular walls. The estimated MVFs of the extracted ISs were calculated between adjacent CG frames. In the previously feature-based cardiac ME method, the IS motion was used in combination with a cardiac motion model to create an initial estimate for the conventional optical-flow ME algorithm. The information was found to reduce the aperture problem effect and provide more accurate cardiac MVF estimate as compared to without the information. In the new algorithm, it was used as an additional constraint to restrict the range of the search for the cardiac MVF estimate. The new approach was evaluated in terms of accuracy of the estimated cardiac MVF and compared with those using the previous methods. The evaluation results showed the estimated cardiac MVF obtained from using the IS as an initial estimate (S-initial) was more accurate than that using no initial estimate (0-initial) and was comparable to that using the truth MVF as the initial estimate (T-initial). The estimation accuracy was further improved with the S-initial and the IS motion as an additional constraint. We developed and evaluated a new constrained feature-based cardiac ME method for cardiac PET. We demonstrated the new method provided more accurate estimation of the cardiac MVF as compared to the conventional and a previously developed feature-based cardiac ME methods for CG-MP PET.

M04E-4: Contrast-Oriented Seed Based Automatic Segmentation Algorithm: Minimizing Effect of Lesion Heterogeneity on Algorithm Response. M. Carles-Fariña ${ }^{1,2}$, T. Fechter ${ }^{1}$, U. Nestle $^{1}$, A. Schaefer ${ }^{3}$

${ }^{I}$ Deparment of Radiation Oncology, University Hospital Freiburg, Freiburg, Germany

${ }^{2}$ Department of Nuclear Medicine, University Hopital La Fe, Valencia, Spain

${ }^{3}$ Department of Nuclear Medicine, Saarland University Medical Center, Homburg, Germany

The primary aim of this work was to evaluate the impact of different parameters on the performance of a contras oriented (COA) seed-based segmentation algorithm for tumor delineation on PET image. In addition, an extended version of the algorithm (2I-A), accounting for lesion heterogeneity, was proposed and preliminarily evaluated. 80 4D-PET images, corresponding to 8 lung cancer patients (10 time-bins/patient), were employed for the analysis. Consensus of three visual PET contours by experts was established as the ground truth surrogate (GTS). The accuracy of the segmented volume by COA was evaluated with respect to GTS using Dice Similarity Coefficient $\left(\mathrm{DSC}_{\mathrm{A}}\right.$ ). Variability among the experts was also assessed (DSC $\mathrm{E}_{\mathrm{E}}$ ). Increasing $\mathrm{DSC}_{\mathrm{A}} / \mathrm{DSC} \mathrm{E}_{\mathrm{E}}$ was employed as an indicator of increasing segmentation accuracy. Dependence of $\mathrm{DSC}_{\mathrm{A}} / \mathrm{DSC}_{\mathrm{E}}$ with respect to different parameters was investigated. $\mathrm{DSC}_{\mathrm{A}} / \mathrm{DSC}_{\mathrm{E}}$ showed linear correlation with respect to lesion activity concentration $\mathrm{A}_{\mathrm{T}}(\mathrm{r}=-$ 0.827, slope of $\mathrm{m}=-0.05)$ and its homogeneity $1 / \mathrm{COV}_{\mathrm{T}}(\mathrm{r}=0.750, \mathrm{~m}=0.22)$. Good volume segmentation accuracy was obtained on average for COA $\left(\mathrm{DSC}_{\mathrm{A}}=0.71 \pm 0.08 \mathrm{vs} \mathrm{DSC}_{\mathrm{E}}=0.78 \pm 0.07\right)$, but was further improved for 5 heterogeneous $(\mathrm{COV}>0.3)$ lesions by use of 2I-A: 2I-A algorithm diminished volume difference from $37 \pm 16$ to $16 \pm 8 \%$ and increased DSC from $0.69 \pm 0.08$ to $0.82 \pm 0.05$. From the results, lesion heterogeneity could be identified as the parameter with strongest effect on COA-algorithm accuracy. Although COA showed good accuracy, implementing a second algorithm iteration (2I-A) is recommended because 2IA further improves volume segmentation accuracy especially in lesions with high uptake heterogeneity.

\section{M04E-5: PETRA: a Web-Based System Supporting Computer Aided Diagnosis of Alzheimer's Disease}




\author{
F. Segovia ${ }^{1}$, I. A. Illán ${ }^{1}$, D. Salas-Gonzalez ${ }^{1}$, F. J. Martínez-Murcia ${ }^{1}$, A. Ortiz ${ }^{2}$, J. M. Górriz ${ }^{1}$, J. Ramírez $^{1}$

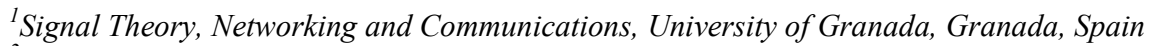 \\ ${ }^{2}$ Communications Engineering, University of Málaga, Málaga, Spain
}

This paper presents PETRA, a Web-based system to assist practitioners in the diagnosis of Alzheimer's Disease (AD).

Particularly, it provides with online tools to carry out multiple advanced analysis on T1 Magnetic Resonance Images (MRI) and Positron Emission Tomographies (PET). The key innovation behind PETRA is that it enables anywhere, anytime access to advanced neuroimaging tools, which previously required specialized machines with high computational resources. Specifically, PETRA allows to automatically segment tissue, white matter, grey matter and cerebro-spinal fluid; to visualize, manipulate and compare MRIs, PETs and already-segmented neuroimages; to manage large sets of images in a simple manner; and to perform an automatized pre-diagnosis through the application of different classification techniques. Apart from the Web interface, mobile applications for iOS and Android have been implemented, to allow receiving notifications in the mobile phones of the practitioners, and to even manage a large subset of the functionalities directly from the phone.

This work was partially supported by the MINECO under the TEC2015-64718-R project and the Consejería de Innovación, Ciencia y Empresa (Junta de Andalucía, Spain) under the P11-TIC-7103 Excellence Project.

\author{
M04E-6: Quantile-Based Classification of Alzheimer's Disease, Frontotemporal Dementia and Asymptomatic \\ Controls from SPECT Data \\ D. Geller ${ }^{1}$, G. Platsch ${ }^{2}$, J. Kornhuber ${ }^{3}$, T. Kuwert ${ }^{4}, \underline{\text { D. Merhof }}^{1}$ \\ ${ }^{1}$ Institute of Imaging \& Computer Vision, Aachen, Germany \\ ${ }^{2}$ Siemens Molecular Imaging EU, Erlangen, Germany \\ ${ }^{3}$ Department of Psychiatry and Psychotherapy, University of Erlangen-Nuremberg, Erlangen, Germany \\ ${ }^{4}$ Clinic of Nuclear Medicine, University of Erlangen-Nuremberg, Erlangen, Germany
}

Nuclear imaging techniques, namely single photon emission computed tomography (SPECT) and positron emission tomography (PET), are commonly used for the study of neurodegenerative diseases such as Alzheimer's disease (AD) and frontotemporal dementia (FTD). Many methods have been proposed to identify different types of dementia based on SPECT and PET images. In order to cope with the low number of datasets compared to the high amount of independent variables (voxels of the dataset), they either perform a dimensionality reduction prior to classification, which implies identical influence of all available datasets, or try to extract the strongest voters for the prediction, which may be affected by statistical fluctuation resulting from mislabeled data or intrinsic noise within data samples. In order to overcome these limitations, this paper presents an alternative method for classification of SPECT image data of asymptomatic controls (HC), AD and FTD participants. The proposed method produces a voxel mask that weights or ignores voxels according to their relevance for classification. The algorithm is based on quantiles and is less sensitive to the non-Gaussian statistical distribution of the classes to separate, which is a very desirable in case of dementia classification. Special care is taken to assess the robustness of the proposed approach. The classification accuracy assessed with bootstrap resampling is presented and the robustness against outliers and misdiagnosed training samples is investigated and compared with a PCA MVA based approach. As a result, the proposed approach shows comparable results with respect to robustness, but better classification accuracy compared to PCA-based approaches.

\title{
M04E-7: Joint Amplitude and Timing Estimation for Scintillation Pulses in GPU
}

M. Ruiz-Gonzalez, L. Caucci, L. Furenlid

Center for Gamma-ray Imaging, The University of Arizona, Tucson AZ, USA

We present an implementation with graphics processing units (GPUs) of a maximum-likelihood (ML) contracting-grid algorithm that performs joint estimation of amplitude and time of scintillation pulses. ML estimation consists in finding the parameters that maximizes the likelihood. We proposed a multivariate normal model for the likelihood, which implies that we need mean pulses and covariance matrices for every parameter variation. In a previous work, we performed a Fisher information analysis to determine the minimum number of samples that contains the most timing information in a digital pulse, for a given acquisition rate. Here, we use just the right amount of samples to reduce the size of mean pulses and covariance matrices. In order to reduce the number of precomputed mean pulses and covariance matrices, we take advantage of the linearity of scintillation pulses, which allows us to scale a normalized pulse to obtain mean pulses of any amplitude. We make use of this information to limit the amount of computation and shared memory used in our GPU code, while preserving full timing performance. We developed our code on a machine with 8 graphics cards, and with a single GPU we were able to process 60,000 events per second. The estimation algorithm and GPU implementation can be used in real-time for systems that require high temporal resolution such as time-of-flight positron emission tomography or in processes that have high gamma-ray count rate.

M04E-8: A High Performance DAQ System for a Free Hand Small-Field Gamma Camera M. Zioga ${ }^{1}$, M. Mikeli ${ }^{1}$, A.-N. Rapsomanikis ${ }^{1}$, E. Stiliaris ${ }^{1,2}$ 
${ }^{I}$ Department of Physics, National \& Kapodistrian University of Athens, Athens, Greece

${ }^{2}$ Institute of Accelerating Systems \& Applications (IASA), Athens, Greece

A high performance software application to control and to collect real time data from a free hand small-field gamma-Camera system developed in our laboratory is presented in this work. This data acquisition (DAQ) system is based on a LabVIEW platform and integrates a fast PCI Analog-to-Digital Converter (ADC) for the readout of the charge signals from a Position Sensitive Photo-Multiplier Tube in resistive chain, as well as basic functions of the Bluetooth protocol for wireless connection of commercially available position and gravitational sensors for the real-time movement reconstruction of the free hand gammaCamera. The recorded signals of these sensors are offline used in an event-by-event reconstruction to automatically correct the position of the detected gamma-ray photons and to produce clear diagnostic planar images of clinical value. The software architecture of this application straightforwardly allows a number of additions in the existing code and parameter modifications to allow its easy adaptation to other, similar movable imaging devices.

M04E-9: Multimodal MRI Radiomics in GBM: a Comparative Investigation of Feature Selection and Classification Techniques for Prognostic Models Including Robustness Assessment T. Upadhaya $^{1,2,3}$, Y. Morvan ${ }^{1}$, E. Stindel ${ }^{2}$, P. J. Le Reste ${ }^{4}$, M. Hatt ${ }^{2}$

${ }^{I}$ b-com Institute of Research and Technologies, Rennes, Bretagne, France

${ }^{2}$ LaTIM, INSERM, UMR 1101, Brest, Bretagne, France

${ }^{3}$ University of Western Brittany, Brest, Bretagne, France

${ }^{4}$ University Hospital Pontchaillou, Rennes, Bretagne, France

The new era of "Radiomics" (image-derived features) have shown potential in prognosis stratification to improve the clinical management of glioblastoma multiforme patients. The large amount of features involved calls for the use of robust machine learning techniques to identify and optimize the combination of the most relevant features with high prognostic value. Benchmarked machine learning techniques such as Support Vector Machines (SVM) and Guided Regularized Random Forest (GRRF) have been extensively exploited for building predictive models, resulting in accurate decision-making. The goal of the present work was to compare these feature selection and classification methods for building a prognostic model in GBM that would combine clinical and contextual features with radiomics features from multimodal MRI. The radiomics features were in addition evaluated for their robustness and models were built using either the entire set of features or by exploiting only the robust ones. SVM obtained a higher accuracy (93\% with all features and $84 \%$ using only the robust ones) compared to GRRF (74\% and $78 \%$ respectively). These results highlight the importance of the features selection and classifier techniques choices, as well as the impact of exploiting only robust features to build the model. Future work will consist in testing in an external validation dataset these models to investigate whether using robust features only improves their validation performance.

M04E-10: MRI Brain Segmentation Using Hidden Markov Random Fields with Alpha-Stable Distributions

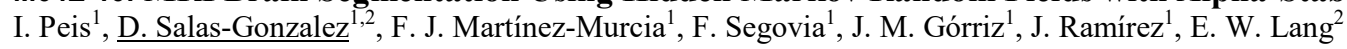

${ }^{1}$ University of Granada, Granada, Spain

${ }^{2}$ University of Regensburg, Regensburg, Germany

In this work, a MRI brain image segmentation method using a hidden Markov random fields with heavy-tailed alpha-stable distributions is presented. Each brain tissue is modelled using an alpha-stable distribution. Then, a HMRF is used to include spatial information in the classification model.

The Gaussian distribution has been widely used for the modelization of the cerebrospinal fluid, white matter and gray matter. Nevertheless, the alpha-stable distribution has been recently proposed as a more accurate alternative for this task. The alphastable distribution is more impulsive and is also able to model the asymmetry and heavy-tails of the histogram of the brain tissues.

We have tested the proposed methodology in $18 \mathrm{MR}$ images from the Internet Brain Segmentation Repository. The proposed methodology outperforms the segmentation results obtained when a Gaussian model for the histogram of the brain tissues is considered. Furthermore, as the Normal distribution is a particulaar case of alpha-stable distribution. Therefore, the proposed approach is also a generalization of the hidden Markov random field segmentation method with Gaussian distributions.

M04E-11: Sub-Regional Pattern Analysis of Heterogeneous PET Tracer Distribution Employed for Disease Assessment

I. S. Klyuzhin ${ }^{1}$, J. Fu$^{1}$, N. Shenkov ${ }^{1}$, A. Rahmim², V. Sossi ${ }^{1}$

${ }^{I}$ Department of Physics and Astronomy, University of British Columbia, Vancouver, BC, Canada

${ }^{2}$ Department of Radiology, Johns Hopkins University, Baltimore, MD, USA 
The mean value of the non-displaceable binding potential $\left(\mathrm{BP}_{\mathrm{ND}}\right)$ within a region of interest $(\mathrm{ROI})$ is the traditionally-employed metric in neurological image analysis. The ability of the mean value to accurately track clinical disease progression may be limited since it does not capture the spatial pattern of tracer distribution. In this work, we employ the principal component analysis (PCA) to quantify the clinically-relevant tracer binding patterns $\left(\left[{ }^{11} \mathrm{C}\right]\right.$ dihydrotetrabenazine $)$ in high-resolution PET images of 37 Parkinson's disease subjects. The principal component (PC) scores that correspond to different binding patterns in the putamen ROIs are combined with the mean $\mathrm{BP}_{\mathrm{ND}}$ and used as the input to several linear models that aim to predict the clinical severity of the disease (disease duration). Multiple regression analysis and LASSO (least absolute shrinkage and selection operator) with cross-validation are used to evaluate the contributions of the PC scores to the accuracy of the tested models. With multiple regression analysis, the value of the adjusted $\mathrm{R}^{2}$ was 0.57 when the mean $\mathrm{BP}_{\mathrm{ND}}$ alone was used as the model input. When the PC scores were included as additional input variables, the value of the adjusted $\mathrm{R}^{2}$ increased to 0.70 . The terms of the model representing the PC scores were statistically significant $(\mathrm{p}<0.01)$. In LASSO analysis, the cross-validated accuracy improved by $25 \%$ when the $\mathrm{PC}$ scores were added to the input (compared to using the mean $\mathrm{BP}_{\mathrm{ND}}$ alone). These results demonstrate that a) the disease- and tracer-specific binding patterns can be identified in sub-cortical brain structures from highresolution PET images, and b) such patterns may facilitate better models of the clinical disease metrics.

\section{M04E-12: Simultaneous Spatiotemporal Tracking of Multiple Positron Sources using Spectral Clustering} H. Li ${ }^{1}$, G. Pratx ${ }^{2}$

${ }^{I}$ Dept. of Electrical Engineering, Stanford University, Stanford, CA, United States

${ }^{2}$ Dept. of Radiation Oncology, Stanford University, Stanford, CA, United States

Tracking point-like objects in optically opaque environments has applications in studying flow in chemical systems, monitoring respiratory motion, and possibly investigating the trafficking of single cell at the whole-body level. One approach is to label the point-like object (particle, fiducial marker, or cell) with positron-emitting tracers. The information contained in the list-mode measurements can be utilized to directly track the motion of a single source, either frame-by-frame or continuously using a trajectory reconstruction algorithm. Applying this concept to multiple simultaneously moving sources is more challenging, in that detected pairs of annihilation photons from different sources are not directly distinguishable. To tackle this problem, we used spectral clustering methods to cluster the lines of response defined by the detected coincidence events, such that each individual source can be independently tracked with existing techniques. Using Monte Carlo simulations, we show that, in a small animal PET system, three static sources can be simultaneously localized with $0.5 \mathrm{~mm}$ accuracy using about 30 lines of response per source. Then, we show that $>95 \%$ clustering accuracy can be achieved for sources of both high and low radioactivity/velocity ratios. Finally, we acquired real data from 4 FDG drops (500Bq/drop) and demonstrated successful clustering. Compared to expectation-maximization approach, spectral clustering is advantageous because (1) it is easy to implement, (2) it can identify the number of objects from the data, and (3) it can handle complex cases through higher-order spectral clustering. This approach could have immediate applications in simultaneously tracking multiple point-like objects in both biological and non-biological settings.

This work was supported by NIH grant R21HL127900

M04E-13: Optimizing 4D-PET/CT Imaging for Heterogeneity Quantification by Texture Features

$\underline{\text { M. Carles }}{ }^{1}$, I. Torres-Espallardo ${ }^{1}$, U. Nestle ${ }^{2}$, L. Martí-Bonmatí ${ }^{1}$

${ }^{I}$ Department of Nuclear Medicine, Hospital Universitario y Politécnico La Fe, Valencia, Spain

${ }^{2}$ Department of Radiation Oncology, University Hospital Freiburg, Freiburg, Germany

The number of time-bins (TB) in retrospectively gated (4D-) PET/CT should be a trade-off between optimizing statistics and minimizing motion effect. The aim of this work was to investigate the impact of TB in heterogeneity quantification by PET texture features (TF) for lesions affected by respiratory motion. Five heterogeneous lesions were simulated by a mixture of radiocompound and alginate. Realistic 2D breathing pattern was applied to the phantoms. PET/CT acquisition (10 min/ $36 \mathrm{~s})$ was retrospectively gated using different TB (4, 6, 8 and 10). Static (S-) PET/CT acquisition (15 min/ $3.7 \mathrm{~s}$ ) was also performed when no movement was applied to the phantoms. Our analysis involved 11 metrics for each of the 5 heterogeneous phantoms (55 data series). Metrics were 3 conventional indexes and $8 \mathrm{TF}$. Impact of TB in PET quantification was evaluated in terms of variability along TB and linear correlation with respect to values derived from ideal contours on S-image, reference values $\left(\mathrm{M}_{\mathrm{ref}}\right)$. From the comparison between different $\mathrm{TB}$, significant worse variability was obtained for $\mathrm{TB}=10$ : coefficient of variance along $\mathrm{TB}$ averaged over the 11 metrics was $\mathrm{COV}_{\text {mean }}=16 \pm 13$ and $11 / 55$ data metrics did not follow a normal distribution along TB. Nonsignificant differences were observed for the variability obtained for $\mathrm{TB}=4,6$ and 8 . The strongest correlation with respect to $\mathrm{M}_{\text {ref }}$ was observed for $\mathrm{TB}=6$. From our results, $\mathrm{TB}<10$ should be preferred for PET quantification of lesions affected by respiratory movement. Although $\mathrm{TB}=6$ could be suggested as optimal $4 \mathrm{D}-\mathrm{PET} / \mathrm{CT}$, future validation with larger sample of heterogeneous inserts and movements is recommended.

M04E-15: Data Driven Respiratory Signal Detection in PET Taking Advantage of Time-of-Flight Data 


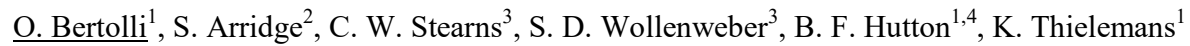

${ }^{I}$ Institute of Nuclear Medicine, University College London, London, United Kingdom

${ }^{2}$ Department of Computer Science, University College London, London, United Kingdom

${ }^{3}$ GE Healthcare, Waukesha, WI, USA

${ }^{4}$ Centre for Medical Radiation Physics, University of Wollongong, Wollongong, Australia

Respiratory gating is a powerful tool for tackling motion-related issues in chest PET imaging. On current scanners the required respiratory signal is obtained from external devices, whereas with Data-Driven (DD) methods (such as Principal Component Analysis, PCA) it can be extracted directly from the data. With the advent of TOF PET scanners, more spatial information has become directly available from the raw data and the performance of DD methods is expected to increase. The aim of this work is to show the increased potential of the application of PCA on TOF data. We propose a DD methodology that retains the TOF information and compare it to the non-TOF method. We unlist the TOF listmode data into 5 dimensional sinograms using $500 \mathrm{~ms}$ time frames, spatial downsampling and a determined number of TOF bins. PCA is then applied and a respiratory signal extracted. We tested the method on 16 FDG oncology patients acquired in 3D listmode on a GE Discovery 690 PET/CT scanner, monitored with an RPM camera. Data were unlisted into non-TOF sinograms, and into 5 or 11 TOF bins sinograms and PCA was applied. To further investigate the benefit of TOF, PCA was selectively applied to sets of TOF bins that correspond to areas equidistant from the center of the scanner. The correlation with the RPM, the level of noise and the respiratory-likeness of the traces were analysed. Using TOF sinograms, the correlation with RPM and the respiratory-likeness of the signals increase significantly compared to non-TOF, whereas the noise level decreased. The central TOF bins alone were able to provide good signals of similar quality to using all bins, whereas the ones corresponding to the external areas of the body provided signals with correlations close to zero and could effectively be disregarded. The application of PCA on TOF data showed promising results: retaining the TOF information into the sinograms considerably increased the quality of the extracted respiratory signals.

This work was supported by EPSRC Industrial CASE studentship (13220093), co-sponsored by GE Healthcare, and by the National Institute for Health Research, University College London Hospitals Biomedical Research Centre.

M04E-16: Comparison of three quantization methods for the calculation of textural features in PET/CT images: impact on prognostic models in Non-Small Cell Lung Cancer.

M.-C. Desseroit ${ }^{1}$, F. Tixier ${ }^{2,1}$, C. Cheze-le-rest ${ }^{2,1}$, R. Perdrisot ${ }^{2}$, R. Guillevin ${ }^{3}$, D. Visvikis ${ }^{1}$, M. Hatt ${ }^{1}$

${ }^{I}$ INSERM UMR 1101, Laboratoire de Traitement de l'Information Medicale (LaTIM), Brest, FRANCE

${ }^{2}$ DACTIM, Nuclear medecine department, University Hospital Milétrie, Poitiers, FRANCE

${ }^{3}$ Department of Radiology, University Hospital Milétrie, Poitiers, FRANCE

Texture analysis is widely used to characterize tumor heterogeneity and this method requires an image pre-processing step beforehand. The main purpose of this study was thus to investigate the impact of the quantization step on texture parameters values and to build a prognostic model. Tumor volumes were delineated on PET and CT images of 116 patients with NSCLC. Three methods of image quantization were applied on resulting volumes: linear quantization and histogram equalization with 64 values and fixed-width bins quantization with bins of $10 \mathrm{HU}$ and $0.5 \mathrm{SUV}$ for CT and PET images respectively. First order, shape metrics, $2^{\text {nd }}$ and $3^{\text {rd }}$ order parameters were then computed and an SVM classification was applied to build a prognostic model. Parameters computed on images quantized with histogram equalization and linear method were found to be more correlated with volume than those assessed after fixed-width bins quantization. Finally, the best prognostic model, built with clinical data and parameters computed on histogram equalized images, showed an accuracy of $80.7 \%$, a specificity of $82.0 \%$ and a sensitivity of $80.2 \%$. Although the three quantization approaches allowed building efficient models with accuracy between 77 and $80 \%$, the exact features (and their number) retained and combined by SVM were different depending on the chosen quantization approach, demonstrating the very important impact of this often overlooked step in the calculation of textural features.

\title{
M04E-17: Loco-Regional Tumour Control of Advanced HNSCC Can Be Predicted Using Pre-Treatment CT
} Images.

\author{
A. Zwanenburg ${ }^{1}$, S. Leger ${ }^{1}$, K. Pilz ${ }^{1,2}$, B. A. W. Hoeben ${ }^{3}$, H. Kaanders ${ }^{3}$, W. J. G. Oyen ${ }^{4}$, M. Baumann ${ }^{1,2,5,6,7,8}$, \\ E. G. C. Troost ${ }^{1,2,5,6,7,8}$, S. Löck ${ }^{1,2,6}$, C. Richter ${ }^{1,2,5,6,7,8}$ \\ ${ }^{I}$ OncoRay - National Center for Radiation Research in Oncology, Dresden, Germany \\ ${ }^{2}$ Dept. of Radiation Oncology, Faculty of Medicine and University Hospital Carl Gustav Carus, Technische Universität Dresden, \\ Dresden, Germany \\ ${ }^{3}$ Dept. of Radiation Oncology, Radboud University Medical Center, Nijmegen, The Netherlands \\ ${ }^{4}$ Dept. of Nuclear Medicine and Molecular Imaging, Institute of Cancer Research, the Royal Marsden Hospital, London, Great \\ Britain \\ ${ }^{5}$ German Cancer Research Center, Heidelberg, Germany \\ ${ }^{6}$ Helmholtz-Zentrum Dresden Rossendorf, Dresden, Germany
}


${ }^{7}$ National Center for Tumour Diseases, Dresden, Germany

${ }^{8}$ German Cancer Consortium, Dresden, Germany

Radiomics is an emerging field of image-based treatment stratification. Medical image characteristics-features - are assessed by machine learning algorithms and related to important outcome variables. In radiation oncology loco-regional tumour control (LC) is an important clinical outcome. A systematic investigation to identify machine learning algorithms for reliably predicting LC as time-to-event data has not been performed. We investigate this issue in two cohorts of patients treated with radiotherapy for advanced head and neck squamous cell carcinoma (HNSCC).

Two cohorts of patients with UICC stage III/IV HNSCC were recruited from University Hospital Dresden (UKD) and Radboud University Nijmegen Medical Centre (RUMC) ( $n=45$ and $n=43$, respectively). Two train-test experiments were performed by using RUMC as training and UKD as test cohort, and conversely. Seven methods for feature selection were implemented to reduce feature redundancy and select the features correlating most with outcome. Five machine learning algorithms were implemented as classifiers to build predictive models based on the training cohort. 100 bootstraps of the training cohort were used for model building. The median prediction of the model array on the test cohort was used as ensemble prediction.

Random forest classifiers demonstrated the best performance in both train-test experiments (concordance index 0.68 and 0.83 ). Concordance indices for training and test cohorts were different, indicating dissimilarities between cohorts.

We demonstrated the feasibility of predicting HNSCC LC as time-to-event data using machine learning algorithms based on pretreatment CT images. Random forests performed well for both train-test experiments. An increase in cohort size is necessary to prevent undersampling issues.

\section{M04E-18: Noise Suppression for Cerebral Perfusion CT via Intrinsic Tensor Sparsity Regularization: Initial} Study

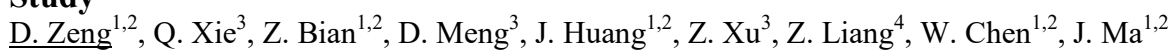

${ }^{I}$ School of Biomedical Engineering, Southern Medical University, Guangzhou, China

${ }^{2}$ Guangdong Provincial Key Laboratory of Medical Image Processing, Guangzhou, China

${ }^{3} X i$ 'an Jiaotong University, Xi'an, China

${ }^{4}$ Departments of Radiology and Biomedical Engineering, State University of New York at Stony Brook, NY, USA

Cerebral perfusion computed tomography (CPCT) provides functional information about capillary-level hemodynamic of brain parenchyma, which is an important tool for evaluating acute stroke, vasospasm and other neurovascular disorders. Meanwhile, the side effect of accumulated radiation, relating to genetic or cancerous diseases due to repeated dynamic scans at the same site, has caused great public concerns. One interesting general approach to counter the effect of overdose of radiation is to develop an iterative image restoration algorithm. In this paper, we focus on presenting a tensor-based restoration approach via fully adopting the intrinsic tensor sparsity (ITS) characteristics of CPCT sequential images. For simplicity, the present approach is termed as "CP-ITS". More specifically, the gains of ITS measure are to adopt the nonlocal self-similarity across space and global correlation along frames, therefore ITS measure can encode both sparsity insights delivered by the most Tucker and CANDECOMP/PAREFAC (CP) low-rank decomposition for a general tensor. Then the ITS measure is introduced into the CPCT images restoration model through characterizing the tensors formed by nonlocal similar patches with in CPCT images. To minimize the associative objective function, we proposed an efficient alternating direction method of multipliers (ADMM) based algorithm. Extensive evaluations on the digital phantom data demonstrate the performance of the present CP-ITS approach for low-dose CPCT imaging.

This work was supported in part by the National Natural Science Foundation of China under Grants 81371544,61571214 , the Science and Technology Program of Guangzhou, China under Grant 201510010039. Zhengrong Liang also was partially supported by the NIH/NCI under grant \#CA143111 and grant \#CA082402.

M04E-19: Enhanced Wiener Filter for Desplecking Ultra-Sound Images $\underline{\text { F. Baselice }}^{1}$, G. Ferraioli ${ }^{2}$, V. Pascazio ${ }^{1}$, G. Schirinzi ${ }^{1}$

${ }^{I}$ Department of Engineering, Universita' di Napoli Parthenope, Napoli, Italy

${ }^{2}$ Dipartimento di Scienze e Tecnologie, Universita' di Napoli Parthenope, Napoli, Italy

Classical Wiener Filter for despeckling Ultra Sound images, which performs the image restoration in the frequency domain, does not take into account the spatial behavior of the image to be restored. An enhanced version of the Wiener Filter able to overcome such limitation by modeling the image as a Markov Random Field is described. The algorithm provides an estimation of the image spatial correlation and automatically tunes for each pixel the intensity of the filter: pixels belonging to smooth areas are filtered with higher intensity, while pixels belonging to edge areas are weakly filtered. The algorithm, tested on realistic images simulated via the widely adopted Field 2 software and compared to other despeckling techniques such as Anisotropic Diffusion and Non-local means, guarantees two main objectives: it keeps the high computational efficiency of Wiener Filter and it provides an accurate restoration of the image, both in terms of smooth areas regularization and edge preservation. Application of the algorithm on larger datasets are required to further assess the potentialities and the impact of the proposed technique. 
M04E-20: An Automatic Segmentation Method for the Measurement of the Functional Volume of Oncological Lesions on MR ADC Maps

\author{
F. Gallivanone $^{1}$, M. Panzeri ${ }^{2}$, C. Canevari ${ }^{3}$, M. Interlenghi ${ }^{1}$, C. Losio $^{2}$, L. Gianolli ${ }^{3}$, F. De Cobelli ${ }^{2,4}$, I. Castiglioni ${ }^{1}$ \\ ${ }^{I} I B F M-C N R$, Segrate (MI), Italy \\ ${ }^{2}$ Radiology Department, IRCCS San Raffaele Scientific Institute, Milan, Italy \\ ${ }^{3}$ Nuclear Medicine Department, IRCCS San Raffaele Scientific Institute, Milan, Italy \\ ${ }^{4}$ Vita-Salute San Raffaele University, Milan, Italy
}

Human cancers frequently display intra-tumor phenotypic heterogeneity, whose nature can have profound implications both for tumor development and therapeutic outcomes. Some recent research efforts have been devoted to develop advanced image processing methods able to extract imaging descriptors characterizing such intra-tumor phenotypic heterogeneity. However, most methods need to accurately define the lesion volume in order to extract imaging descriptors. This work aims at assessing a novel segmentation method to measure the functional volume of lesions on MR ADC maps. The method was validated in advanced breast cancer patients addressed to Neoadjuvant Chemotherapy and surgical intervention, undergoing pre-treatment FDG-PET and multi-parametric MR studies. PET metabolic volume (MTV), SUVmean, SUVmax, and Total Lesion Glycolysis (TLG) of lesions were measured using an already validated segmentation algorithm [Gallivanone et al., J. Instr. 2016]. The MR functional volume of lesions segmented on the ADC map resulted directly correlated to PET MTV. We defined a new parameter characterizing the MR total diffusion of lesions, the Total Lesion Diffusion (TLD) that resulted directly correlated to PET TLG. Furthermore, we assessed an inverse correlation between SUVmax and ADCmin within the PET and MR functional volumes, respectively. Textural indexes were also evaluated. Correlations $(\mathrm{p}<0.05)$ were found among the textural image descriptors related to the spatial distribution of the signal extracted within the PET and MR functional volumes. In conclusion, our segmentation method is effective to define the functional volume of lesions on ADC maps.

M05: MIC Plenary 2

Thursday, Nov. 3 08:00-10:00 Schweitzer

M05-1: Introduction to 2nd MIC plenary session

D. Visvikis ${ }^{1}$, S. Surti ${ }^{2}$

${ }^{I}$ INSERM UMR 1101, CHRU Brest, INSERM, LaTIM, Brest, France

${ }^{2}$ Department of Radiology, University of Philadelphia, Philadelphia, USA

This presentation will concern the introduction of the second MIC plenary session, as well as information related to the rest of the MIC schedule and events.

M05-2: Redefining optical imaging with Multi-Spectral Optoacoustic Tomography. V. Ntziachristos

Faculty of Medicine, Technische Universitat Munchen, Munich, Germany

Optical imaging is unequivocally the most versatile and widely used visualization modality in the life sciences. Yet it has been significantly limited by photon scattering, which complicates imaging beyond a few hundred microns. For the past few years, there has been an emergence of powerful new optical and optoacoustic imaging methods that can offer high resolution imaging beyond the penetration limits of microscopic methods. The talk discusses progress in multi-spectral opto-acoustic tomography (MSOT) that brings unprecedented optical imaging performance in visualizing anatomical, physiological and molecular imaging biomarkers. Some of the attractive features of the method is the ability to offer 10-100 microns resolution through several millimetres to centimetres of tissue and real-time imaging. We demonstrate implementations in the time and frequency domain, showcase how it is possible to accurately solve fluence and spectral coloring issues for yielding quantitative measurements of tissue oxygenation and hypoxia and demonstrate applications in resolving inflammation, metabolism, angiogenesis in label free mode. In parallel we summarize progress with clinical systems that offer to change readings of disease in-vivo and discuss complementarity with ultrasound imaging, fluorescence imaging and other modalities. Finally the talk offers insights into new miniaturized detection methods based on ultrasound detection using optical fibers, which could be used for minimally invasive applications.

M05-3: NMISC awards presentation

D. Visvikis

INSERM UMR 1101, CHRU Brest, INSERM, LaTIM, Brest, France

This presentation will introduce the annual awards associated with the NMISC, their organisation as well as the winners for 2016 
M05-4: Bruce Hasegawa award ceremony

D. Visvikis

INSERM UMR 1101, CHRU Brest, INSERM, LaTIM, Brest, France

This presentation will be given by the person that has nominated the winner of the NMISC Bruce Hasegawa YMIS award

M05-5: Bruce Hasegawa YMIS award winner lecture

D. Visvikis

INSERM UMR 1101, CHRU Brest, INSERM, LaTIM, Brest, France

This will be the presentation given by the Bruce Hasegawa Young Medical Imaging Scientist award winner for 2016

M05-6: EJH NMISC award ceremony

D. Visvikis

INSERM UMR 1101, CHRU Brest, INSERM, LaTIM, Brest, France

This will be the presentation given by the person nominating the 2016 winner of the NMISC EJH scientist award

M05-7: 2016 EJH NMISC scientist award recipient lecture

D. Visvikis

INSERM UMR 1101, CHRU Brest, INSERM, LaTIM, Brest, France

This presentation will be given by the 2016 EJH NMISC scientist award recipient, demonstrating their achievements over the years.

M06: Pre-clinical (small animal) emission/multimodality imaging I

Thursday, Nov. 3 10:30-12:00 Schweitzer

M06-1: Characterization of a PET Prototype Based on Monolithic Detectors

$\underline{\text { P. Rato-Mendes }}{ }^{1}$, J. Marin $^{1}$, M. A. Morcillo ${ }^{1}$, J. Navarrete ${ }^{1}$, J. C. Oller ${ }^{1}$, M. Oteo ${ }^{1}$, J. M. Perez ${ }^{1}$, L. Romero ${ }^{1}$, I. Sarasola ${ }^{2}$, O. Vela

${ }^{I}$ CIEMAT, Madrid, Spain

${ }^{2}$ CERN, Geneva, Switzerland

We have characterized a PET prototype based on monolithic scintillator detectors. The prototype has a modular design with 8 identical detector cassettes, each housing 4 monolithic blocks. The cassettes are mounted on a gantry in 2 groups of 4 cassettes facing each other, totaling 32 detector blocks. Each detector block is composed of a trapezoidal LYSO:Ce scintillator glued to a pair of Hamamatsu S8550-02 APD matrices, which are read out by dedicated VATA 241 ASICs from Gamma Medica Ideas. The ASIC collects and amplifies the charge pulses from the 64 photosensors in each detector block, sums them into 8 rows and 8 column outputs and generates a trigger signal whenever the charge in a channel is above a user-defined threshold. Positioning algorithms based on supervised training neural networks are used for the detector blocks. A rotating platform with precise movement control is aligned with the system axis allowing acquisition of coincidence data along different projection angles. Using this system, Na22 point sources and a NEMA NU4-2008 image quality phantom filled with F18-FDG have been imaged and the images obtained have been characterized and compared with those from a Sedecal rPET small-animal PET/CT scanner, used as reference. Results show that the data acquisition system performs according to specifications and that PET imaging based on monolithic detector blocks is an alternative to designs based on segmented detectors.

CIEMAT is a member of the RD18 Crystal Clear Collaboration at CERN and of COST Action TD1401 "FAST - Fast Advanced Scintillator Timing".

M06-2: The Hardware Architecture of the LabPET II-Mouse, a Highly Integrated APD-Based PET Scanner

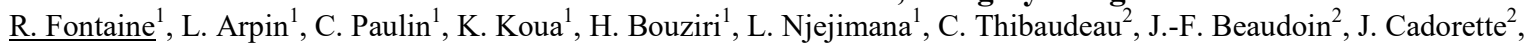
S. Panier ${ }^{1}$, M. Abidi ${ }^{1}$, J. Bouchard ${ }^{1}$, N. Jurgensen ${ }^{1}$, M.-A. Tetrault ${ }^{1}$, M. Bergeron ${ }^{2}$, E. Gaudin ${ }^{2}$, F. Loignon-Houle ${ }^{2}$, J. Charest ${ }^{1}$, M. Paille ${ }^{2}$, A. Samson ${ }^{1}$, P.-Y. Lauzier-Trepanier ${ }^{1}$, W. Ben Attouch ${ }^{1}$, J. Rossignol ${ }^{1}$, M. Gaudreault ${ }^{1}$, K. Forest ${ }^{1}$, N. Viscogliosi ${ }^{1}$, F. Berthelot ${ }^{1}$, C. M. Pepin ${ }^{2}$, J.-B. Michaud ${ }^{1}$, C.-A. Brunet ${ }^{1}$, J.-F. Pratte ${ }^{1}$, R. Lecomte $^{2}$

${ }^{I}$ Dept. Electrical Engineeging and Computer Engineering, Université of Sherbrooke, Sherbrooke, Canada

${ }^{2}$ Dept. of Nuclear Medicine and Radiobiology, Université of Sherbrooke, Sherbrooke, Canada 
The continuous quest for better contrast-to-noise ratio (CNR) in PET imaging mainly follows the paths of better time and spatial resolution. While new detector technologies let us foresee better timing performance, improving spatial resolution remains an overwhelming task due to the high pixel density along with the complex signal readout required to reach the PET resolution limit. The LabPET II-mouse detector and electronic architecture is an answer to the spatial resolution challenge. The scanner was designed with $61441.12 \times 1.12 \times 10.6 \mathrm{~mm}^{3}$ LYSO pixel detectors spread on a $8 \mathrm{~cm}$ diameter $\mathrm{x} 5 \mathrm{~cm}$ axial length cylinder, with the goal of achieving submillimetric reconstructed spatial resolution within a $25 \mathrm{~mm}$ diameter FOV. The scanner architecture relies on detector modules consisting of 4 arrays of 4 x 8 crystals individually coupled to the pixels of avalanche photodiode (APD) monolithic arrays of the same form factor. Each APD pixel has its own Time-over-Threshold based electronic channel implemented in a $4.6 \times 5.9 \mathrm{~mm}^{2}$ ASIC in CMOS $180 \mathrm{~nm}$ technology, flip chipped on a PCB. APD bias, noise and energy thresholds are set in the ASIC through a command link. An FPGA-embedded signal processing unit harvests all detected events from 4 detector modules and corrects, merges and sorts all events prior to transfer to a coincidence engine. A scanner has 12 of these units spread in a star-like fashion. Finally, the coincidence unit implemented in a donut-shaped PCB ensures proper clocking scheme to all embedded signal processing units and ASICs. It also support all data communication through an embedded Ethernet network.

M06-3: INSERT Project: MR Compatible Preclinical SPECT Based on SiPM Photodetectors

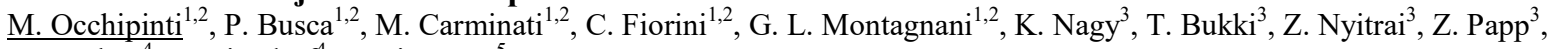
A. Küehne ${ }^{4}$, T. Niendorf ${ }^{4}$, C. Piemonte ${ }^{5}$

${ }^{1}$ Dipartimento di Elettronica, Informazione e Bioingegneria, Politecnico di Milano, Milano, Italy

${ }^{2}$ Sezione di Milano, INFN, Milano, Italy

${ }^{3}$ Mediso Medical Imaging Systems, Budapest, Hungary

${ }^{4}$ MRI.TOOLS GmbH, Berlin, Germany

${ }^{5}$ Fondazione Bruno Kessler (FBK), Trento, Italy

We report on the results achieved with the first SPECT insert for general purpose MR scanners, developed within the INSERT project (INtegrated SPECT/MRI for Enhanced stratification of brain tumors in Radio-chemoTherapy). The present paper focuses on the implementation and performance assessment of a preclinical version of the SPECT. A clinical configuration is currently under development and will be optimized according to the results from the preclinical system. The SPECT is composed by a full ring of gamma detection modules based on compact and MR compatible Silicon PhotoMultipliers (SiPMs). The intrinsic spatial resolution of the detection modules has been measured as $1.0 \mathrm{~mm}$ FWHM over $40 \mathrm{~mm} \times 40 \mathrm{~mm}$ planar field of view (FOV), at $140 \mathrm{keV}$ and at $4{ }^{\circ} \mathrm{C}$. Considering the design of the multi-pinhole collimator (with a sensitivity of $1995 \mathrm{cps} / \mathrm{MBq}$ ), we expect a tomographic resolution of $1.0 \mathrm{~mm}^{3}$ in a $16 \mathrm{~mm}$ trans-axial $\times 11 \mathrm{~mm}$ axial FOV. The energy resolution of the detector is $13.7 \%$ for Tc-99m $\left(140 \mathrm{keV}\right.$, at $\left.4{ }^{\circ} \mathrm{C}\right)$ and has been optimized to permit the simultaneous acquisition of multiple radiotracers. The main components, namely the detection modules, the collimator and custom RF coil, have been realized with a compact design to fit common MR bores. Moreover, mutual compatibility between SPECT system and MR has been studied and optimized for all the SPECT components. The gamma detection modules have been tested in a 7 T MRI (MAGNETOM, Siemens) and the results show satisfactory compatibility. The detection module does negligibly affect MRI imaging. Tests in MR are going to be repeated with the whole SPECT scanner.

M06-4: Evaluation of a Long Axial Field-of-View PET Scanner for Non-Human Primates

E. Berg ${ }^{1}$, X. Zhang ${ }^{1}$, J. Bec ${ }^{1}$, M. S. Judenhofer ${ }^{1}$, Q. Peng ${ }^{1,2}$, M. Kapusta ${ }^{3}$, M. Schmand ${ }^{3}$, M. E. Casey ${ }^{3}$, J. Qi ${ }^{1}$, R. D. Badawi ${ }^{1,4}$, S. R. Cherry ${ }^{1,4}$

${ }^{1}$ Biomedical Engineering, University of California, Davis, CA, USA

${ }^{2}$ Life Sciences Division, Lawrence Berkeley National Laboratory, Berkeley, CA, USA

${ }^{3}$ Siemens Medical Solutions, Knoxville, TN, USA

${ }^{4}$ Radiology, University of California, Davis, CA, USA

In support of the EXPLORER collaboration to build a high sensitivity, total-body PET scanner, we have recently constructed a long axial field-of-view (FOV) PET system for non-human primate imaging. Using the detectors and electronics of a 4 block ring prototype clinical system, we constructed an 8 block ring system ( $453 \mathrm{~mm}$ axial FOV, $320 \mathrm{~mm}$ transaxial FOV), leading to maximum acceptance angle similar to what is expected to be used in EXPLORER $\left(\sim 45^{\circ}\right)$. This work demonstrates the initial scanner performance, following the NEMA NU2-2012 protocol, and presents images of a Derenzo spatial resolution phantom. For NEMA tests, we compared four acceptance angles, representing maximum block ring differences of 2, 4, 6 and 8 rings. NEMA NU-2 sensitivity at the radial center was $20.1 \mathrm{kcps} / \mathrm{MBq}$ with the smallest acceptance angle and $52.1 \mathrm{kcps} / \mathrm{MBq}$ with the full acceptance angle, approximately a five-fold increase from the clinical system. Scatter fraction showed little dependence on acceptance angle, ranging from $32.6 \%$ to $33.9 \%$, and was nearly constant up to the peak NECR activity concentrations. The peak NECR was $337 \mathrm{kcps}(8.9 \mathrm{kBq} / \mathrm{mL})$ and obtained with the 6 block ring acceptance angle. To reduce randoms with a large acceptance angle, a variable coincidence time window computed using the ring difference of each LOR was applied in postprocessing. This increased the peak NECR to $355 \mathrm{kcps}(8.3 \mathrm{kBq} / \mathrm{mL})$ with the full acceptance angle, approximately three-fold higher than the NECR of the 4 block ring at the same activity concentration. A Derenzo phantom $(10 \mathrm{~cm}$ diameter, $9 \mathrm{~cm}$ length) 
was constructed with rod diameters of $2-7 \mathrm{~mm}$. Imaging data containing $\sim 390 \mathrm{M}$ events $(\sim 5 \%$ randoms $)$ were reconstructed using a nonTOF-OSEM algorithm (3 iterations, 20 subsets) with resolution modeling. Attenuation correction based on the expected $511 \mathrm{keV}$ attenuation in a water filled cylinder was applied, however, no other data corrections were used. Rod diameters as small as $3 \mathrm{~mm}$ could be resolved.

M06-5: MAPSSIC, a Novel CMOS Intra-Cerebral Beta+ Probe for Deep Brain Imaging in Awake and Freely Moving Rat: a Monte-Carlo Study.

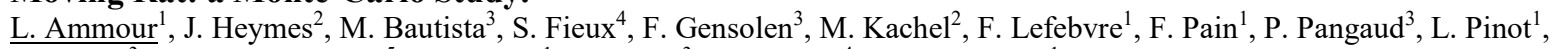
J. Baudot ${ }^{3}$, P. Gisquet-Verrier ${ }^{5}$, P. Laniece ${ }^{1}$, C. Morel $^{3}$, L. Zimmer ${ }^{4}$, M.-A. Verdier ${ }^{1}$

${ }^{I} I M N C$ CNRS/IN2P3, Univ Paris-Sud, Univ Paris Diderot, Université Paris-Saclay, Orsay, France

${ }^{2} I P H C$ CNRS/IN2P3, Univ Strasbourg, Strasbourg, France

${ }^{3}$ CPPM CNRS/IN2P3, Univ Aix-Marseille, Marseille, France

${ }^{4}$ LNRC, CNRS/INSERM, Univ Lyon 1, Lyon, France

${ }^{5}$ NeuroPSI CNRS/INSB, Univ Paris Sud, Université Paris-Saclay, Orsay, France

Among the numerous methods developed to adress neuroscience research needs, the combination of pre-clinical PET with behavioral studies has been recently pointed out as a potential key breakthrough to go further in the understanding of functional processes in the brain. Achieving such a combination is difficult. Anaesthesia or restraints inherent to micro-PET precludes its use for behavior studies. To adress this obstacle, recent approaches have been develsopped but remain affected by important constraints. In that context, we have presented an original strategy using submillimetric pixelated probes to directly measures positrons inside the rat brain. The detection volume around the sensitive area is bounded by positron range, therefore comparable with rat brain loci sizes. Integrated electronics and wireless communication system allows fully freely-moving rats experiments. Former probes have shown promising results but have suffered from various detection limitations. We propose here MAPSSIC, a novel beta probe project benefiting from innovative CMOS sensors to overcome these limitations. Reduced noise, high positrons sensitivity and low gamma rays detection promises relevant detection capabilities. Monte-Carlo simulations with GATE platform have been carried out to investigate probe physical characteristics and performances on the basis of first prototypes $(16 \times 128$ pixels of $30 \times 50 \mu \mathrm{m}^{2}$ on $18 \mu \mathrm{m}$ sensitive layer). Sensitivity to positrons and transparency to gamma rays as well as spatial detection capabilities were determined using specific $18 \mathrm{~F}, 11 \mathrm{C}$ and $15 \mathrm{O}$ phantoms. Results shows a good agreement with the expected performances required for tracers activity measurement in rats brain. In $18 \mathrm{~F}$ studies, the mean deposited energy into pixels is $12,54 \mathrm{keV}$. Positron sensitivity is consistent with former probes $(0.89 \mathrm{evts} /(\mathrm{kBq} / \mathrm{mL}))$ and the probe shows a good transparency to gamma rays. $90 \%$ of the detected positrons comes from less than $1.23 \mathrm{~mm}$ away their detection location.

\section{M06-6: Performance of a Detector Module for a Mouse Brain PET/MRI System}

C. Parl, A. Kolb, F. Schmidt, B. J. Pichler

Werner Siemens Imaging Center, University of Tuebingen, Tuebingen, Germany

The impact of mouse brain imaging studies can be advanced by simultaneously acquired anatomical, functional and molecular in vivo data at a high spatial and temporal resolution. This is provided by the combination of positron emission tomography and magnetic resonance imaging (PET/MRI). However, the restricted spatial resolution and sensitivity in commercial small animal PET systems limits the image quality and quantification accuracy of mouse brain studies. To compensate for this issues we are currently developing a dedicated mouse brain PET/MRI system, which contains small scintillation crystals (LSO) and provides a solid angle coverage of $61 \%$. The system diameter is $28 \mathrm{~mm}$ and the axial length $40 \mathrm{~mm}$. A dual layer scintillator setup provides depth of interaction (DOI) capability. Within the 30 block detectors, the center crystals of the inner layer have a dimension of $0.98 \times 0.98 \times 4.00 \mathrm{~mm}^{3}$, those of the outer layer have $0.98 \times 0.98 \times 8.00 \mathrm{~mm}^{3}$. On the outer rows of the individual detector blocks $1.76 \times 0.98 \times 4.00 \mathrm{~mm}^{3}$ crystals are used for the inner layer and $1.23 \times 0.98 \times 8.00 \mathrm{~mm}^{3}$ for the outer layer to minimize the gaps between the block detectors and maximize the system sensitivity. The LSO stack is coupled to a continuous light guide and read out by a $4 \times 4$ SiPM array. In this study, we evaluated the scintillator setup and front-end electronics in terms of linearity, peak to valley ratio, energy resolution, timing resolution, and temperature dependency. All parameters were determined on the basis of saturation corrected energy values. The peak to valley ratio was $6.88 \pm 2.05$. The energy resolution was $15.52 \pm 1.25 \%$. The timing resolution was $2.29 \pm 0.24 \mathrm{~ns}$. Over a temperature range from $10^{\circ} \mathrm{C}$ to $40^{\circ} \mathrm{C}$, the walk of the full energy peaks of the individual crystals could be reduced from $79.12 \pm 1.14 \%$ to $5.42 \pm 2.94 \%$ by applying a linear voltage correction of $66.1 \mathrm{mV} /{ }^{\circ} \mathrm{C}$. The fully evaluated block detector module represents a milestone to realize our mouse brain PET/MRI insert.

M07: Quantitative imaging techniques \& image analysis II

Thursday, Nov. 3 10:30-12:00 Cassin

M07-1: Atlas-Based Multi-Organ Segmentation for Abdominal PET Using Graph Cuts

$\underline{S . R e n}^{1}$, M. Naganawa ${ }^{2}$, R. E. Carson ${ }^{1,2}$

${ }^{I}$ Department of Biomedical Engineering, Yale University, New Haven, CT, US

${ }^{2}$ Department of Diagnostic Radiology, Yale University, New Haven, CT, US 
In PET studies, to quantitatively analyze functional changes or estimate physiological parameters, regions of interest(ROIs) are applied to extract the underlying tissue time-activity curves(TACs). Manual delineation of ROIs is commonly used, but this process is time consuming and operator dependent. Thus semi- or fully-automated ROI delineation methods have been studied. With a population of images available, it is desirable to include a spatial prior in the segmentation. Atlas-based segmentation is one of the most popular methods to incorporate prior information in the segmentation, and has been employed in PET brain studies. However, abdominal multi-organ segmentation remains a challenging task because of the high variability in shapes and positions of abdominal organs among subjects. There are very few studies on atlas-based abdominal PET segmentation, but several studies have been based on abdominal CT images. In this study, we establish a framework for atlas-based multi-organ segmentation in abdominal PET images, extending the work of van Lijn on MR brain segmentation, incorporating probabilistic atlas information into the segmentation as a spatial prior by using maximum a posterior(MAP) estimation, and determining the optimum segmentation by using graph cuts optimization. The method was evaluated by performing leave-one-out experiments using 12 manually segmented abdominal PET images. The segmentation results compare well with the manual segmentation, with Dice indices of $90 \%, 78 \%, 85 \%$ and $63 \%$ for liver, pancreas, spleen and kidneys, respectively. Additionally, mean image intensity within ROIs were compared between automated and manual segmentation, with a mean percentage error of $-0.6 \%$, $1.4 \%,-0.5 \%$ and $-0.8 \%$ for four abdominal organs.

M07-2: Ant Colony Segmentation Approach for Heterogeneous Volume Delineation in PET A. Ouahabi ${ }^{1}$, V. Jaouen ${ }^{1}$, M. Hatt ${ }^{1}$, D. Visvikis ${ }^{1}, \underline{\text { H. Fayad }}^{1,2}$

${ }^{I}$ INSERM UMR1101, LaTIM, Brest, France

${ }^{2}$ Université de Bretagne Occidentale, Brest, France

Accurate delineation of metabolically active tumor volumes (MATV) in PET imaging is a challenging task, mainly due to low spatial resolution and strong levels of noise. In a previous study, we developed a segmentation approach based on Ant Colony Optimization (ACO), a population-based model that mimics the collective foraging behavior of real ant colonies. Artificial ants explore their environment (PET image) in quest for food (MATV) and exchange information through iterative pheromone update, attracting other ants along their path. Unlike global thresholding algorithms, we exploit local neighborhood analysis to enhance the spatial consistency of the final volume. At convergence, a pheromone map is obtained with highest density around the MATV. The objective of this study will be focused on extending the ACO model to work on 3 tumor classes for the delineation of inhomogeneous tumors. The developed 3 tumor classes ACO algorithm was tested on a 6 real based tumors simulated dataset including different levels of heterogeneities and noise. Results were promising since the segmented images were close to the ground truth with a mean percentage error of $4.03 \%$. Future development will include parameter optimization and automatic estimation of food source to further extend the robustness of the method as well as the use of this algorithm for other applications such as the estimation of 4D PET Kinetics parameters of physiological significance.

M07-3: PCA-Based Approach for Inhomogeneous PSF Estimation and Partial Volume Correction in PET

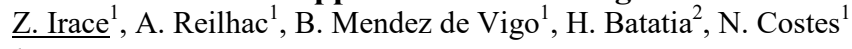

${ }^{I}$ LiLi, CERMEP, Lyon, France

${ }^{2}$ ENSEEIHT, University of Toulouse, Toulouse, France

PET data can be affected by strong Partial Volume Effect (PVE). A good knowledge of the spatially variant Point Spread Function (PSF) is required to correct this artifact. The proposed method relies on the construction of a basis that suits the data at best and assumes that the PSF can be expressed at any point as a linear combination of eigen-PSFs, allowing a fast and accurate iterative-based Partial Volume Correction. Initial results shows accurate PSF estimation as well as significant image restoration.

This work was supported by the following groups: LILI-EQUIPEX Lyon Integrated Life Imaging: hybrid MR-PET ANR project-11-EQPX-0026, CESAME - Brain and Mental Health ANR-10-IBHU-0003, FLI - France Life Imaging and the Neurodis Foundation.

\author{
M07-4: Simultaneous PET Imaging of Liquid Absorption and Mucociliary Transport in the Lungs Based on \\ Triple Coincidences \\ $\underline{\text { J. L. Herraiz }}^{1,2}$, E. Lage ${ }^{3}$, J. Venegas ${ }^{2}$ \\ ${ }^{I}$ Dept. Fisica Atomica. Grupo Fisica Nuclear, University Complutense of Madrid, Madrid, Spain \\ ${ }^{2}$ Dept. Anaesthesia, Massachusetts General Hospital and Harvard Medical School, Boston, MA, USA \\ ${ }^{3}$ Dept. Bioquimica. Facultad de Medicina, University Autonoma of Madrid, Madrid, Spain
}

Triple coincidences in PET can be used to enable the simultaneous imaging and separation of radiotracers labeled with standard positron emitters (such as $13 \mathrm{~N}$ ) and positron-gamma emitters (such as $76 \mathrm{Br}$ ). We have previously shown that multiplexed PET can be achieved without any kinetic modelling or hardware modification, and therefore, it can be used in most current PET scanners. The goal of this study was to demonstrate that this methodology can be applied to address important clinical needs, such as the quantitative measurement of the liquid absorption in the airways, a new biomarker for the treatment response of 
Cystic Fibrosis (CF) patients. To measure LA in the lungs we plan to use simultaneous measurements of the local transport in the airways of a small molecule (13N-NH3), which is cleared by both LA and mucociliary transport (MCT), and a large molecule (Albumin-microagregate,76Br-Alb-Ma) that is transported exclusively by MCT. LA can be then calculated based on the differences in the transport rates of both tracers. We evaluated the performance of the proposed method in a clinical PET/CT scanner with 10 -minutes acquisitions of phantoms filled with $13 \mathrm{~N}-\mathrm{NH} 3$ and $78 \mathrm{Br}(\sim 0.25 \mu \mathrm{Ci} / \mathrm{cc})$. After the simultaneous acquisition, list-mode data were analyzed to classify events into double coincidences (which contains events from both tracers) and triple coincidences (mostly from positron-gamma events). Following the reconstruction of each dataset, an iterative Maximum-Likelihood algorithm provides separated images of each tracer. Images were quantitative in each region containing $76 \mathrm{Br}, 13 \mathrm{~N}$, or both $(<5 \%$ bias respect to the actual values). The image quality and quantitative accuracy obtained in this preliminary experiment are much better than the one achievable with previous techniques such as dual-tracer scintigraphy. Invivo experiments with pigs are currently being performed and the results will be presented at the conference.

This project was supported financially in part by the Comunidad de Madrid through the Madrid-MIT M+Vision Consortium. the COFUND of the Seventh Framework Program of the European Union and the NIH Grant R21-EB020849-01

M07-5: Gradient-Aided Localized Deformable Model for PET Image Segmentation V. Jaouen $^{1}$, M. Hatt ${ }^{1}$, H. Fayad ${ }^{1}$, C. Tauber ${ }^{2}$, D. Visvikis ${ }^{1}$

${ }^{I}$ LaTIM, Université de Bretagne Occidentale, Inserm, Brest, France

${ }^{2}$ Imagerie et cerveau, Université François-Rabelais, Inserm, Tours, France

In this paper, we propose a new deformable model formulation for the segmentation of 3D PET images based on both edge and local region statistics. The originality of our approach lies in the automatic weighting of an edge-based external force field according to a measure of its smoothness and of its spatial configuration. The objective is two fold. Firstly, this technique extends the convergence of local approaches, usually limited by the localization radius. Secondly, it reduces potentially erroneous convergence of edge-based approaches due to either centers of divergence or excessive smoothing of the edge map. The proposed approach was compared to global and local region-based models as well as to the gradient vector flow model. We show example segmentation results for simulated objects of different volumes placed in heterogeneous background (a $59 \mathrm{~cm}^{3}$ and $5.4 \mathrm{~cm}^{3}$ tumor). The proposed model outperformed the other approaches based on sensitivity and positive predictive value in comparison with the known ground truth of the simulated volumes. Further validation will include the use of clinical tumor images with ground truth delineated by experts.

M07-6: Plane-Dependent ML Scatter Scaling: 3D Extension of the 2D Simulated Single Scatter Estimate A. Rezaei ${ }^{1}$, K. Salvo ${ }^{2}$, V. Panin ${ }^{3}$, T. Koesters ${ }^{4}$, M. Casey ${ }^{3}$, F. Boada ${ }^{4}$, M. Defrise ${ }^{2}$, J. Nuyts ${ }^{1}$

${ }^{I}$ Nuclear Medicine, KU Leuven, Leuven, Belgium

${ }^{2}$ Nuclear Medicine, Vrije Universiteit Brussel, Brussels, Belgium

${ }^{3}$ MI, Siemens Medical Solutions, Knoxville, USA

${ }^{4}$ New York University Medical Center, New York, USA

In this work, we propose a plane-dependent maximum likelihood (ML) scatter scale estimation from the emission measurements. The scatter scales obtained are validated using a Monte Carlo simulation of a NEMA-like phantom, and results are shown from two whole-body patient scans.

M08: New radiation detectors / technologies for medical imaging I

Thursday, Nov. 3 14:00-16:00 Schweitzer

M08-1: The Route to 10ps TOFPET Is Open

P. Lecoq ${ }^{1}$, E. Auffray ${ }^{1}$, S. Gundacker ${ }^{1}$, R. Martinez Turtos ${ }^{2}$

${ }^{I}$ EP, CERN, Geneva, Switzerland

${ }^{2}$ University Biccoca, Milano, Italy

A Time-of-Flight resolution of 10ps would introduce a paradigm shift in PET imaging allowing a direct 3D millimeter resolution for each positron annihilation event and therefore a real time visualization of the image during the acquisition. Moreover such a resolution would allow a precise electronic collimation on the region of interest and an improvement of the image $\mathrm{S} / \mathrm{N}$ ratio by a factor more than 10 as compared to non-TOF PET scanners. The potential for a 10-fold dose reduction would ease spreading PET beyond oncology, such as screening, neo-natal and pediatric applications. Our group at CERN has reached a coincidence TOF resolution FWHM of 73ps, 100ps and 122ps for Ce, Ca do-doped LSO of 5mm, 10mm and 20mm respectively, readout by a new generation of SIPM produced by FBK. But we are also actively exploring new ultrafast scintillation mechanisms with subpicosecond rise-time and sub-nanosecond decay time allowing to increase the density of photons in the first 10ps of the light pulse. Evidence will be shown of the generation of prompt photons in different scintillators and the possibility to produce them 
via the HIL (Hot Intraband Luminescnce mechanism). It will be shown that the combination of such scintillators with CdSe nanoplates or $\mathrm{ZnO}: \mathrm{Ga}$ nanocrystals is a promising route to reach a photon density of 100 or more photons in the first 10 picoseconds, largely sufficient to produce a time tag with this precision. Obtaining such a time resolution also implies a good control of the depth of interaction and of the photon travel modes in the crystal. Although this talk is not aiming at concentrating on this aspect in detail some on-going developments in our group will be quickly described.

The authors would like to acknowledge C. Piemonte from FBK (Italy) for providing the last generations of NUV SiPMs, M. Nikl from the Physics Institute and V. Cuba from the Technical University in Prague (Czech Republik) for the procurement of ZnO:Ga nanocrystals as well as J. Grimm and I. Moreels from the Italian Institute of Technology in Genova,(Italy) for the preparation of CdSe nanoplates.

M08-2: Use of the OpenPET Data Acquisition for a strip-line readout TOF PET detector

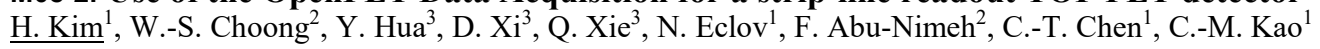

${ }^{I}$ Radiology, University of Chicago, Chicago, IL, United States

${ }^{2}$ Lawrence Berkeley National Laboratory, Berkeley, CA, United States

${ }^{3}$ Biomedical Engineering, Huazhong University of Science and Technology, Wuhan, China

We have developed a signal multiplexing method for a SiPM-based time-of-flight (TOF) positron emission tomography (PET). In the method, multiple SiPM signals are connected to a single strip-line, and the position of hit SiPM is decoded from the arrival time difference measured at the two ends of a strip-line. Since the position resolution on strip-line is directly proportional to the accuracy of the measured time, a high speed (> 1 GSPS) waveform sampling electronics, e.g., Domino Ring Sampler (DRS4), has been used to determine the time stamp of the strip-line signal as precise as possible: e.g., the electronic time resolution of the DRS4 is measured to be $\sim 5$ ps. Although the DRS4 waveform sampling provides a good timing accuracy, its large latency time required for digitizing samples could be a limiting factor for high count operation. Therefore, in an effort to increase the counting performance, we have come up with a new design of the strip-line board that can work with commonly used time-to-digital converters (TDC) with 50-100 ps electronic time resolution, which provide high counting capability. Hamamatsu MPPC 4x4 arrays (S12642-0404PA-50, 3x3 mm^2 area for each) are employed in the new strip-line board; 32 MPPC signals are routed to 4 strip-lines on the board. For verification purpose, we have conducted experiment tests to readout the current strip-line board by using the OpenPET electronics, which is a general purpose data acquisition developed for nuclear medicine research community. In the test, LYSO scintillator arrays ( $\left.3 \times 3 \times 20 \mathrm{~mm}^{\wedge} 3 \mathrm{each}\right)$ are coupled to MPPCs on the strip-line board, and the detector is irradiated by a $22 \mathrm{Na}$ source with $10 \mathrm{uCi}$ activity. The initial results obtained by using OpenPET are very promising: each MPPC on the strip-line is well identified with $1.2 \mathrm{~mm}$ FWHM resolution, and 13.0\% FWHM of energy resolution for $511 \mathrm{keV}$ gamma. The results are comparable to the ones by using DRS4 waveform sampler running at 2.5 GSPS.

\author{
M08-3: Evaluation of the Imaging Performance of Continuous Detectors Etched with Laser Induced Optical \\ Barriers \\ J. Panetta $^{1}$, S. Surti ${ }^{1}$, B. Singh ${ }^{2}$, J. Karp ${ }^{1}$ \\ ${ }^{I}$ Department of Radiology, University of Pennsylvania, Philadelphia, PA, USA \\ ${ }^{2}$ Radiation Monitoring Devices, Watertown, MA, USA
}

In recent years, research into continuous scintillation detectors in PET has demonstrated excellent performance. For use in a clinical PET system that requires thick detectors for high sensitivity, however, achieving excellent spatial resolution and TOF capability is challenging. We recently have evaluated techniques to etch laser induced optical barriers (LIOBs) within a thick continuous detector in order to achieve excellent spatial resolution by modifying the light distribution without pixelating the crystal. Building off of our previous work, here we study the transmission and scattering properties of LIOBs, by etching a single barrier into small cubes of LYSO, varying the parameters of the LIOB in each case, and measuring their properties with optical photons. Preliminary experiments show that the optimized LIOBs can decrease light transmission by as much as $\sim 40 \%$, though the properties of the barriers are dependent on the parameters selected during the etching process (i.e. physical dimensions of the micro-cracks that compose the etchings, the power of the laser beam); the transmission properties additionally are not affected by the angle of incidence. These measurements are then used to update our Monte Carlo model that detects gamma rays and tracks scintillator photons within a designed detector. These simulations are used to optimize the parameters and geometrical pattern chosen to etch a $50 \times 50 \times 25 \mathrm{~mm} 3$ continuous crystal, whose performance is measured and compared to simulations. Finally, EGS4 simulations of a whole-body scanner based on the continuous detector are performed to determine the imaging performance and potential impact of these detectors, and a lesion phantom is used to quantify the performance using the CRC metric. The continuous detector, with an average spatial resolution of 3-mm, leads to higher CRC for small 1-cm diameter lesions, compared to a scanner based on 4-mm pixelated detectors, as in state-of-the-art commercial PET/CT scanners.

M08-4: BGO as a Hybrid Scintillator / Cherenkov Radiator for Cost-Effective Time-of-Flight PET $\underline{\text { S. E. Brunner }}^{1}$, A. Ferri ${ }^{2}$, A. Gola ${ }^{2}$, C. Piemonte ${ }^{2}$, D. R. Schaart ${ }^{1}$ 
${ }^{I}$ Radiation Science \& Technology, TU Delft, Delft, The Netherlands

${ }^{2}$ Center for Materials and Microsystems, Fondazione Bruno Kessler, Trento, Italy

Due to detector developments in the last decade, such as the introduction of silicon photomultipliers (SiPMs), the time-of-flight (TOF) method is now showing its potential for improving the signal-to-noise ratio in positron emission tomography (PET) images. Whole-body systems based on L(Y)SO:Ce crystals read out with SiPMs have recently been developed, offering coincidence resolving times (CRT) of 325-400 ps FWHM. For decades, BGO was used in most PET systems. Besides a relatively low price, BGO offers superior stopping power for annihilation quanta and a higher photoelectric fraction than L(Y)SO. However, the relatively low light yield and slow decay time constants of BGO triggered its replacement by L(Y)SO:Ce in PET systems.

The optical characteristics of BGO, in particular a high transparency that reaches into the UV-range (310 nm) and a high refractive index of 2.15, are expected to make it a good Cherenkov radiator. In recent years, the Cherenkov effect has been investigated for improving TOF properties in PET detectors on the basis of the prompt character of the emission. Nevertheless, the low Cherenkov photon yield in the range of $\sim 10$ photons complicates energy discrimination in PET.

Here, we study the feasibility of combining event timing based on Cherenkov radiation with energy discrimination based on scintillation in BGO, as a potential approach towards a cost-effective TOF-PET detector. CRT measurements were performed with $27 \mathrm{~mm}^{\wedge} 3$ large cubes of BGO coupled to either NUV-HD SiPMs developed by Fondazione Bruno Kessler (FBK) or Digital Photon Counter (DPC) arrays from Philips. CTRs of 350 ps FWHM were obtained with the SiPMs from FBK, whereas, the fastest observed component for the CRT for the DPC arrays was 185 ps FWHM.

Moreover, a setup for time-correlated single photon counting based on DPC arrays was developed to study the rise-time of BGO, revealing a significant and fast rise-time component in the range of $\sim 100 \mathrm{ps}$, attributed to Cherenkov photons.

\author{
M08-5: BGO Coupled with NUV-HD SiPMs for Time-of-Flight PET by Simultaneous Detection of Cerenkov \\ and Scintillation Photons \\ $\underline{\text { S. I. Kwon }}^{1}$, A. Gola ${ }^{2}$, A. Ferri ${ }^{2}$, C. Piemonte ${ }^{2}$, S. R. Cherry ${ }^{1}$ \\ ${ }^{I}$ University of California, Davis, Davis, CA, USA \\ ${ }^{2}$ Fondazione Bruno Kessler, Trento, Italy
}

BGO was a very attractive scintillator in early-generation positron emission tomography (PET) scanners. However, the major disadvantages of BGO are lower light yield and longer rise and decay time than current popular scintillators such as L(Y)SO. These result in poor coincidence timing resolution (CTR; $>1 \mathrm{~ns}$ ). For this reason, it was assumed that BGO is not suitable for time-of-flight (TOF) PET applications. However, when a 511-keV photon interacts in a scintillator, a small number of Cerenkov photons are produced promptly by energetic electrons released by photoelectric or Compton interactions and should provide a better timing trigger for PET. Since BGO has high refractive index (increasing the Cerenkov light yield) and excellent transparency down to $320 \mathrm{~nm}$ (Cerenkov light yield increases at shorter wavelengths), we hypothesized that the CTR of BGO can be significantly improved by efficient detection of the Cerenkov photons. However, since the number of Cerenkov photons are very small compared to scintillation photons, photosensors need to have high UV/blue sensitivity, fast temporal response, and very low noise in order to trigger on the faint Cerenkov signal. In this respect, NUV-HD SiPMs (FBK) are an excellent fit for our approach. In this study, coincidence events were measured using BGO coupled with NUV-HD SiPMs. Scintillation photons were used to select an energy window. The energy resolution of $3 \times 3 \times 20 \mathrm{~mm}^{3} \mathrm{BGO}$ was $16 \%$. The existence and influence of Cerenkov photons in BGO were studied using different configurations to exploit the directionality of the Cerenkov emissions. CTR values (FWHM) of 267 ps from $2 \times 3 \times 2 \mathrm{~mm}^{3}$ BGO and 562 ps from $3 \times 3 \times 20 \mathrm{~mm}^{3}$ BGO were obtained. To our knowledge, these are the best CRTs reported for BGO to date. Consequently, BGO can be considered as a relevant scintillator for TOF PET scanners, especially if photodetectors with even better near UV/blue response can be developed.

\author{
M08-6: First Characterization of the SPADnet-II Sensor: a Smart Digital Silicon Photomultiplier for ToF-PET \\ Applications \\ $\underline{\text { E. Gros-Daillon }}^{1}$, L. Verger ${ }^{1}$, D. A. B. Bonifacio ${ }^{1,2}$, E. Charbon ${ }^{3}$, C. Bruschini ${ }^{4}$, L. H. C. Braga ${ }^{5}$, L. Gasparini ${ }^{5}$, N. Massari ${ }^{5}$, \\ M. Perenzoni ${ }^{5}$, D. Stoppa ${ }^{5}$, R. Walker ${ }^{6}$, A. Erdogan ${ }^{6}$, L. Parmesan ${ }^{6}$, R. K. Henderson ${ }^{6}$, S. Pellegrini ${ }^{7}$, B. Rae ${ }^{7}$ \\ ${ }^{I}$ MINATEC Campus, CEA - LETI, Grenoble, France \\ ${ }^{2}$ Institute of Radioprotection and Dosimetry, Rio de Janeiro, Brazil \\ ${ }^{3}$ Delft University of Technology, Delft, The Netherlands \\ ${ }^{4}$ EPFL, Lausanne, Switzerland \\ ${ }^{5}$ Integrated Radiation and Image Sensors (IRIS), Fondazione Bruno Kessler, Trento, Italy \\ ${ }^{6}$ CMOS Sensors and Systems (CSS) Group, School of Engineering, the University of Edinburgh, Edinburgh, United Kingdom \\ ${ }^{7}$ Imaging Division, STMicroelectronics, Edinburgh, United Kingdom
}

SPADnet-II, a $9.85 \times 9.85 \mathrm{~mm} 2,16 \times 16$ pixels array digital Silicon Photomultiplier has been developed. It is the second generation of the SPADnet sensors based in CMOS technology for Time of Flight Positron Emission Tomography (TOF-PET) imaging, 
with an enhanced pixel fill factor: 55\%. It also includes a number of new features: on-chip frame buffer to decouple pixel array readout from data transmission, revised triggering system (faster, single threshold operation), post-event triggering, and on-chip event centroid calculation. Each pixel contains 672 Single Photon Avalanche Diodes (SPADs) and two Time to Digital Converters. An on-chip discriminator triggers the scintillation integration. The SPADnet-II sensor has been optically coupled to LYSO crystals. The energy resolution at $511 \mathrm{keV}$ was $10 \%$ FWHM with a $3 \times 3 \times 5 \mathrm{~mm} 3$ crystal and $12 \%$ FWHM with a $3.5 \times 3.5 \times 20 \mathrm{~mm} 3$ crystal used for clinical imaging. The imaging performance of the sensor has been assed using a $1.3 \mathrm{~mm}$ pitch LYSO array designed for preclinical imaging.

M08-7: Time-over-Threshold for Pulse Shape Discrimination in a Time-of-fight/Depth-of-Interaction Phoswich PET Detector C.-M. Chang ${ }^{1}$, J. W. Cates ${ }^{2}$, C. S. Levin ${ }^{2,3,4,5}$

${ }^{I}$ Department of Applied Physics, Stanford University, Stanford, California, USA

${ }^{2}$ Department of Radiology, Stanford University, Stanford, California, USA

${ }^{3}$ Department of Bioengineering, Stanford University, Stanford, California, USA

${ }^{4}$ Department of Physics, Stanford University, Stanford, California, USA

${ }^{5}$ Department of Electrical Engineering, Stanford University, Stanford, California, USA

We are developing a PET detector capable of measuring both time-of-fight (TOF) and depth-of-interaction (DOI) with a goal to improve resulting image quality and accuracy. Phoswich designs have been realized in PET detectors to measure DOI for more than a decade. However, PET detectors based on phoswich designs put great demand on the readout circuit, which has to differentiate the pulse shape produced by different crystal layers. A simple and practical pulse shape discrimination approach is required to realize the phoswich design in a clinical PET scanner, which often consists of thousands of crystal elements. In this work, we studied the energy, timing and DOI performance of a phoswich detector design comprising a $3 \times 3 \times 20 \mathrm{~mm}^{3} \mathrm{crystal}$ made from a $3 \times 3 \times 10 \mathrm{~mm}^{3}$ LYSO:Ce crystal epoxied to a $3 \times 3 \times 10 \mathrm{~mm}^{3}$ calcium co-doped LSO:Ce,Ca(0.4\%) crystal and read out by silicon photomultipliers (SiPM). A DOI accuracy of 97.2\% was achieved for photopeak events by using time-overthreshold (ToT) as a pulse shape parameter. The measured energy resolution was $9.7 \pm 0.2 \%$ and $11.3 \pm 0.2 \% \mathrm{FWHM}$ at $511 \mathrm{keV}$ for the LYSO and LSO crystal layers, respectively. The coincidence timing resolution for photopeak events ranges from $164.6 \pm$ 0.9 ps to $183.1 \pm 4.2$ ps FWHM, depending on the layer combinations between two identical, dual layer detector configurations set in coincidence. These results show promise of using ToT for pulse shape discrimination in a TOF-DOI phoswich-based detector since ToT measurement can be easily implemented in readout electronics.

M08-8: A New Four-Layered DOI Detector with Quadrisected Top Layer Crystals

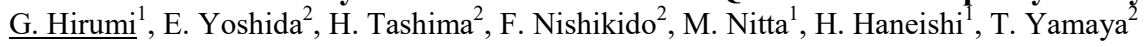

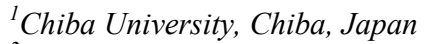

${ }^{2}$ National Institute for Radiological Science, Chiba, Japan

We developed a modified, more practical four-layered depth-of-interaction (DOI) detector based on the light sharing method. Reflectors, which are inserted in every two lines of crystal segments and shifted differently depending on each layer, project 3-D crystal positions onto a 2-D position histogram without any overlapping after applying an Anger-type calculation. The best crystal separation we have ever made based on this method was the 4-layered $32 \times 32$ array of LYSO crystals sized at $1.45 \times 1.45$ $\times 5 \mathrm{~mm}^{3}$. However, assembling crystals of a tiny size tends to cost a lot, and fine tuning of the light guide and the front-end circuit is required to have fine crystal identification from photo sensor signals of coarser pixel pitch. In this paper, therefore, we proposed a more practical 4-layered DOI detector. The key idea is that the crystals in the top layer, which have the highest detection efficiency, mostly contribute to PET spatial resolution. We applied two new ideas: (1) use of $1 / 4$ size crystals only for the 1st (top) layer and (2) inserting a thin light guide between the 1st and the 2nd layers of crystal array. In the developed prototype detector, the 1 st layer used $32 \times 32$ LYSO crystals of quarter size $\left(1.4 \times 1.4 \times 5.0 \mathrm{~mm}^{3}\right)$ compared with the other layers $\left(16 \times 16\right.$ arrays of crystals of $\left.2.8 \times 2.8 \times 5.0 \mathrm{~mm}^{3}\right)$. For better crystal identification of small crystals in the 1 st layer, we optimized the optical condition between crystals such as use of an optical glue or air. Also, a thin light guide was inserted between the 1 st and the 2nd layers for improvement of crystal identification of the 1st layer. With the appropriate insertion of the light guide, all crystals of the 1 st layer were identified as well as the crystals in the other layers.

M09: Image reconstruction techniques I

Thursday, Nov. 3 14:00-16:00 Cassin

M09-1: Direct EM Reconstruction of Parametric Images from List-Mode Brain PET Using a Novel Model Based on Logan Graphical Analysis J.-D. Gallezot ${ }^{1}$, M. K. Germino ${ }^{2}$, R. E. Carson ${ }^{1,2}$

${ }^{I}$ Radiology and Biomedical Imaging, Yale University, New Haven, CT, United States

${ }^{2}$ Biomedical Engineering, Yale University, New Haven, CT, United States 
Direct reconstruction of parametric images from raw dynamic PET data has the potential of producing lower noise images than obtained using intermediate frame-based reconstructed images, due to the accurately characterized statistical properties of raw PET data. The goal of this study was to extend a previous direct parametric reconstruction algorithm (PMOLAR). PMOLAR uses the Expectation Maximization (EM) algorithm to estimate the parametric maps. Previous versions of PMOLAR were based on the one-tissue (1T) compartment model (PMOLAR-1T). The 1T model is suitable for some PET tracers, but most reversible PET radioligand kinetics are better described by more complex models, mainly the two-tissue (2T) compartment model. Alternatively, Logan Graphical Analysis (GA) can be applied to all reversible PET tracers. In this study PMOLAR was adapted to a new model based on GA. PMOLAR-GA was evaluated on human data acquired on the High Resolution Research Tomograph (HRRT) after injection of [11C]PBR28, a radiotracer used to study neuroinflammation. The new model derived from GA was first compared to previous modeling methods (MA1, LEGA, 2T) suitable for [11C]PBR28 on fits of region of interest time-activity curves. Then the new version of PMOLAR was evaluated on a human 4D PET data set. PMOLAR-GA parametric maps were compared to frame-based parametric maps. Frame based parametric maps were visually very noisy and biased (between $66 \pm 10 \%$ and $116 \pm 46 \%$ on a regional level, depending on the modeling method) while the PMOLAR-GA mean regional values were very close to reference values $(-7 \% \pm 7 \%)$, with visually lower noise at the voxel level.

\section{M09-2: Dynamic PET Reconstruction Using the Kernel Method with MRI Information} K. Gong ${ }^{1}$, G. Wang ${ }^{1}$, K. T. Chen ${ }^{2}$, C. Catana ${ }^{2}$, J. Qi $^{1}$

${ }^{I}$ Department of Biomedical Engineering, University of California, Davis, Davis, CA, United States

${ }^{2}$ Athinoula A. Martinos Center for Biomedical Imaging, Department of Radiology, Massachusetts General Hospital and Harvard Medical School, Charlestown, MA, United States

Positron Emission Tomography (PET) is a functional imaging modality widely used in oncology, cardiology, and neuroscience. It is highly sensitive, but suffers from relatively poor spatial resolution, as compared with anatomical imaging modalities, such as magnetic resonance imaging (MRI). With the recent development of combined PET/MR systems, we can improve the PET image quality by incorporating MR information. Previously kernel learning has been successfully embedded into either dynamic PET reconstruction using PET data alone or static PET reconstruction using MRI information. Here we combine the above two approaches to further improve the quality of dynamic PET reconstruction. We examined several different approaches to combine the PET and MRI information in kernel learning to address the issue of potential mismatches between MRI and PET signals. Computer simulations and real patient data acquired on a simultaneous PET/MR scanner were used to evaluate the proposed methods.

This work is supported by the National Institutes of Health under grants R01 EB014894 and R01 EB000194.

\section{M09-3: Multi-Parametric MRI-Guided PET Image Reconstruction}

A. Mehranian, A. J. Reader

Division of Imaging Sciences \& Biomedical Engineering, St Thomas' Hospital, King's College London, London, United Kingdom

This works proposes a novel multi-parametric MRI-guided PET image reconstruction method within a Bayesian estimation framework that addresses the challenges of the conventional anatomically-guided PET reconstructions, namely, mismatches between MRI and PET boundaries and the partial volume averaging effect inherent in MRI images. To exploit all complementary MRI information in the reconstruction of PET images, a non-local prior is proposed in which similarity kernels are jointly calculated from PET and multi-parametric MRI images based on the geometric mean of the Gaussian kernels of each modality. As a result, in the case of unmatched functional and anatomical boundaries, the unique features of the PET image can be preserved. Furthermore, boundary information absent in a given parametric image (e.g. in T1-MRI) can be complemented by other images (e.g. T2-MRI). The proposed prior is studied using a Tikhonov potential function and the resulting reconstruction problem is optimized using the one-step-late MAP-EM algorithm. The BrainWeb phantom was used to create PET, T1-weighted and T2-weighted MRI images with multiple unique lesions and common and un-matched edges and the reconstructions were compared with PSF-MLEM and locally Tikhonov regularized MLEM reconstructions. The simulation results show that the proposed non-local prior can effectively exploit the anatomical boundary information provided by MRI images together with the functional boundary of the PET images being estimated and as such can outperform the local and conventional anatomical priors. In particular it is shown that the proposed prior preserves PET unique features that are otherwise over-smoothed by the conventional regularization methods. It is also demonstrated that the proposed prior outperforms its counterparts in edgepreservation even when using blurred MRI images and thus provides a promising approach toward the clinical adoption of MRIguided PET reconstruction.

This work was supported by the Engineering and Physical Sciences Research Council under grant EPSRC EP/M020142/1.

M09-4: PET Reconstruction with Convex Gradient-Based Priors

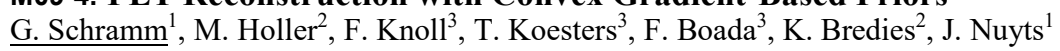


${ }^{I}$ Department of Imaging and Pathology, Division of Nuclear Medicine, KU Leuven, UZ Leuven, Leuven, Belgium

${ }^{2}$ Institute for Mathematics and Scientific Computing, University of Graz, Graz, Austria

${ }^{3}$ Department of Radiology, Bernard and Irene Schwartz Center for Biomedical Imaging, NYU School of Medicine, New York City, US

Introduction: PET imaging is hindered by limited spatial resolution and high Poisson noise. A way to overcome those limitations is the inclusion of anatomical prior knowledge in the image reconstruction process. Recently, two gradient-based anatomical prior functions - Joint Total Variation (JTV) [1] and Parallel Level Sets (PLS) [2] - have been introduced. In both cases the functions are non-smooth which hampers the optimization process. We show how to apply the first-order primal-dual algorithm [3] to solve the PET reconstruction problem with those priors.

Numerical Simulations: We generated a 2D software phantom based on a T1 MR image of a healthy volunteer. Fixed uptake values to grey and white matter with a ratio of 4 to 1 were assigned in the PET ground truth. Several ROIs with increased and decreased uptake were additionally added. Some of those ROIs were only added in one modality to study potential unwanted feature transfer. 10 noise realizations of $6 \mathrm{e} 6$ counts were reconstructed without and with JTV, PLS1, and PLS2 regularization using a fixed prior weight $\beta$.

Results: All gradient-based priors improve the signal to noise ratio and enhance gradients that are common in the PET and MR ground truth. For the chosen value of $\beta$, the reconstructions with JTV show less regional variability in the standard deviation image compared to both PLS methods. Edges between grey and white matter appear sharper in the reconstructions with PLS. All regularized reconstructions suffer from unwanted feature transfer of a simulated signal void in the MR. This artifact is most pronounced in the PLS2 reconstructions.

Outlook: A detailed comparison between the different prior functions including an optimization of the weights $\beta$ in different ROIs is ongoing.

References: [1] E. Haber, M. Holtzman Gazit, Surv Geophys 34:675-695; [2] M.J. Ehrhardt et al., Inverse Problems 31 015001; [3] A. Chambolle, T. Pock, J Math Imaging Vis 40: 120-145; [4] K. Bredies, M. Holler, SIAM 8: 2851-2886

M09-5: Joint Activity/Attenuation Reconstruction in SPECT Using Photopeak and Scatter Sinograms $\underline{\text { A. Bousse }}^{1}$, A. Sidlesky ${ }^{2}$, N. Roth ${ }^{2}$, A. Rashidnasab ${ }^{1}$, K. Thielemans ${ }^{1}$, B. F. Hutton ${ }^{1,3}$

${ }^{I}$ Institute of Nuclear Medicine, University College London, London, UK

${ }^{2}$ Spectrum Dynamics, Caesarea, Israel

${ }^{3}$ Centre for Medical Radiation Physics, University of Wollongong, Wollongong, Australia

This work presents the headlines of a joint activity/attenuation reconstruction scheme in SPECT, based on the maximisation of the scatter and non-scatter data joint log-likelihood. The activity image is updated with standard expectation maximisation (EM) whereas the attenuation is updated with a quasi-Newton line-search. Results on simulation demonstrates that the utilisation of scatter considerably reduces the ill-posedness of the intitial reconstruction problem with non-scatter counts only.

This work was supported by the NIHR-funded UCH Biomedical Research Centre.

M09-6: Hybrid Pre-Log and Post-Log Image Reconstruction for X-Ray Computed Tomography G. Wang ${ }^{1}$, J. Zhou ${ }^{2}$, Z. Yu ${ }^{2}$, W. Wang ${ }^{2}$, J. Qi $^{1}$

${ }^{1}$ University of California, Davis, CA, USA

${ }^{2}$ Toshiba Medical Research Institute, Vernon Hills, IL, USA

Low-dose x-ray computed tomography (CT) is of great interests in clinical applications. Iterative methods have been proposed to reconstruct low-dose CT data using either the pre-log data or post-log data. On one hand, pre-log domain methods have the potential to improve image quality by using an accurate noise model, they often suffer slow convergence in practice due to the nonlinear relationship between the attenuation image and pre-log data. On the other hand, post-log domain methods enjoy fast convergence, but image quality can be suboptimal because the logarithm conversion is sensitive to noise at low dose and cannot properly handle negative measurements. This paper proposes a hybrid method that integrates the pre-log model and post-log model together to take advantages of both pre-log and post-log methods. The hybrid method applies a post-log weighted least squares (WLS) model for measurements greater than a pre-determined threshold and a pre-log shifted Poisson (SP) model for measurements less than the threshold. Computer simulations show that the proposed hybrid method can achieve higher signal-tonoise than the standard post-log WLS method and pre-log SP method, while enjoys faster early convergence similar to the standard post-log WLS method.

M09-7: Accurate Transaxial Region-of-Interest Reconstruction in Helical CT?

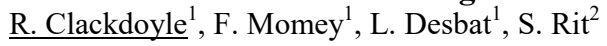

${ }^{I}$ Laboratoire TIMC-IMAG, Université de Grenoble Alpes, Grenoble, France

${ }^{2}$ CREATIS, INSA-Lyon, Lyon, France 
In conventional helical computed tomography (CT), the field-of-view is a cylinder centered on the axis of the helix. In this work we consider region-of-interest (ROI) reconstruction where all measurement lines are blocked except those that intersect a small cylindrical ROI which is not necessarily centered on the axis of the system. Here, we do not address the issue of physical collimation required to achieve such a ROI, nor do we attempt to quantify the possible dose reductions; we are concerned with the question of reconstruction inside the ROI. The patient boundary is assumed known, and we avoid the "interior problem" by focusing on an ROI that includes part of the patient boundary. By applying reconstruction theory, we find that the ROI will contain points for which (ideally) exact image reconstruction is possible, and points for which current theory is not able to establish their reconstructibility (or otherwise). Points in the ROI that are near the patient boundary are always reconstructible, even with heavily truncated projections. Using an iterative algorithm we performed ROI reconstruction from patient data, and compared the results with full-field reconstruction. As predicted, the ROI results were quantitatively accurate near the patient boundary, with gradually increasing bias towards the interior boundary of the ROI. We believe that this behavior may partly be caused by non-uniform convergence of the iterative method, because theory shows increased instability in exactly this direction. In conclusion we have verified that quantitative reconstruction is achievable inside the ROI, with a potential instability at the innermost boundary of the ROI.

This work is partially supported by the Agence Nationale de la Recherche (France), Labex CAMI, number ANR-11-LABX-0004-01 and project DROITE, number ANR-12-BS01-0018.

\author{
M09-8: Atlas-Based Image Reconstruction for Breast CT Imaging Using Non-Isocenteric C-Arm Scanner \\ E. A. Rashed ${ }^{1}$, H. Kudo ${ }^{2}$ \\ ${ }^{I}$ Image Science Lab., Dept. of Mathematics, Faculty of Science, Suez Canal University, Ismailia, Egypt \\ ${ }^{2}$ Division of Information Engineering, University of Tsukuba, Tsukuba, Japan
}

Cone-beam computed tomography $(\mathrm{CBCT})$ is known to provide a high-quality $3 \mathrm{D}$ images for accurate diagnosis of breast cancer compared to conventional 2D mammography. Several CBCT techniques have been developed for breast imaging with different imaging setup and geometry configurations. However, it is more convenient to use a general purpose imaging facility rather than a sophisticated equipment dedicated only for a single imaging application in terms of cost and space usage. This motivate us to study the use of a generic purpose non-isocentric C-arm scanner in 3D breast imaging. The imaging equipment used in this study is known to have several design configurations that limit its use in 3D tomographic imaging. For example, the gantry orbital movement is common to be limited to angular range, which is far below the normal required range in clinical CBCT. Also, the non-isocentric design is known to limit the potential use in 3D imaging as the scanner field-of-view (FOV) is non-stationary and is view-angle dependent. Moreover, it is difficult to acquire enough number of project views with exact angulation value using a manually operated gantry. It is demonstrated that breast tomosynthesis can be successfully achieved using C-arm scanner. Recently, we have developed an image reconstruction method, named PA-MAP algorithm, that incorporate prior information obtained from probabilistic atlas to improve image quality in low-dose CT. The atlas can be generated from a set of earlier scans of different patients and provide a useful prior information in reducing image artifacts in several data limitation problems. In this paper, we study the use of PA-MAP algorithm in CBCT using non-isocentric C-arm scanner for breast imaging.

M10A: Posters: image reconstruction I

Thursday, Nov. 3 16:30-18:30 Etoile

M10A-1: Kernelised EM Image Reconstruction for Dual-Dataset PET Studies

S. Ellis, A. J. Reader

Department of Biomedical Engineering, King's College London, London, UK

In a number of applications, positron emission tomography (PET) requires two or more scans to observe and even quantify changes in function (e.g. tissue metabolism, or receptor binding potentials). Conventionally the raw datasets are reconstructed into images independently, allowing no sharing of information. Kernelised EM (KEM) is a recently proposed PET reconstruction method that utilises one or more sources of prior information to construct spatial basis functions with machine learning techniques for PET reconstruction. Feature vectors are defined for each pixel in the image, and the level of correlation between these feature vectors for different pixels allows spatial basis functions to be created that capture these correlations between similar pixels. This work proposes the use of KEM in dual-dataset PET studies, where the reconstructed image from the primary scan is used to construct spatial basis functions for subsequent scan reconstructions. Feature vectors for each pixel were defined as square patches centred on the pixel in question in the primary scan reconstructions. Using these patch-based feature vectors, basis functions were formed and used to reconstruct the subsequent datasets. Computer simulation studies of a simple dualdataset brain tumour response study with a 50\% change in uptake showed that KEM produces lower errors in the tumour and other regions of structure for the second scan when compared to conventional methods that use no prior information. In addition, visual quality of KEM reconstructions was observed to be superior to that of the competing methods. These results suggest that KEM is applicable to dual-dataset studies, improving reconstructed follow-up images compared to the conventional approaches. 
This work is funded by the King's College London \& Imperial College London EPSRC Centre for Doctoral Training in Medical Imaging (EP/L015226/1)

M10A-2: Improving the Signal-to-Noise Ratio in Static PET Reconstruction Using HYPR-OSEM J.-C. (. Cheng ${ }^{1}$, J. Matthews ${ }^{2}$, R. Boellaard ${ }^{1}$

${ }^{I}$ Department of Radiology and Nuclear Medicine, VU University Medical Center, Amsterdam, The Netherlands

${ }^{2}$ Wolfson Molecular Imaging Centre, The University of Manchester, Manchester, The United Kingdom

We describe an iterative reconstruction algorithm (HYPR-OSEM) which improves the signal-to-noise ratio (SNR) in static imaging (i.e. single frame reconstruction) by incorporating the HighlY constrained back-PRojection (HYPR) de-noising directly within the ordered subsets expectation maximization (OSEM) algorithm. The proposed HYPR operator in this work consists of the image from each subset as the target and the sum of the preceding subset images as the composite which was updated every iteration. 3 strategies were used to apply the HYPR operator in OSEM: i) within the image space modeling component of the system matrix in forward-projection only, ii) within the image space modeling component in both forward-projection and backprojection, and iii) on the image estimate after the OSEM update for each subset (i.e. separated from the update factor) thus generating 3 forms of HYPR-OSEM; namely i) HYPR-F-OSEM, ii) HYPR-FB-OSEM, and iii) HYPR-AU-OSEM. Resolution and contrast phantom simulations with various sizes of hot and cold regions were used to evaluate the performances of various forms of HYPR-OSEM with respect to OSEM and OSEM with post filter. Although the convergence in contrast recovery coefficients (CRC) obtained from all forms of HYPR-OSEM was observed to be slower than that from OSEM (with AU being the fastest among the 3 forms), HYPR-OSEM can achieve a much lower noise level (2-3 times in this case) for a fixed CRC as compared to OSEM. When comparing to the filtered OSEM, HYPR-OSEM images contained similar noise level but with higher CRC due to the degradation of resolution in the filtered OSEM images. In summary, HYPR-OSEM can improve the SNR without degrading the resolution or contrast, and it can achieve better precision than OSEM and better accuracy than filtered OSEM. In addition, the proposed method can be applied to improve the image quality of any frame-based reconstruction for both SPECT and PET. Further optimization and evaluations will be performed.

\section{M10A-3: Compute-Optimised, Direct LOR PET Image Reconstruction for the Siemens Hybrid MR/PET Scanner Exploiting Scanner Symmetries and Rotation-Symmetric Voxel Assemblies}

J. J. Scheins, C. Lerche, N. J. Shah

Forschungszentrum Jülich GmbH, Institute of Neuroscience and Medicine INM-4, Jülich, Germany

Fast PET image reconstruction usually requires a Line-of-Response (LOR) pre-processing step where the detected raw LOR data are interpolated for example to evenly spaced sinogram projection bins or to a generic projection space as proposed for instance by the PET Reconstruction Software Toolkit (PRESTO). Any applied interpolation strategy allows generation of generic projection data which directly supports the usage of scanner-independent reconstruction programs. In this way, speed-optimised, versatile geometrical projectors can be implemented independent of the underlying scanner geometry. Where such optimisations in terms of performance are still insufficient axial and transaxial compression of LOR data is usually applied for an additional reduction of the computational burden.

However, all strategies of projection data interpolation or compression unavoidably lead to a loss of original information and result in some degradation of image quality. Here, direct LOR reconstructions overcome this evident drawback at cost of a massively enhanced calculation effort. Therefore, computational optimisation techniques are essential to make such demanding approaches attractive and economical in the clinical environment.

In this paper, we demonstrate for the Siemens Hybrid MR/BrainPET with 240 million physical LORs that a very fast quantitative direct LOR reconstruction can be realised if utilising the intrinsic scanner symmetries. In general, geometrical symmetries are used within PRESTO to support a highly efficient Single Instruction Multiple Data (SIMD) vectorisation. Now, an extended version of PRESTO is also capable to directly use sets of symmetric physical LORs instead of prior interpolation of LORs to a generic projection space. Exploiting the implemented symmetry-driven SIMD technique and the Simultaneous Multi-Threading (SMT) results in an overall calculation time of 2-3 minutes per frame on a single multi-core machine.

\section{M10A-4: PET 3D Blurring Kernels from Single-Event Coordinates}

R. Taschereau, A. F. Chatziioannou

Crump Institute for Molecular Imaging, University of California Los Angeles, Los Angeles, CA, USA

A method to calculate 3D blurring kernels for iterative PET image reconstruction is presented. The method uses pre-calculated single-event data (obtained from Monte Carlo simulations) to calculate coincidence probabilities in groups of lines of response (kernels). Similar approaches have been explored in the past that typically use segmented crystals and various parameters (angles theta and phi, local coordinates vector ) characterizing the incident photon on the crystal surface. We propose a novel method by which the data kept are coordinates of every single-event, which are obtained from photon interactions with an unsegmented (monolithic) scintillator block. When required for kernel calculations, the distribution of events in crystals from an incident 
photon is obtained from a process we call "dynamic binning". Dynamic binning works by first rotating and translating every coordinate of every event. Next, those transformed events are tallied in a 2D histogram with a bin size equal to the desired crystal size. The benefit of that approach is that only one parameter (theta) is needed and the accuracy on other parameters (phi, $\mathrm{x}$ and $\mathrm{y}$ ) becomes unlimited. The data size and calculation time are also both reduced with respect to a segmented approach. To illustrate the method, blurring kernels have been calculated for the HiPET system, a dual-layer prototype of a preclinical scanner. Reconstructed images of a simulated NEMA NU4-2008 Image Quality Phantom are shown.

\author{
M10A-5: Discrete Iterative Algorithms for Scatter-to-Attenuation Reconstruction in PET \\ $\underline{\text { Y. Berker }}^{1,2}$, V. Schulz ${ }^{2}$, J. S. Karp ${ }^{1}$ \\ ${ }^{I}$ Dept. of Radiology, University of Pennsylvania, Philadelphia, PA, USA \\ ${ }^{2}$ Dept. of Physics of Molecular Imaging Systems, RWTH Aachen University, Aachen, Germany
}

Recently, several groups have proposed the use of scattered coincidences in positron emission tomography (PET), aiming at improved attenuation correction using the PET emission data, e.g., in PET-MRI. In this work, we analyzed the behavior of several algorithms, including reconstruction by two-branch scatter-to-attenuation back-projection (BP) and maximum likelihood expectation maximization with a one-step-late update of the system matrix (MLEM-OSL). A maximum-likelihood gradientascent (MLGA) approach, as previously proposed by us, was tested with four step sizes and several stabilization and acceleration techniques (Armijo step size rule, conjugate gradients, Nesterov acceleration, and subsets). The convergence speed of all algorithms was compared using phantom simulations in fields of view (FOVs) ranging from rat-sized to human-sized. For MLEM-OSL, based on a numerical criterion distinguishing low- and high-attenuation surfaces of response (SOR), the most useful (low-attenuation) SORs were isolated in order to improve convergence speed. We found that the Armijo step size rule improved convergence speed and enabled the use of conjugate gradients, further improving convergence rates. Alternatively, the use of data subsets yielded near-ideal speed-up of MLGA. Even with identical geometries (up to a spatial scale factor), performance of all algorithms depends on the FOV size, suggesting a new kind of scale problem. In particular, shortcomings of MLEM-OSL prevent convergence to the true solution in large FOVs, where MLGA behaves more favorably. Convergence rates of MLEM-OSL were improved by removing high-attenuation SORs, indicating that, opposing intuition, MLEM-OSL convergence can be improved by using less data.

Research reported in this publication was supported by a fellowship within the P.R.I.M.E. program of the German Academic Exchange Service (DAAD), and by the National Cancer Institute of the National Institutes of Health under award number R01CA113941. The content is solely the responsibility of the authors and does not necessarily represent the official views of the National Institutes of Health. Two patent applications relevant to this work have been filed by Koninklijke Philips Electronics N.V. with Y.B. and V.S. as inventors.

\title{
M10A-6: Joint Reconstruction of Activity and Attenuation in Dynamic PET
}

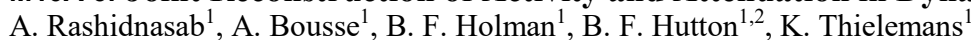

${ }^{I}$ The Institute of Nuclear Medicine, University College London, London, United Kingdom

${ }^{2}$ The Centre for Medical Radiation Physics, University of Wollongong, Wollongong, Australia

In PET imaging, a transmission scan is required for attenuation correction. Alternatively, the attenuation map can be estimated from the emission data. Previously, a reconstruction method based on joint reconstruction of attenuation and emission PET imaging, maximum likelihood activity and attenuation estimation (MLAA), was proposed. However, cross-talk between the activity and attenuation estimation limits the usefulness of MLAA for PET data without time-of-flight (TOF) information. This work introduces dynamic MLAA (dMLAA), an extension of the MLAA algorithm for dynamic data, to jointly reconstruct the activity distributions and a single attenuation map. The hypothesis is that using information from multiple dynamic emission frames improves the estimated single attenuation map compared to using static PET data.

dMLAA consists of two algorithms with an alternating update. In each iteration of dMLAA, the activity distribution for each frame is first updated with maximum likelihood expectation maximisation (MLEM) using the current attenuation map estimate. Second, the attenuation map is updated with maximum likelihood for transmission tomography (MLTR) using the integrated activity distributions estimated in the first step.

dMLAA was tested on phantoms created from reconstructed clinical images of dynamic FDG PET/CT data of brain and thorax using (1) the final dynamic frame, (2) all available dynamic frames to reconstruct the attenuation map and the activity distributions.

Use of multiple dynamic emission frames slightly improved the reconstructed attenuation map especially in bones, cavities, lesion and posterior regions of lung compared to using a single emission frame. However, without TOF, the reconstructed map still suffers from ill-posedness of the problem despite the additional dynamic information. The reconstruction may be improved for tracers that present high variation of contrast between frames and within each frame. 
M10A-7: Direct PET Reconstruction of Regional Binding Potentials P. Gravel ${ }^{1}$, A. Reader ${ }^{2}$

${ }^{I}$ Nuclear Medicine, PERFORM Centre, Concordia University, Montreal, Quebec, Canada

${ }^{2}$ Biomedical Engineering, King's College London, St. Thomas' Hospital, London, UK

This work evaluates a maximum likelihood parameter estimation method for regions-of-interest (ML-ROI) when incorporated in a direct 4D PET image reconstruction framework including the simplified reference tissue model with the basis function method (SRTM-BFM) tracer kinetic model. The ML-ROI algorithm has been evaluated for the usual task of estimating the radioactivity concentration for ROI spatial-bases compared to voxels. We therefore extend the application of this method to include the direct estimation of binding potential (BP) values on simulated 2D+time data sets (with use of [11C]raclopride time-activity curves (TACs) from real data). The performance of the proposed method is evaluated by comparing BP estimates with those obtained from pixel-wise direct reconstruction approaches followed by ROI averaging, as would conventionally be done to obtain regional measures. It is shown that ML-ROI within a direct PET reconstruction framework delivers lower \%BIAS and \%CV, and thus lower \%RMSE, in BP estimates compared to the pixel-wise approach followed by ROI averaging. In addition, the considerable time gained ( 2 orders of magnitude) makes it a potential candidate for routine application.

M10A-8: Performance Improvement and Validation of a New MAP Reconstruction Algorithm

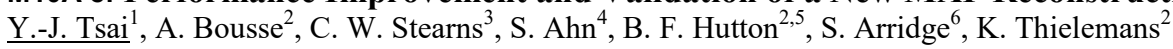

${ }^{I}$ Division of Medicine, University College London, London, UK

${ }^{2}$ Institute of Nuclear Medicine, University College London Hospitals NHS Trust, London, UK

${ }^{3}$ GE Healthcare, GE, Waukesha, US

${ }^{4}$ Global Research, GE, Niskayuna, US

${ }^{5}$ Centre for Medical Radiation Physics, University of Wollongong, Wollongong, Australia

${ }^{6}$ Department of Computer Science, University College London, London, UK

We previously proposed a fast maximum a posteriori (MAP) algorithm, LBFGS-B-PC, combining LBFSG-B with diagonal preconditioning. Previous results have shown in simulations that it converges using around 40 projections independent of many factors. The aim of this study is to improve the algorithm further by using a better initial image and a modified preconditioner that is less sensitive to noise and data scale. By initializing the algorithm with the best initial image (one full iteration of OSEM with 35 subsets), ROI values can converge almost twice as fast for the same computation time. Moreover, the new preconditioner makes the performance more consistent between high and low count data sets. In addition, we have found a means to choose the stopping criteria to reach a desired NMSE level in the reconstructed image. Based on the results with patient data, the optimized LBFGS-B-PC shows promise for clinical imaging.

This project is supported by GE Healthcare, and by the National Institute for Health Research, University College London Hospitals Biomedical Research Centre.

M10A-9: Strategies for Acceleration of a New MLEM Algorithm for Emission-Based Photon Attenuation Correction in PET

$\underline{\text { A. Mihlin }}^{1}$, C. S. Levin ${ }^{2}$

${ }^{I}$ Electrical Engineering, Radiology, Stanford University, Stanford, CA, United States

${ }^{2}$ Radiology, Electrical Engineering, Physics, Bioengineering, Stanford University, Stanford, CA, United States

We report on GPU-based strategies for acceleration of "EMAA" - a maximum likelihood expectation maximization (MLEM) algorithm for joint estimation of emission activity distribution and photon attenuation map from PET emission signal alone. The following strategies are reported: GPU based implementation, ordered subset expectation maximization (OSEM), use of time of flight (TOF) data, and "Adjusted OSEM" - an extension of OSEM for this joint image estimation problem. The strategies are evaluated using a PET signal, computationally generated via Monte-Carlo simulation of a three dimensional XCAT phantom. For a Philips GEMINI LXL system geometry with 400 ps time resolution, these methods accelerate EMAA by six orders of magnitudes compared with a single CPU implementation. Thus, reducing joint estimation duration to just seven minutes, for an emission activity distribution root mean square error of $1 \%$.

This work was partially sponsored by a research grant from Philips Healthcare.

M10A-10: aSRT: A New Analytic Reconstruction Algorithm for SPECT

N. E. Protonotarios ${ }^{1,2}$, A. S. Fokas ${ }^{1,3}$, A. Gaitanis ${ }^{4}, \underline{G}^{\text {. A. Kastis }}{ }^{1}$

${ }^{I}$ Research Center of Mathematics, Academy of Athens, Athens, Greece

${ }^{2}$ Department of Mathematics, National Technical University of Athens (NTUA), Athens, Greece 
${ }^{3}$ Department of Applied Mathematics and Theoretical Physics, University of Cambridge, Cambridge, UK

${ }^{4}$ Center of Clinical, Experimental Surgery, \& Translational Research, Biomedical Research Foundation of the Academy of Athens (BRFAA), Athens, Greece

Introduction: We present the attenuated Spline Reconstruction Technique (aSRT) for single photon emission computed tomography (SPECT). aSRT is an image reconstruction algorithm based on an analytic formulation of the Inverse Attenuated Radon Transform (IART). Our algorithm constitutes a substantial improvement to the Spline Reconstruction Technique (SRT), presented in IEEE NSS/MIC in 2011, due to a new mathematical formulation. The original explicit formulation of IART was proposed by one of the authors in 2006, following the seminal work of Novikov. The aSRT algorithm is an improved mathematical formulation of the original approach. The purpose of this work is (i) to present the mathematics of aSRT, (ii) to reconstruct simulated and real SPECT data using aSRT, in order to evaluate its performance and (iii) to compare aSRT with filtered backprojection (FBP) and ordered subsets-expectation maximization (OSEM).

Materials and Methods: This new mathematical approach to SPECT involves the IART and specifically, the calculation of the Hilbert transform of the attenuation correction coefficient as well as the Hilbert transform of two sinusoidal functions of the attenuated Radon transform of the radioactivity distribution function. We have employed custom-made cubic piecewise polynomials (splines), in order to compute these Hilbert transforms. Comparisons with the industry standards of FBP and OSEM are evaluated using contrast, bias and image roughness. Plots are presented as a function of the number of projections angles. Results: Our new technique can efficiently produce accurate attenuation-corrected reconstructions for simulated phantoms as well as real data. We expect that the aSRT algorithm will provide good quality images with improved performance over FBP and comparable to OSEM. aSRT, by incorporating the attenuation correction within itself, will provide an improved alternative to FBP.

G. A. Kastis acknowledges the financial support of the "Alexander S. Onassis Public Benefit Foundation" under grant No. R ZL 001-0. Furthermore, this work was partially supported by the research program "Inverse Problems and Medical Imaging" (200/842) of the Research Committee of the Academy of Athens.

\section{M10A-11: Initial Validation of Time-of-Flight List-Mode MLEM and OSEM Reconstruction Algorithms in STIR Framework Using Monte Carlo Simulated Data} $\underline{\text { N. Efthimiou }}^{1,2}$, K. Thielemans ${ }^{3}$, C. Tsoumpas ${ }^{1}$

${ }^{I}$ Division of Biomedical Imaging, University of Leeds, Leeds, UK

${ }^{2}$ Biomedical Engineering, Technological Educational Institute of Athens, Athens, GR

${ }^{3}$ Institute of Nuclear Medicine, University College London, London, UK

The most recent Positron Emission Tomography (PET) scanners have reasonably fast time-of-flight (TOF) resolution around $300 \mathrm{ps}$ to $600 \mathrm{ps}$. This offers the opportunity for reconstructing images with higher accuracy and precision. This paper presents a new implementation of a time-of-flight list-mode ordered subsets expectation maximization maximum likelihood reconstruction algorithm (OSEM) in the open source software for tomographic image reconstruction STIR (http://stir.sf.net). The algo-rithm is validated using simulated data. In particular, an acquisition of the NEMA phantom with the mCT Siemens scanner was simulated using the GATE Monte Carlo package. The simulated root file was imported in STIR library as a list-mode file format taking into account all necessary information. The timing resolution of the scanner was set to $600 \mathrm{ps}$. Two challenging datasets of $5 \times 10^{6}$ and $20 \times 10^{6}$ true events were created. Random and scattered coincidences were not considered in this investigation. The validation was made in terms of signal-to-noise ratio (SNR) and contrast-recovery coefficient (CRC). Both SNR and CRC are in good agreement with published literature as it is demonstrated that TOF re-construction converges faster and the peak contrast is better or equal to non-TOF reconstruction. TOF outperforms non-TOF especially when low number of iterations is used. The SNR peaks at about 10 iterations, but without implying that the reconstruction process necessarily stop at this iteration. Contrast recovery is maximized in the first 20 iterations for OSEM and 40 iterations for MLEM, at the ex-pense of lower SNR. Non-TOF demonstrates similar performance, in terms of SNR, to TOF if adequate number of events is provided and a higher number of iteration is performed. In conclusion, we devel-oped a validated list-mode TOF reconstruction feature in the freely distributed STIR framework.

The authors would like to thank Dr. N. Karakatsanis (Mt Sinai Hospital, NY) for the suggestions and fruitful discus-sions. This project was supported by the European Cooperation for Science and Technology Action TD1401: Fast Advanced Scintillation Timing (http://cern.ch/fastcost).

M10A-12: Accelerated Image Reconstruction for Pinhole PET with a Combined Dual-Matrix Dual-Voxel Approach

M. C. Goorden, F. J. Beekman

Radiation, Detection \& Medical Imaging, Delft University of Technology, Delft, Netherlands 
The novel Versatile Emission Computed Tomography (VECTor) technology enables high-energy SPECT and simultaneous SPECT and PET in small animals at sub-mm resolutions using a dedicated pinhole collimator. We recently showed that for optimal resolution and quantitative accuracy of PET images the long tails of the $511 \mathrm{keV}$ Point Spread Functions (PSFs) have to be modelled in image reconstruction. However, as these tails contain many gamma photon paths their inclusion leads to very lengthy reconstructions. Therefore, we propose a combined dual-matrix (DM) dual-voxel (DV) approach to accelerate image reconstruction and thus enable incorporation of full gamma photon transport in the reconstruction process. To accelerate the forward projection step of image reconstruction while still including the PSF tails, a DV method is used: the slowly varying tails are modelled on a two times rougher voxel grid than the central parts of the PSFs. Furthermore, a DM approach is taken in which the backprojection uses a different PSF than the forward projections with a smaller part of the tails included. This DM-DV reconstruction is implemented in OSEM and in recently proposed accelerated similarity-regulated OSEM (SROSEM). Both a visual assessment and a quantitative contrast-noise analysis confirm that images of a hot rod and a uniformity phantom are practically identical when reconstructed with standard OSEM, DM-DV-OSEM or DM-DV-SROSEM. However, compared to OSEM, DM-DV-OSEM and reaches the same contrast 30 and 25 times faster for the resolution and uniformity phantom respectively. With DM-DV-SROSEM these acceleration factors increase to 54 and 30.

\section{M10A-13: Simultaneous Estimation of Activity and Attenuation in Classical PET: Uniqueness Issues} $\underline{\text { M. Defrise }}$

Dept. of Nuclear Medicine, Vrije Universiteit Brussel, Brussels, Belgium

This work revisits the problem of simultaneous reconstruction of activity and attenuation for classical (non-TOF) PET. It is known that in contrast with TOF PET, the non-TOF data do not determine the activity ? even up to a multiplicative factor. However the examples of non-uniqueness are usually restricted to cases where the activity and attenuation are circularly symmetric ("radial"). We prove the existence of large classes of 2D non-radial objects $(\mu, ?)$ such that the activity? is not determined by non-TOF data. Consequently, there are many situations in classical 2D PET where the emission activity distribution? can not be determined from the data unless the attenuation $\mu$ is known.

M10A-14: Simultaneous PET-MR Joint Reconstruction with Information Theoretic Priors

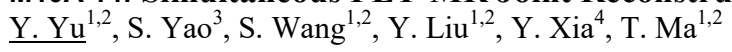

${ }^{I}$ Key Laboratoty of Particle and \& Radiation Imaging (Department of Engineering Physics, Tsinghua), Ministry of Education, Beijing, China

${ }^{2}$ Department of Engineering Physics, Tsinghua University, Beijing, China

${ }^{3}$ Department of Nuclear Medicine, Chinese PLA General Hospital, Beijing, China

${ }^{4}$ Beijing Institute of Spacecraft Environment Engineering, Beijing, China

Utilizing simultaneous functional and anatomic information, PET/MR joint reconstruction can improve images quality of both modalities by exploring the image similarities. Similarity of two images can not only be measured by structure priors such as parallel level set (PLS) or joint total variation (JTV), but also by information theoretic similarity priors such as mutual information (MI) and joint entropy (JE). MI between two random vectors can be interpreted as a similarity metric between two images, which has been successfully applied in image registration between two modalities and also PET-MAP reconstruction with MI based anatomical priors. We propose a novel approach to introduce mutual information into PET/MR joint reconstruction. A numerical phantom, a brain phantom and real human data were used for evaluation with four different priors: proposed MI and JE priors, JTV and quadratic PLS for comparison. In numerical phantom studies, MI and JE both achieved a better resolution than JTV and QPLS. For shared features in MR/PET images, JE gave the smallest bias and variance with $96.3 \%$ reduction of bias compared with JTV and 95\% less than QPLS. Variance was also reduced by $96 \%$ and $92.3 \%$ respectively. MI also gave $77.2 \%$ and $68.7 \%$ reduction in bias compared with JVT and QPLS. The variance reduction was $75 \%$ and $66 \%$ respectively. For other regions, the bias and variance are comparable among JTV, QPLS and MI. JE confounded the value of unique lesions in PET phantom. For undersampled MR data, MI/JE priors obviously reduced the artifacts in MR images and meanwhile obtained better image resolution for PET compared with JTV and QPLS. For brain phantom and patient study, sharpness and visibility of fine image features were also clearly improved for PET images with MI and JE. In conclusion, PET/MR joint reconstruction with information theoretic priors substantially improve resolution and accuracy of both PET and MR images with both simulation and real human data.

M10B: Posters: quantitative imaging I

Thursday, Nov. 3 16:30-18:30 Etoile

M10B-1: MLAA-Based Headphone Attenuation Estimation in Hybrid PET/MR Imaging

T. Heußer ${ }^{1}$, C. M. Rank ${ }^{1}$, M. T. Freitag ${ }^{2}$, M. Kachelrie $\beta^{1}$

${ }^{I}$ Medical Physics in Radiology, German Cancer Research Center (DKFZ), Heidelberg, Germany

${ }^{2}$ Dept. of Radiology, German Cancer Research Center (DKFZ), Heidelberg, Germany 
Purpose: To estimate headphone attenuation in hybrid PET/MR imaging using maximum likelihood reconstruction of attenuation and activity (MLAA).

Materials and Methods: Attenuation of flexible hardware components such as MR body coils and MR-safe pneumatic headphones is currently neglected in clinical routine. Ignoring headphone attenuation has been shown to result in local brain activity underestimation values of up to $15 \%$. To obtain an estimate of the headphone attenuation, we propose to employ the MLAA algorithm to simultaneously estimate attenuation and activity distributions outside the patient body outline. Subsequently, the outline of the headphones is segmented and pre-defined average attenuation coefficients are applied. The average headphone attenuation coefficients were empirically derived performing phantom measurements and chosen such that the activity underestimation when ignoring headphone attenuation could be compensated for. For clinical evaluation, we investigated the proposed headphone attenuation estimation for six PET/MR patient data sets acquired with a Siemens Biograph mMR.

Results: For the headphones used in our mMR system it turned out that an average attenuation coefficient of $\mu=0.009 \mathrm{~mm}^{-1}$ was required to compensate for the activity underestimation in the phantom measurements, reducing the maximum underestimation from $12.7 \%$ to $2.1 \%$. Ignoring headphone attenuation resulted in an average activity underestimation across the six patient data sets of $3.9 \%$ evaluated in the full brain and $8.6 \%$ evaluated in the cerebellum, compared to compensating for headphone attenuation using the proposed method.

Conclusion: We propose a method to estimate PET/MR headphone attenuation making use of the MLAA algorithm. The proposed method was shown to significantly reduce activity underestimation in both phantom and patient data and can, potentially, be readily included into clinical workflow.

M10B-2: Five-Dimensional Respiratory and Cardiac Motion Compensation for Simultaneous PET/MR C. M. Rank ${ }^{1}$, T. Heußer ${ }^{1}$, A. Wetscherek ${ }^{1}$, M. T. Freitag ${ }^{2}$, H.-P. Schlemmer ${ }^{2}$, M. Kachelrie ${ }^{1}$

${ }^{I}$ Medical Physics in Radiology, German Cancer Research Center (DKFZ), Heidelberg, Germany

${ }^{2}$ Radiology, German Cancer Research Center (DKFZ), Heidelberg, Germany

We propose a new method for 5D motion-compensated PET reconstruction, which is based on radial MR data and which compensates for both respiratory and cardiac patient motion. The new method is evaluated for simultaneous ${ }^{18} \mathrm{~F}-\mathrm{FDG}$ PET/MR acquisitions of four patients with a total acquisition time of $5 \mathrm{~min}$ per bed position. PET/MR data are sorted into 20 overlapping respiratory phases and 12 overlapping cardiac phases. Respiratory and cardiac motion vector fields are estimated sequentially from MR data employing a novel algorithm, which alternates between MR image reconstruction and motion estimation. For 5D motion-compensated PET reconstruction, the resulting motion vector fields are incorporated into the system matrix. 5D motioncompensated PET images reveal less motion blurring than 3D motion average PET and considerably lower noise levels than 5D double-gated PET reconstructions. The myocardium of all four patients is evaluated quantitatively in a diastolic cardiac phase. In comparison to 3D PET reconstructions, 5D MoCo PET yields an average increase of $\mathrm{SUV}_{\text {mean }}$ and contrast of $2.8 \pm 1.1 \%$ and 4.8 $\pm 2.0 \%$, respectively.

M10B-3: Quantification Accuracy of a New HRRT High Throughput Rat Hotel Using Transmission-Based Attenuation Correction: a Phantom Study

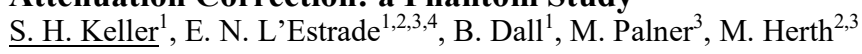

${ }^{I}$ Department of Clinical Physiology, Nuclear Medicine and PET, Rigshospitalet, University of Copenhagen, Copenhagen, Denmark

${ }^{2}$ Department of Drug Design and Pharmacology, Faculty of Health and Medical Sciences, University of Copenhagen, Copenhagen, Denmark

${ }^{3}$ Neurobiology Research Unit, Rigshospitalet, University of Copenhagen, Copenhagen, Denmark

${ }^{4}$ Lund University Hospital, Lund, Sweden

Aim: To parallelize scanning and save time and cut costs of preclinical studies we have designed a new hotel holding 4 rats in the HRRT, which has a spatial resolution close to that of preclinical PET scanners. In this work we test the quantitative accuracy on phantoms in the hotel using different attenuation corrections methods on the HRRT. Material and Methods: The rat hotel has 4 compartments made of acrylic plastic with an $8 \mathrm{~mm}$ base plate and a $3 \mathrm{~mm}$ half-cylinder lid. Four $50 \mathrm{ml}$ syringes filled with $[18 \mathrm{~F}]-F D G$ in water were used as phantoms and scanned in the rat hotel for $20 \mathrm{~min}$. on the HRRT and a high statistics speed 10 transmission scan was acquired. Three $\mu$-map processing/reconstruction methods - MAP-TR with either human head (HH) or water phantom (WP) prior and TXTV - were used and $\mu$-maps and PET images reconstructed with each of the $3 \mu$-maps evaluated. Results: The $\mu$-maps all underestimated the LAC of the acrylic plastic material as compared to CT, and the base plate thickness was underestimated. Activity concentrations were thus also underestimated: $-4.6 \%$ using HH, $-8.7 \%$ using TXTV and $13.8 \%$ with WP. No noteworthy local variations were found. Conclusion: We found a global underestimation of PET activity, which was within a $\pm 5 \%$ acceptance range using MAP-TR with the human head prior and a long transmission scan (speed 10 ). Fine tuning HH or TXTV parameters might give further improvements. 


\title{
M10B-4: Combining CTs in Multi-CT Dynamic PET Acquisitions Increases Reproducibility and Reduces
} Kinetic Parameter Estimate Errors.

B. F. Holman, B. F. Hutton, K. Thielemans

Institute of Nuclear Medicine, University College London, London, UK

Dynamic PET/CT is becoming more popular for imaging diffuse lung diseases. However, to ensure patient comfort, it is often necessary to split the acquisition into multiple sections. As the CT for attenuation correction (AC) of the PET data at each section is subject to respiratory cycle induced density variations, the reconstructed PET quantitation is also affected. This may result in tissue time activity curve (TTAC) errors, decreasing the accuracy of the kinetic analysis. Here we demonstrate the extent of the errors and test a method of combining the CT information to improve kinetic parameter accuracy and reproducibility. Simulated multi-section TTACs were derived from STIR reconstructed dynamic XCAT phantoms. Next, 5 patients with idiopathic pulmonary fibrosis underwent dynamic 18-FDG PET/CT in 3 parts. The PET data was reconstructed using the appropriate CT from each section for $\mathrm{AC}$. The $\mathrm{CT}$ images were then registered and averaged to create a single combined $\mathrm{CT}$ image which was used as the AC map for reconstruction of the PET data. The sections from each set of PET images were registered and lung TTACs determined. The 2-tissue irreversible compartment model and Patlak influx rate constants (Ki) were estimated and the goodness of fit (GoF) errors from each simulation and patient reconstruction method compared. The simulated and original patient reconstruction TTACs displayed displacement between study sections resulting in poor kinetic fits. These displacements were reduced with the combined CT for AC reconstructions. GoF errors in Ki were reduced by a factor of 4 and 28 for the 2TICM and Patlak analysis respectively using the new technique. The difference between the two methods of calculating Ki reduced from $44 \pm 109 \%$ to $28 \pm 14 \%$ when the combined CT for AC was utilised. Using a combined CT for AC of PET data in multi-section protocols for imaging lung tissue significantly reduces errors in kinetic parameter estimates and improves reproducibility.

We acknowledge funding support from GSK (STU100028576) and the EPSRC (EP/K0052781/1). The data for this study was provided by GSK from study NCT01725139. Also, this project is supported by researchers at the NIHR, UCLH Biomedical Research Centre.

\author{
M10B-5: A Method for Thresholds Optimization in Photon Counting Spectral CT

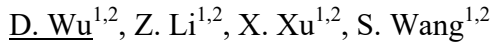 \\ ${ }^{I}$ Key Laboratory of Particle \& Radiation Imaging (Tsinghua University), Ministry of Education, Beijing, China \\ ${ }^{2}$ Engineering Physics, Tsinghua University, Beijing, China
}

Photon counting spectral CT has the potential of quantitative k-edge imaging and reducing patient dose by exploiting the spectral information of incident X-ray photons. One of the key factors that influence the reconstruction noise is the configuration of detector's thresholds. In this paper, we proposed a method for thresholds optimization for optimal reconstruction noise. Rather than an exhaustive search of all the thresholds, approximate numerical models of the scanned object, the source spectrum and the detector's energy response function were established. The material thicknesses were built into probability distributions and Cramer-Rao lower bound was calculated for each thickness sample, and then weighted summed to estimate the overall noise level. The thresholds were optimized via the differential evolution algorithm to minimize the weighted summed Cramer-Rao lower bound. Experiments were carried out with an XCounter Flite X1 detector and a VJ X-ray source of 100kVp. The contrast to noise ratio of a $50 \mathrm{umol} / \mathrm{ml} \mathrm{Gd}$ solution in the Gd image was calculated to evaluate the noise level. The optimized thresholds were in well accordance with the results from exhaustive search, which indicated the effectiveness of the proposed method.

This work is supported in part by National Natural Science Foundation of China (NNSFC) under Grant 11435007, 11235007 , and 11275104.

\author{
M10B-6: Simultaneous Activity and Crystal Efficiencies Reconstruction for Continuous Motion Bed \\ Acquisition \\ V. Y. Panin
}

Molecular Imaging, Siemens Healthcare, Knoxville, TN, USA

PET scanner quality check control is performed on a daily basis. In Siemens scanners, for example, data are acquired from a uniform cylinder and the estimation of a crystal effiencies (CE) normalization component is carried out. Besides producing a normalization array, the $\mathrm{CE}$ analysis provides information about whether or not additional calibration should be performed. In previous work we demonstrated that the CE can be simultaneously reconstructed with activity from TOF patient data. Therefore, scanner performance can be monitored as patient scans occur, eliminating the need for the frequent quality check scan. We extended this work to include Continuous Bed Motion (CBM) acquisition. In CBM data formation, counts from various detector pairs are combined, taking into account the bed motion. While it is beneficial for activity reconstruction, axial CE structure is practically lost in the corresponding computed normalization array due to averaging over motion. We suggest the use of additional data, produced similarly with step by step (stationary) acquisition. In this complimentary data set, activity is integrated over motion; however, the CE structure is preserved. The same rebinner can be used to generate both data sets. Using two 
complimentary data sets, simultaneous reconstruction of activity and CE becomes practical for the CBM case. The algorithm can be interpreted as activity ML reconstruction with a nested loop of CE estimation, which uses the same data but are compressed differently. Patient data from a Siemens mCT Flow scanner were used to validate the algorithm's performance. The proposed method produced crystal efficiencies maps similar to those of the daily scans.

M10B-7: Patient Specific Motion Model for an Optimal Respiratory Motion Correction in PET/MR Imaging H. Fayad $^{1,2}$, H. Schmidt ${ }^{3}$, T. Kustner ${ }^{3,4}$, D. Visvikis ${ }^{1}$

${ }^{I}$ INSERM UMR1101, LaTIM, Brest, France

${ }^{2}$ Université de Bretagne Occidentale, Brest, France

${ }^{3}$ Department of Radiology, University of Tubingen, Tübingen, Germany

${ }^{4}$ University of Stuttgart, Stuttgart, Germany

Positron emission tomography (PET) / magnetic resonance imaging (MRI) is an imaging technique allowing simultaneous PET and MR acquisition and therefore leading to a direct registration and fusion of anatomical and functional information. There are however some challenges in order to ensure an accurate spatial registration of these two types of images. The major one, even in multimodality devices where images are acquired with the patient in the same position, is patient respiratory motion. Respiratory motion is a source of artifacts in PET/MR imaging leading to a reduction of both quantitative and qualitative accuracy of reconstructed PET images. The use of respiratory synchronized PET and MR acquisitions (4D) suffers from low signal to noise ratio which can be increased by including the non-rigid deformations extracted from the anatomical information during the reconstruction process. Four frames have been usually used for the derivation of such deformations which correspond to a temporal resolution of approximately $1250 \mathrm{~ms}$ considering a 5 seconds respiratory average cycle. The goal of this work is to find the ideal temporal resolution of the deformation matrices used in the PET image correction process by using a MR compressed sensing based respiratory model. Results on two patients indicate that the use of such model allows improving the temporal resolution of the deformation matrices used in the PET image correction process and therefore improve the accuracy of the PET respiratory corrected images. The optimal temporal resolution based on this two patients study was approximately $625 \mathrm{~ms}$.

\section{M10B-8: Highly Efficient Motion-Corrected Simultaneous Cardiac PET-MR Imaging}

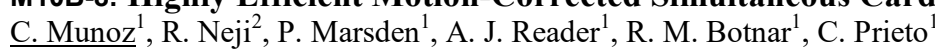

${ }^{I}$ Department of Biomedical Engineering, King's College London, London, United Kingdom

${ }^{2}$ MR Research Collaborations, Siemens Healthcare, Frimley, United Kingdom

MR-based motion correction of PET data has been proposed as a high-impact application for improving image quality and myocardial tissue quantification in cardiac PET-MR imaging. However, current PET-MR motion compensation schemes acquire only motion information from MR data during PET acquisition time and diagnostic MR afterwards, significantly increasing total exam time. Here we propose an efficient acquisition and reconstruction scheme for simultaneous cardiac PET-MR imaging that acquires both diagnostic and motion information from MR, with motion information being used to correct both PET and MR data. The proposed MR acquisition scheme includes acquiring low-resolution 2D image navigators at each heartbeat by spatially encoding the start-up echoes of an ECG-gated gradient echo sequence, allowing for $100 \%$ MR scan efficiency (i.e. all data is accepted for reconstruction, reducing scan time). These 2D image navigators are then used to estimate foot-head and right-left motion of the heart during the respiratory cycle in a beat-to-beat fashion, providing motion estimates to translationally correct and bin the MR data. MR images from each respiratory bin are used to estimate 3D non-rigid motion between respiratory bins. These non-rigid motion fields are then used for correcting both the MR and PET data. Results from healthy volunteers show that motion correction improves MR visualisation of the right and left anterior descending coronary arteries. A simulated PET acquisition showed improvements in contrast of the myocardium and better depiction of papillary muscles when applying motion correction. The presented framework improves image quality and contrast compared with uncorrected images for both MR and PET images.

This work is funded by the King's College London \& Imperial College London EPSRC Centre for Doctoral Training in Medical Imaging $(\mathrm{EP} / \mathrm{L} 015226 / 1)$

M10B-9: The Effect of Isoflurane on 18F-FDG Uptake in the Rat Brain: a Fully Conscious Dynamic Study Using Motion Compensation M. G. Bickell ${ }^{1}$, B. De Laat ${ }^{1}$, R. Fulton ${ }^{2,3}$, G. Bormans ${ }^{4}$, J. Nuyts ${ }^{1}$

${ }^{I}$ Department of Nuclear Medicine, Katholieke Universiteit Leuven, Leuven, Belgium

${ }^{2}$ Brain \& Mind Research Institute and the Faculty of Health Sciences, University of Sydney, Sydney, Australia

${ }^{3}$ Department of Nuclear Medicine, Westmead Hospital, Sydney, Australia

${ }^{4}$ Department of Radiopharmacy, Katholieke Universiteit Leuven, Leuven, Belgium 
In preclinical studies anaesthesia is used to ensure that the animal does not move during the scan. However, the anaesthesia may have confounding effects on the drug or tracer kinetics under study, and the nature of these effects is usually not known. We have implemented a protocol for tracking the rigid motion of the head of a fully conscious rat during a PET scan and performing a motion compensated list-mode reconstruction of the data. Using this technique we have conducted 8 rat studies to investigate the effect of isoflurane on the uptake of $18 \mathrm{~F}-\mathrm{FDG}$ in the brain, by comparing awake and anaesthetised scans. Our results indicate that the isoflurane decreases the relative regional FDG uptake in the cortex and diencephalon, while increasing it in the hindbrain region. Limited effect on the cerebellum was observed.

M10B-10: Garment Compensation in Marker-Less Motion Tracking for Medical Imaging C. Lindsay, M. A. King

Radiology, University of Massachusetts Medical School, Worcester, United States

To mitigate image artifacts resulting from patient motion, detection and tracking of motion is necessary. Existing techniques to track patient motion are either image-derived or utilize systems external to the imaging modality such as visual tracking systems (VTS). VTS methods adopted in clinical research often use optical stereo cameras to track retro-reflective markers placed on the patient to provide a measure of motion for a target structure. However, these systems have important limitations including: high equipment costs, hindrance to patient workflow, marker slippage, and frequent calibration and maintenance. An alternative is to use marker-less VTS, which employ small low-cost depth-sensing optical cameras that can be placed inside the imaging bore and track 1000's of points without markers using disturbances in reflected light patterns. Despite the advantages of size and not needing markers, marker-less VTS cannot directly see the patient's body surface, only the surface of the garments covering their body. In lieu of asking the patients to disrobe, we have developed a method to enable tracking of the body surface of patients despite being fully clothed. Our method is based on physical simulation of Newtonian forces acting on the clothing's fabric to accurately predict how a garment will deform from a given a body shape and motion. This work leverages recent advances in the computer graphics field that now enable robust methods for capturing and modeling the shape, motion, elasticity, and other properties of garments. In simulations, we show the ability to track points on the surface of the torso despite loose and wrinkled garments and our findings confirm that garment compensation will be a necessary and important part of the overall motion tracking methods which utilize marker-less VTS.

This work was supported by the National Institute of Biomedical Imaging and Bioengineering (NIBIB) grant K25EB019032-01A1. The contents are solely the responsibility of the authors and do not represent the official views of the NIBIB.

M10B-11: An Attenuation Method for Reducing Count Rate Losses in Preclinical PET During Intratherapeutic Imaging

M. Dahlbom ${ }^{1}$, E. Mellhammar ${ }^{2}$, S. Evans-Axelsson ${ }^{1}$, T. Tran ${ }^{3}$, J. Axelsson ${ }^{4}$, S.-E. Strand ${ }^{2,5}$

${ }^{I}$ Department of Molecular \& Medical Pharmacology, David Geffen School of Medicine at UCLA, Los Angeles, CA, USA

${ }^{2}$ Department of Clinical Sciences, Lund University, Lund, Sweden

${ }^{3}$ Lund University Bioimaging Center, Lund University, Lund, Sweden

${ }^{4}$ Department of Physics, Lund University, Lund, Sweden

${ }^{5}$ Department of Medical Radiation Physics, Lund University, Lund, Sweden

In preclinical imaging, tumor response to radionuclide therapy can be monitored with PET. Radionuclides used for therapy such as Lu-177 emit a significant amount of low energy gammas. These gammas have an energy high enough to penetrate the imaged object and are likely to be detected. Although these gammas are likely to be rejected electronically, they add dead-time to the system since they need to be processed by the electronics. This is a problem in high-sensitivity pre-clinical PET system with a low number of readout channels, such as the Genisys G4 used in this work. The low energy gammas may also affect image quality due to increased probability of pulse pile-up. The use of lead shields to absorb most of the low energy gamma rays emitted from therapeutic radionuclide were investigated. Cylindrical lead shields were constructed with thicknesses between 1.4 and $7.4 \mathrm{~mm}$. The diameter of the shields was wide enough to fit around a $10 \mathrm{ml}$ plastic vial, which served as the phantom. The attenuation of the shields for annihilation radiation was measured by adding the phantom with $2.5 \mu \mathrm{Ci}$ of F-18. Measurements of the coincidence rate were performed with the lead shields in place. At a thicknesses of 3.6 and $7.2 \mathrm{~mm}$, the coincidence rate was reduced by a factor or 0.52 and 0.25 , respectively. To study the effect of the presence of low energy gammas on the count rate and the efficacy of the lead shields, Tc-99m was added to the vial source. When $100 \mu \mathrm{Ci}$ of Tc-99m was present, the coincidence rate was reduced to $25 \%$ of the original, background free, count rate due to increased dead-time. At 1,000 $\mu \mathrm{Ci}$ the system was paralyzed. Adding $3.6 \mathrm{~mm}$ lead shields, the coincidence count rate was restored to within $3 \%$ in the presence of $100 \mu \mathrm{Ci}$ of Tc$99 \mathrm{~m}$. For the higher activity level, $7.2 \mathrm{~mm}$ was needed to restore the coincidence rate to within $10 \%$ of the original background free. This may be due to dead-time from photons escaping through the ends of the lead cylinders.

M10B-12: A Recovery Coefficient Study Using Micro-Spheres in Clinical PET/CT Scanners

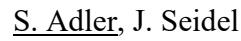


Aim: To measure and study the recovery coefficients of micro-spheres in clinical PET/CT scanners. Materials and Methods: Seven microspheres with inner diameters ranging from $3.95 \mathrm{~mm}$ up to $15.43 \mathrm{~mm}$, were imaged in a Jaszczak ECT Phantom, in air and with sphere to background activity concentration ratios of 15 to 1 for 16 minutes. The images were reconstructed to $2 \times 2 \times 2$ $\mathrm{mm}$ voxel size images using the default clinical reconstruction method supplied by the scanner. The imaging protocol was performed on five different PET/CT scanners; the Gemini TF and Vereos by Philips, the mCT and HRRT by Siemens, and the Discovery 710 by General Electric. Results: The NEMA NU2 recovery coefficient for the $15.43 \mathrm{~mm}$ and $12.43 \mathrm{~mm}$ diameter spheres were about the same within each scanner. The Philips Vereos and HRRT scanners had RC values of $108 \%$ and $104 \%$ respectively, while the GE Discovery 710 was $118 \%$ and the Siemens mCT was $126 \%$. The Gemini TF was $76 \%$. For the smallest micros-sphere of $3.95 \mathrm{~mm}$, the RC were $6.6 \%, 3.6 \%, 13 \%, 8.9 \%$ and $6.1 \%$ for the Gemini TF, Vereos, Discovery 710 , $\mathrm{mCT}$ and HRRT respectively. An anomalous increase in the RC for the Discovery 710 and $\mathrm{mCT}$ was found for sphere sizes 9.89 $\mathrm{mm}$ diameter spheres where the RC was $161 \%$ and $145 \%$ for each respectively. A related recovery coefficient parameter called RC summed which measures the recovery coefficient of the "summed" activity in the spheres did not show this anomaly of increased recovery above $100 \%$ at the $9.89 \mathrm{~mm}$ sphere diameter measurement. Conclusion: The recovery coefficient data resulting from scanning five different scanners is presented. An increase in the recovery coefficient is seen in two scanners which is possibly due to over correction by the iterative reconstruction used to generate the images. This effect is not present when measuring the total activity in the sphere.

\author{
M10B-13: Performance of a Scatter Pre-Calculated Kernel Based System Matrix in Preclinical SPECT \\ Quantification Studies \\ B. Auer, F. Boisson, V. Bekaert, D. Brasse \\ Institut Pluridisciplinaire Hubert Curien (IPHC), Strasbourg, Alsace, France
}

In Single Photon Emission Computed Tomography (SPECT), attenuation and scatter introduce important artifacts in the reconstructed images, which could lead to misdiagnosis for patient's follow-up. Gold standard Monte Carlo Simulation (MCS) is one of the well-established tools that has been used in SPECT image reconstruction due to its ability to accurately model photon transport. However, MCS requires extensive computation time to obtain a low noise system matrix and are therefore inappropriate for the rate of daily exams performed in both clinical and preclinical routine: an improvement in simulation speed is thus mandatory. In this work, we first propose to simplify and accelerate the modeling of the physical effects occurring in the subject in an acceptable time for routine by using a system matrix factorization associated to a scatter pre-calculated database approach. Secondly, a preclinical quantification performance study of the developed subject-dependent attenuation and scatter correction is presented. For that purpose, a modified NU-4 IQ phantom is used to assess the quality of the reconstructed images for each system matrix (no correction, attenuation correction, attenuation and scatter correction). The developed approach, taking into account attenuation and scattering occuring in the subject, allows the users to obtain quantitative data in less than an hour. The reconstruction workflow leads to significant image artifacts reduction as well as a $6 \%$ improvement in terms of recovery coefficients.

M10B-14: System Specific Modeling for Absolute Quantification of 99mTc and 177Lu with SPECT/CT $\underline{\text { H. Ryu }}^{1}$, K. P. Willowson ${ }^{2}$, S. R. Meikle ${ }^{1}$, D. L. Bailey ${ }^{1}$

${ }^{I}$ Faculty of Health Sciences, University of Sydney, Sydney, Australia

${ }^{2}$ Institute of Medical Physics, University of Sydney, Sydney, Australia

The use of 177Lu-DOTATATE is rapidly growing for radionuclide therapy. 177Lu emits B-radiation for the cancer treatment and ?-radiation for localizations. In this study, quantitative SPECT was evaluated for a Symbia Intevo 6 (Siemens Medical Solutions, Germany) SPECT/CT with $177 \mathrm{Lu}$ and $99 \mathrm{mTc}$. The aim of this study was to assess the accuracy of quantitative SPECT images from absolute activity concentrations $(\mathrm{kBq} / \mathrm{ml})$ and as a SUV by using CT-based scatter and attenuation correction for $99 \mathrm{~m}$ Tc with dead time, sensitivity, and scatter measurements. For dead time measurements, a cylindrical phantom with $2 \mathrm{GBq} 99 \mathrm{mTc}$ and $10 \mathrm{GBq} 177 \mathrm{Lu}$ was scanned over different time points. The sensitivity was measured with $30 \mathrm{MBq} 177 \mathrm{Lu}$ and $12 \mathrm{MBq} 99 \mathrm{mTc}$. The Transmission-dependent scatter correction method was use, and the scatter function was measured from a line source in air and water. Scatter fraction was calculated using the build-up function modeled with $200 \mathrm{MBq}$ of $177 \mathrm{Lu}$ and $260 \mathrm{MBq}$ of $99 \mathrm{mTc}$ in different depths of water. The OSEM was used for the reconstruction, and the iterative Chang attenuation correction was performed based on the CT-derived attenuation correction map. The images were converted to absolute activity with $\mathrm{kBq} / \mathrm{ml}$ and SUV using the measured camera sensitivity factor. A NEMA IEC body phantom was used for the validation of the quantitative SPECT methodology. The paralyzable and non-paralyzable constants for dead time correction were $2.84 \times 10-6 \mathrm{~s}$ and $-1.13 \times 10-6 \mathrm{~s}$ for $99 \mathrm{mTc}$, and $9.10 \times 10-7 \mathrm{~s}$ and $-7.08 \times 10-7 \mathrm{~s}$ for $177 \mathrm{Lu}$. The sensitivity factor of the system was $100.43 \mathrm{cps} / \mathrm{MBq}$ for $99 \mathrm{mTc}$ and $12.16 \mathrm{cps} / \mathrm{MBq}$ for $177 \mathrm{Lu}$. The scatter function slope was $0.35 \mathrm{~cm}-1$ for $177 \mathrm{Lu}$ and $0.49 \mathrm{~cm}-1$ for $99 \mathrm{mTc}$. The scatter build-up parameters were $2.15,1.16$ and 0.29 for $177 \mathrm{Lu} ; 1.50,0.513$ and 0.659 for $99 \mathrm{mTc}$, respectively. The activity concentration showed $0.38 \%$ differences from the true concentrations, and the SUV value of the phantom image also matched to the true concentration ratio very well. 
M10B-15: Influence of Respiratory Signal Sampling Rate on Motion Resolution in Respiratory-Gated PET $\underline{\text { F. Buether }}^{1,2}$, M. He $\beta^{2}$, L. J. Frohwein ${ }^{2}$, K. P. Schaefers ${ }^{2}$

${ }^{I}$ Department of Nuclear Medicine, University Hospital of Muenster, Muenster, Germany

${ }^{2}$ European Institute for Molecular Imaging, University of Muenster, Muenster, Germany

During the last years, respiratory gating of PET data has become the established method-of-choice for minimizing breathing motion effects on thoracic and abdominal structures in PET. Additionally, gated PET may serve as the basis for more sophisticated motion correction algorithms. Crucial to all these approaches is the acquisition of respiratory motion signals during the measurement. This can be done either by using additional dedicated hardware, by PET raw data driven methods, or by CT or MRI information. In all approaches, the question arises how good the temporal resolution of the signals have to be to not loose significant amounts of motion resolution. We here investigate the influence of signal sampling on gating success in terms of reconstructed displacements between maximum inspiration and expiration phases. To this end, 14 [F-18]FDG list-mode datasets comprising high-uptake structures were retrospectively analyzed. Respiratory signals were calculated using a data-driven approach with signal sampling rates of $20 \mathrm{~Hz}, 2 \mathrm{~Hz}, 1 \mathrm{~Hz}$, and $0.5 \mathrm{~Hz}$. Additionally, the signal at $0.5 \mathrm{~Hz}$ was interpolated to 20 $\mathrm{Hz}$ by means of a cubic spline algorithm. These signals were used for gated reconstructions (amplitude-based gating, 10 gates), and the apparent respiratory displacements between gate 1 and 10 were determined. We found that motion resolution dropped with decreasing sampling rate (mean decrease at $0.5 \mathrm{~Hz}$ as compared to $20 \mathrm{~Hz}$ : $23 \%$, range: $11 \%$ - 45\%) and increasing respiratory rates of the patients. Spline interpolation of the $0.5 \mathrm{~Hz}$ signal improved motion resolution (mean decrease as compared to $20 \mathrm{~Hz}: 11 \%$, range: $3 \%-15 \%$ ). A sampling rate of $2 \mathrm{~Hz}$ seems to be sufficient to resolve most of the motion (mean decrease as compared to $20 \mathrm{~Hz}: 2 \%$, range: $0 \%-11 \%$ ).

\title{
M10B-16: Quantification of TSPO Expression Using [18F]DPA-714 in a Mouse Model of Epilepsy: Impact of Image Processing

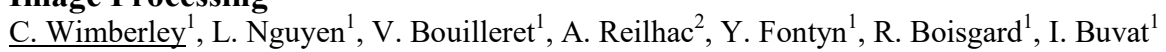 \\ ${ }^{I}$ Laboratoire Imagerie Molecular In Vivo (IMIV), CEA/I2BM/Service Hospitalier Frederic Joliot, Orsay, France \\ ${ }^{2}$ CERMEP, Lyon, France
}

Mesial temporal lobe epilepsy (MTLE) is the most common type of epilepsy. A mouse model of MTLE by injection of kainic acid (KA) can reproduce the pattern of disease process. TSPO PET imaging can follow neuroinflammation associated with MTLE using [18F]-DPA-714, but there are quantification challenges due to the small size of the brain structures in the mouse leading to partial volume effects. The aim of this study was to investigate the impact of spatial resolution recovery and noise removal on binding parameter estimates, and the statistical significance of differences in binding parameters between a group of mice before and after induction of the model of epilepsy.

The model was induced by injection of KA into the right dorsal hippocampus of adult male C57/B16 mice ( $\mathrm{n}=6$ ). A dynamic [18F]DPA-714 PET/CT (60 min) was performed at 2 time points: (i) baseline scan before and (ii) one month after KA injection (KA1m). Post-processing consisting of 4D iterative deconvolution and spectral regularisation was applied to reconstructed images (OSEM2D). The SRTM2 was used to estimate BPnd before and after post-processing, with the striatum as reference region.

Before post-processing, the highest difference of BPnd between baseline and KA1m was in the left occipital cortex (difference of $0.83 \pm 1.6$, not significant between time points), and the only region to show significance was the right occipital cortex $(0.3 \pm 0.32$, $\mathrm{p}=0.033$ ). After post-processing, the left and right hippocampi showed the highest significant differences in BPnd, as expected $(0.28 \pm 0.30$ and $0.58 \pm 0.61 ; \mathrm{p}=0.034$ and 0.034 respectively). The right parietal-temporal cortex also showed a significant difference in BPnd $(0.19 \pm 0.13, \mathrm{p}=0.006)$.

To detect subtle changes of BPnd in the mouse brain related to TSPO expression, and ensure that differences are statistically significant, it's necessary to use partial volume effect correction and noise removal such as those performed by the postprocessing used in this study.

\author{
M10B-17: A Monte Carlo-Based Quantitative Pinhole ML-EM Reconstruction Using a Ray-Driven \\ Backprojection \\ M. I. Peterson, M. Ljungberg \\ Medical Radiation Physics, Lund University, Lund, Sweden
}

MLEM functionality has been added to the Monte Carlo (MC) program SIMIND, using SIMIND as a forward projector and a ray-driven algorithm for the back projection and evaluated for a GE Discovery 530c CZT SPECT system. Using MC allows inclusion of image degenerative effects, like scatter and collimator penetration, in the reconstruction model. SIMIND estimates the probability of a pixel event per $\mathrm{MBq}$ in a given source distribution and the reconstructed data unit is therefore $\mathrm{MBq} / \mathrm{cc}$. Reconstruction has been performed for two measurements with a torso phantom and a 99Tcm-filled cardiac insert with the torso cavity being either water-filled or empty. The accuracy of the myocardial activity quantification increases with the number of iterations and the reconstructed activity stabilizes within $3 \%$ of the known phantom activity beyond seven iterations for both air 
and water-filled torso. The spatial convergence of the myocardial activity concentration in water is somewhat slower than air. Spatial agreement of CT and SPECT reconstructed volume was evaluated visually and deemed satisfactory.

\section{M10B-18: Motion Model Based on Orthogonal 2D MRI Data to Correct Physiological Motion in PET/MRI} $\underline{\mathrm{M} . \mathrm{He}}^{1}$, L. J. Frohwein ${ }^{1}$, F. Büther ${ }^{1,2}$, K. P. Schäfers ${ }^{1}$

${ }^{I}$ European Institute for Molecular Imaging (EIMI), University of Münster, Münster, NRW, Germany

${ }^{2}$ Department of Nuclear Medicine, University Hospital of Münster, Münster, NRW, Germany

In this work we present a novel motion model that provides the basis for correcting PET images for both respiratory and cardiac motion in dynamic PET/MRI studies. The model uses orthogonal 2D MRI images that are combined retrospectively to form 3D volumes that depict specific motion states. While sagittal images from a single slice location are acquired with high temporal resolution to also capture respiratory motion in the early minutes of the dynamic PET scan, high-resolution coronal images from various slice locations are acquired subsequently and assigned to different respiratory and cardiac phases in post processing. To select suited coronal images, we make use of the anatomical information provided by the sagittal images and analyze the similarity of pixel intensity profiles along the intersection lines and additionally utilize ECG information. Choosing a single coronal image per slice location and dual-gate and generating missing ones with optical flow interpolation yields complete 3D volumes with high temporal and spatial resolution. We applied the same dual-gating scheme to simultaneously acquired MRI and PET data and compared the resulting 3D volumes, i.e. MRI volumes based on coronal 2D MRI images and reconstructed PET images, respectively. Visual comparison shows that the volumes match closely and accurately depict the same respiratory and cardiac motion phases. We further found a strong correlation between MRI- and PET-based motion signals, which justifies the application of the same gating scheme to the data from both modalities. By decoupling the acquisition process from forming the model, we bypass the necessity to obtain high-resolution volumes for motion estimation in almost real-time.

\section{M10B-19: Continuous Motion Estimation in Hybrid PET/MRI Using Fast Interleaved 2D MRI Acquisitions} L. J. Frohwein $^{1}$, M. He ${ }^{1}$, F. Büther ${ }^{1,2}$, K. P. Schäfers ${ }^{1}$

${ }^{I}$ European Institute for Molecular Imaging (EIMI), University of Muenster, Münster, NW, Germany

${ }^{2}$ Department of Nuclear Medicine, University Hospital of Münster, Münster, NW, Germany

Respiratory motion during a PET acquisition causes image blurring and thus degrading of tracer uptake quantification. In this work, we present an MRI-based method for the creation of volumes suitable for the motion correction in PET. The method was designed to capture motion during the entire PET acquisition. The advantage of this method over a model-based approach is the ability of acquiring irregular motion patterns. The approach is based on a fast 2D single-shot TurboFLASH sequence capable of acquiring about 28 images per second. For the acquisition, we additionally use an interleaved slice scheme in which a whole volume is divided into two parts being processed separately. Each sub-volume is complemented with slices of the other subvolumes in order to create temporally coherent volumes. The imaging speed of 28 images per second - achieved with the proposed imaging set up - is capable of resolving respiratory motion. Furthermore, the forming of the volumes with the help of the proposed slice-selection approach leads to an increased temporal coherence of the slices. These aspects qualify the method for the usage in the creation of motion vector fields for the PET motion correction.

M10B-20: Partial Volume Effect and Noise Compensation of Dose-Volume Histograms for Y90-PET Based Dosimetry by Means of Region Sub-Segmentation

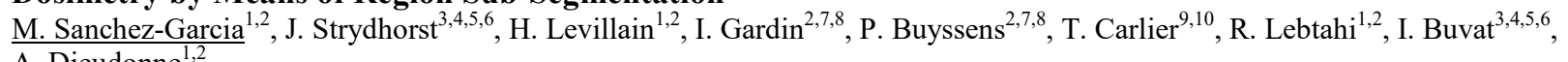
A. Dieudonne $\mathrm{N}^{1,2}$

${ }^{I}$ Nuclear Medicine, Beaujon Hospital, Paris, France

${ }^{2}$ Nuclear Medicine, Henri Becquerel Cancer Center, Rouen, France

${ }^{3} I M I V, C E A$, Orsay, France

${ }^{4}$ University Paris-Sud, Paris, France

${ }^{5}$ CNRS, Orsay, France

${ }^{6}$ Universtity Paris Saclay, Saclay, France

${ }^{7}$ Rouen University, Rouen, France

${ }^{8}$ QuantIF, LITIS, Rouen, France

${ }^{9}$ Nuclear Medicine, University Hospital of Nantes, Nantes, France

${ }^{10}$ CRCNA-UMR 892, INSERM, Nantes, France

Partial volume effects (PVE) and noise in 90Y-PET images propagate in the absorbed dose calculation, degrading dose-volume histograms (DVH). We propose a semi-automated method to compensate for these effects, consisting in segmenting the region of interest into sub-regions (SVs) defined by Eikonal Region Growing. The SVs are generated on a median filtered image and are assumed to contain a homogeneous activity distribution. The optimal number of SVs nopt is defined by the "jump" approach. Geometric Transfer Matrix inversion is then applied for PVE compensation and the DVH is calculated. The resolution was 3 mm 
FWHM. The method was validated on a realistic voxelized phantom. A 30-minute acquisition with a mCT PET scanner was simulated using GATE and reconstructed with Siemens software. Two median filters were tested: $2 \times 2 \times 2(\operatorname{med} 2)$ and $3 \times 3 \times 3$ (med3). For each, the calculation was performed with nopt, nopt-1 and nopt +1 . Theoretical, uncorrected and corrected DVHs were compared in terms of doses covering $5 \%, 20 \%, 50 \%, 80 \%$ and $95 \%$ of the lesion volume. The uncorrected DVH deviated from the theoretical by $15.2 \%,-7.5 \%, 7.1 \%,-20.7 \%$ and $-38.1 \%$ respectively. Using either nopt or nopt +1 resulted in similar improvements with $5.7 \%, 0.4 \%,-6.7 \%, 9.1 \%$ and $-10.2 \%$ errors for the med 2 filter and $5.8 \%, 2.1 \%,-8.3 \%,-9.2 \%$ and $-10.5 \%$ errors for med3. Using nopt-1 resulted in no DVH improvement. The "jump" approach provided a good estimate of the optimal number of SVs, also the results were robust with respect to the pre-filtering.

\section{M10B-21: Towards Better Normalization Using Photopeak Monitoring from Phantom/Patient Data in Positron Emission Tomography (PET)}

M. Aykac, V. Y. Panin

Siemens Molecular Imaging, Knoxville, TN, US

It is essential to have properly tuned PET scanner and data correction methods to obtain accurate quantitative images. Quality control (QC) procedure ensures reproducible performance of a clinical PET scanner. The scope of this study is to observe the stability of a PET scanner by monitoring the $511 \mathrm{keV}$ photopeak during a phantom/patient scan at the crystal level. In addition, new deadtime model will be investigated to provide better normalization by utilizing photopeak monitoring. During the rebinning process of the listmode data, normalization including the crystal-based deadtime model can be implemented for accurate data correction. The method of peak monitoring could be used to adjust crystal sensitivity based on the exact condition of each crystal during the patient scan. This would allow for a "personalized", improved normalization, using data already available during the data acquisition.

\section{M10C: Posters: Radiotherapy II}

Thursday, Nov. 3 16:30-18:30

M10C-1: Study of the Secondary Radiation Produced by He, C and O Ion Beams Impinging on a PMMA Target in the Context of Particle Therapy

I. Mattei $^{1}$, G. Battistoni ${ }^{1}$, F. Collamati ${ }^{2}$, E. De Lucia ${ }^{2}$, R. Faccini ${ }^{3,4}$, P. M. Frallicciardi ${ }^{5}$, C. Mancini-Terracciano ${ }^{3,4}$, M. Marafini ${ }^{4,6}$, S. Muraro ${ }^{1}$, R. Paramatti ${ }^{4}$, V. Patera ${ }^{4,6,7}$, L. Piersanti ${ }^{4,7}$, D. Pinci ${ }^{4}$, A. Rucinski ${ }^{4,7}$, A. Russomando ${ }^{3,4,8}$, A. Sarti $^{2,6,7}$ A. Sciubba ${ }^{4,6,7}$, E. Solfaroli Camillocci ${ }^{3,4}$, M. Toppi ${ }^{2}$, G. Traini ${ }^{3,4}$, C. Voena $^{4}$

${ }^{1}$ INFN - Sezione di Milano, Milano, Italy

${ }^{2}$ Laboratori nazionali di Frascati dell'INFN, Frascati, Italy

${ }^{3}$ Dipartimento di Fisica, Sapienza Università di Roma, Roma, Italy

${ }^{4}$ INFN - Sezione di Roma, Roma, Italy

${ }^{5}$ Istituto di Ricerche Cliniche Ecomedica, Empoli, Italy

${ }^{6}$ Museo Storico della Fisica e Centro Studi e Ricerche, Roma, Italy

${ }^{7}$ Dipartimento di Scienze di Base e Applicate per Ingegneria, Sapienza Università di Roma, Roma, Italy

${ }^{8}$ Center for Life nano Science@Sapienza, Istituto Italiano di Tecnologia, Roma, Italy

Particle Therapy (PT) exploits the dose deposition localization and biological effectiveness of charged hadrons irradiating a tumor volume, sparing healthy tissues and surrounding organs at risk. Protons are the main projectiles used in clinical practice, but recently an interest in heavier ions $(\mathrm{He}, \mathrm{C}, \mathrm{O})$ is growing due to their reduced multiple scattering inside the body and increased Relative Biological Effectiveness and Oxygen Enhancement Ratio. Such interest motivated an experimental campaign performed at the Heidelberg Ion-beam Therapy center (HIT, Germany). The aim of the experiment was the measurement of the production of projectile fragments emitted at small angle and the measurement of prompt photons and secondary charged particles detected at large angles for on-line beam range monitoring purposes.

A PMMA target was exposed to primary $\mathrm{He}, \mathrm{C}$ and $\mathrm{O}$ beams at therapeutic energies. The experimental setup was built in order to contemporary perform the detection of the different secondary radiations, involving scintillating crystals for the energy measurement and using plastic scintillators for the time of flight computation. A multi-wire drift chamber was used to track the secondary charged particles.

The measured yields of the forward peaked fragments will be presented together with their kinetic energy spectra. The production yields of prompt photons and secondary charged particles will be presented and compared to other measurements available from literature. The emission distributions of the tracked secondary charged particles related to the beam range inside the target will be shown. The obtained results have been extrapolated to a real case scenario in view of the development of new detectors in PT clinical application. The achieved outcomes confirmed the feasibility of a real time monitoring technique based on the detection of secondary charged particles and prompt photons in PT treatments with Helium and Oxygen ions.

M10C-2: Beam-on PET Imaging of Very Short-Lived Nuclides During Proton Therapy $\underline{\text { H. J. T. Buitenhuis }}^{1}$, F. Diblen ${ }^{1,2}$, K. W. Brzezinski ${ }^{1}$, S. Brandenburg ${ }^{1}$, P. Dendooven ${ }^{1}$ 
${ }^{I}$ KVI - Center for Advanced Radiation Technology, University of Groningen, Groningen, The Netherlands

${ }^{2}$ Department of Electronics and Information Systems, MEDISIP, Ghent-University, Ghent, Belgium

Proton therapy is more sensitive than photon therapy to differences between the treatment plan and the actual treatment situation. An in-vivo method to daily verify the dose delivery to the patient is needed to fully exploit the dosimetric benefits of protons. PET imaging during the irradiation maximizes the number of detected counts and minimizes biological washout of the positron emitting nuclei. In such a scenario, also (very) short-lived positron emitters will be observed, with a half-life in the order of milliseconds to seconds. One of the most interesting nuclei is $12-\mathrm{N}$, which has a half-life of $11 \mathrm{~ms}$, and is produced at a rate of $9.1 \%$ of the production of $11-\mathrm{C}$ on carbon. The short half-life and relatively high production rate may allow feedback on the dose delivery on a short time scale. In this experiment, we imaged the 12-N nuclei using a prototype PET system to further investigate the use of short-lived PET imaging for "prompt" feedback. The PET system is based on the Module-TEK from Philips Digital Photon Counting, using LYSO scintillators mounted on dSiPM arrays. This system is coupled to the cyclotron RF to do event timing with respect to the RF-phase. Production of 12-N compared to longer-lived nuclei will be shown, as well as a novel method to isolate the PET image of the short-lived nuclei.

M10C-3: The INSIDE in-Beam PET: System Characterization and First Measurements

E. Fiorina, G. Battistoni, N. Belcari, M. G. Bisogni, N. Camarlinghi, P. Cerello, A. Del Guerra, A. Ferrari, V. Ferrero, G. Giraudo, E. Kostara, M. Morrocchi, F. Pennazio, C. Peroni, M. A. Piliero, G. Pirrone, A. Rivetti, M. D. Rolo, V. Rosso, P. Sala, G. Sportelli, R. Wheadon

Physics, University of Turin, Turin, Italy

The INSIDE collaboration has recently completed the construction of an in-beam Positron Emission Tomography (PET) scanner for real-time monitoring of the particle range in hadrontherapy.

The detector features two 10x25 cm2 PET panels, based on segmented Lutetium Fine Silicate scintillating crystals, coupled to Silicon PhotoMultiplier sensors and read out by the TOFPET ASIC.

Since the experimental in-beam PET image is correlated in a complex way to the Bragg Peak positions scanned during treatment sessions, the expected in-beam PET image must be simulated to evaluate the compliance of the delivered treatment with the clinical prescription.

A dedicated simulation was implemented in FLUKA by considering both the beam delivery information and the detector geometry and performance.

The system characterization with proton and carbon ion beams is ongoing at the CNAO synchrotron facility (Pavia, Italy) and first measurements showed very promising results.

Different delivery configurations on phantoms were considered: when analyzing activity profiles along the beam axis, data and simulation are in agreement within less than $1 \mathrm{~mm}$ in the difference between the distal fall-off profiles corresponding to different Bragg Peak positions. Good agreement between data and simulation was also found with the delivery of real treatment sessions. In order to start testing with patients at the CNAO, (1) the system will be further characterized to evaluate its overall uncertainty, (2) the simulation will be improved to perform it on the patient Computed Tomography scans and (3) the quality assurance procedure will be optimized to obtain a useful and reliable compliance index between experimental and simulated images.

\section{M10C-4: Experimental Characterization of Megavoltage Beams for Orthogonal Ray Imaging}

${ }^{I}$ LIP - Laboratório de Instrumentação e Física Experimental de Partículas, Coimbra, Portugal

${ }^{2}$ Physics Department, University of Coimbra, Coimbra, Portugal

${ }^{3} I P O P F G$, EPE - Instituto Português de Oncologia do Porto Francisco Gentil, Porto, Portugal

${ }^{4}$ Radiotherapy Department, CHUC, EPE - Centro Hospitalar e Universitário de Coimbra, Coimbra, Portugal

Radiotherapy (RT) is one of the main procedures for cancer treatment. Modern RT techniques provides increasingly higher conformality to the tumor. To guarantee maximum dose exposure to the tumor and avoid the irradiation of organs at risk or healthy tissues it is important to reduce patient misalignments and to detect patient anatomical morphological alterations. We proposed an imaging system that provides almost no additional dose to the patient, in external X-ray beam RT: OrthoCT (orthogonal computed tomography). OrthoCT focusses on acquiring images of the volume to be irradiated when the patient is already positioned for treatment. It relies on the detection of scattered photons emitted perpendicularly to the beam direction. For that, it is necessary that the beam has an inferior size $(\sim 5 \mathrm{~mm} \times 5 \mathrm{~mm})$ when compared to the typical ones used in patient irradiation. Moreover the scanned profile requires (1) high homogeneity so that variations can be associated only to dose/morphological alterations and (2) high velocity (which favors MLC-based scans in respect to jaw-based scans). Until the moment, we have irradiated an acrylic phantom with beamlets from a True Beam (Varian Medical Systems) machine at Centro Hospitalar e Universitário de Coimbra-EPE. EBT1 Gafchromic films were interleaved with solid water slices at distances before, at, and after the isocenter of such slices. Analysis is being evaluated and transverse profiles will be shown with the aim of defining the best scanning condition for an ORI (orthogonal ray imaging) experiment producing 3D images without rotation of the linac source. 
M10C-5: Monitoring Tumor Head Irradiation with OrthoCT (Orthogonal Ray Imaging): a Full System Simulation Study

P. Crespo ${ }^{1,2}, \underline{\text { H. Simões }}^{1,2}$

${ }^{I}$ Laboratório de Instrumentação e Física Experimental de Partículas, Coimbra, Portugal

${ }^{2}$ Departamento de Física, Universidade de Coimbra, Coimbra, Portugal

The OrthoCT (standing for orthogonal computed tomography) imaging technique consists in detecting radiation dispersed in the patient and emitted at right angles in respect to the beam axis. Since photon scattering in the patient occurs with higher intensity in tissues of higher density, a detection system (constituted by a multi-slice collimator and a photon detector) positioned perpendicularly to the beam axis yields a signal proportional to the photons that escaped the patient (i.e., a signal correlated with patient morphology). This low-dose morphological imaging can potentially be useful to (1) on-board imaging for assisting radiotherapy, or (2) real time radiotherapy monitoring. In this work we report Geant4 simulation results with an anthropomorphic phantom to analyze the capability of OrthoCT to detect pertinent and clinically-relevant morphological variations during head irradiation. The counts distributions obtained show a very high visual correlation both with the simulated, prescribed dose, and with the phantom structures/morphology. The capability of OrthoCT to obtain morphological images with no X-ray source rotation potentially allows to minimize dose in healthy tissues and organs at risk. Therefore, this technique is likely to represent a high potential asset for on-board imaging (i.e. imaging after patient positioning, just before the treatment), or eventually, during the treatment.

M10C-6: Optical Fiber Dosimeter for Real-Time HDR Brachytherapy $\underline{\text { L. M. Moutinho }}^{1}$, H. Freitas ${ }^{1}$, A. J. Gonçalves ${ }^{1}$, I. F. Castro ${ }^{1}$, P. J. Rachinhas ${ }^{2}$, P. C. Simões ${ }^{2}$, J. F. C. A. Veloso ${ }^{1}$

${ }^{I}$ Physics Department, University of Aveiro, Aveiro, Portugal

${ }^{2}$ Serviço Radiologia, CHUC, Coimbra, Portugal

Dose verification and quality assurance in radiotherapy should be assessed in order to provide the best treatment possible and minimize risks for patients. Notwithstanding, due to technical constraints in certain treatments there are no tools capable to perform real-time dose measurement. An ideal dosimeter for prostate brachytherapy should provide real-time and in-vivo dose measurement, present high sensitivity and no dependencies on energy, dose, dose rate or temperature. It should also be detectable in the anatomic volume to check its position, easy to use and calibrate and inexpensive or with disposable use of its implantable part. We developed a fiber optic dosimeter suitable for real-time dose monitoring in breast and prostate brachytherapy, thus opening the possibility for real-time dose correction. The dosimeter comprehends a sensitive optical fiber probe of $1 \mathrm{~mm}$ or 0.5 $\mathrm{mm}$ diameter where a $5 \mathrm{~mm}$ length scintillating optical fiber is coupled. The clear optical fiber provides scintillation light guidance into the photodetectors. The photodetectors used are the Silicon Photomultipliers from Hamamatsu. The first round of in-vitro tests in clinical setting allowed demonstrating that fiber optical based dosimeters are suitable for dosimetry in regimes such as the ones in HDR prostate brachytherapy. The versatility and easiness of use of this kind of device allows application in other radiotherapy modalities. The dosimeter response was evaluated under irradiation with a 10.07 Ci Ir-192 HDR-

brachytherapy source. The dosimeter shows a linear response with dose and is capable of detecting $\mu$ Gy dose variations like an ionization chamber.

\section{M10C-7: High-Granularity Digital Tracking Calorimeter for the Estimation of Proton Energy in Proton} Computed Tomography

H. E. S. Pettersen ${ }^{1,2}$, D. Röhrich ${ }^{2}$, O. H. Odland ${ }^{1}$, H. Wang ${ }^{3}$, C. Zhang ${ }^{3}$, E. Rocco ${ }^{3}$, T. Peitzmann ${ }^{3}$

${ }^{I}$ Dept. of Oncology and Medical Physics, Haukeland University Hospital, Bergen, Norway

${ }^{2}$ Institute of Physics and Technology, University of Bergen, Bergen, Norway

${ }^{3}$ Institute for Subatomic Physics, University of Utrecht, Utrecht, The Netherlands

Proton CT is a proposed modality, measuring the path and energy loss of high energy protons through a patient in order to estimate the patient's volumetric proton stopping power. The goal of proton CT is to provide higher accuracy in the treatment plans during during proton radiation treatment, as well as in-room position verification and day to day online check of anatomical changes.

The Segmented Tracking Detector is a candidate for a high-speed Bragg Peak detector, for use in proton CT. It is a a proposed upgrade to the ALICE experiment at CERN, and is a compact, layered and high-granularity digital tracking detector. Because of the extremely large number of pixels, the device will be able to cope with large particle multiplicities. It is a MIMOSA23-based CMOS digital sampling pixel detector, with 24 layers of $4 \times 4 \mathrm{~cm}^{2}$ with pixels of $30 \mu \mathrm{m}$ pitch. Between each layer is $3.3 \mathrm{~mm}$ tungsten for energy absorption. While it has been optimised for high energy physics, it proves valuable insights into using segmented tracking detectors for proton CT.

Through beam tests performed at KVI Groningen in the Netherlands, key aspects of this detector has been determined. Proton 
beams of $2 \mathrm{kHz}$ intensity ranging from 150 to $188 \mathrm{MeV}$ were used. This prototype has an accurate range estimate at the $2 \%-3 \%$ level through precise Bragg Peak determination, as well a high readout frequency of at least $400 \mathrm{kHz}$.

This PhD project is supported by Helse Vest grant 911933.

M10C-8: The Use of CMOS Detectors for a $24 \mathrm{MeV}$ Proton Beam Dosimetry

J. Constanzo, C. Finck, C. Mathieu, M. Pellicioli, J. Schuler, M. Vanstalle, M. Rousseau

IPHC, UNISTRA, CNRS, 23 rue du Loess, 67037 Strasbourg, France

Animal radiotherapy research platforms mimicking accurately human radiotherapy conditions are needed. Indeed, small animal studies are important to fully understand the therapeutic possibilities and limitations/toxicities of proton therapy. Several experimental proton irradiation platforms are emerging mainly with energy below $70 \mathrm{MeV}$. We developed a $24 \mathrm{MeV}$ proton beam platform dedicated to small animal experiments, mostly for subcutaneous tumors irradiation. At this energy, dosimetric controls remain very challenging. Standard protocols, such as ionizing chamber dosimetry, cannot be performed as the proton beam path in water is about $5 \mathrm{~mm}$. In this study we measured simultaneously the count rate of the CMOS detector placed at the small animal position, and the dose given by Gafchromic ${ }^{\circledR}$ EBT3 films. These preliminary results demonstrated that CMOS detectors enable the fluence measurement at low beam current of about $1.3 \times 10^{\wedge} 6$ protons.cm- 2 , which corresponded to the dose of 60 cGy assessed by EBT3 films.

M10C-9: Dosimetric Evaluation of Proton CT Using a Prototype Proton CT Scanner V. Giacometti ${ }^{1}$, S. Guatelli ${ }^{1}$, A. Zatserklyaniy ${ }^{2}$, R. P. Johnson ${ }^{2}$, H. Sadrozinski ${ }^{2}$, T. E. Plautz ${ }^{2}$, P. Piersimoni ${ }^{3}$, C. E. Ordonez ${ }^{4}$, V. A. Bashkirov", R. W. Schulte ${ }^{5}$, A. B. Rozenfeld ${ }^{1}$

${ }^{1}$ Center for Medical Radiation Physics, Wollongong University, Wollongong, NSW, Australia

${ }^{2}$ Division of Radiation Research, Department of Basic Sciences, Loma Linda University, Loma Linda, CA, USA

${ }^{3}$ Santa Cruz Institute for Particle Physics, Santa Cruz University, Santa Cruz, CA, USA

${ }^{4}$ Department of Computer Science, Northern Illinois University, DeKalb, IL, USA

${ }^{5}$ Department of Radiation Oncology, University of San Francisco, San Francisco, CA, USA

Proton computed tomography has been suggested as an imaging technique alternative to x-ray CT for proton therapy treatment planning and image guidance. Dose, image quality, and range prediction accuracy are closely related and the calculation of the dose delivered during a proton CT scan is crucial for characterizing a proton CT scanner. Here we report on the dosimetric evaluation of proton CT scanners using a prototype proton CT scanner built by the pCT collaboration at Loma Linda and Santa Cruz University. The Catphan CTP554 $16 \mathrm{~cm}$ acrylic dose phantom, representing a human head, was used to measure the dose to water during a typical proton CT scan at the Northwestern Medicine Chicago Proton Center in Warrenville, Illinois. A Farmer ionization chamber was installed in two locations (center and periphery of the phantom) and the accumulated charge was measured with the 35040 Advanced Therapy Dosimeter (Fluke Biomedical). The proton CT scanner was exposed to a uniform beam profile of $200 \mathrm{MeV}$ protons (range in water $26 \mathrm{~cm}$ ) on the clinical scanning proton beam line. At a rate of $1 \mathrm{M}$ protons sec, it takes 7 minutes in order to collect about $400 \mathrm{M}$ protons during a continuous 360 degree rotation. The proton fluence at the level of upstream inner tracker planes during the 7 minutes of exposure was estimated to be $1.4 \mathrm{M}$ protons $/ \mathrm{cm} 2$. The dose to water was $1.46 \mathrm{mGy}$ in the peripheral location and $1.48 \mathrm{mGy}$ in the central location, demonstrating that it is practically uniform across the phantom. Compared to the dose delivered with a $\mathrm{kV}$ x-ray CBCT of the head that achieves soft tissue resolution similar to our proton $\mathrm{CT}$, which is about $10 \mathrm{cGy}$, the dose required for a proton CT scan is thus at least 5 times lower. In addition, experience with head phantom proton CT scans has demonstrated the absence of high-density artefacts. In conclusion, proton CT is a promising modality for low-dose image guidance and adaptive proton therapy.

M10C-10: Scintillator-Based Measurement of off-Axis Neutron and Photon Dose Rates During Proton Therapy C. A. Miller ${ }^{1}$, M. A. Norsworthy ${ }^{1}$, S. D. Clarke ${ }^{1}$, S. A. Pozzi ${ }^{1}$, R. W. Schulte ${ }^{2}$

${ }^{I}$ Department of Nuclear Engineering and Radiological Sciences, University of Michigan, Ann Arbor, MI, USA

${ }^{2}$ Department of Radiation Medicine, Loma Linda University Medical Center, Loma Linda, CA, USA

Dose rates, especially from photons and neutrons, inform safety procedures in any radioactive environment. A method for measuring dose rates from both particles with one device will simplify current methods for surveying dose fields. We have developed a technique using organic scintillators that is based on the definition of effective dose, extracted from energy deposited in the detector. Using pulse shape discrimination, energy deposited from either neutrons or photons can be separated. Radiation weighting factors are applied to obtain a measurement of effective dose from neutrons and photons concurrently. This method was previously demonstrated for organic scintillators with a californium-252 source. This work presents measurements of offaxis secondary particle dose rates from a sub-clinical strength proton beam incident on various targets. The experiment was modeled using the Monte Carlo code MCNPX-PoliMi. Measured proton beam secondary neutron dose rates are shown to agree reasonably with simulated results. The comprehensive analysis of this dose rate technique demonstrates the possibility for a dual 
particle dosimeter to be used in a variety of applications, including surveying, room monitoring, and shielding analysis. Enhancing and simplifying dose rate information will allow for a safer and more productive radiation worker environment.

\author{
M10C-11: An Image Guided and Energy-Multiplexed Combinatorial Therapeutic Delivery System for X-Ray \\ Induced Photodynamic Therapy \\ J. George $^{1}$, L. Giannoni ${ }^{1}$, L. Luo ${ }^{2}$, P. J. La Riviere ${ }^{3}$, L.-J. Meng ${ }^{3}$ \\ ${ }^{I}$ Department of Nuclear, Plasma, and Radiological, University of Illinois at Urbana-Champaign, Urbana, IL, USA \\ ${ }^{2}$ Division of Medical Engineering Research, National History Institute, Taipei, Taiwan \\ ${ }^{3}$ Department of Radiology, University of Chicago, Chicago, IL, USA
}

Our general goal is to explore the feasibility of using X-rays of different energies to selectively active different types of therapeutic nanoparticle to achieve a multiplexed therapeutic delivery for cancer. In recent years, there have been increased interests in nanoparticle-mediated X-ray induced cancer therapy. During the therapeutic delivery process, a high-collimated external X-ray beam is used to irradiate the target. The metal atoms within the NPs preferably absorb the X-rays and induce local therapeutic effect through photosensitization, thermal ablation or other processes.

With this scheme, it is possible to optimize the energy characteristics of the irradiating X-rays, so that one could selectivity activate a specific type of NPs that contain certain metal elements. Furthermore, by fine-tuning the X-ray source (with different filters, monochromators, and tube voltages) and by using designing therapeutic NPs containing different metal elements, one could achieve a multiplexed combinatorial therapeutic delivery in real-time.

In this study, we will experimentally demonstrate the feasibility of this scheme by using a bench-top X-ray imaging/irradiation setup to selectively active two types of nano-particles developed for X-ray PDT that contains Y2O3:Tb and LaF3:Eu nanoscintillators. We will use imaging and quantitative measurements to access specificity for the selective activation, and to confirm the combinatorial therapeutic delivery approach.

Preliminary results show that the use of $17 \mathrm{keV}$ could active the Y2O3: Eu NPs with very high efficiency, which is evident from the luminescence signal observed with the EMCCD. However, the same X-ray beam could not active the LaF3:Eu NPs. These preliminary results indicate that the potential for selective activation of these nanoparticles for therapeutic application.

M10C-12: Pulse by Pulse Timing Analysis in Adaptive Radiotherapy: a Preliminary Study M. Duncan $^{1}$, M. K. Newall ${ }^{1}$, V. Caillet ${ }^{2}$, J. T. Booth ${ }^{2,3}$, P. J. Keall ${ }^{4}$, A. B. Rozenfeld ${ }^{1}$, M. Petasecca $^{1}$

${ }^{I}$ Centre for Medical Radiation Physics, University of Wollongong, Wollongong, NSW, Australia

${ }^{2}$ Royal North Shore Hospital, Northern Sydney Cancer Care Centre, St Leonards, NSW, Australia

${ }^{3}$ Institute of Medical Physics, University of Sydney, Sydney, NSW, Australia

${ }^{4}$ Radiation Physics Laboratory, University of Sydney, Sydney, NSW, Australia

Adaptive radiotherapy (ART) monitors and modulates the beam during treatment in accordance with patient motion. ART is often combined with stereotactic radiotherapy modalities that have a high 'ablative' total dose, small fields and steep dose gradients. To achieve accurate and meaningful quality assurance of ART, both the instantaneous dose distribution throughout treatment and the total integral dose need to be measured. For accurate small field dosimetry, a detector requires high spatial and temporal resolution. Centre for Medical Radiation Physics has developed such a 2D silicon diode array detector with 52x52mm2 total sensitive area with two perpendicular strip arrays of 256 elements. Each diode has an area of $1 \times 0.01 \mathrm{~mm} 2$, with $200 \mathrm{~m}$ pitch between the strips. DUO was used to reconstruct time resolved dose distributions from an ART treatment which used multi-leaf collimator (MLC) tracking in combination with a motion platform and RF tracking array. The timing resolution of the detector allows dose to be recorded for every linac pulse, hence the interplay effect between patient motion and MLC movement can be investigated. We monitor the pixel receiving $50 \%$ of the penumbral dose and plot its response as function of time. Fourier analysis of this response helps quantify the effectiveness of a particular tracking algorithm for the type of motion experienced. We define a 'quality' parameter that reflects this result. Using a sine wave motion, we have seen up to $90 \%$ improvement in the treatment using this analysis with predictive tracking. In future this quality factor can be used to optimize a tracking system for a specific patients breathing and organ motion.

\author{
M10C-13: R\&D of an Ultra-Low Noise (<=1.8 E- ) and High Sensitive (3 Photons ) Light Field Camera for \\ Next-Generation Real-Time Full 3D Scintillation Dosimetry

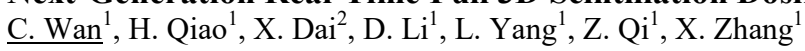 \\ ${ }^{I}$ School of Nuclear Science and Technology, Lanzhou University, Lanzhou, Gansu, China \\ ${ }^{2}$ China institute for radiation protection, Taiyuan, Shanxi, China
}

A real-time and full 3D dosimetry is essential in radiation therapy treatments to examine expected radiation dose distribution inside patient bodies for quality assurance. The next generation 3D dosimetry has two main components: a light field camera with a 3D radiation dose distribution reconstruction algorithm and a body equivalent scintillator In this work, we only focus on the research and development of an ultra-low noise $(<1.8 \mathrm{e}-)$, high sensitivity ( $\sim 3$ photons) custom-made light field camera. The 
developed camera is made out of a custom-made Dhyana 400D camera with a specific microlens. With the help of cooling system, the camera has a low current noise of $0.01 \mathrm{e}-/ \mathrm{s} / \mathrm{p}$, a low readout noise of $1.8 \mathrm{e}-$, a large dynamic range of $85 \mathrm{~dB}$, a low light sensitivity of $\sim 3$ photons, and a deep full well of $48 \mathrm{k}$ electrons. All these features make it be able to gain a very high signalto-noise ratio, a very low dose detection limit, and a very large dynamic range of detected radiation dose. The microlens is made of polymer. It has a pitch of 125 microns and a focal length of 500 microns. The overall system can achieve a lateral resolution of about 40 microns and a depth resolution of about 60 microns for a small volume of $3 X 3 X 1 \mathrm{~cm} 3$. For a large volume of $10 \mathrm{X} 10 \mathrm{X} 10 \mathrm{~cm} 3$, the two of $0.2 \mathrm{~mm}$ and $0.9 \mathrm{~mm}$ respectively can be obtained.

This work is supported by the National Natural Science Foundation of China (Grant No. 11275087, 10875054, and 11475077)

\author{
M10C-14: Timing Performances of a Time-of-Flight Detection System for Fragmentation Cross Section \\ Measurements in Carbon Therapy

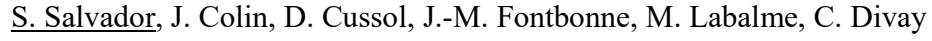 \\ Normandie Univ, ENSICAEN, UNICAEN, CNRS/IN2P3, LPC Caen, Caen, France
}

Carbon ions therapy is a promising tool for treating cancerous tumors when proton therapy is less efficient. However, carbon ions encounter nuclear fragmentation processes that reduce the beam intensity and create a mixed radiation field. This field is composed of faster and lighter fragments that is not fully taken into account in Monte Carlo codes. To overcome this limitation, our group had performed double differential fragmentation cross sections of ${ }^{12} \mathrm{C}$ ions on thin target of medical interest at 95 and $50 \mathrm{MeV} /$ nucleons. The construction of the ARCHADE center in Caen, France, will allow us to measure these cross sections with a beam energy up to $400 \mathrm{MeV}$ /nucleons using the FRACAS detection system, a large acceptance mass spectrometer. The Timeof-Flight capability of the system is investigated in this work through a Parallel Plate Avalanche Counter (PPAC) in coincidence with a scintillation detector composed of a YAP: Ce scintillator and an ultra-bialkali photomultiplier tube. With this system, we measured Coincidence Resolving Times (CRT) lower than 300 ps that will lead to a better particle mass identification and energy resolutions.

M10C-15: Time-of-Flight Surface Imaging for 6 DoF Patient Positioning in Radiotherapy

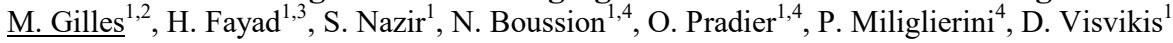

${ }^{1}$ LaTIM, Inserm UMR 1101, Brest, France

${ }^{2}$ CERV, Ecole Nationale d'Ingénieurs de Brest, Brest, France

${ }^{3}$ Université de Bretagne Occidentale, Brest, France

${ }^{4}$ Radiotherapy department, CHRU Morvan, Brest, France

Daily patient positioning accuracy is a major factor in radiotherapy treatment efficiency. During the radiotherapy delivery process at each radiotherapy fraction, the tumor and the organs at risk (OARs) should be placed at the same position as that during the planning process. The actual techniques combine imprecise and irradiant methods with a first positioning using lasers and tattoo points followed by kilovoltage or megavoltage imaging. Existing surface based imaging systems are currently commercialized but present constraints preventing an accurate real-time motion monitoring. A stereo system that uses time-offlight technology has previously been tested for 3 degree of freedom (DoF, 3 translations) treatment couch displacements showing sub-millimeter errors. In this study, we evaluate the accuracy of such a system with 6 DoF couch motion. Phantoms and patients were filmed before and after the table motion. An Iterative Closest Point (ICP) registration was performed between the two point clouds, and the registered points were compared to the acquired points. The comparison was not direct because point clouds are not ordered. Therefore, we first used B-Splines modeling to measure the norm between the points of the registered and acquired data. The second evaluation consisted on creating the final point cloud from the translations and rotations applied on the couch, while adding noise to the data. In this case, the ICP was performed between the acquired initial image and the final created one. After two consecutive ICP, results on phantoms are similar to those obtained for a 3 DoF displacement $(0.8 \pm 0.7 \mathrm{~mm})$ with a mean error of $0.8 \pm 0.5 \mathrm{~mm}$ for B-Splines comparisons and $0.8 \pm 0.2 \mathrm{~mm}$ when using the created images. Results on patients

show an increased error $(1.1 \pm 0.4 \mathrm{~mm}$ and $1.5 \pm 1.2 \mathrm{~mm}$ respectively) probably due to patient motion or reflections of the accelerator arm. Future works will consist on adding motion information to improve the tumor and OARs positioning.

M10D: Posters: CT I

Thursday, Nov. 3 16:30-18:30 Etoile

M10D-1: Prior-Based Metal Artifact Reduction in CT Using Statistical Metal Segmentation on Projection Images

M. A. Hegazy, M. H. Cho, S. Y. Lee

Dept. of Biomedical Engineering, Kyung Hee University, Yongin, S.Korea 
Abstract - Metal objects such as dental fillings, dental implants, and surgical clips generate metal artifacts appearing as streaks and dark shadows. We present a method that can reduce metal artifacts by generating a prior image. The prior image was used to replace the pixel values at the metal region in the projection image. To generate the prior image, we first segmented the metal parts in the projection image using simple global thresholding. We corrected the result of simple global thresholding by applying local thresholding based on statistics of pixel values around the metal boundary. Then, we applied linear interpolation to initially fill the metal region with pixel values similar to the surrounding body tissues. From the interpolated projection data, we reconstructed the first image for generating the prior image. We reconstructed the second image from the original projection data with removing the metal parts. We generated the prior image by weighted summation of the two images. Then, we synthesized a projection data by forward projecting the prior image. For the final image reconstruction, we replaced the metal parts in the original projection data with the ones in the synthesized projection data. The proposed method shows significantly improved performance in reducing streak artifacts and dark shadows in experimental imaging studies of a dental phantom using a microCT. Keywords - Metal artifact reduction, CT artifacts, Statistical segmentation

\title{
M10D-2: Development of a Method to Obtain High-Contrast Photon Counting CT Images in the Case of Arteriosclerosis
}

K. Yokoi, I. Takahashi, K. Amemiya

Research \& Development Group, Hitachi, Ltd., Hitachi-shi, Ibaraki-ken, Japan

Photon counting detectors which can obtain energy information of each incident photon have been developed to be utilized in the range of medical Computed Tomography (CT) flux, that is, over $100 \mathrm{M}$ photons $/ \mathrm{sec} / \mathrm{mm}^{2}$. Photon Counting CT (PCCT) is expected to extend clinical applications because of the fact that each element has different energy dependence on X-ray linear attenuation coefficient. To apply PCCT for arteriosclerosis diagnosis, We evaluated material resolution of conventional CT, dual energy CT and PCCT in basis material decomposition (BMD) images with a numerical simulation. We prepared mixed materials which consist of adipose as atheroma, hydroxyapatite (HAp) as calcified tissues, and blood. The range of density is $0-5 \%$ for HAp, $0-100 \%$ for adipose and $0-100 \%$ for blood. The calcium score threshold, $130 \mathrm{HU}$, corresponds to $1.7 \%$ HAp in this study. In conventional CT, 1\% HAp is cancelled by existence of 35\% adipose. This means that simultaneous progression of atherogenesis and calcification can be invisible with conventional CT. We found that dual energy CT and PCCT can measure adipose density independent of HAp density. Adipose Contrast-to-Noise Ratio (CNR; background = blood) of dual energy CT and PCCT turned out to be 3.4 and 5.9, respectively. So, PCCT improved the CNR by $71 \%$. Adipose and HAp density can be independently measured in PCCT, while each BMD image has poor image quality. We proposed a new method to derive a good quality image from BMD images like the "monochromatic imaging". We found that scatter plot of BMD image values of pixels for each material shows clear ellipse structure, and the axes of the ellipse is generally not parallel to those of basis materials. By setting a new axis being perpendicular to the ellipse, the image of less standard deviation was derived. In the comparison using HAp CNR over the conventional CT, the proposed method gives $19.6 \%$ improvement, while monochromatic (50 keV) imaging shows $17.7 \%$.

\author{
M10D-3: Dosimetry of Dual-Energy CT for the Detection of Acute-Stage Cerebral Infarction : a Phantom \\ Study

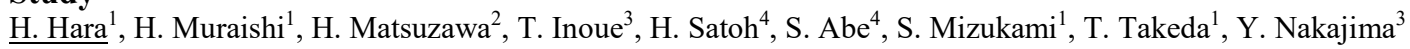 \\ ${ }^{1}$ School of Allied Health Sciences, Kitasato University, kanagawa, Japan \\ ${ }^{2}$ General Medical Center, Saitama Medical University, Saitama, Japan \\ ${ }^{3}$ Department of Radiology, St. Marianna University School of Medicine, Kanagawa, Japan \\ ${ }^{4}$ School of Health Sciences, Ibaraki Prefectural University of Health Sciences, Ibaraki, Japan
}

Purpose: We recently improved the contrast of acute-stage cerebral infarction using virtual monochromatic images obtained by dual-energy CT. We measured radiation doses using the newly developed phantom while assessing its potential clinical application. Methods: Our phantom developed for dose measurement in precisely mimics the head shape and consists of brain and cranium sections with CT values of approximately 36 and 900-1500 HU, respectively. The phantom consists of six slice cross sections obtained from the orbital to the parietal region at an angle along the orbitomeatal line. Each slice cross section has 11 cavities for insertion of a TLD. In this study, the tube voltages with a thin (Sn) filter were $80 \mathrm{kV} / \mathrm{Sn} 140 \mathrm{kV}, 100 \mathrm{kV} / \mathrm{Sn} 140 \mathrm{kV}$, and $140 \mathrm{kV} / 80 \mathrm{kV}$; the tube current time products were 400,600 , and $800 \mathrm{mAs}$; and the scanning range was $120 \mathrm{~mm}$ from the orbitomeatal line to the parietal region. The dose was measured three times using TLD, and the absorbed dose was obtained from the average. Results: Compared with $120 \mathrm{kV}$, the absorbed dose could be reduced by approximately $47 \%$ at $80 \mathrm{kV} / \mathrm{Sn} 140 \mathrm{kV}$, approximately $30 \%$ at $100 \mathrm{kV} / \mathrm{Sn} 140 \mathrm{kV}$, and approximately $22 \%$ at $140 \mathrm{kV} / 80 \mathrm{kV}$, when the tube current time product was 600 mAs. If an absorbed dose of approximately 1.5 times that at $120 \mathrm{kV}$ was deemed acceptable, the contrast was significantly improved in dual-energy CT and effectively detected acute-stage cerebral infarction. Conclusion: Our dose measurement for visualizing acute-stage cerebral infarction revealed that the dose could be reduced with dual-energy CT compared with CT at $120 \mathrm{kV}$. 
This study was partially supported by the Grants for Scientific Research (C) from the Ministry of Education, Culture, Sports, Science and Technology of Japan (Grant-in-Aid No. 26460732).

\author{
M10D-4: Improvement of Spatial Resolution in Two-Dimensional transXend Detector Consisting of Metal \\ Absorbers and Flat Panel Detector \\ I. Kanno, K. Yamauchi, T. Hamaguchi \\ Department of Nuclear Engineering, Kyoto University, Nishikyo, Kyoto, Japan
}

For utilizing the energy information of X-rays in computed tomography (CT), the authors have invented a new detector system, a "transXend" detector. This transXend detector consisted of several segment detectors stacked in the direction of X-ray incidence. The transXend detector measured X-rays as electric current, and gave energy distribution after analysis with using previously obtained response functions. The stacked type transXend detector was developed for the detection of pencil beam X-rays. For the application of the principle of the transXend detectors for cone beam or fan beam X-rays, a two-dimensional transXend detector consisted of lattice shaped metal absorbers and a flat panel detector (FPD) was proposed in IEEE MIC 2014. The pixel size of the transXend detector was $1 \times 1 \mathrm{~mm} 2$ in case of using $0.5 \mathrm{~mm}$ width stripe absorbers. For making the pixel size of the transXend detector smaller, a new absorber configuration is reported in this presentation: band absorber transXend detector. The pixel width is the one of the pixel of the FPD, i.e., $48 \mu \mathrm{m}$. The pixel length in body axis direction is $2 \mathrm{~mm}$. The spatial resolution and contrast-to-noise ratio (CNR) are compared for the CT images obtained by the band and lattice transXend detector. Although the spatial resolution of the band transXend detector is 9 times better than that of lattice one, the CNR is 24 times worse. The inferior CNR of the band transXend detector is the result of smaller pixel area of the band transXend detector. The number of X-rays coming into the pixel is so small that the pixel value has influence of the dark current of the pixel of the FPD. With combining 13 pixels of the band transXend detector in lateral direction, the CNR of band transXend detector shows nearly the same value as with the one of the lattice transXend detector. In this case, the spatial resolution of the band transXend detector shows twice better value than that of lattice transXend detector.

\title{
M10D-5: Semi-Analytic X-Ray Source Model for MARS Spectral CT
}

M. Shamshad $^{1}$, M. Anjomrouz ${ }^{1}$, D. J. Smithies ${ }^{2}$, A. Largeau ${ }^{3}$, G. S. Lu ${ }^{4}$, A. Atharifard ${ }^{1}$, J. Humphrey ${ }^{4}$, L. Vandon Broeke ${ }^{4}$, R. Aamir ${ }^{1,2}$, B. Goulter ${ }^{2}$, M. F. Walsh ${ }^{2}$, R. K. Panta ${ }^{1,2}$, D. Knight ${ }^{4}$, K. Rajendran ${ }^{1}$, S. Bheesette ${ }^{1}$, N. D. Ruiter ${ }^{1,2,5}$, A. Chernoglazov ${ }^{2,5}$, V. B. H. Mandalika ${ }^{5}$, R. M. Doesburg ${ }^{2}$, C. J. Bateman ${ }^{1,2,4}$, S. T. Bell ${ }^{2,6}$, A. P. H. Butler ${ }^{1,2,4,6}$, P. H. Butler ${ }^{2,4,6}$, J. L. Healy ${ }^{4}$

${ }^{I}$ Dept. of Radiology, University of Otago, Christchurch, New Zealand

${ }^{2}$ MARS Bio-imaging Ltd., Christchurch, New Zealand

${ }^{3}$ Télécom Physique Strasbourg, Université de Strasbourg, Strasbourg, France

${ }^{4}$ Dept. of Physics and Astronomy, University of Canterbury, Christchurch, New Zealand

${ }^{5}$ HIT lab NZ, University of Canterbury, Christchurch, New Zealand

${ }^{6}$ European Centre for Nuclear Research (CERN), Geneva, Switzerland

MARS scanners fitted with Medipix3RX energy resolving photon counting detectors are the spectral CT system that can identify and quantify different materials simultaneously. Spectral CT systems are sensitive to the polychromatic structure of the X-ray beams that they use, therefore it is important to use spectrally accurate beam models in spectral reconstruction techniques. Using an inaccurate $\mathrm{x}$-ray model in spectral reconstruction can lead to severe image artifacts, one of the extreme cases of this is the well-known beam-hardening artifacts. An often overlooked spectral feature of x-ray beams in spectral reconstruction models is that shape of their spectrum changes with angle away from the central beam axis. We have developed a parameterized semianalytical x-ray source model in diagnostic range (30-120 kVp) by applying the regression techniques to data obtained from a custom Monte Carlo simulation of the x-ray tube used in several MARS scanners. It provides qualitative and quantitative information about the energy distribution of the $\mathrm{x}$-ray spectrum within $\pm 17^{\circ}$ along ? (vertical direction), $\pm 5^{\circ}$ along $-\mathrm{f}$ (horizontal direction) of the central axis and with the energy resolution of $1 \mathrm{keV}$. Comparisons of our model with those generated by SpekCalc, TOPAS, and IPEM78 at the central axis show a good agreement (within 2\%). Additionally, we also present a comparison of the model with experimental data collected with the MARS scanner.

\section{M10D-6: Estimation of an Atomic Density with a Singular Value Decomposition Method Using a Photon- Counting X-Ray CT

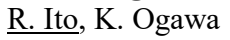

Department of Applied Informatics, Graduate School of Engineering, Hosei University, Tokyo, Japan

In the treatment planning of a heavy ion radiation therapy, an accurate density distribution of each atom in a human body is required. However, it is very difficult to obtain this distribution, and so the distribution of the electron density is estimated with the x-ray CT number of an electron density phantom. The purpose of our research is to measure the density distribution of a specified atom in compounds in a human body. The paper proposed a new method to obtain the density distribution of an atom 
with the singular value decomposition method using a photon counting detector. We conducted simulation using a phantom which was composed of water and ethanol solution, and we calculated the density distribution of hydrogen, carbon and oxygen. The simulation results of a phantom including several compounds showed the feasibility of our proposed method.

M10D-7: CT Auto-Calibration by Consistent Contours

$\underline{\text { S. Maur }}^{1,2}$, D. Stspankou ${ }^{1}$, J. Hesser ${ }^{1}$

${ }^{I}$ University Medical Center Mannheim, Heidelberg University, Experimental Radiation Oncology, Mannheim, Germany

${ }^{2}$ Sirona Dental Systems GmbH, Bensheim, Germany

This paper discusses a novel strategy for estimating projection matrices of a computed tomography using object contours in projection images. We formulate mathematical conditions for projection parameter consistency based on contour detection and tracking in 2D. Hence we derive a global optimization problem and a greedy optimizer herein. Applying the optimizer for motion detection show an average reduction of motion error in object space by $84,2 \%$. The evaluation is performed on simulated data with different motion types and object shapes and considers the influence of noisy contour data. The accuracy of pose estimation is linked to the sample resolution, the number and distinctiveness of imaged objects and the accuracy of input data. Due to an iterative approach our optimizer is robust towards bad initialization if some basic limitations are met.

\section{M10D-8: Implementation of Majorization-Minimization (MM) Algorithm for 3D Total Variation Minimization in DBT Image Reconstruction}

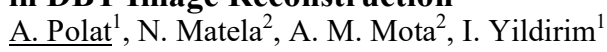

${ }^{I}$ Istanbul Technical University, Faculty of Electrical and Electronics Engineering, Institute of Informatics, Istanbul, Turkey

${ }^{2}$ Universidade de Lisboa, Faculdade de Ciencias, Instituto de Biofisica e Engenharia Biomedica, Lisbon, Portugal

Iterative image reconstruction of Digital Breast Tomosynthesis (DBT) data is a developing modality that produces 3D images of a breast to detect masses and microcalcifications. Algebraic reconstruction technique (ART), an iterative image reconstruction method, reconstructs the 3D model of the breast via projections acquired from a limited view angle scanning. In the last decade, DBT studies have largely addressed the problem of obtaining a reconstructed image from a few projections, which means a highly undersampled data. To achieve this, 3D total variation (TV) which is an operator of compressed sensing (CS) can be applied to the DBT images reconstructed by ART. On the other hand, in the signal processing literature, a new majorizationminimization (MM) algorithm on TV denoising is described for a N-point $\mathrm{x}(\mathrm{n})$ as $1 \mathrm{D}$ signal. According to our literature review, this 1D MM algorithm has not been applied to DBT studies yet. In this paper, we propose an application of MM1D algorithm to the 3D DBT reconstructed ART data with 3D TV minimization (ART+TV3D+MM1D). Both quantitative and qualitative analysis of ART+TV3D and the proposed method ART+TV3D+MM1D are carried out for a phantom that mimics 3D breast. In the algorithm of ART+TV3D+MM1D, MM1D is applied to ART+TV3D volumes in each iteration. To evaluate the performance of the proposed method, metrics such as structural similarity (SSIM) and signal to noise ratio (SNR) of a specific layer of interest (LOI) are examined. ART+TV3D+MM1D shows superior results in terms of SNR\&SSIM values and in smoothing image compared to ART+TV3D. For instance, at the end of the 50th iteration, while ART+TV3D presents SSIM of 0.8912, ART+TV3D+MM1D exhibits a value of 0.9174 .

This work has been supported in part by Fundação para a Ciência e a Tecnologia - Portugal (Project Pest-OE/SAU/IU0645/2013) and TUBITAK, The Scientific and Research Council of Turkey, under the grant 111E086.

M10D-9: Preliminary Study of Quantitative X-Ray Spectral Imaging with Spectral Deconvolution S. Wang, L. Zhang, X. Xu, D. Wu

Dept. of Engineering Physics, Tsinghua University, Beijing, China

The degrading factors of photon counting detectors such as charge-sharing and K-escape, which lead to spectrum distortion, set the crucial limitation for its application in quantitative imaging. We present a method to eliminate the degrading factors by spectral deconvolution. Simulation study and experimental study are carried out to verify its effectiveness and robustness.In simulation study, the method can significantly reduce the deviation of measurement to theoretical value, which implies the effectiveness. Poisson noise is added to test the robustness and we find the result shows no significant difference to noiseless result when up to $10^{6}$ photons are detected. In experimental study, theoretical spectrum is not easily accessible, instead we verify the effectiveness by calculating width of an aluminum plate based on Beer-Lambert Law, which is supposed to be consistent among the whole energy range. An improvement can be seen after deconvolution and the output shows a good agreement with simulation result. Furthermore, we simulate of a CT reconstruction process using the promoted method and find the reconstructed $\mu$ fits the theoretical value very well, which manifests its potential in quantitative X-ray spectral imaging.

M10D-10: An Investigation of the Influence of Scatter in Energy-Resolved Computed Tomography 
T.-H. Tsai, I. Kanno

Graduate School of Engineering, Kyoto University, Kyoto, Japan

Energy-resolved computed tomography (ERCT) is a novel imaging modality which can provide more useful information than conventional X-ray computed tomography (CT). However, in ERCT, the influence of scatter such as cupping artifact may differ from those in conventional CT and should be investigated properly. In this study, we performed Monte Carlo simulations and compared the image contrast and cupping artifact between conventional CT and ERCT, with different contrast agents and different beam qualities. The results show that the influence of scatter in ERCT is highly correlated to energy range. The cupping artifact in ERCT is significantly high in the low-energy range, and using a harder X-ray beam for imaging can further enhance it. Therefore, care should be taken while obtaining low-energy-range ERCT images with an iodine contrast agent. On the other hand, using a contrast agent with K-edge at higher energy, such as gold, may provide good image contrast as well as low cupping artifact. The air scattering also shows some influence in low-energy-range ERCT images. An appropriate air-scattering model may be necessary while developing scatter correction algorithms.

\title{
M10D-11: Empirical Noise Power Spectrum Based on the Image Subtraction in Radiography Imaging
} D. S. Kim, E. Lee

Dept. Electronics Engineering, Hankuk University of Foreign Studies, Geonggi-do, South Korea

In order to measure the noise power spectrum (NPS) of a radiography detector from an acquired image without any object, a subtraction-based algorithm, in which the input image is subtracted from another image acquired at the same exposure condition, is usually used to alleviate the fixed pattern noise influence. However, depending on variations in the x-ray tube and the readout circuits in the detector, the acquired images show nonuniform statistics and thus selecting an appropriate image pair from a given image set is important for a stable measurement of NPS. In order to select a good image pair for measuring an accurate normalized NPS (NNPS), a measurement method is proposed based on selecting an image pair from comparing the signal-tonoise ratios, which are obtained from the subtracted image. For the comparison, a commutative relationship as a sufficient condition for the accurate NNPS is considered. For a given image pair, the proposed method can provide a degree of accuracy of the empirical NNPS. For real x-ray images acquired from an indirect radiography detector, the proposed method is tested and shows stable measuring results for a set of images.

\author{
M10D-12: Multi-View Scatter Estimation for Moving Blocker Scatter Correction of CBCT \\ C. Zhao ${ }^{1}$, L. Ouyang ${ }^{2}$, J. Wang ${ }^{2}$, M. Jin ${ }^{1}$ \\ ${ }^{I}$ Physics, University of Texas at Arlington, Arlington, TX, USA \\ ${ }^{2}$ Radiation Oncology, University of Texas Southwestern Medical Center, Dallas, TX, USA
}

The moving blocker method is economic and effective for scatter correction (SC) of cone-beam computed tomography (CBCT). However, at the regions with large intensity transition in the projection images in the axial blocker moving direction, the estimation of scatter signal from blocked regions in a single projection view can produce large errors, which can cause significant artifacts in reconstructed images and null the usability of these regions. In this work, we propose to use the adjacent multi-view projection images to jointly estimate scatter signal more accurately. The experimental results using CatPhan600 CBCT data show that the new method can effectively suppress the estimation errors of scatter signal and lead to much improved image quality in these regions. This development will expand the utility of moving blocker-based SC for the target with sharp intensity changes in the projection images, such as bone/lung and body/air interfaces.

M10D-13: Beam Profile Assessment in MARS Spectral CT

M. Anjomrouz ${ }^{1}$, M. Shamshad ${ }^{1}$, D. J. Smithies ${ }^{2}$, A. Largeau ${ }^{3}$, L. Vandon Broeke ${ }^{4}$, R. K. Panta ${ }^{1,2}$, R. Aamir ${ }^{1,2}$, A. Atharifard ${ }^{1}$, M. F. Walsh ${ }^{2}$, B. P. Goulter ${ }^{2}$, D. Knight ${ }^{4}$, S. Bheesette ${ }^{1}$, N. D. Ruiter ${ }^{1,2,5}$, A. Chernoglazov ${ }^{2,5}$, V. B. H. Mandalika ${ }^{5}$, C. J. Bateman ${ }^{1,2,4}$, S. T. Bell ${ }^{2,6}$, A. P. H. Butler ${ }^{1,2,4,6}$, P. H. Butler ${ }^{2,4,6}$

${ }^{I}$ Dept. of Radiology, University of Otago, Christchurch, New Zealand

${ }^{2}$ MARS Bioimaging Ltd., Christchurch, New Zealand

${ }^{3}$ Télécom Physique Strasbourg, Université de Strasbourg, Strasbourg, France

${ }^{4}$ Dept. of Physics and Astronomy, University of Canterbury, Christchurch, New Zealand

${ }^{5}$ HIT lab, University of Canterbury, Christchurch, New Zealand

${ }^{6}$ European Centre for Nuclear Research (CERN), Geneva, Switzerland

We present a methodology that uses a combination of experimental and modeled data to assess various properties of x-ray beam measurements obtained from MARS spectral CT scanners. Properties that are assessed include inter-scan and intra-scan beam profile variability; comparing beam profile shape and magnitude between experimental and modeled data; and determination of the experimental the x-ray beam center. We demonstrate this methodology on data collected on a MARS scanner equipped with a 
CdTe Medipix3RX detector. This set of experiments is shown to have inter-scan variability of $<1 \%$ and intra-scan variability of $<0.1 \%$. We have also observed the x-ray beam center offset to vary between $0.1^{\circ}$ 
to $3^{\circ}$ 
on different MARS systems. These beam profile measures can be used to identify and characterise variability within data acquired from spectral scans, in addition to providing x-ray beam information that can be used in spectral reconstruction techniques to improve material discrimination and quantification.

\author{
M10D-14: Application of a Blind Image Deblurring Method Based on Compressed-Sensing (CS) Scheme in \\ Dental Cone-Beam CT: Simulation and Experimental Studies \\ K. Kim, H. Cho, Y. Park, U. Je, C. Park, H. Lim, S. Park, G. Kim, S. Park, H. Lee \\ Dept.of Radiation Convergence Engineering, Yonsei University, Wonju, Gangwon-do, South Korea
}

In cone-beam computed tomography (CBCT), the reconstructed images are inherently degraded, limiting its image performance, mainly due to the finite focal spot size of the x-ray source, aspects inherent to detector such as the detector pixel size and the detector resolution, and the reconstruction procedure as well. Thus the recovery of the reconstructed images from their degraded version is essential for further improving the image characteristics. In this work, as an effective image deblurring method in CBCT, we investigated a compressed-sensing (CS)-based blind deconvolution method in which a two-dimensional (2D) blur kernel is recursively optimized by using the conjugate-gradient (CG) solver for the next updated images in the CS framework. Here the CS is the state-of-the-art mathematical theory for solving the inverse problems, which exploits the sparsity of the image substantially high accuracy. We implemented the proposed deblurring algorithm for CBCT and performed a systematic simulation and experiment to demonstrate its viability. In the experiment, we used a commercially-available dental CBCT system (Expert7TM, Vatech Co., South Korea) which consists of an x-ray tube $(90 \mathrm{kVp}, 5 \mathrm{~mA})$, a CMOS-type flat-panel detector (pixel size: $0.2 \mathrm{~mm}$, active area: $234 \mathrm{~mm} \times 288 \mathrm{~mm}$ ), and a mechanical support for object installation. Our simulation and experimental results indicate that the proposed approach appears to be effective for the blurring problems in CBCT and thus is applicable to improve the present image performance.

This work was supported by the Radiation Technology Development Program of the National Research Foundation (NRF) funded by the Korea Ministry of Science, ICT \& Future Planning under Contract No. 2015-51-0284.

M10D-15: Simultaneous Reconstruction and Separation in a Spectral CT Framework

S. Tairi ${ }^{1}, \mathrm{~S}$. Anthoine ${ }^{2}, \mathrm{C}$. Morel $^{1}, \underline{\text { Y. Boursier }}{ }^{1}$

${ }^{1}$ Aix Marseille Universiti $; 1 / 2$, CNRS/IN2P3, CPPM UMR 7346, Marseille, France

${ }^{2}$ Aix Marseille Universitï $\angle 1 / 2$, CNRS, Centrale Marseille, I2M UMR 7373, Marseille, France

In this paper, we propose a method to simultaneously separate and reconstruct the physical components of an object observed in the context of spectral Computed Tomography (CT). Spectral CT has been made possible by the technological breakthrough of $\mathrm{X}$-ray hybrid pixel detectors in the last years and brings CT imaging from an anatomic to a functional biomedical imaging modality. Our paper considers the polychromatic model and combines it with a low-rank prior on the components. The simultaneous separation and reconstruction of components is done by solving a non-convex ill-posed inverse problem. Promising results are shown on synthetic data with a simple regularization that incorporates positivity contraints.

\author{
M10D-16: Multitarget Data Association with Higher-Order Motion Models for Tracking in Proton CT \\ Instrumentation \\ L. Gong, X. Ye, N. Allinson \\ Computer Science, University of Lincoln, Lincoln, UK
}

PRaVDA is an advanced imaging and dosimetry system for proton therapy with solid-state sensors throughout. The residual energy resolving detector (range telescope) can be composed of multiple layers of CMOS imagers. Such imagers, though operating at relatively low frame rates, can record many protons events per read cycle. The challenge is to identify the non-linear tracks of individual protons through the telescope. A multi-target data association with higher-order motion model (MDAMM) was designed for the proton events in each layer. Such algorithms focus more on motion constraints rather than appearance. We use a cost function based on Gaussian for MDAMM which is suitable for protons as they are laterally scattered. We factor in all trajectories of a protons path (through all layers) into a cost function in order to improve the quality of a candidate trajectory, achieving good accurate and fast proton tracking. Also an extended cost function considers the most likely hypothesis update depending on previously defined tracks has been developed.

M10D-17: Object Removal in Gradient Domain of Cone-Beam CT Projections

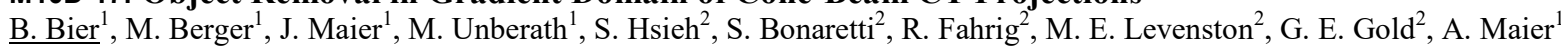
${ }^{I}$ Department of Computer Science, Pattern Recognition Lab, Friedrich-Alexander-University Erlangen-Nuremberg, Erlangen, Germany

${ }^{2}$ Radiological Sciences Laboratory, Stanford University, Stanford, CA, USA 
We propose a method to reduce streak artifacts in cone-beam CT reconstructions that arise from the edges of dense objects outside the 3D field-of-view. To this end, ramp filtering is decomposed into a derivative- and a Hilbert transform step. This allows for spectral inpainting directly in gradient domain, such that only contributions of sharp edges are removed. We applied our approach to weight-bearing knee imaging data, where plastic pipes outside the field-of-view introduce notable streak artifacts. Streak artifacts in the reconstructions are reduced and on semisimulated data the correlation coefficient could be improved from 0.88 to 0.99 . The method is applicable for arbitrary object shapes and can be easily integrated into existing FDK reconstruction algorithms.

M10D-18: Needle Detection in Interventional Pain Management with 3D Image Reconstruction E. A. Rashed ${ }^{1}$, M. al-Shatouri ${ }^{2}$, M. Selim ${ }^{1,3}$, H. Kudo ${ }^{4}$

${ }^{I}$ Image Science Lab., Dept. of Mathematics, Faculty of Science, Suez Canal University, Ismailia, Egypt

${ }^{2}$ Dept. of Radiology, Faculty of Medicine, Suez Canal University, Ismailia, Egypt

${ }^{3}$ Dept. of Mathematics and Computer Science, Faculty of Science, Suez University, Suez, Egypt

${ }^{4}$ Division of Information Engineering, University of Tsukuba, Tsukuba, Japan

Interventional pain management surgery is a clinical application performed though the guidance of x-ray fluoroscopy. Several protocols requires the injection of a needle close to the spinal cord to deliver a medication directly to the nerve system. The needle position information in the 3D space is important to avoid possible damage to the nerve system. It is common to perform the pain management surgery using $\mathrm{C}$-arm scanner to follow up the treatment procedure. In many cases, it is difficult to observe the exact position of the injected needle through $2 \mathrm{D}$ images acquired using conventional $\mathrm{C}$-arm scanner especially with complicated bone structures. It requires several attempts to image the patient from different positions and physician requires a mental process to imagine how the 3D structure looks like before starting the interventional procedure. This process may be repeated several times during a single interventional session, which cause a significant increase of radiation dose given to both patient and surgeon. In this paper, we introduce a method for needle detection in interventional pain management surgery using a clinical C-arm scanner. First, an in-house made gantry control unit (GCU) is mounted to the C-arm gantry to control the scanner orbital rotation. Second, the gantry rotation is traced using inertial measurement unit (IMU) sensor attached. A single cine loop is acquired by automatically rotate the C-arm gantry around the patient using GCU. Geometry information obtained from the IMU sensor is used to define the gantry position in the 3D space and synchronized with detector measurements in cine loop frames. The SCAN algorithm is then adopted for the 3D reconstruction of bone structures and injected needle.

\title{
M10D-19: Bias-Corrected Spectrum Decomposition for X-Ray Computed Tomography (CT): Theory and Method
}

$\underline{\mathrm{H} . ~ G a o}^{1}, \mathrm{G} . \mathrm{Fu}^{2}$, Y. $\mathrm{Jin}^{2}$, P. Edic ${ }^{2}$

${ }^{I}$ GE Healthcare, Waukesha, WI, USA

${ }^{2}$ GE Global Research, Niskayuna, NY, USA

Modern diagnostic X-ray computed tomography (CT) usually provides a limited number of standard X-ray tube operating voltages $(\mathrm{kVp}$ 's) that are well calibrated and optimized. In certain scenarios, however, a spectrum using a non-standard $\mathrm{kVp}$ may have potential of achieving improved image quality and dose efficiency. For a CT scan using a non-standard kVp, estimating an effective spectrum is a problem to be solved. An efficient but not exact method of spectrum estimation in practice is to decompose the non-standard spectrum into a linear combination of spectrua generated using standard kVp's, i.e., spectrum decomposition. This approach works well when the spectrum generated at a non-standard $\mathrm{kVp}$ is reasonably close to that generated by a standard $\mathrm{kVp}$. If the operating voltage is far from a standard $\mathrm{kVp}$, the residual error (systematic bias) could be significant, causing artifact/inaccuracy in CT images. In order to improve spectrum decomposition, we present a bias-corrected spectrum decomposition approach in this work. A lookup table (LUT) is established in advance to store the estimation errors using a set of known spectra generated using known $\mathrm{kVp}$ 's from simulation that do not have to be well calibrated. A reasonable and key assumption here is that the bias of spectrum decomposition in the LUT generation would be close to that of actual spectrum estimation. Simulations of a dual-energy CT scan at non-standard kVp demonstrate the effectiveness of this approach, leading to more quantitative CT images.

\author{
M10D-20: A Framework for Iterative Reconstruction in Phase Contrast Computed Tomography Dedicated to \\ the Breast

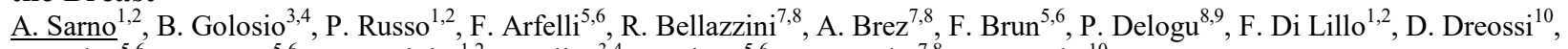

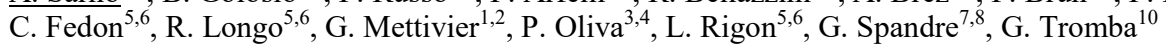 \\ ${ }^{I}$ Dip. di Fisica, Universit? di Napoli, Napoli, Italy \\ ${ }^{2}$ INFN sez. di Napoli, Napoli, Italy \\ ${ }^{3}$ Universit? degli Studi di Sassari, Sassari, Italy \\ ${ }^{4}$ INFN Sez. di Cagliari, Cagliari, Italy \\ ${ }^{5}$ Universit? di Trieste, Trieste, Italy
}


${ }^{6}$ INFN sez. di Trieste, Trieste, Italy

${ }^{7}$ PiXirad Imaging Counters srl, Pisa, Italy

${ }^{8}$ INFN sez. di Pisa, Pisa, Italy

${ }^{9}$ Dipartimento di Scienze Fisiche, della Terra e dell'Ambiente, Universit? di Siena, Siena, Italy

${ }^{10}$ ELETTRA Sincrotrone Trieste, Trieste, Italy

We present the implementation of the CT iterative reconstruction strategy developed within the SYRMA-CT project for in vivo phase contrast CT of the uncompressed breast, ongoing at the ELETTRA synchrotron radiation facility (Trieste, Italy).

Propagation-based phase-contrast imaging exploited the high spatial coherence of the monoenergetic laminar X-ray beam (3-mm high along the chest-wall-to-nipple direction), as well as the large object-to-detector distance $(\sim 2 \mathrm{~m})$ and the use of a prototype of Pixirad-8 high-resolution photon counting CdTe detector (60- $\mu \mathrm{m}$ pitch, eight detector units arranged in a row). The signal in projection views depends on the X-ray absorption as well as on the phase shift introduced by the breast tissue in the beam path. A phase retrieval algorithm allows recovering the projected 2D phase map of the irradiated tissue layer, which were input to the CT reconstruction; then, the 3D image of the breast was reconstructed via a simultaneous algebraic reconstruction technique (SART) algorithm. The developed iterative reconstruction - coupled with a filtering process for reducing the noise level and ring artifacts by preserving edges sharpness - showed better image quality than conventional filtered backprojection (FBP) reconstruction. A phantom study showed that the iterative reconstruction produced images with a contrast-to-noise-ratio up to $65 \%$ and a spatial resolution up to $12 \%$ higher than those obtained with FBP. Finally, the developed algorithm removed ring-like artifacts caused by the detector dead space $(0.16 \mathrm{~mm})$ across adjacent detector units and by no perfect equalization after flat-field correction, without worsening the image quality.

\title{
M10D-21: Extended View Cone-Beam Reconstruction with a Movable Gantry
}

A. V. Bronnikov

Bronnikov Algorithms, Arnhem, Netherlands

The problem of extending the lateral field of view of cone-beam CT systems arises in diverse applications, ranging from small animal imaging to radiation therapy imaging and dental CBCT. The well-known method of using the displaced detector allows for the extension of the field of view roughly in two times. The method of translational shift of the detector allows for larger extensions but suffers from deterioration of image quality at large angles. An alternative approach would be to move the entire gantry in the axial plane so that a larger area could be covered with the given detector. One of the examples of such geometry is provided by the spiral motion of the gantry in the axial plane. Here one can extend the field of view in several times. We propose a backprojection-type 3D image reconstruction algorithm for this geometry and evaluate it with computer-generated data.

M10D-22: An Improved Approach to Calculate the Presampling Modulation Transfer Function Using Edge Samples with Surface Irregularities for X-Ray Imaging Systems

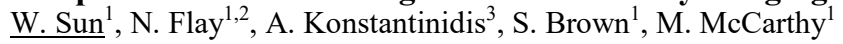

${ }^{I}$ National Physical Laboratory, Teddington, United Kingdom

${ }^{2}$ University of Southampton, Southampton, United Kingdom

${ }^{3}$ Christie NHS Foundation Trust, Manchester, United Kingdom

Analysis based on the use of the modulation transfer function (MTF) has been widely used to assess the spatial resolution of Xray imaging systems. Common MTF calculation methods using slanted edges require an edge sample with high surface quality to ensure robust results. However, in the case where detector pixels are of the order of hundreds of micrometre size and below, small surface irregularities of these edge samples can detrimentally influence the test results.

In this paper, the authors propose a modified approach to calculate the MTF that overcomes these issues related to surface irregularities. The method uses a Gaussian curve-fitting algorithm to estimate the location of an edge, where the relative position of its data to the corresponding edge can then be calculated to produce the presampling MTF. The suggested method has been compared with conventional MTF calculation and the advantages are shown.

\author{
M10D-23: Investigation of Non-Negativity Constraint on Basis Images in Half-Rotation Data Reconstruction in \\ Spectral CT

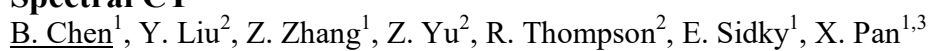 \\ ${ }^{I}$ Department of Radiology, The University of Chicago, Chicago, IL, USA \\ ${ }^{2}$ Toshiba Medical Research Institute USA, Inc., Vernon Hills, IL, USA \\ ${ }^{3}$ Department of Radiation and Cellular Oncology, The University of Chicago, Chicago, IL, USA
}

In this work, we investigate the impact of the non-negativity constraint applied on the basis images with a dual-energy phantom with iodine and calcium inserts. Employing a previously developed one-step reconstruction method, we looked at two 
optimization problems with and without the non-negativity constraint and reconstructed basis images. The results suggest that some streak artifacts that are associated with the half-plus-half rotation scans can be reduced and that the quantitative accuracy for calcium inserts can be improved by the absence of the non-negative constraint and allowing for negative basis components.

M10D-24: An ML-EM Spectrum Reconstruction Method Based on the Detector Response Model Calibrated by XRF Spectrums

R. Li, L. Li

Engineering Physics, Tsinghua University, Beijing, China

Accurate estimation of distortion-free spectrum is important but difficult in various applications including spectral CT systems. To reconstruct the incident spectrum, there are two key problems to solve. One is the acquisition of detector response function. Different from many existing methods that either require detailed Mont-Carlo modeling of the detector system, or require monochromatic light from synchrotron sources or radioactive isotope sources, in this paper we promote a feasible method based on the parametric modeling of XRF spectrums, which is inspired by an existing model based on the XRF spectrums. The other key problem is reconstructing the spectrum based on the obtained detector response. Since the system is severely ill that the condition is up to $? 10 ?^{\wedge} 13$, it is very difficult to obtain an accurate solution from noisy data. Existing method uses a Spectrum Stripping algorithm, whereas with an imperfect assumption that could lead to inaccurate results. We propose to reconstruct the incident spectrum by ML-EM algorithm based on the Poisson noise model of the system. To verify our method, simulation and experiment are conducted with both the ML-EM method and the existing method for comparison. Results show that the proposed method is effective for the reconstruction of incident spectrums, especially for XRF spectrums, for which our method markedly increases the accuracy comparing to the existing method.

\section{M10D-25: XFCT Imaging System with Pinhole Collimation and Attenuation Correction}

S. Zhang, L. Li, Z. Chen

Engineering Physics, Tsinghua University, Beijing, China

The study of gold nanoparticles (GNPs) has been more attractive because of its application for cancer detection and therapy. Xray fluorescence computed tomography (XFCT), which is sensitive to high $\mathrm{Z}$ elements such as gold, is a promising approach to determine the distribution of GNPs in vivo. Several XFCT imaging systems have been designed and developed in recent years. However, as an emission tomography, the limit of fluorescence yield and low detection efficiency brings high statistic noise. The interference of Compton scattering as well as the effect of absorption when photons crossing the phantom should also be considered in data acquisition and image reconstruction. These are the main problems that current XFCT imaging systems confront. In this paper, we present a design of XFCT system with fan beam X-ray source and two photon counting detectors for $\mathrm{XFCT}$ imaging and attenuation correction. One detector with pinhole collimation is placed perpendicular to the beam direction for fluorescence photon and scattering photon collection. The second detector is placed behind the phantom to obtain transmission CT imaging for attenuation correction. The main advantage of using two detectors is that the X-ray fluorescence signal and the attenuation map can be obtained in one time of scanning procedure. Numerical simulations are performed to study the feasibility of the system and its Sensitivity to GNPs.

\section{M10D-26: CT Iterative Reconstruction Based on Both Noise Model and Image Constraint}

T. Zhang, L. Li, Z. Chen

Engineering Physics, Tsinghua University, Beijing, China

This research aims to reduce the effect of noise on reconstructed image of CT. We give a method which adds noise model and image constraint to the CT iterative reconstruction algorithm. The image constraint we use is Total Variation minimization method. The noise model depends on the types of noise. On one hand, the noise in the projection data before logarithmic transformation is approximated by the Poisson distribution. One the other hand, the noise in the projection data after logarithmic transformation is approximated by the Gaussian distribution. Finally, we use the Split-Bregman method to solve our final objective function. In our numerical experiments, we adopt different methods to dispose the projection data in which the noise is already added. And by comparison between the results, we test feasibility of our method. In conclusion, the completed algorithm which is presented in this research is better than other methods. And it can effectively reduce the noise effect and improve the image quality. In the future, we will optimize the algorithm which obtained in our research and extend the methods for 3D construction and apply it for multi-source tomography.

M10D-27: X-Ray Spectral Radiography for Cardiovascular Applications

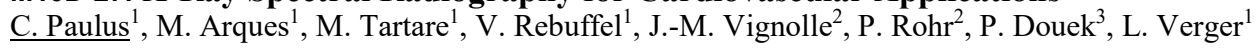


${ }^{I}$ CEA LETI MINATEC Campus, Grenoble, France

${ }^{2}$ Trixell, Moirans, France

${ }^{3}$ Claude Bernard University Lyon 1, Villeurbanne, France

X-ray spectral imaging is raising increasing interest in radiology. Mainly developed in X-ray CT scanners with linear detectors, the work presented here is part of a project aiming at providing similar spectral capabilities to conventional radiography. Two main issues arise. First one is the feasibility of such spectrometric 2D flat panel. For this purpose, a new pixel electronic concept mixing spectrometry and integration modes has been designed. Secondly, we investigated the clinical interest to have such spectrometric detector and the new perspectives opened for x-ray imaging. In this paper, we evaluate the interest of spectral Xray radiography for cardiovascular applications and especially in the coronary angiogram case. A significant capability of spectral imaging is considered to be K-edge imaging that can quantify heavy elements such as iodine, gold, bismuth, or gadolinium with a single shot acquisition image. The proposed study is conducted on simulations using a numerical labellised thorax phantom in which various concentrations of contrast agent inserts were added in lung cavities and behind the spine. The processing method used is a calibration-based Poisson log-likelihood decomposition over three materials: bones, soft tissue and contrast agent. We show that the decomposition processing combined with a 5-channel detector provides good results and makes it possible to both detect and quantify different concentrations of gadolinium in pulmonary cavities.

\author{
M10D-28: Population-Based Scatter Correction Framework for Large FOV CBCT Scanner \\ M. Sibomana $^{1}$, D. Benoit ${ }^{2}$ \\ ${ }^{I}$ ICTEAM/ELEN, Universite Catholique de Louvain, Louvain-La-Neuve, Belgium \\ ${ }^{2}$ LaTIM/UMR 1101 INSERM, CHRU Brest, Brest, France
}

A major limitation of large Field-Of-View (FOV) Cone-Beam CT (CBCT) is the low image contrast due to a large fraction of scatter photons in the acquired data. The aim of this work is to develop a population-based framework to predict the scatter fraction and correct the acquired data using low computation resources for clinical applications. We have used an extra-fast simulation software (GGEMS) on GPU to generate projection data (including scatter) of different regions (head, thorax, pelvis) of an anthropomorphic phantom to create an initial prediction model which will be extended with patient data. The target system is a CBCT (based on Varian G292 X-ray source and Thales RF 4343 detector) with 360 degrees' rotation, a distance from the Xray source focal spot to the rotation axis of $800 \mathrm{~mm}$, and a distance from the focal spot to the flat panel detector of $1300 \mathrm{~mm}$. The $\mathrm{X}$-Ray source has an aperture angle of 13 degrees and the detector size is $430 \mathrm{~mm}$ in both dimensions. The simulated scatter projections were structure less and a linear model was used to predict their average as function of the sum of scatter and primary data. Two scatter subtraction methods (raw simulated scatter and predicted average scatter) from the simulated data were used prior to reconstruction using a general Maximum Maximization for Transmission iterative method. There were no visual differences between the two reconstructed images; a quantitative assessment using regions of interest gave a similar average for both methods with a lower standard deviation for the prediction method.

\author{
M10D-29: A New Material Decomposition Method Eliminating the K-Shell Photoelectric Effect for Dual- \\ Energy CT \\ T. Zhao, L. Li, Z. Chen \\ Dept. Engineering Physics, Tsinghua University, Beijing, China
}

In dual keV energy CT, the photon interacts with matter mainly in two ways, photoelectric effect and Compton scatter. The classic double-effect decomposition method is based on the physical models of these two effects. K-edge, which is usually introduced by contrast agent, makes the physic model not appropriate for classic basis material decomposition (CBMD) method, which means that the result of direct CBMD has relatively large artifacts. We propose a new basis material decomposition method. The k-shell photoelectric effect attenuation contribution is first estimated from a preliminary CBMD result with basis function of components of contrast agent. This contribution is eliminated from the projection data to obtain new CBMD result. The numerical experiment results show that after this elimination, the image of CBMD result has less artifacts. This idea is a new data processing way of DECT and needs more verification.

M10D-30: Analog Non-Linear Transformation-Based Tone Mapping for Image Enhancement in C-Arm CT L. Shi ${ }^{1}$, M. Berger ${ }^{2}$, B. Bier ${ }^{2}$, C. Soell ${ }^{1}$, J. Roeber ${ }^{1}$, R. Fahrig ${ }^{3,4}$, B. Eskofier ${ }^{2}$, A. Maier ${ }^{2}$, J. Maier ${ }^{2}$

${ }^{I}$ Dept. of Electrical Engineering, Institute for Electronics Engineering, Friedrich-Alexander-University Erlangen-Nuremberg, Erlangen, Germany

${ }^{2}$ Dept. of Computer Science, Pattern Recognition Lab, Friedrich-Alexander-University Erlangen-Nuremberg, Erlangen, Germany

${ }^{3}$ Radiological Sciences Laboratory, Stanford University, Stanford, CA, USA

${ }^{4}$ Siemens Healthcare GmbH, Forchheim, Germany 
Flat-Panel C-arm Computed Tomography (CT) suffers from pixel saturation due to the detector's limited dynamic range. We describe a novel approach of analog, non-linear tone mapping (TM) for preventing detector saturation. An analog TM operator (TMO) applies a non-linear transformation in a CMOS (complementary metal-oxide semiconductor) sensor and its inverse TMO based on 14-bit digital raw data. This is done in order to prevent overexposure and to enhance image quality to 32 bits. The method was applied to the cases of low-contrast head imaging and to that of imaging both knees. Cone-beam projection data with and without overexposure was simulated for a 200 degree short-scan of the knees and a 360 degree full-scan of a Forbild head phantom. The results show an increased correlation coefficient with respect to ground truth of 0.99 for TMO compared to 0.96 for overexposed knee data and a higher low-contrast visibility $(\mathrm{CC}=0.99)$ compared to linear quantization $(\mathrm{CC}=0.97)$.

\section{M10E: Posters: preclinical II}

Thursday, Nov. 3 16:30-18:30 Etoile

M10E-1: SAFIR: High Rate Test Inside the MR Bore with the Assessment of a MR-Compatibility R. Becker ${ }^{1}$, A. Buck ${ }^{2}$, C. Casella ${ }^{1}$, V. Commichau ${ }^{1}$, S. Corrodi ${ }^{1}$, G. Dissertori ${ }^{1}$, J. Fischer ${ }^{1}$, A. S. Howard ${ }^{1}$, M. Ito ${ }^{1}$, P. Khateri ${ }^{1}$, K. Kramer ${ }^{1}$, W. Lustermann ${ }^{1}$, J. F. Oliver ${ }^{3}$, C. Ritzer ${ }^{1}$, U. Röser ${ }^{1}$, Q. Wang ${ }^{4}$, G. Warnock ${ }^{5}$, B. Weber ${ }^{5}$

${ }^{I}$ ETH Zürich, Institute for Particle Physics, Zürich, Switzerland

${ }^{2}$ Zürich University Hospital, Division of Nuclear Medicine, Zürich, Switzerland

${ }^{3} U V$-CSIC, Instituto de Fisica Corpuscolar, Valencia, Spain

${ }^{4}$ Tsinghua University, Institute of Medical Physics, Beijing, China

${ }^{5}$ University of Zürich, Institute of Pharmacology and Toxicology, Zürich, Switzerland

The SAFIR (Small Animal Fast Insert for $\mathrm{mRi}$ ) project aims at the development of an innovative high rate PET insert for a preclinical 7T MRI scanner. The system is designed to be used for quantitative dynamic studies with short-lived tracers. In order to achieve good counting statistics and high temporal resolution, extremely high activities up to $500 \mathrm{MBq}$ of tracers are injected into a small animal and data are acquired with ultra-short frame duration of a few seconds. Challenging requirements are count rate capability of the data acquisition (DAQ) electronics and excellent timing resolution of $\sim 300 \mathrm{ps}$ at high activity to reduce random contribution by using a narrow coincidence timing window ( $\sim 400 \mathrm{ps})$. In previous high rate tests performed outside of $\mathrm{MR}$, we obtained the excellent coincidence timing resolution of $\sim 300 \mathrm{ps}$ FWHM at the SAFIR equivalent activity of $500 \mathrm{MBq}$ and a high rate DAQ capability covering the SAFIR requirement of $40 \mathrm{kHz} / \mathrm{ch}$ using two detector matrices of SiPM array and LYSO crystals with the STiC (ver. 3.1) ASIC. The preliminary results on the performance evaluation of PETA modules gave a comparable timing resolution. In this study, we build two independent prototype inserts with the STiC readout and the PETA modules. Their high rate performances will be evaluated inside of the MR bore, including the assessment of a MR-compatibility. We will report the first results on the investigation of interference between the prototype PET inserts involving electronics and MR with the static magnetic field, the radiofrequency (RF) signals, and gradient system.

This work was supported by the ETH Zurich Foundation through ETH Research Grant ETH-30 14-2, and by an SSSTC Exchange Grant EG $02-$ 03201.

\section{M10E-2: Investigation of Factors Affecting a Potential Worldwide Network of Medical PET Scanners to} Monitor the Decay Rate of Lu-176 and Detect Global Radiation Events

M. V. Green $^{1,2}$ J. Seidel $^{1,2}$, J. J. Vaquero ${ }^{3}$, P. L. Choyke ${ }^{1}$

${ }^{1}$ Molecular Imaging Program, National Cancer Institute, Bethesda, Maryland, USA

${ }^{2}$ Laboratory Animal Sciences Program, Leidos Biomedical Research, Inc., Frederick, Maryland, USA

${ }^{3}$ Departamento de Bioingeniería e Ingeniería Aeroespacial, Universidad Carlos III de Madrid, Leganés, Madrid, Spain

Medical PET scanners now exist in relatively large numbers around the world suggesting that certain physics experiments might be carried out during off-hours if these machines were organized into an array of interlinked devices. For example, workers at Purdue (Jenkins, et.al., Astropart. Phys 37 (2012) 81-88) have claimed to detect an annual periodicity in the decay rate of Cl-36 as the Earth moves around the Sun but these claims remain controversial (Norman, et. al. Astropart. Phys 31 (2009) 135-137). A subset array of Lu-based PET scanners might help resolve this matter by measuring for an extended period (years) the decay rate of Lu-176 (38 billion year half life), a radioactive contaminant present in the scintillation crystals of these machines. Night-side PET scanners in time synchrony might also detect and directionally locate previously unrecognized global radiation events of astronomical origin. However, PET scanners are not purpose-built for such experiments and when operated in a medical environment, are subject to many more potential sources of error. Here, we focused on identifying errors that might affect $\mathrm{Lu}$ decay rate measurements by recording for extended periods the total singles and coincidence count rates from Lu-176 between 2 and 3 am local time in two identical small animal Lu-based PET research scanners, one in Bethesda, MD (N=824 days) and one in Madrid, Spain ( $\mathrm{N}=299$ days). Crude comparison of Lu count rates from these two machines suggests that a carefully designed strategy might successfully exploit this unutilized resource and make feasible a worldwide PET scanner array. 
M10E-3: Use of a Resistive Network to Estimate the 3D Positioning of Events in a Monolithic Crystal

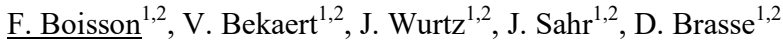

${ }^{I} I P H C$ - CNRS/IN2P3, Strasbourg, France

${ }^{2}$ Université de Strasbourg, Strasbourg, France

Current researches on small-animal PET detectors are based on highly segmented crystal matrices to obtain the best possible spatial resolution. One alternate solution is the use of monolithic crystals read by multi-channel photo-detectors. Here we investigated the performance of a detection module dedicated to preclinical PET imaging. We assess the intrinsic performance of a detection module, which consists in a monolithic $50 \times 50 \times 8 \mathrm{~mm}^{3}$ LYSO crystal coupled to a H9500 PMT directly attached to a resistive network, providing outputs for each row and column of the $16 \times 16$ PMT channels. We first introduce the operating principle and specific properties of the resistive network we developed. Results obtained without the resistive network show an average energy resolution below 20\% FWHM at $511 \mathrm{keV}$, an average intrinsic spatial resolution of $1.61 \pm 0.36 \mathrm{~mm}$ and a DOI uncertainty of $2.2 \mathrm{~mm}$. Future work will evaluate four resistive network impedance values $(180,348,750,1500 \mathrm{O})$ to optimize the performance of a detection module. Based on simulation results, both the (x,y) location and DOI will be estimated for each event using the resistive network and compared to the results obtained without the network. The objective of using a resistive network is to reduce the total number of readout channels while maintaining/improving the overall intrinsic performance of the detection module.

\section{M10E-4: easyPET - a New Approach for Axial Preclinical PET}

P. M. Correia ${ }^{1}$, I. F. Castro ${ }^{1}$, A. L. Silva ${ }^{1}$, N. Romanyshyn ${ }^{1}$, V. Arosio ${ }^{2}$, M. Caccia ${ }^{2}$, R. Santoro ${ }^{2}$, A. C. Santos ${ }^{3}$, P. Sá ${ }^{4}$, N. Matela ${ }^{4}$, P. Almeida ${ }^{4}$, J. F. Veloso $^{1}$

${ }^{I} i 3 N$, Departamento de Fisica, Universidade de Aveiro, Aveiro, Portugal

${ }^{2}$ Universita degli Studi dell'Insubria, Como, Italy

${ }^{3}$ IBILI, Faculty of Medicine of the University of Coimbra, Coimbra, Portugal

${ }^{4}$ Instituto de Biofisica e Engenharia Biomédica, Faculdade de Ciências, Universidade de Lisboa, Lisboa, Portugal

EasyPET is a new concept of PET scanner using an acquisition method based on two rotation axes for the movement of detector pairs*. The concept allows achieving high and uniform position resolution over the whole field of view (FOV), by eliminating parallax errors due to the depth of interaction, typical of ring based PET systems. This characteristic, no DOI effect, does not impose limitations on the proximity of the detector elements to the FOV and therefore favours the sensitivity of the system. Full axial imaging is possible with easyPET using only a small number of detector elements.

A small demonstrator for 3D imaging with $16+16$ detector cells was built, based on LYSO scintillators coupled to SiPMs, covering a $50 \mathrm{~mm}$ diameter $\mathrm{x} 34 \mathrm{~mm}$ long field of view. The detectors run in a $60 \mathrm{~mm}$ diameter circumference, allowing a $50 \mathrm{~mm}$ diameter FOV. A comparison between simulation results obtained with GATE and experimental results will be presented in terms of system performance, namely position resolution and sensitivity, following NEMA NU 4-2008 standard protocols. In addition, simulation of a scaled up version of easyPET for preclinical purposes will be presented and discussed.

*Patent pending, Universidade de Aveiro (PCT/IB2016/051487)

This work was partially supported by project POCI-01-0145-FEDER-016855 and PTDC/BBB-IMG/4909/2014, UID/CTM/50025/2013 and PestOE/SAU/IU0645/2013, through COMPETE, FEDER and FCT (Lisbon) programs

\section{M10E-5: An Efficient Statistical Framework for Optimizing Complex SPECT System Designs} E. M. Zannoni ${ }^{1}$, X.-C. Lai $^{2}$, L.-J. Meng ${ }^{1,2,3}$

${ }^{I}$ Bioengineering, University of Illinois Urbana Champaign, Illinois, USA

${ }^{2}$ Nuclear, Plasma and Radiological Engineering, University of Illinois Urbana Champaign, Illinois, USA

${ }^{3}$ Beckman Institute for Advance Science and Technology, Illinois, USA

The Radiation Detection and Imaging Group at University of Illinois Urbana-Champaign developed a statistical framework for optimizing complex SPECT imaging systems characterized by a very large number of design parameters. This approach relies on an indirect system optimization technique operated on a discrete system parameter space and various performance indices derived from analytical approximations of the first and second order image statistics using the vector-form Modified Uniform CramerRao Bound (MUCRB), to identify the best system configuration from a vast number of possible candidates. This approach offers two key benefits. First, it offers a dramatically improved computation efficiency, which allows us to search through a very large parameter space for complex imaging system designs. Second, this framework allows one to incorporate a wide range of system performance indices, such as resolution variance tradeoffs, local detectability index, ROI quantitation and (potentially) various computer observers into the optimization scheme. These makes it a general and computationally efficient system optimization technique for Nuclear Imaging hardware design problems. We will demonstrate the use of this novel system optimization approach for optimizing the MRC-SPECT-II system, currently under development in our lab, that uses a novel synthetic inverted compound eye (S-ICE) gamma camera design bioinspired by superposition compound eyes to achieve a dramatically improved 
system sensitivity. MRC-SPECT-II represents an ideal and challenging platform for applying this hardware optimization technique, since it consists of $>1500$ independent micro-camera-elements and each could be optimized independently.

M10E-6: DoI Detector Design and Characterization for Open-Field Mouse Brain PET A. Z. Kyme ${ }^{1}$, M. S. Judenhofer ${ }^{1}$, S. R. Meikle ${ }^{2}$, S. R. Cherry ${ }^{1}$

${ }^{I}$ Biomedical Engineering, University of California Davis, Davis, CA, USA

${ }^{2}$ Faculty of Health Sciences and Brain \& Mind Centre, University of Sydney, Sydney, NSW, Australia

'Open-field' PET imaging of freely moving rodents is a powerful paradigm because it enables the correlation of functional and behavioral outputs in response to environmental or pharmacologic stimuli. Use of motion compensation techniques provides a feasible framework for imaging a moving animal. However, to overcome the geometric and performance limitations encountered in adapting motion compensation techniques to existing scanners, we are developing a purpose-built PET imaging system for freely moving mice. Two important innovations of this scanner are panel detectors with thick $(20 \mathrm{~mm})$ scintillators arranged in a tightly packed box geometry to provide high sensitivity $(\sim 15 \%)$ and a motion-controlled sliding gantry moving in synchrony with the animal. Here we describe the design and characterization of the depth-of-interaction (DoI) detector module forming the basis of a prototype system. The detector comprises a $23 \times 23$ LYSO crystal array, $0.85 \mathrm{~mm}$ pitch, $20 \mathrm{~mm}$ length, read out at both ends using 6x6 arrays of through-silicon-via (TSV) silicon photomultipliers. The crystal, photodiode arrays and light guide exactly matched to minimize gaps between neighboring modules. A cross-wire readout with resistive weighting was used to provide 4 position and one timing output per array, i.e. 10 outputs/module. Average energy resolution was $17.6 \pm 5.3 \%$. Average DoI resolution near the center of the crystal $(10 \mathrm{~mm})$ was $2.8 \pm 0.35 \mathrm{~mm}$ and $3.5 \pm 1.0 \mathrm{~mm}$ near the edges. This almost meets our target DoI of $<3 \mathrm{~mm}$ to provide near-isotropic spatial resolution of $\sim 1 \mathrm{~mm}$ throughout the FoV. The timing resolution was $3.7 \pm$ $0.13 \mathrm{~ns}$. Manufacturing flaws in our scintillator array, particularly the integrity of the reflector material and its bonding to the individual crystals, likely compromised light collection and uniformity in our characterization. Therefore, although the results are promising, we expect improved performance in energy resolution, DoI and timing with a new crystal array.

M10E-7: MRC-SPECT Imaging of Neural Stem Cells - An Exploration of Simultaneous SPECT and MR Image Acquisition for Detection and Localization of Small Cell Populations E. M. Zannoni ${ }^{1}$, X.-C. Lai ${ }^{2}$, I. V. Balyasnikova ${ }^{3}$, Q. Li $^{4}$, C.-T. Chen ${ }^{5}$, L.-J. Meng ${ }^{1,2,6}$

${ }^{I}$ Bioengineering, University of Illinois Urbana Champaign, Illinois, USA

${ }^{2}$ Nuclear, Plasma and Radiological Engineering, University of Illinois Urbana Champaign, Illinois, USA

${ }^{3}$ Department of Neurological Surgery, Northwestern University, Illinois, USA

${ }^{4}$ Gordon Center for Medical Imaging, Massachusetts General Hospital, Harvard Medical School, Massachusetts, USA

${ }^{5}$ Department of Radiology, University of Chicago, Illinois, USA

${ }^{6}$ Beckman Institute for Advance Science and Technology, Illinois, USA

There is a strong interest in utilizing Neural Stem Cells (NSCs) as carriers for targeted delivery of therapeutics to brain tumors due to their strong inherent tumor-tropic properties and capability of crossing the brain-blood barrier. Non-invasive imaging approaches for tracking NSCs are essential for investigating tropism, distribution, safety and therapeutic efficacy of NSC as therapeutic carriers. The Radiation Detection and Imaging Group at University of Illinois Urbana-Champaign developed a cutting-edge preclinical Single Photon Emission Tomography (MRC-SPECT-I) for simultaneous Nuclear Molecular Imaging and Magnetic Resonance Imaging. This stationary system consists of forty MR-compatible, energy-resolved, photon counting, and highly pixelated CdTe semiconductor detectors. The high performance detection system provides us a high sensitive and ultrahigh resolution tool to image NSCs labeled with In-111. The preliminary studies have demonstrated the system capability of

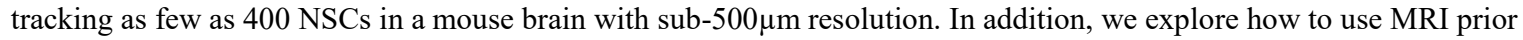
information for SPECT reconstruction and its benefits for detection and localization of small NSC populations.

\author{
M10E-8: Design and Initial Performance of HiPET, a High Sensitivity and High Spatial Resolution DOI PET \\ Tomograph

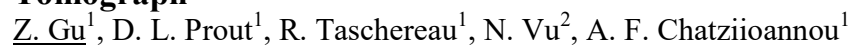 \\ ${ }^{I}$ UCLA, Los Angeles, United States \\ ${ }^{2}$ Sofie Biosciences, Los Angeles, United States
}

A new small animal PET tomograph named HiPET is currently being developed at the Crump Institute for Molecular Imaging, at UCLA. This scanner aims to achieve very high peak absolute sensitivity (up to 19.1\%) and submillimeter spatial resolution. To achieve this, HiPET consists of ten panel detectors, with a crystal ring diameter of $160 \mathrm{~mm}$ and an axial extent of $104 \mathrm{~mm}$. Each panel detector consists of two modules, and each module employs a phoswich depth of interaction (DOI) design composed by two layers of scintillator arrays (LYSO and BGO). The top (gamma ray entrance) layer is a $48 \times 48$ array of $1.01 \times 1.01 \times 6.1$ mm3 LYSO crystals ( $1.09 \mathrm{~mm}$ pitch). The bottom (towards the PMT) layer is a $32 \times 32$ array of $1.55 \times 1.55 \times 8.9 \mathrm{~mm} 3 \mathrm{BGO}$ crystals (1.63 $\mathrm{mm}$ pitch). In order to decrease the electronic channels required by each detector and lower the system cost, a new 
readout circuit has been designed and constructed to achieve simultaneous acquisition of BGO and LYSO events using only three readout channels per detector module (reduced from eight channels when using previous readout). The peak absolute sensitivity measurement was simulated using GATE. As the energy window changes from 350-650 keV to $150-650 \mathrm{keV}$, the peak system sensitivity varies from $13.2 \%$ to $19.1 \%$. Using the fast last dynode signal, previously we have achieved $\sim 1 \mathrm{~ns}$ and $\sim 4 \mathrm{~ns}$ timing resolution for LYSO-LYSO and BGO-BGO coincidence events. In this study, the slower energy signal is used for triggering and timing. As a result, the total readout channels required for the HiPET system can be reduced from 80 to 60 , which significantly reduces the system electronics cost. The timing resolution for LYSO-LYSO, LYSO-BGO and BGO-BGO are $4.8 \mathrm{~ns}, 6.0 \mathrm{~ns}$ and $8.4 \mathrm{~ns}$. The timing resolution is compromised because the energy signal needs to pass through a low pass filter for accurate signal digitization. Nevertheless, the timing resolution is still acceptable for non-TOF PET timing.

\author{
M10E-9: Development of a Prototype SPECT System Using a Variable Pinhole Collimator

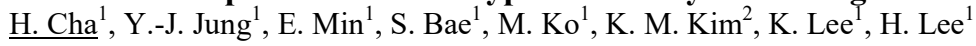 \\ ${ }^{I}$ Dept. of Bio-convergence Engineering, Korea university, Seoul, Korea \\ ${ }^{2}$ Molecular Imaging Research Center, Korea Institute of Radiological and Medical Sciences, Seoul, Korea
}

We designed a novel single-photon emission computed tomography (SPECT) system with a variable pinhole (VP) collimator for obtaining high-sensitivity and high-spatial resolution imaging of region of interest (ROI). Extending the capabilities of conventional SPECT systems, this novel SPECT system with a VP collimator can adjust its driving parameters, such as a collimator diameter, an acceptance angle, and distances between the ROI, collimator, and detector at different rotation angles. Previously, we simulated a VP collimator and demonstrated the superiority of the VP SPECT system in terms of its sensitivity and spatial resolution. Based on these previous results, we developed a prototype SPECT system for performance evaluations of a VP collimator. The collimator was made from $99.9 \%$ pure tungsten layers, with 16 holes of different diameters. The area of the CsI(Tl) scintillator was $45.7 \times 45.7 \mathrm{~mm}^{2}(51 \times 51$ pixels $)$, with pixel dimensions $0.7 \times 0.7 \times 5 \mathrm{~mm}^{3}$. The MPPC photomultiplier was tiled into $2 \times 2$, and its dimensions were $52 \times 52 \mathrm{~mm}^{2}$. The collimator, the detector, and the gantry of this SPECT system were independently controlled. Reconstructed images were acquired for point sources (Co-57) and an ultra-micro phantom (Tc$99 \mathrm{~m})$. The point sources and the phantom were clearly resolved, demonstrating satisfactory detector's response, signal processing, and imaging processing capabilities. The $1.35 \mathrm{~mm}$ phantom was also clearly resolved in the ultra-micro phantom. The performance of ROI-optimized VP SPECT system will be evaluated up to the conference.

M10E-10: Real Time PET Imaging on the SuperArgus Preclinical Scanner

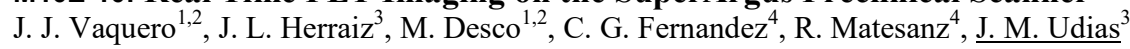

${ }^{I}$ Departamento de Bioingeniería e Ingeniería Aeroespacial, Universidad Carlos III, Leganés, Madrid, Spain

${ }^{2}$ Instituto de Investigación Sanitaria Gregorio Marañón, Madrid, Spain

${ }^{3}$ Nuclear Physics Group, Universidad Complutense, Madrid, Spain

${ }^{4}$ Preclinical Division, SEDECAL, Algete, Madrid, Spain

Recent advances in PET systems have enabled real time PET imaging. We have developed a high performance data acquisition system (DAQ) withstanding rates in excess of $1 \mathrm{Mevent} / \mathrm{s}$ per square inch of detector. Single events from the DAQ boards are funnelled to a workstation were coincidence events are sorted out on the fly by a multithreaded software. In our implementation a single thread can handle up to 9 million single events per second (Msingles/s), while the 8 thread version handles more than 50 Msingles/s. We combine this DAQ to a two, four or six rings system with 24 detectors per ring, each detector comprised by a $13 \times 13$ dual layered LYSO+GSO crystals at $1.55 \mathrm{~mm}$ pitch, with a FOV of $12 \mathrm{~cm}$ (transaxial) x 5, 10 or $15 \mathrm{~cm}$ (axial), resulting in different configurations of the SuperArgus preclinical scanner. This DAQ can acquire, free from electronic deadtime, events originated from $2 \mathrm{mCi}$ of activity in the FOV. Identification of events inside time coincidence window, energy window, and coincidence matrix is performed in real time. The processing computer is able not only of filtering, sorting and casting single events into standard coincidence list files, but also to reconstruct these data sets several times per second. Coincidence events are arranged into a 3Dsinogram file, which can be updated at intervals of 0.1 seconds. Deadtime corrections, scatter and random subtractions are performed on the fly. Working in parallel to the DAQ software, a multithread version of an accelerated FBP algorithm is able of reconstructing 3D images spanning the whole FOV of the scanner at rates of three frames per second. Alternatively projected images can be obtained at any desired angle at 10 frames per second. These short-time projected images contain enough counts, even for modest activities (less than $0.5 \mathrm{mCi}$ ), to guide a probe, to observe dynamic distribution of radiotracers with subsecond sampling or even to scan awake animals.

M10E-11: An Experimental Evaluation of a Hybrid Pixel-Waveform CdTe Based Prototype PET Detector Against Commercial MicroPET for Imaging Tau Protein Pathology in Transgenic Mouse Brain Tissue

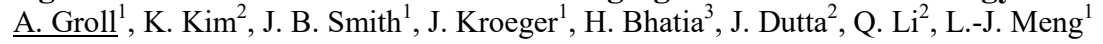

${ }^{I}$ Nuclear, Plasma, and Radiological Engineering, University of Illinois at Urbana-Champaign, Urbana, IL, USA

${ }^{2}$ Radiology, Massachusetts General Hospital and Harvard Medical School, Boston, MA, USA

${ }^{3}$ Electrical Engineering, University of Illinois at Urbana-Champaign, Urbana, IL, USA 
The undeniable impact of neurodegenerative diseases on our society has brought tremendous efforts to explore various means of preclinical investigation to help further the discovery of a cure. PET has identified itself to be a modality of interest for understanding long standing disease such as Alzhiemer's Disease (AD), and the new and emerging athlete associated chronic traumatic encephalopathy (CTE). Our work has focused on developing semiconductor CdTe Hybrid Pixel-Waveform (HPWF) Detectors for application in imaging transgenic mouse models for just such diseases. In this endeavor, we attempt to justify the potential role of sub 400 um resolution CdTe PET for small animal imaging in a field dominated by scintillation based microPET. To do this, comparative studies were carried out to analyse the benefits and drawbacks of both modalities contextualized in the pursuit of imaging the tau protein present in neurological diseases.

M10E-12: Preliminary Results of an Embedded Timing Probe for Calibrating PET Scanner.

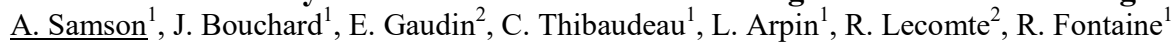

${ }^{I}$ Department of Electrical and Computer Engineering, Université de Sherbrooke, Sherbrooke, Canada

${ }^{2}$ Department of Nuclear Medicine and Radiobiology, Université de Sherbrooke, Sherbrooke, Canada

With the aim of improving the CNR of the LabPET II scanner, a method to correct the channel-to-channel coincidence time difference has been developed. The LabPET II, an avalanche photodiode-based PET scanner, features up to 55000 channels which benefit from precise timing alignment. The correction process is fully automated and embedded in the scanner hardware and firmware. It uses a timing probe designed to reacts with the excess kinetic energy of a positron, enabling an absolute time reference of the positrons emission inside the probe. The time measurement is performed by a 312.5 ps time-to-digital converter (TDC) implemented in the LabPET II coincidence unit FPGA featuring a differential nonlinearity of -0.23 LSB peak and an integral nonlinearity of $0.14 \mathrm{LSB}$ peak. The system computes probe-to-channel coincidences in order to obtain the absolute time difference between each channel of the scanner. The time correction is then applied to each channel to align all coincidence spectra, enhancing time resolution, thus improving CNR. Finally, these corrections are applied in real-time to each event during a typical PET acquisition.

M10E-13: Development of MRC-SPECT-II System for Alzheimer's Disease Mouse Models

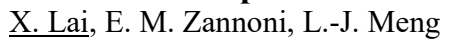

NPRE, University of Illinois at Urbana-Champaign, Urbana, United States

The second-generation MR-compatible SPECT system using the Inverted Compound-Eye Gamma-ray camera is under development in our lab. The system could achieve $>1 \%$ detection efficiency while keeping sub-500um resolution. In this study, we will carry out Monte Carlo simulations to study the benefits of MRC-SPECT-II for beta-amyloid plaques imaging in AD mouse models. To evaluate the design feasibility of ICE gamma camera, the prototype system is being built in our lab. Using this prototype system, we will carried a series of experiment studies, including developing a novel system modeling method, system optimization based on synthetic sampling and comparison of prototype ICE camera with MRC-SPECT-I system.

M10E-14: Tri-Modality X-Ray Luminescence, Fluorescence and Transmission Computed Tomography for Monitoring X-Ray Induced Photodynamic Therapies J. George $^{1}$, L. Giannoni ${ }^{1}$, K. Kim ${ }^{2}$, J. Dutta ${ }^{2}$, S.-H. Cheng ${ }^{3}$, Q. Li ${ }^{4}$, L.-W. Lo ${ }^{3}$, C.-T. Chen ${ }^{2}$, P. La Riviere ${ }^{3}$, L.-J. Meng ${ }^{1}$

${ }^{I}$ Nuclear, Plasma, and Radiological Engineering, University of Illinois at Urbana-Champaign, Urbana, IL, USA

${ }^{2}$ Department of Radiology, Massachusetts General Hospital, Bostin, MA, USA

${ }^{3}$ Department of Radiology, University of Chicago, Chicago, IL, USA

${ }^{4}$ Institute of Biomedical Engineering and Nanomedicine, National Health Research Institutes, Zhunan, Miaoli, Taiwan

In this work, we experimentally explore the use of a XFCT/XLCT/XCT tri-modality imaging system for imaging several nanophosphors, such as Y2O3:Eu3+ and LaF3:Tb3+ developed for X-PDT applications. The geometry of this study will mimic the X-ray microbeam therapy environment, which provides the highly collimated and the highly intense incident X-ray beam needed to produce sufficient fluorescent and luminescent yields for XFCT and XLCT imaging. The irradiated nanoparticles (NPs), themselves would produce therapeutic effects, either through indirect mechanisms such as photosensitization, or directly from low-energy secondary electrons killing the cells. XF signals emanating from the irradiated NP could determine the distribution of the NPs in the object quantify the X-ray energy contributing to photoelectron interactions. The XL signals could provide quantitative information proportional to any kind of therapeutic effects caused by conjugated photosensitizers and spatial mapping of the scintillation process induced by incident X-ray irradiation. The third modality, micro-CT, would provide anatomical or structure information of the subject for precise delivery of the beam to the target region. The combination of these three imaging techniques would provide a tool capable of guiding therapeutic delivery through providing a wide array of spatial and functional information.

Preliminary XRF/XLCT studies demonstrate the ability to acquire images of Y2O3 and LAF3 NP's. For this study, we will acquire XFCT and XLCT 3D images using various nanophosphors including Y2O3:Eu3+ and LaF3:Tb3+ nanoparticles. The minimum detectable concentrations and system sensitivity will also be determined. Furthermore, we will investigate the benefit 
of using both the XF and XL signals to produce the image, using information within both data sets to contribute to an improved image quality when compared to using one modality on its own. The results from these studies will be presented.

\section{M10E-15: Pilot Tests of a PET Insert Based on Monolithic Crystals in a 7T MR}

A. J. Gonzalez ${ }^{1}$, A. Aguilar ${ }^{1}$, P. Conde ${ }^{1}$, A. Gonzalez-Montoro ${ }^{1}$, S. Sanchez ${ }^{1}$, S. Junge ${ }^{2}$, S. Stanculovic ${ }^{2}$, R. Wissmann ${ }^{2}$, T. Bruckbauer ${ }^{2}$, T. Greeb ${ }^{2}$, R. Garcia ${ }^{3}$, J. Barbera ${ }^{4}$, C. Molinos ${ }^{4}$, C. Correcher ${ }^{4}$, F. Sanchez ${ }^{1}$, J. M. Benlloch ${ }^{1}$

${ }^{I}$ Institute for Instrumentation in Molecular Imaging, Valencia, Spain

${ }^{2}$ Preclinical Imaging, Bruker BioSpin, Ettlingen, Germany

${ }^{3}$ Institute of Design and Manufacture, Valencia, Spain

${ }^{4}$ Oncovision, Valencia, Spain

New photosensor developments on solid state technology have significantly pushed the implementation of hybrid and simultaneous PET-MR imaging. In particular, SiPM with TSV (Through Silicon Vias) package are the preferred choice to build new MR compatible PET scanners. In this work we show the pilot results of a small animal PET insert based on such SiPM photodetectors inside a 7 Tesla preclinical MR scanner.

The PET design makes use of high density SiPM arrays of about $51 \times 51 \mathrm{~mm} 2$ active area and $10 \mathrm{~mm}$ thick monolithic, painted black, LYSO crystals. 8 detectors have been mounted in a ring shape defining an axial and transaxial FOV of 46 and $80 \mathrm{~mm}$, respectively. Using the NEMA 22Na source with $0.3 \mathrm{~mm}$ diameter the system shows a resolution around $1 \mathrm{~mm}$ and it hardly degrades moving radially towards the FOV periphery.

PET-MR tests were carried out using the Bruker BioSpec 70/30 USR with a magnetic field of $7 \mathrm{~T}$ and the RF coil 112/86. We have implemented a novel design to shield the RF field to the PET electronics. Very thin layers of carbon fiber composites have been packed following a newly developed curing process. Initial tests of the PET and the carbon fiber screens inside the MR system have successfully shown the RF shielding capabilities of this assembly. Various RF pulse schemes of up to $630 \mathrm{Watts} \mathrm{did}$ not affect the PET performance. PET compatibility tests in the MR were carried out with a $1 \mathrm{~mm}$ diameter $22 \mathrm{Na}$ source. Energy resolution remained stable at $12 \%$. The FWHM of the reconstructed point remained at $1.2 \pm 0.1 \mathrm{~mm}$ (MLEM 15 iterations) in all tested conditions, namely with the PET outside the MR, with the PET inside the 7T MR but in the absence of RF pulses and with single RF pulses. Typical MR image sequences for anatomical and functional imaging did not affect the reported PET performance.

M12: Pre-clinical (small animal) emission/multimodality imaging II

Friday, Nov. $4 \quad$ 08:00-10:00 Schweitzer

M12-1: LabPET II: Initial Results of a Time-over-Threshold APD-Based PET Scanner

E. Gaudin ${ }^{1}$, L. Arpin ${ }^{2}$, C. Thibaudeau ${ }^{2}$, M. Paille ${ }^{1}$, J.-F. Beaudoin ${ }^{1}$, J.-D. Leroux ${ }^{3}$, K. Koua ${ }^{2}$, J. Bouchard ${ }^{2}$, A. Samson ${ }^{2}$, C. M. Pepin ${ }^{1}$, R. Fontaine ${ }^{2}$, R. Lecomte ${ }^{1}$

${ }^{I}$ Department of Nuclear Medicine and Radiobiology, Université de Sherbrooke, Sherbrooke, Canada

${ }^{2}$ Department of Electrical and Computer Engineering, Université de Sherbrooke, Sherbrooke, Canada

${ }^{3}$ Novalgo, Sherbrooke, Canada

The LabPET II is an avalanche photodiode (APD) based PET scanner designed to achieve submillimetric spatial resolution in pre-clinical Positron Emission Tomography (PET). This new generation scanner is based on Time-over-Threshold signal processing with individual detector read-out and highly integrated parallel electronic architecture. The aim of this study is to evaluate the initial performance of the mouse version of this scanner.

The system consists of 192 LabPET II detector arrays. Each array is based on $4 \times 8$ crystal array made of $1.12 \times 1.12 \times 12 \mathrm{~mm} 3$ Lu1.9Y0.1SiO5:Ce (LYSO) scintillator pixels with one-to-one coupling to a $4 \times 8$ pixelated monolithic APD array. Four of these detector arrays are mounted on a daughter-board carrying two flip-chip, 64-channel, mixed-signal application-specific integrated circuits on the backside to independently process the signals from the 128 pixels. Forty-eight of these stand-alone front-end detector modules are used to form a $78.8 \mathrm{~mm}$ diameter ring with an axial field of view (FOV) of $50.4 \mathrm{~mm}$.

With an energy window of $250-650 \mathrm{keV}$, the system detection efficiency is estimated to $12 \%$ and its absolute sensitivity to $3 \%$. The coincidence time resolution between opposing 128-channel modules is typically $3.3 \pm 0.3 \mathrm{~ns}$ FWHM, with pixel pair coincidence resolution as low as $1.9 \mathrm{~ns}$. The maximum NECR reaches $367 \mathrm{kcps}$ at $6.5 \mathrm{MBq} / \mathrm{cc}$. A $0.8 \mathrm{~mm}$ intrinsic FWHM radial resolution is obtained at the center of the FOV. Submillimeter resolving capability is demonstrated up to a diameter of $26.6 \mathrm{~mm}$, which is adequate for whole-body mouse imaging. Coincidence time resolution between opposing 128-channel modules is below 4 ns FWHM.

M12-2: Design of a Dual-Resolution, Rectangular-Pinhole Collimator with Improved Projection Tiling for Small-Animal SPECT L. C. Johnson ${ }^{1}$, Z. Liu ${ }^{2}$, M.-A. Park ${ }^{2}$, S. C. Moore ${ }^{2}$, S. D. Metzler ${ }^{1}$

${ }^{I}$ Dept. of Radiology, University of Pennsylvania, Philadelphia, PA, USA

${ }^{2}$ Dept. of Radiology, Brigham and Women's Hospital and Harvard Medical School, Boston, MA, USA 
Collimators in small-animal SPECT systems play a pivotal role in determining image quality. We are developing a dualresolution collimator for cardiac imaging that utilizes rectangular-pinhole apertures. One section of the collimator tube will have a larger radius of rotation used for scout imaging, while a second section will have a smaller radius of rotation and be utilized for high-resolution, small field-of-view applications. In this work we present a method of multiple-pinhole design that maximizes the use of the detector area. We first determined the location of each projection on the detector face, and then back-calculated the rectangular pinhole position, orientation, and shape of the aperture opening, ignoring penetration. Projections were forced to be rectangular and were tiled on the detector face to maximize coverage. This tiling resulted in apertures that were quadrilateral in shape, but not necessarily rectangular or square. The resulting high-resolution section design was comprised of 27 quadrilateral pinholes per detector ( 81 total) with $\sim 0.3 \mathrm{~mm}$ sides. Apertures were located at a mean and standard deviation of $32.7 \mathrm{~mm}$ and $5.79 \mathrm{~mm}$, respectively, away from the tube's centerline. Despite aperture shape distortion, all apertures had areas within $99.78 \%$ of the central rectangular aperture's area. All projections on the detector face had an axial length of $70 \mathrm{~mm}$, while transaxial lengths ranged from 70 to $140 \mathrm{~mm}$. Pinhole designs are being evaluated for sampling completeness, resolution, and sensitivity. We are also adding features to the collimator tube so that it fits into a U-SPECT (MI Labs) system. This requires a stationary insert in the SPECT system with rails that allow for the collimator to move axially in and out of the system in order to center the high-resolution and scout collimator segments on the detector. Future work will involve manufacturing of the collimator and using it in phantom and animal studies to evaluate image quality.

M12-3: Whole-Body Preclinical SPECT Imaging: Scanning Without Multiplexing vs. Stationary Imaging with Multiplexing

S. C. Moore $^{1}$, M. F. Kijewski ${ }^{1}$, L. C. Johnson ${ }^{2}$, S. D. Metzler ${ }^{2}$

${ }^{I}$ Div. of Nuclear Medicine, Brigham \& Women's Hospital and Harvard Medical School, Boston, MA, USA

${ }^{2}$ Dept. of Radiology, University of Pennsylvania, Philadelphia, PA, USA

We are developing dual-resolution collimation for a large field-of-view triple-camera SPECT system that combines stationary whole-body (WB) imaging of a mouse with high-resolution targeted organ imaging; the WB collimator tube acquires partially multiplexed data through 39 rectangular pinholes with wide opening angles that are reconstructed using a novel iterative demultiplexing algorithm. An alternative, non-multiplexed technique relies on scanning the WB through a target region seen by many more pinholes, each with highly restricted aperture opening angles. These two approaches were compared using activityestimation tasks for equalized 1.5-mm-FWHM system spatial resolution and for identical total scan time. For both systems, we simulated 5, 3.6-mm-diameter spheres embedded within a uniform cylindrical phantom $(2.4 \mathrm{~cm}$ diam. x $6.9 \mathrm{~cm}$ long) containing half the Tc-99m activity concentration of the spheres. All pinholes in the stationary WB imaging tube could accept photons emitted from anywhere in the phantom, while the 69-pinhole scanning system accepted photons only from within a pyramidshaped volume that included a small cylindrical target region ( $9 \mathrm{~mm}$ diam. $\mathrm{x} 7.2 \mathrm{~mm}$ long); the object was stepped through 70 positions to sample the whole phantom. We evaluated performance for different values of total scan time, as well as for the assumed dead-time required to move the phantom between scan positions. For a 5-minute scan, SNR values averaged over the 5 spheres were the same for the WB stationary system as for a scanning system with $\sim 10 \%$ dead-time, based on ideal Cramer-Rao bounds (CRB) on variance of activity estimates computed from projections, or $\sim 34 \%$ dead-time, based on reconstructed image volume-of-interest (VOI) measurements; these cases correspond, respectively, to $0.44 \mathrm{~s}(\mathrm{CRB})$ or $1.5 \mathrm{~s}$ (VOI) time for object motion between positions. The WB system generally outperformed the scanning system for shorter (e.g., dynamic frame) times, or for larger scanning dead times.

This work was supported in part by the U.S. National Institutes of Health under Grant R01-HL111883.

\author{
M12-4: Impact of a MR Transmit/Receive Coil on the Performance of a Dedicated Preclinical MR-Compatible \\ PET-Insert \\ $\underline{\text { C.-C. Liu }}^{1}$, H. F. Wehrl ${ }^{1}$, A. Kolb ${ }^{1}$, C. Parl ${ }^{1}$, M. Rafecas ${ }^{2}$, B. J. Pichler ${ }^{1}$ \\ ${ }^{1}$ Werner Siemens Imaging Center, University of Tuebingen, Tuebingen, Germany \\ ${ }^{2}$ Institut für Medizintechnik, University of Luebeck, Luebeck, Germany
}

Our preclinical PET/MRI system is based on lutetium oxyorthosilicate scintillators and avalanche photodiodes. The simultaneous data acquisition of the device is used in our lab for small animal studies on regular basis. Based upon our previous experience, MR coils could cause a $20 \%$ increase of the $511 \mathrm{keV}$ photon attenuation. The aim of this work was to investigate the impact of a MRI coil (Bruker model T10720) on the performance of the PET-insert and the image quantitation accuracy. The following PET image quality parameters were studied with and without the coil located in the FOV: The spatial resolution and the absolute system sensitivity were measured at the center of the FOV (CFOV) by using a ${ }^{22} \mathrm{Na}$ source with a diameter of $0.25 \mathrm{~mm}$. The noise equivalent count rate (NECR) and scatter fraction (SF) were measured by using two cylindrical phantoms, with the size of a typical mouse and rat respectively. The spill-over ratio (SOR) and recovery coefficient (RC) were measured for the air and water components in a National Electrical Manufacturers Association (NEMA) image quality phantom, which was filled with 18F. By presence of the MRI coil the spatial resolution at CFOV was degraded by $1-6 \%$ and the system sensitivity by $11 \%$. The peak of the NECR of the mouse / rat phantom was degraded by $9.6 \% / 10.6 \%$, and the SF by $15.0 \% / 6.9 \%$. In the air component, the 
SOR was deteriorated by $3.3 \%$ and the RC by $0.4-8.5 \%$. However, the SOR in the water component was improved by $2.5 \%$ and the uniformity by $3.8 \%$. The results show that the MRI coil causes not only additional photon attenuation but photon scatter. In conclusion, scatter correction of MRI coils should be considered with attenuation correction in the image reconstruction for the PET/MRI studies.

\author{
M12-5: Small Animal and Endoscopic PET Detector Modules Based on Multichannel Digital Silicon \\ Photomultipliers \\ E. Venialgo, S. Sinha, A. Carimatto, T. Gong, S. Mandai, E. Charbon \\ Electrical Engineering, Delft University of Technology, Delft, Netherlands
}

In time-of-flight (TOF) and conventional positron emission tomography (PET), the coincidence resolving time (CRT) has a strong influence on the overall performance. Multichannel digital silicon photomultiplier (MD-SiPMs) are able to obtain several timestamps for gamma photon timemark estimation. The CRT can be improved significantly by utilizing multiple photoelectron timestamps. In addition, the PET instrumentation chain is simplified because of the intrinsic digitization and integrated functionality of the MD-SiPM. In this work, we present the implementation of two PET detector modules that are based on MDSiPMs: a small animal and an endoscopic PET detector modules. The small animal PET detector module consists of $2 \$ 1$ times $\$ 4$ monolithic arrays of MD-SiPM. In addition, this module includes a low-cost FPGA, a temperature controlling system and data transfer interfaces. The endoscopic PET detector module comprises a single monolithic array of 9x18 MD-SiPM and a small form-factor FPGA. In this module, a remarkable level of compactness is achieved.

\title{
M12-6: Initial Evaluation of a State-of-the-Art Commercial Preclinical PET/CT Scanner
} T.-S. Lee $^{1}$, A. Rittenbach ${ }^{1}$, C. G. Fernández ${ }^{2}$, J. Lopez-Longas ${ }^{2}$, J. M. Arco ${ }^{2}$, B. M. W. Tsui ${ }^{1}$

${ }^{I}$ Radiology, Johns Hopkins University, Baltimore, MD, USA

${ }^{2}$ SEDECAL Molecular Imaging, Madrid, Spain

We performed an initial evaluation of a state-of-the-art commercial preclinical PET/CT scanner (SuperArgus 4R, SEDECAL, Madrid, Spain). The PET unit consists of 4 rings of detector modules each with an array of $1.45 \mathrm{~mm} \times 1.45 \mathrm{~mm} \times 15 \mathrm{~mm}$ pixelated LYSO and GSO phoswich crystals with DOI information. It has a maximum axial FOV of $100 \mathrm{~mm}$ and transaxial FOV of $120 \mathrm{~mm}$. The CT unit consists of an X-ray source with variable micro focal spot size and a large $229 \mathrm{~mm} \times 145 \mathrm{~mm}$ flat-panel detector that allow 15 micron pixel width at the object at the highest magnification. We measured the sensitivity and uniformity of the PET unit using a calibrated Na-22 point source and a cylindrical phantom filled with a homogeneous FDG solution. The system resolution was determined from the reconstructed images of a FDG-filled capillary tube in air and inside a plastic cylinder, and a hot-rod phantom using the FBP and the 3D iterative reconstruction algorithm at different iteration numbers. A 30slice averages of the reconstructed images at three magnifications of a calibration phantom were used to measure the uniformity of the CT unit. The resolution was determined from the edge functions of the images of a set of precision-machined acrylic rods of different diameters at three magnifications and with different acquired and reconstructed pixel sizes. The dual-modality image co-registration was checked using a set of CT and PET images obtained from a phantom consisting of a Ge-68 annulus ring phantom with two attached Na-22 point sources. Finally, several small animal PET/CT studies were conducted to demonstrate the preclinical imaging performance. Our preliminary results showed the PET unit is capable of achieving a system resolution of $0.75 \mathrm{~mm}$ and sensitivity of $\sim 8 \%$, and the CT unit a highest resolution of $\sim 20$ micron. We conclude the preclinical PET/CT meet the stated specifications and is suitable for high performance preclinical molecular imaging of small to medium size animals.

M12-7: Investigation of Angled Crystals for a Depth-of-Interaction Detector

Y. Valenciaga, D. L. Prout, A. F. Chatziioannou

Crump Institute for Molecular Imaging, UCLA, Los Angeles, CA, United States

Positron Emission Tomography (PET) is based on the detection of annihilation photons through their interactions with scintillator crystals. To obtain a high sensitivity PET scanner, scintillation crystals must be big enough to stop the annihilation gammas. However, narrow crystals are needed for high spatial resolution resulting in thin and long crystals with the goal of simultaneously achieving high sensitivity and spatial resolution. Traditionally, the position assigned to the interaction of an annihilation gamma within a scintillation crystal has corresponded to a single location in the crystal regardless of where the interaction occurred. This method introduces a positioning error known as parallax error. This error becomes larger for longer scintillation crystals and oblique lines of response. This positioning error can be reduced when information on the depth of interaction in the crystal is available. To address this issue, we examine the performance of a slanted crystal geometry in terms of DOI resolution and potential number of DOI bins. In previous studies a change of light output depending on the location of interaction has been observed for slanted crystals. Simulations were performed by a Monte Carlo based software (GATE) and measurements were obtained on a similar set up. Preliminary results from this study indicate the potential of using slanted crystals to gather DOI information. Based on these results, we propose to design a dual-ended readout detectors with two layers of slanted crystals. Measurements to evaluate the performance of this design are ongoing. 
M12-8: phenoPET - Results from the Plant Scanner

M. Streun $^{1}$, K. Borggrewe ${ }^{1}$, A. Chlubek ${ }^{1}$, M. Dautzenberg ${ }^{1}$, C. Degenhardt ${ }^{1}$, R. Dorscheid ${ }^{2}$, D. Durini ${ }^{1}$, A. Erven ${ }^{1}$, L. Jokhovets ${ }^{1}$, L. Meessen ${ }^{2}$, R. Metzner ${ }^{1}$, O. Mülhens ${ }^{2}$, H. Nöldgen ${ }^{1}$, D. Pflugfelder ${ }^{1}$, S. Reinartz ${ }^{2}$, J. Scheins ${ }^{1}$, B. Zwaans ${ }^{2}$, S. Jahnke ${ }^{1}$,

U. Schurr ${ }^{1}$, S. van Waasen ${ }^{1}$

${ }^{I}$ Forschungszentrum Juelich, Juelich, Germany

${ }^{2}$ Philips Digital Photon Counting, Aachen, Germany

Within the German Plant Phenotyping Network (DPPN), we developed a novel PET scanner dedicated for plant research based on Philips DPCs (= Digital Photon Counter, also known as digital Silicon Photomultiplier) [1]. The scanner provides functional information on carbon transport within the plant. The plant takes up radioactively labelled $\left[{ }^{11} \mathrm{C}\right]-\mathrm{CO}_{2}$ by photosynthesis and transports it into the root system where the scanner observes its distribution and dynamics.

The detector ring is oriented horizontally and the Field-of-View measures $18 \mathrm{~cm}$ dia. and $20 \mathrm{~cm}$ in height. The size of the 36,864 LYSO scintillator crystals is $1.85 \times 1.85 \times 10 \mathrm{~mm}^{3}$ each [2]. The read-out electronics [3] cluster hits from different photodetector pixels when they originate from the same scintillation event. These single events are written via USB 3.0 with up to $300 \mathrm{MB} / \mathrm{s}$ to the computer system. Crystal identification, energy discrimination and coincidence detection is realized in software.

The high-resolution image reconstruction is realized with the PET Reconstruction Software Toolkit (PRESTO) [4]. A variancereduced normalization and random correction for each individual LOR have been implemented and they are considered within the iterative reconstruction using the Ordinary Poisson-OSEM algorithm (OP-.OSEM). For proper attenuation correction transmission scans are required. To this end a rotating source will be installed soon.

The spatial resolution in the center Field-of-View (CFOV) could be estimated to $=1.6 \mathrm{~mm}$ from measurements of a dedicated Derenzo phantom. A sensitivity of $4.6 \%(? \mathrm{E}=250-750 \mathrm{keV})$ was measured in the CFOV. The system is now operational and first plant images were obtained with satisfying results.

[1] Y. Haemisch, T. Frach, C. Degenhardt, and A. Thon, "Fully Digital Arrays of Silicon Photomultipliers (dSiPM) - a Scalable Alternative to Vacuum Photomultiplier Tubes (PMT)." Physics Procedia 37, 1546 (2012).

[2] Streun, M., et al. "phenoPET: A dedicated PET Scanner for Plant Research based on digital SiPMs (DPCs) ", IEEE NSS/MIC Seattle 2014 Conf. Rec. M11-18 (2014).

[3] Nöldgen,H., et al. "Read-Out Electronics for Digital Silicon Photomultiplier Modules", IEEE NSS/MIC Seoul 2013 Conf. Rec. M16-46 (2013).

[4] Scheins, J. J., et al. "High performance volume-of-intersection projectors for 3D-PET image reconstruction based on polar symmetries and SIMD vectorisation." Phys. Med. Biol. 60(24) 9349 (2015).

\section{M11: CT imaging technologies}

Friday, Nov. $4 \quad$ 08:00-10:00 Cassin

M11-1: PRaVDA: a New Instrument and New Methods for Proton CT

N. M. Allinson

Lab of Image Engineering, University of LIncoln, Lincoln, UK

On behalf of the PRaVDA Consortium

There were an estimated $14 \mathrm{~m}$ new cancer cases in the world, and in the developed world it is the second commonest cause of death. Again in the developed world, $\sim 40 \%$ of patients will receive radiotherapy as part of their curative treatment: the majority with external x-ray beams. Proton therapy is rapidly gaining use due to how protons loose their energy. Some 60 centres are in operation with more planned. With reducing costs, it is likely a significant proportion of future therapy will utilise protons. New delivery systems are capable in delivering treatments to mm accuracy, but imaging methods upon which treatments are based, delivered and measured do not allow planning of treatments to better than $\sim 5 \mathrm{~mm}$ accuracy. This discrepancy prevents PT attaining full clinical benefit. It can be fulfilled with the provision of proton CT - using the same radiation type to image and to treat. Imaging with protons is challenging as they undergo multiple coulomb scattering with a fraction of particles experiencing non-elastic collisions. PRaVDA is the first attempt to build a fully solid-state proton CT system. Informed by comprehensive Monte Carlo simulations, the instrument consists of 2 pairs of proton trackers (each containing 3 rotated silicon strip sensors) and a multi-layer range telescope (consisting of strip sensors, CMOS imagers or a mixture). Due to the quasi-deterministic manner in which protons interact, it is necessary to record trajectories and residual energies of individual protons. As the normal conditions for CT reconstruction using the popular back-projection algorithm are not fully valid, new methods of image reconstruction are needed. Proton CT based on the stopping power is the most useful in treatment planning, other forms are possible and can result in a much reduced complexity to the instrumentation. The presentation will outline the design philosophy of the system, present results including various forms of $\mathrm{CT}$, and suggest future developments.

PRaVDA is funded by the Wellcome Trust (grant number 098285)

M11-2: Noise Reduction in Low-Dose CT with Stacked Sparse Denoising Autoencoders 
Z. Ma, Y. Zhang, W. Zhang, Y. Wang, F. Lin, K. He, X. Li, Y. Pu, J. Zhou

College of Computer Science, Sichuan University, Chengdu, China

Due to the growing concerns about the X-ray radiation to the patients, low-dose computed tomography (CT) imaging is encouraged to follow the famous as low as reasonably achievable (ALARA) principle. Lowering the milliampere-seconds (mAs) parameter is a common way to reduce the radiation dose. However, this method will unavoidably increase the quantum noise in the projection data and the reconstructed CT images will be severely degraded. Improving the quality of low-dose CT images has been one of the major topics in the CT field. The post-reconstruction restoration approaches, which donï $\ddot{c}^{1 / 2 t}$ rely on the projection data, can be directly applied on low-dose CT and easily integrated into the current CT systems. Inspired by the superior performance achieved by non-linear deep neural networks in the field of image processing, a stacked sparse denoising autoencoders (SSDA) based noise reduction method for low-dose CT imaging is presented in this summary. By training on patches extracted from clinical dataset, the experimental results show that our method is competitive with the state-of-the-art lowdose image denoising methods in both qualitative and quantitative measures.

\author{
M11-3: Improving Basis Material Decomposition in the Presence of X-Ray Scatter with an Energy-Resolved \\ Photon Counting Detector

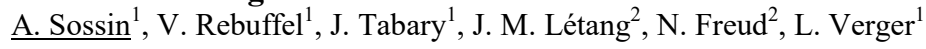 \\ ${ }^{I} D T B S / S T D / L D E T, C E A-L E T I$, Grenoble, France \\ ${ }^{2}$ INSA-Lyon, Université Lyon 1, UJM-Saint Etienne, CNRS, Inserm, CLB, CREATIS UMR 5220, U1206, Univ. Lyon, Lyon, \\ France
}

The emergence of energy-resolved photon counting detectors opens up new perspectives in x-ray imaging. The addition of energy information gives access to novel imaging techniques. By processing a single shot acquisition image, individual material components can be differentiated in terms of their equivalent thicknesses and relative ratios. However, due to scattered radiation, the accuracy required for such techniques is often difficult to achieve. In order to fully exploit multi-energy data, a novel Partial Attenuation Spectral Scatter Separation Approach (PASSSA) has been introduced. The present study aims to analyze the improvement in basis material decomposition of scatter corrupted multi-energy x-ray data in radiography after applying PASSSA. This evaluation was carried out with the aid of simulations provided by a recently introduced simulation tool, SindbadSFFD. A simplified thorax phantom was used. A material decomposition onto a water-bone material basis was performed with aid of a polynomial approach using two energy bins. Bone thickness was chosen as the performance criterion. A scatter-toprimary ratio (SPR) of $60 \%$ and $12 \%$ was considered. Bone thickness profiles from the decomposed PASSSA-corrected images proved to be remarkably close to the ones obtained from scatter free data. Furthermore, after correction of the $60 \%$ SPR case, the average relative error per pixel on bone thickness was reduced from $61 \%$ to $16 \%$ for the uncorrected and corrected data, respectively. For the $12 \%$ SPR case, the error on bone thickness estimation was $27 \%$ and $10 \%$ for the uncorrected and corrected data, respectively.

\title{
M11-4: Direct measurement of the X-ray tube spectrum from the scanning plane of a CT system operating in its nominal configuration
}

N. Shapira, E. Lahoud

Diagnostic Imaging $\mid C T$, Philips Healthcare, Haifa, Israel

With the advent of spectral imaging in Computed Tomography (CT), accurate knowledge of the X-ray tube spectrum becomes ever more important. The spectrum is often simulated to provide an essential input to the two-base model decomposition process as well as to a variety of artifact corrections and processing algorithms during image reconstruction such as beam hardening corrections. In more advanced CT imaging technologies, such as Photon Counting and Phase Contrast, the importance of higher quality estimates of the spectrum will play an even greater role. In recent years, a significant number of attempts have been made to estimate the spectrum mostly employing indirect methods due to the limitations imposed by the high flux emitted by X-ray tubes. Here we present the first direct X-ray spectrum measurement obtained within the scanning plane of a commercially available CT system operating in its nominal configuration using a CdTe solid state detector. Through careful collimation, scatter management and illumination condition selection, we were able to reduce the flux as well as the accompanying scatter radiation. We validated our results by the consistent agreement we obtain when comparing the impact of known filtration layers on the measured spectrum to the expected attenuation.

We acknowledge Ami Altman, Yoad Yagil and Ira Blevis for their support and technical advice.

M11-5: Calibration Free Beam Hardening Correction Using Grangeat-Based Consistency Measure

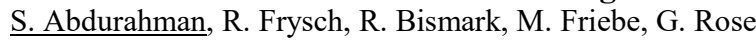

Institute for Medical Engineering, Otto-von-Guericke University, Magdeburg, Germany 
Due to polychromatic nature of the X-ray spectrum, beam hardening artifacts are introduced in cone beam computed tomography reconstructed images. To reduce these artifacts, projection images can be linearized with higher order polynomials. Polynomial coefficients are usually computed with calibration scans of homogeneous phantoms. This can be a challenging task when the objects with different material composition and geometry are scanned with different tube voltages. Here, we present a beam hardening correction method based on a consistency condition derived from Grangeat's formula for cone beam projections. First, an objective function is designed to quantify the inconsistency between two arbitrary projections. It is observed that inconsistency due to beam hardening is significantly high. Thus, by minimizing the objective function, polynomial parameters can be computed without prior knowledge about material composition, material thickness, $\mathrm{x}$-ray spectrum or detector response. Since the consistency conditions based on Grangeat's formula can be applied to two arbitrary cone beam projections, the modeling of polynomials is very robust compared to other consistency conditions. Our preliminary results show that proposed correction significantly reduces beam hardening artifacts like cupping. For the first time, we show that projection linearization optimized by a consistency measure can be applied to real data from a flat panel detector scanner to reduce the beam hardening artifacts. The proposed method can also be extended for multi-material beam hardening correction by using two or more polynomials or by estimating an effective projection linearization for each view.

M11-6: Volume Dose Distribution in Digital Breast Tomosynthesis: a Phantom Study M. Masi ${ }^{1}$, F. Di Lillo ${ }^{1,2}$, G. Mettivier ${ }^{1,2}, \underline{\text { A. Sarno }}^{1,2}$, R. Castriconi ${ }^{1,2}$, P. Russo ${ }^{1,2}$

${ }^{I}$ Dept. of Physics, Universita' di Napoli Federico II, Napoli, Italy

${ }^{2}$ Sezione di Napoli, INFN, Napoli, Italy

Monte Carlo calculations for breast dosimetry in Digital Breast Tomosynthesis (DBT) may require experimental validations, e.g. via the assessment of the calculated and measured dose volume distribution in the breast. This might also take into account any uneven distribution of the beam intensity in the entrance plane, not usually considered in Monte Carlo simulations, e.g. as determined by the heel effect. We measured the 3D dose distribution in a breast phantom, using XR-QA2 radiochromic films calibrated in free-in-air air kerma. Film sheets were positioned at the entrance surface, at the bottom surface as well as at four depths between adjacent slabs in the 5-slabs, 5-cm-thick phantom simulating a compressed breast with $50 \%$ glandular fraction. By varying the irradiation angle, the basic requirements of a DBT scan were simulated. The irradiations were made at $40 \mathrm{kV}$ (HVL $1.1 \mathrm{~mm} \mathrm{Al})$ for three angular positions of the beam central axis $(\theta=? 25 \mathrm{deg}$ and $\theta=0$ deg normal incidence, simulating a craniocaudal view). Results show that it is possible to determine the transverse and longitudinal distribution of the average dose in the phantom (in terms of kerma in simulated breast tissue 50/50 normalized to the entrance kerma), showing the angular dependence of the depth-resolved dose. In the direction of the beam axis, the dose decreases down to about $26 \%$ of the entrance value without the phantom. The backscatter fraction was $8 \%$. In transverse planes the maximum dose variations are between $6 \%$ and $18 \%$ at $\theta=0 \mathrm{deg}$, whereas the dose varies up to $22 \%$ in angular views.

M11-7: Large Area Photon Counting Detector with Cylindrical Surface for Applications in Small Animal CT P. Soukup, J. Jakubek, M. Jakubek, E. Trojanova, D. Turecek

Advacam, Prague, Czech Republic

The excellent imaging quality of particle counting pixel detectors of the Medipix family was already presented many times. The major limitation of this technology has been for long time their relatively small sensitive area. This disadvantage was recently successfully solved in WidePIX devices using tilling method in combination with technology of edgeless sensors without any gaps and insensitive areas between tiles. Each tile consists of single Timepix hybrid detector (256x256 pixels, $55 \ddot{i}_{i}^{1} / 2 \mathrm{~m}$ pitch) with edgeless silicon or CdTe sensor. In this contribution we present redesigned of large area detector WidePIX optimized for applications in computed tomography. The main improvement was changing flat planar shape of the sensitive area to cylindrical surface. The main disadvantage of the flat planar sensor for CT scanner is very unpleasant energy dependent blurring in offcenter regions where the $\mathrm{X}$-ray impact direction differs to much from surface normal vector. The new large area photon counting detector was developed for application in CT of soft biological objects. It consists of up to 5 rows of 5 Timepix tiles with silicon

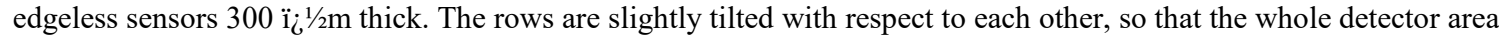
approximates the cylindrical surface with radius of $15 \mathrm{~cm}$. The maximal detector resolution with all five rows is $1280 \mathrm{x} 1280$ pixels (1.6 Mega pixels) and area of roughly $7 \times 7 \mathrm{~cm} 2$. The fast USB 3.0 based read-out electronics allows for maximal speed of 20 frames per second. The detector properties and first tomographic results will be presented during the conference.

M11-8: Low-Frequency Motion Artifact Correction for Myocardial Dual-Energy CT Perfusion Imaging

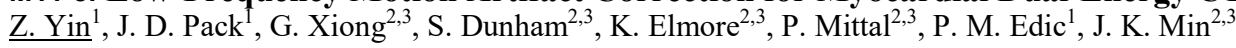

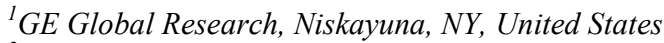

${ }^{2}$ Department of Radiology, Weill Cornell Medical College, New York, NY, United States

${ }^{3}$ Dalio Institute of Cardiovascular Imaging, New York, NY, United States 
Hemodynamic information generated with myocardial Computed Tomography Perfusion (CTP) imaging may facilitate the diagnosis of Coronary Artery Disease (CAD) by augmenting anatomical information provided by cardiac CT angiography (CCTA). However, CT shading artifacts due to the motion of the heart during data acquisition can significantly limit the quantitative accuracy of CTP. We adapted a motion compensation algorithm that originally was designed to correct for motion in coronary arteries and applied the techniques to correct for motion in the entire heart [1]. While this technique demonstrated significant motion artifact reduction in the myocardium, residual low-frequency artifacts still persisted. Motivated by these findings, we investigated a new motion correction algorithm which estimates the low-frequency, motion-induced artifacts and subsequently corrects these artifacts. We applied the new correction algorithm both to CT acquisitions using a 3D left ventricle model and thorax phantom [2] and limited clinical dual-energy CT data sets, and demonstrated that the improved technique effectively removes the motion-induced, low-frequency artifacts in the myocardium. We conclude that the proposed whole-heart motion compensation approach combined with low-frequency artifact correction can improve the quantitative accuracy of dualenergy CT perfusion imaging, thereby providing more accurate hemodynamic information to make better clinical decisions.

M13A: Posters: image reconstruction II

Friday, Nov. $4 \quad$ 10:30-12:00 Etoile

M13A-1: Time-of-Flight Parametric Image Reconstruction from Variable Random Fraction Dynamic PET Data

$\underline{\text { F. A. Kotasidis }}^{1,2,3}$, H. Zaidi ${ }^{3,4,5}$

${ }^{I}$ Faculty of medicine, University of Geneva, Geneva, Switzerland

${ }^{2}$ Wolfson Molecular Imaging Centre, University of Manchester, Manchester, United Kingdom

${ }^{3}$ Department of Nuclear Medicine and Molecular Imaging, Geneva University Hospital, Geneva, Switzerland

${ }^{4}$ Geneva Neuroscience Centre, University of Geneva, Geneva, Switzerland

${ }^{5}$ Department of Nuclear Medicine and Molecular Imaging, University of Groningen, Groningen, The Netherlands

Dynamic PET imaging allows the time course of the activity distribution to be measured and modelled, therefore estimating parametric images of micro- or macro-parameters. Due to the need for increased temporal sampling, frames of low statistics are often reconstructed, leading to noisy dynamic data and subsequently to kinetic parameters of reduced precision and accuracy. TOF image reconstruction can substantially improve upon the kinetic parameter SNR. However variable contributions to TOF variance reduction between true and random events, owing to their spatial distribution, results in SNR improvements depending on the random fraction and leading to variable TOF gains amongst temporal frames within a dynamic study. Such variable gains between early/late frames (high/low random fractions) are also expected to be more pronounced at increasing injected doses. Therefore we hypothesize that certain kinetic parameters receive differential improvements depending upon the part of the timeactivity curve they are estimated from. Using simulated dynamic [15O]H2O datasets at ever increasing doses and random fractions, we investigate the variable TOF gain in dynamic imaging and its effect on the kinetic parameters. Data are presented at improving TOF resolutions and using both indirect and direct methods to kinetic parameter estimation. Initial results suggest that kinetic parameter TOF gain is highly variable (increasing) at ever increasing injected doses, with changes of up to $25 \%$ in TOF gain within the simulated range of doses and random fractions. Furthermore this variable TOF gain is different for each parameter, based on the part of the dynamic data it is derived from and owing to the variable TOF gain within dynamic frames.

M13A-2: A Modified Quantitative Multispectral Weight Reconstruction Approach for Cerenkov Luminescence Tomography

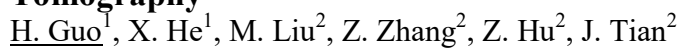

${ }^{I}$ School of Information Sciences and Technology, Northwest University, Xi'an, China

${ }^{2}$ Institute of Automation(IA), Chinese Academy of Sicences(CAS), BeiJing, China

Based on the theory of Cerenkov radiation, Cerenkov luminescence tomography (CLT) can provide a pathway for threedimensional (3D) tomographic radiopharmaceutical. Even more enticing 3D CLT can be used in cancer diagnosis and therapy. Since the weakness and severe scattering of Cerenkov radiation, the reconstruction of Cerenkov luminescence (CL) source is an ill-posed problem. Generally this challenge issue is solved by the multispectral CLT and hybrid-spectral CLT reconstruction method. And for CLT reconstruction, the accuracy of the photon transport model relies on the accuracy of the optical properties of the biological tissue. In this paper, the features of the emission and transmission progress of the Cerenkov radiation were analyzed and a modified multispectral weight reconstruction approach (MWRA) was developed to improve the reconstruction accuracy. The core of MWRA is dividing the multispectral information into several sub-spectral bands according to the CL spectral characteristics and the measured surface imaging. Then the incomplete variables truncated conjugate gradient (IVTCG) method was adopted to restore the 3D CL source distribution of every sub-spectral band. Lastly, the results of every sub-spectral band was weighted to get the averaged results. Through an in vivo experiment which aims at quantify uptake of $18 \mathrm{~F}-\mathrm{FDG}$ in mouse bladder, the results shows that the MWRA is much more accurate to quantify the uptake of radiopharmaceuticals in biological tissues. 
The authors thank Zhenhua Hu, Muhan Liu and Zeyu Zhang for insightful discussions and for sharing their work on Cerenkov luminescence tomography.

M13A-3: The Dual Head Panel PET Image Reconstruction Based on Simulated System Response Matrix Y. Shang, S. Wang, Y. Liu, W. Cao, Q. Xie, P. Xiao

Biomedical Engineering, Huazhong University of Science and Technology, Wuhan, China

Measuring system response matrix (SRM) by Monte Carlo method is time and resource consuming, even with high performance computer nodes. In this paper, we exploited symmetry properties available to drastically reduce the complexity in computing SRM of dual head panel PET, which has two parallel panel detectors. By extending the original system outward at least 1 time and simulating 4 voxels every slice parallel to the PET detector, we can obtain SRM of other voxels on the same image slice by symmetry and translation. Without loss of generality, the voxel size in $\mathrm{Y}$ and $\mathrm{Z}$ direction is one quarter of the crystal pitch size respectively, and they may not be the same. The results show that the noise level and convergence of reconstruction images by Monte Carlo method and symmetry are better than that by calculating SRM on-the-fly like solidangle.

M13A-4: AMIAS: a Novel Statistical Method for Tomographic Image Reconstruction - Application in Thermal Emission Tomography

$\underline{\text { L. Koutsantonis }}^{1}$, C. Papanicolas ${ }^{1,2}$, A.-N. Rapsomanikis ${ }^{2}$, E. Stiliaris ${ }^{1,2}$

${ }^{I}$ Computation-based Science and Technology Research Center, The Cyprus Institute, Nicosia, Cyprus

${ }^{2}$ Department of Physics, National \& Kapodistrian University of Athens, Athens, Greece

This study presents a novel statistical analysis method implemented in the field of tomographic image reconstruction. The method, the Athens Model Independent Analysis Scheme (AMIAS), a well suited analysis method for inverse scattering problems has thus far been applied for analysis of data in nuclear and particle physics. The performance of the AMIAS implementation in image reconstruction was examined by using planar images from thermal emission (IR) in simple phantoms with strong absorption.

\section{M13A-5: Null-Space Smoothing of Tomographic Images Using TV Norm Minimization B. D. Smith}

Electrical and Computer Engineering, University of Texas San Antonio, San Antonio, Texas, USA

When a finite-dimensional linear model is used to describe the formation of the tomographic data, the data does not contain any information about the null-space of the object being imaged. This suggests the following question: How can a reconstruction be made so it produces an image with a non-zero null-space component? A non-zero null-space component can be produced if a criterion for reconstruction is accepted. The conjecture made here is that the row-space component of the reconstruction should be given more credence then the null-space component because the row-space component is based directly upon the measurements made of a given object whereas the null-space component is based upon a criterion that is accepted. This conjecture suggests that if a criterion that does not affect the row-space component of the object is used to produce a non-zero null-space component, may be superior then a criterion that does. An objective of the work presented here is to propose and demonstrate such a criterion; namely, null-space smoothing using TV norm minimization. Methodology. Reconstructions using this criterion and other established criteria are produced and compared. In these comparisons, simulated data that is consistent with a mathematical phantom is used. It is concluded that although null-space smoothing can produce smooth images with nonzero null-space components, more work needs to be done in the future to demonstrate its usefulness with real data.

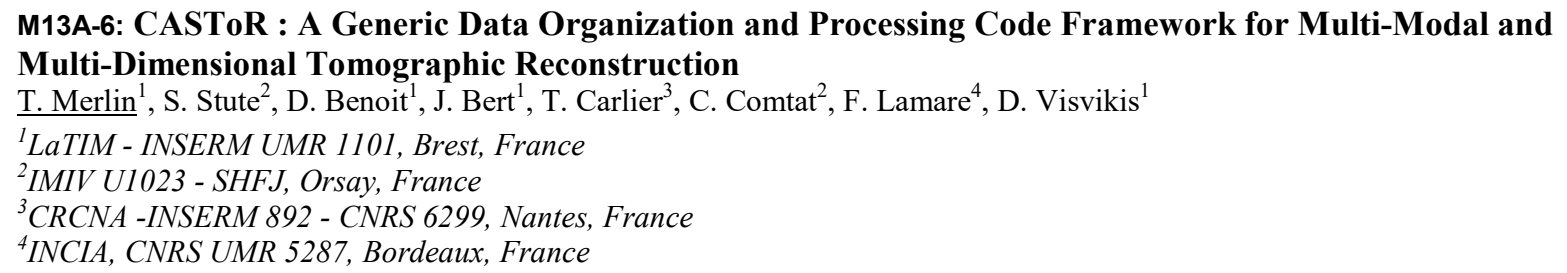

Handling the differences in data acquisitions and organization is a major hurdle for tomographic reconstruction softwares. The implementation of a given methodology is usually limited to a specific set of conditions, depending either on the modality (PET, SPECT, CT), the purpose, or to the reconstruction algorithm itself. However, separate implementations corresponding to each set of conditions can lead to several drawbacks including restricted or limited use of methodologies, differences in duplication of implementations, and potentially impractical code development. This work attempts to address these issues by proposing a unified and generic data organization and processing code framework for multi-modal and multi-dimensional tomographic 
reconstruction. The proposed iterative framework implies that all events composing the data-set are processed separately and sequentially (for both list-mode or histogrammed data). The architecture relies on generic core classes for each main component of the reconstruction process which are inherited by specific classes, allowing to incorporate specific code dedicated to a modality or a particular methodology without sacrificing the generic behavior of the algorithm. Current implementation allows the reconstruction of dynamic datasets including tracer kinetics and two levels of gating (up to 6D). Assessments of the framework implemented in an open-source $\mathrm{C}++$ multithreaded platform indicate that the time loss due to the genericity remains limited, about 1.5 times slower compared with a non-generic algorithm optimized for computational efficiency. The speed-up factor relative to the number of threads is not strictly linear but shows a low dependence with respect to the number of subsets. A speedup factor of 1.2 was also obtained when using matched forward and backward projectors. The proposed framework allows a substantial flexibility for the integration of new reconstruction methodologies while maintaining computation efficiency.

M13A-7: Penalized MLAA with Spatially-Encoded Anatomic Prior in TOF PET/MR $\underline{\text { K. Kim }}^{1}$, J. Yang ${ }^{2}$, G. El Fakhri ${ }^{1}$, Y. Seo ${ }^{2}$, Q. Li $^{1}$

${ }^{I}$ Radiology, Gordon Center for Medical Imaging, Massachusetts General Hospital and Harvard Medical School, Boston, MA, USA

${ }^{2}$ Radiology and Biomedical Imaging, University of California, San Francisco, San Francisco, CA, USA

The PET/MR scanner has been developed as an imaging tool with both molecular and morphological assessment that has great potentials in clinics. The MR-based attenuation correction (MRAC) has been routinely used in clinical PET/MR scanners. However, the MRAC has major challenges such as large bias of attenuation coefficient and the lack of bone signals. Recently, the maximum likelihood reconstruction of activity and attenuation (MLAA) using the time-of-flight (TOF) PET emission data has been developed, and is considered as a potential solution for attenuation correction of TOF PET/MR. However, the MLAA does not always work, because the quality of attenuation image depends on the amount of emission counts in a region and most of the region of the body has limited emission counts since PET tracers is designed to only accumulate in target regions. To address this issue, we propose a penalized MLAA using a MR-based, spatially-encoded anatomic prior in TOF PET/MR scanner, in which the MR dixon images are used as anatomical prior of attenuation estimation, and the patch-based spatially-encoded similarity weight is calculated using both attenuation image and dixon-based MR images in each iterative process to reduced the potential bias caused by anatomical prior. The proposed method iterates two steps: one step is the estimation of activity image using TOFbased separable quadratic surrogate (SQS) with a quadratic penalty; and another step is the estimation of attenuation image using the SQS with the MR-based anatomic prior. In this framework, we can also use multiple MR images in the anatomical prior for the estimation of attenuation image. An alternating direction method of multipliers (ADMM) algorithm is exploited to optimize the cost function with multiple penalty terms. We perform the experiment using the patient data to demonstrate that the proposed method can significantly improve attenuation estimation compared to the conventional methods.

\author{
M13A-8: Stepwise Linear Regression Modeling of the Point Spread Functions of a Multi-Pinhole SPECT \\ Camera for I-123 DaTscan Imaging \\ J. M. Mukherjee, A. Konik, M. A. King \\ Radiology, University of Massachusetts, Worcester, MA, United States
}

In many multi-pinhole (MPH) SPECT imaging systems, improvement in image quality has been demonstrated with accurate modeling of the system point spread function within reconstruction. The point spread function (PSF) is obtained either through measurement during the calibration process by placing a point source at regularly spaced locations in the volume imaged, or through Monte Carlo simulations of the system using the knowledge of system geometry from calibration. Modeling the PSF within reconstruction requires either storage of pre-computed PSF, or computation of the PSF on the fly from stored coefficients. The choice depends upon computation time vs memory requirement tradeoffs. To facilitate faster reconstruction and smaller memory overhead, simpler PSF models are preferred such as a Gaussian. In many geometries a Gaussian model is reasonably accurate for the PSF. However, for different pinhole aperture shapes such as square or rectangular, and angled pinhole aperture planes, the measured PSF including all physical effects such as penetration and parallax can have substantial non-gaussian components. In this work, we illustrate with examples from our MPH SPECT system how stepwise regression analysis can be used to develop a generalized but parsimonious model for more complex PSFs without a-priori knowledge of the exact form of the PSF. The flexibility of the approach comes from the ability to include or exclude as many model terms as is required to fit the measured data based on criteria such as goodness of fit, sum-squared error etc. It also allows the exploration (data-mining) of the measured PSF to determine the impact of including or excluding the higher order polynomial terms if present.

The authors want to thank Professor Lars R. Furenlid of the College of Optical Sciences, University of Arizona for discussions on PSF modeling which led to this work. This work was supported by the National Institute of Biomedical Imaging and Bioengineering (NIBIB) grant R21EB016391, and R01-EB022092. The contents are solely the responsibility of the authors and do not represent the official views of the NIBIB.

\title{
M13A-9: A Direct Image Reconstruction Algorithm for PET Scanners Based on Monolithic Crystals
}


A. Iborra $^{1}$, P. Conde ${ }^{1}$, S. Sanchez ${ }^{1}$, A. J. Gonzalez ${ }^{1}$, M. J. Rodríguez-Álvarez ${ }^{1}$, A. Aguilar ${ }^{1}$, P. Bellido ${ }^{1}$, E. Diaz-Caballero ${ }^{2,1}$, J. J. Garcia-Garrigos ${ }^{1}$, A. Gonzalez-Montoro ${ }^{1}$, D. Grau-Ruiz ${ }^{1}$, L. Hernández ${ }^{1}$, F. Martos ${ }^{1}$, L. Moliner ${ }^{1}$, J. P. Rigla ${ }^{2,1}$, F. Sánchez ${ }^{1}$, M. Seimetz ${ }^{1}$, A. Soriano ${ }^{1}$, J. C. Valderas ${ }^{1}$, L. F. Vidal ${ }^{1}$, J. M. Benlloch ${ }^{1}$

${ }^{I}$ Institute for Instrumentation in Molecular Imaging (i3M), UPV / CSIC, Valencia, Spain

${ }^{2}$ Tesoro Imaging S. L., Alicante, Spain

A direct image reconstruction algorithm for PET is proposed in this work. This algorithm follows naturally form the concept of LOR, and easily admits DOI and TOF information. In addition, this algorithm does not require the computation of sinograms, thus not being restricted in the formation of high resolution images and allowing the image formation in real-time (as the data is collected by the scanner).

When an event is detected by the PET scanner, a new LOR is considered as a line, connecting the two detector impact positions of the back-to-back $511 \mathrm{keV}$ detected photons, and being the probability of the emission location equidistributed trough this line. The algorithm starts casting a line defined from each event's LOR. Then, locates this line in the 3D field of view and assigns to each voxel traversed by the line the same probability of emission, thus drawing the line in the FOV.

One of the key advantages of this algorithm is the preservation of high frequencies, producing fine detailed images that can be generated in high resolution because of the lack of space restrictions imposed by the intermediate generation of sinograms, aside of the native support of real-time image reconstruction. In this work, a C++ implementation of the described algorithm has been developed and image reconstructions of simulated PET events, according to the Albira scanner specifications has been performed. A Derenzo-like phantom has been simulated in GATE, which is made of cylinders of radii ranging from $0.75 \mathrm{~mm}$ to 2 $\mathrm{mm}$, and $20 \mathrm{~mm}$ in height. The scanner geometry is composed of one ring of 8 detectors blocks with a distance of $117 \mathrm{~mm}$ in between opposite detectors. Each detector module includes a monolithic LYSO crystal of $50 \times 50 \times 10 \mathrm{~mm}$.

Image reconstructions have been compared against those obtained with the Filtered Backprojection algorithm which is required by the NEMA NU 4-2008 QA standard. Obtained results show a notable image quality improvement.

\author{
M13A-10: A Novel Approach to Image Reconstruction and Calibration for a Multi-Slit-Slat SPECT System \\ K. Erlandsson $^{1}$, D. Salvado ${ }^{1}$, B. F. Hutton ${ }^{1,2}$ \\ ${ }^{I}$ Institute of Nuclear Medicine, University College London, London, UK \\ ${ }^{2}$ Centre for Medical Radiation Physics, University of Wollongong, New South Wales, Australia
}

In the context of the development of a simultaneous SPECT/MRI system, we have previously proposed a multi-mini-slit-slat (MSS) collimator, with multiple sections of short slits in order to improve the angular sampling [Salvado et al., IEEE TNS 62(4):1716-24, 2015]. The data can be reconstructed using a 3D reconstruction algorithm that models the collimator geometry. However, one drawback is that the projection data with this collimator are difficult to interpret visually, and calibration can be problematic, as each mini-slit only covers part of the object FOV. We have therefore developed an algorithm for transforming the MSS projection data into the traditional sinogram format. These sinogram consist of multiple thin tilted lines with gaps in between due to the lack of rotation of the system. Cross-talk between different MSS sections, caused by blurring of the projection data, is handled by deconvolution before the sinogram transformation. The data can be reconstructed using a standard parallelbeam algorithm, taking into account the missing data. We have shown with simulations that the algorithm can transform complex data, consisting of multiple broken line segments, into a simple sine-curve. This algorithm can be useful for interpreting the acquired MSS data, reconstructing images, and calibrating the system.

Acknowledgments: This work was done as part of the INSERT collaboration, which is supported by the EC: FP7- HEALTH-305311. DS is supported by a PhD Fellowship (FCT:SFRH/BD/88093/2012). UCL/UCLH research is supported by the NIHR University College London Hospitals Biomedical Research Centre.

\title{
M13A-11: Parameter Optimization for Blob-Based Image Reconstruction with Generalized Kaiser-Bessel Radial Functions

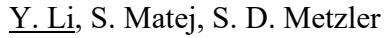

Department of Radiology, University of Pennsylvania, Philadelphia, USA

Incorporation of spherically-symmetric volume elements (blobs), instead of the traditional voxels, into iterative image reconstruction algorithms, can substantially improve the quality of the reconstructed images. Generalized Kaiser-Bessel radial basis functions are frequently used in tomographic reconstructions, and the parameter selection can significantly impact both the qualitative and quantitative image characteristics. Hence, a detailed investigation and optimization of blob parameters is needed to achieve optimal reconstruction results. In this study, we aim at optimizing the parameters of these basis functions for optimal image representation and image reconstruction. We first represent unknown radiotracer activity distribution as the coefficients of blob basis functions on a Bravais lattice from crystallography. We point out that the optimal sampling lattice is just the reciprocal of the lattice that achieves highest packing of equally sized spheres. Based on multidimensional sampling theorem and the Kepler conjecture (proven by Thomas C. Hales), we obtain that body-centered cubic (BCC) lattice is optimal in terms of either reducing 
computational cost or improving image quality at the same computational cost. We formulate total harmonic distortion (THD) based on the theory of approximation to analytically characterize the approximation error between a non-zero uniform object and its blob representation. Further, we derive a new image quality metric, approximation power (AP), to characterize the approximation error between an arbitrary object and its blob representation. The AP is formulated in terms of of the power spectral density of an imaging object, and we use it for object-dependent blob parameter optimizations. We validate the THD and AP through numerical examples using homogeneous and heterogeneous objects, respectively. We also present 3D blob-based image reconstructions with BCC lattice and different shape parameters to evaluate the usefulness of the proposed optimization.

The authors would like to thank Dr. Robert M. Lewitt for valuable comments and suggestions.

M13A-12: Comparison of Two Motion Compensation Models : Adding Ordered Subsets into the Mix M. Toussaint ${ }^{1}$, J.-P. Dussault ${ }^{1}$, R. Lecomte ${ }^{2}$

${ }^{I}$ Dep. of Computer Science, Université de Sherbrooke, Sherbrooke, Québec, Canada

${ }^{2}$ Dep. of Nuclear Medicine and Radiobiology, Université de Sherbrooke, Sherbrooke, Québec, Canada

The motion compensation model was introduced to improve statistical accuracy of iterative tomographic image reconstruction in the presence of periodic motion. However, this is achieved at the expense of substantial extra computational burden to handle all gated histograms simultaneously. An alternative model was proposed by re-interpreting the interdependence of the Poisson processes defining the projection data along the motion axis, thus allowing all gated histograms to be merged into a single one for reconstruction. It was demonstrated that much less computational resources were then required to converge using this Merged model, while achieving equivalent image quality. In this work, we investigate the impact of Ordered Subsets (OS) on the convergence rate and image quality of the Merged model compared to the Classical motion compensated model. Comparison of 1, 2, 4 and 8 OS was performed using simulated and experimental datasets. As expected, the gain in convergence rate was roughly proportional to the number of OS for both models and both datasets. Both models also converged to images that were equally well correlated to the ground truth. However, the Merged model was observed to retain its advantage in computational resources over the Classical model, which amounted to a three fold gain for our experimental dataset. In conclusion, the proposed Merged model is a promising approach for imaging applications involving periodic motion, such as cardiac or respiratory gated studies.

M13A-13: Regularized MLEM Reconstrution with a Strong Anatomical Prior Using Newton Iterative Algorithm

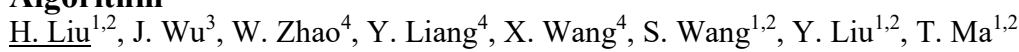

${ }^{\prime}$ department of engineering physics,, Tsinghua University, Beijing, China

${ }^{2}$ Key Laboratory of Particle \& Radiation Imaging (Tsinghua University), Ministry of Education, Beijing, China

${ }^{3}$ Department of Radiology and Biomedical Imaging, Yale University, NEW HAVEN, USA

${ }^{4}$ Departement of Nuclear Medicine, Navy General Hospital, Beijing, China

MAP algorithm has outperformed in medical image reconstruction with noise suppression and edge preservation. However MAPOSL algorithm could hardly apply a strong prior by using a large regularization parameter. In this study we proposed a MAPNewton reconstruction framework to enable a strong prior to be applied in MAP optimization. EM iterative framework was used in MAP-Newton. In the step of maximizing expectation of the complete data log-likelihood function, MAP-Newton solves the non-linear equation accurately with Newton iterative algorithm, which is potentially better than approximate linearization method used in MAP-OSL. MAP-Newton reconstruction algorithm was implemented with both Bowsher's prior and joint total variation (JTV) prior based on anatomical image. 18F PET and CT data of an image-quality phantom were acquired on the small animal PET/SPECT/ CT Iniview 3000 system for performance evaluation. The priors with three different strength (regularization parameter was from 0.01 to1.0) were applied in the list mode reconstruction studies with three noise levels (100 M, $12 \mathrm{M}$ and 2 M LORs). The normalized standard derivation (NSTD) of region of interest was calculated. The results with Bowsher's prior for all noise level cases showed no significant difference between MAP-Newton and MAP-OSL when the regularization parameter was smaller than 1.0. When the parameter was set to be 1.0, the proposed MAP-Newton can greatly reduce NSTD with reasonable image quality although no reasonable images could be obtained with MAP-OSL. The results with JTV prior showed the same trend, except that increased regularization parameter did not reduce NSTD. In conclusion, with a strong prior, MAPNewton can result in better image quality than MAP-OSL in terms of noise suppression, while no significant difference when applying a light prior. It indicated that the MAP-Newton reconstruction with a strong prior could be applied, when we have enough confidence on the prior.

M13A-14: Improvement of Simultaneous Radioactivity and Attenuation Estimation in TOF-PET Using MRBased Attenuation Prior $\underline{\text { P.-H. Hsu }}^{1}$, Y. Hsu ${ }^{1}$, C.-M. $\mathrm{Kao}^{2}$, C.-T. Chen ${ }^{2}$, C.-Y. Chou ${ }^{1}$ 
${ }^{I}$ Department of Bio-Industrial Mechatronics Engineering, National Taiwan University, Taipei, Taiwan

${ }^{2}$ Department of Radiology, The University of Chicago, Chicago, Chicago, IL, USA

In hybrid PET/MR systems, MR images can offer excellent soft tissue contrast and resolution and also be used for attenuation correction. However, MR-based attenuation correction remains a major challenge in such hybrid systems. The enhancement in PET hardware timing resolution enables PET systems to resolve time-of-flight (TOF) information and thus can mitigate noise and artifacts, thereby improving the image quality. Recently, the TOF-PET has been further extended to estimating not only activity distributions, but also attenuation maps simultaneously by use of iterative algorithms. The attenuation map estimated by MR approaches can be employed as prior to improve the TOF-PET attenuation estimation. In this work, the data fidelity term, total variation and MR-derived attenuation prior will be formulated as a composite convex optimization problem. The results will compared with those obtained by use of the maximum-likelihood algorithm for both activity and attenuation estimation. The preliminary computer simulation studies that the application of the proposed algorithm can greatly improve the image quality and reduce noisy appearance.

M13B: Posters: New radiation detectors II

Friday, Nov. $4 \quad$ 10:30-12:00 Etoile

M13B-1: A Novel Multiplexing Method Using Bipolar Pulse

Y. K. Kim, Y. Choi, K. B. Kim, H. T. Leem

Molecular Imaging Research \& Education (MiRe) Laboratory, Department of Electronic Engineering, Sogang University, Seoul, Korea

Although individual channel readout method of pixelated detectors could provide high spatial resolution of radiation detectors, it could lead to significantly increase of the required number of readout electronic channels and the development cost. Various channel reduction methods have been introduced, but they suffer various problems of reduced SNR, position distortion, and complexity for PET system development. In this study, we developed a novel multiplexing concept using bipolar pulse to improve the drawbacks of the conventional multiplexing methods. Each channel of the detector was designed to generate different bipolar pulse having different zero crossing point by adjusting the time constant value of high pass filter, as a function of channel position. It would allow to identify channel position by estimating the width difference between rising edge and zero crossing point of the bipolar pulse. The energy and timing information were measured using the sum signal of cathode output. The developed PET detector module was composed of a $4 \times 4$ matrix of $3 \times 3 \times 20 \mathrm{~mm}^{3}$ LYSO, a $4 \times 4$ array MPPC. The information of position, energy, and time of a detected gamma ray were measured with the high-speed oscilloscope. The average energy resolution was $10 \%$ FWHM and channel position was accurately identified for all measured channels. This study demonstrated that the novel bipolar multiplexing method could be useful to reduce the number of readout channels and to identify pixel position. Further study will be performed to extend the number of channels using the proposed multiplexing method which could be utilized for the development of PET system.

M13B-2: Low-Cost Gamma Detector with Novel Light-Guide-PMT Geometry to Increase Usable Field-of-View B. Wang ${ }^{1}$, R. Kreuger ${ }^{1}$, F. J. Beekman ${ }^{1,2}$, M. C. Goorden ${ }^{1}$

${ }^{I}$ Section Radiation, Detection \& Medical Imaging, Delft University of Technology, Delft, The Netherlands

${ }^{2}$ MILabs B.V., Utrecht, the Netherlands

PMT-based scintillation gamma detectors are still predominant in medical imaging due to their cost-effectiveness. A drawback of these detectors is that the positioning is severely degraded at the edges of the scintillator. Existing solutions that allow to reduce the dead edge problem are often expensive. Here we propose a relatively cost-efficient solution that is based on the use of blackedge scintillators with an unconventional light-guide-PMT layout with the gamma interaction position estimated by a maximum likelihood algorithm. A gamma detector of a monolithic $\mathrm{NaI}(\mathrm{Tl})$ scintillator read out by $3 \times 5$ square PMTs is considered. Instead of attaching a full row of PMTs near the scintillator's edge (Conventional Layout, CL), we propose to insert a row of 5 additional rectangular light-guides with reflecting sides such that they guide the light toward square PMTs placed behind them (Shifted Layout, SL). The shorter length of the second light-guide is in the direction perpendicular to the scintillator's edge thus creating effective "half-sized" PMTs in that direction without adding additional space at the edge. Moreover, instead of replacing the full row of PMTs near the edge, we also propose to replace them alternatingly, such that the PMTs are in an interlock pattern (Alternating Shifted Layout, ASL). The spatial and energy resolution of our designs are tested with the well-validated Monte Carlo simulation toolkit GATE. Simulation results show that the new designs give better spatial resolution (from $3.9 \mathrm{~mm}$ on average for CL reduced to $3.7 \mathrm{~mm}$ and $3.6 \mathrm{~mm}$ for SL and ASL) especially at the edges, and thus a larger usable area (from $74 \%$ for CL increased to $85 \%$ and $92 \%$ for SL and ASL). However, the black-edge gamma detector also shows poorer energy resolution at the edge than conventional white-edge detectors (16\% at the edges compared to $11 \%$ in the center). To conclude, for applications where small dead edges are crucial, our design may be a cost-effective solution. 
M13B-3: Sensitivity and Timing Resolution Improvement of Inter-Crystal Compton Scattered Events for Sub250ps TOF-PET Detector

G. Fu, A. Ivan, H. Qian

GE Global Research Center, Niskayuna, NY, USA

In PET detector designs, high sensitivity is a critical performance parameter required for obtaining images with reasonable signal-to-noise ratio (SNR). The sensitivity of a PET scanner is primarily determined by the efficiency of detection system (e.g. crystal thickness and the solid angle coverage), but could be enhanced by distinguishing and recovering inter-crystal Compton scattering events while maintaining good timing resolution. Generally, the identification of such events is based on the measured energies deposited in each crystal; however, the energy performance of SiPM device is affected by the inherent non-linearity of the SiPM response. The measured electric signal exhibits a nonlinear relationship to the number of detected incident photons due to saturation, crosstalk, and after-pulse. To investigate the sensitivity improvement and timing resolution of inter-crystal Compton scatter events and correct for the presence of saturated SiPM and thus non-linearity in energy, the same experimental setup and procedures would be used for evaluating timing resolution of SiPM devices with different PDE (e.g. SensL B, C and J devices) directly coupled to various length (5 to $25 \mathrm{~mm}$ ) LYSO crystals. The performance of different inter-crystal Compton scatter event recovery methods and timing resolution are also compared for 1-to-1 readout block design.

M13B-4: Energy Discrimination Using First Emitted Photon Timestamps: an Exploratory Study

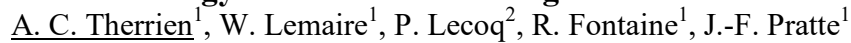

${ }^{I}$ Institut Interdisciplinaire d'Innovation Technologique, Université de Sherbrooke, Sherbrooke, Canada

${ }^{2}$ Physics Department, CERN, Geneva, Switzerland

Single Photon Avalanche Diodes (SPAD) have evolved tremendously in the past decade and the arrays for PET are becoming more sophisticated, including some with a fully digital readout. New methods (MLE, BLUE) use the precise time information on multiple photons to estimate with greater accuracy the timestamp of each event, resulting in excellent time resolution. Since we already have the circuitry to log the timestamps of these photons, can we extract the energy information from them? In this study, we investigated if these first photons also contain enough information for discriminating Compton from photopeak events. The total number of photons generated depends on the deposited energy and, as such, the rate of emission of the initial photons should also increase with energy. By using the information of the first photons rather than the whole event, we can turn off the SPADs in the array for the remaining duration of the event, reducing afterpulsing, power consumption and thermal dark counts. Three methods to extract this information were studied: a linear regression, a time window to count photons and the Ranked Initial Photons Energy Discrimination method. With a LYSO $1 \times 1 \times 10 \mathrm{~mm}^{3} \mathrm{LYSO}$ crystal and a $1 \times 1 \mathrm{~mm}^{2}$ array with $50 \mu \mathrm{m}$ pitch, this third method leads to a $92,6 \%$ successful photopeak discrimination when using the time of arrival of the $64^{\text {th }}$ photon.

M13B-5: Feasibility Study of Direct Beta Particle Detection Using Gas Electron Multiplier

B. Izudike ${ }^{1}$, C. Zhao ${ }^{1}$, J. Yu ${ }^{1}$, W. Chen ${ }^{1}$, X. $\operatorname{Sun}^{2}$, G. Balch ${ }^{3}, \underline{\text { M. Jin }}{ }^{1}$

${ }^{1}$ Physics, University of Texas at Arlington, Arlington, TX, USA

${ }^{2}$ Radiology, University of Texas Southwestern Medical Center, Dallas, TX, USA

${ }^{3}$ Surgery, University of Texas Southwestern Medical Center, Dallas, TX, USA

Portable nuclear imaging devices have been pursed for intraoperative localization and visualization of tumors for a complete removal of malignant tissues. In the proximity of the tumor site, beta imaging is advantageous over gamma imaging due to far less long-range contamination and no need of heavy metal collimation for a compact size. The traditional beta particle detection usually uses a thin scintillation layer coupled with photomultiplier tubes or solid-state detectors, which may lose efficiency due to the multiple conversion steps. In this work, we study the feasibility of direct beta particle detection using gas electron multiplier (GEM) detectors, which are highly sensitive to charged particles. The Geant4 Monte Carlo simulation package is used to simulate Copper-64 (Cu-64) beta particle transportation in different matters. Our simulation results demonstrate that GEM is feasible for direct detection of beta particles and the sensitivity of GEM to beta particles is much higher than gamma photons for certain GEM structures.

This research is supported by NIH under Award Number R15CA199020.

M13B-6: Characterization of 0.5-Mm Lutetium Oxyorthosilicate Detector Arrays for High-Resolution PET Applications

A. A. Refaey ${ }^{1,2}$, M. S. Judenhofer ${ }^{3}$, R. D. Badawi ${ }^{2,3}$

${ }^{I}$ Dept. of Physics, Minia University, EL-Minia, Egypt

${ }^{2}$ Dept. of Radiology, University of California, UC Davis Medical Center, USA

${ }^{3}$ Dept. of Biomedical Engineering, University of California, Davis, USA 
We previously built a high-resolution PET scanner (PawPET) using $8 \mathrm{~mm}$ thick Lutetium Oxyorthosilicate (LSO) detectors, but it suffers from poor sensitivity. In this work, we investigate the performance of two $12 \mathrm{~mm}$ thick, $0.5 \mathrm{~mm}$ pitch LSO arrays with different surface treatment and reflector materials using single-ended and hybrid dual-ended readout methods. We evaluated depth dependence of photopeak position, depth dependence of flood histogram quality, energy resolution and spatial resolution. The arrays consisted of $28 \times 28$ polished LSO elements each of dimensions $0.43 \times 0.43 \times 12 \mathrm{~mm} 3$ with ESR reflectors and $32 \times 32$ polished LSO elements each of dimensions $0.43 \times 0.43 \times 12 \mathrm{~mm} 3$ with Toray reflectors. Crystal decoding is performed using a Hamamatsu M-64 PS-PMT while depth decoding is performed by taking the weighted ratio of the output of an RMD avalanche photodiode and the summed PS-PMT output. Signals are processed using NIM electronics. For single-ended readout mode, the ESR array shows better segmentation than Toray one. The Toray array shows strong dependence of full-energy peak position as a function of depth. The ESR array shows weak dependence, indicating poor depth encoding potential. The mean energy resolution for the ESR array with single ended readout is $13.4 \%$ and that for the Toray array with dual ended readout is $24.8 \%$. The mean depth of interaction resolution (Toray) is $2.14 \mathrm{~mm}$. The optimized mean intrinsic spatial resolution (Toray), integrated over a column of crystals, is $0.57 \mathrm{~mm}$. We plan to start building an ultra-high resolution four-detector PET scanner for small animal imaging based on the Toray array.

\section{M13B-7: Light Transport in PET Scintillator Detectors Fabricated Using Laser Induced Optical Barriers} $\underline{\text { L. Bläckberg }}{ }^{1,2}$, D. Uzun Oszahin ${ }^{1}$, N. Moghadam ${ }^{3}$, G. El Fakhri ${ }^{1}$, H. Sabet ${ }^{1}$

${ }^{I}$ Massachusetts General Hospital and Harvard, Boston, MA, USA

${ }^{2}$ Department of Physics and Astronomy, Uppsala University, Uppsala, Sweden

${ }^{3}$ GRAMS lab. Institut interdiciplinaire d'innovation technologique (3IT), Université de Sherbrooke, Quebec, Canada

The focus of our work is the development of a high resolution LYSO detector with DOI capability and single side readout for small animal PET. This will be realized by using the new promising scintillator processing technique Laser Induced Optical Barriers (LIOB). With LIOB we can place optical structures of varying refractive index inside the bulk of a scintillator crystal. Here we report on light transport simulations performed with the aim of studying the light spread in scintillator detectors fabricated using LIOB. We also investigate the feasibility of achieving DOI capabilities in such detectors with 10-20 mm thickness and sub-mm transverse resolution. An array of 2-3 mm silicon photomultipliers (SiPMs) is used to evaluate the potential performance of the detectors. Results show that the light spread is heavily dependent on the refractive index of the barriers as well as on the characteristics of the interface between the barriers and the crystal. While uniform refractive index throughout the barrier walls, realized in a pixel-like pattern in the crystal, result in limited DOI information based on the light spread function, such information may be achieved by using higher refractive index in the barriers in the top part of the crystal compared to the bottom part. A maximum of $\sim 67 \%$ of the scintillation light can be contained within the same pixel-like element. This fraction gradually decreases with increasing barrier RI. The results also suggest that for the simple pixel-like barrier pattern better DOI information can be achieved when relaxing the transverse spatial resolution and vice versa.

This work was supported in part by the US National Institute of Health under grant 1R21EB020162-01A1 L. Bläckberg acknowledges support from the Swedish Research Council.

\section{M13B-8: Testing and Development of an OWC MRI Compatible PET Insert Front-End} G. D. Konstantinou ${ }^{1}$, W. Ali ${ }^{2}$, R. Chil ${ }^{1,3}$, G. Cossu ${ }^{2}$, E. Ciaramella ${ }^{2}$, J. J. Vaquero ${ }^{1,3}$

${ }^{I}$ Departamento de Bioingeniería e Ingeniería Aeroespacial, Universidad Carlos III de Madrid, Madrid, Spain

${ }^{2}$ Scuola Superiore Sant'Anna, Pisa, Italy

${ }^{3}$ Investigación Sanitaria Gregorio Marañón, Madrid, Spain

We present the design and development of a positron emission tomography (PET) detector module that could be used inside magnetic resonance imager (MRI). Critical factors compromising this combination have been studied and different solutions have been offered. Our design divides the detector module in two sections: one is the insert front-end that is placed inside the MRI and that comprises of a scintillator, a silicon photomultiplier and minimum analog electronics. The analog pulses are sent to the second section, the back-end digitalization and reconstruction module. The analog link is implemented using optical wireless communication (OWC) techniques. In this work we study how such a setting retains all the necessary characteristics for the detection and characterization of gamma scintillation events, providing sufficient communication quality without occupying space for cabling and interconnection and with very low power consumption. Possible multiplexing schemes for achieving the necessary transmission with less communication channels are also proposed and studied. A series of tests and measurements on different settings demonstrate the viability of this technique. When fully developed, it can provide a cost effective alternative for the industrial production of a flexible and customizable modular PET detector insert that can be applied to pre-existing small animal or human MRI settings, only minimally affecting the size of the MRI bore, without compromising the least the PET signal quality.

This work was partially funded by the TEC2014-56600-R from the Spanish Ministerio de Economía y Competitividad, the INFIERI Network (Marie Curie ITN EU FP7, grant agreement no. 317446), the TOPUS S2013/MIT-3024 project from the regional government of Madrid, and the 
RETOS RTC-2015-3772-1 from the Spanish Ministerio de Ciencia e Innovación. G. Konstantinou is with the Departamento de Bioingeniería e Ingeniería Aeroespacial, Universidad Carlos III de Madrid, Madrid, Spain (telephone: +34916248201, e-mail: gkonstantinou@hggm.es). R. Chil and J.J. Vaquero is with the Departamento de Bioingeniería e Ingeniería Aeroespacial, Universidad Carlos III de Madrid, Madrid, Spain, and with the Instituto de Investigación Sanitaria Gregorio Marañón, Madrid, Spain. W. Ali, G. Cossu and E. Chiaramella are with the Scuola Superiore Sant'Anna, Pisa, Italy.

\author{
M13B-9: drimPET: Assessment of DoI in LYSO Crystals Using SiPMs and Wavelength Shifters \\ P. M. M. Correia, I. F. Castro, N. Romanyshyn, P. Quinta, J. F. C. A. Veloso \\ I3N, Physics Department, University of Aveiro, Aveiro, Portugal
}

The determination of the depth-of-interaction (DoI) of $511 \mathrm{keV}$ gamma photons in scintillator crystals is of great importance in small diameter positron emission tomography (PET) systems, in order to achieve high spatial resolution with good uniformity within the entire field-of-view. drimPET is new concept for DoI determination wherein a single layer ring of 128 LYSO crystals is read out on both ends: on the outer end each crystal is directly coupled to a SiPM, while on the inner end groups of several (e.g., 16 or 32) crystals are indirectly coupled to only 1 or 2 SiPMs, through the use of intermediate wavelength-shifters (WLS). This results in a simpler and less expensive readout when compared to the typical dual-ended readout method. Also, less components on the inner side of the ring allow more proximity to the FoV and thus increased sensitivity. Proof-of-concept studies were performed on a single detector cell with electronic collimation. The amount of light detected on each side of the crystal and the achievable DOI resolution with drimPET were measured, as well as the energy resolution, for different positions of a $22 \mathrm{Na}$ source along the crystal depth. The single cell was composed of a LYSO crystal, initially with both ends read by SiPMs and afterwards applying the drimPET method. Experimental studies and GATE simulations of drimPET carried out to evaluate the feasibility of applying the method in full detector rings for a small animal PET system, considering different number and dimension of the wavelength-shifting fibers, will be presented.

This work was partially supported by project POCI-01-0145-FEDER-016855 and PTDC/BBB-IMG/4909/2014, and UID/CTM/50025/2013, through COMPETE, FEDER and FCT (Lisbon) programs. P. M. M. Correia was supported by FCT (Lisbon) grant PD/BD/52330/2013.

\author{
M13B-10: Delay Grid Multiplexing: Light Sharing Capable and Scalable Time-Based Positioning Method \\ $\underline{\text { J. Y. Won }}^{1,2}$, G. B. Ko ${ }^{1,2}$, J. S. Lee ${ }^{1,2}$ \\ ${ }^{I}$ Dept. of Nuclear Medicine, Seoul National University, Seoul, South Korea \\ ${ }^{2}$ Dept. of Biomedical Sciences, Seoul National University, Seoul, South Korea
}

Delay grid multiplexing is the scalable and light sharing capable time-based positioning method. The delay grid consists of traces on a printed circuit board and connects every signal pin of SiPM into four time pickoff channels. The gamma-ray interaction position is identified using the innate and uniquely determined transit times from silicon photomultipliers (SiPMs) to four time pickoff channels. The aim of this study is to verify the scalability and light sharing capability of delay grid multiplexing.

To verify the scalability, we assembled two scintillation detectors with the multiplexing ratios of $16: 4$ and $64: 4$. The $4 \times 4$ and $8 \times 8$ LGSO crystals, each with the dimension of $3 \times 3 \times 20 \mathrm{~mm}^{3}$, were one-to-one coupled with a single and four SiPM arrays (S11064050P, Hamamatsu Photonics). To verify the light sharing capability, we built a scintillation detector that consisted of $5 \times 5$ LGSO crystals coupled with a single SiPM array. The multiplexing ratio was 25:4. The same crystals and SiPM array model were used here as the scalability test. The LGSO/SiPMs were mounted on the delay grid board where four corner nodes of delay grid were fed into the time pickoff device. We employed a 5-GSPS waveform sampler (DT5742B, CAEN) for data acquisition. The sampled waveforms were interpolated and constant fraction discrimination method was applied to interpolated waveforms to pickoff corner node arrival times.

In the flood maps generated using transit time differences, all the crystals were clearly resolved for one-to-one coupled $4 \times 4,8 \times 8$, and light shared $5 \times 5$ crystals. Energy resolutions were $8.0 \pm 0.3,11.4 \pm 0.7$, and $12.7 \pm 0.7 \%$, respectively. CRTs were $314 \pm 21$, $431 \pm 21$, and $439 \pm 36$ ps FWHM, respectively. Energy resolutions and CRTs were uniform because of the minimal signal distortion from a multiplexing circuit.

As the future work, we will replace waveform sampler with time-to-digital converter (TDC) implemented in a FPGA to simplify the readout device.

M13B-11: An Investigation of Crystal Surface Treatment on Timing and DOI Resolution of SiPM Based DualEnded Readout TOF-DOI PET Detector: an Experimental and Optical Simulation Study

H.-G. Kang ${ }^{1}$, S. H. Song ${ }^{1}$, K. M. Kim², Y. B. Han ${ }^{3}$, S. J. Hong ${ }^{3}$

${ }^{I}$ Department of Senior Healthcare, Eulji University, Daejeon, Korea

${ }^{2}$ Molecular Imaging research center, Korea Institute of Radiological and Medical science, Seoul, Korea

${ }^{3}$ Department of Radiological Science, Eulji University, Seongnam, Gyeonggi, Korea 
In the previous work, we developed a silicon photo-multiplier (SiPM) based dual-ended read scintillation detector for time-offlight (TOF) and depth-of-interaction (DOI) positron-emission-tomography (PET). In this paper, we present the effects of LYSO crystal surface treatment on timing and DOI resolution to optimize the performance of the dual-ended readout detector. GATEv6.2 optical simulation was performed to estimate the effect of surface roughness (salpha) on the light output and DOI resolution of the dual-ended readout detector. We irradiated two $511 \mathrm{keV}$ annihilation gamma rays (beam divergence angle $\left.=2.09^{\circ}\right)$ using a $22 \mathrm{Na}$ point source to the LYSO scintillating crystal $(2.9 \times 2.9 \times 20 \mathrm{~mm} 3)$, each end surface of which was coupled to SiPM. Energy, timing and DOI resolution were measured with a $4 \mathrm{~mm}$ DOI step with different crystal surface finish and cross section. When the crystal surface was treated as a saw-cut, the light output increased about $24 \%$ higher as compared to the polished crystal. Consequently, the coincidence timing resolution and DOI resolution could be improved significantly by using a saw-cut crystal $(365 \pm 27 \mathrm{ps}, 2.57 \pm 0.33 \mathrm{~mm})$ as compared to the polished crystal $(393 \pm 10 \mathrm{ps}, 14.35 \pm 0.96 \mathrm{~mm})$ for the LYSO crystal dimension of $2.9 \times 2.9 \times 20 \mathrm{~mm} 3$. Although, the small cross section of crystal $(2.0 \times 2.0 \mathrm{~mm} 2)$ could improve the DOI resolution $(2.22 \pm 0.24 \mathrm{~mm})$ about $0.25 \mathrm{~mm}$, the coincidence timing resolution was degraded (441 $\pm 52 \mathrm{ps})$ about $76 \mathrm{ps}$. The GATEv6.2 optical simulation could be used to model and optimize the scintillation detector performance. The DOI resolution $(2.30 \pm 0.36 \mathrm{~mm})$ obtained by GATEv6.2 optical simulation agreed with the experimental results $(2.57 \pm 0.33 \mathrm{~mm})$. The proposed dual-ended readout TOF-DOI PET detector can be used for brain or breast dedicated PET.

This research was supported by the National R\&D program through the Korea Institute of Radiological and Medical Science funded by the Ministry of Science, ICT\&Future Planning. (No.1711021927)

\author{
M13B-12: PET Detector Using a Ceramic Scintillator Array (GLuGAG:Ce) Coupled with Digital SiPMs

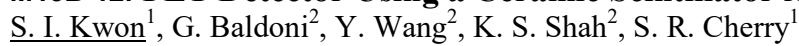 \\ ${ }^{I}$ University of California, Davis, Davis, CA, USA \\ ${ }^{2}$ Radiation Monitoring Devices, Inc., Watertown, MA, USA
}

We have developed a novel rare earth garnet ceramic scintillator $\left(\mathrm{Gd}_{\mathrm{x}} \mathrm{Lu}_{1-\mathrm{x}}\right)_{3}\left(\mathrm{Ga}_{\mathrm{y}} \mathrm{Al}_{1-\mathrm{y}}\right)_{5} \mathrm{O}_{12}: \mathrm{Ce}($ abbreviated as GLuGAG:Ce). Since GLuGAG:Ce has a cubic crystal structure, it can be fabricated using ceramic techniques. The GLuGAG:Ce ceramic scintillator not only has high stopping power for gamma-rays but also high light yield. It shows good transparency even in thick samples and is not sensitive to moisture. Therefore, it is expected that GLuGAG:Ce ceramic scintillator is a better fit for PET applications than previous ceramic scintillators. In this study, we present the first assembly of a PET detector using GLuGAG:Ce ceramics with a relevant size for clinical PET. The GLuGAG:Ce pixel array consisted of $12 \times 12$ pixels. Each pixel has a size of $2.59 \times 2.59 \times 20 \mathrm{~mm}^{3}$ and is optically separated through enhanced specular reflector film. The array size was $32.06 \times 32.06$ $\mathrm{mm}^{2}$ and the pixel pitch was $2.67 \mathrm{~mm}$. The digital SiPM used in this study was a DPC-3200-22-44-M22 module sensor (PDPC). The array perfectly fits with $4 \times 4$ dies on the digital SiPM tile. There was no additional optical light guide between the digital SiPM array and the array. All 144 GLuGAG:Ce pixels were clearly distinguishable in all flood histograms measured with different neighbor logic settings. Coincidence event rate decreased with the higher neighbor logic settings. Average photopeak amplitude was $1202 \pm 129$ and average energy resolution was $14.5 \pm 1.6 \%$. We successfully fabricated large quantities of GLuGAG:Ce ceramics to build a complete $12 \times 12$ pixel array. GLuGAG:Ce pixels showed fairly uniform performance. The performance of GLuGAG:Ce arrays appear promising for PET applications.

M13B-13: Reconstruction of Spatial Response of Compact Gamma Camera from Flood Field Irradiation Data

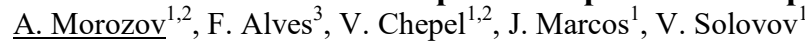

${ }^{1}$ LIP-Coimbra, Coimbra, Portugal

${ }^{2}$ Department of Physics, University of Coimbra, Coimbra, Portugal

${ }^{3}$ ICNAS, University of Coimbra, Coimbra, Portugal

During the last decade development of compact gamma cameras with a square shaped crystal and an array of silicon photomultipliers received much interest. In these cameras reconstruction of scintillation event positions is often performed using statistical algorithms, which require accurate knowledge of the camera spatial response. The response is typically obtained from calibration data recorded by scanning the camera's field of view with a pencil-beam gamma source. An alternative technique, based on iterative procedure and using only flood field irradiation data was already successfully applied for clinical gamma cameras of traditional design, where spatial response of each photosensor can be closely approximated by an axially symmetric function. In this study we show that axially-symmetric light response functions can also be applied for square-shaped compact gamma cameras and the iterative reconstruction of the detector response is a viable option. The feasibility of the approach is demonstrated with Monte Carlo simulations using ANTS2 package and experimentally with a 30x30x2 $\mathrm{mm}^{3}$ camera prototype.

M13B-14: Investigating CeBr3 for Ultra-Fast TOF-PET Detector Designs J.P. Schmall ${ }^{1}$, S. Surti ${ }^{1}$, P. Dokhale ${ }^{2}$, A. Ferri ${ }^{3}$, A. Gola ${ }^{3}$, C. Piemonte ${ }^{3}$, K. Shah ${ }^{2}$, J. S. Karp ${ }^{1}$ 
${ }^{I}$ Radiology, University of Pennsylvania, Philadelphia, USA

${ }^{2}$ Radiation Monitoring Devices, Inc., Watertown, MA, USA

${ }^{3}$ Fondazione Bruno Kessler, Trento, Italy

Here we present results from several productions of Cerium Bromide $(\mathrm{CeBr} 3)$ pixels investigated for use in the design of an ultrafast TOF-PET detector. $\mathrm{CeBr} 3$ has high light output, a short decay time, and a very fast rise time - a combination of properties that should result in excellent timing resolution. Compared to $\mathrm{LSO} / \mathrm{LYSO}, \mathrm{CeBr} 3$ also has the potential to be much less expensive when manufactured in large quantities because of the combination of less expensive raw materials and low melting point, even when considering the additional volume required for similar sensitivity. The $\mathrm{CeBr} 3$ productions were cut into pixels, all with a cross section of $4 \times 4 \mathrm{~mm} 2$ and an assortment of lengths from $3 \mathrm{~mm}$ to $30 \mathrm{~mm}$, and each pixel surface was fully polished. Measurements of rise time and decay time, energy resolution and timing resolution using a PMT readout and also a SiPM readout were performed. The effect of pixel length on the temporal properties of the scintillator emission and timing performance was studied. New productions of $\mathrm{CeBr} 3$ have an improved performance, particularly for longer pixels due to less self-absorption. Coincidence timing resolutions of $69 \mathrm{ps}$ to $126 \mathrm{ps}$ were measured over a range of pixel lengths from $5 \mathrm{~mm}$ to $15 \mathrm{~mm}$ using FBK NUV-HD SiPMs; with a PMT readout the coincidence timing resolutions varied from 96ps to $143 \mathrm{ps}$ over the same range of crystal lengths. Our goal is to use these measurements to determine how best to design a CeBr3-based detector, and these results also serve as a benchmark for evaluating of future advancements in $\mathrm{CeBr} 3$ production.

\author{
M13B-15: Design and Optimization of Direct Conversion Photon-Counting Detector for Dual-Energy CT \\ Imaging \\ Y. Jin 1 , G. Fu ${ }^{1}$, H. Gao ${ }^{2}$, P. M. Edic ${ }^{1}$ \\ ${ }^{I}$ GE Global Research, Niskayuna, NY, USA \\ ${ }^{2}$ GE Healthcare, Waukesha, WI, USA
}

A direct conversion photon-counting detector has great potential in CT imaging and has been intensively studied in recently years. However, the optimization of the detector configuration based on clinically relevant metrics is still lacking. In this study, we developed an optimization scheme for direct conversion photon-counting detector design. The spectral response functions characterizing different detector designs are generated using a Monte Carlo-based approach and evaluated with a dual-energy analysis tool. The results demonstrate the degradation in dose efficiency caused by charge sharing when the detector pixel size is below $\sim 500 \mu \mathrm{m}$. The results also show that thinner sensor material has degraded performance due to the lower absorption efficiency. Based on this analysis, $\sim 500 \mu \mathrm{m}$ pixel size and $2.5 \mathrm{~mm}$ thickness seem to be a good balance between dose efficiency and spatial resolution. The results also show that electronic noise has limited impact on dose efficiency and is not a major risk to the detector development.

Research reported in this publication was supported by the National Institute Of Biomedical Imaging And Bioengineering of the National Institutes of Health under Award Number U01EB017140. The content is solely the responsibility of the authors and does not necessarily represent the official views of the National Institutes of Health.

\author{
M13B-16: Effect of Scintillation Crystal Surface Finish in the Light Sharing TOF PET Detector \\ C. L. Kim, M. Ito, D. L. Mcdaniel \\ Imaging Subsystem, GE Healthcare, Waukesha, Wisconsin, USA
}

The effect of crystal surface finish on PET detector performance has been studied by many researchers. However, most of that research has been done with a single crystal coupled to a single photosensor. In this paper, we studied the effect of crystal surface finishes in crystal arrays. LYSO crystals of $3.95 \mathrm{~mm} \times 5.3 \mathrm{~mm} \times 25 \mathrm{~mm}$ are used to build six crystal arrays. Two $2 \times 3$ crystal arrays were built with all surfaces of all crystals polished, three crystal arrays with one surface of each crystal roughened, and one crystal array with a mixture of all-polished and one-surface-roughened crystals. Since only one surface was lapped on each rectangular crystal, the effect of different orientation of lapped surface was also evaluated. The arrays were directly coupled to SiPM devices and their performance was measured for light collection and timing resolution. Crystals with lapped crystal finish are known to have increased light output. However, it was uncertain if that increased light output would improve timing resolution since spreading of scintillation light over wider photosensor area was expected to degrade timing resolution due to its increased transit time spread. In the measurement, crystal arrays with roughened crystals showed improved light collection of $10 \%$. They also improved the coincidence timing resolution by more than $10 \%$ ( $39 \mathrm{ps}$, from $297 \mathrm{ps}$ to $258 \mathrm{ps}$ ), a much larger effect than predicted by the $10 \%$ increase in light collection. These results demonstrate that, when properly designed, light sharing among photosensors or light spread among crystals does not necessarily imply a degradation in timing resolution and could be designed to improve timing resolution.

M13C: Posters: Application specific

Friday, Nov. $4 \quad$ 10:30-12:00 Etoile 
M13C-1: Development of a Cost-Effective Compton Camera for MeV-Gamma-Ray Imaging Applications Y. Nagao $^{1,2}$, M. Yamaguchi ${ }^{1}$, N. Kawachi ${ }^{1}$, H. Watabe ${ }^{1,2,3}$

${ }^{I}$ Takasaki Advanced Radiation Research Institute, National Institutes for Quantum and Radiological Science and Technology (QST), Takasaki, Gunma, Japan

${ }^{2}$ Graduate School of Biomedical Engineering, Tohoku University, Sendai, Miyagi, Japan

${ }^{3}$ Cyclotron and Radioisotope Center (CYRIC), Tohoku University, Sendai, Miyagi, Japan

We have been studying the applications of semiconductor Compton cameras mainly to the fields of medical imaging. To date, almost all the radioisotopes used in medical imaging emit gamma rays having energies less than or equal to $511 \mathrm{keV}$, such as ${ }^{99 \mathrm{~m}} \mathrm{Tc}(141 \mathrm{keV})$ and ${ }^{18} \mathrm{~F}(511 \mathrm{keV})$. Nowadays, production and use of radioisotopes emitting higher-energy gamma rays are being studied, such as ${ }^{44} \mathrm{Sc}(1157 \mathrm{keV})$ and ${ }^{92} \mathrm{Y}(934 \mathrm{keV})$. Since sensitivity of Compton cameras decrease with increase in gamma-ray energy, higher sensitivity is required to image such radioisotopes. For higher sensitivity with low cost, we developed a Compton camera using thick scintillators instead of thin semiconductor detectors. A conventional circuit for a PET camera was diverted to reduce cost. The camera consisted of two detectors: a scatterer and an absorber. The scintillator material of both the detectors was Ce-doped $\mathrm{Gd}_{3} \mathrm{Al}_{2} \mathrm{Ga}_{3} \mathrm{O}_{12}$ with the density of $6.63 \mathrm{~g} / \mathrm{cm}^{3}$. The thicknesses of the scintillators for the scatterer and the absorber were $5 \mathrm{~mm}$ and $10 \mathrm{~mm}$, respectively. We used a silicon photomultiplier for the scatterer and a flat panel type multianode photomultiplier tube for the absorber. To test imaging capability of the camera, an imaging experiment of a ${ }^{133}$ Ba point source was performed, and the source was successfully imaged. Future research plans include the improvement of energy calibration, optimization of various parameters, and imaging experiments of other radioisotopes emitting higher-energy gamma rays.

M13C-2: Development of a Si-PM Based Intraoperative PET System for Breast Tumor Resection H. Watabe $^{1}$, S. Yamamoto ${ }^{2}$, G. Watanabe ${ }^{1}$

${ }^{I}$ Tohoku University, Sendai, Japan

${ }^{2}$ Nagoya University Graduate School of Medicine, Nagoya, Japan

For an intraoperative evaluation of the spread of breast cancer, functional imaging using such as F-18-FDG during surgery will be a powerful tool. However there is no possible imaging system that can be used during tumor resection. For this purpose, we developed a Si-PM based intraoperative PET system that can be used during tumor resection. The block detector of the PET system consists of a GAGG block optically coupled to a Si-PM array with a $1 \mathrm{~mm}$ thick light guide. The GAGG block used $1.4 \mathrm{~mm} \times 1.4 \mathrm{~mm} \times 15 \mathrm{~mm}$ pixels arranged in $32 \times 32$ matrix with $0.1 \mathrm{~mm}$ thick BaSO4 as a reflector. The size of the GAGG block was $48 \mathrm{~mm} \times 48 \mathrm{~mm}$. The Si-PM array used TSV Si-PM arrays (Hamamatsu, S12642-0404PA-50) arranged in $4 \mathrm{x} 4 \mathrm{matrix}$ (16 x 16 channels) with the size of $52 \mathrm{~mm} \times 52 \mathrm{~mm}$. The thickness of the block detector was $\sim 20 \mathrm{~mm}$, so easy to use during surgery. We made two block detectors and arranged them with $5 \mathrm{~cm}$ apart to form a dual head PET system. We could clearly separate all GAGG pixels in 2-dimentional position histogram for $511 \mathrm{keV}$ gamma photons. The energy resolution was $12 \% \mathrm{FWHM}$. The sensitivity was $\sim 2 \%$ and the spatial resolution of the projection image was $1.3 \mathrm{~mm}$ FWHM at center. Since the temperature dependency of the Si-PM arrays were corrected by controlling the supply voltage for the Si-PM with the temperature, the sensitivity was stable from 20?to 45?. We conclude that the developed Si-PM based intraoperative PET system will be promising for imaging of positron radionuclides during breast tumor resection.

M13C-3: Assessment of shielding materials for the add-on PET at different magnetic field strengths of MRI

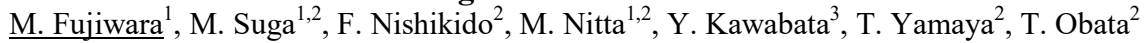

${ }^{\text {I} C h i b a ~ U n i v e s i t y, ~ C h i b a, ~ J a p a n ~}$

${ }^{2}$ National Institute of Radiological Sciences, Chiba, Japan

${ }^{3}$ Takashima Seisakusho Co., Ltd, Tokyo, Japan

We are developing a novel PET/MRI system. In this system, PET detectors are closely located to the MR RF-coil. To reduce the electromagnetic interaction between the PET detectors and the RF-coil, the PET detectors are covered with conductive shield boxes. However, when the magnetic field around the shield box is changed by MRI field gradient pulses, an eddy current is generated in the shield box. The eddy current produces the secondary magnetic field (?B0), resulting in degraded MR image quality. The interference effect may depend on the static magnetic field strength of the MRI. In our previous work, we evaluated the signal-to-noise ratio and the eddy current for several shield materials with a $3 \mathrm{~T}$ clinical MRI system. The results showed that carbon fiber roving had high shielding performance and suppression capacity for the eddy current. In this paper, we evaluated performance of the carbon fiber roving in different static magnetic field strengths of the MRI.

The results showed that carbon fiber roving had high shield performance regardless of the static magnetic field strength. From the experiment using the $3 \mathrm{~T}$ MRI, we clarified that the ?B0 of carbon fiber roving was suppressed in comparison with $\mathrm{Cu}$ foil. The slew rate of the gradient magnetic field of $0.3 \mathrm{~T}$ MRI is lower than that of the $3 \mathrm{~T}$ MRI, therefore, the eddy current and ?B0 of the 0.3 T MRI are lower than those of the 3 T MRI. Thus, we judged that carbon fiber roving was valid as the shield material of shield boxes regardless of magnetic field strength of the MRI. 
M13C-4: Optimization of Pinhole Aperture Size of a Combined MPH/Fan-Beam SPECT System for I-123 DAT Imaging

\author{
$\underline{\text { A. Konik }}^{1}$, J. M. Mukherjee ${ }^{1}$, J. D. Beenhouwer ${ }^{2}$, G. Zubal ${ }^{3}$, M. A. King ${ }^{1}$ \\ ${ }^{I}$ Radiology, Umass Medical School, Worcester, MA, USA \\ ${ }^{2}$ iMinds-Vision Lab, University of Antwerp, Antwerp, Belgium \\ ${ }^{3}$ Z-Concepts LLC, East Haven, CT, USA
}

\begin{abstract}
Introduction: We proposed an inexpensive method to improve the performance of the conventional dual-camera SPECT systems for I-123 dopamine transporter (DAT) imaging for Parkinson Disease. In this method, one of the collimators is replaced with a specifically designed multi-pinhole (MPH) collimator, thus performing combined MPH/fan-beam acquisition. The MPH consists of 9 pinholes focusing to the central brain and covering a cylindrical field of view (diameter: $12 \mathrm{~cm}$ and height: $8 \mathrm{~cm}$ ), which includes the striatum. We present here our preliminary simulation work investigating the optimal aperture size for the striatum activity estimation task. Methods: Projections of the XCAT brain phantom were obtained for a range of aperture sizes. Multiple noise realizations were simulated for each aperture size at realistic count levels. Reconstructions from MPH, fan-beam and combined MPH/fan-beam systems were obtained for various numbers of iterations. The activities were estimated for a range of volumes of interest (VOI) within the striatum. Results: Plots for the normalized root mean square errors (NRMSE) were obtained for the MPH, fan-beam and combined MPH/fan-beam reconstructions at different iterations and VOIs. Conclusion: Our preliminary results suggest that a larger aperture and thus higher sensitivity for the MPH component yields better quantitative estimations for the MPH and combined MPH/fan-beam reconstructions. We will continue our investigation with calculations of the striatum binding ratio (SBR) using clinical software.
\end{abstract}

M13C-5: Performance Evaluation of the MindView PET Using GATE and STIR

$\underline{\text { S. Sanchez }}{ }^{1}$, E. Preziosi ${ }^{2,3}$, C. Correcher ${ }^{4}$, A. J. Gonzalez ${ }^{1}$, P. Conde ${ }^{1}$, A. Gonzalez-Montoro ${ }^{1}$, A. Aguilar ${ }^{1}$, M. J. RodriguezAlvarez $^{1}$, A. Iborra ${ }^{1}$, P. Bellido ${ }^{1}$, D. Grau-Ruiz ${ }^{1}$, E. Diaz-Caballero ${ }^{1}$, M. Seimetz ${ }^{1}$, L. Moliner ${ }^{1}$, F. Sánchez ${ }^{1}$, M. Bettiol ${ }^{5}$, C. Borrazzo ${ }^{2,6}$, A. Soriano ${ }^{1}$, J. P. Rigla ${ }^{1}$, J. J. Garcia-Garrigos ${ }^{1}$, R. Pani ${ }^{7}$, J. M. Benlloch ${ }^{1}$

${ }^{I}$ Institute for Instrumentation in Molecular imaging (I3M), 46022 - Valencia, Spain

${ }^{2}$ SAIMLAL Department, Morphofunctional Sciences - Biophysics, Rome, Italy

${ }^{3}$ Department of Molecular Medicine, Sapienza University of Rome, Rome, Italy

${ }^{4}$ Oncovision (GEM Imaging S.A.), Valencia, Spain

${ }^{5}$ Department of Medico-Surgical, Campus Bio-Medico University, Rome, Italy

${ }^{6}$ Postgraduate School of Medical Physics, Sapienza University of Rome, Rome, Italy

${ }^{7}$ Department of Medico-Surgical Sciences and Biotechnologies, Sapienza University of Rome, Rome, Italy

A series of small size ${ }^{22} \mathrm{Na}$ point sources have been simulated using GATE following the NEMA protocol and applied to the geometry of the brain dedicated PET so-called MindView. The simulated scanner geometry is composed by one ring of 20 detector blocks with a distance of about $330 \mathrm{~mm}$ between opposite detectors. Each detector module includes a monolithic LYSO crystal of $50 \times 50 \times 20 \mathrm{~mm}^{3}$ and a custom $12 \times 12 \mathrm{SiPM}$ array (TSV-type, SensL). The system defines an axial and transaxial FOVs of about $50 \mathrm{~mm}$ and about $240 \mathrm{~mm}$, respectively. Three different approaches for the determination of the impact coordinates within the crystal blocks have been considered. A first approach makes use of the center of gravity (CoG) calculation for the planar XY coordinates but the photon impact depth of interaction (DOI) is not considered. The other methods obtain the XY coordinates using the Raise to Power algorithm with both discrete (second method) and continuous (third one) DoI information. The impacts positions within the crystal blocks were computed from the scintillation light distributions obtained via a Monte Carlo simulation in GEANT4. The data is inserted on the STIR platform for reconstruction using a filtered back projection 3D re-projected algorithm (FBP3DRP). Alternatively to this analytical approach, iterative reconstructions have been used for comparison, such as List Mode (event-by-event) using Tubes of Response, and MLEM implemented on GPUs. We observed a clear improvement of the results both at the CFOV and at one fourth, when the DOI capabilities are enabled. The best performance was obtained with the third method, ranging from $1.4 \mathrm{~mm}$ for the radial component at the center of the FOV to 4 $\mathrm{mm}$ at $10 \mathrm{~cm}$ radial offset. However, very similar results were obtained for the discrete DOI method.

\title{
M13C-6: Contrast Recovery Performance of a $1 \mathrm{~mm}^{3}$ Resolution Clinical PET System
}

D. F. C. Hsu ${ }^{1}$, D. L. Freese ${ }^{1}$, D. R. Innes ${ }^{2}$, C. S. Levin ${ }^{1,2,3,4}$

${ }^{I}$ Electrical Engineering, Stanford University, Stanford, CA, USA

${ }^{2}$ Radiology, Stanford University, Stanford, CA, USA

${ }^{3}$ Physics, Stanford University, Stanford, CA, USA

${ }^{4}$ Bioengineering, Stanford University, Stanford, CA, USA

We are constructing the world's first $1 \mathrm{~mm}^{3}$ resolution clinical PET system dedicated to locoregional imaging (e.g. breast, head/neck, etc). The edge-on layout of densely-packed $8 \times 8$ arrays of $0.9 \times 0.9 \times 1 \mathrm{~mm}^{3}$ LYSO crystal elements provides high sensitivity and the capability to position the 3-D coordinates of one or more interactions per $511 \mathrm{keV}$ photon event. Contrast 
recovery (CR), which measures the accuracy of focal tracer accumulation in the field-of-view (FOV), must be evaluated in order to understand the ability of this system to visualize and quantify smaller lesions. The constructed dual-panel system prototype has a sensitive area of $160 \mathrm{~mm}$ by $33 \mathrm{~mm}$, separated by $64 \mathrm{~mm}$. CR as a function of activity volume was evaluated with the different rod sizes of a micro-Derenzo phantom, yielding CR coefficients of 1.00, 0.96, 1.00, 0.84, 0.67, 0.55 for rod sizes of 4.8, 4.0, 3.2, $2.4,1.8$, and $1.2 \mathrm{~mm}$ respectively. CR as a function of position was evaluated using capillary tubes with a 20:1 activity to warm background concentration ratio, positioned at different positions inside the FOV. Measured contrast between activity and background ranged from 1.63 at the center of the FOV, to 1.48 at $5 \mathrm{~cm}$ away from the center of the FOV. Finally, contrast as a function of activity concentration was evaluated using capillary tubes with different activity to warm background concentration ratios 1000:1, 2000:1, and 4000:1, resulting in measured contrast of 4.86, 13.00, and 34.52 respectively. These results show the outstanding performance of our $1 \mathrm{~mm}^{3}$ resolution locoregional clinical PET imaging system at recovering extremely small contrast structures with realistic background uptake values.

\title{
M13C-7: Hardware Parameter Optimization for a $1 \mathrm{~mm}^{3}$ Resolution Clinical PET System
} D. F. C. Hsu ${ }^{1}$, D. L. Freese ${ }^{1}$, D. R. Innes ${ }^{2}$, C. S. Levin ${ }^{1,2,3,4}$

${ }^{I}$ Electrical Engineering, Stanford University, Stanford, CA, USA

${ }^{2}$ Radiology, Stanford University, Stanford, CA, USA

${ }^{3}$ Physics, Stanford University, Stanford, CA, USA

${ }^{4}$ Bioengineering, Stanford University, Stanford, CA, USA

We are constructing a dual-panel PET system dedicated to locoregional imaging (e.g. breast, head/neck, etc). The modular data acquisition electronics uses the RENA-3 application specific integrated circuit (ASIC) to read out position-sensitive avalanche photodiodes (PSAPDs). The RENA-3's settings must be optimized to extract the best timing resolution and ADC dynamic range mapping performance. In the constructed dual-panel system prototype, which has a sensitive area of $160 \mathrm{~mm}$ by $33 \mathrm{~mm}$ by $64 \mathrm{~mm}$, two PSAPDs are replaced by a board that injects synchronized charge pulses into the readout system to simulate coincident PSAPD hits. A capacitor divider network then splits the charge pulse into a saturated timing trigger channel and an energy channel. We tested the effects of different timing to energy charge division ratios, as well as data acquisition trigger thresholds, on timing resolution and ADC dynamic range. Using a high charge division ratio of 5:100,000 achieved timing resolution improvements of $28 \%$ at $100 \mathrm{keV}, 13 \%$ at $200 \mathrm{keV}$, and $9 \%$ at $300 \mathrm{keV}$, as well as a $30 \%$ improvement in ADC dynamic range usage in comparison to a 27:100 ratio. The measured timing jitter was $4.06 \mathrm{~ns}, 2.61 \mathrm{~ns}$, and $2.33 \mathrm{~ns}$ FWHM for input pulse energies higher than $300 \mathrm{keV}$ when using trigger acquisition thresholds of 40, 65, and $100 \mathrm{keV}$, respectively.

M13C-8: Performances Evaluation of an Intraoperative Positron Imaging Probe with Radioactive Phantoms S. Spadola ${ }^{1}$, C. Esnault ${ }^{1}$, L. Pinot ${ }^{1}$, M.-A. Verdier ${ }^{2}$, N. Dinu ${ }^{3}$, B. Y. Ky ${ }^{3}$, D. Breton ${ }^{3}$, Y. Charon ${ }^{2}$, M.-A. Duval ${ }^{1} \underline{\text { L. Menard }}^{2}$ ${ }^{1}$ Univ. Paris Sud et Paris Diderot, IMNC CNRS/IN2P3, ORSAY, France

${ }^{2}$ Sorbonne Paris Citï ${ }^{1} / 2$, IMNC CNRS/IN2P3, Univ. Paris-Diderot, ORSAY, France

${ }^{3}$ LAL CNRS/IN2P3, LAL CNRS/IN2P3, ORSAY, France

Precise localization of residual tumor after surgical resection of gross tumor mass is a crucial information to improve patients survival and quality of life. Alongside radioguided surgery using tumor-seeking agents labeled with medium and high-energy gamma rays emitters, in situ detection of short range particles, such as positrons, is currently under development to further improve the precise delineation of tumor extend and the post-operative control of the surgical cavity. In that context, we developed a new intraoperative positron imaging probe specifically dedicated to the assessment of tumor resection margins. To achieve high compactness, the detector consists of a very thin p-terphenyl organic scintillator coupled to a SiPM array. This optimized configuration offers a passive rejection of the background noise coming from the annihilation gamma rays and high beta sensitivity and spatial performances (submillimetric spatial resolution and bias). The detection head and the dedicated readout electronics are held in a compact mechanical housing. A temperature-compensated circuit was also implemented to accurately offset the influence of temperature variation on SiPM performances. The positron sensitivity measured with $18 \mathrm{~F}-\mathrm{FDG}$ sources is 305 counts $/ \mathrm{s} / \mathrm{kBq}$. The spatial resolution ranges from $1.08 \mathrm{~mm}$ FWHM to $1.31 \mathrm{~mm}$ FWHM as the source depth increased from 0 to $0.5 \mathrm{~mm}$. The ability of the probe to detect small radiolabeled tumors was quantified with 18F-FDG radioactive phantoms. We showed that the high sensitivity and passive ability of the probe to reject background noise allow to confidently localizing residual tumors as small as $3 \mathrm{~mm}$ for realistic radiotracer uptakes and acquisition times compatible with the surgery duration (less than $10 \mathrm{~s}$ ). Detailed description of the positron imaging probe as well as complete characterization of its performances with realistic phantoms will be presented at the conference.

\author{
M13C-9: Performance Characteristics of Position Sensitive Sparse Sensor (PS3) PET Detectors for Organ \\ Specific PET Systems \\ R. S. Miyaoka, W. C. Hunter, D. Q. DeWitt \\ Radiology/Nuclear Medicine, University of Washington, Seattle, WA, USA
}


Objectives: We investigate performance characteristics of PET detector modules constructed using position sensitive sparse sensor (PS3) arrays. Our overall goal is to develop lower cost, high spatial resolution PET detector modules for neuro-PET imaging.

Methods: We investigated performance of a 14x14 BGO crystal array readout by a $5 \times 5$ element PS3 array (sparsity factor of $\sim 3$ ). The crystal array consisted of $2 \times 2 \times 10 \mathrm{~mm}^{\wedge} 3$ discrete crystals. We also investigated performance of a $15 \times 15$ LYSO crystal array readout by a $4 \times 4$ element PS3 array (i.e., sparsity factor of $>4.8$ ). The crystal array consisted of $1.93 \times 1.93 \times 20 \mathrm{~mm}^{\wedge} 3$ discrete $^{2}$ crystals. For the LYSO crystal array we also explored a dual-sided readout design that enables depth of interaction positioning. A PS3 array consists of a sparse arrangement of silicon photomultiplier (SiPM) dies coupled to a custom light guide and a reflective mask. SiPM dies with $3 \times 3 \mathrm{~mm}^{\wedge} 2$ active area were used. Crystal-of-interaction decoding and energy resolution was assessed using a flood source. Depth of interaction decoding was determined using a collimated point flux stepped along the length of the crystal array.

Results: Initial experimental results indicate excellent crystal decoding performance for the LYSO crystal array. All crystals are easily visualized except along the edge of the array. To improve edge crystal identification, we are presently exploring light guide modifications that help to reduce light spread from edge crystals. Decoding of the BGO crystal array was more challenging; however, most crystals are visible even along the edge of the crystal array. For both BGO and LYSO detector modules there was only about a $33 \%$ difference in light collection efficiency between crystals directly above a sensor and crystals in between sensors.

Conclusions: Using PS3 arrays is a cost effective method to fabricate high spatial resolution PET detectors for human brain and other organ specific imaging systems.

M13C-10: Development of the Second "add-on PET" Prototype: a Head Coil with DOI-PET Detectors for MRI

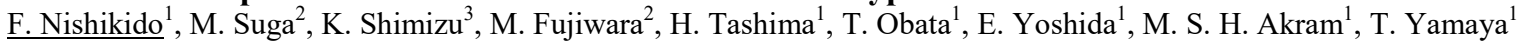

${ }^{I}$ National Institute of Radiological Sciences, Chiba, Japan

${ }^{2}$ Chiba University, Chiba, Japan

${ }^{3}$ Hamamatsu Photonics K. K., Hamamatsu, Japan

We are developing a PET/MRI system based on 4-layered depth-of-interaction (DOI) detectors integrated with a birdcage RFcoil. The PET detectors are placed close to the objective to achieve both high sensitivity and high spatial resolution even at the edge of the field of view (FOV). In a previous study, we reported the first prototype which had a single detector ring to show a proof-of-concept and lower than $1.6 \mathrm{~mm}$ spatial resolution was achieved. However, the axial FOV of the prototype was only 1 $\mathrm{cm}$, which could not be extended due to the size of the readout circuit boards. In this paper, we finally constructed a new RF-coil for the second prototype, which is combined with 24 DOI-PET detectors. We evaluated performance in simultaneous measurements. The second prototype consisted of an RF-coil and 24 PET detector units with two PET detectors each. The detector units were shielded with carbon fiber shielding boxes. Each PET detector consisted of a 14 x 14 x 4-layer array of lutetium fine silicate (LFS) crystals (1.9 mm x $1.9 \mathrm{~mm}$ x $4.0 \mathrm{~mm}$ ), an 8 x 8 multi-pixel photon counter (MPPC, S12641PA-050) array and a readout circuit board with an ASIC. The RF coil was dedicated to a 3T MRI (MAGNETOM Verio, Siemens). We conducted performance tests of the second prototype in simultaneous measurements. As a result, the influence of the MRI measurements on the PET performance was found to be negligible. We expected that imaging performance of the PET system also would not be degraded by the MRI measurements. In addition, the degradation of the signal-to-noise ratio of the magnitude images and eddy current effect were reduced, compared with the previous prototype system. Now, we are evaluating imaging performance of the PET system using PET phantoms and will present results of the imaging performance of the second prototype at the conference.

M13C-11: Detector Size and Geometry Optimization for the Helmet-Chin PET

A. M. Ahmed, H. Tashima, E. Yoshida, T. Yamaya

National Institute of Radiological Sciences, Chiba, Japan

High sensitivity, high spatial resolution and low cost dedicated brain PET scanners are required for early detection of Alzheimer's disease and brain function studies. As an optimal geometry, we proposed and developed the first prototype of the helmet-chin PET scanner. The scanner was constructed from 4-layered DOI detectors constructed from GSO scintillators which were originally developed for the OpenPET. The helmet part of the helmet-chin PET consisted of three ring detectors with different radii arranged in a hemisphere and a top cover. In this study, for our next development, we optimized the size of the detectors to be arranged on the helmet, and compared two types of geometrical arrangements of the detectors on the hemisphere: a spherical arrangement (geom1) in which the centers of the detectors face towards the center of the hemisphere, and a multi-ring arrangement (geom2) which has a similar detector arrangement to that of the first prototype. Geant 4 simulation toolkit was used to model the scanner. The simulated scanners were constructed from LYSO scintillator crystals with a size of $1 \times 1 \times 5$ $\mathrm{mm}^{3}$ (transaxial $\times$ axial $\times \mathrm{DOI}$ ). A dead-space of $2 \mathrm{~mm}$ was assumed in the axial and transaxial directions of the detector such as for wrapping. While fixing the number of the DOI layers to 4 , the size of the detectors was varied by changing the number of the crystals in the transaxial and axial directions. The results showed that, for the helmet detector, a detector with a size of $42 \times 42 \times 20$ $\mathrm{mm}^{3}$ had the highest sensitivity evaluated using a hemispherical phantom. Then, the helmet-chin PET was modeled based on a 
detector whose size is $42 \times 42 \times 20 \mathrm{~mm}^{3}$ with the two geometries (geom1 and geom2). The sensitivities of geom1 and geom2 were compared. For a given number of detectors, the respective sensitivities for geom 1 and geom 2 were $3.4 \%$ and $2.9 \%$ for a hemispherical phantom, and geom1 had higher sensitivity for the point source placed at several positions in the FOV.

M13C-12: Bayesian Tissue Decomposition Method for Spectral Mammography

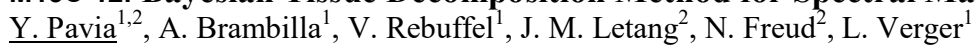

${ }^{I}$ CEA LETI, Grenoble, France

${ }^{2}$ CREATIS, Lyon, France

Breast density evaluation is an active topic in the breast imaging field. This can be accurately measured separating fibroglandular tissue from adipose tissue using material decomposition techniques. In this work, we present a Bayesian approach, based on a Poisson maximum likelihood material decomposition method and that includes an adjustable prior, known from the compressed breast thickness during a screening exam. Since some density variations in biological tissues may appear and the measured compressed thickness is not perfectly known, the proposed method moderates the prior upon the confidence on the thickness value. This work on spectral mammography has been carried out with energy-sensitive photon counting detectors that allow multi-measurements from a single acquisition unlike dual-energy digital mammography. The proposed approach has been benchmarked with a maximum likelihood method through a simulation process that included a detector response function of 60 $\mu \mathrm{m} \mathrm{CdTe} X$-ray detector. Our method has been investigated in severe conditions as in contrast-enhanced mammography. Hence, a 3-material decomposition base has been set up, made of PMMA, water (to mimic adipose and fibroglandular tissues) and iodine. A phantom mimicking breast densities ranging from $10 \%$ to $100 \%$ with an iodine concentration set to $7 \mathrm{mg} / \mathrm{mL}$ was used as test case. In our specific conditions ( $49 \mathrm{kVp}$ tungsten anode, $5 \mathrm{mAs}$, mean glandular dose $=0.93 \mathrm{mGy}, \mathrm{ROI}=1.04 \mathrm{~mm}^{2}$ ), the Bayesian approach reduced bias and noise on both breast density and iodine concentrations. Finally, the error on iodine concentration was below $0.1 \mathrm{mg} / \mathrm{mL}$ and around $5 \%$ on breast density, that is 1.8 times lower compared to standard maximum likelihood approach.

\author{
M13C-13: Design of Wireless Dual-Energy Dual-Source Versatile Pediatric Imaging System Based on CMOS \\ Flat-Panel Detectors \\ Y. Qi, Z. Zhou, Y. Wang, J. Chu, Z. Li, K. Wang \\ Sun Yat-sen University, Guangzhou, Guangdong, China
}

Dose reduction is particularly important for pediatric imaging because children are more susceptible and sensitive to radiation exposure. This work reports on a design of a dual-energy dual-source versatile pediatric imaging system based on sensitive CMOS flat-panel detectors. To improve flexibility and usability, a fully-wireless control and data transfer platform based on WiFi technology was implemented. We also performed a set of post-processing scheme including Laplacian of Gaussian operator and contrast enhancement with reduced noise to process images taken by the CMOS detector at a lower dose. In addition, a dualenergy subtraction technique was used to demonstrate its capability in dual-energy imaging. It is found that such a system is promising to perform imaging such as digital radiography (DR), dual-energy imaging, tomosynthesis, and even computed tomography $(\mathrm{CT})$ dedicated for pediatric patients at potentially lower doses.

\title{
M13C-14: First Clinical Tests of an Intra-Operative ?eta- Detecting Probe for Radio-Guided Surgery in
} Tumour Resection

E. Solfaroli ${ }^{1,2}$, C. Mancini Terracciano ${ }^{1,3}$, V. Bocci ${ }^{1}$, M. Colandrea ${ }^{4}$, F. Collamati ${ }^{1,3}$, M. Cremonesi ${ }^{4}$, R. Donnarumma ${ }^{2}$, M. E. Ferrari ${ }^{4}$, P. Ferroli ${ }^{5}$, F. Ghielmetti ${ }^{5}$, C. M. Grana ${ }^{4}$, M. Marafini ${ }^{1,6}$, S. Morganti ${ }^{1}$, M. Patanè ${ }^{5}$, G. Pedroli ${ }^{4}$, B. Pollo $^{4}$, L. Recchia $^{1}$, A. Russomando ${ }^{1,2,8}$, M. Toppi $^{7}$, G. Traini ${ }^{1,2}$, R. Faccini $^{1,2}$, M. Schiariti $^{5}$

${ }^{I}$ INFN Sezione di Romal, Roma, Italy

${ }^{2}$ Dip. Fisica, Sapienza Univ. di Roma, Roma, Italy

${ }^{3}$ Dip. Scienze di Base e Applicate per l'Ingegneria, Sapienza Univ. di Roma, Roma, Italy

${ }^{4}$ Istituto Europeo di Oncologia, Milano, Italy

${ }^{5}$ Istituto Neurologico Carlo Besta, Milano, Italy

${ }^{6}$ Museo Storico della Fisica e Centro Studi e Ricerche "E. Fermi", Roma, Italy

${ }^{7}$ Laboratori Nazionali di Frascati dellINFN, Frascati, Italy

${ }^{8}$ Center for Life Nano Science@ Sapienza, IIT, Roma, Italy

A Radio-Guided Surgery (RGS) technique exploiting ß- emitting radio-tracers has been suggested, to overcome the impact of the large penetration of? radiation. In order to validate this technique, we developed several probe prototypes with a millimetric core of para-terphenyl, a high light yield and low density plastic scintillator. Feasibilities studies on brain tumours -meningioma and glioma- and abdominal neuro-endocrine tumours have been performed with Monte Carlo simulations based on PET and CT images and in laboratory with specific phantoms. These studies considered finite size lesion remnants, embedded in healthy tissue, reproducing the tumor-to-non-tumor activity ratio as expected in a real clinical case. Moreover, we carried out the first ex- 
vivo tests on meningioma patients administered with 90Y-DOTATOC. All these tests validated this technique yielding a large signal on the bulk tumour, a significant signal on residuals of $\sim 0.2 \mathrm{ml}$ and negligible background from healthy tissues. The tests also confirmed that the medical staff exposure is negligible and that among the biological wastes only urine has a significant activity.

M13C-15: Evaluation of Geometrical Arrangements of High Resolution Sensors in PET Probe Configuration $\underline{\text { A. Studen }}^{1,2}$, V. Cindro ${ }^{1}$, N. H. Clinthorne ${ }^{3}$, H. Kagan ${ }^{4}$, C. Lacasta ${ }^{5}$, G. Llosa ${ }^{5}$, M. Mikuž ${ }^{1,2}$, J. F. Oliver ${ }^{3}$, D. Žontar $^{1}$

${ }^{I}$ Experimental Particle Physics, Jožef Stefan Institute, Ljubljana, Slovenia

${ }^{2}$ Faculty of Mathematics and Physics, University of Ljubljana, Ljubljana, Slovenia

${ }^{3}$ Department of Radiology, University of Michigan, Ann Arbor, MI, USA

${ }^{4}$ Department of Physics, Ohio State University, Columbus, OH, USA

${ }^{5}$ IFIC/CSIC-UVEG, Valencia, Spain

The probe arrangement in positron emission tomography (PET) is a combination of a high resolution detector (probe) placed within a whole body PET scanner. In a subset of events one of the annihilation photons is detected in the probe and the other in the scanner ring. The resolution properties of the resulting line of response are dominated by the properties of the high-resolution detector. A study was designed to evaluate impact of probe geometry and layout on the quality of obtained image. The analysis was performed on data collected by the high-resolution PET prototype assembled at the University of Michigan. The prototype consists of two arcs of standard PET detector elements at $50 \mathrm{~cm}$ radius combined with silicon sensors segmented to $1 \mathrm{~mm}^{3}$ at 5.7 $\mathrm{cm}$ radius. A high-resolution Derenzo phantom filled with ${ }^{18} \mathrm{~F}$-flourodeoxyglucose was rotated during a scan. A subset of highresolution data at select angular positions was chosen to mimic limited angular coverage. The image quality was measured as contrast recovery coefficient (CRC) on the largest, $4.8 \mathrm{~mm}$ rod of the Derenzo phantom. A full coverage low-resolution dataset was mixed with high-resolution events in a regularized maximum-likelihood expectation maximization image reconstruction. The data shows a $7 \%$ increase in CRC if high-resolution event portion is $1 \%$, and a $15 \%$ improvement for $5 \%$ portion.

Qualitatively, data combined with a set of perpendicular angular position subsets reproduces the object with more fidelity than a combination with a single angular position. Quantitatively, impact on CRC is similar for both arrangements.

\section{M13C-16: Developing a Method for Estimation of Internal 131I Contamination in Nuclear Medicine Staff Using Imaging with Gamma Cameras in Emergency and Normal Working Situations Using a Home-Made Thyroid Phantom \\ S. MehdizadehNaderi ${ }^{1}$, F. Lotfalizadeh ${ }^{1}$, M. Karimipoorfard ${ }^{1}$, Z. MolaieManesh $^{1}$, R. Faghihi ${ }^{1}$, S. Sina ${ }^{2}$ \\ ${ }^{I}$ Radiation Medicine department, School of Engineeing, Shiraz university, Shiraz, Iran, Shiraz, Iran \\ ${ }^{2}$ Radiation Research center, School of Engineeing,Shiraz university, Shiraz, Iran, Shiraz, Iran}

The purpose of this study is to develop calibration curves for estimation of activity concentrations inside thyroid gland using male, and female thyroid phantoms filled with 131I, and dual-head gamma camera.In this study, the gamma cameras of two hospitals in Shiraz city were calibrated for estimation of radioiodine activity concentration inside the thyroid. For this purpose, a home-made anthropomorphic neck phantom having both male, and female thyroids were used. The thyroid gland of the phantom was filled with $131 \mathrm{I}$ with $0.444 \mathrm{MBq}$, and thyroid scans were performed by the dual head gamma cameras of two of Shiraz hospitals. The thyroid scans were performed weekly for two months until the activity inside the phantom reaches less than 0.04 micro Curie. It should be mentioned that the radioactive decay of iodine, was also considered for estimation of activity concentration at the time of each scan. The procedures were performed for both female, and male phantoms. The calibration curves of the two phantoms were plotted as the count rate registered by gamma camera, versus activity inside the thyroid. To obtain the values of minimum detectable activity of the method, the neck phantom with water-filled thyroids were imaged with both systems and the average counts of the ROI were used as the MDA of each system. Results: According to the results obtained in this study, the calibration factors for male thyroids were found to be 4.06, and 0.82 for the first and the second hospital respectively. Such values were 4.23 , and 0.84 for female phantom. Calibration curves were obtained for gamma cameras to obtain the activity concentration inside the thyroid glands. By the calibration factors, the iodine activities can be obtained inside the thyroids of the nuclear medicine staff working by 131I when they are suspect of internal contamination during their normal works, and during the accidents. Their contamination is estimated by preparing a single gamma camera image of their thyroids.

M13C-17: PET and MRI-Guided Focused Ultrasound Surgery for Neurologic Applications

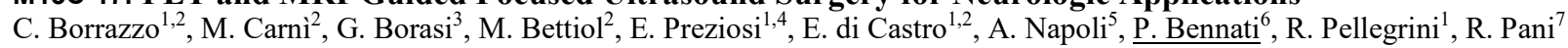

${ }^{I}$ Dept. of Molecular Medicine, Sapienza, Rome, Italy

${ }^{2}$ Medical Physics unit, Policlinico Umberto I, Rome, Italy

${ }^{3}$ Dept. Molecular Bioimaging and Physiology, Bocconi, Milan, Italy

${ }^{4}$ SAIMLAL Dept., Morphofunctional Sciences, Sapienza, Rome, Italy

${ }^{5}$ Dept. Radiological Sciences, Oncology and Anatomical Pathology, Sapienza, Rome, Italy 
${ }^{6}$ School of Technology and health, KTH, Stockholm, Sweden

${ }^{7}$ Dept. Medico-Surgical Sciences and Biotechnologies, Sapienza, Rome, Italy

Magnetic resonance (MR) guided Focused Ultrasound Surgery (MRgFUS) technology combined with a High Intensity Focused Ultrasound (HIFU) beam has opened to new therapeutic protocols for various pathological conditions. The success of this therapy relies on the accuracy of thermal mapping of sonication, which is obtained with MR imaging. Recently, Positron Emission Tomography-Magnetic Resonance Imaging (PET-MRI), hybrid imaging has been developed. It provides both soft tissue morphological imaging and functional imaging. The key concept behind this proposal is the development of a very high resolution and high efficiency brain dedicated PET, able to show cerebral functional signal coming from the tissue undergoing HIFU treatment in real time. The monitoring during the treatment gives the possibility to immediately know the condition of the target tissues, in the search for better diagnose and therefore to a better treatment with ultrasound therapy. The new system is composed of PET-MRI and a customized MRI-compatible focused ultrasound applicator. 18Ffluoromisonidazole (18F-miso) radioactive tracer delineates regions based on a threshold lesion/muscle activity ratio. 18F-miso PET/MRI fused images were used for targeting lesion regions for focused ultrasound ablation. With MRI real-time temperature imaging guidance, PETdetected regions of lesion could be selectively ablated with temperatures $\mathrm{T}>55^{\circ} \mathrm{C}$. PET and MRI guided focused ultrasound surgery of treatment regions is a feasible and innovative therapy for brain diseases that will be potentially useful for preclinical studies using ultrasound.

M13C-18: Motion Correction for 3D PET-MR with High Sensitivity and Resolution G. M. Soultanidis ${ }^{1,2}$, I. Polycarpou ${ }^{1,3}$, P. K. Marsden ${ }^{1}$

${ }^{I}$ Div. of Imaging Sciences and Biomedical Engineering, King's College London, London, United Kingdom

${ }^{2}$ School of Biological, Biomedical and Environmental Sciences, University of Hull, Hull, United Kindom

${ }^{3}$ Department of Health Sciences, European University of Cyprus, Nicosia, Cyprus

Simultaneous PET-MR imaging has recently emerged into clinical and preclinical routine. A promising aspect of MR is the motion caption, which can be used to correct PET motion artefacts. The aim of this study is to evaluate the most common motion correction method, using a high-resolution digital PET insert, and a novel elastic PET-MR phantom. PVA cryogel has been used for motion modeling because of its elastic properties. The phantom consists of a $8 \mathrm{~mm}$ lesion, surrounded by a radioactive background. Motion is transferred with a hydraulic system to the MR room, inside the PET insert. The applied motion is derived by human respiratory motion. This PET insert was equipped with digital silicon photomultipliers and is synchronized with MR for truly simultaneous PET-MR acquisition. The estimated volume of this PVA cryogel phantom does not diverge more than 6.2 $\%$ from the ideal sphere. The reconstructed data are corrected with use of MR derived motion fields. Motion correction restored the lesion intensity, improved volume estimation and restored the lesion to background ratio. In this study we have investigated the effect of motion correction to small animal PET inserts, where the results can be reflected to any investigation under similar circumstances.

This project is supported by SUBLIMA (http://www.sublima-pet-mr.eu), a European Union funded project under the seventh framework program (241711). The presenter has been supported additionally by the Greek State Scholarship Foundation (IKY).

M13C-19: A Multimodal System with Endo-PET/NIRF/visible for Sentinel Lymph Node and Tumor Detection for Laparoscopic Surgery: a Feasibility Study

S. H. Song ${ }^{1}$, H. G. Kang ${ }^{1}$, H.-Y. Lee ${ }^{2}$, K. M. Kim ${ }^{3}$, S. J. Hong ${ }^{1,4}$

${ }^{I}$ Department of Senior Healthcare, Eulji Unversity, Deejon, Korea

${ }^{2}$ Department of Nuclear Medicine, Seoul National University, Seoul, Korea

${ }^{3}$ Molecular Imaging research center, Korea Institute of Radiological and Medical science, Seoul, Korea

${ }^{4}$ Department of Radiological Science, Eulji University, Seongnam, Gyeonggi, Korea

Robotic-assisted or laparoscopic surgery using radio-tracer or fluorescence dye has become a preferred approach for precise detection and efficient dissection of tumor. If the surgeon can operate surgery with a multimodal imaging system that simultaneously provides the gamma, NIR, and visible images around the suspected region, the surgery can become quite efficient with a reduced operation time. We propose a prototype of the endo-PET/NIRF/visible imaging system which provides those images. The endo-PET detector consists of an in-body and an out-body detector. The in-body detector consists of $4 \times 4$ LYSO crystals with a size of $1.5 \times 1.5 \times 7 \mathrm{~mm} 3$. We coupled this crystal array with a SiPM that has $4 \times 4$ channels with a sensitive size of $3 \times 3 \mathrm{~mm} 2$. The out-body detector had an array of $18 \times 17$ crystals with a size of $2.47 \times 2.74 \times 20 \mathrm{~mm} 3$ size and the crystal array was coupled to a H9500 PMT. Coincidence event signals with X-, Y-position information obtained with the Anger-logic were reconstructed into an image by a fast planar reconstruction algorithm using MATLAB 2015a software. To optimize the optical lens system for NIRF and visible lights, Winlens 3D optical simulation software was used. We obtained the image of a $22 \mathrm{Na}$ point source at the center point of the endo-PET detector. Sensitivity and spatial resolution of the endo-PET was 0.34 cps/KBq (12978 counts/374 KBq) and $2.9 \mathrm{~mm}$ respectively. We also obtained an NIRF image using the Winlens 3D optical 
simulation software and a preliminary NIRF image. The prototype showed a feasibility of the endo-PET/NIRF/visible imaging system.

\author{
M13C-20: The Impact of Detector-Detector Sensitivity Variation on a Dedicated Cardiac SPECT Camera \\ $\underline{\text { R. G. Wells }}$ \\ Cardiology, University of Ottawa Heart Institute, Ottawa,ON, Canada
}

Detector-detector sensitivity variation is known to cause image artifacts with traditional cameras. This study evaluates the impact of inter-detector sensitivity variations on cardiac images acquired with a multi-detector cardiac SPECT system. Methods: 15 min of listmode data from a cardiac-torso phantom were acquired on a multi-head pinhole cardiac camera (Discovery NM530c, GE Healthcare). $10 \mathrm{~min}$ of acquisition was used as the baseline for comparison. Listmode data were rebinned to create projection datasets with acquisition times between 5 and $15 \mathrm{~min}$, simulating sensitivity changes from $-50 \%$ to $+50 \%$ of baseline. Variation in the sensitivity of individual detectors was simulated by replacing the corresponding projection in the baseline dataset with that from an acquisition of shorter or longer duration. Also, random distributions of sensitivities were created using a mean duration of $10 \mathrm{~min}$ and a standard deviation (SD) between 5\% and 30\%. For each SD, 10 sets of projection data were generated. The projection data were reconstructed (MLEM, 60 iterations) with and without attenuation correction (AC), converted to polar maps, and compared to baseline using a 17-segment model. The SD of the segments in the normal region of the cardiac image, reconstructed with AC, was used to measure homogeneity. Results: Varying any single projection by up to $50 \%$ caused a change of $<5 \%$ in the uptake measured in individual segments. However, the SD of the detector sensitivities was nearly linearly related to the maximum change in segmental uptake, with a SD of $20 \%$ causing a segmental error of $4 \%$ (5\%) without (with) attenuation correction. The homogeneity of the normal portion of the heart also decreased as a square of the sensitivity SD. Conclusion: Changes in individual-detector sensitivity has only a small effect on the cardiac image. Errors in segmental uptake increase linearly, and homogeneity degrades quadratically, with increasing SD of the detector-detector variations.

M13D: Posters: simulation II

Friday, Nov. $4 \quad$ 10:30-12:00 Etoile M13D-1: Design of a Novel Quantitative Imaging System for Molecular Radiotherapy

$\underline{\text { S. J. Colosimo }}^{1}$, L. J. Harkness-Brennan ${ }^{1}$, D. S. Judson ${ }^{1}$, L. McAreavey ${ }^{1}$, A. J. Boston ${ }^{1}$, H. C. Boston ${ }^{1}$, P. J. Nolan ${ }^{1}$, G. Flux ${ }^{2}$, A. M. Denis-Bacelar ${ }^{2,3}$, M. Carroll ${ }^{4}$, B. Harris ${ }^{5}$, I. Radley

${ }^{1}$ University of Liverpool, Liverpool, $U K$

${ }^{2}$ Royal Marsden Hospital, Sutton, Surrey, UK

${ }^{3}$ The Institute of Cancer Research, London, UK

${ }^{4}$ The Royal Liverpool and Broadgreen University Hospitals, Liverpool, UK

${ }^{5}$ Kromek, Sedgefield, County Durham, UK

We investigated two collimator designs for a CZT-based quantitative SPECT demonstrator. Molecular Radiotherapy (MRT) using I-131 is a well established tool in the treatment of thyroid conditions and cancers. The decay modes of I-131 include emission of ß-particles which deposit locally, whilst ?-rays allow for SPECT imaging to be performed in real time. In order to determine the absorbed dose and therefore efficacy of treatment, real-time dosimetry would ideally be performed [1],. However, limitations in capabilities of standard systems, dead time arising from high count rates (3-10GBq I-131 initial activity) and limited energy resolution have meant that accurately determining the quantity and distribution of the radioisotopes in MRT treatments is not possible. Utilising commercially available Cadmium Zinc Telluride (CZT) detectors as well as custom collimators, we aim to create an imaging system capable of dosimetry for MRT measurements. The CZT detector, supplied by Kromek [2], has been commissioned with count rate capabilities up to $160 \mathrm{kcps}$. The energy resolution has been measured to be $3 \%$ at $356 \mathrm{keV}$, which is superior to that of scintillator detectors commonly used in SPECT imaging $(\sim 10 \%)$. A simulation of the system has been written using GAMOS [3], a GEANT4 framework, and has been experimentally validated. Two collimator types are currently being investigated, one parallel hole and one pinhole. The designs were studied through the parameters of sensitivity and spatial resolution and were optimised for the therapeutic radioisotope I-131. In order to determine the collimator parameters, transmission has been studied. The final collimator designs yielded $\sim 0.04 \%$ transmission at $10 \mathrm{~cm}$ for $364 \mathrm{keV}$, which is within our requirement for the system. The collimator optimisation will be presented as well as initial imaging results.

[1] Glenn D. Flux, Masud Haq, Sarah J. Chittenden, Susan Buckley, Cecilia Hindorf, Kate Newbold, and Clive L. Harmer, "A dose-effect correlation for radioiodine ablation in differentiated thyroid cancer," Eur J Nucl Med Mol Imaging, vol. 37, pp. 270-275, 2010

[2] Kromek, "D-MATRIX", http://www.kromek.com/index.php/medical/medical-technology/czt/dmatrix

[3] P. Arce, P. Rato Mendes, and J.I. Lagares, "GAMOS: a Geant4-based easy and flexible framework for Nuclear Geant4 simulations," IEEE Proc. Nuc. Sci. Symp. Conf. Rec., pp. 3162 - 3168, 2008

M13D-2: Non-Diverging Analytical Expression for the Sensitivity of Converging SPECT Collimators J. van Roosmalen ${ }^{1}$, F. J. Beekman ${ }^{1,2,3}$, M. C. Goorden ${ }^{1}$ 
${ }^{I}$ Section of Radiation, Detection and Medical Imaging, Applied Sciences, Delft University of Technology, Delft, the Netherlands ${ }^{2}$ MILabs B.V., Utrecht, the Netherlands

${ }^{3}$ Department for Translational Neuroscience, Brain Center Rudolf Magnus, University Medical Center Utrecht, Utrecht, the Netherlands

Accurate analytical expressions for sensitivity of astigmatic, fan- or cone-beam collimators are important tools in the optimization of SPECT. Presently known expressions for sensitivity either diverge near the focal point or line(s), or are only valid on the collimator axis. As a result, these expressions are unsuitable to calculate volumetric sensitivity for e.g. short-focal length collimators that focus inside the object to enhance sensitivity. We developed non-diverging sensitivity formulas that are applicable over the full collimator's field-of-view. These are calculated by integrating previously derived collimator response functions over the full detector surface. Contrary to common approximations, the variation of solid angle of pixels over the detector is fully taken into account. We validated our expressions using ray-tracing simulations and found very close agreement: a maximum error of $1.6 \%$ was found which can be attributed to discretization effects in the simulations. We conclude that our new expression is an accurate generalization of previous formulas over the full field-of-view. This may enable the further optimization of collimators, in particular when the focus is close to, or in, the object of interest.

M13D-3: The Use of a Fast Monte Carlo Tool for Dose Verification in Image-Guided Proton Therapy T. V. R. Almeida ${ }^{1,2}$, M. Senzacqua ${ }^{3}$, F. R. Cassetta Jr. ${ }^{2}$, D. Iuso ${ }^{3}$, V. Denyak ${ }^{1}$, M. Riboldi ${ }^{2}$, A. Pella ${ }^{4}$, G. Magro ${ }^{4}$, G. Baroni $^{2,4}$, V. Patera $^{3}$, A. Schiavi ${ }^{3}$

${ }^{I}$ Medical Physics Unit, Faculdades Pequeno Príncipe \& Instituto Pele Pequeno Príncipe, Curitiba, Paraná, Brazil

${ }^{2}$ Dipartimento di Elettronica, Informazione e Bioingegneria, Politecnico di Milano, Milan, Lombardy, Italy

${ }^{3}$ Department of Basic and Applied Sciences for Engineering, Università di Roma "La Sapienza", Roma, Lazio, Italy

${ }^{4}$ Bioengineering Unit, Centro Nazionale di Adroterapia Oncologica, Pavia, Lombardy, Italy

FRED is a fast Monte Carlo (MC) tool, which has been developed for a hybrid CPU+GPU architecture. The tool is able to track proton beams through a voxel 3D reconstruction grid by using simplified physical models and fast-MC algorithms. Moreover, FRED exploits anatomical information from the computerized tomography (CT) by reconstructing and simulating the atomic composition of each crossed tissue. The input/output interfaces have been adapted to the "Centro Nazionale di Adroterapia Oncologica" (CNAO) environment. All results have been checked for efficacy and accuracy in comparison with the generalpurpose full-MC code, FLUKA. The positioning deviations between the treatment planning and the treatment irradiation characterize the residual positioning errors in Image-guided Proton Therapy (IGPT). Usually, an IGPT system is composed by Xray tubes and silicon detectors, producing orthogonal images to be registered with the treatment planning CT, allowing the verification of the treatment isocenter position. In this study, the FRED code was applied for head \& neck treatments in order to compare the dose-volume histogram (DVH) of the original treatment planning versus the DVH simulating the residual positioning errors, relying on a roto-translational matrix description. FRED allows performing a typical plan recalculation within minutes, depending on the number of primaries. Considering treatment dose calculations with 25 million of primaries, using FRED-GPU it was possible to carry out the entire dose calculation in less than 1 min, enabling the analysis of the DVH to judge the suitability of current patient position for actual irradiation.

Acknowledgments to Coordination for the Improvement of Higher Education Personnel (CAPES - Brazil).

\author{
M13D-4: Characterisation of Angular Detection Dependence of Prompt Gamma-Rays with Respect to the \\ Bragg Peak in a Water Phantom Using Proton Beam Irradiations \\ M. Zarifi ${ }^{1}$, S. Guatelli ${ }^{1}$, D. Bolst ${ }^{1}$, B. Hutton ${ }^{2}$, A. Rosenfeld ${ }^{1},{\mathrm{Y} . \mathrm{Qi}^{1}}^{1}$ \\ ${ }^{I}$ Centre for Medical Radiation Physics, University of Wollongong, Wollongong, NSW, Australia \\ ${ }^{2}$ Institute of Nuclear Medicine, University College London, London, UK
}

The rationale for the use of the prompt gamma (PG) signal for in vivo verification of the proton beam range is that the PG falloff distribution along the beam path is correlated with the dose profile in the Bragg peak (BP) distal falloff region. However, this correlation has complicated characteristics. Quantitative characterization of this correlation, particularly with respect to the BP, is of great importance to assess its limitation and helps to design a clinically reliable PG imaging system to maximize the PG detection. In this work we investigate the angular dependence of PG detections with respect to the BP for in vivo beam range verification in proton radiation therapy. Geant4 Monte Carlo simulations have been used to study the energy spectral and spatial characteristics of the PG signal from high-energy proton irradiations. A cylindrical water phantom (F30 cmx50cm) with an ideal detection sensitive cylinder $(\mathrm{F} 100 \mathrm{~cm} \times 50 \mathrm{~cm})$ coaxially surrounding the phantom has been used in the simulation. The angular dependences of PG detection as a function of beam energy and PG energy windows have been characterised with respect to the BP positions. Our preliminary results show that there exists an angular preference for PG detection, which has a strong dependence on the beam energy. As the beam energy increases, the longitudinal angular preferences are forwardly decreased in the lower beam energies of $100-130 \mathrm{MeV}$ but backwardly increased at the higher energies of $140-200 \mathrm{MeV}$, with respect to their 
BP positions. The azimuthal distributions of PG emissions are isotropic regardless of beam energy. The angular preferences also show significant variations with PG energy windows depending on the PG emission sources. At beam energy of $200 \mathrm{MeV}$, the longitudinal angular preferences are $-10^{\circ},-15^{\circ}$ and $-17^{\circ}$ from $\mathrm{PG}$ energy windows of $4.2-4.6 \mathrm{MeV}, 5.0-5.4 \mathrm{MeV}$ and $5.9-6.3 \mathrm{MeV}$, respectively. These results indicate that there is unlikely to be a simple solution to maximise the PG detection.

\section{M13D-5: Time of Flight Impact in Brain Studies}

$\underline{\text { P. Solevi }}^{1}$, L. Caldeira ${ }^{2}$, J. E. Gillam ${ }^{3}$, C. Hoeschen ${ }^{1}$, C. Lerche ${ }^{2}$, I. Torres-Espallardo ${ }^{4}$

${ }^{I}$ Institut für Medizintechnik, Otto-von-Guericke University, Magdeburg, Germany

${ }^{2}$ Institute of Neuroscience and Medicine, Forschungszentrum Jülich GmbH, Jülich, Germany

${ }^{3}$ Brain \& Mind Centre, University of Sydney, Sydney, Australia

${ }^{4}$ Nuevo Hospital La Fe, Valencia, Spain

Time Of Flight (TOF) capability in Positron Emission Tomography (PET) can improve signal to noise ratio of reconstructed images. The impact of TOF at the image level has been extensively studied with timing resolutions down to hundreds of ps, which is the state-of-the-art in existing TOF-PET devices. In the last years, however, sub-100 ps Coincidence Time Resolutions (CTR) have been measured. These new achievements open an interesting field of study shifting the focus of TOF gain from whole-body towards smaller targets. The purpose of the present work is to study - through simulations - the potential impact of TOF in future systems capable of achieving extremely good CTR values, with special focus on brain studies. The Gemini TF PET scanner was simulated by means of GATE and image reconstruction was performed through the Origin Ensemble technique. The Hoffman brain phantom was modelled and acquired according to clinical standard protocols and different figures of merits were studied for CTR values down to $50 \mathrm{ps}$. The reconstructed images were evaluated using NeuroQ, a PET analysis software that register and compare images to reference values from a control group made of 50 normal brain PET studies. The image quality provided by the $50 \mathrm{ps}$ TOF reconstruction was superior to the other images, and reflected in a significant improvement in the correlation with ground truth images.

M13D-6: Scattering proton CT using filtered backprojection along most likely paths C. T. Quiñones, J. M. Létang, S. Rit

Université de Lyon, CREATIS, CNRS UMR5220, Inserm U1206, INSA-Lyon, Université Claude Bernard Lyon 1, Centre Léon Bérard, France, Lyon, France

This work investigates the multiple Coulomb scattering (MCS) of protons to reconstruct the relative scattering power map of the object which is related to the relative radiation length of the material. The exit angular deviation of each proton is binned at different distances from the source by taking into account a curved proton path. The angular variance of each pixel as a function of depth was then converted to the water equivalent path length (WEPL) of the MCS angle. The virtual projections at the center for 360 projection angles at 1 degree intervals were considered for reconstruction by implementing the filtered back-projection (FBP) algorithm. The results on phantoms show that the spatial resolution of scattering proton CT (pCT) is comparable with that of energy-loss pCT in the studied situations. The noise in the reconstructed scattering pCT slices is however much larger for the same dose. While conventional energy-loss pCT already provides the information for range calculations in proton therapy treatment planning, the preliminary results show that scattering $\mathrm{pCT}$ could provide a complementary information about the object.

M13D-7: In Beam Prompt Gamma Proton-Range Monitoring During Proton Therapy G. Lönn $^{1}$, P. Bennati ${ }^{1}$, D. Larsson ${ }^{1}$, A. Dasu ${ }^{2}$, M. Colarieti-Tosti ${ }^{1}$

${ }^{I}$ School of Technology and Health, Royal Institute of Technology, KTH, Stockholm, Huddinge, Sweden

${ }^{2}$ Department of Medical and Health Sciences, Linköping University, LIU, Linköping, Sweden

A knife-edge slit system for prompt gamma imaging of in beam proton range is described and evaluated with GATE Monte Carlo simulations. A reference phase space file storing all outgoing particles from a cylindrical PolyMethylMethAcrylate (PMMA) phantom was acquired for $2 \times 109150 \mathrm{MeV}$ protons and was used as simulation input. Two random slit positions were chosen and simulated 25 times each with individual seeds. In addition, a background profile (knife-edge slit substituted by solid lead block, $10 \mathrm{~cm}$ thick) was simulated at each position. Each of the 50 detector profiles were subtracted by the background profile acquired at the corresponding position. Following subtraction, the profiles were oversampled and fitted with a six segment linear approximation with points selected to minimize the root mean square error. The system was able to resolve the proton range at the two investigated positions with a promising precision of $\pm 1 \mathrm{~mm}$ and $\pm 1.5 \mathrm{~mm}$, and with mean profile range shifts of -12 and $-14 \mathrm{~mm}$, respectively, following prompt gamma production energy thresholds. Future work includes more extensive Monte Carlo simulations and experimental measurements at the Skandion clinic.

M13D-8: Validation of the Poisson Nature of PET Simulations Performed Using GATE E. K. Leung ${ }^{1,2}$, J. Qi $^{1}$, R. D. Badawi ${ }^{1,2}$ 
${ }^{I}$ BIOMEDICAL ENGINEERING, UC DAVIS, DAVIS, UNITED STATES

${ }^{2}$ RADIOLOGY, UC DAVIS MEDICAL CENTER, SACRAMENTO, CA, UNITED STATES

Abstract - A Poisson validation protocol based on reduced chi-square statistics was developed to determine whether Monte Carlo PET simulations produce statistically reliable results. The protocol is based around simulations of an mCT-like detector and on NEMA-like measurements on an mCT-like scanner. Different GATE versions (v7.0 and v7.1) were compared. In particular, GATE v7.1 was compiled with the available GEANT4 BUILD MULTITHREADED $=$ OFF (abbreviated as GATE v7.1s) and = ON (abbreviated as GATE v7.1m). The results suggested that GATE v7.0 produces statistically accurate results, while GATE v7.1 with GEANT4 BUILD MULTITHREADED = ON (not currently recommended by the GATE consortium) results in lower variance than is consistent with the Poisson distribution. We also find that prompt and random coincidence rates have greater variance than would be predicted by Poisson statistics. Further investigation revealed that up to $33 \%$ of prompt and delayed coincidences are classified as multiple coincidences at $50 \mathrm{kBq} / \mathrm{cc}$. Since multiple coincidences are correlated in time, this may explain the non-Poisson variance. This may have implications for noise estimations using NEC methods, especially on large axial FOV scanners where high rates of multiple coincidences are expected.

M13D-9: Influence of Tissue Non-Homogeneities on the Accuracy of 3-D Dose Distribution Monitoring During Gamma-Ray Radiotherapy

M. Miklavec ${ }^{1,2}$, S. Sirca ${ }^{1,3}$, D. Savran ${ }^{4}$, M. Vencelj $^{1}$

${ }^{I} F 2$, Jozef Stefan Institute, Ljubljana, Slovenia

${ }^{2}$ LGA, Higher Education Centre Sezana, Sezana, Slovenia

${ }^{3}$ Faculty of Mathematics and Physics, University of Ljubljana, Ljubljana, Slovenia

${ }^{4}$ GSI Helmholtzzentrum für Schwerionenforschung, Darmstadt, Germany

Delivering precisely the desired dose distribution to the patient during radiotherapy is of crucial importance for treatment success and monitoring the actual distributed dose opens the possibility to correct for deposition inaccuracies and thus achieve better conformance to the treatment plan.

Positron emission tomography has already been successfully used to measure the residual radioactivity from (gamma,n) reactions in the patient after the treatment with hadron therapy or high-energy gamma-ray radiotherapy (above $20 \mathrm{MV}$ ). When trying to use a similar approach for gamma-rays of lower energies - most commonly 6 to $18 \mathrm{MV}$ - the only source of positrons is prompt pair production in the beam, calling for measurements while the linac is operating and saturating the detector. The problems with saturation were alleviated to a formidable extent by using advanced digital processing techniques to measure energies of incident particles at rates beyond 10 Mcps per scintillation crystal.

The approach suggested by [1] builds on the discovery that there is a strong correlation between the delivered dose and the density of pair production. Our research confirmed the findings, but only as long as homogeneous objects were involved.

In an inhomogeneous object, the annihilation density was no longer proportional to the dose, but rather to the density of deposited energy. This means that the intimate knowledge of the material density is required for proper reconstruction of the dose field image from the PET measurement. Additionally, one gets $2-4 \mathrm{~mm}$ thick regions at material boundaries where the charge particle equilibrium gets broken and the correlation no longer holds. This is, in principle, possible to account for based on CT data that is always available in such cases, but definitely calls for further work.

[1] Kormoll, T. et al; 3D in-vivo dosimetry for photon radiotherapy based on pair production, NSS/MIC Conference Record, 2009 IEEE, pp.2969-2975, 2009

\author{
M13D-10: Feasibility of On-line Multiple Scanning Beam Range Verification with PET Imaging: Monte Carlo \\ Simulation Studies \\ Y. Zhong, W. Lu, X. Jia, Y. Shao \\ Radiation Oncology, UT Southwestern Medical Center, Dallas, US
}

We investigate the feasibility for intra-fraction on-line multiple scanning proton beam range verifications (BRVs) with in-situ PET imaging, which is beyond the current single-beam BRV. We simulated various 110-MeV proton beams with 4-mm diameter and different beam-separations irradiating PMMA phantoms by GATE simulation, which generated nuclear interaction-induced positrons which, distributed in either a planar or a curved activity range (AR) profile, were imaged by a PET ( 2 -mm resolution, $10 \%$ sensitivity, 250x250x128 mm^3 FOV) with different acquisition times. We calculated the ARs from reconstructed PET images and compared them with the corresponding ARs of original positron distributions. Preliminary studies show: 1) for two separated beams with overlapped positrons, errors of AR measurement for each beam varied from $0.2 \mathrm{~mm}$ to $2.3 \mathrm{~mm}$ as centerto-center beam separation and AR difference were in the range of 8-12 $\mathrm{mm}$ and 2-8 $\mathrm{mm}$ respectively. It is feasible to achieve $=$ 1.0-mm accuracy for two beams with 100-second PET acquisition and 12-mm beam-separation; 2) for densely distributed beams with a continuous profile of AR distributions, PET image was blurred further at non-planar AR region than planar region which led to worsened AR measurement accuracy at non-planar region. By applying a simple de-convolution based modeling PET resolution response, the accuracy of AR measurement can be slightly improved. Further studies will focus on identifying key 
factors that impact the AR measurement with multi-beam irradiations, such as irradiations with different beam and phantom setups, PET resolution/sensitivity/configuration, image process and enhancement to improve BRVs>

\author{
M13D-11: Montecarlo Simulation to Evaluate Factors Affecting Imaging Performances of Monolithic \\ Scintillation Gamma Camera

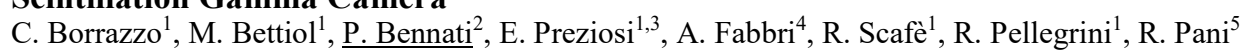 \\ ${ }^{I}$ Dept. of Molecular Medicine, Sapienza, Rome, Italy \\ ${ }^{2}$ School of Technology and health, KTH, Stockholm, Sweden \\ ${ }^{3}$ SAIMLAL Dept. Morphofunctional Sciences, Sapienza, Rome, Italy \\ ${ }^{4}$ INFN Sezione Roma III, Roma Tre, Rome, Italy \\ ${ }^{5}$ Dept. Medico-Surgical Sciences and Biotechnologies, Sapienza, Rome, Italy
}

In gamma cameras based on continuous scintillation crystals, intrinsic Spatial Resolution (SR) is mainly affected by two factors: scintillation light yield/collection capability and overall crystal thickness. The first affects the counting statistics, the latter impacts on the light distribution width. In recent days, a new generation of compact gamma cameras, based on monolithic crystal, becomes increasingly widespread. The main advantages of a small FoV gamma camera with respect to standard one are high portability, low cost and weight, therefore enabling their clinical applications, from scintimammography to intraoperative tumor localization. To fully exploit the potentiality of these devices we should start from the beginning: Monte Carlo simulations can be a valuable tool to physically characterize the imaging systems and to establish a priori reference values. GEANT4 toolkit allows to fully describe the phenomenon of light emission, providing control to all second-order factors present in real systems. In this way, the imaging capabilities of innovative detectors based on new scintillation crystals and solid-state detectors and position sensitive photomultipliers can be assessed and evaluated. We have simulated a set of 50x50 mm2 crystals of different materials, with different refractive index, light yield and surface treatment, coupled to a multi-anode photodetection system. A 64 independent channel electronic readout is simulated. In addition, it was also studied the effect of light distribution enlargement, due to the increasing of optical guide thickness, on the SR. Our results clearly show that SR is dependent from the number of photoelectrons and from the light spread. Furthermore, the role of refraction index was unambiguously identified as an important factor affecting light collection and so SR. Our model makes easy to identify the predominant factors that influence the SR in order to better design a new gamma camera with high imaging performances.

\title{
M13D-12: Implementation of a Lens on GATE Monte-Carlo Software for Optical Imaging Application and Its Validation Using ZEMAX \\ S. J. Hong ${ }^{1}$, H.-G. Kang ${ }^{2}$, S. H. Song ${ }^{2}$, H.-Y. Lee ${ }^{3}$, K. M. Kim ${ }^{4}$ \\ ${ }^{I}$ Department of Radiological Science, Eulji University, Seongnam, Gyeonggi, Korea \\ ${ }^{2}$ Senior Healthcare, Eulji University, Daejeon, Korea \\ ${ }^{3}$ Department of Nuclear Medicine/College of Medicine, Seoul National University, Seoul, Korea \\ ${ }^{4}$ Molecular Imaging research center, Korea Institute of Radiological and Medical science, Seoul, Korea
}

The optical simulation parameters such as Rayleigh scattering coefficient and Mie scattering coefficient of the GATE (Geant4 Application for Emission Tomography) have been validated using an established Monte-Carlo optical simulation software called MCML (Monte Carlo modeling of light transport in Multi-Layered tissues) by Vesna Cuplov et al in 2014. However, optical lens has not been implemented into the GATE optical simulation for bioluminescence or fluorescence simulation. In this paper, we investigated the feasibility of an optical lens design using GATEv7.0 Monte-Carlo toolkit for optical imaging application. A biconvex spherical lens with focal length of $10.15 \mathrm{~mm}$ was designed according to the Lensmaker's equation. In order to build the lens on GATE, two sphere geometries were used. The radii of curvature of the lens were set to $-10 \mathrm{~mm}$ and $10 \mathrm{~mm}$ for left and right side respectively. The thickness and diameter of the lens were $2 \mathrm{~mm}$ and $6.4 \mathrm{~mm}$ respectively. The material and refractive index of the lens were glass and 1.51 respectively. Two optical photon sources were located in left side of the lens with $30 \mathrm{~mm}$ distance. The optical photon was irradiated onto the lens with a cone angle of $3^{\circ}$. The image was formed on the right side of the lens with a $15.33 \mathrm{~mm}$ distance. The ZEMAX ray tracing optical simulation was performed with the identical lens geometry. The ray diagrams and the image of the GATE optical simulation agreed with the ZEMAX optical simulation results. The implementation of a lens on GATE could extend its application for bioluminescence and fluorescence optical simulation.

This work has supported by grants from the Korea Evaluation Institute of Industrial Technology funded by the Ministry of Trade, Industry and Energy of the Korean Government (2015-4-10051988)

M13D-13: Tomographic Imaging with Carbon Ion Beams

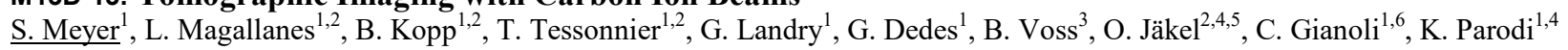

${ }^{I}$ Department of Medical Physics, Ludwig-Maximilians Universität München, Munich, Germany

${ }^{2}$ Department of Radiation Oncology and Radiation Therapy, Heidelberg University Hospital, Heidelberg, Germany

${ }^{3}$ Department of RBDL, GSI Helmholtz Centre for Heavy Ion Research, Darmstadt, Germany 
${ }^{4}$ Heidelberg Ion Beam Therapy Center, Heidelberg, Germany

${ }^{5}$ German Cancer Research Center, Heidelberg, Germany

${ }^{6}$ Department of Radiation Oncology, Klinikum Ludwig-Maximilians-Universität München, Munich, Germany

The sensitivity of ion therapy to inaccuracies in treatment planning and delivery procedures demands the development of imaging techniques capable of accurate assessment of the relative (to water) ion stopping power (RSP).

The investigated tomographic imaging setup consists of a prototype integration-mode detector working as range telescope using active scanning ion beam delivery to image phantoms of tissue-like materials placed on a rotating table [1]. Extensive MonteCarlo (MC) simulations using the FLUKA code [2,3] were performed, to investigate the features of the tomographic reconstruction of RSP (iCT). The results were compared to experiments performed at HIT (Heidelberg). The influence of acquisition parameters was investigated to optimize the imaging dose. Dedicated post-processing relying on the linear decomposition of the integrated signal in individual Bragg curves was applied to solve ambiguities in the presence of material inhomogeneities due to the finite beam size. As comparison the performance of a list-mode detector for single ion tracking was examined.

For raw iCT images, on average median errors of $2.1 \%$ were observed, consistent with experimental data. Optimization of acquisition parameters reduced the imaging dose exposure by a factor of 50, down to around 50mGy without accuracy degradation, and further reduction can be achieved if using proper post-processing. Signal decomposition showed a considerable benefit reducing the average error to around $0.9 \%$, due to a correct determination of the individual Bragg curves. Simulations verified that list-mode provides accuracy improvements comparable to the decomposition method.

The presented study quantitatively underlines the promise of carbon iCT for range verification, showing the performance of different detectors for direct RSP assessment at acceptable dose levels. Post-processing enables the possibility of using MC priors for experimental data reconstruction.

Acknowledgement:

BMBF (Project SPARTA); DFG (Projects MAP, KFO214 and "Ionenstrahlbasierte Computertomographie")

[1] Rinaldi et al. 2013, Physics in Medicine and Biology 58(3), 413

[2] Ferrari et al. 2005, CERN-2005-10 (2005), INFN/TC 05/11, SLAC-R-773

[3] Böhlen et al. 2014, Nuclear Data Sheets 120, 211-214 (2014)

\author{
M13D-14: Including Inter Crystal Scattering Data in PET Image Reconstruction: a Monte Carlo Study for \\ MADPET4 \\ $\underline{\text { N. Omidvari }}^{1}$, J. Cabello ${ }^{1}$, F. R. Schneider ${ }^{1}$, S. Paul ${ }^{2}$, S. I. Ziegler ${ }^{1}$ \\ ${ }^{I}$ Nuklearmedizinische Klinik und Poliklinik, Klinikum rechts der Isar, Munich, Germany \\ ${ }^{2}$ Physik Department E18, Technischen Universität München, Garching, Germany
}

Including inter crystal scattering (ICS) data in PET reconstruction can offer sensitivity increase, image quality enhancement, and consequently less dose or shorter scan time. This is of great interest for PET scanner designs such as MADPET4, in which the quantity of ICS events in data is high. Previous approaches on including ICS data were focused on either selecting the primary crystal from a probable ICS sequence or including triples based on the probabilities of the two most probable LORs. In this work an alternative approach is proposed and studied using Monte Carlo simulations, in which triples add new indices of probabilities in the system response matrix (SRM). Furthermore, the best achievable image quality using the selection approaches was studied by identifying the primary crystal in ICS sequence based on the data from GATE simulations. The images obtained with the two approaches were compared to the images with only doubles at different energy thresholds, SRM statistics, and phantom statistics. The results showed a 54\% increase in sensitivity by including the ICS data, compared to having only doubles with an energy threshold of $50 \mathrm{keV}$. The selection approach was shown to offer limited enhancements in image quality, with increased noise in image due to the absence of ICS data in the SRM. The new inclusion approach on the other hand was largely affected by the statistics in the SRM, but preliminary results obtained for spatial resolution suggest that potential improvements in image quality can be achieved as the statistics in the SRM increase.

This work was supported by the European Commission Seventh Framework Programme (FP7), project number 294582: Multimodal Molecular Imaging (MUMI).

M13E: Posters: Other imaging modalities

Friday, Nov. $4 \quad$ 10:30-12:00 Etoile

M13E-1: Formation of T2* Mapping Using Mixed-Effects Model

H.-M. Huang 
Medical Physics Research Center, Institute of Radiological Research, Chang Gung University and Chang Gung Memorial Hospital, Taoyuan, Taiwan

Using ultrashort echo time (UTE) magnetic resonance imaging (MRI), T2* can be measured and used to evaluate cartilage degradation. In general, high-quality MRI is required for accurate T2* measurement. However, such high-quality MRI requires long scan times which make the clinical utility of $\mathrm{T} 2 *$ measurement infeasible. Thus, a method that can produce satisfactory $\mathrm{T} 2 *$ maps within reasonable scan times (i.e. limited data) is required. Non-linear mixed-effects model (NLME) is a powerful tool for analyzing incomplete longitudinal data. Since NLME contains both fixed and random effects, it is preferred over more traditional methods. The aim of this study is to investigate whether T2* map can be generated using NLME. The simulation results show that NLME is a feasible method for T2* measurement. Moreover, NLME can improve the accuracy and precision of parameter estimates compared to the traditional non-linear least square fit.

M13E-2: Dose Distribution in Phase-Contrast Tomography

L. Xu ${ }^{1}$, A. Pan $^{1}$, G. Barbastathis ${ }^{2}$

${ }^{1}$ Institute of Medical Engineering and Science, MASSACHUSETTS INSTITUTE OF TECHNOLOGY, Cambridge, MA, USA

${ }^{2}$ Mechanical Engineering, MASSACHUSETTS INSTITUTE OF TECHNOLOGY, Cambridge, MA, USA

We examine the effect of dose distribution on 3D reconstruction quality in the context of propagation-based phase-contrast tomography. We explore different imaging schemes in which dose is distributed variably between acquiring less noisy projection images and acquiring more numerous projections. Results from simulations and experiments show that distributing the dose to acquiring more projections at the expense of higher noise levels, provides improvements in image quality by overcoming undersampling artifacts. However, we also find that once the number of projections is sufficient to over oversampling, no additional benefits to image quality is gained by distributing dose to even more projections. These insights can help inform efficient scanning techniques that optimize image quality while minimizing data size and computation time.

\title{
M13E-3: PhoSim: a Software Simulation Package Designed for Macroscopic and Microscopic Studies in the Time-Resolved Optical Tomography
}

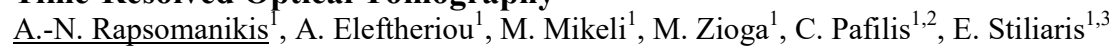

${ }^{I}$ Department of Physics, National \& Kapodistrian University of Athens, Athens, Greece

${ }^{2}$ EEAE, Greek Atomic Energy Commission, Aghia Paraskevi, Athens, Greece

${ }^{3}$ IASA, Institute of Accelerating Systems \& Applications, Athens, Greece

PhoSim is an optical ray tracing Monte-Carlo simulator capable of reproducing the physical processes taking place in a tissue environment when illuminated by Near-Infrared radiation. From a macroscopic point of view this can be accomplished by the proper manipulation of the Henyey-Greenstein phase parameter g, which represents a simple and analytical solution for the fast generation of a random scattering angle photon distribution. Microscopically, the program can simulate certain biological structures by placing a proper density of subcellular organelles inside the volume of interest, proportional to the wavelength of the radiation used at the study $(\sim 750-1000 \mathrm{~nm})$. The new version of this software package is able to create different type of phantoms in multilayer environments and it is also equipped with a detailed Fate and Time of Flight information of each traveling photon. PhoSim is a simple and useful tool for Time-Resolved Optical Tomographic studies; its basic functions and capabilities with optical tomographic examples are presented in this work.

\author{
M13E-4: A Combined Partial Volume Reduction and Super-Resolution Reconstruction for Magnetic \\ Resonance Images \\ $\underline{\text { F. Fallah }}^{1,2}$, B. Yang ${ }^{1}$, F. Schick ${ }^{2}$, F. Bamberg ${ }^{2}$ \\ ${ }^{I}$ Institute of Signal Processing and System Theory, University of Stuttgart, Stuttgart, Germany \\ ${ }^{2}$ Department of Diagnostic and Interventional Radiology, University of Tübingen, Tübingen, Germany
}

Super-resolution reconstruction is proposed to overcome the compromises between the acquirable spatial resolutions, signal-tonoise ratio, and scan time efficiency of magnetic resonance imaging. This technique acquires multiple sub-voxel-shifted lowresolution images to estimate the underlying high-resolution image. However, it involves an ill-posed inverse problem whose solution can be uniquely and stably identified only by incorporation of some prior knowledge. Such incorporation can be done either by searching the solution in a smaller convex set that satisfies certain properties, or by minimizing a regularized objective function. In this work, we propose an approach for a combined partial volume reduction and super-resolution reconstruction of magnetic resonance images. This approach reconstructs high-resolution images from multiple sub-voxel shifted low-resolution images by minimizing an augmented penalized objective function that incorporates a half-quadratic regularizer and some auxiliary variables. The employed regularizer enables faster convergence to the solution, while the auxiliary variables provide a means to precondition the solution space. The proposed scheme employs a constrained reverse diffusion process to update those 
auxiliary variables simultaneously with the iterative super-resolution reconstruction. This process aims at reduction of partial volume effects in the reconstructed high-resolution images by defining the inward and outward signal flows between sub-voxels of the acquired low-resolution images. Comparing the reference and the reconstructed images, quantitative evaluations were done according to the fidelity metrics of mean structural similarity index and relative signal-to-noise ratio. These evaluations revealed superiority of the proposed preconditioner to a threshold-based edge-preserved preconditioner, with regard to both performance and convergence speed.

This work was financially supported by German Research Foundation (DFG) with the grant numbers of SCHI 498/6-2, YA 28/6-2, and BA 4233/4-1.

\author{
M13E-5: New Thermal Breast Phantom Dedicated to Experimentally Test a Novel Approach to 3D Dynamic \\ AngioThermography (DATG)

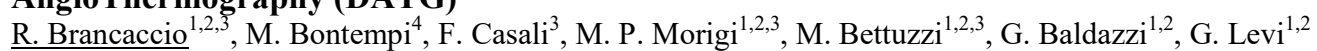 \\ ${ }^{I}$ Department of Physics and Astronomy, University of Bologna, Bologna, Italy \\ ${ }^{2}$ Section of Bologna, INFN (National Institute for Nuclear Physics), Bologna, Italy \\ ${ }^{3}$ Centro Studi e Ricerche Enrico Fermi, Rome, Italy \\ ${ }^{4}$ Istituto Ortopedico Rizzoli, Bologna, Italy
}

The Dynamic Angiothermography (DATG), is a plate-contact diagnostic technique, non-invasive and painless which provides an imaging of the vascularity of a body, mainly in human female breasts. As a detector, the DATG, which should not be confused with the mammary thermography, makes use of a plate covered with cholesteric liquid crystals. The crystals orientate themselves in different way according to their temperature. For the DATG application the temperature range in which liquid crystal can work is designed to be that of the human skin $\left(32^{\circ} \mathrm{C}-38^{\circ} \mathrm{C}\right)$. At room temperature only the infrared radiation is reflected and the crystals appear brown. Temperature variations modify their structure so that the reflected radiation shifts successively to red, yellow, green, blue, until returning to brown in correspondence to the reflection of UV radiation. In this way, putting the plaque on the patient's skin, the image is formed in a "dynamic way". The image formation time depends on the intensity of the heat source and on its depth in the breast. For the same source the image of a deep source appears later than a surface one. The heat diffusion is described by the Fourier heat equation. By solving the equation (depth as function of time) it is possible to infer the depth of the heat source. An approximate solution based on the conduction shape factor is proposed here and a dedicated algorithm is developed. Some phantoms have been manufactured to test the mathematical model. One of them makes use of Plexiglass and an electric wire as heat source; another one is made of material simulating the breast tissue, with thin channels of warm water simulating blood. The results were encouraging. Clinical trials are now necessary to validate the method.

The authors would like to thank Dr. Francesca Draetta for her valuable discussions on DATG peculiarities and Dr. Silvio Marino (working at the ANT foundation, Italian National Foundation for Cancer Research and Patient Care) for the in vivo measurements performed. Lastly, we fondly remember Dr. Daniele Montruccoli, who died prematurely during the course of our research; he was the greatest expert in DATG and introduced this technique even to the poorest countries.

\author{
M13E-6: Compensation of Periodic Motion for Averaging of Magnetic Particle Imaging Data \\ M. Schlüter ${ }^{1,2}$, N. Gdaniec ${ }^{1,2}$, A. Schlaefer ${ }^{3}$, T. Knopp ${ }^{1,2}$ \\ ${ }^{I}$ Institute for Biomedical Imaging, Hamburg University of Technology, Hamburg, Germany \\ ${ }^{2}$ Section for Biomedical Imaging, University Medical Center Hamburg-Eppendorf, Hamburg, Germany \\ ${ }^{3}$ Institute of Medical Technology, Hamburg University of Technology, Hamburg, Germany
}

The temporal resolution of magnetic particle imaging (MPI) is sufficiently high to capture dynamic processes like cardiac motion. The achievable spatial resolution of MPI is closely linked to the signal-to-noise ratio of the measured voltage signal. Therefore, in practice it can be advantageous to improve the signal-to-noise ratio by block-wise averaging the signal over time. However, this will decrease the temporal resolution such that cardiac motion is not resolved anymore. In the present work, we introduce a framework for averaging MPI data that exhibit periodic motion induced by e.g. respiration and/or the heart beat. The frequency of motion is directly derived from the MPI raw data without the need for an additional navigator signal. The short time Fourier transform is used for this purpose, because each of these periodic movements will have a frequency varying over time. In order to average the captured frames corresponding to the same phase of the motion, one has to calculate virtual frames since the data acquisition and the periodic motion are not synchronized. In a phantom study it is shown that the developed method is capable of averaging experimental data without introducing any motion artifacts.

M13E-7: X-Ray Fluorescence Computed Tomography with a Compton Camera for a Clinical Application D. Vernekohl $^{1}$, M. Ahmad ${ }^{2}$, G. Chinn ${ }^{2}$, X. Lei ${ }^{1}$

${ }^{1}$ Department of Radiation Oncology, Stanford, Stanford, USA

${ }^{2}$ Department of Radiology, Stanford, Stanford, USA 
XFCT is a promising new molecular imaging modal- ity which is able to measure non-radioactive tracer molecules even in deep tissues. Unrestricted spatial resolution imaging with pencil beam x-ray excitation is possible but cannot be translated easily to clinical applications as scanning times are at least several hours. The spatial information accessed with Compton cameras (CCs) overcomes this drawback as fan or cone-beams can boost the measurement process. The spatial resolution and sensitivity performance of this new approach are studied on the basis of Monte-Carlo simulations. The results show that molecular sensitivities of $400 \mathrm{fM}$ are accessible at $15 \mathrm{mGy}$ for the demonstrated lung scan scenario and FWHM below $7 \mathrm{~mm}$ for lesion diameters of $0.5 \mathrm{~mm}$. The scanning time was reduced by a factor of 30 for the given example.

M13E-8: Comparison of a Large Area CZT Detector to a Spectroscopic CdTe Detector for X-Ray Fluorescence Computed Tomography.

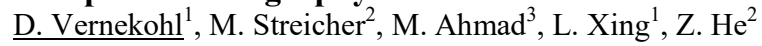

${ }^{I}$ Department for Radiation Oncology, Stanford, Stanford, USA

${ }^{2}$ Department of Nuclear Engineering and Radiological Science, Michigan, USA

${ }^{3}$ Department of Radiology, Stanford, USA

X-ray fluorescence CT (XFCT) imaging is a promis- ing molecular imaging modality with high spatial resolution and nonradioactive high atomic number tracers for medical imaging. While dozens of bench-top experiments proofed the concept of the approach, scalability for clinical systems with high molecular sensitivity at acceptable doses is not yet accomplished. This study compares a spectroscopic CdTe detector which was used for XFCT measurements beforehand to a CZT detector with scalable active area. The results show that the CZT detector is able to provide better image quality due to higher detection efficiency even with a significantly reduced energy resolution and absorbing enclosure.

M13E-9: Investigation of the Image Characteristics in Single Grid-Based Phase Contrast X-Ray Imaging (PCXI) by Simulation and Experimental Studies

H. Lim, H. Cho, Y. Park, U. Je, C. Park, K. Kim, G. Kim, S. Park, S. Park, H. Lee

Dept. of Radiation Convergence Engineering, Yonsei University, Wonju, Gangwon-do, South Korea

In this work, we investigated a simplified approach to phase-contrast X-ray imaging (PCXI) by using a single antiscatter X-ray grid and a conventional polychromatic x-ray source, which has potential to open the way to further widespread use of PCXI into the related application areas. We performed a systematic simulation to demonstrate its viability for PCXI. We also performed an experiment by using a table-top setup we established which consists of a focused-linear grid having a strip density of 200 lines/in, an x-ray tube having a focal-spot size of $5 \mu \mathrm{m}$, and a CMOS-type flat-panel detector having a pixel size of $48 \mu \mathrm{m}$, respectively. By using our prototype PCXI system and the Fourier demodulation technique, we successfully obtained attenuation, scattering, and differential phase-contrast images of improved visibility from the raw images of several selected samples at $\mathrm{x}$-ray tube conditions of $90 \mathrm{kVp}$ and $0.1 \mathrm{mAs}$. Our simulation and experimental results indicate that single-grid-based approach seems a useful method for PCXI with great simplicity and minimal requirements on the setup alignment.

Radiation Technology Development Program of the National Research Foundation (NRF)

\author{
M13E-10: Brain Extraction from MR Images Using a Combination of Segmentation Fusion and Marker- \\ Controlled Watershed Transform \\ A. K. Thanellas, M. Pollari, T. Alhonnoro, M. Lilja \\ Dept. of Neuroscience and Biomedical Engineering, Aalto University, Espoo, Finland
}

A new brain extraction method for MR images is presented that combines segmentation fusion with an active segmentation step. Three common brain extraction methods (Brain Extraction Tool (BET), 3DSkullStrip and FreeSurfer) were used to provide the input segmentations. The areas where the input segmentations agreed were fused normally, while the areas of disagreement were left to be handled by an active segmentation in a marker-controlled watershed framework. The performance of the proposed algorithm was compared with the input segmentations as well as with the majority voting and staple meta-algorithms. Three evaluation criteria related to the overlap error, average distance and volume di?erences were used on two datasets. The results showed that the proposed method outperformed the input segmentations as well as the meta-algorithms on all the evaluation criteria for both datasets. It is concluded that the proposed method appears highly suitable for large studies involving brain extraction, where full automation and robust segmentation results are needed for all datasets.

M13E-11: Comparison of Sensitivity Models for Image Reconstruction of a Compton Telescope $\underline{\text { P. G. Ortega }}{ }^{1}$, E. Muñoz ${ }^{2}$, A. Etxebeste ${ }^{2}$, J. Barrio ${ }^{2}$, C. Lacasta ${ }^{2}$, G. Llosá ${ }^{2}$, C. Solaz ${ }^{2}$, J. F. Oliver ${ }^{2}$

${ }^{I}$ EN, European Organization for Nuclear Research (CERN), Geneva, Switzerland

${ }^{2}$ Instituto de Física Corpuscular (IFIC)/Universidad de Valencia-CSIC, Valencia, Spain 
In this work we present a systematic comparison of different parametrizations of sensitivity matrices commonly employed in the literature for a two and three-layer Compton Telescope. A more complete analytical method to evaluate the sensitivity matrix for iterative reconstructions is presented. The proposed method takes into account all the relevant physics in the system.

The obtained analytical model is then validated against simulated events generated with FLUKA Monte Carlo code, indicating that previous methods are not well suited for our Compton Telescope prototype. The influence of the sensitivity correction in the final reconstructed relative intensities is compared for all the methods.

The studied sensitivity matrix is able to correct the loss of intensity, improving the quality of the reconstructed images both for two and three layer interactions.

M13E-12: Prototype of a Handheld Laparoscopic Compton Camera for Radio Guided Surgery

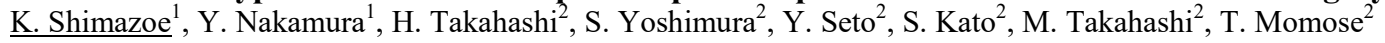

${ }^{I}$ School of Engineering, The University of Tokyo, Tokyo, Japan

${ }^{2}$ University Hospital, The University of Tokyo, Tokyo, Japan

Intra-operative localization of the tracer is important to identify local margins for less-invasive surgery, especially FDG/radioguided surgery. The objective of this paper is to develop a laparoscopic Compton camera and system aimed at use for intraoperative FDG imaging for accurate and less-invasive dissections. The laparoscopic Compton camera consists of four layers of a 12-pixel cross-shaped array of GFAG crystals $(2 \times 2 \times 3 \mathrm{~mm} 3)$ and through silicon via multi-pixel photon counter(MPPC)s and dedicated individual readout electronics based on a dynamic time-over-threshold method. Experimental results yielded a spatial resolution of $4 \mathrm{~mm}(\mathrm{FWHM})$ for a $10-\mathrm{mm}$ working distance and an absolute detection efficiency of $0.11 \mathrm{cps} / \mathrm{kBq}$, corresponding to an intrinsic detection efficiency of $\sim 0.18 \%$. In an experiment using a NEMA-like well-shaped FDG phantom, a ?5 $\times 10 \mathrm{~mm}$ cylindrical hot spot was clearly obtained even in the presence of a background distribution surrounding the Compton camera and the hot spot. We successfully obtained reconstructed images of a resected lymph node and primary tumor ex vivo after FDG administration to a patient having esophageal cancer. These performance characteristics indicate a new possibility of FDGdirected surgery by using a Compton camera intra-operatively.

\section{M13E-13: Quantitative Cherenkov Luminescence Imaging: Measurements and Simulations} E. Ciarrocchi $^{1,2}$, N. Belcari ${ }^{1,2}$, A. G. Cataldi ${ }^{3}$, P. A. Erba ${ }^{4}$, A. Del Guerra ${ }^{1,2}$

${ }^{I}$ Department of Physics, University of pisa, Pisa, Italy

${ }^{2}$ INFN Section of Pisa, Istituto Nazionale di Fisica Nucleare, Pisa, Italy

${ }^{3}$ UO Medicina Nucleare, Azienda Ospedaliera Universitaria Pisana, Pisa, Italy

${ }^{4}$ Department of Translational Research and New Technologies in Medicine and Surgery, Azienda Ospedaliera Universitaria Pisana, Pisa, Italy

In preclinical imaging and with a farther perspective in clinical applications there is an interest in detecting $\ddot{i}_{i}^{1} / 2$-emitting radiotracers with optical techniques. Several groups have started investigating the possibility of Cerenkov light emission, which is produced when the velocity of the charged $\ddot{i}_{\dot{\zeta}}{ }^{1 / 2}{ }^{+}$or $\ddot{i} \dot{\zeta}^{1 / 2}$ particle is higher than the velocity of light in the medium. We are looking for a method for Cerenkov luminescence imaging to image radionuclides of biomedical interest, providing also the absolute quantitative information on the source activity concentration. We believe that this goal can be obtained with the combination of a quantitative detection system and a Monte Carlo model to reconstruct the source activity from the measured signal. To this aim we are performing Cerenkov luminescence measurements with simple geometries that can be easily simulated. A radioactive source diluted in water is used as a Cerenkov radiator and is placed with the detector in a light-tight box. The experimental results are compared with the simulation output, obtained considering the radioactive decay, the Cerenkov radiation production and transport, and the detector response. Preliminary results show that, with F-18 diluted in water, activity levels lower than $0.5 \mathrm{MBq}$ produce a signal that depends on the acquisition settings. For activity levels higher than this, simulation results overestimate the experimental measurements by a factor 1.3. The differences are probably due to experimental factors that were not modelled (like the detector glass entrance window), and to uncertainties on other factors like the lens transmission curve and the sensor quantum efficiency. We will use spectral acquisitions made with a high energy pure $\ddot{i}_{i}^{1 / 2}$ emitter such as ${ }^{90} \mathrm{Y}$ and with opaque tissue to provide more insight on the potential of Cerenkov luminescence imaging to be quantitative in real applications.

M13E-14: Feasibility Study of a Gradient Coil for a Dedicated and Portable Single-Sided MRI System D. Grau-Ruiz ${ }^{1}$, J. P. Rigla ${ }^{1,2}$, E. Diaz-Caballero ${ }^{1,2}$, A. Nacev ${ }^{3}$, A. Aguilar ${ }^{1}$, P. Bellido ${ }^{1}$, P. Conde ${ }^{1}$, A. González-Montoro, A. González ${ }^{1}$, L. Hernández ${ }^{1}$, A. Iborra ${ }^{1}$, L. Moliner ${ }^{1}$, M. J. Rodriguez-Álvarez ${ }^{1}$, S. Sanchez Goez ${ }^{1}$, M. Seimetz ${ }^{1}$, A. Soriano $^{1}$, L. F. Vidal ${ }^{1}$, I. N. Weinberg ${ }^{3}$, F. Sanchez ${ }^{1}$, J. M. Benlloch ${ }^{1}$

${ }^{I}$ Institute for Instrumentation in Molecular Imaging, I3M-CSIC, Valencia, SPAIN

${ }^{2}$ Tesoro Imaging S.L., Alicante, Spain

${ }^{3}$ Weinberg Medical Physics, Bethesda, MD, USA 
The aim of gradient coils in a Magnetic Resonance Imaging (MRI) system is to provide linear magnetic fields to spatially encode the volume of interest (VOI). In order to obtain an optimized design of a gradient coil, we must take into account three important factors: linearity, inductance and resistance. The considered gradient coils are capable of generating ultra-steep and ultra-fast magnetic field gradients avoiding peripheral nerve stimulation.

A feasibility study of these gradient coils for a dedicated and single-sided MRI system has been made by performing electromagnetic and thermal simulations and comparing these simulations results with experimental data.

Two gradient coils have been designed according to the above factors and manufactured on Printed Circuit Boards.

Electromagnetic (EM) and thermal simulations have been performed in order to study its feasibility.

On the one hand, EM experimental data show a high linearity in the VOI. On the other hand, the thermal simulation shows the need to implement a cooling system to decrease the gradient coils temperature.

EM results show the possibility of using the studied gradient coil in order to assemble it in the open MRI system. Thermal simulation shows the viability of using an air cooling system. Moreover, a train of pulses typical of a MRI pulse sequence will be used in order to obtain more realistic thermal results.

Funding received under the "Retos-Colaboración" and "Retos-Investigacion" Programme from the Spanish Ministry of Economy and Competitiveness and "Adquisición de pequeño equipamiento" Programme (European Regional Development Fund) from the Generalitat Valenciana.

\author{
M13E-15: The Influence of Field Distortions on Multi-Patch Image Reconstruction in Magnetic Particle \\ Imaging

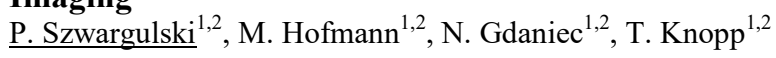 \\ ${ }^{1}$ University of Technology, Institute for Biomedical Imaging, Hamburg, Germany \\ ${ }^{2}$ University Medical Center Hamburg-Eppendorf, Section for Biomedical Imaging, Hamburg, Germany
}

Magnetic Particle Imaging (MPI) is a highly sensitive imaging modality which is currently used for preclinical studies. The purpose of this technique is the visualization of nanometer sized super-paramagnetic particles in tissue by using magnetic fields for excitation and selection. Due to physiological and technical constraints the field of view in MPI is limited to a few centimetres. To enlarge the region of interest, additional fields can be used to shift the field of view in space. However, due to inhomogeneous and non-linear magnetic fields an application of these focus fields lead to distortions in the reconstructed images if these are not taken into account. Within this work, we investigate the impact of the focus-field shift on the MPI system function and the resulting distortions. We propose a zero order correction scheme that leads to a substantial improvement in image quality when it is taken into account during image reconstruction.

M13E-16: Tumor Microvasculature in Lung Cancer and DW-MRI

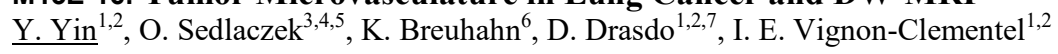

${ }^{I}$ INRIA, Paris, France

${ }^{2}$ Sorbonne Universités UPMC Univ. Paris 6, Laboratoire Jacques-Louis Lions, Paris, France

${ }^{3}$ Translational Lung Research Center Heidelberg (TLRC), member of the German Centre for Lung Research (DZL), Heidelberg, Germany

${ }^{4}$ Department of Diagnostic \& Interventional Radiology, University Hospital of Heidelberg, Heidelberg, Germany

${ }^{5}$ Department of Diagnostic and Interventional Radiology with Nuclear Medicine, Thoraxklinik at University of Heidelberg, Heidelberg, Germany

${ }^{6}$ Institute of Pathology, University Hospital Heidelberg, Heidelberg, Germany

${ }^{7}$ IZBI, University of Leipzig, Leipzig, Germany

Angiogenesis is critically important in invasive tumor growth and metastasis. Many recent studies have focused on the microvasculature in tumor. However, the most often used tumor tissues are from animals; rarely human tumors are selected. Besides, the microvasculature may provide useful information for probing the microperfusion of blood in tumor. In this work, we aim to achieve the quantitative analysis of microvasculature in human tumor and attempt to compare the vessel features with blood perfusion in tumor. We present an automatic procedure to extract blood vessels and reconstruct the 3D vessel structures in lung tumor based on serial histological tumor tissue slices. The digitized histological tissue slice is in 2D with fine resolution up to cellular level. The proposed method was tested on a stack of 288 serial slices of a human lung tumor. It has the potential to work on different tumor types. We compare the vessel area fraction in specific lung tumor regions with the perfusion-related parameter from IVIM (intravoxel incoherent motion) of the diffusion-weighted MRI. High perfusion was observed in tumor sample with high vessel area fraction.

M13E-17: Total Variation Assisted Fourier Shift Manipulation to Remove Gibbs' Artifacts in Compressive Sensing Techniques

P. Adibpour, M. Smith 
Gibbs' distortions are common artifacts in magnetic resonance (MR) applications where discrete Fourier transform (DFT) based algorithms are applied to finite length data. Compressive sensing (CS) techniques emerged in MR applications to meet high temporal resolution necessity using sparsity of medical images to recover the full resolution data from under sampled k-space data set through nonlinear algorithms. While CS removes most of noise-like under sampling artifacts using recursive nonlinear algorithm, it fails to fully recover the regions of interest affected by Gibbs' distortions due to the blurriness imposed to inherently filtered high frequency components inside CS algorithm. We propose Fourier shift manipulation (FSM) approaches to CS reconstructions which use total variation metric to correct Gibbs' artifacts before low pass filtering of k-space data through recursive optimization algorithm. We discuss the proposed approaches in functional magnetic resonance imaging context which starts with an initial scan followed by a time series of CS data. We provide the theory of the alternative FSM approach for removal of Gibbs' artifacts and then outline the steps of an automated FSM technique to mitigate Gibbs' artifacts in CS reconstructions. The application of the proposed method is presented on simulation and clinical brain data with visual and numerical comparison of the accuracy of common and proposed techniques.

\author{
M13E-18: Improving RF Field (B1) Penetration Efficiency by Implementing Concept of Multiple Full-Ring \\ PET Insert for MRI System \\ M. S. H. Akram ${ }^{1}$, C. S. Levin ${ }^{2}$, T. Obata ${ }^{1}$, T. Yamaya ${ }^{1}$ \\ ${ }^{I}$ Department of Radiation Measurement, Imaging and Dosimetry, National Institute of Radiological Sciences, Chiba, Japan \\ ${ }^{2}$ Stanford University, Stanford, CA, USA
}

In case of RF field penetrable PET inserts for the existing MRI system, for the case of full-ring PET, it is very challenging to get efficient RF field (B1) in the imaging region (ROI) inside the ring. Our experimental results confirmed that. The aim of this study is to improve the RF field penetration efficiency by introducing multi-ring PET concept. We performed initial study by using two complete cylindrical PET rings. In each ring we implemented 16 shield boxes with $1 \mathrm{~mm}$ gap between the shield boxes and each ring diameter was $210 \mathrm{~mm}$. The gap between the two rings was $20 \mathrm{~mm}$. We also made one ring by combining the two rings. Experimental results showed, for one full-ring case, B1 homogeneity reduces from $79 \%$ (for without shield case) to $44 \%$ in the central coronal plane $(\mathrm{X}-\mathrm{Z})$ of a $100 \mathrm{~mm}$ by $180 \mathrm{~mm}$ area. Also the average field strength reduced by $25 \%$ compare to that of without PET insert. In case of two-ring insert, for the same coronal plane, B1 homogeneity improves to $64 \%$ and average field reduction from without case was 14\%. Most interestingly, we have found that for $20 \mathrm{~mm}$ ring gap RF field extends farther (about $120 \mathrm{~mm}$ ) along the axial direction. These findings confirmed that, for an extended PET FOV and for full-ring PET geometry, by introducing multiple full-rings instead of one long ring we can have efficient RF penetration inside the PET rings and can have quality MR images.

M13E-19: Simulation of a Table-Top Analyzer Based Phase Contrast Imaging System O. Caudevilla $^{1}$, W. Zhou ${ }^{1}$, S. Stoupin ${ }^{2}$, J. G. Brankov ${ }^{1}$

${ }^{I}$ ECE, Illinois Institute of Tehnology, Chicago Illinois, USA

${ }^{2}$ Argonne National Laboratory, Argonne, Illinois, USA

Analyzer-based X-ray phase contrast imaging (ABI) belongs to a broader family of phase-contrast (PC) X-ray imaging modalities. Unlike the conventional X-ray radiography, which measures only X-ray absorption, in PC imaging one can also measure the X-rays refraction and scatter properties of the imaged object. It has been shown that refraction imaging provide better contrast when imaging the soft tissue. In this paper, we introduce a simulation tool specifically designed to simulate the ABI systems with a conventional polychromatic X-ray source. By utilizing ray tracing and basic physical principles of diffraction theory our simulation tool can predict the X-ray beam profile shape, the energy content and the total throughput (photon count) at the detector. In addition, we can evaluate imaging system point- spread function for various system configurations.

\title{
M14: New radiation detectors / technologies for medical imaging II
}

Friday, Nov. $4 \quad$ 14:00-16:00 Schweitzer

M14-1: Demonstration of Multi-Color 3D Imaging of Gamma Rays Based on Ultra-Compact Compton Camera

$\underline{\text { A. Kishimoto }}^{1}$, J. Kataoka ${ }^{1}$, Y. Iwamoto ${ }^{1}$, S. Ohsuka ${ }^{2}$

${ }^{I}$ Waseda University, Tokyo, Japan

${ }^{2}$ Hamamatsu Photonics, Shizuoka, Japan

The Compton camera, which shows gamma-ray distribution utilizing the kinematics of Compton scattering, is a promising detector capable of imaging across a wide range of energy. In this study, we aim to construct a small-animal molecular imaging system in a wide energy range by using the Compton camera. We developed a compact medical Compton camera based on a Ce- 
doped Gd3Al2Ga3O12 (Ce:GAGG) scintillator and 8x8 multi-pixel photon counter (MPPC). A basic performance confirmed that for $662 \mathrm{keV}$, the typical energy resolution was 7.4\% (FWHM) and the angular resolution was 4.5 degree (FWHM). We then used the medical Compton camera to conduct imaging experiments based on a 3-D imaging reconstruction algorithm. In the result, we succeeded in several 3-D imaging such as syringe phantom imaging based on 18F and 3-D multi-color imaging of different simultaneous energy sources $(22 \mathrm{Na}, 137 \mathrm{Cs}, 54 \mathrm{Mn})$, both under short measurement time of 15 and 6 min, respectively. In this study, we will present results of precise detector performances and its imaging capability.

M14-2: Design Concepts and Characterization of a Next Generation Clinical PET Detector J. W. Cates ${ }^{1}$, C. S. Levin ${ }^{1,2,3,4}$

${ }^{I}$ Radiology, Stanford University, Stanford, CA, USA

${ }^{2}$ Bioengineering, Stanford University, Stanford, CA, USA

${ }^{3}$ Electrical Engineering, Stanford University, Stanford, CA, USA

${ }^{4}$ Physics, Stanford University, Stanford, CA, USA

An important and long-standing milestone for the time-of-flight positron emission tomography (TOF-PET) community is achieving 100 ps FWHM coincidence timing resolution (CTR) in a clinical system. However, the high-aspect ratio (HAR) scintillation crystal element geometry employed in a traditional PET detector configuration has poor light collection efficiency (LCE) and increases the scintillation light transit time variance, increasing the temporal variance on the earliest arriving scintillation photons, thus degrading the resulting CTR. A novel approach to achieve near-complete LCE with low variation and minimize scintillation photon transit time variance is to read the scintillation light from a HAR crystal from its long side. With this approach, $3 \times 3 \times 11 \mathrm{~mm}^{3}$ LGSO:Ce $(0.025 \mathrm{~mol} \%)$ scintillators coupled on their $3 \times 11 \mathrm{~mm}^{2}$ sides to arrays of analog SiPMs, making a $3 \times 3 \times 22 \mathrm{~mm}^{3}$ pixel volume (via two separate experiments), yielded $103 \pm 1$ ps and 106 \pm 2 FWHM CTR versus identical crystal pairs by using simple, leading edge time pickoff. In addition to excellent CTR, this detector also can position the $511 \mathrm{keV}$ interaction coordinates in three-dimensions (3D). This ability enables two important features (1) more accurate positioning of one or more annihilation photon interactions ( $=2$-interaction inter-crystal scatter events are much more probable than singleinteraction photoelectric events) and (2) an estimate of the incoming annihilation photon incident angle for $=2$-interaction intercrystal scatter events - which provides an opportunity to retain a high fraction of photon events that are normally rejected. These innovations can be packaged into a practical clinical detector module with mixed analog-digital multiplexing schemes that require a low number of readout channels. We present the design and proof-of-concept measurements for this advanced clinical PET detector module concept capable of 3D positioning and $\sim 100$ ps FWHM coincidence time resolution performance.

M14-3: Development of a 3D Silicon Coincidence Avalanche Detector for Charged Particle Tracking in Medical Applications

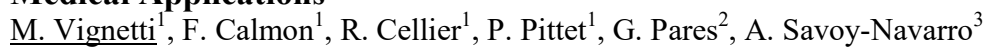

${ }^{I} I N L$ - Institut des Nanotechnologies de Lyon, Lyon, France

${ }^{2}$ CEA - LETI, Grenoble, France

${ }^{3}$ Laboratoire d'AstroParticule et Cosmologie, Paris, France

This work describes the development of a novel position sensitive detector suitable for nuclear physics applications, in the field of High Energy Physics experiments and emerging medical applications such as hadrontherapy and Proton Computed Tomography (pCT). The "3D Silicon Coincidence Avalanche Detector - 3DSiCAD" pixel consists of a pair of 3D vertically aligned Geiger-mode avalanche diodes working in time-coincidence mode. This novel detector provides: i) single charged particle detection capability thanks to the high intrinsic gain of each diode, ii) compatibility with standard CMOS technology and commercially available 3D integration techniques, iii) very low dark count noise mainly due to fake coincidences occurring within a very short time-window. The main focus of the present work is to demonstrate this latter point. A silicon test-chip has been designed and fabricated for the realization of a first 3DSiCAD prototype, ensuring compatibility with 3D flip-chip assembly. Measurements performed over a specific test structure consisting on two "on-plane" avalanche diodes have demonstrated a very high noise rejection enhancement thanks to the coincidence detection mode ( 3 to 4 orders of magnitude lower than the intrinsic dark count rate). This measured performance with a low cost standard technology is equal or even lower than the dark count rate achievable with expensive dedicated detector technologies. 3DSiCAD detectors target several important applications in the field of proton-therapy and proton tomography, such as: i) proton beam monitoring for quality assurance, ii) real-time control of the ion range by means of prompt-gamma radiations, iii) proton radiography and proton Computed Tomography (pCT). The first two applications require fast response times with high counting rates which are intrinsically featured by SPAD devices while a very low noise is mandatory for the last application; these specifications are achievable with the proposed 3DSiCAD detector.

This project is funded by the EU's 7th Framework Program for research, technological development and demonstration under grant agreement $\mathrm{n}^{\circ}$ 317446. 
M14-4: Geant4 Simulation and Measurements of the GEMPix Detector as Beam Monitor for Carbon Ions at CNAO

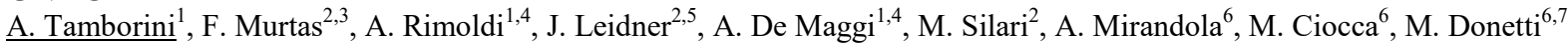 \\ ${ }^{I}$ INFN Section of Pavia, Pavia, Italy \\ ${ }^{2}$ CERN, Geneve, Switzerlandand \\ ${ }^{3} L N F-I N F N$, Frascati, Italy \\ ${ }^{4}$ University of Pavia, Pavia, Italy \\ ${ }^{5}$ Rheinisch-Westfaelische Tech. Hoch., Aachen, Germany \\ ${ }^{6}$ Medical Physics Unit, National Center for Oncological Hadrontherapy (Fondazione CNAO), Pavia, Italy \\ ${ }^{7}$ INFN Section of Torino, Torino, Italy
}

This work discusses the full simulations and measurements of a GEMPix (Gas Electron Multiplier) detector for a possible application as monitor for beam verification at CNAO (National Centre for Oncological Hadrontherapy) in Pavia, Italy. The Monte Carlo Geant4 10.01 patch 03 toolkit was used to simulate the complete CNAO extraction beamline and beam delivering system.

The triple GEMPix detector was fully simulated: a homogeneous electric field was implemented to take into account the drift of secondary particles in the gas gap. An ArCO2CF4 gas mixture was simulated to reproduce the GEMPix response. The complete measurement setup was simulated with the GEMPix placed in a water phantom and irradiated with carbon ions at different energies. Important beam parameters such as the transverse FWHM were compared with experimental measurements at CNAO. A triple GEM detector prototype read by 4 Timepix chips ( $55 \mu \mathrm{m}$ pitch pixelated ASIC for the readout) was tested for a detailed characterization and measurements of energy deposition inside the water phantom. The energy deposition was measured at different positions in depth allowing a 3D reconstruction of the beam inside the phantom.

The simulation results are very encouraging since they reproduce the experimental data within a few percent. Measurements were carried with different parameters settings. Experimental measurements are still ongoing.

Further validations are needed, but nonetheless the good results obtained so far confirm that GEMPix has a good potential as beam monitor in hadrontherapy.

This work was supported by the Istituto Nazionale di Fisica Nucleare (INFN) within the IRPT Project and the Fondazione CNAO - Pavia, Italy

\author{
M14-5: Comprehensive and Fast Pulse-Shape Characterization in Segmented HPGe Detectors for Gamma- \\ Ray Tracking and Imaging \\ M. Ginsz ${ }^{1}$, G. Duchêne ${ }^{2}$, B. Pirard ${ }^{1}$, F. Didierjean ${ }^{2}$ \\ ${ }^{I}$ Canberra Specialty Detectors, Lingolsheim, France \\ ${ }^{2}$ IPHC, UNISTRA, CNRS, Strasbourg, France
}

A new setup for the 3D pulse-shape characterization of multi-segmented High Purity Germanium (HPGe) detectors has been developed and tested. It allows for the first time to perform a complete experimental pulse-shape characterization of large volume detectors in only 3 weeks. First experimental results of the scan of a coaxial segmented HPGe detector are presented in this paper. They show promising results for further improvement of Pulse-Shape Analysis Techniques used for gamma-ray tracking and imaging devices.

\author{
M14-6: Characterization of SiPM Non-Linearity and Energy Resolution for Prompt Gamma Imaging \\ Applications

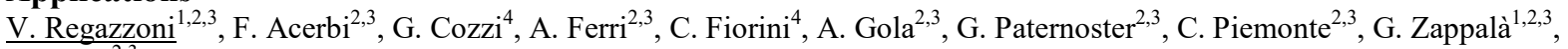 \\ N. Zorzi ${ }^{2,3}$ \\ ${ }^{I}$ University of Trento, Trento, Italy \\ ${ }^{2}$ Fondazione Bruno Kessler, Trento, Italy \\ ${ }^{3}$ Trento Institute for Fundamental Physics and Applications, Trento, Italy \\ ${ }^{4}$ Politecnico di Milano, Milano, Italy
}

Fondazione Bruno Kessler has recently introduced Ultra High-Density (UHD) RGB-SiPMs, featuring a minimum cell size of $5 \mathrm{x}$ $4.3 \mu \mathrm{m}^{2}$ and a maximum cell density of 46200 cells $/ \mathrm{mm}^{2}$. The high cell density is very important for improving the linearity of the SiPM in high-dynamic-range applications, such as the scintillation light readout in high-energy Gamma-ray spectroscopy and in prompt gamma imaging (PGI) in proton therapy. However, non-linearity (NL) is not the only parameter that defines performance in these applications: indeed the energy resolution in the scintillation light readout at high energies is a trade-off between the excess noise factor (ENF) caused by the non-linearity of the SiPM and its photon detection efficiency (PDE). To study these effects, we developed a new setup that simulates the LYSO light emission in response to gamma photons up to 30 $\mathrm{MeV}$, using a pulsed light source. With the setup, we measured the non linearity and energy resolution vs. energy of the UHDSiPM technology and of the more conventional HD-SiPM technology. Up to now, we considered five different cell sizes, ranging 
from $10 \mu \mathrm{m}$ up to $25 \mu \mathrm{m}$. With the UHD technology, we were able to observe a remarkable reduction of the SiPM non-linearity, measuring a NL of less than $5 \%$ at $5 \mathrm{MeV}$ with $10 \mu \mathrm{m}$ cells, which should be compared to a NL of $50 \%$ with the more conventional $25 \mu \mathrm{m}$-cell HD-SiPMs. With the same setup, we also measured the different components of the energy resolution (intrinsic, statistical and electronic noise) vs. cell size, over-voltage and energy and we calculated the different components of the ENF.

\author{
M14-7: Calibration Method for Time Measurement with the Best Linear Unbiased Estimator for Digital \\ Silicon Photomultipliers \\ W. Lemaire, A. Corbeil Therrien, J.-F. Pratte, R. Fontaine \\ Department of Electrical and Computer Engineering, Université de Sherbrooke, Sherbrooke, Québec, Canada
}

In detectors capable of timestamping individual photons, such as digital silicon photomultipliers (DSiPMs), the timing resolution can be improved by combining multiple timestamps of a scintillation event with statistical methods. Integrating a time estimator directly in a multichannel photodetector would not only improve its timing resolution but would also considerably lower the bandwidth requirements. The maximum likelihood estimation (MLE) method provides a significant improvement, but at the cost of a high computational burden. As an alternative, the best linear unbiased estimator (BLUE) has demonstrated similar improvements in simulations, while requiring significantly fewer resources. Calculating the BLUE weights requires a set of data in which all the timestamps are relative to the time of interaction. However, the time of interaction can only be known precisely in simulations. To circumvent the aforementioned problem, we propose and compare two methods, a differential estimator, and an iterative estimator, for estimating the BLUE weights using only data from a pair of photodetectors in coincidence. The scintillation data is generated with Geant4 and run through a model simulating the DSiPM and the readout electronics. Iteratively estimating the BLUE weights provides the best results converging to the same performance improvement as if the BLUE coefficients were calculated with the information of the time of arrival. Using a simulated $1 \times 1 \times 5 \mathrm{~mm}^{3}$ scintillator, the iterative method improves the timing resolution from 122 ps to 103 ps FWHM, reaching the same performance as when the time of arrival is known.

\author{
M14-8: Investigation of Electron Multiplication Effect in Optical Property Modulation-Based Radiation \\ Detection Method for PET \\ $\underline{\text { L. Tao }}^{1}$, H. M. Daghighian ${ }^{2}$, C. S. Levin ${ }^{1,2,3,4}$ \\ ${ }^{I}$ Department of Electrical Engineering, Stanford University, Stanford, USA \\ ${ }^{2}$ Department of Radiology, Stanford University, Stanford, USA \\ ${ }^{3}$ Department of Physics, Stanford University, Stanford, USA \\ ${ }^{4}$ Department of Bioengineering, Stanford University, Stanford, USA
}

In this paper, we further explore the optical property modulation-based method for ionizing radiation photon detection in PET as a potential new direction to dramatically improve the coincidence time resolution. We compare the performance of three detector crystals for this method including two types of cadmium telluride (CdTe) crystals and one bismuth silicon oxide (BSO) crystal under high bias voltages up to $3500 \mathrm{~V}$. We first show that the induced current flow in the detector crystal determines the strength of the optical property modulation signal due to ionization. A larger resistivity is favorable for reducing the dark current (noise) in the crystal and facilitates the detection of weak optical property modulation signals. In addition, we show that BSO is a potential candidate detector material. When biased at $3500 \mathrm{~V}$, it has comparable modulation signal sensitivity as CdTe biased at $1000 \mathrm{~V}$, but with higher resistivity (lower noise), larger $511 \mathrm{keV}$ photon attenuation coefficient, lower price, better crystal surface finish quality, and less toxicity. By studying the dependence of modulation signal amplitude on crystal bias voltage, we show that the modulation signal amplitude (induced by both UV laser diode and Ge-68 source) is linearly proportional to crystal bias voltage with a linear fit $\mathrm{R}$ factor of around 0.95 . The modulation signal amplitude induced by UV laser diode irradiation increases from $0 \%$ to $2 \%$ (normalized to the average signal level) for both CdTe and BSO under crystal bias voltage from $0 \mathrm{~V}$ to $3500 \mathrm{~V}$. The modulation signal amplitude induced by Ge- 68 irradiation increases from $0 \%$ to $12 \%$ for CdTe under crystal bias voltage from $0 \mathrm{~V}$ to $1500 \mathrm{~V}$, and increases from $0 \%$ to $10 \%$ for BSO under crystal bias voltage from $0 \mathrm{~V}$ to $3500 \mathrm{~V}$. Therefore the electron multiplication effect (with high crystal bias) shows promise to significantly boost the modulation signal amplitude with the ultimate goal to achieve single $511 \mathrm{keV}$ photon detection.

M15: Image reconstruction techniques II

Friday, Nov. $4 \quad$ 14:00-16:00 Cassin

M15-1: Pitfalls in MLAA and MLACF

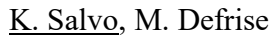

$B E F Y$, Vrije Universiteit Brussel, Brussels, Belgium 
In time-of-flight (TOF) positron emission tomography (PET) imaging, it is possible to correct for attenuation using only the TOF-PET data. Currently two main iterative algorithms exist for this purpose: MLAA and MLACF. In addition to reconstructing the activity image ?, MLAA reconstructs the attenuation map $\mu$, whereas MLACF reconstructs the attenuation correction factors a. While implementing MLAA and MLACF, one has to be careful. Possible pitfalls are: (i) obtaining slice dependent scale factors, (ii) enforcing a physical constraint on the attenuation without proper scaling of the activity, (iii) not initialising the activity and attenuation properly, (iv) using an incorrect or inefficient update scheme, and (v) not handling divisions by zero. We will discuss those pitfalls and provide solutions where possible.

\section{M15-2: Synergistic Longitudinal PET Image Reconstruction}

S. Ellis, A. J. Reader

Department of Biomedical Engineering, King's College London, London, UK

Positron emission tomography (PET) is regularly used to measure changes in physiological and molecular processes at multiple time points, for example in oncological treatment response studies or in brain activation studies. Conventionally, the datasets from these time points are used independently to reconstruct images, and post-reconstruction measurements made and compared. However, this independent dataset approach fails to consider that much of the image detail between scans remains unchanged. By exploiting this inherent similarity between the images, image reconstruction and quantification can be improved. This work proposes to achieve this by incorporating two datasets into a joint reconstruction framework, linked by a regularisation term that penalises differences between the scans. Two such penalty terms were considered, the L1-norm and a non-convex function. Optimisation of the joint objective function was sought using a one-step-late approach. For comparison of methods, a dual time point brain cancer treatment response study was simulated, including three regions of change. Longitudinally penalised reconstructions were compared to conventional maximum likelihood expectation-maximisation (MLEM), and a benchmark method that assumes perfect knowledge of areas of change and averages MLEM reconstructions where no change occurs. Results show that the proposed penalised reconstruction methods achieved a lower error level across the whole brain when compared to MLEM, and error levels comparable to the benchmark method. While the L1 penalised method lost accuracy in regions of change due to over-penalisation of large differences, the non-convex prior maintained and even improved performance in these regions compared to the benchmark method, highlighting the potential of longitudinal reconstruction methods in improving the quantification of multi-dataset PET studies.

This work is funded by the Kingï $i^{1} / 2$ S College London \& Imperial College London EPSRC Centre for Doctoral Training in Medical Imaging $(\mathrm{EP} / \mathrm{L} 015226 / 1)$.

\section{M15-3: Accurate Detector Response Modeling in PET Reconstruction for Systems Using Monolithic} Scintillators $\underline{\text { A. Autret }}^{1}$, J. Bert ${ }^{1}$, E. Preziosi ${ }^{2}$, A. J. González ${ }^{3}$, J. M. Benlloch ${ }^{3}$, D. Visvikis ${ }^{1}$

${ }^{I}$ INSERM UMR1101, LaTIM, Brest, France

${ }^{2}$ Dept. of Molecular Medicine, Sapienza University of Rome, Rome, Italy

${ }^{3}$ Institute for Instrumentation in Molecular Imaging, Valencia, Spain

The detectors used in Positron Emission Tomography (PET) systems have undergone multiple developments over the years to improve both the qualitative and quantitative accuracy of the reconstructed images. One such detector design concept involves the use of monolithic scintillator crystals instead of the classic pixelated scintillators. The monolithic crystal design permits the estimation of the Depth of Interaction (DOI) using the measured scintillation light distribution, which is of particular interest for organ specific PET systems that usually involve small detector rings and as such are associated with large parallax errors that can significantly impact overall system spatial resolution. Unlike in the case of the pixelated scintillator design, not many works have addressed the reconstruction of data from monolithic crystal based PET scanners and more specifically the modeling of the detector response within this context. In this work we propose a model of the detector response for a PET scanner using monolithic crystals, based on a multi-ray approach. In order to evaluate this model, three different approaches were used to estimate the position of interaction in the monolithic crystals, the Center of Gravity (CoG), the Raised To the Power (RTP) and a Gaussian function fitting method. These methods were used to generate list-mode datasets of a point sources phantom using Monte Carlo simulation. The proposed model allows for an improved resolution and intensity homogeneity in the reconstructed images across the FOV. The greatest improvements were achieved when the POI uncertainty was the highest. To further evaluate the expected advantages we will present reconstructed image contrast recovery and noise characterization results using a NEMA image quality phantom. Finally, further improvements in the detector model will be undertaken to include the SiPMs' dark current noise characteristics.

M15-4: Preliminary Study of TV-Constrained-Likelihood-Maximization Image Reconstruction from ListMode TOF-PET Data

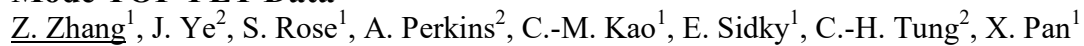


${ }^{1}$ University of Chicago, Chicago, IL, USA

${ }^{2}$ Philips Healthcare, Cleveland, OH, USA

We investigate optimization-based image reconstruction from list-mode TOF-PET data collected with a digital PET system under clinical evaluation. In the study, the reconstruction problem is formulated as an image-TV-constrained data-likelihoodmaximization program. We employ a primal-dual algorithm to reconstruct the image through solving the proposed program. In an attempt to evaluate the reconstruction design, we have performed investigation on reconstructions in computer-simulation study and in real-data study in which data were collected from the standard IEC phantom by use of a digital PET system. Results reveal that the proposed algorithm yields image reconstruction with suppressed noise, enhanced contrast, and sharper boundaries than does a conventional reconstruction algorithm under the study conditions.

\author{
M15-5: Imaging of Freely-Moving Mice in PET Using Partially Rigid Transformations During List-Mode \\ Reconstruction

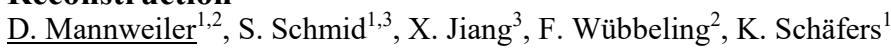 \\ ${ }^{I}$ European Institute for Molecular Imaging - EIMI, University of Münster, Germany \\ ${ }^{2}$ Institute for Computational and Applied Mathematics, University of Münster, Germany \\ ${ }^{3}$ Department of Computer Science, University of Münster, Germany
}

Noninvasive functional imaging of awake and freely- moving mice is a challenging task in preclinical imaging but potentially of interest, as anesthetizing the mouse is no longer required. In order to depict the natural metabolism or the behav- ior response to administered drugs, a non-anesthetized mouse is required. We propose a method to estimate external motion with a four-camera setup observing an infrared illuminated mouse chamber with a frame rate of $100 \mathrm{~Hz}$. Based on captured images synchronized with the PET scanner, we compute rigid transformations for every time step. Based on feature points we acquire series of transformations for the head and for the hip of the mouse. Thus, we compute a motion-corrected PET-image by incorporating these transformations within the reconstruction framework. We performed a proof-of-concept study with [ 18 F]- FDG and [ 18 F]-fluoride.

M15-6: Calibration of a Micro CT System Based on Data Consistency Conditions

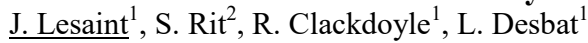

${ }^{I}$ TIMC IMAG, Université Grenoble Alpes, Grenoble, France

${ }^{2}$ CREATIS, Lyon, France

In cone-beam computed tomography (CT), imprecise knowledge of the acquisition geometry can lead to low quality reconstructions. This work investigates geometric calibration using data consistency conditions (DCCs). We assume a circular trajectory with eight global geometric parameters to estimate. The estimation was achieved by minimizing a cost function built on pairwise quantification of the consistency between two projections. By considering pairs of projections, we were able to apply previously published cone-beam consistency conditions for a linear source trajectory. To simplify the implementation of of the DCCs, we backprojected pairs of projections onto a virtual plane parallel to the effective linear source trajectory. A proof of concept was obtained on simulated projection data of a Shepp-Logan phantom. The method was then applied to real data acquired on a micro CT system. Our method significantly improved the quality of the CT image and gave comparable results with a marker-based calibration method. The criterion for success of the calibration was the quality of reconstructed images obtained with the estimated parameters. Although the two methods gave comparable results, the estimated parameters were considerably different. We conclude that the DCC method can achieve effective calibration even though the physical parameters may be poorly estimated.

This work is partially supported by the Agence Nationale de la Recherche (France), Labex CAMI, number ANR-11-LABX-0004-01 and project "DROITE", number ANR-12-BS01-0018.

\title{
M15-7: Composite System Modelling for High Accuracy Brain PET Image Reconstruction using GATE M. A. Belzunce, A. J. Reader \\ Division of Imaging Sciences \& Biomedical Engineering, King's College London, London, United Kingdom
}

High resolution and good quantification is needed in specific regions of the brain in a number of PET brain imaging applications. An improvement in the spatial resolution and in the quantification of the tracer uptake can be achieved by using statistical reconstruction methods with an accurate model of the scanner acquisition process. This model is represented by a system response matrix and needs to include all the factors that contribute to the degradation of the reconstructed images. Monte Carlo simulations are the best method to model the complex physical processes involved in PET, but they have an extremely high computational cost and the system matrix needs to be recomputed for every new scan. Furthermore, for 3D PET the system 
matrix can have billions of elements, therefore at present it is impossible to store in memory during the iterative reconstruction. Consequently, on-the-fly Monte Carlo modelling of the system matrix has been previously proposed by other authors, where a Monte Carlo simulation is used in the forward projector and a simpler analytic model in the backprojector. In this work, we propose a different approach, where a composite system matrix is used, with a complete Monte Carlo model computed with GATE for a small region of interest and a simpler analytic model for the voxels outside that region. We evaluated the feasibility of the method using 2D brain simulations. A Monte Carlo system matrix was generated with GATE for a region of interest centred in the striatum. The brain simulations were reconstructed using the proposed method and compared with the standard reconstruction used clinically, with and without resolution modelling. The proposed method showed an improvement of the contrast and better definition of the structures in the caudate region.

M15-8: Respiratory Gated PET Image Reconstruction Using Composite Image Prior M. Zhang ${ }^{1}$, W. Qi ${ }^{2}$, J. Zhou ${ }^{2}$, G. Wang ${ }^{1}$, M. Teshigawara ${ }^{3}$, T. Kawano ${ }^{4}$, M. Ogawa ${ }^{4}$, E. Asma ${ }^{2}$, W. Wang ${ }^{2}$, J. Qi $^{1}$

${ }^{I}$ Department of Biomedical Engineer, UC davis., Davis, CA, USA

${ }^{2}$ Toshiba Medical Research Institute USA, Inc, Vernon Hills, IL, USA

${ }^{3}$ Toshiba Medical Systems Corporation, Otawa-Shi, Tochigi, Japan

${ }^{4}$ Yokohama City University Hospital, Yokohama-Shi, Kanagawa, Japan

Respiratory motion is a limiting factor of PET image quality in whole-body PET imaging, particularly in the regions near the lungs. Respiratory gating can be used to reduce motion blur by dividing PET data into multiple datasets based on the respiratory phase. However, each gated dataset has less number of coincidence events and hence suffers from higher noise. In comparison, an ungated PET image has much lower noise. In this study, we use a composite image prior that is derived from the ungated image to guide the reconstruction of the gated data so that the noise in gated PET image can be reduced. The method was previously developed for cardiac imaging and was demonstrated using simulated data. Here we apply the method to respiratorygated patient data. Quantitative comparisons show that the method can reduce background noise in gated PET images without sacrificing lesion contrast.

M16A: Posters: signal processing II

Friday, Nov. $4 \quad$ 16:30-18:30 Etoile

M16A-1: Texture-Preserved Low-Dose CT Reconstruction Using Region Recognizable Patch-Priors from Previous Normal-Dose CT Images

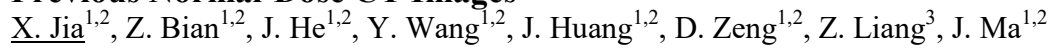

${ }^{1}$ School of Biomedical Engineering, Southern Medical University, Guangzhou, China

${ }^{2}$ Guangdong Provincial Key Laboratory of Medical Image Processing, Guangzhou, China

${ }^{3}$ Departments of Radiology and Biomedical Engineering, State University of New York at Stony Brook, NY, USA

Low-dose computed tomography (LdCT) has been widely used in clinical applications including image-guided biopsy needle and lung screening. With the low radiation dose in data acquiring during the scans, the image quality will degrade due to the excessive quantum noise if there is no adequate noise treatment in processing the data for image reconstruction. For conventional low-dose CT (LdCT) reconstruction, much effort has been spend on the preservation of edges while removing the noise, and not enough attention is paid to texture preserving. However, the image textures are usually recognized as imaging biomarker and play an important role for the tasks, such as lung cancer and nodule detections. In this paper, a texture-preserving method is proposed in LdCT reconstruction. The proposed method consists of the following steps: (1) decompose the normal-dose CT (NdCT) image from FBP reconstruction into overlapping patches. Then, classify these patches into different clusters by Gaussian mixture model (GMM); (2) extract the texture-preserving priors for each cluster from the NdCT image patches with the classified patches into different clusters; (3) decompose the LdCT image from FBP reconstruction into overlapping patches, and then classify these patches by the same GMM in (1); (4) apply the priors in LdCT reconstruction under penalized weighted least-squares criteria. The experimental results show that the proposed method can achieve promising results for LdCT reconstruction in terms of edge and texture preserving.

This work was supported in part by the National Natural Science Foundation of China under Grants 81371544, 61571214, the Science and Technology Program of Guangzhou, China under Grant 201510010039. Zhengrong Liang also was partially supported by the NIH/NCI under grant \#CA143111 and grant \#CA082402.

M16A-2: MRI Multicomponent Relaxometry Based on Compressive Sensing

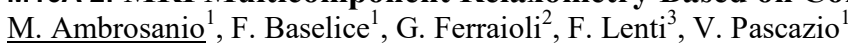

${ }^{1}$ Department of Engineering, Universita' di Napoli Parthenope, Napoli, Italy

${ }^{2}$ Dipartimento di Scienze e Tecnologie, Universita' di Napoli Parthenope, Napoli, Italy

${ }^{3}$ IRIT, Université de Toulouse, Toulouse, France 
In magnetic resonance images, each pixel represents a volume of the imaged body that can contain different tissues undistinguishable in the acquired image. A new technique aimed at evaluating the presence of multiple contributions within each image voxel exploiting relaxometry is described. The methodology discriminates the different tissues by detecting their number and estimating their spin-spin relaxation times. Due to the nature of the acquisition model, the observation matrix is badly conditioned, thus a robust inversion technique, based on Compressive Sensing theory, is applied. A phantom composed of one beaker filled with manganese chloride aqueous solution and oilseed has been specifically built in order to generate volumes containing two different contributions and has been imaged using a Philips Achieva 3.0T scanner. The methodology, even if applied to a limited number of Spin Echo images (five) of the phantom with different Echo Times, is able to discriminate the two contributions and estimate their spin-spin relaxation times, providing values in accordance with existing literature. Further studies will be focused on the extension of the algorithm to other MRI sequences, and on the validation of the methodology on clinical datasets to assess its application impacts.

\author{
M16A-3: Effective Dose Kernel, a Concept for Partial Volume Effect Restoration in Voxel Dosimetry: \\ Introduction and Limitations \\ M. Sanchez-Garcia, R. Lebtahi, A. Dieudonne \\ Nuclear Medicine Dept., Beaujon Hospital, Clichy, France
}

Voxel dosimetry in internal radionuclide therapy is biased by the partial volume effect (PVE) resulting from the finite resolution of imaging systems. The absorbed dose can be calculated from a cumulated activity image by convolving it with a dose-pointkernel (DK). This work proposes compensating the PVE by substituting the DK by an effective one (DKeff); which implicitly deconvolves the system point spread function (PSF) from the input image. The Fourier transform of DKeff is the ratio of Fourier transforms of DK (FDK) and PSF (MTF). It may diverge depending on the combination of DK and PSF. The frequency at which the FDK and MTF intersect gives an indication of the maximum recoverable frequency, Fmax. Noise present in the original image at frequencies above Fmax would be amplified, potentially drowning the signal. In that case, additional regularization may be needed, for example with a Butterworth filter, precluding full PVE compensation. Fmax was calculated for a beta isotope used in therapy (Y90) and for mono-energetic gamma photons of $100 \mathrm{keV}$ (G100) with respect to 3 gaussian PSFs (7, 4 and $2 \mathrm{~mm}$ ). Fmax for Y90 was $\sim 0,0.1$ and $0.4 \mathrm{~mm}-1$ for 7, 4 and $2 \mathrm{~mm}$ FWHM. For G100, Fmax was $0.17,0.33$ and $>0.5 \mathrm{~mm}-1$ for 7,4 and $2 \mathrm{~mm}$ FWHM. In theory, the effective DPK should allow to fully or partially recover spatial resolution, however with current system resolutions (FWHM $>4 \mathrm{~mm}$ ) the usefulness of the method is limited for noisy images.

M16A-4: Segmentation and Kinetic Modeling of Human Arteries in PET/CT Imaging M. Alenezi $^{1}$, M. Bentourkia ${ }^{1}$, A. Khalil ${ }^{2}$

${ }^{I}$ Nuclear Medicine and Radiobiology, Université de Sherbrooke, Sherbrooke, Canada

${ }^{2}$ Medicine, Université de Sherbrooke, Sherbrooke, Canada

Purpose: Human arteries in elderly are subject to accumulation of fatty deposits forming atheromatous plaques. The plaque can develop into calcifications and provoke rigidity of the arteries or it can rupture and occlude peripheral arteries leading to vascular complications. Calcifications were measured with CT, and the high PET-FDG metabolism was associated with inflammation, a possible precursor of plaque rupture. In this work we present repeated measurements with $\mathrm{CT}$ and PET at $\mathrm{T}=0$ and $\mathrm{T}=12$ months later in three groups of elderly subjects: normal $(\mathrm{N})$, hypercholesterolemic $(\mathrm{H})$ and with stable angina $(\mathrm{A})$. Methods: CT and PET-FDG images were first coregistered for calcification and inflammation localization, and then the arteries were segmented on CT and PET images using active contours, Chan-Vese, thresholding and factor analysis approaches. The segmentation allows to demonstrate if the calcification is accompanied with the inflammation and its progression during 12 months. Results: The calcifications were present at some portions of the arteries in all the subjects even in the normal group. The results showed that the most noticeable SUV changes between T0 and T12 were in non-calcified arteries of the normal group. In the three groups, the calcified arteries showed no significant differences between T0 and T12 while significant differences were observed for the noncalcified arteries. In non-calcified arteries at T0, N presents a low FDG uptake when compared to A and H. However, at T12, the SUV in N increased significantly when compared to A and reached values comparable to $\mathrm{H}$. Conclusions: The quantitative analysis with FDG-PET/CT is efficient in the localization of the atheromatous plaque and evaluation of its progression instead of global evaluations with systemic inflammatory biomarkers. This technique is able to detect the progress of the disease within one year in order to prevent subsequent complications and to potentially assess the effects of medication.

M16A-5: Automated Multiscale 3D Feature Learning for Vessels Segmentation in Thorax CT Images $\underline{\text { T. Konopczynski }}^{1,2,3}$, T. Kröger ${ }^{3}$, L. Zheng ${ }^{1}$, C. S. Garbe ${ }^{2}$, J. Hesser ${ }^{1,2}$

${ }^{I}$ Experimental Radiation Oncology, Dept. of Radiation Oncology,

University Medical Center Mannheim, Heidelberg University, Heidelberg, Germany

${ }^{2}$ Interdisciplinary Center for Scientific Computing (IWR), Heidelberg University, Heidelberg, Germany

${ }^{3}$ Volume Graphics GmbH, Heidelberg, Germany 
We address the vessel segmentation problem by building upon the multiscale feature learning method of Kiros et al., which achieves the current top score in the VESSEL12 MICCAI challenge. Following their idea of feature learning instead of handcrafted filters, we have extended the method to learn 3D features. The features are learned in an unsupervised manner in a multiscale scheme using dictionary learning via least angle regression. The 3D feature kernels are further convolved with the input volumes in order to create feature maps. Those maps are used to train a supervised classifier with the annotated voxels. In order to process the $3 \mathrm{D}$ data with such a large number

of filters a parallel implementation has been developed. The algorithm has been applied on the example scans and annotations provided

by the VESSEL12 challenge. Each scan is of dimension $512 \times 512 \times 512$. Annotations contain in total 882 annotated voxels (vessel/non vessel). We have compared our setup with Kiros et al. by running their implementation. Our current results show an improvement of classification accuracy from $96.66 \pm 1.10 \%$ to $97.24 \pm 0.90 \%$.

This work has received funding from the European Union's Seventh Framework Programme under grant agreement No. 607817.

M16A-6: Bayesian MRI Noise Filtering in Complex Domain

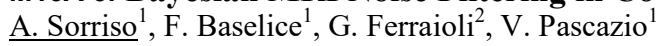

${ }^{I}$ Department of Engineering, Universita' di Napoli Parthenope, Napoli, Italy

${ }^{2}$ Dipartimento di Scienze e Tecnologie, Universita' di Napoli Parthenope, Napoli, Italy

A novel Bayesian filter for Magnetic Resonance Imaging denoising, based on the maximization of the a posteriori distribution of the image to be restored, is proposed. The novelty of the approach consists in working in the complex domain, allowing the joint regularization of the real and the imaginary parts of the acquired signal. In order to reduce the amount of noise while preserving the details, Markov Random Field theory has been exploited for statistically modeling the imaged slice without any external constraint. The specific adopted Local Gaussian Markov Random Field allows to tune the filter strength to the local behavior of the image, preserving the edges and the small details while strongly regularizing smooth areas. The methodology has been tested on a $466 \times 405$ pixels Spin Echo brain image acquired using a Philips Achieva 3.0T scanner and compared to other widely adopted approaches, such as Linear Minimum Mean Square Estimator and Nonlinear Anisotropic Diffusion filters, highlightening the strengths of the proposed filter: details are well preserved and smooth areas are well restored. The methodology will be further compared to other recently proposed noise filters, such as those based on non-local means, and it will be tested on several datasets with different characteristics to asses its potentialities.

M16A-7: 3D Denosing of Magnetic Resonance Images Exploiting Bayesian Estimation Theory F. Baselice $^{1}$, G. Ferraioli ${ }^{2}$, V. Pascazio ${ }^{1}$

${ }^{I}$ Department of Engineering, Universita' di Napoli Parthenope, Napoli, Italy

${ }^{2}$ Dipartimento di Scienze e Tecnologie, Universita' di Napoli Parthenope, Napoli, Italy

A novel noise filtering statistical technique in the magnetic resonance imaging field, based on Bayesian estimation theory and Markov Random Fields, applied to 3D stacks, is presented. For each pixel of the image stack, Markov Random Fields model the three dimensional neighborhood in order to estimate the correlation among pixels and to spatially adapt the filtering intensity to the local behavior of the image. The correlation values, estimated in the Bayesian framework, are exploited to distinguish smooth areas, where a strong filtering is suitable, and sharp areas, where a weak filtering is recommended in order to preserve edges and small details. First results on a realistic simulated dataset, together with a comparison with the widely adopted Nonlinear Anisotropic Diffusion filter, are reported, showing the effectiveness of the proposed approach in restoring the image, well balancing noise reduction and details preservation. The visual results are confirmed by the Mean Square Error and Structural Similarity Index, adopted as synthetic quality metrics. The methodology will be tested on real datasets acquired with different imaging sequences and configurations, and compared to other filtering approaches, for a wider validation and to asses its practical potentialities.

M16A-8: FDG-PET and the Assessment of Spinal Cord Metabolism in Amyothrophic Lateral Sclerosis (ALS) A. M. Massone ${ }^{1}$, C. Campi ${ }^{2}$, M. C. Beltrametti ${ }^{3}$, C. Marini $^{4}$

${ }^{I}$ CNR - SPIN, Genova, Italy

${ }^{2}$ Dipartimento SBAI, Università degli Studi di Roma, Roma, Italy

${ }^{3}$ Dipartimento di Matematica, Università degli Studi di Genova, Genova, Italy

${ }^{4}$ Sezione di Genova, CNR - IBFM, Genova, Italy

Synaptic activity in the nervous system consumes glucose. Therefore FDG-PET may in principle provide iconographic representations of glucose utilization impairment in neurodegenerative diseases and, specifically, in Amyothrophic Lateral Sclerosis (ALS). We developed a computational method that applies a modern generalization of the Hough transform to identify the spinal canal and the spinal cord in X-ray Computed Tomography images of ALS patients and combines this information with 
the functional data provided by FDG-PET to measure the spinal marrow metabolism in detail. We used this method to evaluate differences in all spinal cord volumes between patients and normal subjects and to assess possible correlations between disease severity and metabolism functional data.

M16A-9: Noise rejection in monolithic SiPM based PET detector

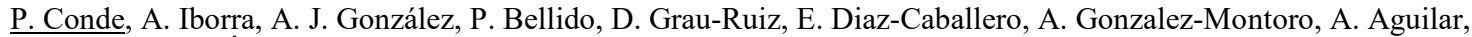
M. J. Rodriguez-Álvarez, M. Seimetz, L. Moliner, S. Sánchez, F. Sánchez, A. Soriano, J. P. Rigla, J. M. Benlloch

Institute for Instrumentation in Molecular Imaging (I3M), Valencia, Spain

PET detectors based on monolithic scintillators in combination with Silicon Photomultiplier as photodetectors have larger influence of dark counts because of the larger area required for detecting one event. This typically implies increasing the presence of electronic noise from the readout. These factors can degrade the signal to noise ratio (SNR) of the detector block and, as a result, the SNR of the reconstructed PET image. Since the SNR is significantly affected by counting statistics, it could be improved by measuring longer or to some extent by increasing the activity, but in practice this is usually not desirable. Instead of increasing the acquiring time, noisy events can be filtered in a detector basis. In this work we have simulated a PET detector block, formed by a thick LYSO scintillator crystal $\left(50 \times 50 \times 20 \mathrm{~mm}^{3}\right)$ and the custom SiPMs parts from SensL for the MindView collaboration. The photo-conversion process of the incident gamma-ray has been simulated using the GEANT4 framework. The optical behaviour of the scintillator has been modeled with the computer graphics algorithm called photon mapping. Finally, the SiPM response has been simulated with the GosSiP framework. The obtained simulated data is then filtered by means of a matched filter to a theoretical model. Moreover, the matched filter in the spatial domain corresponds to the cross-correlation between the measured data and the LD model allowing to define a metric suitable for assessing the similarity between the two LDs. Using this metric a threshold filter can be defined to only allow signals that resemble the validated LD model and disregard signals that having passed all the pre-filters do not follow the LD model, as pure dark noise events, multi-interaction events with long travelled distance, pileup events, etc. Rejected events represents a $4 \%$ of the simulated data. This method allows increasing either the scintillation volume or the system rates without worsening the reconstructed image SNR.

\author{
: A Random Location Index Classifier for Computer-Aided Diagnosis (CADx) of Colorectal Polyps \\ Y. Hu ${ }^{1,2}$, P. J. Pickhardt ${ }^{3}$, W. Zhu ${ }^{1}, \underline{\text { Z. Liang }}{ }^{2}$ \\ ${ }^{I}$ Department of Applied Mathematics and Statistics, Stony Brook University, Stony brook, NY, USA \\ ${ }^{2}$ Department of Radiology, Stony Brook University, Stony brook, NY, USA \\ ${ }^{3}$ Department of Radiology, University ofWisconsin School of Medicine and Public Health, Madison, WI, USA
}

Feature classification plays a very important role in computer-aided diagnosis (CADx) of suspicious lesions. As an expansion of k-nearest neighbor (KNN), location index (LI) algorithm has a distinguished efficiency performance for CADx tasks compared with other widely used classifiers like SVM and random forest (RF). Bootstrap aggregating (bagging), on the other hand, is a well-known technique for reducing classifiersï $i \frac{1}{2}$ variance, which is one component of the error in classification. In this paper, the bagging technique was combined with the LI algorithm as a new integrated classifier for achieving a higher AUC measure. Experiments were performed by the use of two polyp databases: one dataset contains 153 lesions (polyps), including 116 neoplastic lesions and 37 hyperplastic lesions and the other dataset contains 384 lesions, including 332 neoplastic lesions and 52 hyperplastic lesions. Noticeable gains by the proposed new integrated classifier were quantified by the AUC measure on both datasets. Keywords: CADx, classification, bagging, location index, ROC analysis

\author{
M16A-11: Statistical Feature Selection and Classification Models for Alzheimer's Disease Progression \\ Assessment \\ A. Domínguez, J. Ramírez, J. M. Górriz, F. Segovia, D. Salas-Gonzalez, F. J. Martínez-Murcia, I. A. Illán \\ Signal Theory, Networking and Communications, University of Granada, Granada, Spain
}

Alzheimer's disease (AD) is a progressive course neurodegenerative disease and the most common cause of dementia. Prediction of mild-cognitive impairment (MCI) to $\mathrm{AD}$ is still a challenging problem. As the disease progresses, well defined brain areas are affected and neuropsychological clinical scores such as the Mini Mental State Examination (MMSE) and cognitive assessment subscale (ADAS-Cog) Alzheimer's disease reveals cognitive decline in MCI patients. This paper shows statistical feature selection and classification models for tissue-segmented T1 magnetic resonance images (MRI) in order to predict whether an MCI patient will evolve to AD within 6-12 months. It was found that, independently of the system tested, using MRI data and cognitive assessments of two sessions improves significantly the classification accuracy. Moreover, the atlas-based t-test support vector machine (SVM) system showed its ability to correctly identify brain areas affected by the progression of the disease yielding the best results when compared to principal component analysis (PCA) and partial least squares (PLS) feature reduction methods. 
This work was partially supported by the MINECO under the TEC2015-64718-R project and the Consejería de Innovación, Ciencia y Empresa (Junta de Andalucía, Spain) under the P11-TIC-7103 Excellence Project.

\author{
M16A-12: A Novel Fast PCA Based Denoising Technique for Ultra-High-Rate Computed Tomography \\ M. Taki ${ }^{1}$, K. Zarei $^{2}$, Z. Mortezai ${ }^{1}$ \\ ${ }^{I}$ Electrical engineering, university of qom, Qom, Iran \\ ${ }^{2}$ iMinds-VisionLab, University of Antwerp, Wilrijke, Belgium
}

Increasing the X-ray tomography acquisition rate is of high importance especially in medical applications as well as dynamic processes investigation. Thanks to the high brilliance of synchrotron radiation, it is possible to reduce the exposure time and do a tomographic scan with a sub-second temporal resolution which allows following dynamic processes in 4D (3D space + time). On the other hand, increasing the acquisition rate leads to more background noise and afterglow artifacts which strictly limit the advantages of high rate scan. We apply a new fast denoising technique using universal properties of eigen-spectrum of random covariance matrices. Our proposed technique is established based on the principal component analysis (PCA) of redundant data which shows that most of the signal-related variance is contained in a few components, whereas the noise is spread over all components. Extensive numerical evaluations of the proposed technique on a real dataset, were acquired at the TOMCAT beamline with its ultra-fast endstation, show significant improvement in the quality of reconstructed images and elimination of noise.

\author{
M16A-13: Development and Evaluation of Two Interventricular Sulcus Extraction Methods for Cardiac PET \\ J. Wang, T. Feng, B. Tsui \\ Radiology, Johns Hopkins University, Baltimore, MD, USA
}

The goal is to develop and evaluate two methods to extract interventricular sulcus (IS) from 4D cardiac gated (CG) myocardial perfusion (MP) PET images for use in quantitative cardiac motion estimation (ME). In Method 1, the entire myocardium was first segmented from the 3D MP PET image of each CG frame by $3 \mathrm{D}$ region growing. The left ventricle (LV) was then extracted by 3D image erosion and dilation, and the right ventricle (RV) by subtracting the LV from the myocardium. The IS was finally identified from the overlap of the extracted LV and RV. In Method 2, the inner boundary (InB) of the RV was first extracted by segmenting the blood pool (BP) within the RV using 3D region growing. The septal side of the BP boundary, or the LV outer boundary (OutB) within the RV, was separated out. We then identified segments of the LV OutB on the anterior (Ant) and posterior (Post) sides of the RV. The three extracted line segments were fitted with a B-spline curve while the lateral side of the RV InB was extrapolated using B-spline. The intersection points of the fitted curves were identified as the Ant and Post IS which, when assembled from all short-axis images, formed entire IS. The two IS extraction methods were applied to realistic CG MP PET images simulated from the 4D XCAT phantom at PET system resolution from $0.6 \mathrm{~mm}$ to $4.5 \mathrm{~mm}$ for 4 CG frames. The accuracy of the extracted IS were compared with the true IS from the XCAT. For both methods, the errors of the extracted IS locations increased with poorer PET system resolution with the Ant IS showing lower accuracy than the Post IS. Method 1 achieved lower accuracy than Method 2 and failed to provide reliable estimates at $4.5 \mathrm{~mm}$ system resolution. We conclude the Bspline based interpolation and extrapolation curve fitting method was capable of extracting the IS with high reliability and accuracy from the 4D CG MP PET images obtained from state-of-the-art and PET systems and will be useful in the cardiac ME from CG PET images.

\author{
M16A-14: Three-Dimensional Blood Vessels Detection from Small Number of Views CT Reconstruction \\ E. A. Mohamed, E. A. Rashed \\ Image Science Lab., Dept. of Mathematics, Faculty of Science, Suez Canal University, Ismailia, Egypt
}

Accurate and efficient detection of blood vessels in medical images is important in several applications such as accurate diagnosis and surgical planning. Computed Tomography (CT) is a common imaging modality used for blood vessels imaging. It can provide a high quality images for both diagnosis and treatment purposes. However, CT is known as a source of high radiation dose especially for cases required a follow up scans in short time. Reducing the CT dose is known to produce statistical noise and artifacts that significantly reduce the image quality. So, the detection of blood vessels from CT images reconstructed from lowdose acquisitions is challenging due to noise, small artifacts and low-resolution of vessel structures. In this paper, we propose a method for three-dimensional (3D) blood vessels detection from low-dose CT images. The proposed method is implemented in two stages. First, is a preprocessing stage to reduce noise. In the second stage, we use skeletonization method to extract the centerline of 3D tubular object. Then, we use the blood vessels skeleton to construct a graph model for 3D vascular network. Finally, we use the graph model to detect the blood vessels. The proposed method is tested using 3D blood vessel-like phantom and achieved results are promising.

M16A-15: Indirect Bilateral Filtering to Sharpen and Denoise Dynamic PET Images

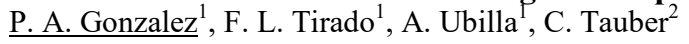


${ }^{I}$ DCI, Universidad Católica del Maule, Talca, Chile

${ }^{2}$ UMRS INSERM U930, Université Francois Rabelais Tours, Tours, France

Positron Emision Tomography (PET) images are inherently affected by noise and low spatial resolution that may lead to incorrect estimations of the real uptake of the radiotracer in tissues. In this work, a new method for enhancing the signal-to-noise ratio (SNR) of dynamic PET images is presented. The proposed method consists in a sharpening and edge preserving filter based upon local structure analysis. We use an indirect structure tensor that embeds local orientations of features in the image. We exploit the spatial and temporal information along the entire sequence to adapt the bilateral filter to denoise while sharpening edges between functional regions. Comparative experimentations on realistic simulations validate the potential of the proposed method.

M16A-16: PET Image Denoising Using Anatomically Guided Non-Local Euclidean Medians $\underline{\text { J. Dutta }}^{1,2}$, G. El Fakhri ${ }^{2}$, Q. Li ${ }^{2}$

${ }^{I}$ Electrical and Computer Engineering, University of Massachusetts Lowell, Lowell, MA, USA

${ }^{2}$ Gordon Center for Medical Imaging, Massachusetts General Hospital, Boston, MA, USA

Qualitative and quantitative interpretation of PET images is often a challenging task due to high levels of noise in the images. This often necessitates a denoising step following PET reconstruction. Non-local means (NLM) denoising, which computes similarities between non-neighboring image patches, has been successfully applied to PET images. In this work, we utilize the Euclidean median to enhance the denoising performance of NLM along tissue and organ boundaries in PET images. The underlying idea behind this approach is to replace the L2 norm in NLM with more robust L1 regression. To further improve denoising performance along sharp edges, we use anatomical guidance to limit the spatial window for non-local similarity computation. We tested this anatomically guided non-local Euclidean medians (ANLEM) denoising technique by performing simulations on the BrainWeb digital phantom. ANLEM was compared to traditional NLM and NLEM denoising. Our results show the ANLEM and NLEM are robust and particularly effective for high noise levels. Furthermore, ANLEM leads to noticeable improvement in denoising accuracy along tissue boundaries.

\section{M16A-17: Applying J-Optimal Channelized Quadratic Observers (J-CQO) to a Clinical Imaging Study for} Ovarian Cancer Detection

M. Kupinski, A. Rouse, A. Gmitro

Medical Imaging, University of Arizona, Tucson, AZ, USA

A dataset of 206 clinical images were acquired with a novel confocal fluorescence microlaparoscope to test the imaging system's ability to detect ovarian cancer in vivo. Standard histologic evaluation of extracted tissue samples was performed and used as the gold standard of disease diagnosis. Trained human observers from different specialties viewed the microlaparoscopic images. Receiver operating characteristic (ROC) curves were generated using these scores and the gold standard histopathologic diagnosis. Using LABROC software the area under the ROC curve (AUC) was calculated as a performance metric. For comparison AUC estimates were evaluated mathematically using the J-optimal Channelized Quadratic Observer (J-CQO). To calculate the J-CQO, normal likelihoods are assumed and the first- and second-order statistics of the channelized image data are estimated from training images. The novelty and primary advantage of J-CQO is that second-order statistics can be reliably estimated from a small quantity of training images. This is accomplished by reducing the dimensionality of the image data through a linear transform, i.e. a channel matrix. The J-CQO method optimizes the channel matrix for the detection task by using J-divergence as a surrogate figure of merit for AUC. The J-CQO AUC was 0.90 for four channels, 91 images used to optimize the channel matrix, and 12 images used for a leave-one-out testing and training AUC estimate. This AUC value is very similar to those obtained in prior human observer studies. The visible difference between the healthy and cancerous images can be qualitatively explained as a texture difference. This AUC comparison between human and mathematical observers indicates the importance of using second-order statistics to mimic human observers. The J-CQO method offers a unique and novel approach to estimating second-order statistics from a small quantity of high-dimensional image data.

This material is based upon work supported by the National Institutes of Health under R01-EB000803 and P41-EB002035 and the National Science Foundation under CHE-1313892.

M16A-18: MR-Guided PET Image Restoration for Neurological Applications

M. S. Tahaei ${ }^{1,2}$, A. J. Reader ${ }^{3}$, D. L. Collins ${ }^{1,2}$

${ }^{I}$ Dept. of Biomedical Engineering, McGill University, Montreal, Canada

${ }^{2}$ McConnell Brain Imaging Centre, Montreal Neurological Institute, Montreal, Canada

${ }^{3}$ Dept. of Biomedical Engineering, Divi. of Imaging Sciences and Biomedical Engineering, King's College London, St. Thomas'

Hospital, London, Uk 
With the growing interest in conducting multi-centre and multi-modality studies on neurological disorders, post-reconstruction PET image enhancement methods that take advantage of available anatomical information are becoming more important. In this work, a novel method for denoising PET images using the subject's registered T1-weighted MR image is proposed. The proposed method combines the non-local means approach with the twicing strategy from the image denoising literature to restore a reconstructed PET image. Preliminary analysis shows promising improvements in peak signal to noise ratio (PSNR) and contrast recovery coefficients (CRC) of the lesions when denoising simulated images reconstructed using the MLEM algorithm.

M16A-19: Spatial Response of Double-Sided Strip High-Purity Germanium Detectors for SPECT Imaging $\underline{\text { R. S. Perea }}^{1,2}$, D. L. Campbell ${ }^{2,3}$, S. Shokouhi ${ }^{2,3}$, T. E. Peterson ${ }^{2,3}$

${ }^{I}$ Physics and Astronomy, Vanderbilt University, Nashville, TN, USA

${ }^{2}$ Institute for Imaging Sciences, Vanderbilt University Medical Center, Nashville, TN, USA

${ }^{3}$ Radiology and Radiological Sciences, Vanderbilt University Medical Center, Nashville, TN, USA

We are developing a dual-headed small-animal SPECT system using double-sided strip HPGe detectors. HPGe provides superior energy resolution (2@131 keV) at the Advanced Photon Source. These data reveal efficiency losses that extend more than 100 micrometers from the strip edges as well as variations in spatial resolution across the strip area using our current processing techniques. From these data we will develop distortion and efficiency correction techniques to improve our current position estimation method, as well as explore advanced signal processing methods to improve performance. Improvements in event estimation should remove artifacts and lead to better contrast and sensitivity in SPECT images.

M16A-20: Rapid Measurement of Fluorescence Lifetimes Using SiPM Detection and Waveform Sampling H.-M. Tsai ${ }^{1}$, J. S. Souris ${ }^{1}$, H. Kim ${ }^{1}$, S.-H. Cheng ${ }^{1}$, C.-T. Chen ${ }^{1}$, L.-W. Lo ${ }^{1,2}$, L. Chen ${ }^{3}$, C.-M. Kao ${ }^{1}$

${ }^{I}$ Department of Radiology, The university of chicago, Chicago, United States

${ }^{2}$ Institute of Biomedical Engineering and Nanomedicine, National Health Research Institute, Miaoli, Taiwan

${ }^{3}$ Department of Surgery, University of Illinois at Chicago, Chicago, United States

In this work we demonstrate, for the first time, the feasibility of making rapid fluorescence lifetime measurements with analog silicon photomultipliers (SiPMs) and waveform sampling techniques. Fluorophore excitation was provided by means of a lowpower fiber-coupled pulsed laser diode operating at $405 \mathrm{~nm}$, whose beam had been expanded to a diameter of $2 \mathrm{~mm}$ before impinging upon the sample. A portion of the sample's isotropic fluorescent emission was collected orthogonal to the incident excitation beam via an analog SiPM and analyzed by waveform sampling at $2.5 \mathrm{GS} / \mathrm{s}$. Fluorophores for system evaluation were selected such that their fluorescence lifetimes differed significantly from one another but their excitation and emission wavelength's maxima did not, and included Coumarin 6, Coumarin 314, and Coumarin 343, each dissolved in ethanol. Measurements made of a non-fluorescent isotropically-scattering specimen were used to derive the system's response function for subsequent deconvolution with fluorescent dye measurements. The resulting single-exponential decay functions revealed fluorescence lifetimes that closely correlated with lifetime measurements made of the same samples on commercially available instruments.

M16A-21: Atlas-Based Myocardial Segmentation Using ${ }^{18} \mathrm{~F}$ Myocardial PET J. W. Kim ${ }^{1}$, S. Seo ${ }^{1}$, H. S. Kim², D.-Y. Kim², J.-J. Min², J. S. Lee ${ }^{1}$

${ }^{I}$ Nuclear Medicine, Seoul National University, Seoul, South Korea

${ }^{2}$ Nuclear Medicine, Chonnam National University Hwasun Hospital, Hwasun, Jeonnam, South Korea

In this study, we proposed a new atlas-based segmentation for ${ }^{18} \mathrm{~F}$ - labeled myocardial PET, where one atlas template with the most similar distribution as a source PET image is selected from a pool of template candidates and then pre-build myocardial ROIs of the template is used to segment the source PET image. We first evaluated the proposed method by comparing its results with original ROI. The correlation $\left(r_{2}\right)$ between the normalized source image and the best selected template were average 0.93 in the template-space evaluation and 0.96 in the individual-space evaluation. The ROIs of the selected template were well matched to the myocardium regions of the normalized source image. Further evaluation using perfusion parametric image showed similar polarmaps and highly-correlated average values $\left(r^{2}=0.91\right)$ in 17-segment ROIs. Accordingly, the proposed atlas-based segmentation showed its potential as an adequate method for segmenting myocardial image sufficiently well and appropriate for building polarmap in ${ }^{18} \mathrm{~F}$-labeled myocardial PET imaging. Therefore, the atlas-based segmentation would be useful for analyzing various state of myocardium with high accuracy.

M16A-22: Medical Image Processing Using Brain Emulation $\underline{\text { C.-L. Sotiropoulou }}^{1,2}$, P. Luciano ${ }^{3,2}$, S. Gkaitatzis ${ }^{4}$, M. Viti ${ }^{5}$, A. Giuliano ${ }^{2}$, A. Retico ${ }^{2}$, S. Citraro ${ }^{1,2}$, P. Giannetti ${ }^{2}$, M. Dell'Orso ${ }^{1,2}$ ${ }^{I}$ University of Pisa and INFN Pisa Section, Pisa, Italy

${ }^{2}$ INFN Pisa Section, Pisa, Italy 
${ }^{3}$ University of Cassino and Southern Lazio, Cassino, Italy

${ }^{4}$ Aristotle University of Thessaloniki, Thessaloniki, Greece

${ }^{5}$ Universite' Pierre et Marie Curie, Paris, France

We propose the use of an algorithm based on the learning process of the human vision as an edge-enhancing filter for medical images. We set up an experiment to test its impact on the performance of decision-making algorithm working on brain magnetic resonance data. The execution times of similar filters can become unpractical on real 3D or higher dimensional data, if implemented on CPU. We developed an innovative and high performance embedded system for real-time pattern matching. The design uses Field Programmable Gate Arrays (FPGAs) and the powerful Associative Memory chip (an ASIC) to achieve realtime performance. The implementation of this edge-enhancing filter on FPGAs and Associative Memories is expected to positively impact on medical devices for real-time diagnosis (e.g. diagnostic ultrasound) and for image processing steps in medical image analysis where computing power is a limiting factor.

This work is partly funded by European Commission FP7 People grant FTK 324318 FP7-PEOPLE-2012-IAPP.

M16A-23: Novel Active Contour Model-Based Automated Segmentation of PET Images M. Zhuang ${ }^{1}$, R. A. Dierckx ${ }^{1}$, H. Zaidi ${ }^{2}$

${ }^{I}$ Nuclear Medicine and Molecular Imaging, University of Groningen, Groningen, Netherlands

${ }^{2}$ Nuclear Medicine and Molecular Imaging, Geneva University Hospital, Geneva, Switzerland

Although positron emission tomography (PET) has shown potential to improve the accuracy of targeting in radiation therapy planning and assessment of response to treatment, the boundaries of tumours are not easily distinguishable from surrounding normal tissue owing to the low spatial resolution and inherent noisy characteristics of PET images. In this work, a novel method for automatic segmentation using an active contour model is presented. The algorithm incorporates histogram fuzzy C-means clustering and localized and textural information to constrain the active contour to detect boundaries in an accurate and robust manner. Moreover, the lattice Boltzmann method is used as an alternative approach for solving the level set equation to make it faster and suitable for parallel programming. The proposed method was compared with the contourlet-based active contour algorithm and Schaefer's thresholding method using phantom and clinical studies. For the simulated phantom studies, MASAC and CAC provide similar segmentations of the different lesions, while ST fails to achieve reliable results. For the clinical datasets ( 2 cases with connected high-uptake regions excluded), CAC provides for the lowest mean RE ( $-8.38 \% \pm 27.49 \%)$, while MASAC achieves the best mean DSC $(0.71 \pm 0.09)$ and mean CE $(53.92 \% \pm 12.65 \%)$, respectively. Our results demonstrate that the developed novel PET segmentation algorithm is applicable to various types of lesions and is capable of producing accurate and consistent target volume delineations, potentially resulting in reduced intra- and inter-observer variability commonly observed when using manual delineation.

\author{
M16A-24: Iterative Structural Functional Synergistic Resolution Recovery (iSFS-RR) for Improving the \\ Performance of PET Quantification on Focal Cortical Dysplasia \\ J. Silva-Rodríguez $^{1,2}$, J. Cortés ${ }^{2}$, X. Rodríguez-Osorio ${ }^{3}$, Á. Ruibal ${ }^{2}$, C. Tsoumpas ${ }^{4}$, P. Aguiar ${ }^{1,2}$ \\ ${ }^{I}$ Molecular Imaging, Health Research Institute, Santiago de Compostela, Galicia, Spain \\ ${ }^{2}$ Nuclear Medicine, University Hospital, Santiago de Compostela, Galicia, Spain \\ ${ }^{3}$ Neurology, University Hospital, Santiago de Compostela, Galicia, Spain \\ ${ }^{4}$ Div. of Biomedical Imaging, University of Leeds, Leeds, United Kingdom
}

Statistical Parametric Mapping (SPM) is a widely used quantification technique in brain imaging modalities as Positron Emission Tomography (PET), but its sensitivity in epilepsy has been reported to be relatively low $(60 \%)$. This may be related with the low resolution of PET images and its inability for detecting small lesions, such as those observed in focal cortical dysplasia (FCDs), a common cause of epilepsy. Image processing techniques that can provide improvements on PET resolution are promising in order to improve the localization of these focal hypometabolisms. In this study, we investigate the impact of the recently developed iSFS-RR on the sensitivity of SPM for the localization of small focal hypometabolisms associated with FCD. Fourteen patients previously diagnosed with epilepsy caused by FCD were evaluated using 18F-FluoroDeoxyGlucose (FDG) PET images. The analysis was complemented with SPM comparing the patient to a database of 87 controls. PET images were corrected for the Partial Volume Effect (PVE) using iSFS-RR. The SPM results with and without the PVE correction were compared. On noncorrected images, SPM reported the location of the epileptic focus on $64.3 \%$ of the patients. After the correction, the SPM statistical analysis showed a sensitivity of $85.7 \%$, which implies an improvement of a $19.4 \%$. iSFS-RR improved the SPM analysis as the results had better sensitivity compared with visual inspection $(+28.6 \%)$ and SPM maps without PVC $(+19.4 \%)$. The observed improvements were compatible to those previously obtained for temporal lobe epilepsy. The PVE correction with the iSFS-RR method showed very good potential to improve the sensitivity of SPM on the detection of the hypometabolisms associated with FCD in refractory epilepsy. 
This work was supported in part by public Fondo de Investigaciones Sanitarias (DTS14/00158). P. Aguiar is awarded a public fellowship from Xunta de Galicia (POS-A/2013/00). J. Pardo-Montero acknowledges the support of Instituto de Salud Carlos III (Miguel Servet grant CP12/03162).

M16A-25: Multiple Timestamp Estimation with Analog Silicon Photomultipliers

$\underline{\text { E. Venialgo }}^{1}$, K. O'Neill' ${ }^{2}$, S. Gnecchi ${ }^{2}$, C. Jackson ${ }^{2}$, E. Charbon ${ }^{1}$

${ }^{I}$ Electrical Engineering, Delft University of Technology, Delft, Netherlands

${ }^{2}$ SensL Technologies Ltd., Cork, Ireland

Abstract - In 2011 we introduced the concept of timestamping multiple visible light photons from a scintillation pulse. Until now, this required the use of multichannel digital silicon photomultipliers (MD-SiPMs). In this work, we show how to transform an analog SiPM (A-SiPM) into a MD-SiPM and we demonstrate the advantages of doing so. We develop the theoretical framework supporting this analysis and we verify our results with Monte Carlo simulations and with experimental characterization

M16B: Posters: quantitative imaging II

Friday, Nov. $4 \quad$ 16:30-18:30 Etoile

M16B-1: First Clinical Results of a CZT-Based Blood Counter for Quantitative Molecular Imaging Studies $\underline{\text { R. Espagnet }}^{1}$, A. Frezza ${ }^{1}$, J.-P. Martin ${ }^{2}$, L.-A. Hamel ${ }^{2}$, J.-M. Beauregard ${ }^{3,4}$, P. Després ${ }^{1}$

${ }^{I}$ Département de physique, de génie physique et d'optique, Université Laval, Québec, Qc, Canada

${ }^{2}$ Département de physique, Université de Montréal, Montréal, Qc, Canada

${ }^{3}$ Department of Radiology and Nuclear Medicine and Cancer Research Center, Université Laval, Québec, Qc, Canada

${ }^{4}$ Div. of Nuclear Medicine, Dept of Medical Imaging and Oncology Axis of CHU de Québec Research Center, CHU de QuébecUniversité Laval, Québec, Qc, Canada

In Positron Emission Tomography (PET), a semi-quantitative analysis of uptake is possible with Standardized Uptake Values. To obtain a more robust quantitative analysis, the blood time-activity curve can be used as an input function for pharmacokinetic modeling of tracer uptake. For this purpose, a new device for the determination of blood activity as a function of time was developed. The device was designed to be compact, easy to use and reliable. It relies on a peristaltic pump for continuous blood

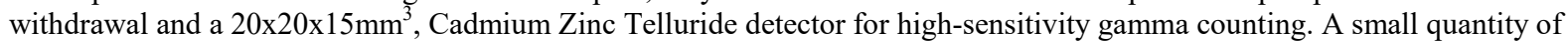
blood from the patient flows in a tubing that enters a shielded enclosure where counting is performed. This paper presents typical time-activity curves obtained with this device and reports on its use in a clinical setting. More specifically, time-activity curves from prostate cancer patients undergoing dynamic ${ }^{18} \mathrm{~F}$-fluoromethylcholine PET studies are presented. The device was used during the first 12 minutes post-injection and withdrew blood at a rate of $2 \mathrm{ml}$ per minute. Results suggest that the device performs as expected in terms of signal to noise. Its use adds a small overhead of approximately 5 min to the imaging study. These results suggest that the device can be used routinely and reliably to extract more quantitative and potentially better imaging biomarkers from molecular imaging studies.

M16B-2: Ultra Efficient and Robust Estimation of the Attenuation Map in PET Imaging

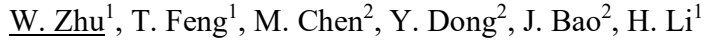

${ }^{1}$ UIH America Inc, Houston, United States

${ }^{2}$ MI, Shanghai United Imaging Healthcare, Shanghai, China

Time-of-Flight (TOF) PET data determines the attenuation map up to a constant. MLAA (Maximum Likelihood Activity and Attenuation Estimation) was proposed for this purpose. However, in real systems the estimated attenuation map usually results in bias and artifacts, due to various factors such as non-uniform timing resolution, detector timing drift, and biased scatter estimation. Moreover, MLAA has much higher computational cost than conventional PET reconstruction. Improving the practical performance of MLAA is important.

We proposed an efficient and robust emission-attenuation joint estimation framework, based on the condition that regions with almost uniform attenuation coefficients are segmented. Following the derivation, the update of attenuation map only requires a few weighted additions in sinogram space, which reduces overall computational cost significantly compared with conventional MLAA, as the latter demands at least two backward projections in each iteration. Furthermore, as the parameter space for the attenuation map is reduced and that the math model encourages an averaging effect in reach region, the bias and artifacts due to various factors above can be reduced.

We used clinical TOF PET data to evaluate the performance of our proposed method. In each iteration, the computation time for updating the attenuation map was less than $20 \%$ of that for conventional MLAA, leading to significant improvement in overall computation efficiency (twice as fast as conventional MLAA). More importantly, the method resulted in high quantitative accuracy. SUV computed with the estimated attenuation map and the CT based attenuation map had relative error less than $4 \%$ in multiple VOIs including the spine, liver, kidney, and heart. 
Our method can be used as a robust and efficient solution to estimate the attenuation map for quantitative PET image reconstruction, based on a single assumption that the attenuation coefficients are similar in each segmented regions.

\author{
M16B-3: Investigation of Sub-Centimeter Nodule Quantification Using PET/CT

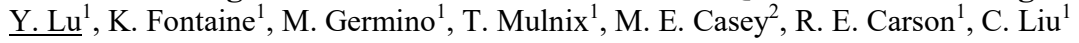 \\ ${ }^{I}$ Radiology and Biomedical Imaging, Yale University, New Haven, CT, USA \\ ${ }^{2}$ Siemens Medical Solutions, Knoxville, Tennessee, USA
}

With the recent development of high resolution TOF-PET scanner and resolution recovery made in the reconstruction algorithm, sub-centimeter nodules might become feasible to be detected and accurately quantified. In this study, we performed a comprehensive simulation and mini-Derenzo phantom study to explore the quantitative accuracy of sub-centimeter nodules using the Siemens Biograph $\mathrm{mCT}$ scanner. We simulated nodules ranging from 4 to $10 \mathrm{~mm}$ in diameter, 2:1 to 8:1 contrast level, $1 \%$ to 100\% (50 min human $10 \mathrm{mCi}$ FDG scan) count-level, and with respiratory motion amplitudes of 5/3 mm to 20/12 mm, in SI/AP directions based on traces obtained in human studies. Images were reconstructed using MOLAR (Motion-compensation OSEM List-mode Algorithm for Resolution-Recovery Reconstruction). We also investigated different reconstruction voxel-size of 0.5 , 1.0 and $2.0 \mathrm{~mm}$ for both simulation and phantom studies. We found that nodules larger than $6 \mathrm{~mm}$ with contrasts higher than $4: 1$ resulted in a bias of SUVmean smaller than $20 \%$ even when the count-level dropped to $4 \%$. A larger SUVmean performance drop was found between the static and $5 \mathrm{~mm}$ motion blurred data than was found between $5 \mathrm{~mm}$ motion and larger motion amplitudes. Images reconstructed with voxel-size of 1.0 and $0.5 \mathrm{~mm}$ resulted in more accurate quantification and reduced distortion than those reconstructed with $2 \mathrm{~mm}$ voxel-size. The results indicated that it is feasible to achieve accurate quantification for nodules $=6 \mathrm{~mm}$ using low-dose PET, with respiratory motion correction and fine reconstruction voxel-size. Partial volume correction might be needed to further improve the quantitative accuracy.

\author{
M16B-4: Robust Optimization of Coincidence Timing Resolution for PET Using a Huber Penalty \\ D. L. Freese $^{1}$, D. F. C. Hsu ${ }^{1}$, D. Innes ${ }^{2}$, C. S. Levin ${ }^{1,2,3,4}$ \\ ${ }^{\text {I}}$ Electrical Engineering, Stanford University, Stanford, United States \\ ${ }^{2}$ Radiology, Stanford University, Stanford, United States \\ ${ }^{3}$ Physics, Stanford University, Stanford, United States \\ ${ }^{4}$ Bioengineering, Stanford University, Stanford, United States
}

Positron Emission Tomography (PET) relies on accurate timing information to pair two $511 \mathrm{keV}$ photons into a coincidence event. Calibration of time delays between detectors becomes increasingly important as detector technology improves, because calibration error can quickly become a dominant source of error. Previous work has shown the maximum likelihood estimate of these delays can be calculated by least squares estimation, but is not tractable for complex systems and the solution becomes suboptimal in the presence of randoms. We modify the original problem to be solvable iteratively using the Preconditioned Conjugate Gradient (PCG) method. Using PCG we solve for 62,343 per-crystal delays using 2,185,139 coincidence events in $15.1 \mathrm{~s}$ for a two panel system dedicated to loco-regional imaging. We then extend the original least squares problem to be robust to randoms coincidences by implementing a Huber fit using the Alternating Direction Method of Multipliers (ADMM) algorithm. The ADMM algorithm converges after 5 iterations, or $36.6 \mathrm{~s}$, and improves the timing resolution from $68 \pm 1 \mathrm{~ns}$ FWHM to $14 \pm 1 \mathrm{~ns}$ FWHM. We also implement this algorithm for a previously calibrated GE Discovery PET/CT 690 system by scanning a rotating transmission source. After subtracting the $511 \mathrm{keV}$ photon time-of-flight due to the source position we calculate 13,824 percrystal delays and show a timing resolution improvement of $50 \pm 17 \mathrm{ps}$ from a default calibration of $659 \pm 11 \mathrm{ps}$ FWHM to $609 \pm 10 \mathrm{ps}$ FWHM.

This work is supported in part by NIH-NCI grant R01CA119056, DOD grant W81XWH-10-1-03-93. D. L. Freese is supported by a Stanford Graduate Fellowship and a National Science Foundation Fellowship. The authors would like to thank Judit Lantos for pro- viding the GE data and Steve Ross for support with the GE data format.

\author{
M16B-5: Performance of an Image-Based Motion Compensation Algorithm for the HRRT: a Striatum- \\ Phantom Study with True Motion \\ J. J. Johansson $^{1}$, S. H. Keller ${ }^{2}$, J. Tuisku ${ }^{1}$, M. Teräs ${ }^{1}$ \\ ${ }^{I}$ Turku PET Centre, Turku University Central Hospital, Turku, Finland \\ ${ }^{2}$ Department of Clinical Physiology, University of Copenhagen, Copenhage, Denmark
}

Performance of the current image-based motion compensation algorithm for the HRRT brain-studies was investigated. In this study, the striatum phantom was exposed to controlled motion pattern during PET scanning and motion compensation algorithm was employed using various parameters. In particular, the impact of allowed within frame motion threshold was investigated using thresholds relative to HRRT resolution $(1.25 \mathrm{~mm}, 2.5 \mathrm{~mm}, 3.75 \mathrm{~mm}$ and $5 \mathrm{~mm})$, and attenuation-map registration was implemented during reconstruction. Results showed significant impact (up to 50\% improvement) in image quantification when 
frame-by-frame registration was employed, while motion-based framing and attenuation-map registration showed somewhat smaller impact. We conclude that current image-based motion compensation provides a robust framework for HRRT brain studies and it should be routinely employed in studies targeting small brain nuclei and/or cortical binding.

\author{
M16B-6: Elastic Motion Correction for Continuous Bed Motion Whole-Body PET/CT \\ I. Hong, J. Jones, J. Hamill, C. Michel, M. Casey \\ Molecular Imaging, Siemens Healthcare, Knoxville, United States
}

The Continuous Bed Motion (CBM) acquisition mode in PET imaging, recently introduced commercially by Siemens and marketed as FlowMotion, has the potential advantages compared to the conventional step and shoot (S\&S) mode. With CBM, the image quality at different axial regions of interest can be adjusted based on clinical need by using variable bed speed acquisitions. This flexibility cannot be accommodated by S\&S mode since discrete bed positions are acquired. The respiratory motion in wholebody PET studies degrades image quality, introduces bias and partial volume artifacts, which are critical considerations for obtaining quantitative imaging with high resolution scanners. Elastic motion correction is needed to correct for respiratory motion artifacts. Elastic Motion DeBlurring (EMDB) was previously introduced by the authors. It was shown that it does not depend on the number of gates. Instead, a Mass Preservation Optical Flow (MPOF) algorithm was used to calculate a blurring kernel between the reference gate and the static (motion blurred) image. This approach reduces the processing time and hardware requirements for an iterative EMC reconstruction. In this work, EMDB is applied to CBM PET studies acquired with variable bed speed.

\author{
M16B-7: Exploring the relationship between MR ZTE Intensity and bone density: Application to MR \\ Attenuation Correction in PET/MR. \\ M. M. Khalifé ${ }^{1}$, C. C. Nioche ${ }^{1}$, B. B. Fernandez ${ }^{2}$, I. I. Buvat ${ }^{1}$, M. M. Soussan ${ }^{1}$, S. S. Desarnaud ${ }^{1}$, C. C. Comtat ${ }^{1}$ \\ ${ }^{I} I M I V$, UMR 1023 Inserm/CEA/Université Paris Sud - ERL 9218 CNRS, CEA/I2BM/SHFJ, Orsay, France \\ ${ }^{2}$ Applications and Workflow, GE Healthcare, Orsay, France
}

Accounting for the bone density in PET/MR attenuation correction can be important for accurate PET quantification. Several techniques for attenuation correction using MR images are implemented on PET/MR systems, based either on an atlas or on the segmentation of MR images to create a CT-like attenuation map. However, bone density changes in the same patient and between patients and thus, cannot be considered as constant. A few studies have studied the relationship between CT measured tissue density in Hounsfield Units (HU) and R2* maps generated from Ultrashort Echo Time sequence (UTE) in the bones. Previously, we proposed to use the Zero Echo Time sequence (ZTE) to compute a continuous bone attenuation map in the head using $\mu$ values scaled from corresponding measured CT attenuation map. Here, we explored an MR-CT patient database in search of a relationship between normalized ZTE image intensity and corresponding CT measured bone intensity in HU. ZTE image intensity was normalized such as zero corresponds to air and one to soft tissues, allowing for the comparison of different ZTE images. ZTE intensity for bone tissues is between air and soft tissues. A 2D voxel-to-voxel joint histogram was created between the bone CT intensities ([700, 2000] HU) and the normalized ZTE image intensities ([0, 1]). A relationship between CT HU and ZTE was visually observed on the resulting scatter plot. A principal component analysis was applied on the joint histograms. The first principal component represented more than $99 \%$ of the variance of the histogram data. Based on this first component, the following linear relationship between normalized ZTE intensity and CT was found for the bone tissues within a $10 \%$ error margin: $\mathrm{CT}[\mathrm{HU}]=1990-2130$.normZTE. In future work, the interest of this relationship will be evaluated for pediatric PET/MR brain scans where bone density distribution is thought to be lower than the one in adults.

M Khalifé, C Nioche, I Buvat, M Soussan, S Desarnaud and C Comtat are with IMIV, UMR 1023 Inserm/CEA/Université Paris Sud -ERL 9218 CNRS, Orsay, France. B Fernandez is with GE Healthcare, Applications and Workflow, Orsay France. This work is supported by a public grant overseen by the French National Research Agency (ANR) as part of the "Investissement d'Avenir" program, through the "Lidex-PIM" project funded by the IDEX Paris-Saclay, ANR-11-IDEX-0003-02. This work was performed on a platform of France Life Imaging network partly funded by the grant "ANR-11-INBS-0006". We thank GE Healthcare for providing access to research tools and ATSM pulse sequences.

\author{
M16B-8: X-Ray Scatter Correction Method for Planar Radiography Based on a Beam Stopper: a Simulation \\ Study

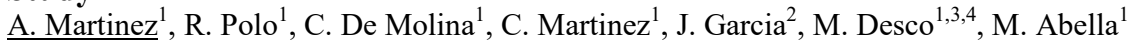 \\ ${ }^{I}$ Departamento de Bioingeniería e Ingeniería Aeroespacial, Universidad Carlos III de Madrid, Madrid, Spain \\ ${ }^{2}$ Arquitectura de Computadores, Comunicaciones y Sistemas (ARCOS), Universidad Carlos III de Madrid, Madrid, Spain \\ ${ }^{3}$ Instituto de Investigación Sanitaria Gregorio Marañón (IiSGM), Madrid, Spain \\ ${ }^{4}$ Centro de Investigación en Red de Salud Mental (CIBERSAM), Madrid, Spain
}

X-Ray scatter causes a decrease in image contrast in planar radiography that can reduce its diagnostic utility. The use of antiscatter grids allows the partial removal of the scattered photons reaching the detector at the cost of a 3-6 times increase in 
radiation dose. Digital radiography has allowed the development of post-processing methods to correct the effects of scatter, avoiding the use of anti-scatter grids. Most proposed methods focus on computer tomography, combining scatter estimation with iterative reconstruction algorithms. In this work we present a method for scatter correction in planar radiography based on the estimation of the scatter field from a second acquisition with a beam stopper placed between the X-Ray source and the patient. The beam stopper is a thin plate of highly absorbent material with a rectangular matrix of holes that allow the passing of the $\mathrm{x}$-ray beam. The scattered radiation can be sampled in the shadowed zones of the projection image and used to estimate the scatter field by means of two-dimensional interpolation. The corrected image is finally obtained by subtraction of a weighted version of the estimated scatter field from the projection image. Preliminary results for chest radiography using simulations from a full-body anthropomorphic phantom showed a noticeable contrast enhancement with around $80 \%$ of scatter reduction based on measured profiles compared to the non-grid acquisition. The proposed method implies up to 4 times of dose reduction compared to the use of anti-scatter grids, while removing acquisition geometry limitations.

This work was funded by the Spanish Ministerio de Economía y Competitividad (www.mineco.gob.es/) with projects IDI-20130301, TEC201347270-R, IPT-2012-0401-300000, RTC-2014-3028-1, and RD12/0042/0057.

M16B-9: Effects of the Collimator Magnification Factor in the Geometrical Calibration of SPECT Systems D. Salvado $^{1}$, K. Erlandsson ${ }^{1}$, B. F. Hutton ${ }^{1,2}$

${ }^{I}$ University College London, Institute of Nuclear Medicine, London, United Kingdom

${ }^{2}$ University of Wollongong, Centre for Medical Radiation Physics, Wollongong, Australia

Motivated by the need for compact systems, advances in detector technology with high intrinsic resolution allow small cameras to image large FOVs, trading off system resolution. However, with minification, precise measurement in the projection space, required for calibration purposes, may be compromised. The objective of this study is to investigate the impact of minification in a standard model-based calibration procedure. Measured pinhole data were created from ideal projections of three point sources, blurred according to the system resolution and parallax effects. Data were then scaled for the count level and Poisson noise added. This method was repeated for 100 noise realizations and three count levels. In the calibration method, seven parameters were defined to describe the pinhole aperture. Estimation of these parameters was obtained by minimizing the distance between true and measured projection data from the three point sources. The same method was applied to a single slit-slat collimator, where the number of parameters in the transaxial direction can be reduced to five. A Derenzo phantom was reconstructed with true and worst-case estimated calibration parameters. Comparing the estimations for the two pinhole collimators, the one with minification shows higher bias and standard deviation, suggesting that calibration is less robust when minification is present. When calibrating the slit-slat collimator, the lower number of estimated parameters reduces the bias, but precision is compromised at low counts. Despite the low bias, differences in the FWHM of the reconstructed rods are still present. Geometric calibration for SPECT requires high precision estimation of the model parameters, in order to avoid deterioration in image quality.

D Salvado is supported by a PhD Fellowship (FCT: SFRH/BD/88093/2012). This work was done as part of the INSERT collaboration, which is supported by the EC: FP7-HEALTH-305311. UCL/UCLH research is supported by the NIHR University College London Hospitals Biomedical Research Centre.

\section{M16B-10: Attenuation Correction in PET Using LSO Background}

A. R. Selfridge, E. Berg, M. Judenhofer, J. Qi, S. Cherry

Biomedical Engineering, University of California, Davis, Davis, California, USA

The development of large axial field-of-view (FOV) PET systems, such as the proposed total-body EXPLORER scanner, has stimulated growing interest in the possibilities associated with improved scanner sensitivity and solid angle coverage. At UC Davis, development of a large axial FOV primate scanner has allowed for assessment of the potential improvements that such a scanner offers. Simultaneous measurement of attenuation and emission using lutetium-oxyorthosilicate (LSO) background activity is of particular interest due to its potential to decrease patient radiation dose. Trace amounts of 176Lu in LSO detectors result in prompt beta and gamma emissions which often register as a coincidence event. Since the resultant gamma ray must traverse the FOV of the scanner in order to be recognized as a valid coincidence, its associated time-of-flight (TOF) is particularly long, approximately 1 to $2 \mathrm{~ns}$ depending on the scanner diameter. By applying TOF and energy thresholds, coincidences originating due to LSO background radiation can be differentiated from emission events, allowing for attenuation measurement without the need for an additional transmission or CT scan. We have performed a simulation study in GATE demonstrating the ability of this method to correctly measure attenuation. The quantitative accuracy of the attenuation measurement was assessed at various timing resolutions and TOF threshold values, as were the effects of cross-talk due to an emission source. Methods for improving accuracy in spite of these sources of error were considered and tested. The optimal TOF threshold, based on comparison of simulated and known attenuation values, was between 0.9 and 1.3 ns for the primate scanner. The measured attenuation maps had a reconstructed spatial resolution of better than 1 centimeter with a timing resolution of 500 ps, indicating that this approach is feasible on modern TOF scanners. 
M16B-11: Quantifying Pulmonary Strain During Respiration by Dynamic 4D-CT Scans M. J. Pomeroy ${ }^{1,2}$, Z. Liang ${ }^{2}$, Y. Hu ${ }^{2,3}$, A. Brehm ${ }^{1}$

${ }^{I}$ Medicine, SUNY Stony Brook, Stony Brook, NY, USA

${ }^{2}$ Radiology, SUNY Stony Brook, Stony Brook, NY, USA

${ }^{3}$ Applied Mathematics and Statistics, SUNY Stony Brook, Stony Brook, NY, USA

Idiopathic Pulmonary Fibrosis (IPF) is an interstitial lung disease that causes fibrosis and scarring of the lung parenchyma, resulting in suffocation and death due to respiratory failure. Currently there is no cure for IPF and limited treatment options that aim to slow the progression of the disease. In this paper, we adapt a mathematical model and further develop the model for early detection of fibrotic changes, typical of IPF. Using 4D-CT images and an accurate registration algorithm, we track the respiratory motion of segments of a healthy lung and convert this information into a hexagonal lattice. We then adapt the mathematical model to approximate the alveolar clusters as a hexagonal lattice to simulate breathing by adding an effective outward pressure force. Mapping the respiratory motion to the real image data through alignment of specific vertices, we use the forces predicted by the adapted model to determine the distribution of forces throughout a lung segment usually afflicted the most by this disease. The adapted and developed model for early detection of IPF was tested by several segments from the volunteer 4D-CT scans and differences among the segments were observed. It is conjectured that the observed difference indicates the potential to show the difference between a healthy lung and fibrotic lung at different stages of the disease. This difference between a healthy lung and fibrotic lung can be a biomarker to measure the success of already available treatments, as well as drugs in development.

\author{
M16B-12: Towards Personalized Injected Patient Doses for Cardiac Perfusion SPECT Imaging: a \\ Retrospective Study

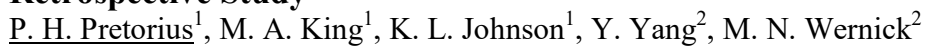 \\ ${ }^{I}$ Department of Radiology/Division of Nuclear Medicine, University of Massachusetts Medical School, Worcester, MA, USA \\ ${ }^{2}$ Department of Electrical and Computer Engineering/Medical Imaging Research Center, Illinois Institute of Technology, \\ Chicago, IL, USA
}

Reduction of the injected radionuclide dose in myocardial perfusion imaging (MPI) has become a major effort among clinicians and researchers alike. The aim of this study was to retrospectively determine the relationship between injected radioactivity and total projection counts in the heart region of clinically acquired Tc-99m sestamibi patients in an effort to formulate a strategy to better calculate individual and personalized doses prior to injection using easily measured metrics From a pool of 601 patients (304 female) 101 patients that undergone exercise stress testing and were read as having a normal perfusion distribution or small perfusion defects were included for the initial investigation. For all patients, injected activity at the time of the stress myocardial perfusion imaging (MPI) were determined. All patient BMI's were calculated and circumference measurements of the chest, abdomen and hips obtained. For all patients, list mode acquisitions were available and attenuation maps were derived from conebeam CT acquisitions (BrightView SPECT/CT scintillation camera, Philips, Cleveland, $\mathrm{OH}$ ). Total projection counts in the heart region were determined using a fixed region-of-interest (ROI) and counts per injected activity plotted against either BMI or chest circumference. Correlations were calculated. Using this information, 25 patients' list mode data were resampled randomly to create acquisition with only a fraction of the counts in the original study such that the heart region counts in all of the patients were equal to $\sim 750 \mathrm{~K}$. Correlations with BMI were again determined using the reduced radioactive dose. The proposed method reduced the radioactive dose by between $29 \%$ and $66 \%$ while maintaining image fidelity. Therefore, we established an initial strategy to calculate an individual and personalized injected dose for every patient prior to showing up at the MPI clinic using the correlation between BMI and count level in the heart region.

Acknowledgments: Research reported in this publication was supported by the National Heart, Lung, and Blood Institute of the National Institutes of Health under Award Number R01 HL122484. The content is solely the responsibility of the authors and does not necessarily represent the official views of the National Institutes of Health.

\author{
M16B-13: MEMS Gating: A new dual gating technique for eliminating motion-related inaccuracies in PET \\ imaging \\ $\underline{\text { M. Jafari Tadi }}{ }^{1,2}$, J. Teuho $^{1}$, E. Lehtonen ${ }^{2}$, A. Saraste ${ }^{1}$, T. Koivisto ${ }^{2}$, M. Pänkäälä ${ }^{2}$, M. Teräs ${ }^{3,4}$ \\ ${ }^{I}$ Turku PET center, University of Turku, Turku, Finland \\ ${ }^{2}$ Technology Research Center, University of Turku, Turku, Finland \\ ${ }^{3}$ Department of Medical Physics, Turku University Central Hospital, Turku, Finland \\ ${ }^{4}$ Institute of Biomedicine, University of Turku, Turku, Finland
}

Respiratory and cardiac motions may cause quantitative inaccuracies and hinder visual interpretation in positron emission tomography (PET) imaging. In practice, concurrent respiratory and cardiac gating is required to reliably image and identify small structures such as vulnerable coronary plaques. This study proposes a new mirco electromechanical (MEMS)-based gating method for eliminating motion-related artefacts. We used a miniaturized tri-axial accelerometer and gyroscope sensors attached 
to the patients' chest to extract seismocardiographic (SCG) and gyrocardiographic (GCG) signals. Dual gating in cardiac patients was performed using 18F-?uorodeoxyglucose (FDG) as a tracer. The MEMS dual gating was con?gured and studied parallel to clinically approved gating equipment, i.e. electrocardiography (ECG) and Real-time Position Management (RPM) systems, in PET acquisition of two atherosclerosis patients. Moreover, the radiopacity of the sensor readout-electronics was tested using a Ge-68 phantom before the clinical examinations. Accordingly, no image artifacts were detected either in CT or PET images due to the motion sensor board, as investigated by two independent operators. Therefore, the MEMS gating board does not contain materials that could affect the visual or quantitative accuracy of PET images or CT-based attenuation correction (CTAC). The MEMS-gated images from the patient study were compared to non-gated images and images that were gated with the reference methods. In conclusion, these results from the small patient group imply that implementation of the MEMS gating approach is applicable in dual gated PET imaging. Thus, the ?rst clinical experiences using MEMS cardiac-respiratory dual gating were promising and warrant for further investigations in PET imaging studies.

\section{M16B-14: Validation of 3D Model-Based Maximum-Likelihood Estimation of Normalisation Factors for Partial Ring Positron Emission Tomography}

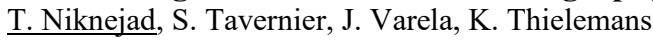

Laboratory of Instrumentation and Experimental Particles Physics, Lisbon, Portugal

In previous work, we have proposed a new design for positron emission mammography (PEM) using a stationary partial ring geometry. In this work a component-based ML estimation of normalisation factors for 3D PET data reconstruction is evaluated and validated for such a system. The model includes the estimation for crystal efficiencies and geometric factors. The algorithm is validated using Gate simulation and 3D reconstruction with MLEM in STIR with both full ring and partial ring scanners. The uniformity of the reconstructed images of cylinder phantoms before and after normalisation in both scanners is assessed. The results show that uniform images in both axial and transaxial directions are obtained after applying the estimated normalisation factors. The accuracy of the algorithm is validated by comparing the normalisation factors between the full and partial ring systems. We show that the estimated normalisation factors are almost identical, even though the separate components are not. This proves that the ML estimation of the $3 \mathrm{D}$ normalisation factors is valid and can be applied to the partial ring scanner.

M16B-15: Quantitative Accuracy of Time-of-Flight PET at High Count Rates

M. E. Daube-Witherspoon, V. Viswanath, S. Surti, S. Matej, J. S. Karp

Dept. of Radiology, University of Pennsylvania, Philadelphia, PA, USA

The benefit of time-of-flight (TOF) in PET reconstruction has been established for clinical imaging and studied using phantoms at higher activities. While TOF information mitigates and reduces image artifacts arising from errors in the corrections, it may not account for all changes with count rate. Standard performance measures of image quality are carried out at low or clinical count rates for whole-body scanning, but the dependence of TOF quantitative performance on count rate has not been wellstudied. We investigated the quantitative accuracy of TOF PET using cardiac and uniform phantoms imaged over a range of activities. At very high count rates the cardiac phantom showed significantly degraded image quality and quantification errors without TOF; TOF reconstruction was more stable to image artifacts and biases at high activities. For the uniform phantom, when the same number of prompt-delay events (trues+scatters) were reconstructed at different activities, a systematic negative bias in a large region was observed with increasing activity for all TOF reconstructions; this bias was significantly larger than that for nonTOF reconstructions. This correlates with a cool center seen in the TOF images and suggests an overcorrection for scatter. At high count rates, object scatter modeled by the TOF-modified single scatter simulation (SSS) does not change, but detector pileup leads to an increase in detected scatter with a wider TOF spread than at low activities, which is not modeled by TOF-SSS and impacts the accuracy of tail fitting. Deadtime correction factors implicitly include these sources of bias but depend on the algorithm (TOF vs. non-TOF) and possibly the activity distribution. Measurements of image quality and quantitative performance of TOF PET systems as a function of activity will be included in our study, as these will be used to determine the optimal range of activities for dynamic imaging and the quantitative accuracy of the reconstructed images.

M16B-16: Real-Time Data-Driven Respiratory Gating with Optimized Automatic VOI Selection

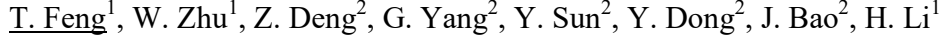

${ }^{I}$ United Imaging Healthcare America, Inc, Houston, US

${ }^{2}$ United Imaging Healthcare, Shanghai, China

Data-driven respiratory gating was previous developed to extract respiratory information directly from PET listmode data. It was also shown that different regions may contribute differently to the accuracy of the motion signal, affecting the success rate of this method. The goal of this study is to develop and evaluate a method that automatically determine the optimum regions to acquire respiratory motion signal. Maximum likely annihilation point was used to map each event into 3D volume space. Parametricbased volume of interests (VOI) was used for selection of events. For a fixed VOI, the center of mass (COM) within each fixed time interval was calculated to generate a motion signal. The signal to noise ratio (SNR), which was defined as the energy of 
signal over energy of noise in Fourier domain, of the acquired motion signal was calculated. The optimum VOI was determined by acquiring the motion signal which has the maximum SNR. Twenty patients with 18F-FDG injection were included in this study to evaluate this method. Conventional method which calculates the motion signal using COM without the selection of VOI was also implemented for comparison. Measurement using external device were also included for several patients to validate our methods. Fourier analysis of the acquired signal shows that while in only $50 \%$ of the patients the respiratory signal peaks were clearly visible in Fourier analysis using conventional method, more than $90 \%$ of the patients show clear respiratory signal peaks using the proposed method. High correlation was achieved with signal measured using external device. Gated reconstructed images using the acquired motion signal also showed much reduced motion blurring and attenuation-activity mismatch artifacts. The results suggested that the success rate of data-driven respiratory gating improved dramatically using the new methods without user intervention and can be achieved real-time during scan, making it viable for clinical application.

\section{M16B-17: Quality Control Algorithms Studies for a PMT-Based Time-of-Flight PET System $\underline{\mathrm{H} . \mathrm{Du}}$, K. C. Burr}

Toshiba Medical Research Institute USA, Inc., Vernon Hills, IL, United States

We developed a modular and scalable PMT-based detector for a Time-of-Flight PET system. The detector is divided into 5 segments. The position within each segment is determined by applying Anger logic to 4 PMTs which read out the segment and using a crystal look-up table (LUT) to convert the position to a specific crystal location. Routine quality controls (QC) are necessary to keep the system performance stable over time. Our approach is to keep the crystal LUT unchanged while compensating gain change for each PMT. We evaluated two algorithms for compensating gain changes. Algorithm 1 requires reference data when the PET system is freshly calibrated (including photopeaks of crystals in the center of PMTs, counts-percrystal and position map value on crystal segmentation boundaries). During QC PMT gains are adjusted to minimize the difference between new data and reference data. Algorithm 2 adjusts PMT gains to minimize the energy resolution for all crystals within each segment. The algorithms were validated by applying simulated gain changes to PMT signals measured using a calibration acquisition mode. They are evaluated by the accuracy in estimating simulated gain changes for each PMT channel under 3 conditions: moderate and large gain changes that are equivalent or larger than what might occur in a real system; extreme gain changes to test algorithm robustness. Then the estimated gain compensation was applied to each PMT and the resulting system performance (energy resolution and total energy-qualified counts) was calculated. We evaluated performance in 5 cases: 1) from initial data, 2) after simulated PMT gain changes, 3 5) after simulated gain changes and applying a simple energy drift correction, Algorithms $1 \& 2$ respectively. The two algorithms are similar in terms of gain change estimation accuracy and recovering system performance for moderate to large gain changes. However, when PMTs have extreme gain changes, Algorithm 1 clearly outperforms Algorithm 2.

M16B-18: Maximum Likelihood Activity and Attenuation Estimation with LSO Background Radiation L. Cheng ${ }^{1,2}$, T. $\mathrm{Ma}^{2}, \mathrm{~J} \cdot \mathrm{Qi}^{1}$

${ }^{I}$ Biomedical Engineering, University of California, Davis, Davis, CA 95616, USA

${ }^{2}$ Engineering Physics, Tsinghua University, Beijing 100084, China

Attenuation correction is essential for quantitative positron emission tomography (PET) imaging. The correction factors are often obtained using a CT scan in modern PET/CT scanners. With the development of the extreme performance long axial field-ofview PET scanner (EXPLORER), which enables ultra-low dose PET imaging, it is desirable to find a low-dose approach to attenuation correction. One method is to perform joint estimation of emission and attenuation images from time-of-flight (TOF) emission data, but theoretical analysis has shown that the attenuation sinogram can be only determined up to a constant.

Researchers have also used intrinsic background radiation from lutetium-based scintillator obtain an initial attenuation map for the joint estimation. However, this does not solve the scaling problem. Here we combine the TOF emission data and LSO background radiation. We form a joint likelihood function that includes both emission data and transmission data to solve the scale problem. We derived a monotonically convergent algorithm for the joint estimation. Computer simulations were conducted to evaluate the performance of the proposed method.

\section{M16B-19: Simulating the Accuracy of the Fotonic E Serie Range Imager for Respiratory Motion Tracking} E. Golkar, A. A. Abd. Rahni

Department of Electrical, Electronic \& Systems Engineering, Universiti Kebangsaan Malaysia, Bangi, Selangor, Malaysia

Radiotherapy, either alone or with other forms of treatment, is recognised as an important means of treating cancer. Diagnosis and treatment of tumours in the thoracic-abdominal region is especially affected by respiratory motion. One method which has been recently gained attention is to deduce internal motion by using distance camera systems that images the surface of the body during diagnosis and treatment. This is possible via correspondence models which relate the externally detected motion by the cameras to internal motion found from 4D imaging methods. In this paper, we present a preliminary study on assessing the accuracy of the Fotonic E Serie camera via simulation. Our simulation assumes the use of the camera during diagnostic imaging 
for instance in treatment planning. We simulately accurately the geometry of the scanner at our centre (Siemens Somatom Sensation 64) as well as a realistic positioning of the camera considering the scanner room. The estimated motion from the built correspondence model is then compared to motion found from deformable image registration which is assumed to be the ground truth. Using a linear multivariate correspondence model, our preliminary results have an average accuracy in the order of $1.5 \mathrm{~mm}$. We will show a more thorough analysis in the final paper as well as consider non-linear correspondence models.

\section{M16B-20: Direct Regional Quantification and Uncertainty Estimation Using Origin Ensembles} J. E. Gillam, G. I. Angelis, S. R. Meikle

Brain and Mind Centre, Faculty of Health Sciences, The University of Sydney, New South Wales, Australia

Regional quantification is often considered the end-point of many studies in Positron Emission Tomography (PET). However, in neurological small animal imaging, features can be sufficiently small that device resolution and voxel granularity inhibit the definition of segmentation boundaries that closely conform to organs or regions of interest. Even if well defined, estimation of uncertainty over small regions is problematic, particularly given that in some cases - such as in awake animal imaging - studies are unique and non-repeatable. The Origin Ensembles algorithm is an approach to image reconstruction based on Bayesian inference which utilises Monte-Carlo driven sampling to determine the posterior distribution of the emission counts given the measured data. The algorithm explores the full posterior and so can be used to estimate measures of uncertainty in quantitative parameters drawn from the data. However, being inference based, Origin Ensembles is sensitive to the dimension of the parameter space over which estimates are made. In this investigation the dimension of the image space is reduced using a regional segmentation based on an initial ML-EM reconstruction of detected data. Origin Ensembles is used to reconstruct data directly to the regions of interest over which quantification is desired providing estimation of the full posterior distribution for each region. Origin Ensembles sampling allows the uncertainty in quantitative values to be determined both in terms of parameter variance and the covariance between recovered regional intensities. Direct regional reconstruction was employed in this study using simulated data and was shown to enhance the precision and accuracy of recovered values.

\section{M16B-21: Optimization of Supplemental Transmission Source Imaging for Joint Transmission-Emission Scanning on an Integrated PET-MR System \\ $\underline{\text { S. L. Bowen }}$}

Virginia Tech Carilion Research Institute, Roanoke, VA, USA

The addition of supplemental transmission sources (TX) for simultaneous transmission-emission (EX) imaging has been shown to significantly improve the performance of $\mu$-map estimation compared to EX-alone reconstruction schemes. It is not clear; however; how TX distribution and acquisition parameters should be configured to maximize TX count rates while minimizing the degradation to reconstructed EX image quality. This problem is further complicated on the Siemens Biograph mMR PET-MR scanner due to space constraints caused by the relatively small diameter scanner bore and patient table. We aimed to optimize TX source geometry and activity for improved attenuation correction on the Biograph mMR during joint TX-EX imaging. Through a Monte Carlo simulation and projection model we examined count rate and tomographic sampling performance of full axial length ring, multiple parallel line ( $n$-line), and variable pitch helix alone or with a torus at each axial extreme (tori-helix). Attenuation map reconstructions of a structured sphere phantom demonstrated that stand-alone helix and $n$-line configurations produced reconstructed image artifacts in the absence of EX activity compared to the ring geometry, which were not evident utilizing a tori-helix source with a pitch set to the full scanner axial FOV. Noise equivalent count rate (NECR) estimates from joint TX-EX anthropomorphic pelvis simulations were the greatest for the tori-helix configuration (1.00 kcps at 14\% EX NECR degradation); four times that of the full axial length ring. For optimal TX activity, it was determined that utilizing a TX source strength that produces a $10 \%$ reduction in EX NECR at relatively high $(18.3 \mathrm{mCi})$ total subject activity can lead to a drop in EX NECR by $37 \%$ at low subject dose $(1.7 \mathrm{mCi})$. Use of a tori-helix geometry in combination with appropriate TX activity can lead to maximized TX and EX count rate performance compared with competing configurations.

M16B-22: Implications of Bias-Correction on Mean-Squared Error for Optimizing Reconstruction S. D. Metzler, M. E. Daube-Witherspoon, J. S. Karp, S. Matej

Radiology, University of Pennsylvania, Philadelphia, PA, USA

Iterative reconstruction algorithms contain many parameters. Adjusting these parameters can substantially impact the quantitative accuracy of regions of interest (ROIs) within the reconstructed volume, particularly for small lesions. A common way to optimize the reconstruction is to use an ensemble of lesions and find the parameter values (e.g., the number of iterations (NI)) that minimize the mean-squared error (MSE) of their uptake estimate, which can be separated into two components: bias and variance. If the bias is accurately known, a bias correction (BC) can be applied to the ROI, effectively reducing MSE to just the variance component; the optimal NI is then most probably 1 , where noise is minimized. However, the BC has uncertainty due to factors such as uncertainty in the lesion boundaries, heterogeneity, or limited ensemble statistics. Our approach to addressing these issues is to incorporate both $\mathrm{BC}$ and bias uncertainty into a bias-corrected MSE and then to optimize reconstruction 
parameters per lesion by minimizing this quantity. To test this approach, we considered 3 sizes of lesions measured with a smallanimal scanner: 1.0, 1.2, and $1.4 \mathrm{~mm}$. We determined the BC, MSE, and bias-corrected MSE for these lesions as a function of NI. We then applied weightings to these 3 ensembles to mimic a $1.2 \mathrm{~mm}$ lesion with boundary uncertainty. We found the appropriate $\mathrm{BC}$ based on the input weightings and applied it to statistically independent data sets. Our results confirm that if the $\mathrm{BC}$ were perfectly known, the optimal NI would be 1 , where noise is minimized. They also show that the weighted correction performs better than the individual corrections assuming 1.0,1.2, and $1.4 \mathrm{~mm}$ lesions, when the reconstruction parameters are separately optimized for each case. In conclusion, the introduction of uncertainty into the technique for quantifying ROIs leads to different reconstruction parameters and corrections and yields a better result as measured by MSE.

\section{M16B-23: Uniform acquisition modelling across PET imaging systems: unified scatter modelling} $\underline{\text { P. J. Markiewicz }}{ }^{1}$, M. J. Ehrhardt ${ }^{2}$, N. Burgos ${ }^{1}$, D. Atkinson ${ }^{3}$, S. R. Arridge ${ }^{4}$, B. F. Hutton ${ }^{5}$, S. Ourselin ${ }^{1}$

${ }^{I}$ Translational Imaging Group, CMIC, Dept. of Medical Physics and Biomedical Engineering, University College London, London, UK

${ }^{2}$ Dept. of Applied Mathematics and Theoretical Physics, University of Cambridge, Cambridge, UK

${ }^{3}$ Centre for Medical Imaging, University College London, London, UK

${ }^{4}$ Centre for Medical Image Computing, University College London, London, UK

${ }^{5}$ Institute of Nuclear Medicine, University College London, London, UK

PET imaging is an important tool commonly used for studying disease by research consortia which implement multi-centre studies to improve the statistical power of findings. The UK government launched the Dementias Platform UK to facilitate one of the world's largest dementia population study involving national centres equipped with state-of-the-art PET/MR scanners from two major vendors. However, the difference in PET detector technology between the two scanners involved makes the standardisation of data acquisition and image reconstruction necessary. We propose a new approach to PET acquisition system modelling across different PET systems and technologies, focusing in particular on unified scatter estimation across TOF (timeof-flight) and non-TOF PET systems. The proposed scatter modelling is fully 3D and voxel based, as opposed to the popular lineof-response driven methods. This means that for each emitting voxel an independent 3D scatter estimate is found, inherently preserving the necessary information for TOF calculations as well as accounting for the large axial field of view. With adequate sampling of the input images, the non-TOF scatter estimate is identical to the summed TOF estimates across TOF bins, without an additional computational cost used for the TOF estimation. The model is implemented using the latest NVIDA GPU CUDA platform, allowing finer sampling of image space which is more essential for accurate TOF modelling. The high accuracy of the proposed scatter model is validated using Monte Carlo simulations. The model is deployed in our stand-alone image reconstruction pipeline for the Biograph $\mathrm{mMR}$ scanner, demonstrating accurate $3 \mathrm{D}$ scatter estimates resulting in uniform reconstruction for a high statistics phantom scan.

Funding was received from the National Institute for Health Research University College London Hospitals Biomedical Research Centre (NIHR BRC UCLH/UCL High Impact Initiative BW.mn.BRC10269), the EPSRC (EP/K005278/1) and the CMIC-EPSRC platform grant.

\section{M16B-24: Impact and Correction of the Bladder Uptake on Tumor Quantification}

M. I. Oloniyo ${ }^{1}$, N. Efthimiou ${ }^{1,2}$, P. Wadhwa ${ }^{1}$, J. Silva-Rodriguez ${ }^{3}$, C. Tsoumpas ${ }^{1}$

${ }^{I}$ Biomedical Imaging, University of Leeds, Leeds, United Kingdom

${ }^{2}$ Biomedical Engineering, Technological Educational Institute of Athens, Athens, Greece

${ }^{3}$ Health Research Institute (IDIS), Galicia, Spain

A well-known drawback of $18 \mathrm{~F}$-based radiotracers is their urinary excretion with variable and high accumulation in the bladder. Previous studies have demonstrated that bladder activity affects quantification around the pelvic region, hence resulting in inaccurate diagnosis and staging. Nevertheless, factors which could affect quantification remain to be studied. Such factors are: variation in bladder volume and shape, as well as distance between the tumor and the bladder. This research is aimed at investigating the possible effects of bladder volume and activity variation on the surrounding tissues, taking into account tumor sizes and distance from bladder. The study used simulated data with GE SIGNA PET/MR scanner. Eight tumors were placed in different planes around the bladder to mimic different tumor cases. Bladder activities and tumor uptake from 18F-FDG distribution were obtained, which was used to simulate different combinations of bladder and tumor radiotracer concentrations. Analytical simulations and reconstructions were carried out using the open source STIR package. Scatter and attenuation corrections were included within the OSEM algorithm. For mitigating the bladder effect, a recently proposed methodology based on the introduction of physiological background terms on the reconstruction was used. We segmented the bladder which was then forward-projected and used as a background term for reconstruction. SUVmax is the main parameter of interest which was measured in several regions of interest. It was deduced that tumor uptake increases when bladder activity and/or tumor size increase. Tumors close to the bladder has the highest SUV variation, which could typify high sensitivity to bladder activity. There is no noticeable effect of bladder volume on tumor quantification. Bladder correction was able to effectively remove the background contribution from bladder and SUV variability. The bias was also significantly reduced, thereby improving quantification. 
M16B-25: Analysis of SPECT/CT Quantitation in Soft Tissue and Lung Density Phantoms

W. C. J. Hunter, R. S. Miyaoka, T. K. Lewellen, R. Harrison, W. McDougald

Radiology, University of Washington, Seattle, WA, USA

Objectives: We examine sources of uncertainty affecting quantitative performance of SPECT/CT imagery in objects with heterogeneous activity and attenuation (i.e. soft tissue and lung). Understanding the cause and amount of uncertainty in SPECT quantitation is a necessary step towards multicenter SPECT studies and for improving the consistency and standards of care. While quantitation has been studied using water filled phantoms, we seek to extend this effort to lung phantoms of varying densities spanning norm lung tissue (i.e., $-750 \mathrm{HU}$ ) to diseased lung tissue (i.e., -950 HU). Methods: A series of images of a prototype lung phantom were acquired with a GE Discovery NM/CT 670. This phantom consists of a repeatable structure of two or three water-filled blocks and two expandable polyurethane foam (UEF) cylinders with activity mixed in the foam and one of the water blocks. Contributions of uncertainty due to VOI selection, intrinsic scanner repeatability, setup repeatability, phantom density, and scatter profile were examined. Our preliminary study consists of 3 samples per configuration and aims to identify dominant effects on quantitative performance. Follow-on studies will be made to more accurately measure uncertainty of dominant effects on quantitative SPECT imaging. Results: Initial experimental results have been acquired for intrinsic scanner repeatability $(\sim \pm 0.9 \%)$ and setup repeatability $(\sim \pm 1.5 \%)$. A more complete analysis of quantitative SPECT errors and dependencies, including effects of intrinsic scanner repeatability, VOI selection repeatability, phantom setup repeatability, effects of density variation, and effects of scatter profile variation will be presented.

\section{M16B-26: Noise-Weighted FBP Algorithm for Uniformly Attenuated SPECT Projections} G. L. Zeng ${ }^{1,2}$

${ }^{I}$ Electrical Engineering, Weber State University, Ogden, Utah, USA

${ }^{2}$ Radiology, University of Utah, Salt Lake City, Utah, USA

Noise-weighted FBP (filtered backprojection) algorithm and Bayesian FBP algorithm were developed recently for un-attenuated Radon transform, which have applications in x-ray CT (computed tomography). This paper extends the noise-weighted FBP algorithm to the case of uniformly attenuated Radon transform, and this extended FBP algorithm can be applied in uniformly attenuated SPECT (single photon emission computed tomography). Computer simulations and experimental data demonstrate that the proposed FBP algorithm has similar noise control capability as the iterative ML-EM (maximum likelihood expectation maximization) algorithm. In practice, the attenuator is rarely uniform. A stable FBP algorithm must be developed for nonuniform attenuators before the FBP algorithm can be applied in clinics when attenuation correction is required.

M16C: Posters: CT II

Friday, Nov. $4 \quad$ 16:30-18:30 Etoile

M16C-1: Statistical Image Reconstruction for Low-Dose Dual Energy CT Using Alpha-Divergence Constrained Spectral Redundancy Information

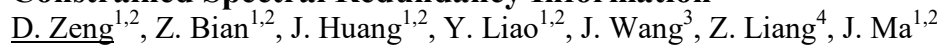

${ }^{I}$ School of Biomedical Engineering, Southern Medical University, Guangzhou, China

${ }^{2}$ Guangdong Provincial Key Laboratory of Medical Image Processing, Guangzhou, China

${ }^{3}$ Department of Radiation Oncology, University of Texas Southwestern Medical Center, Dallas, Texas, USA

${ }^{4}$ Departments of Radiology and Biomedical Engineering, State University of New York at Stony Brook, NY, USA

Dual energy computed tomography (DECT) has improved capability of differentiating different materials compared to conventional CT. However, due to non-negligible radiation exposure to patients, dose reduction has recently become a critical concern in CT imaging field. Moreover, direct material decomposition techniques such as numerical inversion can yield significantly amplified noise in the basic material images, and this is another common tissue in DECT imaging. In this work, to address the two issues, we present an iterative algorithm combining the image reconstruction and material decomposition procedures. More specifically, the DECT images are reconstructed by minimizing one objective function consisting a datafidelity term using Alpha-divergence to describe the statistical distribution of the DE sinogram data and a regularization term utilizing redundant information within DECT images. For simplicity, the present algorithm is termed as "AlphaD-aviNLM". To minimize the associative objective function, a modified proximal forward-backward splitting algorithm is proposed. Digital phantom was utilized to validate and evaluate the present AlphaD-aviNLM algorithm. The experimental results characterize the performance of the present AlphaD-aviNLM algorithm. 
This work was supported in part by the National Natural Science Foundation of China under Grants 81371544,61571214 , the Science and Technology Program of Guangzhou, China under Grant 201510010039. Zhengrong Liang also was partially supported by the NIH/NCI under grant \#CA143111 and grant \#CA082402.

\author{
M16C-2: Four Dimensional Cone-Beam Computed Tomography Reconstruction Using Multi-Phase \\ Projections \\ H. Zhang, Y. Liu, X. Tao, Z. Bian, J. Ma, W. Chen \\ Guangdong Provincial Key Laboratory of Medical Image Processing, Southern Medical University, Guangzhou, China
}

Restricted by the hardware, the projection number at a single phase for 4D-CBCT imaging is very low or even less than 10 , thus the associated reconstruction by using conventional reconstruction algorithms will be constrained by serious streak artifacts and noises. To address this problem, in this paper, we are aiming to develop an approach to reconstruct the 4D-CBCT image with multi-phase projections, which means that when the images at one phase were estimated, not only the projection data of the current phase but also the projections at the other phases were used. The proposed approach is based on the assumption that the image at one phase can be viewed as the motion-compensated image of another phase. Specifically, in this work, we formulate a cost function using multi-phase projections to construct the fidelity term and the TV regularization method was adopted. The Gradient-Projection-Barzilai-Linesearch (GPBL) method was used to optimize the complex cost function. Physical phantom and real patient data were used to evaluate the proposed algorithm. Results show that the proposed approach can effectively reduce the noise and artifacts, which suggest that the introduction of additional temporal correlation (along the phase direction) can improve the 4D-CBCT image quality.

This work was partially supported by the National Natural Science Foundation of China, No.81501466, No.61571214 and the Natural Science Foundation of Guangdong Province, No. 2015A030310018.

M16C-3: The Use of a Contactless ToF Camera for 4D CT Binning

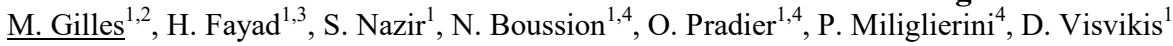

${ }^{1}$ INSERM UMR1101, LaTIM, Brest, France

${ }^{2}$ Ecole Nationale d'Ingénieurs de Brest, Brest, France

${ }^{3}$ Université de Bretagne Occidentale, Brest, France

${ }^{4}$ CHRU Morvan, Brest, France

Respiratory motion is a major concern in radiotherapy reducing the treatment performance. Indeed, the incertitude in tumor location leads to increased margins and dose limitation in order not to damage healthy tissue. This motion is more and more studied when planning a treatment especially with the expansion of 4D CT acquisitions and the corresponding 4D radiotherapy. In this case the CT is synchronized with the respiratory phase or amplitude of a respiratory signal acquired using an external device such as the pressure belt or the Respiratory Position Management system (RPM). In this work, we evaluate the possibility of using a signal extracted from patient surfaces acquired using a time of flight (ToF) camera having some practical advantages such as being contactless, light and portable. We propose first a comparison of the respiratory signals measured simultaneously with the pressure belt, the RPM and the SR4000 ToF camera. Then, we compare 4D CT's reconstructed using one of the acquired signals. We studied the breathing signals and the 4D CT images of one patient. The superimposition of the breathing curves acquired by the different systems show a good correspondence. Obtained correlation coefficients between the 22 reconstructed CT volumes show similarities when using the RPM or the ToF camera with a mean \pm standard deviation of $0.96 \pm 0.12$. This value was $0.93 \pm 0.03$ between 4D CT images binned using the ToF and the pressure belt signals. This prospective study is a proof of concept that the ToF signals can be used for 4D CT reconstructions. Future work consists on comparing signals and 4D CTs of more patients.

M16C-4: TV Constrained CT Image Reconstruction with Discretized Natural Pixels S. D. Rose, E. Y. Sidky, X. Pan

Radiology, University of Chicago, Chicago, IL, United States

In this work we perform a preliminary investigation of a discretized natural pixel expansion set for image representation in optimization-based image reconstruction for X-ray computed tomography. In particular, we focus our attention on total variation regularized image reconstruction in pixel and discrete natural pixel expansion sets. The work is motivated by two observations: (1) under certain conditions, total variation regularization in a pixel basis has been seen to introduce an undesirable patchy texture in reconstructed images, and (2) use of different expansion sets has been demonstrated to have a significant effect on image texture. Our results demonstrate the feasibility of sparse-view reconstruction with total variation regularization in the discretized natural pixel expansion set and indicate that the expansion set has the potential to yield images with texture more similar to filtered back projection than a square pixel basis. 


\title{
M16C-5: Reconstruction and Multi-Material Decomposition of Spectral CT in Dynamic-Threshold-Based
} Counting and Integrating Modes

\author{
Z. Chen ${ }^{1}, \mathrm{~L}$. Li \\ ${ }^{I}$ Engineering Physics, Tsinghua University, Beijing, China
}

For conventional spectral CT, it's difficult to balance the statistical noise and the number of energy bins. A method to cut the statistical noise without reducing the number of energy bins is to use the spectral CT with hybrid detectors in dynamic-thresholdbased counting and integrating modes that we proposed before. In this work, we adopted ART-TV method to solve the reconstruction problem, and gained a decent result. However, in the further work, we used the reconstruct result for multimaterial decomposition. We found the decomposition result was influenced hugely by even a little error of reconstruct. To improve the result of multi-material decomposition, we need to find an iteration method to decompose directly, which is our next research direction.

M16C-6: New Generation of Photon Counting Pixelized Detector, Medipix3RX, Used in Mammography. J. P. Idarraga Munoz ${ }^{1}$, J. Visser ${ }^{1}$, A. Mischke' ${ }^{2}$, H. W. A. M. de Jong ${ }^{3}$

${ }^{I}$ R\&D, Nikhef, Amsterdam, Netherlands

${ }^{2}$ Physics Department, Utrecht University, Utrecht, Netherlands

${ }^{3}$ Image Sciences Institute, University Medical Centre Utrecht, Utrecht, Netherlands

Mammography is an X-ray imaging technique used to screen women for breast cancer. Current Mammography systems employ high-resolution full field detectors using low energy X-rays. 10-30\% of malign masses escape screening due to limitation in contrast. The Medipix3RX chip features spectroscopic capabilities clearing the path to fast energy resolved X-ray. We will present the results from a prototype based on the Medipix3RX technology, with which we performed a one-to-one comparison with a commercial mammography system used currently at the University Medical Centre Utrecht (UMC, the Netherlands). We also motivate the use of the Medipix3RX data as input for Color X-ray reconstruction, successfully performing material identification in a test sample.

M16C-7: Atlas Based Interior Tomography M. Selim ${ }^{1,2}$, E. A. Rashed ${ }^{2}$, H. Kudo ${ }^{3}$

${ }^{I}$ Dept. of Mathematics and Computer Science, Faculty of Science, Suez University, Suez, Egypt

${ }^{2}$ Image Science Lab., Dept. of Mathematics, Faculty of Science, Suez Canal University, Ismailia, Egypt

${ }^{3}$ Division of Information Engineering, University of Tsukuba, Tsukuba, Japan

Image reconstruction of a region of interest (ROI) located inside a scanned object is called interior tomography or local tomography. In interior tomography, the projection data is limited because x-ray beams is directed only to ROI inside the object and the beam portion located left and right of the ROI are truncated in all view angles. Interior tomography has great benefits in reducing the x-ray radiation dose that the patient expose because it focus the x-ray beams to a limited region of the whole object. Therefore, decreasing the risk that the patient can expose where regular CT scans may lead to a malignancy. Also, it has a benefit in minimizing the necessary detector size. Using the conventional analytical reconstruction method such as filtered backprojection (FBP) algorithm for interior problem is known to result in DC-shift and low-frequency artifacts in the reconstructed image. Several previous studies investigated the interior problem in computed tomography. In this paper, we introduce the using of prior knowledge from probabilistic atlas in solving the interior problem. The atlas is constructed using a set of CT images previously scanned for different patients and fitted using Laplacian mixture model (LMM). Then, expectation maximization (EM) algorithm is used to estimate the mixture parameters. We use a statistical reconstruction method and the cost function is designed to incorporate the information of the probabilistic atlas. Experimental results indicate that the proposed method can remarkably improve the image quality and minimize the effect of DC-shift. Surprisingly, we observe a high quality reconstruction concerning the region outside the ROI.

M16C-8: Impact of a Data-Derivative Fidelity on Truncation-Data Reconstruction in CBCT $\underline{\text { D. Xia }}^{1}$, D. A. Langan ${ }^{2}$, S. B. Solomon ${ }^{3}$, H. Lai ${ }^{2}$, Z. Zhang ${ }^{1}$, B. Chen ${ }^{1}$, E. Y. Sidky ${ }^{1}$, X. Pan ${ }^{1}$

${ }^{I}$ Radiology, The University of Chicago, Chicago, IL, U.S.A.

${ }^{2}$ GE Global Research Center, Niskayuna, NY, U.S.A.

${ }^{3}$ Memorial Sloan Kettering Cancer Center, New York, NY, U.S.A.

In practical CBCT imaging such as that in interventional procedures, data collected are truncated due to the limited detector size that cannot cover the imaged subject and especially the involved interventional devices. We study optimization-based image reconstruction directly from truncated data, with an aim to reduce truncation artifacts observed in practical or clinical reconstructions. In the study, the reconstruction problem is formulated as a constrained optimization program in which a data- 
derivative fidelity term was introduced for effective suppression of the truncation artifacts. In this work, we carry specifically out a quantitative investigation of impact of the data-derivative term on the artifact reduction. In the investigation, the contribution of the data-derivative term is characterized by a weighting parameter, and the investigation is enabled subsequently by tailoring the Chambolle-Pock (CP) algorithm to solve the optimization program containing the data-derivative fidelity term. The results of the study suggest that an appropriately designed data-derivative fidelity can be exploited for yielding image reconstruction with reduced artifacts directly from truncated data.

\author{
M16C-9: Reconstructing Dynamic Magnification CBCT Scans with Optimization-Based Reconstruction \\ A. M. Davis, X. Pan, C. A. Pelizzari \\ Department of Radiation and Cellular Oncology, University of Chicago, Chicago, IL, USA
}

In image guided radiation therapy (IGRT), certain patient treatment positions can lead to collisions of the patient with the linac gantry. When such collisions arise, it prevents the gantry from rotating around the patient and acquiring sufficient projection information for current analytic-based reconstruction methods such as FDK. One way to alleviate such a collision would be to move the patient or imaging arms at the offending gantry position changing the magnification. In this work, we investigate if optimization-based reconstruction methods can reconstruct from data acquired from such dynamic-magnification trajectories. Using real data from Varian's TrueBeam $2.0 \mathrm{kV}$ imaging system, we found that these methods can reconstruct from trajectories where different magnifications are used in a single scan. This suggests these optimization-based reconstruction methods have the potential to enable collision avoiding imaging trajectories, improving patient comfort and reducing the costly clinical delays that currently arise from these collisions.

Funding was provided in part by Varian Medical Systems, the Lawrence H. Lanzl Fellowship (to A. D.), and NIH Grants R01 CA182264, R01 EB018102, S10 RR021039 and P30 CA14599. The contents of this work are solely the responsibility of the authors and do not necessarily represent the official view of any of the supporting organizations.

M16C-10: In-Line Phase Contrast Tomography of the Breast with a Dedicated Micro-CT Scanner

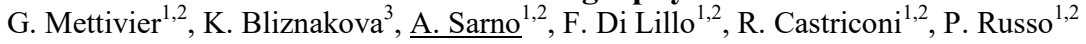

${ }^{I}$ Dept. of Physics, Università di Napoli Federico II, Napoli, Italy

${ }^{2}$ Sezione di Napoli, INFN, Napoli, Italy

${ }^{3}$ Department of Software and Internet Technology, Technical University of Varna, Varna, Bulgaria

We performed laboratory investigations of the image quality for in-line phase contrast micro tomography dedicated to the breast, using a 3D breast phantom. We employed the cone-beam, microfocus (7-micrometer focal spot size) computed tomography (CT) scanner developed in-house for investigation of high resolution, absorption based, and phase contrast based, X-ray imaging of the breast for cancer diagnosis, at dose levels comparable to two-view mammography. CT slices were reconstructed (FDK algorithm) from phase contrast projection images as well as from phase retrieved projections (ANKAphase algorithm), under the assumption of spatially uniform distribution in the object volume of the ratio of the X-ray optical coefficients of the sample material, at the effective energy of the X-ray beam $(80 \mathrm{kV}, \mathrm{HVL}=6.11 \mathrm{~mm} \mathrm{Al})$. Microcalcifications were best visible after phase retrieval, down to $0.23 \mathrm{~mm}$ size.

\author{
M16C-11: Evaluation of an Image-Based Dual-Energy CT Material Characterization Method \\ K. Grogg, X. Zhu, G. El Fakhri, N. M. Alpert \\ Gordon Center for Medical Imaging, Radiology, Massachusetts General Hospital/Harvard Medical School, Boston, MA, USA
}

Dual-energy CT (DECT) can be used to determine both the relative electron density (RED) and effective atomic number (Zeff) of tissues, which can potentially improve the accuracy of treatment planning and monitoring of proton radiation therapy. Both planning and verification of proton therapy rely on correct information about the elemental composition of the patient tissues, which is more accurately determined from RED and Zeff than from single energy CT numbers. An image-based approach has previously been proposed to use a single reference material to calibrate a scanner system to calculate RED and Zeff. We improved this approach by using multiple reference materials and a least-squares method to determine the calibration parameters. We have also evaluated the performance of the method using in-house made phantom materials with tissue-like elemental compositions. Whereas previous studies have relied on commercial phantoms with higher carbon to oxygen ratios than are typically found in human tissue, we have created phantom materials with higher oxygen content, which translate to an additional set of more realistic RED and Zeff combinations. The phantom was scanned on a Siemens Somatom Definition Flash scanner at $100 \mathrm{kVp}$ and $140 \mathrm{kVp}+\mathrm{Sn}$ filter. We used 4 calibration materials to calculate the RED and Zeff of 15 materials. We compared the results to the theoretical values determined by known elemental compositions, and to the original approach of using only one reference material. Using multiple calibration points resulted in more stable calculated values. The range of deviations was 3\% for RED and 1\% for Zeff. The accuracy is sufficient for an improvement in treatment planning and verification. 
M16C-12: Model Based Iterative Reconstruction of Ultra Low Dose CT Scans for PET Attenuation Correction $\underline{X . R u i}^{1}$, T.-C. Lee ${ }^{2}$, A. M. Alessio ${ }^{2}$, P. E. Kinahan ${ }^{2}$, B. De Man ${ }^{1}$

${ }^{I}$ Image Reconstruction Laboratory, General Electric - Global Research, Niskayuna, NY, USA

${ }^{2}$ Department of Radiology, University of Washington, Imaging Research Laboratory, Seattle, WA, USA

PET/CT imaging has become a standard tool for oncology diagnosis and staging. For PET imaging, CT scans are used for anatomical localization and attenuation correction. To improve the quantitative accuracy of respiratory-gated PET images, phasespecific attenuation images are desired which requires extended-duration respiratory-gated CT scans. To reduce the accumulated CT scan dose, ultra-low dose (ULD) CT scans are needed. We have shown with simulation studies that CT images used for PET attenuation correction can tolerate resolution loss and a noise increase while preserving the quantitative accuracy of the PET images. We wanted to evaluate with measured data if CT scans useable for PET attenuation correction could be acquired at ULD levels, i.e. 10x lower in dose compared to standard low-dose diagnostic CT scans. Our studies demonstrate the feasibility of using ULD CT for PET imaging on a GE discovery STE scanner. The results show that using model based iterative reconstruction for ULD CT data reduces bias and noise in the corresponding PET image. This approach has the potential to enable extendedduration CT scanning for compensation of respiratory motion in PET/CT scanning.

The project described was supported by NIH Grant Number R01 CA160253 and its contents are solely the responsibility of the authors and do not necessarily represent the official views of the NIH.

\author{
M16C-13: An Empirical Material Decomposition Method for Spectral CT \\ C. Feng, Q. Shen, K. Kang, Y. Xing \\ Department of Engineering Physics, Tsinghua University, Beijing, China
}

Photon counting detectors (PCDs) with pulse height analysis provide spectral information in contrast to energy integrating detectors (EIDs), which can be used in material discrimination in spectral Computed Tomography (CT). Most material decomposition algorithms need to use detected spectral data. Affected by the performance of PCDs, such as charge sharing, pulse pileup, k-escape and other physical effects, it may cause serious spectra distortion that leads to material decomposition errors, which should be taken into account inevitably. Based on the calibration produced by Marc in dual energy CT (DECT), we propose an empirical material decomposition method (EMDM) for spectral CT. Without the requirements of the knowledge of spectra, we use the attenuation coefficients of the basis materials to discriminate substances in image domain by polynomial fitting and least square deviation. A series of experiments were carried out to validate EMDM and the results show good performance in material discrimination with specific concentration of different solution.

\author{
M16C-14: Few-View CT Reconstruction Method Based on Deep Learning \\ J. Zhao, Z. Chen, L. Zhang \\ Ministry of Education, Key Laboratory of Particle and Radiation Imaging (Tsinghua University), Beijing, China
}

The importance of performing exact image reconstruction from the minimum amount of data has been recognized for a long time. We propose a deep learning method for few-view CT reconstuction. Our method directly learns an end-to-end mapping between the full-view/few-view reconstruction. The mapping is represented as a deep convolutional neural network (CNN) that takes the few-view reconstruction image as the input and outputs the full-view one. We further show that traditional Dictionary Learning based reconstruction methods can also be viewed as a deep convolutional network. But unlike traditional methods that handle each component separately, our method jointly optimizes all layers. Our deep CNN has a lightweight structure, yet demonstrates state-of-the-art reconstruction quality, and achieves fast speed for practical on-line usage. We explore different network structures and parameter settings to achieve trade-offs between performance and speed.

This work was supported by a grant from the National Natural Science Foundation of China (No. 11235007) and a Tsinghua University Independent Research Project Grant, "Research on Key Technologies and CT Reconstruction methods of multi-energy X-ray imaging". All authors are with Key Laboratory of Particle and Radiation Imaging (Tsinghua University), Ministry of Education, Beijing 100084, China and the Department of Engineering Physics, Tsinghua University, Beijing 100084,China (Corresponding author: Zhiqiang Chen,

czq@tsinghua.edu.cn)

M16C-15: Dual-Energy CT Reconstruction Using Guided Image Filtering H. Yang ${ }^{1}$, K. Kim ${ }^{2}$, G. El Fakhri ${ }^{2}$, K. Kang ${ }^{1}$, Y. Xing ${ }^{1}$, Q. Li $^{2}$

${ }^{I}$ Department of Engineering Physics, Tsinghua University, Beijing, China

${ }^{2}$ Radiology, Gordon Center for Medical Imaging, Massachusetts General Hospital and Harvard Medical School, Boston, MA, USA 
Dual-energy computed tomography (CT), which uses two X-ray spectra enabling material differentiation by analyzing materialdependent photo-electric and Compton effects, has become more widely used in various medical applications such as material decomposition and k-edge subtraction imaging. In most image reconstruction methods using Gaussian filtering, total variation (TV) for dual-energy CT, two CT images of high and low energies are separately reconstructed and utilized in applications. To improve the image quality by considering redundant information of both the high and low energy images, we propose a novel dual-energy CT reconstruction method using the guided image filtering algorithm, which jointly reconstructs two images of different energy spectra simultaneously. In our computer simulation, we demonstrate that the image quality can be significantly improved, which can be potentially exploited in material decomposition and radiation dose reduction. The proposed method will be validated using real experimental data of dual-energy CT.

\author{
M16C-16: CT Reconstruction from Truncated Projections Based on Prior Knowledge of Histogram \\ J. Zhao, Z. Chen, L. Zhang \\ Ministry of Education, Key Laboratory of Particle and Radiation Imaging (Tsinghua University), Beijing, China
}

The importance of performing exact image reconstruction from the minimum amount of data has been recognized for a longtime. Yangbo Ye et al. has proved that the interior problem can be exactly and stably solved if a subregion in an ROI/VOI in the FOV is known from fan-beam/cone-beam projection data sets using the analytic continuation technique. But the analytic continuation is very ill-posed question. The conventional methods are iterative or not suited to multi-region-known situation.

As the problem is ill-posed, we need to apply some prior knowledge to regularize. As in some special applications (e.g. medical imaging), the CT images at different times may have different shapes, but the histogram is the same. In this work we proposed a novel regularization method on prior knowledge of histogram. We applied this method on reconstruction method we developed before based on MSF analytic continuation.

M16D: Posters: Parametric imaging

Friday, Nov. $4 \quad$ 16:30-18:30 Etoile

M16D-1: Regularizing Direct Parametric Reconstruction for Dynamic PET with the Method of Sieves L. Szirmay-Kalos, Á. Kacsó

Budapest University of Technology and Economics, Budapest, Hungary

This paper proposes regularization methods for direct parametric dynamic PET reconstruction, when the space-time activity function needs to be recovered from measurements. In case of high spatial and temporal resolution, the reconstruction is statistically poorly defined, requiring the inclusion of a priori information in the form of a penalty term or filtering. The method of sieves executes filtering in each iteration step, i.e. projects the actual estimate into the subspace of acceptable solutions, and has been successful in reconstructing static data. The objective of this paper is to generalize the filtering scheme for spatiotemporal reconstruction, taking into account that accurate kinetic models describing the temporal behavior are non-linear. Fast changes are impossible to distinguish from noise if only a small temporal window is examined, thus the simple extension to 4D does not provide acceptable results. We show that efficient filtering can be obtained if voxel based model parameters are modified according to the time activity functions of neighboring voxels belonging to the same anatomic region. As the dependence of the time activity function on the model parameters is non-linear for sophisticated kinetic models, the filtering step involves a non-linear parameter fitting, which can be solved analytically for the two-tissue compartment model. The presented method is compared to the application of a TV penalty of the voxel activities. The presented model is built into the TeraTomo system.

M16D-2: Image Derived Input Function for Rapid PET Measurement of CBF, OEF, and CMRO N. Kudomi ${ }^{1}$, Y. Maeda ${ }^{2}$, H. Yamamoto ${ }^{1}$, Y. Yamamoto $^{3}$, T. Hatakeyama ${ }^{4}$, Y. Nishiyama ${ }^{3}$

${ }^{I}$ Dept of Medicine, Kagawa University, Facl Of Medicine, Kagawa University, Kagawa, Japan

${ }^{2}$ Hospital, Kagawa University, Clinical Radiology, Kagawa, Japan

${ }^{3}$ Dept of Medicine, Kagawa University, Radiology, Kagawa, Japan

${ }^{4}$ Dept of Medicine, Kagawa University, Neurological Surgery, Kagawa, Japan

Aim: Parametric CBF, OEF and $\mathrm{CMRO}_{2}$ images can be measured using ${ }^{15} \mathrm{O}-\mathrm{PET}$. Recently, we have developed a novel protocol allowing an extremely short PET scan time of $<10 \mathrm{~min}$. In that protocol, two tracers of ${ }^{15} \mathrm{O}_{2}$ and $\mathrm{H}_{2}{ }^{15} \mathrm{O}$ are sequentially administrated during a single PET scan (DBFM). One of issues to be solved is invasive arterial blood withdrawal for input function. The aim of the present study was to develop a new method for image-derived input function (IDIF). Methods: Our technique consisted of formula based on the single tissue compartment model for expressing inputs from a measured tissue curve with parameters of rate constants: $\mathrm{K}_{1}{ }^{\mathrm{w}}$ and $\mathrm{K}_{1}{ }^{\mathrm{o}}$ for water and oxygen, respectively. For multiple tissue curves from brain regions in a PET image, the rate constants were estimated so as to minimize a sum of square differences for expressed inputs for the tissue curves. For the estimated rates, IDIFs were reconstructed, and mean of reconstructed inputs was estimated as IDIF. To test 
validity, 20 subjects with suspected cerebrovascular disorders were studied with DBFM protocol. Arterial blood was sampled during scan time to serve as reference. The parametric images were computed by the IDIF and measured input functions. Results: The estimated IDIFs were well reproduced compare to the measured ones. The mean of calculated CBF, OEF, and $\mathrm{CMRO}_{2}$ values were $0.46 \pm 0.16$ and $0.46 \pm 0.16 \mathrm{ml} / \mathrm{min} / \mathrm{g}$ by the measured and IDIF, $0.46 \pm 0.08$ and $0.44 \pm 0.08$, and $0.037 \pm 0.014$ and $0.035 \pm 0.013 \mathrm{ml} / \mathrm{min} / \mathrm{g}$, respectively and differences were $\pm 7.1 \%, \pm 6.7 \%$ and $\pm 8.3 \%$ for those functions, respectively. Regression analysis showed tight correlation between the input methods as: $r=0.97,0.93$, and 0.97 , for those functions, respectively. Conclusion: The presented results demonstrate that IDIF can be estimated directly from tissue curves from dynamic PET image for DBFM, suggesting a possibility of noninvasive $\mathrm{CBF}, \mathrm{OEF}$ and $\mathrm{CMRO}_{2}$ assessments.

This work for NK, YY, and YN were supported by the Ministry of Education, Science, Sports and Culture of Japan, a grant-in-aid for KAKENHI (C) (grant number 23590675 2014-2016).

M16D-3: CBV Computation from a Rapid PET Scan Data with Sequential Administration of ${ }^{15} \mathrm{O}_{2}$ and $\mathrm{C}^{15} \mathrm{O}_{2}$ N. Kudomi ${ }^{1}$, Y. Maeda ${ }^{2}$, H. Yamamoto ${ }^{1}$, Y. Yamamoto ${ }^{3}$, T. Hatakeyama ${ }^{4}$, Y. Nishiyama ${ }^{3}$

${ }^{I}$ Medical Physics, Facl of Medicine, Kagawa University, Kagawa, Japan

${ }^{2}$ Clinical Radiology, Hospital, Kagawa University, Kagawa, Japan

${ }^{3}$ Radiology, Facl of Medicine, Kagawa University, Kagawa, Japan

${ }^{4}$ Neurological Surgery, Facl of Medicine, Kagawa University, Kagawa, Japan

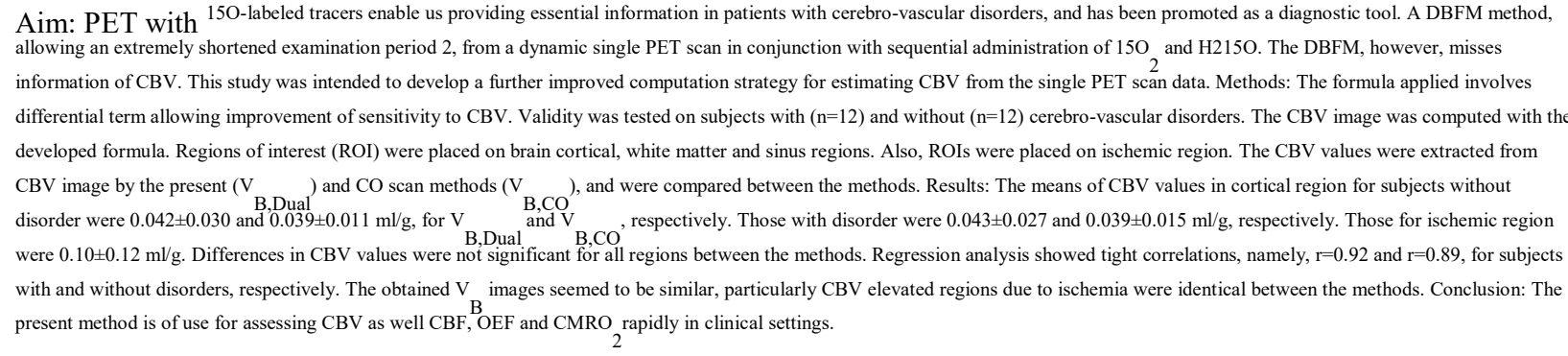

This work for NK, YY, and YN were supported by the Ministry of Education, Science, Sports and Culture of Japan, a grant-in-aid for KAKENHI (C) (grant number 23590675 2014-2016).

M16D-4: Novel quantitative whole-body parametric PET imaging utilizing multiple clustering realizations

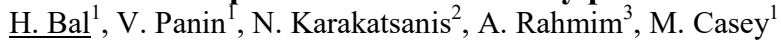

${ }^{I}$ Siemens Medical Solutions USA, Inc, Knoxville, TN, U.S.A

${ }^{2}$ ICAHN School of Medicine at Mount Sinai, New-York, NY, U.S.A

${ }^{3}$ Johns Hopkins University School of Medicine, Baltimore, MD, U.S.A

Quantitative whole-body parametric PET imaging utilizing Patlak graphical analysis has the potential to provide improved diagnosis, prognosis and treatment response assessment. However, a voxel-based fitting approach for short dynamic scan protocols results in high statistical noise in the parametric images. The objective of our study is to present the framework of a novel clustering based method for estimating parametric images with low statistical noise. The method relies primarily on using standard k-means clustering for segmenting the time-activity curves within the whole-body volume. In addition, in order to obtain improved accuracy without increasing noise, multiple realizations of clustering are performed. During each realization, cluster centers are selected from a unique ordered set of time-activity curves within the whole body volume. All the remaining data are classified into the cluster centers based on the minimum Euclidean distance measure. Patlak analysis is subsequently performed on the cluster average to form the slope and intercept images which are then averaged for all realizations to form the final images. XCAT phantom based simulations representing dynamic acquisition were performed by creating 5 frames each representing FDG activity distribution starting 60 minutes post injection with 7 minute intervals. In addition, 5 clinical wholebody dynamic FDG patient datasets with image-derived blood input function and whole-body dynamic data measurements similar to the simulated data were also used. All dynamic data were reconstructed using OSEM applying corrections for imagedegrading factors. Parametric image noise in a liver region of interest increased as a function of the number of clusters but decreased as the number of clustering realizations increased without increase in bias. Image quality for the simulated and patient datasets improved over voxel-fitting approach. Multiple clustering realizations method has the potential to provide improved parametric image quality for short scan whole-body parametric PET imaging.

M16D-5: Influence of Heating Conditions on 18F-FDG PET Imaging Quantification and Kinetics: Results on Biodistribution in Mice 


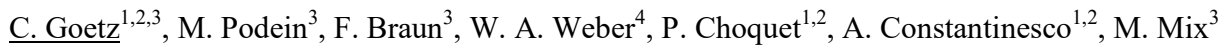

${ }^{I}$ UF6237 Imagerie Preclinique, Pole dimagerie, CHU Hautepierre, Hopitaux Universitaires de Strasbourg, Strasbourg, France

${ }^{2}$ ICube, Departement Mecanique, CNRS, Strasbourg, France

${ }^{3}$ Department of Nuclear Medicine, University of Freiburg, Faculty of Medicine, University of Freiburg, Freiburg, Germany

${ }^{4}$ Molecular Imaging and Therapy Service, Memorial Sloan-Kettering Cancer Center, New York, USA

Anesthesia and heating parameters modifications may lead in unstable homeostatic conditions in mice thus modifying the uptake of molecular imaging tracers also potentially inducing quantification errors. The objective of our work was to evaluate the impact of heating conditions on PET imaging quantification. Methods and procedures: xenograft tumors were implanted subcutaneously in 6 adult female mice. After tumor growth, animals were administered twice with 3.2 +/- 0.4 MBq of 18F-FDG through a tail vein inside an imaging holder (Minerve, Esternay, France). A PET dynamic acquisition followed each FDG administration. Both acquisitions covered the first 45 minutes after injection (R4 microPET, Concorde Microsystems, US). Heating temperature of the animal holder was changed between acquisitions leading to either optimal or sub-optimal heating conditions. Time-activity curves were obtained for each animal from ROIs placed over the brain, heart, liver and implanted tumors. Bi-exponential fitting conducted to calculation of uptake and washout time constants for each ROI (proFit, Quantumsoft, Switzerland). Outcomes and results: optimal animal heating showed stable heart rates during acquisition $(537+/-55 \mathrm{bpm})$ whereas sub-optimal heating leads to lower heart rates with higher standard deviation $(442+/-96 \mathrm{bpm})$. Both uptake and washout time constants were faster $(\mathrm{p}<$ 0.01 ) in animals maintained under optimal heating conditions. Conclusions: different heating conditions during anesthesia led to slight but statistically significant differences in heart rates during PET imaging. Despite these slight differences optimal heating showed different distribution with faster kinetics of the radiotracer in mice.

\author{
M16D-6: Joint Direct Dynamic Analysis in Dual-Tracer PET Imaging

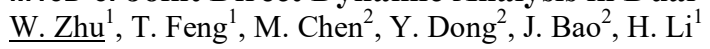 \\ ${ }^{I}$ UIH America Inc, Houston, United States \\ ${ }^{2}$ MI, Shanghai United Imaging Healthcare, Shanghai, China
}

Dual-tracer PET imaging may improve overall lesion detectability due to different tracer kinetics. However, separating two tracers in the mixed acquired data is difficult because of unknown individual activity change over time. We proposed an effective and robust method to separate two tracers dynamically, by introducing joint Patlak and Logan analysis in dual-tracer PET imaging.

Several patients underwent dual-tracer brain PET/CT scans. The entire scan time was $100 \mathrm{~min} .13 \mathrm{~N}$ ammonia was injected at $\mathrm{t}=0$ $\mathrm{min}$ and $18 \mathrm{~F}-\mathrm{FDG}$ was at $\mathrm{t}=20 \mathrm{~min}$. The dual blood input functions were separated and estimated non-invasively from static frame reconstructions, assisted with an exponential model to fit the blood input function after certain elapse of time. Direct Logan analysis was performed for data 0 20min to generate Logan parametric images for 13N. For the 20 100 min data, direct Patlak estimation from raw data was performed to generate Patlak parametric images for FDG, with a modified iterative algorithm including the contribution of $13 \mathrm{~N}$ activities.

Results showed that the proposed method is robust and applicable to dual-tracer PET imaging in separation of two tracers. In addition, dynamic images were generated to assist lesion detection. Specifically, in simulation the estimated Logan slope and the Patlak slope yielded relative error $<2 \%$ for four rods in the NEMA-like phantom. In the application on clinical data, the estimated Logan parametric images and Patlak parametric images revealed distinguished contrasts. The Patlak parametric images estimated with our method also resulted in significantly less noise than conventional image based method, and at the same time preserved the quantitative accuracy, with $<6 \%$ difference from the ones estimated with the conventional image based estimation method. The proposed Logan and Patlak joint estimation method can be used in dual tracer imaging to robustly obtain parametric images with higher SNR than conventional methods and may be used to improve lesion detectability.

M16D-7: MRI-Guided PET Kinetic Modeling Pipeline in a Rat Brain Tumor Model

M. A. Richard, J. P. Fouquet, R. Lebel, M. Lepage

Centre d'imagerie moléculaire de Sherbrooke, Université de Sherbrooke, Sherbrooke, Québec, Canada

Voxel-wise kinetic modeling of PET tracers is often limited by statistical noise, especially in early time frames. Moreover, in the 2-tissue compartment model, perfusion, permeability and metabolism can be hard to decouple based solely on positron emission tomography (PET) data. To circumvent these problems, a pipeline of image denoising and kinetic modeling based on dynamic contrast-enhanced magnetic resonance imaging (DCE-MRI) is introduced. This pipeline is tested on data sets of glioblastomabearing rats imaged by MRI (dynamic sequence with Gd-DTPA enhancement) and PET (dynamic sequence with FDG or FET). Registered MRI and PET concentration maps are denoised using a MR-guided non-local means filter and compartment modeling is performed for all imaging probes. Denoising decreases the appearance of artefacts on the kinetic parameter maps and allows for voxel-wise comparison between the perfusion and elimination of FDG or FET and Gd-DTPA. A significant correlation is found between the perfusion of FET and Gd-DTPA in accordance with previous region-of-interest-based observation. 
This work was supported by National Science and Engineering Research Council of Canada under Grant No. RGPIN-2014-05386. ML is member of the FRQS-funded Centre de recherche du Centre hospitalier universitaire de Sherbrooke.

M16D-8: Automated Time-Activity Curve Extraction Using a Novel PET Rat Brain Template

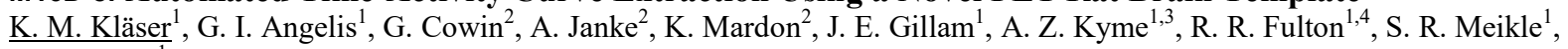
W. J. Ryder ${ }^{1}$

${ }^{I}$ Brain and Mind Centre, The University of Sydney, Sydney, NSW, Australia

${ }^{2}$ Centre for Advanced Imaging, University of Queensland, Brisbane, Queensland, Australia

${ }^{3}$ Department of Biomedical Engineering, University of California Davis, Davis, California, USA

${ }^{4}$ School of Physics, The University of Sydney, Sydney, NSW, Australia

A rat brain atlas can be used for automated extraction of regions of interest (ROIs) from positron emission tomography (PET) images. When the atlas is spatially co-registered with the reconstructed PET volumetric data, it can be used to accurately delineate anatomical structures and extract corresponding regional kinetic parameters without operator bias. To develop the rat brain atlas, we performed rat brain studies and acquired PET and MR data simultaneously. Post-image reconstruction, the Johnson MR histology template was spatially registered to the MR images resulting in a PET/MR atlas in a common space. We demonstrated the capability of the template for the registration of both MR and PET images and the automated extraction of timeactivity curves (TACs) in the striatum and cerebellum. Future work includes the evaluation of the new PET/MR templates, simulated data studies and testing of image registration algorithms.

M16D-9: Spectral Analysis of [18F]ML-10 Time Activity Curves in Glioblastoma Multiforme Subjects. M. J. Oborski ${ }^{1}$, C. M. Laymon ${ }^{1,2}$, F. S. Lieberman ${ }^{3}$, J. Drappatz ${ }^{3}$, J. M. Mountz ${ }^{2}$

${ }^{I}$ Bioengineering, University of Pittsburgh, Pittsburgh, PA, USA

${ }^{2}$ Radiology, University of Pittsburgh, Pittsburgh, PA, USA

${ }^{3}$ Neurology, University of Pittsburgh, Pittsburgh, PA, USA

18F-labeled 2-(5-fluoro-pentyl)-2-methyl-malonic acid ([18F]ML-10) has been proposed as a PET tracer of apoptosis. Initial studies of [18F]ML-10 have focused on assessing voxel-wise changes of standardized uptake values pre- and post-therapy in glioblastoma multiforme (GBM). However, no studies have been reported that characterize [18F]ML-10 pharmacokinetics in GBM. The purpose of this study is to evaluate the degree of heterogeneity in GBM uptake profiles of [18F]ML-10 using spectral analysis (SA), a data driven method used to decompose tissue time activity curves (TACs) into a sum of component exponentials. $\mathrm{N}=6$ GBM subjects were enrolled in this study. The study was designed such that subjects would receive three total scanning sessions: baseline (BL; prior to therapy start), early-therapy assessment (EA; 16 days post therapy start), and followup assessment (FA; 72 days post therapy start). However, 2 subjects did not receive a FA scan, and one subject received only a BL scan. PET scans were performed on either a Siemens HR+ PET scanner or an integrated PET/MR scanner. Each scanning session consisted of 2 dynamic scans, a $45 \mathrm{~min}$ scan initiating with injection of $10 \mathrm{mCi}$ of [18F]ML-10, followed by a second $30 \mathrm{~min}$ scan initiating $120 \mathrm{~min}$ post-injection. Image based input functions (IBIF) were obtained from the internal carotid arteries using a threshold based method, which allowed only voxels with values exceeding $90 \%-95 \%$ of the maximum voxel to contribute to the IBIF. Tumor TACs were obtained using a similar threshold method. SA was performed using two methods: nonnegative least squares (NNLS) and basis pursuit (BP).

As few as 1 and as many as 4 spectral peaks were observed using both BP and NNLS. A strong, fast component was observed in all tumor spectra, indicating that the inclusion of a quickly equilibrating compartment in any compartmental modeling may be appropriate. No peaks were observed at the $18 \mathrm{~F}$-decay rate suggesting reversible uptake of [18F]ML-10.

US National Institutes of Health research grants U01 CA140230, UL1 RR024153, and UL1TR000005. This project used the University of Pittsburgh Cancer Institute In Vivo Imaging Facility that is supported in part by award P30CA047904.

\author{
M16D-10: Direct 4D Slice-Wise Whole-Body Parametric PET Image Reconstruction for Continuous Bed \\ Motion Acquisitions \\ N. A. Karakatsanis ${ }^{1,2}$, A. Mehranian ${ }^{3}$, M. E. Casey $^{4}$, H. Zaidi ${ }^{2,5,6}$ \\ ${ }^{I}$ Translational and Molecular Imaging Institute, Icahn School of Medicine at Mount Sinai, New York, NY, USA \\ ${ }^{2}$ Division of Nuclear Medicine and Molecular Imaging, Geneva University Hospital, Geneva, Switzerland \\ ${ }^{3}$ Department of Biomedical Engineering, King's College London, London, UK \\ ${ }^{4}$ Siemens Molecular Imaging, Knoxville, TN, USA \\ ${ }^{5}$ Geneva Neuroscience Center, University of Geneva, Geneva, Switzerland \\ ${ }^{6}$ Department of Nuclear Medicine and Molecular Imaging, University of Groningen, Groningen, Netherlands
}

Whole-body (WB) parametric PET imaging has recently demonstrated its clinical potential in enhancing quantification by employing robust dynamic analysis on a series of fast WB passes obtained either with step-and-shoot (SS) or continuous bed 
motion (CBM) acquisition mode. In particular, the CBM method has been associated with enhanced lesion detectability over SS in bed overlap regions for indirect Patlak influx rate constant (Ki) images. However, indirect Patlak is not very robust to the high noise levels often encountered in clinical WB dynamic PET imaging, thus limiting its clinical adoption. In addition, the CBM data at each transaxial slice are acquired within different time frames, while the SS data from all slices of a bed can be assigned a single average scan time. Thus, in the absence of list-mode data, accurate time data management may be challenging for dynamic CBM data analysis. In this study, we introduce a novel direct 4D WB parametric PET sinogram-based ordered subsets expectation maximization (OSEM) reconstruction algorithm specifically designed for the robust generalized Patlak analysis of dynamic CBM clinical PET data. The 4D algorithm employs the optimization transfer method to nest multiple faster Patlak subiterations within each of the slower global iteration steps and, thus, accelerate the convergence rate. Furthermore, an average scan time is assigned to each transaxial slice to construct an accurate set of slice-dependent nested Patlak model matrices and therefore permit a highly quantitative and efficient slice-wise $4 \mathrm{D}$ reconstruction of the CBM data. The clinical evaluation of the 4D CBM versus the 4D SS method was performed on a WB dynamic (12 passes) 18F-FDG PET/CT clinical study by switching the two modes between the passes. The quantitative analysis of a suspected high uptake foci located in a bed overlap region indicated an increase of $40-50 \%$ in $\mathrm{Ki}$ target values and 35-45\% in Ki target-to-background scores for the CBM 4D Patlak method.

This work was supported by the Swiss National Science Foundation under Grant SNSF 31003A-149957.

M16E: Posters: Clinical emission

Friday, Nov. $4 \quad$ 16:30-18:30 Etoile M16E-1: A High Resolution Clinical PET/MR System with Optimized Design: Simulation and Preliminary Result

$\underline{\mathrm{X} . \mathrm{CaO}}^{1}, \mathrm{~W} . \mathrm{Xie}^{1}, \mathrm{~L} . \mathrm{Hu}^{2}, \mathrm{H} . \mathrm{Li}^{2}$

${ }^{1}$ United Imaging Healthcare, Shanghai, China

${ }^{2}$ UIH America, Houston, USA

Introduction: Clinical adoption of simultaneous PET/MR has been growing in the past five years. We aimed to evaluate a new clinical PET/MR system (with TOF capability) which is optimally designed for image quality matching between MRI and PET sub-system. The simultaneous whole body PET/MR is equipped with SiPM based on PET detectors and a 3T MRI (with $50 \mathrm{mT} / \mathrm{m}, 200 \mathrm{~T} / \mathrm{m} / \mathrm{s}$ gradient coil).

Method: Compared to commercially available PET/MR scanner, the system was optimized by (1) increasing the PET axial FOV to $32 \mathrm{~cm}$; (2) reducing crystal size to $2.76 \mathrm{~mm}$; (3) speeding up MRI scan. GATE simulation was used to investigate the system architecture and the essential performance characteristics of the design. We also performed spatial resolution measurement and animal imaging to assess the image quality of the system.

Result: Spatial resolution was measured as $2.8 \mathrm{~mm}$ at the center of FOV. DOI effect was significantly mitigated by reducing the crystal size to $2.76 \mathrm{~mm} \times 2.76 \mathrm{~mm} \times 15.5 \mathrm{~mm} 3$. The $32 \mathrm{~cm}$ long axial FOV results in reduced total number of PET scanning beds and increased sensitivity greater than $17 \mathrm{cps} / \mathrm{kBq}$. Animal study was performed on a rabbit injected with $1 \mathrm{mCi}$ FDG.

Simultaneous imaging of PET and T1W MRI sequence with 5 minutes/bed demonstrates good PET/MR registration and clear visualization of fine structure due to the optimized spatial resolution. Because of the large $32 \mathrm{~cm}$ axial FOV, only two scanning bed were needed to cover the whole imaging area. PET performance was not affected by the MRI sequence because of the complete shielding between the two sub-systems.

Conclusion: High resolution PET and MRI was demonstrated to image simultaneously in an optimized whole body PET/MR system. High spatial resolution with minimal DOI effect allows MRI-matching PET image quality in the system. A MRImatching (typically $30-40 \mathrm{~cm}$ ) PET axial FOV not only accelerates the PET acquisition but also reduces the total number of scanning beds.

M16E-2: Multi-Pinhole SPECT System with a Triple Head Gamma Camera H. Kubota $^{1}$, Y. Hemuki ${ }^{1}$, N. Motomura ${ }^{2}$, K. Ogawa ${ }^{1}$

${ }^{I}$ Department of Applied Informatics, Graduate School of Engineering, Hosei University, Tokyo, Japan

${ }^{2}$ Toshiba Medical Systems, Tochigi, Japan

The purpose of our research is to develop a new multi-pinhole SPECT system using a triple head gamma camera system. The proposed data acquisition geometry used a multi-pinhole collimator attached to each gamma camera. We made a multi-pinhole SPECT system with a commercially available gamma camera (GCA-9300R). Eight pinholes were evaluated in this study and the diameter of a pinhole was $5 \mathrm{~mm}$. Acquired data were reconstructed with the OS-EM method. In the experiment we used a three hot-rod phantom and myocardial phantom. As the reference we acquired projection data with the parallel hole collimator. The results of the experiment demonstrated the feasibility of our proposed multi-pinhole SPECT system with a triple head gamma camera. 
M16E-3: Positron Emission Tomography with Additional ?-Ray Detectors for Multiple Probe Imaging

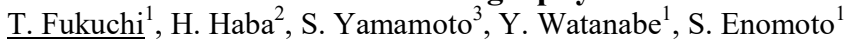

${ }^{I}$ Center for Life Science Technologies, RIKEN, Kobe, Hyogo, Japan

${ }^{2}$ Nishina Center for Accelerator-Based Science, RIKEN, Wako, Saitama, Japan

${ }^{3}$ Department of Radiological and Medical Laboratory Sciences, Nagoya University Graduate School of Medicine, Nagoya, Aichi, Japan

For the feasibility study of the 3-dimensional multiple probe imaging using a positron emission tomography (PET) with additional ?-ray detectors, we have developed a prototype PET system. This system consists of a gadolinium orthosilicate (GSO) ring-type PET and 8 large volume $(50 \times 50 \times 30 \mathrm{~mm} 3)$ bismuth germinate $(\mathrm{BGO})$ additional ?-ray detectors. The ring-type PET has $95 \mathrm{~mm}$ inner diameter and $37.5 \mathrm{~mm}$ of axial field-of-view (FOV). Our developed system identifies different probes based on detection of de-excitation ?-rays, which are successively emitted after the positron, by the additional BGO detectors. To evaluate basic performance of our developed system, we measured the position dependence of the de-excitation ?-ray efficiencies using a $\mathrm{Na}-22$ point source. The detection efficiency for a $1275-\mathrm{keV}$ de-excitation ?-ray of $\mathrm{Na}-22$ was about $7.0 \%$ at the center position, and the minimum efficiency was about $4 \%$ at the edge of axial FOV. In order to test imaging performance, we carried out a rods phantom imaging with dual isotopes. Three of $10 \mathrm{~mm}$ diameter rods had activities of Na-22 (560 kBq) $+\mathrm{F}-18(600 \mathrm{kBq}), \mathrm{Na}-22$ $(560 \mathrm{kBq})$, and F-18 $(600 \mathrm{kBq})$ in the beginning of measurement. As the result of the 30 minutes measurement and image reconstruction containing the normalization of position dependence of de-excitation ?-ray efficiencies, we succeeded in reconstruct clear images with and without de-excitation ?-ray detection. This result indicates our developed system has the ability to analyze the distribution of multiple probes.

M16E-4: Detector Shielding Design of a PET Insert in a 3T MRI System B. J. Lee ${ }^{1}$, R. D. Watkins ${ }^{2}$, C.-M. Chang ${ }^{3}$, I. Kwon ${ }^{2}$, C. S. Levin ${ }^{2}$

${ }^{I}$ Mechanical engineering, Stanford University, Stanford, CA, USA

${ }^{2}$ Radiology, Stanford University, Stanford, CA, USA

${ }^{3}$ Applied physics, Stanford University, Stanford, CA, USA

The combination of positron emission tomography (PET) and magnetic resonance imaging (MRI) provides a powerful tool for disease characterization as it enables the simultaneous measurement of molecular, physiological and anatomical information for the patient. For a PET insert to be placed inside an MR system, shielding for the detector modules is crucial as otherwise there can be mutual interference between the two modalities. In this work, we have assessed various shielding designs for both RF shielding effectiveness and gradient-induced eddy current performances. We designed six shielding configurations with various thicknesses, patterns and materials: 36,18 and $9 \mu \mathrm{m}$ thick solid copper (solc); $36 \mu \mathrm{m}$ thick segmented copper(segc); $36 \mu \mathrm{m}$ thick phosphor bronze mesh (pbm); and $0.8 \mathrm{~mm}$ thick carbon fiber composite ( $\mathrm{cfc}$ ). To evaluate the shielding performance, we performed transmission measurements (S21) and acquired the induced voltage from a MRI spin echo sequence using an RF probe. The eddy current performance was evaluated by the reflection measurement (S11) and observing the ghosting artifact and temperature rise from a MRI echo planar imaging (EPI) sequence. For the shielding effectiveness in both in/outside the MR system, the S21 magnitude for the solc $(-43.1 \pm 1.7,-42.5 \pm 3.4$, and $-42.3 \pm 2.6 \mathrm{~dB})$ and pbm $(-37.6 \pm 3.6 \mathrm{~dB})$ configurations performed significantly better compared to other configurations (cfc $-29.2 \pm 1.5 \mathrm{~dB}$ and segc $-11.0 \pm 0.7 \mathrm{~dB}$ ). For the eddy current performance, the phase delay acquired from the $\mathrm{S} 11$ measurement was less than $45^{\circ}$ for segmented copper, phosphor bronze mesh and carbon fiber composites and significantly higher at $\sim 120^{\circ}$ for the solid copper configurations. The EPI ghosting artifact and temperature measurements showed similar trends inside the MR system as seen in the outside. In summary, we have evaluated the shielding effectiveness and the eddy current performances of several shielding designs, with the pbm showing the best overall outcomes.

M16E-5: Investigation of Cooling Structure Design for PET Detector Thermal Regulation Methods B. J. Lee ${ }^{1}$, C.-M. Chang ${ }^{2}$, I. Kwon ${ }^{3}$, C. S. Levin ${ }^{3}$

${ }^{I}$ Mechanical engineering, Stanford University, Stanford, CA, USA

${ }^{2}$ Applied physics, Stanford University, Stanford, CA, USA

${ }^{3}$ Radiology, Stanford University, Stanford, CA, USA

Temperature is an essential factor for stable performance of the positron emission tomography (PET). Some of the PET detector components produce significant heat (e.g. readout integrated circuits) that affects its performance. The temperature alters the breakdown voltage of photodetectors, which in turn affects the gain, cross talk, dark count rate and after-pulsing. One of the thermal regulation methods is to use an air/liquid cooling pipe embedded in a cold plate broadly distributing the temperature to the PC board; the gold vias in the board then conduct the temperature to the other side of the board. This paper concentrates on investigating the temperature distribution from various cooling structure configurations. The simulation software COMSOL was used with heat transfer and pipe flow modules to assess the thermal behavior of the cooling system. Two types of cooling pipe arrangements were simulated; a simple and a more complex curve arrangement. For the cold plates, a ceramic $(92 \mathrm{~W} / \mathrm{m} \cdot \mathrm{K})$ and a 
thermally conductive plastic $(10 \mathrm{~W} / \mathrm{m} \cdot \mathrm{K})$ were simulated. Full as well as "patchy" gold via configurations were also simulated. The simulation was performed for 300 seconds (real time) and the average and the standard deviation temperature of each SiPM was analyzed. With the complex cooling pipe arrangement, the temperature variation throughout all SiPMs was on average $37.9 \pm 5.2 \%$ higher for ceramic cold plates. For the cold plate materials, the ceramic showed $33.9 \pm 15.4 \%$ smaller thermal variation and $-1.1 \pm 0.6 \%$ lower temperature compared to the thermally conductive plastic. The patchy vias resulted in $218.2 \pm 74.9 \%$ larger thermal variation when compared to the patchy vias. In summary, we have simulated various cooling pipe designs, cold plates and gold via distribution configurations to analyze the temperature variation across a PET detector PC board. The thermally conductive ceramic cold plate with as many vias as possible resulted in the most stable temperature variation.

\author{
M16E-6: The "bed-PET": a Proposed Geometry for a Highly Sensitivite and Affordable Whole Body PET \\ Scanner \\ A. M. Ahmed, H. Tashima, E. Yoshida, T. Yamaya \\ National Institute of Radiological Sciences, Chiba, Japan
}

Continuous efforts are being made to increase the sensitivity and achieve high spatial resolution of positron emission tomography (PET) scanners. Current clinical PET scanners have a cylindrical shape with a diameter of about $800 \mathrm{~mm}$. For PET scanners, it is essential to arrange the detectors close to the subject to increase the sensitivity and decrease the angular deviation effect. However, the parallax error caused by the thickness of the scintillator degrades the spatial resolution of the system. To address this problem, advanced depth-of-interaction (DOI) detectors can be used. Another way of increasing the sensitivity is to increase the number of detector rings. Although this arrangement increases the sensitivity, the cost of the scanner is the limiting factor. In this study, we propose a PET scanner with a half-ring and a bed detector, referred to as the "bed-PET". In this configuration, half of the detectors in a full ring detector were replaced with a rectangular shaped detector. The basic idea is to reduce the unused space under the patient's bed; this area is usually not in use in clinical diagnosis. We used the geant 4 simulation toolkit to construct the bed-PET. In the simulation, the bed-PET was constructed from $82 \%$ of the detectors used in the cylindrical PET. Each detector was composed of a $16 \times 16 \times 4$ array of GSO crystals with dimensions of $2.8 \times 2.8 \times 7.5 \mathrm{~mm}^{3}$. The sensitivity and the spatial resolution were evaluated using a point source placed at several positions in the field-of-view. The results showed that the bed-PET could achieve a maximum of $177 \%$ gain in sensitivity and $18 \%$ reduction in the number of detectors with respect to the cylindrical PET. The spatial resolution of the bed-PET with DOI detectors was slightly improved due to a significantly minimized non-collinearity effect. In conclusion, the bed-PET can provide high sensitivity and affordable cost with a slightly improved spatial resolution.

M16E-7: Time-Based Signal Sampling Using Saw-Tooth Shaped Threshold

G. B. Ko, J. S. Lee

Department of Nuclear Medicine, Seoul National University, Seoul, South Korea

The simplicity of time-over-threshold (ToT) is attractive for SiPM-based TOF PET systems having large numbers of readout channels, because ToT reduces readout cost, power consumption, and complexity compared to pulse height analysis which uses an analog to digital converter (ADC). However, relatively poor energy resolution and energy nonlinearity of ToT results in degradation of detector performance making it not suitable to achieve high performance. In this study, we propose a new timebased signal sampling method for the scintillation detector using an adaptive saw-tooth shaped threshold (called saw-tooth threshold sampling, STS) to address these problems. The STS was implemented with a circuit composed of a comparator and several digital logic components. A digital pulse train is generated by comparing the scintillation pulse with the saw-tooth threshold. Using the time information at several leading and trailing edges of the digital pulse train, time and voltage of several sampling points were calculated and the bi-exponential model was then fitted to these sampling points to reconstruct the original scintillation pulse. The energy is calculated by integrating the reconstructed pulse and the arrival time was extracted from the first rising edge of digital pulse. Coincidence data from two identical 1:1 coupled SiPM detectors consisting of LGSO crystals $(3 \times 3$ $\times 20 \mathrm{~mm}^{3}$ ) and FBK NUV-HD SiPMs were acquired to assess the performance of STS. We found that there is a strong linear relationship between the energy acquired using STS and that measured using waveform sampling. The energy resolution and time resolution of $10.3 \%$ and 198.7 ps were obtained using STS, which are similar to those obtained using waveform sampling $(9.8 \%$ and $193.0 \mathrm{ps}$ ) but better than those obtained using simple ToT method (16.4\% and $223.5 \mathrm{ps})$. The results prove that the proposed method is promising cost-effective solution for high performance PET systems.

The authors would like to thank FBK for the SiPM samples used in this work.

M16E-8: Performance of the MediPROBE Compact Gamma Camera for Coded Aperture Imaging

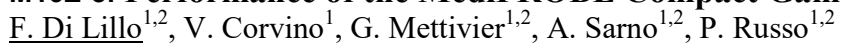

${ }^{I}$ Dept. of Physics, Universita' di Napoli Federico II, Napoli, Italy

${ }^{2}$ Sezione di Napoli, INFN, Napoli, Italy 
The aim of this work is to assess the performance of a prototype compact gamma camera (MediPROBE) based on a CdTe semiconductor hybrid pixel detector for coded aperture imaging. This probe can be adopted for various tasks in nuclear medicine such as preoperative sentinel lymph node localization, breast imaging with 99mTc-sestamibi and thyroid imaging with iodinated radioisotopes. The hybrid detector is an assembly of a $1 \mathrm{~mm}$ thick CdTe semiconductor detector (sensitive area 14.08?14.08 $\mathrm{mm} 2$ ) bump-bonded to a photon-counting CMOS readout circuit of the Medipix2 series (256?256 square pixels, $55 \mu \mathrm{m}$ pitch) or with a photon counting, energy-sensitive Timepix detector (128?128 square pixels, $110-\mu \mathrm{m}$ pixel pitch). MediPROBE is equipped with a set of three interchangeable knifeedge pinhole collimators (hole aperture of $0.78 \mathrm{~mm}, 1 \mathrm{~mm}$, or $1.9 \mathrm{~mm}$ ) and a set of two coded aperture masks with $0.070-\mathrm{mm}$ or $0.080-\mathrm{mm}$ round holes. We performed measurements of field of view (FOV), system spatial resolution, and signal-difference-tonoise ratio (SDNR) by using a point-like gamma-emitting $241 \mathrm{Am}$ source (60 $\mathrm{keV})$. The detector is operated at a single low-energy threshold of about $5 \mathrm{keV}$. At $60 \mathrm{keV}$ the sensitivity is $0.0085 \mathrm{cps} / \mathrm{kBq}(\mathrm{w} /$ $0.78 \mathrm{~mm}$ pinhole). The system spatial resolution was $2.57 \mathrm{~mm} \mathrm{FWHM} \mathrm{(w/} 0.78 \mathrm{~mm}$ pinhole) at a source-collimator distance of $45 \mathrm{~mm}$, and $0.560 \mathrm{~mm} \mathrm{FWHM} \mathrm{(w/} \mathrm{coded} \mathrm{aperture} 0.080 \mathrm{~mm}$ ) at a source-collimator distance of $50 \mathrm{~mm}$. We observed greater values of SDNR with the use of the coded aperture and the Timepix detector. A clinical trial is ongoing to evaluate the performance of MediPROBE for gamma-ray nuclear imaging.

\author{
M16E-9: An RF-Penetrable Oval Body PET Insert for MRI: Initial Experimental Study for Efficient MR \\ Imaging Performance \\ M. S. H. Akram ${ }^{1}$, C. S. Levin ${ }^{2}$, T. Obata ${ }^{1}$, T. Yamaya ${ }^{1}$ \\ ${ }^{I}$ Department of Radiation Measurement, Imaging and Dosimetry, National Institute of Radiological Sciences, Chiba, Chiba, \\ Japan \\ ${ }^{2}$ School of Medicine, Stanford University, Stanford, California, USA
}

The complementary advantages of PET/MRI body imaging like, for lung cancer, esophageal cancer, etc. are immense. To utilise these opportunities with existing MRI systems, we are working on development of an oval shape body PET insert. The oval shape matches human body shape and it will be suitable to insert inside MRI bore. For feasibility study, we developed a smaller prototype oval insert (major and minor axis of $210 \mathrm{~mm}$ and $140 \mathrm{~mm}$ ) with 16 shield boxes. All experiments were conducted in a 3 T clinical MRI system by using uniform phantom. For MR imaging, we used MRI body RF coil as transceiver. To let the RF transmission field pass through the gaps between shielded PET modules, PET modules were kept floating from RF ground. For such RF-penetrable PET inserts, it is very challenging to get efficient RF field (B1) and high SNR MR signals inside the PETring. Experimental results showed, for all shield box condition, B1 homogeneity reduced from $79 \%$ (for without shield) to $36 \%$ and, SNR values for spin-echo (SE) image reduced from 219 to 82 within the $70 \%$ central circular transverse plane. To get efficient RF field and high SNR images, we removed shield boxes from different positions. Two particular positions showed remarkable results: removing shield boxes from near (a) minor and (b) major axis-ends. We got $66 \%$ and $75 \%$ RF field homogeneities respectively for case (a) and (b), and corresponding SE SNR were 133 and 125 for the central transverse plane. This study proves that with full-ring RF-penetrable PET insert MR imaging performances degrades to much lower level whereas removing PET detector modules from different positions would improve MR imaging performances even close to without PET insert case.

\author{
M16E-10: Development of the Second Prototype of RF Coil Integrated DOI-PET Insert: MRI Compatibility \\ Study \\ M. S. H. Akram ${ }^{1}$, T. Obata ${ }^{1}$, M. Suga ${ }^{2}$, F. Nishikido ${ }^{1}$, E. Yoshida ${ }^{1}$, K. Shimizu ${ }^{3}$, M. Fujiwara ${ }^{1}$, A. Mohammadi ${ }^{1}$, T. Yamaya $^{1}$ \\ ${ }^{I}$ Department of Radiation Measurement, Imaging and Dosimetry, National Institute of Radiological Sciences, Chiba, Japan \\ ${ }^{2}$ Research Center for Frontier Medical Engineering, Chiba University, Chiba, Japan \\ ${ }^{3}$ Development Center, Hamamatdu Photonics K.K., Hamamatsu, Japan
}

As the commercial PET/MRI systems are highly costly, for loco-regional imaging, like, brain imaging, different groups are working on development of PET inserts for the existing MRI systems. In our lab we are working on the development of an MRI radiofrequency $(\mathrm{RF})$ coil integrated PET modality for simultaneous brain imaging with a $3 \mathrm{~T}$ clinical MRI system. This study corresponds to the development and MRI compatibility study of the second prototype of this integrated PET/RF-coil concept. Compare to first prototype we have made several important design modifications: (a) implementing carbon-fiber as shielding instead of copper shield as it is outperformed $\mathrm{Cu}$ in terms eddy current effects; (b) PET axial FOV is $55 \mathrm{~mm}$ instead of $12 \mathrm{~mm}$; (c) MPPC array contains temperature control sensor to provide common bias voltage to all MPPC channels; (d) PET read out circuit board implements ASIC preamplifiers; (e) data read out cables are coaxial instead of twisted pair; (f) 24 PET detector modules were integrated with an 8-element RF coil whereas it was 8 PET modules with 8-element RF coil; (g) data acquisition system was inside the MRI room and so on. Initial MRI compatibility study of this system showed maximum RF homogeneity of $85 \%$ (and minimum 70\%), static field inhomogeneity of $2 \mathrm{ppm}(6 \mathrm{~T})$, and very negligible SAR for a $170 \mathrm{~mm}$ diameter by $80 \mathrm{~mm}$ axial-length cylindrical FOV at the center. The overall results show a greater promise for using this multimodal system for simultaneous human brain imaging with higher DOI-PET resolution. 
M16E-11: Characterization and Quantitation of Emission Contamination of Dedicated Transmission Images in Breast SPECT-CT

\author{
${ }_{\text {F. A. McDougal }}^{1}$, J. P. Shah ${ }^{2,3}$, M. P. Tornai ${ }^{1,2,3}$ \\ ${ }^{I}$ Medical Physics, Duke University Medical Center, Durham, NC, USA \\ ${ }^{2}$ Biomedical Engineering, Duke University, Durham, NC, USA \\ ${ }^{3}$ Radiology, Duke University Medical Center, Durham, NC, USA
}

An advanced dual-modality SPECT-CT system for dedicated functional and structural breast imaging was developed for diagnostic imaging. The SPECT and CT subsystems have a common FOV, where images could be measured simultaneously or sequentially, and each subsystem can move independently of the other in complex trajectories. A patient undergoing a SPECT scan will necessarily have $140 \mathrm{keV}$ gammas emanating from their breast (and torso), and these could contaminate the $40 \times 30 \mathrm{~cm} \wedge 2$ large-area, CsI(Tl) flat-panel CT detector. As previously shown with a smaller flat-panel detector, this contamination can increase the projection image noise, as well as lower the measured reconstructed attenuation values associated with potential lesions, leading to lower image contrast and lesion detectability. Several different sized cylinders with simulated glandular and fatty content (water and methanol mixtures, from $340-3800 \mathrm{~mL}$ ) with/without radioactivity $(0,1 \mathrm{mCi} / \mathrm{mL}, 10 \mathrm{mCi})$ are measured by the CT subsystem in projection and tomographic modes using the various angle-of-incidence vantages possible $\left( \pm 12.5^{\circ}\right.$ polar $)$ with the fully-3D acquisition system. Anthropomorphic phantoms are also imaged. Initial results indicate that the phantom size has a greater affect on measured background signal level than the stochastic gamma emissions from the phantoms. Histograms of ROIs within the projection images also show differences under the different radioactive contamination conditions. Characterizing any emission contamination during a CT scan is important for better corrections and more quantitative CT images, which can lead to more accurate diagnoses of breast disease.

\author{
M16E-12: Characterizing CNR of Super-Resolution and Sub-Resolution PET \\ G. Chinn, J. W. Cates, C. S. Levin \\ Radiology, Stanford School of Medicine, Stanford, CA, USA
}

In this work, we study the effect of intrinsic detector spatial resolution on the contrast-to-noise ratio (CNR) of reconstructed images. Super-resolution PET uses patient or gantry motion to reconstruct images at a higher resolution than the intrinsic detector resolution. Sub-resolution PET reconstructs images at a lower resolution than the intrinsic detector resolution. Super-resolution can increase the image resolution at lower cost, but at a penalty of CNR loss. Sub-resolution is more expensive, but improves the CNR of reconstructed images. In this study, we characterize the CNR for super- and sub- resolution PET using simulated 2-D non-TOF ML-EM, 3D non-TOF ML-EM, and 3D TOF data sets. We show that a $1 \mathrm{~mm}$ non-TOF sub-resolution system with 2-D data acquisition improves the CNR of 1-3 mm lesions by 2-3 fold compared to a system with $3 \mathrm{~mm}$ intrinsic resolution. Meanwhile, super-resolution has a 4-9 fold CNR penalty.

\title{
M16E-13: Geometry Optimization of Dual-Layer Offset Detectors for Compact Ring Diameter PET Systems M. Teimoorisichani ${ }^{1}$, A. L. Goertzen ${ }^{1,2}$ \\ ${ }^{I}$ Dept. of Physics and Astronomy, University of Manitoba, Winnipeg, Manitoba, Canada \\ ${ }^{2}$ Dept. of Radiology, University of Manitoba, Winnipeg, Manitoba, Canada
}

Compact ring diameter PET systems suffer degradation in spatial resolution with increasing radial offset due to the parallax error caused by depth of interaction (DOI) effects. In this work, we study the problem of optimizing layer thicknesses for a detector that uses a dual-layer offset (DLO) scintillator array, in which there is a half-crystal offset between layers. The goal of this optimization is to minimize parallax related event mispositioning for fixed total detector thicknesses and crystal pitches. GATE Monte Carlo simulations are used to investigate detector geometries for a dedicated human brain PET system with diameter 343 mm using LYSO DLO detectors. Total crystal thicknesses range from 10 to $30 \mathrm{~mm}$, with front-to-back layer ratios of $10 / 90$ to $100 / 0$ (i.e. single layer) and crystal pitches of 2 and $4 \mathrm{~mm}$. Collectively these options define 60 system geometries. For each total thickness, the layer thickness ratio was found such that the radial displacement between the first point of interaction in the scintillator array and the assigned position of the event is minimized. Coincidence response functions are generated by simulating measurements with a finely stepped point source for crystal pitches of 2 and $4 \mathrm{~mm}$. Results show that the radial blurring is minimized for front layer thicknesses of approximately 5,8 and $9 \mathrm{~mm}$ for total crystal thicknesses of 10,20, and 30 mm, respectively. For these layer thicknesses and a $2 \mathrm{~mm}$ crystal pitch, radial displacement decreased by $29.2 \%, 37.5 \%$ and $43.6 \%$, respectively, vs. single layer arrays for a $45^{\circ}$ angle of incidence. For total detector thicknesses greater than $10 \mathrm{~mm}$, the optimal geometry was always one that had a thinner front layer.

\section{M16E-14: Development of Tachyon Time-of-Flight PET Cameras} Q. Peng, W. W. Moses

Department of Molecular Biophysics and Integrated Bioimaging, Lawrence Berkeley National Laboratory, Berkeley, USA 
The signal-to-noise ratio (SNR) in PET is dominated by counting statistics (i.e., PET is starved for events), but incorporating the additional Time-of-Flight (TOF) information into the reconstruction increases the statistical quality of the acquired data. The increased statistical efficiency gained with TOF information can be used to decrease acquisition time, decrease injected dose, or improve image quality. We are designing Tachyon TOF PET cameras (Tachyon I, II and III) to: (1) Construct "demonstration" TOF PET cameras that achieve significantly improved timing resolution; (2) Quantitatively measure the benefit for clinically relevant tasks as a function of timing resolution.

Tachyon I is a single-ring TOF PET scanner constructed with LYSO crystals and Hamamatsu R-9800 PMTs. The ring diameter of the scanner is $76.6 \mathrm{~cm}$. Time resolution of the system (defined as of each crystal-crystal combination) is $314 \mathrm{ps} \pm 20 \mathrm{ps}$.

Tachyon I is currently in clinical test stage.

Tachyon II is a single-ring SiPM-based TOF PET scanner currently under construction. The ring diameter of the scanner is still $76.6 \mathrm{~cm}$. LaBr3 scintillator crystals will be used in Tachyon II. The target system-level CRT timing resolution is 100ps or better. We are currently testing the performances of the photo sensors and electronics using LYSO crystals. A 144ps CRT timing resolution has been achieved with $5 \mathrm{~mm} \times 5 \mathrm{~mm} \times 5 \mathrm{~mm}$ LYSO crystals and $6 \mathrm{~mm}$ SiPMs.

Tachyon III is a practical multiple-ring SiPM-based TOF PET scanner for real clinic applications. Tachyon III is currently in system design stage. We have completed the detector design and simulation. p-PET v1.0 electronics, a novel high-performance, low-cost, compact and low power consumption electronics system, will be used to construct Tachyon III camera.

M16E-15: Progresses of designing a high-sensitivity high-resolution dodecahedron PET for brain imaging J. Xu ${ }^{1}$, Q. Huang ${ }^{2}$, J. Chen ${ }^{1}$, Z. Zhao ${ }^{2}$, S. Xie ${ }^{1}$, Q. Peng ${ }^{3}$

${ }^{I}$ School of Mechanical Science and Engineering, Huazhong University of Science and Technology, WuHan, China

${ }^{2}$ Shanghai Jiaotong University, Shanghai, China

${ }^{3}$ Department of Molecular Biophysics and Integrated Bioimaging, Lawrence Berkeley National Laboratory, Berkeley, USA

Dedicated brain PET has the potential to achieve better sensitivity, spatial resolution and image quality than conventional whole body PET cameras in brain tomography. Spherical PET (S-PET), that can achieve a higher geometrical sensitivity and a lower parallax error than conventional cylindrical ring PET scanners, is a good candidate for high performance dedicated brain imaging. Our simulation studies show that the S-PET has a local geometric efficiency 2.7 times higher than the $30 \mathrm{~cm}$ cylinder PET, and 19 times higher than the $76 \mathrm{~cm}$ cylinder PET in the zones around the cerebral cortex. However, it is very challenging to design detectors with curved surface for S-PET. Convex polyhedron PETs, such as dodecahedral PET, is easier to build and has the potential to achieve performances equivalent to those of S-PETs. We are building a high-sensitivity and high-resolution $(1 \sim 1.5 \mathrm{~mm})$ dodecahedron PET for brain imaging. In this paper, we report our progresses in: (1) design, simulation and fabrication of a pentagon-shaped detector module, (2) design, fabrication and test of high-performance readout electronics (pPET v1.0 electronics), and (3) design and fabrication of the mechanic gantry for the dodecahedron PET.

M16F: Posters: Image Quality

Friday, Nov. $4 \quad$ 16:30-18:30 Etoile

M16F-1: Comparison Study of Noise Reduction Algorithms in Dual Energy Chest Digital Tomosynthesis D. Lee ${ }^{1}$, Y.-S. Kim ${ }^{2}$, S. Choi $^{2}$, H. Lee ${ }^{2}$, S. Choi ${ }^{1}$, H.-J. Kim ${ }^{1,2}$

${ }^{I}$ Radiation Convergence, Yonsei University, Wonju, Korea

${ }^{2}$ Radiological Science, Yonsei University, Wonju, Korea

Dual energy chest digital tomosynthesis (DECDT) is recently developed new medical device that contained advantages of both tomosynthesis and dual energy x-ray images. However, the quantum noise, which occurs in the process of dual energy x-ray images, strongly interfered diagnose in various clinical situations. For this reason, the noise reduction in DECDT is necessary. In this study, different dual energy techniques including simple log subtraction (SLS), simple smoothing of the high energy image (SSH), and anti-correlated noise reduction (ACNR) were evaluated in terms of image noise property in DECDT by experimental studies. The CDT was operated in linear movement and 21 projections were obtained over 30-degree angular range with 1.5 degree angular interval. For DECDT, $80 \mathrm{kVp}$ and $140 \mathrm{kVp}$ x-ray energy spectrum were used. The results showed that DECDT achieved anatomical noise suppressed results by simplifying chest structure with dual energy techniques, while the image quality according to dual energy techniques was different. For example, SLS technique showed high image noise characteristics compared to SSH and ACNR techniques. Likewise the quantitative evaluation with coefficient of variation (COV) indicated that SSH and ACNR technique achieved much less COV than SLS technique. Especially, ACNR technique showed the best result ofnoise reduction in CDT system. In conclusion, DECDT with ACNR techniques provided improved image quality regarding both anatomical and quantum noise. The results of this study can contribute to better diagnostic DECDT images in various clinical situations.

This research was financially supported by the Ministry of Trade, Industry \& Energy(MOTIE), Korea Institute for Advancement of Technology(KIAT) and Gangwon Institute for Regional Program Evaluation(GWIRPE) through the Economic and Regional Cooperation Industry 
M16F-2: Impact of Time-of-Flight and Scanner Sensitivity on Lesion Recovery in PET $\underline{X}^{\text {. Jin }}{ }^{1}$, S. G. Ross ${ }^{1}$, W. T. Peterson ${ }^{1}$, H. Qian ${ }^{2}$, G. Fu ${ }^{2}$, C. W. Stearns ${ }^{1}$

${ }^{I}$ GE Healthcare, Waukesha, WI, USA

${ }^{2}$ GE Global Research Center, Niskayuna, NY, USA

Introduction - SiPM-based PET detectors have provided the capability of improved time resolution in time-of-flight (TOF) PET scanners. Another key factor that drives PET image quality is the number of counts, which is especially important in clinical studies for dose reduction and short scans. Methods - We investigated the impact of time resolutions from non-TOF to 200 ps, and a wide range of scanner sensitivities between $5.5 \mathrm{cps} / \mathrm{KBq}$ and $21 \mathrm{cps} / \mathrm{KBq}$ on lesion recovery in simulation studies using the SimSET package. We designed 30 lesions of $4 \mathrm{~mm}$ to $16 \mathrm{~mm}$ in diameter and 2:1, 4:1 and 8:1 in contrast. The scanner was defined based on a GE prototype PET/CT scanner with SiPM-based detectors. PET data were simulated for 40M, 56M, 70M, 100M and $153 \mathrm{M}$ events with 550ps, 380ps, 340ps, 270ps and 200ps time resolutions. Relative visual conspicuity of the lesions in the reconstructed images was assessed across various time resolution and count levels. This subjective assessment was augmented by the contrast-to-noise ratio (CNR). Results - With 8:1 contrast, the $4 \mathrm{~mm}$ lesion is much better recovered with $>100 \mathrm{M}$ counts than $40 \mathrm{M}$ counts, in which the $4 \mathrm{~mm}$ lesion was not visible. Improved time resolution did not substantially improve the recovery of the $4 \mathrm{~mm}$ lesion. With 4:1 contrast, the four $6 \mathrm{~mm}$ lesions can only be clearly detected with $100 \mathrm{M}$ or more events and some level of TOF. There is a clear trend of progressively improved CNR with more counts. The CNR for $153 \mathrm{M}$ events approximately doubles that for $40 \mathrm{M}$ events. Better time resolution also improves CNR, as $200 \mathrm{ps}$ time resolution gives 30\%-50\% increase in CNR compared to non-TOF. However, the improvement with better TOF is less pronounced than the improvement with more counts. Conclusion - Better time resolution is constrained by the inherent noise in PET isotope annihilation and iterative image reconstruction. On the other hand, increased scanner sensitivity can substantially reduce image noise and enhance lesion conspicuity.

\author{
M16F-3: The Importance of Specifying Detector Sensitivity and Quantum-limited Dose in DQE \\ Characterizations of Flat Panel Detectors to Ensure High-quality Images \\ M. J. Petrillo ${ }^{1}$, I. D. Job ${ }^{1}$, I. Cunningham ${ }^{2}$ \\ ${ }^{I}$ Imaging Components, Varian Medical Systems, Salt Lake City, UT, United States \\ ${ }^{2}$ Robarts Research Inst, Western University, and DQE Instruments, Inc., Toronto, ON, Canada
}

In general, Detection Quantum Efficiency, DQE, is the standard metric for assessing image quality of a flat panel detector (FPD). However, a single descriptor is insufficient for describing the image quality of an FPD used for clinical imaging tasks that involve complex anatomical structures. Most clinical imaging applications study anatomy with varying x-ray attenuation properties which creates a wide range of entrance dose to the FPD and it is important to understand the limiting dose level, or Quantum Limited Dose (QLD). For example, lung studies may have X-ray attenuation differences as high as two orders of magnitude. This paper seeks to demonstrate DQE usually presented at optimal x-ray exposure levels can mislead expectations of image quality delivered at lower exposures. This paper proposes including QLD and DQE in order to understand the dose range of a FPD.

\title{
M16F-4: Evaluation of Imaging Accuracy for Limited Field of View (LFOV) in Digital Tomosynthesis System D. Kim ${ }^{1}$, B. Jo ${ }^{2}$, H. Lee ${ }^{2}$, C. Zhen ${ }^{1}$, H.-J. Kim ${ }^{1,2}$ \\ ${ }^{I}$ Dept of Radiation Convergence Engineering, College of Health Science, Yonsei University, Wonju, Korea \\ ${ }^{2}$ Dept of Radiological Science, College of Health Science, Yonsei University, Wonju, Korea
}

Limited field of view (LFOV) reconstruction is a technique to reduce radiation dose in CT (Computed tomography) application. Digital tomosynthesis is a method for offering high-quality image at radiographic dose levels. Applying LFOV reconstruction method to digital tomosynthesis system could expect a better dose reduction effect. The aim of this study was to evaluate the improvement of image accuracy by LFOV reconstruction using conventional extrapolation and a prior image method in digital tomosynthesis system. Digital tomosynthesis system was simulated by using matrix programming language. The distance from source and object to detector were $900 \mathrm{~mm}$ and $100 \mathrm{~mm}$, respectively. Forty one projection data were acquired over a $40^{\circ}$ angular range. Shepp-logan and computational human chest phantom with lung nodule were used in this simulation study. Region of interest (ROI) was selected on the 64th slice of reconstructed images obtained using extrapolation and a prior image method. The root mean square error (RMSE) of ROI was calculated to measure differences between the reference data and the restored data which were obtained using extrapolation and a prior image method. In these results, image accuracy of the reconstructed images has been improved using a prior image method and extrapolation. The results showed that a prior image method resulted in the lowest RMSE value. In conclusion, the truncated portion can be restored by extrapolation and a prior image method in LFOV data. A prior image may provide the most accurate method for LFOV imaging technique in digital tomosynthesis system. 
This research was financially supported by the Ministry of Trade, Industry \& Energy(MOTIE), Korea Institute for Advancement of Technology(KIAT) and Gangwon Institute for Regional Program Evaluation(GWIRPE) through the Economic and Regional Cooperation Industry

M16F-5: Performance analysis of ML and ART methods in newly developed chest digital tomosynthesis(CDT) $\underline{\text { H. Lee }}^{1}$, Y.-S. Kim ${ }^{1}$, S. Choi ${ }^{1}$, D. Lee ${ }^{2}$, D. Kim ${ }^{2}$, S. Choi ${ }^{2}$, H.-J. Kim ${ }^{1,2}$

${ }^{I}$ Department of Radiological Science, Research Institute of Health Science, Yonsei University, Wonju, Korea

${ }^{2}$ Department of Radiation Convergence Engineering, Research Institute of Health Science, Yonsei University, Wonju, Korea

In chest digital tomosynthesis (CDT), algorithm development is challenging in that a limited number of projections are acquired over a limited angular range. To confirm the feasibility of maximum likelihood (ML) and algebraic reconstruction technique (ART) methods under variations in key imaging parameters, quality metrics were conducted using LUNGMAN phantom included grand-glass opacity (GGO) tumor. The reconstructed images ware acquired from the 41 projections over 40 degrees angular range using a prototype CDT. To investigate comparison of the limited-angle cone beam reconstruction methods for $\mathrm{CDT}$, the projection data were reconstructed using ML and ART algorithm. We investigated contrast-to-noise ratio (CNR) to evaluate the quality of tomosynthesis image in the reconstruction plane (XY) and artifact spread function (ASF) to quantify the magnitude of artifacts in z-direction. Optimization of ART relaxation parameter could yield considerably improved reconstruction quality from the same projection data. Back-projection (BP) initial guess also improved quality of image in comparison of uniform (UI) initial guess. ART method with proper values of reconstruction parameters could give higher quality of image than ML method. Our results using these algorithms and considering reconstruction parameters may provide information to improve performance for nodule detection in CDT system.

ACKNOWLEDGEMENT This research was financially supported by the Ministry of Trade, Industry \& Energy(MOTIE), Korea Institute for Advancement of Technology(KIAT) and Gangwon Institute for Regional Program Evaluation(GWIRPE) through the Economic and Regional Cooperation Industry.

\author{
M16F-6: Comparative Evaluation of Image Reconstruction Methods for the Siemens PET-MR Scanner Using \\ STIR Library \\ D. Deidda $^{1,2}$, N. Efthimiou ${ }^{1}$, R. Manber ${ }^{3}$, P. Markiewicz ${ }^{4}$, K. Thielemans ${ }^{3}$, R. Aykroyd ${ }^{2}$, C. Tsoumpas ${ }^{1}$ \\ ${ }^{I}$ Division of Biomedical Imaging, University of Leeds, Leeds, United Kingdom \\ ${ }^{2}$ Department of Statistics, University of Leeds, Leeds, United Kingdom \\ ${ }^{3}$ Institute of Nuclear Medicine, University College London, London, United Kingdom \\ ${ }^{4}$ Centre for Medical Image Computing, University College London, London, United Kingdom
}

With the introduction of PET-MR scanners a comparison of the performance of different reconstruction algorithms and the characteristics of the reconstructed images data is relevant. In this work, we perform a quantitative assessment of the currently used ordered subset (OS) algorithms for both standard and lower count PET-MR data taken from a Siemens Biograph mMR scanner using the Software for Tomographic Image Reconstruction (STIR, stir.sf.net). A comparison has been performed in terms of bias and coefficient of variation (CoV). Within the STIR library different algorithms are available, such as OSEM, OS Maximum A Posteriori One Step Late (OSMAPOSL) with Quadratic Prior (QP) and with Median Root Prior (MRP), OS Separable Paraboloidal Surrogate (OSSPS) with QP and Filtered Back-Projection (FBP). In addition, List Mode (LM) reconstruction is available. Corrections for attenuation, scatter and random event are performed using STIR instead of using the scanner. Data from the Hoffman brain phantom are acquired, processed and reconstructed. Clinical data from the thorax of a patient have also been reconstructed with the same algorithms. The reconstructed images of the Hoffman's brain phantom show that OSEM has small bias, $1 \%$, on standard counts images. The number of subsets does not appreciably affect the bias nor the Coefficient of variation $(\mathrm{CoV}=11 \%)$ at a fixed sub-iteration number. The percentage relative bias and $\mathrm{CoV}$ maximum values for OSMAPOSL-MRP are $10 \%$ and $15 \%$ at $360 \mathrm{~s}$ acquisition and $12 \%$ and $15 \%$ for the 36 secs, whilst for OSMAPOSL-QP they are $6 \%$ and $16 \%$ for 360 secs acquisition and $11 \%$ and $23 \%$ at 36 secs and for OSEM $6 \%$ and $11 \%$ for the 360 secs acquisition and $10 \%$ and $15 \%$ for the 36 secs. Our findings demonstrate that when it comes to shortage of counts, noise and bias become significant. The methodology for reconstructing Siemens mMR data with STIR is included in the CCP-PET-MR website (ccppetmr.ac.uk).

Daniel Deidda is supported by the University Research Scholarship, University of Leeds. This collaboration is supported by the EPSRC (EP/M022587/1) Collaborative Computational Project in Positron Emission Tomography and Magnetic Resonance imaging (CCP-PET-MR): (www.ccppetmr.ac.uk)

M16F-7: First Experimental Comparison Between the Cartesian and the Lissajous Trajectory for Magnetic Particle Imaging $\underline{\text { F. Werner }}^{1,2}$, T. Knopp ${ }^{1,2}$ 
${ }^{1}$ Institute for Biomedical Imaging, University Medical Center Hamburg-Eppendorf, Hamburg, Germany

${ }^{2}$ Institute for Biomedical Imaging, University of Technology, Hamburg, Germany

Magnetic Particle Imaging (MPI) is a quantitative imaging modality that allows distributions of superparamagnetic nanoparticles to be visualized. Sampling is achieved by moving a field-free point (FFP) along a specific trajectory through the volume of interest. The magnetic material that lies along the path or in the close vicinity of the FFP changes its magnetization, which induces a voltage in surrounding receive coils. Various trajectories for the FFP are conceivable, but all experimental MPI scanners either use a Cartesian or a Lissajous sampling trajectory. This study for the first time compares both sampling methods experimentally using an MPI scanner that allows to implement both trajectories. By default the scanner is capa- ble of scanning 2D and 3D field of views using a Lissajous trajectory. But since it also has a 1D mode, we were able to perform Cartesian measurements by shifting the 1D scanline in perpendicular direction to the FFP movement using the static focus field. These line scans were jointly reconstructed to arrive at a 2D image representation. In a further step, the ordinary Cartesian trajectory was improved by interchanging excitation and focus field direction, which we named bidirectional Cartesian trajectory. Our initial findings reveal similar results for the bidirectional Cartesian and the Lissajous trajectory, whereas the ordinary Cartesian trajectory does not yield sufficient image quality.

We gratefully acknowledge funding and support of the German Research Foundation (DFG, grant number AD 125 / 5 - 1).

\title{
M16F-8: Improving the Contrast-to-Noise Ratio by Averaging in Scintillation Detectors
} M. Vopalensky, I. Kumpova, D. Vavrik

Centre of Excelence Telc, Institute of Theoretical and Applied Mechanics, Academy of Sciences of the Czech Republic, Telc, Czech Republic

From the point of view of the image quality, the signal-to-noise ratio (SNR) and contrast-to-noise ratio (CNR) are crucial parameters. In case of the scintillation detectors, which are widely used in X-ray radiography and computed tomography, these two parameters are strongly determined by the inherent noise of the detector and the X-ray beam fluctuation. Both parameters can be lowered by averaging of images from the same projections, but this approach leads to an increased time of the tomography, which can be an undesirable effect. This paper shows a method that allows determination of a minimum number of averaged images for required SNR or CNR based on two initial projections. The results are intended to be used for optimized setting of the detector parameters and X-ray source parameters for a tomography to obtain the best possible results in a reasonable time.

\author{
M16F-9: Low Trues Statistics and High Randoms Rates Degrade Quantitative Accuracy in PET Iterative \\ Reconstruction Methods \\ $\underline{\text { N. M. Maughan }}^{1}$, P. J. Parikh ${ }^{2}$, R. Laforest ${ }^{3}$

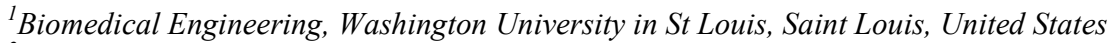 \\ ${ }^{2}$ Radiation Oncology, Washington University in St Louis, Saint Louis, United States \\ ${ }^{3}$ Radiology, Washington University in St Louis, Saint Louis, United States
}

Recently, post-radioembolization dosimetry of yttrium-90 (Y-90) microspheres with PET has proven effective in predicting individual lesion response in patient studies. However, these images suffer from low quantitative accuracy, likely due to low positron statistics and high Randoms rates. In this study, we investigate the effects of reducing Trues statistics and increasing Randoms rates on the quantitative accuracy of iterative PET reconstructions. We imaged a NEMA IEC Body Phantom, filled with $2.28 \mathrm{mCi}$ of fluorine-18 (F-18), on a Siemens Biograph mMR PET/MRI scanner for 15 minutes in listmode. We imaged the same phantom with $81.8 \mathrm{mCi}$ of Y-90 for 30 minutes in listmode. PET images were reconstructed using the OSEM algorithm (3 iterations, 21 subsets, $5 \mathrm{~mm}$ Gaussian post-reconstruction filter, and resolution recovery). F-18 data was reconstructed with various frame durations of 3-900 seconds to simulate different levels of Trues statistics. Varying levels of Randoms:Prompts ratios were simulated by adding an array of random numbers to the sinogram matrices. Recovery coefficients decreased on average by $6.93 \%$ when we used F-18 data that offered the same amount of Trues statistics as Y-90 ( 3 second frames). When we simulated the amount of Randoms typically seen in Y-90 PET imaging ( 77\% Randoms:Prompts), recovery coefficients decreased by $9.62 \%$, on average. Decreasing the Trues statistics and increasing the Randoms together reduced recovery coefficients by $16.4 \%$, on average. Imaging with Y-90 resulted in an average reduction of $25.2 \%$ in recovery coefficients compared to the original F-18 data. These differences increased with a decrease in sphere size. In conclusion, the low positron count rate and high Randoms fraction significantly degrade the quantitative accuracy of PET images. Careful consideration when measuring and estimating Randoms, Trues, and Prompts events is necessary for improving quantitative accuracy of iterative reconstruction algorithms.

M16F-10: Dynamic PET Reconstruction Using the Split Bregman Formulation

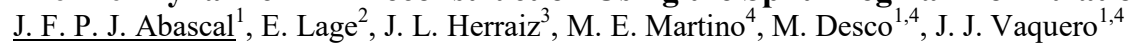


${ }^{I}$ Dept. Bioingenieria e Ingenieria Aeroespacial, Universidad Carlos III de Madrid, Leganes, Spain

${ }^{2}$ Biochemistry Dept., Medical School, Universidad Autonoma de Madrid, Madrid, Spain

${ }^{3}$ Fisica Atomica Molecular y Nuclear, Universidad Complutense de Madrid, Madrid, Spain

${ }^{4}$ IISGM, Hospital G. U. Gregorio Maranon, Madrid, Spain

In this work, we propose a novel 4D reconstruction method for PET which is based on spatiotemporal total variation (ST-TV). The 4D ST-TV method takes advantage of image redundancies in 4D and was efficiently implemented using the split Bregman formulation, which has been shown to be optimal for decreasing noise while maintaining image quality. To evaluate the proposed approach we simulated a dynamic phantom with different number of counts to mimic high and low Signal-to-Noise Ratio (SNR) scenarios. Data were reconstructed with the proposed methodology and results were compared with applying a reconstruction algorithm based on Spatial TV (without using the timing information), and with well known PET reconstruction methods (2DOSEM and FBP algorithms). Our proposed 4D reconstruction led to major improvements in the recovery of time activity curves and image SNR while preserving spatial resolution. Futhermore, 4D ST-TV also demonstrated to be less sensitive to reduction in data SNR than any other method, suggesting that this approach holds the potential to provide significant improvements in image quality for dynamic PET imaging.

E. Lage and J.L. Herraiz are supported by the UE cofund Fellowship (7th frame program) and by the Madrid-MIT Mvision Consortium

M16F-11: Towards Truly Comprehensive Quality Assurance to Optimize Detectability in Digital Radiography I. A. Cunningham, T. R. Escartin, T. Nano

Robarts Research Institute, Western University, London, Ontario, Canada

An effective quality assurance program must identify potential system weaknesses or points of failure that may affect image quality or key aspects of system performance, and assess these potential failures and the impact they may have using quantitative and meaningful metrics. We suggest that a truly comprehensive QA program should go beyond this and determine how effectively a system performs relative to an objective concept of ideal performance on an absolute vendor-neutral scale. This work looks at the science of image quality and the link between performance metrics and image perception in terms of the detectability index to establish meaningful measures of system performance on an absolute scale with minimal risk of unintended or erroneous missrepresentation of test results.

We propose a program consisting of the following points. 1) All Fourier-based metrics require a linear system response and tests must verify sufficient linearity. Some systems tested have local slope deviations of $4 \%$ in the response curve that result in $8 \%$ errors in the DQE. 2) MTF measurements must be correctly normalized to avoid errors of $4-5 \%$ in the MTF, and $8-10 \%$ in the DQE. 3) The area under the DQE curve is a good indicator of the detectability index for the detection of objects with a flat spatial-frequency spectrum. We propose using the DQE area as the most applicable single-value indicator of system performance, supplemented by full DQE and MTF information. 4) Reporting DQE curves at standard exposure levels can be misleading if the levels do not cover actual use. We suggest reporting the DQE at a nominal level and also reporting a quantum-limit exposure that characterizes the detector. 5) Exposure technique charts should be developed based on the quantum-limit exposure.

M16F-12: Evaluation of Time-of-Flight Benefit on Clinical Imaging Using the Tachyon PET Scanner $\underline{\text { X. Zhang }}{ }^{1}$, Q. Peng ${ }^{2}$, J. Zhou ${ }^{1}$, J. S. Huber ${ }^{2}$, R. H. Huesman ${ }^{2}$, W. W. Moses ${ }^{2}$, J. Qi $^{1}$

${ }^{I}$ Department of Biomedical Engineering, University of California, Davis, Davis, CA, USA

${ }^{2}$ Lawrence Berkeley National Laboratory, Berkeley, CA, USA

The objective of this study is to evaluate the benefit of improved time-of-flight (TOF) capability for lesion detection and quantification using the demonstration TOF PET scanner (Tachyon), which has a coincidence timing resolution of $314 \mathrm{ps}$. We performed 18F-FDG scan using the Tachyon scanner on twelve healthy volunteers to obtain normal background images. Attenuation factors were measured using a rotating point source. To mimic hot lesions, we scanned a point source and hot spheres of different sizes at various locations. The point source and sphere data were superimposed onto the human volunteer data after accounting for the subject attenuation. We used a bootstrap method to generate multiple independent noisy datasets with and without a lesion present. To simulate a TOF scanner with $500 \mathrm{ps}$ timing resolution, the measured time difference of each list-mode event was artificially blurred by a Gaussian distribution. The signal-to-noise ratio (SNR) of a channelized Hotelling observer $(\mathrm{CHO})$ was calculated for each lesion size and location combination. The resulting CHO-SNR showed improved performance in lesion detection with better timing resolution, while the SNR gain is greater for smaller lesions. The detection performance is also dependent on the lesion size and location. We further examined the lesion quantification performance. The results of bias versus noise tradeoff show that the noise (standard deviation) reduction ratio is about $\sim 1.25$ over the $500 \mathrm{ps}$ TOF and 1.7 2.0 over the nonTOF mode, which are similar to the SNR gain for lesion detection. 
This work was supported in part by the Director, Office of Science, Office of Biological and Environmental Research, Biological Systems Science Division, U.S. Department of Energy under contract DE-AC02-05CH11231, and in part by the National Institutes of Health, National Institute of Biomedical Imaging and Bioengineering, under Grant R01EB006085.

\title{
M17: Application specific emission imaging
}

Saturday, Nov. 5 08:30-10:30 Schweitzer

M17-1: J-PET: a Novel TOF-PET Detector Based on Plastic Scintillators

P. Moskal

Jagiellonian University, Cracow, Poland

On behalf of the J-PET Collaboration

The Jagiellonian Positron Emission Tomograph (J-PET) is built out of 192 scintillator strips arranged axially in three layers forming a cylindrical diagnostic chamber with the diameter of $85 \mathrm{~cm}$ and axial field-of-view of $50 \mathrm{~cm}$, and it is characterized by $300 \mathrm{ps}$ coincidence resolving time. The novelty of the concept lies in employing long strips of polymer scintillators instead of crystals as detectors of annihilation quanta, and in using predominantly the timing of signals instead of their amplitudes for the reconstruction of Lines-of-Response. To take advantage of the superior timing properties of plastic scintillators a novel front-end electronics was developed allowing for sampling in a voltage domain. An axial arrangement of long strips of plastic scintillators, and their small light attenuation allows us to make a TOF-PET scanner with a long axial field-of-view. The presented solution opens unique possibilities of combining PET with CT and PET with MRI for scanning the same part of a patient at the same time with both methods. The relative ease of the cost effective increase of the axial field-of-view makes the J-PET tomograph competitive with respect to the current commercial PET scanners as regards sensitivity and time resolution. The talk will include presentation of the J-PET scanner along with the description of its characteristics estimated according to NEMA-NU-2 standards.

\section{M17-2: First Clinical Test of the Helmet-Chin PET Prototype}

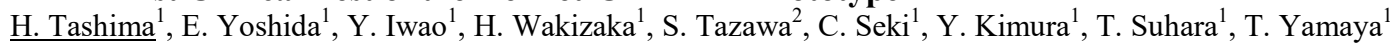

${ }^{I}$ National Institute of Radiological Sciences, Chiba, Japan

${ }^{2}$ ATOX Co. Ltd, Tokyo, Japan

Dementia is a severe problem in aging societies. There is a strong potential demand for high-sensitivity, high-resolution, and lowcost brain positron emission tomography (PET) imaging for its early diagnosis. Therefore, we have proposed the helmet-chin PET, a novel geometry for high-sensitivity brain imaging based on a hemispheric arrangement of detectors. For a proof-ofconcept, we developed the first prototype using 4-layered depth-of-interaction (DOI) detectors. The helmet-chin PET prototype used 54 DOI detectors, each of which consisted of $16 \times 16 \times 4 \mathrm{Zr}$-doped GSO crystals $\left(2.8 \times 2.8 \times 7.5 \mathrm{~mm}^{3}\right)$ and a high sensitivity 64 channel flat-panel photomultiplier tube. In the prototype, 47 detectors were used to form a hemisphere of $25 \mathrm{~cm}$ inner diameter and $50 \mathrm{~cm}$ outer diameter, and 7 detectors were used for the chin detector. In this study, we conducted the first clinical test with a healthy volunteer. First, we compared sensitivity with a whole-body time-of-flight-PET (Biograph mCT Flow 64-4R PET/CT system, Siemens Medical Solutions USA, Inc.) to determine injection activity. As a result, the sensitivity of the helmet-chin PET prototype for the brain region was almost twice as high as that of the $\mathrm{mCT}$, even though the number of detectors was less than $30 \%$ that of the $\mathrm{mCT}$. Then a healthy volunteer was measured by the $\mathrm{mCT}$ for $15 \mathrm{~min}$ beginning $60 \mathrm{~min}$ after ${ }^{18} \mathrm{~F}$-FDG injection of $70 \mathrm{MBq}$ and by the helmet-chin PET for $18 \mathrm{~min}$ beginning $85 \mathrm{~min}$ after the injection. For the first clinical test, we could obtain a clear image of the brain with low radioactivity injection. We analyzed region of interest (ROI) values based on the anatomical structure. As a result, ROI values measured by the helmet-chin PET prototype and the mCT were in good agreement. The first clinical test showed promising performance of the helmet-chin PET for highly sensitive brain imaging.

This work was partially supported by the Japan Society for the Promotion of Science (KAKENHI Grant Number 26870864) and ATOX Co. Ltd.

\author{
M17-3: Design Study of a Practical-Entire-Torso PET (PET-PET) with Low-Cost Depth-of-Interaction \\ Detectors \\ W. H. Wong, Y. Zhang
}

Cancer Systems Imaging, The University of Texas M. D. Anderson Cancer Center, Houston, Texas, USA

This is a design and Monte Carlo (MC) study of a Practical Entire Torso PET (PET-PET) with 1-meter axial field-of-view (AFOV) for imaging the head-\&-torso simultaneously. Our studies show that PET-PET would image the entire body in 3-4 minutes with a 3 mCi FDG dose, which would reduce whole-body cancer-imaging cost and together with the low radiation dose may justify its use for cancer screening. It can capture dynamic whole-body time-activity and arterial input from the heart to create quantitative metabolic rate-constant images of the entire body. It uses low-cost BGO crystals with depth-of-interaction (DOI) detection using sparsely packed SiPM to reduce SiPM-related cost, thus enabling the cost to approach that of current clinical PET with LSO with a 25-30 cm AFOV. Two sparse SiPM readout options are available for DOI detection: (a) the 
Dichotomous Offset-Quadrant-Sharing (DO-QS) design with a crystal-to-SiPM ratio of 2:1, and (b) The Dichotomous Orthogonal Symmetry (DOS) design with a crystal-to-SiPM ratio of 3.2:1. There is a third lower cost non-DOI option using 4 SiPM (3mm) at the corner of a 6x6 BGO array to achieve a crystal-to-SiPM ratio of 9:1. For the DO-QS design, MC studies showed DOI resolution of $5 \mathrm{~mm}$ with $3.6 \times 3.6 \times 30 \mathrm{~mm}$ BGO crystals, with each crystal divided into 6 segments along the 30-mm depth; the crystal-position decoding is unique (perfect) without using analog decoding; the timing FWHM is $1.6 \mathrm{~ns}$. For the DOS design, the DOI resolution is $5.5 \mathrm{~mm}$, with a timing FWHM of $1.9 \mathrm{~ns}$ and analog decoding error of 16\% in an $8 \times 8$ array of $2.4 \times 2.4 \times 30 \mathrm{~mm}$ BGO crystals. Resolution and NES characteristics were estimated with MC simulations for point source, NEMA NES phantom and whole-body Turkington phantom, which showed the transaxial resolution to be $3.2 \mathrm{~mm}$ (DO-QS). The NES results on the Turkington and NEMA NES phantoms showed a 3-4 min whole-body imaging with 3 mCi FDG dose would be comparable to current clinical TOF PET with 10-mCi dose and 15-20-min scans.

\section{M17-4: A New Brain PET Insert MR Compatible: Final Design and First Results}

A. J. Gonzalez ${ }^{1}$, A. Gonzalez-Montoro ${ }^{1}$, A. Aguilar ${ }^{1}$, P. Conde $^{1}$, G. Cañizares ${ }^{1}$, L. Hernandez ${ }^{1}$, L. F. Vidal ${ }^{1}$, S. Sanchez ${ }^{1}$, R. Garcia ${ }^{2}$, J. Barbera ${ }^{3}$, C. Correcher ${ }^{3}$, S. Aussenhofer ${ }^{4}$, D. Gareis ${ }^{4}$, M. Galasso ${ }^{5}$, A. Fabbri ${ }^{5}$, F. Sanchez ${ }^{1}$, J. M. Benlloch ${ }^{1}$

${ }^{I}$ Institute for Instrumentation in Molecular Imaging, Valencia, Spain

${ }^{2}$ nstitute of Design and Manufacture, Valencia, Spain

${ }^{3}$ Oncovision, Valencia, Spain

${ }^{4}$ Noras, Hochberg, Germany

${ }^{5}$ INFN Sezione Roma III, Rome, Italy

The first prototype of the MindView project, a brain PET insert MR compatible, is currently being assembled. The scanner is composed of 3 rings of 20 detector blocks each. The detector block includes a monolithic LYSO crystal with 50x50x20 mm3 and a 12x12 SiPM array. The system defines an axial and transaxial FOV of $150 \mathrm{~mm}$ and $240 \mathrm{~mm}$, respectively. Detector blocks are kept at a stable temperature in the range of $20-25^{\circ} \mathrm{C}$. The $\mathrm{X}$ and $\mathrm{Y}$ scintillation light projections of each detector are measured and from them the planar and DOI photon impact coordinates estimated. Novel retroreflector layers coupled to the entrance crystal surface are used. Thus, the light distribution remains preserved, but light collection significantly improves. The detector XY impact positions are obtained by an improved center of gravity approach, whereas the DOI is estimated through the ratio of the energy to the light distribution maximum, with a resolution of about $5 \mathrm{~mm}$. This DOI capability allows us to divide the monolithic crystal in four "virtual" layers. An average detector spatial resolution (without source finite size corrections) of about $1.7 \mathrm{~mm}$ is obtained for the whole crystal volume. Average energy resolutions of $12 \%$ are determined also for the whole crystal. The scintillation light distribution was modeled starting from the Fresnel, Snell-Descartes, Lambert and Lambert-Beer laws. The resulting light distribution was integrated on the sensitive areas of the SiPMs to get the measured light profile. A very good agreement is shown for the experimental and modeled results. Taking into account the source dimensions, average spatial resolution FWHM at the entrance layer of $1.6 \mathrm{~mm}$ was obtained, improving to $1.4 \mathrm{~mm}$ and $1.0 \mathrm{~mm}$ at the second and third layers. Parts of the detector ring have been successfully tested for RF shielding and eddy currents. This was carried out by using a radio frequency screen structure based on carbon fiber composites with specific thickness and orientations.

\section{M17-5: Development of a Prototype OpenPET-Guided Surgery System}

H. Tashima, Y. Yoshii, Y. Iwao, E. Yoshida, H. Wakizaka, H. Takuwa, T. Yamaya

National Institute of Radiological Sciences, Chiba, Japan

Tumors diagnosed as malignant are generally removed surgically. However, in cases where the tumors are widely and complexly distributed and they move with organs or they are located on the backside of the organs, it is challenging to remove all the tumors in one surgery. To deal with this problem, we are developing the OpenPET-guided surgery system which can provide sufficient open space for surgical operation during PET measurement. In this study, we developed a small prototype and demonstrated a tumor extirpation surgery of a mouse. In order to display images immediately after the data acquisition, we developed a real-time imaging system by implementing the one-pass 3D list-mode DRAMA on the GPU. Because it is difficult to find tumors in images reconstructed with too short time frames due to low count statistics while the time required for identifying tumor locations is desired to be short, we implemented the system so that the displayed images became clearer gradually as the data accumulation time increased. For a demonstrational surgery, human colon carcinoma HCT116-RFP cells had been intraperitoneally transplanted into a mouse. One hour after ${ }^{18} \mathrm{~F}$-FLT injection of $3.7 \mathrm{MBq}$, the mouse was set onto the operation table inside the FOV of the OpenPET for an abdominal operation. Tumor locations were identified before and after the isolation. Measurement time required to acquire sufficient numbers of data to visually identify the tumors from background radioactivity with clear contrast was about 20-30 s. Images were reconstructed with the computational time of less than $1 \mathrm{~s}$, and displayed images became gradually clearer every second. We could observe the tumors were successfully isolated from the body. The surgery demonstrated that the system allowed us to confirm tumor positions anytime during the operation. We concluded that the proposed system was effective in preventing any tumors, especially those located behind organs, from being left after the surgery. 
M17-6: A Novel Approach for an Integrated NIR/Gamma/Visible Imaging System Using a Single Endoscopic Fiber Bundle for Laparoscopic Surgery

\author{
H.-G. Kang ${ }^{1}$, S. H. Song ${ }^{1}$, H.-Y. Lee ${ }^{2}$, K. M. Kim³ ${ }^{3}$, S. J. Hong ${ }^{4}$
}

${ }^{I}$ Department of Senior Healthcare, Eulji University, Daejeon, Korea

${ }^{2}$ Department of Nuclear Medicine/College of Medicine, Seoul National University, Seoul, Korea

${ }^{3}$ Molecular Imaging research center, Korea Institute of Radiological and Medical science, Seoul, Korea

${ }^{4}$ Department of Radiological Science, Eulji University, Seongnam, Gyeonggi, Korea

We present a novel approach for an integrated NIR/gamma/visible imaging system using a single endoscopic fiber bundle for single- or multi-port laparoscopic surgery. The proposed imaging system consists of a custom-made endoscope head, an endoscopic fiber bundle (Schott, IG-154, format $=4 \times 4 \mathrm{~mm} 2$, length=122 cm), and a proximal-end part. The endoscope head consists of a tungsten pinhole collimator, plastic focusing lens, BGO crystal $(11 \times 11 \times 2 \mathrm{~mm} 3)$, and a fiber optic taper. The endoscope head was specially designed to form an NIR, gamma, and visible image on the same image plane of the entrance surface of the fiber optic taper. Subsequently, the three images can be transferred to the relay lens via the endoscopic fiber bundle and imaged on the cooled CCD camera sequentially. In order to evaluate the performance of the proposed imaging system, a custom-made Derenzo-like phantom (outer diameter $=28 \mathrm{~mm}$, height $=10 \mathrm{~mm}$ ) filled with a mixture of $99 \mathrm{mTc}(7.6 \mathrm{MBq})$ and ICG $(0.1 \mathrm{ml})$ was imaged. To obtain the NIR image, a collimated excitation light $(723-758 \mathrm{~nm})$ was irradiated onto the ICG fluorphore by using a LED. Subsequently, the emission NIR light passed through a band pass filter $(780-820 \mathrm{~nm})$, was detected on the CCD camera for $50 \mathrm{~ms}$. The visible image was obtained after removing the band pass filter from proximal end of the endoscope for $50 \mathrm{~ms}$. The gamma image was obtained for $3 \mathrm{~min}$ after closing a dark box which mimics an abdominal cavity. The NIR, gamma, and visible images of the Derenzo-like phantom showed a good spatial correlation. We demonstrated the feasibility of an integrated NIR/gamma/visible imaging system using a single endoscopic fiber bundle for laparoscopic surgery.

This work has supported by grants from the Korea Evaluation Institute of Industrial Technology funded by the Ministry of Trade, Industry and Energy of the Korean Government (2015-4-10051988)

M17-7: Markerless Motion Tracking of Awake and Unrestrained Rat Brain PET $\underline{\text { A. Miranda }}^{1}$, S. Staelens ${ }^{1}$, S. Stroobants ${ }^{2}$, J. Verhaeghe ${ }^{1}$

${ }^{I}$ Nuclear Medicine, University of Antwerp, Antwerp, Belgium

${ }^{2}$ Nuclear Medicine, Antwerp University Hospital, Antwerp, Belgium

To avoid the influence of anesthesia and stress in rat brain PET tomography, motion correction methods are used to perform scans of awake and unrestrained rats. In these methods the motion of the rat head must be measured during the PET scan to compensate the data for motion. In this work we aim to track the motion of the rat head using a structured infra-red light stereo camera which gives 3D data in the form of point clouds. Using this camera no markers or texture information are needed to perform the motion tracking. The tracking is performed using the Point Cloud Library to process the point cloud scene obtained from the camera. A microDerenzo phantom and an awake [18F]FDG rat brain experiments were performed to validate the technique. The phantom was manually moved during 10 minutes and the rat remained awake inside the scanner in a cylinder plastic container while their motion was tracked. In the phantom motion scan, after motion correction, the 3,2 and $2.5 \mathrm{~mm}$ rods were recovered with some loss of spatial resolution in comparison with a motion-free scan ( 2.75 and 3.25 mm FWHM for the 3 $\mathrm{mm}$ rods in the motion-free and motion corrected scans respectively). In the awake rat brain reconstruction no brain structure could be identified. After motion correction structures as the brain cortex and hippocampus are clearly identified although with some loss of spatial resolution. The motion tracking performed with the proposed stereo camera is uninvasive and imperceptible for the rat avoiding perturbation of the rat behavior by the tracking system.

\author{
M17-8: Fine-Grained Temporal Measurement of Volume Activation with the DoPET-L Particle Therapy \\ Monitoring System

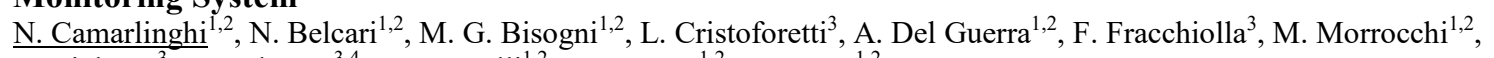 \\ R. Righetto ${ }^{3}$, M. Schwarz ${ }^{3,4}$, G. Sportelli ${ }^{1,2}$, E. Zaccaro ${ }^{1,2}$, V. Rosso ${ }^{1,2}$ \\ ${ }^{I}$ Department of Physics, University of pisa, Pisa, Italy \\ ${ }^{2}$ INFN, Pisa, Italy \\ ${ }^{3}$ Proton Therapy department, Trento Hospital, Trento, Italy \\ ${ }^{4}$ TIFPA-INFN, Trento, Italy
}

One of the most consolidated methods for particle range monitoring in particle therapy is by means of Positron Emission Tomography (PET). PET monitoring is based on detecting the annihilation photons from the $\beta+$ emitting isotopes produced during the irradiation of the target volume. The advantage of PET is that it allows for three dimensional imaging of the induced activity in the irradiated volume. When the PET scan is performed in-beam, the imaging procedure can be performed during dose delivery and thus provide a prompt feedback. For this reason we developed an in-beam dual head planar tomograph named 
DoPET-L. In this work, we analyse the response of the DoPET-L system when used in the Trento Proton Therapy Center (TPTC) with particular focus on the quality of acquired data with respect to the temporal micro-structure of the beam. In order to finely resolve in time the particle spills delivered during the treatment, we have upgraded the DoPET-L acquisition system so as to record timestamps of the acquired events with a precision of hundreds of nanoseconds. Results show that the DoPET-L system is capable of acquiring coincidence data also during the irradiation in a clinical cyclotron-based facility and to distinguish events in the different phases of the beam micro-structure. This capability opens the possibility of studying the properties of short-lived isotopes produced by the irradiation and as such to optimise the reconstruction based on the prior knowledge available from the treatment plan.

\section{M18: Radiotherapy imaging and dosimetry}

Saturday, Nov. 5 08:30-10:30 Cassin

M18-1: Proof-of-Principle Results of Proton Computed Tomography

M. Bruzzi ${ }^{1}$, C. Civinini ${ }^{2}$, M. Scaringella ${ }^{2}$, D. Bonanno ${ }^{3}$, M. Brianzi ${ }^{2}$, M. Carpinelli ${ }^{4,5}$, G. A. P. Cirrone ${ }^{5}$, G. Cuttone ${ }^{5}$, D. Lo Presti ${ }^{3}$, G. Maccioni ${ }^{4}$, S. Pallotta ${ }^{1,2}$, N. Randazzo ${ }^{3}$, F. Romano ${ }^{5}$, V. Sipala ${ }^{4,5}$, C. Talamonti ${ }^{1,2}$, E. Vanzi ${ }^{6}$

${ }^{1}$ University of Florence, Firenze, Italy

${ }^{2}$ INFN Firenze, Firenze, Italy

${ }^{3}$ INFN Catania, Catania, Italy

${ }^{4}$ University of Sassari, Catania, Italy

${ }^{5}$ Laboratori Naz Sud INFN, Catania, Italy

${ }^{6}$ Fisica Sanitaria, Azienda Ospedaliero-Universitaria Senese, Siena, Italy

A proof-of-principle proton Computed Tomography $(\mathrm{pCT})$ system based on a silicon tracker and a YAG:Ce calorimeter has been developed. Tests with a $175 \mathrm{MeV}$ proton beam, aiming at collecting data for reconstructing a tomographic image, have been carried out at The Svedberg Laboratory ( TSL, Uppsala, Sweden). Algebraic iterative reconstruction methods (ART), together with most likely path calculation, have been used to obtain non-homogeneous phantom images and to extract density and spatial resolutions. A Filtered Back Projection algorithm has been applied to the same data-set to obtain tomographic images used as an initial seed for the ART algorithm, a process that fasten ART convergence, significantly reducing reconstruction times. The heavy computation load required by the algebraic algorithms has been approached within the CUDA environment to fully exploit the high calculation parallelism of Graphics Processing Units. This computation scheme substantially reduces the image processing time. Values of density ans spatial resolution obtained are within medical requirements settled for this technique, proving that $\mathrm{pCT}$ will be beneficial if used instead of X-CT in hadrontherapy. A pre-clinical pCT system with extended field-ofview, able to reconstruct objects of the size of a human head is now at an advanced construction stage: first results on its characterization under proton beams will be also presented.

This work is supported by INFN CSN5 RDH and IRPT MIUR

M18-2: Clinical Validation of a New Digital Spectrometer System for Range Verification in Proton Therapy $\underline{\text { T. Werner }}{ }^{1}$, J. Petzoldt ${ }^{1}$, F. Hueso-González ${ }^{2}$, K. Römer ${ }^{2}$, J. Berthold ${ }^{3}$, A. Rinscheid ${ }^{4}$, W. Enghardt ${ }^{1,2,5,6}$, G. Pausch ${ }^{1}$

${ }^{l}$ Faculty of Medicine and University Hospital Carl Gustav Carus, OncoRay, Dresden, Germany

${ }^{2}$ Institute of Radiooncology, Helmholtz-Zentrum Dresden-Rossendorf, Rossendorf, Germany

${ }^{3}$ Particle and Nuclear Physics, Technische Universität Dresden, Dresden, Germany

${ }^{4}$ Institute of Physics, Martin Luther University Halle-Wittenberg, Halle, Germany

${ }^{5}$ German Cancer Consortium, Dresden, Germany

${ }^{6}$ German Cancer Research Center, Heidelberg, Germany

A major challenge for quality assurance in particle therapy is to ensure the precise irradiation of the tumor volume in the patient body. One promising approach for in vivo range verification during the irradiation of patients is the novel technique of prompt gamma-ray timing (PGT). The energy-resolved timing spectra of promptly emitted photons encode the range information of the primary protons and allow a real time range verification. The concept of PGT was developed and tested in first proof- ofprinciple experiments, which confirm that PGT is a promising method for range assessment. Especially the simplicity of the concept according to the experimental implementation justifies a further development with special focus on clinical applications, but challenges the detectors, electronics and data acquisition. To fulfill the requirements for typical treatment conditions a novel compact detection system was developed and tested. The PGT unit consists of a suitable scintillation crystal coupled to a common photomultiplier tube with a compact digital energy and time spectrometer as readout electronic. The first uncollimated measurements show the general applicability of the PGT method in a clinical scenario with realistic treatment plans and beam currents.

M18-3: Improved Laboratory and in-Beam Results of a Compton Telescope with LaBr3 and SiPMs

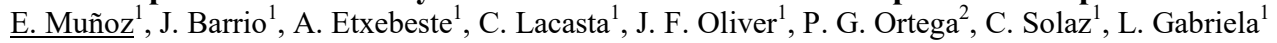


${ }^{I}$ Fisica Experimental, Instituto de Física Corpuscular, Valencia, Spain

${ }^{2}$ CERN, Geneva, Switzerland

A fully functional prototype of a three-layer Compton Telescope for hadron therapy treatment monitoring, based on continuous $\mathrm{LaBr} 3$ crystals coupled to Silicon Photomultipliers (SiPMs), has been developed at IFIC - Valencia. Coincidence measurements have been carried out in the laboratory using either two or three planes with Na-22 and a Y-88 sources. The device has also been evaluated in beam tests. In all cases, images of the emission distribution have been successfully reconstructed.

A coincidence board which allows the simultaneous acquisition of two- and three-interaction events is operational and has already been tested. Ongoing work is mainly focused on the combination of both types of events in a simultaneous image reconstruction, and on performance improvement of the detectors. Simulations with GATE are being carried out to optimize telescope layout and geometry and to characterize the detector response under different conditions.

M18-4: Absorbed Energy Monitoring During Hadrontherapy via Prompt Gamma Detection $\underline{\text { J. Krimmer }}^{1}$, L. Balleyguier ${ }^{1}$, D. Dauvergne ${ }^{1,2}$, M. Fontana ${ }^{1}$, N. Freud ${ }^{3}$, J. Herault ${ }^{4}$, J. M. Létang ${ }^{3}$, H. Mathez ${ }^{1}$, M. Pinto ${ }^{5}$, E. Testa ${ }^{1}$, Y. Zoccarato ${ }^{1}$, C. Koumeir ${ }^{6}$

${ }^{I} I P N L$, Lyon, France

${ }^{2}$ LPSC, Grenoble, France

${ }^{3}$ CREATIS, Lyon, France

${ }^{4}$ CAL, Nice, France

${ }^{5}$ LMU, Munich, Germany

${ }^{6}$ ARRONAX, Nantes, France

An independent monitoring system aiming for the integrated absorbed energy in the patient is presented. The system is based on prompt gamma-ray detection via scintillation detectors and the use of time-of-flight information. Test measurements at cyclotrons show the influence of degraders used in passive beam delivery systems. A dedicated data acquisition card has been developed which is suitable for clinical beam intensities.

\author{
M18-5: Prostate Brachytherapy Optimization Using GPU Accelerated Simulated Annealing and Monte Carlo \\ Dose Simulation

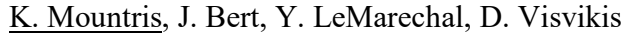 \\ UMR1101, INSERM, LaTIM, Brest, France
}

We present a prostate brachytherapy optimization framework aiming to predict the optimal radioactive seeds' distribution for a given configuration of inserted needles. A GPU accelerated version of the simulated annealing (SA) method is proposed for the minimization of a cost function describing the deposited dose of the seeds distribution. The deposited dose is evaluated on the prostate contour and on uniformity measurement points located between all the possible seeds locations. The accuracy of the cost function construction is ensured using GPU accelerated Monte Carlo (MC) simulation for the dose calculation within the previously proposed GGEMS-Brachy framework. For each possible seed position a dose map is recorded considering the tissue heterogeneities and the inter-seed interactions. During the SA optimization a random initial configuration of seeds is chosen. The dose maps of the seeds belonging to the initial configuration are summed in parallel to a total dose map using CUDA and the cost function is constructed. For the next possible configuration a seed is randomly discarded from the initial configuration and another available seed is added. The total dose map is updated and the new cost function is calculated. The simulated annealing algorithm stops when the configuration with the minimum cost function is found. Using the GPU capabilities a standard optimization procedure of 20000 iterations needs $24 \mathrm{~s}$ on a single GPU (1.2 ms per iteration). The seeds dose maps calculation needs $9.5 \mathrm{~s}$ or $2.5 \mathrm{~s}$ per seed for one or four GPUs respectively. The proposed method combines the MC simulation accuracy and the SA efficiency for the optimization of the prostate brachytherapy procedure with execution times compatible with an intraoperative usage.

This work was partly supported by the French ANR within the Investissements d'Avenir program (Labex CAMI) under reference ANR-11LABX-0004 (Integrated Project CAPRI).

M18-6: An Assessment of Photoacoustic and Photon Counting Multispectral X-Ray Imaging Techniques for Imaging GNRs in vivo as Part of Predicting Dose Enhancing Effects. O. L. P. P. Scienti, A. J. Shah, J. C. Bamber, D. G. Darambara

Multi-modality Molecular Imaging, Institute of Cancer Research and Royal Marsden NHS Foundation Trust, London, England, United Kingdom 
Radiotherapy, whilst useful in the treatment of many cancers, suffers from the drawback of unavoidably delivering radiation doses to healthy tissue. Gold nanoparticles (GNPs) have been the subject of much interest as they are able to generate a local dose enhancing effect (DEE), allowing lower intensity beams to achieve a given effect, sparing healthy tissue. It is important that GNP distributions can be ascertained before radiotherapy begins so that accurate treatment plans can be made. Two imaging modalities that show great promise in being able to identify these GNPs in vivo without delivering excessive DEEs are photoacoustic imaging $(\mathrm{PAI})$ and photon counting multi-spectral $\mathrm{x}$-ray imaging (x-CSI). This work forms part of a larger project to overcome current limitations with these techniques that prevent their widespread clinical use with GNPs. Silica coated gold nanorods (GNRs) have been studied to prevent GNR degradation due to the laser light used in PAI. This coating is also hoped to prevent spectral broadening when the GNRs are endocytosed, allowing PAI to confirm uptake of GNRs by cells. This work also uses a combination of Monte Carlo and Finite Element modelling techniques to more accurately model cylindrical phantoms containing spherical GNR inclusions, in order to determine a maximum useful x-ray flux and minimum detectable GNP concentration and to demonstrate the potential improvements in imaging that $\mathrm{x}$-CSI can produce over traditional energy integrated x-ray imaging techniques. Results on the assessment of both techniques are discussed.

The silica coated GNRs used in this project were graciously provided by Joan Comenge and Raphael Levy at the University of Liverpool.

\author{
M18-7: Construction of personalized computational phantoms of pregnant patients for assessment of CT \\ radiation dose \\ $\underline{\text { T. Xie }}^{1}$, P.-A. Poletti ${ }^{1}$, C. Becker ${ }^{1}$, H. Zaidi ${ }^{1,2}$ \\ ${ }^{I}$ Division of Nuclear Medicine and Molecular Imaging, Geneva University Hospital, Geneve, Switzerland \\ ${ }^{2}$ Department of Nuclear Medicine and Molecular Imaging, University Medical Center Groningen, Groningen, Netherlands
}

The use of x-ray computed tomography (CT) has increased drastically worldwide during the last two decades and is nowadays playing a pivotal role in clinical diagnosis and radiation therapy planning. The radiation dose delivered to the fetus during CT imaging procedures of pregnant patients raised fhealth concerns because of the high radiosensitivity of the developing embryo/fetus. To assess the diagnostic benefits considering the radiation risks, the radiologist needs reasonably accurate and detailed estimates of the fetal dose from diagnostic CT imaging protocols. To produce realistic biological and physical representations of pregnant patients and embedded fetus, we developed a methodology for construction of patient-specific voxelbased computational phantoms based on existing standardized hybrid computational pregnant female phantoms. We estimated the maternal absorbed dose and fetal organ dose for seven pregnant patients referred to the emergency unit of Geneva University Hospital for abdominal CT scans. The N-Particle eXtended (MCNPX) general purpose Monte Carlo code was used for radiation transport simulation. The obtained results were compared to those reported by RadimetricsTM (Bayer Healthcare) dose tracking software. The estimated absorbed dose to the maternal body varied between $3.8 \mathrm{mSv}$ and $4.9 \mathrm{mSv}$. The calculated mean fetal effective dose is $4.6 \pm 1.0 \mathrm{mSv}$, about $78 \%$ higher than the one estimated by RadimetricsTM. The methodology developed for construction of personalized computational models can be exploited to estimate patient-specific radiation dose from CT imaging procedures. The generated dosimetric data can be used for accurate assessment of the radiation risks to the pregnant patient and fetus from various CT scanning protocols, thus guiding the decision making process.

Computational phantoms, radiation dosimetry, Monte Carlo, x-ray CT imaging

\title{
M18-8: An Analytical Treatment Plan for Proton Preclinical Irradiation
}

M. Vanstalle, Y. Karakaya, J. Constanzo, C. Finck, M. Rousseau

IPHC, Strasbourg, France

There is nowadays a growing interest for proton therapy, with an increase of treatment facility number in the world. Compared to conventional radiotherapy (photons), physical properties (Bragg peak) of proton allow high doses deposition in the target while sparing surrounding healthy tissues. Considering the expansion of proton therapy, additional pre-clinical studies are needed to fully understand the therapeutic possibilities and limitations of this new modality, and consequently proton irradiation platforms for small animals are emerging. These platforms require development of dedicated tools adapted to specific constraints imposed by small dimensions of the targeted zones.

This study proposes a treatment plan for preclinical irradiation, based on an analytical formulation of the Bragg peak using PSTAR tabulated range values of protons. The chosen method was validated by comparison with the PSTAR reference and with Monte Carlo simulations performed with GEANT4. This validation will be presented, as well as the impact of different parameters of interest (energy straggling, beam scattering...) on the accuracy of the delivered dose. The obtained results demonstrate the interest of the analytical choice rather than a Monte Carlo based solution. The analytical modelisation limit the errors (on the ranges and on the delivered doses) induced by Monte Carlo misestimation of the stopping power of low energy protons $(\mathrm{E}<50 \mathrm{MeV})$, by using appropriate database.

M19: Assessment \& comparison of image quality 
Saturday, Nov. $5 \quad$ 11:00-12:30 Schweitzer

M19-1: Evaluation of Penalized Maximum-Likelihood PET Image Reconstruction for ROI Quantification

L. Yang, J. Zhou, E. Asma, W. Wang

Toshiba Medical Research Institute USA, Inc., Vernon Hills, IL, USA

Region of interest (ROI) quantification is one of the important tasks in molecular imaging using positron emission tomography (PET). Statistical reconstruction methods based on the penalized maximum-likelihood (PML) principle have been developed to improve image quality. The penalty function that controls the image spatially smoothness plays an important role in PML reconstruction. The commonly used quadratic penalty function often over-smooths the sharp edges in reconstructed images. While the edge-preserving non-quadratic penalty functions can produce sharp intensity transitions across boundaries, it also introduces blocky artifacts which limits its clinical widespread use. Recently a patch-based edge-preserving penalty has been proposed, which is able to preserve sharp edges and locally smoothness while reducing blocky artifacts at the same time. However, the benefit of these penalty functions on clinical task is less obvious. In this work, we analyzed the performance of ROI quantification for these three representative penalty functions, and also compared them with the standard ordered subsets expectation maximization (OSEM) reconstructions. We acquired a standard NEMA phantom scan and a whole-body patient scan with both realistic lesions and artificial inserted lesions. The hot and cold sphere contrast recovery coefficient (CRC) versus the background variability was calculated to quantify the phantom data following the standard NEMA procedure. The mean of lesion standard uptake value (SUV) versus liver background variability was used for quantifying patient data. The results show that the patch-based edge-preserving penalty function can achieve better quantification performance compared to other penalty functions and the conventional OSEM reconstructions.

\section{M19-2: Ex Vivo and in Vivo Study Evaluating Edge-Preserving and Anatomical Priors for Partial Volume Correction in Cardiac PET}

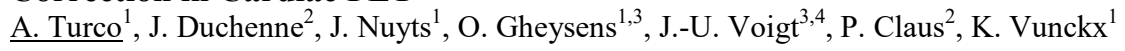

${ }^{I}$ Dept. of Imaging and Pathology, Nuclear Medicine and Molecular Imaging, KU Leuven, Leuven, België

${ }^{2}$ Dept. of Cardiovascular Sciences, KU Leuven, Leuven, Belgie

${ }^{3}$ Dept. of Nuclear Medicine, UZ Leuven, Leuven, België

${ }^{4}$ Dept. of Cardiovascular Diseases, UZ Leuven, Leuven, België

In this study, we evaluate the effect of partial volume correction (PVC) in cardiac 18F-FDG PET during iterative reconstruction, by performing resolution modelling in combination with different noise suppressing priors. In particular, we compare anatomical priors based on a high-resolution CT to edge-preserving priors such as total variation (TV) and the relative difference prior (RD). The effect of PVC is studied both for absolute quantification of the tracer uptake (Bq/cc, against a ground truth dataset) and for relative quantification (ratios between the septal and the lateral activities of the left ventricle). Ex vivo scans of animal (sheep) hearts with dilated-cardiomyopathy were performed on a clinical and a preclinical (ground-truth) PET scanner, to obtain reference data unaffected by respiratory and cardiac motion. After having determined the best reconstruction algorithm in the ex vivo case, we evaluate which reconstruction of corresponding, in vivo datasets would produce the most similar results. From this analysis, we aim at getting a better insight into the best algorithm to use both for cardiac motion correction and for PVC and noise suppression.

\section{M19-3: PET Imaging Lesion Detection Study for Penalized Likelihood Image Reconstruction with Relative} Difference Penalty

$\underline{\text { K. A. Wangerin }}^{1,2}$, S. Ahn ${ }^{2}$, S. D. Wollenweber ${ }^{3}$, S. Ross ${ }^{3}$, P. E. Kinahan ${ }^{1}$, R. M. Manjeshwar ${ }^{2}$

${ }^{1}$ University of Washington, Seattle, WA, United States

${ }^{2}$ General Electric Global Research, Niskayuna, NY, United States

${ }^{3}$ General Electric Healthcare, Waukesha, WI, United States

Ordered Subset Expectation Maximization (OSEM) is currently the most widely used image reconstruction algorithm for clinical PET. However, OSEM does not necessarily provide optimal image quality, and a number of alternative algorithms have been explored. We have previously shown that a penalized likelihood (PL) image reconstruction algorithm using the relative difference penalty (RDP) achieves more accurate lesion quantitation than OSEM, and importantly, shows improved visual image quality including lesion conspicuity in clinical whole-body PET. However, lesion detection of the PL algorithm has not been extensively evaluated yet. The goal of this work was to evaluate lesion detectability using both OSEM and PL image reconstruction methods. We performed a two-alternative forced choice $\mathrm{CHO}$ model observer study, which was previously developed and validated against human observers. At matched image noise, the two algorithms showed equivalent detectability in the lungs and improved detectability for PL in the liver for a subset of tested lesion properties. TOF improved lesion detectabilities with increasing benefit for decreasing lesion size and contrast as well as lesion location in the liver compared to the lung. These results suggest our PL algorithm with RDP provides not only improved quantitation and clinically acceptable visual image quality as previously shown but also provides equivalent or improved lesion detectability compared to OSEM. 
M19-4: Effect of Reducing PET Injected Activity for the Assessment of Non-Lesional Epilepsy with PET/MR. J. Cal-Gonzalez $^{1}$, K. Vunckx ${ }^{2,3}$, I. Rausch ${ }^{1}$, J. Nuyts $^{2,3}$, T. Traub-Weidinger ${ }^{4}$, T. Beyer ${ }^{1}$

${ }^{I}$ QIMP group, Center for Medical Physics and Biomedical Engineering, Medical University of Vienna, Vienna, Austria ${ }^{2}$ Department of Imaging \& Pathology, Nuclear Medicine \& Molecular Imaging, KU Leuven, Leuven, Belgium

${ }^{3}$ University Hospitals Leuven, Medical Imaging Research Center (MIRC), KU Leuven, Leuven, Belgium

${ }^{4}$ Division of Nuclear Medicine, Department of Biomedical Imaging and Image-guided Therapy, Medical University of Vienna, Vienna, Austria

The aim of this study is to evaluate the effect of reducing the ${ }^{18} \mathrm{~F}$-FDG injected activity on the quantitative evaluation of the brain PET images of patients with non-lesional epilepsy. Four patients with non-lesional epilepsy have been scanned on a Siemens Biograph mMR PET/MR system. Acquisitions were performed in LM for $50 \mathrm{~min}$ immediately after injection of about $240 \mathrm{MBq}$ of $\left[{ }^{18} \mathrm{~F}\right]$-FDG. The virtual reduction of the injected dose levels was performed by randomly removing counts from the listmode (LM) data prior to image reconstruction. The following activities were simulated: $50 \%, 35 \%, 20 \%$ and $10 \%$ of the injected FDGactivity. We used LM-data from 40-50 min post injection with and without post-filtering of the reconstructed data. In addition to the standard (OSEM) and resolution recovery (PSF) reconstructions available with the vendor tools, we evaluated two maximuma-posteriori (MAP) algorithms: the A-MAP and aBOWSHER methods. Four different white matter (WM) and gray matter (GM) volumes-of-interest (VOI) were defined for the quantitative evaluation of the reconstructed images. In addition, we evaluated a VOI in the hypometabolic region. Mean activity concentration in the GM and WM, the GM-to-WM ratio, uniformity within the WM (measured as the SD within the WM ROIs) and hypometabolic region-to-GM ratios were evaluated. We observed higher GM-to-WM and lower hypometabolic-to-GM ratio values for the MAP reconstructions, although these differences became slightly smaller for reduced activity levels. The uniformity within the WM was degraded in all cases when reducing the activity. This dependence was reduced when applying a post-filter to the images or when increasing the prior weights in the MAP reconstructions. In conclusion, we observed that post-filtered images and MAP reconstructions with high prior weights permit the reduction of injected PET activity levels to $35 \%$ of the standard dose without substantial losses in quantitative accuracy.

M19-5: Dose Optimization of SPECT-MPI Reconstruction Algorithms for Perfusion-Defect Detection

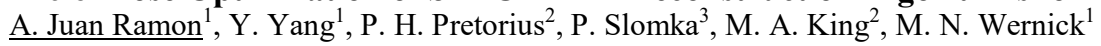

${ }^{I}$ Illinois Institute of Technology, Chicago, IL, USA

${ }^{2}$ University of Massachusetts Medical School, Worcester, MA, USA

${ }^{3}$ Cedars-Sinai Medical Center, Los Angeles, CA, USA

In SPECT-myocardial perfusion imaging (MPI), as in any imaging modality that involves ionizing radiation, minimization of radiation dose to the patient is an important objective. This paper reports the first of a series of studies we are performing to optimize the dose for various reconstruction algorithms, the ultimate aim being to provide dose recommendations, corresponding to optimized algorithm parameters, and performance comparisons across algorithms. Dose optimization involves determination of the extent to which administered activity can be reduced without sacrificing diagnostic accuracy. An important consideration is to conduct the optimizations in a principled way so as to avoid overfitting of the algorithm parameters during optimization. Using clinical SPECT-MPI data with realistic simulated lesions we optimized each reconstruction algorithm for maximum perfusiondefect detection performance, in which each study was evaluated for the presence of perfusion defects by using the total perfusion deficit score computed by the clinically validated Quantitative Perfusion SPECT (QPS) software package. Preliminary results suggest that tracer dose (and hence radiation dose) can be greatly decreased from current clinical dose levels without significant loss of diagnostic performance. We found this to be the case for both filtered backprojection (FBP) and ordered subsets expectation-maximization (OS-EM). By the time of the conference we expect to have evaluated further algorithms in this regard.

This research was supported by NIH grant R01 HL122484. P.S. was also supported by NIH grant R01 HL089765.

\author{
M19-6: Study of [11C]-PE2I Binding Potential Accuracy and Precision for Various Iterative Image \\ Reconstruction Algorithms

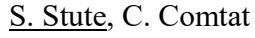 \\ IMIV - Service Hospitalier Frédéric Joliot, Orsay, France
}

In dynamic brain PET studies, the reconstruction task can be challenging due to the low count statistics of the time frames. In such cases, standard EM-ML based iterative reconstructions suffer from bias, but they benefit from PSF modeling that unbiased analytical algorithms cannot. Alternative iterative algorithms allowing for negative image values (NEGML and AML), while still benefiting from PSF modeling, were evaluated for low counts static acquisitions where they exhibit much less bias than EM-ML (Van Slambrouck et al 2015). In this work, we study the effect of such algorithms on dynamic DAT scans using the [11C]-PE2I radiotracer, looking at the impact on the extraction of the binding potential (BP) using the simplified reference tissue model (SRTM). These algorithms were applied to data acquired with healthy volunteers on the HRRT. Lacking a reference value to 
objectively analyze the results, we went through replicated analytical simulations of realistic [11C]-PE2I scans in order to explain the results. From the simulations, we observed that BP values extracted from EM-ML reconstructions suffer from negative bias (about 10\%), while those from alternative algorithms are almost bias-free, thought with an increased standard-deviation leading to a RMSE that can be worse than for EM-ML at high numbers of iterations (70 iterations with 16 subsets). In the same way as EM-ML is often stopped in practice before convergence, the alternative algorithms could benefit from a similar use. From the acquisitions, we observed that the BP values as a function of iterations are very similar than those obtained from the simulations. This drives us to the conclusion that the alternative algorithms are more robust than EM-ML for evaluating the BP in such clinical studies (especially while comparing two populations).

\section{M20: Other imaging modalities}

Saturday, Nov. 5 11:00-12:30 Cassin M20-1: Tabletop MRI System Development for Intraoperative Biopsy Analysis J. P. Rigla ${ }^{1,2}$, D. Grau-Ruiz ${ }^{2}$, E. Diaz-Caballero ${ }^{1}$, A. Nacev $^{3}$, P. Stepanov ${ }^{3}$, R. Hilaman ${ }^{3}$, L. Mair ${ }^{3}$, I. N. Weinberg ${ }^{3}$, E. Anasking ${ }^{3}$, S. T. Fricke ${ }^{4}$, L. Hernandez ${ }^{2}$, A. Aguilar ${ }^{2}$, A. J. Gonzalez ${ }^{2}$, C. D. Vera-Donoso ${ }^{5}$, J. M. Benlloch ${ }^{2}$

${ }^{I}$ Tesoro Imaging S.L., Alicante, Spain

${ }^{2}$ Institute for Instrumentation in Molecular Imaging, Valencia, Spain

${ }^{3}$ Weinberg Medical Physics, Bethesda, MD, USA

${ }^{4}$ Children's National Medical Center, Washington, DC, USA

${ }^{5}$ Department of Urology, La Fe University and Polytechnic Hospital, Valencia, Spain

A histological intraoperative analysis of tissue specimen is usually required for evaluation of sentinel lymph node and determination of margins of surgical resection. Currently, this analysis is performed by Touch Imprint Cytology (TIC), Frozen Section (FS) analysis, scrape cytology or a combination of these methods. We propose an alternative approach based on Magnetic Resonance Imaging (MRI). In particular, in this contribution we present the development, components, characterization as well as the first images obtained with our tabletop and transportable MRI system for histological analysis. A biplanar permanent magnet, together with the gradient coils and inside a Faraday cage, was characterized. We found a homogenous magnetic field region (20 ppm) using two magnetic test benches (3D Hall probes). The developed gradient system is capable to generate strong $(0.2 \mathrm{~T} / \mathrm{m})$ and fast (rise time $<30 \mu \mathrm{s})$ magnetic fields.

The results obtained during the experimental evaluation show that we can get images with high image spatial resolution (about 30 $\mu \mathrm{m})$ and good signal-to-noise ratio in only few seconds. First ex-vivo tests using the tabletop MRI have been performed using human tissue (prostate and skin) and the results indicate the ability to obtain images of good quality using fast pulse sequences.

Funding received under the "Retos-Colaboración" Programme from the Spanish Ministry of Economy and Competitiveness and "Adquisicion de pequeño equipamiento" Programme (European Regional Development Fund) from the Generalitat Valenciana.

M20-2: Improved Resolution in Positron Attenuation Tomography Using Multiple Views C. C. Watson

Siemens Healthcare Molecular Imaging, Knoxville, TN, USA

Positron attenuation tomography (PAT) is a new modality for imaging the linear attenuation coefficients (LACs) of magnetically constrained beta-ray beams in matter. A complete 3D LAC image of an object can be made by measuring the positron annihilation rate density within it at a single orientation relative to the beam, and then applying the PAT transformation. The spatial resolution of this image is limited by the resolution of the positron emission tomography (PET) system used to acquire the data as well as the gyroradii of the positrons in the magnetic field. Due to the non-linear nature of the imaging process, PAT images acquired at different view angles carry unique information on the object's structure. This can be exploited to improve the spatial accuracy of the LAC image. This paper describes a forward model of PAT image formation and a back-propagation algorithm that, when used together to iteratively combine the data from multiple views, can improve the spatial resolution of PAT LAC images beyond the limit of the PET system. The technique is demonstrated on empirical and simulated data.

\section{M20-3: Feasibility of Marker-Free Motion Tracking for Motion Corrected MRI and PET-MRI} A. Z. Kyme $^{1}$, J. Maclaren ${ }^{2}$, M. Aksoy ${ }^{2}$, R. Bammer ${ }^{2}$

${ }^{I}$ Biomedical Engineering, University of California Davis, Davis, CA, USA

${ }^{2}$ Radiology, Stanford University, Palo Alto, CA, USA

Prospective motion correction based on optical tracking is a very promising means of improving image quality in standalone MR brain imaging and also advanced hybrid imaging modalities such as PET-MR. To simplify optical tracking, improve its accuracy, and expedite clinical deployment, it is desirable to avoid attaching optical markers to the patient's head. Here we demonstrate proof-of-principle marker-free tracking using an MR-compatible stereo camera and head coil configuration. We tested the 
method outside the MR environment using a 6-axis robot to control a head phantom with $20 \mu \mathrm{m}$ motion repeatability. A realistic forehead image with and without additional small feature patches applied directly to the skin surface was used for feature detection and matching within the pose estimation algorithm. There was sub-millimeter agreement between the majority of pose estimates and the applied robot motion. Most of the residual error observed in this experiment was likely the result of error in the cross-calibration used to relate the robot and camera measurements. We also tested the marker-free tracking method using a phantom inside the bore of a 3T MRI scanner. Out-of-plane rotations were estimated to within $0.2 \mathrm{deg}$. and $0.2 \mathrm{~mm}$ of ground truth measurements obtained using wireless active MR markers. In conclusion, we demonstrate that very small feature rich patches applied directly to the skin surface - avoiding traditional non-rigid attachment mechanisms such as goggles, neoprene caps and glue - allows highly accurate pose estimation within the extremely tight geometric constraints of the MRI head coil and bore. We are currently investigating the use of rubber stamps and skin safe ink to generate small feature patches on the forehead and estimate human head motion in vivo using this technique.

\section{M20-4: Deconvolution-Based Reconstruction for Selective-Plane X-Ray Induced Luminescence Imaging C. D. Smith, B. Quigley, P. La Rivière}

Department of Radiology, University of Chicago, Chicago, IL, 60615

We have been exploring a novel imaging approach called selective-plane x-ray induced luminescence imaging (SPXIL) in which a sheet of x-rays illuminates a plane through a sample containing luminescent nanoparticles. A camera placed at 90 degrees captures an image of the selected plane, albeit one whose resolution is severely limited by diffuse optical propagation. In contrast to most diffuse optical reconstruction problems, the depth of the plane of interest is determined by the selective illumination. This transforms an ill-posed 3D diffuse optical tomography reconstruction problem into a better-conditioned 2D problem. In the limiting case of homogeneous optical properties with a semi-infinite planar surface, the problem reduces to a 2D deconvolution. Using an analytic model that describes the surface radiance as the point spread function of the imaging system along with a shifted Poisson noise model, we apply Richardson-Lucy deconvolution to experimentally acquired data. After deconvolution, the resolution of the nanophosphor distributions was improved by over $50 \%$ at multiple depths in a tissue equivalent phantom.

M20-5: Calibrating and Validating a Predictive Model for X-Ray Induced Luminescence (XIL) Imaging Using Lanthanide-Doped Nanophosphors and an Optical Gel Phantom B. Quigley $^{1}$, C. Smith ${ }^{1}$, S.-H. Cheng ${ }^{1}$, J. Souris ${ }^{1}$, C.-T. Chen ${ }^{1}$, C. Pelizzari ${ }^{1}$, S. Kron ${ }^{1}$, L.-W. Lo ${ }^{1}$, R. Wiersma ${ }^{2}$, P. La Riviere ${ }^{1}$

${ }^{I}$ Radiology, The University of Chicago, Chicago, IL, United States

${ }^{2}$ Radiation and Cellular Oncology, The University of Chicago, Chicago, IL, United States

Europium-doped nanophosphors emit near-infrared light under x-ray exposure. This luminescence has the potential to be exploited in a clinical setting for high dose x-ray regimes employed by radiation therapy. Possible applications include using the particles as nanodosimeters for real-time dose feedback, imaging radiosensitizers to confirm localization to treatment volumes, or using the nanophosphors as a light source for photosensitizers to achieve photodynamic therapy in tandem with conventional radiotherapy. Our work is concerned with modeling the physics of radiation dose deposition, x-ray induced luminescence generation, and optical imaging in the challenging environment of turbid materials such as human tissue. We constructed homogenous optical gel phantoms using 1\% agar along with Intralipid and black ink in order to give the material optical scattering and absorption coefficients similar to mammalian tissue $\left(\mu_{\mathrm{a}}=0.30 \mathrm{~cm}^{-1}, \mu_{\mathrm{s}}^{\prime}=10 \mathrm{~cm}^{-1}\right)$. XIL images were acquired with a cooled CCD camera and macro lens within a small animal therapy unit. Nanophosphors in a capillary tube were placed at varying depths below the imaging surface in order to evaluate the signal level and resolution detected at the surface of the gel phantom. We then modeled the diffuse optical imaging using the extrapolated boundary condition for a luminescent point source at depth. This was then compared with the experimental data to develop a predictive model in an attempt to validate and calibrate the simulation.

M20-6: Tumor Cell Load Estimation Based on Diffusion-Weighted MRI and Histology Data

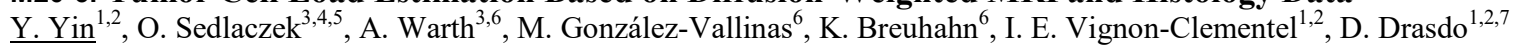

${ }^{I}$ INRIA Paris, Paris, France

${ }^{2}$ Sorbonne Universités UPMC Univ. Paris 6, Laboratoire Jacques-Louis Lions, Paris, France

${ }^{3}$ Translational Lung Research Center Heidelberg (TLRC), member of the German Centre for Lung Research (DZL), Heidelberg, Germany

${ }^{4}$ Department of Diagnostic \& Interventional Radiology, University Hospital of Heidelberg, Heidelberg, Germany, Heidelberg, Germany

${ }^{5}$ Department of Diagnostic and Interventional Radiology with Nuclear Medicine, Thoraxklinik at University of Heidelberg, Heidelberg, Germany

${ }^{6}$ Institute of Pathology, University Hospital Heidelberg, Heidelberg, Germany

${ }^{7}$ IZBI, University of Leipzig, Leipzig, Germany 
Tumor cellularity is an important tissue microstructural feature, which is useful for cancer diagnosis and cell number related treatment such as targeted cancer therapies. Diffusion-weighted MRI (DW-MRI) is a clinically key and non-invasive imaging technique for cancer diagnosis and tumor treatment assessment, which measures the water molecule mobility in tissues and provides an indirect measure of tumor cellularity. Histopathological examination of tissues reveals the tissue microstructure, but is usually available as a small sample or after resection. How to quantitatively estimate the total tumor cell load is still a challenging work. In order to link low-resolution clinical data with high-resolution histological information for tumor cell load estimation, we proposed a pipeline based on image processing techniques. The main pipeline steps include: 1) adaptive cell nuclei segmentation; 2) model based 3D cell density estimation; 3) correlation identification between the DW-MRI diffusion coefficient (D value) and the underlying tumor cellularity; 4) cell number calculation based on tumor D values and the identified correlation between diffusion and cellularity. The proposed cell segmentation algorithm performs detection with a very high accuracy $(0.95)$ on selected tumor tissue samples. For a specific patient with NSCLC, the estimated total cell number of the tumor $(6.5 \mathrm{~cm}$ in diameter $)$ is $3.64 \times 10^{10}$. In conclusion, results presented in this study represent a novel and innovative approach for the estimation of tumor cell numbers based on DW-MRI. These results might be of importance for the personalized adjustment of therapies and can be applied to many different solid tumors.

\title{
RTSD Program
}

\section{R01: RTSD Opening}

\author{
Monday, Oct. $31 \quad$ 08:00-10:00 Schuman \\ R01-1: Introductory and Welcoming Remarks \\ $\underline{\text { R. B. James }}{ }^{1}$, M. Fiederle ${ }^{2}$ \\ ${ }^{I}$ Science and Technology Directorate, Savannah River National Laboratory, Aiken, SC, USA \\ ${ }^{2}$ University of Freiburg, Freiburg, Germany
}

The talk will provide a welcome to the RTSD attendees and brief introductory comments about the conference.

\author{
R01-2: Can 3-D Position Sensitive Detectors Enable a Drastically Increased Photon Sensitivity in Positron \\ Emission Tomography?
}

C. S. Levin

Radiology/Nuclear Medicine, Stanford University, Stanford, CA, USA

Despite increases in positron emission tomography (PET) coincidence photon detection efficiency (a.k.a. "photon sensitivity") over the years, the fraction of detected coincidence photons from a patient in a clinical PET/CT study is typically at most $1 \%$ ( $\sim 400$ counts/sec/microCi). Most of the detected events such as single (unpaired), tissue-scattered photon coincidences, and multiple-photon coincidences are discarded from the data set. This talk asks the basic question: Is it possible to recover a significant fraction of these "bad" events, and instead position them and include them in image reconstruction? We discuss two novel prototype PET systems we have developed and describe associated event processing algorithms that, if successful, enable us to retain rather than discard a high fraction of events that are normally rejected during PET data acquisition, with a goal to boost PET system photon sensitivity by greater than 20 -fold.

\author{
R01-3: Large Volume, High Performance CZT for Nuclear, Medical, and Security Applications - Recent \\ Breakthroughs \\ H. Chen, M. Prokesch, A. Sundaram, M. Reed, S. Soldner, H. Li, J. Eger, J. W. Hugg
}

eV Products division, Kromek USA, Saxonburg, PA, USA

High quality (high resistivity, high mu-tau, great crystallinity, and material uniformity) large single-crystal $\left(>700 \mathrm{~cm}^{3}\right) \mathrm{CZT}$ has been consistently produced by us in large commercial quantities by the traveling heater method (THM) for fabrication of radiation sensors used in nuclear, medical, and homeland security applications. Sub-1\% FWHM energy resolution at Cs-137 (662 $\mathrm{keV}$ ) has been obtained on a square array of $22 \times 22 \times 15 \mathrm{~mm}^{3}$ detectors fabricated from THM CZT with 2 mm pitch pixels. 100$200 \mu \mathrm{m}$ position resolution has also been achieved on $15 \times 15 \times 10 \mathrm{~mm}^{3}$ detectors with $500 \mu \mathrm{m}$ pixel pitch.

Our THM CZT crystal has excellent crystallinity and material uniformity evidenced by 30 arc second FWHM from X-ray rocking curve measurements while IR microscopy shows low density of Te-precipitates/inclusions of size $<4 \mu \mathrm{m}$ throughout the boule of "as grown" material. Bulk resistivity is consistently in the $10^{10} \mathrm{Ohm}-\mathrm{cm}$ range with electron-mu-tau $>10^{-2} \mathrm{~cm}^{2} / \mathrm{V}$. Photon counting spectral X-ray detectors fabricated from THM CZT material can respond to more than 100 million $\mathrm{cps} / \mathrm{mm}^{2}$ input flux without polarization. X-ray spectral response of every pixel will also be presented and discussed. These recent breakthrough trends indicate that our CZT material has great potential to revolutionize the room temperature nuclear radiation detection field. 
R01-5: Analysis of Strategies to Decrease Tellurium Inclusions in CZT via Bridgman Crystal Growth and the Accelerated Crucible Rotation Technique

\author{
M. S. Divecha ${ }^{1}$, S. K. Swain ${ }^{2}$, S. Kakkireni ${ }^{2}$, J. J. McCoy ${ }^{2}$, K. G. Lynn ${ }^{2}$, J. J. Derby ${ }^{1}$ \\ ${ }^{I}$ Chemical Engineering and Materials Science, University of Minnesota, Minneapolis, MN, USA \\ ${ }^{2}$ Mechanical and Materials Engineering, Washington State University, Pullman, WA, USA
}

Cadmium zinc telluride (CZT) is currently used for advanced gamma ray detection due to its high resolution, wide band gap, and ability to operate at room temperature. The Bridgman process is widely employed to grow CZT crystals. One advantage is that growth rates are quite high, at least an order of magnitude faster than in the traveling heater method (THM); however, detector yield is often limited by significant second-phase particle populations and the loss of crystallinity during growth. Strategies that suppress these problems in the Bridgman growth method would represent a major advance toward decreasing the very high cost of this material for use in large-volume gamma-ray spectrometers with high energy resolution.

In this presentation, we describe how rigorous crystal growth models developed at the University of Minnesota (UMN) are being used to assess the promise of the accelerated crucible rotation technique (ACRT) on CZT Bridgman growth. ACRT dramatically increases mixing of the melt, which stabilizes morphological instabilities of the growth interface and, in turn, reduces the potential for inclusion processes that lead to the formation of tellurium-rich particles. ACRT flows may also suppress new grain nucleation at the crucible walls to improve crystallinity. Indeed, preliminary growths of CZT under ACRT at Washington State University (WSU) have demonstrated more uniform radial zinc distribution, reductions in the mean second-phase particle size, and improved crystallinity. We will discuss metrics that allow us to employ UMN computations to optimize the effects of ACRT on reducing tellurium-rich second-phase particles in grown material. Concurrent experiments conducted at WSU will assess different ACRT cycles designed by computational optimization. Ultimately, we believe great advances in process yield and material quality will enable significant cost reduction of this important material.

This work is supported by the Department of Energy, National Nuclear Security Administration, under Award DE-NA0002565; no official endorsement should be inferred.

R01-6: A Full Energy Resolved Photon-Counting Chip for High Count Rate X-Ray Detectors

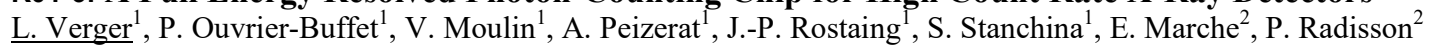

${ }^{I}$ CEA-LETI MINATEC Campus, F-38054 Grenoble, France

${ }^{2}$ MULTIX, F-38430 Moirans, France

There is a great excitement in X-ray systems for either luggage control or CT application with the recent development of energysensitive photon-counting X-ray detectors mainly based on $\mathrm{Cd}(\mathrm{Zn}) \mathrm{Te}$ semi-conductor technology. Such innovative X-ray detectors count individual photons and sort them into selected energy bins. Most of available detectors \& electronics architectures have been designed to ensure both a high count rate and a few selected energy bins. Although results are encouraging, there are still some remaining issues related to combine charge sharing correction, pile up and induction rejection, high count rate electronic integration and capability to adapt thresholds according to the scanned object. Aiming at providing answers to the challenge listed above, CEA-Leti has developed a two side buttable full energy resolved photon counting readout electronics circuit with $4 \times 8$ pixels based on the concept "spectrum inside pixel": each pixel ( $0.8 \mathrm{~mm}$ pitch scalable to $0.5 \mathrm{~mm}$ pitch) comprises a charge sharing correction, a pile up \& induction rejection, an ADC and a spectrum builder to provide the full 128 bins $(\sim 1 \mathrm{keV}$ width) information at high count rate (10Mcps/pixel) in real time. These developments will be presented and illustrated by experimental measurements obtained with a 5.2x6.2 x3 mm3 Cd(Zn)Te under X-and gamma-ray.

\author{
R01-7: Localization and Identification of Radiological/Nuclear Material Dispersed in the Environment by \\ Means of Unmanned Aerial Vehicle Equipped with Spectroscopic CdZnTe Detector \\ $\underline{\text { M. Bettelli }}{ }^{1}$, G. Micconi ${ }^{2}$, J. Aleotti ${ }^{2}$, S. Caselli ${ }^{2}$, N. Zambelli ${ }^{3}$, G. Benassi ${ }^{3}$, D. Calestani ${ }^{1}$, A. Zappettini ${ }^{1}$ \\ ${ }^{I}$ IMEM-CNR, Parma, Italy \\ ${ }^{2}$ Ingegneria dell'Informazione, University of Parma, Parma, Italy \\ ${ }^{3}$ due2lab s.r.l., Parma, Italy
}

Recently we had developed a haptic teleoperated UAV carrying a CZT-based nuclear radiation sensor for detection of radiating substances in industrial plants. The use of haptic feedback was motivated by the lack of situation awareness of the operator when teleoperating an UAV at a distance using standard remote control devices. A haptic feedback has been used as an additional sensory channel to guide the exploration of the environment helping the operator to focus on relevant areas. In particular, an attractive force feedback is generated when the UAV is close to the location of the most intense detected radiation, thereby reducing operator's perceptual load. As a result, the operator does not rely only on direct visual feedback of the UAV but rather he takes advantage of information from the onboard radiation sensor. The developed prototype is innovative compared to the state of the art and it has been tested in preliminary experiments to assess the feasibility of the approach. The CZT detector has a 
2 cubic centimeter volume and good spectroscopic performances (about $2 \%$ at $662 \mathrm{keV}$ ). In this work, we show the demonstration of the prototype in relevant and operational environments. These experiments have been carried out to demonstrate the effective sensitivity of the system to weak nuclear sources, the localization capability of the prototype, the capability of the system to discriminate between two different nuclear sources at different distances. A second set of experiments has been carried out in operational environments, such as landfills metallic waste disposals in cooperation with the local agency for environmental control ARPA E-R.

\title{
R02: Applications1
}

\author{
Monday, Oct. $31 \quad$ 10:30-11:50 Schuman \\ R02-1: Arrays of Position-Sensitive Virtual Frisch-Grid CdZnTe \\ A. E. Bolotnikov ${ }^{1}$, G. S. Camarda ${ }^{1}$, Y. Cui ${ }^{1}$, G. De Geronimo ${ }^{1}$, R. Gul ${ }^{1}$, J. Fried ${ }^{1}$, A. Hossain ${ }^{1}$, E. Vernon ${ }^{1}$, G. Yang ${ }^{1}$, \\ R. B. James ${ }^{2}$ \\ ${ }^{1}$ Brookhaven National Laboratory, Upton, NY, USA \\ ${ }^{2}$ Savannah River National Laboratory, Aiken, SC, USA
}

High-energy resolution position-sensitive CdZnTe (CZT) drift detectors offer unique capabilities for imaging gamma rays and correcting the response non-uniformities caused by crystal defects. The position sensing allows for segmenting the detectors' volumes into large numbers of small voxels and equalizing the responses generated by the incident photons in each of them. Arrays of such detectors can be integrated into compact hand-held instruments for many practical applications, including international safeguards, nonproliferation, arms control, and homeland security. Here, we present the results from testing of such detectors integrated in small arrays coupled with its front-end ASIC chip, which can capture signals from up to 36 anodes and 9 cathodes. Each detector is encapsulated into a polyester shell with $5 \mathrm{~mm}$-wide charge-sensing pads placed near the anode. The pad signals provide X-Y position information, which allow for more accurate corrections of the charge losses caused by crystal defects. The basic array (module) consists of a number of detectors grouped into $2 \times 2$ sub-arrays, each having a common cathode made by connecting together the cathodes of the individual detectors. The cathode signals provide position information along the $\mathrm{Z}$ coordinate. As we will describe in the talk, these features can significantly improve the performance of detectors fabricated from typical CZT material and, thus, extend their acceptance boundaries, leading to an increase in the yield and an expected decrease in cost.

\section{R02-2: Recent Improvements to HiSPECT Imaging Module}

G. Montémont, O. Monnet, S. Stanchina, M. Bernard, L. Verger

CEA, LETI, Minatec campus, Grenoble, France

We introduced in 2014 a four-side buttable CdZnTe detection module using sub-pixel positioning. It is based on standard SPECT module specifications: $40 \times 40 \mathrm{~mm}$ area, $5 \mathrm{~mm}$ thickness and $2.5 \mathrm{~mm}$ anode pitch. By using signal processing techniques, we showed it was possible to improve spectral performance, allowing an average resolution of $2.5 \%$ at $122 \mathrm{keV}$ and to locate an interaction in $3 \mathrm{D}$ inside the detector. A $2.5 \times 2.5 \times 5 \mathrm{~mm}$ electrode volume can be in practice subdivided in $8 \times 8 \times 8$ voxels. This device enables the use of different gamma imaging techniques: collimator oversampling, coded aperture imaging and Compton imaging. We presents here recent work carried out on this device in order to make it operate faster. First, the 3D positioning technique is now fully implemented in hardware to simplify software and speed up the processing. Second, our reconstruction techniques have evolved to list-mode to fully take profit of the precise 3D positioning and allow dynamic imaging. Third, we have carried out new tests of the device with diverse imaging scenarios and on a broad range of energy (from 14 to $1400 \mathrm{keV}$ ) and a broad range of source dose rate (from $1 \mathrm{nSv} / \mathrm{h}$ to $100 \mathrm{mSv} / \mathrm{h}$ ). Main strength of the device is its ability to combine spectral, spatial resolution, compact modular design and cost effectiveness.

\author{
R02-3: Investigation of High-Z Sensors with Charge Integrating Pixel Detectors with a High Spatial \\ Resolution \\ D. Greiffenberg, B. Schmitt, A. Bergamaschi \\ SLS Detector Group, Paul-Scherrer-Institut (PSI), Villigen PSI, Switzerland
}

The SLS Detector Group has a long history in developing pixel detectors for the needs of synchrotron sources. Over the last year's charge integrating dynamic gain switching pixel detectors have been developed to suit the needs of the upcoming FELs. Due to the charge integrating nature of the preamplifier, each pixel will give a direct measure of the collected charge of a well defined area, which allows a deep insight into what happens within the sensor (e.g. fluorescence effects). This presentation will show results from the charge integrating pixel detector JUNGFRAU with a pixel pitch of 75 um in combination with CdTe and GaAs sensors

\section{R02-4: Study on Achievable Energy Resolution of 3-D CdZnTe Gamma-Ray Detectors}


Z. He, Y. Zhu, M. Streicher, J. Xia, B. Williams

Nuclear Engineering and Radiological Sciences Department, University of Michigan, Ann Arbor, Michigan, USA

Three dimensional position sensitive CdZnTe detectors have demonstrated sustained improvement on energy resolution over the past two decades, from about 1.7\% FWHM for single-pixel events in 1996 on CZT detectors of $1 \times 1 \times 1$ cubic centimeter to just under $0.4 \%$ FWHM at $662 \mathrm{keV}$ for single-pixel events recently on CZT detectors of $2 \times 2 \times 1.5$ cubic centimeters. This work is to study on the best achievable energy resolution on large volume CZT detectors, using commercially available CZT detectors, latest low-noise digital application specific integrated circuitry (ASIC) and various CZT cutting, polishing, etching and attachment techniques. Contributions of bulk material properties, ASIC readout noise and effects of device fabrication processes are analyzed to guide further improvement and advances on CZT detector technology.

This material is based upon work supported by the DOD Defense Threat Reduction Agency under Contract \# HDTRA1-15-C-0049

\title{
R03: Pixel Detectors
}

\author{
Monday, Oct. $31 \quad$ 14:00-16:05 Schuman \\ R03-1: Fast and Spectroscopic X-ray Imaging with Timepix and Timepix3 Detectors with CdTe and CZT \\ Sensors \\ $\underline{\text { J. Jakubek }}^{1}$, M. Jakubek ${ }^{1}$, P. Soukup ${ }^{1}$, E. Trojanova ${ }^{1}$, D. Turecek ${ }^{1}$, S. Vahanen ${ }^{2}$ \\ ${ }^{I}$ ADVACAM Cameras, Prague, Czech Republic \\ ${ }^{2}$ ADVACAM Semiconductors, Espoo, Finland
}

\begin{abstract}
The newly developed fast USB 3.0 interface AdvaPIX for hybrid particle counting pixel detectors of Medipix family significantly extents their application field. The AdvaPIX interface is compact, fast (1700 fps with Timepix), plug-and-play and easy to use. Fast readout allows imaging in so called event-by-event mode processing each detected particle (e.g. X-ray photon) individually. Especially the Timepix3 detector, which is optimized for this mode, provides very complete information for every detected particle. Every pixel measures time and deposited energy. It can be exploited with great advantage in the field of spectroscopic and high resolution X-ray imaging especially in case of new high-Z sensor materials such as GaAs, CdTe or CZT. The X-ray imaging based on photon counting with these sensors faces several complications distorting their spectral response and degrading their spatial resolution. There are basically three reasons: (i) Charge sharing among small pixels and (ii) generation of an internal $\mathrm{X}$-ray fluorescence (XRF) signal and (iii) incomplete charge collection. All these issues can be reduced using event-by-event measurement approach along with technique of spatial and temporal coincidence and simple model of charge collection. The principles will be described along with several examples of spectral X-ray radiography.
\end{abstract}

R03-2: Comparison of CdZnTe and CdTe Schottky Pixel Sensors for Construction of a High Spatial Resolution Spectroscopic Photon Counting Large Area Camera J. J. Kalliopuska $^{1}$, J. Jakubek ${ }^{2}$, D. Turecek ${ }^{2}$, P. Soukup ${ }^{2}$, M. Jakubek ${ }^{2}$, S. Vähänen ${ }^{1}$, J. Salmi $^{1}$

${ }^{I}$ Semiconductors, Advacam Oy, Espoo, Finland

${ }^{2}$ Cameras, ADVACAM s.r.o, Prague, Czech Republic

During the past two years Advacam has developed a spectral photon counting camera for inspection of static or moving objects. In the previous IEEE RTSD 2015 workshop we presented a spectral imaging single row camera using five Ohmic CdTe pixel sensors of $1 \mathrm{~mm}$ thick flip chip bonded to Timepix readout ASICs and placed next to each other. The constructed CdTe camera showed outstanding properties for X-ray imaging and feasibility to extend the imaging area of the camera was proven. The approach of the camera is based on a novel row structure that can be extended indefinitely or to cover a large area with a full field of view

Recently we have custom fabricated edgeless $1 \mathrm{~mm}$ thick Schottky CdTe and $2 \mathrm{~mm}$ thick CdZnTe pixel sensors with $55 \mu \mathrm{m}$ and $110 \mu \mathrm{m}$ pitch, respectively. The sensors were flip chip bonded to Timepix readout ASICs using low temperature InSn solder micro bumps. Comparison of imaging and spectroscopic performance of the two sensor materials will be presented and compared.

In addition, we have further demonstrated the capability to scale up the imaging area by tiling CdTe sensor assemblies as continuous full field of view double row camera. X-ray imaging properties of the new Schottky CdTe and CdZnTe row cameras will be demonstrated on various scientific and industrial samples. The compared properties include long term sensor stability, material homogeneity, charge sharing between the pixels and high flux operation.

R03-3: Characterization of High-Z Sensor Materials with the IBEX ASIC

V. Radicci, P. Zambon, C. Disch, M. Rissi, T. Sakhelashvili, M. Schneebeli, P. Trueb, C. Broennimann 
Thanks to their high atomic number, GaAs $(Z=32), \mathrm{CdZnTe}\left(\mathrm{Zi}_{i}{ }^{1 / 249.1)}\right.$ ) and $\mathrm{CdTe}(\mathrm{Z}=50)$ offer the unique possibility to widen the detectable $x$-ray energy range up to levels unattainable with Silicon sensors. We carried out a comprehensive characterization of an innovative hybrid photon counting (HPC) detector featuring High-Z sensor materials $\ddot{i}_{i} 1 / 2 \mathrm{GaAs}, \mathrm{CdZnTe}$ and $\mathrm{CdTe} \ddot{i}_{i}{ }^{1 / 2}$ bump bonded to the new generation IBEX ASIC developed at DECTRIS Ltd. for spectral imaging applications. We investigated detectors of $75 \ddot{i}_{i}^{1 / 2 \mathrm{mi}} \ddot{c}^{1 / 2} 75 \ddot{i}_{i} 1 / 2 \mathrm{~m}$ pixel size both in our in-house laboratory and at synchrotron facilities over a wide range of energies. In order to compare the different materials and to define their range of applications, we measured the quantum efficiency (QE), the energy and spatial resolution and the count rate capabilities. The QE shows values close to $100 \%$ for energies below the K-edges of the elements constituting the sensor, where they suddenly decrease. The QE of CdTe $1 \mathrm{~mm}$-thick is however still $60 \%$ at $95 \mathrm{keV}$. The energy resolution at $20 \mathrm{keV}$ lies at $2.3 \mathrm{keV} \mathrm{FWHM}$ and worsens slightly for incoming energies above the K-edges. In this energy region, the spectra become structured due to the presence of fluorescence photons. Modulation transfer functions (MTF) were measured with direct x-ray W-tube beam in the range $40-60 \mathrm{kVp}$ using the slantededge technique. In virtue of the direct photo-conversion and despite of the conspicuous charge sharing the spatial resolution remains excellent. Finally, the instant retrigger technology $\ddot{i}_{i} 1 / 2$ which allows for a non-paralyzable counting mode $\ddot{i}_{i} 1 / 2$ extends a prompt count rate above $10^{7} \mathrm{cts} / \mathrm{s} /$ pixel $\left(1.7 \mathrm{i}_{i} 1 / 210^{9} \mathrm{cts} / \mathrm{s} / \mathrm{mm}^{2}\right.$ for $75 \ddot{i}_{i} 1 / 2 \mathrm{~m}$ pixels).

\section{R03-4: Spectroscopic Performance of Timepix Chips with Different CdTe Sensor Configurations}

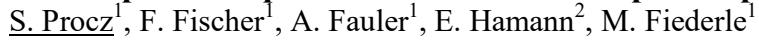

${ }^{I}$ FMF University Freiburg, Freiburg, Germany

${ }^{2}$ KIT Karlsruhe Institute of Technology, Karlsruhe, Germany

The photon counting semiconductor detectors Timepix and Timepix3 [1] have the capability of measuring the energy of each incident X-ray and ?-photon by the so called time-over-threshold (ToT) method. In combination with the high spatial resolution of the Timepix detectors and the high efficiency sensor material CdTe it is possible to develop spectroscopic ?-cameras. The native pixel pitch of Timepix detectors is $55 \times 55 \mu \mathrm{m}^{2}$, in sum possessing $256 \times 256$ pixels, but detectors with a larger pixel pitch like $110 \times 110 \mu \mathrm{m}^{2}$ can be produced by bump-bonding only every second pixel. This minimizes charge-sharing effects and the amount of counting pixels in a cluster, but also diminishes the spatial resolution of ?-camera applications.

Different sensor thicknesses and pixel pitch configurations have been produced to analyze the influence of the CdTe sensor thickness and detector pixel pitches on the energy information and detection efficiency for high energy ?-photons. A comparison of detector efficiency will be shown as well as the energy information of incident ?-photons that was obtained by ToT analysis from clusters.

[1] X.Llopart et al., Timepix, a 65k programmable pixel readout chip for arrival time, energy and/or photon counting measurements, NIM A Vol. 581, Issue 1-2, pages 485-494, Oct. 2007.

\section{R03-5: Characterisation and Development of GaAs:Cr High Frame Rate X-Ray Imaging Systems}

M. C. Veale ${ }^{1}$, B. Cline ${ }^{1}$, J. Coughlan ${ }^{1}$, M. Hart ${ }^{1}$, J. Lipp ${ }^{1}$, T. Nicholls ${ }^{1}$, M. French ${ }^{1}$, A. Schneider ${ }^{1}$, P. Seller ${ }^{1}$, P. Sellin $^{2}$, I. Paper ${ }^{3}$, K. Swahney $^{3}$, E. Gimenez-Navarro ${ }^{3}$, I. Horswell ${ }^{3}$, N. Tartoni ${ }^{3}$, A. Lozinskaya ${ }^{4}$, V. Novikov ${ }^{4}$, O. P. Tolbanov ${ }^{4}$ A. Tyazhev $^{4}$, A. Zarubin ${ }^{4}$

${ }^{I}$ Technology, STFC Rutherford Appleton Laboratory, Didcot, Oxfordshire, UK

${ }^{2}$ Department of Physics, University of Surrey, Guildford, Surrey, UK

${ }^{3}$ Diamond Light Source, Didcot, Oxfordshire, UK

${ }^{4}$ Siberian Physical-Technical Institute, Tomsk State University, Tomsk, Russia

The STFC Rutherford Appleton Laboratory (UK) and Tomsk State University (Russia) have been working to develop and characterise detector systems based on chromium-compensated gallium arsenide (GaAs:Cr) material for high frame rate X-ray imaging. Previous work has demonstrated the excellent spectroscopic performance of the material and its resistance to damage induced by high fluxes of X-rays. In this paper, the role of the charge transport properties of electrons and holes and material uniformity in the high frame rate imaging performance of the material will be presented.

GaAs:Cr detectors were fabricated by Tomsk and have been hybridized to HEXITEC, PIXIE, Medipix and LPD ASICs at STFC using a combination of epoxy-stud and indium bump bonding techniques. Measurements with HEXITEC and PIXIE detectors have been used to characterise the charge transport properties of electrons and holes in the GaAs:Cr material. These measurements have shown the electron mobility to have a value of the order $1000 \mathrm{~V} \mathrm{~cm}^{2} \mathrm{~V}^{-1} \mathrm{~s}^{-1}$ and a lifetime of $30 \mathrm{~ns}$. Measurements of the transport properties of holes have shown the lifetime to be $<10 \mathrm{~ns}$.

The role of these carrier properties in the performance of high frame rate imaging detectors produced from GaAs:Cr have been investigated using the Medipix and LPD ASICs. These detectors have been characterised at the Diamond Light Source synchrotron (UK) at X-ray fluxes $>1 \times 10^{8}$ photons s $\mathrm{mm}^{-2}$. Measurements have shown that while it's possible to produce single 
photon counting performance with these devices, small scale variations in the detector properties lead to variations in gain from pixel to pixel that require correction.

\title{
R03-6: Evaluation of Semi-Insulating GaAs Radiation Detectors with a Novel Electrodes Concept
} F. Dubecký ${ }^{1}$, B. Zatko ${ }^{1}$, P. Bohácek ${ }^{1}$, M. Sekácová ${ }^{1}$, V. Necas ${ }^{2}$

${ }^{I}$ Dept. of Microelectronics and Sensors, Inst. of Electrical Engineering, Slovak Academy of Sciences, Bratislava, Slovakia

${ }^{2}$ Dept. of Nuclear Engineering, Inst. of Nuclear and Physical Engineering, Slovak University of Technology, Bratislava, Slovakia

Prefered electrode technology of a radiation detector based on semi-insulating (SI) GaAs includes a blocking Ti/Pt/Au barier electrode coupled with an ohmic $\mathrm{N}+$ contact on the opposite side of the substrate. A novel low work function $\mathrm{Mg}$ contact comparing with a Ti/Pt contact gives almost two orders of magnitude lower current in a low bias saturation region [1]. Recent study [2] of SI GaAs device with two Mg contacts demonstrates more than an order of magnitude lower current at high electric field $>10^{\wedge} 4 \mathrm{~V} / \mathrm{cm}$, which is typical for operation of radiation detectors. Such contact forms a heterojunction $\mathrm{MgO}-\mathrm{SI} \mathrm{GaAs}$ with a high conduction and valence bands offsets as follows from the X-ray photoelectron spectroscopy [2].

Present work is devoted to fabrication and testing of Gd-SI GaAs-Gd and Mg-SI GaAs-Mg radiation detectors. The used low work function $\mathrm{Mg}$ and $\mathrm{Gd}$ contacts form heterojunctions and such present novel electrodes concept of SI GaAs-based radiation detector. Fabricated detectors are characterized by current-voltage measurements and detection performance testing using Am241 and Co-57 photons. The charge collection efficiency and the energy resolution are evaluated and compared with a standard Pt-SI GaAs-N+ detector based on the same material with the same geometry.

[1] F. Dubecký, M. Dubecký, P. Hubík, D. Kindl, E.Gombia, M. Baldini, V. Necas, Unexpected current lowering by a low workfunction metal contact: Mg/SI-GaAs, Sol. St. El. 82 (2013) 72-76.

[2] F. Dubecký, D. Kindl, P. Hubík, M. Micušík, M. Dubecký, P. Bohácek, G. Vanko, E. Gombia, V. Necas, J. Mudron, A comparative study of Mg and Pt contacts on semi-insulating GaAs: Electrical and XPS characterization, Appl. Surf. Sci. (2016), in press.

R03-7: D2R1: a 2D ASIC for CdTe Based Fine Pitch and High Energy Resolution Imaging Spectrometer

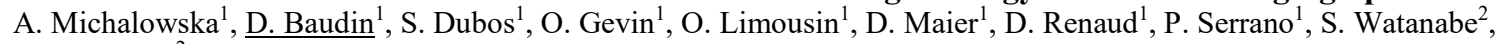
T. Takahashi ${ }^{2}$

${ }^{I}$ DRF/IRFU, CEA Saclay, GIF-SUR-YVETTE, FRANCE

${ }^{2} I S A S, J A X A$, Tokyo, Japan

This paper describes D2R1, a new 2dim full custom ASIC developed at CEA-Saclay for the read out of fine pitch $\mathrm{CdTe}(\mathrm{Cl})$ based detector system. The chip prototype is made of a $16 \times 16$ pixel array with 300 -micron pitch. The detector aims at being operated in space for hard X-ray astronomy or Solar Physics with excellent spectral response and good timing capabilities. The combination of very low noise and appropriate direct Indium Gold Stud Bump bonding of CdTe Schottky diodes on top of the D2R1 ASIC have permitted to reach promising energy resolution measured to $478 \mathrm{eV}, 584 \mathrm{eV}, 817 \mathrm{eV}$ FWHM at 13.94, 59.54, and $122.06 \mathrm{keV}$, respectively. The wide energy range of 4-250 keV and the fast timing capabilities complete D2R1 to suite a variety of different applications like future missions in X-ray astrophysics, medical applications, or nuclear safety. The paper introduces the concepts of the D2R1 ASIC and presents a detector performances.

\author{
R03-8: Study of Experimental and Simulated Performances at Various Fluence Rates on CdTe Spectrometric \\ Imaging Detector

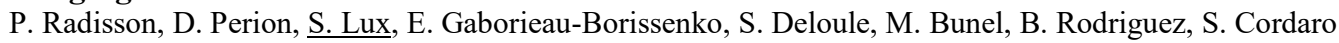 \\ MULTIX, Moirans, France
}

This paper presents the performances of the ME100 detector under monochromatic photon beam at various fluence rates. The ME100 is a multi-channel high count rate x-ray spectrometric detector commercialized by Multix detection (Moirans, France). Measurements were performed at the European Synchrotron Radiation Facility (Grenoble, France). Detector energy resolution and linearity for various energy beams and fluence rates are studied. The effects of the pile-up as well as the charge sharing have been investigated and will be presented. Experimental results compared to the simulation ones will be given.

R04: Crystal Growth

Monday, Oct. $31 \quad$ 16:30-18:30 Schuman
R04-1: Melt Growth of Detector Grade CdZnTe: Challenges and Prospects
K. G. Lynn, S. K. Swain
Center For Material Research, Washington State University, Pullman, WA, USA


Two major scientific and technological challenges currently of significance in CdTe and CdZnTe is the issue of single crystal yield and the elimination or minimization of second phase extended defects and associated defects. Historically, the adopted crystal growth technologies using various doping ideas have not been able to completely solve these problems simultaneously; both have difficulties as will be discussed. For example THM technology can produce large single crystals, however the time scale involved in growth cycle coupled with the necessity of post growth processing to eliminate large second phase particles does not permit a favorable cost structure for large detectors from a large-scale commercialization standpoint. Despite decades of research, the yield of device grade CZT is still quite low. Similarly, melt growth techniques, although possess the advantage of faster growth rates and provision for stoichiometry control, suffer from polycrystallinity (i.e. yield issues). We will discuss the current status of melt growth of CdZnTe with emphasis on strategies to minimize second phase particles and approaches towards improved high-purity single crystal yield. Effects of crucible material selection on grain structure and the prospects of seeding in melt growth in order to improve single crystal yield will be discussed. Additionally, there is an ongoing effort on the implementation of accelerated crucible rotation technique in the author's laboratory. Recent results on the influence of forced melt convection on second phase particle distribution as well as the homogeneity in terms of $\mathrm{Zn}$ and dopants will be discussed.

This work is supported by the Department of Energy, National Nuclear Security Administration, under Award DENA0002565; no official endorsement should be inferred.

R04-2: Using a Focused Laser Beam to Investigate the Feasibility of Achieving Sub-Pixel Resolution with Time-Correlated Transient Signals in Pixelated CdZnTe Detectors

L. A. Ocampo Giraldo ${ }^{1}$, A. E. Bolotnikov ${ }^{2}$, G. S. Camarda ${ }^{2}$, Y. Cui ${ }^{2}$, S. Cheng ${ }^{2}$, G. De Geronimo ${ }^{2}$, A. McGilloway $^{2}$, R. Gul $^{2}$,

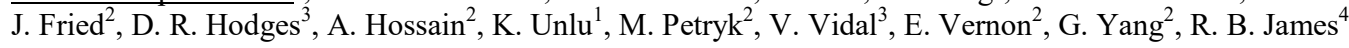

${ }^{I}$ Pennsylvania State University, University Park, PA, USA

${ }^{2}$ Brookhaven National Laboratory, Upton, NY, USA

${ }^{3}$ University of Texas - El Paso, El Paso, TX, USA

${ }^{4}$ Savannah River National Laboratory, Aiken, SC, USA

Cadmium Zinc Telluride (CZT) crystal defects are a major problem causing response non-uniformity across the detector. To address this challenge, high-granularity position-sensitive detectors have been proposed to correct response non-uniformities by virtually subdividing the detectors area into small voxels and equalizing responses from each voxel. 3D pixelated detectors coupled with multichannel readout electronics are the most advanced type of CZT devices offering many options in signal processing and enhancing detector performance. One recent innovation proposed for pixelated detectors is to use the induced (transient) signals from neighboring pixels to achieve high sub-pixel position resolution while keeping the large pixel sizes. The main hurdle in achieving this goal is the relatively low signal induced on the neighboring pixels because of the electrostatic shielding effect caused by the collecting pixel. In addition, to achieve high position sensitivity one should rely on time-correlated transient signals, which means that digitized output signals must be used. We present the results of our studies to measure the amplitude of the pixel signals so that these can be used to measure positions of the interaction points. This is done with the processing of digitized correlated time signals measured from several adjacent pixels taking into account rise time and charge sharing effects. In these measurements we used a focused pulsed laser to generate a 50 micron beam at one milliwatt (650nm) over the detector surface while the collecting pixel was moved in cardinal directions. The results include measurements that present the benefits of combining conventional pixel geometry with digital pulse processing for the best approach in achieving sub-pixel position resolution with the pixel dimensions of 2-3 $\mathrm{mm}$. Using large pixel sizes offers the opportunity of using a lesser number of the readout channels and, on the other hand, it gives opportunity to achieve the position resolution of $\sim 100$ microns within footprints of individual pixels.

Acknowledgements: The work was supported by the Office of Nonproliferation and International Security, Office of Nuclear Safeguards \& Security, Next Generation Safeguards Initiative's Safeguards Technology Development Team (NA-241), and the work to develop CZT detectors with thickness up to $50 \mathrm{~mm}$ was supported by the U.S. Department of Energy, Office of Defense Nuclear Nonproliferation Research \& Development (NA-22). The manuscript has been authored by Brookhaven Science Associates, LLC under Contract No. DE-AC02-98CH1-886 with the U.S. Department of Energy. The United States Government retains, and the publisher, by accepting the article for publication, acknowledges a world-wide license to publish or reproduce the published form of this manuscript, or allow others to do so, for the United States Government purposes.

R04-3: Cd1-xZnxSeyTe1-Y: a Potential Candidate for Low Cost Alternative to CdZnTe U. N. Roy ${ }^{1}$, G. Camarda ${ }^{1}$, Y. Cui ${ }^{1}$, R. Gul ${ }^{1}$, A. Hossain ${ }^{1}$, G. Yang ${ }^{1}$, P. Vanier ${ }^{1}$, J. Zazvorka ${ }^{2}$, V. Dedic ${ }^{2}$, J. Franc ${ }^{2}$

${ }^{I}$ Non-proliferation and National Security, Brookhaven National Laboratory, Upton, NY, US

${ }^{2}$ Department of Physics, Charles University, Prague, Czech Republic

$\mathrm{CdZnTe}$ is one of the most widely used materials for radiation detector applications including homeland security, astrophysics and medical imaging. Over the past years, the quality of CdZnTe crystals improved drastically at a steadily reduced cost. However, due to its poor thermo-physical properties, the yield of large-volume high-quality detector-grade CZT continues to be 
an issue. The main issue with CZT is the high segregation coefficient of $\mathrm{Zn}$, which causes a composition variation along the length of the ingot and reduces the yield. The presence of a high concentrations of sub-grain boundaries and their networks also hinder the yield of high-quality large-volume CZT detector-grade material. In order to address these issues, we are developing Cd1-xZnxSeyTe1-y in search of a low-cost alternative to CZT. Selenium also plays a vital role in reducing the detrimental defects such as reduction of sub-grain boundaries and their networks, better compositional homogeneity, and lower concentration of secondary phases in the material. Our initial findings are very encouraging, and Cd1-xZnxSeyTe1-y appears to be a promising candidate to achieve low-cost detector-grade material.

R04-4: Recent Progress in CdTe/n+-Si Epitaxial Layer Based Heterojunction Diode-Type Gamma Detectors M. Niraula, K. Yasuda, M. Kojima, S. Kitagawa, S. Tsubota, T. Yamaguchi, J. Ozawa, Y. Agata

Graduate School of Engineering, Nagoya Institute of Technology, Nagoya, Japan

We are developing room-temperature gamma ray detectors for spectroscopy and imaging applications with working photon energies up to $100 \mathrm{keV}$, using metalorganic vapor-phase epitaxy (MOVPE) grown thick single crystal CdTe epilayers on Si substrates. We have previously reported on growth details and gamma ray detection properties of the $\mathrm{p}-\mathrm{CdTe} / \mathrm{n}-\mathrm{CdTe} / \mathrm{n}+-\mathrm{Si}$ heterojunction diode detectors ${ }^{1}$. We further developed an $(8 \mathrm{x} 8)$ imaging array using CdTe layer grown on a $12 \times 12 \mathrm{~mm}^{2} \mathrm{Si}$ substrate, and demonstrated its spectroscopic imaging capability ${ }^{2}$. To achieve larger arrays with uniform properties we have been optimizing our crystal growth and detector array fabrication techniques. Recently we achieved uniform growth of single crystal CdTe on $25.4 \times 25.4 \mathrm{~mm}^{2}$ sized Si substrates with high material quality, and developed a (20x20) pixel array having $1.12 \times 1.12$ $\mathrm{mm}^{2}$ size pixel in a $1.27 \mathrm{~mm}$ pitch. The detector shows highly uniform distribution of leakage currents throughout the pixels. Moreover the spectroscopic performance of the detector improved significantly. When compared to our previous detectors, these recent detectors can withstand higher bias voltages $(\sim 150 \mathrm{~V})$, and resolve all energy peaks associated with ${ }^{241} \mathrm{Am}$ gamma isotope at room temperature. Detail results of our recent detectors together with a review of our epitaxial growth based detector development technique will be presented.

1. M. Niraula et al., IEEE RTSD Workshop 2006, 2008. 2. M. Niraula et al., IEEE RTSD Workshop 2011, 2013.

\section{R04-5: Control of Te Inclusion Size Distribution in CdZnTe Crystals Through Exogenous Modification of Convection During THM Growth}

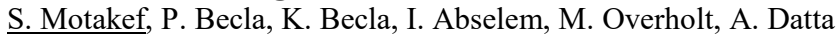

CapeSym, Inc., Natick, MA, US

One of the major factors controlling the yield of high quality CdZnTe crystals grown by THM is the presence of Te inclusions with a broad size distribution, exceeding the 1-3 micron threshold required for high resolution spectroscopy. At present the standard approach to overcoming this deficiency is a two-step annealing process to remove the Te inclusions while achieving high resistivity in doped crystals. We report on an alternative approach where convection in the solution zone is modified to influence the population density of Te inclusions. Two stirring mechanisms will be discussed: electron-magnetic stirring by travelling magnetic fields (TMF) and Accelerated Crucible Rotation Technique (ACRT), each leading to distinctively different flow patterns in the solution zone. Numerical simulation and experimental results on THM-grown crystals under TMF stirring are presented. TMF is shown to have a dramatic effect on the reduction of the Te inclusion size distribution, with inclusion diameters reduced to 1-3 microns. A range of spin-up and spin-down acceleration rates during ACRT were experimentally investigated. It is observed that that at above a critical acceleration rate, substantial modification to the Te inclusion size distribution is achieved. Whereas, appreciable change to the diameter distribution of Te inclusions is obtained by both exogenous stirring mechanisms, only a modest change in total volume of inclusions is observed.

R04-6: First-Principles Investigations of the Electronic Properties and Thermodynamic Stability of CdZnTeSe Alloys for Room Temperature Radiation Detectors

J. B. Varley ${ }^{1}$, V. Lordi ${ }^{1}$, U. N. Roy ${ }^{2}$, R. B. James ${ }^{2,3}$

${ }^{I}$ Materials Science Division, Lawrence Livermore National Laboratory, Livermore, CA, USA

${ }^{2}$ Brookhaven National Laboratory, Upton, NY, USA

${ }^{3}$ Savannah River National Laboratory, Aiken, SC, USA

Cadmium Zinc Telluride $\left(\mathrm{Cd}_{1-x} \mathrm{Zn}_{x} \mathrm{Te}\right.$ or $\left.\mathrm{CZT}\right)$ alloys are currently the state-of-the-art choice for room-temperature radiation detectors owing to a number of favorable properties, including a suitably large band gap and high carrier mobility-lifetime product $(\mu \mathrm{t})$, that facilitate efficient charge collection. Unfortunately, the widespread adoption of CZT detectors has been limited by high material costs due to the difficulty in growing large, uniform CZT single crystals. Recently, the inclusion of Se has also garnered interest for room-temperature gamma detection due to experimental demonstrations of very high $\mu \mathrm{t}\left(\mathrm{up} \mathrm{to} 10^{-2} \mathrm{~cm}^{2} / \mathrm{V}\right)$ in $\mathrm{CdTe}_{1-y} \mathrm{Se}_{y}$ (CTS) alloys with modest amounts of incorporated Se. While fabricated CTS alloys display a very high $\mu \mathrm{t}$, the band gap is lower than CdTe, resulting in a reduced signal to noise for gamma detection at room temperature. Hence, adding some $\mathrm{Zn}$ may be desirable to increase the band gap while hopefully maintaining the excellent charge carrier transport. Here we 
use hybrid density functional calculations to computationally assess the properties of $\mathrm{Cd}_{1-x} \mathrm{Zn}_{x} \mathrm{Te}_{1-y} \mathrm{Se}_{y}$ (CZTS) alloys as a function of composition to improve the development of alloys with favorable electronic properties. Our calculations characterize the thermodynamic stability and band gaps of solid solution alloys over the entire quaternary space and identify compositions that exhibit favorable properties for alternatives to pure CZT or CTS.

Prepared by LLNL under Contract DE-AC52-07NA27344 and funded by the U.S. DOE/NNSA Office of Defense Nuclear Nonproliferation R\&D.

R04-7: Analysis of Strategies to Increase Growth Rates of CZT via the Traveling Heater Method J. H. Peterson, J. J. Derby

Chemical Engineering and Materials Science, University of Minnesota, Minneapolis, MN, USA

The traveling heater method (THM) has enjoyed particular success for the growth of large, high-quality bulk crystals of cadmium telluride $(\mathrm{CdTe})$ and cadmium zinc telluride (CZT) for radiation detector applications. However, the THM suffers from growth rates that are an order of magnitude slower than competing methods, such as Bridgman growth. Increasing THM growth rates would represent a major advance toward decreasing the very high cost of this material for use in large-volume gamma-ray spectrometers with high energy resolution.

In this presentation, we apply a rigorous, comprehensive computational model that couples phase equilibria with heat and species transfer, fluid mechanics, and interfacial dissolution and growth phenomena to faithfully describe the THM crystal growth process. We explain mechanisms that drive supercooling instabilities that limit THM growth rates. Specifically, we discuss the formation of melt flow structures (lee-wave vortices) near the growth interface that influence lateral transport in the melt and, in turn, promote concentration gradients in excess of the limit for morphological stability. Significantly, this mechanism results in growth rate limitations far below those predicted by the classic analysis of Mullins and Sekerka. In this presentation, we build on previous work to more carefully analyze the role of the lee-wave vortex in the onset of constitutional supercooling and investigate strategies for mitigation. Specifically, we consider the application of the accelerated crucible rotation technique (ACRT) to disrupt solute transport within the liquid zone that may alleviate constitutional supercooling and allow for faster growth rates.

This work has been supported in part by the National Science Foundation, under DMR-1007885, and no official endorsement should be inferred.

\section{R05: Materials1}

Tuesday, Nov. 1 08:00-10:00 Schuman

R05-1: Perovskite Semiconductor Materials for Hard Radiation Detection B. W. Wessels

Materials Science and Engineering, Northwestern University, Evanston, Illinois, USA

On behalf of the Kanatzidis-Freeman-Wessels collaboration

There has been widespread interest in perovskite semiconductors for optoelectronic device applications. We have been investigating cesium lead bromide and methylammonium lead bromide for potential use as room temperature hard radiation detectors. The synthesis, electronic properties and hard radiation detector response of these semiconductors will be presented.

R05-2: High Temperature Measurements of N-Type 4H-SiC Epitaxial Schottky Barrier Radiation Detectors K. C. Mandal, R. O. Pak, C. Oner, T. A. Chowdhury

Electrical Engineering Department, University of South Carolina, Columbia, SC, USA

High resolution Schottky barrier alpha detectors have been fabricated on $50 \mu \mathrm{m}$ n-type $4 \mathrm{H}$-SiC epitaxial layers. The junction properties of the detectors were examined by temperature dependent $(300-875 \mathrm{~K})$ current-voltage (I-V) and capacitance-voltage $(\mathrm{C}-\mathrm{V})$ measurements. A thermionic emission model applied to the forward I-V characteristic revealed an effective surface barrier height of $1.72 \mathrm{eV}$ and a diode ideality factor of 1.08 at $300 \mathrm{~K}$ suggesting uniform spatial distribution of surface barrier height. The high frequency $(1 \mathrm{MHz}) \mathrm{C}-\mathrm{V}$ measurements at $300 \mathrm{~K}$ revealed a doping concentration of $1.8 \times 10^{14} \mathrm{~cm}^{-3}$. Alpha spectroscopy measurements revealed an energy resolution of $0.38 \%$ for $5.48 \mathrm{MeV}$ alpha particles. Deep level transient spectroscopy (DLTS) studies revealed the presence of $\mathrm{Ti}, \mathrm{Z}_{1 / 2}$, and $\mathrm{Cil}$ defect centers. Cathodoluminescence (CL) studies revealed three photon peaks with energies centered on $3.16 \mathrm{eV}, 2.32 \mathrm{eV}$, and $1.66 \mathrm{eV}$ respectively. The presence of green luminescence (GL) at $2.32 \mathrm{eV}$ suggests the presence of boron impurities, and it is argued that the peak centered at $1.66 \mathrm{eV}$ is related to carbon vacancies. Correlation of EBIC contrast with extended defects in $4 \mathrm{H}-\mathrm{SiC}$ epitaxial layers have been studied using a new oxidizing chemical etching salt in molten $\mathrm{KOH}$. EBIC results under bias stress of the Schottky devices showed that the screw dislocations produce characteristic bright halos with the EBIC current higher than that of the background. The mechanism of EBIC contrast has been 
investigated in detail by changing electron beam energy and thus beams penetration depth. A significant improvement in detector resolution to $\sim 0.28 \%$ was observed after a new edge termination while evaluating effect of surface defects on detector performance at high temperatures $(300-875 \mathrm{~K})$.

R05-3: Advances in CdZnTe and CdMnTe Semiconductors for Detection of Radiological and Nuclear Threats $\underline{\text { S. U. Egarievwe }}{ }^{1}$, R. B. James ${ }^{2}$, U. N. Roy ${ }^{3}$, A. E. Bolotnikov ${ }^{3}$, E. D. Lukosi ${ }^{4}$, G. S. Camarda ${ }^{3}$, G. Yang ${ }^{3}$, A. Hossain ${ }^{3}$

${ }^{I}$ Nuclear Engineering and Radiological Science Center, Alabama A\&M University, Normal, AL, USA

${ }^{2}$ Savannah River National Laboratory, Aiken, SC, USA

${ }^{3}$ Department of Nonproliferation and National Security, Brookhaven National Laboratory, Upton, NY, USA

${ }^{4}$ Nuclear Engineering Department, University of Tennessee, Knoxville, TN, USA

Cadmium zinc telluride (CdZnTe) X-rays and gamma-rays detectors have found applications in radiological and nuclear security, environmental protection, astrophysics, and nuclear medical imaging. Cadmium manganese telluride (CdMnTe) has the potential for similar success as CdZnTe in the detection of ionizing radiations. A key advantage of CdZnTe and CdMnTe detectors is the ability to operate at room-temperature without cryogenic cooling. The detection of radiological and nuclear threats would greatly benefit from detector wafers with large volume, high performance (energy resolutions of 3\% to less than $1 \% \mathrm{FWHM}$ at $662 \mathrm{keV}$ ), and low-cost of production. This paper presents recent advances in the development of CdZnTe and CdMnTe nuclear detectors with focus on the detection of radiological and nuclear threats. Results from efforts on improving the performance of CdZnTe and CdMnTe detectors will be presented, covering three major areas: crystal growth, post-growth annealing, and device fabrication. A discussion on cost reduction with respect to crystal growth yield and energy resolution will also be presented.

This work has been supported by the U.S. Department of Homeland Security, Domestic Nuclear Detection Office, under Award 2012-DN-077ARI065-04; the U.S. Nuclear Regulatory Commission under Award NRC-27-10-514; and the U.S. Department of Energy Office of Defense Nuclear Nonproliferation R\&D, NA-22.

R05-4: Towards a High-Z Room Temperature GaSb/GaAs APD - Investigation of the Gamma-Ray Response of GaSb

B.-C. Juang $^{1}$, D. L. Prout ${ }^{2}$, B. Liang ${ }^{3}$, A. F. Chatziioannou ${ }^{2,4}$, D. L. Huffaker ${ }^{1,5}$

${ }^{I}$ Dept. of Electrical Engineering, University of California at Los Angeles, Los Angeles, CA, United States

${ }^{2}$ Crump Institute for Molecular Imaging, University of California at Los Angeles, Los Angeles, CA, United States

${ }^{3}$ California NanoSystems Institute, University of California at Los Angeles, Los Angeles, CA, United States

${ }^{4}$ Dept. of Molecular and Medical Pharmacology, University of California at Los Angeles, Los Angeles, CA, United States

${ }^{5}$ School of Physics and Astronomy, Cardiff University, Cardiff, Wales, United Kingdom

Room temperature semiconductor detectors with high energy resolution are attractive for identifying hazardous elements and nuclear threats for environmental protection and homeland security. The high atomic number and small bandgap of GaSb suggests a high stopping power and a low predicted PCE, favorable in achieving high energy resolution. However, the small bandgap of GaSb inevitably leads to high dark leakage noise. This can be addressed by using a separate absorption and multiplication avalanche photodiode (SAM-APD), with a GaSb-based absorber monolithically integrated to a GaAs-based multiplication region using interfacial misfit (IMF) arrays. The GaSb absorber can offer enhanced ?-ray stopping power, and the low-Z GaAs junction promises low dark current as well as avalanche multiplication for improved signal-to-noise ratio. In this work, we demonstrated the $\mathrm{x}$ - and ?-ray response of $\mathrm{GaSb}$, and investigated the absorption behavior of GaSb by energy spectra from Am-241 radioactive sources. The primary photopeaks up to $59.5 \mathrm{keV}$ were clearly identified, and the device showed good detection linearity. We also present analysis of the obtained photopeaks and measured energy resolutions.

The authors acknowledge the financial support by the Defense Threat Reduction Agency (DTRA) through award number HDTRA1-14-1-0035.

\section{R05-5: High Linearity Silicon Carbide Detectors for Medical Applications}

$\underline{\text { N. S. Mohamed }}^{1,2}$, N. Wright ${ }^{1}$, A. Horsfall ${ }^{1}$

${ }^{I}$ School of Electrical and Electronic Engineering, Newcastle University, Newcastle Upon Tyne, UK

${ }^{2}$ Faculty of Health Sciences, Universiti Sultan Zainal Abidin, Kuala Terengganu, Malaysia

Silicon carbide is well known as a radiation hard semiconductor, that has been demonstrated in a range of detector structures for deployment in application where the ability to tolerate high radiation dose is imperative. This includes applications in space and nuclear environments, where the ability to detect highly energetic radiation is important. In contrast, medical treatment uses a range of radiation dose rates and energies and here we investigate the response and linearity of a highly radiation tolerant detector fabricated using silicon carbide to dose rates in the range of $30 \mu \mathrm{Gy} \cdot \mathrm{min}-1$. This dose rate is typical of those used for medical imaging purposes, rather than radiotherapy treatment. The data show that the generated current originates within the depletion region of the detector and that the response is linearly dependent on the volume of the space charge region. The realization of a 
vertical detector structure, coupled with the high quality of epitaxial layers, has resulted in a sensitivity of the detector that is significantly higher than those published previously for moderate dose rate testing.

Keywords—-silicon carbide, linearity, low dose rate

R05-6: Evaluation of Suitability of GaAs:Cr Sensors for X-Ray Transmission Technology of Diamond-Bearing Ore Enrichment

A. V. Tyazhev ${ }^{1}$, A. N. Zarubin ${ }^{1}$, O. P. Tolbanov ${ }^{1}$, M. S. Skakunov ${ }^{1}$, S. A. Ryabkov ${ }^{2}$, E. N. Vladimirov ${ }^{3}$, T. E. Romanovskaya ${ }^{3}$, S. R. Belotserkovskii ${ }^{4}$

${ }^{\text {I}}$ Functional Electronics Laboratory of Tomsk State University, Tomsk, Russia

${ }^{2}$ XDiCon LLC, Tomsk, Russia

${ }^{3}$ Bourevestnik, Inc., Saint Petersburg, Russia

${ }^{4}$ ALROSA Co., Ltd, Mirny, Russia

The paper demonstrates the experimental results of testing of multichannel GaAs: Cr detector prototypes for X-ray transmission separators. Two GaAs: Cr detector prototypes were tested: line and pixel prototypes operating in integrating and counting mode, respectively. The line detector prototype consists of 256 channel GaAs: Cr sensors with 200 um pitch with 500 and 700 microns thickness of sensitive layers. The pixel detector prototype has active area of $14 \mathrm{~mm}$ by $14 \mathrm{~mm}$ and bases on 500 um thick GaAs: $\mathrm{Cr}$ pixel sensor with $55 \mathrm{um}$ pitch. The results of investigations of X-ray image quality dependence on X-ray intensity, sensor temperature and bias voltage value are presented. It was demonstrated that application of $\mathrm{GaAs}$ : $\mathrm{Cr}$ sensors for X-ray transmission separation allows detection of class $+3-6 \mathrm{~mm}$ (size from 2 to $8 \mathrm{~mm}$ ) diamonds at conveyor belt speed of $1 \mathrm{~m} / \mathrm{c}$.

The investigation was financially supported with grant of Russian Federation \# 14.587.21.0003 (RFMEFI58714X0003)

R05-7: Laser-Induced Formation of Surface State and Highly Doped Layer in CdTe for Diode Type Detectors V. A. Gnatyuk ${ }^{1,2}$, S. N. Levytskyi ${ }^{1}$, O. I. Vlasenko ${ }^{1}$, T. Aoki ${ }^{2}$

${ }^{1}$ Institute of Semiconductor Physics of the National Academy of Sciences of Ukraine, Kyiv, Ukraine

${ }^{2}$ Research Institute of Electronics, Shizuoka University, Hamamatsu, Japan

To decrease charge losses caused by incomplete collection of nonequilibrium carriers and achieve quite low leakage current in $\mathrm{X} /$ ?-ray detectors, we have developed CdTe-based diode structures with a $p$ - $n$ junction. Using laser processing of CdTe crystals, it was possible to select the ranges of energy density of nanosecond laser pulses and conditions of irradiation to optimize the surface state, decrease the surface recombination velocity, increase the photosensitivity, modify the photoconductivity spectrum profile and provide heavy doping of the thin surface region of $p$-like CdTe crystals with In atoms. Laser-assisted techniques were applied for semi-insulating CdTe crystals to fabricate diode structures with high rectification properties. The electrical and photoelectric characteristics of the CdTe crystals before and after laser irradiation and formed In/CdTe/Au diode detectors were studied. Irradiation with nanosecond ruby laser pulses resulted in an increase in the photosensitivity and decrease in the slope of the photoconductivity spectrum on the high-energy side and rise of the high-energy wing of the spectrum. This was attributed to a decrease in surface recombination velocity as result of improvement of the morphology and structure of a thin surface layer because of laser-induced annealing of residual structural imperfections and surface cleaning. To provide high doping of a thin CdTe surface layer with In and form a built-in $p$ - $n$ junction, a KrF excimer laser was used. The shift of the positions of the high energy edge of the photoresponse spectrum of the $\mathrm{In} / \mathrm{CdTe} / \mathrm{Au}$ diodes was close to the transition energy level of a donor $\mathrm{In}_{\mathrm{Cd}}$. The narrowing of the semiconductor band gap was evidence of high doping of the CdTe surface region with In under KrF laser irradiation of CdTe crystals pre-coated with an In film. The In/CdTe/Au diode detectors have shown suitable electrical and spectral characteristics $(\mathrm{FWHM}<1 \%$ at $662 \mathrm{KeV})$.

This research was supported by the NATO Science for Peace and Security Programme (Project SENERA, SfP-984705).

R06: RTSD Award

Tuesday, Nov. 1 10:30-11:10 Schuman

R06-1: RTSD Scientist Award Presentation

$\underline{\text { R. B. James }}{ }^{1}$, M. Fiederle ${ }^{2}$

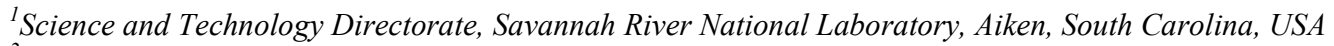

${ }^{2}$ University of Freiburg, Freiburg, Germany

The RTSD Scientist Award for the 23rd International Conference on Room-Temperature Semiconductor Radiation Detectors will be presented. 
R06-2: Photon Counting Detectors for X-Ray Spectral Imaging

J. S. Iwanczyk

DxRay, Inc., Northridge, CA, USA

The paper will give a historical perspective on development of semiconductor detectors for x-ray applications with a particular emphasis on a development of cadmium telluride (CdTe) and cadmium zinc telluride (CZT) detector arrays toward photon counting spectral imaging uses. The use of photon counting detectors in x-ray imaging is very new because of technical challenges related to recording very high flux of x-rays, relatively small signals generated by the individual photons and requirements for low electronic noise and good energy resolution. The development of an innovative photon counting detector technology have been possible due to recent progress in material/detector technologies combined with the availability of application specific integrated circuits (ASIC) readout electronics. The $3 \mathrm{~mm}$ thick CdTe and CZT detector arrays with $16 \times 16$ pixels and a $0.5 \mathrm{~mm}$ pixel pitch interconnected to our ASIC electronics were used for construction of computed tomography modules. The above described detector arrays were tested with radiation from an x-ray generator and their characteristic were measured in terms of the achievable energy resolution, throughput and linearity of the energy response. The experimental results will be presented in the paper.

\section{R07: Imaging1}

Tuesday, Nov. $1 \quad$ 16:30-18:20 Schuman

R07-1: Gamma-Ray Imaging and Spectroscopy for Nuclear-Power Applications

C. G. Wahl, W. R. Kaye, F. Zhang, W. Wang, Y. A. Boucher, J. M. Jaworski, K. Moran, D. Tefft, H. Yang, B. Kitchen, M. Ulrich, A. King, T. Slatina, T. Matthews, Z. He

H3D, Inc., Ann Arbor, MI, USA

In the three years since the introduction of H3D's Polaris-H CZT imaging spectrometer, it has been adopted by more than a third of US nuclear plants. With its more widespread use, the applications for which gamma-ray imaging and spectroscopy within nuclear power plants are most advantageous have become clear. The most valuable and prevalent uses of this instrument for radiation protection and plant operations will be discussed, along with operational lessons. Additionally, users have identified some limitations of Polaris-H in measurements they wish to perform. Work to alleviate these limitations will be discussed, including a new design to image a source in the presence of a much stronger source at the same energy.

\section{R07-2: COMPTON AND CODED APERTURE RADIATION IMAGE RECONSTRUCTION USING STOCHASTIC ORIGIN ENSEMBLES IN 3-D POSITION SENSITIVE CdZnTe DETECTORS D. I. Goodman, Z. He}

Nuclear Engineering and Radiological Sciences, University of Michigan, Ann Arbor, MI

Stochastic Origins Ensembles (SOE) is Bayesian image reconstruction technique that can be applied to both Compton and coded aperture gamma-ray reconstructions in $3 \mathrm{D}$, position sensitive CdZnTe. Careful consideration of detector physics such as attenuation or event sequencing in origin sampling used to generate the Monte Carlo Markov Chain improves reconstruction accuracy. Differing levels of system matrix realism were considered while reconstructing Cs-137 and Co-57 point sources via Compton and coded aperture imaging using the Polaris II system. Efficient origin sampling schemes implemented on a graphical processing unit (GPU) alongside resolution gains from differing levels of system matrix detail, with the associated computational trade-offs, are described.

R07-3: Hybrid Imaging Reconstruction Method at Intermediate Energy Range

$\underline{\mathrm{Y}} \mathrm{Liu}^{1,2}, \mathrm{Y} . \mathrm{Li}^{1,2}, \mathrm{~J} . \mathrm{Fu}^{1,2}, \mathrm{Y} . \mathrm{Li}^{1,2}, \mathrm{Y} . \mathrm{Xing}{ }^{1,2}$

${ }^{I}$ Department of Engineering Physics, Tsinghua University, Beijing, China

${ }^{2}$ Key Laboratory of Particle \& Radiation Imaging, Ministry of Education, Beijing, China

3D Pixel CZT detector using Compton imaging is an ideal candidate at energy above $250 \mathrm{keV}$, mechanical collimation technique must be used at lower energy range where the Compton scattering probability is low. We propose a hybrid imaging method to reconstruct gamma-ray source distribution at intermediate energy range. In this new method, both single and two interaction events are used, and a coded mask is brought into the system to concentrate the possible source direction of the single interaction event. The feasibility of this method is verified by the data generated from the Monte Carlo simulation program of a CZT detector system, and better imaging resolution and efficiency are reached than single method.

\section{R07-4: Performance Improvement of an X-Ray Diffraction Imaging System Using Sub-Pixel Positioning} Within CZT-Detectors 


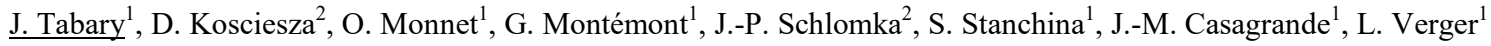

${ }^{I}$ CEA LETI MINATEC Campus, F-38054 Grenoble, France

${ }^{2}$ Morpho Detection Germany GmbH, 22453 Hamburg, Germany

Energy dispersive X-ray diffraction (EDXRD) is known to be a very effective method for explosive and narcotic threat detection in baggage screening. Morpho Detection has built an innovative X-ray diffraction imaging (XDi) system which enables EDXRD screening of cabin baggage under reasonable throughput. This system is equipped with 17 pixelated Cadmium-Zinc-Telluride (CZT) detector modules, called IMADIF modules, which has been specifically developed by CEA-Leti to meet the performances required by the EDXRD technique in terms of energy resolution, detection efficiency, noise and stability. Recently, a new version of the module firmware has been developed in order to enable subpixelation inside the IMADIF modules, particularly along the direction corresponding to the scattering angle. Each line can be now virtually sampled in 8 sublines. Subpixelation along the scatter angle is interesting for different reasons: it can improve the trade-off between angular resolution and sensitivity and also improve the spatial resolution along the depth direction of the imaged baggage. A simulation study has been carried out to optimize the secondary collimation of the XDi system in order to take maximum advantage of the subpixelation. Several more opened collimations are proposed, which enable a gain of sensitivity between $30 \%$ and $50 \%$ without angular resolution degradation, and induce an oversampling of the imaged volume. Preliminary experimental tests performed on simple objects tend to confirm the promising results of the simulation.

R07-5: CdZnTe Detector Prototype for Boron Imaging by SPECT During BNCT Treatment: Simulations and Measurements in a Neutron Field

M. Bettelli ${ }^{1}$, S. Fatemi ${ }^{2,3}$, S. Altieri ${ }^{2,3}$, S. Bertolussi ${ }^{2,3}$, I. Postuma ${ }^{2,3}$, N. Protti ${ }^{2,3}$, A. De Bari ${ }^{2,3}$, G. Benassi ${ }^{4}$, N. Zambelli ${ }^{4}$, A. Zappettini ${ }^{1}$

${ }^{I}$ IMEM-CNR, Parma, Italy

${ }^{2}$ Physics, University of Pavia, Pavia, Italy

${ }^{3}$ Pavia, INFN, Pavia, Italy

${ }^{4}$ due2lab s.r.l., Parma, Italy

Boron Neutron Capture Therapy (BNCT) tumor treatment effectiveness strongly depends on the radiation dose deposited locally by the $10 \mathrm{~B}(\mathrm{n}, \mathrm{a}) 7 \mathrm{Li}$ reaction in the tumor; however, the local and real time measurement of this quantity during the neutron irradiation is a big challenge, not yet solved by the BNCT researchers' community. The deposited dose evaluation needs a correct knowledge of the $10 \mathrm{~B}$ concentration spatial distribution and the thermal neutron flux in the tissue at the irradiation time. In order to solve this problem it is possible to exploit the $470 \mathrm{keV}$ dis-excitation photons of the 7Li and as such avoid the difficulty of measuring the Boron concentration and the neutron flux as separated quantities. Room-temperature semiconductor detectors, such as CZT, have favourable physical characteristics for medical application, moreover the material is present on the market at affordable prices and generally shows very good energy resolution. Therefore our project aims to develop a Single Emission Computed Tomography (SPECT) system based on a CdZnTe (CZT) semiconductor detector to be installed at a new BNCT clinical facility. In this work, we show the exploitation of a $5 \times 5 \times 20 \mathrm{~mm} 3 \mathrm{CZT}$ stripe detector. Characterization measurements carried out on our prototype estimated an energy resolution of $2.6 \%$ at $661 \mathrm{keV}$ energy. Then, we measured the detector's performances in presence of a neutron field, such measurements were conducted in the thermal column of the Triga Mark II reactor. The $470 \mathrm{keV}$ line due to the $10 \mathrm{~B}(\mathrm{n}, \mathrm{a}) 7 \mathrm{Li}$ reaction was clearly evidenced from the background. In both cases the experimental set up was also simulated by the means of the MCNP Monte Carlo code. Moreover to study the pre-clinical application of the CZT SPECT system we simulated, using GEANT4, the setup needed for measurements on small animals using a PMMA phantom and setting the detector at different distances from the PMMA phantom axis. Preliminary results and the possibilities for further studies are shown.

R07-6: Digital CZT Detector System for High Flux Energy-Resolved X-Ray Imaging L. Abbene $^{1}$, F. Principato ${ }^{1}$, G. Gerardi ${ }^{1}$, G. Benassi ${ }^{2}$, N. Zambelli ${ }^{2}$, A. Zappettini ${ }^{3}$, M. Bettelli ${ }^{3}$, P. Seller ${ }^{4}$, M. C. Veale ${ }^{4}$

${ }^{I}$ Dipartimento di Fisica e Chimica, University of Palermo, Palermo, Italy

${ }^{2}$ due2lab s.r.l., Reggio Emilia, Italy

${ }^{3}$ IMEM/CNR Parma, Parma, Italy

${ }^{4}$ Rutherford Appleton Laboratory, Oxfordshire, U.K.

Photon counting detectors with energy resolving capabilities are desired for high flux X-ray imaging. In this work, we present the performance of CZT pixel prototypes, with different thicknesses and pixel pitches (500 $\mu \mathrm{m}$ and $250 \mu \mathrm{m})$, coupled to a fast and low noise ASIC, characterized by only the preamplifier stage. A 16-channel digital readout electronics was used to continuously digitize and process the signals from each pixel (ASIC), performing multi-parameter analysis (event arrival time, pulse shape, pulse height, pulse time width, etc.) even at high fluxes and at different throughput and energy resolution conditions. X-ray measurements, at both low and high fluxes, will be presented, taking into account the mitigation of incomplete charge collection, pile-up and charge sharing effects through time coincidence and pulse shape analysis. 
R07-7: Experimental Material Discrimination in Spectral Tomography

C. Lacroix, V. Rebuffel, C. Paulus, $\underline{\text { V. Moulin, L. Verger }}$

DTBS/STD, CEA, LETI, MINATEC Campus, 38054 Grenoble, France

The new technology of spectral detectors opens new perspectives in a lot of fields, especially for security application with luggage inspection. The photon counting detector (up to 128 energy bins of $1 \mathrm{keV}$ width) ME100 provided by Multix already improves material discrimination in LineScan inspection. This paper shows the discrimination performance with a "one shot" tomography. To discriminate critical materials the density is not enough, indeed explosives are still active in the form of flakes (wide range of density), we will used the spectral information to estimate the effective atomic number of critical materials. Some luggage with materials are acquired with two ME100. A material basis will be used to compute a bi-material projection-based decomposition with a classic polynomial method. Once the two sinograms in basis materials (polypropylene and polyvinyl chloride) obtained, we reconstruct them with a filtered back-projection algorithm. From this slices, density and effective atomic number of each critical material are estimated. A comparison with a classical bi-energy discrimination highlights the necessity of a density/effective atomic discrimination. For example, our discrimination allows to differentiate a baby food jar (authorized in cabin luggage) from nitromethane (explosive) by the effective atomic number while they have the same density.

\title{
R08: Neutron Detectors
}

\section{Wednesday, Nov. $2 \quad$ 08:00-10:05 Schuman}

R08-1: Development of the Semiconductor 6LiInSe2 as a Neutron Detector

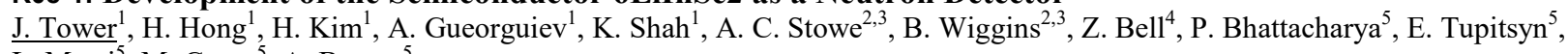
L. Matei ${ }^{5}$, M. Groza ${ }^{5}$, A. Burger ${ }^{5}$

${ }^{1}$ Radiation Monitoring Devices, Inc., Watertown, MA, United States

${ }^{2}$ Vanderbilt University, Nashville, TN, United States

${ }^{3}$ Y-12 National Security Complex, Oak Ridge, TN, United States

${ }^{4}$ Oak Ridge National Laboratory, Oak Ridge, TN, United States

${ }^{5}$ Fisk University, Nashville, TN, United States

The wide-bandgap semiconductor LiInSe2 has been developed for use as a room temperature neutron detector. Its properties include very high sensitivity to thermal neutrons when enriched with the $6 \mathrm{Li}$ isotope. Considerable progress has been achieved in the growth of high quality crystals and subsequent fabrication of semiconductor devices that can be used for neutron detection. Control of crystal defects (precipitates and native point defects) through the growth process is crucial for detector performance. We will review the development path of LiInSe2 and report on significant new progress in the following areas:

- Single-crystal growth with well-controlled defects and scale-up to 1-inch diameter. - Increased detector size (up to 1 square centimeter) and tiling multiple detectors. $\bullet$ Device fabrication and long-term stability studies. $\bullet$ Large-batch purification of enriched 6Li metal to 5-nines purity. Development of a compact neutron pager instrument.

This work has been supported by the US Department of Homeland Security, Domestic Nuclear Detection Office, under competitively awarded contracts 2013-DN-077-ER00002 and HSHQDC-16-C-00041. This support does not constitute an express or implied endorsement on the part of the Government.

\section{R08-2: Low Energy Threshold Measurements Using Digital 3-D CdZnTe Spectrometers for Fast Neutron} Detection

M. W. Streicher, D. Goodman, Y. Zhu, Z. He

Nuclear Engineering \& Radiological Sciences, University of Michigan, Ann Arbor, Michigan, USA

Fast neutrons are an important signature of special nuclear materials. Some fissile materials spontaneously emit neutrons and all will upon active interrogation. Furthermore, fast neutrons have a low natural background rate and readily penetrate shielding materials, especially materials with high atomic numbers which easily shield gamma-ray signatures. A backscattered $1 \mathrm{MeV}$ neutron in CdZnTe will deposit only $30 \mathrm{keV}$ in the detector; however, due to recombination and quenching, only about $10 \mathrm{keVee}$ (electron equivalent energy) is induced from a backscattering interaction. In order to record this event, the low energy threshold of the detectors must be reduced. The threshold has been reduced to less than $5 \mathrm{keVee}$. The $5.9 \mathrm{keV}$ x-ray from Fe-55 has been resolved from electronic noise at these settings. Preliminary results indicate that neutron scattering interactions are observable in digital CdZnTe spectrometers. A DD neutron generator was used to estimate the quenching factor in CdZnTe. The quenching factor was found to be close to 0.45 , higher than previous theoretical estimates of 0.3 . Estimates of position resolution for low energy events are presented as well as a statistical method to alert a user of the presence of fast neutrons.

\author{
R08-3: Ultra-High Resolution Semiconductor Detectors for Neutron Imaging Based on the Timepix \\ Technology
}


Typical state of the art detectors for neutron imaging with timing resolution are presently limited in spatial resolution to approximately $1 \mathrm{~mm}$. So far no time resolved detectors are commonly used with a position resolution between 100 micron and 1 $\mathrm{mm}$. At ESS, the Neutron Macromolecular Spectrometer (NMX), an instrument for magnetic single crystal diffraction and macromolecular crystallography, requires a position resolution of at least $200 \mathrm{um}$. The Timepix series of readout chips have a pixel size of $55 \times 55$ um2. By using the Timepix 3 chip, time of arrival and deposited energy can be measured simultaneously. In this paper we present initial measurements using Gadolinium as a converter on Timepix and Timepix 3 readout chips.

\section{R08-4: Multi Modal CZT Detector - Neutron/Gamma}

M. J. Anderson

Engineering, University of Lancaster, Lancaster, UK

This paper will describe the current state of development of a semiconductor based hybrid pixelated multi modal spectroscopic radiation detector specifically to simultaneously detect gamma and neutron emissions using CZT and Gadolinium. The method being developed is anticipated to assist in debris identification in mixed field scenarios

\section{R08-5: Solid-State Neutron Detectors Based on Hexagonal Boron Nitride Epilayers} A. Maity, T. C. Doan, J. Li, J. Lin, H. Jiang

Dept. of Electrical and Computer Engineering, Texas Tech University, Lubbock, TX, USA

Solid-state neutron detectors with improved detection efficiencies are highly sought after for the detection of fissile materials. Wafer scale (up to 4-inches in diameter) natural and isotopically B-10 enriched h-BN epilayers with large thicknesses have been successfully synthesized. The measured pulse-height spectra revealed distinguishable peaks corresponding to the product energies of B-10 and thermal neutron reaction, implying that h-BN detectors are very sensitive in resolving energies of specific reaction products. Furthermore, it was shown that detectors incorporating B-10 enriched h-BN epilayers of $36 \mu \mathrm{m}$ in thickness exhibit an overall detection efficiency of $45 \%$ for thermal neutrons, a charge collection efficiency of $90 \%$, and gamma rejection of better than 10-6. These results represent a significant step toward the realization of practical BN semiconductor based neutron detectors for passive and active interrogation detection applications. Our results indicate that h-BN are highly promising for realizing sensitive solid-state neutron detectors with expected advantages resulting from semiconductor technologies, including no pressurization, fast response time, ruggedness, compact size, light weight, ability of wafer-scale processing allowing scale to large detectors at low cost, and easy to integrate with other electronics, and low maintenance cost.

R08-6: Dual Detection Charge Collection and Light Emission in LiInSe2 and ZnSe

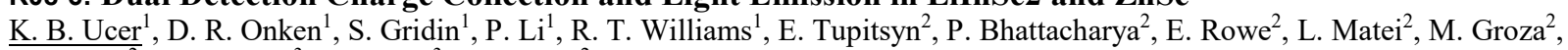
V. Buliga ${ }^{2}$, B. Wiggins ${ }^{3}$, A. Stowe ${ }^{3}$, A. Burger ${ }^{2}$

${ }^{I}$ Department of Physics, Wake Forest University, Winston-Salem, NC, USA

${ }^{2}$ Department of Life and Physical Sciences, Fisk University, Nashville, TN, USA

${ }^{3}$ Y-12 National Security Complex, Oak Ridge, TN, USA

It has previously been shown by members of this collaboration that LiInSe2 is promising as a semiconductor detector of neutrons, and that it produces scintillation response under alpha, neutron, and gamma excitation. In the present work, simultaneous detection in semiconductor and scintillator modes in the same material is studied under alpha particle and femtosecond laser excitation. It is found that the electric field normally applied to collect charge has only a small effect on scintillation light yield in LiInSe2 so that dual detection without significant interference is feasible. From a more fundamental perspective, the effect of applied electric field on scintillation can be used to probe processes in the track and potentially extract more information from, e.g., charge/light ratio than is contained in simple pulse height. Confocal two-photon excitation by a femtosecond laser is used in this work to create overlapping electron $\&$ hole distributions of variable diameter in the few hundred nanometers range, approaching comparablility to electron tracks after hot electron diffusion. The electron and hole ellipsoidal distributions are pulled apart in the applied electric field until re-trapping (time t) occurs. The light loss is analyzed to yield $\mu$ t without measuring current directly. Modeling of the laser-induced carrier distribution and electron tracks under applied electric field allows extracting more parameters from these experiments and could lead to a tool for characterizing track structure.

Acknowledgment: WFU and Fisk acknowledge support from the US Department of Homeland Security, Domestic Nuclear Detection Office (DNDO), Academic Research Initiatives 2014-DN-077-ARI077-03 and 2014-DN-077-ARI076-03. This support does not constitute an express or implied endorsement on the part of the Government. We thank Alan Janos for helpful discussions. 
Nuclear Engineering and Radiological Sciences, University of Michigan, Ann Arbor, United States

In previous works, maximum-likelihood expectation-maximization (MLEM) in the spatial-energy domain was implemented, which considers both fully-deposited Compton events and partially-deposited Compton events, and reconstructs energy spectrum as a function of incident angle or image slides in different energy bins. However, due to the slow convergence rate of EM algorithm, the application of MLEM in the spatial-energy domain is quite limited. To accelerate convergence, we propose an energy-block subsets algorithm, which uses the properties of system responses in the spatial-energy domain, and decrementally updates images in energy-block subsets. This process can also be further accelerated by ordered subsets and/or parallel computing techniques. With the energy-block subsets algorithm, the convergence rate of reconstruction is significantly improved. This algorithm is applicable to any Compton imagers with the capability of recording 3D location and energy deposition for each interaction.

\title{
R09-2: Noise Reduction for Pixelated Room-Temperature Detectors Using VAD_UM V2.2 Digitizer ASIC Y. Zhu, Z. He \\ Department of Nuclear Engineering and Radiological Sciences, University of Michigan, Ann Arbor, MI, USA
}

Pixelated CdZnTe gamma detectors are well-known candidate of room-temperature spectrometer. It is promising to provide near HPGe performance in the near future. VAD UM ASICs are 130-channel digitizer chip, designed to pursue the optimal performance for pixellated semiconductor detectors. VAD_UM v2.2 is the latest version of the family. It contains four dynamic ranges: $700 \mathrm{keV}, 3 \mathrm{MeV}, 7 \mathrm{MeV}$ and $9 \mathrm{MeV}$. This article presents the performance of this ASIC. The electronic noise of the system is analyzed and studied. Several approaches to reduce the electronic noise are undertaken. The lowest electronic noise of below $1.5 \mathrm{keV}$ FWHM with 700-keV dynamic range has been achieved.

\author{
R09-3: Energy Resolution Improvement Through Digital Pulse Shape Analysis at CdZnTe-Cross-Strip- \\ Detectors \\ D. Weinberger $^{1}$, T. Kormoll ${ }^{2}$, F. Fiedler ${ }^{1}$ \\ ${ }^{I}$ Institute of Radiation Physics, HZDR - Helmholtz-Zentrum Dresden-Rossendorf e.V., Dresden, Saxony, Germany \\ ${ }^{2}$ OncoRay-National Center for Radiation Research in Oncology, Dresden, Saxony, Germany
}

$\mathrm{CdZnTe}$ is a semiconductor material with very good properties for gamma-ray detection. A relatively large band gap of $1.57 \mathrm{eV}$ allows the use at room temperature without any additional cooling. Since holes are nearly immobile compared to electrons in $\mathrm{CdZnTe}$, spectroscopic readout requires special techniques which reduce the depth dependence of the signal. One possibility is to apply an anode pattern which exploits the small pixel effect which induces a signal as the charge cloud is very close to the anode. However, a small current is already induced as the charge cloud travels the bulk. The additional drift current will be accumulated at the charge sensitive preamplifier and influences the base line determination algorithm and results in an additional energy input. We present a simple approach to eliminate the influence of this drift current with the use of pulse shape discrimination implemented in an FPGA of a sampling ADC board. With this algorithm and the known transfer function of the preamplifier a proper depth correction is possible to improve the spectral resolution.

\section{R09-4: Weighting Potential Cross-Talk Correction for Charge Sharing Events in Pixelated CZT Detectors} J. Xia, Z. He

Nuclear Engineering and Radiological Sciences, University of Michigan, Ann Arbor, United States

Multiple pixel events in 3D position sensitive CdZnTe gamma-ray detectors may result from true multisite interactions (i.e. Compton scattering events followed by a photoelectric absorption) or from a single photoelectric absorption where the resulting electron cloud is shared between two pixel anodes. A method to partially distinguish these interaction mechanisms based on the source geometry, the interaction location, and the energy deposited was developed using the H3Dv4 ASIC. Energy of the likely 
charge sharing events was overestimated due to transient signal induction. A correction method was developed to account for the excess signal induced due to weighting potential cross-talk. The energy resolution of all two-pixel events improved by $0.05 \%$ FHWM at $662 \mathrm{keV}$ using this method.

The authors wish to thank Michael Streicher, Dr. Yuefeng Zhu and other co-workers for providing valuable comments and suggestions.

R09-5: Recent Results from Pixelated TIBr Detectors with Tl Electrodes Operated at Room-Temperature $\underline{\text { C. Leak }}^{1}$, W. Koehler ${ }^{1}$, S. O'Neal ${ }^{1}$, Z. He ${ }^{1}, \mathrm{~K}_{\text {. Hitomi }}{ }^{2}$

${ }^{I}$ Nuclear Engineering and Radiological Sciences, University of Michigan, Ann Arbor, MI, USA

${ }^{2}$ Quantum Science and Energy Engineering, Tohoku University, Sendai, Japan

Thallium-bromide (TlBr) is considered a viable alternative to CZT for room-temperature gamma ray spectroscopy because of its high effective atomic number and density. Sub-1\% FWHM energy resolution at $662 \mathrm{keV}$ has been achieved on $5 \mathrm{~mm}$ thick pixelated devices. However, these results are mostly limited to stable operation at $-20^{\circ} \mathrm{C}$. During room-temperature operation, the devices degrade with time, a phenomenon known as polarization. Work over the last five years has concluded that polarization is likely caused by bulk-to-contact interactions facilitated by ionic conduction. In 2008, Hitomi et al. found that the roomtemperature lifetime could be extended by applying Tl electrodes. In this work, we verify stable room-temperature performance of nominally $5 \mathrm{~mm}$ thick pixelated TlBr detectors with $\mathrm{Tl}$ electrodes. Energy resolution, mobility-lifetime product, resistivity, and hole performance are characterized and presented. Resolutions of 1.6\% and 1.2\% FWHM are achieved for all depths and cathode-side events only, respectively. A very high electron mobility-lifetime product is calculated: $3.8 \pm 0.3 \times 10^{-2} \mathrm{~cm}^{2} / \mathrm{V}$. Additionally, the effect of different 'charging' (applying reverse bias after forward bias) times is explored.

\section{R09-6: Electric Field Profile in CdZnTe Coplanar Grid Detectors Examined by Laser Induced Transient Current Waveforms: Compared to Theoretical Calculation}

P. Praus, E. Belas, R. Grill, J. Kunc, J. Pekarek

Institute of Physics, Charles University in Prague, Prague, Czech republic

Laser induced ( $\left.\ddot{L}_{i} 1 / 2 \mathrm{TCT}\right)$ electron current waveform (CWF) measurement on collecting and nonï ${ }^{1} 1 / 2$ collecting anode grid of the coplanar structure $\mathrm{CdZnTe}$ detector $(\mathrm{CPG})$ under various cathode bias and intergrid voltage are presented. Our $\mathrm{Li}_{i}^{1} / 2 \mathrm{TCT}$ setup is based on the direct high frequency amplification of CWF flowing on the respective AC coupled detector anode. CWF shape is recorded by using an ultrafast digital sampling oscilloscope. Supplementary voltage between the collecting and nonï $i^{1 / 2}$ collecting anode grid is introduced by an additional adjustable power supply. Both collecting and nonï $i^{1} / 2$ collecting grid CWF is recorded in the setup independently. We use optical pulses ( $i_{i} 1 / 25 \mathrm{~ns}$ as FWHM, $660 \mathrm{~nm}$ wavelength) of the laser diode that is powered by an ultrafast pulse generator with adjustable pulse width and repetition rate. Collimation and imaging optics and a translation stage is used to adjust the laser beam onto the examined spot of $\sim 3 \mathrm{~mm} 2$ on the cathode. Neutral density disc filter is placed in the beam line for variable intensity attenuation of the laser pulse. Deconvolution procedure that removes influencing by the electronic setup transfer function is routinely applied to obtain original measured CWF shapes. Electron CWFs are recorded on the collecting grid and the nonï $i^{1 / 2}$ collecting grid, respectively; in a bias range from $1000 \mathrm{~V}$ to $1700 \mathrm{~V}$. $\mathrm{Li}_{i}{ }^{1 / 2 \mathrm{TCT}}$ enables us to characterize the time resolved charge collection process and to evaluate the electric field profile inside the detector under various intergrid voltage. Theoretical simulations of the detector internal electric field profile with using of Shockleyï $i 12$ Ramo theorem in a

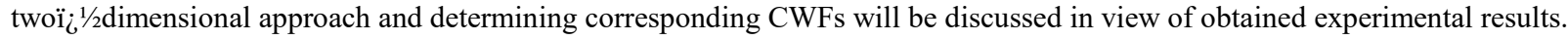
Influencing of the electric field profile by a cathode equipotential shielding and the laser spot position dependent CWF mapping will also be shown in the paper.

\section{R09-7: Characterization of High Mobility Transistor Channels for Terahertz Detectors Applications}

\section{F. Z. Mahi}

science and technology/University of bechar, university of Algeria, bechar, Algeria

In the last years, High Mobility Transistors (HEMT) have become interesting devices for terahertz (THz) applications (detectors or emitters) working at room temperature [1]. In particular, modern HEMTs have emerged as important competitors due to the existence of $\mathrm{THz}$ plasma wave oscillations in the channel $[2,3]$. One of the most important microwave characterizations of HEMT transistors is based on the determination of the small-signal equivalent circuit elements such as the admittance parameters of their three-terminals. We analyze the amplitude and phase of the admittance in high mobility channel transistors by using an analytical model. The analytical approach takes into account the longitudinal and transverse electric fields through a pseudo twodimensional approximation of the Poisson equation. The frequencydependent small-signal admittance response is determined by the total currents and the potentials matrix relation between the gate and the drain terminals. The analytical results show that the admittance spectrum exhibits a series of resonant peaks corresponding to the excitation of plasma waves. The appearance of the resonance is discussed and analyzed as functions of the channel length and the relaxation rate. The model can be used, on one 
hand, to control the appearance of plasma resonances, and on the other hand, can give a significant information about the admittance phase frequency dependence. and the temperature. Key words

\author{
R09-8: Imaging from Polycrystalline HgI2 Film Flat Panel Detector \\ L. Zhang, X. Ma, B. Li, X. Cao, W. Wang \\ $R \& D$ Institute, Nuctech Company Limited, Beijing, China
}

Direct detection method of flat panel detectors provides higher efficiency and better imaging. Compared to a-Se and CZT flat panel detectors polycrystalline $\mathrm{HgI} 2$ film possess higher atomic number and better temperature stability. We deposited HgI2 film on TFT substrate by PVD method and set up a two dimension imaging system. The imaging from the system is shown below. The film thickness is about $124 \mu \mathrm{m}$. The gold was evaporated on the HgI2 film as the contact for bias. We tested the leakage current. Due to the thin film the bias should not be so high for low noise. The $\mathrm{X}$ ray tube output is $45 \mathrm{keV}$ and $3 \mathrm{~mA}$. The bias is $50 \mathrm{~V}$.Other primary results are provided here. Figure 1. Imaging with a $\mathrm{HgI} 2$ film flat panel detector

\title{
R09-9: Structural Peculiarities of CdTe Crystals Doped with Rare Earths
}

N. V. Sochinskii, S. Rubio, J. L. Plaza, E. Diéguez

Department of Materials Physics, UAM, Madrid, Spain

The effect of rare earth impurities (REI) on crystal structure and composition was studied in the CdTe crystals doped with ytterbium, erbium and dysprosium. The crystals were grown by the vertical Bridgman method from the $\mathrm{Cd} 1-\mathrm{xMxTe}(\mathrm{M}=\mathrm{Yb}, \mathrm{Er}$, Dy) pre-synthesized feeds with the charge composition in the range of $\mathrm{x}=0.001-0.05$. The CdTe:M crystals were studied comparatively by scanning electron microscopy, energy dispersive X-ray analyses, X-ray diffractometry and optical transmittance spectroscopy. It was demonstrated that the REI cause the self-structuration effect into CdTe bulk. The type of REI impurity is important for the type of self-structuration. In particular, the $\mathrm{Yb}$ and $\mathrm{Dy}$ impurities are distributed in the form of lineal textures while the Er impurity induces the formation of cellular structure. It was found that the precipitation of $\mathrm{M} 2 \mathrm{Te} 3$ second phase starts in CdTe:M crystals when the feed composition exceeds $x=0.01$. At higher compositions $x>0.03$, the crystals become a mixture of several phases such as CdTe host, M2Te3 textures and MTe-rich inclusions. Experimental findings testify that homogeneous CdTe:M crystals can be grown with the M concentration below the value of (1-5)?1019 cm-3 which is estimated to be a limit for the REI solubility in CdTe crystals grown by Bridgman method. These experimental findings correlate well with the early-reported data on electrical and cathode-luminescence characterization of REI-doped CdTe crystals.

\section{R09-10: Deep Levels in High Resistive CdTe and CdZnTe Explored by Photo-Hall Effect Spectroscopy with Double Wavelength Illumination \\ A. Musiienko, R. Grill, P. Moravec, J. Zázvorka, G. Korcsmáros, J. Franc, I. Vasylchenko \\ Institute of Physics, Charles University in Prague, Prague, Czech Republick}

Cadmium Telluride (CdTe) and Cadmium Zinc Telluride (Cd1-xZnxTe) are well known as important semiconductors with applications in X-and gamma-ray detection, solar cells and electro-optic modulators. Many of their physical properties are controlled by energy levels within the bandgap, deep levels (DLs), associated with native defects, impurities, and defect complexes. Such defects create recombination and trapping centers in the energy band gap capturing electrons and holes created by radiation and depreciating the performance of the detector. In this presentation we introduce a novel spectroscopy method, Photo-Hall Effect spectroscopy with double wavelength illumination (DWPHES). The method is derived from well-known Photo-Hall effect measurements, where additional laser illumination with different wavelength is used. DWPHES allows us direct observation of DLs which cannot be observed from PHES, furthermore, it was ascertained that additional laser light exposure improves the quality of the Hall voltage. A number of DLs was discovered by DWPHES and interpreted, either levels above or below Fermi energy. After analyzation of numerous Hall signal spectra and flux dependencies, the key principles of PHES and DWPHES measurements were formulated. The most of DLs observed by DWPHES were consistently verified by PL spectroscopy.

The study was supported by the Charles University in Prague, project GAUK No. 8515

R09-11: Influence of Guard Ring Electrode on Performance of (CdZn)Te Pixel Detector Characterized by Laser-Induced Transient Current Technique

I. Vasylchenko, R. Grill, E. Belas, P. Praus, A. Musiienko

Faculty of Mathematics and Physics, Charles University in Prague, Prague, Czech Republic

In our presentation we introduce investigation of guard ring influence on performance of commercially available Redlen $(\mathrm{CdZn}) \mathrm{Te} 8 \times 8$ pixel detector. By means of laser induced transient current technique it was shown that guard electrode acts by the 
same way as non-collecting grid in coplanar detectors when guarded pixel is illuminated by the laser diode. This phenomenon is also observed when we apply interelectrode voltage between the guard ring and the pixel. Moreover, in this particular case the interelectrode voltage polarity has a weak impact on electrodes behavior. Therefore, the guard ring not only reduces the dark current, but also improves the charge carriers transient time and the charge collection efficiency. Illumination of the other spots (guard ring, spots between guard and pixel, another pixel outside the ring) and simultaneous measurement of signals on both electrodes allowed us to investigate the charge sharing effects. By varying positive interelectrode voltage applied to the guard ring we also managed to control charge collection on pixel. Measured data showed that the pixel can be effectively shielded from charge carrier clouds generated under the pixels outside of the guard ring.

The study was supported by the Charles University in Prague, project GAUK No. 8515 and GAUK No. 2052214.

\author{
R09-12: A Study on a Photon-Counting Detector Applying Pixel Shift Technology and Charge Sharing \\ Correction

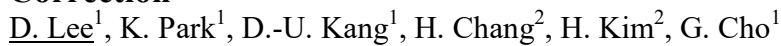 \\ ${ }^{I}$ nuclear and quantum engineering, KAIST, daejon, republic of Korea \\ ${ }^{2}$ Institute for information technology, KAIST, daejon, republic of Korea
}

Charge sharing limits detector contrast and spatial resolution in photon-counting sensors. Because the charge cloud generated by a single X-ray photon or gamma ray photon can induce multiple counts in not only a dedicated pixel but also adjacent pixels. The pixels influenced by charge sharing around the target pixel dramatically increases as pixel pitch decreases. A further reduction of pixel pitch is limited now due to charge sharing and not enough area for ASIC. For improvement of the spatial resolution without reducing the pixel pitch in this work, we adopted correction algorithm of charge sharing using ToT mode and pixel shift technology (PST). And those are applied to the images with XRI-UNO detector using Medipix2 ASIC which are bump-bonded to $1 \mathrm{~mm}$ thick CdTe with a pixel pitch of $55 \mathrm{um}$. A simulation was performed reflecting the performance of the XRI-UNO detector. The results of the simulation and experiments show improvement of the image quality factor in MTF, DQE, and CNR without changing the detector design. The spatial resolution of the image applying charge sharing correction and PST increased dramatically. Those results are compared to simulated image from a half-pixel pitch (27.5 um pixel pitch) detector. The CNR result of image applied charge sharing algorithm and PST shows about $30 \%$ improved the CNR value at the same X-ray flux. This study gives an alternative method to achieve high spatial resolution without a detector redesign.

Charge Shairng, Contrast, DQE, NPS, MTF, Photon-counting detector, CNR.

R09-13: Improvement of the Spatial Resolution using Charge Sharing in X-Ray Photon Counting Detectors K. Park ${ }^{1}$, D. Lee ${ }^{1}$, D.-U. Kang ${ }^{1}$, G. Cho ${ }^{1}$, H. Kim ${ }^{2}$, H. Chang ${ }^{2}$

${ }^{I}$ Nuclear and Quantum Engineering, Korea Advanced Institute of Science and Technology, Daejeon, Republic of Korea

${ }^{2}$ Institute for Information Technology Convergence, Korea Advanced Institute of Science and Technology, Daejeon, Republic of Korea

The photon counting x-ray detectors consisting of pixel array sensor with bump bonded to an application specific integrated circuit (ASIC) chip directly provide several advantages over conventional x-ray detectors using the scintillators. They have a high noise immunity, charge to noise ratio (CNR), signal to noise ratio (SNR), spatial resolution and charge collection efficiency. The small pixel size, however, leads to induce the electrical pulses on neighboring pixels and distortion of the image because of occurring the charge sharing and K-escape fluorescence x-ray. In this work, we find out the precise pixel position of the incident radiation in clusters obtained by the time-over-threshold (TOT) mode using the calculation for resolving the charge sharing. In addition, we include the method to increase spatial resolution of the image without reducing the pixel size physically. When we scan the image using the TOT mode, it can be seen pixel clusters consisting of the magnitude of the signal caused by the radiation. Therefore, it is possible to find out the pixel position of the incident radiation in the pixel clusters accurately using the calculation of the proportional relationship. Furthermore, dividing into 4-quarters or more which means increasing the spatial resolution by the virtual pixel offered more accurate the position through the calculation in the same way. In the experiment, we used the detector consisting of Timepix chip with CdTe sensor and x-ray source as an $80 \mathrm{kV}, 5 \mathrm{uA}$. For comparisons of the SNR, MTF and DAQ, we used tungsten edge and lead line phantoms. The final image is combined in 50,000 images during $30 \mathrm{us}$ per 1 image. It is possible to confirm that the arrangement of the line image obtained using the calculation is sharper than obtained by the counting mode. According to this research, we expect that distorted images will not occur by using the aforementioned idea to reduce charge sharing even if the spatial resolution increased.

Acknowledgments: This work is supported by ITC and KKI, KAIST

R09-14: Improved Charge Collection in Large-Volume CZT Detectors Using a Modified Contact Configuration 


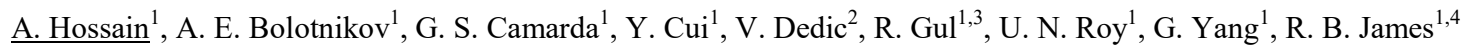

${ }^{I}$ Brookhaven National Laboratory, Upton, NY, USA

${ }^{2}$ Charles University, Prague, Czech Republic

${ }^{3}$ Alabama A\&M University, Normal, AL, USA

${ }^{4}$ Savannah River National Laboratory, Aiken, SC, USA

A uniform distribution of the internal electric field (E-field) is vital for high charge collection efficiency in large-volume CZT detectors. The electric-field profile depends on the electrode configuration, crystalline defects, surface properties, among other factors. For example, the E-field lines may deviate towards dead regions due to the presence of extended defects and residual strains in the crystals, which leads to the drifting of charge carriers away from the collecting electrode. Such phenomena results in incomplete charge collection and distortion of the pulse-height spectra. In this work, we reconfigured the electrodes of a quasihemispheric and Virtual Frisch-Grid detector to guide the E-field lines towards the collecting anode, thus improving electron charge collection. We mapped the distribution of the internal E-field using Pockels effect measurements before and after modifying the electrodes of the devices. We also collected pulse-height spectra using different radioactive sources, and employed a highly collimated x-ray beam to study the spatial variations of the detector response with micron-scale resolution. The detector performance was compared for different electrode configurations. Eventually, based on the acquired data, we modified the electrode configuration of devices to achieve a more optimal design.

R09-15: Electrical and detecting properties of undoped ?-?d?? crystals $\underline{\text { P. Fochuk }}^{1}$, V. Sklyarchuk ${ }^{1}$, Z. Zakharuk ${ }^{1}$, Y. Nykoniuk ${ }^{2}$, A. Bolotnikov ${ }^{3}$, R. James ${ }^{3}$

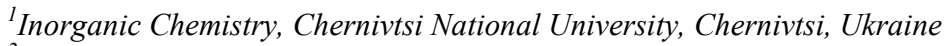

${ }^{2}$ Physics Department, National University of Water Management and Natural Resources Application, Rivne, Ukraine

${ }^{3}$ Non-proliferation Department, Brookhaven National Laboratory, Upton, USA

The goal of this work was produce the detector grade material using undoped CdTe. Synthesized CdTe was twice purified by vacuum distillation and then vertical zone melting (zone 4 passes ). ?-?d?? crystals were grown by modified Bridgman method. Current-voltage characteristics $(\mathrm{I}-\mathrm{V})$ of $\mathrm{Au} / \mathrm{CdTe} / \mathrm{Au}$ structure were linear to a certain voltage, then there was a deviation from linearity and current depended on the voltage by quadratic law (I $\sim \mathrm{V} 2)$. The crystal resistivity at $300 \mathrm{~K}$ was $\sim 2 * 10^{\wedge} 9 \mathrm{Ohm}$, $\mathrm{Nd} / \mathrm{Na}=30 \%, \mathrm{Ea}=0.7 \mathrm{eV}$. The dark conductivity was determined by the deep acceptor level. The temperature dependence of voltage of the ohmic area transition to quadratic Vo was practically absent. To evaluate the detecting properties of ?-?d?? crystals the rectifying contacts were produced. The obtained $\mathrm{Cr} / \mathrm{CdTe} / \mathrm{Au}$ structures possess by the rectifying properties. The $\mathrm{Cr} / \mathrm{CdTe} / \mathrm{Au}$-based detector reliably records the americium radiation. FVHM resolution $\sim 7,5-8,0 \mathrm{keV}$ that worse than in the best CdTe-based detectors. We attribute this result by the relatively high concentration of uncompensated impurities of $\mathrm{N} \sim 10^{\wedge} 12 \mathrm{~cm}-$ 3. The analysis of electrons' and holes' statistics based on the decision of electroneutrality equation and experimental measurement of the temperature dependence of the material resistivity has showed that the observed features of the electrical properties of the studied crystals are explained by the specific compensation processes of semiconductor material. The assessment method of uncompensated impurity concentration $\mathrm{N}$, which is an important parameter of ionizing radiation detector and determines the thickness of the crystal depleted region with the rectifying contact, is offered. Unsatisfactory detecting properties of structure with the rectifying contact are explained by the relatively high concentration of uncompensated impurities in the studied crystals.

\section{R09-16: Cd(Mn)Te-Based Diode Structures}

V. Sklyarchuk ${ }^{1}, \underline{\text { P. Fochuk }}^{1}$, Z. Zakharuk ${ }^{1}$, Y. Nykoniuk ${ }^{2}$, O. Kopach ${ }^{1}$, O. Sklyarchuk ${ }^{1}$, A. Bolotnikov ${ }^{3}$, R. James $^{3}$

${ }^{I}$ Inorganic Chemistry, Chernivtsi National University, Chernivtsi, Ukraine

${ }^{2}$ Physics Department, National University of Water Management and Natural Resources Application, Rivne, Ukraine

${ }^{3}$ Non-proliferation Department, Brookhaven National Laboratory, Upton, USA

$\mathrm{Cd}(\mathrm{Mn}) \mathrm{Te}$ crystals with $\mathrm{Mn}$ content of $20 \%(\mathrm{x}=0.2)$ for use as photodiodes of visible radiation range and detectors of ionizing radiation were investigated. The crystal resistivity was $\sim 4.5^{*} 10^{\wedge} 5 \mathrm{O} \times \mathrm{cm}$. The temperature dependence of the resistivity were described by an exponential dependence with activation energy of $0.45 \mathrm{eV}$. From the temperature dependence of the resistivity and analysis of electrons and holes statistics in a compensated semiconductor it has been found the energy position of the deep acceptor level, responsible for the dark conductivity, as well as its compensation degree, which was equal to $\mathrm{Nd} / \mathrm{Na}=0,12$. A relatively small crystal resistivity $\left(4.5 \times 10^{\wedge} 5 \mathrm{O} \times\right.$ ? $\left.\mathrm{m}\right)$ is due to the fact that partially compensated acceptor level, responsible for the electrical conductivity, was significantly removed from the middle of the semiconductor band gap. Diode structures were created by thermal deposition of aluminum. Rectifying contact diameter of $2.0 \mathrm{~mm}$, ohmic contact was applied to the whole back surface. Obtained structures have been showed the pronounced diode characteristics. Rectification coefficient was $\sim 10^{\wedge} 6$ at $1 \mathrm{~V}$, the dark currents at the reverse voltage of $1 \mathrm{~V}$ were no more than 10-20 pA. Photodiodes' I-V curves are well described by the theory of Sah-Noyce-Shockley adapted for metal-semiconductor contact. Photosensitivity spectra of Al-Cd(Mn)Te-Ni photodiodes cover the range of 0,3-0.9 $\mu \mathrm{m}$ with monochromatic current sensitivity of $\sim 1.0 \mathrm{~A} / \mathrm{W}$. Small dark currents of 
$\mathrm{Cd} 0,8 \mathrm{Mn} 0,2 \mathrm{Te}$ diodes, monochromatic current sensitivity $(\sim 1.0 \mathrm{~A} / \mathrm{W})$ indicate the possibility to use them as photodiodes in $0,3-$ $0.9 \mu \mathrm{m}$ range. For use of such structures as ionizing radiation detectors it is necessary, primarily, to increase the resistivity and significantly reduce the concentration of uncompensated impurities in the initial crystals. This will increase the depletion region thickness at the reverse bias and hence the semiconductor layer thickness absorbing an ionizing radiation.

R09-17: State Stabilization of CMT Crystals' Defect-Dopant System

$\underline{\text {. Fochuk }}^{1}$, Z. Zakharuk ${ }^{1}$, Y. Nykoniuk ${ }^{2}$, A. Bolotnikov ${ }^{3}$, R. James ${ }^{3}$

${ }^{I}$ Inorganic Chemistry, Chernivtsi National University, Chernivtsi, Ukraine

${ }^{2}$ Physics Department, National University of Water Management and Natural Resources Application, Rivne, Ukraine

${ }^{3}$ Non-proliferation Department, Brookhaven National Laboratory, Upton, USA

The temperature dependencies of electro-physical characteristics (conductivity, Hall) of Cd1-xMnxTe $(\mathrm{x}=0,05 ; 0,1)$ crystals as the low-resistance (I), p-type, specially undoped ( $\mathrm{T}=160-420 \mathrm{~K}$ ) and the semi-insulating (II): n-type, In-doped ( $\mathrm{T}=290-420 \mathrm{~K}$ ) were investigated. It have been established that samples' keeping in the range of 320-390 K was accompanied by isothermal changes of electrical parameters. In 1st type samples the conductivity and hole concentration increases, in 2nd type samples the conductivity and electron density decreases. With temperature decreasing the isothermal changes occur in the opposite direction, that is, the relaxation of hysteresis type is observed. But after a prolonged (hours) samples' holding at $420 \mathrm{~K}$ the hysteretic effects disappear, electrical parameters in repeated "heating-cooling" cycles are reproduced, i.e. the state of crystals' defect-dopant system (DDS) is stabilized. It is proposed to explain the described effects by dissociative mechanism of uncontrolled copper impurity incorporation into the crystal lattice of crystals. The temperature dependence of the Hall constant RH for stabilized samples of 2 nd type is described by two areas with different activation energy: high-temperature (HT) and low temperature (LT). The temperature dependence of electron mobility for HT-area is weak, for LT-section - strong, exponential, with activation energy eb. The activation energy of RH(?) in HT-area corresponds to the ionization energy of donors (eD) which control the nconductivity. The activation energy (e1) of LT-area is related with eD and eb by ratio e1=eD-ae?, where a is close to 1 . In this area the carrier velocity is limited by drift barriers eb. They are due to the potential relief fluctuations, caused by material microheterogeneities. It is important to note the lack of photochemical reactions in the semi-insulating $\mathrm{Cd}(\mathrm{Mn}) \mathrm{Te}$-In crystals, unlike of $\mathrm{Cd}(\mathrm{Zn}) \mathrm{Te}-\mathrm{In}$ crystals.

R09-18: Fabrication of Organic Radiation Detector with Ink-Jet Printing Techonlogy

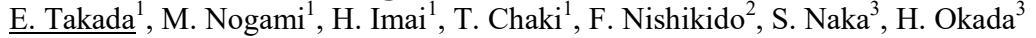

${ }^{I}$ National Institute of Technology, Toyama College, Toyama, Japan

${ }^{2}$ National Institute of Radiological Sciences, National Institutes for Quantum and Radiological Science and Technology, Chiba, Japan

${ }^{3}$ University of Toyama, Toyama, Japan

In order to develop a radiation detector which does not disturb inspection result in Interventional radiography (IVR), the authors have been developing a new radiation detector where organic photodiodes (OPDs) are fabricated directly on plastic scintillator. Fundamental possibility of applying the detector to X-ray measurements have been shown in the previous studies. In the present study, the authors have fabricated the detector with the technology of Ink-Jet printing. Different from the spin coating method, the organic regions can be fabricated independently with each other. The device structure of bulk hetero junction OPD was plastic scintillator plate $(1 \mathrm{~mm}) /$ IZO $(100 \mathrm{~nm}) /$ PEDOT: PSS $(30 \mathrm{~nm}) / \mathrm{PCBM}$ : P3HT $(200 \mathrm{~nm}) / \mathrm{Al}(70 \mathrm{~nm})$. For the devices with different pixel size, response to the white X-rays were measured. It has been shown that the devices fabricated by ink-jet printing technique could produce comparable current with those by spin coating technique. However, the properties were relatively unstable, which might be because the optimization of the fabrication process was not enough. Further results of experiments will be reported at the conference with changing the solvent and the fabrication procedures.

\author{
R09-19: Surface Processing of CdTe Crystals in $\mathbf{H}_{2}$ /Ar Electron Cyclotron Resonance Plasma \\ M. Niraula, K. Yasuda, S. Kitagawa, M. Kojima, T. Yamaguchi, J. Ozawa, S. Tsubota, Y. Agata \\ Graduate School of Engineering, Nagoya Institute of Technology, Nagoya, Japan
}

Surface processing of (111) CdTe detector grade crystals was performed in $\mathrm{H}_{2} / \mathrm{Ar}$ ECR plasma to study its effect on the surface chemistry and also how these surface states control the electrical and gamma ray detection properties of the detectors. The plasma parameters were selected in such as a way that there was no physical damage on the CdTe surface due to the sputtering effect. All crystals studied were cut from a larger CdTe wafer, and we further maintained the process consistency to ensure that the obtained results do not get affected due to the process related factors. These crystals were chemically etched in a $1 \% \mathrm{Br}$-methanol solution before loading into the plasma chamber. X-ray photoelectron spectroscopy (XPS) was used to study the surface chemistry, while surface and the bulk leakage currents were investigated by fabricating detectors. XPS results revealed that the plasma processing removed the excess Te from the CdTe surface. Moreover, both the surface and the bulk leakage currents decreased which led to 
improved gamma detection properties in the plasma processed detectors when compared to unprocessed one. Details results on plasma parameters, surface properties as well as properties of the detectors will be presented.

\section{R09-20: Peculiarities of Melting and Crystallization Processes in $\mathbf{C d}_{1-\mathbf{x}} \mathbf{M n}_{\mathbf{x}}$ Te Solid Solutions} P. Fochuk, Z. Zakharuk, S. Dremlyuzhenko, V. Deibuk, I. Yuriychuk

Chernivtsi National University, Chernivtsi, Ukraine

$\mathrm{Cd}_{1-\mathrm{x}} \mathrm{Mn}_{\mathrm{x}} \mathrm{Te}$ crystals were obtained by the Bridgman method. It was found that quality of monocrystalline blocks depends on homogeneity of manganese distribution along the ingot and on the duration of synthesis and growth. Experimental dependence of microhardness on composition of $\mathrm{Cd}_{1-\mathrm{x}} \mathrm{Mn}_{\mathrm{x}} \mathrm{Te}$ solid solutions has two peaks at 0.14 and $0.46(\mathrm{~T}=300 \mathrm{~K})$. Theoretical calculations have shown that this is due to spinodal decomposition of the solid solution and is caused by redistribution of electronic charge density between Cd-Te and Mn-Te atoms, that leads to a change in the charge asymmetry coefficient. Studies of melting and crystallization of solid solutions by thermogram method have revealed a smooth bend on melting line that is typical for the melting of solid solutions. Crystallization of the samples containing more than 10 mol.\% MnTe takes place with overcooling. Crystallization curves on thermograms are more sharper in this case, indicating a spontaneous crystallization. Overcooling was not observed for $\mathrm{Cd}_{0.9} \mathrm{Mn}_{0.1}$ Te solid solutions, crystallization curves are smooth, indicating an activation nature of the melting.

\section{R09-21: Charge Transport Properties and Detector Performance of CdZnTe Sample with an Inhomogeneous} Distribution of Resistivity J. Pekarek, J. Zazvorka, E. Belas, P. Praus, R. Grill, J. Bok

Institute of Physics, Charles Univerzity in Prague, Prague, Czech Republic

The charge transport properties and the detector performance were investigated on semi insulating indium doped n-type CdZnTe sample with inhomogeneous distribution of resistivity. In general, high resistivity is desired for a better signal-to-noise ratio but the homogeneity of the detector material is also wanted for a stable detector performance. The main effort of this work was to characterize this inhomogeneous sample by various methods and explain the different detector performance throughout the sample. The map of diverse resistivity of this sample was compared with the photoconductivity map measurement, the photoluminescence, the current-voltage characteristic, the alpha- and gamma-spectroscopy and with the laser induced transientcurrent-technique (L-TCT). By these methods it was possible to summarize different charge transport and spectroscopic properties towards the distribution of resistivity. Even though the high resistive part of the sample shows a higher mobilitylifetime product assessed by alpha-spectroscopy, it has rather lower electron mobility and a high space charge density. That has a negative impact in final spectroscopic properties when the high resistive part of the sample has worse gamma spectral resolution and worse charge collection efficiency then the low resistive sample part. It can be explained with the theory of Fermi level shift. This effect changes the charge compensation inside the bandgap and lead to variation in detector parameter, e.g. mobility, space charge density. The region with the maximal value of the resistivity has a deep level inside the band gap where the interaction with conduction band plays a major role. In the part of the sample with lower resistivity the interaction of the deep level with valence band has the main influence. The most likely reason is a varied distribution of defects concentration in the sample which induce the different deep levels inside the band gap or a different capture cross-section.

\section{R09-22: Space Charge Limited Photocurrents in CdZnTe/CdTe Detectors in the Case of Strongly Absorbed Light \\ K. Ridzonova, E. Belas, R. Grill, P. Praus, J. Pekarek \\ Charles University in Prague, Prague, Czech Republic}

Measurements of DC photocurrent-voltage characteristic, photocurrents spectral response and laser induced transient current technique enable investigation of surface recombination centers, bulk trap levels and distribution of the inner electric field. All these effects significantly contribute to the transport properties of semiconductors (i.e. mobility and lifetime of free carriers). In the presented work, the $\mathrm{n}$ - type planar CdZnTe detectors with quasi Ohmic and Schottky contacts were studied by above mentioned methods. It has been shown that in the case of strongly absorbed light under the DC regime of illumination not only surface recombination influences the detector's transport properties. The effect of the space charge, created as a consequence of carriers trapped by the impurity levels, must be taken into account. Therefore new theoretical model, based on classical MottGuerney law was created in order to describe measured photocurrent-voltage dependencies. Obtained data were fitted with the new theory and the mobility and surface recombination velocity for both electrons and holes were determined. Finally, measured values of the mobility by DC photoconductivity technique were correlated with the results obtained from the laser induced transient current technique.

R09-23: Basic Study of a Compton Scattering Camera Using a Pixelated TIBr Detector 
$\underline{\text { M. Matsumura }}^{1}$, K. Watanabe ${ }^{1}$, A. Yamazaki ${ }^{1}$, S. Yoshihashi ${ }^{1}$, A. Uritani ${ }^{1}$, H. Sunaba ${ }^{2}$, N. Nagano ${ }^{2}$, K. Hitomi $^{2}$

${ }^{I}$ Nagoya University, Nagoya,Aichi, Japan

${ }^{2}$ Tohoku University, Rokkasho-mura,Aomori, Japan

Compton scattering cameras have exciting potentials for wide applications such as the medical imaging, radioactive decontamination and so on. Thallium bromide ( $\mathrm{TlBr}$ ) is one of the promising candidates for detectors in the Compton scattering cameras because of operating stably at room temperature and exhibiting good energy resolutions for gamma rays.

The requirements for constructing Compton scattering cameras include the good energy resolutions and the good position resolutions for incident gamma-rays. In case of pixelated detectors, the energy and position resolutions for near-anode events become worse because the pulse height decreases due to the weighting potential distortion. Charge-sharing events in adjacent pixels contribute also to deteriorate the energy and position resolutions. Therefore, development of pulse waveform analysis methods is necessary for using the pixelated $\mathrm{TlBr}$ detectors for the Compton scattering cameras.

In this paper, we discuss the basic issues for using pixelated $\mathrm{TlBr}$ detectors as the Compton scattering cameras. We have already demonstrated the depth-sensing capability in $\mathrm{TlBr}$ pixelated detectors by using the cathode to anode signal ratio. However, the depth-of-interaction information for multi-pixel events cannot be obtained using the cathode to anode ratio. We, therefore, adopt a new depth-sensing method, which derives the depth-of-interaction from the electron drift time extracted from waveform processing. We confirmed that the depth-of-interaction estimated with the proposed procedure is well consistent with expectation from Compton scattering kinematics.

\section{R09-24: Determination of the Charge Collection Efficiency in Pixelated TIBr Detectors for Accurate} Measurement of Ionization Energy

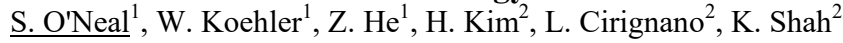

${ }^{I}$ Nuclear Engineering and Radiological Sciences, University of Michigan, Ann Arbor, MI, United States

${ }^{2} R M D$, Inc., Wattertown, MA, United States

Thallium-bromide $(\mathrm{TlBr})$ is currently under investigation for use as an alternative room-temperature semiconductor gamma-ray spectrometer. Performance of better than $1 \%$ FWHM at $662 \mathrm{keV}$ can be achieved when $\mathrm{TlBr}$ detectors are cooled to $-20^{\circ} \mathrm{C}$. The theoretical limit of energy resolution is determined by the ionization energy in semiconductor detectors, and accurately measuring it is important for determining the best possible performance. One method to determine the ionization energy of semiconductor detectors compares pulse heights obtained from the semiconductor to pulse heights from a silicon detector. Due to their higher trapping, the charge collection efficiency (CCE) of $\mathrm{TlBr}$ is significantly lower than it is in silicon, therefore a correction to the ionization energy must be made. In this work, we present the theory and measurement for accurately determining the CCE using the Shockley-Ramo theorem and apply it to measurement of ionization energy in pixelated detectors.

R09-61: Growth of (Cd,Zn)Te by THM under Microgravity

M. Fiederle $^{1}$, A. Fauler ${ }^{1}$, A. Senchenkov ${ }^{2}$, A. Egorv ${ }^{1}$

${ }^{I}$ Freiburger Materialforschungszentrum, Freiburg, Germany

${ }^{2} 2$ Research and Development Institute for Launch Complexes NIISK, Moscow, Russia

The Travelling Heater Method THM is a solution growth technique, which allows the preparation of bulk monocrystalline mixedcompound semiconductors. Since the process is conducted below the congruent melting point of the semiconductor, relatively low temperatures are involved. These low temperatures have the important advantages in reduction of contamination and vapour pressure related problems, reduction of intrinsic defects like vacancies, interstitials and antisites. This leads to bulk crystals with improved dopant homogeneity, reduced dislocation densities, and excellent electrical properties. THM provides a uniform crystal composition of ternary materials such as $(\mathrm{Cd}, \mathrm{Zn}) \mathrm{Te}$ that cannot be achieved by the melt techniques. Recently, the travelling heater method is gaining acceptance as a viable production process for the growth of large diameter single crystals of CdTe and $(\mathrm{Cd}, \mathrm{Zn}) \mathrm{Te}$ by commercial companies. Several experiments with $\mathrm{CdTe}$ and $(\mathrm{Hg}, \mathrm{Cd}) \mathrm{Te}$ have been carried out in close collaboration between European and Russian scientists using the FOTON satellite with the Zona 4 facility. The reduced gravity opens the possibility to study the influence of forced mixing by external fields. The use of magnetic fields e.g a static or rotating magnetic field in combination with microgravity is further tool for investigations of formation of defects and inhomogeneities. THM experiments for $\mathrm{CdTe}, \mathrm{Cd}(\mathrm{Se}, \mathrm{Te})$ and $(\mathrm{Cd}, \mathrm{Zn}) \mathrm{Te}$ were performed in three Russian missions FOTON 7 - 9 in the Zone 4 facility and in 2013 in the FOTON M4 mission. In all configurations a rotating magnetic field was applied to the solution zone. All crystals were doped to obtain high resistivity, detector grade material. The latest results of the FOTON M4 mission yield a improved $(\mathrm{Cd}, \mathrm{Zn})$ Te crystal with a large single crystal part and showed an improvement of the homogeneity of the electrical properties.

R09-26: Ionizing Radiation Detectors Comprised of Micrometer-Scale PbS Star-Shaped Dendrites and Laminar Nanocubes Exhibiting Strong-Confinement Effects

M. D. Hammig, B. Davis, M. Jeong, B. Van 
Nanosemiconductors provide one pathway through which one can realize high energy resolution at room temperature while utilizing a low-cost solution-based fabrication procedure. One of the challenges of forming nanosemiconductor-based colloidal arrays across large areas is to minimize the formation of colloidal gaps that arise during solidification because the ratio of the inter-particle to the Brownian driving force is too large. That challenge can be mitigated if one can form single-crystalline microstructures comprised of stars or cubes possessing dendrites and laminated layers that retain the strong-confinement effects of smaller discrete nanoparticles. In this paper, we describe the fabrication of well-defined single-crystalline PbS microstructures that are formed into colloidal solids, and subsequently configured in the form Schottky diode sensors. The optical characteristics of the microstructures show that they increase the band-gap from the bulk level of $0.41 \mathrm{eV}$ to $1.85 \mathrm{eV}$, and the radiation response of the structures reveals that they have energy-conversion characteristics similar to common single-crystalline materials.

\section{R09-27: A Study on Improvement in Material Decomposition Capability for Photon Counting Detectors} D.-U. Kang, D. Lee, K. Park, M. S. Kim, G. Cho

Department of Nuclear and Quantum Engineering, KAIST, Daejeon, Republic of Korea

Recently, photon counting X-ray detectors has been receiving more and more attention as next generation of X-ray image detectors due to its low noise operation and energy separation capability. More energy bins in a pixel provide better material decomposition with multiple K-edge images. However, more energy bins require bigger pixel size. Pixel size is limited due to maximum counting rate. Therefore, the number of energy bins in each pixel is limited. In this research, we proposed the patterned pixel array that improves material decomposition capability of 2D photon counting detectors without more comparators and counters in a pixel. Each pixel has a common threshold to represent the number of incident photons regardless of the energy of incident photons and a specific threshold according to its pixel type to separate incident photon energy. Although each pixel of the detector, used in this research, has only two comparators and counters, five energy bins are obtained by interpolation with its neighbor pixels. In order to evaluate the proposed method, Monte Carlo simulation (MCNP6) was performed. The Monte Carlo simulation results with two energy bins in each pixel show five images from five energy bins instead of two images without additional circuit blocks. The spatial resolution is not decreased by interpolation since every pixel has common threshold at a comparator. Detailed simulation results and measurement results from the readout ASIC that we designed with $1 \mathrm{~mm}$ thick CdZnTe sensor will be presented at the conference.

\section{R09-28: Dose Evaluation for Medipix Based $\mu \mathrm{CT}$}

F. Fischer, S. Procz, M. Fiederle, A. Fauler

Material Characterization and Detector Technology, Freiburg Materials Research Center (FMF), Freiburg, BadenWürttemberg, Germany

Computed micro tomography $(\mu \mathrm{CT})$ is an X-ray imaging tool used in medical diagnostics and non-destructive material analysis alike. Over the last years, hybrid semiconductor photon-counting detectors of the Medipix family have proven themselves beneficial for the acquisition of the projections for this imaging technique. An advantage of the Medipix detectors is their hybrid structure which allows the use of various sensor materials such as $\mathrm{Si}, \mathrm{GaAs}$ or CdTe. Especially materials with high atomicnumbers like GaAs and CdTe are materials of interest, as they allow the effective detection of mid to high energy photons. Furthermore, the single photon-counting abilities of the Medipix enable a dose reduction compared to integrating detector systems which is further improved by the use of iterative reconstruction. The purpose of this work is the simulation and measurement of the radiation dose in Medipix based computed micro tomography ( $\mu \mathrm{CT})$. Additionally, the knowledge of the absorbed dose in the scanned object provides an opportunity to optimize scanning protocols accordingly. Given that the dose determination of a laboratory X-ray tube can be an error-prone task, several techniques like Monte Carlo simulation and X-ray flux determination with different systems are compared to identify the best match.

\section{R09-29: Influence of Low-Temperature Annealing on Bulk and Surface Properties of CdZnTe Detector M. Rejhon, J. Franc, V. Dedic, J. Zázvorka Institute of Physics, Charles University in Prague, Prague, Czech republic}

We have studied an influence of low-temperature annealing on detector-grade CdZnTe samples, especially the effect on their surface and bulk properties. The dimensions of studied n-type CdZnTe detectors were $5.4 \times 5.4 \times 2 \mathrm{~mm}^{3}$. The surfaces were just optically polished without any further chemical treatment. Gold and Indium contacts were deposited on the large opposite sides by evaporation. The In electrode was planar and opposite Au contact was formed by a central electrode and a guard-ring, both set to the same potential. This configuration of contacts allowed us to measure bulk and leakage currents separately. Studied detector samples were annealed in the air in the temperatures ranging between 70 and $100^{\circ} \mathrm{C}$ with a step of $10^{\circ} \mathrm{C}$. We have measured I-V curves of the bulk and leakage currents before and after annealing after cooling down to the room temperature. Initial findings were that the leakage current was much higher than the bulk current. While the leakage current significantly decreases after the 
annealing, the bulk current slightly increases. Another observation shows the ohmic behavior of the surface, while the bulk was Schottky at low bias according to the assumptions based on used contact metals (Au slightly blocking for holes, In blocking for electrons). In addition to the of I-V curves we will present the results of gamma spectroscopy and Pockels effect measurements supported by surface composition analyses such ellipsometry and TEM in order to discuss in detail an influence of lowtemperature annealing on $\mathrm{CdZnTe}$ detectors.

\author{
R09-30: Exploiting Subpixel Positioning in CZT-Detectors for Baggage X-Ray Diffraction Imaging: an \\ Experimental Implementation \\ J. Tabary, C. Paulus, G. Montémont, L. Verger \\ CEA LETI MINATEC Campus, F-38054 Grenoble, France
}

X-Ray Diffraction is becoming a prevailing technique to detect illicit materials (explosives or drugs) both under polycrystalline form (solid explosives or drugs) and under liquid or amorphous state (liquids, amorphous and gels (LAGs)). For security applications, Energy Dispersive X-Ray Diffraction (EDXRD) is preferred: advantages are the possible use of powerful X-ray tubes and the limited size of the system since angular scanning is unnecessary. Parallelization of detectors is then possible to inspect an entire object in a reasonable time. However, this technique requires the use of good energy-resolved detectors to measure the diffracted spectra. For this purpose, CZT detectors are well suited to EDXRD systems since they combine good efficiency, energy resolution, compactness and working at room temperature. Moreover, some specific detector-level signal processing can be implemented in the diffraction detector module in order to optimize energy resolution and spatial resolution with subpixel positioning. Subpixelation along the scatter angle is interesting for different reasons: it can improve the trade-off between angular resolution and sensitivity and also improve the spatial resolution along the depth direction of the imaged baggage. Based on its own developed technologies of spectrometric CZT detectors, the CEA-Leti is designing EDXRD systems. In this paper, we present an original experiment in which subpixelation is combined with an open collimation to oversample the inspected volume in several subvolumes in the depth direction. Our system is associated to some specific iterative inversion algorithms to correct the different factors such as incident spectrum shape, object attenuation, energy and angular resolution and to finally restore the proper diffraction signature of the different materials included in the baggage. Thereby, with only one single pixel from our own CZT based MINIGAMI imager, we achieve to perform 2D EDXRD images of realistic baggage.

\title{
R09-31: Monte Carlo Simulation of a CZT 3D Spectro-Imager for Scattering Polarimetry
}

E. Caroli ${ }^{1}$, G. De Cesare ${ }^{1}$, R. M. Curado da Silva ${ }^{2}$, N. Auricchio ${ }^{1}$, C. Budtz-Jørgensen ${ }^{3}$, S. Del Sordo ${ }^{4}$, P. Ferrando ${ }^{5}$, J. L. Galvèz ${ }^{6}$, M. Hernanz ${ }^{6}$, J. Isern ${ }^{6}$, I. Kuvvetli ${ }^{3}$, P. Laurent ${ }^{5}$, O. Limousin ${ }^{5}$, J. M. Maia ${ }^{2}$, A. Meuris ${ }^{5}$, M. Moita ${ }^{2}$, N. Produit ${ }^{7}$, J. B. Stephen ${ }^{1}$, A. Zappettini ${ }^{8}$

${ }^{I}$ INAF/IASF-Bologna, Bologna, Italy

${ }^{2}$ LIP-Coimbra, Coimbra, Portugal

${ }^{3}$ DTU Space, Lyngby, Denmark

${ }^{4}$ INAF/IASF-Palermo, Palermo, Italy

${ }^{5}$ CEA-Saclay, Gif-sur-Yvette, France

${ }^{6}$ IEEC-CSIC/UAB, Bellaterra, Spain

${ }^{7}$ ISDC/University of Geneva, Versoix, Switzerland

${ }^{8} I M E M / C N R$, Parma, Italy

The measurement of the polarization status of hard X- and soft gamma-ray emission from cosmic sources is recognized as a key observational parameter to improve our understanding of active production mechanisms and geometries. The capability to provide high sensitivity for polarimetric measurements is a mandatory requirement for new instrumentation operating in this energy regime. In this perspective, we are studying the concept of a small high-performance spectro-imager optimized for polarimetry between 100 and $600 \mathrm{keV}$ suitable for a stratospheric balloon-borne payload and as a focal plane pathfinder for a high energy focussing satellite mission like a broad-band Laue based telescope. We propose a detector with 3D spatial resolution based on highly segmented CZT spectrometer units designed to operate simultaneously as a high performance scattering polarimeter and a high performance spectro-imager. Herein, we report on the results of a Monte Carlo study devoted to optimize the configuration of the detector for polarimetry. In particular, we will present the polarimetric sensitivity for different 3D spatial resolution scales ( 2 and $0.5 \mathrm{~mm}$ ) and with different detector response models. Furthermore, to assess the reliability of the numerical model, we compare Monte Carlo results with experimental data obtained with a high performance pixel spectrometer.

\section{R09-32: Passive Algebraic Tomography of Nuclear Fuel with Electronically Collimated CdZnTe-Detectors} O. V. Maslov, V. A. Mokritsky, N. N. Kornieva

Department of Physics, Odessa National Polytechnic University, Odessa, Ukraine

Passive algebraic tomography is applied for measurement of distribution burnup, initial enrichment, and cooling time, detection of facts of damage of fuel assemblies or unauthorized removal of nuclear material. For tomography the big quantity of 
measurements and special algorithms for processing experiment data are needed. Sufficient quantity of experiments can be obtained due to usage of numerous detectors or long-time investigations. Array of CdZnTe- detectors, which are applied for tomographic measurements FA can be installed on the fuel-handling machine mast or on the cooling pond walls. Applying several methods of detectors' disposition causes significant difficulties during realization of control of volume distribution of fission products in FA on the basis of passive algebraic tomography using CdZnTe-detectors. One of the methods to improve the quality of measurements is the method of computing matrix of the contributions of fuel pin based on the technology of electronic collimation. Investigations have been carried out in order to optimize the measurement time, the number and design of collimator detectors, the FA activity volume distribution reconstruction algorithms. The earlier passive algebraic tomography methods developed for a few gamma radiation energies have been used for estimation of the distribution. Also the older criteria have been used to estimate the quality of fission products distribution reconstruction (it was decided to investigate restored tomograms from the point of view of a relative dispersion of a deviation activity fuel pin within the limits of the tomogram, a relative dispersion of a deviation activity fuel pin within the limits of one lines, the maximal deviation of activity within the limits of one lines and the histogram of a deviation activity of fuel pin). The analysis has shown that when using electronic collimation the single fuel pin activity deviation of $20 \%$ can be marked out in the interior of FA.

\author{
R09-33: A Simulation Tool to Explore Next Generations of Hybrid Pixel Detectors for Synchrotron \\ Applications \\ T. Johng-ay, P. Fajardo, T. Martin, C. Ponchut, P.-A. Douissard, M. Ruat \\ ISDD/DETECTOR UNIT, European Synchrotron (ESRF), Grenoble, France
}

The upcoming new ultra-bright synchrotron source EBS at the European Synchrotron Radiation Facility will increase the intensity of the current X-ray beams by several orders of magnitude, opening new research opportunities in many scientific fields. To take full benefit of this new photon source, scientists will need X-ray detectors with enhanced counting rate capabilities, substantially improved time and spatial resolution. Hybrid pixel detectors have demonstrated their adequacy for many synchrotrons applications and in the case of many of the ESRF experiments that target rather high photon energy up to $100 \mathrm{keV}$, this technology is the most promising to satisfy the upcoming challenges. The development of hybrid new pixel detectors requires long-term projects with substantial amount of resources. The optimal configuration (materials, geometry, readout technology...) is a critical matter that can be fundamentally assisted by simulations tools. This in mind, we have undertaken the development of an efficient and reliable tool for the simulation of the complete detection chain from the generation of the incident X-ray photon distributions to the production of the final detector data. The main goal of this simulation tool is to characterize the various detector configurations under consideration at ESRF for future developments, evaluate the effect of critical design parameters and then assist in decision making for the choice of final concepts and architectures. The approach that we have adopted is based on the use of Monte Carlo with optimised calculation efficiency by carefully selecting the numerical models adapted to our specific purposes. One of the particular aspects is the implementation of efficient semi-empirical models that are being parameterized from actual results obtained via experimental measurements. The purpose of the poster is to present the specificities of our particular concept and implementation, its feasibility and the first simulation results.

\author{
R09-34: Measurement and Simulation of the Response to Fast Neutrons of VPE GaAs Detectors with a \\ Polyethylene Converter \\ S. V. Chernykh ${ }^{1}$, A. V. Chernykh ${ }^{1}$, F. M. Baryshnikov ${ }^{1}$, S. I. Didenko ${ }^{1}$, N. Burtebayev ${ }^{2}$, G. I. Britvich ${ }^{3}$, A. P. Chubenko ${ }^{4}$, \\ Z. Kerimkulov ${ }^{2}$, T. Zholdybayev ${ }^{2}$, M. Nassurlla $^{2}$, M. Nassurlla ${ }^{2}$ \\ ${ }^{I}$ National University of Science and Technology $\ddot{i}_{i}^{1 / 2 M I S I S i ̈} \ddot{i}^{1 / 2}$, Moscow, Russia \\ ${ }^{2}$ Institute of Nuclear Physics, Almaty, Kazakhstan \\ ${ }^{3}$ Institute of High Energy Physics, Protvino, Moscow Region, Russia \\ ${ }^{4}$ Lebedev Physical Institute of the Russian Academy of Sciences, Moscow, Russia
}

Surface-barrier structures based on thin $\left(=50 \ddot{i}_{i}^{1 / 2 \mathrm{~m}}\right)$ HP VPE GaAs epilayers were proposed as a sensor for fast neutron detection by the recoil proton method $[1,2]$. The current work is a continuation of this research and reports the results of characterization and simulation of fast neutron VPE GaAs detectors with an increased area $\left(80 \mathrm{~mm}^{2}\right)$. The detectors are intended for neutron yield measurements from various targets in accelerator physics experiments.

The recoil-proton surface-barrier sensor was fabricated on $45 \ddot{i}_{i} 1 / 2 \mathrm{~m}$ HP GaAs epilayers implementing the process flow presented in [2]. The neutron detection efficiency measured using a ${ }^{241} \mathrm{Am}-\mathrm{Be}$ source was $1.30 \mathrm{i}_{\mathrm{i}}^{\mathrm{i}} / 210^{-3} \mathrm{puls}$./neutr. The signal-to-?background ratio was at the level of 45 .

The response to fast neutrons of the detectors were simulated in Geant4 in order to analyse the experimental results and optimize the design of the detector. Simulation has showed a good agreement with the experimental data and allowed to estimate the maximum theoretical detection efficiency of the detector which is determined by the PE converter $\ddot{i}_{i}{ }^{1 / 2} 1.37 \ddot{i}_{i} 1 / 210^{-3}$ puls./neutr. The difference between measured and simulated values of detection efficiency is due to the fact that events with energies below $0.5 \mathrm{MeV}$ were not taken into account during the experimental measurements.

The optimum active layer thickness of the GaAs sensor was determined during the simulation and was $25 \ddot{i}_{i}^{1 / 230} \ddot{i} i^{1 / 2} \mathrm{~m}$. In this 
case: the recoil proton detection threshold is reduced; the signal from recoil protons is high enough that allows of good discrimination between the signal and low energy background; the signal-to-background ratio increases up to $55 \ddot{i}_{i} 1 / 260$; the detector can operate without any external bias with CCE close to $100 \%$.

1. A.V. Chernykh et al. Fast neutron detector with a surface-barrier VPE GaAs sensor. iWoRID $201422 \mathrm{i} \iota 1 / 226$ June 2014 , Trieste, Italy.

2. A.V. Chernykh et al. 2015 JINST 10 C01021.

R09-35: A Portable Position Sensitive Neutron Detecting Device Based on a Multilayer Silicon Strip Detector $\underline{\text { T. Slavicek }}^{1}$, Z. Kohout ${ }^{1}$, P. Masek ${ }^{1}$, S. Pospisil ${ }^{1}$, D. Meier ${ }^{2}$, A. Kok ${ }^{3}$, T.-E. Hansen ${ }^{3}$, O. Koybasi ${ }^{3}$

${ }^{I}$ IEAP CTU in Prague, Prague, Czech Republic

${ }^{2}$ Integrated Detector Electronics AS, Oslo, Norway

${ }^{3}$ SINTEF, Oslo, Norway

A demonstrator of the portable position sensitive neutron detecting device for neutron imaging in a mixed radiation field will be presented. A detector of the device is based on multilayer perpendicularly crossed strip sensors interlaced with neutron converter. A $6 \mathrm{LiF}$ converter producing energetic alpha particles and tritons is used. The converter permits precise determination of a position of the neutron interaction even in a radiation fields with a high gamma background. The neutron detection efficiency is increased with a 3D structured surface of the multilayer silicon strip sensor. The demonstrator is comprised of the neutron sensitive multilayer silicon strip detector, a 128 channel general purpose charge sensitive amplifier ASICs IDEAS VATAGP8 with nominal dynamic range of $+/-250 \mathrm{fC}$, a 13 bit analogue to digital conversion and an FPGA control. Basic characteristics of the amplifier like amplifier linearity probed with an external charge generator will be shown. Moreover, results of experimental tests of the demonstrator with alpha, beta, gamma sources and neutrons, which include determination of neutron detection efficiency will be demonstrated. The device may be used for neutron imaging via position sensitive detection of particular neutrons on the basis of coincidence detection of neutron conversion products or on the basis of response comparison of individual strips. An effect of charge sharing between individual strips on position resolution will be elaborately discussed. Imaging performance of the demonstrator will be compared with a pixel neutron camera (Timepix based).

The research leading to these results has received funding from the Norwegian Financial Mechanism 2009-2014 and the Ministry of Education, Youth and Sports Project under Contract no. MSMT-28477/2014

\section{R09-36: Space-Charge Limited Transport in CdTe-Based X- and G-Ray Detectors}

O. L. Maslyanchuk ${ }^{1}$, M. M. Solovan ${ }^{1}$, V. V. Kulchynsky ${ }^{1}$, V. A. Gnatyuk ${ }^{2,3}$, T. Aoki ${ }^{3}$

${ }^{\text {I}}$ ury Fedkovych Chernivtsi National University, Chernivtsi, Ukraine

${ }^{2}$ Institute of Semiconductor Physics of NAS of Ukraine, Kyiv, Ukraine

${ }^{3}$ Research Institute of Electronics, Shizuoka University, Hamamatsu, Japan

The principle feature limiting spectrometric performance of CdTe and CdMnTe-based X/?-ray detectors is the impossibility to increase the operating voltage to provide sufficient collection of charge carriers because of raising leakage current. We have carried out the detailed investigation of electrical characteristics of CdTe and CdMnTe-based detectors with two Ohmic contacts. It is shown that a rapid increase in leakage current at applying high bias voltage in the CdTe and CdMnTe crystals is caused by the current limited by space charge (SCL?). The same activation energy for the current of equilibrium holes and the current caused nonequilibrium holes at high voltages confirms the fact that SCLC in the Ni/CdMnTe/Ni detector formed by injection of majority carriers (holes) due to lowering the barrier at an imperfect Ohmic contacts. This fact distinguishes $\mathrm{Ni} / \mathrm{CdMnTe} / \mathrm{Ni}$ detector from $\mathrm{Pt} / \mathrm{CdTe} / \mathrm{Pt}$ detector where SCLC formed by tunnel transitions of minority carriers (electrons) from the Fermi level in the metal (or slightly below it) to the semiconductor through a thin insulating film between the crystal and metal contact. Comparison the measured and calculated current-voltage characteristics of CdMnTe crystal at different temperatures taking into account SCLC according to the Mott-Gurney theory allowed to determine density of discrete trapping centers $\left(1.4 \times 10^{\wedge} 13 \mathrm{~cm}^{\wedge}\right.$ $3)$ and energy of holes traps $(0.39 \mathrm{eV})$ in the $\mathrm{Ni} / \mathrm{CdMnTe} / \mathrm{Ni}$ detector.

This research was supported by the NATO Science for Peace and Security Programme (Project SENERA SfP-984705).

R09-37: Diffusion Coefficient and Drift Parameter Evaluation in Telluride Solid-State Detectors

A. Santi ${ }^{1}$, M. Bettelli ${ }^{2}$, A. Zappettini ${ }^{2}$, M. Zanichelli ${ }^{1}$, M. Pavesi ${ }^{2}$

${ }^{I}$ Phys. Dept., University of Parma, Parma, Italy

${ }^{2} I M E M-C N R$, Parma, Italy

The photoinduced current transients contain a lot of information about the transport properties of the sample. They show a typical behavior consisting in a sharp rise, an intermediate decaying region due to the electron drift-diffusion, and a final diffusion- 
related tail. Values of mobility and lifetime of charge carriers can be found and furthermore the profile of electric field can be reconstructed handling the current transients under the hypothesis in which detrapping and diffusion phenomena are negligible. The tail profile is related instead to the finite collection time of the charge density. The barycenter of photoinduced charge cloud moves inside the crystal following the law of motion while the charge carriers are spatially dispersed around the barycenter and move away from it with a thermic velocity proportional to the diffusion coefficient. The spread of the charge cloud does not change at first sight the times of flight, but it determines the final part of the transient signal, as effect of those carriers collected after the barycenter arrival. We suggest a new method to obtain the diffusion coefficient from photoinduced transient current profiles in the case of linear electric field profiles and, in addition, a different way to find the value of carrier mobility. The model is based on the expression for the carrier current density, containing the drift term due to the electric field and the diffusive contribution due to the carrier density gradient, and the differential equation of continuity for the carrier density. The procedure, tested on several telluride planar detectors, gives results in good agreement with literature data.

\section{R09-38: Tuning Optical and Scintillation Properties of 6LiInSe2: a Dopant Study} A. C. Stowe $^{1,2}$, B. Wiggins ${ }^{1,2}$, K. Stassun ${ }^{2,3}$, A. Burger ${ }^{2,3}$

${ }^{I}$ Physical Sciences, CNS Y-12 National Security Enterprise, Oak Ridge, TN, USA

${ }^{2}$ Physics, Vanderbilt University, Nashville, TN, USA

${ }^{3}$ Physics, Fisk University, Nashville, TN, USA

The Li-containing chalcogenide class of materials has been studied extensively for their radiation detection and non-linear optical properties. They are discrete semiconducting compounds which can be grown by the vertical Bridgman technique into cubic centimeter sized crystals. When enriched in ${ }^{6} \mathrm{Li}$, the crystal performs as both a semiconducting (charge collection) and scintillation (photon collection) neutron sensor at room temperature. As a scintillator, ${ }^{6} \mathrm{LiInSe}_{2}$ emits $512 \mathrm{~nm}$ photons which can easily be coupled to a SiPM for low voltage detection. The light yield is $\sim 21,000$ photons/thermal neutron with a decay time of less than $150 \mathrm{~ns}$. In order to improve the light yield, detection efficiency, and prevent self-activation from In-115, a series of quaternary compositions was synthesized in which indium in ${ }^{6} \mathrm{LiInSe}_{2}$ was substituted with $\mathrm{Ga}$. The crystals of each resultant composition was grown with the optical band gap tuned from 2.8-3.4 eV dependent on Ga concentration. Additionally, the photoemission changed from $512 \mathrm{~nm}$ to $650 \mathrm{~nm}$ proportional to the Ga concentration. ${ }^{6} \mathrm{LiInSe}_{2}: 5 \% \mathrm{Ga}$ shows the most promising response to date of doped ${ }^{6} \mathrm{LiIn}_{1-\mathrm{x}} \mathrm{Ga}_{\mathrm{x}} \mathrm{Se}_{2}$ compounds.

R09-39: Possibilities of CdTe-Based ?/?-Ray Detectors with MoO Contacts $\underline{\text { O. L. Maslyanchuk }}^{1}$, M. M. Solovan ${ }^{1}$, P. D. Maryanchuk ${ }^{1}$, V. V. Kulchynsky ${ }^{1}$, V. A. Gnatyuk ${ }^{2,3}$, T. Aoki $^{3}$

${ }^{I}$ Yury Fedkovych Chernivtsi National University, Chernivtsi, Ukraine

${ }^{2}$ Institute of Semiconductor Physics of NAS of Ukraine, Kyiv, Ukraine

${ }^{3}$ Research Institute of Electronics, Shizuoka University, Hamamatsu, Japan

Cadmium telluride (CdTe) based detectors are very attractive devices for high-resolution spectroscopic imaging widely used in astronomy, industry, medicine, science, nuclear safeguard, etc. For obtaining high-resolution and time stability of electrical and spectral properties, the creation of high quality noninjecting contacts to CdTe crystals is one of the main challenges in the detector design. Transition metal oxides, in particular molybdenum oxide (MoOx), represent one of the most promising classes of electronic materials due to its high workfunction (5.2-6 eV). Transparent conductive layers are widely used for the fabrication of electronic and optoelectronic devices, in particular CdTe-based solar cells. In this paper we present the analysis of electrical properties of $\mathrm{MoOx} / \mathrm{p}-\mathrm{CdTe}$ structures prepared by the deposition of molybdenum oxide thin films by the DC reactive magnetron sputtering onto semi-insulating p-type single-crystal CdTe wafers produced by Acrorad Co., Ltd. To determine the electrical parameters of MoOx thin films simultaneously with the deposition on the p-CdTe substrates, the similar films were deposited on glass and ceramic substrates. The values of the conductivity $\left(150 \mathrm{Ohm}^{\wedge}-1 \mathrm{~cm}^{\wedge}-1\right)$ and carrier concentration $\left(8.47 \times 10^{\wedge} 17 ? \mathrm{~m}^{\wedge}-3\right)$ for the MoOx thin films was determined. Spectrometric properties of MoOx/p-CdTe structures have also been analyzed. It is shown that CdTe crystal with MoOx contacts can be used as X-/?-ray detector in the spectrometric mode.

This research was supported by the NATO Science for Peace and Security Programme (Project SENERA SfP-984705).

\section{R09-40: Theoretical Approach to the Energy Resolution of Semiconductor Detectors} V. V. Samedov

National Research Nuclear University MEPhI (Moscow Engineering Physics Institute), Moscow, Russian Federation

There are a number of processes in semiconductor detectors that lead to peak broadening. In many papers, semi-empirical approach is used to take into account the contribution of one or another factor to the signal variance of a semiconductor detector. The correct formula for the signal variance cannot be semiempirical at the researcher's option. It must result strictly from exact mathematical description of the particle registration by detector. In this work, using the theory of branching cascade processes the general expression for the energy resolution of semiconductor detectors was derived. It was shown that the general formula 
encloses all specific contributions of the peak broadening published in literature and has additional contributions to the energy resolution fluctuations of the electron and hole lifetimes caused by nonuniformity of the trap density, and fluctuations of the electronic gain.

R09-41: On-the-Fly Fast X-Ray Tomography Inspection of the Three Point Bending Test I. Kumpova ${ }^{1}$, T. Fila ${ }^{2}$, D. Kytyr ${ }^{2}$, J. Jakubek ${ }^{1}$, V. Vesely ${ }^{3}$, D. Vavrik ${ }^{2}$, M. Vopalensky ${ }^{1}$

${ }^{I}$ The Institute of Theoretical and Applied Mechanics AS CR, v. v. i, Centre of Excellence Telc, Telc, Czech Republic

${ }^{2}$ The Institute of Theoretical and Applied Mechanics AS CR, v. v. i, Prague, Czech Republic

${ }^{3}$ Brno University of Technology, Faculty of civil engineering, Institute of Structural Mechanics, Brno, Czech Republic

Measurement and visualization of the fracture process zone (FPZ) in silicate composites is essential for understanding how crack is formed and how it is propagating through the material. Although there have been already realized several time-lapse radiography and tomography experiments during the three point bending of silicate composites with conventional imaging techniques in the past, crack initiation and propagation is so fast that it is very difficult to capture sufficient spatial information in the exact moment when this process is occurring. Nowadays, progress in X-ray imaging instrumentation, especially single photon counting CdTe Timepix detectors with a fast parallel readout, allows to highly speed up the acquisition of the tomographic data while keeping the intrinsic resolution of tens of micrometres and high efficiency even for higher energies. Not surprisingly, also the time lapse tomography is moving towards this direction with the expectation of better results in shorter time. Tomography of relatively fast processes is becoming realistic even in laboratory conditions using commercially available micro-focus X-ray tubes. As an example of such a fast tomography, results of on-the-fly series of tomographic experiments performed on the specimen subjected to continual three point bending test allowing monitoring of the three-dimensional spatial distribution of a crack initiation and propagation and evaluation of FPZ models is presented.

The research has been supported by the project No. GA15-07210S of the Grant Agency of the Czech Republic.

\title{
R09-42: Bias Dependence of the Energy Resolution of Semiconductor Hemispherical Detectors V. V.Samedov
}

National Research Nuclear University MEPhI (Moscow Engineering Physics Institute), Moscow, Russian Federation

Now hemispherical geometry is used for designing insensitive to the hole transport single polarity CdZnTe detectors. In this work, theoretical consideration of the processes in semiconductor hemispherical detectors for low energy X-rays was considered. The formulae for the mean amplitude and the variance of the signal at the output of the semiconductor hemispherical detector were derived. It was shown that the power serial expansions of the detector amplitude and the variance in terms of the inverse bias voltage allow determining the Fano factor, electron mobility lifetime product, and the nonuniformity level of the trap density of the semiconductor material.

\author{
R09-43: KALYPSO: a Linear Array Detector with a MHz Line-Rate for Real-Time Beam Monitoring \\ Applications \\ $\underline{\text { L. Rota }}^{1}$, M. Caselle ${ }^{1}$, M. Balzer ${ }^{1}$, S. Kudella ${ }^{1}$, M. Weber ${ }^{1}$, A. Mozzanica ${ }^{2}$, N. Hiller ${ }^{2}$, M. J. Nasse ${ }^{1}$, P. Schönfeldt ${ }^{1}$, S. Walther ${ }^{3}$, \\ B. Steffen ${ }^{3}$, C. Gerth ${ }^{3}$, D. Makowski ${ }^{4}$, A. Mielczarek ${ }^{4}$ \\ ${ }^{1}$ Karlsruhe Institute of Technology (KIT), Eggenstein-Leopoldshafen, Germany \\ ${ }^{2}$ Paul Scherrer Institute (PSI), Villigen, Switzerland \\ ${ }^{3}$ DESY, Hamburg, Germany \\ ${ }^{4}$ University of Lodz, Lodz, Poland
}

Electro-Optic Spectral Decoding (EOSD) experiments are installed in several accelerator machines to measure the time structure of the longitudinal bunch profile. In order to improve the temporal resolution of EO experimental setups, we have developed a novel linear array detector with a continuous line rate of up to $2.7 \mathrm{MHz}$. The detector board mounts a InGaAs or Si linear array sensor, two front-end chips (GOTTHARD version 1.6) and a high-speed ADC. A readout card based on an FPGA controls and synchronizes the detector with the accelerator machines. Continuous and real-time data elaboration has been achieved by integrating the detector with a custom data-acquisition system based on PCI-Express 3.0 and Graphical Processing Units (GPU). We present the experimental measurements taken with different laser sources and we discuss the first preliminary results obtained at different accelerator machines. The current limitations of the system are also discussed, together with the improvements planned for the future version.

\section{R09-44: First Steps in the Deposition of Heavy Metal Iodides Layers by Spin Coating}


${ }^{I}$ GDMEA, Departamento de Desarrollo Tecnologico, Centro Universitario Regional del Este, Universidad de la Republica, Rocha, Uruguay

${ }^{2}$ GDMEA, Catedra de Radioquimica, Facultad de Quimica, Universidad de la Republica, Montevideo, Uruguay

With the aim of controlling the first steps in layer growth onto amorphous substrates, we employed $\mathrm{HgI}_{2}$ and $\mathrm{BiI}_{3}$ nanostructures for nucleation. Nanostructures were synthesized from solution with and without hydrothermal treatment, and by the hydrothermal method, from different precursors, in some cases. $\mathrm{HgI}_{2}$ and $\mathrm{BiI}_{3}$ were confirmed by powder X-ray diffraction. Nanostructures were characterized in their size and morphology by transmission electron microscopy. Depending on the procedure, nanometer sizes were from 4 to $50 \mathrm{~nm}$; also morphologies varied, but nanoparticles were crystalline (verified by XRD and electron diffraction). Nanostructures were deposited onto floating silica glass by spin coating; the best conditions were a speed of 1100 $\mathrm{rpm}$ during $15 \mathrm{~s}$ and then a speed of $4000 \mathrm{rpm}$ during $20 \mathrm{~s}$. Some $\mathrm{BiI}_{3}$ depositions were also subjected to a post annealing process. Layers morphology was studied by atomic force microscopy. The results we obtained will lead to the first heavy metal iodide films grown from spin coating nucleations, procedure which arises as a possible first step for nucleation. Results are very promising for improving the film growth and therefore the current applications of these materials, as well as for opening new ones.

\section{R09-45: Interaction Position-Sensing in CZT Detectors Using Proximity Electrodes}

A. M. Alhawsawi, A. T. Farsoni, L. Ranjbar, E. M. Becker

School of Nuclear Science and Engineering, Oregonstate University, Corvallis, Oregon, USA

Proximity charge sensing is a technique used in semiconductor detectors to read out detector output after incident radiation interacts with the crystal. This technique has several advantages over traditionally directly-deposited electrodes, including easier, lower-cost fabrication and better position sensitivity. Additionally, proximity charge sensing reduces the leakage current associated with directly depositing the electrodes on the crystal surface. While the proximity-sensing technique has been implemented in Ge and Si-based detectors, it has not been demonstrated with room-temperature detectors, which more often use complicated electrode designs. Room-temperature detectors with proximity-sensing have an additional advantage over HPGe detectors because they do not require maintenance-intensive cooling systems. This study investigates the use of proximity charge-sensing on CZT crystals to demonstrate and evaluate the performance of the technique on a room-temperature material. The weighting potentials of proximity-sensing and directly-deposited electrode designs were generated using ANSYS Maxwell, and the best pixilated design was chosen for implementation. Three pixel sizes were simulated with three different pitch sizes to assess the effect on position sensitivity within the detector. Energy resolution and position sensitivity of proximity-sensing are comparable to directly-deposited designs.

This work was funded in-part by the Consortium for Verification Technology under Department of Energy National Nuclear Security Administration award number DE-NA0002534 and supported by Sunstone Circuits.

R09-46: Response to Alfa-Particles of SiC UV Sensors

$\underline{\text { A. Fazzi }}^{1,2}$, G. Bertuccio ${ }^{3,2}$, D. Giove ${ }^{2}$

${ }^{I}$ Dept. of Energy, Politecnico di Milano, Milano, Italy

${ }^{2}$ Milan Section, INFN, Milano, Italy

${ }^{3}$ DEIB Dept., Politecnico di Milano, Milano, Italy

\section{R09-47: Diamond-Based Neutron Scatter Camera}

A. Alghamdi, E. Lukosi

University of Tennessee, Knoxville, TN, USA

For neutron spectrometry, diamond has several promising features. It is fast, low-Z, and is small. The low- $Z$ of carbon has resulted in many publications on the use of diamond for neutron detection. However, in-combination with the superior timing performance of diamond detectors, we have evaluated the potential of a diamond scatter neutron camera (DNSC). In the results presented, implications of system design on DNSC performance will be discussed, including array design and intrinsic limitations in timing performance (including deposited energy for deep epithermal neutron detection). First results indicate excellent reconstruction of the source neutron spectrum is possible.

R09-48: Detectors Based on Wide Bandgap Semiconductors (CdTe, CdZnTe) in the Post-Soviet Space $\underline{\text { Y. Petukhov }}$

Center of Radiation and Nuclear Safety Technologies, Riga, Latvia 
On behalf of the Y. Petukhov Mulivanov S. (RNIIRP Riga) Khrunov V. Fedorkov V. Smirnov Al., Gazizov I, Kharitonov Yu (Dubna IFTP)

Detectors based on wide bandgap semiconductors (CdTe, $\mathrm{CdZnTe})$ in the post-Soviet space

In recent years, very rapidly it was the development of a detection instrument engineering worldwide. Including this trend has developed in the territory of Russia. In Russia, with the participation of employees RNIIRP (RIGA) different detectors (countable and spectrometric) and blocks on the basis of their detection were created. We have studied the detectors with different structures, the metal-semiconductor-metal (MSM) and rectifying structures (PIN). Detectors investigated in operation and as a straight room temperature and at reduced temperatures. We give them the characteristics of the registration study in a wide range of spectra of radiation (gamma and X-ray) from 6 to $662 \mathrm{keV}$. Specific examples of possible practical application of these detectors

R09-49: First Imaging Measurements of a Timepix3 ASIC Bump Bonded to a 1mm GaAs:Cr Sensor $\underline{\text { M. Zuber }}{ }^{1}$, E. Hamann ${ }^{1}$, L. Tlustos ${ }^{2}$, T. König ${ }^{1,3}$, M. Fiederle ${ }^{1,2}$, T. Baumbach ${ }^{1}$

${ }^{I} I P S / L A S$, Karlsruhe Institute of Technology, Karlsruhe, Germany

${ }^{2}$ FMF, Universität Freiburg, Freiburg, Germany

${ }^{3}$ Ziehm Imaging GmbH, Nürnberg, Germany

Within the family of Medipix single photon counting detectors, the Timepix3 opens the possibility to read-out single photon hits event by event. This allows to perform multiple data-processing steps during post processing, such as the detection and correction of charge sharing and reconstruction of the real charge, which otherwise has to be realized by the analog part of the ASIC prior to performing event counting. In this event driven mode, a pixel individually sends data packages containing the time-overthreshold (that relates to the energy), the time of arrival and the interaction location. This high data transfer sets a limit to the maximal count rate of the detector system and requires a fast read-out. Additionally, the Timepix 3 can also be operated in the conventional frame based counting mode. We will present first results of such a Timepix 3 detector featuring a $1 \mathrm{~mm}$ chromium compensated GaAs sensor, designated for high energy imaging applications. Both its spectroscopic capabilities and spatial resolution of the detector assembly have been measured for the event driven and the counting modes. In addition, we will present first examples of imaging applications, in particular low flux applications like micro-CT. Here, we can choose the effective pixel size and the energy binning after the data was acquired, allowing the optimization of the image quality for purposes that are not known prior to performing a scan.

\author{
R09-50: Laser-Based Technique of Formation of CdTe-Metal Diode Structures for High Energy Radiation \\ Detectors \\ $\underline{\text { K. Zelenska }}^{1}$, D. Gnatyuk ${ }^{2}$, T. Aoki ${ }^{3}$ \\ ${ }^{I}$ Faculty of Physics, Taras Shevchenko National University of Kyiv, Kyiv, Ukraine \\ ${ }^{2}$ Graduate School of Science and Technology, Shizuoka University, Hamamatsu, Japan \\ ${ }^{3}$ Research Institute of Electronics, Shizuoka University, Hamamatsu, Japan
}

The primary objective of the research is to develop the advanced laser-based technique of formation of CdTe-metal diode structures which can be suitable for high energy resolution X-and gamma-ray detectors. Semi-insulating p-like CdTe crystals were used for the diode type detector fabrication. The semiconductor surface was processed using chemical etching in $5 \% \mathrm{Br}$ methanol or Br-ethanol solution before deposition of In or Al (both as dopant and electrode) and Au (electrode) metal films, respectively. The diode structures were created by the laser-induced doping of a thin surface region of CdTe crystals pre-coated by an In or Al metal film (thickness of 0.1-1 $\mu \mathrm{m}$ ). The experimental setup of the optical instruments for laser-induced modification of the semiconductor-metal interface and doping of CdTe crystals was elaborated. The key feature of the proposed technique consisted of the direct impact of nanosecond laser pulses on the semiconductor-metal interface. The CdTe-metal (In or Al) interface was irradiated through the CdTe crystal employing different numbers of YAG:Nd laser pulses $(?=1064 \mathrm{~nm}, \mathrm{t}=8$ ns) with power density of 1.4-20 MW/cm2 in water environment. Computer simulation of laser heating of the 3-layer CdTe-metal (In or $\mathrm{Al}$ )-water structure irradiated through the CdTe crystal was used to study the optimal laser power densities which corresponded to different temperatures of the CdTe-metal ( $\mathrm{In}$ or $\mathrm{Al}$ ) interface region and different temperature distributions inside the 3-layer structure. The obtained $\mathrm{In} / \mathrm{CdTe} / \mathrm{Au}$ and $\mathrm{Al} / \mathrm{CdTe} / \mathrm{Au}$ diodes with a p-n junction were tested by $\mathrm{I}-\mathrm{V}$ and isotope spectral measurements. Steep rectification, low leakage current and high energy resolution were obtained for the diode detectors formed by the laser-induced doping with a sequence of tens of laser pulses of moderate power density which was enough to heat the CdTe-metal (In or $\mathrm{Al}$ ) interface up to the corresponding metal melting point.

This work was supported by the NATO Science for Peace and Security Programme (Project SENERA, SfP-984705).

R09-51: Development of CdZnTe Position-Sensitive Detector Module for Integration into Handheld Spectrometer 
Y. Cui $^{1}$, A. E. Bolotnikov ${ }^{1}$, H. Brands ${ }^{2}$, G. S. Camarda ${ }^{1}$, R. Gul ${ }^{1}$, G. De Geronimo ${ }^{1}$, J. Fried ${ }^{1}$, A. Hossain ${ }^{1}$, L. Hoy ${ }^{2}$, F. Liang ${ }^{2}$, J. Preston ${ }^{2}$, E. Vernon ${ }^{1}$, G. Yang ${ }^{1}$, R. B. James ${ }^{1}$

${ }^{I}$ Brookhaven National Laboratory, Upton, NY, USA

${ }^{2}$ FLIR Systems, Inc., Oak Ridge, TN, USA

$\mathrm{CdZnTe}(\mathrm{CZT})$ is an attractive detecting medium for room-temperature operating gamma-ray detectors used in many applications, including nonproliferation, safeguards, national security, medical imaging and astrophysics. Some designs of CZT detectors operate like gas ionization chambers with virtual Frisch-grids, in which the electron clouds generated by the incident photons drift in the applied electric field towards the collecting electrode and generate output signal pulses used for spectral analysis of the detected photons. The carriers generated in the ionization process could be trapped by crystal defects, which are present at high concentrations in CZT. To overcome this issue, we have recently developed position-sensitive detectors that allow us to segment detectors' volumes into large numbers of small voxels and equalize the responses to incident photons in each of them. This technique has demonstrated high energy-resolution spectroscopic capability in the laboratory environment. The encouraging results motivated us to move this technology to field applications. For this purpose, we are designing a compact detector module that includes a 2x2 array of position-sensing CZT detector array packaged with compact readout electronics. Such a module can be easily integrated into a handheld device or portable instrument for field use. In this presentation, we will discuss the design of this module and present test results with sealed source.

* J. Preston's current address: CNS LLC, Y-12 National Security Complex, Oak Ridge, TN, 37831-6501 ** Ralph B. James' current address of : Savannah River National Laboratory, Aiken, SC 29808

R09-52: Characterization of 3 and 4 Inch GaAs:Cr Wafers

A. Tyazhev, D. Budnitskyi, V. Novikov, A. Zarubin, A. Lozinskaya, I. Kolesnokiva, A. Shemeryankina, T. Mikhailov, M. Skakunov, O. Tolbanov, I. Chsherbakov

Functional Electronics Laboratory of Tomsk State University, Tomsk, Russia

This article presents the investigation results of electrophysical characteristics of three and four inch chromium compensated gallium arsenide (GaAs:Cr) wafers. Characteristics of X-ray pad sensors based on GaAs:Cr is also demonstrated. The resistivity mapping of wafers was carried out via contactless method and by analyzing of current-voltage characteristics of sensors. It was found that the resistivity distribution in HR GaAs material essentially depends on the distribution of the shallow impurity in the $\mathrm{n}$-GaAs wafers. Therefore there is a variation of resistivity across the wafer in the range from 0.55 to $1.25 \mathrm{GOhm} \cdot \mathrm{cm}$. It was shown that IV characteristics have close to linear dependence on bias within operating voltage. Pulse height distribution and charge collection efficiency measurements were done using $241 \mathrm{Am}$ radiation source. The estimation of $\mu$ ete based on it has shown quite high values reaching $7 \cdot 10^{-5} \mathrm{sq} . \mathrm{cm} / \mathrm{V} \cdot \mathrm{s}$. These facts state GaAs:Cr wafers of different diameter as a high-potential material in pixel sensors' construction.

The investigation was financially supported with grant of Russian Federation \# 14.587.21.0003 (RFMEFI58714X0003)

R09-53: Surface Passivation and Contacts in CdZnTe X-Rays and Gamma-Rays Detectors $\underline{\text { S. U. Egarievwe }}{ }^{1}$, E. D. Lukosi ${ }^{2}$, O. K. Okobiah ${ }^{1}$, A. Hossain ${ }^{3}$, U. N. Roy ${ }^{3}$, A. L. Adams ${ }^{1}$, R. Gul ${ }^{1}$, R. B. James ${ }^{4}$

${ }^{I}$ Nuclear Engineering and Radiological Science Center, Alabama A\&M University, Normal, AL, USA

${ }^{2}$ Nuclear Engineering Department, University of Tennessee, Knoxville, TN, USA

${ }^{3}$ Department of Nonproliferation and National Security, Brookhaven National Laboratory, Upton, NY, USA

${ }^{4}$ Savannah River National Laboratory, Aiken, SC, USA

Contacts deposition and surface passivation are important processes in the fabrication of cadmium zinc telluride $(\mathrm{CdZnTe}) \mathrm{X}$ rays and gamma-rays detectors. Surface passivation is used to reduce the effects of fabrication induced surface defects. It is also used to reduce defects that could result from aging of the detector. Another important factor in charge collection is the interface between the electrical contacts and the detector wafer. In this paper, we will present results of surface passivation in two experiments: full passivation and masked passivation (where the contacts are protected from the passivation chemical). We will also characterize and discuss the effect of the contact-detector interface in terms of charge collection and detector efficiency. The effects of the expansion coefficients of the contacts and the detector wafer will also be discussed.

This work has been supported by the U.S. Department of Homeland Security, Domestic Nuclear Detection Office, under Award 2012-DN-077ARI065-04; the U.S. Nuclear Regulatory Commission under Award NRC-27-10-514; and the U.S. Department of Energy, Office of Defense Nuclear Nonproliferation R\&D, NA-22.

R09-54: Batch Annealing of CdMnTe Wafers for Nuclear Detector Applications

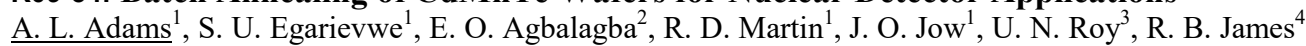


${ }^{I}$ Nuclear Engineering and Radiological Science Center, Alabama A\&M University, Normal, AL, USA

${ }^{2}$ Department of Physics, Federal University of Petroleum Resources, Effurun, Delta State, Nigeria

${ }^{3}$ Department of Nonproliferation and National Security, Brookhaven National Laboratory, Upton, NY, USA

${ }^{4}$ Savannah River National Laboratory, Aiken, SC, USA

Post-growth annealing is used to eliminate performance-limiting Te inclusions and related defects from cadmium manganese telluride $(\mathrm{CdMnTe})$ wafers for nuclear detector applications. These defects could cause problems in applications that require large-volume detectors, where they trap charges that are generated by nuclear radiation. The trapping of charges degrades the charge transport properties and reduce their carrier lifetime, thereby limiting the overall performance for the detector. We will present results of batch annealing of CdMnTe wafers using a three-zone furnace. The annealing processes compared include temperature-gradient and constant-temperature annealing in $\mathrm{Cd}$ vapor, in $\mathrm{Mn}$ vapor, and in a combination of $\mathrm{Cd}$ and $\mathrm{Mn}$ vapors.

This work has been supported by the U.S. Department of Homeland Security, Domestic Nuclear Detection Office, under Award 2012-DN-077ARI065-04; the U.S. Nuclear Regulatory Commission under Award NRC-27-10-514; and the U.S. Department of Energy Office of Defense Nuclear Nonproliferation R\&D, NA-22.

R09-55: Thermally Evaporated B- and Li-Doped Amorphous Selenium Alloy Based Radiation Detectors K. C. Mandal, R. O. Pak, C. Oner, T. A. Chowdhury

Electrical Engineering Department, University of South Carolina, Columbia, SC, USA

Amorphous selenium (a-Se) alloy materials with doping elements of boron and lithium were synthesized for room temperature nuclear radiation detector applications. A multi-step synthetic process has been followed to synthesize Se-As and Se-Cl master alloys from zone-refined selenium (Se), and then synthesized the final alloys for thermally evaporated large-area thin-film deposition on oxidized aluminum $\left(\mathrm{Al} / \mathrm{Al}_{2} \mathrm{O}_{3}\right)$ and indium tin oxide (ITO) coated glass substrates. Morphology and compositional characteristics of the as-synthesized alloy materials and B- and Li-doped films were examined using glow discharge mass spectroscopy (GDMS), differential scanning calorimetry (DSC), x-ray photoelectron spectroscopy (XPS), x-ray diffraction (XRD), scanning electron microscopy (SEM), and energy-dispersive analysis by x-rays (EDAX). Current-voltage (I-V) measurements were carried out to confirm high resistivity of the deposited films with very low leakage currents. We have further investigated the junction properties of the alloy films with selective metals of varying work functions ( $\mathrm{Au}, \mathrm{Pt}, \mathrm{Ni}, \mathrm{W}, \mathrm{Pd}, \mathrm{Al}, \mathrm{Mo}$, In, and $\mathrm{Sn}$ ). The aim was to investigate whether the choice of metals can improve the performance of fabricated detectors by minimizing the dark leakage current, and it was found that the performance of the fabricated detectors were dependent on the presence of macroscopic and microscopic defects affecting the charge transport properties adversely. Capacitance mode deep level transient studies (DLTS) on boron doped alloy detector revealed for the first time the presence of shallow level defects (Ec$0.18 \mathrm{eV}$ ) with concentration $\sim 10^{12} \mathrm{~cm}^{-3}$ and capture cross-section $7.2 \times 10^{-14} \mathrm{~cm}^{2}$.

\section{R09-56: Characterization of Large-Area, High-Resolution $\mathrm{Cd}_{0.9} \mathrm{Zn}_{0.1}$ Te Nuclear Radiation Detectors} K. C. Mandal, R. O. Pak, C. Oner, T. A. Chowdhury

Electrical Engineering Department, University of South Carolina, Columbia, SC, USA

High barrier cadmium zinc telluride $\left(\mathrm{Cd}_{0.9} \mathrm{Zn}_{0.1} \mathrm{Te}, \mathrm{CZT}\right)$ Schottky diodes with very low reverse leakage current have been fabricated and characterized for high resolution nuclear radiation detectors. The diodes were fabricated using $\mathrm{Cd}_{0.9} \mathrm{Zn}_{0.1} \mathrm{Te}$ detector grade crystals grown from zone refined $\mathrm{Cd}, \mathrm{Zn}$, and $\mathrm{Te}(7 \mathrm{~N})$ precursor materials using low temperature tellurium solvent method. Various crystallographic defects and electrical activity of dislocations including Te-inclusions/precipitates were identified and characterized using electron beam induced current (EBIC) measurements. It was observed that EBIC contrast depends on the type of defects or dislocations. The dependency was attributed to spatial inhomogeneities in the electrical properties of the CZT crystals around the dislocations. Chemical etching of the CZT crystals was used to define the nature of the extended defects or dislocations observed by EBIC imaging. It was found that electrical breakdown of the Schottky diodes occurs mostly at the location of larger size Te-precipitates. Further characterization by the thermally stimulated current (TSC) spectroscopy revealed shallow and deep level centers with activation energies $0.22-0.45 \mathrm{eV}$ and $0.62-0.85 \mathrm{eV}$ respectively, which was attributed to intrinsic defects associated with Te inclusions/precipitates and in the vicinity dislocation cores. Pulse height spectra (PHS) measurements were carried out using ${ }^{137} \mathrm{Cs}(662 \mathrm{keV})$ radiation source and energy resolution of $\sim 1.0 \%$ FWHM was obtained from the as-grown CZT boule.

\section{R09-25: Organic Diodes for Neutron Detection}

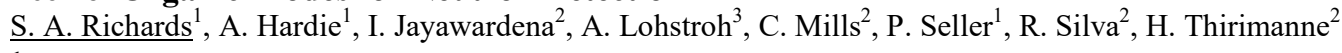

${ }^{I}$ Detector Development, STFC-Rutherford Appleton Laboratory, Didcot, Oxfordshire, United Kingdom

${ }^{2}$ Advanced Technology Institute, University of Surrey, Guildford, Surrey, United Kingdom

${ }^{3}$ Department of Physics, University of Surrey, Guildford, Surrey, United Kingdom 
The Science and Technology Facilities Council's Rutherford Appleton Laboratory and the University of Surrey's Advanced Technology Institute have been developing organic diodes loaded with boron nanoparticles for use as neutron detectors. The diodes, which use thin film organic solar cell technology can be made into layers with thicknesses of several micrometers are insensitive to the ?-ray background typically associated with neutrons. The diodes can be made sensitive to neutrons by incorporating 10B based nanoparticles into the conjugated polymer blend. The diodes have been shown to have a fast response at $90 \mathrm{~ns}$ and low leakage current. Simulations have been conducted that show that the range of the a-particle released by the 10B neutron reaction is shorter than the achievable diode thickness.

\title{
R10: Detector Devices1
}

\author{
Wednesday, Nov. $2 \quad$ 16:30-18:30 Schuman \\ R10-1: Measurements on the Physical Properties of Semiconductor and Scintillator Detectors at the Advanced \\ Light Source (ALS) \\ G. S. Camarda, A. E. Bolotnikov, Y. Cui, R. Gul, A. Hossain, U. Roy, G. Yang, R. James
}

Brookhaven National Lab, Upton, NY, USA

Recently, a new multi-channel acquisition system was installed at the LBNL's ALS Beamline 3.3.2 to carry out X-ray Detector Response Mapping. Measurements performed at this synchrotron facility also include White Beam X-ray Diffraction Topography (WBXDT), Micro X-ray Fluorescence ( $\mu$-XRF), among others. We will provide an update on the capabilities of the Beamlines and present our most recent results obtained on selected compound semiconductors and scintillators. One of the objectives of the studies on CdZnTe is to understand the origin and effects of subgrain boundaries and help to visualize the presence of a higher concentration of impurities, which might be responsible for the deterioration of the energy resolution and response uniformity in the vicinity of the sub-grain boundaries. We also investigated the dependence of the detector performance on the X-ray flux. The results will be discussed in greater detail in the presentation.

CdZnTe detectors, scintillators, crystal defects, impurities, NSLS, ALS, CZT

R10-3: Micro-Bump Connections for 3D Stacked Detectors and Sensors

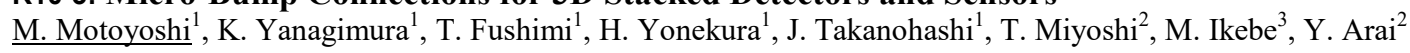

${ }^{1} H Q$, Tohoku-MicroTec Co., Ltd., Sendai, Japan

${ }^{2}$ Institute of Particle and Nuclear Studies, High Energy Accelerator Research Organization, Tsukuba, Japan

${ }^{3}$ Graduate School of Information Science and Technology, Hokkaido University, Sapporo, Japan

A 3D IC structure with micro-bump connections enables considerable improvement of the time-space resolution for detectors and array sensors. This paper presents two types of Au micro-bump connections. An improvement with respect to the bump junction resistance of a $3 \mu \mathrm{mf}$ Au conical bump is realized by adding pre-treatment for removing organic contamination. The bump resistance is reduced to $0.25 \mathrm{O}$, and this value is less than $1 / 20$ of that reported previously. A cylindrical bump has also been developed for bonding with soft and fragile compound semiconductor materials such as CdTe. Heterogeneous stacking with CdTe and Si device TEGs are successfully bonded with low-pressure thermal compression bonding. The same level of bump resistance as that in the case of $\mathrm{Si}-\mathrm{Si}$ bonding is obtained.

\section{R10-4: The New Generation 3DCZT High Resolution Detector with Improved Position Sensitivity Developed at DTU Space \\ I. Kuvvetli, C. Budtz-Jørgensen}

Astrophysics, DTU Space National Space Institute, Technical University of Denmark, Kngs. Lyngby, Denmark

At DTU Space we have developed a high resolution three dimensional (3D) position sensitive CZT detector for high energy astronomy. The design of prototype 3D CZT detectors $(20 \mathrm{~mm}$ x $20 \mathrm{~mm}$ x $5 \mathrm{~mm}$ ) is based on the CZT Drift Strip detector principle. Detectors contain 12 drift cells, each comprising one collecting anode strip with 4 drift strips, biased such that the electrons are focused and collected by the anode strips. The anode pitch is $1.6 \mathrm{~mm}$. The position determination perpendicular to the anodes, the $\mathrm{X}$-direction, is performed using a novel interpolating technique from a/c coupled drift strips. The position determination along the detector depth direction, Y-direction, is made using the depth sensing technique. The position determination along the anode strips, Z-direction is made with the help of 10 cathode strips orthogonal to the anode strips. The detector provides 3D position as well as high resolution energy information and is therefore a well suited candidate e.g. as a Compton telescope detector. The 3D CZT prototype was characterized with a fine collimated high-energy (Cs137) monochromatic beam $(0.15 \mathrm{~mm} \times 40 \mathrm{~mm})$ using digitizer in order to analyze the pulse shapes of the bi-polar signals from all noncollecting electrodes (drift strips). The pulse shapes provide information for the interaction position and thus make it possible for $3 \mathrm{D}$ correction for the residual electron trapping and also allow detection and characterization of multiple interaction events occurring in the same detector cell, e.g. a Compton interaction. We performed 1D position scans in the XY- XZ- and ZY-plane 
with beam energy of $662 \mathrm{keV}$. We measured position resolutions of $<0.4 \mathrm{~mm}$ FWHM in the $3 \mathrm{D}$. The measured energy resolution was $\sim 6 \mathrm{keV}$ FWHM at $662 \mathrm{keV}$. The electronic noise of the detector setup was $\sim 4 \mathrm{keV} \mathrm{FWHM}$. The 3D position capability allowed the energy response to be corrected for position dependent differential nonlinearities throughout the detector volume.

\author{
R10-5: Discrimination of Single-Site and Multi-Site Events in CZT-CPG Detectors for the COBRA \\ Experiment \\ $\underline{\text { S. Zatschler }}$
}

Institute of particle and nuclear physics, TU Dresden, Dresden, Germany

On behalf of the COBRA Collaboration

The aim of the COBRA experiment is to search for the existence of neutrinoless double beta-decay (0?ßß-decay) with CZT semiconductor detectors. One key instrument to reach the required half-life sensitivity of several $10^{26}$ years is to differentiate between signal and background events. The 0 ? ßß-signal is expected to be almost always a single-site event (SSE) within only one detector. Hence, all multi-site events (MSEs) can be vetoed as background. Recently, an identification routine has been developed to distinguish between signal-like SSEs and background-like MSEs based on pulse-shape analysis. For the verification of this method a laboratory experiment was performed which makes use of coincident Compton scattering to build up a library of definite SSEs. The results of this investigation, the analysis behind it as well as the application of the MSE identification algorithm will be presented.

The COBRA experiment is funded by the German Research Foundation DFG.

\title{
R10-6: Spatially Resolved Investigation of Large CdZnTe Detectors with a Coplanar Quad-Grid
} $\underline{\text { R. Temminghoff }}$

Experimental Physics 4, TU Dortmund, Dortmund, Germany

On behalf of the COBRA Collaboration

The COBRA experiment uses CdZnTe coplanar-grid (CPG) detectors to search for neutrinoless double beta-decay. The current demonstrator phase with 64 detectors with a volume of $(1 \times 1 \times 1) \mathrm{cm}^{3}$ showed that a long-term low-background experiment using CdZnTe is feasible. In the next stage, $(2 \times 2 \times 1.5) \mathrm{cm}^{3}$ detectors with a coplanar quad-grid (CPqG) will be used. The use of larger devices will not only lead to a higher detection efficiency, but can also help to suppress surface related backgrounds and allow for easier instrumentation. A CPqG is used to maintain good energy resolution. Currently several detectors are investigated in various laboratory setups at TU Dortmund and TU Dresden. Among the techniques used are spectroscopic measurements with several sources, measurements of the leakage currents and spatially resolved scanning with a collimated $137 \mathrm{Cs}$-source. A beam with a diameter of about $1 \mathrm{~mm}$ is used to map the detector performance depending on the source position. With this technique we found that the $\mathrm{CPqG}$ does not lead to severe non-uniformities even in parts of the detector where two anode grids are adjacent. We were also able to verify the reconstruction of the interaction depth in $\mathrm{CPqG}$ devices and found that its performance is comparable to single grid detectors. We conclude that $(2 \times 2 \times 1.5) \mathrm{cm}^{3} \mathrm{CPqG}$ detectors made from CdZnTe are suitable to be used in the future of COBRA.

COBRA is funded by the German Research Foundation DFG

R10-7: Conception and Validation of a Virtual Coplanar Grid for a 11x11 Pixelated CZT Detector $\underline{\text { R. Espagnet }}^{1}$, L. Lechippey ${ }^{1,2}$, A. Frezza ${ }^{1}$, J.-P. Martin ${ }^{3}$, L.-A. Hamel ${ }^{3}$, P. Després ${ }^{1}$

${ }^{I}$ Département de physique, de génie physique et d'optique, Université Laval, Québec, Qc, Canada

${ }^{2}$ CEA saclay, Institut national des sciences et techniques nucléaires, Gif-sur-yvette, France

${ }^{3}$ Département de physique, Université de Montréal, Montréal, Qc, Canada

Due to the low mobility of holes in CZT, commercially available detectors with a relatively large volume typically use a pixelated anode structure. They are used mostly in imaging applications and often require a dense electronic readout scheme. These large volume detectors are also interesting for high-sensitivity applications and a CZT-based blood gamma counter was developed from a $20 \times 20 \times 15 \mathrm{~mm}^{3}$ crystal available commercially and having a $11 \times 11$ pixelated readout scheme. A method is proposed here to reduce the number of channels required to use the crystal in a high-sensitivity counting application, dedicated to pharmacokinetic modeling in PET and SPECT in this case. Inspired by a classic coplanar anode, an implementation of a virtual coplanar grid was done by connecting the 121 pixels of the detector to form alternating bands. The layout, the front-end electronics and the characterization of the detector in this 2-channel anode geometry is presented. The coefficients required to compensate for electron recombination in CZT were determined experimentally to improve the performance. The resulting virtual coplanar detector has an intrinsic efficiency of $30 \%$ and an energy resolution of $9 \%$ at $662 \mathrm{keV}$. The detector's response 
was linear between $80 \mathrm{keV}$ and $1372 \mathrm{keV}$. This suggests that large CZT crystals offer an excellent alternative to scintillation detectors for some applications, especially those where high-sensitivity and compactness are required.

\author{
R10-8: Improvement of Properties of CdZnTe Coplanar Grid Detector by Tuning Electric Field and Weighing \\ Potential

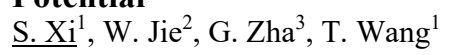 \\ ${ }^{\text {I}}$ State Key Laboratory of Solidification Processing, Northwestern Polytechnical University, xi'an, China \\ ${ }^{2}$ Key Laboratory of Radiation Detection Materials and Devices, Northwestern Polytechnical University, xi'an, China \\ ${ }^{3}$ Imdetek Co., Ltd, Xianyang, China
}

The Aim of this paper is to enhance the energy resolution of CdZnTe coplanar grid detector for Cs137 ? ray by tuning the hole collection and electron collection. The hole collection are tuned through changing the electric field in the near anode region. The electron collection are tuned by correcting the weighing potential of non collection region by weighing factor. The $\mu$ te can be evaluated by the weighing factor.

\title{
R11: Defects1
}

Thursday, Nov. 3 08:00-09:55 Schuman

R11-1: Laser Induced Transient Current Technique as a Powerful Tool to Determine Charge Transport Properties, Electric Field and Weighing Field Distribution in CdZnTe Detectors

$\underline{\text { A. Zappettini }}{ }^{1}$, A. Santi ${ }^{2}$, M. Bettelli ${ }^{1}$, G. Piacentini ${ }^{2}$, M. Zanichelli ${ }^{2}$, M. Pavesi ${ }^{2}$

${ }^{I}$ IMEM-CNR, Parma, Italy

${ }^{2}$ Physics Dept, University of Parma, Parma, Italy

We have recently shown that , by the use of the laser Induced Transient Current Technique (LI-TCT), it is possible to determine, at the same time, charge transport properties (namely carrier lifetime and mobility) and the electric field distribution in the direction parallel to the applied electric field direction, in detectors with two planar contacts. The method was founded on a procedure of minimization built up from the R-S theorem and few physical constraints. This last procedure was tested to determine electron transport properties and electric field distribution on a set of planar detectors with different thicknesses, and the results compared with the results obtained by other techniques. In this work we show that the method is applicable also to situations where a more complex contact geometry makes the induced charge affected also by the so-called weighing field. In these conditions, the current transients result to be affected not only by the transport parameters and by the electric field distribution, but also by the weighing field distribution. A method to deduce the weighing field is obtained and applied to characterize the weighing potential profile in the case of typical single charge carrier CdZnTe detectors. Moreover, results concerning the hole transport properties are also shown.

\section{R11-2: Analysis of Trapping and De-Trapping in CdZnTe Detectors by Pockels Effect} J. Franc, M. Rejhon, V. Dedic, R. Grill

$M F F$, Institute of Physics, Charles University, Prague, Czech Republic

We introduce a method of deep level spectroscopy in semi-insulating semiconductors demonstrated on detector-grade bulk $\mathrm{CdZnTe}$. The method is based on the measurements of time and temperature evolution of electric field in studied samples sensitive to a change of occupancy of deep levels. The measurement of the electric field is based on linear electro-optic (Pockels) efect using InGaAs avalanche photodiode with fast response. The internal electric field profile in studied samples significantly changes under various external conditions represented by application of the bias and pulsed illumination with below-bandgap light. From the knowledge of the electric field behavior and using standard analysis based on thermally induced transitions of electrons and holes from the deep levels to the conduction and valence bands, respectively, it is possible to get activation energies of the energy levels, their types (donor or acceptor) and corresponding capture cross-sections. This analysis determines the thermally activated transitions. We can distinguish the type of the transition based on the sign of the change of the space charge. If the internal electric field under the cathode increases it means that electron transitions from the deep level to the conduction band take place, because of the increase of positive space charge. On the other hand a decrease of the internal electric field under the cathode corresponds to electron transitions from the valence band to the deep level (de-trapping of holes). We identified four levels responsible for trapping and de-trapping of the charge carriers in the CZT material. The deep levels Ev $+0.40 \mathrm{eV}, \mathrm{Ev}+$ $0.77 \mathrm{eV}$ and $\mathrm{Ev}+0.94 \mathrm{eV}$ capture the photo-generated holes and thus form a positive space charge. Electrons are trapped at deep level Ec - 0:90 eV after application of the bias. We determined the Schottky barrier height of the CZT/Au $=0.91 \mathrm{eV}$ and we found the electron trap Ec $-0.65 \mathrm{eV}$ responsible for space charge limited current.

\section{R11-3: Investigation of the Electric Field of CdZnTe and CdZnTeSe Radiation Detectors with Different Electrode Materials and Configurations}


G. Yang $^{1}$, A. E. Bolotnikov ${ }^{1}$, Y. Cui ${ }^{1}$, G. S. Camarda ${ }^{1}$, A. Hossain ${ }^{1}$, U. N. Roy ${ }^{1}$, R. Gul ${ }^{1}$, S. Sun ${ }^{1,2}$, R. B. James ${ }^{1,3}$

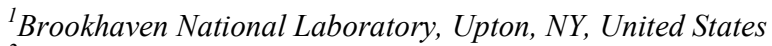

${ }^{2}$ Shanghai Institute of Technical Physics, Shanghai, PRC

${ }^{3}$ Savannah River National Laboratory, Aiken, SC, United States

$\mathrm{CdZnTe}(\mathrm{CZT})$ radiation detectors have attracted a sustained research interest due to their great potential for room-temperature radiation detection applications. More recently, a new quaternary compound semiconductor CdZnTeSe (CZTS) has been developed as another promising candidate material for these applications, mainly due to its potential to grow crystals with a lower concentration of material defects. An important parameter that substantially affects the transport behavior of electrical charge and, therefore, the performance of CZT and CZTS detectors, is the distribution of the internal electrical field. A uniform distribution is always desirable. Here, we use Pockels effect measurements as an approach to measure the change of the internal electric field of CZT and CZTS detectors, which are equipped with different electrode materials and configurations. The distribution of the internal electrical field can be changed significantly by the build-up of space charge, which could result in degrading the charge-collection properties, as well as causing polarization effects. We use an external light illumination to tune the internal electric field of CZT and CZTS detectors. The electrons and holes trapped at certain deep levels can be released due to the de-trapping energy provided by external light illumination of wavelength corresponding to the specific defect level, thus changing the distribution of space charges and modifying the internal electric field. We observed that the internal electric field behaves in a totally different way under the same illumination conditions, depending on the type of detector materials and the choice of electrode materials. From these findings, we note that the specific configuration of the external light illumination needs to be customized to CZT and CZTS detectors of different quality. Such optimization can have a big impact on the electric field and can be potentially used to focus the internal electric field.

R11-4: Characterization of Crystallographic Defects in Detector-Grade CdTe-Based Crystals $\underline{\text { A. Hossain }}^{1}$, A. E. Bolotnikov ${ }^{1}$, G. S. Camarda ${ }^{1}$, Y. Cui ${ }^{1}$, R. Gul ${ }^{1,2}$, U. N. Roy ${ }^{1}$, G. Yang ${ }^{1}$, R. B. James ${ }^{1,3}$

${ }^{I}$ Brookhaven National Laboratory, Upton, NY, USA

${ }^{2}$ Alabama A\&M University, Normal, AL, USA

${ }^{3}$ Savannah River National Laboratory, Aiken, SC, USA

Large-scale deployment of CZT detectors mostly depends on the availability of high-quality crystals at an acceptable cost. Over the past decade, many problems have been identified and resolved, taking this emerging technology to a more advanced stage. The remaining critical defects in CZT crystals can mostly be attributed to stress-related sub-grain boundaries or micro-twins and crystallographic strains, which cause non-uniformities in the detector response and degrade the performance of the fabricated detectors, especially ones with high aspect-ratio. We employed various state-of-the-art techniques, e.g. high spatial resolution Xray spectroscopy, micro-diffraction and micro-fluoroscopy to identify and understand the roles of these defects in CZT and CZTS crystals. We have also chemically revealed the defects on the crystal's surfaces, analyzed the geometry, sizes, and densities of etch pits and correlated them with the nature of the crystal's defects. We evaluated the areal densities and distributions of the etch pits associated with dislocations and other crystallographic defects, as well as the elemental homogeneity using a scanning electron microscopy (SEM) combined with wavelength dispersive spectroscopy (WDS). In this presentation, we describe in detail our acquired data of the material defects and their influence on the detector's performance, and discuss possible resolutions.

\author{
R11-5: Influence of IR Depolarization on CdZnTe Spectroscopic Detector Operating under High Flux of X- \\ Rays

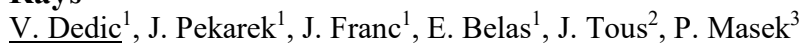 \\ ${ }^{I}$ Institute of Physics of Charles University, Prague, Czech Republic \\ ${ }^{2}$ Crytur Ltd., Turnov, Czech Republic \\ ${ }^{3}$ Institute of Technical and Experimental Physics, Czech Technical University, Prague, Czech Republic
}

CdZnTe detectors operating under high radiation fluxes usually suffer from polarization effect due to a screening of internal electric field by a positive space charge caused by photogenerated holes trapped at deep level. Previously, we showed an IR depolarization method by which it is possible to restore the electric field in indium doped CdZnTe detector using IR light with a wavelength ranging around $1200 \mathrm{~nm}$ due to an optical transition of valence electrons to the deep level decreasing the positive space charge present under high flux of X-rays. We studied a spectroscopic behavior of CdZnTe under X-ray flux up to $10^{6} \mathrm{photons} /\left(\mathrm{mm}^{2} . \mathrm{s}\right)$ generated by X-ray tube operated at $50 \mathrm{kV}-120 \mathrm{kV}$. Beside a presence of pile-up events negatively affecting measured spectra there was a significant negative influence of polarization of the detector blurring and shifting spectral data towards lower channels compared to low flux measurements. Using simultaneous illumination of the detector by depolarizing LED at $1200 \mathrm{~nm}$ it was possible to almost recover X-ray spectra originally deformed by polarization effect. X-ray spectra measured under low and high radiation fluxes with and without depolarizing LED at $1200 \mathrm{~nm}$ will be presented and the discussion will be supported by results of TCT measurements. 
R11-6: Space-Charge-Limited Photocurrent in Semi-Insulating Semiconductors

R. Grill, E. Belas, K. Ridzonová, J. Pekárek, J. Zázvorka, P. Praus, J. Franc, A. Musiienko, I. Vasylchenko, P. Höschl

Institute of Physics, Charles University, Prague, Czech Republic

The formation of space charge (SC) in semiconductors represents an important difficulty at their application for radiation detectors. The ordinary SC is built-up in biased samples equipped with blocking or injecting contacts due to the carrier's depletion or injection. The high photon flux entails the SC formation by the trapping of photo-excited carriers. The sub-band-gap illumination may be also used for the SC tuning by a direct trap-to-band excitation. While SC is mostly considered undesirable for the detector's performance, the possibility to study SC by multiple techniques enables us to identify principal charging entities, mostly deep defect levels, and analyze their properties and the possibility to suppress the $\mathrm{SC}$ and improve the quality of detectors.

In this contribution we report on the comprehensive research of SC formation by the measurement of the space-charge-limited photocurrent (SCLP). The principle of the method consists in the measurement of the photocurrent in planar samples and its extensive analysis within versatile setting of parameters - bias, illumination intensity and wavelength, and temperature. Both steady-state and pulse modes are used and combined. The theory of SCLP is revisited on simple models solved analytically and extended with refined approaches based on numerical simulations describing realistically the charge dynamics in the sample. Coupled one-dimensional drift-diffusion and Poisson's equations including different types of photo-excitation are solved in the steady state and in the pulsed regimes. Free carriers trapping/detrapping is described by the Shockley-Read-Hall model. Theoretical results are compared with experiment and the validity of the model is demonstrated. Properties of defect states responsible for SC formation are evaluated. We also critically address previous approaches used for the derivation of detector's properties by the photoconductivity measurements and define limits of their applicability.

\author{
R11-7: A Compensation Model for Material Resistivity and Electric Fields in Semi-Insulating CdTe Radiation \\ Detectors

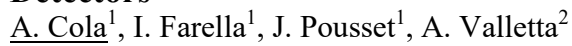 \\ ${ }^{I} I M M$, Unit Lecce, CNR, Lecce, Italy \\ ${ }^{2} I M M$, Unit Rome, CNR, Rome, Italy
}

A compensation model based on a single dominant deep level is proposed for semi-insulating $\mathrm{CdTe}: \mathrm{Cl}$. The model is able to account for both the thermal behaviour of the electrical resistivity and the stationary electric field in Ohmic and Schottky radiation detectors. Within the model, we have developed an ambipolar analysis able to describe resistivity measurements as a function of temperature, controlled by a single activation energy. The non uniform electric field distributions in the Ohmic $\mathrm{Pt} / \mathrm{CdTe} / \mathrm{Pt}$ and Schottky $\mathrm{In} / \mathrm{CdTe} / \mathrm{Pt}$ detectors are discussed considering their hole injecting and blocking contacts, respectively and successfully compared with numerical results. Experimental data are consistent with an energy level $0.725 \mathrm{eV}$ above the valence band, a concentration in the range $2-4 \times 10^{12} \mathrm{~cm}^{-3}$, comparable electron/hole capture cross sections, and a compensation ratio $\mathrm{R}^{\sim} 2.1$. The approach elucidates the role of electrical contacts and compensation levels in controlling the electric fields in radiation detectors based on compensated semi-insulating crystals.

R12: Special Tribute to Paul Siffert

Thursday, Nov. 3 10:30-12:00 Schuman

R12-1: Growth of (Cd,Zn)Te

M. Fiederle, A. Fauler

Freiburger Materialforschungszentrum, Freiburg, Germany

The Travelling Heater Method THM is a solution growth technique, which allows the preparation of bulk monocrystalline mixedcompound semiconductors. Since the process is conducted below the congruent melting point of the semiconductor, relatively low temperatures are involved. These low temperatures have the important advantages in reduction of contamination and vapour pressure related problems, reduction of intrinsic defects like vacancies, interstitials and antisites. This leads to bulk crystals with improved dopant homogeneity, reduced dislocation densities, and excellent electrical properties. THM provides a uniform crystal composition of ternary materials such as $(\mathrm{Cd}, \mathrm{Zn}) \mathrm{Te}$ that cannot be achieved by the melt techniques. Recently, the travelling heater method is gaining acceptance as a viable production process for the growth of large diameter single crystals of CdTe and $(\mathrm{Cd}, \mathrm{Zn}) \mathrm{Te}$ by commercial companies.

\author{
R12-2: Developments of Germanium Spectroscopy Detectors :from Pioneering Times to the Current Most \\ Challenging Applications \\ M. Fiederle ${ }^{1}$, M. O. Lampert ${ }^{2}$ \\ ${ }^{I}$ Freiburger Materialforschungszentrum, Freiburg, Germany \\ ${ }^{2}$ GM Lingolsheim Specialty detector site, Canberra, Tanneries Cedex Lingolsheim, France
}


We will explore the significant milestones in the development of Germanium crystals and of technologies for Germanium spectroscopy detectors and present some examples of scientific and industrial breakthroughs enables by these developments"

\section{R12-3: 30 Years of Cdte and Cdznte Detectors Medical Applications}

\section{Scheiber}

nuclear medicine, Hospices Civils de Lyon, Lyon, France

Medical applications of CdTe and/or CdZnTe RTSD were initiated in the 90's as nuclear probes for sentinel node detection (oncology) owing to their good spectrometric properties and small size compared to conventional INa probes. Almost 20 years of joint effort of the scientific community were needed to allow routine dedicated nuclear mï $i^{1 / 2}$ dicine devices (gamma-cameras) to be available. Today more than 100 dedicated ultra-fast cardiac systems are running in the world some mammography dedicated systems as well. Recently, april 2016 the first large field whole body gamma-camera has been commercialized. The design of this new generation is one step to the next generation which is already studied in the R\&D labs. Moreover other medical Imaging applications have emerged in parallel such as the spectral computed tomograph. The talk will review the CdTe and CdZnTe story which is still on its way to help the medical community to improve patient care.

R12-4: Crystals Based on (Cd,Mn)Te with Mg and Se as Materials for X- and Gamma Ray Detectors. A. P. Mycielski ${ }^{1}$, D. D. Kochanowska ${ }^{1}$, M. D. Witkowska-Baran ${ }^{1}$, M. D. Szot $^{1}$, M. P. Moszynski ${ }^{2}$, J. D. Domagala ${ }^{1}$, R. D. Jakiella ${ }^{1}$, B. E. Witkowska ${ }^{1}$, W. E. Kaliszek ${ }^{1}$

${ }^{I}$ Institute of Physics, Polish Academy of Sciences, Warsaw, Warsaw, Poland

${ }^{2}$ National Centre for Nuclear Research, Swierk, Poland

The $(\mathrm{Cd}, \mathrm{Mn})$ Te based crystals could be materials for $\mathrm{X}$ - and gamma ray detectors. The (Cd,Mn)Te, $(\mathrm{Cd}, \mathrm{Mn}, \mathrm{Mg}) \mathrm{Te}$ and $(\mathrm{Cd}, \mathrm{Mn})(\mathrm{Te}, \mathrm{Se})$ crystals were studied. Investigated crystals were grown from stoichiometric melt. Part of the crystals were grown with dopant of vanadium or chlorine. The magnesium and selenium content used in the growth process was in the range from $0,01(1018 \mathrm{~cm}-3)$ to 5 at. \%. After crystallization by the Bridgman method the material was annealed in cadmium atmosphere. Annealing reduces by several orders of magnitude: the $\mathrm{Cd}$ vacancies (VCd) concentration, the tellurium inclusion/precipitation concentration (Infrared microscopy observations) and the concentration of structural defects determined from the Etch Pit Density (EPD). Annealing in cadmium vapors of the samples from stoichiometric ingots causes an increase in the sample resistivity from $105 ? \mathrm{~cm}$ to $108 ? \mathrm{~cm}$. We will present results (resistivity maps, $\mu$ ? parameter maps) provided by the contactless method, Time Dependent Charge Measurements (TDCM), for the monocrystalline samples. For the investigated crystals the results of the Low Temperature Photoluminescence (PL) measurements will be presented. The Full Width at Half Maximum (FWHM) of the rocking curve measured on the samples will be shown. The ohmic contacts to the samples were done by a deposition in high vacuum of an amorphous layer of ZnTe:Sb covered by thin layer of Au. The current-voltage (I-V) characteristics for the investigated samples will be shown. The drift velocity and $\mu$ ? product obtained on the samples by the measurements of the Time of Flight (TOF) will be presented. The possibility of using the samples as detectors will be tested. Some of the results from different radiation sources (93Mo - KX - 16,6 keV, 133Ba - KX - 30,9 keV, 241 Am - 59,6 keV, $57 \mathrm{Co}-$ $122 \mathrm{keV}, 133 \mathrm{Ba}-276$ and $302 \mathrm{keV}$, and $137 \mathrm{Cs}-662 \mathrm{keV}$ ) will be presented.

\section{R13: Imaging2}

\section{Thursday, Nov. 3 14:00-15:50 Schuman}

R13-1: High Frame Rate CdTe Flat Panel Detector for Application to Material Identification CT

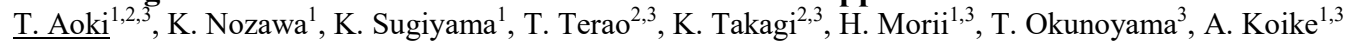

${ }^{1}$ Research Institute of Electronics, Shizuoka University, Hamamatsu, Shizuoka, Japan

${ }^{2}$ Graduate School of Science and Technology, Shizuoka University, Hamamatsu, Shizuoka, Japan

${ }^{3}$ ANSeeN Inc., Hamamatsu, Shizuoka, Japan

We have developed high-frame rate CdTe flat panel detector. The pixel pitch is $100 \mathrm{um}, \sim 50 \mathrm{~mm}$-square imaging area, and 100 frame / $\mathrm{s}$ with 10 line-pair / $\mathrm{mm}$ resolution driven by current integrated mode. This CdTe detector device is thin with USB3.0 interface, therefore, we can apply to portable imaging, embedded devices for inspection machines. Motion images can be detected in X-ray penetration imaging, and it is suitable for non-destructive CT imaging. Moreover, the detector can be used for line-sensor with quasi-energy discrimination put 90-degree angle of X-ray beam for material identified CT system. We can estimate quasi-energy of X-ray from depth of X-ray penetration in flat panel detector without photon counting. It can not measure exact energy of photon, but we can demonstrate easy material identification in this CT system.

R13-2: Multimodal Analysis of the Cultural Herritage Artefacts Utilizing Computed Tomography and X Ray Flourescence Imaging

D. Vavrik ${ }^{1}$, J. Jakubek ${ }^{1}$, J. Zemlicka ${ }^{2}$ 
${ }^{I}$ Institute of Theoretical and Applied Mechanics, v. v. i., Prague, Czech Republic

${ }^{2}$ Institute of Experimental and Applied Physics, CTU in Prague, Prague, Czech Republic

Multimodal device combining X-ray CT (XCT) and X-ray Fluorescence (XRF) imaging instrumentation was developed for inspection of valuable cultural heritage artefacts. Dual source XCT scanner with adjustable irradiation geometry and exchangeable X-ray imagers (standard Flat panels Perkin Elmer or outstanding 6.5 MPx large area single photon counting detector) was equipped with large goniometer holding XRF camera. CT rotational stage is installed inside of the goniometer aperture. This arrangement allows finding optimal angle between X-ray beam and XRF camera axes, while CT data acquisition can be done concurrently with XRF imaging. Moreover, XRF camera can be placed behind inspected object to record small angle scattering images, which are beneficial for inspection of the material with high granularity (the ceramic for instance). XRF camera consists of two Timepix detectors with 300 um thick Silicon and 1000 um thick CdTe sensor respectively. It brings wide energy range sensitivity $3-1400 \mathrm{keV}$, with energy resolution $3 \mathrm{keV}$ (standard deviation for $60 \mathrm{keV}$ photons). Data acquisition of $\mathrm{XRF}$ camera is realized via USB 2.0 port with frame rate up to $30 \mathrm{fps}$. Results demonstrating beneficial fusion of the CT and $\mathrm{XRF}$ data for cultural heritage artefacts inspection will be presented.

The research was supported by Project No. DG16P02M022 of the Applied Research and Development of National and Cultural Identity Programme.

\author{
R13-3: 3D Non-Destructive Fluorescent X-Ray Computed Tomography (FXCT) with a CdTe Array \\ C. Yoon, Y. Kim, W. Lee \\ Health \& Environmental Science, Korea Univ., Seoul, Korea
}

In this study, we develop 2D and 3D tomographic non-destructive tests for detecting fluorescence X-rays using a 2D CdTe array. Experiments were conducted using various phantoms and image-reconstruction methods. In general, conventional computed tomography analyzes materials according to attenuation coefficients and is highly dependent on the densities of the materials; thus, it is difficult to discriminate materials that have similar densities, even if their atomic numbers differ. In our research, materials were exposed to X-rays, and both conventional transmission images and fluorescent X-ray images were reconstructed using the information from characteristic X-rays detected using a 2D CdTe planar detector array. Since atoms have their own characteristic X-ray energies, our system was able to discriminate materials of the same or similar density if the materials had different atomic numbers. Additionally, the transmission and characteristic X-ray images were combined to analyze the positions, densities, and atomic numbers of the unknown materials. Several image-reconstruction methods were applied; the reconstructed images were compared to determine an optimized algorithm for fluorescence X-ray computed tomography. If pitch between detector pixel size and collimator hole size did not match, shadow of collimator would be shown in reconstructed images. For this reason, image quality of reconstructed images was decreased. Therefore, we conducted experiments about effect of collimator moving.

R13-4: ORIGAMIX: Current State and Results of a Technology Transfer Project from High Energy Astrophysics Space Grade Detector to Nuclear Safety Gamma Camera

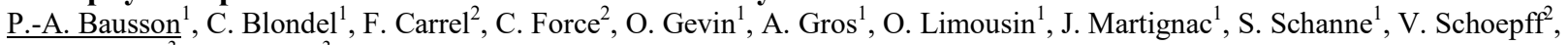
M.-C. Vassal ${ }^{3}$, F. Soufflet ${ }^{3}$

${ }^{I}$ DRF/IRFU, CEA Saclay, GIF-SUR-YVETTE, FRANCE

${ }^{2}$ DRT/LIST/D2MI, CEA Saclay, GIF-SUR-YVETTE, FRANCE

${ }^{3} 3 D$ PLUS, BUC, FRANCE

After Chernobyl and -sadly recent- Fukushima nuclear accidents, nuclear safety became a major issue. Localization and spectroidentification of radioactive sources can give crucial information about the current accident's stage and how to act on it. Besides, astrophysics does ask for spectro-imaging instruments development for years, and now such technologies are mature and ready to serve other domains. Our team is currently in the process of using a high-energy photon spectro-imaging technology initially designed for astrophysics, the CALISTE-HD camera, for use in nuclear safety applications. The ORIGAMIX project is funded thanks to a nuclear safety project call from the Investissements d'Avenir program. Its aim is to develop a commercial grade nuclear safety camera, able to localize and spectro-identify radioactive sources in nuclear accident scenes. In this paper, we will present the result of ORIGAMIX development and we will demonstrate the excellent performance in terms of spectral response into a portable coded mask aperture system.

R13-5: Material Discrimination and Imaging Improvement Using High Count Rate X-Ray CdTe Spectrometric Detector for Non-Destructive Testing and Security Applications

D. Perion, E. Gaboriau-Borissenko, P. Radisson

MULTIX, Moirans, France 
The requirements of security screening and Non-Destructive Testing (NDT) applications become more stringent, the demand for high-performance radiation detectors has intensified and interest in multi-energy x-ray imaging methodologies has grown. To answer to these above application demands, Multix has developed a linear array spectrometric detector called ME100, able to provide a fully resolved energy spectrum over 128 energy bins, in real time and under high photon flux. The aim of this work is to present the results obtained with theME100 detector showing the benefits of using a multi-energy for both security and NDT applications. Three examples will be presented the Separability Criterium according to the number of energy bands, improvement in material discrimination as well as highly precise fat in meat quantification for non-destructive testing (NDT). The detector performances used for this study will also be detailed.

R13-6: An Energy-Independent Gamma Camera

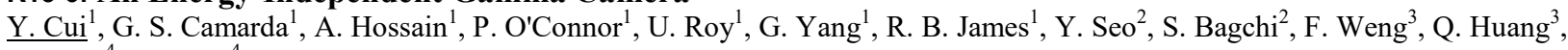
Z. Deng ${ }^{4}$, Y. Chen ${ }^{4}$

${ }^{I}$ Brookhaven National Laboratory, Upton, NY, USA

${ }^{2}$ University of California at San Francisco, San Francisco, CA, USA

${ }^{3}$ Shanghai Jiao Tong University, Shanghai, Shanghai, China

${ }^{4}$ Tsinghua University, Beijing, Beijing, China

Conventional single photon emission computed tomography (SPECT) system requires changes of collimators for different gamma-ray photon energies and different purposes of studies. To eliminate this time-consuming procedure, we are developing an energy-independent SPECT system. This technology is based on high energy-resolution cadmium zinc telluride (CZT) detectors, customized application-specific integrated circuit (ASIC), innovative collimator design, and ultrafast graphics processing unit (GPU) based image reconstruction. Recently we finished the design of a new 128-channel low-power photon-counting ASIC for this application. As a proof-of-concept, we designed and assembled a gamma camera coupled to a smaller version of the novel collimator. The camera has a field of view (FOV) of $5 \times 5 \mathrm{~cm} 2$ with $1.6-\mathrm{mm}$ pixel. The whole system is controlled by a Cypress FX3 microcontroller and communicates with computer via USB3.0 protocol. In this presentation, we will introduce the ASIC and camera system design and present test results demonstrating its energy-independent imaging capability.

R13-7: Metrological Characterization of the GAMPIX Gamma Camera

F. Carrel, G. Amoyal, T. Branger, C. Force, H. Lemaire, V. Lourenco, V. Schoepff

CEA LIST Institute, CEA, Gif-sur-Yvette, France

Localization of radioactive hot spots is a major issue for several applications related to the nuclear industry (NPP measurements, decommissioning applications, post-accidental events like Fukushima or Homeland Security applications). Gamma cameras are powerful tools to address this localization challenge. These systems enable to superimpose a gamma-ray image with a visible image in order to locate radioactive hot spots present in their field-of-view. Compton or coded aperture gamma-ray imagers were developed by several research teams in the past years and recently industrialized (for instance, iPIX by CANBERRA, POLARIS by H3D and ASTROCAM by Mitsubishi). In addition to the qualitative information provided by gamma cameras, a quantitative information on the dose rate generated by a given hot spot can be extracted from the gamma-ray pictures. The latter can be very useful to define intervention scenarios for decommissioning operations and minimize the dose received by the first responders. For this reason, performances of gamma cameras according to the nature and geometry of the source need to be carefully calibrated to provide an accurate dose rate value. Accuracy and uncertainties associated to dose rates provided by gamma camera were not extensively considered in the past literature. In the frame of the METRODECOM project, an extensive study was initiated to validate quantitative performances of the GAMPIX system in a metrological way. Different reference sources were especially produced by the Henri Becquerel National Laboratory. In the frame of this study, punctual or extended sources were produced to validate the GAMPIX performances on a large range of applications. Moreover, as GAMPIX is a coded aperture imager, impact of the decoding algorithm on the results will also be studied (correlation function or specific ML-EM approach). This article gives an overview of experimental results obtained in the frame of the METRODECOM project.

\section{R14: Detector Devices2}

Thursday, Nov. 3 16:30-18:10 Schuman

R14-1: GAMPIX Gamma Camera: Past, Present and Future

V. Schoepff, F. Carrel, C. Force, O. Gal, M. Gmar, F. LAINi i $i \frac{1}{2}$, H. Lemaire

CEA, LIST, 91191 GIF-SUR-YVETTE Cedex, FRANCE

From radiation protection of operational workers in Nuclear Power Plants to dismantling of installations, where operating records are not always well-preserved, the nuclear industry is highly concerned by the mapping of radiological activities. In this context, a great interest is shown for gamma cameras. These systems allow the visual localization of gamma hot spots in a remote scene, and endless developments have been carried out in this technologically-challenging field over the past decades. The recent 
accident at Fukushima Daiichi NPP, combined with the constant terrorist threat and the subsequent eventuality of a malevolent use of radiological material, significantly intensified researches in gamma imaging in the last few years. From the early 1990 s, CEA carried out researches on first generation of gamma cameras, based on scintillator technology. These gamma cameras were proven efficient but their size and weight were found out perfectible for easy deployment on site. In the 2000s, the technological breakthrough generated by the use of semi-conductor sensors led to a significant decrease in weight and size of gamma imaging systems, along with an improvement of performances and ease-of-use. Imagers resulting from these developments are called second generation gamma cameras. Constant evolution of radiological detection electronics and ASICS opened new research paths. Current electronics along with pixelated CdTe or CdZnTe sensors allow implementing spectrometric capabilities to imaging systems. These developments enable not only the localization of hot spots but also an identification of radionuclides in the imaged scene. This work leads to third generation gamma cameras, also known as imaging spectrometers. Finally, the use of a combination of both coded-mask method and Compton imaging is currently being studied. Such a technique should result in new improvements, especially in terms of sensitivity, over the whole range of energy covered by gamma cameras.

Authors want to acknowledge colleagues from CEA DEN, CEA Tech LETI and CEA DRF/IRFU for the fruitful collaborations that resulted in important technological advances in the frame of gamma imaging developments. A special acknowledgment to CANBERRA for their support in developing state of the art systems as part of common Partnership Agreement with CEA LIST. Authors also acknowledge Medipix Collaboration, and especially CERN development team, for the continuous work on detection chips that allow technological breakthrough in gamma imaging field. Part of this work was carried out in the frame of the ANR/RSNR Project ORIGAMIX, partially funded by a Grant from the French Government, coordinated by the French National Research Agency as part of the "Investissements dâ€ ${ }^{\mathrm{TM}}$ Avenir" program, under the reference ANR-11-RSNR-16.

R14-2: CZT Detector Assemblies for OEM Applications in Medical, Security, and Nuclear Power Markets J. W. Hugg ${ }^{1}$, B. W. Harris ${ }^{1}$, A. Emerick ${ }^{1}$, S. Soldner ${ }^{1}$, F. Walker ${ }^{2}$, D. Kuhn ${ }^{2}$, B. McVay ${ }^{1}$, J. Samstag ${ }^{1}$, M. Prokesch ${ }^{1}$, H. Li $^{1}$, A. Cherlin ${ }^{3}$, A. Sundaram ${ }^{1}$, M. Reed ${ }^{1}$, C. Jones ${ }^{1}$, H. Chen ${ }^{1}$

${ }^{1}$ eV Products division, Kromek USA, Saxonburg, PA, USA

${ }^{2}$ Nova R\&D division, Kromek USA, Riverside, CA, USA

${ }^{3}$ Kromek Group, PLC, Sedgefield, County Durham, UK

We have consistently grown by the traveling heater method (THM) large commercial quantities of high quality large singlecrystal $\left(>700 \mathrm{~cm}^{3}\right)$ CZT for fabrication of radiation sensors used in medical, homeland security, and nuclear power applications. Our custom integration of CZT detectors with advanced ASICs and readout electronics improves system performance. In some applications, our software becomes a key ingredient for the success of the OEM customer. We have designed many customized detector assemblies for OEM customers and will discuss the performance characteristics of representative examples.

In the medical application field, intra-operative probes, bone mineral densitometry (BMD), SPECT, and CT applications require differentiated CZT characteristics and matched electronics designs. In the homeland security field, linear array baggage scanning, $\mathrm{x}$-ray diffraction, focal-plane arrays, and CT are example applications to be discussed. In the nuclear power field, high resolution spectroscopy and isotope identification are essential capabilities. There are very few customers whose needs are satisfied with simple CZT sensors that they must themselves integrate into full detection systems. Most customers rely upon us to design and manufacture the advanced CZT detector assemblies for their OEM applications.

\section{R14-3: High Bias Voltage CZT Detectors for High-Flux Measurements}

N. Zambelli ${ }^{1}$, L. Abbene ${ }^{2}$, G. Gerardi ${ }^{2}$, A. A. Turturici ${ }^{2}$, G. Benassi ${ }^{1}$, M. Bettelli ${ }^{3}$, F. Principato ${ }^{2}$, A. Zappettini ${ }^{3}$

${ }^{I}$ due2lab s.r.l., Reggio Emilia, Italy

${ }^{2}$ Dipartimento di Fisica e Chimica, University of Palermo, Palermo, Italy

${ }^{3}$ IMEM/CNR Parma, Parma, Italy

In this work, we present the results of electrical and spectroscopic investigations on THM grown CZT detectors characterized by new gold electroless contacts (due2lab and IMEM-CNR, Parma, Italy). The detectors ( $3 \mathrm{~mm}$ thick) allow very low leakage currents at high bias voltages even at room temperature: $29 \mathrm{nA} / \mathrm{cm} 2\left(\mathrm{~T}=25 \mathrm{i}_{i} 1 / 2 \mathrm{C}\right)$ at $2000 \mathrm{~V}$, making them very attractive for high flux X-ray measurements, where high bias voltage operation is required. The spectroscopic response at different temperatures and fluxes, will be measured. Performance improvements by using digital pulse height and shape analysis will be also presented.

R14-4: Performance Assessment of CdZnTe Detectors with ZnO:Al Contacts $\underline{\text { U. N. Roy }}{ }^{1}$, G. Camarda ${ }^{1}$, Y. Cui ${ }^{1}$, R. Gul ${ }^{1}$, A. Hossain ${ }^{1}$, G. Yang ${ }^{1}$, P. Vanier ${ }^{1}$, R. James ${ }^{1}$, A. K. Pradhan ${ }^{2}$, R. Mundle ${ }^{2}$, M. Roul $^{2}$, J. Skuza ${ }^{2}$

${ }^{I}$ Nonproliferation and National Security, Brookhaven National Laboratory, Upton, NY, US

${ }^{2}$ Department of Engineering \& Center for Materials Research, Norfolk State University, Norfolk, VA, US 
The poor adhesion of common metals to $\mathrm{CdZnTe}(\mathrm{CZT}) / \mathrm{CdTe}$ surfaces has been a long-standing challenge for radiation detector applications. In addition to this problem, there is a large mismatch of the coefficients of thermal expansion (CTE) between the metal contact and CdZnTe, which exerts thermal stress at the metal/CZT interface, causing mechanical degradation of the contact. To form a reliable and stable interface, the contact material should have excellent adhesion and a close match of the coefficients of thermal expansion with $\mathrm{CZT} / \mathrm{CdTe}$. We found $\mathrm{ZnO}: \mathrm{Al}$ (AZO) to be a highly suitable contact material for $\mathrm{CdZnTe} / \mathrm{CdTe}$ crystals used for radiation detector applications. ZnO has a close match of its CTE with CdTe, and it offers higher hardness compared to the conventional metals used for contacts. Due to the oxide nature of the contact, the AZO can assure better interface adhesion with $\mathrm{CdTe} / \mathrm{CZT}$. The presentation will compare the charge transport characterization of CZT detectors with gold contacts and detectors with AZO contacts. An assessment of device performance will also be discussed.

R14-5: Hybrid Direct X-Ray Detectors

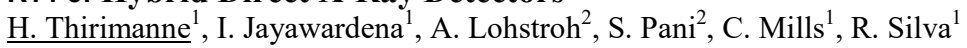

${ }^{I}$ Advanced Technology Institute, University of Surrey, Surrey, UK

${ }^{2}$ Department of Physics, University of Surrey, Surrey, UK

Semiconducting organic polymer X-ray detectors are emerging as flexible, low cost technology complementary to current inorganic and gas-filled X-ray detectors. However, such organic diodes suffer from low responsivity to X-rays due to the low Z number of active organic materials. In this work, novel 'inorganics-in-organics' hybrid semiconductor detectors using a conjugated polymer blend with the inclusion of high atomic number $(Z)$ nanoparticles have been optimized. The introduction of the high $\mathrm{Z}$ nanoparticles improves X-ray attenuation compared to the polymer blend alone. The semiconductor diodes exhibit a low dark leakage current proving its suitability as a solid-state X-ray detector. More importantly, the hybrid detectors lead to real time radiation monitoring with the added advantage of being processed at room temperature and potentially over large areas overcoming some of the limitations of current inorganic X-ray detectors.

R14-6: Demonstration of a High Spectroscopic Range CdTe Detector M. D. Wilson ${ }^{1}$, W. H. Baumgartner ${ }^{2}$, S. D. Christe ${ }^{2}$, D. D. Duarte ${ }^{1}$, J. A. Gaskin ${ }^{3}$, P. S. Grant ${ }^{4}$, L. L. Jones ${ }^{1}$, E. Liotti ${ }^{4}$, A. Lui ${ }^{4}$, M. Panessa ${ }^{2}$, M. Prydderch ${ }^{1}$, P. Seller ${ }^{1}$, A. Y. Shih ${ }^{4}$, S. Thomas ${ }^{1}$, M. C. Veale ${ }^{1}$

${ }^{I}$ STFC, Hawell, Oxfordshire, UK

${ }^{2}$ NASA Goddard Space Flight Centre, Greenbelt, MD, USA

${ }^{3}$ NASA Marshall Space Flight Centre, Huntsville, AL, USA

${ }^{4}$ Departement of Materials, University of Oxford, Oxford, UK

The HEXITEC detector has previously demonstrated fully spectroscopic imaging from $10 \mathrm{keV}$ to $200 \mathrm{keV}$ with sub $1 \mathrm{keV}$ (FWHM) energy resolution and count rates of $>106$ photons/s per 80x80 pixel module when used with $1 \mathrm{~mm}$ thick Schottky anode CdTe. The ability to tile the HEXITEC detectors on three sides with a dead space of $<2$ pixels and the pixel size of $250 \mu \mathrm{m}$ make it a good candidate to be used with the latest generations of grazing incident X-ray optics used for solar and astrophysics. Using a new compact data acquisition system, the low energy performance of the HEXITEC detector has been studied. X-ray fluorescence measurements showed that the detector was able to resolve the $2.3 \mathrm{keV}$ L-line from Mo when operating at room temperature and a bias of $-500 \mathrm{~V}$. The low energy measurements were made with the same conditions used to measure the $122 \mathrm{keV} \mathrm{Co-57} \mathrm{line} \mathrm{with} \mathrm{an} \mathrm{average} \mathrm{energy} \mathrm{resolution} \mathrm{of} 800 \mathrm{eV}$ FWHM. This ability to simultaneously measure the energy of Xrays from $2 \mathrm{keV}$ to $200 \mathrm{keV}$ will reduce the low energy shoulder in the spectral peaks following charge sharing corrections and allow X-ray fluorescence imaging to sample a broader range of elements from their $\mathrm{K}$ and $\mathrm{L}$ line emissions. This spectroscopic imaging performance over a high dynamic range is also important for space born applications. The low energy range will allow a greater number of solar flares to be observed in the proposed FOXSI and SuperHERO missions and the high dynamic range will increase the overlap in performance with other astrophysics instruments.

\section{R15: Materials2}

Friday, Nov. $4 \quad$ 08:00-10:00 Schuman

R15-1: X-Ray Transparent Multi-Channel Dosimeter Based on Organic Photodiodes and Plastic Scintillators for Real-Time IVR Monitoring

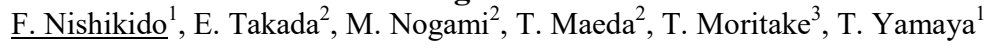

${ }^{I}$ National Institute of Radiological Sciences, Chiba, Japan

${ }^{2}$ National Institute of Technology, Toyama College, Toyama, Japan

${ }^{3}$ University of Occupational and Environmental Health, Fukuoka, Japan

Interventional radiology (IVR) is a medical subspecialty of radiology to realize image-guided surgical procedures using imaging modalities, such as x-ray fluoroscopy. However, skin injuries by prolonged x-ray exposure in duration of procedure have been reported. Therefore, monitoring of skin dose is desired in clinical sites for reduction of excessive x-ray exposure. We are 
developing a real-time dose distribution monitoring system for the IVR in which only radiolucent materials were in the field of view not to interfere with the IVR procedure. The organic photodiode (OPD) is mainly composed of organic materials, which has good transparency for x-ray. As a result, the detector using the OPDs combined with a plastic scintillator connected to thin flexible cable is expected to have transparency to x-rays. In this paper, therefore, we developed a new detector based on the OPD connected to the thin flexible cable for the real-time monitoring of skin dose distribution in IVR. The device structure of the OPD was plastic scintillator $(\mathrm{BC}-408,10 \mathrm{~mm} \times 10 \mathrm{~mm} \times 1 \mathrm{~mm}) / \mathrm{IZO}(100 \mathrm{~nm}) /$ PEDOT: PSS $(30 \mathrm{~nm}) /$ PCBM: P3HT $(200 \mathrm{~nm}) / \mathrm{Al}$ $(70 \mathrm{~nm})$. The size of a sensing area is $6 \mathrm{~mm} \times 4 \mathrm{~mm}$ on the plastic scintillator. The readout flexible cable was made of $25 \mu \mathrm{m}$ polyimide board and $12 \mu \mathrm{m}$ copper line. The length of the flexible cable is $30 \mathrm{~cm}$ and there are three readout electrodes to connect to the OPD electrode. We evaluated performance of the x-ray detectors for with a micro CT (R_mCT2, RIGAKU). As a result, the proposed $\mathrm{x}$-ray detector had a enough transparency to be used in the IVR dosimeter. In addition, we obtained the sufficient detector responses for the various tube current, tube voltage and irradiation angle for x-ray irradiation in real-time with $1 \mathrm{~s}$ interval.

\section{R15-2: Flexible, Ultra-Low Voltage, Fully Printed Radiation Detectors Based on Organic Semiconductors B. Fraboni \\ Department of Physics and Astronomy, University of Bologna, Bologna, Italy}

A new generation of ionizing radiation sensors based on organic materials is attracting a large attention exploiting appealing features of such as ease of processing, low power supply and mechanical flexibility. Moreover, the equivalence of the typical density of organic molecules to that of human tissue makes them very suitable for medical X-ray direct dosimetry. Our approach is based on the use of organic semiconductors as the active material for the direct detection of ionizing radiation, implementing real-time and room temperature operating sensors. We fabricated fully printed detectors based on micro-crystalline thin films of TIPS-pentacene deposited by drop casting onto flexible substrates and we assessed their high X-ray sensitivity (Figure 1). We investigated the direct X-ray photo-conversion process and we developed a kinetic model that gives an important insight into the physical process that leads low-Z organic materials to sucha highly sensitive response. This investigation allowed us to realize, by printing processes only, a flexible, $2 \times 2$ pixelated X-ray detector based on organic thin films, operating at very low voltage (below 1V) (Figure 2). Moreover, we assessed how detectors based on solution-grown Organic Semiconducting Single Crystals (OSSCs) are capable to detect charged particles with very good peak discrimination and an Hecht-like behavior. It has been possible to estimate the mobility-lifetime product obtaining values comparable to polycrystalline inorganic detectors [3]. The good detection efficiency and peak discrimination observed for charged particle detection in organic materials (hydrogen-rich molecules) are encouraging for their further exploitation in the detection of thermal and high-energy neutrons. These results open the way for novel flexible, large area and low voltage ionizing radiation detection systems, capable of providing quantitative and real time information on dose rate and spatial distribution of impinging radiation.

References [1] Fraboni, B. et al. Adv. Mater. 24, 2289-2293 (2012); Basirico, L. et al. IEEE Trans. Nucl. Sci. 62, 1791-1797 (2015); Ciavatti, A. et al. Adv. Mater. 27, 7213-7220 (2015). [2] Intaniwet, A et al. Nanotechnology 23, 235502 (2012); Mills, C. A. et al. J. Phys. Appl. Phys. 46, 275102 (2013). [3] Ciavatti A. et al. Appl.Phys.Lett.108 153301(2016)

R15-3: X-Ray Imaging with Scintillator-Sensitized Hybrid Organic Photodetectors

O. Schmidt, P. Büchele, S. F. Tedde, R. Fischer, J. Hürdler

Technology Center, Siemens Healthcare GmbH, Erlangen, Germany

Organic semiconductors provide exiting new opportunities for the realization of flat panel X-ray detectors as they can be processed from the solution phase on large areas at low cost. Here, we demonstrate a disruptive approach that improves the resolution of such X-ray imaging detectors by incorporating terbium-doped gadolinium oxysulfide (GOS:Tb) scintillator particles into an organic photodetector matrix. Light emission from the scintillating particles is detected by the surrounding organic photodetection matrix. Optical cross-talk is minimized as the particles are small $(1-5 \mu \mathrm{m})$ and the penetration depth of the photons is short $(100-500 \mathrm{~nm})$ compared to the pixel size of the X-ray detector. We will discuss various options to process the hybrid material on a 256x256 pixel detector and will demonstrate a working device with a thickness of $170 \mu \mathrm{m}$. From this demonstrator we estimate an electron-hole pair generation energy of $\sim 70 \mathrm{eV}$ and achieve a resolution of $4.75 \mathrm{lp} / \mathrm{mm}$ at a MTF of 0.2 .

We would like to acknowledge funding by German Federal Ministry for Education and Research (Project HOP-X, 13N12377) and the European Union (Project DiCoMo, 643920). Further we would like to thank all partners of the above mentioned projects for their contributions.

\section{R15-4: Performance of Thallium Bromide devices for Room Temperature Field Applications}

A. Datta, J. Fiala, P. Becla, K. Becla, S. Motakef

CapeSym, Inc., Natick, MA, US 
Thallium Bromide (TlBr) is a wide bandgap, compound semiconductor with high gamma-ray stopping power and promising physical properties. However, performance degradation and the eventual irreversible failure of $\mathrm{TlBr}$ devices can occur due to "polarization", caused by the electro-migration of $\mathrm{Tl}+$ and $\mathrm{Br}$ - ions to the electrical contacts across the device. With recent developments, we fabricated $\mathrm{TlBr}$ devices which gives reliable detection for more than a year at room temperature under continuous bias and gamma irradiation. These results are reproducible, and shows highly reliable performance of $\mathrm{TlBr}$ devices. The evidence and statistics of these developments will be demonstrated in the paper. Effects of ambient atmosphere and applied detector bias on the long-term detector performance are also explored. PRD prototypes were prepared using these devices. The performance of these PRDs are comparable to the COTS scintillator-based units, but with much higher efficiency and lower cost.

This work was supported in part by the U.S. Department of Homeland Security, Domestic Nuclear Detection office contract SBIR HSHQDC-15C-00044. This support does not constitute an express or implied endorsement on the part of the Government.

R15-5: Direct Gamma-Ray Detection with Strip TIBr Detectors for Nuclear Medicine Applications

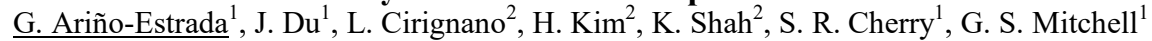

${ }^{I}$ Department of Biomedical Engineering, University of California Davis, Davis, CA, USA

${ }^{2}$ Radiation monitoring Devices, Inc., Watertown, Watertown, MA, USA

Thallium bromide $(\mathrm{TlBr})$ is a promising semiconductor material for use in positron emission tomography (PET) because of its very high detection efficiency, excellent spatial resolution, low noise, and high energy resolution. We report on the fabrication process and performance of two thin orthogonal strip $\mathrm{TlBr}$ detectors. $\mathrm{TlBr}$ ingots have been grown at Radiation Monitoring Devices (RMD) by the travelling molten zone method and show high material purity, high resistivity, and potential long term stability (up to one month continuously biased) at room temperature. The detectors have approximate dimensions of $10 \mathrm{~mm} \times 10$ $\mathrm{mm} \times 0.75 \mathrm{~mm}$ and on each side their electrodes are divided into 9 strips with $1 \mathrm{~mm}$ pitch $(0.9 \mathrm{~mm}$ width and $0.1 \mathrm{~mm}$ inter-strip space). Initial studies include the pulse height spectra of the detector strips for the radioactive sources Am-241 and Cs-137 at different bias voltages. A multi-channel readout board has each of the strips (18 per detector) coupled to a charge sensitive preamplifier. The preamp output is shaped with a 2-pole slow spectroscopy amplifier to measure the deposited energy. The same preamp output feeds a fast shaper and is used to obtain the trigger signal. Coincident events between the two sides of a detector are observed and used to localize the interaction region to the intersection of the orthogonal strips. The two detectors will be used to evaluate detector performance with $511 \mathrm{keV}$ gamma rays, with the eventual design goal of using a stack of thin detectors to build a three-dimensional detector block of T1Br material.

R15-6: Characterization of pin GaN diodes radiation detection for a-ray

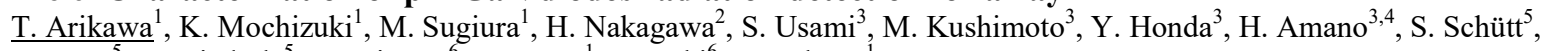

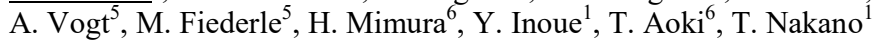

${ }^{I}$ Dept. of Engineering, Shizuoka University, 3-5-1 Johoku, Hamamatsu, Japan

${ }^{2}$ Dept. of Nanovision Technology, Shizuoka University, 3-5-1 Johoku, Hamamatsu, Japan

${ }^{3}$ Dept. of Engineering and Institute of Materials and Systems for Sustainability, Nagoya University, Furo-cho Chikusa-ku, Nagoya, Japan

${ }^{4}$ Akasaki Research Center, Nagoya University, Furo-cho Chikusa-ku, Nagoya, Japan

${ }^{5}$ Freiburg Materials Research Center, University of Freiburg, Stefan-Meier-Str. 21, Freiburg, Germany

${ }^{6}$ Research Institute of Electronics, Shizuoka University, 3-5-1 Johoku, Hamamatsu, Japan

We focused $\mathrm{B}$, which have large neutron cross-section area, to suggest a new $\mathrm{GaN}$ based semiconductor neutron detector. In this system, the characteristics of a-particle detection for GaN are important, but the evaluation of radiation detection property of GaN has not been sufficiently addressed so far. In this study, we estimate the range distance of a-particles in GaN by measurements and calculations. We fabricated GaN pin-diodes with two patterns of electrodes, which are planar type and mesh type. We used ${ }^{241} \mathrm{Am}$ as a-particle source and the energy spectrum measurement was performed by using multi-channel analyser. We evaluated the characterization of radiation detection for a-particles by changing the distance from the diode to the a-particle source. a-particle energy, which attenuated with air dumping, was estimated in the simulation. In the result of the energy spectrum measurement using planar electrode, when the incident energy of a-particles is $3.25 \mathrm{MeV}$, the detected peak energy attains a maximum value. In the region of less than $3.25 \mathrm{MeV}$, the detection energy was attenuated by the reduction of a-particle energy. This result indicates that the Bragg peak of a-particles is present in the detection area. In the region of more than 3.25 $\mathrm{MeV}$, the detection energy is slowly attenuated due to an increase of a-particles. This result indicates that Bragg peak of aparticles is not present in the detection area and that the a-particles with energies over $3.25 \mathrm{MeV}$ penetrate through the GaN diodes. In the case of mesh pattern electrode, detection peak position becomes maximum value when the energy is $3.14 \mathrm{MeV}$. This phenomenon means that the incidence of the a-particles from the electrode-free part is affected. If the energy of a-particles from electrode-free part becomes dominant, the peak position changes. From these results, we calculated the range of $1.47 \mathrm{MeV}$ a-particles which are generated by B(n, a)Li reaction, and we estimated it to be about $0.9 \mu \mathrm{m}$. 
R15-7: Growth of TIBr Crystals by the Vertical Bridgman Method and the Traveling Molten Zone Method for Gamma-Ray Detector Applications

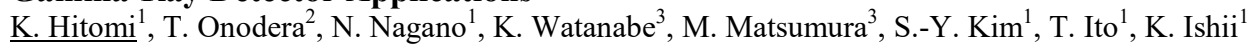

${ }^{I}$ Tohoku University, Sendai, Japan

${ }^{2}$ Tohoku Institute of Technology, Sendai, Japan

${ }^{3}$ Nagoya University, Nagoya, Japan

Thallium bromide $(\mathrm{TlBr})$ is a promising semiconductor material for gamma-ray detector fabrication. Growing large crystals is indispensable for using $\mathrm{TlBr}$ detectors for practical applications including gamma-ray spectrometers, gamma cameras and Compton cameras. In this study, two crystal growth methods including the vertical Bridgman method and the traveling molten zone method were employed for growing large TlBr crystals. The starting materials for both crystal growth methods were commercially available $\mathrm{TlBr}$ with $99.999 \%$ purity. Further purification of the starting materials was performed by the zone refining method. Growth of TlBr crystals by the traveling molten zone method was performed using the same system as the zonepurification method with a slower furnace movement speed. The advantage of the traveling molten zone method is that no extrinsic impurities are introduced in the crystal during the process because material purification and crystal growth can be performed in the same ampule. For growing TlBr crystals by the vertical Bridgman method, the pure part of the zone-purified ingot was loaded into another ampule. A TlBr crystal with 1-inch diameter was grown successfully in this study by the vertical Bridgman method. Pixelated detectors and strip detectors were fabricated from the TlBr crystals. Very large mobility-lifetime products for electrons and holes were observed from the crystals. The crystal growth methods, the detector fabrication processes, and the detector performance will be discussed in detail.

\section{R16: Defects2}

Friday, Nov. 4 10:30-12:15 Schuman

R16-1: The Effects of Low and High Temperature Annealing in CdZnTe Detector $\underline{\text { K. Kim }}^{1}$, S. Hwang ${ }^{1}$, A. E. Bolotnikov ${ }^{2}$, R. B. James ${ }^{3}$

${ }^{\text {I} R a d i o l o g i c ~ S c i e n c e, ~ K o r e a ~ U n i v e r s i t y, ~ S e o u l, ~ R e p u b l i c ~ o f ~ K o r e a ~}$

${ }^{2}$ Noproliferlation and National Security, Brookhaven National Laboratory, Upton, USA

${ }^{3}$ Science \& Technology, Savannah River National Laboratory, Aiken, USA

Te secondary phase defects are most abundant and responsible for the incomplete charge collection in CdTe compounds. One of the useful ways to remove Te secondary phase defects is to anneal CdTe compounds under Cd overpressure. Although Cd overpressure annealing remove Te secondary phase defect completely, it leaves another defects, that is, star-shaped prismatic punching defects and causes a loss of initial resistivity. With the help of in-situ monitoring system of electrical resistivity, we will report the phenomenon happened in CdTe compounds while making the low and high temperature annealing and also provide a clue to restore its resistivity.

This work was supported by the National Research Foundation of Korea (NRF) grant funded by the Korean government (MSIP) (NRF2015M2B2A9032788 and NRF-2015M2A2A4A01045094) and by the U.S. DOE/NNSA Office of Defense Nuclear Nonproliferation R\&D.

R16-2: CdTe/CdZnTe Detector Crystals Quality Search by Using Nondestructive Methods M. Sowinska ${ }^{1}$, G. Hennard ${ }^{1}$, L. Mengus ${ }^{1}$, P. Siffert ${ }^{2}$

${ }^{I}$ EURORAD, Eckbolsheim, France

${ }^{2}$ E-MRS Headquarters, Strasbourg, France

Despite intensive research for the improvement of the quality of II-VI crystals, until now no clear method permits to determine quickly the performance of the final detector, directly on the as-grown crystal or on the wafers. This situation induces excess cost, which hinders the development of detectors based on these binary and ternary semiconductors. The physical properties of both CdTe and CdZnTe make these materials among the most suitable for their application as semiconductor radiation detectors, especially, for high spatial resolution imaging applications. Indeed, the today request for imaging systems are: a good spectral resolution, a high spatial definition, a high image contrast quality as well as a good homogeneity over the whole sensor area. The challenge is particularly important for the crystals grownsince the quality of semiconductor material wafers used for sensors production determines the uniformity and performances of the final instrument. Fast characterisation methods become, therefore, imperative to keep the production costs in industry acceptable. The aim of the present study is to evaluate CdTe (THM) and CZT (VB) crystals by using a number of different non destructive methods of material characterization: (i) X-ray topography method for the crystals uniformity determination, (ii) X-ray fluorescence for the material composition distribution uniformity for $\mathrm{Zn}$, Cd or Te, (iii) resitivity and charge carrier mobility mapping over large crystals area, (iv) infrared microscopy mapping for Te precipitates, inclusions, dislocations and subgrain boundaries, (v) Finally detectors production and characterization (electrical and 
spectroscopic properties) on the characterized wafers, in their capacity to determine a quick and routine manner of the materials selection for further devices production. The main results will be presented and discussed.

\author{
R16-3: Interaction of Point Defects with Dislocations in CdTe and Nucleation of Te Precipitates \\ K. Kweon, V. Lordi \\ Material Science Division, Lawrence Livermore National Lab, Livermore, USA
}

Cadmium telluride (CdTe) and its alloy cadmium zinc telluride (CZT) are among the highest performing semiconductor materials for room-temperature X-ray and ?-ray detectors. However, their performance has been limited by spatially non-uniform carrier trapping by deep-level defects. Dislocations are of particular interest, since point defects, impurities, and Te-rich secondary phases that are known to trap charge may be associated with dislocations, and dislocations also may trap carriers themselves. The distribution of dislocations in CdTe or CZT crystals often has been observed to be very non-uniform. In this work, we determine the interaction of various point defects $\left(\mathrm{Cd} / \mathrm{Te}\right.$ vacancies, interstitials, and anti-site defects) with $30^{\circ} \mathrm{Cd}-/ \mathrm{Te}$-core partial dislocations in CdTe using density functional theory (DFT). Our calculations predict that most of the defects strongly bind to the dislocation cores and largely modify the defect states associated with the dislocations. Furthermore, the $\mathrm{Cd}$ vacancies at the $\mathrm{Cd}-$ core and the Te interstitials at the Te-core are predicted to be very mobile along the dislocation line, which can facilitate defect clustering within or near the dislocations. To demonstrate the Te clustering/precipitation near dislocations, we performed semiempirical molecular dynamics simulations with large numbers of excess Te added to the defect-dislocation systems, based on results from our DFT calculations. We show the role of dislocations on Te precipitation by comparing to the dynamics in the absence of dislocations. Better understanding of the properties of dislocations decorated with various point defects and Te precipitates can help devise growth and processing procedures for improved detector performance.

R16-4: Twin-Shaping Filter Technique Applied to CZT Detectors Grown by the Vertical Bridgman Method N. Auricchio ${ }^{1}$, E. Caroli ${ }^{1}$, F. Schiavone ${ }^{1}$, A. Basili ${ }^{1}$, J. B. Stephen ${ }^{1}$, A. Zappettini ${ }^{2}$

${ }^{I}$ IASFBO, INAF, Bologna, Italy

${ }^{2} I M E M, C N R$, Parma, Italy

$\mathrm{CdTe} / \mathrm{CZT}$ is an attractive and consolidated material with which to realize detectors with good efficiency and energy resolution, operating at room temperature for a large variety of applications such as astrophysics, medical imaging and security. However, this kind of material suffers from the low mobility of the charge carriers (particularly the holes), which are trapped and so degrade the detector response in terms of charge collection efficiency, energy resolution and photopeak efficiency. The response of a planar $\mathrm{CdTe} / \mathrm{CZT}$ detector, which depends on the distance between the charge formation position and the collecting electrodes, can be improved by using two kinds of techniques, based on the optimization of the electrode geometry and/or signal compensation methods. We are studying the feasibility and the reliability of a bi-parametric method that uses a twin pulse shaping active filter to analyze each signal from the detector twice: one "slow", which is proportional to the energy of the incident photon, and one "fast", which depends on the position of the interaction with respect to the collecting electrode. In this paper we describe the bi-parametric technique applied to planar CZT detectors grown by the Vertical Bridgman method at CNR/IMEM and report the experimental results and the compensated spectra obtained as a function of the bias voltage, photon energy and shaping time pairs. Furthermore, this technique could be implemented in an array of detectors, whose front-end electronics is composed of ASICs, where the shaping time can be selected for each channel, like the RENA-3 IC (NOVA R\&D).

R16-5: A Comparison of Point Defects and Their Effects on Transport Properties in CdTe- Based Crystals Grown by the Bridgman and Traveling Heater Methods

$\underline{\text { R. Gul }}^{1,2}$, A. E. Bolotnikov ${ }^{1}$, G. S. Camarda ${ }^{1}$, Y. Cui ${ }^{1}$, S. U. Egarievwe ${ }^{2}$, A. Hossain ${ }^{1}$, U. N. Roy ${ }^{1}$, G. Yang ${ }^{1}$, P. Vanier $^{1}$, R. B. James ${ }^{1,3}$

${ }^{I}$ Nonproliferation National Security, Brookhaven National Laboratory., Upton, NY, United States

${ }^{2}$ Alabama A\&M University, Normal, AL, United States

${ }^{3}$ Savannah River National Laboratory, Aiken, SC, United States

Point defects and their concentrations play an important role in limiting the electrical and spectral properties of crystals. It was observed that the crystal-growth process causes the generation of different types of point defects, and these defects create trapping centers and non-uniformities that can be detrimental to device performance. Point defects are characterized by techniques such as photo-induced transient spectroscopy (PICTS), current- deep level transient spectroscopy (i-DLTS), thermal emission electron spectroscopy (TEES), and thermally stimulated current (TSC) spectroscopy. These techniques are mostly based on transient current signals as a function of temperature, time and applied bias, which provide the foot prints for the presence of point defects. In this research work the CdTe-based detectors, including CZT, CMT, CTS, and CZTS crystals grown by Bridgman and Travelling Heater Methods, were characterized for their point defects by using i-DLTS. Point defects including shallow impurity-related electron donors $(<100 \mathrm{meV})$, medium energy traps including A-centers, cadmium vacancies (VCd)-0.11-0.40 eV, and deep traps ( $0.50-1.1 \mathrm{eV})$ were identified, and the raw data are further analyzed for their capture cross-sections 
for trapping of electrons and holes, concentration of individual traps, and the trapping times / lifetime of the carriers. To support and correlate the i-DLTS results with other properties and to study the effects of point defects on the lifetime of charge carriers, other techniques such as Transient Current Technique (TCT), X-ray Photo Current Technique (XPCT), and Infrared Photo Current Technique (IRPCT) were employed.

R16-6: Combined Computational/Experimental Study of Semiconductor-Metal Contact Interfaces in TIBr Radiation Detectors

K. G. Ray ${ }^{1}$, J. B. Varley ${ }^{1}$, A. M. Conway ${ }^{1}$, A. J. Nelson ${ }^{1}$, L. F. Voss ${ }^{1}$, E. L. Swanberg ${ }^{1}$, R. T. Graff ${ }^{1}$, S. A. Payne ${ }^{1}$, H. Kim $^{2}$, L. Cirignano ${ }^{2}$, K. Shah ${ }^{2}$, Z. Dai ${ }^{1}$, V. Lordi ${ }^{1}$

${ }^{I}$ Lawrence Livermore National Laboratory, Livermore, CA, USA

${ }^{2}$ Radiation Monitoring Devices, Inc., Watertown, MA, USA

$\mathrm{TlBr}$ is a promising semiconductor material for room temperature radiation detection, which has achieved an energy resolution as high as $1-2 \%$ at $662 \mathrm{keV}$, as well as a high absorption cross-section. However, the performance is observed to degrade over time. There are many possible causes of the degradation, including the incorporation of metal impurities from the contacts and the accumulation of charged vacancies at the ends of the device. Experimentally it has been shown that $\mathrm{TlBr}$ surface treatments and the choice of metal contact affect device longevity. Here we report on a first principles density functional theory study of intermetallic compounds that may form between $\mathrm{TlBr}$ and the contacts, along with experimental characterization of the interface using transmission electron microscopy. We discuss the effects of these contact-related interphase compounds on device performance and longevity, including their impact on vacancy migration and concentration, which we study using time dependent finite element simulations of the ionic and electronic transport in devices.

This work was performed under the auspices of the U.S. Department of Energy by Lawrence Livermore National Laboratory under contract DEAC52-07NA27344.

R16-7: Concluding Comments

R. B. James, M. Fiederle

Science and Technology Directorate, Savannah River National Laboratory, Aiken, South Carolina, USA

Brief comments pertaining to the 2016 RTSD, including a summary of some highlights and expression of gratitude to all organizers, will be provided at the end of the workshop.

\section{WKSP Program}

\section{Satellite Workshops}

(WKSP) Instrumentation and Measurement in Nuclear Environments (Reactors, Fuel Cycles and Safeguards)

NE1 Advanced Measurements and Instrumentation for Reasearch Reactors

Sunday, Oct. $30 \quad$ 08:30-11:15, Londres

NE1-1: Key Technologies Challenges and Prospective for Nuclear Measurements in Reactors and Fuel

Facilities

M. Morichi

$R \& D$, International Expert in Nuclear Instrumentation and Measurements, Paris, France

Nuclear installations (Nuclear Power Plants and Fuel Cycle facilities), projects as well as nuclear management activities are facing nowadays a unique combination of parallel events that are influencing, in a unique manner, the roadmap of nuclear instrumentation and measurements and open challenges are offering an opportunity for significant innovation.

Five main areas are identified as key challenger and opportunity for innovation: - The management of the nuclear facility assets and operations (long term in operation) - The nuclear plant renovations and their life extension: requiring a significant effort towards the evaluations/assessments and the licensing process; • The nuclear dismantling and decommissioning of obsolete nuclear installations; • The arrival of a new-wave of emerging projects for future advanced nuclear reactors; $\bullet$ The management of SNM/Spent Fuel and the management along the fuel cycle of the non-proliferation and the safeguards aspects;

Nuclear Instrumentation and Measurements are representing a critical and unique asset of knowledge, technologies methods and 
multidisciplinary competences that will be essential for operators, to be deeply studied and addressed to secure operational performances, provide ultimate safety and support to operations challenges providing innovative solutions to support new coming reactor concepts. The presentation will describe the open challenges, offering an overview of the relevant actual nuclear measurements and instrumentation solutions "state of the art" on the above points, while is proposing a vision for a future evolution to overcome the challenges, mitigate the risks of operations and projects and offering an innovative vision for the future.

\author{
NE1-2: State of the Art of the CEA R\&D for Instrumentation and Measurements in Experimental Nuclear \\ Reactor \\ C. Destouches \\ DEN/DER/SPEX, CEA-Cadarache, Cadarache, France
}

Reliable and accurate measurements (neutron, gamma flux, dimensions, temperatures, chemical properties) are key stakes in the field of the nuclear reactor science. In addition to the primary need of instrumentation for a safe nuclear reactor operating (control command), specific instrumentation is developed for experimental studies of the properties of the reactor structure materials and of the nuclear fuel elements. Generally speaking, experimental sensors and measurement systems have to satisfy several severe criteria due to the harsh environment encountered: high gamma and neutron radiation flux levels (up to $1 \mathrm{E} 14 \mathrm{n} / \mathrm{cm}^{2} \mathrm{~s}$ ) and doses (up to $1 \mathrm{E} 21 \mathrm{n} / \mathrm{cm}^{2}$, several GGy), variable temperature gradient (up to $700^{\circ} \mathrm{C}$ ), miniaturization,... In addition, as no repairing is possible after the beginning of the irradiation, a high level of reliability is required. And finally, the measurement accuracy has to be better than $10 \%$ (even 5\%) to allow validation or improvement of the calculation schemes. After a summary of their goals and requirements (locations, ranges, uncertainties ...) a description of the main sensor developments performed by the CEA is given: Neutron Self power Detector, Sub-Miniature Ionization/Fission chambers, Calorimeters, extensometers, Optical fiber based sensors (Bragg gratings, Pyrometry techniques, optical extensometer,...), solid state dosimeters, sensors for dimensional measurements (LVDT), ... The calibration issues (prior, during and after irradiation) of these measurement systems are then discussed introducing associated modelling issues. Computational nuclear, thermos-hydraulic and mechanical codes as well as quality of the basic data (mainly nuclear data) are analyzed in order to highlight needs for improvements. Finally, a conclusion on the state of the art of the measurement techniques in experimental reactor is drawn and the main future improvements axes are presented.

\title{
NE1-3: Energy Rate Induced by Nuclear Ray-Matter Interactions. How to Improve Its Measurement Inside MTRs \\ C. Reynard-Carette
}

Aix Marseille Université, CNRS, Université de Toulon, IM2NP UMR 7334, 13397, MARSEILLE, FRANCE

Energy deposition rate due to nuclear ray-matter interactions, is a crucial parameter to design irradiation experiments carried out inside Material Testing Reactors: for the choice of apparatus (exchangers, shields/screens), the establishment of suitable temperatures, the control of flow regimes, phase-change and mechanical points. This energy rate in such reactors has to be measured with a high accuracy. At present it is done with calorimeters which consist of sensors containing a specific sample, exchanging with their external surroundings and based on temperature measurements. Nuclear heating determination requires three main steps: out-off reactor calibrations, in-pile measurements with dedicated procedures, and calculations thanks to analysis methods. The talk will concern works realized in the framework of a joint laboratory between CEA and Aix-Marseille University, named LIMMEX and including international collaborations. The aim of these works is to improve sensors by a thermal approach from laboratory conditions without nuclear flux to irradiation conditions [1-3]. The enhancements are based on complementary axis: development of new laboratory test facilities to reproduce special conditions during calibration experiments (such as a bench simulating thermal/hydraulic conditions expected into irradiation channels of the Jules Horowitz reactor core), parametric studies of the response of usual sensors, design of innovative sensors by simulations, studies of prototypes, studies of analysis methods and numerical thermal models representing the whole process, calorimeter inter-comparisons and prospects for reducedsize sensors. After a state of the art and a description of the studied differential calorimeters, the talk will consist of a review of results obtained since 2010. Drawbacks and advantages of calorimeters will be discussed. Some recommendations to improve calorimetry for high levels of nuclear heating (inside JHR channels in particular) will be given.

References: [1] D. Fourmentel et al., Nuclear Heating Measurements in Material Testing Reactor: a Comparison Between a Differential Calorimeter And a Gamma Thermometer, IEEE Transactions on Nuclear Science Nuclear Science, Volume 60, Issue:1, Part:2, Publication Year: 2013 , Page(s) : 328 - 335 http://dx.doi.org/10.1109/TNS.2012.2232304. [2] J. Brun et al., Comparison of the Thermal Response of Two Calorimetric Cells Dedicated to Nuclear Heating Measurements during Calibration, IEEE Transactions on Nuclear Science, Vol. 61, N4, pp. 2254-2261, 2014. [3] C. De Vita et al., Study of the Influence of Heat Sources on the Out-of-Pile Calibration Curve of Calorimetric Cells Used for Nuclear Energy Deposition Quantification, Http://dx.doi.org/10.1109/TNS.2016.2572605. [4] J. Brun et al., Responses of Single-cell and Differential Calorimeters: from Out-of-Pile Calibration to Irradiation Campaigns, IEEE Transactions On Nuclear Science, 2016, Http://dx.doi.org/10.1109/TNS.2016.25649234. 
NE1-4: Neutron Flux Monitoring Using Self-Powered Neutron Detectors: Modeling and Validation $\underline{\text { L. Vermeeren }}$

ANS/NSD/ICR, SCK・CEN, Mol, Belgium

Self-powered neutron detectors (SPND) are routinely used for the on-line monitoring of the thermal neutron flux in many research and power reactors. Activation dosimetry can provide valuable reference values for the flux, but only time-integrated and a posteriori. Instantaneous flux information is crucial for a detailed follow-up of the irradiation history of samples in research reactors. For power reactor applications, SPNDs are mainly used for neutron flux profile monitoring, also requiring on-line data recording. An SPND consists of a cylindrical metallic emitter surrounded by an insulator (usually $\mathrm{MgO}$ or $\mathrm{Al} 2 \mathrm{O} 3$ ) and a metallic sheath. Impact of neutrons or gammas leads to the creation of energetic electrons, a fraction of which is sufficiently energetic to cross the insulator. These electrons constitute a current which can be measured by connecting a current meter between the emitter and the sheath. SPNDs can be classified in two groups: delayed SPNDs (relying on the production of betas upon decay of unstable isotopes formed after neutron capture) and prompt SPNDs (where capture gamma rays create energetic electrons). In the past, SPND signals were interpreted only in a relative way. SPNDs can be calibrated against activation dosimetry data, but since the detector sensitivity depends on various parameters, such calibrations are only valid for these specific conditions. From the theoretical side, analytical models were developed already in the 1970 s, but they rely partly on experimental input. In parallel, a Monte Carlo approach for the SPND sensitivity calculation was proposed, but because of the limited computer capacity in those days, several important processes were neglected. More recently, an MCNP(X) based model has been developed for the calculation of the absolute neutron sensitivity of SPNDs. In this workshop contribution we will explain the calculation approach, apply it for some specific cases and present some experimental data for model validation, showing the capabilities and limitations of the model.

\author{
NE1-5: Recent Achievements in Single Neutron Counting Using CMOS Cameras and Future Developments \\ P. Mutti, E. Ruiz Martinez, P. Van Esch \\ Institut Laue-Langevin, Grenoble, France
}

With the present paper we report the first results of a new development in which we aim at counting individual neutrons with CMOS cameras. We explore theoretically a technique that allows us to use imaging detectors as counting detectors at lower counting rates, and transits smoothly to continuous imaging at higher counting rates. As such, the hope is to combine the good background rejection properties of standard neutron counting detectors with the absence of dead time of integrating neutron imaging cameras as well as their very good spatial resolution. Compared to X-ray detection, the essence of thermal neutron detection is the nuclear conversion reaction. The released energies involved are of the order of a few MeV, while X-ray detection releases energies of the order of the photon energy, which is in the $10 \mathrm{KeV}$ range. Thanks to advances in camera technology which have resulted in increased quantum efficiency, lower noise, as well as increased frame rate up to 100 fps for CMOS-type cameras, this more than 100-fold higher available detection energy implies that the individual neutron detection light signal can be significantly above the noise level, as such allowing for discrimination and individual counting, which is hard to achieve with X-rays. The time scale of CMOS-type cameras, so far, doesn't allow one to consider time-of-flight measurements, but kinetic experiments in the $10 \mathrm{~ms}$ range are possible. The possibility to extend this technique to new type of CMOS cameras and to achieve frame rates up to $100 \mathrm{kHz}$, necessary for ToF measurements, will be also discussed.

\title{
NE2 Non Destructive Assay for material, fuel and radiactive wastes Characterization and Control
}

Sunday, Oct. $30 \quad$ 10:30-12:00, Londres

NE2-1: Developments of Nondestructive Nuclear Measurements in the Nuclear Industry and for Homeland Security

B. Pérot

Nuclear Measurement Laboratory, CEA, DEN, Cadarache, F-131085 Saint-Paul-lez-Durance, France

The Nuclear Measurement Laboratory of CEA Cadarache, France, develops nuclear nondestructive assay for the nuclear fuel cycle, ranging from uranium mining to radioactive waste characterization, including fuel fabrication and reprocessing, contamination assay in nuclear power plants, nondestructive exams for the JHR reactor, dismantling of nuclear facilities, nuclear accident studies, and for CBRNE threats detection (homeland security). LINAC X-ray imaging has replaced gamma imaging to improve measurement time, as well as size and density of the inspected waste packages, allowing also photofission with X-ray energies larger than $6 \mathrm{MeV}$ to characterize nuclear materials with delayed neutrons and gamma rays. Corium-water interactions can also be visualized by X-ray radioscopy with up to 200 pictures/s. Gamma-ray spectroscopy is widely studied, e.g. to improve uranium gamma logging. In the field of radioactive waste passive neutron coincidence counting, plastic scintillators are studied to replace $3 \mathrm{He}$ detectors, too costly because of $3 \mathrm{He}$ gas shortage. Active neutron interrogation with the differential die-away technique (DDT) is also implemented with the detection of fission prompt neutrons to control fissile materials $(235 \mathrm{U}, 239 \mathrm{Pu}$, $241 \mathrm{Pu}$ ) in radioactive waste or nuclear processes. The alpha activity is also estimated by combining prompt and delayed neutron signals. For historic waste, a matrix effect correction based on a flux monitor (close to the drum) has been developed to 
compensate the limited knowledge of waste composition, thus reducing DDT uncertainties. Finally, fast neutron interrogation with the associated particle technique has been developed to detect illicit materials (explosives, narcotics, chemical warfare, and nuclear materials) in cargo containers, or to inspect unexploded ordnance on the seafloor with a compact system embedded in a remotely operated vehicle, to improve the security of maritime areas.

\author{
NE2-2: Imaging of Special Nuclear Material Inside and Outside of Nuclear Reactors by Muon Scattering \\ Tomography

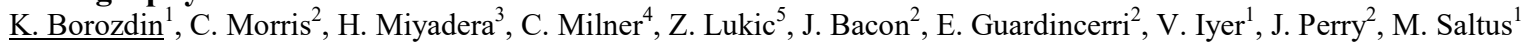 \\ ${ }^{I}$ Headquarters, Decision Sciences International Corporation, Poway, CA, United States \\ ${ }^{2}$ Physics, Los Alamos National Laboratory, Los Alamos, NM, United States \\ ${ }^{3}$ Quantum Beam Technology, Toshiba Corporation Power Systems Company, Yokohama, Japan \\ ${ }^{4}$ Physics, Southern Methodist University, Dallas, TX, United States \\ ${ }^{5}$ Computational Research, Lawrence Berkeley National Laboratory, Berkeley, CA, United States
}

Empowered by penetrating capability of cosmic-ray muons, robust position-sensitive charge particle detectors and state-of-the-art methods of image reconstruction and data analysis, muon scattering tomography provides revolutionary capabilities for a variety of special nuclear material (SNM) detection and imaging applications. For this workshop presentation, we focus on several of these applications, including imaging of reactor fuel in Fukushima reactors, in situ monitoring of the reactor fuel burn-out process and inspection of dry casks used for reactor spent fuel storage. We describe SNM detection capabilities of commercially available Multi-Mode Passive Detection System (MMPDS) as relevant to nuclear safeguards, non-proliferation, homeland security and radiation waste monitoring. Experimental results are discussed to illustrate successful performance of drift tube detectors in high radiation fields typical for nuclear environments. Looking forward, we will present recent improvements in detector hardware, electronics and analysis algorithms that promise to further improve muon tomography technological capabilities in near future.

NE2-3: Preliminary Results on the Irradiation Test for Innovative Proliferation Resistant U-Mo/Mg Fuel V. Golovko, P. Pfeiffer, J. Budgell, X. Wang

Dept. of Reactor and Applied Sciences, Canadian Nuclear Laboratories, Chalk River, Ontario, Canada

Irradiation tests of U-Mo/Al reactor fuel were performed in the National Research Universal (NRU) reactor located at Chalk River, Ontario in the past. Recently, it has been proposed to perform similar tests on innovative Proliferation Resistant $\mathrm{U}-\mathrm{Mo} / \mathrm{Mg}$ fuel, of which magnesium is considered to have a better material characteristic than aluminium. For the preparation of the irradiation test an assessment of the power history of two types of molly content samples, $\mathrm{U}-10 \% \mathrm{Mo} / \mathrm{Mg}$ and $\mathrm{U}-7 \% \mathrm{Mo} / \mathrm{Mg}$ with different Mo-concentration, at a required linear power rating has been done. In addition, the Reactor Detector Network REDNET at NRU has been modified to obtain data for new fuel rods in real time. On January 14-th 2016 the experiment began with the installation of Proliferation Resistant Fuel UMO-1, containing the first U-7\%Mo/Mg fuel rod, into a reactor position. Preliminary results show good agreement between predicted and observed absolute power values for the $\mathrm{U}-7 \% \mathrm{Mo} / \mathrm{Mg}$ rod and neighboring sites, re-assuring safe execution of the irradiation experiment with a new and innovative fuel. The experiment will continue for a few hundred days to reach a desired burnup range for both fuel rods.

We would like to acknowledge the support from NRU personnel in starting this experiment and performing it safely.

NE3 Innovative Sensors and Detection Devices

Sunday, Oct. $30 \quad$ 14:00-16:30, Londres

NE3-1: Thermal and Fast Neutron Detectors : a Comparison Between Silicon-Carbide and Diamond

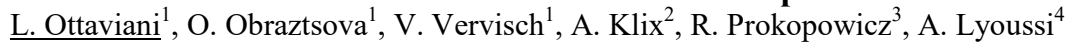

${ }^{1}$ IM2NP, Aix Marseille University, Marseille, France

${ }^{2}$ Institute of Neutron Physics and Reactor Technology, KIT, Eggenstein-Leopoldshafen, Germany

${ }^{3}$ NCBJ, Otwock-Swierk, Poland

${ }^{4}$ CEA Cadarache, INSTN, Cadarache, France

The principal role of neutron detectors in nuclear applications is to get informations about the actual neutron yield and reactor environment. Harsh radiation environments near the nuclear reactor core require the radiation detectors to be resistant to high temperature (up to $500^{\circ} \mathrm{C}$ ) and radiation level. Currently, Si is the most used semiconductor material but its drawbacks make it impossible for harsh media applications. Firstly, the leakage current of a Si diode limits it to operate at temperature not higher than $30^{\circ} \mathrm{C}$, due to the small energy band gap $(1.12 \mathrm{eV}$ at RT). Additionally, the Si based devices suffer from radiation damage which causes the degradation of their performance. Silicon Carbide is one of the most attractive materials for neutron detection. Thanks to its outstanding properties, such as high displacement threshold energy (22-35 eV), wide band gap energy (3.27 eV) and 
high thermal conductivity $(4.9 \mathrm{~W} / \mathrm{cm} \cdot \mathrm{K}), \mathrm{SiC}$ can operate in harsh environment (high temperature, high pressure and high dose rate) without additional cooling system. Our analyses reveal that $\mathrm{SiC}$ detectors, under irradiation and at elevated temperature, respond to neutrons showing consistent counting rates as function of external reverse bias voltages and radiation intensity. The counting-rate of the thermal neutron-induced peak increases with the area of the detector, and appears to be linear with respect to the reactor power. Diamond is another semi-conductor considered as one of most promising materials for radiation detection. Diamond possesses several advantages in comparison to other semiconductors such as a wider band gap (5.5 eV), higher threshold displacement energy $(40-50 \mathrm{eV})$ and thermal conductivity $(22 \mathrm{~W} / \mathrm{cm} \cdot \mathrm{K})$, which leads to low leakage current values and make it more radiation resistant that its competitors. A comparison is proposed between these two semi-conductors for the ability and efficiency to work under harsh environment.

NE3-2: Modelling of a Self-Powered Detector for Fast Neutrons P. Raj

Institute for Neutron Physics and Reactor Technology, Karlsruhe Institute of Technology, Eggenstein Leopoldshafen, Germany

Self-powered detector is a compact and reliable choice for neutron or gamma flux monitoring in fission reactor cores. In this work, experiments and calculations are done to investigate the possibilities of using this principle of measurement for fast neutron field in the test blanket modules of ITER, the fusion reactor under construction in south of France. Constraints on the physical and nuclear properties are identified for an emitter material responsive to fast neutrons. A few materials including beryllium, chromium, vanadium, silver etc. are selected for test, based on these constraints. A test device in flat geometry is constructed in-house and tested with a $14 \mathrm{MeV}$ neutron generator. It has been found in the experiments that the purely neutroninduced effect is low compared to other effects. Other main contributors to the current signal are parasitic reactions due to high energy gamma and neutrons in emitter or other parts of the detector. These effects are studied with computational simulations. A multi-step Monte-Carlo particle transport method is employed to calculate the response of SPND to relevant neutron and/or gamma spectra. Values calculated using this method for gamma sensitivity of SPND in cylindrical geometry agree well with previously conducted experiments. A combination of emitter and collector (e.g. beryllium and stainless steel) in representative dimensions is modeled and its sensitivity to neutrons and gammas are calculated separately. The effect of change of dimensions of various parts is calculated and compared to find the most neutron-responsive configuration of a combination. A comparison is done between results of experiments and Monte-Carlo simulations.

NE3-3: Neutron Measurement Instrumentation Development at KIT for the EU ITER TBM $\underline{\text { A. Klix }}{ }^{1}$, M. Angelone ${ }^{2}$, U. Fischer ${ }^{1}$, D. Gehre ${ }^{3}$, A. Lyoussi ${ }^{4}$, P. Raj ${ }^{1}$, T. Reimann ${ }^{1}$, D. Szalkai ${ }^{1}$, K. Tian ${ }^{1}$

${ }^{I}$ Institute of Neutron Physics and Reactor Technology, Karlsruhe Institute of Technology, Eggenstein-Leopoldshafen, Germany

${ }^{2}$ ENEA C.R., Frascati, Italy

${ }^{3}$ Institute for Nuclear and Particle Physics, Technical University of Dresden, Dresden, Germany

${ }^{4}$ Instrumentation Sensors and Dosimetry Laboratory, CEA, Cadarache, France

Test Blanket Modules (TBM) will be installed in ITER with the aim to investigate the nuclear performance of different breeding blanket concepts. Currently there is no fully qualified nuclear instrumentation available for the measurement of neutron fluxes and tritium production which can withstand the harsh environment conditions in the TBM such as high temperature (up to 650 oC) and, depending on the operation scenario, intense radiation levels. As partner of the European Consortium on Nuclear Data and Measurement Techniques in the framework of several F4E specific grants and contracts, KIT and ENEA have jointly studied the possibility to develop and test detectors suitable to operate in the EU ITER-TBMs. Here we present an overview of ongoing work on three types of neutron flux monitors under development for the TBMs with focus on the KIT activities.

A neutron activation system (NAS) with pneumatic sample transport provides absolute neutron flux measurements. A test system was constructed at the DT neutron generator laboratory of Technical University of Dresden to investigate the neutronics aspects. Tests were done focussing on the simultaneous measurement of the extracted activated probes. An engineering assessment of a TBM NAS in the conceptual design phase was done which considered issues of design requirements and integration. Within the I_SMART project, KIT is developing an online detector based on silicon carbide electronics. The operation of such detectors at TBM relevant temperatures is expected to incur lower accumulated radiation damage to them than at room temperature due to annealing effects. Detectors of several designs have were irradiated with DT neutrons. Tests at elevated temperatures have been done and further tests are currently underway. Self-powered neutron detectors (SPND) are widely applied in fission reactor monitoring. We are investigating novel materials for SPND which would be sensitive also to the fast neutron flux expected in the TBMs.

The work leading to this publication has been funded partially by Fusion for Energy under the Specific Grant Agreement F4E-FPA-395-1. This publication reflects the views only of the authors, and Fusion for Energy cannot be held responsible for any use which may be made of the information contained therein.

NE3-4: Room Temperature Large Sensitive Area High Resolution Spectroscopic Silicon Drift Detectors A. G. Vacchi 
Trieste section, INFN - Trieste Italy, Trieste, Italy

On behalf of the REDSOX

A long progression involving all aspects of the detection system based on the majority carriers drift in silicon, Silicon Drift Detectors (SDD) has been carried ahead since about thirty years focusing at various challenging basic science applications requiring sensitive areas up to few square meters and at the same time high position and energy resolution. Relevant systems have been realized that have given proof of stability and reliability for time laps of many years. This consistent effort has lead to front edge results in the performances as well as in the design production and assembly of detectors, front end electronics and readout systems whose quality can certainly be considered for the realization of high sensitivity, robust, light weight applications in spectroscopic systems. A non secondary aspect of this effort is the possibility to realize custom detectors design engineering and integration adaptable to each specific use with relevant gain in speed and efficiency. The state of the art will be reported.

\author{
NE3-5: Diamond-like Heat Spreaders in the Form of Cheap Synthetic Graphite Tape for Cooling of \\ Instrumentation in Radiation Intense Environments \\ A. Dierlamm, S. Maier, T. Barvich, C. Beskidt, W. de Boer \\ Physics, KIT, Karlsruhe, Germany
}

Silicon based particle detectors in high radiation environments need efficient cooling to reduce the leakage currents. This can be efficiently done with synthetic graphite tape, which approaches closely the performance of ideal diamond heat spreaders with the additional advantage of being flexible and having adhesives on both sides. It is nowadays used in practical all mobiles phones, LED screens, etc. to remove the heat from the chips. Hence, the very thin (40-100 um) synthetic tapes are available for a tiny fraction of the cost of diamond heat spreaders, but shows a similar performance. We built realistic silicon detector modules with it, which were mechanically robust and radiation hard and showed a far superior thermal behavior than present detector modules planned for the LHC upgrade at CERN. It seems to be first time, that this common cooling practice in industry by synthetic graphite is applied in our field of instrumentation for nuclear and particle physics.

(WKSP) Detectors for Ultra-Rare Event Processes

UR1 Light detection in Noble Gas Experiment (Part I)

Sunday, Oct. $30 \quad$ 08:30-10:10, Madrid

UR1-1: Photodetectors for the XENON1T Dark Matter Experiment

$\underline{\text { Y. Wei }}$

Physik-Institut, Zurich University, Zurich, Switzerland

On behalf of the XENON1T Collaboration

The XENON1T experiment employs 248 Hamamastu R11410-21 photomultiplier tubes (PMTs) in its time projection chamber (TPC) to detect scintillation light induced by a possible WIMP interaction. These PMTs are specifically developed for XENON1T with $\sim 32.5 \%$ typical quantum efficiency (QE) at $178 \mathrm{~nm}$ xenon scintillation light and low radioactivity which was evaluated through a screening campaign performed by the XENON1T collaboration. Important features, such as the gain, dark count rate, afterpulses and light emission, were evaluated at liquid xenon temperature for selecting the best PMTs for the XENON1T TPC. The gain stability was measured over a period of 5 month within $\pm 2 \%$ fluctuation. An average dark count rate of $\sim 40 \mathrm{~Hz}$ was measured with all selected PMTs. The afterpulses were measured for analysing the vacuum quality and residual gas content inside the PMTs. The light emission was measured with a "face to face" PMT setup. The performance of PMTs in XENON1T current operation will be presented. Novel photosensors, such as SiPM, Gaseous Photomultipliers, will be discussed for potential application in the future experiment.

\author{
UR1-2: The PandaX Project for Dark Matter and Neutrinoless Double Beta Decay \\ $\underline{\text { K. Giboni }}$ \\ Physics and Astronomy, Shanghai Jiao Tong University, Shanghai, China \\ On behalf of the PandaX Collaboration
}

After the completion of PandaX I the PandaX project comprises two experiments in the deep underground JinPing lab. PandaX II is a data taking experiment to search for Dark Matter (DM) in the form of WIMPs, and PandaX III is still under development to detect neutrinoless double beta decays in the new CJPL II, soon to be available at the JinPing site. PandaX II is a LXe TPC in double phase technology with an active mass of $500 \mathrm{~kg}$. For some time it was the largest operating DM detector in the world. Initial results from a commissioning run are very promising and were recently published. PandaX III is under design and expected to search for neutrinoless double beta decays from 136Xe starting at the end of next year. Both experiments search for very rare events. After optimization, however, they are entirely different. PandaX III is a $200 \mathrm{~kg}$ gaseous TPC under $10 \mathrm{bar}$, filled 
with $90 \%$ enriched 136Xe. The charge read out is accomplished with two micro bulk MicroMega planes. The added topological information allows reconstructing the tracks of the electrons. This provides an additional background reduction by a factor 100 . Thus the experiment becomes nearly background free. For DM we also work on the next generation experiment. Besides being larger, we want to replace the PTFE reflectors by active photo sensors. Then Pulse Shape Discrimination (PSD) can be used to distinguish nuclear recoil events from ?-rays. In this case the additional timing information will reduce the background by a factor up to 100. Thus, in both cases added information is used to significantly increase the sensitivity for a given detector size.

UR1-3: Development of Photomultipliers for the XMASS Experiment $\underline{\text { S. Moriyama }}$

Institute for Cosmic Ray Research, The University of Tokyo, Higashi Mozumi, Kamioka cho, Hida city, Gifu prefecture, Japan On behalf of the XMASS Collaboration

A series of low background photomultipliers (PMTs) sensitive to the scintillation light of liquid xenon was developed for the XMASS experiment. In the running detector XMASS-I, 2-inch PMTs with hexagonal shaped photocathodes are used. This special shape enables us to realize unprecedented high light yields ( $\sim 14$ photoelectrons $/ \mathrm{keV}$ at $122 \mathrm{keV})$ and low energy threshold based on the liquid xenon scintillator. For future generations of XMASS detectors, a new type of 3-inch PMTs with a convex-shaped photocathode with lower radioactivity than the 2-inch PMTs is currently being developed. Features of these PMTs will be presented in this study.

UR1-4: Light Detection Technologies for the DarkSide Experiment $\underline{\mathrm{G}}$. Fiorillo $^{1,2}$

${ }^{\text {I} P h y s i c s, ~ U n i v e r s i t a ̀ ~ d e g l i ~ S t u d i ~ d i ~ N a p o l i ~ F e d e r i c o ~ I I, ~ N a p o l i, ~ I t a l y ~}$

${ }^{2}$ INFN, Napoli, Italy

On behalf of the DarkSide Collaboration

WIMP dark matter could be detected through observation of nuclear recoils induced by elastic collisions with a sensitive target. The DarkSide program in the underground Laboratori Nazionali del Gran Sasso has tailored two-phase argon time projection chamber technology for this purpose by choosing radio-pure structural and target materials, constructing neutron and muon vetoes, and utilizing efficient discrimination techniques to reject electron recoils. These are based on the collection of argon scintillation light by low radioactivity photosensors optimized to operate in cryogenic environments.

We will review the studies we carried out on 3 inches cryogenic photomultipliers of the type R11065 from Hamamatsu, currently used by the DarkSide-50 detector, discussing problems and limitations of these devices.

The next step in the DarkSide program is DarkSide-20k, having a fiducial mass of 20 tonnes. This next generation experiment is designed to attain a factor of 1000 improvement over currently-published results for spin-independent cross sections of WIMPs with $1 \mathrm{TeV} / \mathrm{c}^{\wedge} 2$ mass, covering a large fraction of the mass-cross section parameter space currently preferred by supersymmetric models.

Like its processor, DarkSide-20k design aims at ultra-low background and a very efficient light collection.

A striking innovation of the detector is the replacement of photomultiplier tubes with SiPM arrays specially designed for cryogenic operation and high quantum efficiency. The top and the bottom of the TPC will be instrumented with more than 5000 SiPM tiles, totalling $14 \mathrm{~m}^{\wedge} 2$ of sensitive surface area. In this talk, an update to the SiPM R\&D being carried out within the DarkSide collaboration will be presented, including the characterization at cryogenic temperatures of several SiPM and the study of their performance in prototype detectors.

UR2 Light detection in Noble Gas Experiment (Part II)

Sunday, Oct. $30 \quad$ 10:30-12:10, Madrid UR2-1: ArDM, a Ton-Scale Liquid Argon Detector for Direct Dark Matter Searches $\underline{\text { C. Regenfus }}$

IPP, ETH Zurich, Zurich, Switzerland

On behalf of the ArDM collaboration

The ArDM experiment, the first ton-scale Liquid Argon detector for direct Dark Matter searches, was recently commissioned in a 6 month long physics data run at full target size in single phase operational mode. The results give a promising perspective for a planned double phase operation of ArDM in 2016. Various experimental upgrades are planned for the future, in particular we are evaluating alternative light read out solutions based on SiPMs or low background cryogenic PMTs.

UR2-2: Single-Photon Counting in Dark Matter Search: DEAP-3600 Achievements and Promising Light Detector for the Next Generations. 
$\underline{\text { P. Gorel }}$

SNOLAB, Sudbury, ON, Canada

On behalf of the DEAP-3600 Collaboration

First ton-scale single-phase detector using liquid argon, DEAP-3600 is now fully engaged in the search for the Weakly Interacting Massive Particles of mass $100 \mathrm{GeV}$. Three years will be required to attain the expected sensitivity of $10-46 \mathrm{~cm} 2$ for the spin-independent elastic scattering.

DEAP-3600 is also the first detector using an acrylic cryostat, leading to unprecedented low levels of background. This detector and its acquisition have been designed to maximize the light collection and the single-photon counting and timing in order to optimize the pulse shape discrimination, which is crucial for its success.

The next generation of detector will need to go even further, either to characterize the newly detected WIMP, or to improve the sensitivity down to the neutrino floor. The replacement of the classic photomultipliers by new light detectors, such as silicon photomultipliers, offers new possibilities for the single-photon counting and timing. More specifically, large area sensors such as the 3DdSiPM could be used to provide unprecedented sensitivity for the new generation of detector.

UR2-3: The Low-Background Detector EXO-200

$\underline{\text { T. Brunner }}$

McGill University, Montreal, Canada

On behalf of the EXO-200 Collaboration

The low-background detector EXO-200 is searching for $0 ? ß ß$ decays in ${ }^{136} \mathrm{Xe}$. If this decay was observed it would violate lepton number conservation and determine the Majorana nature of the neutrino. About $175 \mathrm{~kg}$ of xenon enriched at $\sim 81 \%$ in the isotope ${ }^{136} \mathrm{Xe}$ are deployed in the EXO-200. The detector is a liquid xenon time-projection chamber (TPC) consisting of two halves sharing a central cathode. Scintillation light and ionization signal are simultaneously read out by large area avalanche photodiodes (LAAPDs) and a wire grid, respectively. This information is used to reconstruct energy and position of each event. Thorough calibration of the detector is required to create a light map of the response function of the LAAPDs and to measure the electron life time inside the liquid xenon. Very sensitive measurement campaigns have been performed focusing on but not limited to the search for 0 ? $3 ß$. Among those are measurements are of ion mobility in the detector, measurement of background rates, and measurements of cosmogenic backgrounds.

UR2-4: Status and Prospects of the NEXT Experimental Program J. J. Gomez-Cadenas

IFIC (CSIC-UV), Valencia, Spain

On behalf of the NEXT collaboration

The Neutrino Experiment with a Xenon TPC (NEXT) is an experimental program to develop the technology of electroluminescent High Pressure gas Xenon TPCs for neutrino less double decay searches. The project started in 2008 as a collaboration between Iberian (Spain \& Portugal) and American (USA and Colombia) universities. After successfully completing the initial R\&D period, a first radio pure detector, called NEXT-White (NEW) is being currently commissioned at the Canfranc Underground Laboratory (LSC) in Spain. NEW will operate during 2016 and 2017 and will allow a detailed studies of the already demonstrated excellent energy resolution and topological signatures (the salient features of the technology) as well as a data-driven assessment of the experiment background model. A $100 \mathrm{~kg}$ detector, NEXT-100, is schedule to go online in 2018 . In its current configuration, NEXT-100 will reach a sensitivity of $5 \mathrm{x} 10^{\wedge} 25 \mathrm{y}$ in a 3 years run, with an expected background of less than one event per year.

While NEXT-100 is being built and operated, the NEXT collaboration aims to demonstrate the feasibility of expanding the technology to the ton scale, building 3 or more modules hosting 300-500 $\mathrm{kg}$ of xenon each. Improvements of the topological signature and optimisation of energy resolution and radioactive budget may allow the NEXT technology to achieve less than one background event per ton and year of operation.

Last but not least, the collaboration is developing a new Ba-tagging concept, based in Scintillation Molecular Fluorescence (SMF), which could result ultimately in a "background free" $(<0.1$ counts per ton and year) experiment.

This talk will review the status and prospects of the NEXT program.

The NEXT program is supported by an Advanced Grant of the ERC

UR3 Light detection in Other Technique Experiments

Sunday, Oct. $30 \quad$ 14:00-14:50, Madrid UR3-1: The liquid argon scintillation light instrumentation of GERDA Phase II 


\section{$\underline{\text { J. Janicsko }}$}

Technische Universität München, Garching, Germany

The GERmanium Detector Array (GERDA) at the Laboratori Nazionali del Gran Sasso of INFN, Italy, searches for neutrinoless double beta decay of Ge-76. Bare germanium detectors, enriched in $\$^{\wedge}\{76\} \mathrm{Ge} \$$, are operated in liquid argon serving simultaneously as a coolant, a high purity shield against external radiation, and in GERDA Phase II, as an active veto system. To reach unprecedented low background levels recently presented at the Neutrino 2016 conference, the GERDA collaboration has developed a modular system which is deployed together with the germanium detector array in order to detect the scintillation light of liquid argon and to discriminate background events efficiently. The design and performance of the liquid argon system is presented together with the results obtained so far in GERDA Phase II.

\section{UR3-2: The Central Detector of JUNO}

\section{Y. Heng}

Experimental Physics Center, Institute of High Energy Physics, Beijing, China

On behalf of the JUNO

Jiangmen Underground Neutrino Observatory (JUNO) is under constructing in China which aims to measure the neutrino mass hierarchy, neutrino oscillation parameters, supernova neutrino, atmosphere neutrino, sun neutrino and earth neutrino. JUNOï $i^{1 / 2} \mathrm{~S}$ central detector is designed to measures neutrinos by inverse decay with $20 \mathrm{k}$ tons of liquid scintillator of target and with $3 \% / \mathrm{vE}$ of the energy resolution. The detector consists of an inner transparent sphere of $35.5 \mathrm{~m}$ in diameter and an outer support structure of $39.5 \mathrm{~m}$ in diameter. The inner sphere is made of acrylic and holds $20 \mathrm{k}$ tons of liquid scintillator. About 17000 photomultiplier tubes (PMTs) are carried on the outer structure, covering $75 \%$ of the acceptance angle of the scintillating photons. The central detector is placed in $44 \mathrm{~m}$ deep water pool, which is underground $700 \mathrm{~m}$ deep. Such a big detector with such a precise energy resolution is a challenge. Here the design and $R \& D$ progress of the central detector are introduced.

\section{UR4 Light detection: new directions (Part I)}

Sunday, Oct. $30 \quad$ 14:50-15:40, Madrid UR4-1: Silicon Photomultipliers for nEXO and Other Rare Event Searches I. Ostrovskiy

Department of Physics and Astronomy, University of Alabama, Tuscaloosa, AL, USA

On behalf of the nEXO collaboration

$\mathrm{nEXO}$ is planning to search for neutrinoless double beta decay using a 5 tonne liquid xenon time projection chamber. $\mathrm{nEXO}$ borrows basic approach from the predecessor experiment, EXO-200, but several improvements need to be made to achieve the sensitivity goal of completely covering the inverted hierarchy region of the allowed parameter space for neutrino masses after 10 years of data. One of the several needed improvements is lowering the energy resolution compared to what has been demonstrated with EXO-200. This will allow one to keep 2?ßß decay from becoming a significant background. To achieve this nEXO plans to increase the light collection and reduce noise, by covering up to 4-5 $\mathrm{m}^{2}$ with light detectors with higher gain than avalanche photo-diodes used in EXO-200, and by maximizing reflectivity of internal components. Silicon Photomultipliers (SiPMs) are attractive candidates for light detectors for $\mathrm{nEXO}$ and other similar rare event searches due to their light weight, expected intrinsic radiopurity, and high gain. At the same time, the sensitivity for vacuum ultra-violet (VUV) light and scalability to large surface areas are still challenging. This talk gives general overview of the nEXO experiment and discusses the development of the conceptual design, with the particular emphasis on the light collection and readout. Highlights of the broad characterization effort by nEXO collaboration of SiPMs manufactured by Fondazione Bruno Kessler (FBK), Hamamatsu Photonics, and KETEK are also presented.

\section{UR4-2: New Directions in Noble-Liquid Detectors of Rare Events} L. Arazi

Particle Physics \& Astrophysics, Weizmann Institute of Science, Rehovot, Israel

Large-volume noble-liquid TPCs, particularly dual-phase devices, are leading tools in rare-event searches and neutrino-physics experiments. Their scaling up in future experiments, however, calls for innovative detector solutions. We will present and discuss some new concepts of charge and light recording in noble liquid systems. The recently introduced bubble-assisted Liquid HoleMultiplier (LHM) is a $\ddot{i}_{i}^{1} / 2$ local dual-phasei $i^{1 / 2}$ detection element, designed for recording both ionization electrons and primary scintillation photons induced by particle interactions within the noble liquid. The LHM comprises a perforated electrode (e.g. Thick Gas Electron Multiplier $\ddot{i}_{\zeta}^{1} \frac{1}{2}$ THGEM, or Gas Electron Multiplier - GEM) immersed in the liquid, with a bubble of the noble gas supported underneath. Ionization electrons deposited in the liquid and scintillation-induced photoelectrons extracted 
from a CsI UV-photocathode, deposited on the electrode, are focused into the electrode's holes; they induce electroluminescence (EL) in the bubble, with up to several hundred EL photons emitted per drifting electron. Precise reconstruction of the event topology can be performed with pixilated photon detectors, e.g. SiPMs or cryogenic Gaseous Photomultipliers $\ddot{i} i^{1 / 2}$ GPMs. Another new concept is that of ionization-charge recording in the saturated vapor of a dual-phase TPC, with Resistive-Plate WELL detectors. They comprise of a single-THGEM $\ddot{i}_{i} 1 / 2$ blindi $i 1 \frac{1}{2}$ electrode coupled to a patterned readout anode through a high bulk-resistivity plate; the latter has shown to quench eventual discharges, leading to stable large dynamic-range operation at superior gains. The operation characteristics and performances of the LHM and GPM in LXe and that of the RPWELL in Xe vapor phase will be presented, with their potential applications in DM searches and neutrino physics.

UR5 Light detection: new directions (Part II)

Sunday, Oct. $30 \quad$ 16:10-17:25, Madrid

UR5-1: Low Dose 3 Gamma Medical Imaging for Small Animals with the XEMIS2 Single- Phase Liquid Xenon Compton Camera

L. Gallego $^{1}$, J. P. Cussonneau ${ }^{1}$, D. Thers ${ }^{1}$, L. Scotto Lavina ${ }^{1}$, J. Masbou ${ }^{1}$, E. Morteau ${ }^{1}$, N. Beaupere ${ }^{1}$, L. Virone ${ }^{1}$, P. Leray $^{1}$, J.-

S. Stutzmann $^{1}$, S. Bouvier ${ }^{1}$, S. Acounis ${ }^{1}$, F. Lefevre ${ }^{1}$, D. Roy ${ }^{1}$, O. Lemaire ${ }^{1}$, S. Bassetto ${ }^{2}$, P. Briend ${ }^{2}$, M. Staempflin ${ }^{2}$,

J. Butterworth ${ }^{2}$, T. Carlier ${ }^{3}$, M. Cherel ${ }^{4}$, F. Kraeber-Bodere ${ }^{3}$, H. Mathez ${ }^{5}$, L. Royer ${ }^{5}$, Y. Xing ${ }^{1}$, A. Bongrand ${ }^{1}$, N. Pillet $^{5}$,

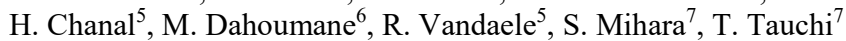

${ }^{1}$ Subatech, Ecole des Mines de Nantes, CNRS/IN2P3, Nantes, France

${ }^{2}$ Air Liquide Advanced Technologies, 2 rue Clémencière, F-38360 Sassenage, France

${ }^{3}$ Centre Hospitalier Universitaire de Nantes, 1 place Alexis-Ricordeau, 44093 Nantes, France

${ }^{4}$ équipe 13, INSERM U892, 8 quai Moncousu, 44000 Nantes, France

${ }^{5}$ LPC, Clermont-Ferrand, 24 Avenue des Landais, Clermont-Ferrand, France

${ }^{6}$ CNRS/IN2P3 UMR5822, IPNL Université de Lyon, Lyon, France

${ }^{7}$ High Energy Accelerator Research Organization (KEK), Tsukuba, Japan

The 3 ? imaging technique is an innovative functional medical imaging modality, which is based on two new concepts: the joint use of a new technology based on a liquid xenon Compton camera, and a new radiopharmaceutical labeled with 44Sc. The benefit of this new technique is expressed directly in terms of reducing the number of disintegrations necessary for obtaining the image and therefore, the activity injected to the patient is significantly reduced. A successful R\&D program has provided an experimental demonstration of the use of a liquid xenon Compton camera for 3 ? imaging. This initial phase was the starting point of the XEMIS project (XEnon Medical Imaging System), which involves both fundamental research and the development and implementation of innovative technologies. A first prototype of a single-phase liquid xenon Compton telescope, called XEMIS1, was successfully developed and tested at Subatech laboratory. A second phase dedicated to small animal imaging, XEMIS2, is now under qualification. This larger scale prototype is a monolithic liquid xenon cylindrical camera that holds around $200 \mathrm{~kg}$ of liquid xenon. The geometry of XEMIS2 is optimized to provide a full coverage of the small animal thanks to its $24 \mathrm{~cm}$ axial field of view. To detect both the ionization signal and the VUV scintillation photons produced in the liquid xenon after the interaction of ionising radiation, the active volume of the detector is covered by Hamamatsu PMTs and two end segmented anodes with a total number of 20000 pixels. XEMIS2 has been designed for preclinical applications in hospital centers. It includes a very compact liquid xenon cryogenics infrastructure and a fast data acquisition system with new front-end electronics. The XEMIS2 camera should be completely qualified this year and it will be operational and available from 2017 for preclinical research at the Center for Applied Medical Research (CIMA) located in the Nantes Hospital.

\author{
UR5-2: Innovative Devices for Amplification of Ionisation Charge in Liquid Argon Time Projection Chamber \\ Detectors \\ $\underline{\text { A. Fava }}^{1}$, F. Pietropaolo ${ }^{2}$ \\ ${ }^{I}$ Fermilab, Batavia, Illinois, United States \\ ${ }^{2}$ CERN, Geneva, Switzerland
}

The groundbreaking idea we are proposing is the development of innovative devices to multiply and collect electron charge generated by ionizing particles passing through liquid Argon (LAr) medium.

The primary motivation is to make single-phase Liquid Argon Time Projection Chamber detectors (LAr-TPCs) sensitive to events with energy deposition of the order of $10 \mathrm{keV}$ or less, 100 times smaller than present state of the art. The concept is to seek the multiplication of ionization electrons directly in LAr at the end of their drift path by dimensioning and realizing a Micro Strip anodic plane capable of generating an electric field locally large enough $(\sim 1 \mathrm{MV} / \mathrm{cm})$ to trigger the proportional multiplication of charge carriers. The feasibility of the project is supported by the positive results of pioneering attempts to multiply charge in LAr in proximity of micrometric wires, yet too fragile to be exploited in TPC detectors.

The impact on Particle Physics of low energy rare events is potentially dramatic, mainly in searches for Dark Matter interactions and coherent neutrino scattering characterized by $\mathrm{O}(100 \mathrm{keV})$ experimental signature and a $\mathrm{O}\left(10 \mathrm{E}-40 \mathrm{~cm}^{\wedge} 2\right) \mathrm{cross}$ section. 
UR5-3: The Performance of ABALONE Photosensors for the Detection of Ultra-Rare Processes D. Ferenc $^{1}$, A. Chang ${ }^{1}$, M. Segedin Ferenc ${ }^{2}$

${ }^{I}$ University of California, Davis, Davis, CA, USA

${ }^{2}$ PHOTONLAB, Inc., Davis, CA, USA

The fields of astroparticle physics, medical imaging and nuclear security — which all rely on large-area photosensors- have been stuck with the expensive 80-year old photomultiplier tube (PMT) manufacture. PMTs comprise more than 180 hand-made components, created out of $\sim 8$ materials. That complexity necessitates a very slow, labor-intensive vacuum processing, incompatible with low-cost mass-production. Our search for a modern mass-production technology has therefore started with a fundamental redefinition of allowed processes and materials. The patented — and by now fully proven - ABALONE technology for inexpensive mass-production of large-area photosensors is based on only 3 vacuum-processed components-exclusively mass-producible of glass, or ultrapure fused silica - when low radioactivity is important. The 3 glass components are processed by the common thin-film deposition technique (equivalent to $\mathrm{CDs}$ ), and bonded using our novel vacuum encapsulation method. Hereby we report on the unique performance of ABALONE Photosensors, specifically for the detection of ultra-rare processes. The purity of the materials used lead to some of the lowest achievable levels of radioactivity. Also thanks to the purity of the materials and processes, as well as to the patented ABALONE photosensor design, the rate of ion feedback after-pulses in ABALONE has been by two orders of magnitude lower than in the best PMTs. The single-photon sensitivity allows one to resolve even the (non-returning) back-scattered photoelectrons. Thanks to the simple and symmetric geometry, and the strong photoelectron focusing (factor 10,000), which allows the application of a very small and thus fast (and cheap) Geiger Avalanche Diode (G-APD), the timing resolution of a 10-cm prototype has been $\sim 900 \mathrm{ps}$ fwhm, even without any optimization of the geometry or the readout. The robustness of ABALONE photosensors is unprecedented, including the survival of exposures to strong light.

(WKSP) Technology Frontier for Single Photon Detection and Advanced Scintillator Timing

SP1 Challenges and Demands on fast and large area Photon Detection Devices: the point of industrials

Friday, Nov. $4 \quad$ 08:00-09:50, Londres

SP1-1: Fast Timing with Scintillators: Towards 10ps Coincidence Time Resolution for Pet? P. Lecoq

EP, CERN, Geneva, Switzerland

There is an increasing demand on timing performance for a wide range of applications, such as time of flight (TOF) techniques for PET cameras and particle identification in particle physics detectors, precise event time tagging at high luminosity accelerators and a number of photonic applications based on single photon detection. An objective of 10ps for a scintillator-based detector requires the precise localization of the gama conversion as well as an optimization of the whole detection chain, i.e. light generation in the scintillator, light transport from the interaction point to the photodetector, light extraction efficiency, photoconversion in the photodetector, electronic readout. The time resolution of a scintillator-based detector is driven by the density of photoelectrons generated in the photodetector at the detection threshold. For the scintillator it is related to the intrinsic light yield, the pulse shape (rise time and decay time) and the light transport from the gamma-ray conversion point to the photodetector. When aiming at 10ps time resolution fluctuations in the thermalization and relaxation time of hot electrons and holes generated by the interaction of ionization radiation with the crystal become important. The light transport in the crystal is also an important source of time jitter. Light bouncing within the scintillator must be reduced as much as possible as it spreads the arrival time of photons on the photodetector and strongly reduces the light output by increasing the effect of light absorption within the crystal. The photodetector has also an important contribution, that can be evaluated through its single photon time response. A fully digital photon counting scheme could allow approaching the theoretical limit given by the photostatistics. This talk will review the different processes at work, identify the important parameters at play and evaluate the conditions for reaching 10 ps coincidence time resolution in Time-of-Flight PET.

SP1-2: Silicon Photomultipliers (SiPM) for Timing and Scintillation Applications $\underline{\text { J. Murphy }}^{1}$, C. Jackson, D. Herbert

${ }^{I}$ SensL Technologies Ltd., Cork, Ireland

Recent developments have lead to a variety of improvements that allow Silicon Photomultiplier (SiPM) sensors to compete with the PMT for the purpose of large-area detection and timing applications. J-Series SiPM sensors from SensL have been developed with high-performance timing applications in mind. Larger SiPM sensors can suffer from timing degradation due to the transit time spread (TTS) that results from the variation in the track lengths from different parts of the sensor. SensL's J-Series sensors feature multiple topside anodes and signal track length equalization in order to minimize the TTS, preserving the fast timing performance of larger sensors. J-Series sensors also benefit from a chip-scale TSV package that has the highest fill factor on the market, allowing large-area arrays to be formed with over $90 \%$ fill factor. In addition, the TSV glass entrance window allows 
good detection in the UV, with a PDE of $5 \%$ even at $250 \mathrm{~nm}$.

All SensL SiPM sensors feature a unique 'fast output' as a third terminal (in addition to the anode and cathode), which is capacitively coupled to each microcell providing an ultra-fast signal. The fast output also has considerably lower capacitance, around $1 \mathrm{pF}$ for a $1 \mathrm{~mm}$ sensor, compared to $\sim 100 \mathrm{pF}$ for the standard output, leading to simpler electronics design. Another unique opportunity afforded by the fast output is the use of a technique called signal driven multiplexing to readout large-area arrays of SiPM. This technique uses Schottky diode pairs to avoid the issues of a large amount of summed dark noise and capacitance load when many readout channels are multiplexed together.

Timing applications are at the fore-front of much of SensL's current development. We will present ToF-PET CRT results with JSeries and also give an update on our new red sensitive R-Series SIPM sensors that is focussed on ToF LiDAR applications.

SP1-3: Introduction of KETEK's latest SiPM generation and Evaluation Kits

W. Hartinger, C. Dietzinger, T. R. Ganka, F. Schneider, P. Iskra, E. Engelmann, S. Loebner, W. Gebauer, A. Márquez Seco, F. Düsberg, F. Wiest

KETEK GmbH, Munich, Germany

KETEK's Silicon Photomultipliers (SiPMs) are state-of-the-art low level light detectors for applications like fast scintillator or fiber read out, time of flight measurements and low-level-light detection. With the latest SiPM generation, KETEK introduces a new chip and package design with significant improvements of essential technical features. In addition the new PACKAGE 2.0 is the key technology for highly compact and application specific sensor arrays and due to its MSL1-compliance, handling and processing is very simplified. An overview about sensor and package characteristics and the SiPM portfolio will be given. Besides the new sensors, KETEK offers a full Evaluation Kit for SiPM performance measurements. Due to the modular concept of the Evaluation Kit based on well adapted pre-amp, bias source and pre-mounted SiPMs, application specific configurations can be quickly and easily realized and tested. Information about the EVAL-Kit and test results will be presented.

SP1-4: New Silicon Photomultiplier Technologies and Time-Resolution Measurements Techniques F. Acerbi, A. Ferri, A. Gola, G. Zappala, C. Piemonte, N. Zorzi

Center for Materials and Microsystems, FBK, Trento, Italy

Silicon Photomultipliers are multi-cell photodetectors used in many applications. They have single-photon sensitivity, they are compact and require low bias voltage and have good time resolution. They are used in many application field, to detect from single-photon to many photons from a gamma-ray scintillation events. Many different technologies have been developed for SiPM. In FBK (Fondazione Bruno Kessler, Trento, Italy) during last years "high-density" (HD) SiPM technology proved good results in terms of photon detection efficiency and time resolution. HD-SiPM features narrow trenches to separate the SiPM microcells, obtaining small cells with high fill-factor (FF). In this contribution this new technologies will be described and along with the procedures to extract the best time resolution from these photodetectors. For SiPMs, the time resolution it is usually not straightforward to measure, and it can be divided into: i) single-photon time resolution, ii) few-photon time resolution or iii) coincidence time resolution. Differently from the first two, the last one is used, and it is an important parameter, when measuring the time-of-arrival of a two gamma-photons (in coincidence), as in the case of TOF-PET. It can depends from many factors: SiPM properties, ii) read-out electronics, iii) SiPM and scintillator crystal packaging, etc.

\author{
SP1-5: Fast Advanced Scintillator Timing - Overview of the Working Group Photodetectors \\ E. Charbon \\ TU Delft, Delft, Netherlands \\ On behalf of the FAST network a COST TDP Action
}

The activities of the FAST (Fast Advanced Scintillator Timing) working group on Photodetectors at the European Trans Domain COST Action TD1401 are presented as an overview. The overall focus of the working group is on cutting edge technologies addressing the issues that currently limit the use of single photon detectors, including in particular large area readout issues, ultrafast timing better than $100 \mathrm{ps}$, radiation hardness, and Ultra-Violet and Vacuum Ultra-Violet sensitivity. The technologies that are of particular interest include analog SiPMs, SiPMs with embedded digital electronics (monolithic digital SiPMs, 3D-integration, etc.), as well as non-solid state based solutions such as Micro-Channel plates, hybrid photodetectors or gas-based solutions.

\author{
SP1-6: Progress in Development of a Solar-Blind Photosensor for Readout of the Fast Scintillation Component \\ of Barium Fluoride

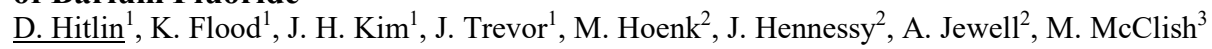 \\ ${ }^{I}$ Caltech, Pasadena, CA, USA \\ ${ }^{2}$ Jet Propulsion Laboratory, Pasadena, CA, USA \\ ${ }^{3} R M D$, Inc., Watertown, MA, USA
}


Recent progress on the development of a large area UV-sensitive, solar-blind, high gain avalanche photodiode suitable for the readout of the fast $220 \mathrm{~nm}$ scintillation component of barium fluoride, while being insensitive to the slow $300 \mathrm{~nm}$ component, is presented. The APD incorporates an interference filter and a superlattice layer to produce high quantum efficiency at $220 \mathrm{~nm}$, strong rejection at $300 \mathrm{~nm}$ and improved timing characteristics. Optimization studies and measurements of the filter and superlattice layer characteristics will be discussed.

\section{SP2 Performance Measurements of new Single Photon Detection Devices}

Friday, Nov. $4 \quad$ 10:30-12:25, Londres

SP2-1: Influence of SiPM Single Photon Timing Resolution on Coincidence Timing Resolution with Fast Scintillator

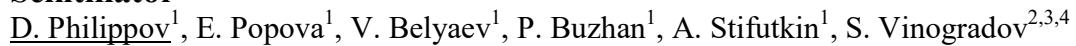

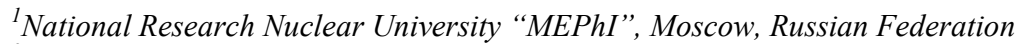

${ }^{2}$ Lebedev Physical Institute of the Russian Academy of Sciences, Moscow, Russian Federation

${ }^{3}$ Department of Physics, University of Liverpool, Liverpool, United Kingdom

${ }^{4}$ Cockcroft Institute of Accelerator Science and Technology, Warrington, United Kingdom

A coincidence timing resolution (CTR) of scintillation detectors based on SiPMs depends on both photodetector and scintillator properties. The photodetector-related contribution is very important and has a considerable impact on the CTR. The CTR dependence on photon detection efficiency (PDE) is well studies and clarified, but as soon as advances in developments of fast scintillators and fast SiPMs make CTR approaching to a fundamental limits, a role of a single photon timing resolution (SPTR) is increased, and it requires more attention and studies. This study presents and discusses results of SPTR and CTR measurements of long and short LYSO crystals and a series of timing optimized KETEK SiPM samples of 3x3 mm2 size as well as their processing algorithms and theoretical analysis based on the analytical model of SiPM time resolution.

This work was supported in part by Megagrant program of Russia under Grant Agreement 14.?12.31.0006 and the additional agreement ?2 from 03.03.2016, COST-STSM-TD1401-33279 FAST COST Collaboration Grant and EC under Grant Agreement 329100 "SiPM in-depth".

\section{SP2-2: Active SiPM- Fast Analogue CMOS SiPM Prototypes with Integrated Amplifiers}

E. Popova $^{1}$, S. Ageev ${ }^{2}$, D. Philippov ${ }^{1}$, P. Buzhan ${ }^{1}$, A. Stifutkin ${ }^{1}$, S. Klemin ${ }^{1}$, P. Iskra ${ }^{3}$, W. Butler ${ }^{3}$, E. Engelmann ${ }^{4}$, F. Wiest ${ }^{3}$, R. Fojt ${ }^{3}$, F. Kayumov ${ }^{5}$

${ }^{1}$ SiPM laboratory, MEPhI, Moscow, Russia

${ }^{2}$ EXELTEK, Moscow, Russia

${ }^{3}$ KETEK GmbH, Munich, Germany

${ }^{4}$ Department of Electrical Engineering and Information Technology, Universitaet der Bundeswehr Muenchen, Munich, Germany

${ }^{5}$ P.N. Lebedev Physical Institute of the Russian Academy of Sciences, Moscow, Russia

Silicon Photomultipliers (SiPMs) are well-known low level light detectors. Besides a high photon detection efficiency they demonstrate quite nice timing properties due to the high electric fields inside of the depletion region. SiPMs might potentially replace Photomultiplier Tubes (PMT) for many applications where large areas have to be covered by photodetectors. However, conventional SiPMs have a significant drawback for such applications, because their output capacitance rapidly grows with increasing device area.

As a consequence of an increased capacitance, the amplitude of the output SiPM signal decreases, the leading edge becomes slower and the signal/noise ratio is deteriorated. This leads to a worsening of the single photon time resolution (SPTR) and the photon-number-resolving performance.

In order to improve the SiPM parameters for large area devices we have developed an active analogue SiPM approach. Produced in the framework of a standard CMOS technology, this prototype of active SiPM consists of an usual SiPM photosensitive part and an integrated fast amplifier located at the periphery of the sensor. The active SiPM operates now as a high impedance pure current source, like anode of PMT.

Measurement results of active SiPMs prototypes, recently produced on a CMOS foundry, will be presented and discussed.

Work has been supported by Megagrant 2013 program of Russia Agreement 14.A12.31.0006 from 24.06.2013 and the additional agreement ?2 from 03.03.2016.

SP2-3: Precise Metrology of SiPM: Measurement and Reconstruction of Time Distributions of Single Photon Detections and Correlated Events $\underline{\text { S. Vinogradov }}^{1,2,3}$ 
${ }^{I}$ Lebedev Physical Institute, Moscow, Russia

${ }^{2}$ University of Liverpool, Liverpool, UK

${ }^{3}$ Cockcroft Institute of Accelerator Science and Technology, Warrington, UK

Recent advances in developments of fast scintillators and fast silicon photomultipliers (SiPM) resulted in significant improvements in a time resolution of TOF PET and similar applications. A coincidence time resolution of $10 \mathrm{ps}$ is considered as an ultimate goal of these improvements. Approaching to fundamental timing limits in TOF applications, all essential SiPM parameters responsible for true and false detection events initiated by photons and dark carriers, and accompanied by crosstalk and afterpulsing correlated events, should be measured, reconstructed and identified in their timing dependencies with a high precision. This study is based on a probabilistic consideration of key SiPM parameters as temporal distributions of corresponding events. A single photon (true) detection is represented either by a probability density function (PDF) which width relates to a single photon time resolution (SPTR) and the area defines a photon detection efficiency (PDE) or by incomplete cumulative distribution function (CDF) approaching to the PDE at long times. A false detection is a probabilistic event combined from dark counts and correlated events, which can be specified and separated by corresponding CDFs. Proper extraction of pure photon detection and correlated event distributions is especially valuable in case of large area SiPMs with a high dark noise affecting quality and precision of standard measurement techniques. Representative examples of measurements of the time distributions as well as analytical models and reconstruction algorithms are presented and discussed.

This work was supported in part by the EC under Grant Agreement 329100.

\section{SP2-4: 3D Digital SiPM for Precise Single Photon Timing Resolution}

\section{J.-F. Pratte}

Interdisciplinary Institute for Technological Innovation - 3IT, Universite de Sherbrooke, Sherbrooke, QC, Canada

On behalf of the Groupe de Recherche en Appareillage Médical de Sherbrooke (GRAMS)

This presentation aims at stimulating discussion on single photon timing resolution and how 3D digital SiPM can contribute to improve it. The design and optimization of each building block of the acquisition chain will be discussed. Now that we have working 3D digital SiPMs and that the proof of concept is established, we will discuss future plans toward sub $10 \mathrm{ps}$ single photon timing resolution.

\section{SP2-5: Radiation Damage Studies of SiPMs at Low Temperatures} T. Tsang ${ }^{1}$, T. Rao ${ }^{1}$, C. Woody ${ }^{2}$, S. Stall ${ }^{2}$, M. Chiu ${ }^{2}$

${ }^{I}$ Instrumentation, BNL, Upton, NY, USA

${ }^{2}$ Physics, BNL, Upton, NY, USA

We characterized the performance of Silicon Photomultipliers (SiPMs) before and after exposure to $10^{\wedge} 12 \mathrm{neutron} / \mathrm{cm}^{\wedge} 2 \mathrm{dosage}$. Despite the typical orders of magnitude increase of dark current upon neutron irradiation that loss SiPM's photon number resolving capability and deem unusable, we show that the elevated dark current can be suppressed and single-photoelectron detection can be restored by operating it at a lower temperature. The required operating temperature depends on the dosage received. Furthermore, after thermal annealing at high temperature, there is strong evidence that the extrinsic dark current is lowered and the single-photon detection performance are to some extent revived at ambient temperature.

\section{SP2-6: Single Photon Detection by Gaseous Counters: Status and Perspectives}

$\underline{\text { S. Dalla Torre }}$

Sezione di Trieste, INFN, Trieste, Italy

The development of gaseous detectors for single photon detection has been mainly triggered by the requirements of the Cherenkov imaging counters and three generation of detectors have been developed: the gaseous detector with converting vapours included in the gas atmosphere, the open geometry detectors with solid state CsI photocathodes, where no ion back flow limitation can be envisaged, and the MPGD-based detectors with solid state CsI photocathodes, where the reduction of the ion back flow can be effectively introduced. The use in gaseous counters of solid state photoconverters in the visible range is desirable, even if challenging due to the fragility and chemical reactivity of the converters: nevertheless, it is being persuaded. Moreover, nowadays, the use of gaseous photon detectors is not restricted to Cherenkov counters: they are proposed for cryogenic noble liquid detectors in rare-event experiments and they can detect the luminescent light produced in multiplication processes in gas in a variety of applications in science and beyond.

After a short historical overview, the status and perspective of gaseous detectors of single photons are analyzed in detail, including application examples. 
SP2-7: Development of Cherenkov Detectors for PET

V. Sharyy ${ }^{1}$, E. Ramos ${ }^{1}$, O. Kochebina ${ }^{1,2}$, C. Canot $^{1}$, M. Alokhina ${ }^{1,3}$, X. Mancardi ${ }^{1}$, P. Abbon ${ }^{1}$, D. Desforge ${ }^{1}$, C. Flouzat ${ }^{1}$, J.- $^{-}$ P. Mols ${ }^{1}$, S. Jan ${ }^{2}$, P. Starzinski ${ }^{1}$, P. Verrecchia ${ }^{1}$, G. Tauzin ${ }^{1}$, D. Yvon ${ }^{1}$

${ }^{I} I R F U, C E A$, Gif-sur-Yvette, France

${ }^{2}$ SHFJ, CEA, Orsay, France

${ }^{3}$ Nuclear Physics Department, Taras Shevchenko National University of Kyiv, Kyiv, Ukraine

In this presentation we are investigating the feasibility of the Cherenkov detectors to enhance the TOF performance of PET scanners. We discuss the results of the experimental tests and simulation expectations for two Cherenkov detectors. The first detector uses an innovative liquid, TriMethyl Bismuth, as a Cherenkov radiator, which plan to be used in the high precision brain and pre-clinical PET (CaLIPSO project). The second project, PECHE, develops the full body PET scanner using the crystalline lead fluoride to radiate the Cherenkov light. In both project we are using the micro-channel-plate PMT to obtain the highest possible resolution in time. Our results are showing that we can detect the Cherenkov lights with a good efficiency. Using the dedicated GATE simulations we estimated the several main parameters for the brain-oriented and full body scanners: spatial resolution, coincident resolving time and noise equivalent count rate, modified for account the TOF potential.

\section{SP3 Large area photodetectors}

\section{Friday, Nov. $4 \quad$ 14:00-16:00, Londres}

SP3-1: Fast Timing for Dark Matter Search in LXe with Pulse Shape Discrimination

$\underline{\text { K. L. Giboni }}{ }^{1}$, H. Kusano ${ }^{1}$, A. Banjongkan ${ }^{2}$, P. Namwongsa ${ }^{2}$, S. Rujirawat ${ }^{2}$

${ }^{I}$ Physics and Astronomy, Shanghai Jiao Tong University, Shanghai, China

${ }^{2}$ School of Physics, Suranaree University of Technology, Nakhon Ratchasima, Thailand

The strongest background in Dark Matter (DM) direct detection experiments are ?-rays. In LXe detectors such electron recoil events (ER) can be distinguished from nuclear recoils (NR) of DM by their different ionization densities, most often expressed the ratio of Charge Signal (S2) to the direct scintillation light signal (S1). The difference in ionization density is also reflected in different amounts of S1 signal. Direct scintillation and light produced by recombination of free charges have different decay constants, $2.2 \mathrm{~ns}$ and $27 \mathrm{~ns}$, respectively. Several attempts to reject background based on the scintillation pulse shape were limited in the past by the not too fast response of the used photo-sensors. At the low light levels typical in DM detectors, the timing information is smeared out by slow photon detectors and a background discrimination is not efficient. With a LXe test set up and simulations we are investigating if a new PMT from Hamamatsu can provide better results. This is a 2" x 2" metal channel PMT with a transit time spread $<0.3 \mathrm{~ns}$, compared to $7 \mathrm{~ns}$ for the commonly used PMTs. . This study is part of the detector development within the PandaX project in the Chinese CJPL laboratory.

\section{SP3-2: Understanding SiPM Instrumentation and Why SiPMs May Not Replace PMTs}

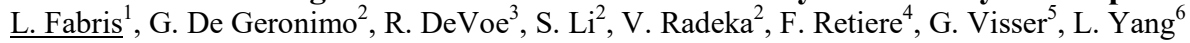

${ }^{I}$ NS\&ITD, Oak Ridge National Laboratory, Oak Ridge, TN, USA

${ }^{2}$ Instrumentation Division, Brookhaven National Laboratory, Upton, NY, USA

${ }^{3}$ Physics, Stanford University, Stanford, CA, USA

${ }^{4}$ Science Technology, TRIUMF, Vancouver, BC, Canada

${ }^{5}$ Cntr For Explor Of En \& Matter, Indiana University Bloomington, Bloomington, IN, USA

${ }^{6}$ Physics, University of Illinois Urbana-Champaign, Urbana, IL, USA

Advancements in integrated silicon technology over the last decades have enabled significant progress in all fields of engineering and science, and radiation detection is no exception. Several large-scale experiments have been made possible by the development of new ways to make circuits ever smaller, with good resistance to damaging radiation. Detectors, especially those for high-energy physics experiments, have also seen benefits from the processing of large silicon substrates into viable particle trackers. On the other hand, devices such as photomultiplier tubes (PMTs) have been used for three quarters of a century and are still the workhorse of radiation detection in many applications. Only in recent years a new device has become attractive in overcoming some undesired shortcomings of PMTs. The new devices, called silicon-photomultipliers, or SiPMs in short, seem to have all of the features of PMTs without the same constraints of large bulk volumes, need for high voltage bias, sensitivity to magnetic fields, and lack of ruggedness. For these reasons, SiPMs have recently become very popular, and it is no wonder they are considered direct replacements for PMTs from the electronics design point of view. This has been demonstrated to be true for some applications where the amount of signal is large. However, when the signal becomes smaller, such as in large-area experiments or when observing weak interactions, the analogy breaks down in such a way that SiPMs have to be treated much differently than PMTs.

We will discuss what happens when SiPMs are used in applications where the analogy with PMTs no longer holds, and we will supply a theoretical basis to generalize the observations made. Specifically, the influence of SiPMs' large capacitance on system 
sensitivity and signal integrity will be discussed. Also practical design criteria and a solution for large (100 $\mathrm{cm} 2 \mathrm{and}$ above) SiPM arrays will be given.

This manuscript has been authored by UT-Battelle, LLC under Contract No. DE-AC05-00OR22725 with the U.S. Department of Energy.

\author{
SP3-3: Large Area VUV-Sensitive MPPCs for Liquid Xenon Detector in MEG II Experiment \\ S. Ogawa \\ The University of Tokyo, Tokyo, Japan \\ On behalf of the MEG II Collaboration
}

The MEG II experiment is an upgrade of the MEG experiment to search for the charged lepton flavor violating decay of muon, $\mu^{+}$? $\mathrm{e}^{+}$?. The performance of the liquid xenon (LXe) ?-ray detector will be greatly improved with a highly granular scintillation readout realized by replacing 216 photomultiplier tubes (PMTs) on the ?-ray entrance face with 4092 Multi-Pixel Photon Counters (MPPCs). For this purpose, we have developed a new type of large area MPPC which is sensitive to the LXe scintillation light in vacuum ultraviolet (VUV) range, in collaboration with Hamamatsu Photonics K.K. The MPPC has been tested in LXe, and an excellent performance has been confirmed including high photon detection efficiency ( $>15 \%)$ for LXe scintillation light. This MPPC is a discrete array of four $6 \times 6 \mathrm{~mm}^{2}$ chips, and a sufficiently short time constant for a single photoelectron waveform is achieved by connecting them in series. A good energy and timing resolution have been confirmed for the LXe scintillation light. For further understanding of the MPPC, we are also planning a new performance test in the xenon gas. In this talk, the performance of our VUV-sensitive MPPC obtained from these tests will be reported.

\title{
SP3-4: Silicon Photomultipliers in Astroparticle Experiments
}

$\underline{\text { N. Otte }}$

Georgia Institute of Technology, Atlanta, GA, USA

The majority of astroparticle detectors are photon hungry and use large-area photon detectors. While the classical photomultiplier tube still plays a dominant role in the field, SiPMs are on the rise and are being used in a number of experiments already. With the recent boost in performance of SiPMs it is to be expected that SiPMs will outnumber PMTs in astroparticle experiments in the not so distant future. In this presentation I discuss the requirements of photon detectors in astroparticle experiments, how existing SiPMs meet these requirements, and what improvements remain to be done. I focus on imaging atmospheric Cherenkov telescopes like the planned Cherenkov Telescope Array but will also address the needs of other techniques in astroparticle physics, which use large numbers of photon detectors.

\section{SP3-5: The DarkSide Photodetection Program}

\section{G. Paternoster}

Fondazione Bruno Kessler, Trieste, Italy

On behalf of the DarkSide Collaboration

The DarkSide collaboration is developing a large argon experiment for dark matter search. The primary detector, a dual phase Time Projection Chamber, will be equipped with about $14 \mathrm{~m}^{2}$ of photo sensors sensitive to the scintillation light emitted by target mass, 20 tonnes of depleted argon. The project includes an extensive program for the realization of large area SiPM based units, $5 \times 5 \mathrm{~cm}^{2}$, operated as aggregated single photon counters at $88 \mathrm{~K}$. These units exhibit an overall photo-detection efficiency in excess of $40 \%$ at $400 \mathrm{~nm}$ with a primary dark-noise lower than $10 \mathrm{cps} / \mathrm{cm}^{2}$ in liquid argon. Moreover the photo-detector module includes a local low noise cryogenic pre-amplifier designed to aggregate the signals from the SiPMs into a single output channel with a signal to noise better than $8 \mathrm{~V} / \mathrm{V}$ and a dynamic range higher than 30 photo-electrons. Finally an innovative signal extraction strategy is under study: a cryogenic analog optical transmission couples the high radio-purity of optical fibers with their electromagnetic noise immunity.

\section{SP3-6: Large Area Low Power Photodetector Based on 3D Digital SiPM}

S. A. Charlebois

Interdisciplinary Institute for Technological Innovation - 3IT, Université de Sherbrooke, Sherbrooke, Canada

On behalf of the Groupe de Recherche en Appareillage Médical de Sherbrooke (GRAMS)

Solid state photodetectors such as SiPMs are interesting for large area detector for sensitivity, timing resolution, robustness and cost. We will discuss the advantages of using 3D digital SiPMs (3DdSiPM) for large area systems. In a 3DdSiPM, each single photon avalanche diode (SPAD) is read individually and converted to a digital signal. This mitigates most of the SPADs variations between various units and simplifies drastically the calibration requirement. Furthermore, the " $3 \mathrm{D}$ " architecture of the 
detector allows for an independent optimization of the photosensing layer (the SPAD array) and the electronic readout layer. 3DdSiPM can therefore be designed to be sensitive to different parts of the light spectrum. Similarly, the readout electronics can be tailored for low power consumption and/or fast timing. We will present our proposal for a large area low power fully digital photon detector made of 3DdSiPMs for noble liquid experiments. We will discuss the 3DdSiPM architecture, the readout and trigger algorithm, the digital and system integration being considered and comment on thermomechanical, background and contamination issues.

\section{SP4 Projects Perspectives for Young Researchers}

Friday, Nov. $4 \quad$ 16:30-16:30, Londres

(WKSP) Academia meets industry forum

Al1 Academia Industry Matching Forum I

Tuesday, Nov. $1 \quad$ 08:00-10:00, Varsovie

Al1-1: Modular High Energy X-Ray Imagers

$\underline{\text { P. Seller }}^{1}$, M. D. Wilson ${ }^{1}$, D. D. Duarte ${ }^{1}$, L. L. Jones ${ }^{1}$, M. Prydderch ${ }^{1}$, S. L. Thomas ${ }^{1}$, M. C. Veale ${ }^{1}$, I. Sedgwick ${ }^{1}$, S. Richards ${ }^{1}$, R. Turchetta ${ }^{1}$, B. Marsh ${ }^{1}$, G. Royle ${ }^{2}$

${ }^{I}$ Detector Development, STFC-Rutherford Appleton Laboratory, Didcot, Oxfordshire, United Kingdom

${ }^{2}$ Medical Physics and Biomedical Engineering, University College London, London, United Kingdom

The Detector and Electronics Division at RAL have built many X-ray imaging systems over the last decade. Many of them are very application specific but two in particular have wider application possibilities to industrial, security and medical fields. The HEXITEC detector has demonstrated fully spectroscopic imaging from $2 \mathrm{keV}$ to $200 \mathrm{keV}$ with sub $1 \mathrm{keV}$ (FWHM) energy resolution and count rates of $>10^{6}$ photons/s per $80 \times 80$ pixel module when used with $1 \mathrm{~mm}$ thick Schottky anode CdTe. The ability to tile the HEXITEC detectors on three sides with a dead space of detectors on three sides with a dead space of $<2$ pixels and the pixel size of $250 \mu \mathrm{m}$ make it a good candidate to be used as a single module or in tiled arrays. The LASSENA CMOS sensor is our first wafer scale imager. This sensor has 6.7 million pixels, each $50 \mu \mathrm{m}$ square giving an active area of $120 \mathrm{~mm} \mathrm{x}$ $140 \mathrm{~mm}$ which can be readout at $34 \mathrm{fps}$. We have attached scintillator layers to this device to make X-ray sensitive detectors. An STFC funded program called XNEXT tiles these already large modules to create larger area X-ray imager with very small dead regions between individual tiles. Currently we have a $240 \mathrm{~mm}$ x $250 \mathrm{~mm}$ active array but the modular nature allows larger arrays if needed. We have demonstrated its use with several different scintillators for different applications but of particular interest is a $1 \mathrm{~mm}$ thick CsI structured scintillator which allows high spatial resolution, fast readout speed and good efficiency for X-ray tube energies up to $160 \mathrm{keV}$.

Al1-2: Development of a MR-Compatible DOI-TOF Detector Module for PET Imaging Systems

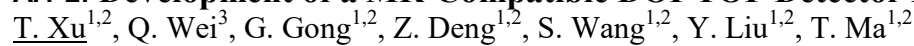

${ }^{I}$ Department of Engineering Physics, Tsinghua University, Beijing, China

${ }^{2}$ Key Laboratory of Particle \& Radiation Imaging (Tsinghua University), Ministry of Education, Beijing, China

${ }^{3}$ School of Automation and Electrical Engineering, University of Science and Technology Beijing, Beijing, China

PET systems are widely used in clinical and preclinical molecular imaging applications. Various PET systems, including wholebody human and small animal PET, and dedicated systems such as breast and brain PET have been developed. A Standardized PET detector module with good performance will make it much easier and more efficient for different kind of PET scanner instrumentation. In this paper, we developed a compact MR-compatible PET detector with DOI and TOF capability. The detector module has staggered $15 \times 15+16 \times 16$ LYSO crystal array with single crystal size of $2 \times 2 \times 7 \mathrm{~mm} 3$. The detector is coupled to SiPM array (MicroFJ-30035-TSV, SensL). The output signals of SiPMs are multiplexed by 64-channel ASIC chips and then digitized by $80 \mathrm{MHz} 12$-bit ADC chips and TDC implemented inside FPGA to generate energy and timing information. The performance of the detector was preliminarily evaluated. A flood image was acquired and all crystals were clearly identified with an average energy resolution of $12.7 \%$ for $15 \times 15$ array and $14.2 \%$ for $16 \times 16$ array. About 300 ps coincidence timing resolution was achieved for a pair of single crystal. More accurate energy resolution and coincidence timing resolution for the detector module will be evaluated in the future. And the MR compatibility evaluation of this module is underway. Large quantities of detector blocks will be made and tested to verify the performance stability and reproducibility. Furthermore, we are developing standardized power and signal interface for better usability.

Al1-3: easyPET: a Novel Concept for an Affordable Tomographic System

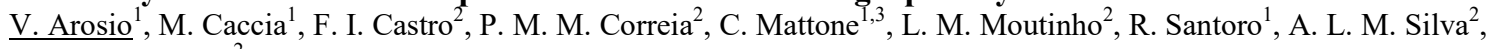
J. F. C. A. Veloso ${ }^{2}$ 
${ }^{I}$ Dipartimento di Scienze ed Alta Tecnologia, Università degli Studi dell'Insubria, Como, Italy

${ }^{2}$ i3n, Departamento de Fisica, Univerdisade de Aveiro, Campus Universitario de Santiago, Aveiro, Portugal

${ }^{3}$ CAEN S.p.a., Viareggio, Italy

easyPET is a novel PET scanner concept, patented by University of Aveiro, aiming to reduce complexity and cost of conventional preclinical Positron Emission Tomography (PET) scanners. This original principle is based on a pair of detectors and exploits a rotating mechanism with two degrees of freedom to reproduce the functionalities and cover the same field of view of an entire PET ring. A prototype providing two dimensional real-time image reconstruction has been designed, engineered and commissioned. A spatial resolution of $1 \pm 0.1 \mathrm{~mm}$ and a sensitivity of $0.1 \%$ with an energy threshold of $80 \mathrm{keV}$ have been measured. Thanks to these good performances, combined with the simple design, the affordability and the portability, the easyPET constitutes an asset in high level educational laboratories. A great outcome that has been reached is the commercialization on behalf of CAEN S.p.A. of the easyPET prototype as a didactic PET system.

Al1-4: MMPDS: First Commercially Available System for Muon Scattering Tomography K. N. Borozdin Headquarters, Decision Sciences International Corporation, Poway, CA, United States

On behalf of the Decision Sciences

Decision Sciences' Multi-Mode Passive Detection System (MMPDS) combines technology invented by scientists at Los Alamos National Laboratory with considerable private sector investment, to deliver totally safe, effective and reliable automated detection techniques to speedily detect both shielded and unshielded nuclear and radiological threats. Advanced image reconstruction and data analysis algorithms enable explosives and contraband detection in addition to nuclear threat detection. A key technology behind the success of Decision Sciences' MMPDS is a scattering muon tomography. Muons are naturally occurring cosmic ray-induced particles that continuously rain down from the Earth's upper atmosphere, harmlessly penetrating everything in their path. Decision Sciences' MMPDS tracks these muons, detecting and recording their deflection in different materials to provide signatures for material density and atomic number, uniquely sensitive to high-Z, high-density materials. The system produces no ionizing radiation, meaning it is completely safe for people, animals, plants and food. Harnessing natural flux of cosmic-ray muons and high-energy electrons produced in the atmosphere, MMPDS detectors track these charged particles through even heavily shielded SNM materials. Based on these tracks the system computes a color-coded 3-D image of scanned objects. Combining automatic detection and 3-D imaging with its gamma radiation detection capabilities, MMPDS provides efficient and safe scanning while facilitating the flow of commerce. Depending on customer requirements and configurable threat levels, a typical 40-foot shipping container can be cleared fast when no threat is detected, at the same time automatically alerting the operator to more complicated scenes requiring extended scan time up to several minutes for reliable threat detection. MMPDS modular construction enables the system to be scaled up or down to scan any type of vehicle, rail cars and cargo containers.

Based on marketing materials of Decision Sciences International Corporation http://decisionsciencescorp.com/

Al2 Academia Industry Matching Forum II

Tuesday, Nov. $1 \quad$ 10:30-12:30, Varsovie

Al2-1: A Modulated X-Ray Generator for Possible Industrial Applications

T. Tamagawa

Nishina Center, RIKEN, Wako, Saitama, Japan

On behalf of the CCNS-MXS collaboration

We invented a modulated X-ray generator (CCNS-MXS) by using gas electron multiplier (GEM) foils combining with the coniferous carbon nano structure (CCNS). The CCNS-MXS is a compact but high-power X-ray generator, controlled by a low voltage $(\sim 100 \mathrm{~V})$ applied to the GEM foil.

The GEM foil, originally invented at CERN by F. Sauli, is a typical micro pattern gaseous detector, but we use it in vacuum as a metal-mesh extraction-electrode of electrons from CCNS. The extracted electrons are accelerated by a high voltage and bombard on a metal target generating high flux X-rays.

We employed CCNS as the field emission device, in which carbon nanotube (CNT) roots to a substrate with some bulky structures of carbon, forming tight connection between CNT and substrate. We just put the GEM foil onto the CCNS substrate and applied the gate voltage to the GEM electrodes. Thanks to the thinness (50-100um) of the GEM foil, the CCNS+GEM device becomes an efficient electron emitter working with a low $(<100 \mathrm{~V})$ gate voltage.

We can turn on and off the generator quickly ( $<1 \mathrm{us)} \mathrm{by} \mathrm{switching} \mathrm{the} \mathrm{gate} \mathrm{voltage;} \mathrm{it} \mathrm{is} \mathrm{not} \mathrm{necessary} \mathrm{to} \mathrm{switch} \mathrm{the} \mathrm{high} \mathrm{voltage}$ applied to the target metal. The CCNS-MXS can issue trigger timing, i.e. can emit X-rays with synchronous to the data acquisition timing of an imaging system. This character implies that the generator becomes a good device for which the industrial 
imaging for fast-rotating devices such as engines, turbo fans, etc.

We will present the design and performance of the X-ray generator at the conference.

\section{Al2-2: RHIP, a Radio-Controlled High-Voltage Insulated Picoammeter}

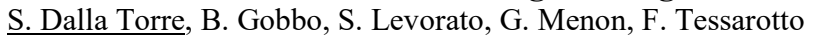

Sezione di Trieste, INFN Trieste, Trieste, Italy

A picoammiter system has been developed by prototyping and engineering. It consists in a current-voltage converter, based on an operational amplifier with very low input current, a high precision ADC, a radio controlled data acquisition unit and the computer-based control, visualization and storage. The picoammiter is characterized by a precision of the order of a tenth of picoamperes and it can measure currents between points laying at potentials of the order of a ten of kilovolts. The current-voltage converter and the radio transmitter are battery powered and a number of strategies have been implemented to limit the power consumption. The system is designed for multichannel application and up to 256 parallel channels can be controlled. The overall implementation is cost-effective to make the availability of multichannel setups easily affordable.

The design, implementation and performance of the picoammiter system are described in detail.

\section{A12-3: Technology Frontier for Fast Advanced Scintillator Timing}

\section{K. Ziemons}

Faculty of Medical Engineering a. Technomathematics, FH Aachen University of Applied Sciences, Aachen, Germany On behalf of the COST Action FAST Collaboration

Scintillator based detectors have been very successful in high energy physics (HEP) calorimetry, nuclear physics, medical imaging, and many other applications. Technologies for single photon detection are rapidly evolving, with silicon photomultipliers (SiPMs) replacing vacuum photomultiplier tubes in many applications. In particular, the potential of such detectors to achieve precise timing information better than $10 \mathrm{ps}$ is of increasing importance for those applications. The implications of such a radical improvement in time resolution come with huge benefits in many domains. HEP will profit from a significant increase in detection efficiency and the health sector from an unprecedented improvement in imaging quality and image reconstruction time. Such a 'paradigm' change, however, must go hand-in-hand with a similar break in the interdisciplinary domain of photon detection. Therefore, new expertise must be gained in the fields of scintillators, photo detectors, as well as electronics read out systems to develop ultrafast timing scintillator-based detectors. The Fast Advanced Scintillation Timing (FAST) at the European Trans Domain COST Action TD1401 is more than a technology shift. It represents a technical revolution with profound impact on feasible applications in particle physics, accelerator physics, medical \& biological imaging, non-destructive industrial processing and electronic design issues. An important objective that this action also embarks on is training early stage researchers in a very innovative approach. We need change agents from every facet of industry, government, academia and healthcare to harness the full potential of FAST and to define what is possible. Responsible transformation will be ushered through an alliance of industry and academia.

European Trans Domain COST Action TD1401 COST is supported by the EU Framework Programme Horizon 2020

Al2-4: Ultrafast Radiotherapy Device

G. M. Grittani, T. Levato, C. M. Lazzarini, G. Korn

Particle acceleration by laser, ELI-Beamlines, Dolni Brezany, Czech Republic

Radiotherapy is one of the most efficient treatment for prostate, breast and lung tumors. One of the main limiting factors in the accuracy of the treatment is the lack of knowledge about the position of the tumor during the treatment. This is due to the fact that the tumor can move during the treatment, which lasts several minutes. Reducing the treatment time will thus lead to a better accuracy, but conventional rf linear accelerators can hardly accomplish this goal due to their technology. Our innovative ultrafast radiotherapy device can deliver the treatment dose in just few seconds. It is based on laser-plasma linear accelerator technology, which is highly suited to deliver high amount of dose in deeply lying tissue in very short time (more than 100 mGy in less than 1 ns). Moreover, our device can, due to the laser technology, perform imaging of the planned target volume during each radiation pulse with a time resolution better than $100 \mathrm{~ms}$. This leads to a series of snapshots that are used to reconstruct with high accuracy the position and the movement of the tumor. This information is implemented in a software that creates an accurate map of the deposited dose. The deposited dose map is used to check the treatment planning and, if needed, to modify it during the treatment. Besides these clear advantages, our ultrafast radiotherapy devices presents additional benefits when compared to conventional medical linear accelerators, such as easier patient alignment or easier disposal.

\section{Al3 Green Particle Physics}

Tuesday, Nov. 1 14:00-16:20, Varsovie 
Al3-1: Energy Sustainability at the European Spallation Source D. McGinnis

Accelerator, European Spallation Source, Lund, Sweden

The European Spallation Source (ESS) is currently under construction in Lund Sweden and will be the world's brightess neutron source when completed in 2023. From wind power to hydro-power to bio-waste, Sweden is a world leader in renewable energy and one of the constraints for hosting ESS in Sweden is that ESS must be a truly energy sustainable facility. The cornerstones of energy sustainability at ESS is a superconducting linac in which waste heat from the radio frequency power sources is recycled into Lund's district heating system. Also ESS has embarked in research into high efficient RF sources based on multi-beam induction output tubes. Finally, of the long pulse nature of the ESS facility, special power converters for the radio frequency systems have had to be developed that takes advantage of the latest advances in high power solid state technology. These advances open up the possibility of directly utilizing solar energy into the linear accelerator. This talk describes ESS and the current plans and status for energy sustainability at ESS.

\section{Al3-2: Particle Colliders: the Green-ILC, an Industry-Research Endeavor D. Perret-Gallix}

LAPP/IN2P3/CNRS-KEK, Annecy-Le-Vieux, France

A general introduction to issues related to energy consumption in large accelerator/collider infrastructures. It will cover 3 main topics: energy saving, energy recovery and recycling and the gradual use of renewable energies.

The ILC (International Linear Collider) is the most advanced project proposed as the next global infrastructure going beyond the CERN LHC (Large Hadron Collider) achievements. These fundamental research infrastructures are heavy energy consumers.

The LHC electrical consumption is similar to an European city of 250.000 people. The ILC, proposed to be hosted by Japan, will have a similar consumption, however much lower than other alternative projects also in discussion.

Any reduction or reuse of the energy consumed as well as any energy running cost reduction through the use of renewable energies will also find applications in the society and in the industrial/medical use of particle accelerators. The large technological infrastructures like the high-energy particle colliders are built thanks to the strong involvement and expertise of many industrial sectors (civil engineering, cryogenics, superconducting cavities, high RF power, electronics, computing, ...). All of them have interest in reducing the energy consumption in their processes and of their products. The Green-ILC project has been set to team up the research and industry communities so as to meet the energy consumption challenge currently limiting the reach to higher energies.

As proposed by the Green-ILC project, a working group under the aegis of ICFA (International Committee for Future Accelerators) has been set to address these issues.

Al3-3: Development of Platform for Total Energy Managment: Proposal for Total Energy Solution M. Miyamoto ${ }^{1}$, S. Ishii ${ }^{1}$, T. Saeki ${ }^{2}$, D. Perret-Gallix $^{3}$

${ }^{I}$ Misubishi Heavy Industries, Ltd (MHI), Kobe, Japan

${ }^{2}$ AAA Green-ILC-WG-KEK, Tokyo, Japan

${ }^{3}$ LAPP/IN2P3/CNRS-KEK, Annecy-Le-Vieux, France

In response to the need for liberalization and the stable supply of energy, energy supply systems have been diversified and many options are now available. On the other hand, the prompt proposal and evaluation of large-scale energy supply systems consisting of various pieces of energy equipment and related operating procedures has become difficult. Mitsubishi Heavy Industries, Ltd. (MHI) is working on the development of a platform for offering a total energy solution that realizes efficient energy utilization under normal circumstances and ensures business continuity in times of emergency such as power outages or equipment failure. This paper describes an overview, applications, and the future development of the planning system, which is a part of the platform.

\section{Al3-4: Approach to Automated Demand Response}

T. Furuya

Fine Ceramics Sales Department, KYOCERA Corporation, Kyoto, Japan

As enhancement of incentive for energy saving, facilitation of Japan's "negawatt" exchange market is planned to open in 2017. We develop a negawatt exchange demonstration system with automated demand response in grocery stores where gateway turns down air-conditioning equipment and discharge power from storage system to reduce electricity used. We report the demonstration results that show reserve capacity.

\section{Al3-5: Superconductor Yttrium-Based Superconducting Wire}


K. Kikuchi ${ }^{1}, \underline{\text { T. Saeki }}^{2}$

${ }^{I}$ New Business Center, Fujikura Ltd., Sakura,Chiba, Japan

${ }^{2}$ KEK, Tsukuba,Ibaragi, Japan

Fujikura Ltd., has been the global leader of developing Yttrium-based 2nd Generation High Temperature Superconducting wires over 20 years. We sustain this title by producing best perfomance wire with higher critical current, longest piece length and higher uniformity for longitudinal critical current distribution. Prospective applications of our superconducting wires are now widely spread from power cables, electrical/industrial equipment to medical and measuring instruments. Our Superconducting wires are committed to not only be improving quality of human life but also have great potential for industrial innovation and saving natural resources.

Al3-6: Study on the beam dump of the International Linear Collider by plasma-wakefield deceleration T. Tajima ${ }^{1}$, D. M. Farinella ${ }^{1}$, X. Zhang ${ }^{1}$, T. Saeki ${ }^{2}$, H. Hayano $^{2}$, K. Yokoya ${ }^{2}$, M. Zeng ${ }^{3}$, D. Perret-gallix ${ }^{4}$, J. Fujimoto ${ }^{5}$, A. Chao ${ }^{6}$ ${ }^{I}$ Irvine, UCI, California, USA

${ }^{2}$ Accelerator, KEK, Tsukuba, Japan

${ }^{3}$ ELI-NP, Bucharest, Romania

${ }^{4}$ IN2P3, Paris, France

${ }^{5}$ Institute of Particle and Nuclear Science, KEK, Tsukuba, Japan

${ }^{6}$ SLAC, California, USA

As the scales of accelerator projects and the amounts of their electric powers increase, improving the power efficiency and the power recovery of accelerator systems become immediate and important requirement and goal. Moreover, the radiation level in the accelerators should be kept to a minimum extent to cope with environmental consideration. In the design of the next future largest accelerator, the International Linear Collider (ILC), the power consumption is estimated to be $161 \mathrm{MW}$. Important efforts are being undertaken to reduce the electricity consumption and to lower the radiation level in the current study of its implementation plans. Consequently, we proposed a new system of beam dump which is based on the wakefield deceleration of beams in plasma, where the radiation level is far less than in the conventional designs and where the beam power might be recovered. We started the study in early 2015 and first focused on simulation where the ILC electron beams at $250 \mathrm{GeV}$ is dumped into a gas chamber of an appropriate pressure and size so as to create a plasma and therefore to achieve efficient deceleration. We will also consider energy recovery from the created plasma in the gas chamber. The resulting radioactive products are expected to be reduced drastically when compared to the conventional designs. The article presents the current status of these studies.

This work is supported by GrantinAid for Challenging Exploratory Research, JSPS KAKENHI Grant Number 15K13494.

Al3-7: Round-Table: Green Particle Physics, Energy for Innovation, Innovation in Energy

D. Perret-Gallix ${ }^{1}$, A. Yamamoto ${ }^{2}$, T. Saeki ${ }^{2}$

${ }^{I} L A P P / I N 2 P 3 / C N R S-K E K$, Annecy-Le-Vieux, France

${ }^{2} \mathrm{KEK}, \mathrm{Tsukuba}$, Japan

Industry-Research: Building up a stronger partnership The discussion will address some of the key issues for developing a stronger partnership between basic research and industry R\&D in the energy sector. After a short introduction, the session's speakers will be invited to comment and the floor will be open for discussion. 